\title{
Development of the West Virginia University Small Microgravity Research Facility (WVU SMiRF)
}

Kyle G. Phillips

West Virginia University

Follow this and additional works at: https://researchrepository.wvu.edu/etd

\section{Recommended Citation}

Phillips, Kyle G., "Development of the West Virginia University Small Microgravity Research Facility (WVU SMiRF)" (2014). Graduate Theses, Dissertations, and Problem Reports. 484.

https://researchrepository.wvu.edu/etd/484

This Thesis is protected by copyright and/or related rights. It has been brought to you by the The Research Repository @ WVU with permission from the rights-holder(s). You are free to use this Thesis in any way that is permitted by the copyright and related rights legislation that applies to your use. For other uses you must obtain permission from the rights-holder(s) directly, unless additional rights are indicated by a Creative Commons license in the record and/ or on the work itself. This Thesis has been accepted for inclusion in WVU Graduate Theses, Dissertations, and Problem Reports collection by an authorized administrator of The Research Repository @ WVU. For more information, please contact researchrepository@mail.wvu.edu. 


\title{
Development of the West Virginia University Small Microgravity Research Facility (WVU SMiRF)
}

\author{
Kyle G. Phillips
}

Thesis

\author{
In submission to \\ West Virginia University's \\ Benjamin M. Statler College of Engineering and Mineral Resources' \\ Department of Mechanical and Aerospace Engineering in \\ Morgantown, WV in the year 2014
}

\author{
In partial fulfillment for the degree of \\ Master of Science in Aerospace Engineering \\ Advisory and Examining Committee Members: \\ John Kuhlman, Ph.D. (Chair) \\ Majid Jaridi, Ph.D. \\ Patrick Browning, Ph.D.
}

Keywords: West Virginia University, Small Microgravity Research Facility, WVU SMiRF, SMiRF, Drop Tower, Microgravity, Reduced Gravity, NonNewtonian Fluid, Shear-Thickening Fluid, Die Swell, Extrudate Swell 


\title{
Abstract \\ Development of the \\ West Virginia University Small Microgravity Research Facility (WVU SMiRF)
}

\author{
Kyle G. Phillips
}

West Virginia University (WVU) has created the Small Microgravity Research Facility (SMiRF) drop tower through a WVU Research Corporation Program to Stimulate Competitive Research (PSCoR) grant on its campus to increase direct access to inexpensive and repeatable reduced gravity research. In short, a drop tower is a tall structure from which experimental payloads are dropped, in a controlled environment, and experience reduced gravity or microgravity (i.e. "weightlessness") during free fall. Currently, there are several methods for conducting scientific research in microgravity including drop towers, parabolic flights, sounding rockets, suborbital flights, NanoSats, CubeSats, full-sized satellites, manned orbital flight, and the International Space Station (ISS). However, none of the aforementioned techniques is more inexpensive or has the capability of frequent experimentation repeatability as drop tower research. These advantages are conducive to a wide variety of experiments that can be inexpensively validated, and potentially accredited, through repeated, reliable research that permits frequent experiment modification and re-testing.

Development of the WVU SMiRF, or any drop tower, must take a systems engineering approach that may include the detailed design of several main components, namely: the payload release system, the payload deceleration system, the payload lifting and transfer system, the drop tower structure, and the instrumentation and controls system, as well as a standardized drop tower payload frame for use by those researchers who cannot afford to spend money on a data acquisition system or frame. In addition to detailed technical development, a budgetary model by which development took place is also presented throughout, summarized, and detailed in an appendix. After design and construction of the WVU SMiRF was complete, initial calibration provided performance characteristics at various payload weights, and full-scale checkout via experimentation provided repeatability characteristics of the facility. Based on checkout 
instrumentation, Initial repeatability results indicated a drop time of 1.26 seconds at an average of $0.06 \mathrm{~g}$, with a standard deviation of $0.085 \mathrm{~g}$ over the period of the drop, and a peak impact load of $28.72 \mathrm{~g}$, with a standard deviation of $10.73 \mathrm{~g}$, for a payload weight of $113.8 \mathrm{lbs}$.

In order to thoroughly check out the facility, a full-scale, fully operational experiment was developed to create an experience that provides a comprehensive perspective of the end-user experience to the developer, so as to incorporate the details that may have been overlooked to the designer and/or developer, in this case, Kyle Phillips. The experiment that was chosen was to determine the effects of die swell, or extrudate swell, in reduced gravity. Die swell is a viscoelastic phenomenon that occurs when a dilatant, or shear-thickening substance is forced through a sufficient constriction, or "die," such that the substance expands, or "swells," downstream of the constriction, even while forming and maintaining a free jet at ambient sea level conditions. A wide range of dilatants exhibit die swell when subjected to the correct conditions, ranging from simple substances such as ketchup, oobleck, and shampoo to complex specially-formulated substances to be used for next generation body armor and high performance braking systems. To date, very few, if any, have researched the stabilizing effect that gravity may have on the phenomenon of die swell. By studying a fluid phenomenon in a reduced gravity environment, both the effect of gravity can be studied and the predominant forces acting on the fluid can be concluded. Furthermore, a hypothesis describing the behavior of a viscoelastic fluid particle employing the viscous Navier-Stokes Equations was derived to attempt to push the fluid mechanics community toward further integrating more fluid behavior into a unified mathematical model of fluid mechanics. While inconclusive in this experiment, several suggestions for future research were made in order to further the science behind die swell, and a comprehensive checkout of the facility and its operations were characterized. As a result of this checkout experience, several details were modified or added to the facility in order for the drop tower to be properly operated and provide the optimal user experience, such that open operation of the WVU SMiRF may begin in the Fall of 2014. 
Together, in the infinite pursuit of transgression beyond the contemporary for all of humanity 


\section{Acknowledgements}

Mom - for remaining steadfast in your belief of me, despite the odds, and always lending and ear

Dad - for your genuine interest, for brainstorming and keeping me in check, for floating me the $\$ 11,500$ for my experiment, for loaning me office space to write my thesis, for your unwavering support

Nana - for the ability to go after my dreams carefree, for your honest curiosity, for giving me the challenge of completing the family's first graduate degree, and for giving me inspiration from Grandpa Jack

Melissa Holmes - for doing whatever needed to be done, when it needed to be done, for taking the risk of being the first-trained operator, for being my sounding board to make sure I wasn't insane, for keeping my eyes on the future and the important things

Koren \& Mike - for always being my intent champions and my respite

Family \& Friends - for your inquisitive support

\section{In Memoriam}

Grandpa Jack - for your inspiration

Gram \& Uncle Rocky - for your resolute support (both of whom passed while I was in pursuit of this degree) 
Dr. John Kuhlman - for your initial inspiration, guidance, and enthusiasm, for sticking in there when there was obvious reason for doubt, for giving me the latitude to pursue such things

Dr. Majid Jaridi - for your genuine generosity, and for your complete support you have shown toward the student experience

Dr. Pat Browning - for your morale support, intuition, and random discussions in the shop

Mike Nussbaum - for helping me with project development and memory foam material test analysis

Chuck Coleman - for your continuous support, site identification, facility fabrication, and master procurement haggling skills

Josh O'Connell - for being my geotechnical consultation, facility fabrication assistant, and for helping me to prepare my initial experiment

Cliff Judy - for fabricating my experiment frame

Emily Calandrelli \& Jackie Grimes - for helping to write the PSCoR grant that kicked this whole thing off

Mehran Mohebbi - for letting me crash on your couch and for helping me with component checkout tests

Debbie Willis - for helping me get through school with my head above water 
WVU Research Foundation - PSCoR Grant

Statler College of Engineering and Mineral Resources - additional funding

CEMR MAE Department - additional funding and labor support

NASA WVSGC - graduate school Employment

Sierra Lobo, Inc. - employment and facility modification support

Student Partnership for the Advancement of Cosmic Exploration (SPACE) - donations toward student research

NASA Glenn Research Center - payload frame designs and drop tower consultation

Doug West \& Grainger - gracious discounts on the primary structure

National Instruments - huge discounts on the data acquisition system

Columbia Winch \& Hoist - and affordable, custom lift system design

Dynamic Systems, Inc. - discounted memory foam deceleration system

WVU MRT - lab space for experiment and future use

WVU Baja Team - shop space to fabricate lift support 


\section{Table of Contents}

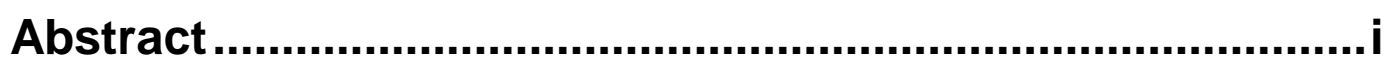

Acknowledgements ...................................................... iv

1.0 Compendium of Figures ...........................................

2.0 Compendium of Tables............................................ xii

3.0 Compendium of Symbols ........................................ xiii

4.0 Compendium of Abbreviations .................................. XV

5.0 Project Identification .................................................. 1

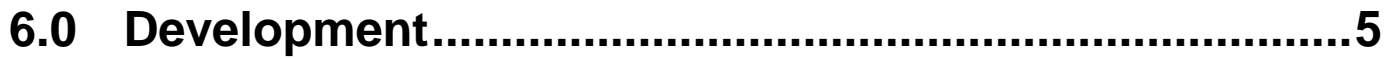

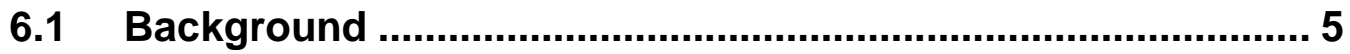

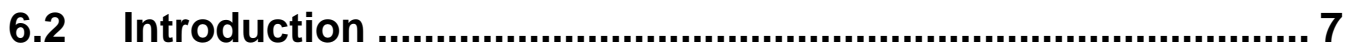

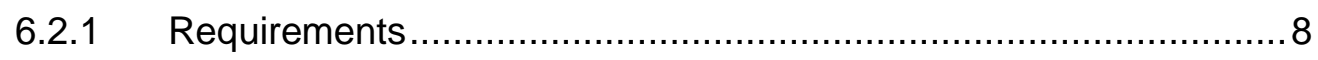

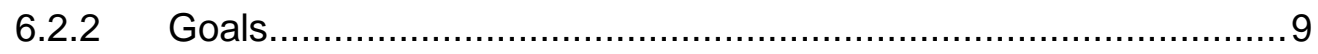

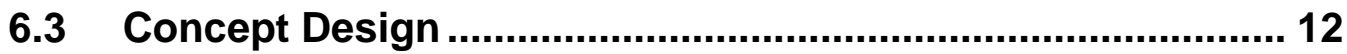

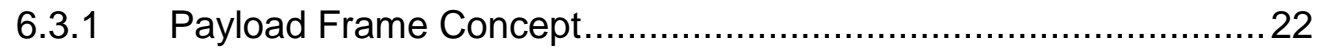

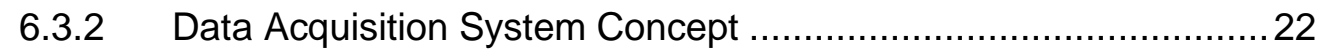

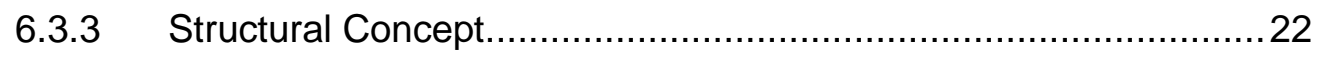

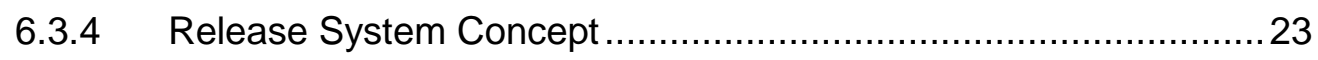

6.3.5 Deceleration System Concept ………………......................... 24

6.3.6 Lift/Retrieval System Concept .............................................. 24

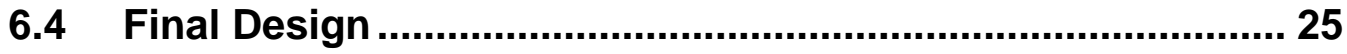

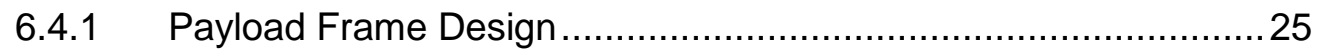

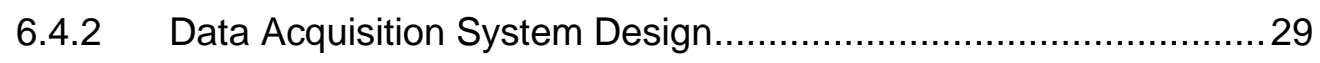

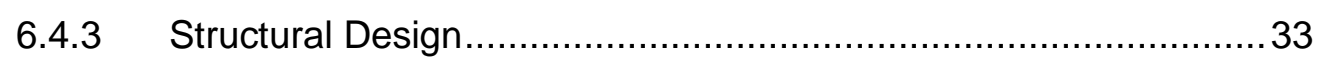

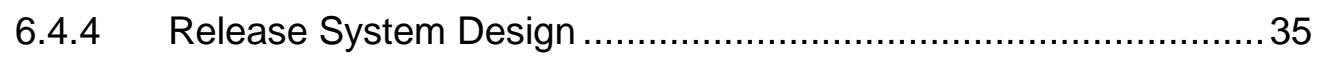

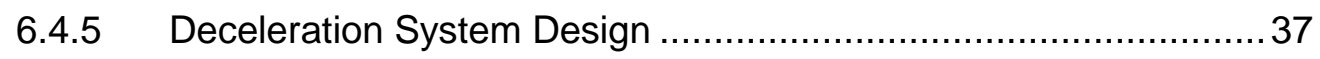

6.4.6 Lift/Retrieval System Design ................................................. 42

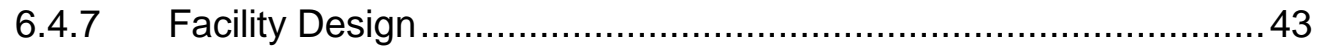




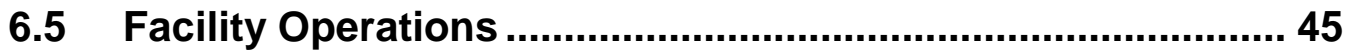

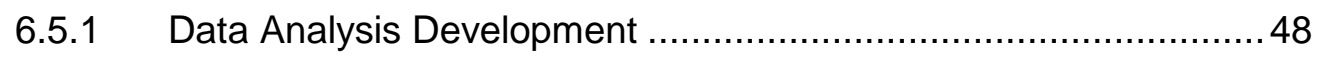

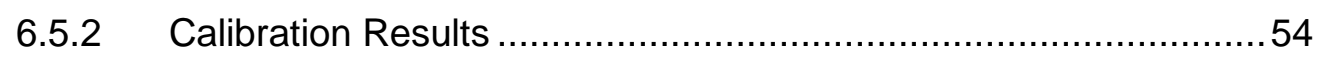

7.0 Experiment/Checkout of Facility ..............................64

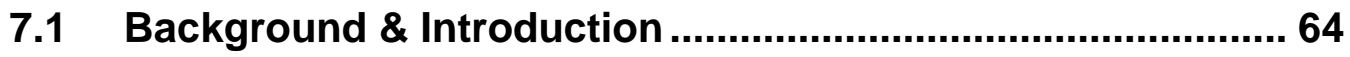

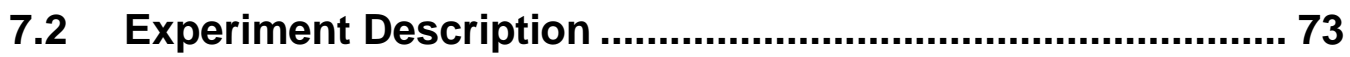

7.3 Experiment/Facility Checkout Operations ........................... 75

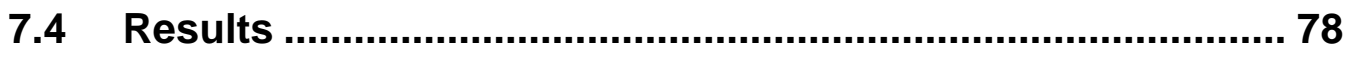

7.5 Experiment Summary \& Conclusions................................... 84

7.6 Suggestions for Future Research .......................................... 85

8.0 Facility Summary \& Conclusions ...............................86

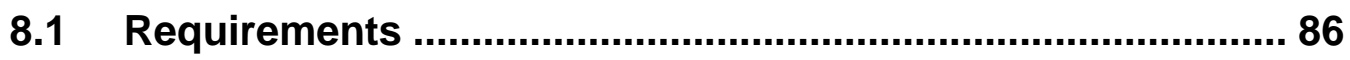

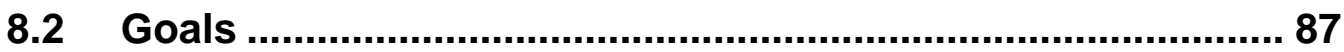

8.3 Suggestions for Future Research / Facility Improvements .. 90

9.0 Experiment Bibliography ........................................93

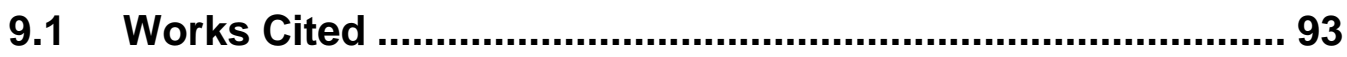

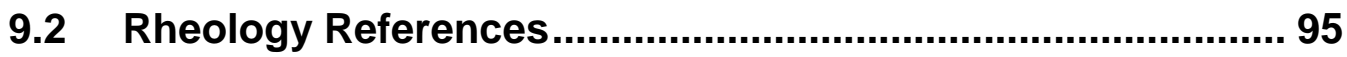

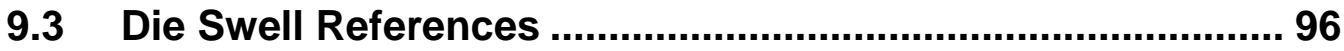

10.0 Drop Tower Bibliography........................................98

10.1 Works Cited (Drop Tower History) …................................ 98

10.2 Drop Tower References .................................................... 104

11.0 Appendix A ....................................................... 107

A Historical Compilation of the Drop Tower ................................. 107

12.0 Appendix B ....................................................... 115

WVU SMiRF Concept Analysis..................................................... 115

13.0 Appendix C ............................................................. 124

Payload Frame Information ........................................................... 124

14.0 Appendix D ........................................................... 128 
Data Acquisition System Information. 128

15.0 Appendix E. 396

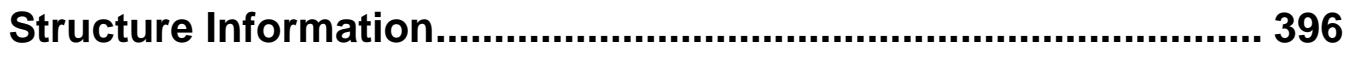

16.0 Appendix F ...........................................................423

Release System Information ....................................................... 423

17.0 Appendix G ........................................................ 434

Deceleration System Analysis \& Information ............................. 434

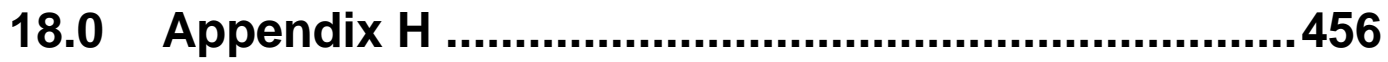

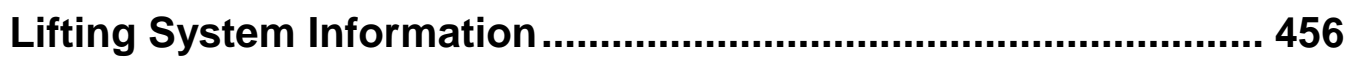

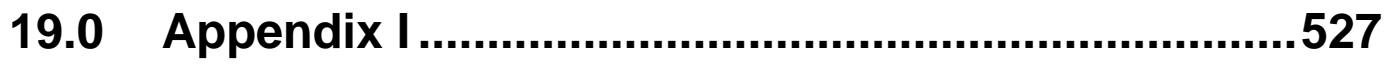

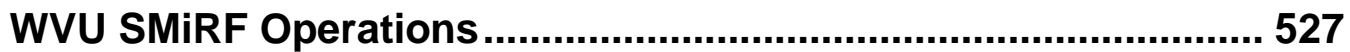

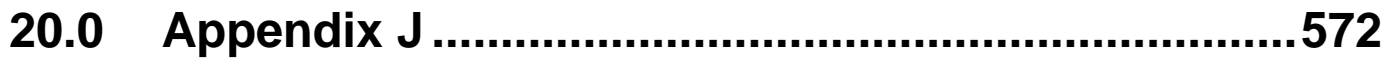

Ongoing Operational Accelerometer Analysis ........................... 572

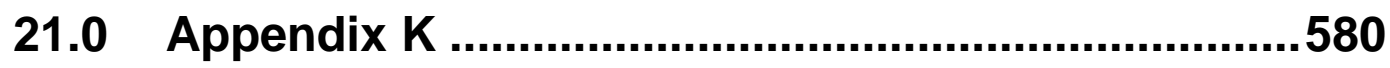

WVU SMiRF Initial Calibration....................................................... 580

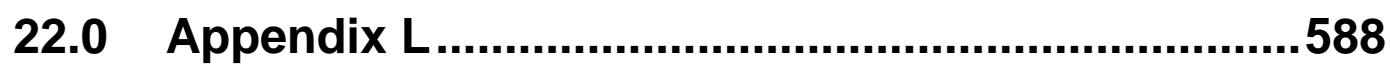

Non-Newtonian Navier-Stokes Derivation..................................... 588

23.0 Appendix M .......................................................638

Die Swell Experiment System Information.................................... 638

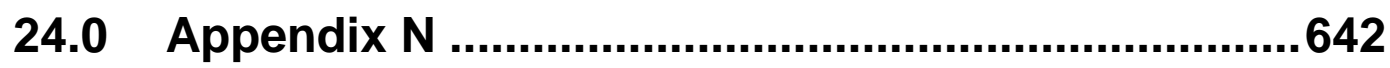

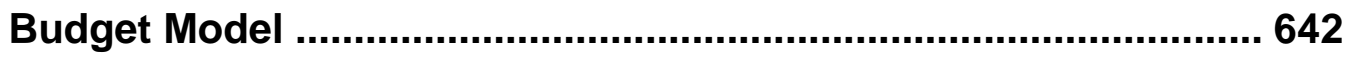

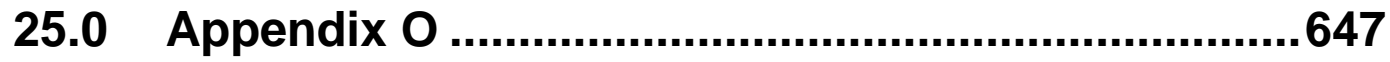

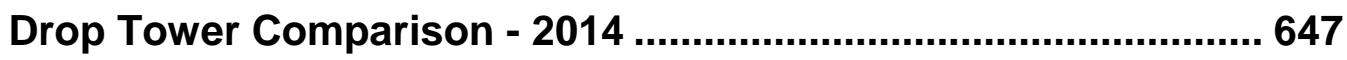

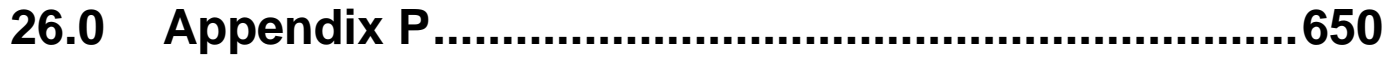

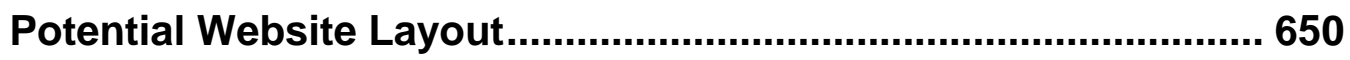




\subsection{Compendium of Figures}

Figure 6-1: Concept Design Drop Time vs. Drop Height.................... 21

Figure 6-2: Concept Design Drop Time vs. Payload Velocity............ 21

Figure 6-3: WVU SMiRF Payload Frame .............................................. 27

Figure 6-4: Payload Frame Interface Table...................................... 28

Figure 6-5: Data Acquisition System Drop Box, Including EMI Shield and Supplemental Padding.............................................................. 32

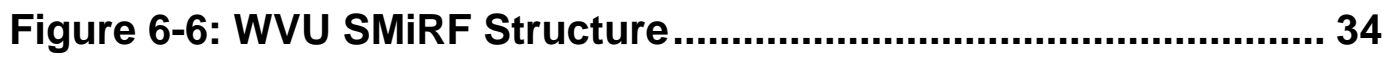

Figure 6-7: Structure Stabilizing Arms ............................................... 34

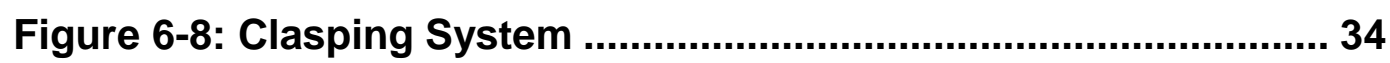

Figure 6-9: Balance Boom .................................................................. 35

Figure 6-10: Payload Frame Crown.................................................. 36

Figure 6-11: Release System Final Design - Depicting Electromagnet and Payload Frame Crown.................................................................. 36

Figure 6-12: Release System with Attached Payload ......................... 37

Figure 6-13: Compiled Stress/Strain Curves for Various Open-Celled

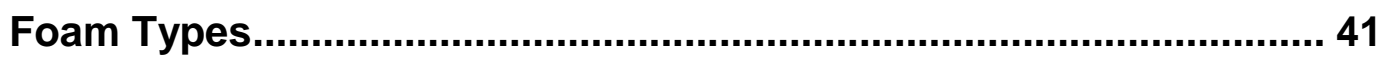

Figure 6-14: Deceleration System ................................................... 41

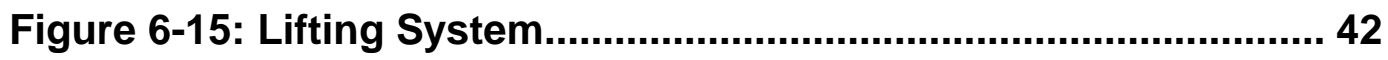

Figure 6-16: Full-Length Photo of the WVU SMiRF ............................ 46

Figure 6-17: Pre-Drop Payload Configuration .................................... 47

Figure 6-18: 25 lb. Calibration Drop Raw Data (Corrected)................ 55

Figure 6-19: 25 lb. Calibration Drop Lift \& Prep Data ......................... 56

Figure 6-20: $25 \mathrm{lb}$. Calibration Drop Low-g Data............................... 56

Figure 6-21: 25 lb. Calibration Impact Data ........................................ 57

Figure 6-22: $25 \mathrm{lb}$. Calibration Drop Rebound Data............................ 57

Figure 6-23: 25 lb. Calibration Drop Recover Data ........................... 58

Figure 6-24: 25 lb. Calibration Drop Low-g Probability Curve ........... 58

Figure 6-25: Compiled Weight vs. Acceleration Lift \& Prep Data...... 61 
Figure 6-26: Compiled Weight vs. Acceleration Low-g Data ............. 61

Figure 6-27: Compiled Weight vs. Acceleration Impact Data ............ 62

Figure 6-28: Compiled Weight vs. Acceleration Rebound Data ........ 62

Figure 6-29: Compiled Weight vs. Acceleration Recover Data.......... 63

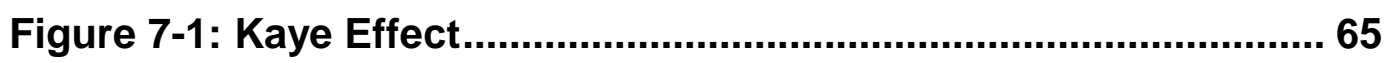

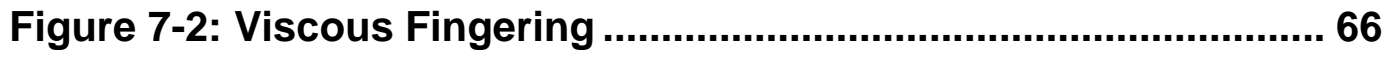

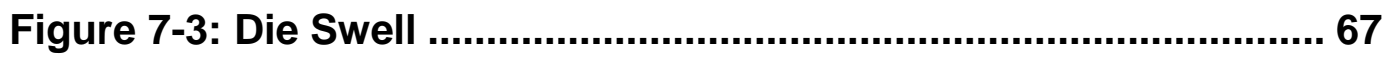

Figure 7-4: Original Combined Experiment Concept Layout............. 67

Figure 7-5: Die Swell Experimental Payload (Front View)................. 75

Figure 7-6: Die Swell Experimental Payload (Back View) .................. 75

Figure 7-7: Loaded Payload Sitting on Temporary Tower Preparation

Surface (TTPS) Above Deceleration System...................................... 77

Figure 7-8: Example Image Sequence as Generated from a Converted

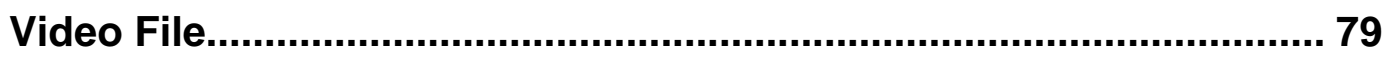

Figure 7-9: Edge Finding Algorithm Applied to Example Image

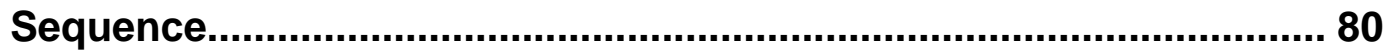

Figure 7-10: Pixel Measurement Applied to Edge-Generated Image

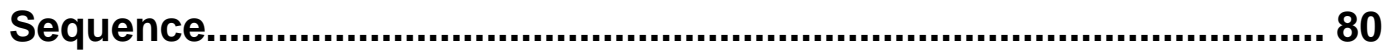

Figure 7-11: Actual Drop Time Comparison with Conceptual Design

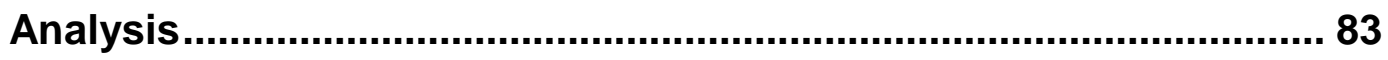

Figure 7-12: Calculated Actual Payload Velocity Comparison with Conceptual Design Analysis ................................................................. 84 


\subsection{Compendium of Tables}

Table 6-1: $25 \mathrm{lb}$. Calibration Statistical Data Summary ....................... 55

Table 6-2: 50 lb. Calibration Statistical Data Summary ..................... 59

Table 6-3: 75 lb. Calibration Statistical Data Summary ...................... 59

Table 6-4: $100 \mathrm{lb}$. Calibration Statistical Data Summary ..................... 60

Table 6-5: 125 lb. Calibration Statistical Data Summary ...................... 60

Table 7-1: Non-Newtonian Fluid Classification Brief.......................... 69

Table 7-2: Statistical Repeatability Data Analysis Summary ............. 83

Table 8-1: Facility Improvement Checklist ....................................... 90 


\subsection{Compendium of Symbols}

\section{English}

\section{Symbol}

$A_{\text {ref }}$

$a_{\text {deceleration }}$

$a_{\text {gravity }}$

$a_{\text {payload }}$

$B$

$B^{2}$

$B_{1}$

$\mathrm{B}_{2}$

$C_{D}$

$D_{e}$

$D_{\text {pad }}$

$D_{0}$

$F_{\text {impact }}$

$H_{\text {bag }}$

$H_{\text {pad }}$

$H_{\text {payload }}$

$J_{s}$

$L_{\text {payload }}$

$m_{\text {payload }}$

$S_{R}$

$S_{w}$

$t$

\section{Description}

Aerodynamic frontal area of the payload

Deceleration of the payload

Acceleration due to gravity

Acceleration of the payload

Maximum swell

Creep recovery function

Diameter swell

Thickness swell

Coefficient of drag

Extrudate diameter

Diameter of a cylindrical deceleration pad

Orifice diameter

Force due to impact with deceleration system

Height of the pneumatic deceleration bag

Height of the deceleration pad

Height of the payload

Steady shear compliance

Length of the payload

Mass of the payload

Elastic strain recovery

Die swell

Time 


\section{Symbol}

$t_{\text {deceleration }}$

$t_{n}$

$t_{n-1}$

$V_{\text {cube }}$

$V_{\text {cylinder }}$

$v_{d r a g}$

$v_{k}$

$v_{\text {terminal }}$

$W_{\text {payload }}$

$w_{\text {payload }}$

$z_{\text {drag }}$

$z_{k}$

\section{English (cont'd)}

\section{Description}

Time elapsed during deceleration

Time at present

Time at one time-step previous

Volume of a cubic deceleration pad

Volume of a cylindrical deceleration pad

Velocity taking drag into account

Velocity due solely to kinematics

Velocity at terminal speed

Width of the payload

Weight of the payload

Z-location taking drag into account

Z-location (or vertical location) due solely to kinematics

\section{Greek}

\section{Symbol}

$\eta_{0}$

$\dot{\gamma}$

$\gamma_{R}$

$\rho_{\text {air }}$

$\tau_{11}$

$\tau_{12}$

$\zeta$

\section{Description}

Zero shear viscosity

Shear rate

Recoverable shear strain at the die wall

Density of air

Fluid particle normal stress

Fluid particle shear stress

Characteristic time 


\subsection{Compendium of Abbreviations}

\begin{tabular}{|c|c|}
\hline Abbreviation & Description \\
\hline CAD & Computer-Aided Design \\
\hline CEMR & $\begin{array}{l}\text { WVU's Benjamin M. Statler College of Engineering } \\
\text { and Mineral Resources }\end{array}$ \\
\hline $\mathrm{CHJ}$ & Circular Hydraulic Jump \\
\hline EMI & Electromagnetic Interference \\
\hline FEA & Finite Element Analysis \\
\hline Fos & Factor of Safety \\
\hline FPGA & Field-Programmable Array \\
\hline GRC & Glenn Research Center \\
\hline ISS & International Space Station \\
\hline JSC & Johnson Space Center \\
\hline MAE & $\begin{array}{l}\text { WVU CEMR Mechanical And Aerospace Engineering } \\
\text { Department }\end{array}$ \\
\hline MRT & Microgravity Research Team \\
\hline MS & Microsoft \\
\hline NASA & National Aeronautics and Space Administration \\
\hline $\mathrm{NI}$ & National Instruments \\
\hline OSHA & Occupational Safety and Health Administration \\
\hline PPE & Personal Protective Equipment \\
\hline PSCoR & Program to Stimulate Competitive Research \\
\hline TTPS & Temporary Tower Preparation Surface \\
\hline SMiRF & Small Microgravity Research Facility \\
\hline WVU & West Virginia University \\
\hline
\end{tabular}


Development of the West Virginia University Small Microgravity Research Facility (WVU SMiRF)
Copyright $^{\odot} 2014$

Kyle G. Phillips 


\subsection{Project Identification}

Most people do not often consider that gravity plays a large role in all aspects of life. It has a significant role in everything from bone structure and bone growth to how flames and fluids behave. Due to its overarching influence, gravity shapes the majority of the experiences we have, thereby influencing our observations and how we interpret the world and the universe around us. Removing gravity from experiments allows researchers to mitigate its effects on a wide variety of scientific phenomena. By removing gravity, it is as if one was able to wipe the fog off a mirror - you can see your reflection through the mirror, and know it is there, but once the fog is removed, you can clearly see details. Researchers are able to do the same by removing gravity - they can see the fundamental effects that are at the heart of fluid behavior, fundamental physics research, etc. Additionally, by removing gravity, researchers and engineers are able to simulate the environment of space more closely, enabling research and development of space-ready technologies, potentially to study everything from the effects that space may have on the human body to fluid mechanics and flame behavior - all without leaving the ground.

Platforms for performing reduced gravity research include, but are not limited to (in order of increasing complexity):

- computer simulation

- drop tower

- parabolic aircraft flight

- sub-orbital rocket

- CubeSat

- NanoSat

- full-sized satellite

- government-funded orbital flight

- commercially-funded orbital flight

- the International Space Station

Future additional reduced gravity research platforms may include, but are not limited to (in order of increasing complexity): 
- high-altitude balloon drop

- commercial sub-orbital flight

- commercial orbital flight

- commercial space station

- deep-space missions

In 2001, WVU professor Dr. John M. Kuhlman established the WVU Microgravity Research Team (MRT) in order to introduce reduced gravity research to undergraduate students through opportunities via parabolic aircraft flight. The WVU MRT program has been so successful, in fact, that nearly every team since 2001 has flown a reduced gravity experiment on a parabolic flight, gaining a host of data of a variety of various fluid phenomena. However, since its inception, many of the WVU MRTs have encountered a fundamental problem - even though an experiment apparatus may work in the lab, no team can guarantee that it will work as intended in microgravity, even if the experiment and its design is based on sound scientific and engineering principles. Reduced gravity can present an abundance of practical obstacles that can cause fatal issues to an experimental apparatus. For example, in 2007, as WVU MRT Team Lead, the author of this thesis and his team encountered an issue with a water pump on a parabolic flight. The pump lost its prime in microgravity and the experiment would not function on the first day of flight. The manufacturer indicated that the pump could be mounted in any direction. However, after the first flight a decision was made to remount the pump in a different orientation, which eliminated the problem for the second day of flight. This issue did not arise until the experiment was in microgravity. The manufacturer may have meant that under normal $1 \mathrm{~g}$ conditions on Earth's surface that the pump could be mounted in any orientation and still function as intended. In an environment where gravity is nearly nonexistent, things change. Many components and engineered systems intended for $1 \mathrm{~g}$ use are used in a modified state in reduced gravity. However, in order to know which modifications to make, several engineered iterations may be required. Hence, several WVU MRTs have had experimental issues during parabolic flight that were unforeseen in lab testing. The WVU MRT program was in need of a better method to develop and validate their experiments in order to resolve issues - a facility such as the WVU SMiRF. 
Thus, in 2009, a group of former MRT members began to investigate better methods to develop MRT experiments for flight, consequently discovering the use of drop towers as one such method. Under the guidance of Dr. John Kuhlman, Emily Calandrelli and Jackie Grimes sought out funding sources and visited NASA Glenn Research Center (GRC) and Purdue University, both sites of drop towers, during the 20092010 academic year. Concurrently, Dr. Kuhlman was able to solicit and obtain \$28,000 of funding from West Virginia University's Research Corporation through their Program to Stimulate Competitive Research (PSCoR) grant on July 1, 2009 with matching $\$ 9,100$ funds (i.e. a total of $\$ 18,200$ ) coming from both the Mechanical \& Aerospace Engineering (MAE) Department and the Statler College of Engineering and Mineral Resources (CEMR). Later, $\$ 10,000$ in funding would be provided by the Statler CEMR's Dean's Office, for the facility structure, to bring the total project budget to $\$ 56,200$. However, due to graduate education and career aspirations at other institutions, Calandrelli and Grimes were unable to continue on the project. Another WVU MRT alumnus, Kyle Phillips, the author, (also known henceforth in this thesis as the graduate student, designer, developer, researcher, and/or operator) was seeking a graduate thesis topic, to which Dr. Kuhlman introduced the possibility of creating a drop tower at WVU. Primarily, identifying the need for MRT experiment development, prior to flight, as a necessary resource for the MRT, Phillips (team lead), Dr. Kuhlman (team faculty advisor), Calandrelli, and Mike Nussbaum (an undergraduate MRT member at the time) all set out to brainstorm possibilities, based on available funding levels. The team quickly realized that a drop tower could have a significant impact well beyond that of MRT development, and have an impact at the college, university, state, and national levels, not to mention on the scientific community at large.

After initial discussions between Phillips and Dr. Kuhlman, it was decided that in order to comprehensively develop a research facility as sophisticated as a drop tower, the scope of work would need to include everything from conceptual design through checkouts and validation of the facility and its characteristics, including:

- Project management

o Obtaining additional funding 

o Budgeting
o Sourcing \& procurements
o Scheduling
o Project requirements development \& management

- Concept design

o Initial aerodynamic calculations

- Preliminary indications of reduced gravity quality

o Estimated payload impact loads

o Facility structural design

o Research \& development involving subsystem concepts

- Final Design

o Budgeting \& sourcing of all major subsystems

o Ensuring proper interfacing and integration

o Design documentation

- Facility construction

- Facility verification

o Independent subsystem checkouts

o Integrated full system checkout, without drops

o Pre-operation system checkout - drop of an empty payload

o System adjustments (as needed)

- Facility validation

o Calibration - payloads of increasing weight

o Initial full-scale operations - operation of full-scale experiment in facility

- Preparation for open use

Hence, this thesis addresses all aspects in the scope of work for the developer to obtain his degree and the detailed effort taken to create the West Virginia University Small Microgravity Research Facility (WVU SMiRF). In doing so, Phillips will provide a model by which others may follow to create similar facilities, in order to increase affordable, frequent reduced gravity access to the scientific community. 


\subsection{Development}

\subsection{Background}

In order to properly develop anything, one must learn from its history. The researcher took the time to comprehensively research past and present drop towers, in order to incorporate good aspects and best practices from other towers, and to benefit from those lessons that have been learned from both success and failure. Citations and references for this historical investigation are provided in Appendix A: A Historical Compilation of the Drop Tower.

Towers first began their use as something other than a residential or wartime element in 1575 when Girolamo Borro, a philosophy teacher in Pisa, Italy dropped wooden and lead balls from the highest window of his house. He would incorrectly conclude that the wooden ball reaches the ground faster, because it was thought at the time that air was a "heavy" element, and the wood sphere contained more air than the lead sphere. Subsequent research would follow in the years to come by Moletti, Stevin, Grotius, and Galileo from 1575-1782, in what the researcher here terms as the "Initial Scientific Studies Era." However, after the initial period of using towers for scientific purposes, others began to find manufacturing uses in drop towers. In 1782, William Watts created the first shot tower at his residence in Redcliffe, Bristol, UK. He poured molten lead through a screen at the top of the tower, thereby letting the lead cool into spheres over the course of the drop (now known to occur because the directional effects of gravity momentarily disappear while the lead is coincidentally in a reduced gravity whilst cooling). The invention of a shot tower revolutionized ammunition manufacturing. The "Shot Tower Era" would last through 1969. However, the "Drop Tower Research Facility Era" would begin in 1956 when the first documented modern drop tower research facility was built for NASA Glenn Research Center at Lewis Field. Since 1956, approximately 17 documented drop tower research facilities have been created worldwide, including the United States of America (11), Germany (1), Japan (3), China (1), and Australia (1), of which at least 9 are still operational - West Virginia University's Small Microgravity Research Facility (WVU SMiRF) is the latest in what history proves to be a long line of tower facilities [ Appendix A]. 
In a modern drop tower research facility, an experimental payload is elevated to the top of a tower, where it is subsequently held, and then dropped onto or into a pad or similar component that catches and decelerates the payload without destroying it, allowing for repeated subsequent research. In essence, the drop tower functions as a controlled malfunctioning elevator. If an elevator were to fall, with an unfortunate passenger along for the ride - a very improbable event, as elevators have several redundant safety systems - if the passenger weren't holding on to anything, they would begin to float inside of the elevator, similar to what a skydiver would feel if there was no air resistance. In the case of a drop tower research facility, the elevator car is the payload and the passenger is the experiment inside the payload. The experiment briefly experiences artificial weightlessness during the drop, thereby allowing researchers to perform experiments in a weightless environment, at a reduced level of gravity, for a temporary period of time. Essentially, a drop tower research facility temporarily removes gravity!

Drop towers have many fundamental advantages in comparison to other forms of research facilities. At present, drop towers are used as research facilities for a wide variety of scientific research applications experimental work for everything from fundamental physics and materials science to space technology development and medical research can be performed in a drop tower research facility. Additionally, the environment and functionality of drop tower research facilities offer a facility within which a wide variety of frequently repeatable, relatively inexpensive, credible experimental research can be performed in a controlled environment at reduced gravity levels. As one may note from the aforementioned lists, drop towers are the second least-complex form of reduced gravity research, next to computer simulations, and are the least complex reduced gravity method that involves practical experimental research. It may serve to develop and validate computer simulations, and therefore, may even serve as the fundamental level of reduced gravity research, depending on the application. The primary disadvantage of drop towers is the amount of time at reduced gravity conditions (i.e. 5-10 seconds maximum). However, a drop tower can serve as the first line, in what can be a long line, of accreditation toward space-flight ready 
technology development to serve applications such as human space exploration, as one specific example of its many uses.

\subsection{Introduction}

It was the goal of the graduate student, under the guidance of Dr. John Kuhlman, with the help of so many others in a variety of capacities (please see the Dedication \& Acknowledgements Section at the beginning of this thesis), to provide a drop tower research facility to WVU to enhance its reduced gravity research capabilities, primarily for the WVU MRT, and in doing so provide a model by which the larger scientific community may gain affordable, increased access to reduced gravity research. However, any successful project is based on a solid foundation that includes both scope of work (see Project Identification Section) and a good set of project requirements.

To obtain the requirements of the system, the team would conduct several meetings over approximately one month. Initially, those discussions were based on idealistic goals, which would drive the desires of the team to achieve the best end result possible. Some of those goals included long drop times with heavy, large-dimensioned payloads, with very light impact loads and very little drag to achieve the lowest reduced gravity conditions as possible. The team also desired self-sustained payloads, with onboard power and data acquisition that would stream wireless, real-time video and data, remotely accessible via the internet. Even a facility that would allow for motorized floors used for preparation of the payload near the top and bottom of the tower was considered. An entire array of possibilities was discussed for each of the subsystems of the tower (more on that in the Concept Design Section). Over the course of that month, however, the discussions evolved into considering practical achievement of those goals. Other aspects had to be considered, such as long facility latency times between projects, without the facility deteriorating or malfunctioning when the next user may want to use it, weeks, months, or possibly even years after the previous use. Well aware that budget is always a concern, the team maintained that the project should maintain as low of a budget as possible, not only for obvious financial reasons, but to make it a priority to show others it can be done affordably, thereby increasing access to other institutions and entities for the development of such facilities and the 
increased proliferation of reduced gravity research. That priority also led to the realization that with a limited budget, we may not be able to get everything we desired after this phase of the project was complete, and to design a system that could be upgraded as additional funds became available. Ongoing discussions such as these led to requirements - those aspects that were non-negotiable, and goals - those aspects that the team should strive toward, aspects that the team should give a best effort to meet or exceed. The result of those discussions formed the other half of the foundation of the project, resulting in the following lists of requirements and goals - requirements representing those aspects that are unwavering and mandatory, while goals represented those aspects the team should strive toward to make the facility that much better:

\subsubsection{Requirements}

1. Ensure personnel safety throughout all levels of the project

- Rationale: People come first. All other priorities are secondary.

2. Keep end-user safety paramount throughout the design

- Rationale: People come first. All other priorities are secondary.

3. Provide a system with long-term reliability

- Rationale: There may be long periods between use and the facility must be able to perform without doubt and to maintain safety over the lifetime of the facility. This requirement also implies minimal capital investment in required maintenance over the lifetime of the facility.

4. Provide a system that requires very infrequent maintenance

- Rationale: This requirement couples with requirement 3 , but implies minimal human intervention is required to maintain reliability, as resources can be scarce and maintenance increases safety risk for those maintaining the facility. This requirement also implies 
minimal capital investment in required maintenance over the lifetime of the facility.

5. Provide a system that can be upgraded over time

- Rationale: Designing a versatile system allows for flexibility in technology to maintain relevance of the facility and extend capital investments to increase the viability of the facility over time

6. Provide a system as inexpensively as possible

- Rationale: Reduces the initial investment in the facility, thereby regaining value on investment as quickly as possible. Additionally, by providing a viable inexpensive model, it may increase access to reduced gravity research via the possibility for other users in the larger scientific community to make a more affordable initial capital investment.

\subsubsection{Goals}

1. The facility should be OSHA-certified to ensure safety

- Rationale: OSHA certification implies an industryaccepted standard of quality and safety that may account for aspects that may not have otherwise been considered. As a result of following those standards in addition to self-imposed standards, an even greater level of end-user safety may be achieved

2. Design the facility such that it may be operated by a single person

- Rationale: If the facility may be safely operated by a single operator, it increases the possibility for versatility in productivity, based on potential sporadic operator availability, and reduces the cost of operation, if one is associated with such a facility. It also implies increased safety if more than one operator is available. 
3. Design the facility with quick turnaround times

- Rationale: The capability of quick activation, preparation, and deactivation times leads to more frequent use of the facility, increasing productivity and value on investment

4. Provide common hardware and setup facilities for users

- Rationale: Providing users common mechanical integration interface, reduces wear on facility equipment, thereby reducing ongoing operational costs. Additionally, by providing users setup facilities, a common readiness level is imposed for operation within the facility and ensures that all experiment payloads are properly prepared for integration into this facility

5. Provide a tower tall enough to meet or exceed 1 second of drop time

- Rationale: The longer the drop time, the more time at reduced gravity, increasing the capabilities of the facility and giving users more value on time and experiment investment.

6. Provide a facility that meets or exceeds experiment weight allowances on parabolic flights from NASA JSC

- Rationale: The larger the design range for weight limits increases the capability of the facility. With allowances at or above those for parabolic flights, it ensures that future WVU MRTs can properly prepare and fully develop experiments for parabolic flight.

7. Provide a facility and method that allows for near maximum payload stability prior to drop

- Rationale: Payload stability prior to a drop ensures safety of personnel and safety of facility and experimental equipment. It also increases quality of drop data. 
8. Provide a facility with impact loads as low as possible

- Rationale: With lower impact loads, the experiment design may be more affordable and may lead to less stress on the facility.

9. Provide a facility that supports an affordable experience for the end-user

- Rationale: Providing an affordable experience for the end-user encourages more frequent use of the facility and provides a better opportunity for end-users. Providing users with commonly available hardware and software analysis tools increases experiment affordability and reduces time, effort, and investment for experimentation, allowing for capital investments in other aspects of a project. It also permits ongoing data collection to increase facility data and understanding to provide to users to provide more accurate data to users.

10. Create a website for the facility

- Rationale: An internet presence allows for increased exposure that may lead to increased use and return on investment.

11. Provide end-users web-based access to the facility and data

- Rationale: Web-based access to the facility and data produced may lead to decreased costs on the user, allowing even more affordable experimentation, or increased capital investment into other areas of the user's project.

12. Create $P R$ and marketing material to market to as many possible entities at the University, surrounding industry, and via the internet

- Rationale: Marketing the facility may increase return on investment through more frequent use, while also providing the information to users who may not have 
otherwise known about the facility, its capabilities, or potential experimental applications. The more people that know about the facility and its capabilities, the more that may benefit from it, thereby benefitting the scientific community.

\subsection{Concept Design}

After the foundational guidelines of requirements and goals were set by the team, the concept design could begin. To initiate the project and brainstorming, the team visited the NASA Glenn Research Center (NASA GRC) to see their $2.2 \mathrm{~s}$ and 5.0s drop towers to expand upon the previously completed background research. Seeing those towers and their operations was an invaluable experience to the direction, design, and construction of the WVU SMiRF.

The NASA GRC's 5.0s drop tower provides a payload frame that resides inside a cylindrical capsule with an aerodynamic cone on one end. Final payload preparations can occur onsite in a large lab space adjacent to the drop tube that tunnels vertically into the ground. During standard, presentday operations, an overhead crane can lift an encapsulated payload into the drop tube, where it is mechanically affixed to a beam via a customized dog-boned bolt. The tube is then evacuated to a hard vacuum to remove air resistance during the drop. The bolt is then explosively sheared at its narrowest thickness. The encapsulated payload drops into a pit filled with small polystyrene spheres that arrest the payload by its transference of energy into the compression and kinetic motion of the spheres. The payload is then automatically recovered using the overhead crane, and the polystyrene spheres are collected and reused for the next drop.

The team also visited the NASA GRC's 2.2s drop tower. The 2.2s drop tower is a stand-alone structure with an entrance at street level, while the majority of the tower resides within a ravine that runs near to the street, allowing for a tall tower. It too has a preparation shop, although much smaller than the 5.0s drop tower. However, they also provide payload frames to users. They allow the users to borrow the frames to construct payloads off-site. Once users assemble their payload, it may be brought into the first floor and into the tower, where it is integrated into a drag 
shield. Once the payload is prepared for a drop, data acquisition systems are enabled via a fiber optic line connected to the facility. The payload is raised to the top of the tower, while the preparation level's floor is opened as a two-door hatch, exposing the deceleration pad many floors below. Once the payload is raised to the top, a small wire is screwed into the top of the payload frame, while the other side of the wire, outfitted with a very smooth metal cap is clamped via a pneumatic vise. The deceleration pad is also inflated pneumatically. Once the payload is ready for a drop, the supply air to the pneumatic vise is turned off. The pneumatic vise slowly loses gripping power, and allows the small wire and payload to slip smoothly from its grips. As the payload falls, all of the aerodynamic loads are transferred into the drag shield that surrounds the payload, while the experiment payload slowly descends toward the front vertical bow of the drag shield, as it does not experience any of the aerodynamic drag and falls relatively faster than the drag shield. It then experiences a controlled arrest via the pneumatic air bag that employs a custom-engineered set of louvers that control the rate of airflow from the air bag. The payload is then automatically retrieved via the overhead crane that raises it.

Both towers were seen to have had advantages and disadvantages. The 5.0s drop tower provided a large preparation space, and enabled users to have very large experimental payloads with onboard data acquisition and power. It also provided a drop devoid of aerodynamic loads. However, turnaround times were slow due to clean up of the polystyrene spheres, and evacuation of the tube. Furthermore, each bolt is a custom-made bolt that cannot be reused. Operations required several personnel. Meanwhile, the 2.2s drop tower also provided a large preparation area and support staff. Additionally, the location of the preparation floor was convenient and unique in design, allowing dropping payload to fall through hatched doors. The use of pneumatics throughout the system truly did provide a very smooth release and deceleration of payloads. It also required fewer personnel for operations. However, it did not have onboard data acquisition. The fiber optic cable had to unspool while the payload dropped. Additionally, the pneumatic systems add some complexity and maintenance. All of these advantages and disadvantages could be applied to a relatively more affordable system from an initial and ongoing investment perspective. 
After the conceptual visioning of the design and operations of a drop tower, and the visitation experience that would prove as an invaluable benchmark for the WVU SMiRF, the designer began work on a conceptual performance analysis for the WVU SMiRF. In order to meet Goal \#9, the designer would create all design and analysis tools in a widely-accessible software platform by employing Microsoft Office products. Therefore, an Excel spreadsheet was created to perform the initial concept design calculations. The spreadsheet allows users to input desired payload specifications of weight, length, width, height, desired drop time, and the desired deceleration specifications of peak impact load, and deceleration pad specifications of length and width, assuming a pneumatic pad similar to that used in the NASA GRC's $2.2 \mathrm{~s}$ drop tower.

Hence, one is able to conceptually design both the height of the tower desired, or estimate drop conditions, based on a limited height of a drop site. One is also capable of estimating a required minimum deceleration performance, including estimations of the peak deceleration forces required. Both are key factors at the conceptual phase in order to properly baseline the minimum performance requirements. With 9 user inputs and 3 input constants that account for drag conditions, all other calculations can lead to a conceptual design that results in estimated outputs that include:

- Kinematic and drag-adjusted payload locations vs. time (i.e. height vs. drop time)

- Payload impact force

- Necessary deceleration forces and deceleration thicknesses

The following 9 user inputs leading to those outputs are:

- Desired drop time

- Payload weight

- Payload length

- Payload width

- Payload height

- Deceleration diameter

- Deceleration length

- Deceleration width 
- Desired peak g-loads (i.e. max payload g-forces during deceleration)

The following 3 input constants are also necessary to adjust for aerodynamic drag:

- Gravitational constant

- Appropriate drag coefficient for the payload shape

o It should also be noted that the designer assumed constant drag for the initial analysis - an assumption that may or may not be true. The true drag should be calculated from the data collected during drops to increase the accuracy of aerodynamic and impact load analyses.

- Air density

Given those inputs, the desired outputs can be calculated against increasing time, from the release of the payload through deceleration, in order to estimate facility performance characteristics. It is also pertinent to note that every variable output would be primarily calculated in SI units and converted to English units. Hence, the spreadsheet also includes conversion factors against which conversions can be made. Furthermore, all calculations are assumed to be estimates, as real-world conditions would inevitably vary from the ideal calculation assumptions. First, several input constants would be calculated from user and constant inputs, including:

- Cross-Sectional frontal area of the payload, as seen by the aerodynamic streamlines

$$
A_{\text {ref }}=L_{\text {payload }} W_{\text {payload }}
$$

- Volume of the deceleration system

$$
\begin{gathered}
V_{\text {cylinder }}=\pi D_{\text {pad }} H_{\text {pad }} \\
V_{\text {cube }}=L_{\text {payload }} W_{\text {payload }} H_{\text {payload }}
\end{gathered}
$$


- Payload mass

$$
m_{\text {payload }}=\frac{w_{\text {payload }}}{a_{\text {gravity }}}
$$

- Terminal velocity

o Based on a free body diagram of a generically shaped object, two predominant forces prevail, specifically gravity and drag. The designer assumes throughout the analysis that both the local gravity gradient, and thus the local gravitational acceleration, is constant and equivalent to the average global gravity constant of $9.8 \mathrm{~m} / \mathrm{s}^{2}\left(32.2 \mathrm{ft} / \mathrm{s}^{2}\right)$. The designer also assumes that due to the short distance and the fact that the facility is located on the land mass surface of Earth that the facility is close enough to the core of the Earth to use Newtonian equations for gravity, rather than Einsteinian equations. Furthermore, $A_{\text {ref }}$ is the frontal reference area of the payload, as seen by the streamlines, most predominantly along the gravity gradient, assuming the payload is dropped evenly. Additionally, when the payload reaches terminal velocity, the net force on the payload is zero, by definition, as the effect of gravity and the effect of drag are equal. Assuming the local gravity gradient and drag are constant and the direction opposite of the gravity gradient is positive:

$$
\begin{gathered}
F=\text { Drag }-m_{\text {payload }} a_{\text {gravity }}=0 \\
\text { Drag }=\frac{1}{2} C_{D} \rho_{\text {air }} v_{\text {terminal }}^{2} A_{\text {ref }}=m_{\text {payload }} a_{\text {gravity }} \\
\therefore v_{\text {terminal }}=\sqrt{\frac{2 m_{\text {payload }} a_{\text {gravity }}}{\rho_{\text {air }} A_{\text {ref }} C_{D}}}
\end{gathered}
$$


Next, a column of increasing time was created, with an arbitrary time step of $0.1 \mathrm{~s}$. The resolution of the calculations and graphs may be adjusted, by adjusting the time step. Using the time column, and previously calculated and converted inputs, and assuming a constant drag coefficient, one could then estimate, based on the resolution of the time step, all necessary drop outputs in sequential order:

- Kinematic drop distance (i.e. payload z-location, height, or drop distance away from the release mechanism)

$$
z_{k}=\frac{1}{2} a_{\text {gravity }} t^{2}
$$

- Kinematic payload velocity without drag

$$
v_{k}=a_{\text {gravity }} t
$$

- Drag-inclusive payload velocity

From first principles of a free body diagram, with drag oriented opposite of gravitational acceleration, away from the surface of the Earth, and Newton's $2^{\text {nd }}$ Law:

From Newton's $2^{\text {nd }}$ Law:

$$
\begin{gathered}
\sum F=m \sum a \\
F=m \frac{d v}{d t}
\end{gathered}
$$

From the FBD:

$$
\begin{gathered}
\sum F=\text { Drag }+ \text { Weight } \\
\sum F=-\frac{1}{2} C_{D} A_{\text {ref }} \rho_{\text {ar }} v_{\text {drag }}^{2}+m_{\text {payload }} a_{\text {gravity }}
\end{gathered}
$$


Substituting for the force from Newton's $2^{\text {nd }}$ Law:

$$
m_{\text {payload }} \frac{d v}{d t}=m_{\text {payload }} a_{\text {gravity }}-\frac{1}{2} C_{D} A_{\text {ref }} \rho_{\text {ar }} v_{\text {drag }}^{2}
$$

Combining masses and approximating:

$$
\begin{gathered}
\left.\frac{\Delta v}{\Delta t}\right|_{\lim t \rightarrow \infty}=a_{\text {gravity }}-\frac{1}{2 m_{\text {payload }}} C_{D} A_{\text {ref }} \rho_{\text {ar }} v_{\text {drag }}^{2} \\
\frac{v_{\text {final }}-v_{\text {initial }}}{t_{\text {final }}-t_{\text {initial }}}=a_{\text {gravity }}-\frac{1}{2 m_{\text {payload }}} C_{D} A_{\text {ref }} \rho_{\text {ar }} v_{\text {drag }}^{2}
\end{gathered}
$$

Where, since the payload is at rest, just prior to drop:

$$
\begin{gathered}
v_{\text {initial }}=t_{\text {initial }}=0 \\
\therefore \frac{v}{t}=a_{\text {gravity }}-\frac{1}{2 m_{\text {payload }}} C_{D} A_{\text {ref }} \rho_{\text {ar }} v_{\text {drag }}^{2}
\end{gathered}
$$

Combining and moving all terms to one side to create a quadratic form to solve for velocity

$$
\left(\frac{t}{2 m_{\text {payload }}}\right)\left(C_{D} A_{\text {ref }} \rho_{\text {ar }}\right) v_{\text {drag }}^{2}+v_{\text {drag }}-a_{\text {gravity }} t=0
$$

Letting:

$$
\begin{gathered}
a=\left(\frac{t}{2 m_{\text {payload }}}\right)\left(C_{D} A_{\text {ref }} \rho_{a r}\right) \\
b=1 \\
c=-a_{\text {gravity }} t
\end{gathered}
$$


And substituting into a quadratic form

$$
v_{\text {drag }}=\frac{-1 \pm \sqrt{1-4\left(\frac{t}{2 m_{\text {payload }}}\right)\left(C_{D} \rho_{\text {air }} A_{\text {ref }}\right)\left(-a_{\text {gravity }} t\right)}}{2\left(\frac{t}{2 m_{\text {payload }}}\right) C_{D} \rho_{\text {air }} A_{\text {ref }}}
$$

Based on common sense, the negative term would never make practical sense, and therefore, we only keep the positive term after simplification

$$
\therefore v_{\text {drag }}=\frac{-1+\sqrt{1+\left(\frac{2 t^{2}}{m_{\text {payload }}}\right) a_{\text {gravity }} C_{D} \rho_{\text {air }} A_{\text {ref }}}}{\left(\frac{t}{m_{\text {payload }}}\right) C_{D} \rho_{\text {air }} A_{\text {ref }}}
$$

- Drag-inclusive payload drop distance

Simplifying and rearranging equation 6.3-8 and taking the indefinite integral

$$
\begin{gathered}
\int d v=\int\left(a_{\text {gravity }}-\frac{1}{2 m_{\text {payload }}} C_{D} A_{\text {ref }} \rho_{a r} v^{2}\right) d t \\
v=\left(a_{\text {gravity }}-\frac{1}{2 m_{\text {payload }}} C_{D} A_{\text {ref }} \rho_{\text {ar }} v^{2}\right) t+C_{1}
\end{gathered}
$$

When $t=0, v=0$, and therefore $C_{1}=0$

Furthermore, using the fact that:

$$
v=\frac{d z}{d t}
$$


Substituting, rearranging to isolate $d z$ and taking the indefinite integral

$$
\begin{gathered}
\int d z=\int\left(a_{\text {gravity }}-\frac{1}{2 m_{\text {payload }}} C_{D} A_{\text {ref }} \rho_{a r} v^{2}\right) t d t \\
z_{\text {drag }}=\left(a_{\text {gravity }}-\frac{1}{2 m_{\text {payload }}} C_{D} A_{\text {ref }} \rho_{a r} v^{2}\right) \frac{t^{2}}{2}+C_{2}
\end{gathered}
$$

Using the same boundary conditions as used in solving for $C_{1}$, we arrive at

$$
z_{\text {drag }}=\frac{1}{2}\left[a_{\text {gravity }}-\left(\frac{C_{D} A_{\text {ref }}}{m_{\text {payload }}}\right)\left(\frac{1}{2} \rho_{\text {air }} z_{k}^{2}\right)\right] t^{2}
$$

- Minimum deceleration height

$$
H_{\text {bag }}=\frac{v_{\text {drag }}{ }^{2}}{2 a_{\text {deceleration }}}
$$

- Payload deceleration

$$
a_{\text {payload }}=\frac{\left(\frac{v_{\text {drag }^{2}}}{2 a_{\text {deceleration }}}\right)}{a_{\text {gravity }}}
$$

- Payload deceleration time

$$
t_{\text {deceleration }}=\frac{2 H_{\text {bag }}}{v_{\text {drag }}}
$$

- Payload impact force

$$
F_{\text {impact }}=m_{\text {payload }}\left(\frac{v_{\text {drag }_{t_{n}}}-v_{\text {drag }_{t_{n-1}}}}{t_{n}-t_{n-1}}\right)
$$


From those calculations, drop time was calculated against drop height and payload velocity, as shown in Figure 6-1 and Figure 6-2 for the assumed values found in Appendix $B$.

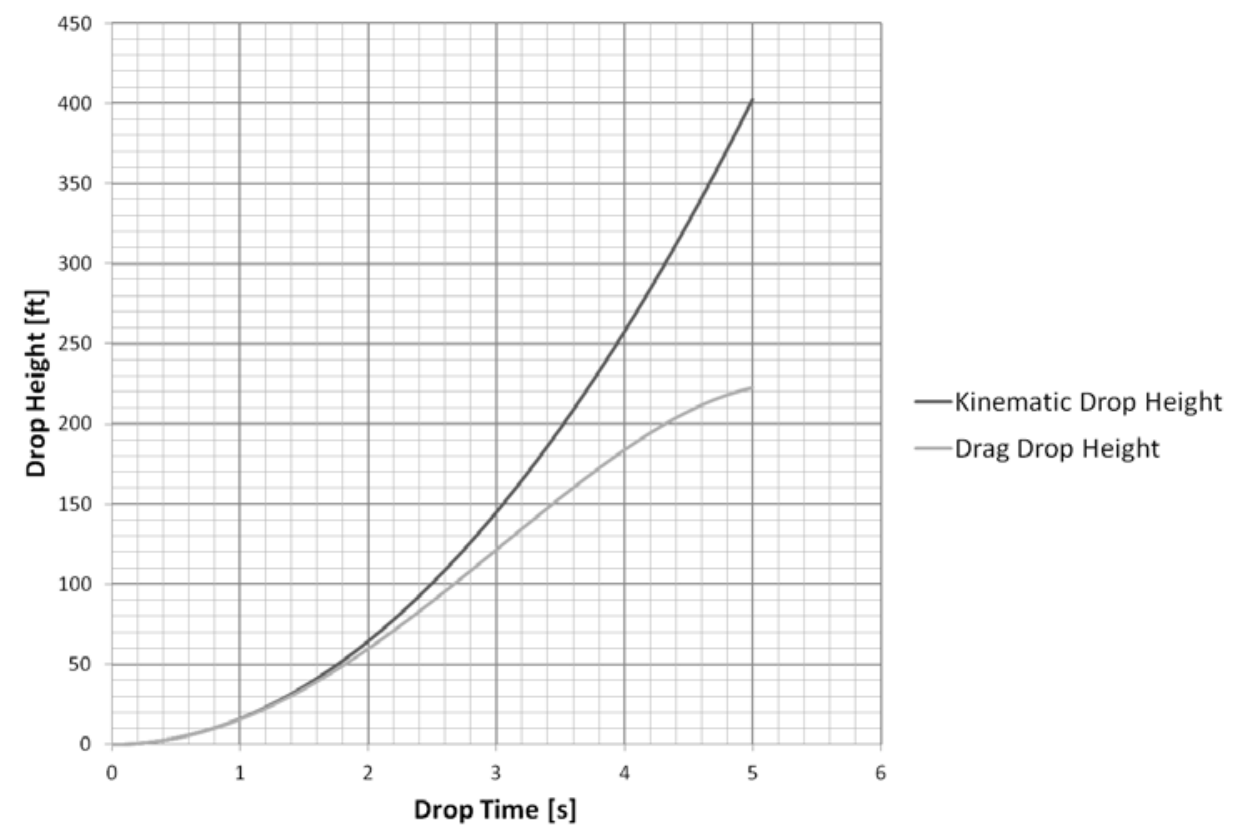

Figure 6-1: Concept Design Drop Time vs. Drop Height

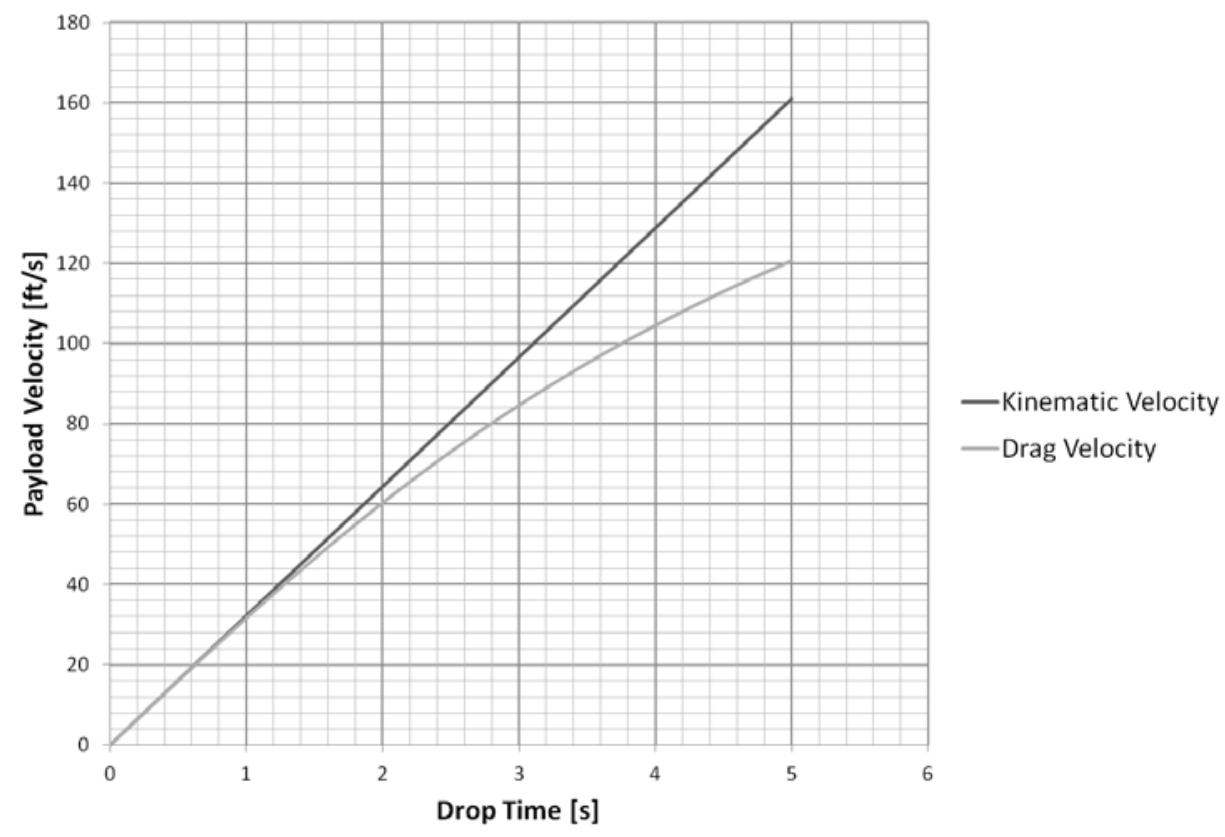

Figure 6-2: Concept Design Drop Time vs. Payload Velocity 


\subsubsection{Payload Frame Concept}

After visiting the NASA GRC's drop towers, the team and the designer were encouraged by the drop heritage that their frames had endured over their long years of operation. The initial idea that the designer had was to build a frame very similar to some of those used at NASA GRC's 2.2s drop tower. The initial decision to provide a payload frame to users stems from Goal \#9. Providing as much of the equipment common to reduced gravity research, provides users with a relatively more affordable experience, allowing them to use their funds in other areas of their projects, leading to better project quality. It also encourages more frequent use of the facility itself, regaining value on capital investment. However, to remain as versatile as possible, we also wanted to design the facility such that it could accept other payload frames, allowing users that remain within design and interface limits to bring their own payload frames, as desired.

\subsubsection{Data Acquisition System Concept}

In addition to providing a payload frame, the designer continued to strive for Goal \#9 and provide a data acquisition system for the user. However, unlike the NASA GRC 2.2s drop tower, the designer wanted to have a standalone, ride-along data acquisition system, so as not to induce vibrational loads from fiber optics, added drag due to the unspooling friction, or to take on the risk of a snag during unspooling of the fiber optics. Furthermore, the designer wanted to provide the user with an onboard power supply system to support both the data acquisition system and the experiment. At the most basic level, the system would have to be capable of measuring acceleration along 3 axes, but the designer would also strive to provide the most versatile, and therefore, affordable system as possible.

\subsubsection{Structural Concept}

The facility structure is the most important subsystem of the entire facility. Without it, the WVU SMiRF would not exist. Originally, several concepts were considered, including one without a full structure. The team had considered a system where a chain hoist, outfitted with a release mechanism, suspended from a ceiling could be raised and lowered from 
the ground, and the release mechanism could be operated from the ground, very similar to the very first drop tower research facility built at the NASA Lewis Research Center. Even in that case, the ceiling structure would still prove to be the most important subsystem. Without a structure to support the payload prior to a reduced gravity operation, a reduced gravity facility could not exist. Even if one were to catapult a payload into the air, the foundation from which the payload was launched would form the structure.

The designer quickly realized that the structural system would be highly dependent on the site chosen for the facility. Ideally, the facility could be located at a pre-existing structure that could be retrofitted to form the facility's structure. Several sites were considered including existing agricultural silos, the exterior of tall buildings, elevator shafts, atriums, and vacant high-bay spaces. Eventually, after speaking with several parties about potential sites, the team found an acceptable, existing interior highbay space that was tall enough to achieve Goal \#5, based on the originally developed concept analysis spreadsheet, and would provide protection from the elements, driving toward Requirement \#3.

Once the site was determined, finalized concept designs began. Initially, it was thought that the walls of the high-bay building could be utilized as part of the structure. A 9-month effort led to the development of an Excel spreadsheet, an accompanying CAD model, and an FEA analysis, to ensure designs were in compliance with OSHA regulations, in order to design a wooden platform and staircase structure to access and operate at the top of a pre-fabricated steel structure that would form the drop tower structure. Those analyses were performed to help ensure Requirements $\# 1$ and \#2. It is the primary function of the structure to provide safety to personnel and users, while all other functions are secondary. Therefore, it must have an abundant Factor of Safety (FoS).

\subsubsection{Release System Concept}

After visiting the NASA GRC's drop towers and realizing the basic fact that there was more than one possibility for releasing a payload, the team and designer considered several release concepts, including a mechanical release mechanism, shearing of a piano wire, shearing of a bolt, 
pneumatics, and electromagnets. All of these concepts would require mounting the release system to the structure. The primary driver of the release system was a goal (i.e. Goal \#7) that involved achieving a very safe and stable holding period prior to release. As a result, the team also considered a two step process of lifting the payload and then transferring to a separate release system, so as not to take on a combined risk of a singular system lifting, holding, and releasing a payload, which would help achieve Requirements \#3 and \#5 and Goal \#7 through reduced reliance on a singular system, thereby decreasing subsystem complexity and increasing reliability. As the release system is an inherent point of risk during operation, its absolute reliability could not be understated. That system must have a large FoS. Additionally, the release system must have a smooth, symmetrical, stable release so as not to impart asymmetric inertia into the payload at the time and point of drop. Doing so would cause the payload to rotate during drop causing an asymmetric landing that could be catastrophic to the experiment, the facility equipment, the facility itself, and most importantly to the people and operators. That is an absolutely unacceptable scenario.

\subsubsection{Deceleration System Concept}

As with the release system, several various options were considered for the deceleration system, including a polystyrene pit, a pneumatic air bag, a series of nets controlled by torque-resistant pulleys, and a foam pad. The primary consideration for the design of the deceleration system is determining the maximum payload weight limit, considering the budget and Goal \#8. Driving toward Goal \#8 allows for less expensive payload frame design and manufacturing. It also drives toward a more versatile range of experiments. Lower impacts loads require less robust experiment hardware. However, with some clever engineering, even those loads can be reduced within the payload frame, so long as the payload frame is versatile enough.

\subsubsection{Lift/Retrieval System Concept}

As discussed in other subsystem concept sections, several options were considered for the lifting/retrieval subsystem. Questions arose to whether the release mechanism should be integrated with the lifting/retrieval 
system, or even if a previously existing system (e.g. a high-bay crane) could be used for this purpose. There was also discussion as to whether the system could automatically retrieve the payload, or if that process had to be a manual process. Using a triggered claw-like system that is triggered by a protrusion on the payload frame, a system could be engineered that automatically closes as the lifting/retrieval system is lowered onto the payload, allowing that subsystem to clasp onto the payload without human intervention, decreasing risk, and increasing turnaround time, driving toward Requirements \#1 and \#2 and Goals \#2 and \#3.

\subsection{Final Design}

To continue to deliver performance over the life of the facility, all subsystems had to strive to meet all of the aforementioned Requirements and Goals set forth. Final designs for each subsystem were developed, based on the conceptual designs.

\subsubsection{Payload Frame Design}

The payload frame serves as the primary interface between the facility and the user. Thus, it has to provide a versatile, universal mechanical interface between the experiment and the facility. As the primary interface, it is also the most likely subsystem to undergo use and encounter wear. Therefore, its design has to remain as simple as possible, thereby increasing reliability and affordability, whilst not imposing on capability. Furthermore, the payload frame also has to be able to withstand the impact force up to the rated payload weights of the facility, with an ample FoS.

Based on past drop heritage, simplistic design, and relatively large dimensional volume, the developer chose the NASA GRC's 2.2s rectangular frame design as the preferred frame of choice. Graciously, the NASA GRC provided us with the drawings necessary, and Wilson Works, Inc. of Morgantown, WV built the frame to those specifications for $\$ 1,557.00$. Its physical dimensions, open interior design without preformed shelving and/or other physical features, and structural performance were key technical drivers of the decision, while expedience 
of schedule and cost of development, or lack thereof due to the provisions of drawings from NASA GRC, were key administrative decision drivers.

Its simplistic design and fabrication affords it the best odds of long-term reliability, low-maintenance, and structural performance. The payload frame is fabricated from as few pieces of AL6061-T6 as possible. By breaking the aluminum at the corners to form a bent aluminum shell, welded to two aluminum plates that form the top and bottom, the structural design utilizes continuity of material to increase structural performance, as indicated by its long drop heritage in the NASA GRC 2.2s drop tower. Gusset plates are used in the corners and riveted between the top and bottom plates and the bent center section. This form created a completely open interior, measuring 37" long x 15" deep x 32" tall (exterior dimensions are an additional 1" per dimension), allowing for versatility of experimental payloads dimensions, with maximum dimensional size, allowing for larger, heavier, and a wider variety of experiments. The payload frame itself weighs $60.2 \mathrm{lbs}$. The term payload weight refers to the full weight being dropped in the tower, thus representing the weight of the payload frame supplemented by the additional weight of the experimental payload. Additionally, a C-channel runs the depth of the frame, bolted to the top plate at its geometric center, increasing structural support at what is considered to be the best location for a single-point-ofsupport for drops, as most drop towers would most likely interface at a singular point to increase the quality and reliability, and thus safety, of a drop. A depiction of the fully-outfitted payload frame is presented in Figure 6-3.

After fabrication and delivery of the payload, the developer made several modifications to the payload frame, in order to meet Requirements \#3, \#4, and $\# 5$, as well as Goals \#3, \#4, \#6, \#7, \#8, and \#9. The developer was striving for a universal interface, such that users would mechanically interface with a pre-determined bolt pattern, structurally sufficient up to, and beyond, the payload weight limits and impact loads created at those weight limits, while also being simplistic, symmetric, and universal for each user. Six $1 / 4$ " countersunk clearance holes were drilled into the bottom plate of the payload frame. The countersink allows panhead screws to stay flush with the bottom plate as the payload drops onto the deceleration system. The user may use those six holes, or they may use a provided 


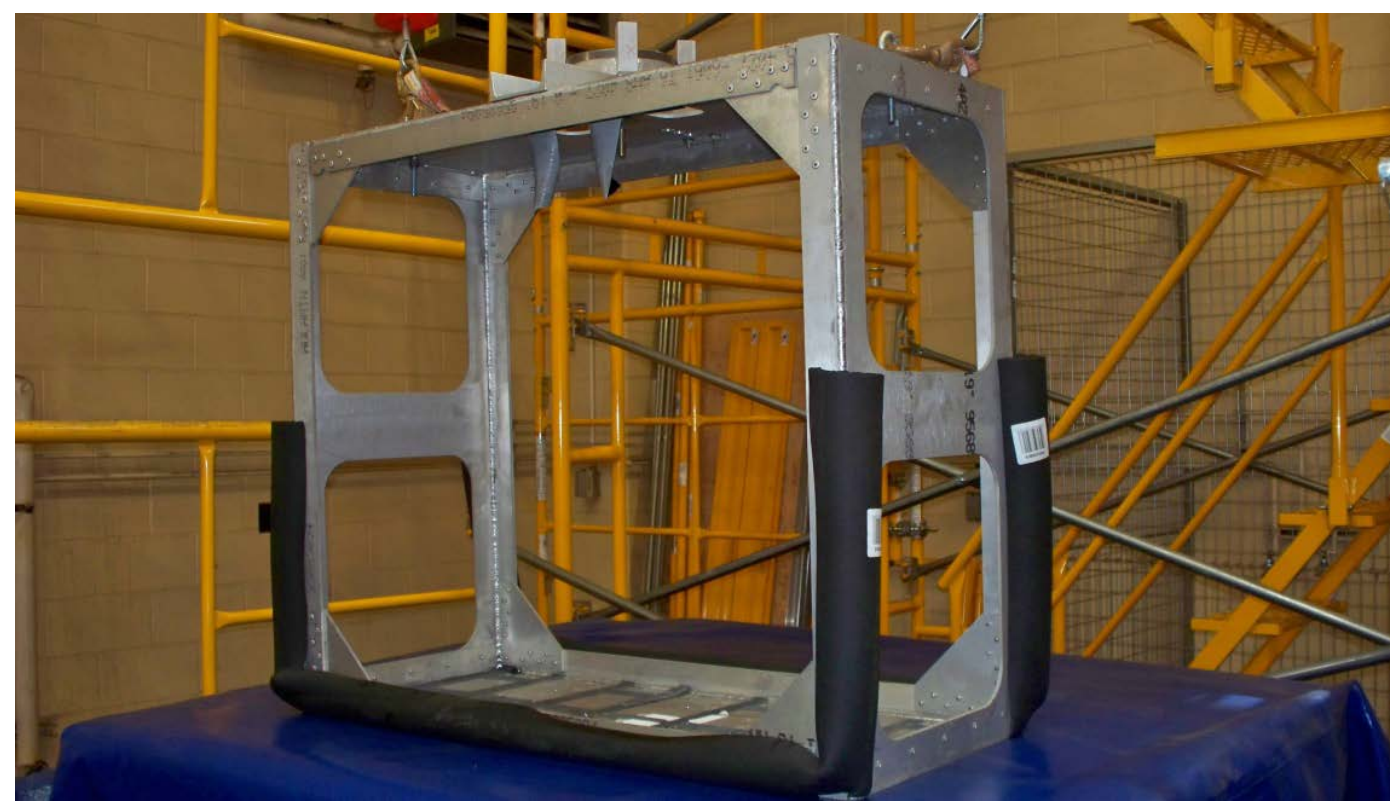

Figure 6-3: WVU SMiRF Payload Frame

"interface table" that was designed by the developer and produced by the WVU MAE shop. That interface table employs foot plates, 1"x1" square tubing, and a 24" x 12" plate, forming a table 7.5" in height, as shown in Figure 6-4. The table surface may be drilled with any custom hole pattern desired by the user, allowing for maximum versatility. Eventually, that table surface would have to be replaced after the structural integrity or usable capability has been exceeded. However, the height of the table reduces the usable space on the interior of the payload frame. Furthermore, a grid of Velcro strips was adhered to the interior of the bottom plate. These Velcro strips are used with WVU SMiRF-provided counterweights to properly balance the payloads prior to integration into the facility, thereby ensuring a clean, balanced drop. Third, a $1 / 2$ " clearance hole was drilled into the C-channel at the geometric center of the payload frame. A grade- $81 / 2$ " bolt was attached to the payload frame with a nylon-insert locknut and large diameter washers, through a polished steel plate, which sits atop an aluminum plate with four stabilizing prongs that do not allow the steel plate to physically move from the release system once attached. That simplistic system provides the interface between the payload frame and the facility. Additionally, it is the system that any user must use in order to use their own payload frame in the facility. Lastly, to reduce wear, foam padding was added to all edges and 
corners of the payload frame that impact the deceleration system. Userproduced payload frames must also have similar features in order to use the facility. This universal simplistic interface ensures minimal wear on both the payload frame and the facility, while remaining versatile enough to accept a wide variety of experimental payloads and deliver safe, reliable testing with low-maintenance and few ongoing costs. Moreover, it also allows the user a custom interface option, or for the user to use their own payload frames, so long as they meet the required interface points with the facility. Appendix $\mathrm{C}$ provides developers and users with the drawings and interface points of the payload frame.

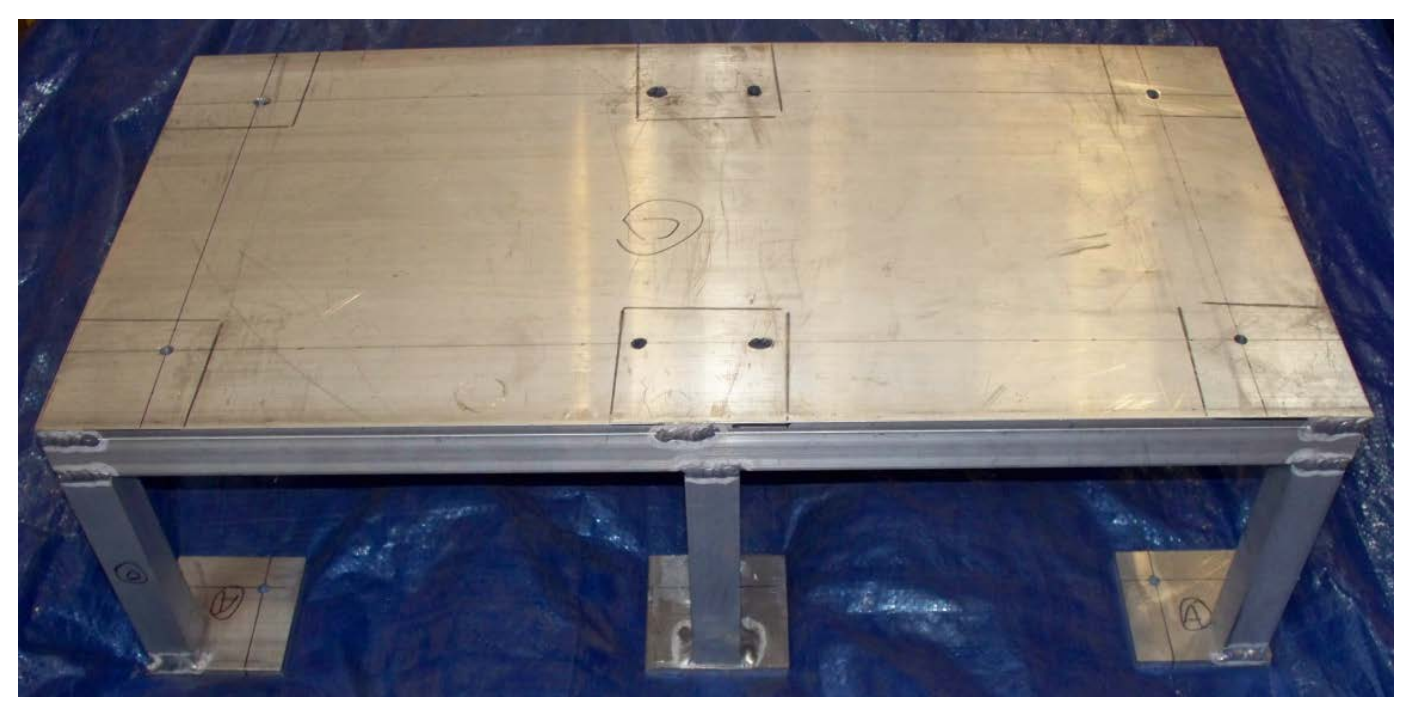

Figure 6-4: Payload Frame Interface Table

Lastly, a drag shield was not included in the initial development of the WVU SMiRF. During initial development, the cost and time associated with designing and developing a drag shield was prohibitive. Moving forward, the developer suggests that a drag shield be developed for permanent employment at the facility. When properly designed, a drag shield will increase the reduced gravity-level (g-level) quality of the drops. The typical design of a drag shield incorporates a metal structure with a fixed nose section that serves as the aerodynamic element, cutting down on the overall drag of the falling payload. The payload is mounted within the drag shield on a set of rails, that allow relative movement between the payload and the drag shield during the drop, as the drag shield will inevitably fall relatively slower than the internal payload, while the shield 
experiences the aerodynamic drag from which the payload is shielded. The design of such a system can get very creative, however. Things such as collapsible pneumatic boots may be fitted onto a relatively simply shaped drag shield, allowing future tailoring of drag shapes and easier handling of the shield upon impact. By incorporating such a design, the deceleration system may also be more simplistically designed for longer reliability of both the drag shield and the deceleration system. Furthermore, removable polycarbonate panels could be affixed to the inner wall surfaces to further reduce drag and aerodynamic transference to experimental payload, while providing pleasing, professional aesthetics to the payload frame.

\subsubsection{Data Acquisition System Design}

In order to provide a comprehensive experience, thereby increasing affordability for the user, the developer decided to procure a data acquisition system capable of providing onboard, real-time data recording. However, to do so required a great deal of forethought into the types of research that may eventually be performed at the WVU SMiRF, and the types of instrumentation and/or control that would be required of the data acquisition system for those endeavors. As drop tower research affords wide flexibility as to the type of data that may be acquired, so too must the data acquisition system.

Additionally, as the WVU SMiRF is, at a minimum, a university asset, it is also an extremely valuable educational opportunity. As such, the experience of not only developing research, but also the execution of that research via experimentation could serve to be very valuable to student education. In order for the experience to prove valuable, it is not only desired to simply have the experience, but also to provide a tangible skill set that may be applied in further academic endeavors and postgraduation experiences in industry. In order to accomplish that level of experience, equipment providing similar skill sets that may be expanded on, or the same equipment used, in industry or advanced research should be purchased, in order to teach those skills needed, if possible.

With all of that in mind, the developer decided to procure a National Instruments system, as it is used in applications from basic to advanced 
research and is commonly used in industry. A National Instruments (NI) Compact RIO system was purchased that has the capability to handle real-time data collection. The choice of the NI cRIO-9074 provides the foundation for current research and future expandability. It features:

- A ruggedized, embedded control system in an 8-slot chassis

- A $400 \mathrm{MHz}$ real-time processor capable of data logging, control, and analysis

- A field-programmable array (FPGA) for Input/Output (I/O) timing of sensing, control, and processing

- Ports for several types of peripherals

Furthermore, in order to provide for a very comprehensive initial experience at the WVU SMiRF, several modules were also procured to populate the slots of the $\mathrm{NI}$ cRIO-9074, in addition to providing accompanying LabView software. These modules included:

- NI-9205 Analog input module

o 32 single-ended channels or 16 differential channels

o 16-bit resolution at $250 \mathrm{kS} / \mathrm{s}$

$0 \pm 200 \mathrm{mV}, \pm 1 \mathrm{~V}, \pm 5 \mathrm{~V}, \pm 10 \mathrm{~V}$ programmable input ranges

o NIST-traceable calibration

- NI-9221 Analog Input Module
o 8 channels
o 12-bit resolution at $800 \mathrm{kS} / \mathrm{s}$
$0 \quad \pm 60 \mathrm{~V}$ range
o NIST-traceable calibration

- NI-9421 Digital Input Module
o 8 channels
o $100 \mu$ s sinking digital input
o $12 \mathrm{~V}-24 \mathrm{~V}$ range

- NI-9472 Digital Output Module

o 8 channels

- $100 \mu$ s sourcing digital output

- $6 \mathrm{~V}-30 \mathrm{~V}$ range 
- $\quad \mathrm{NI}-9234$ IEPE Module

o 4 channels

o BNC connectors

$0 \pm 5 \mathrm{~V}$ input

o 24-bit resolution at $51.2 \mathrm{kS} / \mathrm{s}$

o $102 \mathrm{~dB}$ dynamic range

o Software selectable AC/DC coupling $(0.5 \mathrm{~Hz})$, IEPE signal conditioning (0 or $2 \mathrm{~mA}$ )

o NIST-traceable calibration

- $\quad$ NI-9977 Filler Module (qty = 3)

o Used to fill in empty slots in NI cRIO-9074 to protect slots for future expandability

As one may note, this system would provide a fairly comprehensive experience to the user by providing a variety of sensing and control options for a multitude of experimentation, hence fulfilling Requirement \#5 and Goals \#4 and \#9. Yet, for as much versatility as this initial system has, it is the most highly specified, and hence the least versatile system of the WVU SMiRF, and therefore must be upgraded over time to maintain the versatility of the facility in keeping with the overall requirements set forth for the facility.

However, as the aforementioned data acquisition system will serve as the primary data acquisition system, a simplified, secondary data acquisition system had to be used for system checkouts, primarily due to the uncertainty in the performance characteristics of the facility. The primary unknown performance characteristic was the g-load shock upon impact with the deceleration system. This resultant physical threat led to the selection of a relatively inexpensive data logger that could be replaced if damaged. After researching a wide array of simplified data loggers to record accelerations and thus calibrate the facility, the developer selected a Vernier LabQuest 2 data logger ${ }^{1}$. The primary features of the LabQuest 2 included:

${ }^{1}$ The purchase of that data logger required the stipulation that it was purchased for educational purposes, which was appeased through the training of a select number of students at the drop tower and through the demonstration of actual experimental drop data to a high school student that the author mentored throughout the initial calibration process. 
- A standalone power supply (Li-on battery)

- A touch screen user interface

- Embedded data logging software

- Free analysis and conversion software

- 12-bit resolution at $100 \mathrm{kS} / \mathrm{s}$

- Maximum Sampling Rate with $2+$ sensors $=10 \mathrm{kS} / \mathrm{s}$

- 5 sensor channels

The developer also procured three 25-g accelerometers, each with an accuracy of $\pm 0.25 \mathrm{~g}$, mounted in the orthogonal coordinate system of the payload frame at the geometric center of the bottom plate. The data logger was then mounted inside of a customized housing for drops. The housing included a memory foam liner, to further reduce impact loads on that component, and a Faraday cage to reduce any EMI from outside sources. A photo of the customized housing is presented in Figure 6-5. Any effects from the Faraday cage on data collection are unknown, but do not seem to have affected the desired sampling rate input prior to the drops. This data logging system, along with its software and customized housing, performed well and proved that the data acquisition housing could perform to adequately block EMI and provide impact support, thereby allowing the data acquisition system to survive current impact loads of the checkout configuration. Additional information about both data acquisition systems may be found in Appendix D.

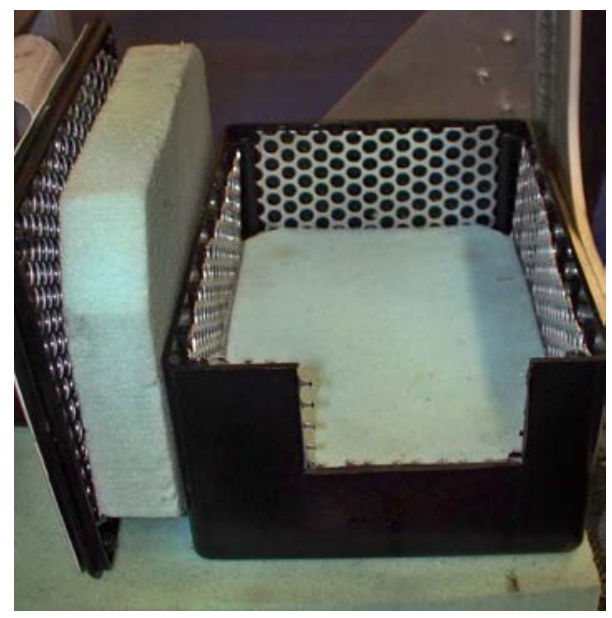

Figure 6-5: Data Acquisition System Drop Box, Including EMI Shield and Supplemental Padding 


\subsubsection{Structural Design}

As the fundamental system of the facility, the structure should also serve to meet all of the Requirements and Goals 1-9. As such, to obtain the versatility required, the design had to be simplified, while meeting those Requirements and Goals. The fact that a simplified system would ultimately lead to increased versatility and reliability, while reducing ongoing maintenance, led the developer to determine that a unified, prefabricated system would accomplish those Requirements and Goals better than a custom system. A custom system, when well designed and fabricated, may perform even better than a mass-produced pre-fabricated system and could cost less. However, custom systems also take a great deal of design and fabrication resources, as well as a thorough and potentially elaborate inspection process for safety certification. Alternatively, by choosing to construct a pre-fabricated, OSHA-certified system, many of those hurdles could be surpassed in favor of expediency, and potentially unforeseen fabrication and maintenance costs. After researching several pre-fabricated options, a scaffolding system was decided to be a versatile solution to form the various necessary elements of the structure, while also capable of being assembled by a minimal number of personnel, thereby increasing safety during construction. A BilJax Sectional Scaffold system was purchased after cost negotiations with Grainger, who accommodated our requests generously. Rather than just one drop tower, two supporting towers would also be erected. One tower would serve to produce access via staircase to the top of the tower, while the other served to provide personnel access to a second side of the drop tower at the top of the tower. The towers were then secured to each other and to two walls of the building's structure within which it was located, every 20 vertical feet, per OSHA standards, all of which can be seen in Figure 6-6 through Figure 6-8. Several extra parts would also be procured for any future part replacements.

However, even with a pre-fabricated solution, some customization was necessary. An I-beam support system had to be custom-fabricated to integrate into the scaffold framing to transfer loads from hanging payloads into the structure, while serving the purposes of hanging the release system from the geometric center of the drop tower and providing a mounting surface for the hoist to lift payloads. The loading scenarios were fairly simple. The I-beam support structure would be symmetrical, and 
thus the calculations could be simplified. Simple point supports at four corners, with point loads for two scenarios: 1) a lifting scenario with the hoist transferring loads into the I-beam structure, and 2) a holding scenario of the payload prior to release from the release system. Including a FoS of 6 , the calculations were completed prior to fabrication. Lastly, a small preparation boom, allowing payloads to hang from the drop tower, was fabricated using extra parts, allowing users to balance their payloads prior to drop, which is shown in Figure 6-9. Further information about the structure may be found in Appendix E.

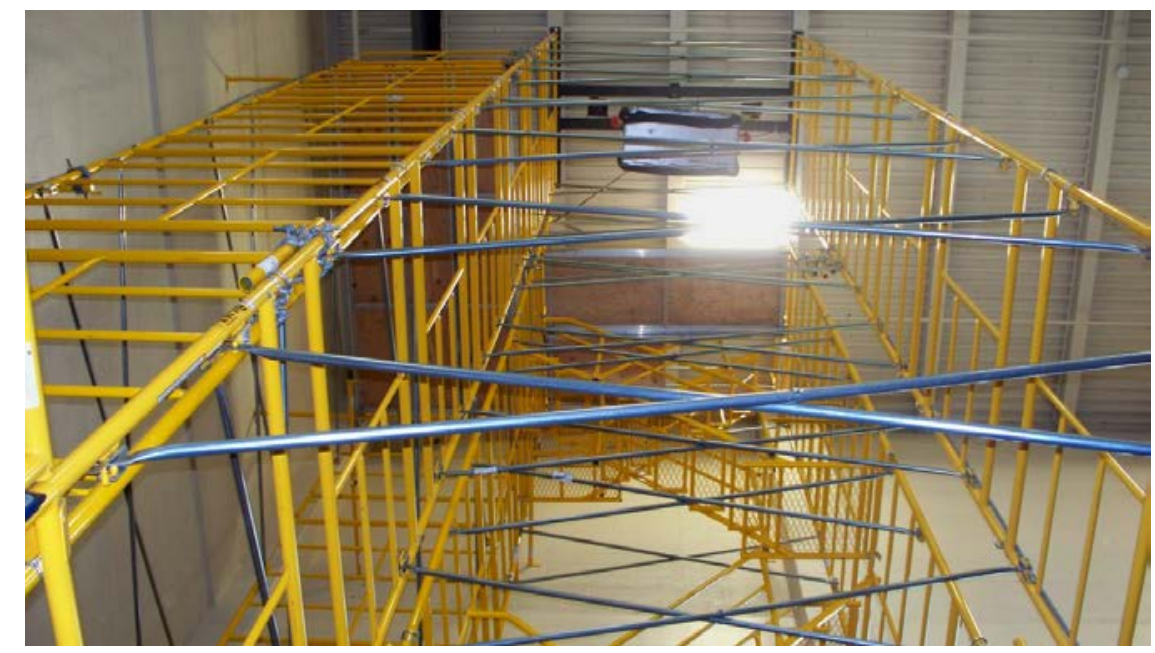

Figure 6-6: WVU SMiRF Structure (Center - Drop Tower; Behind - Staircase Tower; Left- Additional Access Tower)

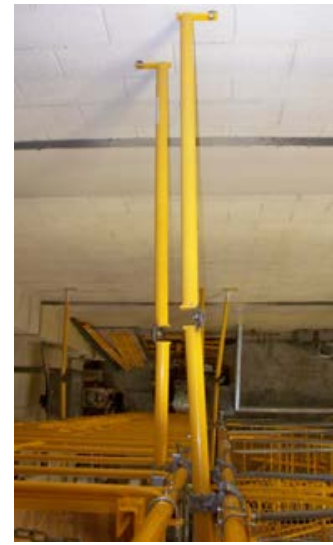

Figure 6-7: Structure Stabilizing Arms (as required by OSHA)

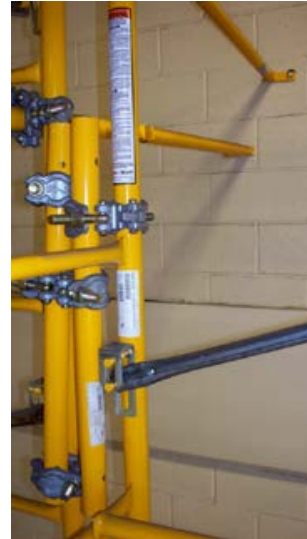

Figure 6-8: Clasping System (used to connect towers together for added stability) 


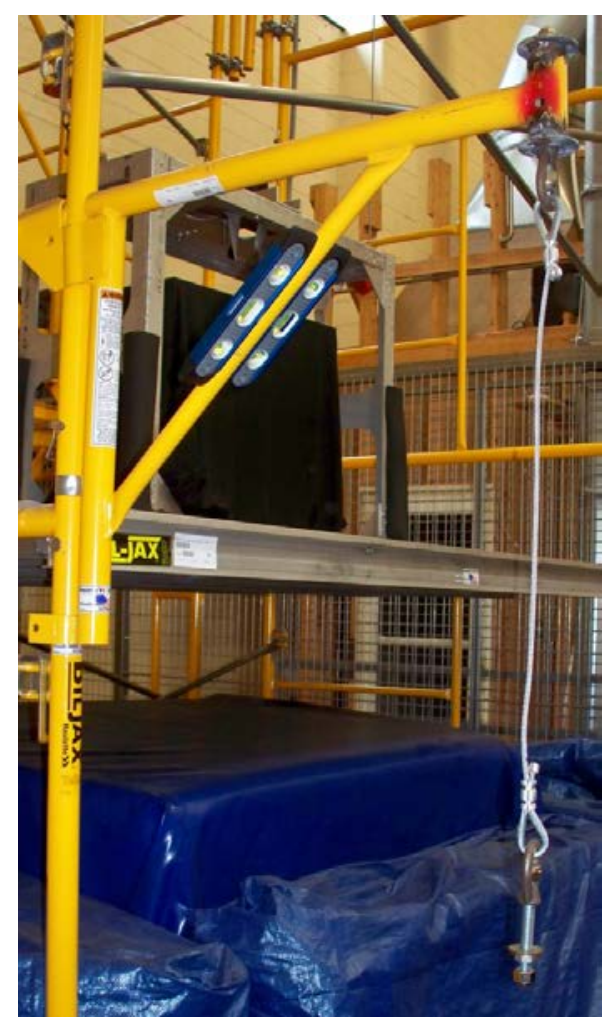

Figure 6-9: Balance Boom

\subsubsection{Release System Design}

As the second of two points of primary risk, and arguably the riskiest safety point of the entire drop tower, the release system must be safe. After safety concerns are addressed, the system must perform to produce a smooth, even release with very little to no resistance. Therefore, after taking into account all of the Requirements, and Goals \#2, \#3, \#4, \#6, and $\# 7$, the developer chose an electromagnetic release method. The primary downsides to an electromagnetic release are that the electromagnet produces electromagnetic interference (EMI) that may interfere with electronics on user payloads, and that it increases the complexity of the drop tower by adding more electrical work. However, an electromagnetic release provides a very smooth, even, nearly resistance-free release across the surface of the ferrous plate to which it must interface. By hanging the electromagnet from a singular point on the I-beam structure, using a $1 / 2$ " cable, the system may also be upgraded at a later time. However, the Magnetool electromagnet chosen should last the life of the drop tower, if there is not a need to increase the capabilities of the WVU 
SMiRF. In addition to the electromagnet, a common interface system that attaches to payloads had to be developed, which included a stabilizing crown, consisting of 4 aluminum prongs to prevent the electromagnet from sliding off the ferrous steel plate contained within the crown, as shown in Figure 6-10 through Figure 6-12. A 1/2" bolt attaches a polished steel plate to the payload frame. This system has since produced very safe, repeatable, stable drops. Additional information about the release system is presented in Appendix $\mathrm{F}$.

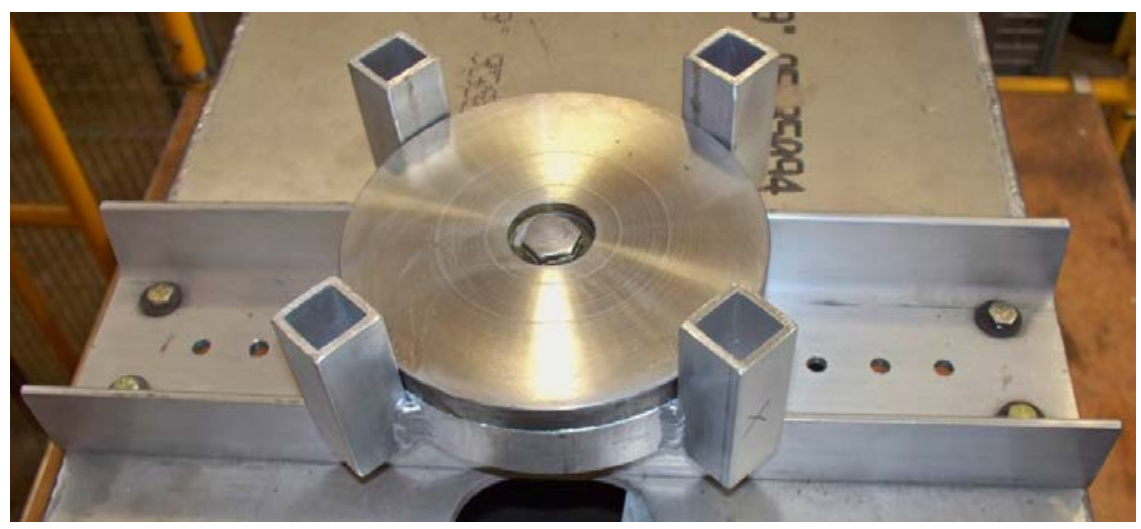

Figure 6-10: Payload Frame Crown

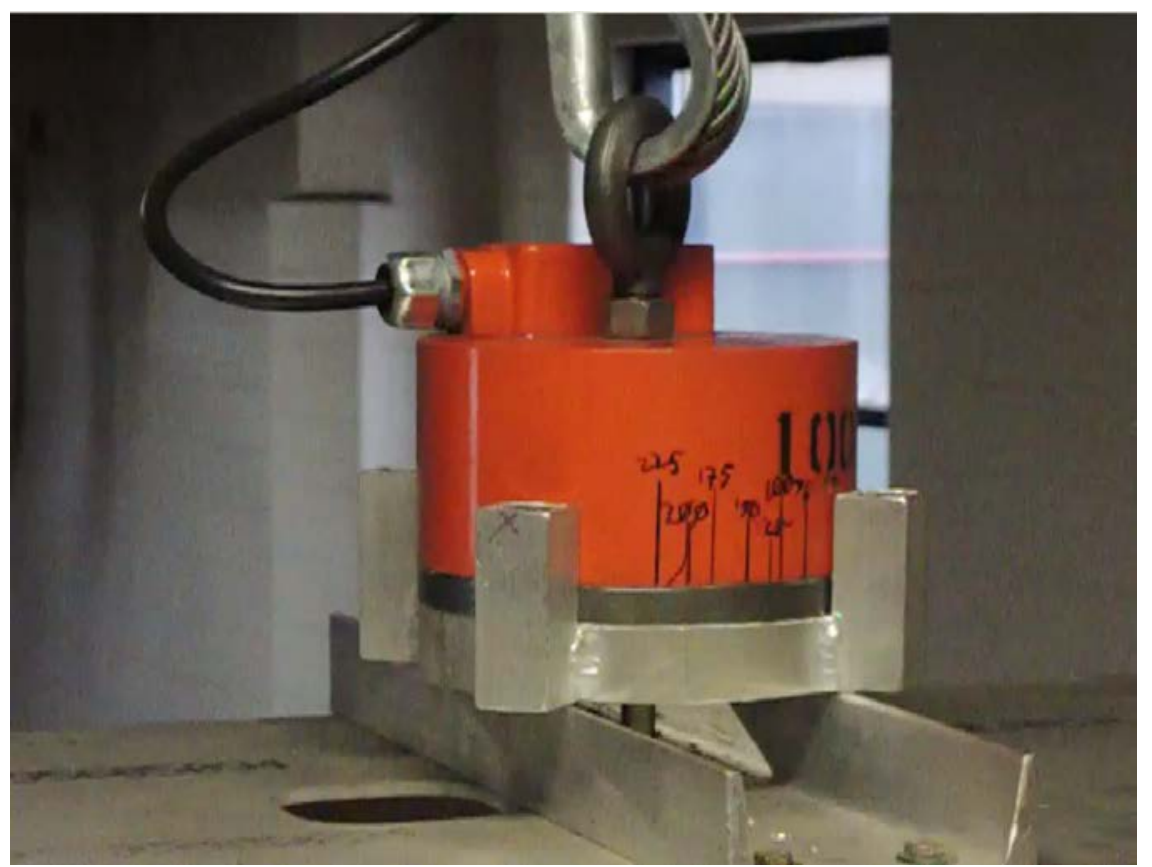

Figure 6-11: Release System Final Design - Depicting Electromagnet and Payload Frame Crown 


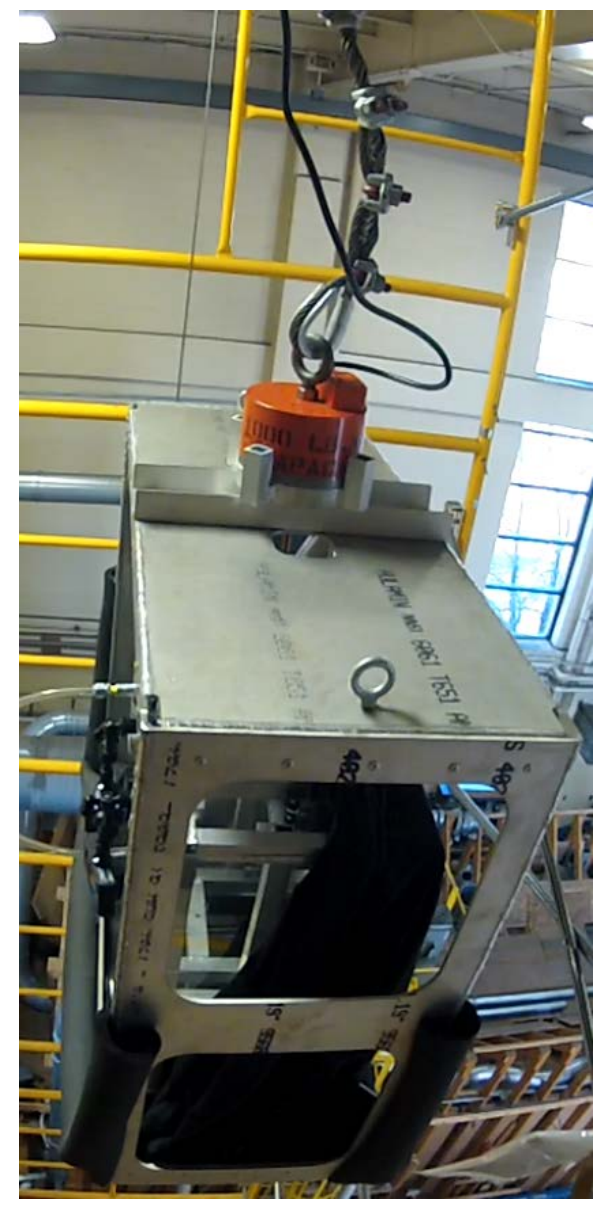

\section{Figure 6-12: Release System with Attached Payload}

\subsubsection{Deceleration System Design}

In partnership with the release system, the deceleration system is the second of two very risky points in the system, that required very careful consideration in addition to meeting the Requirements and Goals set forth. If the risk from the release system is that it may release the payload unevenly causing an abnormal and possibly dangerous drop, it is the responsibility of the deceleration system to perform, even during the worst of scenarios. Its primary function is to protect personnel from dropping payloads. Its secondary function is to protect the building and facility. Its tertiary function is to protect payloads. If the facility performs as intended and personnel follow operational instructions, only the tertiary function is 
ever employed. On the line of thinking that the initial investment must have long-term value, the deceleration system, the system assumed to take the most physical abuse, and therefore have the shortest physical lifetime, must be robust and have very low-maintenance, with an extremely high record of reliability. Simplicity would again be a key design feature for this system. With that in mind, the developer chose memory foam padding as the solution for the deceleration system. The open-cells of the memory foam would allow the system to continue to rebound between drops and continue to perform over long lengths of time. To this end, the team would begin to perform material testing in order to determine the type of memory foam and thickness.

The goal of the design of the deceleration system then turned to determining the proper combination of foam stiffnesses to employ in order to get the proper deceleration profile, based on the results from the concept design. From Newton's $2^{\text {nd }}$ Law we know that

$$
F=m a
$$

If we can assume that the force could be translated into a pressure, using the frontal reference area of the payload from the concept design, then

$$
P=\frac{m a}{A_{\text {ref }}}
$$

Furthermore, we need to determine the amount of deflection through each ply of foam, which can be given by

$$
\delta=\frac{P L}{A_{p l y} E}
$$

where $L$ here describes the height or thickness of an individual ply of foam. Solving for pressure and substituting into the modified version of Newton's $2^{\text {nd }}$ Law

$$
P=\frac{\delta A_{p l y} E}{H_{p l y}}=\frac{m a}{A_{\text {ref }}}
$$


and solving for acceleration to determine the deceleration profile based on an individual ply of open-celled foam and converting to units of "g's" by dividing the deceleration by gravitational acceleration

$$
\frac{a_{\text {deceleration }}}{a_{\text {gravity }}}=\frac{\delta A_{p l y} A_{\text {ref }} E}{m H_{p l y}}
$$

Note here that the deceleration equation would later be applied to a MATLAB code in which various configurations of different foam stiffnesses would be combined. In order to practically implement such a code, it was approximated that the foam ply beneath the current ply of interest would fully deflect prior to the subsequent ply beneath it beginning to deflect. Therefore, when

$$
\begin{gathered}
\delta=H_{\text {ply }} \\
\frac{a_{\text {deceleration }}}{a_{\text {gravity }}}=\frac{A_{\text {ply }} A_{\text {ref }} E}{m}
\end{gathered}
$$

thus, the deceleration profile is primarily based on mass and dimensions of a payload and on the characterization of the elastic modulus of the foam.

To ensure that the designed system adheres to the concept design analysis, one must characterize the elastic modulus of the various plies of memory foam. To generate a stress/strain curve from which the elastic modulus could be derived, the typical pull test would not work, as it would tear apart the foam with no appreciable resistance. Therefore, a different standard method had to be sought. Fortunately, the vast knowledge within the material science and testing fields prevailed to produce the standard ASTM D3574-08 that describes the "Standard Test Methods for Flexible Cellular Materials - Slab, Bonded, and Molded Urethane Foams." Using that standard a test was set up on an Instron material testing apparatus. Using SunMate cushion samples sent from the manufacturer, Dynamic Systems Inc. (DSI), 1" x 1" x 1" (qty = 5) samples were taken from each foam type for characterization. Each sample underwent both compressive and decompressive test analysis. Furthermore, 8 different types of foam would be tested in order of less stiff to more stiff: extra soft, soft, medium soft, medium, medium firm, firm, extra firm, and T50E; the 
latter of which we were told by DSI is used in aircraft ejection seats for high g-load support.

However, only the compressive data sets were retained, as most of the samples lifted off from the load cells during decompression, due to the slow response time of the foam. The results were compiled into a singular graph, including error bars, as shown in Figure 6-13, to determine elastic moduli for each foam type, which were then used in the MATLAB program to determine the necessary foam types and thicknesses required for the desired deceleration profile [Appendix G].

The final configuration would be determined by the MATLAB program, with some final as-built modifications. T50E would be employed as the bottom ply in order to provide the stiffest resistance to impact and protect the building's concrete floor. Furthermore, the final configuration of the foam pad featured stacked plies without adhesion between plies. It was thought that less degradation would occur over time due to the chemical interaction between the glue and the foam plies, allowing for natural degradation times of the foam, which are currently unknown, but are assumed to be many years. The degradation of the plies will show up as varying deceleration performance over time, based on the data that is obtained from individual experiments. The degradation may be tracked using the software developed by the graduate student, and compiling into a master database. Moreover, leaving the foam simply stacked allows for greater energy dissipation through the relatively small translational movement of the plies and the individual ply deflections. It also allows for less ply-to-ply stress in comparison to if they were adhered. In that case, the ply above would inevitably be undergoing a different amount of deflection as the ply below causing a greater shearing stress between adhered plies. However, as it turned out, a closed-cell foam pad was added to the top of the plies of open-celled foam to soften the fall of the payloads. In hindsight, thicker plies of soft foam seemed to work best to arrest the fall of payloads. To finish the configuration, a heavy mil tarp was laid on the ground prior to insertion of the foam plies. Another heavy mil tarp was then draped over top and tucked on the sides to allow for movement during payload impact. Other tarp-enveloped plies were tucked on the sides of the primary pad to fill in the space in case of a 
payload tip-over. A full photograph of the final deceleration system configuration is presented in Figure 6-14.

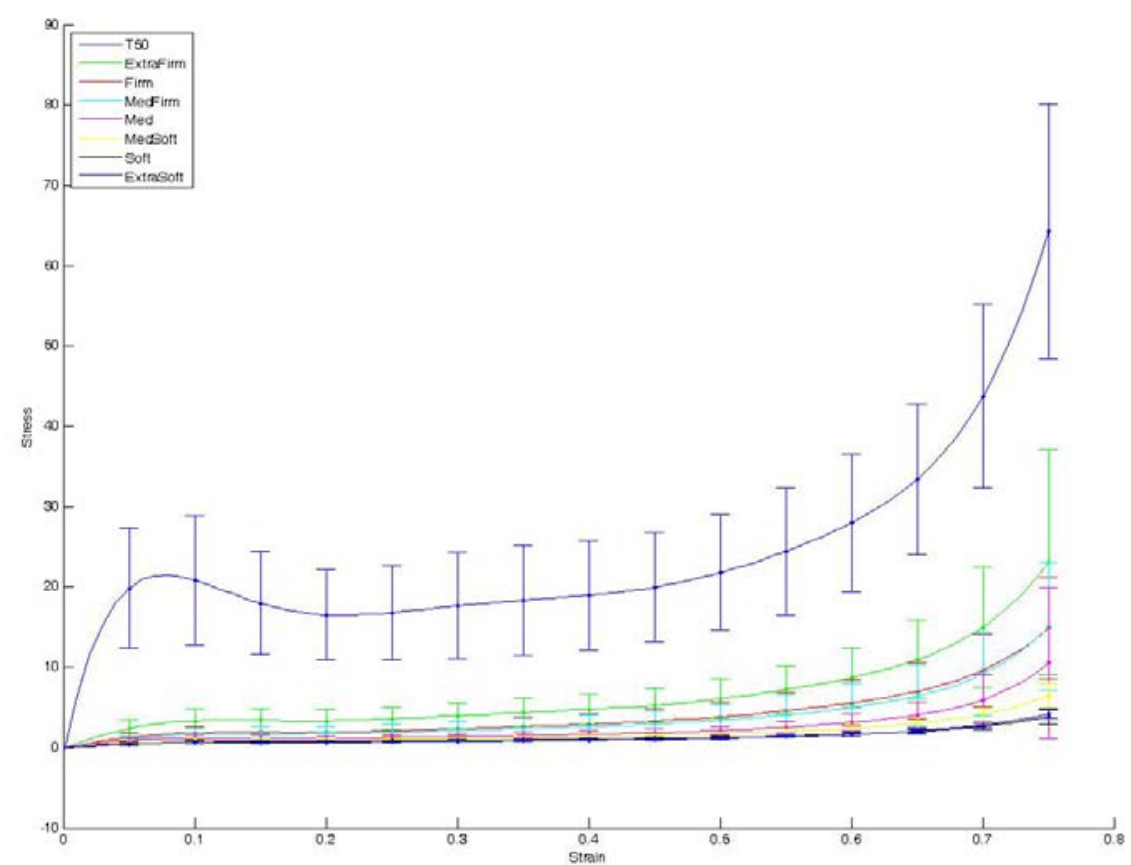

Figure 6-13: Compiled Stress/Strain Curves for Various Open-Celled Foam Types

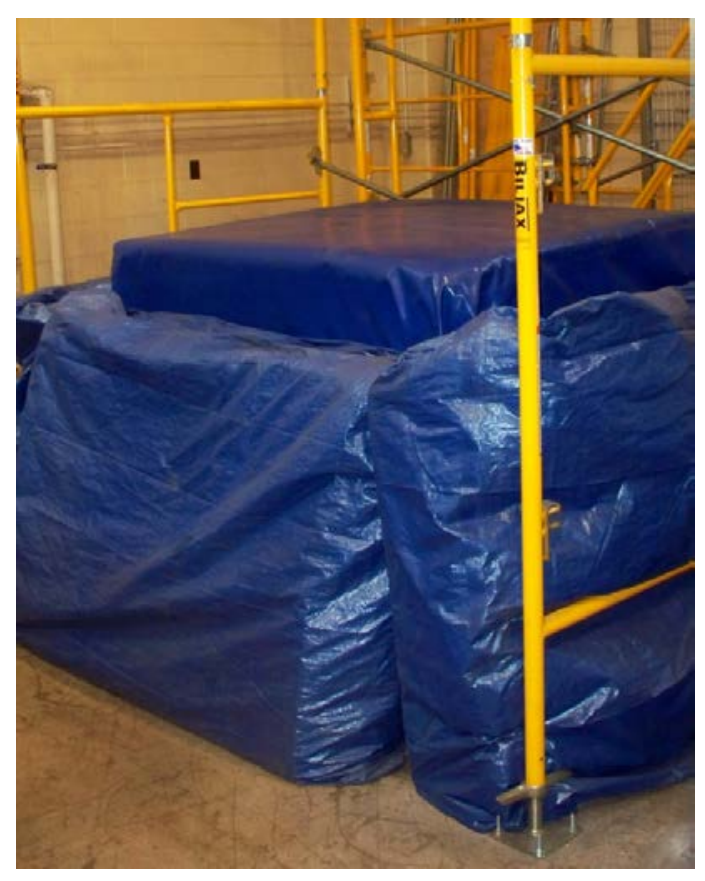

Figure 6-14: Deceleration System 


\subsubsection{Lift/Retrieval System Design}

Given the fact that the developer decided to create a system with separate release and lifting systems, therefore leaving the geometric center of the drop tower unavailable for a singular line lifting path, a unique solution had to be developed. In conjunction with Columbia Winch \& Hoist, a solution was developed such that a singular motor could drive a singular split drum, allowing two cables to be spooled onto a single drum, thereby ensuring an equal rate of lifting on two points connected to the payload frame. Furthermore, lift lines were intentionally left much longer than necessary, in order to allow the lift lines to easily reach down to the floor with plenty of slack to account for payload integration into the facility. Technical data on the selected hoist, the design of the hoist frame, and angles of the sheaves, may be found in Appendix $\mathrm{H}$, the final installation of which is shown in Figure 6-15.

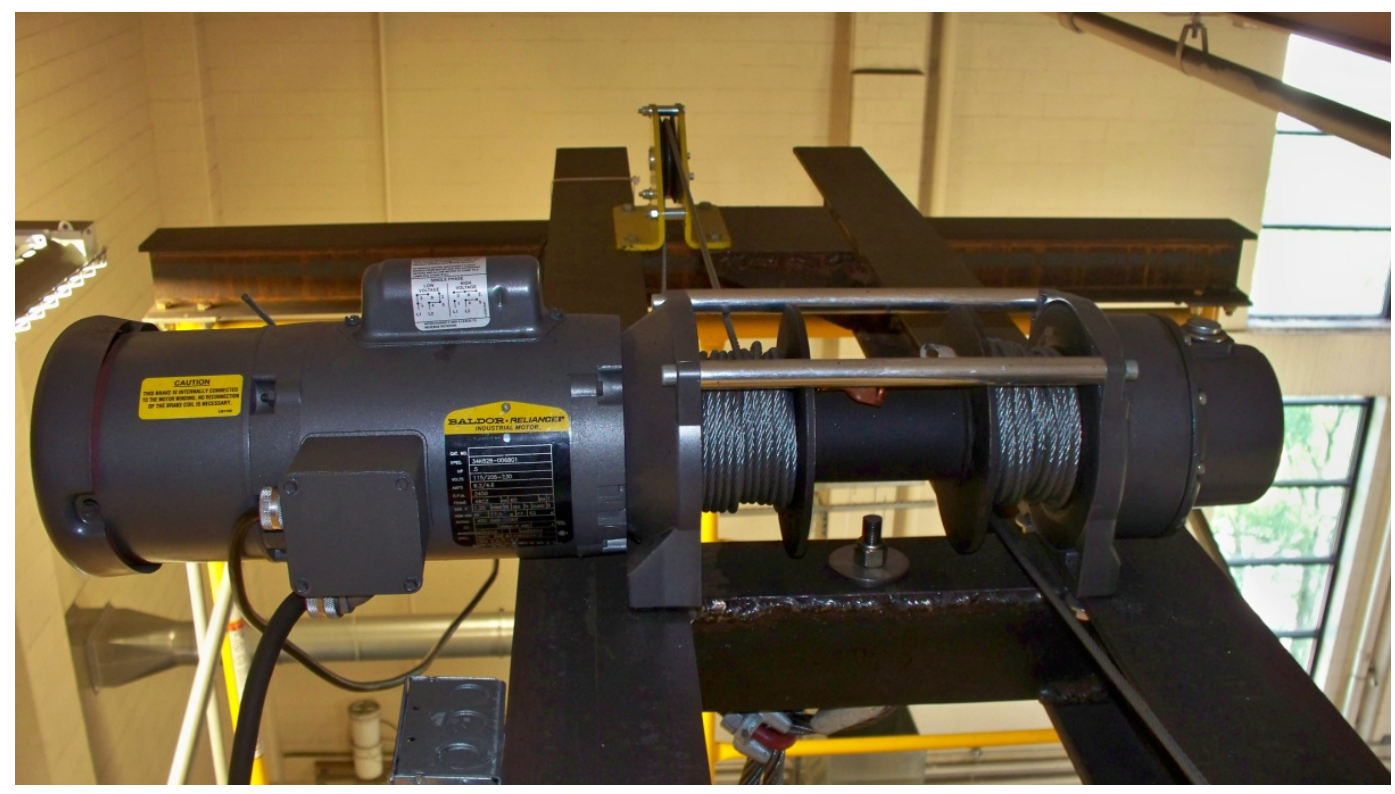

Figure 6-15: Lifting System

(featuring custom structure, dual drum hoist with under/over wrapping lift lines, custom-designed sheave spacing, and release system attachment point under center of hoist) 


\subsubsection{Facility Design}

The design of the WVU SMiRF took into account its operations from the onset of the project, as alluded to in the Requirements and Goals, for both short-term (e.g. Goals \#2 \& \#3) and long-term operations (e.g. Requirements \#3 \& \#4). However, safety at the facility is the most paramount of the Requirements and Goals and should continue to provide the foundation for all operations. For that purpose, the developer has used common sense and precautionary measures to prove the safety of the facility thus far, and common sense safety should continue to dictate all operations. Steel-toed footwear is suggested for all on-site operations. Ear protection should be used when any use of the hoist is possible. Appropriate eyewear should be employed at all times for good measure, whether experimentation is being completed or not. Thick leather gloves should be used for facility operations. Nitrile or latex gloves should be used around experiments. Masks and other appropriate clothing (e.g. long sleeves, aprons, etc.) should be used around experimentation when necessary. A hard hat may be necessary for certain ongoing maintenance activities (e.g. those activities near the ceiling or in tight spaces). Above all, fall protection should be worn by all facility operators any time operators climb above ground level. New operators should undergo fall protection training, how to appropriately employ all personal protective equipment (PPE), and for any and all safety precautions, prior to operator training for the facility. Whenever personnel are not on-site, the facility should be secured using a key-locked cage that surrounds the base of the facility. Furthermore, to charge the electromagnet, the operator must have key-access for power.

Once all safety concerns have been addressed, and operators have been properly trained on PPE and facility operations, the facility also comes equipped with several other amenities. To address ongoing maintenance of the facility, the WVU SMiRF was designed with common hardware, available at most local hardware stores. Additionally, spare parts of larger components that may need replaced or maintained, are currently stored, and should remain stored, at the facility. The facility also provides the user with readable diagnostics that include an activation light to indicate power to the electromagnet, ambient pressure and temperature at both the top and bottom of the tower, and time readouts according to the U.S Naval Observatory Master Clock, currently the most advanced atomic 
clock system, incorporating hydrogen masers and mercury ion frequency standards.

Lastly, prior to any drop testing, all users must properly ensure that their payload is balanced. For this purpose, the developer has provided a balancing boom that hangs off the tower structure at the base of the drop tower. From this boom hangs a cable outfitted with a bolted end connection, which affixes through the same hole used to attach the payload's electromagnet interface. As a bonus, the bolt used to interface with the payload is identical to the one used to interface the payload frame with the electromagnet interface, thus ensuring a proper interface while also balancing the payload. Once attached to the payload via a bolted connection, the conveyance used to transport the payload to the facility may be slowly removed from underneath the payload, being careful to arrest any swinging motion and stored aside, leaving the payload to hang freely. Two bubble levels are also provided to measure the payload's levelness in two horizontally orthogonal directions. The levels should be moved as far away from the bolted connection as possible, without influencing the results of the balancing. The payload itself may be moved around the interior of the supplied payload frame, so long as it can be interfaced with the payload frame, without direct damage to the master payload frame. As previously described, a small interface table may also affix within the payload frame to provide an interface that may be modified at will, whose surface may be replaced appropriately. If users would rather not move their payloads within the provided frame, or if they cannot get the payload to balance within the frame, counterweights are also provided to the user, to be affixed to a Velcro grid on the bottom interior of the payload frame. If the user decides to use their own frame, balance must still be ensured. Once balancing has been completed, the conveyance may again support the weight of the payload, and the balancing hardware removed and stored. Checkouts on the functioning of the experiment and general facility inspections should occur prior to facility operations. Experiments may be stored, integrated within the WVU SMiRF over the allotted period of research. A full-length photo of the WVU SMiRF with Phillips operating the facility is presented in Figure 6-16, while a pre-drop configuration shown in Figure 6-17 presents an internal perspective of the WVU SMiRF. 


\subsection{Facility Operations}

While the WVU SMiRF was designed such that a single operator can operate the facility, requiring minimal overhead for operations, the procedures developed for the facility should be followed by any and all operators at the facility at all times, while the current procedures may be potentially improved upon and documented over time, with safety at the forefront. Although not necessary, it is further suggested that more than one person be present at the facility during maintenance and operations. The detailed operational procedures are provided in flowcharts located in Appendix I.

During development of the facility, several unforeseen abnormalities occurred during operations. Within the first 10 drop attempts, the researcher noticed the payload slipping laterally once interfaced with the electromagnet, leading to unstable landings. Stability tabs were placed on the payload's electromagnet interface to prevent that lateral slipping. However, the researcher also discovered that if the payloads are not stabilized from even slightly noticeable swinging movements that may occur at the top of the tower, that too may result in unstable landings, which may only be currently prevented through operator training and proper execution. Furthermore, due to a current lack of funds and the hoist manufacturer neglecting to provide in-line turnbuckles, the payloads are currently raised in a tilted orientation due to mismatched line length and an over/under spooling method employed by the hoist. As proven by operations thus far, this presents no safety concerns, but is something of note for operations. 


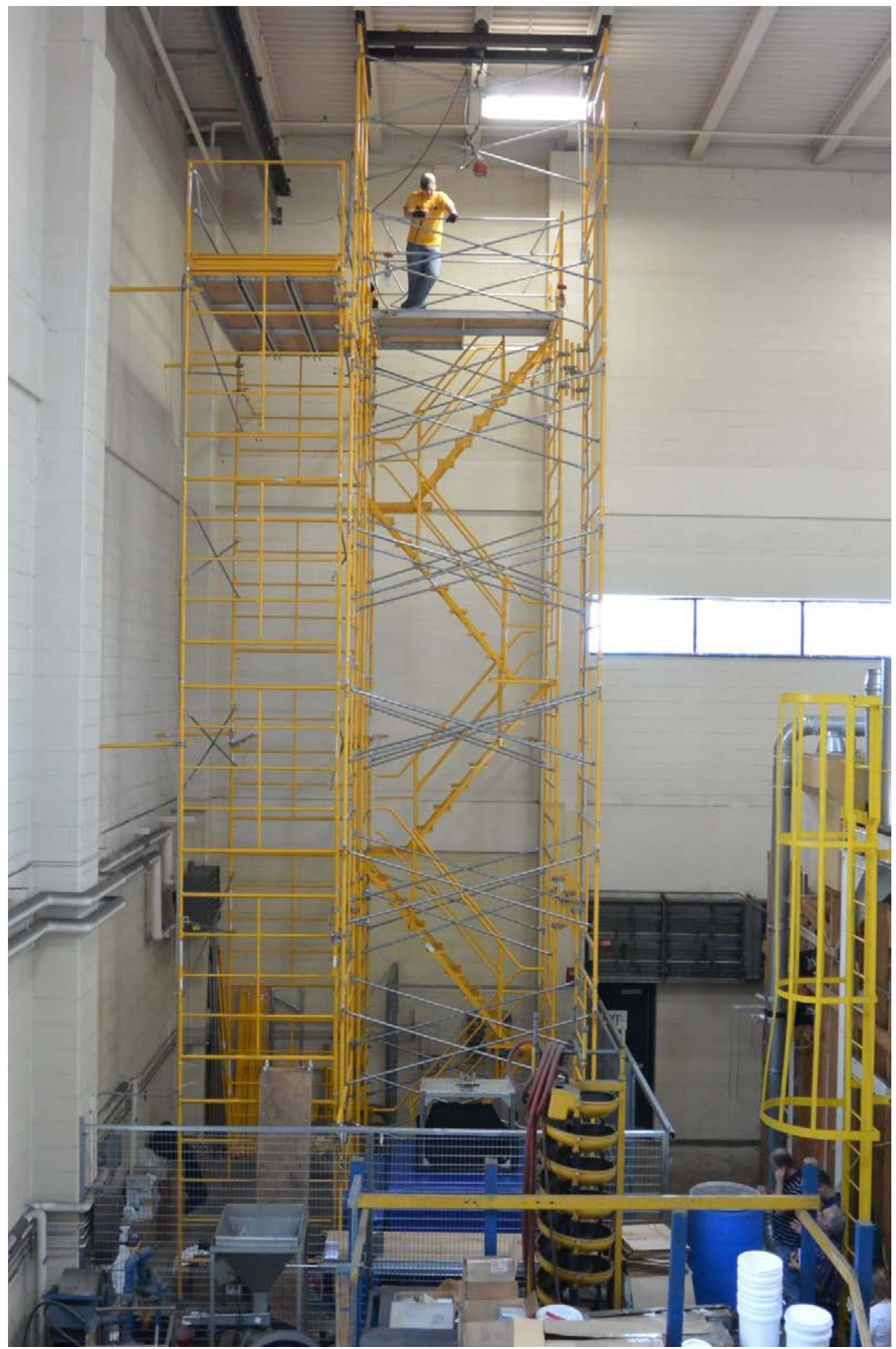

Figure 6-16: Full-Length Photo of the WVU SMiRF 
Development of the West Virginia University Small Microgravity Research Facility (WVU SMiRF)
Copyright $^{\circledR} 2014$

Kyle G. Phillips

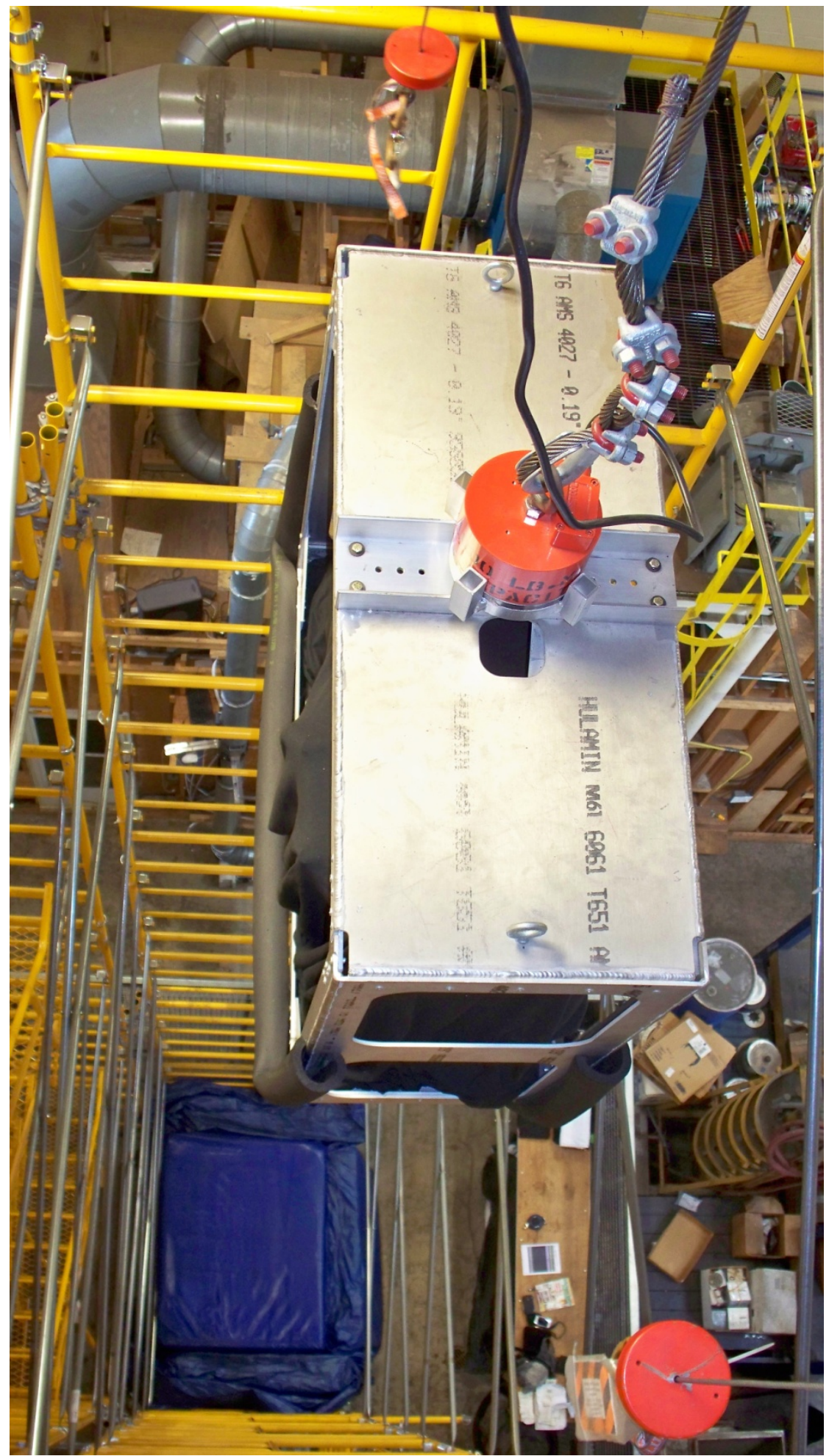

Figure 6-17: Pre-Drop Payload Configuration 


\subsubsection{Data Analysis Development}

Once a drop test has been completed and the electronic data successfully transferred from the payload, the data is ready to be analyzed. In keeping with Goal \#9, for the purpose of data analysis, the researcher also developed a semi-automated process using commonly available software. Using freely downloadable software (from Vernier or proprietarily-provided software when using the $\mathrm{NI}$ data acquisition system), the software may be converted from a proprietary file format into a comma separated value (i.e. .csv) format. Once a .csv file has been generated, it may then be imported into spreadsheet software, such as Microsoft Excel or even other equivalent free solutions, such as the currently available OpenOffice Calc or Google Sheets.

Within a spreadsheet application, the designer has developed a semiautomated data analysis software. The software was developed in MS Excel, but is also available in other spreadsheet formats. Within the analysis spreadsheet workbook, resides 13 spreadsheets, represented as tabs at the bottom of the window:

- Import

- Raw Data

- Raw Data Plot

- Lift \& Prep Data

- Lift \& Prep Plot

- Low-g Data

- Low-g Plot

- Impact Data

- Impact Plot

- Rebound Data

- Rebound Plot

- Recover Data

- Recover Plot

The latter 10 sheets represent the 5 phases of a drop:

- Lift \& Preparation Phase

o The phase of lifting the payload from rest on the deceleration system up to the top of the tower. There, 
the payload is connected to the electromagnet and final preparation of the payload takes place, until the payload is released. With more advanced electronics, that enable faster, more frequent readings, this phase may be broken down into 3 sub-phases:

- Lift phase

- To study the effects and vibrations a more sensitive payload may undergo to transport the system to the top of the tower. While it is not recommended for heavy payloads, smaller, lighter payloads with more sensitive equipment may be carefully carried to the top of the tower, after thorough review and approval.

- Preparation phase

- To study the effects of handling and transferring the load onto the electromagnet. Data may also be taken here to study the effect that the electromagnet has on equipment and payloads. This also represents a potential opportunity for a researcher to log some data, prior to drop, in a 1-g environment, using the exact same experimental configuration as that in the drop phase, except for the additional EMI field that may be present due to the electromagnet, and the potential destabilized motions, thereby providing the best opportunity for comparison between 1-g and reduced-g data.

- Release phase

- The release phase could be studied for vibrational loads and to determine the cleanliness of the drop. If it is found that 
some drops produce better results based on the cleanliness of the release, perhaps future modifications could include coating the outside of the magnet and inside of the prongs of the crown with a thin film of Teflon or low-resistance equivalents.

- Low-g Phase

o The low-g phase represents the payload in freefall during a drop, where gravitational effects are reduced to nearly zero-g levels. In reduced gravity research, this phase would represent the phase of interest. The data in this phase represents the quality of the drop and could be used to study aerodynamic effects. Oscillations within instrumentation or experimental conditions may also be observed in this phase.

- Impact Phase

o The impact phase represents the downward motion and deceleration of the payload within the deceleration system. This phase also could represent an area of interest, as an elevated level of gravity could also be of interest to the user. Reduced gravity researchers may also want to study the effect of increased gravity to emphasize what happens in contrast to a 1-g environment. Other users may want to perform impact research. Those users could potentially replace the deceleration system with an equivalent impact surface, so long as they can guarantee no damage to permanent WVU property. This phase had to be characterized to specify structural loads that must be endured by the payload and experimental frames, electronics, and other equipment in compression. The deceleration system also had to be characterized as to its effectiveness, which could open up other areas of research as far as material impacts and resistance to material impacts. 
- Rebound Phase

o The rebound phase represents the upward motion of the payload, once it was fully decelerated, due to the material response of the deceleration system springing back to its original form. This could be a phase of interest as well, as it represents a negative gravitational load on the payload. This phase also had to be characterized to specify structural loads that must be endured by payloads in negative compression transitioning into tension at the peaks of rebound oscillations. This phase also may be further broken down by repeated oscillations, oscillation count, and the loads encountered as a payload moves through the various diminishing amplitude oscillations.

- Recover Phase

o The recover phase represents the period after the payload has come to what would be considered a stable, steady-state resting period, where it has once again come to experience a 1-g gravitational load. From this phase, the payload may once again be recovered and prepared for further subsequent drops. During this phase, testing could continue to study the effects within a 1-g environment for comparison to pre-drop conditions, depending on the levelness of the payload as it sets on the deceleration system surface.

Based on the observation of the data naturally delineating itself into the aforementioned phases, the data could be filtered into those phases and analyzed. Under the Import tab, the .csv could be imported. The imported data would represent the unfiltered raw data. That data could then be copied, by value only, into the Raw Data sheet in the appropriate columns. In that way, the raw data could remain untouched and serve as a reference point that stays with each analysis for future verification purposes. Furthermore, the Raw Data sheet also has pre-formatted conditional formatting that indicates the various phases for the user, and 
once data has been copied into it from the import sheet, a plot is automatically generated in the following sheet for visual verification.

The conditional formatting serves as a visual filter to aid the user in phase determination. The designer found it prudent for initial analysis and phase-breakdown to allow the determination of the various phases to be made by the human user, rather than an automated filtering process. Initially, this was to the user's benefit, as the designer came to gain experience with phase determination. Additionally, if a singular filter was produced for all users, the resolution of different data acquisition systems may lead to different levels of filtering. Based on extensive experience during calibration and initial checkout results, which will be presented later in this thesis, the designer arbitrarily determined the g-levels within which filters could be applied for conditional formatting, based on the checkout data acquisition system results. Thus, a green cell represents a cell in reduced gravity, between the g-levels of $\pm 0.21 \mathrm{~g}$. A gray cell represents $\mathrm{g}$ levels greater than or less than $1.2 \mathrm{~g}$ to indicate the impact phase. An orange cell represents g-levels of $\pm 0.75 \mathrm{~g}$, indicating all non- $1 \mathrm{~g}$ loads outside of low-g and high-g levels. By assigning priority to each of the conditional formats, with the impacts as the highest priority and low-g as the second highest priority, the orange cells would only indicate g-levels other than non-1-g loads, thereby indicating the instrumentation fluctuations on initial release, initial impact, and rebound phases.

With these indicators, the user was more readily able to identify and separate phases for analysis. First, the user must find the cells indicated in gray to copy and paste the impact period into its pre-defined data section. Next, the user can copy from the cell immediately time-stamped prior to those copied for the impact period, back in time to the point at which the payload was released, as indicated by more frequently consistent green cells. The point at which reduced gravity is considered, based on input about instrumentation fluctuations and cleanliness of release, should be chosen by the user, using best judgment based upon gravity levels attributed to the various phases. All cells prior to those chosen for the low-g phase can be allocated for the lift \& prep phase. Then, the user must specify the cell range for the rebound phase. This phase is also arbitrarily chosen by the user. This range extends from the cells immediately proceeding those chosen for the impact phase until 
oscillations have settled into some average post-impact value near $1 \mathrm{~g}$. It should be noted that the consistent value might not be exactly $1 \mathrm{~g}$, as sensors have experienced an impact and may have settled offset from its calibrated position. Hence, sensors should be calibrated prior to every drop. However, once it has been decided that the oscillations have been arrested, and values are stable, a cell should be chosen to represent the end of the rebound phase. All data after that cell are then allocated for the recover phase, thus completing the data separation into phases.

Once the values have been separated into their pre-allocated areas on the raw data sheet, the values for each phase may be copied into individual sheets with pre-allocated areas for the appropriately copied data. Each individual phase sheet then automatically statistically analyzes each phase to produce the following outputs:

- Mean

- \pm 3 standard deviations and ranges

0 Indicating that $68.27 \%$ of values lie within the $1^{\text {st }}$ standard deviation range, $95.45 \%$ of values lie within the $2^{\text {nd }}$ standard deviation range, and that $99.73 \%$ of values lie within the $3^{\text {rd }}$ standard deviation range

- Median

- Minimum

- Maximum

- $1^{\text {st }}$ and $3^{\text {rd }}$ quartiles

- Upper and lower outliers

o Indicating those values that may be filtered out to give a more accurate analysis

- Frequency and probability tables

o Defines ranges that are used in histograms and probability curves, which may employ increased resolution as desired

- Histogram and probability curves

o Indicating counts at various levels and the probability that a value may fall within a certain range

Once these analyses are completed for each individual drop, they may be compiled into a separate, singular workbook to statistically analyze the 
drop data collectively. Additionally, drop times may also be statistically analyzed. Thus, as a more comprehensive dataset evolves, precisions are increased, and comparisons are more accurate as additional drops are performed. An example of this analysis, as performed for initial checkout operations at WVU SMiRF can be found in Appendix J.

\subsubsection{Calibration Results}

Initially, the preparation flooring outfitted with plies of memory foam was raised up to the levels immediately adjacent to the top of the tower, using the scaffolding system, in order to test out the payload frame release and impact. Then, the previously described procedure was performed for 5 calibration drops at increasingly heavy payload weights, ranging from 25 lbs to $125 \mathrm{lbs}$ in $25 \mathrm{lb}$ increments, in addition to the $60.2 \mathrm{lb}$. weight of the payload frame. It should be noted that the payload weights presented represent the weight of the payload carried within the payload frame, excluding the weight of the payload frame itself. When performing a prediction analysis of impact loads and the like, one should be sure to include the additional weight of the payload frame. Each of those drops was individually analyzed, and then comparatively and comprehensively analyzed to determine any prevailing trends. The following presents zaxis acceleration statistical analysis summary tables for each calibration drop in order of increasing weight. Full calibration data sets and graphs may be found in Appendix $\mathrm{K}$, while a sample of a calibration data set is shown in Table 6-1 and Figure 6-18 through Figure 6-24 for a $25 \mathrm{lb}$ payload, while Table 6-2 through Table 6-5 represent the remainder of the calibration data summaries. The drop distance, as measured from the bottom of the payload frame to the top of the deceleration pad, is approximately 30 feet. All values are presented in units of g's unless stated otherwise.

The raw calibration data had to be corrected in accordance with the procedure described in Appendix I in order to compensate for set up error. The corrected data represents the proper orientation of the sensor, using global data manipulation, consistent across all data points, without manipulation of individual data points or ranges of data. The instructions provided with the checkout data acquisition system were not appropriate for a reduced gravity environment and would produce data and the 
associated graph of Figure 6-18 as though $1 \mathrm{~g}$ was reduced gravity. Eventually a pre-drop instrumentation calibration technique was developed in order to collect true raw data, by simply orienting the z-axis down and calibrating at $1 \mathrm{~g}$, while the payload was at rest, while up was considered $-1 \mathrm{~g}$, thereby setting the orientation or the sensor correctly.

Table 6-1: 25 lb. Calibration Statistical Data Summary

\begin{tabular}{|l|l|l|l|l|l|}
\hline & $\begin{array}{l}\text { Lift \& } \\
\text { Prep } \\
\text { Phase }\end{array}$ & $\begin{array}{l}\text { Low-g } \\
\text { Phase }\end{array}$ & $\begin{array}{l}\text { Impact } \\
\text { Phase }\end{array}$ & $\begin{array}{l}\text { Rebound } \\
\text { Phase }\end{array}$ & $\begin{array}{l}\text { Recover } \\
\text { Phase }\end{array}$ \\
\hline -3 SD & 0.78 & -0.22 & -21.28 & 0.38 & 0.92 \\
\hline -2 SD & 0.84 & -0.12 & -11.38 & 0.57 & 0.94 \\
\hline -1 SD & 0.91 & -0.02 & -1.47 & 0.76 & 0.96 \\
\hline Mean & 0.97 & 0.08 & 8.44 & 0.94 & 0.99 \\
\hline +1 SD & 1.04 & 0.19 & 18.34 & 1.13 & 1.01 \\
\hline +2 SD & 1.10 & 0.29 & 28.25 & 1.31 & 1.03 \\
\hline +3 SD & 1.17 & 0.39 & 38.15 & 1.50 & 1.05 \\
\hline Minimum & 0.32 & 0.00 & 1.43 & -0.16 & 0.93 \\
\hline Median & 0.98 & 0.06 & 8.44 & 0.98 & 0.98 \\
\hline Maximum & 1.61 & 0.53 & 15.44 & 1.16 & 1.05 \\
\hline
\end{tabular}

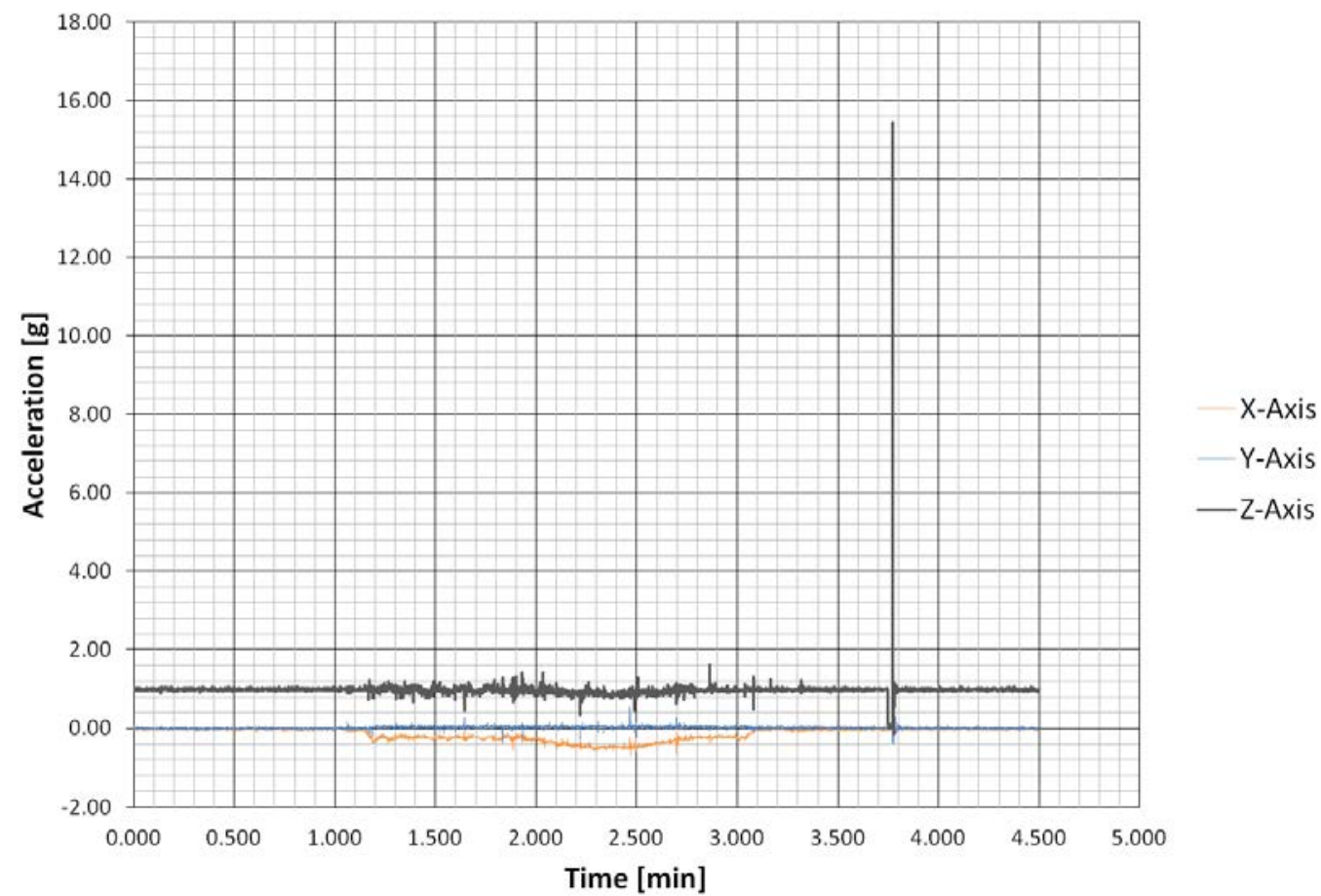

Figure 6-18: 25 lb. Calibration Drop Raw Data (Corrected) 
Development of the West Virginia University Small Microgravity Research Facility (WVU SMiRF)
Copyright $^{\odot} 2014$

Kyle G. Phillips

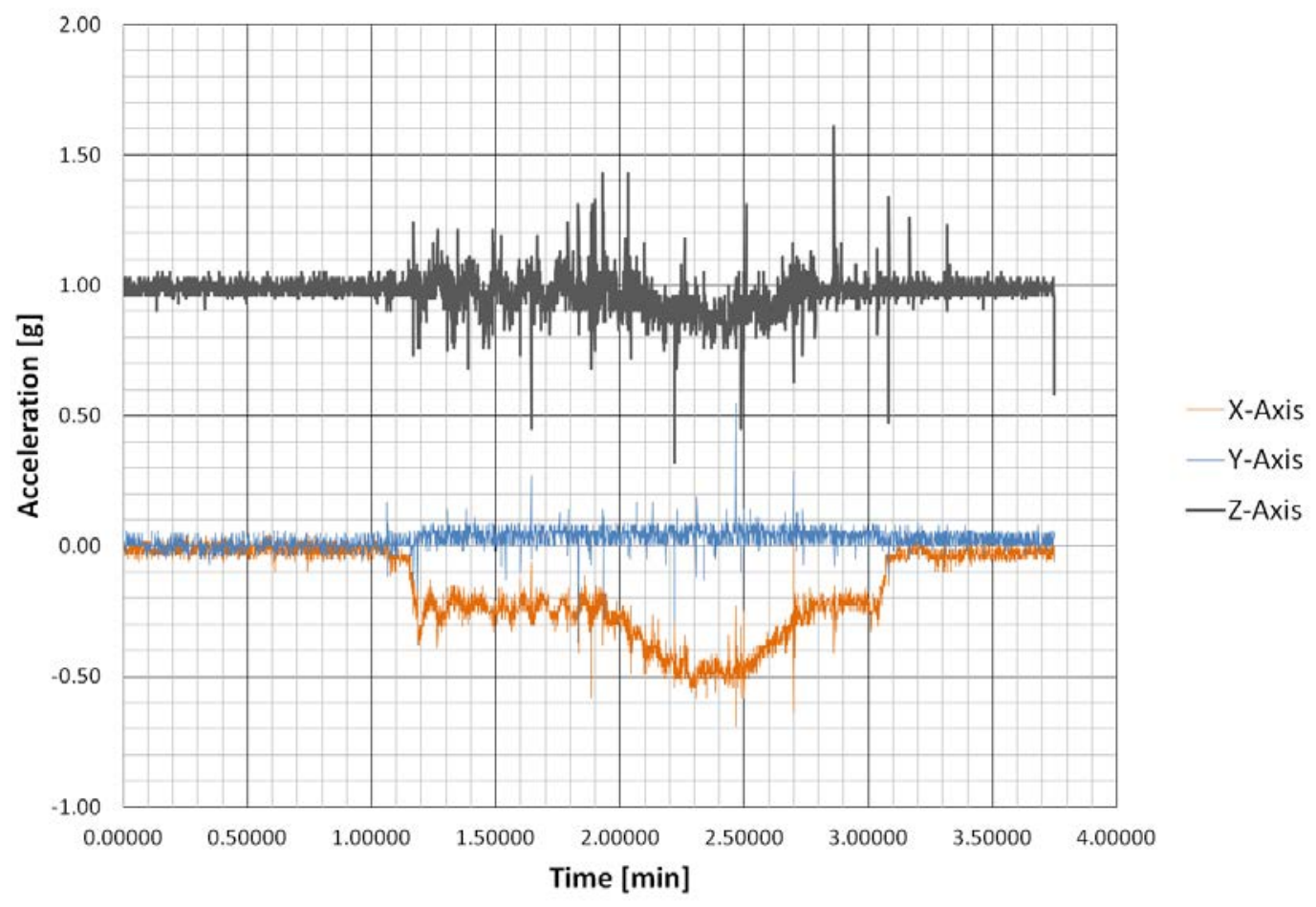

Figure 6-19: 25 lb. Calibration Drop Lift \& Prep Data

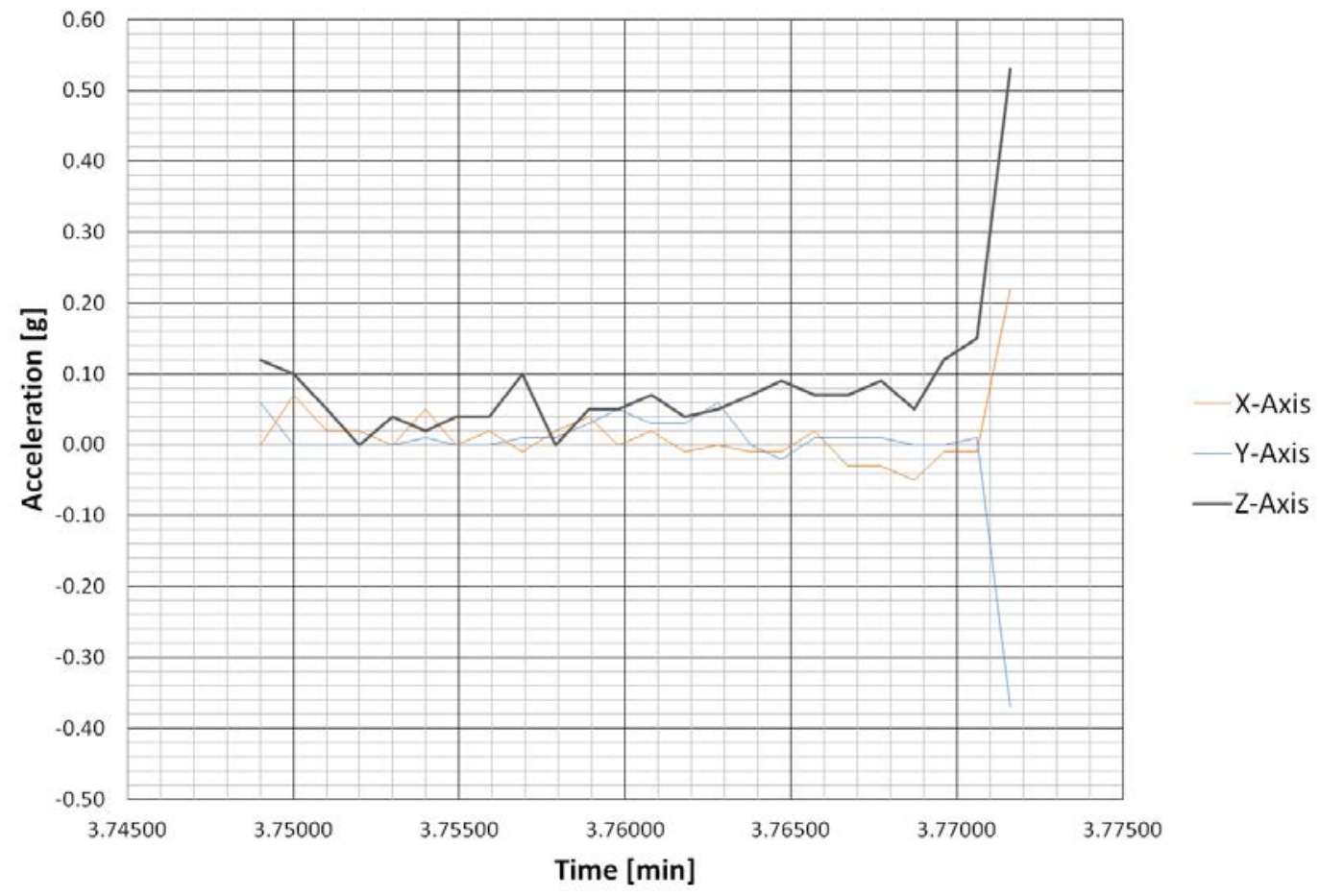

Figure 6-20: 25 lb. Calibration Drop Low-g Data 


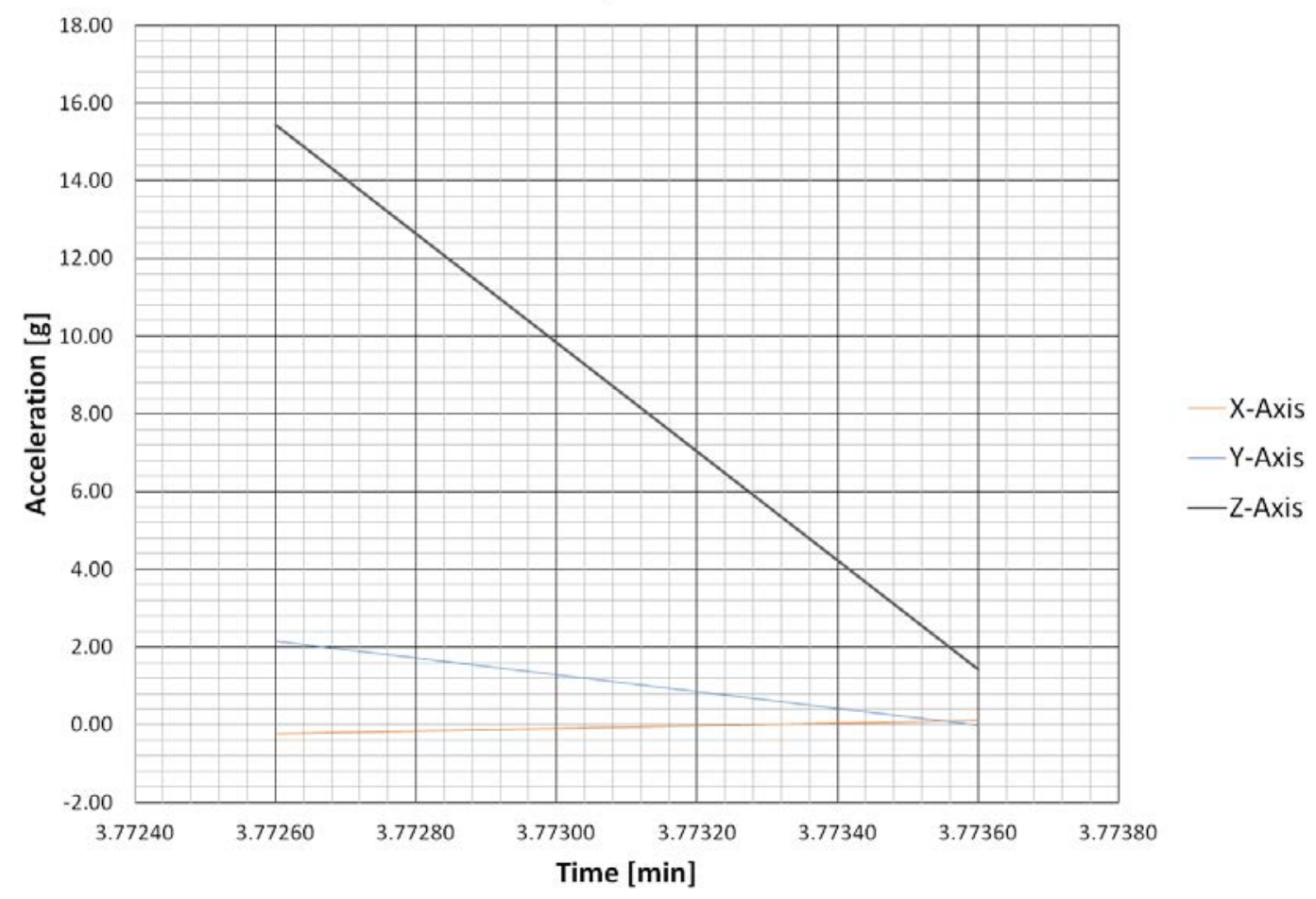

Figure 6-21: $25 \mathrm{lb}$. Calibration Impact Data

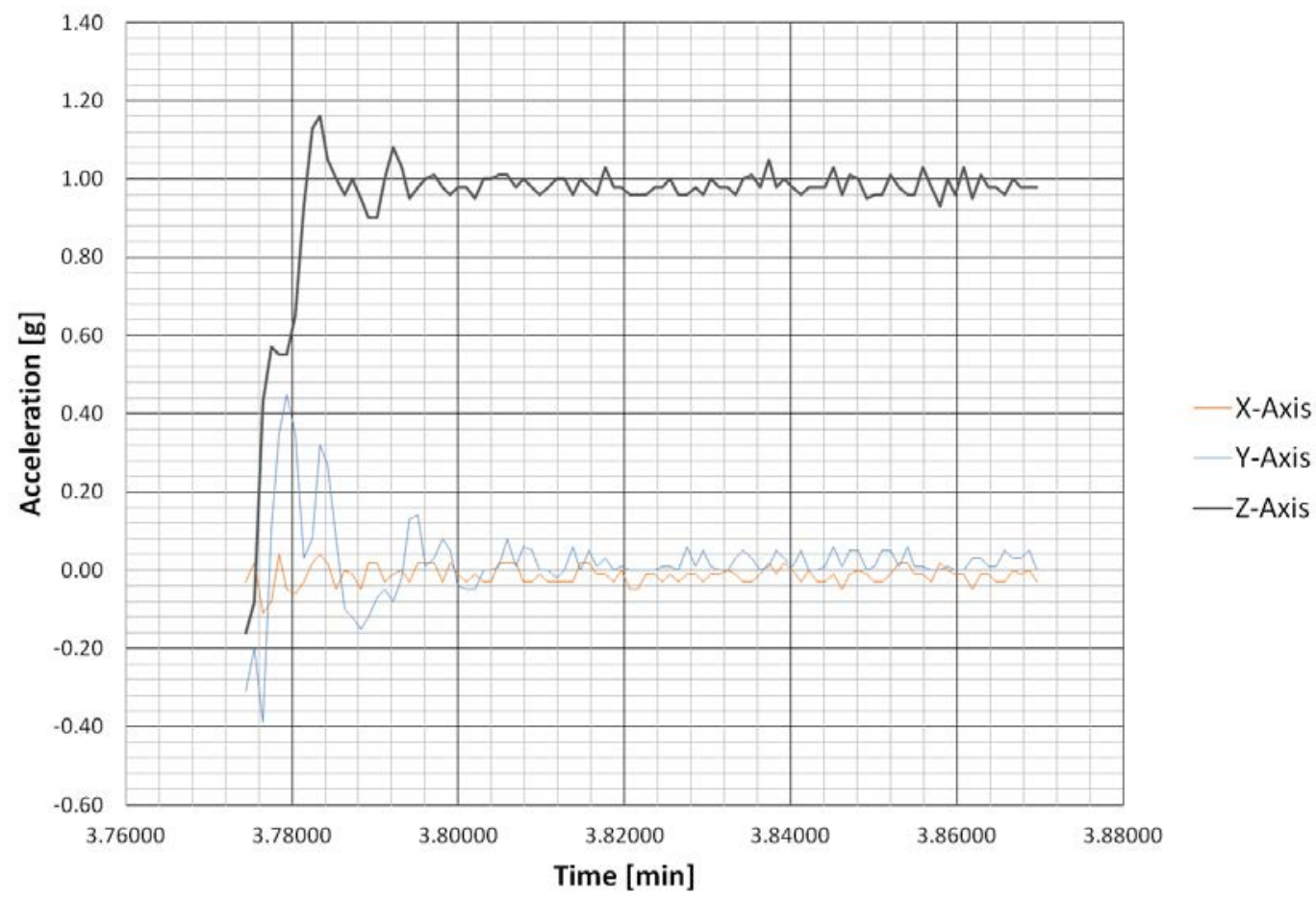

Figure 6-22: $25 \mathrm{lb}$. Calibration Drop Rebound Data 
Development of the West Virginia University Small Microgravity Research Facility (WVU SMiRF)

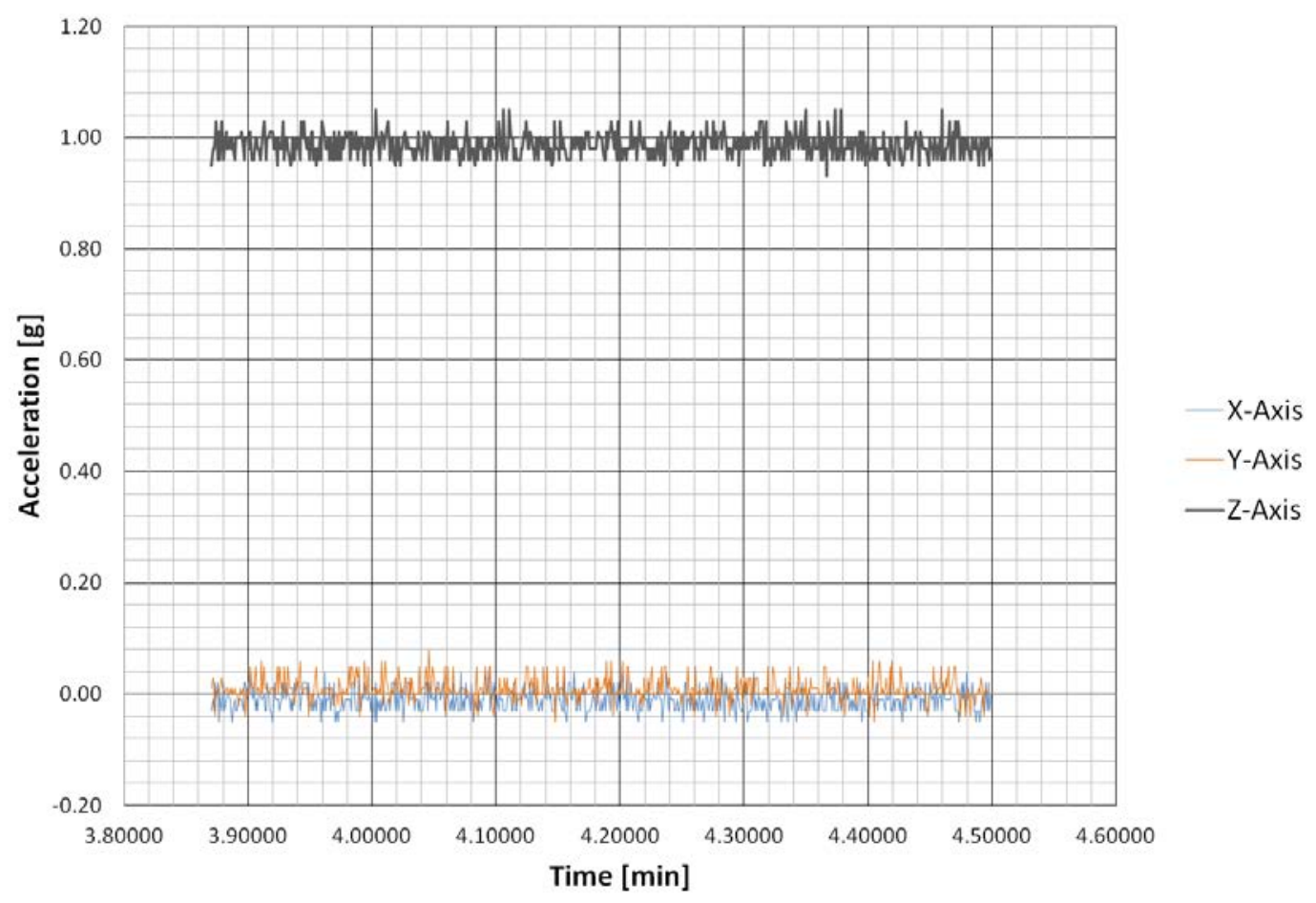

Figure 6-23: 25 Ib. Calibration Drop Recover Data

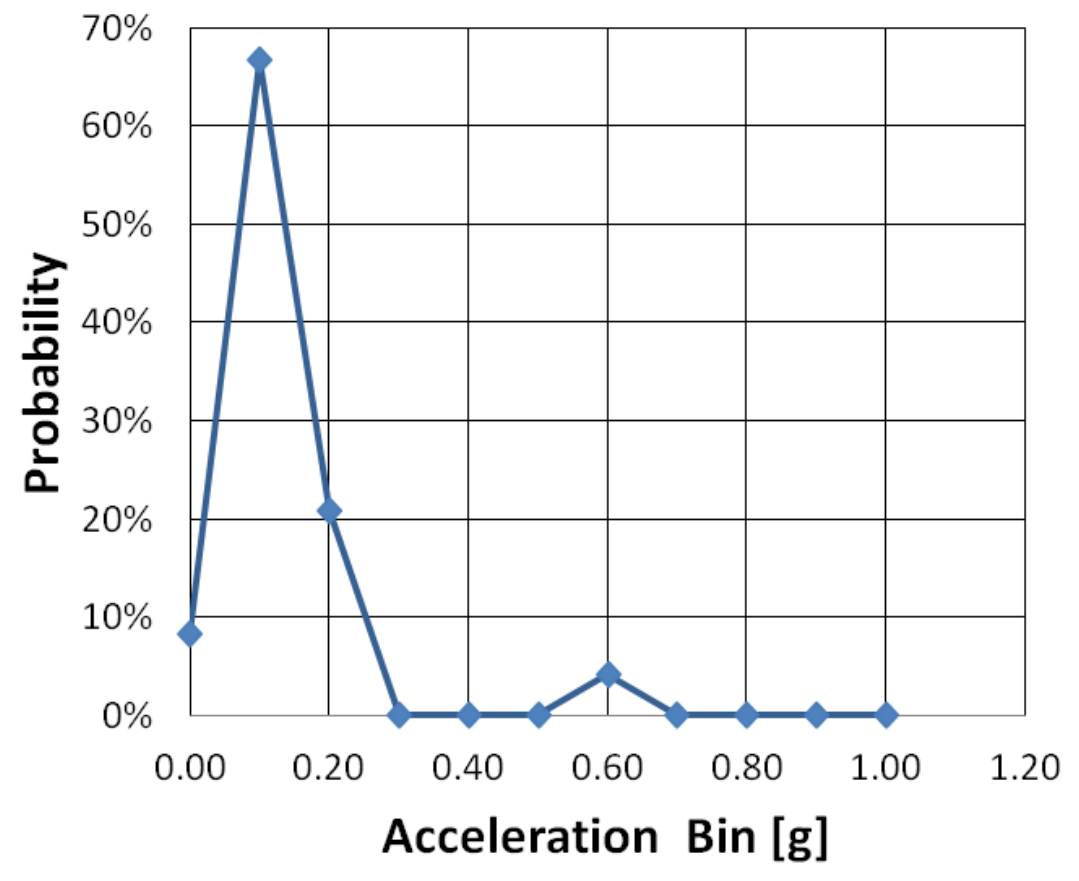

Figure 6-24: 25 lb. Calibration Drop Low-g Probability Curve 
Table 6-2: 50 lb. Calibration Statistical Data Summary

\begin{tabular}{|l|l|l|l|l|l|}
\hline & $\begin{array}{l}\text { Lift \& } \\
\text { Prep } \\
\text { Phase }\end{array}$ & $\begin{array}{l}\text { Low-g } \\
\text { Phase }\end{array}$ & $\begin{array}{l}\text { Impact } \\
\text { Phase }\end{array}$ & $\begin{array}{l}\text { Rebound } \\
\text { Phase }\end{array}$ & $\begin{array}{l}\text { Recover } \\
\text { Phase }\end{array}$ \\
\hline -3 SD & 0.78 & -0.03 & -36.30 & -0.12 & 0.94 \\
\hline -2 SD & 0.85 & 0.00 & -22.87 & 0.21 & 0.96 \\
\hline -1 SD & 0.93 & 0.03 & -9.43 & 0.53 & 0.98 \\
\hline Mean & 1.01 & 0.06 & 4.00 & 0.86 & 1.00 \\
\hline +1 SD & 1.08 & 0.09 & 17.43 & 1.19 & 1.02 \\
\hline +2 SD & 1.16 & 0.13 & 30.87 & 1.51 & 1.04 \\
\hline +3 SD & 1.23 & 0.16 & 44.30 & 1.84 & 1.06 \\
\hline Minimum & -0.29 & -0.01 & -8.51 & -0.40 & 0.94 \\
\hline Median & 1.00 & 0.07 & 2.31 & 0.99 & 1.00 \\
\hline Maximum & 2.54 & 0.13 & 18.20 & 1.14 & 1.07 \\
\hline
\end{tabular}

Table 6-3: 75 Ib. Calibration Statistical Data Summary

\begin{tabular}{|l|l|l|l|l|l|}
\hline & $\begin{array}{l}\text { Lift \& } \\
\text { Prep } \\
\text { Phase }\end{array}$ & $\begin{array}{l}\text { Low-g } \\
\text { Phase }\end{array}$ & $\begin{array}{l}\text { Impact } \\
\text { Phase }\end{array}$ & $\begin{array}{l}\text { Rebound } \\
\text { Phase }\end{array}$ & $\begin{array}{l}\text { Recover } \\
\text { Phase }\end{array}$ \\
\hline -3 SD & 0.71 & -0.04 & 10.57 & 0.33 & 0.95 \\
\hline -2 SD & 0.81 & 0.00 & 15.52 & 0.52 & 0.97 \\
\hline -1 SD & 0.90 & 0.03 & 20.47 & 0.72 & 0.99 \\
\hline Mean & 1.00 & 0.06 & 25.42 & 0.92 & 1.01 \\
\hline +1 SD & 1.10 & 0.09 & 30.37 & 1.12 & 1.03 \\
\hline +2 SD & 1.20 & 0.12 & 35.32 & 1.32 & 1.05 \\
\hline +3 SD & 1.30 & 0.16 & 40.27 & 1.51 & 1.07 \\
\hline Minimum & -1.19 & -0.03 & 21.92 & 0.21 & 0.98 \\
\hline Median & 1.01 & 0.08 & 25.42 & 0.99 & 1.01 \\
\hline Maximum & 3.26 & 0.12 & 28.92 & 1.19 & 1.07 \\
\hline
\end{tabular}


Table 6-4: $100 \mathrm{lb}$. Calibration Statistical Data Summary

\begin{tabular}{|l|l|l|l|l|l|}
\hline & $\begin{array}{l}\text { Lift \& } \\
\text { Prep } \\
\text { Phase }\end{array}$ & $\begin{array}{l}\text { Low-g } \\
\text { Phase }\end{array}$ & $\begin{array}{l}\text { Impact } \\
\text { Phase }\end{array}$ & $\begin{array}{l}\text { Rebound } \\
\text { Phase }\end{array}$ & $\begin{array}{l}\text { Recover } \\
\text { Phase }\end{array}$ \\
\hline -3 SD & 0.80 & -0.11 & 1.72 & 0.40 & 0.94 \\
\hline -2 SD & 0.86 & -0.06 & 4.67 & 0.58 & 0.96 \\
\hline -1 SD & 0.93 & -0.01 & 7.62 & 0.76 & 0.98 \\
\hline Mean & 0.99 & 0.05 & 10.57 & 0.94 & 1.00 \\
\hline +1 SD & 1.06 & 0.10 & 13.51 & 1.12 & 1.02 \\
\hline +2 SD & 1.12 & 0.15 & 16.46 & 1.30 & 1.04 \\
\hline +3 SD & 1.18 & 0.20 & 19.41 & 1.48 & 1.06 \\
\hline Minimum & 0.22 & -0.11 & 8.48 & -0.04 & 0.94 \\
\hline Median & 0.98 & 0.06 & 10.57 & 0.98 & 1.00 \\
\hline Maximum & 1.41 & 0.16 & 12.65 & 1.23 & 1.07 \\
\hline
\end{tabular}

Table 6-5: 125 lb. Calibration Statistical Data Summary

\begin{tabular}{|l|l|l|l|l|l|}
\hline & $\begin{array}{l}\text { Lift \& } \\
\text { Prep } \\
\text { Phase }\end{array}$ & $\begin{array}{l}\text { Low-g } \\
\text { Phase }\end{array}$ & $\begin{array}{l}\text { Impact } \\
\text { Phase }\end{array}$ & $\begin{array}{l}\text { Rebound } \\
\text { Phase }\end{array}$ & $\begin{array}{l}\text { Recover } \\
\text { Phase }\end{array}$ \\
\hline -3 SD & 0.70 & -0.12 & -73.04 & -0.88 & - \\
\hline -2 SD & 0.80 & -0.07 & -47.26 & -0.34 & - \\
\hline -1 SD & 0.90 & -0.02 & -21.49 & 0.20 & - \\
\hline Mean & 1.00 & 0.03 & 4.29 & 0.74 & - \\
\hline +1 SD & 1.10 & 0.08 & 30.06 & 1.28 & - \\
\hline +2 SD & 1.20 & 0.13 & 55.83 & 1.82 & - \\
\hline +3 SD & 1.30 & 0.18 & 81.61 & 2.36 & - \\
\hline Minimum & -2.55 & -0.12 & -13.94 & -0.16 & - \\
\hline Median & 1.00 & 0.03 & 4.29 & 0.77 & - \\
\hline Maximum & 2.26 & 0.13 & 22.51 & 2.04 & - \\
\hline
\end{tabular}

Once all of the calibration data was collected and statistically analyzed at each individual weight, using the same process as was presented for the $25 \mathrm{lb}$. case, those datasets could be combined to gain insight into any trends that could be occurring to characterize the facility further. The plots presented in Figure 6-25 through Figure 6-29 include error bars indicating 
the range of the third standard deviation of each dataset, as indicated in their individual data analyses.

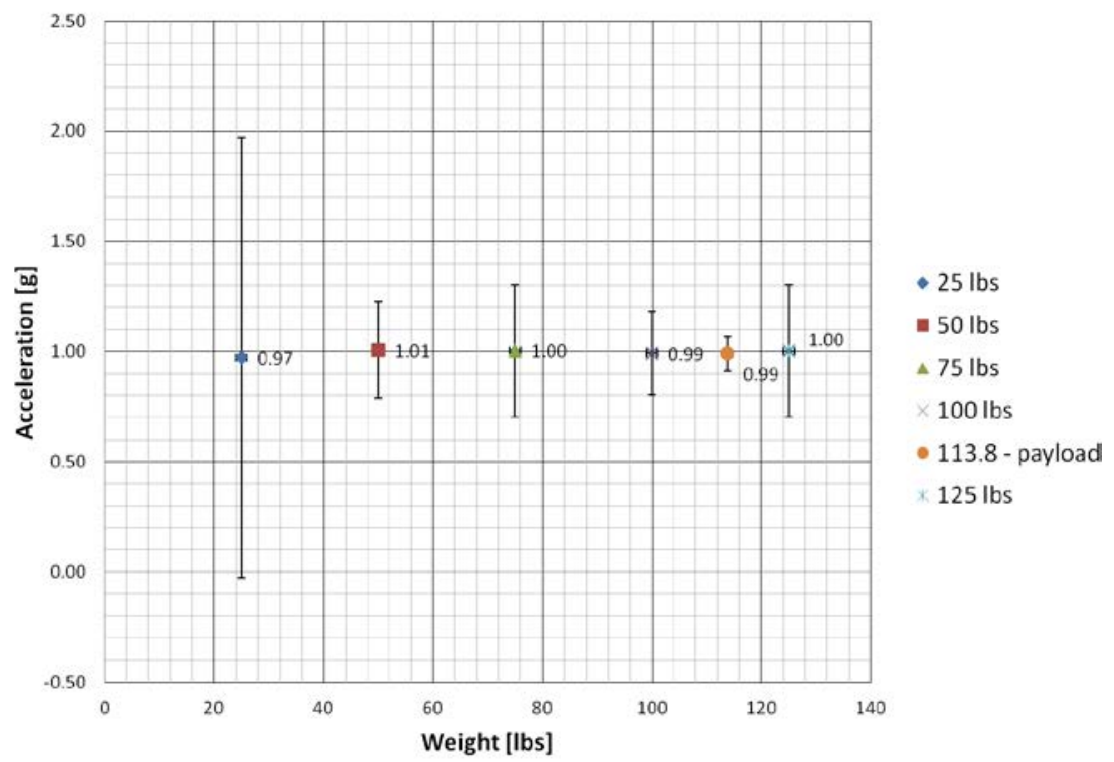

Figure 6-25: Compiled Weight vs. Acceleration Lift \& Prep Data

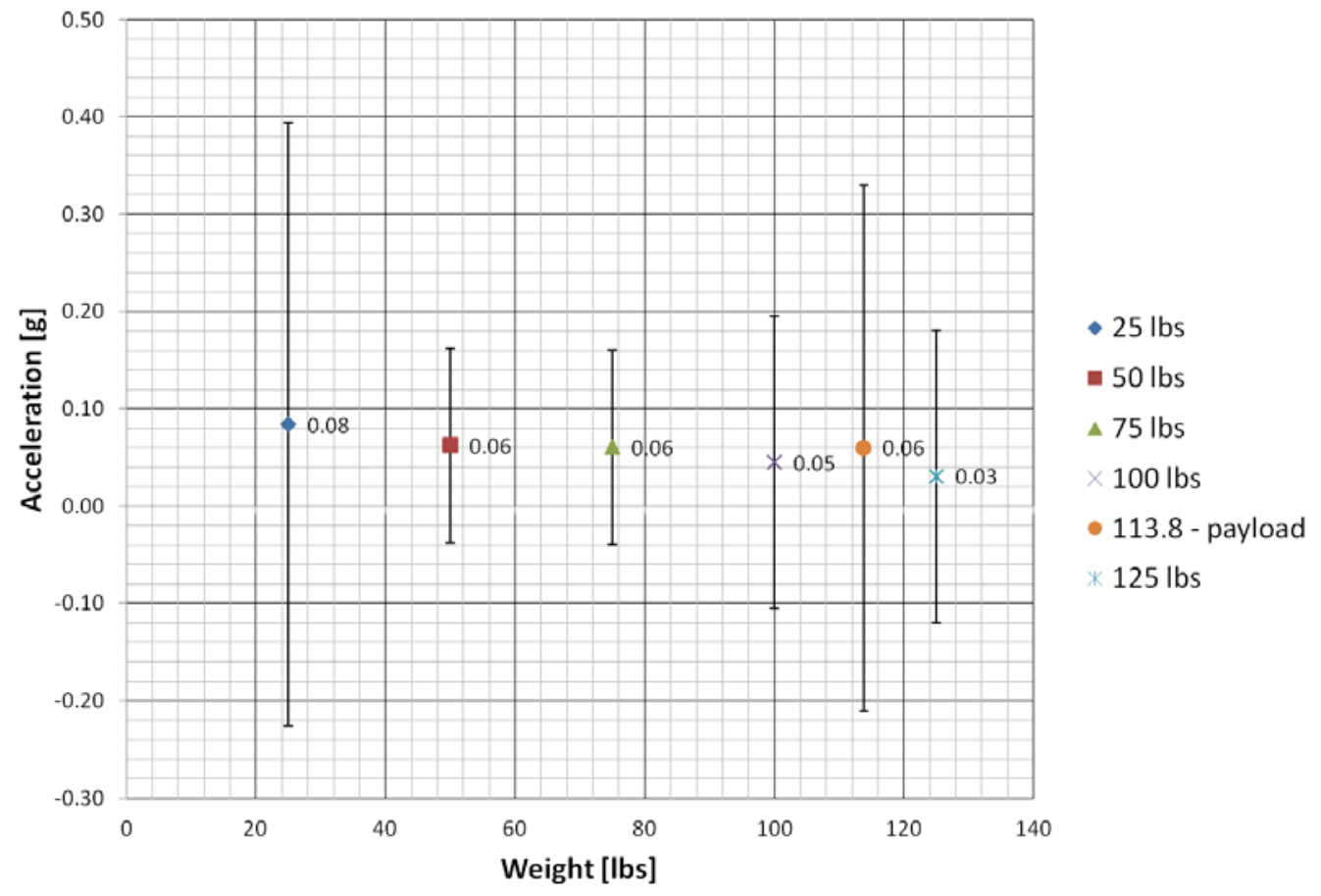

Figure 6-26: Compiled Weight vs. Acceleration Low-g Data 
Development of the West Virginia University Small Microgravity Research Facility (WVU SMiRF)
Copyright $^{\odot} 2014$

Kyle G. Phillips

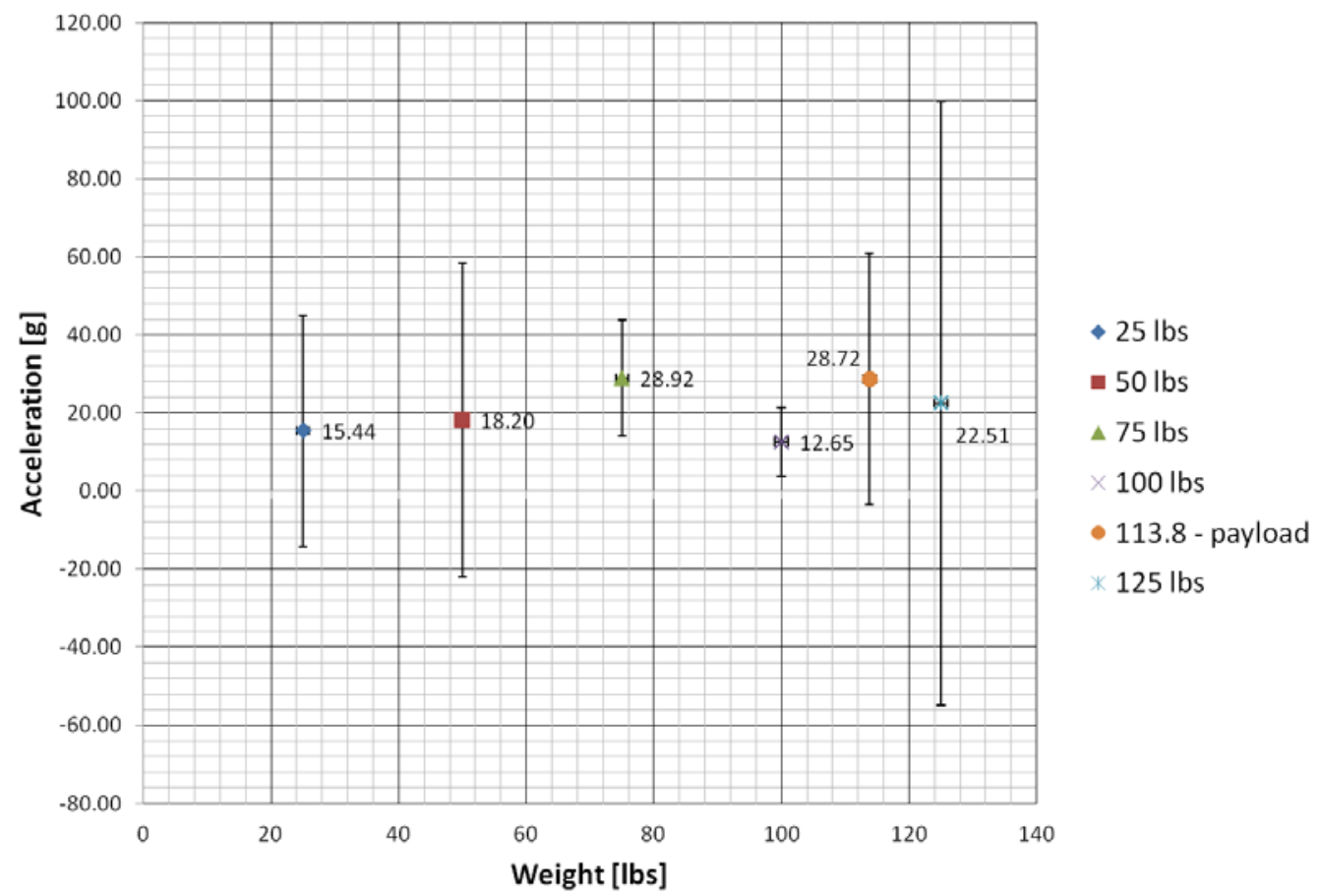

Figure 6-27: Compiled Weight vs. Acceleration Impact Data

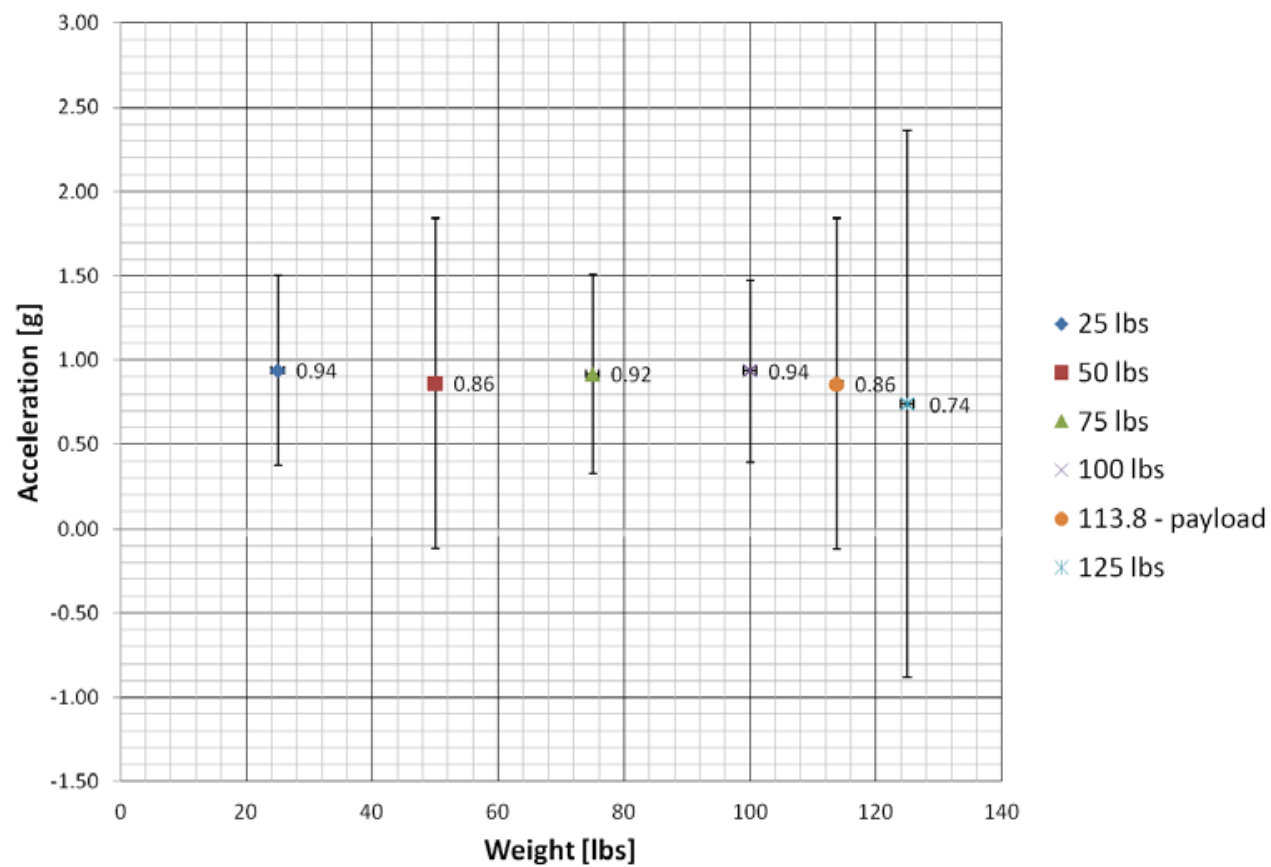

Figure 6-28: Compiled Weight vs. Acceleration Rebound Data 


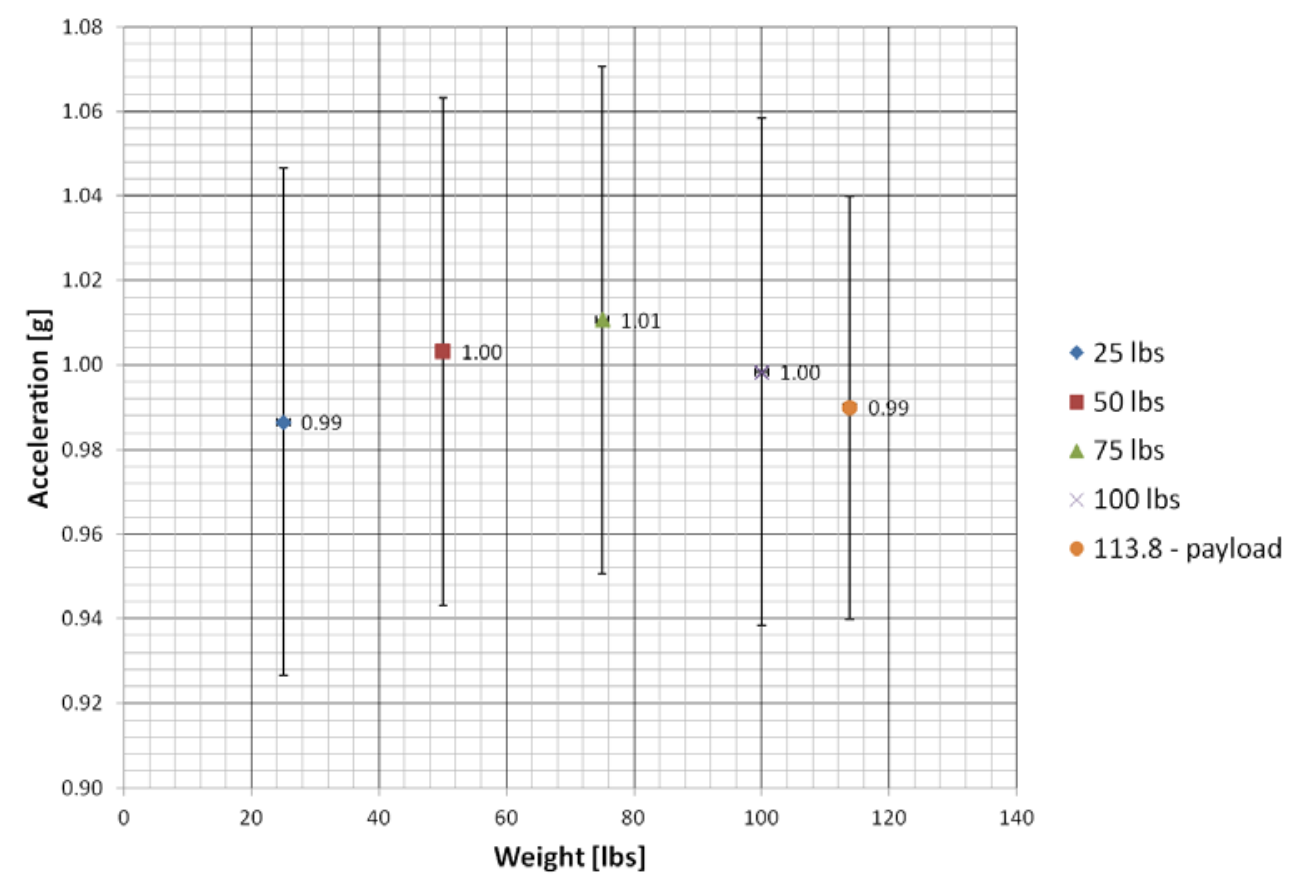

\section{Figure 6-29: Compiled Weight vs. Acceleration Recover Data}

Given the presented comparative results, the majority of phases do not seem to follow a trend when compared with weight. However, some, if not the majority, of the scatter may be due to checkout instrumentation and data acquisition frequency and resolution limitations, as illustrated by impact load data. As previously mentioned, the designer decided to use that data acquisition system due to the risk of damaging the primary data acquisition system from National Instruments. It is difficult to determine the trend of the impact loads, as related to the payload weight, as the memory foam has a non-linear Elastic Modulus. In fact, the only one that may be displaying a trend with respect to weight is the low-g phase. According to the data presented here, the low-g data shows a decreasing trend toward values approaching zero with heavier payloads. Although, given the ranges of the standard deviations, that remains to be confirmed. Additionally, the frequency and resolution of the checkout electronics limited the designer from collecting true g-load peaks during impact. With the improvement of operational data acquisition processors and instrumentation, one would expect this data to begin to indicate clearer trends. 


\subsection{Experiment/Checkout of Facility}

\subsection{Background \& Introduction}

Once the WVU SMiRF was constructed, and empty payloads had been used for calibration, such that the core systems of the facility were initially tested and calibrated, graduate student and his mentor wanted to take an additional step to ensure the operations of the facility were known, in order to train others to operate the facility on an ongoing basis and to amend any unforeseen abnormalities that may arise with full-scale operations. The most comprehensive way to accomplish the checkout was to create and drop a fully-operational microgravity experiment within the facility to produce data. The process would thereby generate additional insight about the Requirements and Goals and their true impact and comprehensiveness, as well as to the end-use, regarding everything from concept through operation and data analysis.

Several experimental topics were considered for the initial experiment, including pool boiling, hydraulic jump, tank sloshing, die swell, Kaye effect, viscous fingering, and flame propagation. Each of these was thought to readily exhibit noticeable behavior in microgravity, within the timeframe of a drop at WVU SMiRF. After long consideration of the type of experiment desired during the final construction of the WVU SMiRF, the field was narrowed to die swell, Kaye effect, and flame propagation. It was thought that flame propagation, while a vital field for research and space exploration moving forward, may be too dangerous for initial experimentation at the WVU SMiRF, as unforeseen operational unknowns may have still been lingering. The concept then evolved into considering a combination of Kaye effect (Figure 7-1), viscous fingering (Figure 7-2), die swell (Figure 7-3), and hydraulic jump in a singular package, as illustrated in the concept sketch depicted in Figure 7-4 on page 67. However, as funding was determined, many aspects of the combined approach had to be de-scoped for practicality. In-line viscous fingering may have caused too many technical challenges leading to a prolonged experimental timeframe. Kaye effect's conditions are such that it was thought to be marginal as to whether an entire experiment could be set up around that particular phenomenon, when we wished to show that the facility does indeed reach a reasonable level of microgravity. Therefore, it 
was thought that if the project evolved in such a way so that it could be included, it would be a bonus. By placing a clear plate beneath a free jet exhibiting die swell, however, a hydraulic jump may also be combined within the same experimental apparatus. Initially, it was thought that if two experiments could be performed at the initial checkout of the facility, that if one failed, for any unforeseen reason, a redundant experiment may succeed, hence still accomplishing the intended objective of displaying an experiment in microgravity. The following is a list of the variables that could be incorporated into the various phenomena in the combined experiment concept:

- Kaye Effect Variables (Figure 7-1)
o Fluid properties
o Initial jet shape
o Initial jet diameter
o Initial jet flow rate/velocity
o Free jet flow rate/velocity
o Free jet diameter
o Fluid bed depth
o Fluid bed inclination
o Height from nozzle exit to fluid bed surface

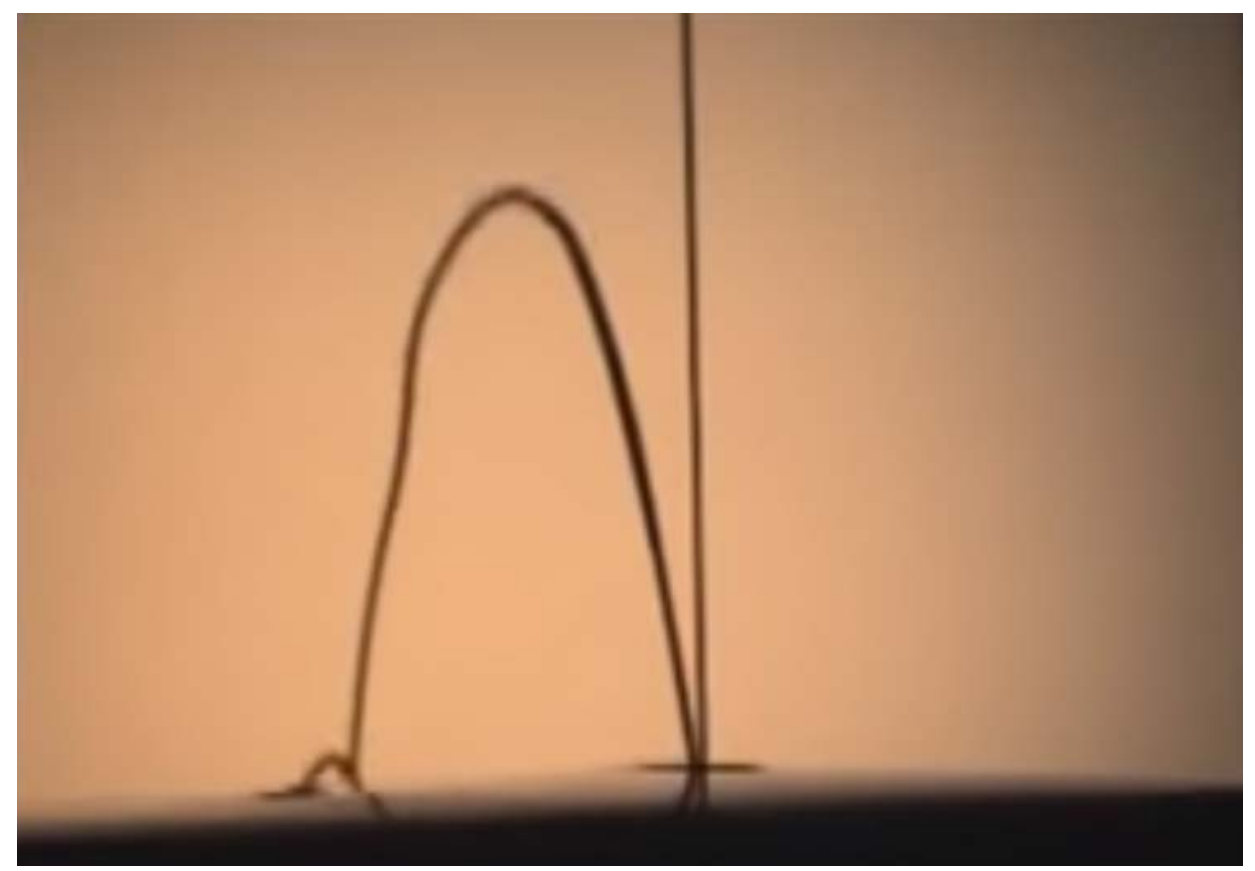

Figure 7-1: Kaye Effect [1] 
- Viscous Fingering Variables (Figure 7-2)

o Cell size/shape

o Cell fluid properties (fluid into which other fluid is injected)

o Injecting fluid properties

o Injection flow rate

o Outlet flow rate

o Inlet dimensions

o Inlet shape

o Quantity of inlets

o Outlet dimensions

o Outlet shape

o Quantity of outlets

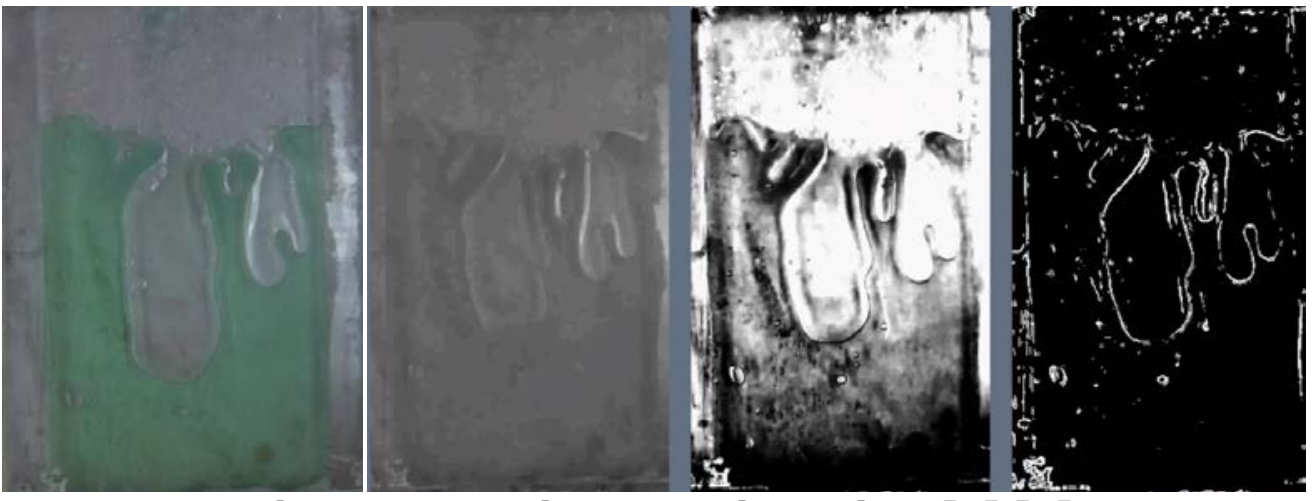

Figure 7-2: Viscous Fingering [2] [3]

(Glycerine/ Water -L to R: Original, Processed Images, Edge Detection Analysis)

- Die Swell Variables (Figure 7-3)

o Fluid properties

o Pre-experimentation fluid resting time

o Original container dimensions

o Upstream flow rate

o Nozzle flow rate

o Ejection tube dimensions

o Ejection tube temperature

o Nozzle dimensions

o Nozzle shape 


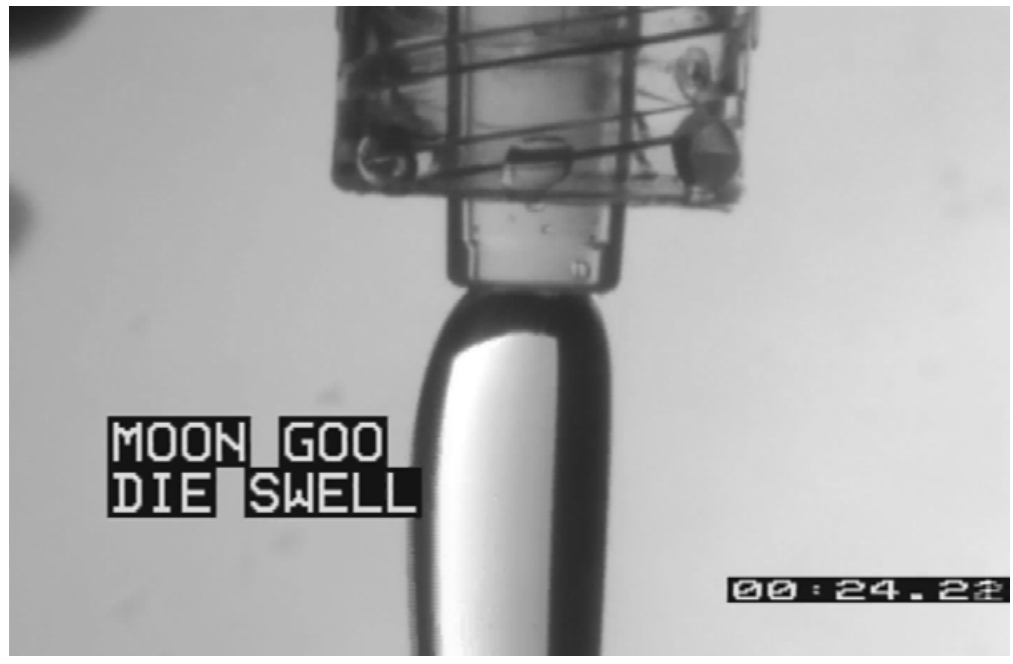

Figure 7-3: Die Swell [4]

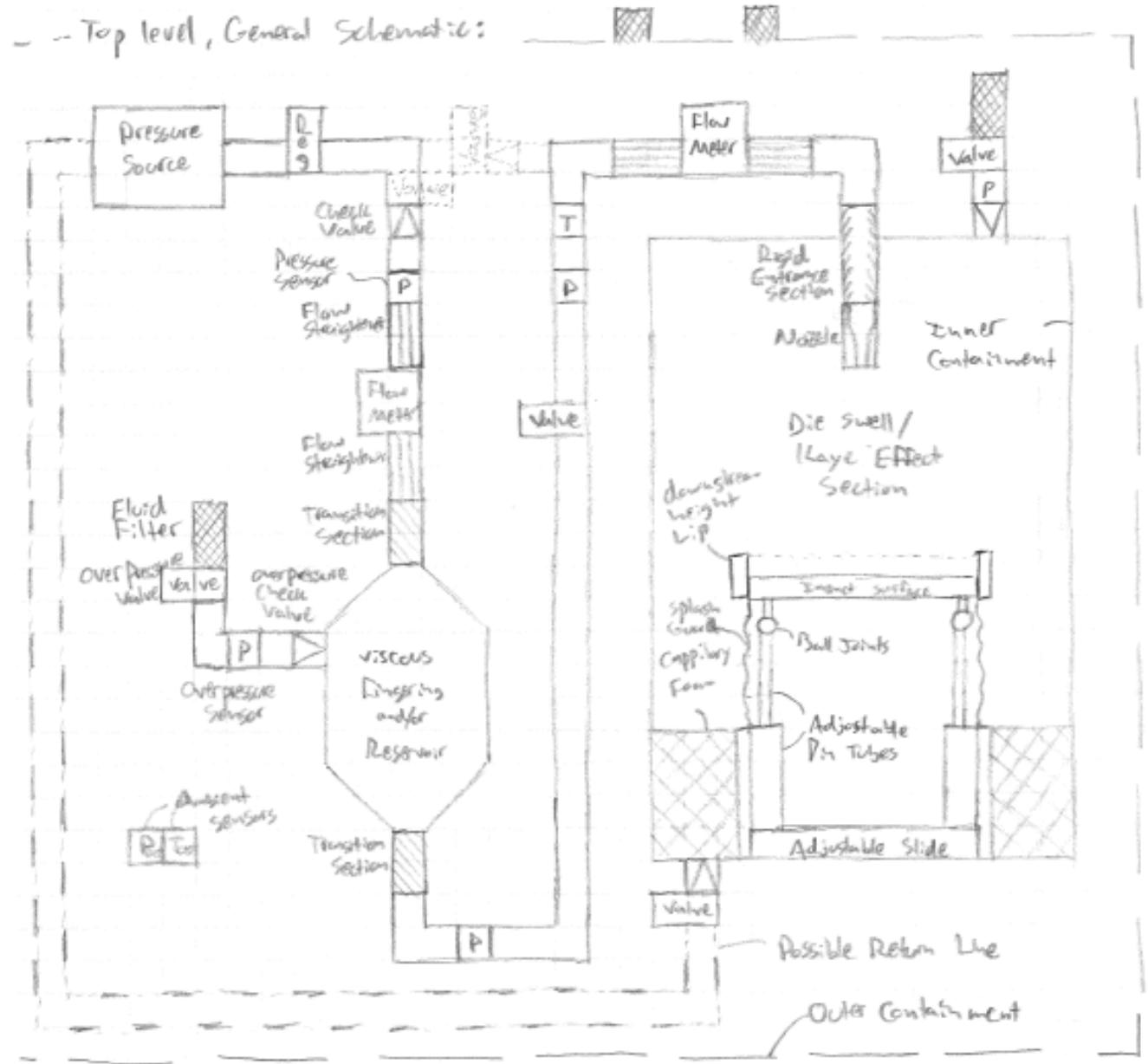

Figure 7-4: Original Combined Experiment Concept Layout 
Over the past century, many investigators have studied the behavior of the circular hydraulic jump (CHJ) under a wide range of conditions, including a pair of experiments that were carried out in a reduced gravity environment. Study of the circular hydraulic jump in a reduced gravity environment remains important in a practical sense, with wide ranging space applications, especially in the area of heat rejection in space. Moreover, as with any new technology, it has the potential to influence other industries, such as the cooling of aircraft generation coils, materials manufacturing processes in space, laser mirrors, etc. [5] Avedisian and Zhao (2000). Previous experiments and analyses have been performed by Watson (1964) [6], Liu and Lienhard (1993) [7], Middleman (1995) [8], Avedisian and Zhao (2000) [5], and Bush and Aristoff (2003) [9], to name a few. Watson compiled past data from planar hydraulic jump experiments and wrote the first significant paper explaining several of the governing equations, including the momentum balance, which provides the foundation for quantitative understanding concerning the circular hydraulic jump [6]. Liu and Lienhard used these foundations and applied them to heat transfer cases. They found that extremely high heat transfer occurs upstream of the circular hydraulic jump [7]. Middleman produced mathematical models of the circular hydraulic jump, through which he successfully compared his results to those of Watson [8] [6]. Avedisian and Zhao compiled the aforementioned bodies of work and analyzed the equations involved with the circular hydraulic jump, realizing that the jump location varies with gravity [5]. With this knowledge, they devised and conducted an experiment in a drop tower, under 1.2 seconds of reduced gravity. Finally, Bush and Aristoff further investigated the circular hydraulic jump phenomenon by analyzing the role that surface tension plays in the circular hydraulic jump [9]. In addition to these previous bodies of circular hydraulic jump studies by other researchers, the 20052006 West Virginia University Microgravity Research Team (WVU MRT) also developed a circular hydraulic jump experiment that was flown aboard the NASA C-9 Weightless Wonder aircraft during July 2006 [10], which formed the basis for the WVU MRT's upgraded circular hydraulic jump experiment, under the team lead of Phillips [11] [12] [13]. While the hydraulic jump phenomenon was to form the second of 3 experiments, occurring upon impact with a clear plate, the first was to occur at the nozzle exit as a die swell of a viscoelastic fluid, while Kaye effect would be a bonus phenomenon, if it manifested. 
Viscoelasticity is a fluid behavioral property that is exhibited through fluids that undergo both viscous and elastic effects during deformation from a resting shape; while rheology, the study of the non-linear viscoelastic flow of matter, has been studied as a specific area of research since the early 1900s [14]. Since then, the collective database of knowledge on various viscoelastic materials/substances, those that exhibit both viscous and elastic behaviors when acted upon, has continued to grow through observation, experimentation, and even application. Table 7-1 displays a high-level of detail about various types of viscoelastic fluids [15] [16].

\section{Table 7-1: Non-Newtonian Fluid Classification Brief}

\begin{tabular}{|c|c|c|c|}
\hline Category & Type & Description & Examples \\
\hline Viscoelastic & $\begin{array}{l}\text { Kelvin/Voigt } \\
\text { substance, } \\
\text { Maxwell } \\
\text { substance }\end{array}$ & $\begin{array}{l}\text { Linearly-dependent } \\
\text { combination of viscous } \\
\text { and elastic effects }\end{array}$ & Lubricants \\
\hline \multirow{4}{*}{$\begin{array}{l}\text { Non-time- } \\
\text { dependent } \\
\text { viscous } \\
\text { behavior }\end{array}$} & $\begin{array}{l}\text { Newtonian } \\
\text { substances }\end{array}$ & $\begin{array}{l}\text { Constant viscosity, while } \\
\text { elasticity depends on } \\
\text { normal stresses and } \\
\text { shear strains }\end{array}$ & $\begin{array}{l}\text { Water, most } \\
\text { common } \\
\text { fluids and } \\
\text { gases }\end{array}$ \\
\hline & $\begin{array}{l}\text { Dilatant (shear- } \\
\text { thickening) }\end{array}$ & $\begin{array}{l}\text { Viscosity proportional to } \\
\text { stress/strain }\end{array}$ & $\begin{array}{l}\text { Quicksand, } \\
\text { oobleck, } \\
\text { mustard }\end{array}$ \\
\hline & $\begin{array}{l}\text { Pseudoplastic } \\
\text { (shear-thinning) }\end{array}$ & $\begin{array}{l}\text { Viscosity inversely } \\
\text { proportional to } \\
\text { stress/strain }\end{array}$ & Paint, Blood \\
\hline & Bingham Plastic & $\begin{array}{l}\text { Viscosity inversely } \\
\text { proportional to stress } \\
\text { after a yield value is } \\
\text { exceeded }\end{array}$ & $\begin{array}{l}\text { Toothpaste, } \\
\text { Sludge }\end{array}$ \\
\hline \multirow{2}{*}{$\begin{array}{l}\text { Time- } \\
\text { dependent } \\
\text { viscous } \\
\text { behavior }\end{array}$} & Rheopectic & $\begin{array}{l}\text { Viscosity proportional to } \\
\text { time duration of } \\
\text { stress/strain }\end{array}$ & Cream \\
\hline & Thixotropic & $\begin{array}{l}\text { Viscosity inversely } \\
\text { proportional to time } \\
\text { duration of stress/strain }\end{array}$ & $\begin{array}{l}\text { Coal } \\
\text { Slurries, } \\
\text { Mayonnaise }\end{array}$ \\
\hline
\end{tabular}

Die swell is a viscoelastic phenomenon that occurs when a dilatant, or shear-thickening, substance is forced through a sufficient constriction, or 
"die," such that the substance expands, or "swells," downstream of the constriction, even while forming and maintaining a free jet at ambient sea level conditions. A wide range of dilatants exhibit die swell when subjected to the correct conditions, ranging from simple substances such as ketchup, oobleck, and shampoo to complex specially-formulated substances to be used for next generation body armor and high performance braking systems.

For all of the interesting and observable behaviors that dilatants exhibit, and all of the research that is spawned by the interest, there are also realworld, industrial applications that are inhibited by die swell. Industries ranging from food processing to plastics manufacturing and even the human body and various medical fields are affected by die swell and the effects of shear-thickening fluids.

Several have attempted to describe the fluid phenomenon, with varied disagreement among scientists as to the mathematical model with which to describe die swell. In its most simplistic forms, die swell may be described as a function of the constriction through which the fluid is forced via a ratio between the entrance and exit diameters, as described by Campanella [17]

$$
\frac{D_{e}}{D}=\left[1+\frac{1}{8}\left(\frac{\tau_{11}-\tau_{22}}{\tau_{12}}\right)\right]^{1 / 6} \quad \text { 7.1- } 1
$$

or as [18]

$$
\begin{array}{cc}
S_{R}=\frac{1}{2} \frac{N_{1}}{\tau} & 7.1-2 \\
S_{w}=\left(1+\frac{1}{2} S_{R}^{2}\right)^{1 / 6} & 7.1-3
\end{array}
$$

However, as in more complicated attempts, Koopmans describes die swell using the following time-dependent mathematical model [19]:

$$
B=0.1+\left[1+0.5\left(\frac{\tau_{11}-\tau_{22}}{2 \tau_{21}}\right)^{2}\right]^{1 / 6} \quad 7.1-4
$$


where

$$
B^{2}=\frac{2}{3} \gamma_{R}\left[\left(1+\frac{1}{\gamma_{R}^{2}}\right)^{2 / 3}-\frac{1}{\gamma_{R}^{3}}\right] \quad 7.1-5
$$

$$
\begin{array}{cc}
\gamma_{R}=J_{S} \eta_{0} \dot{\gamma} & 7.1-6 \\
B_{1}(t)=B_{1, \infty}-\left(B_{1, \infty}-B_{1,0}\right) e^{1 / \zeta_{1}} & 7.1-7 \\
B_{2}(t)=B_{2, \infty}-\left(B_{2, \infty}-B_{2,0}\right) e^{1 / \zeta_{2}} & 7.1-8
\end{array}
$$

The point here is not to criticize or to point to correctness or a "best model." The point here is to demonstrate the disparate mathematical models and the clear disagreement with which the scientific community describes die swell. The researcher here believes that a more sustainable approach to the theoretical and empirical method for describing die swell and other fluid phenomena must be taken.

To date, very few, if any, have researched the stabilizing effect that gravity may have on the phenomenon of die swell [20]. Currently, the predominant theory hypothesizes that the normal stresses within a viscoelastic fluid attempt to expand the fluid back to the diameter of the container from which it was stored, with some variation due to the relaxation time of the fluid, thereby suggesting that all viscoelastic fluids are at least somewhat time-dependent. Thus far, surface tension and the elastic effects of the fluid have been hypothesized to be the predominant forces, with secondary forces attributed to relaxation time, which is still at least loosely associated with elasticity and the normal forces. While several references have used those effects to numerically compare against experimental results, the researcher has not found any experimental data that attempts to study die swell in a reduced gravity environment. By studying a fluid phenomenon in a reduced gravity environment, both the effect of gravity can be studied and the predominant forces acting on the fluid can be concluded. Therefore, the researcher has decided to make the attempt of studying die swell in a reduced gravity environment the primary topic of experimental focus, thereby contributing to a full system checkout of the WVU SMiRF. 
Additionally, the researcher has taken an alternative approach to describing the behavior of a viscoelastic fluid particle using the viscous Navier-Stokes Equations. Furthermore, using the fact that all substances are the same at a fundamental level (i.e. the level of particle physics, such as quarks), and are only differentiated as a categorization at more macro levels, the researcher has chosen to employ solid mechanics with fluid mechanics by attempting to incorporate Hooke's Law with the NavierStokes equations to describe all fluid flow, including that of viscoelastic fluids. A relatively long derivation with several assumptions was performed (see Appendix $L$ ) to arrive at the following proposed constitutive relation:

$$
\begin{gathered}
\tau_{x x}=-p+\mu^{\prime} \nabla \cdot \vec{v}+2 \mu \frac{\partial v_{x}}{\partial x}+E\left(\frac{\partial v_{x}}{\partial x}\right) \Delta t \ldots \\
\ldots \sum_{n=0}^{\infty}\left\{(-1)^{n}\left[\left(\frac{\partial v_{x}}{\partial x} \Delta t+1\right) x_{o}-a\right]^{n}\right\} \ldots \\
\ldots \sum_{i=0}^{n}\left\{\left(\frac{\partial v_{x}}{\partial x} x_{o} \Delta t+1\right)^{n-i}\left[\left(\frac{\partial v_{x}}{\partial x} \Delta t+1\right) x_{o}\right]^{i}\right\}^{-1}
\end{gathered}
$$

The primary point of discussion here is not that the derivation is allinclusive for any non-Newtonian fluid, but to simply point to the fact that the scientific community, especially those related to the fluid sciences, should be attempting to describe fluid behavior into a predominant mathematical model, whenever possible. A discussion should commence to include non-Newtonian effects into the Navier-Stokes equations. The researcher here believes that Newtonian fluids are a special subset of fluids that exhibit so little non-Newtonian effects under most conditions that the assumption can be made that they exhibit no non-Newtonian behavior, whereas the majority of fluids exhibit at a minimum a small amount of non-Newtonian behavior. As more fluid behaviors are observed, the fluid mechanics community should continue to strive toward an inclusive mathematical model to attempt to describe the universal behavior of fluids - a unified theory of fluid mechanics, and to consider many of the effects that surround us as a matter of fluid flow - whether solid mechanics or gas dynamics. 


\subsection{Experiment Description}

To perform a full-scale checkout of the WVU SMiRF and to test the proposed hypothesis, the developer set forth to design and develop an experimental apparatus that would primarily test and demonstrate die swell, while secondarily testing and demonstrating the circular hydraulic jump. Meanwhile, continuous collection of drop data could prove the performance characteristics of the WVU SMiRF for use by future users and researchers. Based upon both budget and schedule, the scope of the checkout experiment would have to remain relatively modest, while continuing to advance the field and maintain the true spirit of scientific research. To that end, the bulk of the budget would be equally invested into non-expendables, such as the experimental structure and electronics - both of which could be used in future research, and into expendables hardware such as tubing, orifices, fittings, etc.

The first aspect to be designed was the general flow path, which had to be designed to operate in isolation during the drop. Thus, the general flow path was developed from the initial concept presented in the previously presented Figure 7-4, omitting the viscous fingering section of the experiment. Thus, a $48 \mathrm{in}^{3}$ air tank outfitted with a 3000 psi Myth Regulator from Guerilla Air would be controlled by both a brass, handloaded 0-500 psi downstream pressure regulator and a Stainless Steel 20turn precision needle valve, with a $\mathrm{C}_{\mathrm{v}}$ of 0.08 and orifice diameter of 0.063 ", while being measured by both a 0-10 scfm and a 0-25 scfm flowmeter. After the gaseous control section of the flow path, the fluid supply section would allow for removal and replacement of the working fluid of choice. Downstream of the fluid supply section, would be a short section to form the entrance to the orifice nozzles, ranging in size from 0.016 " to 0.125 ", which would be the point of focus for the die swell experiment. After the free jet had formed, downstream would be a transparent plate, surrounded by gutters to collect spent fluid.

In order to operate the experiment such that it could be reused for multiple drops, a sufficiently strong experiment frame had to be designed and constructed. So as not to spend much time on the technical design of the frame, it would be over-designed and constructed from 1"x1" square aluminum tubing. Half of the volume of the frame would be dedicated to the test section, enclosed by Plexiglas to contain any stray fluid. The 
other half of the experiment frame's volume would be dedicated to hardware, gauges, and stowage of the air tank. A shelf would be constructed along with a padded stowage cylinder for the air tank. A subshelf would be constructed to serve as a panel for the gauges. The test section would feature a cubic volume that extended up into the test section to form the raised plate. Once enclosed with Plexiglas, that volume could house a video camera to record the radius of the hydraulic jump for later analysis and measurement. The top half of the test section would include vertical rails to adjust the height of the nozzle, while horizontal rails would allow for the stabilization of the nozzle during a drop. Mounted to the top rail on the same side as the gauges, over the test section would be mounted two Israeli arms to hang two Nikon Coolpix P310 point and shoot cameras to record the experiment. The right hand camera was used for macro video observation of the nozzle exit, nearly at full optical zoom with frame rate of $120 \mathrm{fps}$ at a resolution of $640 \times 480$ pixels, while the left hand camera was used for full test chamber viewing with no zoom, but the same settings otherwise. Additionally, the researcher attempted the use of a 3D GoPro Hero 2 system, which was abandoned due to the fish eye lens and the distortion it caused when viewing the experiment. ISO-B Zinc-Plated hose coupling plugs and associated sleeve-lock socket quick disconnect fittings would be used upstream of the entrance section to the nozzle, where needed, while the entrance section would employ an NPT fitting in order to ensure a uniform fluid flow profile. Due to a lack of correct check valve sizing, the check valve would eventually be removed. Full photos of both a front and back view of the experiment apparatus are presented in Figure 7-5 and Figure 7-6, while a rough bill of materials may be observed in Appendix $M$.

The researcher chose glycerine, and later polypropylene glycol as the working fluid in the experiment. Neither fluid was as relatively susceptible to commercial alteration, as ketchup and mustard would both be inconsistently produced by different vendors. At the time, glycerine was thought to be a shear-thickening fluid that was readily available, affordable, and widely used throughout industrial and other applications. Polypropylene glycol would also be a commercially/industrially-produced fluid with consistent properties, also considered a shear-thickening fluid in wide use by industry. Polypropylene glycol would serve as the better working fluid, as glycerine has mixed behavior as a shear-thickening fluid. 


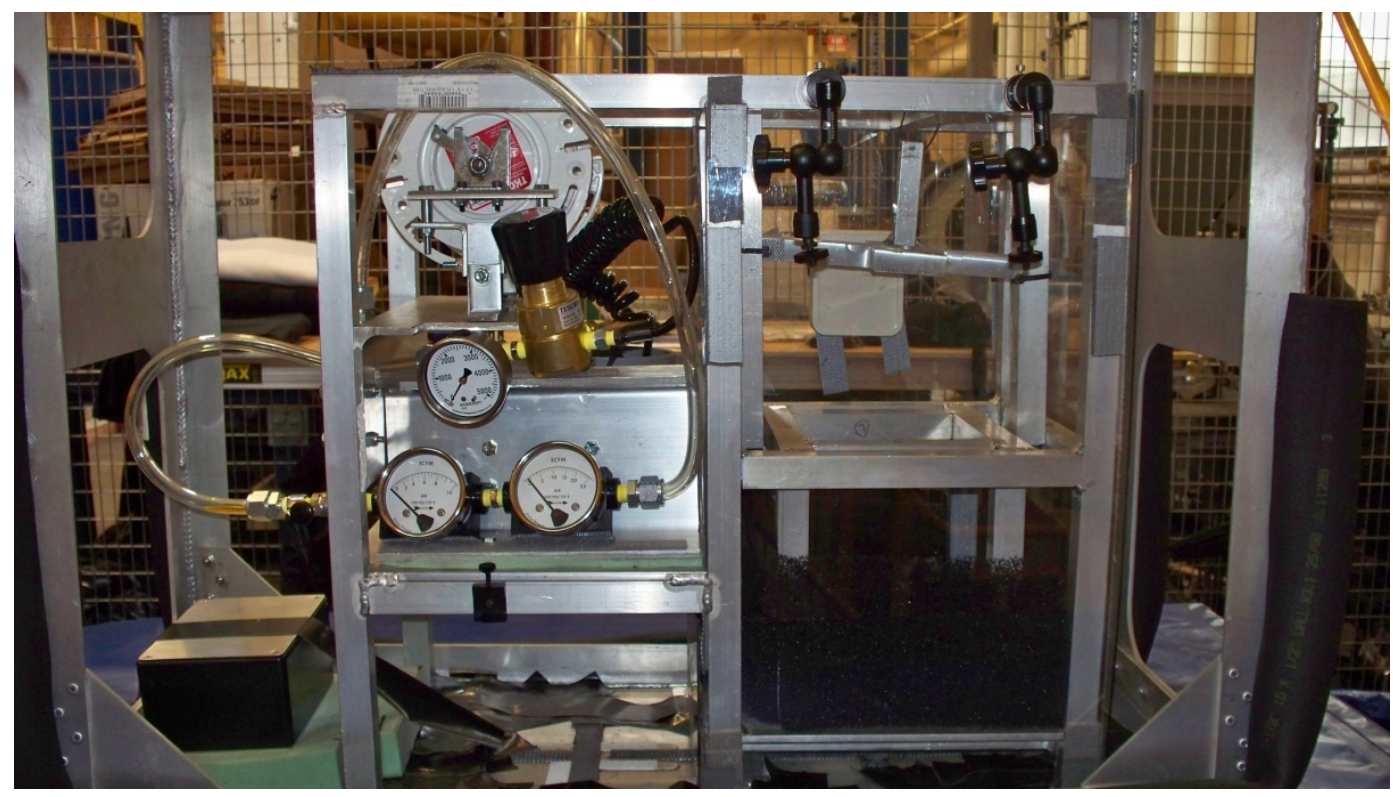

Figure 7-5: Die Swell Experimental Payload (Front View)

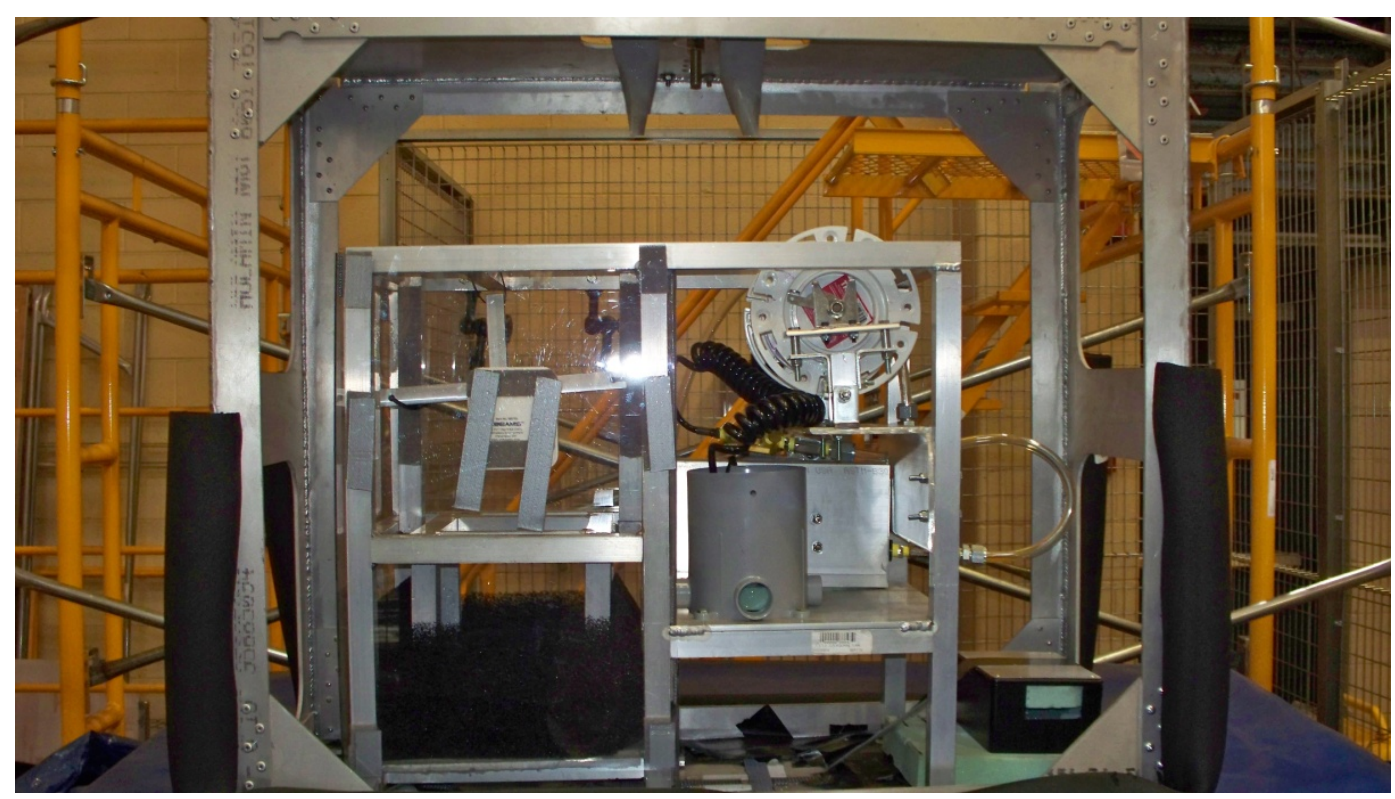

Figure 7-6: Die Swell Experimental Payload (Back View)

\subsection{Experiment/Facility Checkout Operations}

Once the fully-developed experiment was installed onto the frame, and the experiment frame integrated into the payload frame, several subsystem 
checkouts had to be performed on the experiment, prior to integration into the WVU SMiRF. The air tank's preparations and operations both had to be determined prior to integration and operations, and even additional equipment had to be procured for these purposes (i.e. a mobile air compressor, custom air lines, etc.). The gauges, pressure regulator, needle valve, and quick disconnect fittings were checked using a pressurized tank and cycling the fittings. Full system dry runs (using air) and wet runs (using water) were also lab-tested. The working fluids, glycol-water mixtures, were then tested multiple times, adjusting both pressure, via the regulator, and flow, via the valve. Once the system was optimized for operation, it was loaded onto a cart and transported to the WVU SMiRF.

Once the system was at the WVU SMiRF, the specified pre-operations checkouts had to be performed, prior to integration into the facility, in accordance with Appendix I. First, all hardware was connected to the system, including cameras, lighting, the air tank, and a container of test fluid. Once the experiment had been pre-assembled in an operational configuration, the experiment had to be balanced, using the WVU SMiRF balancing boom. The balancing cable was connected through the appropriate hole at the center of the top C-channel of the payload frame, and the cart was removed, allowing the payload to hang freely. The bubble levels were placed orthogonally toward the top edges of the payload frame, and laid on their sides. The diagonal levels were used to obtain a true level. The experiment was spatially adjusted within the payload frame to obtain a true balance in both directions. Once the experiment was secured to the payload frame, the cart was placed underneath the payload, and the balancing cable was removed.

Once the payload was ready to be integrated into the facility, the payload was placed in front of the deceleration system and sensitive components were removed. The lifting lines were lowered to extend passed the deceleration system down to be connected to the payload frame. Once the lines were securely hooked onto the payload frame, using two people (one operating the hoist, and one guiding the payload), the payload was carefully and slowly guided up onto the deceleration pad. Once there, the person at the bottom of the tower cleared the area and stowed the conveyance cart. The payload was then temporarily hoisted 8 feet up 
from the deceleration system's surface. The temporary tower preparation surface (TTPS) was replaced, and the payload lowered onto the temporary floor, consisting of flooring panels that attach to the scaffolding, supplied by the scaffold manufacturer, as shown in Figure 7-7. Once the payload was stable on the surface, the payload's electromagnet interface, also known as the "crown," was attached, and its surface cleaned with acetone. Sensitive components removed prior to integration were replaced to finish the integration process. At this point, the operator could either proceed with drop testing, or temporarily store the experiment in place, by covering the experiment with black cotton sheets.

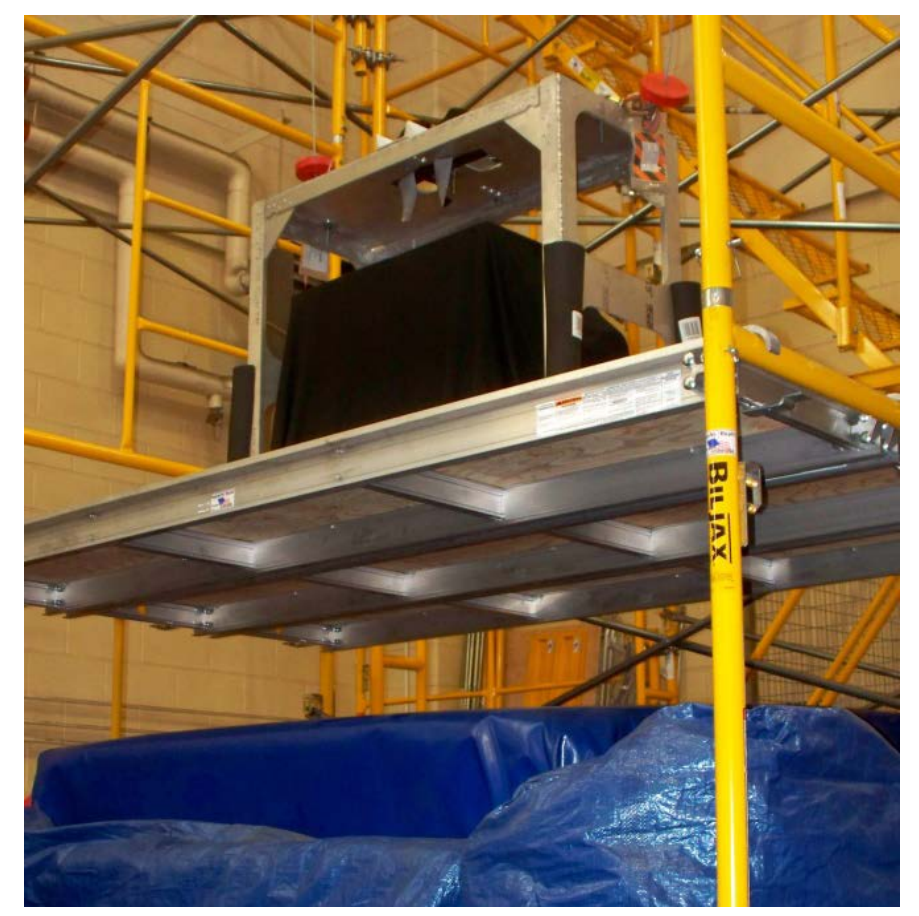

Figure 7-7: Loaded Payload Sitting on Temporary Tower Preparation Surface (TTPS) Above Deceleration System

Once the experiment was integrated into the facility, experimental operations could begin. First, the electromagnet was energized via key toggle on the red button at the bottom of the facility. The air tank was pressurized with an onsite portable compressor. The checkout accelerometers and data logger were attached to the payload frame and integrated into the protective data logging box, and the accelerometers were calibrated. A fluid container was attached in-line. The cameras and 
lighting were activated and focused on the die swell experiment orifice nozzle.

Once the experiment was prepared for test, the operator raised the payload 5 feet, removed the TTPS, and lowered the payload onto the deceleration system. Once there, the data logger was started and secured. The operator disconnected and retrieved the air tank from the air compressor, walked to the top of the tower and lifted the payload to the top of the tower using the lifting system. Once the payload was at the top, the electromagnet was attached, the load transferred onto the electromagnet, and the payload lowered until it was hanging on the magnet. However, the hoist lines were left attached to the payload as a safety precaution, until the payload is ready to drop. At this point, the air tank was attached, the cameras were refocused and began recording. The lift lines were removed, the payload carefully stabilized by hand, and the pressure regulator turned to the pre-determined position to start flowing fluid at the pre-determined flow rate. A countdown was performed and the payload was released by turning power off to the magnet via a switch at the top of the tower.

A little over one second later, the payload touched down on the deceleration pad, and the payload was then stabilized by the operator by deactivating electronics and closing the pressure regulator. The lift lines were lowered and reattached to the payload frame, the payload was raised back up above the temporary preparation surface level, and the TTPS was replaced. The payload was then lowered back onto the TTPS, where the operator retrieved the electronic data, removed the air tank, removed the fluid container, and cleaned up any stray fluid. Once this was complete, the experiment could be prepared for the next drop. A new nozzle could be replaced, a new fluid container inserted, and the process was repeated for the number of tests required to characterize the facility.

\subsection{Results}

Once the experimental checkout was complete, after more than 20 drops, the data was collected and compiled to determine both acceleration repeatability results and die swell experimental results. Due to budgetary concerns, electronic, in-line hardware was outside the scope of the 
project. Therefore, other than accelerometer data, the die swell experiment was a highly qualitative design. However, despite visual data being the primary data, the researcher attempted to use pre-programmed visual analysis software, provided by Fiji, known as ImageJ.

Using the ImageJ software, which was originally developed as open source software for the medical community to compile and analyze digital CT scan slices, high-speed video from camera footage, provided by the Nikon CoolPix P310 cameras at $120 \mathrm{fps}$, could be quantitatively analyzed. Once the post-drop data was retrieved from the cameras, the video data then had to be converted from its native format assigned to it by the cameras to the .avi format, using Any Video Converter, a free video file conversion software package. Once in the avi format, the video could then be imported into Fiji's version of ImageJ. From there, the Fiji software could convert the avi into an image sequence, based on the individual frames of the video file. From there, the entire image sequence could be batch-processed together. For example, if one wished to sharpen the image on each frame in the sequence, that request would be processed on all of the individual frame images simultaneously. Furthermore, the Fiji software also has an edge-finding algorithm that can be batch-processed. Once the edge detection algorithm has been run, Fiji has a built-in measuring function that may be used to determine the intensity of lines, and thereby determine a pixel count between edges. Using that method, it was thought that the presence and width of die swell could be quantitatively measured and subsequently statistically analyzed. The process results are demonstrated in Figure 7-8 through Figure 7-10.

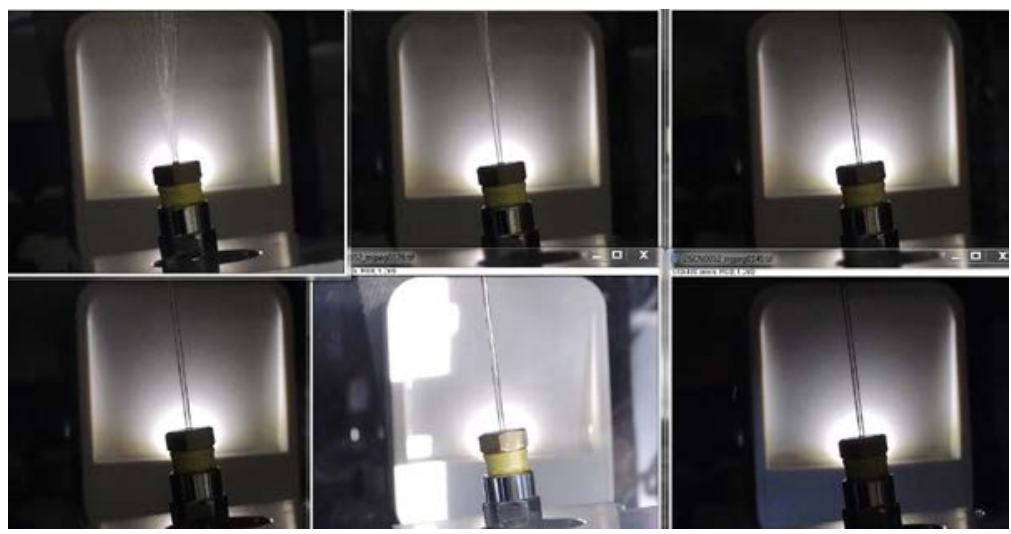

Figure 7-8: Example Image Sequence as Generated from a Converted Video File 

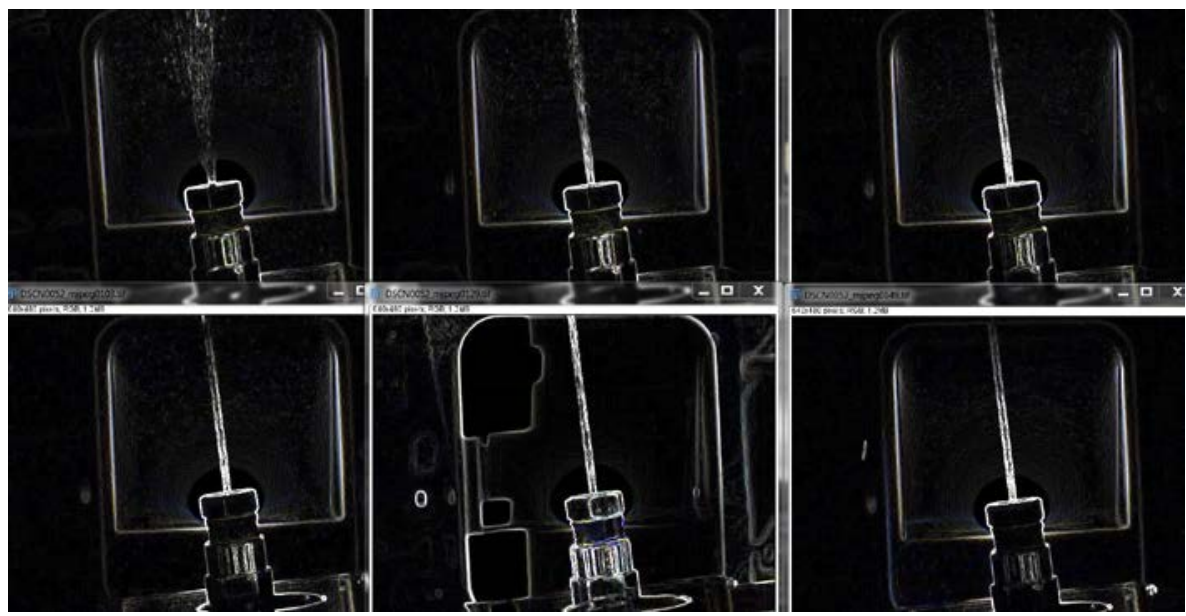

Figure 7-9: Edge Finding Algorithm Applied to Example Image Sequence

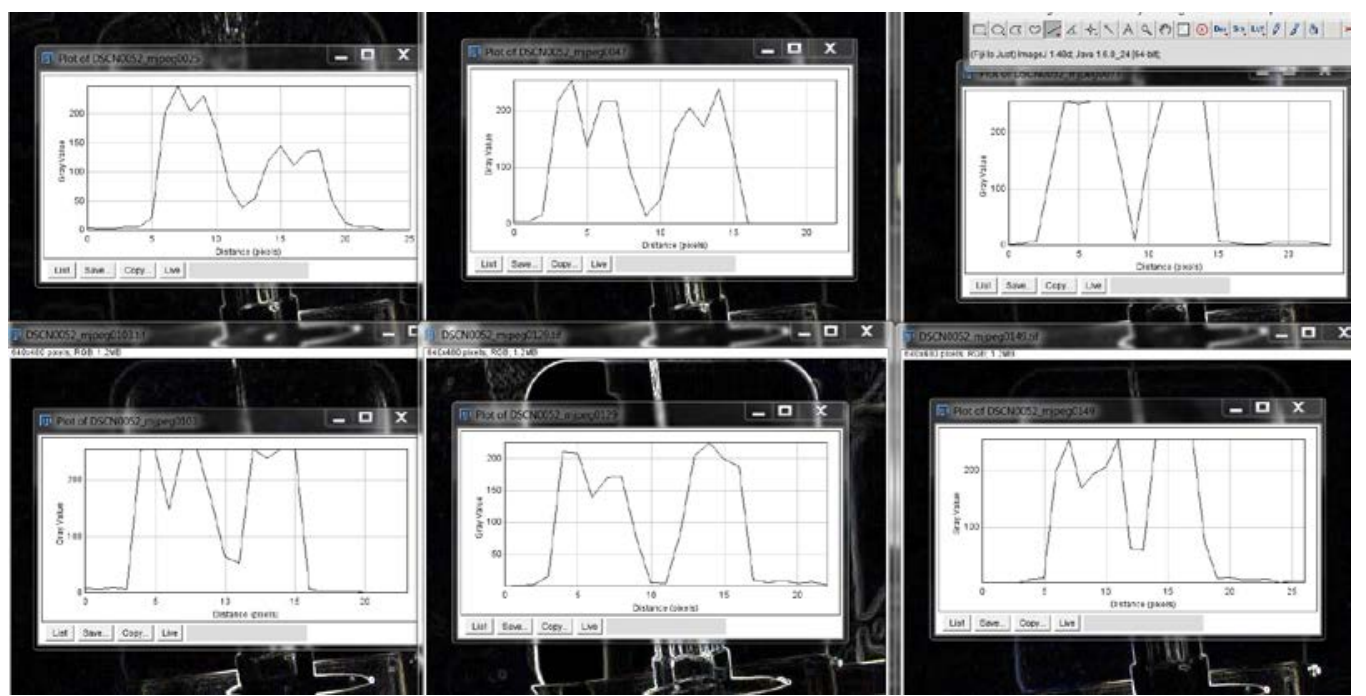

Figure 7-10: Pixel Measurement Applied to Edge-Generated Image Sequence

However, after the analysis of several drops worth of datasets with various test configurations, no appreciable difference was found in the exit jet diameter at the nozzle exit. It is believed that the die swell phenomenon did occur, especially with polypropylene glycol as the working fluid, but could not be recognized by the software due to the physical configuration of the experiment or the flow rate of the fluid (i.e. delayed onset die swell 
may have been occurring) and a relatively low video resolution of the cameras at the high-speed setting. However, without the high-speed camera setting, a sufficient data set could not have been collected. The resultant uncertainty of the resolution, coupled with the designed physical distance from the nozzle exit, may have resulted in such large measurement uncertainty that it may not have yielded accurate results, even if the phenomenon had been observed. In retrospect, given the obtained experience, the researcher would recommend a different design, different electronics, or both, as the optics were the primary limiting factor to the experiment uncertainty and thus the experiment's primary objective. With that said, the experiment successfully operated and gave the developer an opportunity to fully test the operations of the developed facility.

Several lessons were learned while operating the facility. The inclusion of the TTPS was a direct result of full-scale testing, as was the payload interface plate crown modification. Even an unforeseen positive safety aspect of the tower was noticed when each drop causes the outer secondary pad of the deceleration system to tip over onto the floor to prevent even a stray payload from damaging the site. Techniques used to shield and cushion the data logger also stemmed from full-scale testing. Operational techniques will have to be included during future training, especially that of determining when the payload is stable enough to drop, so as to get a smooth, repeatable landing each drop.

Additionally, further compilation of accelerometer data was produced to determine repeatability characterization of the WVU SMiRF. A total of 22 drops were performed during checkout testing. If well prepared, a single person could drop this particular experiment 6 times per day, with an average period between drops of approximately 1 hour and 15 minutes. However, the turnaround time is more a function of the turnaround time of a particular experiment, rather than the facility. For example, if an experiment could run continuously for the entire day, dropping and retrieving would be a matter of minutes, potentially as low as 10 minutes or less, without having to prepare for each drop.

Once it was determined that the experiment had collected sufficient information for what would be analyzed, as well as enough technique and 
sufficient experience developed to determine the performance characteristics and operations of the facility, the data was compiled using the same process as that used to analyze the calibration data, and statistically analyzed drop test data was generated. As the data generated would create too long of a section to present here, only the compiled data comparing the 22 drops will be presented, while individual drop data will be documented in Appendix $\mathrm{J}$.

A comparison dataset was generated in order to determine the repeatability characteristics of the facility. To create such a dataset, the same spreadsheet software that was developed for individual drops was employed. However, in this case, the researcher had to also note the drop number for reference. These data were sequentially compiled in the raw data sheet of the analysis software by drop phase. Each phase was then copied into the appropriate area of each phase's analysis sheet. In doing so, the statistical analysis section for each phase acted on the entire compilation of data to form a statistical analysis for each phase. Additionally, for the low-g data, drop times were extracted. The following presents the collective repeatability data categorized by phase.

Based on the results presented in Table 7-2, the results seem favorable to the Goals set forth at the beginning of the project. Drop times average well over $1.0 \mathrm{~s}$ as documented in Appendix J. Drop quality seems sufficient, given the relatively low resolution of the checkout instrumentation, as further evidenced by the comparison against the conceptual analysis, presented in Figure 7-11 and Figure 7-12, based on actual drop times and heights. Impact data indicate that payload should be able to withstand a peak impact load of more than $42 \mathrm{~g}$, as indicated by the $3^{\text {rd }}$ standard deviation, or be supplemented by secondary dampening, as illustrated by the technique used to protect the checkout data acquisition system. The rebound phase does not seem to put too much force on rebounding payloads. Lift and Recover phases also indicate a stable lift and settling of payloads. 
Table 7-2: Statistical Repeatability Data Analysis Summary

\begin{tabular}{|l|l|l|l|l|l|l|}
\hline & $\begin{array}{l}\text { Lift \& } \\
\text { Prep } \\
\text { Phase }\end{array}$ & $\begin{array}{l}\text { Low-g } \\
\text { Phase }\end{array}$ & $\begin{array}{l}\text { Impact } \\
\text { Phase }\end{array}$ & $\begin{array}{l}\text { Rebound } \\
\text { Phase }\end{array}$ & $\begin{array}{l}\text { Recover } \\
\text { Phase }\end{array}$ & $\begin{array}{l}\text { Drop } \\
\text { Time }\end{array}$ \\
\hline -3 SD & 0.91 & -0.20 & -21.69 & -0.12 & 0.94 & 1.24 \\
\hline -2 SD & 0.93 & -0.11 & -10.96 & 0.20 & 0.96 & 1.25 \\
\hline -1 SD & 0.96 & -0.02 & -0.22 & 0.53 & 0.98 & 1.26 \\
\hline Mean & 0.99 & 0.06 & 10.52 & 0.86 & 0.99 & 1.26 \\
\hline +1 SD & 1.02 & 0.15 & 21.25 & 1.19 & 1.01 & 1.27 \\
\hline +2 SD & 1.04 & 0.24 & 31.99 & 1.51 & 1.03 & 1.28 \\
\hline +3 SD & 1.07 & 0.33 & 42.72 & 1.84 & 1.04 & 1.28 \\
\hline Minimum & -2.93 & -0.93 & -36.87 & -3.08 & -2.28 & 1.18 \\
\hline Median & 0.99 & 0.07 & 10.24 & 0.97 & 1.00 & 1.26 \\
\hline Maximum & 5.09 & 1.16 & 28.72 & 2.24 & 4.47 & 1.36 \\
\hline
\end{tabular}

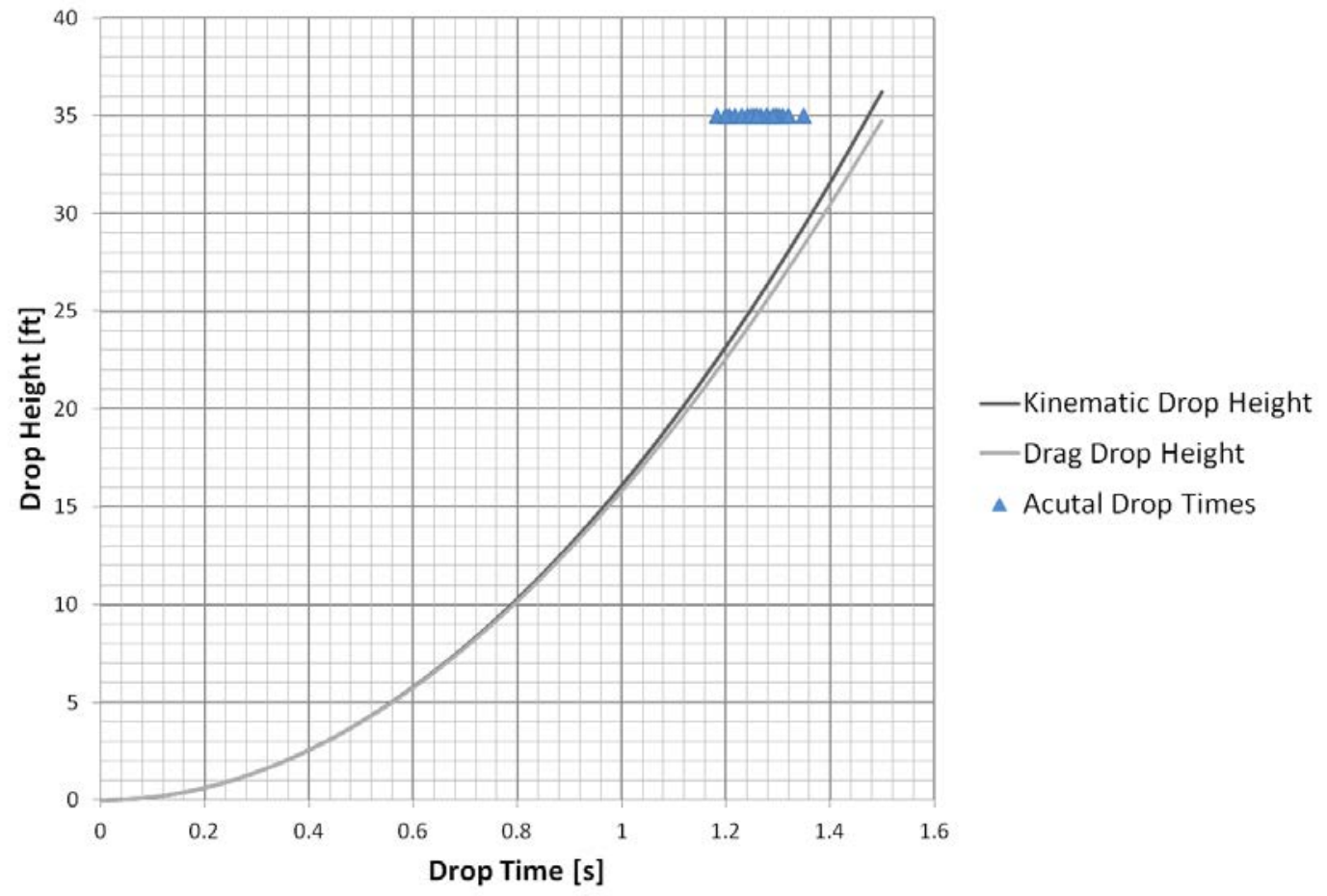

Figure 7-11: Actual Drop Time Comparison with Conceptual Design Analysis 


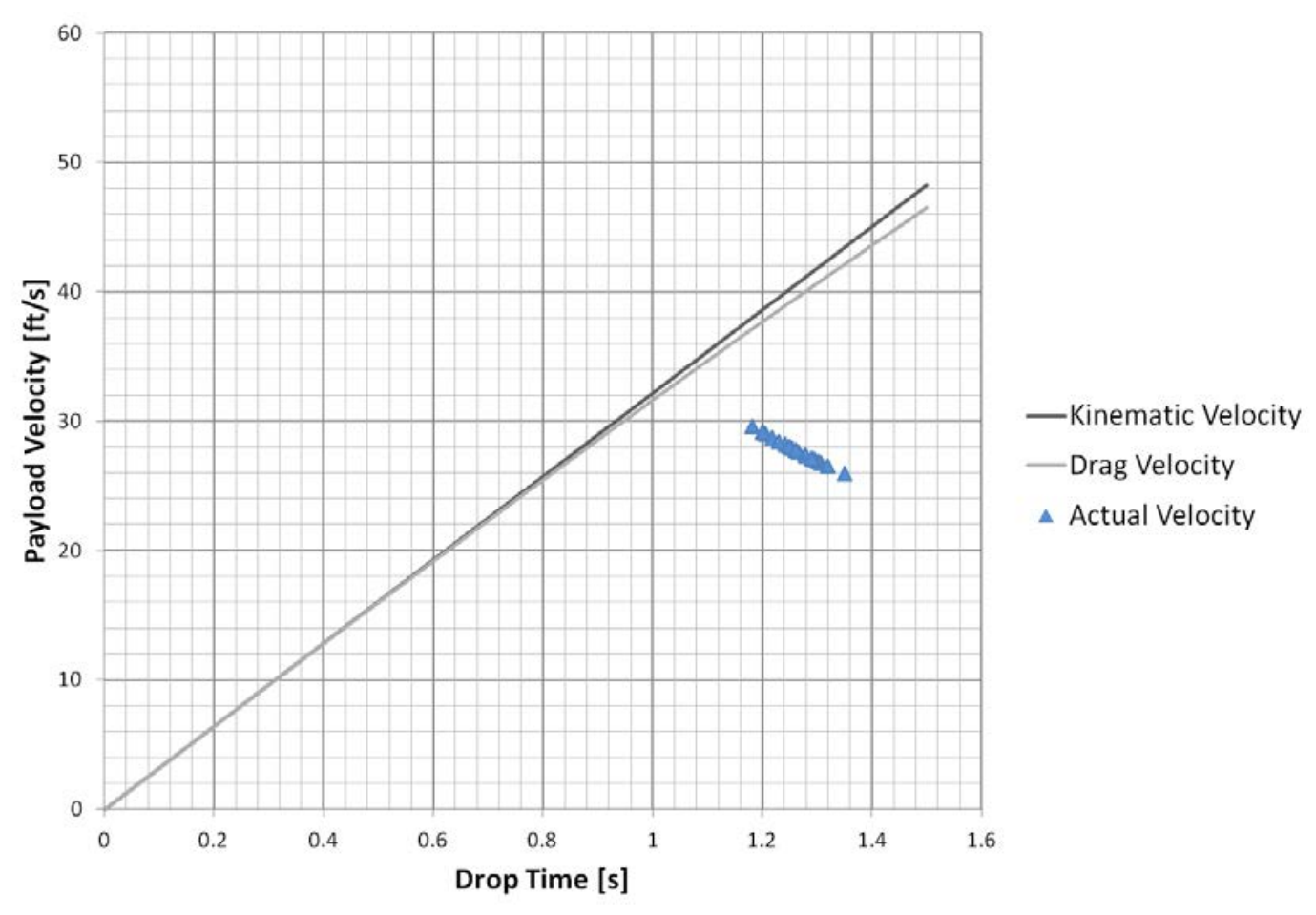

Figure 7-12: Calculated Actual Payload Velocity Comparison with Conceptual Design Analysis

\subsection{Experiment Summary \& Conclusions}

Despite showing promise during prototyping and even during initial lab optimization, the die swell experiment did not quite deliver the desired scientific results. The primary shortcoming of the experiment was the design of the physical optics setup for the imaging and video subsystem. The equipment available within the budget just could not match the performance needed to maintain the necessary resolution at the speed required to obtain meaningful data during the 1.2s drop times. As the optics served as the primary source of data collection, the lack of resolution at high speed, coupled with the physical distance from the nozzle and a potentially inconsistent optical zoom distance, prevented optical data with sufficient resolution from being acquired and analyzed. Furthermore, more analysis should be put forth toward the process system of the experiment design to ensure proper conditions in future experimentation. 
However, regardless of the fact that scientific results fell short of expectations, many scientific endeavors serve to guide engineering endeavors to improve on the equipment used for those endeavors. A process has been established for affordably analyzing visual data for future research. A flow path was developed that has successfully performed within the conditions provided by the WVU SMiRF, which may also be used and improved upon for future research. With a better physical and optical design, the same affordable cameras could be used for future research. Furthermore, it was proven that such electronics could survive the drops within the WVU SMiRF. With the aforementioned being tested, an experiment has been successfully operated within the WVU SMiRF, which stands ready for a variety of future full-scale use.

\subsection{Suggestions for Future Research}

Several improvements could be made to both the experiment itself and the apparatus used. If the budget had allowed and potential student discounts employed, an 80/20-like frame could have been used in place of the tubular aluminum frame. If an 80/20-like frame, such as T-Slots, had been used, adjustments could have been made more easily to correct for the physical disparity between the capabilities of the cameras and the distance to the nozzle. The designer had considered such a frame so as to provide future users with the option of an experiment frame, but could not afford such a system within the budget. The low mass of the cameras used was most likely to the benefit of their functioning during repeated impacts and would be chosen again in the future. With additional labor resources or a longer schedule, an improved flow path could have been designed, prototyped, and refined to tune the system with upgraded components. However, the budget did not allow for a longer timeline. With this experiment as a baseline, future experiments can improve upon the parts used. The air tanks used did not show any signs of fatigue, and the stowage method for drops worked well, as did the reinforcement for the data logger. In retrospect, the researcher would have finished the necessary provisions for the secondary Kaye effect or hydraulic jump experiment, increasing redundancy and the odds for successful scientific results. More emphasis would have been placed on a high-pressure supply with a larger volume to provide a more consistent ongoing pressure differential, regulated by a better pressure regulator and needle valve. 
Furthermore, despite the mass-produced fluid container designed for quick turnaround times, the researcher would have included even more features to reduce turnaround times for scheduling efficiency, which should be taken into account by future designers. Lastly, more time and budget should have been allocated for prototyping and experiment refinement prior to testing. Future users should account for such things and fine-tune experiments prior to drop tests to save time and potential frustration during execution. Proper orientation and training at the WVU SMiRF, prior to experiment design, could help those future users who could afford such a visitation, more appropriately design experimental apparatuses with such insight.

\subsection{Facility Summary \& Conclusions}

Regardless of experimental scientific results, the primary mission of developing a reduced gravity research facility, meeting the Requirements and Goals set forth, was very successful. A comparison amongst various modern era drop towers may be surveyed in Appendix $O$, while the budgetary model is summarized in Appendix $\mathrm{N}$.

\subsection{Requirements}

1. Ensure personnel safety throughout all levels of the project

- Validation: No personnel were injured during the development of the WVU SMiRF.

2. Keep end-user safety paramount throughout the design

- Validation: The WVU SMiRF has been successfully operated without incident during drop test sessions.

3. Provide a system with long-term reliability

- Validation: Including calibration testing, initial operations occurred consistently from July 2012 - January 2014. Only time can prove future reliability, but most systematic problems should have occurred during initial operations, until long-term maintenance issues become predominant. 
4. Provide a system that requires very infrequent maintenance

- Validation: Since initial operations have started, no maintenance has been required, and very little, if any, deterioration of the facility has been observed.

5. Provide a system that can be upgraded over time

- Validation: As is noted in the following section and in previous sections, the system has been designed for simplicity and extensibility.

6. Provide a system as inexpensively as possible

- Validation: A reduced gravity research facility was developed for less than the price of a luxury sedan or most research-grade experiments that may involve travel to such facilities.

\subsection{Goals}

1. The facility should be OSHA-certified to ensure safety

- Validation: West Virginia University Safety \& Health was included from the design phase of the project. The facility has been constructed so as to comply with OSHA regulations. Proper egress has been designed into the system. Proper distance between the facility and preestablished fire pulls was established. Lastly, access to all pre-existing building amenities has been maintained.

2. Design the facility such that it may be operated by a single person

- Validation: Over the period during calibration and initial operations, the facility has been operated by a single operator, the developer.

3. Design the facility with quick turnaround times

- Validation: As previously noted, facility turnaround times are primarily a function of experiment turnaround times. Even if the experiment has a zero-second turnaround time, the facility can perform with a turnaround time of less than 10 minutes. 
4. Provide common hardware and setup facilities for users

- Validation: A common procedure of preparing payloads via the balancing boom, interface preparation guidelines, and facility capabilities was developed.

5. Provide a tower tall enough to meet or exceed 1 second of drop time

- Validation: Average drop times exceed 1.2s.

6. Provide a facility that meets or exceeds experiment weight allowances on ZeroG flight from NASA JSC

- Validation: Weight allowances for the WVU SMiRF are designed to be set at $300 \mathrm{lbs}$, meeting those limits set by NASA for parabolic flight. Thus far, the maximum payload weight that has been dropped is $225 \mathrm{lbs}$.

7. Provide a facility and method that allows for near maximum payload stability prior to drop

- Validation: Modifications were made to produce the payload stabilization crown that prevents electromagnetic slip. Further training to operators, based on initial experience will be provided to ensure maximum payload stability prior to drop.

8. Provide a facility with impact loads as low as possible

- Validation: Average impact loads were maintained below $50 \mathrm{~g}$ at a $113.8 \mathrm{lb}$ payload weight.

9. Provide a facility that supports an affordable experience for the end-user

- Validation: The end-user shall be provided with opportunities to employ an existing payload frame, data acquisition capabilities, and data analysis software, all of which can prove to be very expensive and timeconsuming to develop. The user needs nothing more than to develop their experiment and purchase sensors, capable of physically and electronically interfacing, and 
potentially control software. Essentially, the facility is nearly plug-and-play.

10. Create a website for the facility

- Validation: A sitemap has been developed, as presented in Appendix $\mathrm{P}$, to provide a skeleton for a future website development effort.

11. Provide end-users web-based access to the facility and data

- Validation: This goal has not yet been addressed and remains for future upgrades and development efforts.

12. Create PR and marketing material to market to as many entities at the University, surrounding industry, and via the internet

- Validation: Upon completion of this thesis, Phillips will have the time and latitude to complete PR materials for distribution.

As noted here, all of the Requirements and Goals, with the exception of one Goal, have been successfully accomplished within the current effort. Furthermore, a new reduced gravity facility has been provided that offers a model for future low-budget drop tower development efforts, and an affordable experience for end-users, with world-class capabilities, as shown in the comparison in Appendix O. The WVU SMiRF provides a drop duration within the top 10 of drop towers worldwide. Gravitational accelerations average $0.06 \mathrm{~g}$ over the drop period. Peak decelerations average less than $50 \mathrm{~g}$, at the current payload weight of $113.8 \mathrm{lbs}$. While the original design for the payload frame was $6,000 \mathrm{lbs}$ at $10 \mathrm{~g}$, and payloads are exhibiting much higher g-loads, thereby reducing the FoS from the originally expected FoS of 20 down to a FoS of 5 or less. The payload frame should continue to be monitored, or steps should be taken to modify the deceleration pad to reduce the impact g-loads. Payload weights of $300 \mathrm{lbs}$ and payload footprint dimensions of nearly $7 \mathrm{ft} \times 5 \mathrm{ft}$ can be accommodated, amongst the largest in facilities of its size. Perhaps most importantly, the WVU SMiRF readily provides access to reduced gravity research to even those researchers with low budgets, allowing them to gain credibility for future funding and even greater advancement. 


\subsection{Suggestions for Future Research / Facility Improvements}

Table 8-1 provides a checklist of current thoughts for future upgrades and development efforts for the WVU SMiRF:

Table 8-1: Facility Improvement Checklist ${ }^{2}$

\begin{tabular}{|c|c|c|l|}
\hline $\begin{array}{c}\text { Status } \\
(\checkmark / \mathbf{X})\end{array}$ & Priority & Type & \multicolumn{1}{|c|}{ Task } \\
\hline & 1 & UI & Provide facility-dedicated fall protection \\
\hline & 2 & UI & Provide facility dedicated ear protection \\
\hline & 3 & UI & $\begin{array}{l}\text { Provide facility-dedicated respiratory } \\
\text { protection }\end{array}$ \\
\hline & 4 & UI & Provide facility-dedicated eye protection \\
\hline & 5 & UI & Provide facility-dedicated hard hats \\
\hline & 7 & UI & Provide facility-dedicated hand protection \\
\hline & 8 & UI & Provide facility-dedicated foot protection \\
\hline & 9 & UI & Improve hand rail stability \\
\hline & 10 & UI & $\begin{array}{l}\text { Finprove top floor access door } \\
\text { top and bottom of tower }\end{array}$ \\
\hline & 11 & UI & $\begin{array}{l}\text { Cap off and lock-out/tag-out unused on-site } \\
\text { building outlets }\end{array}$ \\
\hline & 12 & UI & $\begin{array}{l}\text { Ground WVU SMiRF structure to reduce } \\
\text { attraction of coal dust from other } \\
\text { experimental work performed in the high- } \\
\text { bay area in which the drop tower is located }\end{array}$ \\
\hline & UI & $\begin{array}{l}\text { Create air-tight electromagnet cover to } \\
\text { prevent corrosion on EM face }\end{array}$ \\
\hline & 13 & &
\end{tabular}

${ }^{2}$ Legend:

- $\quad \mathrm{UI}=$ Urgent Improvement

- $\quad$ SATR = Second Article Test

Requirement
- $\quad F F U=$ Future Facility Upgrade

- $\quad F C S U$ = Future Computer Science Upgrade 


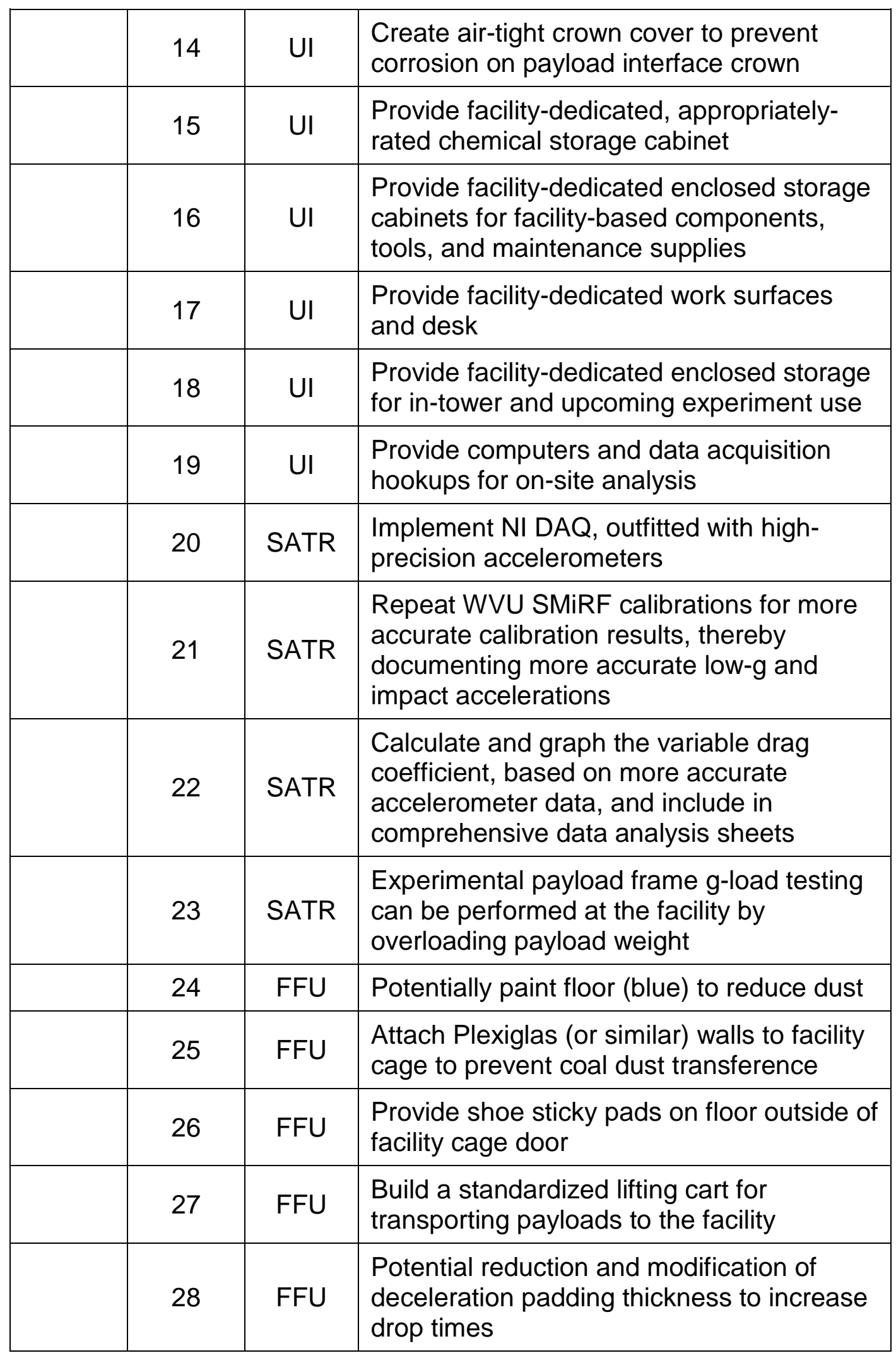




\begin{tabular}{|c|c|c|}
\hline 29 & FFU & $\begin{array}{l}\text { Create video mounting points for on-site } \\
\text { video recording (on WVU SMiRF and } \\
\text { throughout high-bay area) }\end{array}$ \\
\hline 30 & FFU & $\begin{array}{l}\text { Create a black and white background to } \\
\text { facility to measure payload speeds via } \\
\text { video }\end{array}$ \\
\hline 31 & FFU & $\begin{array}{l}\text { Enclose drop tower via clear walls to } \\
\text { prevent any cross-breeze or wind }\end{array}$ \\
\hline 32 & FFU & $\begin{array}{l}\text { Re-rig electromagnet to reduce release } \\
\text { system support line length }\end{array}$ \\
\hline 33 & FFU & $\begin{array}{l}\text { Implement a drag shield solution for } \\
\text { payload to improve the quality of reduced } \\
\text { gravity levels }\end{array}$ \\
\hline 34 & FFU & $\begin{array}{l}\text { Potentially fully enclose facility with clear } \\
\text { walls for pumped evacuation and upgrade } \\
\text { facility components as necessary (e.g. } \\
\text { foam and vacuum don't mix) }\end{array}$ \\
\hline 35 & FFU & $\begin{array}{l}\text { Provide some climate control for summer } \\
\text { heat (e.g. fans, etc.) }\end{array}$ \\
\hline 36 & FFU & $\begin{array}{l}\text { Provide student morale measures - radio } \\
\text { and/or speakers capable of being hooked } \\
\text { up to web, refrigerator, etc. (make WVU } \\
\text { SMiRF a place that people enjoy doing } \\
\text { research) }\end{array}$ \\
\hline 37 & FFU & $\begin{array}{l}\text { Building enhancement: Updated air filter for } \\
\text { high-bay room }\end{array}$ \\
\hline 38 & FCSU & $\begin{array}{l}\text { Provide online scheduling, etc. (see } \\
\text { website suggestions) }\end{array}$ \\
\hline 39 & FCSU & $\begin{array}{l}\text { Develop web-based applications and } \\
\text { remote access to tower for remote } \\
\text { experimentation and real-time } \\
\text { monitoring/operations }\end{array}$ \\
\hline 40 & FFU & $\begin{array}{l}\text { Create video mounting points for remote } \\
\text { monitoring and payload monitoring } \\
\text { throughout facility and building }\end{array}$ \\
\hline 41 & FFU & Build an even bigger, better facility \\
\hline
\end{tabular}




\subsection{Experiment Bibliography}

\subsection{Works Cited}

[1] M. Versluis, C. Blom, R. M. van der Meer, K. van der Weele and D. Lohse, "Leaping Shampoo and the Stable Kaye Effect," 2006.

[Online]. Available: http://doc.utwente.nl/57531/.

[2] M. Nussbaum, "Viscous Fingering (Vid 1 pre-edit)," 8 December 2011. [Online]. Available:

https://www.youtube.com/watch?v=Mrd9gZ01jyw. [Accessed 2014].

[3] M. Nussbaum, "Viscous Fingering - Video Analysis 1," 7 December 2011. [Online]. Available:

https://www.youtube.com/watch?v=Vi_Ifx8XmQk. [Accessed 2014].

[4] C. W. MacMinn and G. H. McKinley, "Extrudate Swell Demonstration," Massachusetts Institute of Technology, [Online]. Available: http://web.mit.edu/nnf/research/phenomena/demos.html. [Accessed 2012].

[5] C. T. Avedisian and Z. Zhao, "The Circular Hydraulic Jump in Low Gravity," in Royal Society of London Proceedings Series A:

Mathematical, Physical, and Engineering Sciences, London, U.K., 2000.

[6] E. J. Watson, "The Radial Spread of a Liquid Jet Over a Horizontal Plane," Journal of Fluid Mechanics, vol. 20, no. 3, pp. 481-499, 1964.

[7] X. Liu and J. H. Lienhard, "Extremely High Influxes Beneath Impinging Liquid Jets," Journal of Heat Transfer, vol. 115, pp. 472476, 1993.

[8] S. Middleman, Modeling Axisymmetric Flows: Dynamics of Films, Jets, and Drops, San Diego, California: Elsevier Science \& Technology Books, 1995, pp. 125-159.

[9] J. W. M. Bush and J. M. Aristoff, "The Influence of Surface Tension on the Circular Hydraulic Jump," Journal of Fluid Mechanics, vol. 489, pp. 229-238, 2003. 
[10] K. Phillips, J. Gross, A. Feathers, T. Blair-Sheppard, T. Wolfe and J. Grimes, "Study of the Hydraulic Jump of Water on a Plat Plate in Microgravity," NASA Reduced Gravity Student Flight Opportunities Program (RGSFOP), Morgantown, WV, 2006.

[11] E. D. Calandrelli, M. Mohebbi, K. G. Phillips, J. M. Kuhlman and D. D. Gray, "Circular Hydraulic Jump Behavior in Microgravity," in 83rd Annual Meeting of the West Virginia Academy of Sciences, Fairmont, West Virginia, 2008.

[12] M. Mohebbi, K. G. Phillips and J. Painter, "Circular Hydraulic Jump Behavior in Microgravity," in 21st Canadian Congress of Applied Mechanics, Toronto, Ontario, 2007.

[13] K. G. Phillips, J. M. Kuhlman, M. Mohebbi, E. D. Calandrelli and D. D. Gray, "Investigation of Circular Hydraulic Jump Behavior in Microgravity," in AIAA 38th Fluid Dynamics Conference, Seattle, Washington, 2008.

[14] D. Doraiswamy, "The Origins of Rheology: A Short Historical Excursion," DuPont iTechnologies, Wilmington, Delaware.

[15] "Dynamic, Absolute and Kinematic Viscosity," [Online]. Available: http://www.engineeringtoolbox.com/dynamic-absolute-kinematicviscosity-d_412.html. [Accessed 2011].

[16] "Non-Newtonian Fluid," Wikimedia Foundtion, Inc., [Online]. Available: http://en.wikipedia.org/wiki/Non-Newtonian_fluid. [Accessed 2011].

[17] O. H. Campanella, P. X. Li and K. A. Ross, "Chapter 24: The Role of Rheology in Extrusion," in Engineering and Food for the 21st Century, J. Welti-Chanes, G. V. Barbosa-Canovas and J. M. Aguilera, Eds., Boca Raton, Florida, CRC Press, 2002, pp. 393-413.

[18] K. P. Menard, "Chapter 2: Basic Rheological Concepts Stress, Strain, and Flow," in Dynamic Mechanical Analysis: A Practical Introduction (2nd Edition), CRC Press, 2008, pp. 15-36.

[19] R. J. Koopmans, "Die Swell or Extrudate Swell," in Polypropylene: An A-Z Reference, J. Karger-Kocsis, Ed., Kluwer Academic Publishers, 1999, pp. 158-162.

[20] L. Brandt and C. Duwig, "On Swell of Viscoelastic Fluids," (Presentation), 1999. 


\subsection{Rheology References}

G. V. Barbosa-Canovas and A. Ibarz, "Chapter 6: Rheology of Food Products," in Unit Operations in Food Engineering, CRC Press, 2002, pp. 89-142.

C. Barry, A. M. Baker and J. Mead, "Chapter 1: Introduction to Polymers and Plastics," in Handbook of Plastics Technologies: The Complete Guide to Properties and Performance, New York, New York, McGraw-Hill, 2006.

R. P. Chhabra, "Chapter 2: Non-Newtonian Fluid Behavior," in Bubbles, Drops, and Particles in Non-Newtonian Fluids, Boca Raton, Florida, CRC Press, 2007, pp. 9-49.

R. P. Chhabra, "Chapter 7: Non-Newtonian Fluid Flow in Porous Media and Packed Beds," in Bubbles, Drops, and Particles in Non-Newtonian Fluids, Second Edition, CRC Press, 2006, pp. 279-394.

Y. Donggang and Y. Wang, "Ultra-Fine Filament Warns Made by Supersonic Jet Splitting," National Textile Center, 2007.

D. Doraiswamy, "Chapter 37: The Rheology of Non-Newtonian Fluids," in The Engineering Handbook, Second Edition, R. C. Dorf, Ed., CRC Press, 2004.

H. Huang, "Non-Newtonian Effect on Ink-Jet Droplet Formation: A Mathematical Model," in Proceedings of the Forty-Fifth European Study Group with Industry, Leiden, The Netherlands, 2003.

H. Hui, "Viscosity of Silicate Melts," Ann Arbor, Michigan, 2008.

A. C. Jain, "Study of Oscillating Jets Extruded from Rectangular Slots," Philadelphia, Pennsylvania, 2001.

E. F. Matthys, "Chapter 56: Heat Transfer to Non-Newtonian Fluids," in The Engineering Handbook, Second Edition, R. C. Dorf, Ed., CRC Press, 2004. 
M. O. McLinden and J. D. Splett, "A Liquid Density Standard Over Wide Ranges of Temperature and Pressure Based on Toluene," Journal of Research of the National Institute of Standards and Technology, vol. 113, no. 1, pp. 29-67, January-February 2008.

PerkinElmer, Inc., "Mechanical Properties of Films and Coatings," PerkinElmer, 2011. [Online]. Available:

http://www.perkinelmer.com/CMSResources/lmages/4474269APP_Relaxation ofPVC.pdf.

M. Roxas and S. Ju, "Fluid Dynamics Analysis of Desktop-Based Fused Deposition Modeling Rapid Prototyping," Toronto, Ontario, 2008. G. Russo, "Spectral Element Methods for Predicting the Die-Swell of Newtonian and Viscoelastic Fluids," Cardiff University, Cardiff, Wales, 2009.

S. R. Shukla, "Rheological Scaling and Bubble Nucleation of a PolymerDiluent Solution in Extrusion Foaming," The Ohio State University, Columbus, Ohio, 2007.

J. F. Swindells, C. F. Snyder, R. C. Hardy and P. E. Golden, "Viscosities of Sucrose Solutions at Various Temperatures: Tables of Recalculated Values," National Bureau of Standards Circular, vol. 440, 31 July 1958.

\subsection{Die Swell References}

S. Blairs, "Correlation Between Surface Tension, Density, and Sound Velocity of Liquid Metals," Journal of Colloid and Interface Science, vol. 302, pp. 312-314, 2006.

S. Bunditsaovapak, T. Fagon and S. Thenissara, "Surface Tension Effect to Die-Swell Extrusion of Viscoelastic Fluid," in $5^{\text {th }}$ International Conference on Complex Systems, Sendai, Japan, 2008.

P. Cassagnau and C. Barres, "Chapter 14: Rheological Behavior of Rubber Nanocomposites - Extrudate Swell," in Rubber Nanocomposites: Preparation, Properties, and Applications, S. Thomas and R. Stephen, Eds., John Wiley \& Sons, 2010, p. 384 
J. den Doelder, M. Mangus and M. B. Kapur, "Correlations Between HighDensity Polyethylene Viscoelasticity and Annular Extrudate Swell," in The $X V$ International Congress on Rheology: The Society of Rheology $80^{\text {th }}$ Annual Meeting, Monterey, California, 2008.

E. J. Hinch, "Conceptual Model of the Climate: Non-Newtonian Geophysical Fluid Dynamics Lecture 1: Introduction," Woods Hole Oceanography Institute, Cambridge, United Kingdom, 2004.

W. R. Hu and Q. Hu, "Influence of Thermocapillary Flow in a Liquid Film Jet," Acta Astronautica, vol. 54, no. 1, pp. 53-59, 2002.

D. D. Joseph, J. E. Matta and K. Chen, "Delayed Die Swell," Journal of Non-Newtonian Fluid Mechanics, vol. 24, pp. 31-65, 1987.

M. Kaseem, K. Hamad and F. Deri, "Rheological and Die Swell Measurements of Thermoplastic Starch/Acrylonitrile-Butadiene-Styrene Blends," Malaysian Polymer Journal, vol. 7, no. 1, pp. 22-27, 2012.

M. M. Kostic and L. G. Reifschneider, "Design of Extrusion Dies," in Encyclopedia of Chemical Processing, S. Lee, Ed., New York, New York, Taylor \& Francis Group, 2006, pp. 633-650.

A. Naveenji, S. Malavarayan and M. Kaushik, "CFD Analysis on Discharge Coefficient During Non-Newtonian Flows Through Orifice Meter," International Journal of Engineering Science and Technology, vol. 2, no. 7, pp. 3151-3164, 2010.

T. R. Salamon, D. E. Bornside, R. C. Armstrong and R. A. Brown, "The Role of Surface Tension in the Dominant Balance in the Die Swell Singularity," Physics of Fluids, vol. 7, no. 10, 1995.

K. Wang, "Chapter 4: Die Swell of Complex Polymeric Systems," in Viscoelasticity - From Theory to Biological Applications, J. de Vicente, Ed. 2012, pp. 77-96. 
M. Yao, G. McKinley and B. Debbaut, "Extensional Deformation, Stress relaxation, and Necking Failure of Viscoelastic Filaments," Journal of NonNewtonian Fluid Mechanics, vol. 79, no. 2, pp. 469-501, 1 November 1998.

\subsection{Drop Tower Bibliography}

\subsection{Works Cited (Drop Tower History)}

[21] R. Scha, "to drop [to release an object in a gravitational field]: scientific demonstrations and experiments," [Online]. Available: http://radicalart.info/physics/gravity/Drop/MotionLawExperiments.htm I. [Accessed 14 July 2011].

[22] G. Sarton, "Simon Stevin of Burges," Isis, vol. 21, no. 2, pp. 241-303, July 1934.

[23] "Simon Stevin," 27 April 2010. [Online]. Available: http://en.citizendium.org/wiki/Simon_Stevin. [Accessed 14 July 2011].

[24] "Leaning Tower of Pisa," Wikimedia Foundation, Inc., 13 July 2011. [Online]. Available:

http://en.wikipedia.org/wiki/Leaning_Tower_of_Pisa. [Accessed 14 July 2011].

[25] E. A. Moody, "Galileo and Avempace," Journal of the History of Ideas, vol. 12, no. 2, pp. 163-193, April 1951.

[26] M. Bellis, "Galileo Galilei," The New York Times Company, [Online]. Available:

http://inventors.about.com/od/gstartinventors/a/Galileo_Galilei.htm. [Accessed 14 July 2011].

[27] A. Martinez, Science Secrets, 1 ed., Pittsburgh, Pennsylvania: University of Pittsburgh Press, 2011.

[28] A. Einstein and L. Infeld, The Evolution of Physics: The Growth of Ideas from Early Concepts to Relativity and Quanta, C. Snow, Ed., Cambridge, England: Cambridge University Press, 1938. 
[29] J. H. Lienhard, "No. 422: Shot Tower," University of Houston, 1997. [Online]. Available: http://www.uh.edu/engines/epi422.htm. [Accessed 14 July 2011].

[30] "Shot Tower," Wikimedia Foundation, Inc., 2 June 2011. [Online]. Available: http://en.wikipedia.org/wiki/Shot_tower. [Accessed 14 July 2011].

[31] I. Garman, "loE Number: 458483," English Heritage, 22 December 2004. [Online]. Available:

http://www.imagesofengland.org.uk/details/default.aspx?id=458483. [Accessed 14 July 2011].

[32] J. L. Wishlade, "IoE Number: 469839," English Haritage, 12 April 2001. [Online]. Available:

http://www.imagesofengland.org.uk/details/default.aspx?id=469839. [Accessed 14 July 2011].

[33] "Chester Shot Tower," Wikimedia Foundation, Inc., 26 June 2011. [Online]. Available: http://en.wikipedia.org/wiki/Chester_Shot_Tower. [Accessed 14 July 2011].

[34] "Jackson Ferry Shot Tower," Wikimedia Foundation, Inc., 29 June 2011. [Online]. Available:

http://en.wikipedia.org/wiki/Jackson_Ferry_Shot_Tower. [Accessed 14 July 2011].

[35] "Sparks Shot Tower," Wikimedia Foundation, Inc., 20 June 2011. [Online]. Available: http://en.wikipedia.org/wiki/Sparks_Shot_Tower. [Accessed 14 July 2011].

[36] J. H. Lienhard, "No. 954: Moses Austin's Lead," University of Houston, 1997. [Online]. Available:

http://www.uh.edu/engines/epi954.htm. [Accessed 14 July 2011].

[37] Shot Tower - Potosi, Missouri. [Art]. Brazoria County Historical Museum.

[38] R. Avery, "Sparks Shot Tower," Independence Hall Association, 4 July 1995. [Online]. Available:

http://www.ushistory.org/oddities/sparks.htm. [Accessed 14 July 2011]. 
[39] "Lambeth Shot Tower," Wikimedia Foundation, Inc., 3 June 2011. [Online]. Available:

http://en.wikipedia.org/wiki/Shot_Tower,_Lambeth. [Accessed 14 July 2011].

[40] J. Constable, Artist, The Opening of Waterloo Bridge. [Art]. Tate Britain, 1832.

[41] H. Roberts and W. H. Godfrey, Eds."Shot Tower and Lead Works, No. 62 Belvedere Road," Survey of London, vol. 23, p. 47, 1951.

[42] W. L. Wyllie, Artist, Shot Tower (1303). [Art]. National Maritime Museum, Early 20th Century.

[43] "Phoenix Shot Tower," Wikimedia Foundation, Inc., 17 June 2011. [Online]. Available: http://en.wikipedia.org/wiki/Phoenix_Shot_Tower. [Accessed 14 July 2011].

[44] "Baltimore's Phoenix Shot Tower," Beaches and Towns Network, Inc., 2011. [Online]. Available: http://www.baltimore.to/ShotTower/. [Accessed 14 July 2011].

[45] "Tower Hill State Park," 9 December 2004. [Online]. Available: http://lwr.state. wi.us/subcategory. asp?linksubcatid $=354 \&$ linkcatid $=48$ 3\&linkid $=$. [Accessed 14 July 2011].

[46] "Tower Hill State Park," Wikimedia Foundation, Inc., 3 March 2011. [Online]. Available: http://en.wikipedia.org/wiki/Tower_Hill_State_Park. [Accessed 14 July 2011].

[47] "History of 4708 South Broadway on the Mississippi River Bluffs," [Online]. Available: http://www.thebluffsonbroadway.com/history/history.html. [Accessed 14 July 2011].

[48] "Dubuque Shot Tower," Wikimedia Foundation, Inc., 11 July 2011. [Online]. Available: http://en.wikipedia.org/wiki/Shot_Tower_(Dubuque). [Accessed 14 July 2011]. 
[49] "Clifton Hill Shot Tower," Wikimedia Foundation, Inc., 8 May 2011. [Online]. Available:

http://en.wikipedia.org/wiki/Clifton_Hill_Shot_Tower. [Accessed 14 July 2011].

[50] "Coops (Melbourne Central) Shot Tower," Wikimedia Foundation, Inc., 1 July 2011. [Online]. Available:

http://en.wikipedia.org/wiki/Coops_(Melbourne_Central)_Shot_Tower . [Accessed 14 July 2011].

[51] J. H. Black, "loE Number: 303353," English Heritage, 23 August 1999. [Online]. Available:

http://www.imagesofengland.org.uk/details/default.aspx?id=303353. [Accessed 14 July 2011].

[52] M. Jones, "Colonial Ammunition Company Shot Tower," The New Zealand Historic Places Trust, 21 August 2001. [Online]. Available: http://www.historic.org.nz/en/AboutUs.aspx. [Accessed 14 July 2011].

[53] G. V. Sanders, "Gravity Molds Shot in a Modern Tower," Popular Science Magazine, vol. 145, no. 4, pp. 123-127, October 1944.

[54] The First Drop Tower Built in 1956. [Art]. NASA Glenn Research Center, 1956.

[55] L. C. Elonen-Wright, "NASA GRC 2.2 Second Drop Tower," NASA, 1 February 2008. [Online]. Available:

http://facilities.grc.nasa.gov/drop/index.html. [Accessed 14 July 2011].

[56] "Wireless Drop Tower for Microgravity Demonstrations Educational Brief," NASA Headquarters, Washington, D.C., 2003.

[57] L. C. Elonen-Wright, "Zero Gravity Research Facility," NASA, 1 February 2008. [Online]. Available: http://facilities.grc.nasa.gov/zerog/index.html. [Accessed 14 July 2011].

[58] "Drop Tower Bremen," ZARM; University of Bremen, 2009. [Online]. Available: http://www.zarm.uni-bremen.de/drop-tower. [Accessed 14 July 2011]. 
[59] C. Eigenbrod, "Drop Tower," European Space Agency, 9 November 2010. [Online]. Available:

http://www.esa.int/SPECIALS/HSF_Research/SEMDQZSTGOF_0.ht ml. [Accessed 14 July 2011].

[60] E. Ceglia, European Users Guide to Low Gravity Platforms, W. Carey, Ed., Noordwijk, The Netherlands: Erasmus User Centre and Communication Office (HME-HE), European Space Agency, 2005, pp. 3-1 through 3-22.

[61] T. J. Rathz and M. B. Robinson, "The NASA/Marshall Space Flight Center Drop Tube User's Manual," National Aeronautics and Space Administration, Huntsville, Alabama, 1990.

[62] M. L. Tuma and D. J. Chenevert, "Ensuring Safe Exploration: Ares Launch Vehicle Integrated Vehicle - Ground Vibration Testing," National Aeronautics and Space Administration, Huntsville, Alabama.

[63] M. F. Fisher and J. J. Martin, "Analysis of Cryogenic Propellant Behaviour in Microgravity and Low Thrust Envrionments," Cryogenics, vol. 32, no. 2, 1992.

[64] "Microgravity," University of Colorado, 2003. [Online]. Available: http://www.colorado.edu/engineering/ASEN/asen5519/02microgravit y.html. [Accessed 14 July 2011].

[65] "Making a Living Underground," ProBoards, Jul. 2009. [Online]. Available:

http://www.japanalicious. proboards.com/index.cgi?board=others\&act ion=display\&thread=277. [Accessed 14 July 2011].

[66] "JAMIC," Wikimedia Foundation, Inc., 17 June 2011. [Online]. Available: http://en.wikipedia.org/wiki/JAMIC. [Accessed 14 July 2011].

[67] Onuki, Misuzu; Lauer, Charles;, "Sources and Uses of Short Duration Microgravity Experiments in Japan," in Next-Generation Suborbital Researchers Conference, Orlando, Florida, 2011.

[68] T. Tsujino, "Trends in Research on the Utilization of Microgravity Competition and Collaboration Between Research in Space and Research on the Ground," Quarterly Review, no. 21, October 2006. 
[69] V. A. Thomas, N. S. Prasad and C. A. M. Reddy, "Microgravity Research Platforms - A Study," Current Science, vol. 79, no. 3, pp. 336-340, 10 August 2000.

[70] T. Nakanomori and S. Asano, "Status Reviews of the Dropshaft in Japan," Tokyo, 1995.

[71] M. Reluga, B. Selles and J. Chung, "Boiling Revisited," National Science Foundation Summer Science and Engineering Program, 1995.

[72] W. Struble, "Fluid Flow in Microgravity," National Science Foundation Summer Science and Engineering Program, 1997.

[73] T. J. Snyder, "WSU 2.1 Second Microgravity Drop Tower Design, Construction, and Performance," Washington State University, Pullman, Washington, 1993.

[74] J. N. Chung, "Bubble Dynamics, Two-Phase Flow, and Boiling Heat Transfer in a Microgravity Environment," in Second Microgravity Fluid Physics Conference, Cleveland, Ohio, 1994.

[75] Drop Shaft and Aircraft Experiment Guidebook, 1 ed., Japan: Japan Space Forum and Japan Aerospace Exploration Agency, 2005.

[76] "National Microgravity Laboratory," Institute of Mechanics, Chinese Academy of Sciences, 2009. [Online]. Available: http://english.imech.cas.cn/rh/rd/nml/. [Accessed 10 July 2014].

[77] A. Marchese, J. Chen, M. Hammill, T. Vaughn, F. Bellottie, S. Jurewicz, G. Soriano, C. Boland and J. Pusey, "Development of a 11 Second Drop Tower Apparatus for Fundamental Combustion and Fluid Dynamics Research," Colorado State University, 5 August 2004. [Online]. Available:

http://www.engr.colostate.edu/ marchese/droptower.htm. [Accessed 14 July 2011]. 
[78] A. Marchese, J. Chen, M. Hammill, T. Vaughn, F. Bellottie, S. Jurewicz, G. Soriano, C. Boland and J. Pusey, "Development of a 11 Second Drop Tower Apparatus for Fundamental Combustion and Fluid Dynamics Research," Rowan University, 5 August 2004. [Online]. Available:

http://users.rowan.edu/ marchese/droptower.html. [Accessed 14 July 2011].

[79] "MG Facility at Akabira," University of Hokkaido, 4 September 2005. [Online]. Available: http://york-me.eng.hokudai.ac.jp/MGfacility.html. [Accessed 14 July 2011].

[80] "Microgravity Drop Tower Facility," Queensland University of Technology, 10 March 2011. [Online]. Available: http://www.bee.qut.edu.au/research/projects/microgravity/. [Accessed 14 July 2011].

[81] "Flowfield Imaging Lab," The University of Texas at Austin, 2007. [Online]. Available: http://research.ae.utexas.edu/FlolmLab/drpt.php. [Accessed 14 July 2011].

[82] "The Neil Armstrong Hall of Engineering," Purdue University, 2011. [Online]. Available:

https://engineering. purdue.edu/Engr/AboutUs/Facilities/ArmstrongHa II/Features/. [Accessed 14 July 2011].

[83] "Dryden Drop Tower," Portland State University, 2010. [Online]. Available: http://www.ddt.pdx.edu/node/599. [Accessed 14 July 2011].

[84] K. Phillips, E. Calandrelli, M. Nussbaum and J. Kuhlman, "Short Microgravity Research Facility," West Virginia Academy of Sciences 85th Annual Meeting, 10 April 2010.

\subsection{Drop Tower References}

"Dropping In a Microgravity Environment (DIME): Educator's Resource Guide," The National Aeronautics and Space Administration Glenn Research Center, Cleveland, Ohio, 2009. 
Drop Shaft and Aircraft Experiment Guidebook, 1 ed., Japan: Japan Space Forum and Japan Aerospace Exploration Agency, 2005.

"NASA Reduced-Gravity Carriers for Experiment Operations," The National Aeronautics and Space Administration, Marshall Space Flight Center, Microgravity Research Program Office, Huntsville, Alabama.

Y. Takagi, "The Drop Tower Experiments as a Tool for Studies of Small Body Surface Phenomena and for Spacecraft Design of Small Body Explorations," in The First Meeting of The International Primitive Body Exploration Working Group (IPEWG), Okinawa, Japan, 2008.

"Zero Gravity Research Facility," The National Aeronautics and Space Administration Glenn Research Center, [Online]. Available: http://facilities.grc.nasa.gov/zerog/. 
Development of the West Virginia University Small Microgravity Research Facility (WVU SMiRF)
Copyright $^{\odot} 2014$

Kyle G. Phillips 


\subsection{Appendix A}

\section{A Historical Compilation of the Drop Tower}

Appendix A: A Historical Compilation of the Drop Tower presents the chronological history, from 1575 through 2014, of the evolution of the tower as a scientific and engineering tool. Based on the Kyle Phillips' extensive research, to his knowledge, this history is comprehensive to the date of publication. The intent was to historically document the evolution of the drop tower, thereby learning from past lessons for the purpose of the current effort, and to provide a reference for those to come on that history. We may learn from our history, both strengths and weaknesses, without which guidance we are more likely to fall short of advancement. 
1575 Girolamo Borro, a philosophy teacher in Pisa, Italy, dropped wooden and lead balls from the highest window of his house, incorrectly concluding that the wooden ball reaches the ground faster, because it was thought at the time that air was a "heavy" element, and the wood sphere contained more air than the lead sphere [21]

1576 Giuseppe Moletti, a mathematics professor in Padua, Italy, releases experiments similar to Borro's in 1575 from a high tower, but correctly observed that the two differing spheres reach the ground simultaneously [21]

1586 Simon Stevin and Hugo Grotius' Father performed a recorded and peer-reviewed falling bodies experiment at the New Church in Delft, dropping lead and wood spheres in separate experiments (2 lead balls, one 10x heavier than the other, as well as one wood and one lead ball of the same size) from a height of $30 \mathrm{ft}$., and concluded that no matter what the experimental constraints, the balls landed at the same time after hearing only one, simultaneous impact [21] [22] [23]

Circa 1589 According to the famous legend, Galileo performed a peer-reviewed falling bodies experiment at the Tower of Pisa, similar to the one performed by Stevin. Galileo, just as Stevin did, went on to legitimately perform recorded inclined plane experiments and "thought experiments," which were later recognized as revolutionary by both Newton and Einstein, and were central to their mathematical formulations of gravity [21] [24] [25] [26] [27] [28]

1589 - 1782 During this period, it is assumed here that various other free falling body and inclined plane experiments were carried out from the windows of buildings, church towers, and other locations to confirm and advance the study of falling bodies [21] 
1782 William Watts created the first shot tower by building a large, very tall addition onto his house, pouring lead through a sieve, and allowing the molten lead to drop the full length of his new addition in Redcliffe, Bristol, UK. During the length of the drop, the molten lead assumes the shape with the least amount of tensile stress, a sphere, and cools during the drop to a point at which the surface of the lead balls has cooled enough to keep its spherical shape when dropped into a water basin, where the spherical lead balls finish cooling. These lead balls were then used as lead shot for military ammunition [29] [30] [31] [32] [33] [34]

Circa 1790s Jackson Ferry Shot Tower was built in Wythe County, VA by John Robinson and William Byrd III, while designed by William Herbert, a Welshman employed by William Watts - the original inventor and patentee of the shot tower, after being recruited by Col. John Chiswell to protect the lead mines owned by Robinson, Byrd, and Moses Austin, father of Stephen F. Austin. The mines and the shot tower later provided lead and shot for the Revolutionary and Civil Wars and is now the centerpiece of Shot Tower Historical State Park [30] [33] [34] [35] [36]

Circa 1798 - 1819 Potosi Shot Tower was built in Potosi, Missouri, most likely by Moses Austin's group [36] [37]

1799 Walker Parker \& Co. created a shot tower in Chester City, UK to produce lead shot for the Napoleonic wars [30] [32] [33]

1808 Sparks Shot Tower was built in Philadelphia, PA to produce shot for hunters, and later provided shot for the War of 1812 and the Civil War. It was operated until 1903 and was later bought by the city of Philadelphia [30] [35] [38] 
1826 Lambeth Lead Works, operated by Thomas Maltby \& Co. constructed the Lambeth Shot Tower on the South Bank of the River Thames in London, England for making large and small shot. It produced shot until 1949 and was later used for the Festival in Britain in 1951, and then destroyed to make room for Queen Elizabeth Hall [39] [40] [41] [42]

1828 Phoenix Shot Tower (AKA Merchants' Shot Tower, or Old Baltimore Shot Tower) was built by Jacob Wolfe for the Merchant's Shot Tower Co., and its cornerstone laid by Charles Carroll, a signer of the Declaration of Independence. It remained the tallest building in the US until the Trinity Church in New York was built in 1846, and produced shot until 1892, and is now owned by the city of Baltimore [43] [44]

1833 Daniel Whitney built a shot tower near Spring Green, Wisconsin that operated until 1860, and later became a summer resort, run by Frank Lloyd Wright's father, and is currently Tower Hill State Park [30] [45] [46]

Circa 1843 Charles Loveland Tucker allowed St. Amant Michau to build a shot tower on his property overlooking the Mississippi River in St. Louis, Missouri, which provided shot during the Civil War [47]

1856 Dubuque Shot Tower was erected in Dubuque, lowa to provide shot for the military, and was later repurposed as a watchtower for the nearby lumberyard, and is now part of ongoing renovations on the riverfront by the city of Dubuque [30] [48]

1882 Clifton Hill Shot Tower was built in Melbourne, Australia [30] [49]

1888 Coops Shot Tower was built near the center of Melbourne, Australia and now sits under the Melbourne Central Cone as an anchor to Melbourne's City Center Business District [50] 
1916 Tynemouth Shot Tower was built in Tynemouth, UK to produce shot and doubled as a lookout post for range finding of artillery in Tynemouth Battery to resist German forces [33] [51]

Circa 1916 Colonial Ammunition Company constructed a shot tower in New Zealand for shotgun cartridges and later supplied shot for World War I [30] [52]

1944 Popular Science magazine reported on the Winchester Repeating Arms Co.'s shot tower in New Haven, Connecticut [53]

1956 The First Drop Tower, built specifically for reduced gravity research, was developed at NASA Glenn Research Center at Lewis Field [54]

1959 NASA GRC's 2.2-Second Drop Tower was constructed to support the US side of the Space Race to understand how technologies would perform onorbit. Located on the edge of a ravine in Brookpark, Ohio, the NASA GRC 2.2s Drop Tower is still in operation at the time of this publication, and has been a model for many of the drop tower research facilities around the world ever since [55] [56]

1966 NASA GRC's Zero Gravity Facility opened with a 5second drop tube, capable of doubling the free-fall length via catapult, and remains one of the longest such facilities in the world to the present day, at time of this publication [57]

1969 In Bristol, England, Seldon Bush and Patent Shot Company built a replacement shot tower to William Watts' original shot tower to continue to produce lead shot [31] 
1990 The Center of Applied Space technology and Microgravity (ZARM) opened the Bremen Drop Tower for the University of Bremen in Germany, within which scientific payloads can experience nearly 5 or 10 seconds of microgravity, similar in concept to the NASA GRC Zero Gravity Facility, and is still in operation [58] [59] [60]

Circa 1990 NASA Marshall Space Flight Center added drop tower and drop tube capabilities to the historic Test Stand 4550 , which was originally developed to perform vibration testing for the Apollo program and the Saturn $\checkmark$ rocket, which was later outfitted to do the same for the Shuttle program, allowing nearly 4.5 seconds of drop, primarily used for material testing [61] [62] [63] [64]

Circa 1990 Japanese Microgravity Center (JAMIC) utilized an abandoned vertical mine shaft near Hokkaido, Japan to achieve approximately 10 seconds of drop using gas thrusters and a double-chambered payload frame/drag shield design to overcome air resistance and properly accelerate payloads down the vertical shaft [65] [66] [67] [68] [69] [70]

Circa 1993 Washington State University constructed two drop tower research facilities, 0.6-second and 2.1-second facilities, to perform experimental research in Pullman, Washington [71] [72] [73] [74]

1995 Micro-Gravity Laboratory of Japan (MGLAB) located near Toki, Japan opened to provide 4.5 seconds of freefall [70] [75]

Circa 2004 National Microgravity Laboratory (NML) is founded in Beijing, China as the Key Laboratory of Microgravity of the Chinese Academy of Sciences to aid China in developing technologies for their manned space program. [76] 
Circa 2004 Colorado State University, in Fort Collins Colorado, built a 1.1-second drop tower research facility, modeled after the NASA GRC 2.2s Tower, in conjunction with Rowan University through a National Science Foundation grant [77]

Circa 2004 Rowan University built a 1.1-second drop tower research facility, in Glassboro, New Jersey, modeled after the NASA GRC 2.2s Tower, in conjunction with Colorado State University through a National Science Foundation grant [78]

Circa 2005 Akabira drop tower was built near the Akabira district in Japan, with the support of both academic and commercial institutions for Hokkaido University, capable of 3 seconds of free fall, which remains in operation at the time of this publication [79]

Circa 2005-2010 Queensland University of Australia built a 2-second drop tower research facility near Brisbane, Australia, which remains in operation at the time of this publication [80]

Circa 2007 The University of Texas Drop Tower Facility was constructed near Austin, Texas to provide 1.1 seconds of near microgravity conditions for experimentation, which remains in operation at the time of this publication [81]

Circa 2008 In West Lafayette, Indiana, Purdue University constructed a 4-story tall drop tower integrated into their new engineering building to study two-phase flow and other fluid dynamics phenomena, after previously constructing a 1.1-second drop tower some years previous [82]

2010 Portland State University opened its Dryden Drop Tower in Portland, Oregon, providing 2.13 seconds of microgravity conditions for experimental research, which remains in operation at the time of this publication [83] 
2014 West Virginia University opened its Small Microgravity Research Facility (SMiRF) drop tower, designed by Kyle Phillips, providing over 1 second of near microgravity conditions for cost-effective, frequently repeatable experimentation in and around the region surrounding Morgantown, West Virginia [84] 


\subsection{Appendix B}

\section{WVU SMiRF Concept Analysis}

Appendix B: WVU SMiRF Concept Analysis presents images of the concept analysis spreadsheet developed to compute initial calculations, based on theoretical equations and user input. The intent is to show the reader a visual layout of the spreadsheet and to indicate the order of calculations through which the researcher progressed in order to provide initial concept estimates of the drop tower performance. 


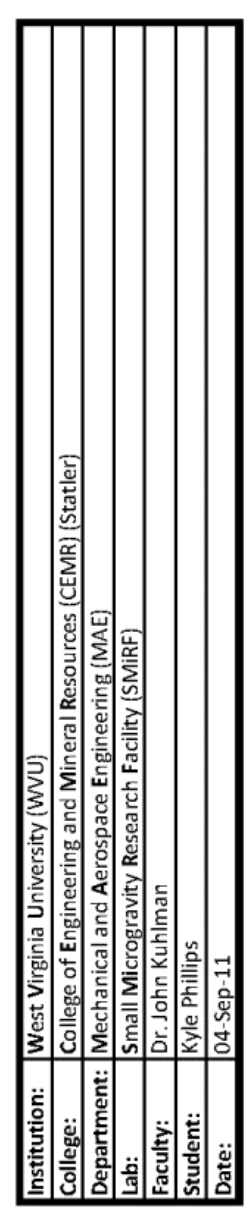

\begin{tabular}{|c|c|c|c|c|c|c|c|}
\hline 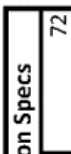 & $\sqrt{2}$ & \begin{tabular}{|l|} 
go \\
\end{tabular} & 9 & 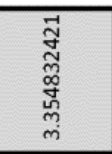 & 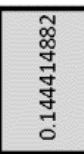 & 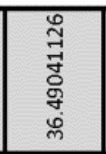 & 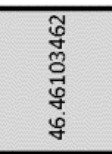 \\
\hline 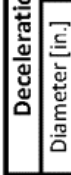 & 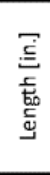 & 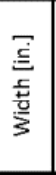 & 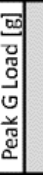 & 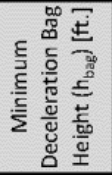 & 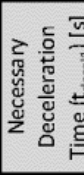 & 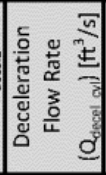 & 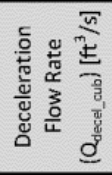 \\
\hline
\end{tabular}

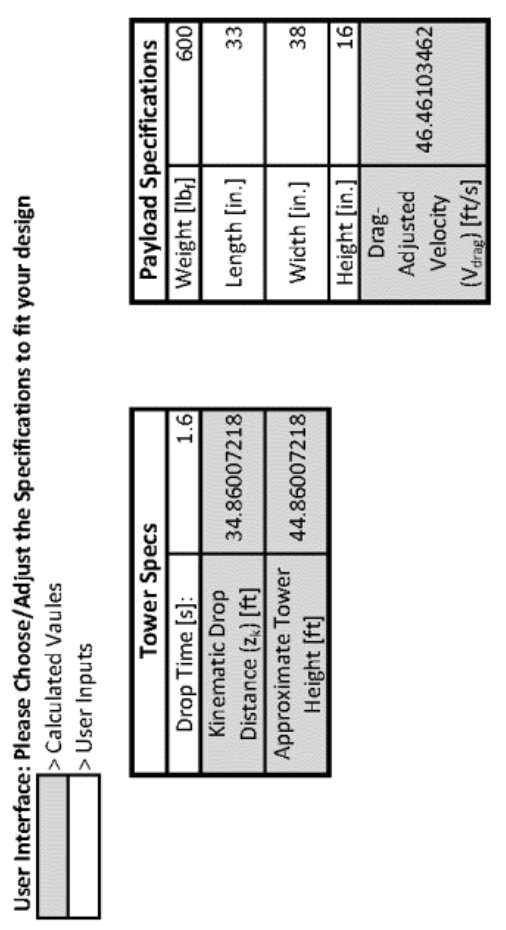




\begin{tabular}{|c|c|c|c|c|c|c|}
\hline Institution: & \multicolumn{5}{|c|}{ West Virginia University (WVU) } & \\
\hline College: & \multicolumn{5}{|c|}{ College of Engineering and Mineral Resources (CEMR) (Statler) } & \\
\hline Department: & \multicolumn{5}{|c|}{ Mechanical and Aerospace Engineering (MAE) } & \\
\hline Lab: & \multicolumn{5}{|c|}{ Small Microgravity Research Facility (SMiRF) } & \\
\hline Faculty: & \multicolumn{5}{|c|}{ Dr. John Kuhlman } & \\
\hline Student: & \multirow{2}{*}{\multicolumn{5}{|c|}{\begin{tabular}{|l|} 
Kyle Phillips \\
O4-Sep-11
\end{tabular}}} & \\
\hline \multirow[t]{25}{*}{ Date: } & & & & & & \\
\hline & \multicolumn{2}{|c|}{ User Input Variables } & \multicolumn{2}{|c|}{ User Input Calculations } & \multicolumn{2}{|c|}{ Constants } \\
\hline & \multicolumn{2}{|c|}{ Payload Specs } & \multicolumn{2}{|c|}{ Payload } & \multicolumn{2}{|c|}{ Drag Constants } \\
\hline & Weight $\left[\mathrm{lb}_{\mathrm{f}}\right]$ & 600 & Weight $[\mathrm{N}]$ & 2668.93296 & $\mathrm{E}\left[\mathrm{m} / \mathrm{s}^{2}\right]$ & 9.806 \\
\hline & Length [in.] & 33 & Length $[\mathrm{m}]$ & 0.8382 & $C_{D}$ & 1 \\
\hline & Width [in.] & 38 & Width [m] & 0.9652 & $\rho_{\text {air }}\left[\mathrm{kg} / \mathrm{m}^{3}\right]$ & 1.225 \\
\hline & Height [in.] & 16 & Height $[\mathrm{m}]$ & 0.4064 & $A_{\text {ref. }}\left[m^{2}\right]$ & 0.809031 \\
\hline & \multicolumn{2}{|c|}{ Deceleration Specs } & $A_{\text {frontal }}\left[\mathrm{m}^{2}\right]$ & 0.80903064 & Mass [kg] & 272.1735 \\
\hline & Diameter [in.] & 72 & $A_{\text {frontal }}\left[\mathrm{in}^{2}\right]$ & 1254 & a & 9.806 \\
\hline & Length [in.] & 72 & \multicolumn{2}{|c|}{ Deceleration } & $\mathrm{b}$ & -0.00182 \\
\hline & Width [in.] & 48 & Diameter $[\mathrm{m}]$ & 1.8288 & $\mathrm{P}$ & 0.133616 \\
\hline & Height [ft.] & 3.354832 & Length $[\mathrm{m}]$ & 1.8288 & $V_{\text {teminal }}[\mathrm{m} / \mathrm{s}]$ & 73.38939 \\
\hline & \multirow[t]{13}{*}{ Peak G Load [g] } & 10 & Width $[\mathrm{m}]$ & 1.2192 & \multicolumn{2}{|c|}{ Conversion Factors } \\
\hline & & & Height $[\mathrm{m}]$ & 0.085212743 & $11 b_{f}-N$ & 4.448222 \\
\hline & & & Diameter [ft.] & 6 & 1 in $-m$ & 0.0254 \\
\hline & & & Length [ft.] & 6 & $1 \mathrm{in}-\mathrm{ft}$ & 0.083333 \\
\hline & & & Width [ft.] & 4 & $1 \mathrm{~m}-\mathrm{ft}$ & 3.28084 \\
\hline & & & Height [ft.] & 0.279569368 & $1 \mathrm{~N}-\mathrm{Ib}_{\mathrm{f}}$ & 0.224809 \\
\hline & & & Vol $_{\mathrm{O} y}\left[\mathrm{~m}^{3}\right]$ & 0.48957658 & & \\
\hline & & & $\mathrm{Vol}_{\mathrm{cub}}\left[\mathrm{m}^{3}\right]$ & 0.18999655 & & \\
\hline & & & $\mathrm{Vol}_{\mathrm{Q} \mid l}\left[\mathrm{in}^{3}\right]$ & 758.845216 & & \\
\hline & & & $V_{\mathrm{c}} \mathrm{I}_{\mathrm{cub}}\left[\mathrm{in}^{3}\right]$ & 11594.30085 & & \\
\hline & & & $\mathrm{Vol}_{\mathrm{O}, 1}\left[\mathrm{ft}^{3}\right]$ & 5.269758444 & & \\
\hline & & & $V_{o l} I_{\text {cub }}\left[\mathrm{ft}^{3}\right]$ & 6.709664842 & & \\
\hline & & & Deceleration $\left[\mathrm{m} / \mathrm{s}^{2}\right]$ & 98.06 & & \\
\hline
\end{tabular}




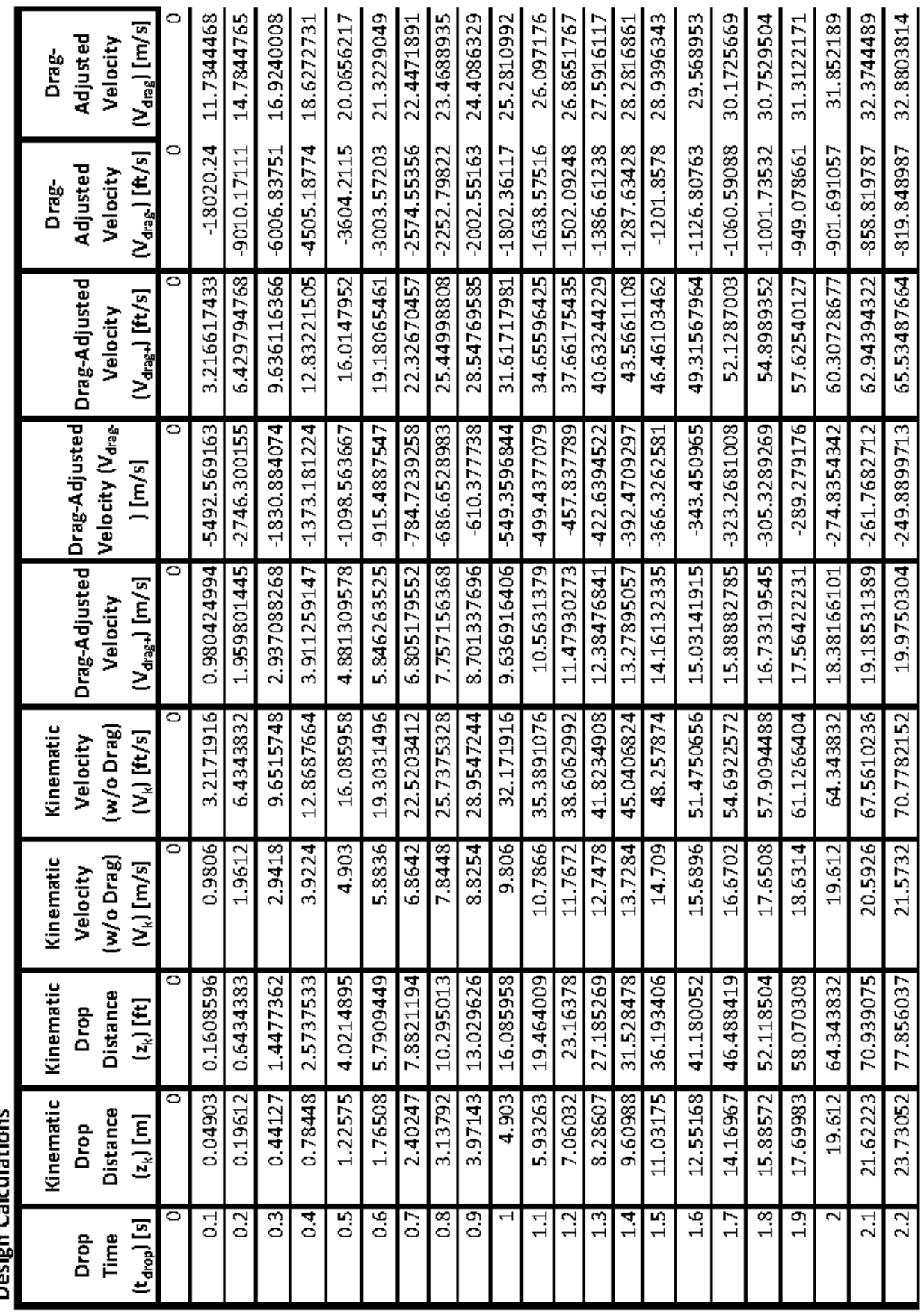




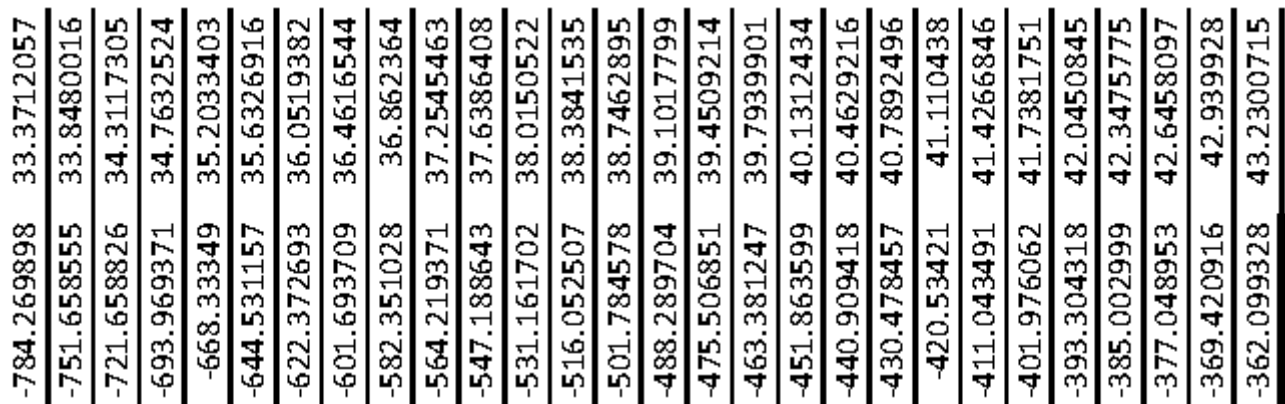

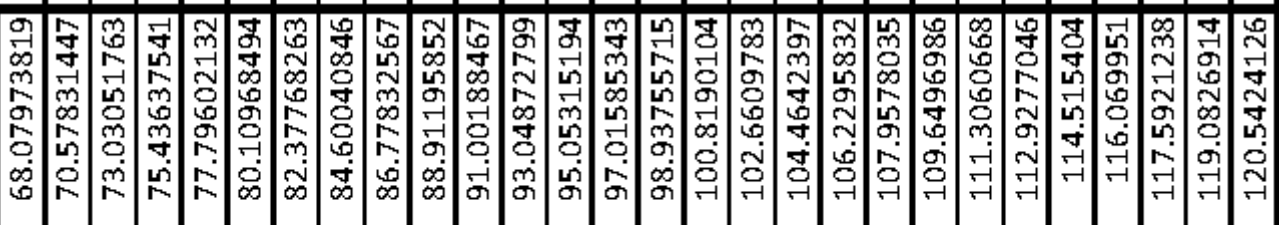

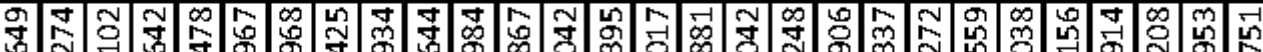

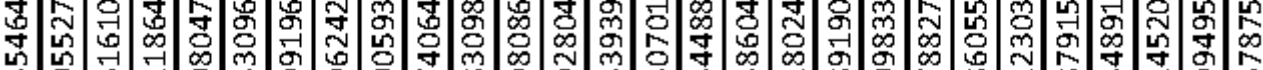

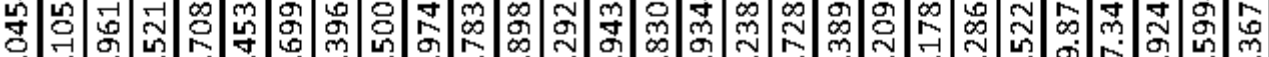

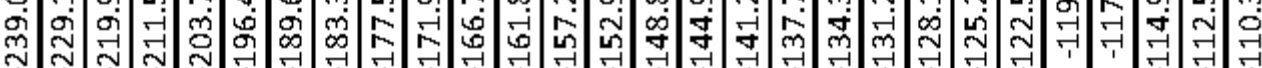

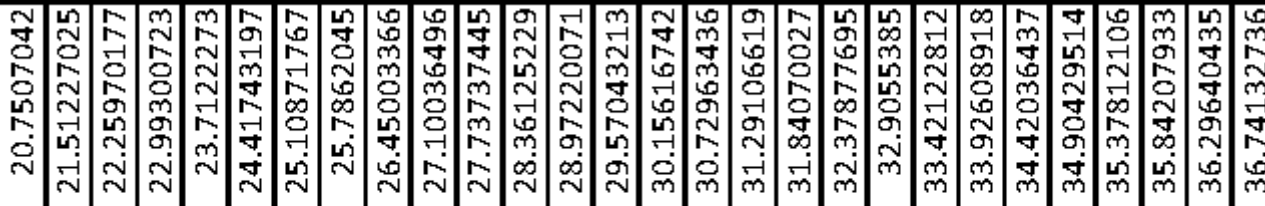

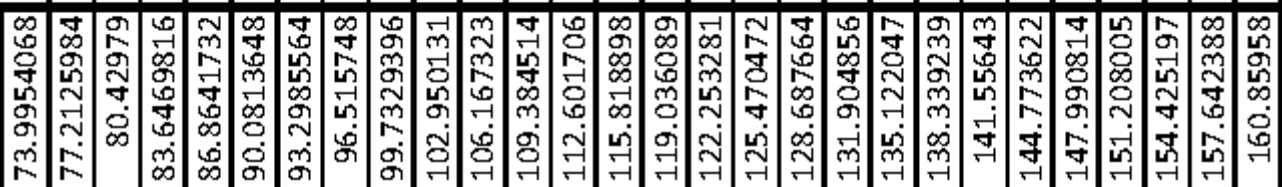

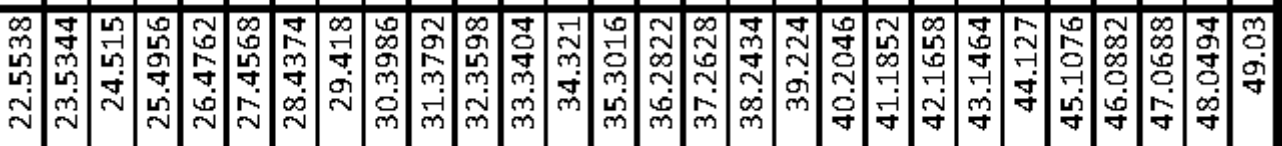

क

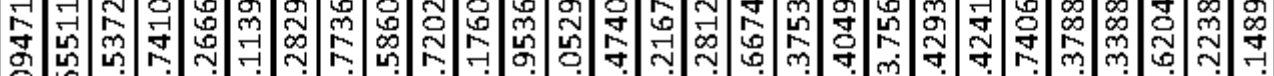
峁

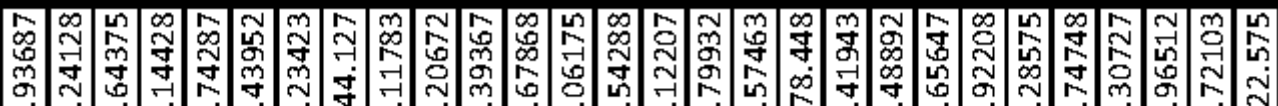

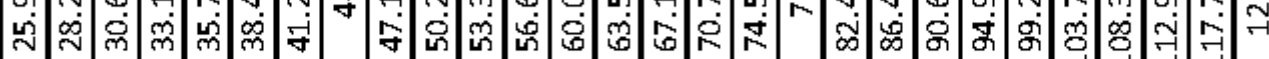


Development of the West Virginia University

Small Microgravity Research Facility (WVU SMiRF)

Copyright $^{\oplus} 2014$

Kyle G. Phillips

\begin{tabular}{|c|c|c|c|c|c|c|c|c|c|c|c|c|c|c|c|}
\hline 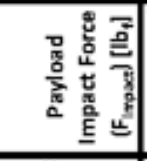 & 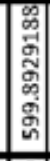 & 闹 & సิ & 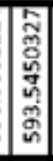 & 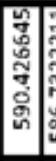 & & 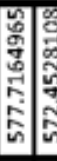 & 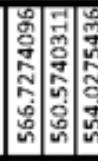 & 总: & 2 & & & & & \\
\hline 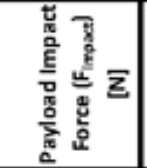 & 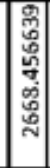 & 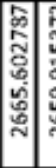 & 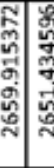 & స్. & & & \begin{tabular}{l|l} 
: \\
:
\end{tabular} & 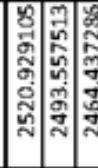 & 素: & స్ & & & & & \\
\hline 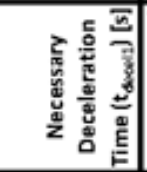 & 兽 & 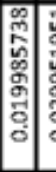 & 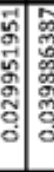 & 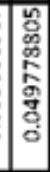 & $\mid \begin{array}{l}2 \\
0 \\
0 \\
0 \\
0 \\
0 \\
0 \\
0\end{array}$ & & 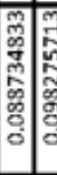 & & 兽: & 政 & & & & & \\
\hline 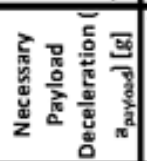 & 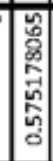 & 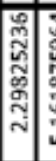 & 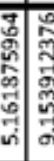 & 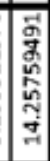 & 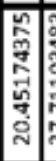 & & 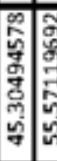 & 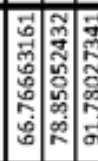 & : & $\begin{array}{l}\text { कू } \\
\text { के } \\
\text { 의 }\end{array}$ & 5 & & & & \\
\hline 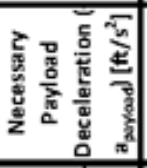 & 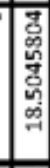 & 离 & 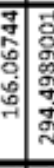 & 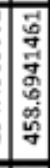 & \begin{tabular}{|c|c}
0 \\
0 \\
$\vdots$ \\
$\vdots$ \\
0 \\
0
\end{tabular} & & 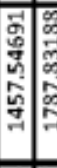 & & 施: & 总 & 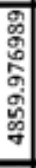 & & & & \\
\hline 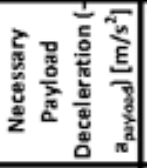 & 兽 & 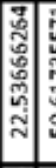 & 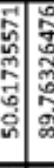 & 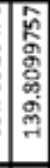 & 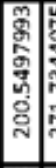 & & 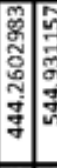 & & 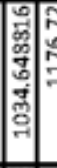 & 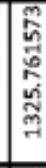 & స్ల్ & 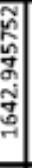 & & & \\
\hline 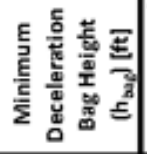 & 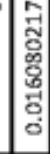 & 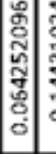 & | & 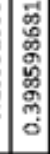 & $\mid \begin{array}{c}0 \\
0 \\
0 \\
0 \\
0 \\
\vdots \\
\vdots \\
0 \\
0\end{array}$ & & 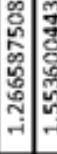 & 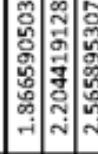 & & 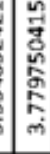 & : & & & & \\
\hline 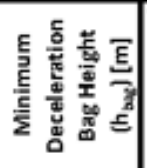 & $g$ & 部 & $\begin{array}{l}0 \\
0 \\
0\end{array}$ & 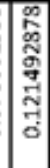 & 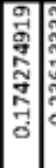 & & & 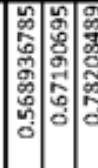 & : & 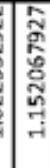 & & & & & \\
\hline 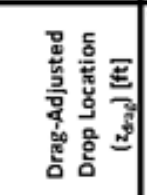 & 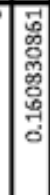 & 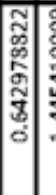 & 过 & 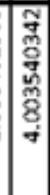 & $\mid \begin{array}{c}0 \\
0 \\
0 \\
0 \\
0 \\
0 \\
0 \\
\vdots\end{array}$ & & 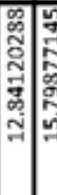 & 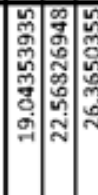 & 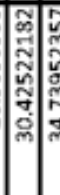 & 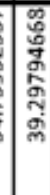 & 总 & & & & \\
\hline 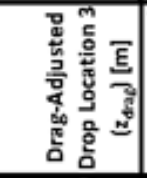 & 总 & 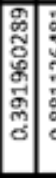 & 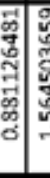 & 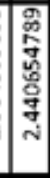 & 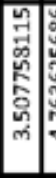 & 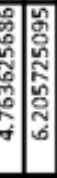 & 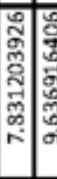 & 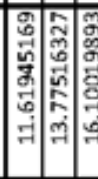 & : & $\begin{array}{l}\text { ơं } \\
\text { ్ㅐㅇ } \\
\text { d. }\end{array}$ & & & & & \\
\hline 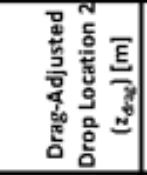 & & के & 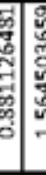 & 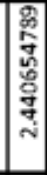 & 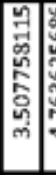 & 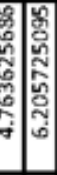 & 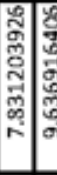 & 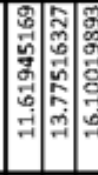 & 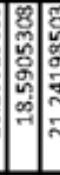 & 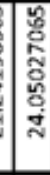 & & & & & \\
\hline 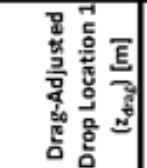 & & 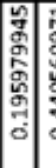 & 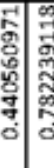 & 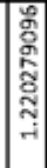 & 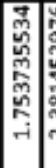 & 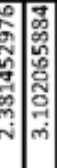 & 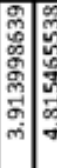 & 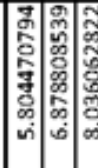 & . & $\begin{array}{l}5 \\
\text { o. } \\
\text { o. }\end{array}$ & & & & & \\
\hline
\end{tabular}


Development of the West Virginia University

Small Microgravity Research Facility (WVU SMiRF)

Copyright $^{\oplus} 2014$

Kyle G. Phillips

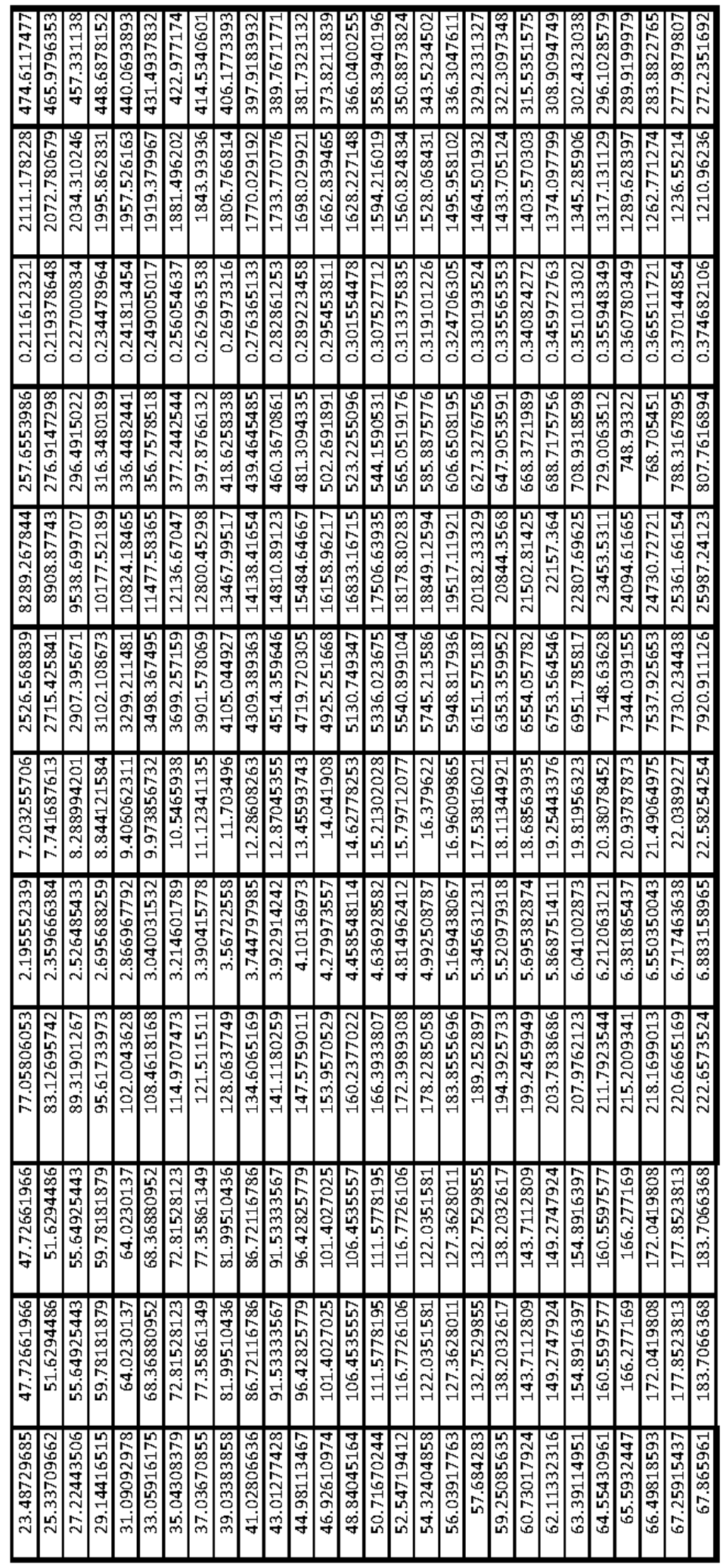




\begin{tabular}{|c|c|c|c|c|c|c|c|}
\hline \multicolumn{6}{|c|}{ Institutior West Virginia University (WVU) } & & \\
\hline College: & \multicolumn{5}{|c|}{ College of Engineering and Mineral Resources (CEMR) (Statler) } & & \\
\hline Departme & \multicolumn{5}{|c|}{ Mechanical and Aerospace Engineering (MAE) } & & \\
\hline & \multicolumn{5}{|c|}{ Small Microgravity Research Facility (SMiRF) } & & \\
\hline Faculty: & \multirow{2}{*}{\multicolumn{5}{|c|}{ Dr. John Kuhlman }} & & \\
\hline Student: & & & & & & & \\
\hline \multirow[t]{24}{*}{ Date: } & \multirow{2}{*}{\multicolumn{4}{|c|}{ 04-Sep-11 }} & & & \\
\hline & & & & & \multicolumn{3}{|c|}{ Deceleration Analysis } \\
\hline & \multicolumn{2}{|c|}{ Deceleration Inputs } & \multicolumn{2}{|c|}{ Deceleration Calculations } & $\begin{array}{c}\text { Deceleration } \\
\text { Time }\left(t_{\text {deoses }}\right)[s]\end{array}$ & $\begin{array}{c}\text { Deceleration } \\
\text { Velocity } \\
\left(\mathrm{V}_{\text {deoe: } 11}\right)[\mathrm{m} / \mathrm{s}]\end{array}$ & $\begin{array}{c}\text { Deceleration } \\
\text { Position } \\
\left(z_{\text {deos } 11}\right)[\mathrm{m}]\end{array}$ \\
\hline & $\begin{array}{c}\text { In intial Payload Velocity } \\
\left(\mathrm{N}_{\text {paylond }}[\mathrm{m} / \mathrm{s}]\right.\end{array}$ & 14.16132335 & $\begin{array}{c}\text { Deceleration Flow } \\
\text { Rate }\left(Q_{\text {orsen_cy }}\right) \\
{\left[\mathrm{m}^{3} / \mathrm{s}\right]}\end{array}$ & 3.39007 & 0 & 14.16132335 & 1.022552922 \\
\hline & $\begin{array}{c}\text { Minimum Deceleration } \\
\text { Bag Height }\left(\mathrm{h}_{\mathrm{bap}}\right\}[\mathrm{m}]\end{array}$ & 1.022552922 & $\begin{array}{c}\text { Deceleration Flow } \\
\text { Rate }\left(Q_{\text {deosi_abib }}\right) \\
{\left[\mathrm{m}^{3} / \mathrm{s}\right]}\end{array}$ & 1.31563 & 0.05 & -44.67467665 & 1.785386754 \\
\hline & $\begin{array}{c}\text { Necessary Payload } \\
\text { Deceleration }\left(-\mathrm{a}_{\text {payload }}\right) \\
{\left[\mathrm{m} / \mathrm{s}^{2}\right]}\end{array}$ & 1176.72 & $\begin{array}{c}\text { Deceleration Flow } \\
\text { Rate }\left(Q_{\text {docat_cy }}\right) \\
{\left[\mathrm{in}^{3} / \mathrm{s}\right]}\end{array}$ & 5254.619 & 0.1 & -103.5106766 & 5.490020587 \\
\hline & $\begin{array}{l}\text { Necessary Deceleration } \\
\text { Time }\left(t_{\text {decesen }}\right)[s]\end{array}$ & 0.144414882 & $\begin{array}{c}\text { Deceleration Flow } \\
\text { Rate }\left\{Q_{\text {dedeot_cubid }}\right\} \\
{\left[\mathrm{in}^{3} / \mathrm{s}\right]}\end{array}$ & 80284.67 & 0.15 & -162.3466766 & 12.13645442 \\
\hline & & & $\begin{array}{c}\text { Deceleration Flow } \\
\text { Rate }\left(\mathrm{Q}_{\text {ocked_cy }}\right) \\
{\left[\mathrm{ft}^{3} / \mathrm{s}\right]}\end{array}$ & 36.49041 & 0.2 & -221.1826766 & 21.72468825 \\
\hline & & & $\begin{array}{c}\text { Deceleration Fow } \\
\text { Rate }\left[\mathrm{Q}_{\text {dedeat_aub }}\right) \\
{\left[\mathrm{ft}^{3} / \mathrm{s}\right]}\end{array}$ & 46.46103 & 0.25 & -280.0186766 & 34.25472208 \\
\hline & & & & & 0.3 & -338.8546767 & 49.72655592 \\
\hline & & & & & 0.35 & -397.6906767 & 68.14018975 \\
\hline & & & & & 0.4 & -456.5266767 & 89.49562358 \\
\hline & & & & & 0.45 & -515.3626767 & 113.7928574 \\
\hline & & & & & 0.5 & -574.1986767 & 141.0318912 \\
\hline & & & & & 0.55 & -633.0346767 & 171.2127251 \\
\hline & & & & & 0.6 & -691.8706767 & 204.3353589 \\
\hline & & & & & 0.65 & -750.7066767 & 240.3997927 \\
\hline & & & & & 0.7 & 809.5426767 & 279.4060266 \\
\hline & & & & & 0.75 & -868.3786767 & 321.3540604 \\
\hline & & & & & 0.8 & -927.2146767 & 366.2438942 \\
\hline & & & & & 0.85 & 986.0506767 & 414.0755281 \\
\hline & & & & & 0.9 & -1044.886677 & 464.8489619 \\
\hline & & & & & 0.95 & -1103.722677 & 518.5641957 \\
\hline & & & & & & -1162.558677 & 575.2212296 \\
\hline
\end{tabular}


Development of the West Virginia University Small Microgravity Research Facility (WVU SMiRF)
Copyright $^{\odot} 2014$

Kyle G. Phillips
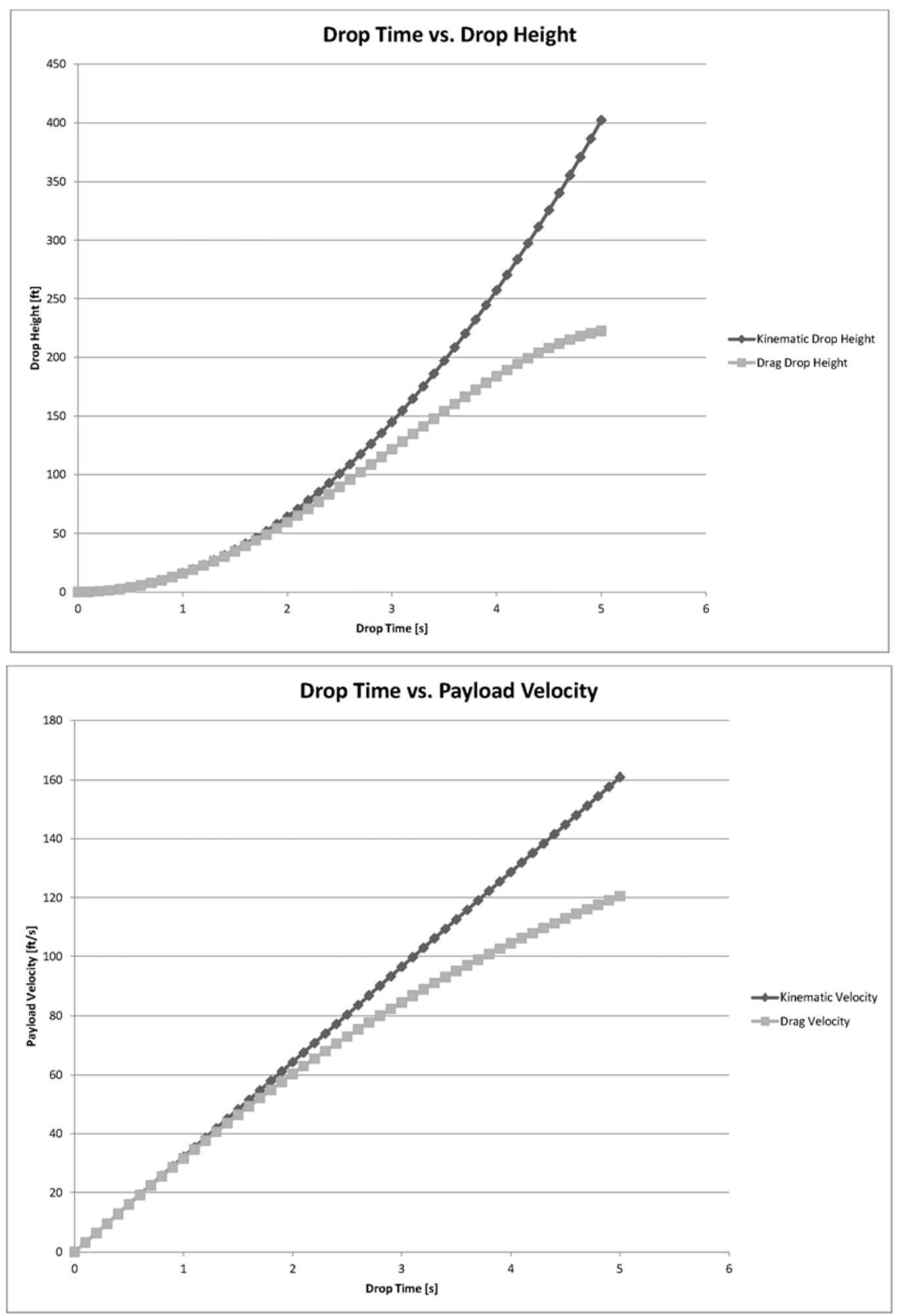


\subsection{Appendix C}

\section{Payload Frame Information}

Appendix G: Payload Frame Information presents the technical fabrication drawings of the primary payload frame, which is to remain unmodified, except for maintenance and repairs, for the life of the WVU SMiRF, provided by NASA Glenn Research Center (Cleveland, Ohio), to which all rights are reserved. 
Development of the West Virginia University

Small Microgravity Research Facility (WVU SMiRF)
Copyright $^{\circledR} 2014$

Kyle G. Phillips
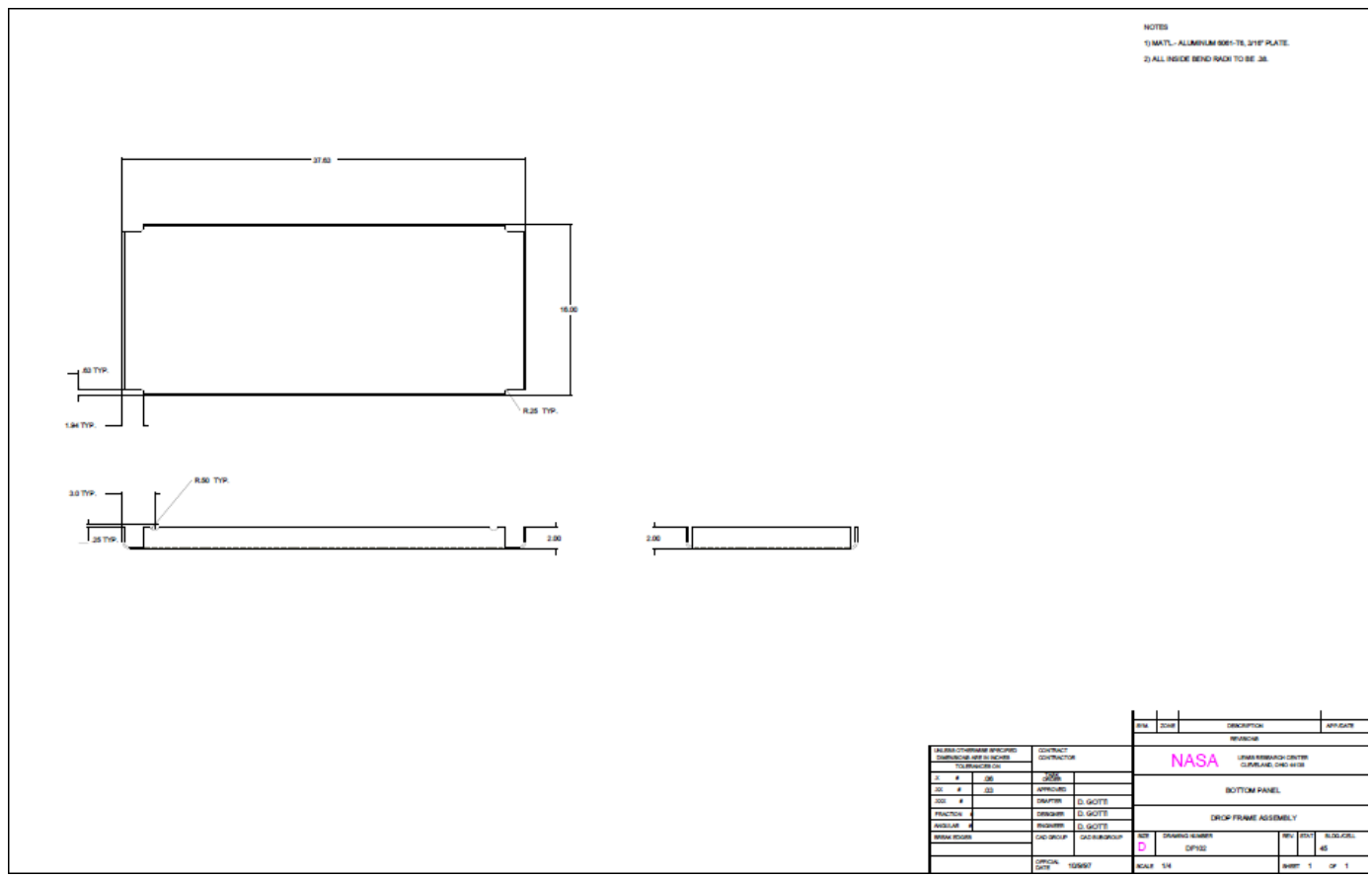

tom
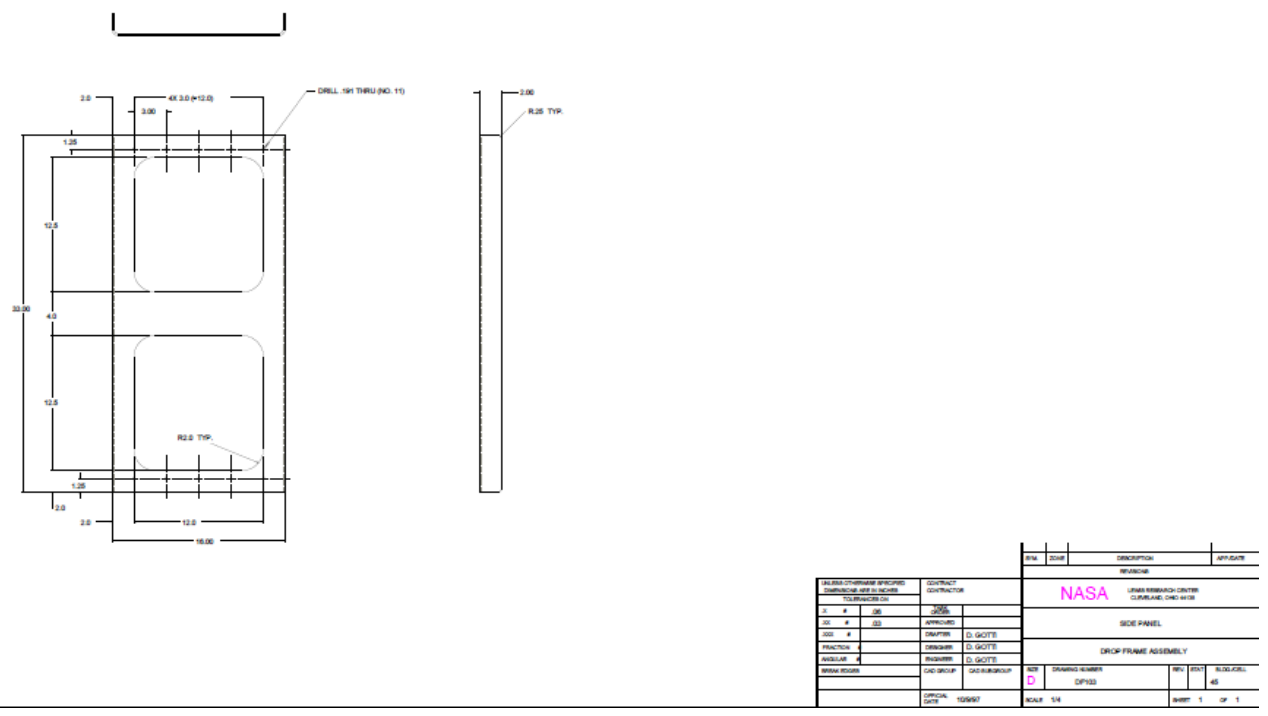
Development of the West Virginia University

Small Microgravity Research Facility (WVU SMiRF)
Copyright $^{\circledR} 2014$

Kyle G. Phillips
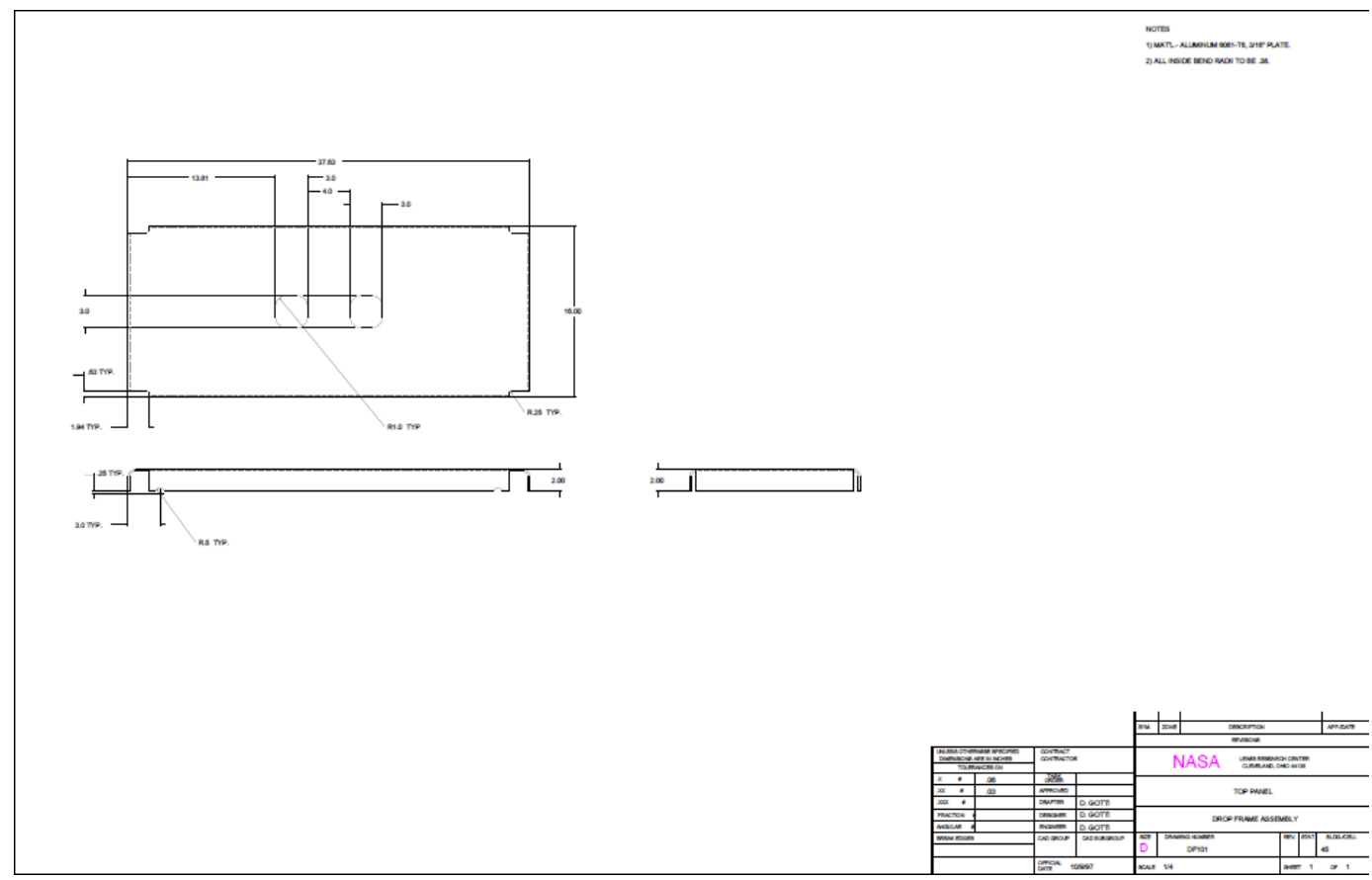

sers
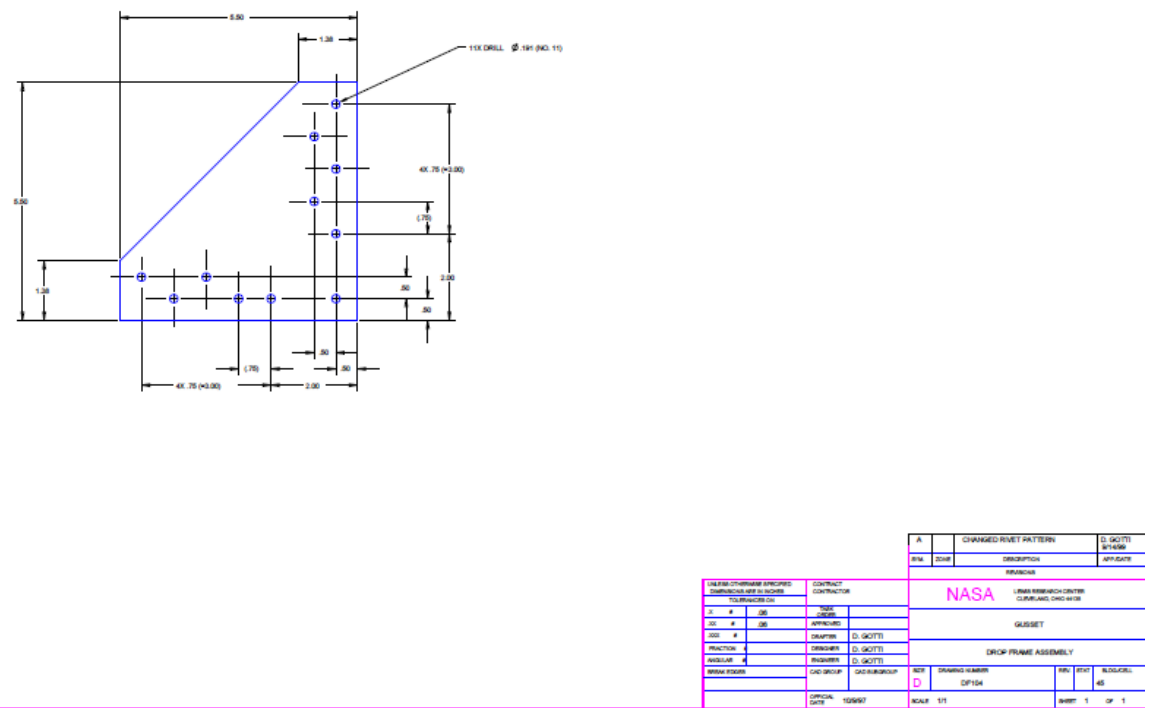
Development of the West Virginia University

Small Microgravity Research Facility (WVU SMiRF)
Copyright $^{\odot} 2014$

Kyle G. Phillips
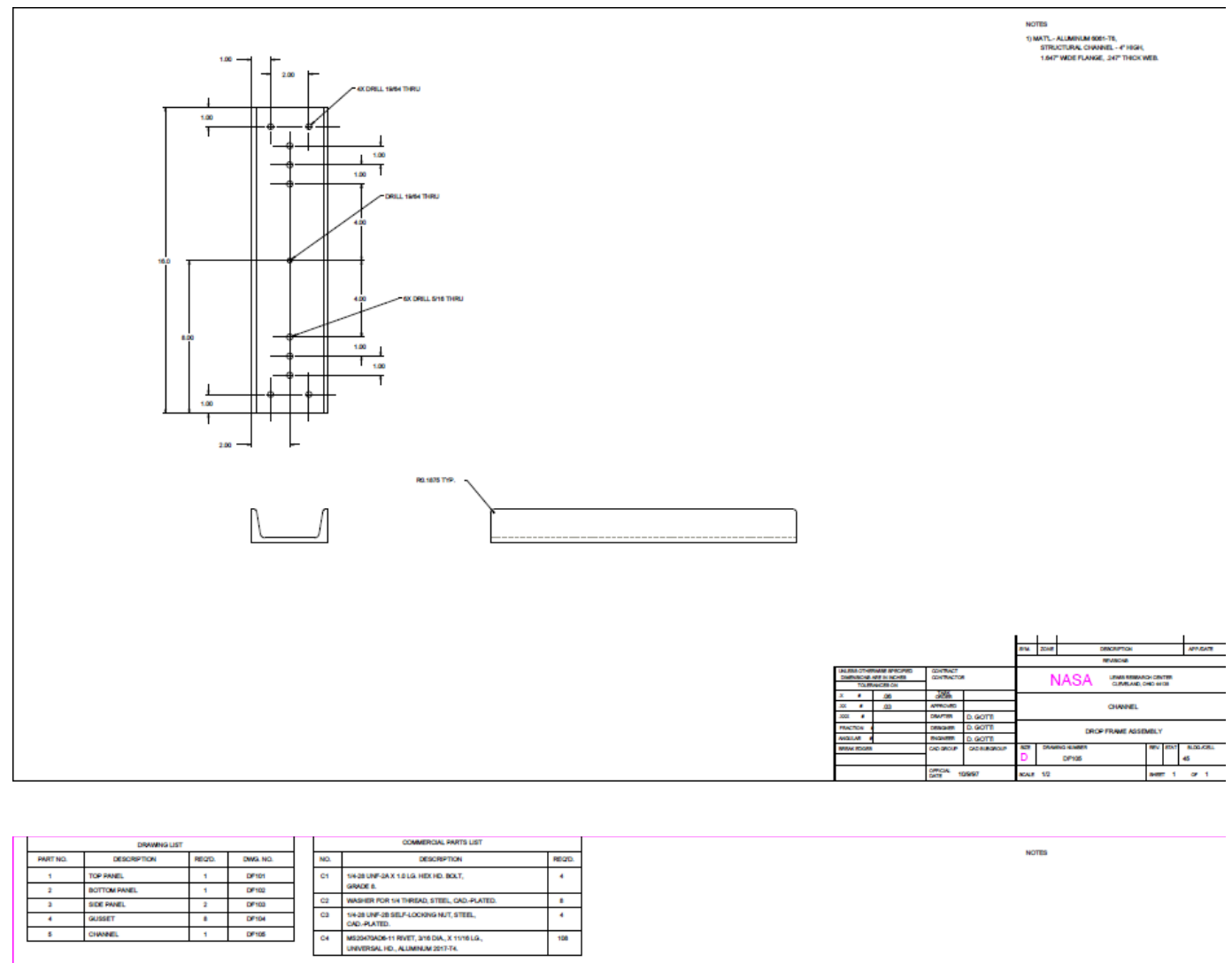

nors
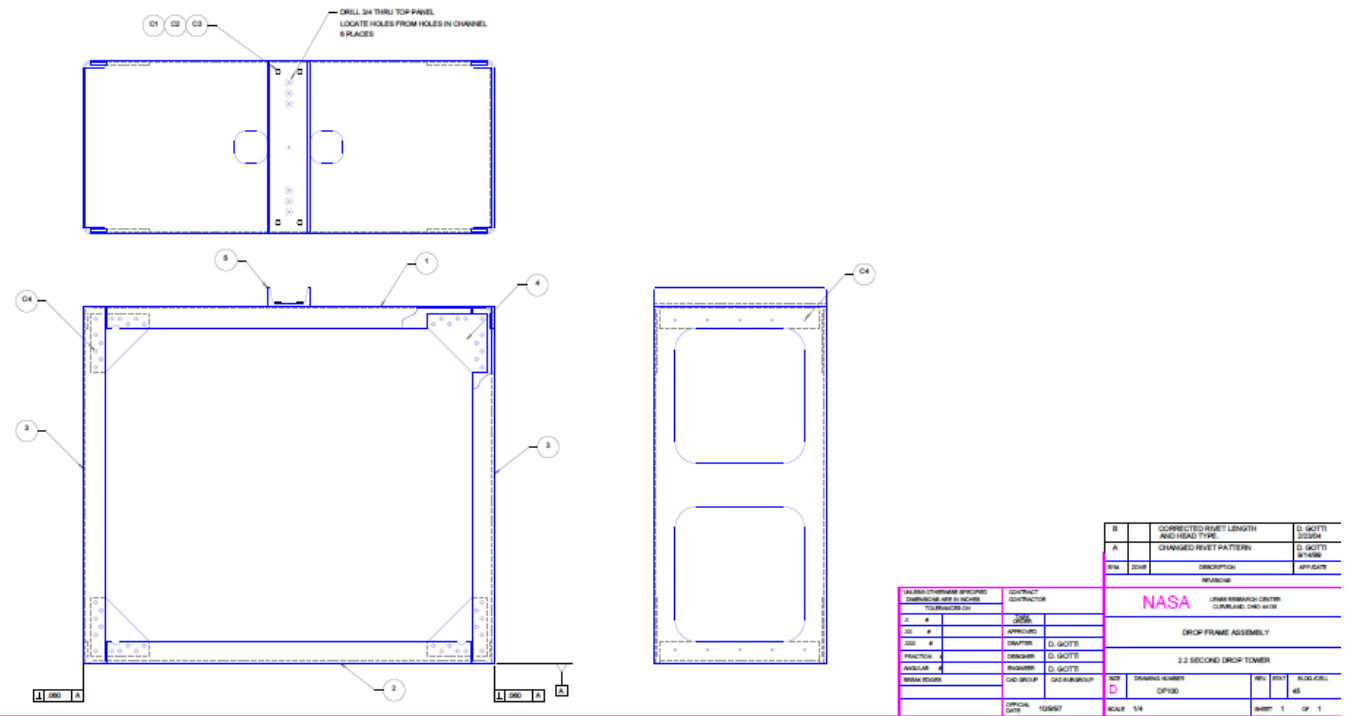


\subsection{Appendix D}

\section{Data Acquisition System Information}

Appendix H: Data Acquisition System Information presents technical specifications and information about the two different data acquisition systems used at WVU SMiRF. The initial portion of this appendix presents information from National Instruments, to which all copyrights are reserved, about the primary data acquisition system to be employed by future researchers, if they so choose, for information at the WVU SMiRF. That system is to remain with the facility at WVU SMiRF for the benefit of researchers using that facility, and shall not be used for any other research. The second system presented is one from Vernier that was used for initial checkouts of the facility, in order to protect the primary National Instruments system in case of unforeseen off-nominal conditions leading to destruction of sensitive electronics. The Vernier system was also used to train initial operators and provide demonstrations to youth visitations. All rights granted by copyright information produced by Vernier are reserved by Vernier. 


$\begin{array}{lr}\text { DNATIONAL } & \text { Technical Sales } \\ \text { United States } \\ \text { (866) 531-6285 } \\ \text { info@ni.com }\end{array}$

Requirements and Compatibility | Ordering Information | Detailed Specifications | PinoutsifFront Panel Connections

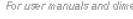

Last Revised: 2011-05-18 10: 19:47.0

CompactRIO Integrated Systems with Real-Time Controller and Reconfigurable Chassis NI cRIO-907x
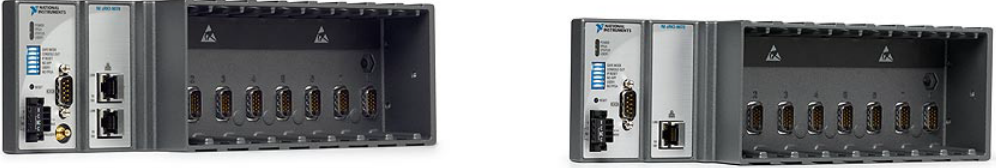

- Integrated CompactRIO sy stems with a reconfigurable FPGA chassis and embedded . Up to $400 \mathrm{MHz}$ realtime processor

. Up to $256 \mathrm{MB}$ DRAM memory, $512 \mathrm{MB}$ of norwolatile storag

- Up to two 101000 ASE-TX Ethernet ports with buitt-in FTPIHTTP servers and
LabVIEN remote panel Web sever

- Up to $2 \mathrm{M}$ gate reconfigurable $F P O A$

- 4 or 8 slots for $C$ Series lio modules

- RS232 serial port and available USB port for peripheral devices

Overview

NII cRIO-907x integrated systems combine an industrial real-time controller and reconfigurable field-programmable gate array (FPGA) chassis for industrial machine control and monitoring applications. The NI cRIO-9074 integrated sy stem features an industrial $400 \mathrm{MHz}$ real-time processor and an eight-slot chassis with an embedded, reconfigurable $2 \mathrm{M}$ gate FPGA chip. The new NI CRIO-9076 integrated sy stem contains a $400 \mathrm{MHZ}$ real-time processor, a four-slot chassis with an embedded, reconfigurable LX45 FPGA chip, and a high-speed USB port. Both systems feature built-in nonvolatile memory and a fault tolerant file system. The new four-slot NI cRIO-9075 and NI cRIO-9076 systems prowide a cost-optimized solution for high volume deployments and OEM application

Back to Top

Requirements and Compatibility

os Information

Software Compatibility

- vxworks

$$
\cdot \text { NIRIO }
$$

- LabVIEW

- LabVIEW FPGa Module

- LabVIEW Professional Development System

- LabVIEN Real-Time Modul

\section{Comparison Tables}

\begin{tabular}{|c|c|c|c|c|c|c|c|c|}
\hline Product $\begin{array}{c}\text { Module } \\
\text { Slots }\end{array}$ & $\begin{array}{l}\text { Processor } \\
\text { Speed (MHz) }\end{array}$ & FPGA & $\begin{array}{l}\text { DRAM } \\
\text { (MB) }\end{array}$ & $\begin{array}{l}\text { Internal Nonvolatile } \\
\text { Storage (MB) }\end{array}$ & $\begin{array}{l}\text { 10/100BASE-TX } \\
\text { Ethemet Port }\end{array}$ & $\begin{array}{c}\text { RS232 } \\
\text { Serial Port }\end{array}$ & $\begin{array}{l}\text { Power Supply } \\
\text { Input Range }\end{array}$ & $\begin{array}{l}\text { USB } \\
\text { Port }\end{array}$ \\
\hline 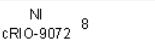 & 266 & $\begin{array}{l}\text { Spartan-3 } \\
1 \mathrm{M}\end{array}$ & 64 & 128 & yes & yes & 19 to $30 \mathrm{VDC}$ & no \\
\hline $\begin{array}{c}\mathrm{Nl} \\
\mathrm{CRlO}-9073\end{array}$ & 266 & $\begin{array}{l}\text { Spartan-3 } \\
2 M\end{array}$ & 64 & 128 & yes & yes & 19 to $30 \mathrm{VDC}$ & no \\
\hline${ }_{\text {CRIO-9074 }}^{\mathrm{NI}} 8$ & 400 & $\begin{array}{l}\text { Spartan-3 } \\
2 M\end{array}$ & 128 & 256 & yes (Dual) & yes & 19 to $30 \vee D C$ & no \\
\hline${ }_{\mathrm{CRIO}-9075}^{\mathrm{NI}}$ & 400 & $\begin{array}{l}\text { Spartan-6 } \\
\text { LX25 }\end{array}$ & 128 & 256 & yes & yes & 9 to $30 \mathrm{VDC}$ & no \\
\hline
\end{tabular}




\begin{tabular}{|c|c|c|c|c|c|c|c|c|c|}
\hline Product & $\begin{array}{l}\text { Module } \\
\text { Slots }\end{array}$ & $\begin{array}{c}\text { Processor } \\
\text { Speed (MHz) }\end{array}$ & FPGA & $\begin{array}{l}\text { DRAM } \\
\text { (MB) }\end{array}$ & $\begin{array}{c}\text { Internal Nonvolatile } \\
\text { Storage (MB) }\end{array}$ & $\begin{array}{l}\text { 10/100BASE-TX } \\
\text { Ethernet Port }\end{array}$ & $\begin{array}{c}\text { RS232 } \\
\text { Serial Port }\end{array}$ & $\begin{array}{l}\text { Power Supply } \\
\text { Input Range }\end{array}$ & $\begin{array}{l}\text { USB } \\
\text { Port }\end{array}$ \\
\hline $\begin{array}{c}\mathrm{NI} \\
\text { CRIO-9076 }\end{array}$ & & 400 & $\begin{array}{l}\text { Spartan-6 } \\
\text { LX45 }\end{array}$ & 256 & 512 & yes & yes & 9 to $30 \mathrm{VDC}$ & yes \\
\hline
\end{tabular}

Application and Technology

System Configuration

These NI CompactRIO real-time controllers combine a four- or eight-slot reconfigurable chassis into an integrated system. The user-defined FPGA circuitry in the chassis controls each I/O module and passes data to the controller through a local PCI bus using built-in communication functions.

\begin{tabular}{|c|c|c|c|c|}
\hline Product & FPGA & Logic Cells & Multipliers & RAM (Kb) \\
\hline NI cRIO-9073 & Spartan-3 2M & 46080 & 40 & 720 \\
\hline NI cRIO-9074 & Spartan-3 2M & 46080 & 40 & 720 \\
\hline NI cRIO-9075 & Spartan-6 LX25 & 24051 & 38 & 936 \\
\hline NI cRIO-9076 & Spartan-6 LX45 & 43661 & 58 & 2088 \\
\hline
\end{tabular}

FPGA Resource Comparison

These systems also accept up to eight $\mathrm{NI} C$ Series I/O modules. A variety of I/O modules are available including voltage, current, thermocouple, RTD, accelerometer, and strain gage inputs; up to $160 \mathrm{~V}$ simultaneous sampling analog I/O; 12, 24, and $48 \mathrm{~V}$ industrial digital I/O; $5 \mathrm{~V} / \mathrm{TTL}$ digital I/O; counter/timers; pulse generation; and high voltage/current relays.

The 10/100 Mbits/s Ethernet port allows for programmatic communication over the network and the cRIO-9074 features dual Ethernet ports, which allows for the use of one port for network communication to a host PC or enterprise system and the other port for expansion 10 (easily connect another CompactRIO system or another Ethernet-based device for additional //O). The new cRIO-9076 also features a USB 2.0 port for data storage and connection to peripheral devices.

NI CompactRIOS have the ability to by synchronized with an SNTP time server on a network and the CRIO-9072, CRIO-9073, and cRIO-9074 also feature a built-in backup battery to maintain operation for the Real-Time Clock when external power is removed. The cRIO-9075 and cRIO-9076 do not contain a backup battery for the Real-Time Clock. Embedded Software

You can synchronize embedded code execution to an FPGA-generated interrupt request (IRQ) or an internal millisecond real-time clock source. The LabVIEW Real-Time ETS OS provides reliability and simplifies the development of complete embedded applications that include time-critical control and acquisition loops in addition to lower-priority loops for

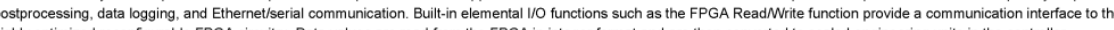
(1)

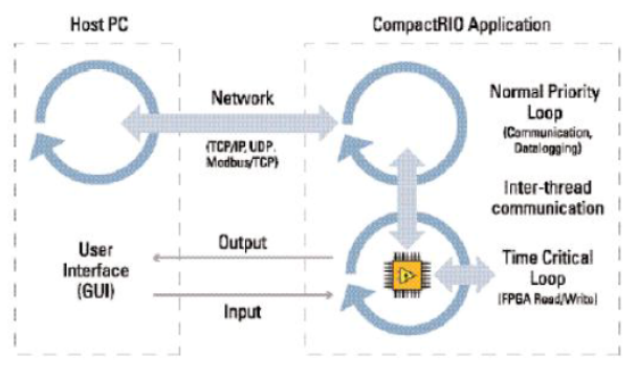

CompactRIO Software Architecture

Note: NI Scan Engine is not supported on the cRIO-9075 and cRIO-9076. Built-In Servers

In addition to programmatic communication via TCPIP, UDP, Modbus/TCP, IIDA, and serial protocols, the CompactRIO controllers include built-in servers for Virtual Instrument Software Architecture (VISA), HTTP, and FTP. The VISA server provides remote download and communication access to the reconfigurable I/O (RIO) FPGA over Ethernet. The server provides access to logged data or configuration files. 


\begin{tabular}{|c|c|c|c|}
\hline Products & $\begin{array}{l}\text { Part } \\
\text { Number }\end{array}$ & Recommended Accessories & $\begin{array}{l}\text { Part } \\
\text { Number }\end{array}$ \\
\hline \multicolumn{4}{|l|}{ NI cRIO-9072 } \\
\hline $\begin{array}{l}\text { cRIO-9072 8-Slot Integrated } 266 \mathrm{MHz} \text { Real-Time CtrIr, } \\
\text { 1M Gate FPGA } \\
\text { Requires: }\end{array}$ & 779998-01 & $\begin{array}{l}\text { Connector Block: Screw Terminal - NI } 9978 \text { 4-pos screw terminal power supply } \\
\text { plugs (quantity 5) }\end{array}$ & $196938-01$ \\
\hline \multicolumn{4}{|l|}{ NI CRIO-9073 } \\
\hline \multirow[t]{2}{*}{$\begin{array}{l}\text { cRIO-9073 8-Slot Integrated } 266 \text { MHz Real-Time Ctrlr, } \\
\text { 2M Gate FPGA } \\
\text { Requires: }\end{array}$} & 780471-01 & $\begin{array}{l}\text { Connector Block: Shielded - NI PS-15 Power Supply, } 24 \text { VDC, } 5 \text { A, } \\
\text { 100-120/200-240 VAC Input } \\
\text { "“Also Avaliable: Screw Terminal }\end{array}$ & 781093-01 \\
\hline & & $\begin{array}{l}\text { Connector Block: Screw Terminal - NI } 9979 \text { Strain relief kit for 4-pos power } \\
\text { connector }\end{array}$ & $196939-01$ \\
\hline \multicolumn{4}{|l|}{ NI CRIO-9076 } \\
\hline $\begin{array}{l}\text { cRIO-9076 4-Slot Integrated } 400 \mathrm{MHz} \text { Real-Time CtrIr, } \\
\text { LX45 FPGA } \\
\text { Requires: } 1 \text { Connectivity Accessory }\end{array}$ & 781716-01 & $\begin{array}{l}\text { Connectivity Accessory: Shielded - NI PS-15 Power Supply, } 24 \text { VDC, } 5 \text { A, } \\
\text { 100-120/200-240 VAC Input }\end{array}$ & 781093-01 \\
\hline \multicolumn{4}{|l|}{ NI CRIO-9075 } \\
\hline $\begin{array}{l}\text { cRIO-9075 4-Slot Integrated } 400 \mathrm{MHz} \text { Real-Time CtrIr, } \\
\text { LX25 FPGA } \\
\text { Requires: } 1 \text { Connectivity Accessory }\end{array}$ & 781715-01 & $\begin{array}{l}\text { Connectivity Accessory: Shielded - NI PS-15 Power Supply, } 24 \text { VDC, } 5 \text { A, } \\
\text { 100-120/200-240 VAC Input }\end{array}$ & 781093-01 \\
\hline \multicolumn{4}{|l|}{ NI cRIO-9074 } \\
\hline $\begin{array}{l}\text { cRIO-9074 8-Slot Integrated } 400 \text { MHz Real-Time CtrIr, } \\
\text { 2M Gate FPGA } \\
\text { Requires: } 1 \text { Connectivity Accessory }\end{array}$ & 779999-01 & $\begin{array}{l}\text { Connectivity Accessory: Shielded - NI PS-15 Power Supply, } 24 \text { VDC, } 5 \text { A, } \\
\text { 100-120/200-240 VAC Input }\end{array}$ & 781093-01 \\
\hline
\end{tabular}

Software Recommendations

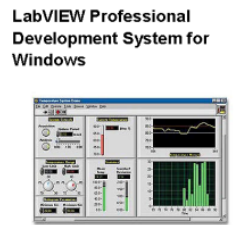

- Advanced software tools for large project development

- Automatic code generation using DAQ

- Tight integration with a wide range of

Advanced measurement analysis and digita

signal processing

Open connectivity with DLLs, ActiveX, an

- Capability to build DLLs, executables, and
MSl installers

NI LabVIEW FPGA Module

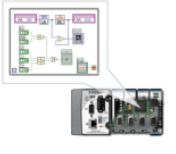

ifing
- Create your own I/O hardware without VHDL design

- Graphically configure FPGAs on NI Define your own control algor Define your own control algonth
rates up to $300 \mathrm{MHz}$

- Execute multiple tasks simultaneously and deterministically

- Implement custom timing and triggering logic,
digital protocols, and DSP algorithms - Incorporate existing HDL code and third-party
IP including Xilinx CORE Generator functions

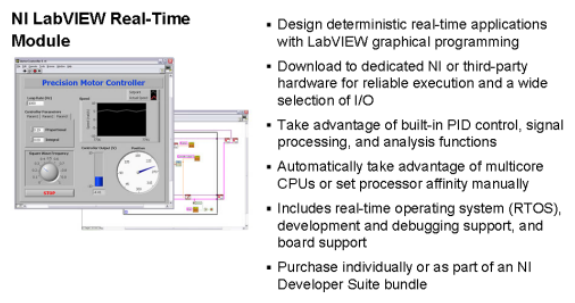




\section{Support and Services \\ System Assurance Programs}

$\mathrm{NI}$ system assurance programs are designed to make it even easier for you to own an NI system. These programs include corfiguration and deployment services for your NI PXI, CompactRlO, or Compact FieldPoint system. The NI Basic System Assurance Program prowides a simple integration test and ensures that your system is delivered completely development erwion When you order your sy stem with the standard, program, you al so receive system-specific documentation including a bill of materials, an integration test report, a recommended maintenance plan, and recuenty asked cuestion documents Finally, the standard program reduces the total cost of owning an NI system by providing three years of warranty coverage and calibration service. Use the online product advisors at ni.comiadvisor to find a system assurance program to meet your needs

Calibration

NI measurement hardware is calibrated to ensure measurement accuracy and verify that the device meets its published specifications. To ensure the ongoing accuracy of your measurementhardware, NI offers basic or detalled recalibration sevvice that provides ongoing ISO 9001 audit compliance and confidence in your measurements. To learn more about NI calibration services or to locate a qualified service center near you, contact your local sales office or visit ni. comicalibration.

\section{Technical Support}

Get answers to your technical questions using the following National Instruments resources

- Support - Visit ni.comisupport to access the NI KnowledgeBase, example programs, and tutorials or to contact our applications engineers who are located in NI sales

Discussion Forums - Vist forums.nic com for a dwerse set of discussion boards on topics you care about.

- Online Community - Visit community.ni.com to find, contribute, or collaborate on customer-contributed technical content with users like you

\section{Repair}

While you may never need your hardware repaired, Nl understands that unexpected events may lead to necessary repairs. Nl offers repair services performed by highly trained technicians who quickly return your device with the guarantee that it will perform to factory specifications. For more information, visit ni.comirepair.

\section{Training and Certifications}

The $\mathrm{N}$ training and certification program delivers the fastest, most certain route to increased proficiency and productivity using $\mathrm{NI}$ software and hardware. Training builds the skills to more efficiently develop robust, maintainable applications, while certification validates your knowledge and ability.

- Classroom training in cities worldwide - the most comprehensive hands-on training taught by engineers,

- On-site training a your facility - an excellent option to train multiple employees at the same time.

- Online instructor-led training - lower-cost, remote training fif classroom or or-site courses are not possible

- Course kits - lowest-cost, self-paced training that you can use as reference guides.

- Training memberships and training credits - to buy now and schedule training later. Visit ni.comitraining for more information

Extended Warranty

NI offers options for extending the standard product warranty to meet the life-cycle requirements of your project. In addition, bec ause Nll understands that your requirements may change, the extended warranty is flexible in length and easily renewed. For more information, visit ni.comwwarranty.

OEM

NI offers design-in consulting and product integration assistance if you need $\mathrm{NI}$ products for $\mathrm{OEM}$ applications. For information about special pricing and services for $\mathrm{OEM}$ customers, visit ni.comioem.

Alliance

Our Professional Services Team is comprised of NI applic ations engineers, NI Consulting Services, and a worldwide National Instruments Alliance Partner program of more than 700 independent consultants and integrators. Services range from start-up assistance to turnkey System integration. Visit ni. comballiance

Detailed Specifications

The following specific ations are typical for the -20 to $55^{\circ} \mathrm{C}$ operating temperature range unless otherwise noted.

\section{Network}

Network interface

10BaseT and 100日aseTX Ethernet

Compatibility

IEEE 802.3

Communication rates

10 Mbps, 100 Mbps, auto-negotiated

Maximum cabling distance

$100 \mathrm{~m} / \mathrm{segment}$

RS-232 Serial Port

Maximum baud rate 


\begin{tabular}{|c|c|}
\hline Data bits & $5,6,7,8$ \\
\hline Stop bits & 1,2 \\
\hline Parity & Odd, Even, Mark, Space \\
\hline Flow control & RTSICTS, XONIXOFF, DTRIDSR \\
\hline \multicolumn{2}{|l|}{ SMB Connector (cRIO-9074 Only) } \\
\hline \multicolumn{2}{|l|}{ Output Characteristics } \\
\hline \multicolumn{2}{|l|}{ Minimum high-level output voltage } \\
\hline With -100 $\mu \mathrm{A}$ output current & $2.9 \mathrm{~V}$ \\
\hline With -16 mA output current & $2.4 \mathrm{~V}$ \\
\hline With -24 mA output current & $2.3 \mathrm{~V}$ \\
\hline \multicolumn{2}{|l|}{ Maximum low-level output voltage } \\
\hline With $100 \mu \mathrm{A}$ output current & $0.10 \mathrm{~V}$ \\
\hline With 16 mA output current & $0.40 \mathrm{~V}$ \\
\hline With 24 mA output current & $0.55 \mathrm{~V}$ \\
\hline Driver type & CMOS \\
\hline Maximum sinkisource current & $\pm 24 \mathrm{~mA}$ \\
\hline Maximum 3-state output leakage current & $\pm 5 \mu \mathrm{A}$ \\
\hline \multicolumn{2}{|l|}{ Input Characteristics } \\
\hline Minimum input voltage & ov \\
\hline Minimum low-level input voltage & $0.94 \mathrm{~V}$ \\
\hline Maximum high-level input voltage & $2.43 \mathrm{~V}$ \\
\hline Maximum input voltage & $5.5 \mathrm{~V}$ \\
\hline Typical input capacitance & $2.5 \mathrm{pF}$ \\
\hline Typical resistwe strapping & $1 \mathrm{k} \Omega$ to $3.3 \mathrm{~V}$ \\
\hline \multicolumn{2}{|l|}{ Mem ory } \\
\hline \multicolumn{2}{|l|}{ CRIO-9072, cRIO-9073 } \\
\hline Nonvolatile & $128 \mathrm{MB}$ \\
\hline System memory & $64 \mathrm{MB}$ \\
\hline \multicolumn{2}{|l|}{ cRlO-9074 } \\
\hline Nonvolatile & $256 \mathrm{MB}$ \\
\hline System memory & $128 \mathrm{MB}$ \\
\hline \multicolumn{2}{|l|}{ Reconfigurable FPGA } \\
\hline \multicolumn{2}{|l|}{ CRI0-9072 } \\
\hline Number of logic cells & 17,280 \\
\hline Avallable embedded RAM & 432 kbits \\
\hline \multicolumn{2}{|l|}{ cRlO-9073, cRIO-9074 } \\
\hline Number of logic cells & 46,080 \\
\hline Available embedded RAM & 720 kbits \\
\hline \multicolumn{2}{|l|}{ Intemal Real-Time Clock } \\
\hline Accuracy & $200 \mathrm{ppm} ; 35 \mathrm{ppm}$ at $25^{\circ} \mathrm{C}$ \\
\hline \multicolumn{2}{|l|}{ Power Requirements } \\
\hline \multicolumn{2}{|c|}{1 Caution You must use a National Electric Code (NEC) UL Listed Class 2 power supply with the cRlo-9072/3/4. } \\
\hline Recommended power supply & $48 \mathrm{~W}, 24 \mathrm{VDC}$ \\
\hline Power consumption & 20 W maximum \\
\hline
\end{tabular}




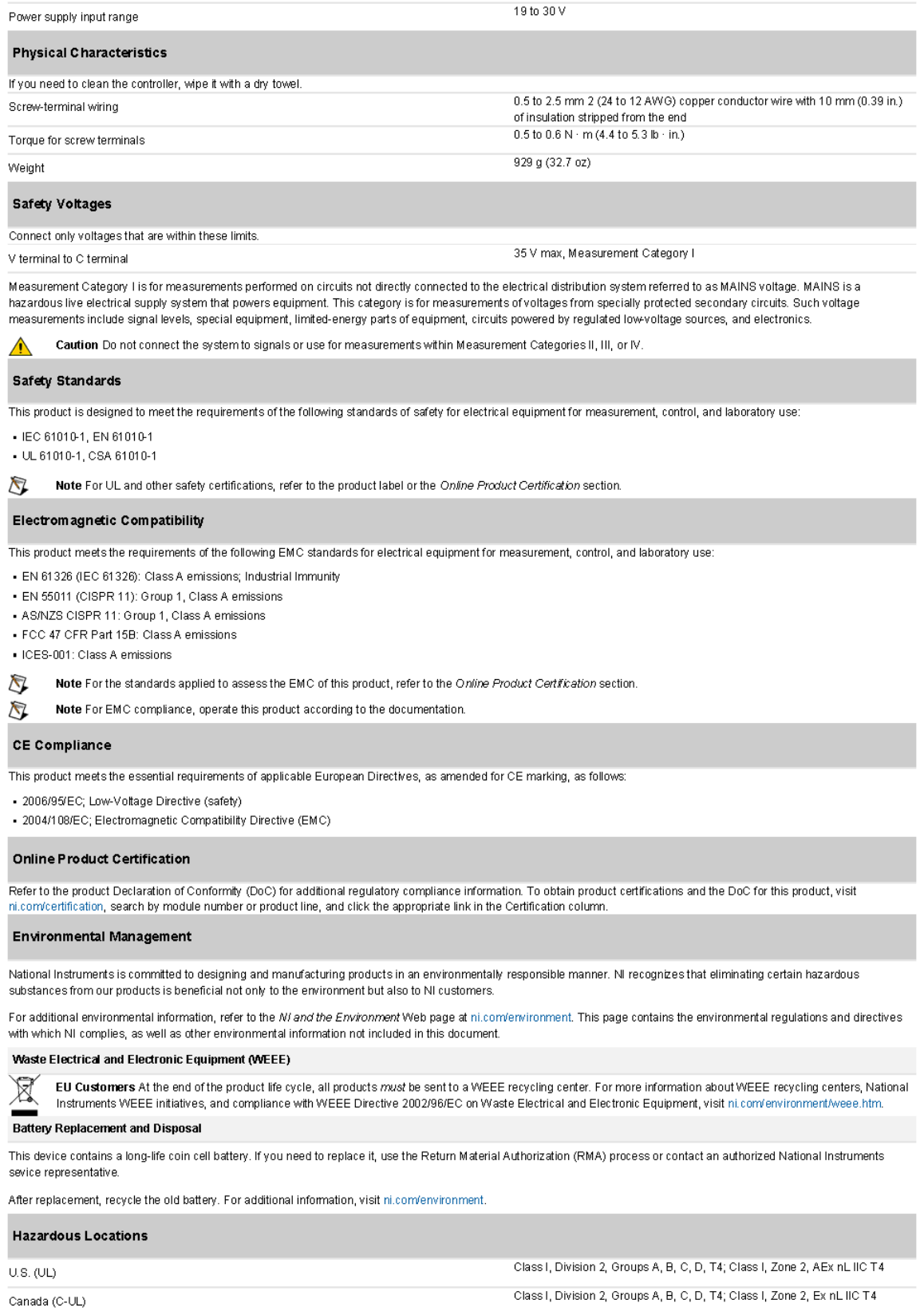




\begin{tabular}{|c|c|c|c|}
\hline Europe (DEMKO) & & & Ex nL IIC T4 (part numbers b \\
\hline Environmental & & & \\
\hline The cRl0-9072/3/4 is intended for indoor use only, but it & it may be used ou & tdoors if mounted in a suit & itably rated enclosure. \\
\hline Operating temperature (IEC 60068-2-1, IEC 60068-2-2) & & & -20 to $55^{\circ} \mathrm{C}$ \\
\hline E Note To meet this operating temperature rang & gge, follow the guide & elines in the installation in & nstructions for your CompactRII \\
\hline Storage temperature (IEC 60068-2-1, IEC 60068-2-2) & & & -40 to $85^{\circ} \mathrm{C}$ \\
\hline Ingress protection & & & IP 40 \\
\hline Operating humiditty (IEC 60068-2-56) & & & 10 to $90 \% \mathrm{RH}$, noncondensin \\
\hline Storage humidity (IEC 60068-2-56) & & & 5 to $95 \% \mathrm{RH}$, noncondensing \\
\hline Maximum altitude & & & $2,000 \mathrm{~m}$ \\
\hline Pollution Degree (IEC 60664) & & & 2 \\
\hline Shock and Vibration & & & \\
\hline To meet these specific ations, you must panel mount the & he compactRIO sys & stem and affic ferrules to $t$ & the ends of the power terminal \\
\hline Operating shock (IEC 60068-2-27) & & & $30 \mathrm{~g}, 11 \mathrm{~ms}$ half sine $50 \mathrm{~g}, 3$ \\
\hline Operating vibration, random (IEC 60068-2-64) & & & $5 \mathrm{~g}_{\mathrm{tms}}, 10$ to $500 \mathrm{~Hz}$ \\
\hline Operating vibration, sinusoidal (IEC 6006\&-2-6) & & & $5 \mathrm{~g}, 10$ to $500 \mathrm{~Hz}$ \\
\hline Cabling & & & \\
\hline The following table shows the standard Ethernet cable $\mathrm{v}$ & wiring connections & s for both normal and cros & sssover cables. \\
\hline & & hernet Cable Wiring Cor & nnections \\
\hline & \begin{tabular}{l|l|} 
Pin & Connector 1 \\
\end{tabular} & Connector 2 (Nomal) & Connector 2 (Crossover) \\
\hline 1 & 1 white orange & white/orange & whiteigreen \\
\hline 2 & 2 orange & orange & green \\
\hline 3 & \begin{tabular}{l|l}
3 & whitel green \\
\end{tabular} & white' green & whitef orange \\
\hline 4 & \begin{tabular}{l|l}
4 & blue \\
\end{tabular} & blue & blue \\
\hline 5 & 5 whiteiblue & white'slue & whiterblue \\
\hline 6 & \begin{tabular}{l|l}
6 & green \\
\end{tabular} & green & orange \\
\hline 7 & 7 whiterbrown & whiteibrown & whites brown \\
\hline 8 & 8 brown & brown & brown \\
\hline
\end{tabular}


Development of the West Virginia University Small Microgravity Research Facility (WVU SMiRF)
Copyright $^{\odot} 2014$

Kyle G. Phillips

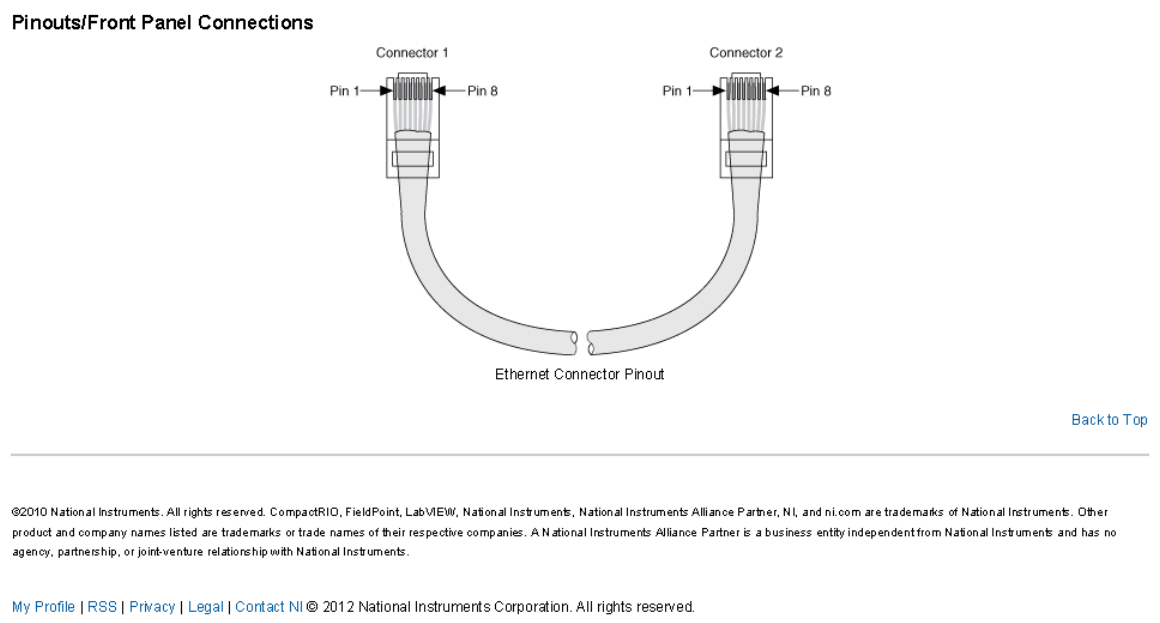




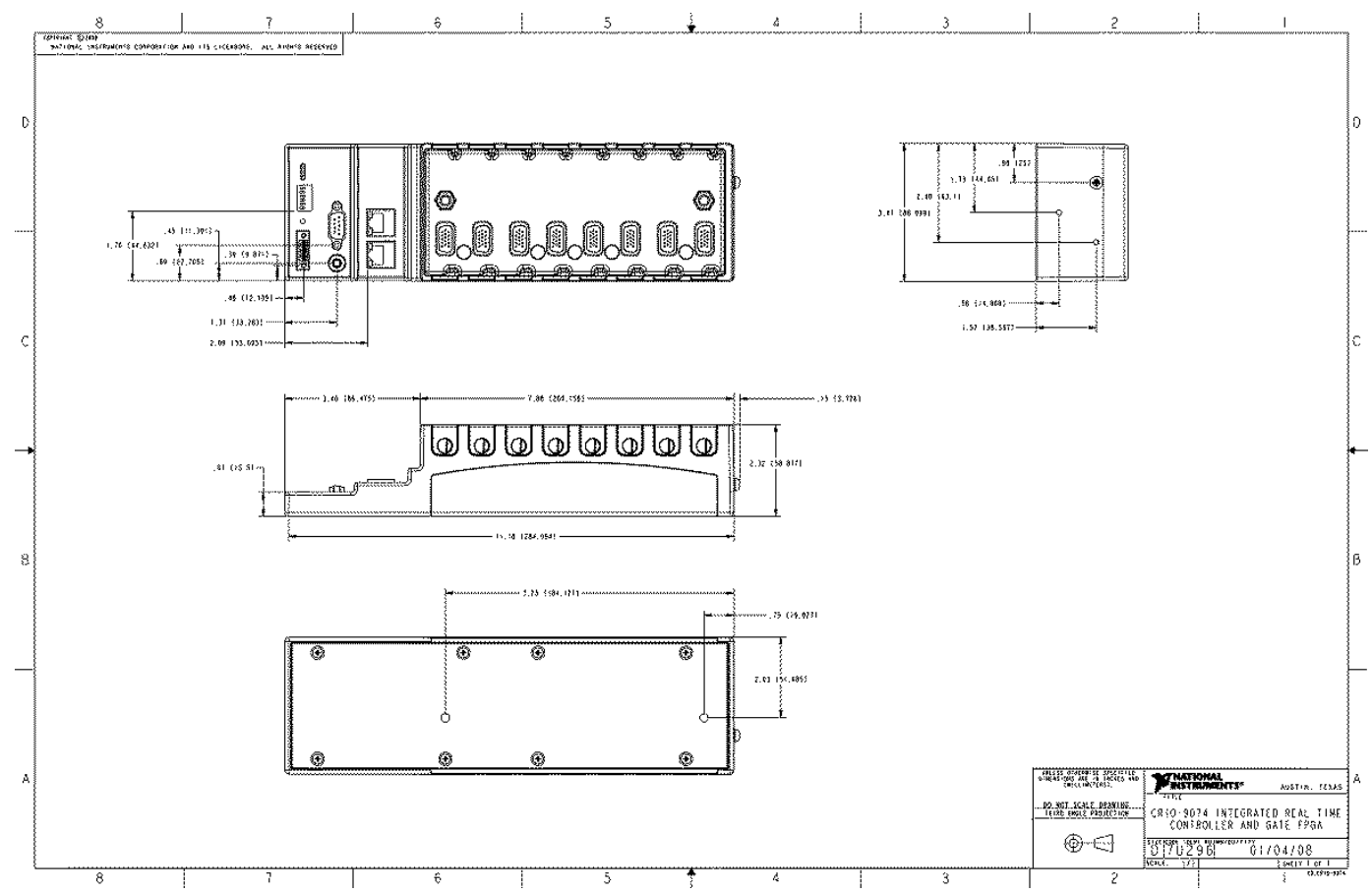


Development of the West Virginia University

Small Microgravity Research Facility (WVU SMiRF)
Copyright $^{\odot} 2014$

Kyle G. Phillips

\section{OPERATING INSTRUCTIONS AND SPECIFICATIONS CompactRIO"' cRI0-9072/3/4}

Reconfigurable Embedded Chassis with Integrated Intelligent Real-Time Controller for CompactRI0

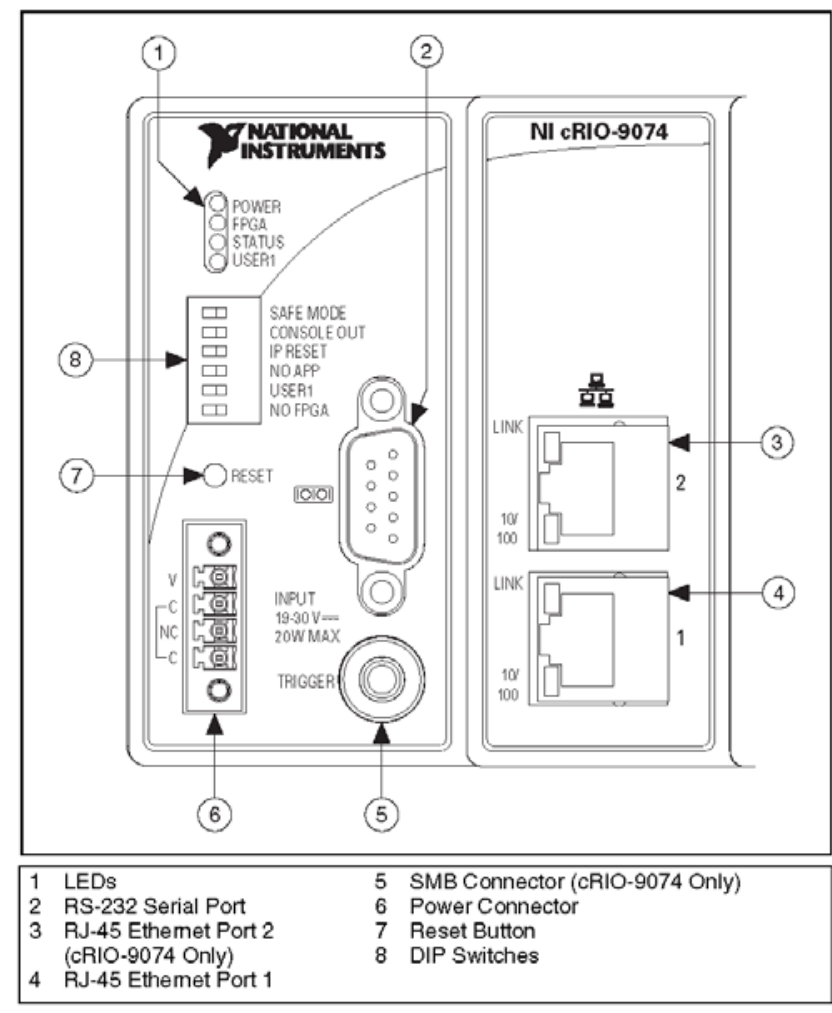

Figure 1. CompactRIO cRI0-9072/3/4 
This document describes how to connect the cRIO-9072/3/4 to a network and how to use the features of the cRIO-9072/3/4. This document also contains specifications for the cRIO-9072/3/4

\section{Safety Guidelines}

Operate the cRIO-9072/3/4 only as described in these operating instructions.

\section{Safety Guidelines for Hazardous Locations}

The cRIO-9072/3/4 is suitable for use in Class I, Division 2, Groups A, B, C, D, T4 hazardous locations; Class 1, Zone 2, AEx nL IIC T4 and Ex nL IIC T4 hazardous locations; and nonhazardous locations only. Follow these guidelines if you are installing the cRIO-9072/3/4 in a potentially explosive environment. Not following these guidelines may result in serious injury or death.

1 Caution Do not disconnect the power supply wires and connectors from the controller unless power has been switched off.

A Caution Substitution of components may impair suitability for Class I, Division 2.

1 Caution For Zone 2 applications, install the CompactRIO system in an enclosure rated to at least IP 54 as defined by IEC 60529 and EN 60529 .

\section{Special Conditions for Hazardous Locations Use in Europe}

Some chassis have been evaluated as Ex nL IIC T4 equipment under DEMKO Certificate No. 07 ATEX 0626664X. Each such chassis is marked (2) II $3 \mathrm{G}$ and is suitable for use in Zone 2 hazardous locations, in ambient temperatures of $-20 \leq \mathrm{Ta} \leq 55^{\circ} \mathrm{C}$.

\section{Special Conditions for Marine Applications}

Some chassis are Lloyd's Register (LR) Type Approved for marine applications. To verify Lloyd's Register certification, visit ni.com/ certification and search for the LR certificate, or look for the Lloyd's Register mark on the chassis. 


\section{What You Need to Install CompactRIO Reconfigurable Embedded Hardware}

CompactRIO reconfigurable embedded chassis with integrated intelligent real-time controller

$\square$ C Series $\mathrm{I} / \mathrm{O}$ modules

DIN rail mount kit (for DIN rail mounting only)

Two M4 or number 10 panhead screws (for panel mounting only)

A number 2 Phillips screwdriver

Power supply

27 Notes Visitni.com/info and enter the Info Code rdsoftwareversion to determine which software you need to use the cRIO-9072/3/4.

The cRIO-9072/3/4 may be shipped with a clear protective film cover on the front panel. You can remove the film cover before installing the cRIO-9072/3/4.

\section{Mounting the CompactRIO Reconfigurable Embedded Chassis}

You can mount the chassis in any orientation on a $35 \mathrm{~mm}$ DIN rail or on a panel. Use the DIN rail mounting method if you already have a DIN rail configuration or if you need to be able to quickly remove the CompactRIO chassis. Use the panel mount method for high shock and vibration applications.

\footnotetext{
1. Caution Your installation must meet the following requirements for space and cabling clearance:

- Allow $25.4 \mathrm{~mm}$ ( $1 \mathrm{in}$.) on the top and the bottom of the chassis for air circulation.

- Allow $50.8 \mathrm{~mm}$ ( $2 \mathrm{in}$.) in front of modules for cabling clearance for common connectors, such as the 10-terminal, detachable screw terminal connector, as shown in Figure 2.
} 


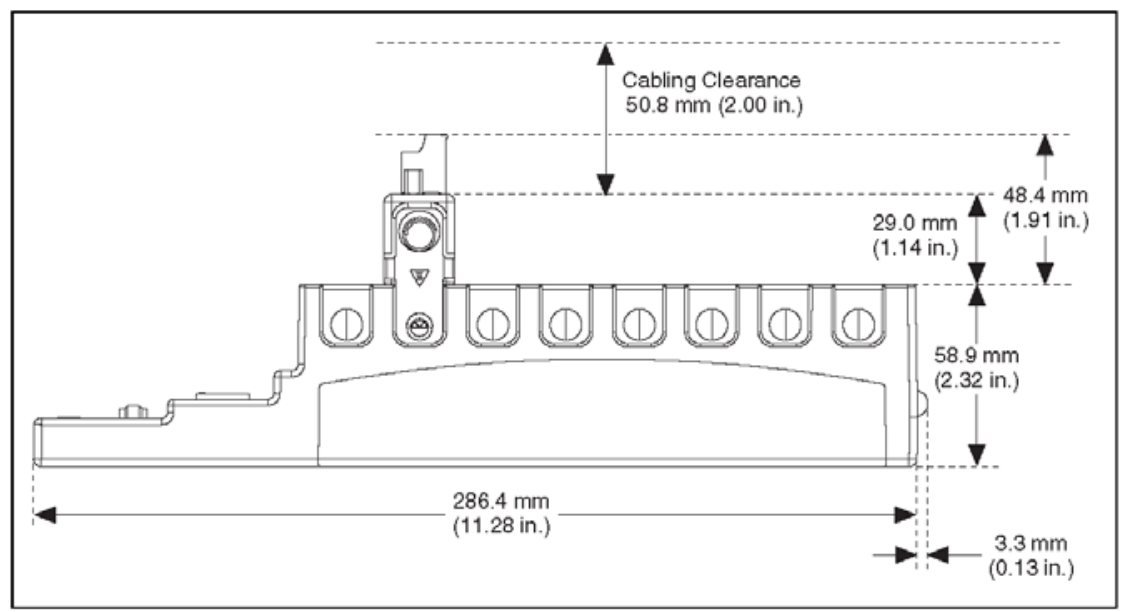

Figure 2. cRI0-9072/3/4, Bottom View with Dimensions

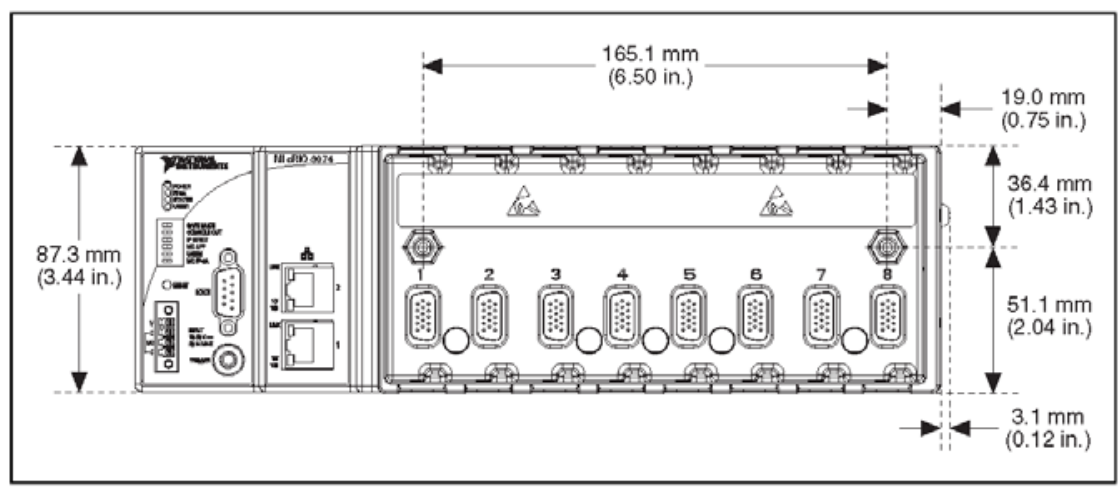

Figure 3. cRIO-9072/3/4, Front View with Dimensions 


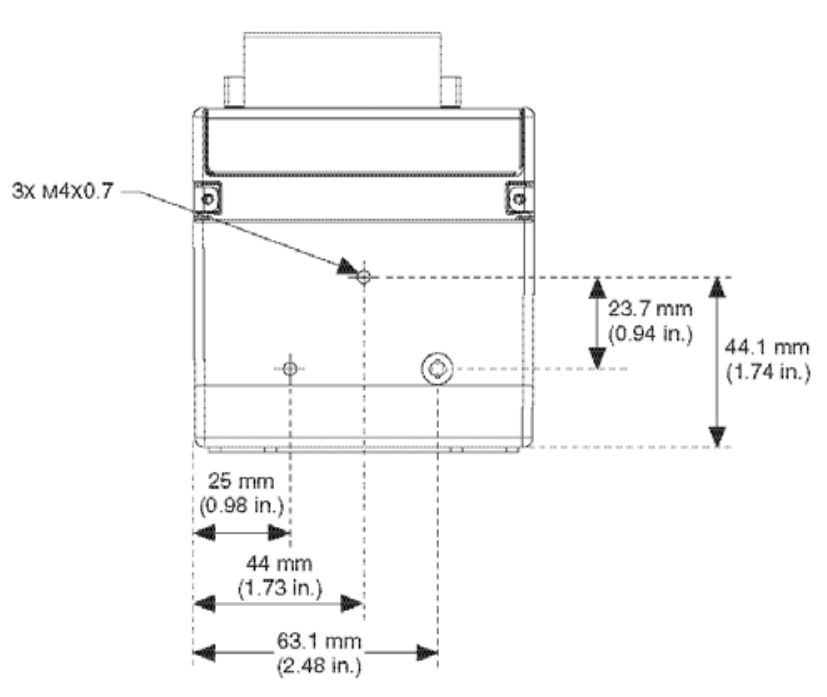

Figure 4. cRI0-9072/3/4, Side View with Dimensions

The following sections contain instructions for the mounting methods. Before using any of these mounting methods, record the serial number from the back of the chassis. You will be unable to read the serial number after you have mounted the chassis.

A Caution Make sure that no I/O modules are in the chassis before mounting it.

\section{Mounting the Chassis on a Panel}

You can use the NI 9905 panel mount kit to mount the cRIO-9072/3/4 on a flat surface. Complete the following steps.

1. Fasten the chassis to the panel mount kit using a number 2 Phillips screwdriver and two M4 $\times 16$ screws. National Instruments provides these screws with the panel mount kit. You must use these screws because they are the correct depth and thread for the panel. 


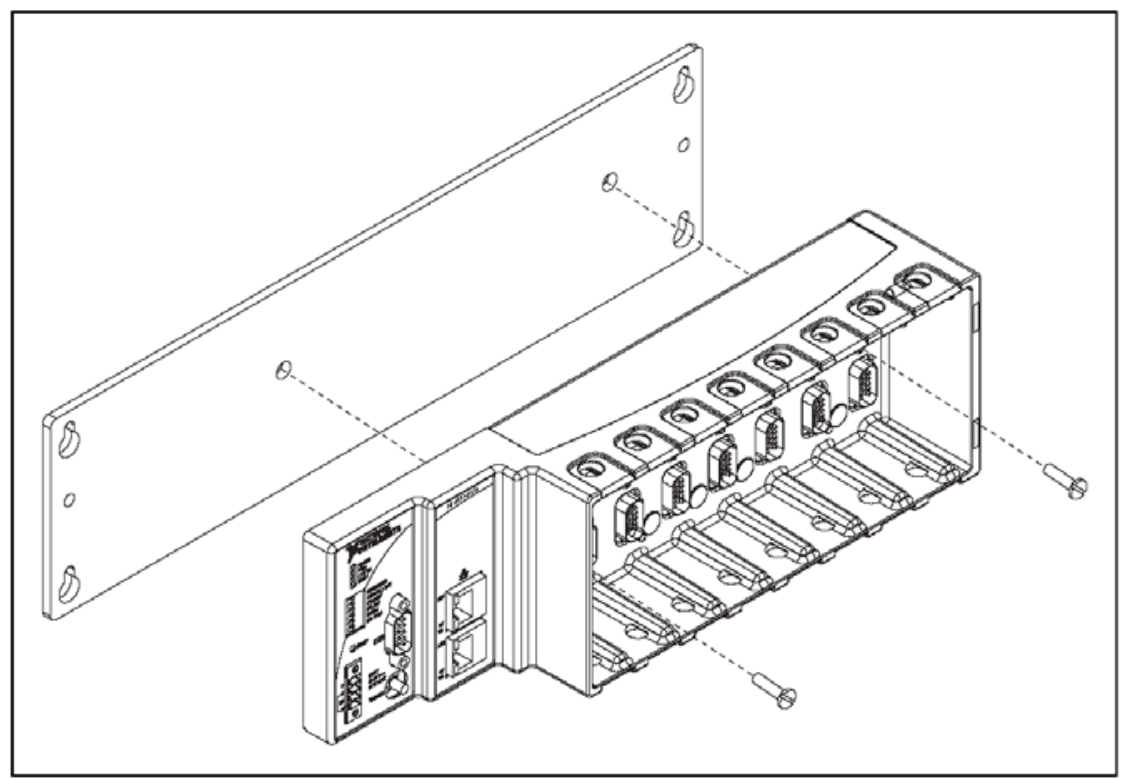

Figure 5. Installing the Panel Mount Accessory on the cRI0-9072/3/4

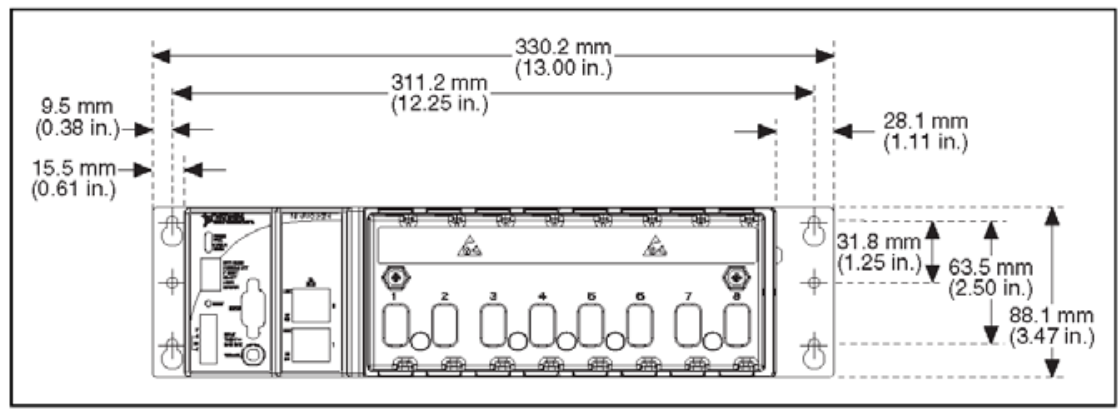

Figure 6. Dimensions of cRI0-9072/3/4 with Panel Mount Accessory Installed

2. Fasten the NI 9905 panel to the wall using the screwdriver and screws that are appropriate for the wall surface.

Caution Make sure that no I/O modules are in the chassis before removing it from the
panel. 


\section{Mounting the Chassis on a DIN Rail}

You can order the NI 9915 DIN rail mount kit if you want to mount the chassis on a DIN rail. You need one clip for mounting the chassis on a standard $35 \mathrm{~mm}$ DIN rail. Complete the following steps to mount the chassis on a DIN rail.

1. Fasten the DIN rail clip to the chassis using a number 2 Phillips screwdriver and two M4 $\times 16$ screws. National Instruments provides these screws with the DIN rail mount kit.

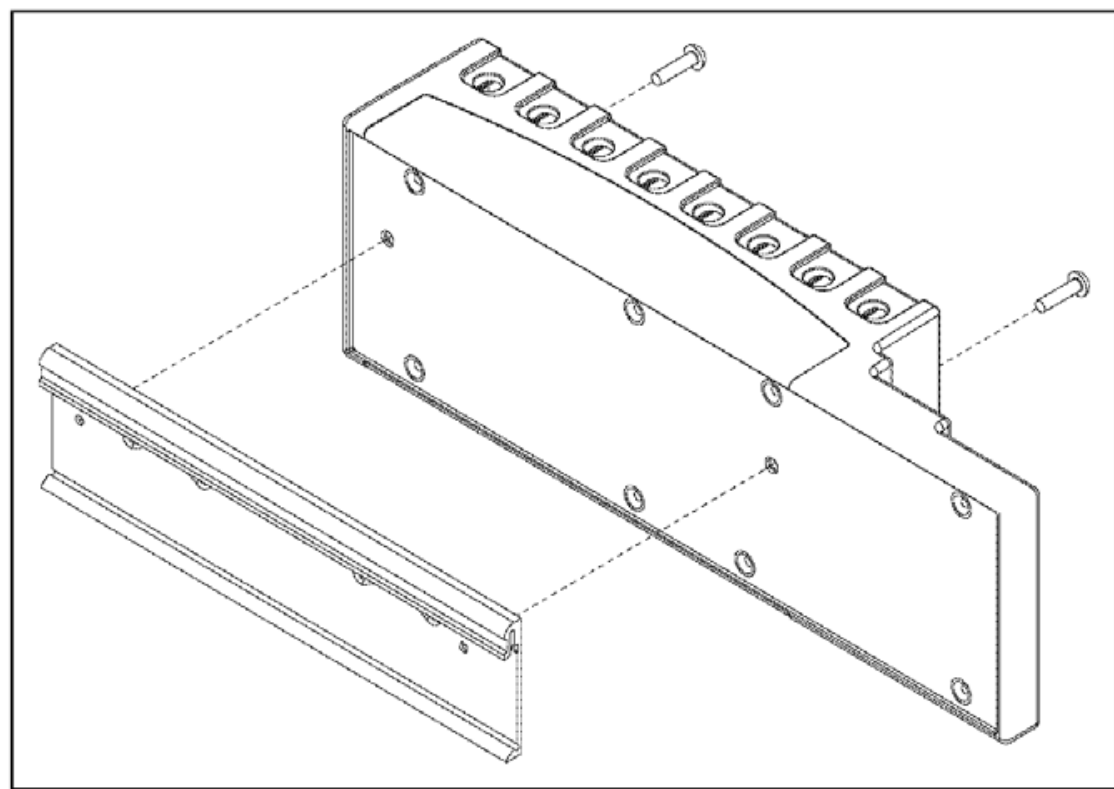

Figure 7. Installing the DIN Rail Clip on the cRI0-9072/3/4 
2. Insert one edge of the DIN rail into the deeper opening of the DIN rail clip, as shown in Figure 8.

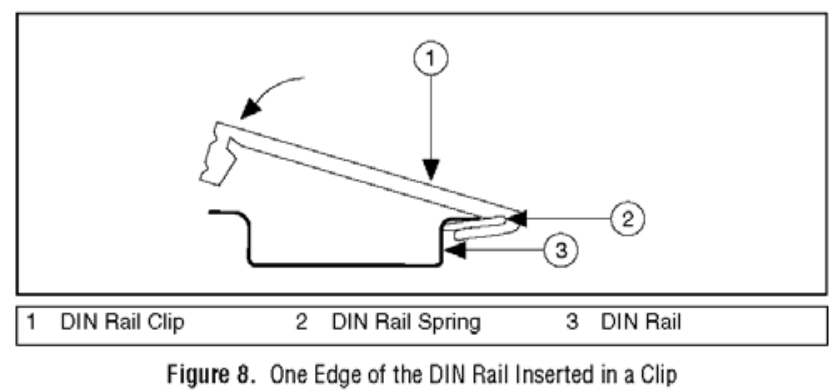

3. Press down firmly on the chassis to compress the spring until the clip locks in place on the DIN rail.

1! Caution Make sure that no I/O modules are in the chassis before removing it from the DIN rail.

\section{Installing C Series I/O Modules in the Chassis}

Figure 9 shows the mechanical dimensions of C Series I/O modules.

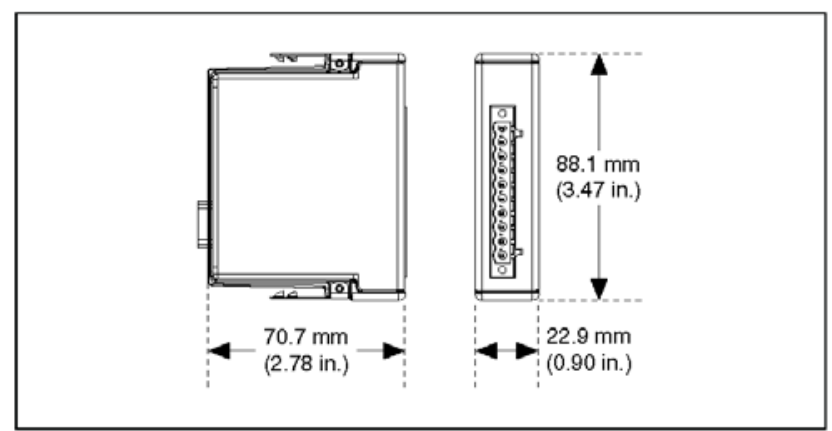

Figure 9. C Series 1/0 Module, Front and Side View with Dimensions 
Complete the following steps to install a C Series $\mathrm{I} / \mathrm{O}$ module in the chassis.

1. Make sure that no $\mathrm{I} / \mathrm{O}$-side power is connected to the $\mathrm{I} / \mathrm{O}$ module. If the system is in a nonhazardous location, the chassis power can be on when you install $\mathrm{L} / \mathrm{O}$ modules.

2. Align the $\mathrm{I} / \mathrm{O}$ module with an $\mathrm{H} / \mathrm{O}$ module slot in the chassis as shown in Figure 10. The module slots are labeled 1 to 8 , left to right.

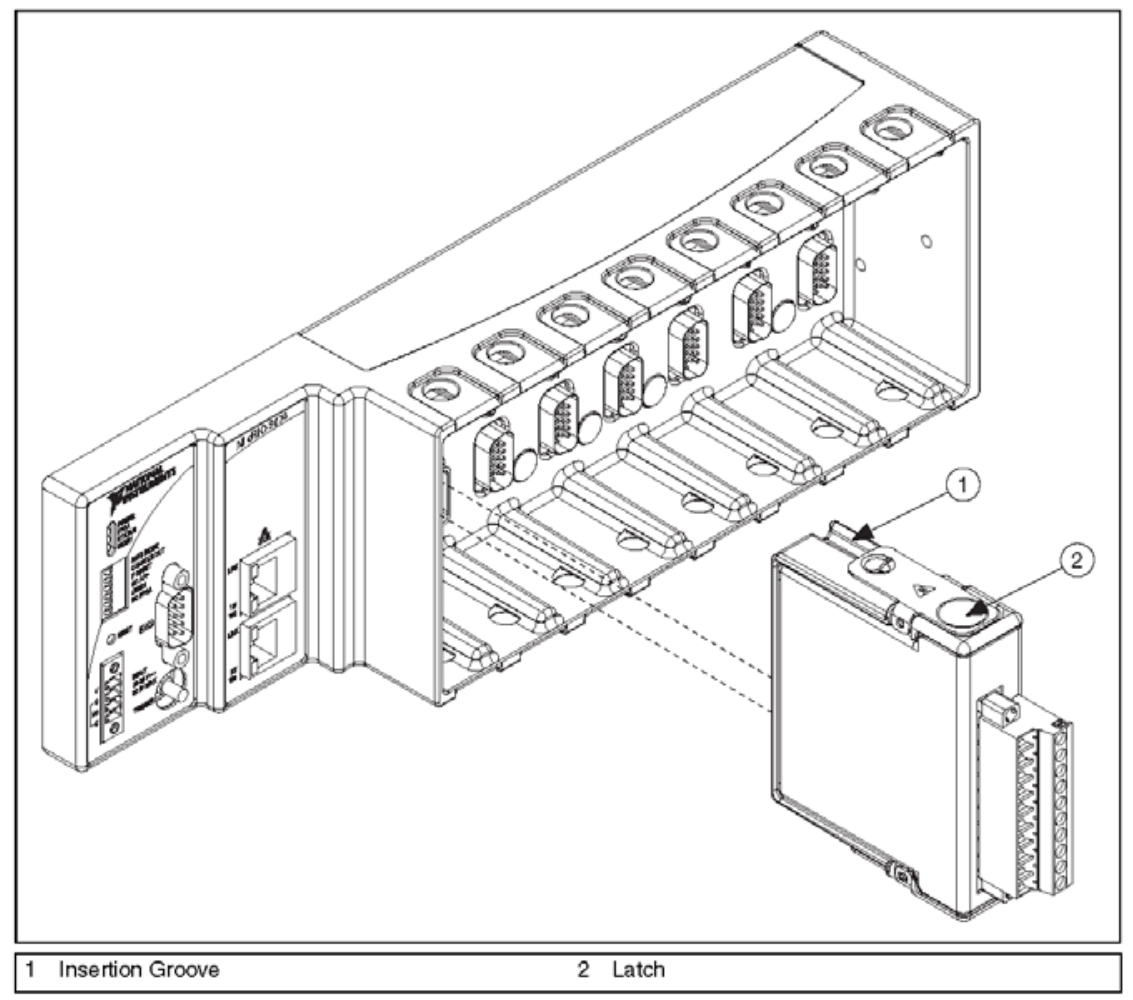

Figure 10. Installing an I/O Module in the Chassis

3. Squeeze the latches and insert the $\mathrm{I} / \mathrm{O}$ module into the module slot.

4. Press firmly on the connector side of the $\mathrm{I} / \mathrm{O}$ module until the latches lock the $\mathrm{I} / \mathrm{O}$ module into place.

5. Repeat these steps to install additional I/O modules. 


\section{Removing $1 / 0$ Modules from the Chassis}

Complete the following steps to remove a C Series I/O module from the chassis.

1. Make sure that no $\mathrm{I} / \mathrm{O}$-side power is connected to the $\mathrm{I} / \mathrm{O}$ module. If the system is in a nonhazardous location, the chassis power can be on when you remove $\mathrm{I} / \mathrm{O}$ modules.

2. Squeeze the latches on both sides of the module and pull the module out of the chassis.

\section{Connecting the Chassis to a Network}

Connect the chassis to an Ethernet network using RJ-45 Ethernet port 1 on the controller front panel. Use a standard Category 5 (CAT-5) or better shielded, twisted-pair Ethernet cable to connect the chassis to an Ethernet hub, or use an Ethernet crossover cable to connect the chassis directly to a computer.

A Caution To prevent data loss and to maintain the integrity of your Ethernet installation, do not use a cable longer than $100 \mathrm{~m}$.

If you need to build your own cable, refer to the Cabling section for more information about Ethernet cable wiring connections.

The first time you power up the chassis, the BIOS network settings determine the initial IP settings and other network behavior. After powerup, you must install software on the chassis and configure the network settings in Measurement \& Automation Explorer (MAX).

Note Installing software may change the network behavior of the chassis. For information about network behavior by installed software version, go to $\mathrm{ni}$. com/info and enter the Info Code ipconfigcrio.

The BIOS network settings of the cRIO-9072/3/4 depend on the part number of the chassis. The part number is located on the bottom of the chassis.

\section{BIOS Network Settings of cRI0-9072/3/4 with Part Number Beginning 192172}

If the part number of the cRIO-9072/3/4 begins with 192172, the IP address, subnet mask, DNS address, gateway, and Time Server IP are all set to 0.0 .0 .0 at powerup. 
The host computer communicates with the chassis over a standard Ethernet connection. If the host computer is on a network, you must configure the chassis on the same subnet as the host computer. If neither the host computer nor the chassis is connected to a network, you can connect the two directly using a crossover cable.

If you want to use the chassis on a subnet other than the one the host computer is on, first connect the chassis on the same subnet as the host computer. Use DHCP to assign an IP address or reassign a static IP address for the subnet where you want it to be and physically move it to the other subnet.

\section{BIOS Network Settings of cRI0-9072/3/4 with Part Number Beginning 198944}

If the part number of the cRIO-9072/3/4 begins with 198944 , the chassis attempts to initiate a DHCP network connection at powerup. If the chassis is unable to initiate a DHCP connection, it connects to the network with a link-local IP address with the form 169.254.x.x.

The host computer communicates with the chassis over a standard Ethernet connection. If neither the host computer nor the chassis is connected to a network, you can connect the two directly using a crossover cable.

\section{Wiring Power to the Chassis}

The cRIO-9072/3/4 requires an external power supply that meets the specifications in the Power Requirements section. The cRIO-9072/3/4 filters and regulates the supplied power and provides power for all of the I/O modules. The cRIO-9072/3/4 has one layer of reverse-voltage protection. Complete the following steps to connect a power supply to the chassis.

1. Connect the positive lead of the power supply to the $\mathrm{V}$ terminal of the COMBICON power connector shipped with the cRIO-9072/3/4, and tighten the terminal screw. Figure 11 shows the terminal screws, which secure the wires in the screw terminals, and the connector screws, which secure the power connector on the front panel. 


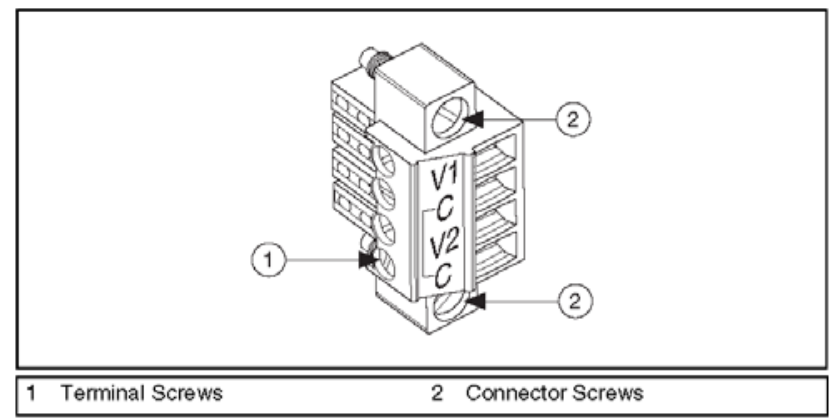

Figure 11. COMBICON Power Connector

2. Connect the negative lead of the power supply to one of the $\mathrm{C}$ terminals of the power connector and tighten the terminal screw.

3. Optionally, you can connect the positive lead of another power supply to the other $\mathrm{V}$ terminal and the negative lead to one of the $\mathrm{C}$ terminals.

4. Install the power connector on the front panel of the cRIO-9072/3/4 and tighten the connector screws.

1) Caution The $\mathrm{C}$ terminals are internally connected to each other.

\section{Powering On the cRI0-9072/3/4}

When you apply power to the cRIO-9072/3/4, the controller runs a power-on self test (POST). During the POST, the Power and Status LEDs turn on. The Status LED turns off, indicating that the POST is complete. If the LEDs do not behave in this way when the system powers on, refer to the Understanding LED Indications section.

You can configure the cRIO-9072/3/4 to launch an embedded stand-alone LabVIEW RT application each time you boot the controller. Refer to the Running a Stand-Alone Real-Time Application (RT Module) topic of the LabVIEW Help for more information. 


\section{Chassis Reset Options}

Table 1 lists the reset options available on CompactRIO systems such as the cRIO-9072/3/4. These options determine how the chassis behaves when the controller is reset in various conditions. Use the RIO Device Setup utility to select reset options. Access the RIO Device Setup utility by selecting Start»All Programs»National Instruments»NI-RIO» RIO Device Setup.

Table 1. CompactRiO Reset Options

\begin{tabular}{|l|l|}
\hline \multicolumn{1}{|c|}{ Chassis Reset Option } & \multicolumn{1}{c|}{ Behavior } \\
\hline \hline Do not autoload VI & Does not load the FPGA bit stream from flash memory. \\
\hline Autoload VI on device powerup & $\begin{array}{l}\text { Loads the FPGA bit stream from flash memory to the FPGA } \\
\text { when the controller powers on. }\end{array}$ \\
\hline Autoload VI on device reboot & $\begin{array}{l}\text { Loads the FPGA bit stream from flash memory to the FPGA } \\
\text { when you reboot the controller either with or without cycling } \\
\text { power. }\end{array}$ \\
\hline
\end{tabular}

\section{Connecting Serial Devices to the $\mathrm{CRI0-9072/3/4}$}

The cRIO-9072/3/4 has an RS-232 serial port to which you can connect devices such as displays or input devices. Use the Serial VIs to read from and write to the serial port from a LabVIEW RT application. For more information about the Serial VIs, refer to the LabVIEW Help.

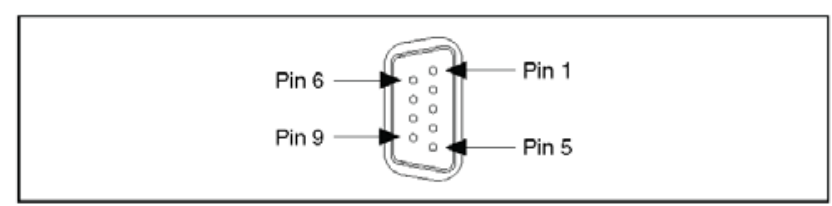

Figure 12. Controller Serial Port 
Table 2. DB-9 Pin Descriptions

\begin{tabular}{|c|c|}
\hline Pin & Signal \\
\hline \hline 1 & DCD \\
\hline 2 & RXD \\
\hline 3 & TXD \\
\hline 4 & DTR \\
\hline 5 & GND \\
\hline 6 & DSR \\
\hline 7 & RTS \\
\hline 8 & CTS \\
\hline 9 & RI \\
\hline
\end{tabular}

\section{Using the Internal Real-Time Clock}

The system clock of the cRIO-9012/9014 is synchronized with the internal high-precision real-time clock at startup. This synchronization provides timestamp data to the controller. You can also use the internal real-time clock to correct drift of the system clock. Refer to the Internal Real-Time Clock specification in the Specifications section for the accuracy specifications of the real-time clock.

\section{Using the SMB Connector for Digital I/0 (cRI0-9074 Only)}

You can use the SMB connector of the cRIO-9074 to connect digital devices to the controller. For example, if you connect the pulse-per-second output of a GPS device to the SMB connector of the cRIO-9074, you can use the GPS device to correct for drift of the system clock.

For software that supports GPS drift-correction and other digital I/O through the SMB connector, go to ni. com/info and enter the Info Code criosmb. 


\section{Configuring DIP Switches}

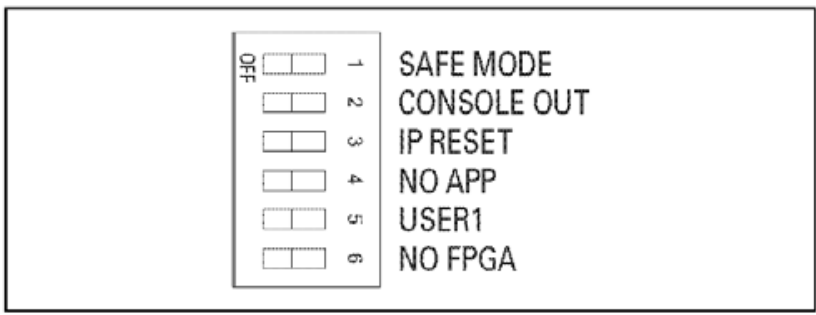

Figure 13. DIP Switches

All of the DIP switches are in the OFF position when the chassis is shipped from National Instruments.

\section{SAFE MODE Switch}

The position of the SAFE MODE switch determines whether the embedded LabVIEW Real-Time engine launches at startup. If the switch is in the OFF position, the LabVIEW Real-Time engine launches. Keep this switch in the OFF position during normal operation. If the switch is in the ON position at startup, the cRIO-9072/3/4 launches only the essential services required for updating its configuration and installing software. The LabVIEW Real-Time engine does not launch.

If the software on the controller is corrupted, you must put the controller into safe mode and reformat the controller drive. You can put the controller into safe mode by powering it up either with the SAFE MODE switch in the ON position or with no software installed on the drive. Refer to the Measurement \& Automation Explorer Help for more information about installing software on a controller and reformatting the drive on the controller.

\section{CONSOLE OUT Switch}

With a serial-port terminal program, you can use the CONSOLE OUT switch to read the IP address and firmware version of the controller. Use a null-modem cable to connect the serial port on the chassis to a computer. Push the switch to the ON position. Make sure that the serial-port terminal program is configured to the following settings:

- 9,600 bits per second

- Eight data bits

- No parity 
- One stop bit

- No flow control

The serial-port terminal program displays the IP address and firmware version of the chassis. Keep this switch in the OFF position during normal operation.

\section{IP RESET Switch}

Push the IP RESET switch to the ON position and reboot the controller to reset the IP address and other TCP/IP settings of the controller to the factory defaults. Refer to the Troubleshooting Network Communication section for more information about resetting the IP address. You also can push this switch to the ON position to unlock a chassis that was previously locked in MAX.

\section{NO APP Switch}

Push the NO APP switch to the ON position to prevent a LabVIEW RT startup application from running at startup. If you want to permanently disable a LabVIEW RT application from running at startup, you must disable it in LabVIEW. To run an application at startup, push the NO APP switch to the OFF position, create an application using the LabVIEW Application Builder, and configure the application in LabVIEW to launch at startup. If you already have an application configured to launch at startup and you push the NO APP switch from ON to OFF, the startup application is automatically enabled. For more information about automatically launching VIs at startup and disabling VIs from launching at startup, refer to the Running a Stand-Alone Real-Time Application (RT Module) topic of the LabVIEW Help.

\section{USER1 Switch}

You can define the USER1 switch for your application. To define the purpose of this switch in your embedded application, use the RT Read Switch VI in your LabVIEW RT embedded VI. For more information about the RT Read Switch VI, refer to the LabVIEW Help.

\section{NO FPGA Switch}

Push the NO FPGA switch to the ON position to prevent a LabVIEW FPGA application from loading at startup. The NO FPGA switch overrides the CompactRIO reset options described in the Chassis Reset Options section. After startup you can download to the FPGA from software regardless of switch position. 


\section{Using the RESET Button}

Pressing the RESET button resets the processor in the same manner as cycling power.

Note The FPGA continues to run unless you select the Autoload on Any Device Reset boot option. Refer to the Chassis Reset Options section for more information.

\section{Understanding LED Indications}

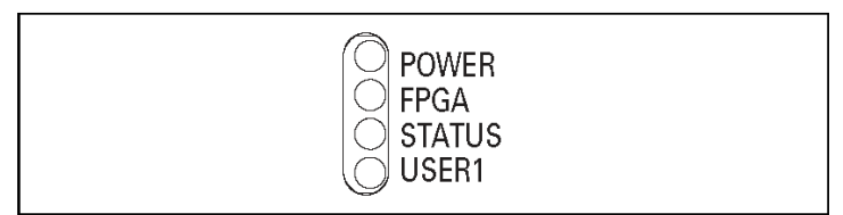

Figure 14. cRI0-9072/3/4 LEDs

\section{POWER LED}

The POWER LED is lit while the cRIO-9072/3/4 is powered on. This LED indicates that the power supply connected to the chassis is adequate.

\section{FPGA LED}

You can use the FPGA LED to help debug your application or easily retrieve application status. Use the LabVIEW FPGA Module and NI-RIO software to define the FPGA LED to meet the needs of your application. Refer to LabVIEW Help for information about programming this LED. 


\section{STATUS LED}

The STATUS LED is off during normal operation. The cRIO-9072/3/4 indicates specific error conditions by flashing the STATUS LED a certain number of times every few seconds, as shown in Table 3.

Table 3. Status LED Indications

\begin{tabular}{|c|c|}
\hline $\begin{array}{l}\text { Number of Flashes } \\
\text { Every Few Seconds }\end{array}$ & Indication \\
\hline 1 & $\begin{array}{l}\text { The chassis is unconfigured. Use MAX to configure the chassis. Refer } \\
\text { to the Measurement \& Automation Explorer Help for information about } \\
\text { configuring the chassis. }\end{array}$ \\
\hline 2 & $\begin{array}{l}\text { The chassis has detected an error in its software. This usually occurs } \\
\text { when an attempt to upgrade the software is interrupted. Reinstall } \\
\text { software on the chassis. Refer to the Measurement \& Automation } \\
\text { Explorer Help for information about installing software on the chassis. }\end{array}$ \\
\hline 3 & $\begin{array}{l}\text { The chassis is in safe mode because the SAFE MODE DIP switch is in } \\
\text { the ON position or there is no software installed on the chassis. Refer to } \\
\text { the Configuring DIP Switches section for information about the Safe } \\
\text { Mode DIP switch. }\end{array}$ \\
\hline 4 & $\begin{array}{l}\text { The software has crashed twice without rebooting or cycling power } \\
\text { between crashes. This usually occurs when the chassis runs out of } \\
\text { memory. Review your RT VI and check the memory usage. Modify } \\
\text { the VI as necessary to solve the memory usage issue. }\end{array}$ \\
\hline Continuously flashing & $\begin{array}{l}\text { The chassis has detected an unrecoverable error. Contact National } \\
\text { Instruments. }\end{array}$ \\
\hline $\begin{array}{l}\text { Continuously flashing } \\
\text { or solid }\end{array}$ & $\begin{array}{l}\text { The device may be configured for DHCP but unable to get an IP address } \\
\text { because of a problem with the DHCP server. Check the network } \\
\text { connection and try again. If the problem persists, contact National } \\
\text { Instruments. }\end{array}$ \\
\hline
\end{tabular}

\section{USER1 LED}

You can define the USER1 LED to meet the needs of your application. To define the LED, use the RT LEDs VI in LabVIEW. For more information about the RT LEDs VI, refer to the LabVIEW Help. 


\section{Troubleshooting Network Communication}

If the cRIO-9072/3/4 cannot communicate with the network, you can perform the following troubleshooting steps.

1. Move the IP RESET switch to the ON position.

2. Push the RESET button to cycle power to the chassis.

3. Configure the IP and other network settings in MAX.

4. Move the IP RESET switch to the OFF position.

2 Note The network behavior of the chassis after powering up with the IP RESET switch on depends on the version of LabVIEW RT installed. For information about the different network behaviors with different software versions, go to ni , com / info and enter the Info Code ipconfigcrio.

If you are unable to fix network communication with the LabVIEW RT network settings restored, you can restore the BIOS network settings of the chassis. Refer to the Connecting the Chassis to a Network section of this document for information about the BIOS network settings for different chassis revisions. Complete the following steps to restore the BIOS network settings of the chassis.

1. Move the IP RESET and SAFE MODE switches to the ON position.

2. Push the RESET button to cycle power to the chassis.

3. Configure the IP and other network settings in MAX.

4. Move the IP RESET and SAFE MODE switches to the OFF position.

Q7 Note If the chassis is restored to the BIOS network settings, the LabVIEW run-time engine does not load. You must reconfigure the network settings and restart the chassis for the LabVIEW run-time engine to load. 


\section{Specifications}

The following specifications are typical for the -20 to $55^{\circ} \mathrm{C}$ operating temperature range unless otherwise noted.

Network

Network interface .............................10 BaseT and 100BaseTX
Ethernet
Compatibility ...................................EEE 802.3
Communication rates ........................10 Mbps, $100 \mathrm{Mbps}$ auto-negotiated
Maximum cabling distance..................100 m/segment

RS-232 Serial Port

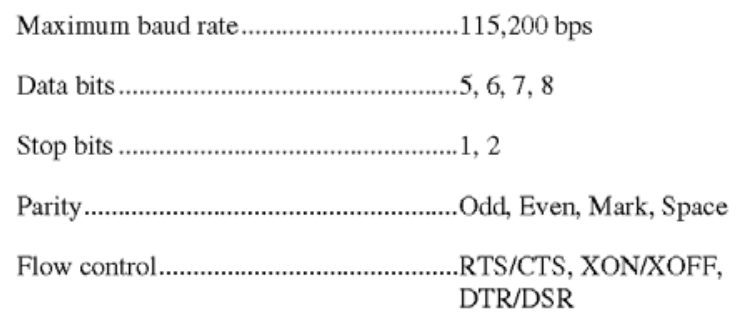

SMB Connector (cRI0-9074 Only)

Output Characteristics

Minimum high-level output voltage With $-100 \mu \mathrm{A}$ output current ..........2.9 V With -16 mA output current............2.4 V With -24 mA output current.............2.3 V

Maximum low-level output voltage With $100 \mu \mathrm{A}$ output current .............0.10 V With 16 mA output current...............0.40 V With 24 mA output current...............0.55 V

Driver type. CMOS 


Maximum sink/source current .............. $\pm 24 \mathrm{~mA}$
Maximum 3-state output
leakage current ................................ $\pm 5 \mu \mathrm{A}$
Input Characteristics
Minimum input voltage..................... $\mathrm{V}$ V
Minimum low-level input voltage........... $0.94 \mathrm{~V}$
Maximum high-level input voltage......... $2.43 \mathrm{~V}$
Maximum input voltage ......................... $5.5 \mathrm{~V}$
Typical input capacitance......................... $2.5 \mathrm{pF}$
Typical resistive strapping ...................... $1 \mathrm{k} \Omega$ to $3.3 \mathrm{~V}$

\section{Memory}

cRIO-9072, cRIO-9073

Nonvolatile... $128 \mathrm{MB}$ minimum

System memory $64 \mathrm{MB}$

cRIO-9074

Nonvolatile. $256 \mathrm{MB}$ minimum

System memory $128 \mathrm{MB}$

For information about the life span of the nonvolatile memory and about best practices for using nonvolatile memory, go to ni.com/info and enter the Info Code SSDBP.

\section{Reconfigurable FPGA}

cRIO-9072

Number of logic cells 17,280

Available embedded RAM 432 kbits

cRIO-9073, cRIO-9074

Number of logic cells. 46,080

Available embedded RAM 720 kbits

For information about the life span of the nonvolatile memory and about best practices for using nonvolatile memory, go to ni. com/info and enter the Info Code SSDBP. 


\title{
Internal Real-Time Clock
}

\author{
Accuracy
} $.200 \mathrm{ppm} ; 35 \mathrm{ppm}$ at $25^{\circ} \mathrm{C}$

\section{Power Requirements}

A Caution You must use a UL Listed ITE power supply marked LPS with the cRIO-9072/3/4.
Recommended power supply.
$48 \mathrm{~W}, 24 \mathrm{VDC}$
Power consumption
$.20 \mathrm{~W}$ maximum
Power supply input range
19 to $30 \mathrm{~V}$

\section{Physical Characteristics}

\author{
If you need to clean the controller, wipe it with a dry towel. \\ Screw-terminal wiring ............................ 0.5 to $2.5 \mathrm{~mm}^{2}$ (24 to $12 \mathrm{AWG)}$ \\ copper conductor wire with \\ $10 \mathrm{~mm}(0.39 \mathrm{in}$.) of insulation \\ stripped from the end \\ Torque for screw terminals \\ 0.5 to $0.6 \mathrm{~N} \cdot \mathrm{m}$ \\ $(4.4$ to $5.3 \mathrm{lb} \cdot \mathrm{in}$.) \\ Weight \\ $.929 \mathrm{~g}(32.7 \mathrm{oz})$
}

\section{Safety Voltages}

Connect only voltages that are within these limits.
$\mathrm{V}$ terminal to $\mathrm{C}$ terminal..........................35 V max, Measurement Category I

Measurement Category I is for measurements performed on circuits not directly connected to the electrical distribution system referred to as MAINS voltage. MAINS is a hazardous live electrical supply system that powers equipment. This category is for measurements of voltages from specially protected secondary circuits. Such voltage measurements include signal levels, special equipment, limited-energy parts of equipment, circuits powered by regulated low-voltage sources, and electronics.

1 Caution Do not connect the system to signals or use for measurements within Measurement Categories II, III, or IV. 


\section{Safety Standards}

This product meets the requirements of the following standards of safety for electrical equipment for measurement, control, and laboratory use:

- IEC 61010-1, EN 61010-1

- UL 61010-1, CSA 61010-1

Q3 Note For UL and other safety certifications, refer to the product label or the Online Product Certification section.

\section{Electromagnetic Compatibility}

This product meets the requirements of the following EMC standards for electrical equipment for measurement, control, and laboratory use:

- EN 61326 (IEC 61326): Class A emissions; Industrial immunity

- EN 55011 (CISPR 11): Group 1, Class A emissions

- AS/NZS CISPR 11: Group 1, Class A emissions

- FCC 47 CFR Part 15B: Class A emissions

- ICES-001: Class A emissions

Note For the standards applied to assess the EMC of this product, refer to the Online Product Certification section.

Q7 Note For EMC compliance, operate this product according to the documentation.

\section{CE Compliance $C \epsilon$}

This product meets the essential requirements of applicable European Directives as follows:

- 2006/95/EC; Low-Voltage Directive (safety)

- 2004/108/EEC; Electromagnetic Compatibility Directive (EMC)

\section{Online Product Certification}

Refer to the Declaration of Conformity (DoC) for this product for additional regulatory compliance information. To obtain product certifications and the DoC for this product, visit ni.com/ certification, search by model number or product line, and click the appropriate link in the Certification column. 


\section{Environmental Management}

National Instruments is committed to designing and manufacturing products in an environmentally responsible manner. NI recognizes that eliminating certain hazardous substances from our products is beneficial to the environment and to NI customers.

For additional environmental information, refer to the $N I$ and the Environment Web page at ni.com/environment. This page contains the environmental regulations and directives with which NI complies, as well as other environmental information not included in this document.

\section{Waste Electrical and Electronic Equipment (WEEE)}

EU Customers At the end of the product life cycle, all products must be sent to a WEEE recycling center. For more information about WEEE recycling centers, National Instruments WEEE initiatives, and compliance with WEEE Directive 2002/96/EC on Waste and Electronic Equipment, visit ni.com/envi ronment/weee.

\section{电子信息产品污染控制管理办法（中国 RoHS）}

(e) 中国客户 National Instruments 符合中国电子信息产品中隄制使用某些有害物质指令 (RoHS) , 关于 Notional Instruments 中国 RoHS 合规珄信息, 请登录 ni.com/environment/rohs_china。 (For information about China RoHS compliance, go to ni. com/environment/rohs_china.)

\section{Battery Replacement and Disposal}

This device contains a long-life coin cell battery. If you need to replace it, use the Return Material Authorization (RMA) process or contact an authorized National Instruments sevice representative.

After replacement, recycle the old battery. For additional information, visit ni.com/environment.

\section{Hazardous Locations}

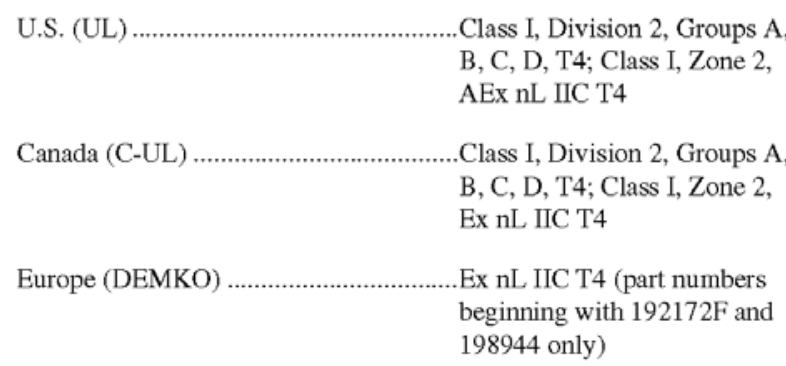




\section{Environmental}

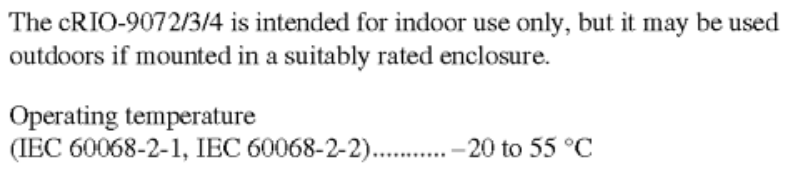

0 Note To meet this operating temperature range, follow the guidelines in the installation instructions for your CompactRIO system.

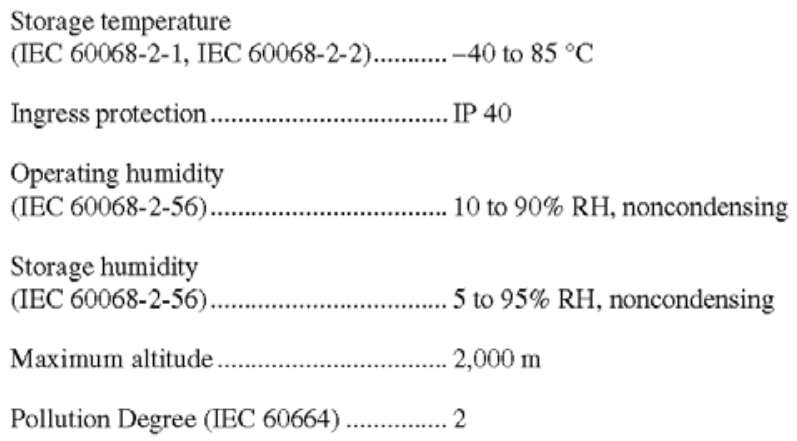

\section{Shock and Vibration}

To meet these specifications, you must panel mount the CompactRIO system and affix ferrules to the ends of the power terminal wires.

Operating shock

(IEC 60068-2-27) .................................... $30 \mathrm{~g}, 11 \mathrm{~ms}$ half sine

$50 \mathrm{~g}, 3 \mathrm{~ms}$ half sine,

18 shocks at 6 orientations

Operating vibration, random

(IEC 60068-2-64) ……......................... $5 \mathrm{~g}_{\mathrm{m} m s}, 10$ to $500 \mathrm{~Hz}$

Operating vibration, sinusoidal

(IEC 60068-2-6) ................................... $5 \mathrm{~g}, 10$ to $500 \mathrm{~Hz}$




\section{Cabling}

Table 4 shows the standard Ethernet cable wiring connections for both normal and crossover cables.

Table 4. Ethernet Cable Wiring Connections

\begin{tabular}{|c|l|l|l|}
\hline Pin & \multicolumn{1}{|c|}{ Connector 1 } & \multicolumn{1}{|c|}{$\begin{array}{c}\text { Connector 2 } \\
\text { (Normal) }\end{array}$} & \multicolumn{1}{|c|}{$\begin{array}{c}\text { Connector 2 } \\
\text { (Crossover) }\end{array}$} \\
\hline \hline 1 & white/orange & white/orange & white/green \\
\hline 2 & orange & orange & green \\
\hline 3 & white/green & white/green & white/orange \\
\hline 4 & blue & blue & blue \\
\hline 5 & white/blue & white/blue & white/blue \\
\hline 6 & green & green & orange \\
\hline 7 & white/brown & white/brown & white/brown \\
\hline 8 & brown & brown & brown \\
\hline
\end{tabular}

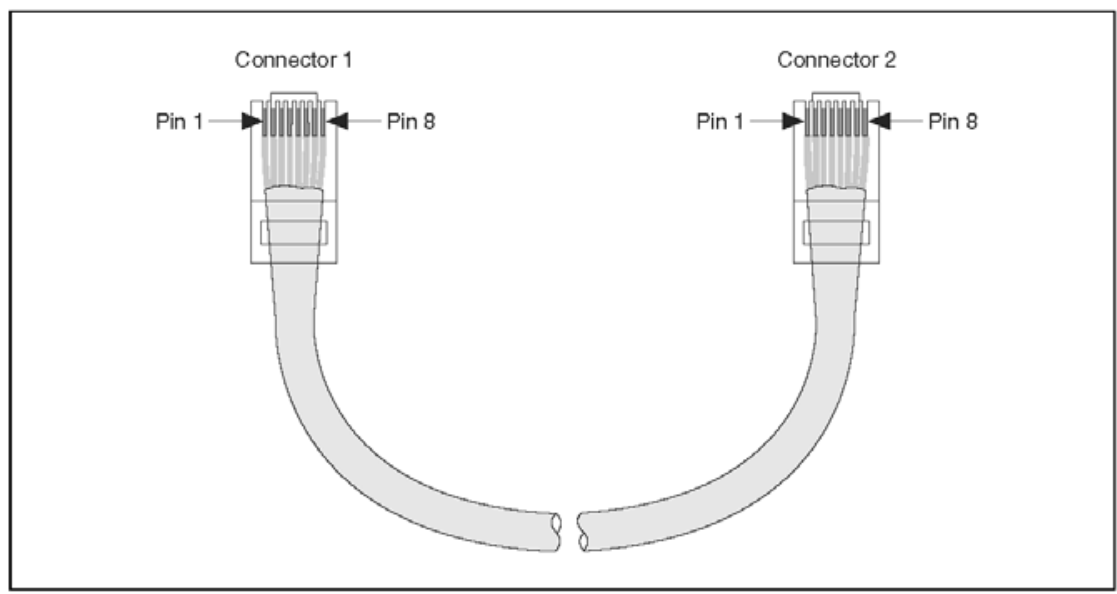

Figure 15. Ethernet Connector Pinout 


\section{Where to Go for Support}

The National Instruments Web site is your complete resource for technical support. At ni. com/ support you have access to everything from troubleshooting and application development self-help resources to email and phone assistance from NI Application Engineers.

National Instruments corporate headquarters is located at 11500 North Mopac Expressway, Austin, Texas, 78759-3504.

National Instruments also has offices located around the world to help address your support needs. For telephone support in the United States, create your service request at $\mathrm{ni}$.com/support and follow the calling instructions or dial 5127958248 . For telephone support outside the United States, contact your local branch office:

Australia 1800300800 , Austria $43662457990-0$,

Belgium 32 (0) 2757 0020, Brazil 551132623599 , Canada 800433 3488, China 862150509800 ,

Czech Republic 420224235774 , Denmark 4545762600 , Finland 358 (0) 9725 72511, France 0157662424 ,

Germany 49897413130 , India 918041190000 , Israel 97236393737 , Italy 390241309277 , Japan 0120-527196, Korea 820234513400 ,

Lebanon 961 (0) 13328 28, Malaysia 1800887710 ,

Mexico 01800010 0793, Netherlands 31 (0) 348433 466,

New Zealand 0800553 322, Norway 47 (0) 66907660 ,

Poland 482232890 10, Portugal 351210311210

Russia 7495783 6851, Singapore 18002265886 ,

Slovenia 386342542 00, South Africa 270118058197 Spain 34916400085 , Sweden 46 (0) 858789500 ,

Switzerland 4156 2005151, Taiwan 8860223772222 ,

Thailand 6622786777 , Turkey 902122793031 ,

United Kingdom 44 (0) 1635523545 
Development of the West Virginia University

Copyright $^{\odot} 2014$

Small Microgravity Research Facility (WVU SMiRF)

Kyle G. Phillips

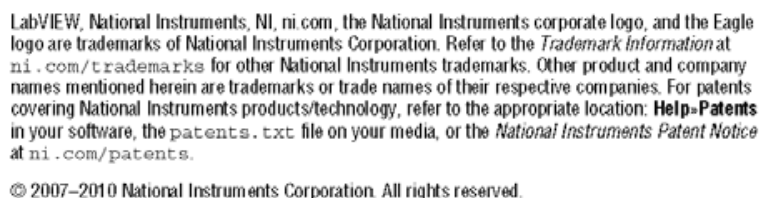

Q 2007-2010 National Instruments Corporation All rights reserved.

374639E-01

Jun10 


$\begin{array}{lr}\text { T NATIONAL } & \text { Technical Sales } \\ \text { United States } \\ (866) 531-6285 \\ \text { info@nic com }\end{array}$

Requirements and Compatibility I Ordering Information I Detailed Specifications

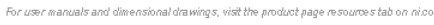

Last Revised: 2010-06-04 1530:49.0

$32 \mathrm{Ch}, \pm 200 \mathrm{mV}$ to $\pm 10 \mathrm{~V}, 16-\mathrm{Bit}, 250 \mathrm{kS} / \mathrm{s} \mathrm{C}$ Series Analog Input Module NI 9205

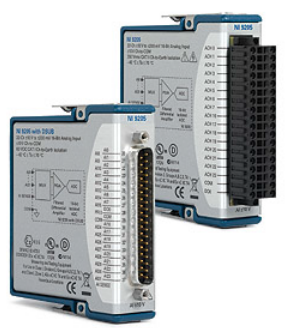

- 32 single-ended or 16 differential analog inputs

- 16-bit resolution, $250 \mathrm{kS} / \mathrm{s}$ aggregate sampling rate

- Hot-swappable operation; overvoltage protection; isolation; NIST-traceable calibration

- $\pm 200 \mathrm{mV}, \pm 1 \mathrm{~V}, \pm 5 \mathrm{~V}$, and $\pm 10 \mathrm{~V}$ programmable input ranges

-40 to $70^{\circ} \mathrm{C}$ operating range

- Spring terminal or D-Sub connectivity

Overview

The NII 9205 is a C Series analog input module for use with NI CompactDAQ and CompactRlO chassis. It features 32 single-ended or 16 differential analog inputs, 16-bit resolution, and a maximum sampling rate of $250 \mathrm{kS} / \mathrm{s}$. Each channel has programmable input ranges of $\pm 200 \mathrm{mV}, \pm 1 \mathrm{~V}, \pm 5 \mathrm{~V}$, and $\pm 10 \mathrm{~V}$. To protect against signal transients, the NI 9205 includes up to $60 \mathrm{~V}$ of overvoltage protection between input channels and common (COM). In addition, the NI 9205 includes a channel-to-earth ground double-isolation barrier for safety, noise immunity, and high common-mode voltage range. It is rated for 1,000 V rms transient overvoltage protection.

Requirements and Compatibility os Information
Driver Inform ation

- NI-DAQmx

- ReatTime os

- Windows
Software Compatibility

- LabVIEW

- LabWindowsicVI

- Measurement Studio

- SignalExpress

- Visual C++

- visual Studio

- visual Studio .NET

Back to Top

Comparison Tables

\begin{tabular}{|c|c|c|c|c|}
\hline Module & Signal Type & Channels & Sample Rate & Resolution (bits) \\
\hline 9201 & Voltage & 8 & $500 \mathrm{kS} / \mathrm{s}$ & 12 \\
\hline 9203 & Current & 8 & $200 \mathrm{kS} / \mathrm{s}$ & 16 \\
\hline 9205 & Voltage & $32 \mathrm{SE} / 16 \mathrm{DI}$ & $250 \mathrm{kS} / \mathrm{s}$ & 16 \\
\hline 9206 & CATI isolated voltage & $16 \mathrm{DI}$ & $250 \mathrm{kSis}$ & 16 \\
\hline 9215 & Voltage & 4 & $100 \mathrm{kS} / \mathrm{s}$ per channel & 16 \\
\hline 9217 & RTD & 4 & $400 \mathrm{~S} / \mathrm{s}$ & 24 \\
\hline 9221 & Voltage & 8 & $800 \mathrm{kS} / \mathrm{s}$ & 12 \\
\hline
\end{tabular}

$1 / 9$ 


\begin{tabular}{|c|c|c|c|c|}
\hline Module & Signal Type & Channels & Sample Rate & Resolution (bits) \\
\hline 9227 & Current & 4 & $50 \mathrm{kS} / \mathrm{s}$ per channel & 24 \\
\hline 9233 & IEPE & 4 & $50 \mathrm{kS} / \mathrm{s}$ per channel & 24 \\
\hline 923559236 & Quarter-Bridge & 8 & $10 \mathrm{kS} / \mathrm{s}$ per channel & 24 \\
\hline 9237 & Bridge & 4 & $50 \mathrm{kS} / \mathrm{s}$ per channel & 24 \\
\hline
\end{tabular}

\section{Application and Technology}

High-accuracy NIC Series analog input modules for NI CompactDAQ and CompactRIO prowide high-performance measurements for a wide variety of industrial, in-vehicle, and tory sensors and signal types. Each module includes built-in signal conditioning and an integrated connector with screw terminal or cable options for flexible and low-cost signal wiring All modules feature CompactRIO Exxtreme Industrial Certifications and Ratings

c Series Compatibility

The $c$ Series hardware family features more than 50 measurement modules and several chassis and carriers for deployment. With this variety of modules, you can mix and match measurements such as temperature, acceleration, flow, pressure, strain, acoustic, voltage, current, digital, and more to create a custom system. Install the modules in one of several carriers to create a single module USB, Ethernet, or Wi-Fi sy stem, or combine them in chassis such as NI CompactDAQ and CompactRIO to create a mived-measurement system with synchronized measurements. You can install up to eight modules in a simple, complete NI CompactDAQ USB data acquisition system to synchronize all of the analog output, analog input, and digtial Iio from the modules. For a System without a PC, CompactFIO holds up to eight modules and features a built-in processor, RAM, and storage for an embedded data logger or control unit. For higher-speed control, CompactRIO chassis incorporate a field-programmable gate array (FPGA) that you can program with NI LabVIEW software to achieve silicon-speed processing on IVO data from C Series modules.

Advanced F eatures

When used with CompactRIO, C Series analog input modules connect directly to recorfigurable IIO (RIO) FPGA hardware to create high-performance embedded systems. The reconfigurable FPGA hardware within CompactRIO provides a variety of options for custom timing, triggering, synchronization, fittering, signal processing, and high-speed decision making for all $\mathrm{C}$ Series analog modules. For instance, with CompactRIO, you can implement custom triggering for any analog sensor type on a per-channel basis using decision making for all C Series analog modules. For instance, with CompactRIO, you can implement custom triggering for any analog sensort
the flexibility and performance of the FPGA and the numerous arthmetic and comparison function blocks buit into the LabVIEW FPGA Module.

Key Features

- High-accuracy, high-performance analog measurements for ary CompactRIO embedded System, R Series expansion chassis, or NI CompactDAQ chassis - Screw terminals, BNC, D-Sub, spring terminals, strain relief, high voltage, cable, solder cup backshell, and other connectivity options - Available channel-to-earth ground double-isolation barrier for safety, noise immunity, and high common-mode voltage range - CompactRlo Extreme Industrial Certifications and Ratings

- Built-in signal conditioning for direct connection to sensors and industrial devices

Visit ni.comicompactrio or ni.comicompactdaq for up-to-date information on module availability, example programs, application notes, and other developer tools. Connectivity Accessories

There are two connector options for the $\mathrm{N} 19205$ : a 36-position spring terminal connector for direct connectivity or a 37-position D-Sub connector. To add strain relief and high-yoltage protection to the 36-position terminal of the NI 9205, NI recommends the NI 9940 strain-relief connector accessory.

The NI 9205 with D-Sub option has an industry-standard 37-position D-Sub connector that provides a low-cost cabling option to a wide variety of accessories from NI or other vendors. A number of vendors who offer custom D-Sub cable fabrication services can provide cables with a pinout that matches your exact application needs. The NI 9933 (o backshell for creating custom cable assemblies

The NI 9933 includes a screw-terminal connector with strain relief as well as a D-Sub solder cup backshell for creating custom cable assemblies for any module with a 37-pin D-Sub connector.

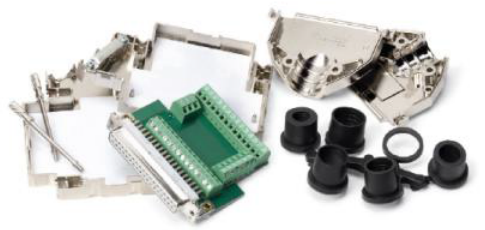

Figure 2. NI 9933 37-Pin D-Sub Connector Kit with Strain Relief and D-Sub Shell

The NN 9940 connector kit provides strain relief and operator protection from high-yoltage signals for any 36-position spring terminal module. 


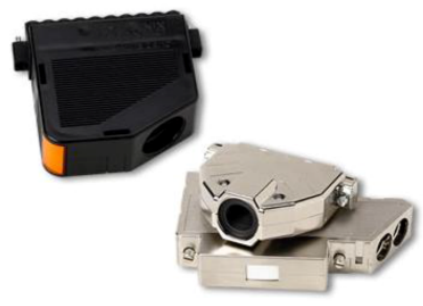

NI 9940 Connector Kit

For a complete list of accessories, visit the product page on ni com.

\begin{tabular}{l|lll} 
Products & Part Number & Recommended Accessories & Part Number \\
\hline N1 9205 Voltage & & & \\
\hline $\begin{array}{l}\text { N1 9205 with Spring Terminals } \\
\text { Requires: } 1 \text { Connectivity Accessories; }\end{array}$ & $779519-01$ & Connectivity Accessories: screwTerminal - N1 9940 Strain relief, operator protection (aty 1) & $779567-01$ \\
\hline
\end{tabular}

Software Recommendations

LabVIEW Professional Development System for Windows

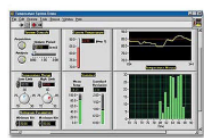

- Advanced software tools for large project - Automatic code generation using DAC - Tight integration with a wide range of

- Advanced measurement analysis and digital signal processing

- Open connectivity with DLLs, ActiveX, an

- Capability to build DLLs, executables, and

NI LabWindows ${ }^{\mathrm{Tm}} / \mathrm{CVI}$ for Windows

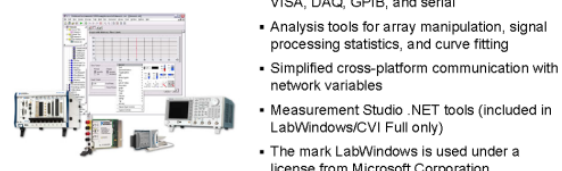
- The mark LabWindows is used under a
SignalExpress for Windows - Quickly configure projects without programming - Control over $400 \mathrm{PC}$-based and stand-alone - Log data from more than 250 data acquisition

- Perform basic signal processing, analysis
and file $1 / O$

- Scale your application with automatic

- Create custom reports or easily export data

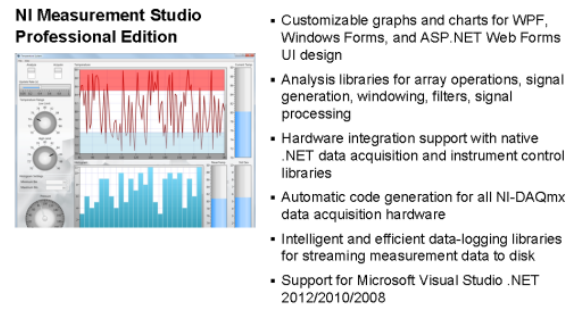


Calibration

NII measurement hardware is calibrated to ensure measurement accuracy and verify that the device meets its published specific ations. To ensure the ongoing accuracy of your measurement hardware, NI offers basic or detailed recalibration service that provides ongoing ISO 9001 audit compliance and confidence in your measurements. To learn more about NI calibration services or to locate a qualified service center near you, contact your local sales office or visit nic comic alibration

\section{Technical Support}

Get answers to your technical questions using the following National Instruments resources

- Support - Visit ni.comisupport to access the NI KnowledgeBase, example programs, and tutorials or to contact our applications engineers who are located in NI sales offices around the world and speak the local language.

- Discussion Forums - Visit forums.ni. com for a diverse set of discussion boards on topics you care abou

- Online Community - Visit community. ni.com to find, contribute, or collaborate on customer-contributed technical content with users like you.

\section{Repair}

While you may never need your hardware repaired, Nl understands that unexpected events may lead to necessary repairs. Nl offers repair services performed by highly trained technicians who quickly return your device with the guarantee that it will perform to factory specifications. For more information, visit ti.comirepair.

\section{Training and Certifications}

The N training and certification program delivers the fastest, most certain route to increased proficiency and productivity using NI software and hardware. Training builds the skills to more efficiently develop robust, maintainable applications, while certficication validates your knowledge and ability.

- Classroom training in cities worldwide - the most comprehensive hands-on training taught by engineers

- On-site training at your facility - an excellent option to train multiple employees at the same time.

- Online instructor-led training - lower-cost, remote training fif classroom or or-site courses are not possible

- Course kits - lowest-cost, self-paced training that you can use as reference guides.

- Training memberships and training credits - to buy now and schedule training later. Visit ni.comitraining for more information

Extended Warranty

NI offers options for extending the standard product warranty to meet the life-cycle requirements of your project. In addition, bec ause NI understands that your requirements may change, the extended warranty is flexible in length and easily renewed. For more information, visit ni.comwwarranty.

OEM

NI offers design-in consulting and product integration assistance if you need NI products for OEM applications. For information about special pricing and services for OEM customers, visit ni.comioem.

\section{Alliance}

Our Professional Services Team is comprised of NI applications engineers, NI Consulting Services, and a worldwide National Instruments Alliance Partner program of more than 700 independent consultants and integrators. Services range from start-up assistance to turnkey sy stem integration. Visit ni.comialliance

Detailed Specifications

The following specifications are typical for the range -40 to $70^{\circ} \mathrm{C}$ unless otherwise noted. All voltages are relative to com unless otherwise noted

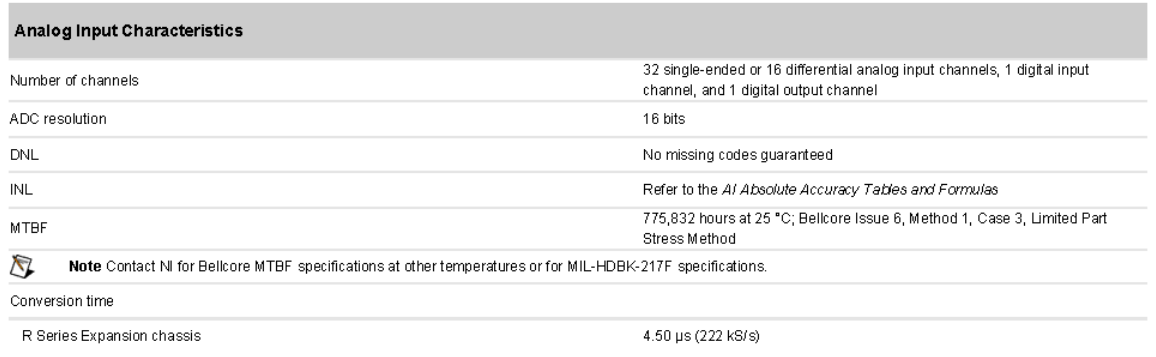




\begin{tabular}{|c|c|}
\hline All other chassis & $4.00 \mu \mathrm{s}(250 \mathrm{kS} / \mathrm{s})$ \\
\hline Input coupling & DC \\
\hline Nominal input ranges & $\pm 10 \mathrm{~V}, \pm 5 \mathrm{~V}, \pm 1 \mathrm{~V}, \pm 0.2 \mathrm{~V}$ \\
\hline Minimum overrange (for $10 \mathrm{~V}$ range) & $4 \%$ \\
\hline Maximum working voltage for analog inputs (signal + common mode) & Each channel must remain within $\pm 10.4 \mathrm{~V}$ of common \\
\hline \multicolumn{2}{|l|}{ Input impedance (Al-to-COM) } \\
\hline Powered on & $>10 \mathrm{G} \Omega$ in parallel with $100 \mathrm{pF}$ \\
\hline Powered offioverload & $4.7 \mathrm{k} \Omega \mathrm{min}$ \\
\hline Input bias current & $\pm 100 \mathrm{pA}$ \\
\hline \multicolumn{2}{|l|}{ Crosstalk (at $100 \mathrm{kHz}$ ) } \\
\hline Adjacent channels & $-65 \mathrm{~d} \theta$ \\
\hline Non-adjacent channels & $-70 \mathrm{~d} \theta$ \\
\hline Analog bandwidth & $370 \mathrm{kHz}$ \\
\hline \multicolumn{2}{|l|}{ Overvoltage protection } \\
\hline Al channel (0 to 31) & $\pm 30 \mathrm{~V}$ (one channel onv) \\
\hline AISENSE & $\pm 30 \mathrm{~V}$ \\
\hline CMRR (DC to $60 \mathrm{~Hz})$ & $100 \mathrm{~d} \theta$ \\
\hline Typical Al+ to Al-CMRR graph & \\
\hline
\end{tabular}

Al $<0 . . .31>$ CMRR

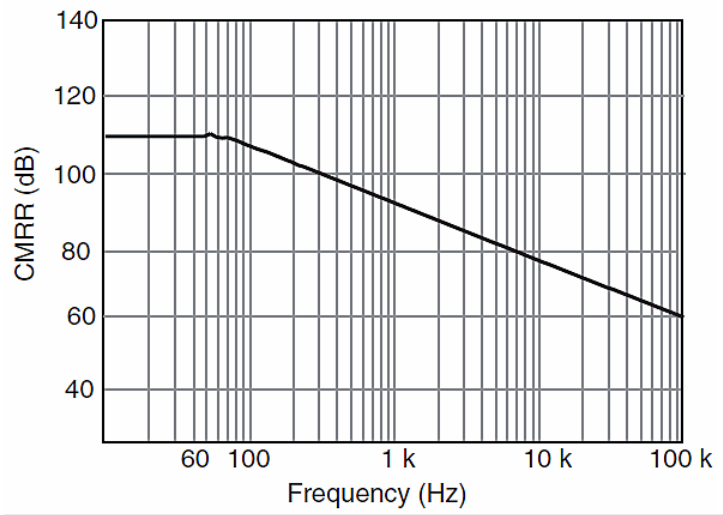

\begin{tabular}{|c|c|c|}
\hline \multicolumn{3}{|c|}{ Settling time for multichannel measurements, accuracy, all ranges } \\
\hline \multicolumn{2}{|l|}{ $\pm 120 \mathrm{ppm}$ of full-scale step ( \pm 8 LSB) } & $4 \mu$ s convert interval \\
\hline \multicolumn{2}{|l|}{ $\pm 30 \mathrm{ppm}$ of fullscale step $( \pm 2 \mathrm{LSB}$ ) } & $8 \mu \mathrm{s}$ convert interval \\
\hline \multicolumn{3}{|l|}{ Analog triggers } \\
\hline \multicolumn{2}{|l|}{ Number of triggers } & 1 \\
\hline \multicolumn{2}{|l|}{ Resolution } & 10 bits, 1 in 1,024 \\
\hline \multicolumn{2}{|l|}{ Bandwidth $(-3 \mathrm{~d} \theta)$} & $370 \mathrm{kHz}$ \\
\hline \multirow[t]{5}{*}{ Accuracy } & & $\pm 1 \%$ of full scale \\
\hline & \multicolumn{2}{|r|}{ Scaling coefficients } \\
\hline & Nominal Range $(M)$ & Typical Scaling Coefficient $(\mu \mathrm{V} / \mathrm{LSB})$ \\
\hline & \pm 10 & 328 \\
\hline & \pm 5 & 164.2 \\
\hline
\end{tabular}




\begin{tabular}{|l|l|}
\hline \multicolumn{2}{|c|}{ Scaling coefficients } \\
\hline Nominal Range M) & Iypical Scaling Coefficient (jVVISB) \\
\hline \pm 1 & 32.8 \\
\hline \pm 0.2 & 6.57 \\
\hline
\end{tabular}

Al Absolute A ccuracy Tables and Formulas

The values in the following tables are based on calibrated scaling coefficients, which are stored in the onboard EEPROM.

\begin{tabular}{|l|l|l|l|}
\hline \multicolumn{4}{|c|}{ Accuracy summary } \\
\hline Nomina Range $(\mathbf{M})$ & Absolute Accuracy at F ull Scale ${ }^{1}(\boldsymbol{\mu})$ & Random Noise, $\boldsymbol{\sigma}\left(\boldsymbol{\mu \mathbf { V } _ { \mathrm { rms } } )}\right.$ & Sensitvity ${ }^{2}(\boldsymbol{\mu})$ \\
\hline \pm 10 & 6,230 & 240 & 96.0 \\
\hline \pm 5 & 3,230 & 116 & 46.4 \\
\hline \pm 1 & 690 & 26 & 10.4 \\
\hline \pm 0.2 & 174 & 4.0 \\
\hline
\end{tabular}

\begin{tabular}{|c|c|c|c|c|c|c|}
\hline \multicolumn{7}{|c|}{ Accuracy details } \\
\hline $\begin{array}{l}\text { Nominal } \\
\text { Range }(M)\end{array}$ & $\begin{array}{l}\text { Residual Gain Error (ppm of } \\
\text { Reading) }\end{array}$ & $\begin{array}{c}\text { Gain Tempco } \\
\left(\mathrm{ppm}^{\circ} \mathrm{C}\right)\end{array}$ & $\begin{array}{l}\text { Reference } \\
\text { Tempco }\end{array}$ & \begin{tabular}{|c|}
$\begin{array}{c}\text { Residual Offset Emor (ppm of } \\
\text { Range) }\end{array}$ \\
\end{tabular} & $\begin{array}{l}\text { Offset Tempco (ppm of } \\
\text { Range } / \mathrm{C} \text { ) }\end{array}$ & $\begin{array}{c}\text { INL Error (ppm of } \\
\text { Range) }\end{array}$ \\
\hline \pm 10 & 115 & \begin{tabular}{|l|}
11 \\
\end{tabular} & 5 & 20 & 44 & 76 \\
\hline \pm 5 & 135 & 11 & 5 & 20 & 47 & 76 \\
\hline \pm 1 & 155 & 11 & 5 & 25 & 66 & 76 \\
\hline \pm 0.2 & 215 & 11 & 5 & 40 & 162 & 76 \\
\hline
\end{tabular}

Absolute accuracy formulas

AbsoliteAccuracy $=$ Reacing $\cdot$ GainError + Range $\cdot$ OffsetError + NoiseUncertainty

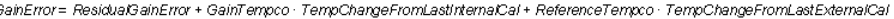

OffisetError = ResidualoffsetEror + OffisetT empco. TemoChangefromLastintemaCal + iNL_Error

Noise Uncertainty $=($ RandomNoise $\cdot 3) / 100$ for a coverage factor of $3 \sigma$ and averaging 100 points

Absolute accuracy at full scale on the analog input channels is determined using the following assumptions:

TemoChangeFromLastExterna $\mathrm{C} a l=70^{\circ} \mathrm{C}$

TemochangeFromLastirternaCal= $1^{\circ}$

Numberot Readings $=100$

Coveragefactor $=$

For example, on the $10 \mathrm{~V}$ range, the absolute accuracy at full scale is as follows:

GainError $=115 \mathrm{ppm}+11 \mathrm{ppm} \cdot 1+5 \mathrm{ppm} \cdot 70$

GainE rror $=476 \mathrm{ppm}$

OffsetE $r$ ror $=20 \mathrm{ppm}+44 \mathrm{ppm} \cdot 1+76 \mathrm{ppm}$

OffsetError $=140 \mathrm{ppm}$

Noiseuncertainty $=(240 \mu \mathrm{V} \cdot 3) / 100$

Noise Uncertainty $=72 \mu \mathrm{V}$

AbsoluteAccuracy $=10 \mathrm{~V} \cdot 476 \mathrm{ppm}+10 \mathrm{~V} \cdot 140 \mathrm{ppm}+72 \mu \mathrm{V}$

Absolite Accuracy $=6,232 \mu \mathrm{V}$ (rounds to 6,230 $\mathrm{\mu}$ )

Digital $\mathrm{C}$ haracteristics

\begin{tabular}{|c|c|c|}
\hline \multicolumn{3}{|c|}{ Digital input logic levels } \\
\hline Level & Min & Max \\
\hline Input high voltage $\left(\mathrm{V}_{\mathbb{H}}\right)$ & $2.0 \mathrm{~V}$ & $3.3 \mathrm{~V}$ \\
\hline Input low voltage $\left(\mathrm{N}_{\mathrm{LL}}\right)$ & $0 \mathrm{~V}$ & $0.34 \mathrm{~V}$ \\
\hline
\end{tabular}




\begin{tabular}{|c|c|c|}
\hline \multicolumn{3}{|c|}{ Digital output logic levels } \\
\hline Level & Min & Max \\
\hline Output high voltage $\left(\mathrm{N}_{\mathrm{OH}}\right.$ ), sourcing $75 \mathrm{\mu A}$ & $2.1 \mathrm{~V}$ & $3.3 \mathrm{~V}$ \\
\hline Output low voltage $\left(\mathrm{V}_{\mathrm{OL}}\right.$ ), sinking $250 \mathrm{\mu A}$ & $0 \mathrm{~V}$ & $0.4 \mathrm{~V}$ \\
\hline
\end{tabular}

\begin{tabular}{|c|c|}
\hline Overvoltage protection & $\pm 30 \mathrm{~V}$ \\
\hline \multicolumn{2}{|l|}{ External digital triggers } \\
\hline Source & PFI0 \\
\hline Delay & $100 \mathrm{~ns} \max$ \\
\hline E. Note The digital outp & \\
\hline \multicolumn{2}{|l|}{ Power Requirements } \\
\hline \multicolumn{2}{|l|}{ Power consumption from chassis } \\
\hline Active mode & $625 \mathrm{~mW} \max$ \\
\hline Sleep mode & $15 \mathrm{~mW}$ \\
\hline \multicolumn{2}{|l|}{ Thermal dissipation (at $70^{\circ} \mathrm{C}$ ) } \\
\hline Active mode & $625 \mathrm{miN} \max$ \\
\hline Sleep mode & $15 \mathrm{~mm}$ \\
\hline \multicolumn{2}{|l|}{ Physical Characteristics } \\
\hline Spring-terminal wiring & $\begin{array}{l}18 \text { to } 28 \text { AWG copper conductor wire with } 7 \mathrm{~mm} \text { ( } 0.28 \mathrm{in} \text {.) of insulation stripped } \\
\text { from the end }\end{array}$ \\
\hline \multicolumn{2}{|l|}{ Weight } \\
\hline NI 9205 with spring terminal & $158 \mathrm{~g} \mathrm{(5.80z)}$ \\
\hline NN 9205 with DSUB & $148 \mathrm{~g}(5.3 \mathrm{oz})$ \\
\hline \multicolumn{2}{|l|}{ Safety } \\
\hline \multicolumn{2}{|c|}{ If you need to clean the module, wipe it with a dry towel. } \\
\hline \multicolumn{2}{|l|}{ Maximum Voltage $^{3}$} \\
\hline \multicolumn{2}{|c|}{ Connect only voltages that are within the following limits. } \\
\hline Al, PFIO, and DO-to-COM & $\pm 30 \mathrm{VDC}$ \\
\hline \multicolumn{2}{|c|}{ NI 9205 with Spring Terminal Isolation Voltages } \\
\hline Channel-to-channel & None \\
\hline \multicolumn{2}{|l|}{ Channel-to-earth ground } \\
\hline Continuous & $250 \mathrm{~V}_{\text {tms' }}$ Measurement Category II \\
\hline Withstand & $2,300 V_{\text {Ims }}$ verfified by a $5 \mathrm{~s}$ dielectric withstand test \\
\hline
\end{tabular}

Measurement Category II is for measurements performed on circuits directly connected to the electrical distribution System. This category refers to locallevel electrical distribution, such as that provided by a standard wall outlet (e.g. $115 \mathrm{~V}$ for U.S. or $230 \mathrm{~V}$ for Europe). Examples of Measurement Category II are measurements performed on household appliances, portable tools, and similar products.

1 Caution Do not connect the $\mathrm{NI} 9205$ with spring terminal to signals or use for measurements within Measurement Categories III or IV

NI 9205 with DSUB Isolation Voltages

Channel-to-channel

None

Channel-to-earth ground

Continuous $\quad 60 \mathrm{VDC}$, Measurement Category 1

Withstand $\quad 1,000 \mathrm{~V}_{\mathrm{rms}}$ verfified by a $5 \mathrm{~s}$ dielectric withstand test

Measurement Category I is for measurements performed on circuits not directly connected to the electrical distribution sy stem referred to as MAINS ${ }^{4}$ voltage. This category is for measurements of voltages from specially protected secondary circuits. Such voltage measurements include signal levels, special equipment, limited-energy parts of equipment, circuits powered by regulated lowwyoltage sources, and electronics.

1. Caution Do not connect the NI 9205 with DSUB to signals or use for measurements within Measurement Categories II, III, or IV.

Safety Standards

This product is designed to meet the requirements of the following standards of safety for electrical equipment for measurement, control, and laboratory use: 
- IEC 61010-1, EN 61010-1

- UL 61010-1, CSTH

Q. Note For UL and other safety certifications, refer to the product label or the Onine Product Certification section.

Hazardous Locations

U.S. (UL)

Class I, Division 2, Groups A, B, C, D, T4; Class I, Zone 2, AEx nC IIC T4

Canada (C-UL)

Class I, Division 2, Groups A, B, C, D, T4; Class I, Zone 2, Ex nC IIC T4

Europe (DEMKO)

EEx nC II T4

\section{Environmental}

National Instruments $C$ Series modules are intended for indoor use only but may be used outdoors if installed in a suitable enclosure. Refer to the manual for the chassis you are using for more information about meeting these specifications.

Operating temperature (EC 60068-2-1, IEC 60068-2-2)

Storage temperature (IEC 60068-2-1, IEC 60068-2-2) $\quad-40$ to $85^{\circ} \mathrm{C}$

Ingress protection $\quad$ IP 40

\begin{tabular}{ll}
\hline Operating humidity (IEC 60068-2-56) & 10 to $90 \% \mathrm{RH}$, noncondensing \\
\hline Storage humidity (EC 60068-2-56) & 5 to $95 \%$ RH, noncondensing
\end{tabular}

Storage humidity (IEC 60068-2-56)

$2,000 \mathrm{~m}$

Maximum attiude

Pollution Degree (IEC 60664)

Shock and Vibration

To meet these specifications, you must panel mount the system and use a backshell kit or shielded cable to protect the connections. Use the NI 9940 backshell for the N 9205 with spring terminal and a 37-pin shielded cable or the N1 9933 backshell for the N 9205 with DSUP.

Operating vibration

Random (IEC 60068-2-64) $\quad 5 \mathrm{~g}_{\mathrm{ImE}}, 10$ to $500 \mathrm{~Hz}$

Sinusoidal (IEC 60068-2-6) $\quad 5 \mathrm{~g}, 10$ to $500 \mathrm{~Hz}$

Operating shock (IEC 60068-2-27) $\quad 30 \mathrm{~g}, 11 \mathrm{~ms}$ half sine, $50 \mathrm{~g}, 3 \mathrm{~ms}$ half sine, 18 shocks at 6 orientations

\section{Electrom agnetic Compatibility}

This product is designed to meet the requirements of the following standards of EMC for electrical equipment for measurement, control, and laboratory use:

- EN 61326 EMC requirements; Industrial Immunity

- EN 55011 Emissions; Group 1, Class A

- CE, C-Tick, ICES, and FCC Part 15 Emissions; Class A

Q. Note For EMC compliance, operate this device with shielded cables

\section{CE Compliance}

This product meets the essential requirements of applicable European Directives, as amended for CE marking, as follows

- 2006/95/EC; Low-Vottage Directive (safety)

- 2004:108:EC; Electromagnetic Compatibility Directive (EMC)

8. Note For the standards applied to assess the EMC of this product, refer to the Online Product Certycation section

\section{Online Product Certification}

Refer to the product Declaration of Conformity ( $\mathrm{DoC}$ ) for addtional regulatory compliance information. To obtain product certficications and the Doc for this product, vist ni.comicertification, search by module number or product line, and click the appropriate link in the Certific ation column.

\section{Environmental Management}

National Instruments is committed to designing and manufacturing products in an environmentally responsible manner. NN recognizes that eliminating certain hazardous substances from our products is beneficial not onty to the erwironment but also to NI customers.

For additional environmental information, refer to the $N /$ and the Emwionment Web page at ni.comverwironment. This page contains the environmental regulations and directives with which NII complies, as well as other environmental information not included in this document.

Waste Electrical and Electronic Equipment (MEEE)

Q EU Customers At the end of their life cycle, all products must be sent to a WEEE recycling center. For more information about WEEE recycling centers and National

Instruments WEEE intitiatives, visit ni.comienvironment'iweee. htm.

\section{电子信息产品污染控制管理办法（中国 RoHS）}

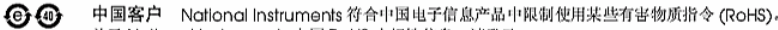

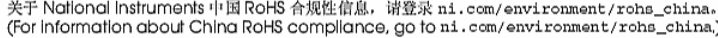


Calibration (A nalog Input)

You can obtain the calibration certificate and information about calibration services for this device at ni. comvicalibration.

Calibration interval

2 years

Absolute accuracy values at full scale on the analog input channels assume the device is operating within $70^{\circ} \mathrm{C}$ of the last external calibration and are valid for averaging 100 samples immediately following internal calibration. Refer to the Absolite accuracy formulas for more information.

${ }^{2}$ Sensitivity is the smallest voltage change that can be detected. It is a function of noise.

${ }^{3}$ The maximum voltage that can be applied or output between AI and COM without creating a safety hazard.

${ }^{4}$ MAINS is defined as the (hazardous live) electrical supply system to which equipment is designed to be connected for the purpose of powering the equipment. Sultably rated

measuring circuits may be connected to the MAINS for measuring purposes.

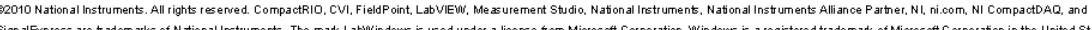

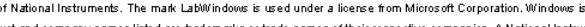
Irstruments and has no agency, partnership, or joint venture relationship with National listrtuments.

My Profile |RSS | Privacy | Legal| Contact N|| 2012 National Instruments Corporation. All rights reserved 

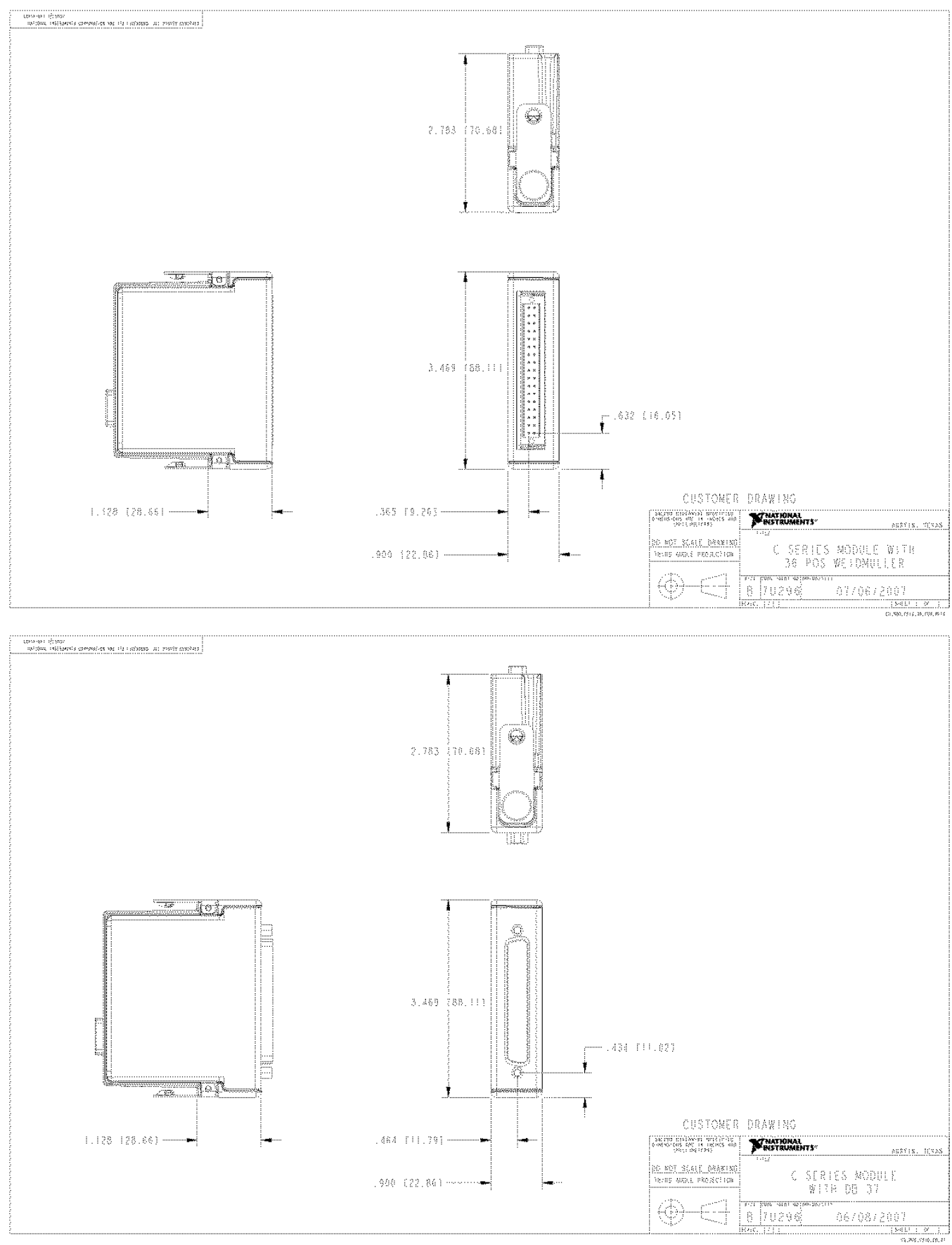


\section{NI 9205/9206 Calibration Procedure}

This document contains information about calibrating National Instruments 9205/9206 modules using NI-DAQmx. This calibration procedure is intended for metrology labs.

This document does not discuss programming techniques or compiler configuration. The NI-DAQmx driver contains online help files that have compiler-specific instructions and detailed function explanations. You can install these help files when you install NI-DAQmx on the calibration computer.

The NI 9205 and NI 9206 should be calibrated at a regular interval as defined by the measurement accuracy requirements of your application. National Instruments recommends that you routinely perform a complete calibration at least once every two years. You can shorten this interval based on the accuracy demands of your application or requirements of your processes. Self-calibration can be performed as needed or when the temperature varies by $5{ }^{\circ} \mathrm{C}$ or more from the last external calibration.

\section{Contents}

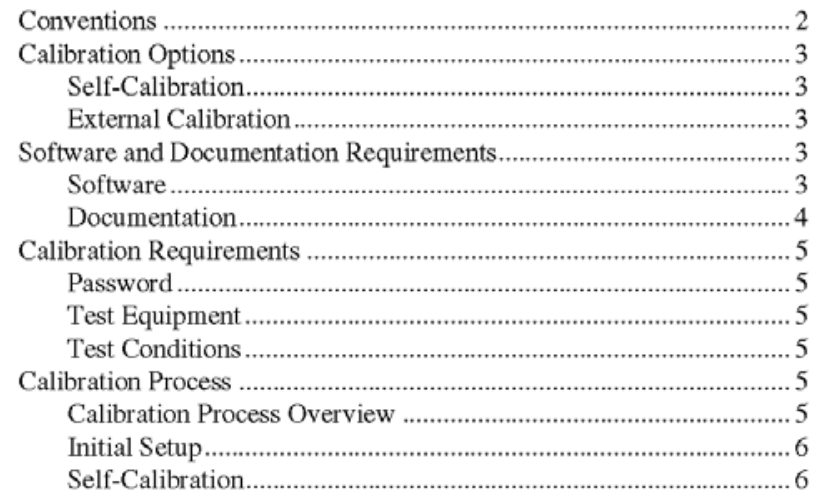




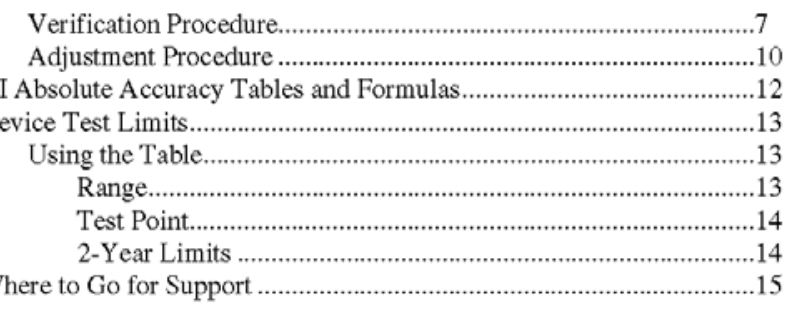

\section{Conventions}

The following conventions appear in this manual:

The $n$ symbol leads you through nested menu items and dialog box options
to a final action. The sequence File»Page Setup»Options directs you to
pull down the File menu, select the Page Setup item, and select Options
from the last dialog box.
This icon denotes a note, which alerts you to important information.
Bold text denotes items that you must select or click in the software, such
as menu items and dialog box options. Bold text also denotes parameter
names and hardware labels.
Italic text denotes variables, emphasis, a cross-reference, or an introduction
to a key concept. Italic text also denotes text that is a placeholder for a word
or value that you must supply.
italic
Monospace text denotes text or characters that you should enter from the
keyboard, sections of code, programming examples, and syntax examples.
This font is also used for the proper names of disk drives, paths, directories,
programs, subprograms, subroutines, device names, functions, operations,
variables, filenames, and extensions.

NI 9205/9206 NI 9205/9206 refers to the NI 9205 and NI 9206. 


\section{Calibration Options}

The NI 9205/9206 support two types of calibration: self-calibration and external calibration.

\section{Self-Calibration}

Self-calibration, also known as internal calibration, uses a software command and requires no external connections. Self-calibration improves measurement accuracy by compensating for variables such as temperature that might have changed since the last external calibration. Self-calibration retains the traceability of the external calibration and can be performed as necessary to compensate for environmental changes.

\section{External Calibration}

External calibration is generally performed with a high-precision calibrator at either NI or a metrology lab. This procedure replaces all calibration constants in the EEPROM and is equivalent to a factory calibration at NI. Because the external calibration procedure changes all EEPROM constants, it invalidates the original calibration certificate. If an external calibration is done with a traceable signal generator source, a new calibration certificate can be issued. This document describes specific programming steps for writing an external calibration procedure for the NI 9205/9206.

\section{Software and Documentation Requirements}

This section describes the software and documentation required for both self-calibration and external calibration.

\section{Software}

Install NI-DAQmx 8.1 or later on the calibration computer. NI-DAQmx includes high-level function calls to simplify the task of writing software to calibrate devices. You must have the proper device driver installed on the calibration system before calibrating the device.

17 Note NI recommends that you install the NI-DAQmx driver software before physically installing the NI 9205/9206. NI-DAQmx, available at ni.com/downloads, configures and controls the NI 9205/9206.

NI-DAQmx supports a number of programming languages, including LabVIEW, LabWindows ${ }^{\mathrm{TM}} / \mathrm{CVI}^{\mathrm{m}}$, Microsoft Visual C++ 6.0, Microsoft Visual Basic 6.0, Microsoft .NET, and Borland C++. 
You can access the NI-DAQmx header file, NIDAQmx. h, like any standard library. You can find examples of how to use the NI-DAQmx driver in the Program Files $\backslash$ National Instruments $\backslash N I-D A Q \backslash$ Examples directory.

\section{Documentation}

You might find the following documentation helpful as you write the calibration procedure:

- NI-DAQmx Help - This help file contains general information about measurement concepts, key NI-DAQmx concepts, and common applications that apply to all programming environments. To access this help file, select Start»All Programs»National Instruments» NI-DAQ*NI-DAQmx Help.

- NI-DAQmx C Reference Help — This help file contains C reference and general information about measurement concepts. To access this help file, select Start»All Programs»National Instruments»NI-DAQ" NI-DAQmx C Reference Help.

- DAQ Getting Started Guide for NI-DAQ 8.0 or later-This guide describes how to install NI-DAQmx for Windows software and NI-DAQmx-supported DAQ devices, and how to confirm that your device is operating properly. To access this guide, select Start \% All Programs»National Instruments»NI-DAQ»DAQ Getting Started Guide.

17 Note The documents above are installed with NI-DAQmx. You can also download the latest versions from the NI Web site at ni. com/manuals.

- NI 9205 Operating Instructions/NI 9206 Operating InstructionsThese documents contain information about specific device features. The limits you use to verify the accuracy of the devices are based on the specifications found in this document. You can download the latest versions of these documents from the NI Web site at ni. com/ manuals. 


\section{Calibration Requirements}

Password

The default password for password-protected operations is NI.

\section{Test Equipment}

National Instruments recommends that you use the following equipment for calibrating the NI 9205/9206:

- Fluke $5700 \mathrm{~A}$ calibrator. If that instrument is unavailable, use a high-precision voltage source with an accuracy of at least $10 \mathrm{ppm}$.

- NI cDAQ-9172 chassis.

\section{Test Conditions}

Follow these guidelines to optimize the connections and the environment:

- Keep connections to the device as short as possible. Long cables and wires act as antennae, picking up extra noise that can affect measurements.

- Use shielded copper wire for all cable connections to the device. Use twisted-pair wire to eliminate noise and thermal offsets.

- Maintain an ambient temperature of $23 \pm 10^{\circ} \mathrm{C}$. The device temperature will be greater than the ambient temperature.

- Keep relative humidity below $80 \%$.

- Allow a warm-up time of at least 10 minutes to ensure that the measurement circuitry is at a stable operating temperature.

\section{Calibration Process}

This section provides instructions for verifying and adjusting the NI 9205/9206.

\section{Calibration Process Overview}

The calibration process consists of the following steps:

1. Initial Setup - Configure the device in NI-DAQmx.

2. Self-Calibration-Measure the onboard reference voltage of the device and adjust the self-calibration constants.

3. Verification Procedure - Verify the existing operation of the device. This step confirms whether the device was operating within its specified range prior to calibration. 
4. Adjustment Procedure-Ferform an extemal calibration that. adjusts the device calibr ation constants with respect to a known voltage source.

5. Perform another verification to ensure that the device oper ates within its specifications after adjustment.

The first four steps are explained in the following sections.

\section{In itial Setup}

You must configure the device in Measurement \& Automation Explorer (MAX) to communic ate with NI-DAQmx.

Complete the following steps to configure a device in MAX:

1. Install the NI-DAQmx diver software.

2. Make sure that no power is connected to the module terminals. If the system is in a nonhazardous location, the chassis power can be on when you install the module.

3. Insert the module into an available slot in the cDAQ-9172 chassis.

4. Launch MAX.

5. Right-click the device name and select Self-Test to ensure that the device is working properly.

Nole When adevice is configured with MAX, it is assigned a device name. Each function call uses this device name to determine which DAQ device to calibrate. This document uses dev1 to refer to the device name. In the following procedures, use the device name as it appears in MAX.

\section{Self-Calibration}

Self-calibrate the device before completing the first verification, after the device has warmed up for the recommended time period. DAomsSelfCal (DAQmx Self Calibrate VI), as shown in the following table, measures the onboard reference voltage of the device and adjusts the self-calibration constants to account for any errors caused by short-termfluctuations in the operating environment. When you self-calibrate a device, no extemal signal connections are necessary.

\begin{tabular}{|c|c|}
\hline NI-DAQTnx Functimn Call & LabVIEW Block Diagram \\
\hline $\begin{array}{l}\text { Call DAonkselfcal with the } \\
\text { following parameters: } \\
\text { deviceName: dev1 }\end{array}$ & $\begin{array}{l}\text { device in } \\
\text { I/or } \\
\text { error in }\end{array}$ \\
\hline
\end{tabular}




\section{Verification Procedure}

Verification determines how well the device is meeting its specifications. By completing this procedure, you can see how the device has drifted over time, which helps you determine the appropriate calibration interval for your application. Table 4 in the Device Test Limits section shows all acceptable settings for the device type. Throughout the verification process, use Table 4 to determine if the device is operating within its specified range prior to calibration. Because the NI 9205/9206 has many different ranges, you must verify measurements for each available range on these modules.

Complete the following steps to test the performance of the device:

1. Refer to Table 1 for a list of the NI connection accessories for the NI 9205/9206 and instructions on connecting the calibrator to the device.

Table 1. NI Connection Accessories

\begin{tabular}{|l|l|l|}
\hline \multicolumn{1}{|c|}{ Device } & \multicolumn{1}{|c|}{ Connection Accessory } & \multicolumn{1}{c|}{ Connection Instructions } \\
\hline \hline NI 9205/9206 & $\begin{array}{l}\text { NI 9940 36-position strain } \\
\text { relief \& high voltage connector } \\
\text { kit }\end{array}$ & $\begin{array}{l}\text { Connect the positive output of the calibrator to } \\
\text { AI0 (pin 1) and the negative output of the } \\
\text { calibrator to AI8 (pin 19) and COM (pin 17). If } \\
\text { the calibrator has a guard connection, connect } \\
\text { that terminal to COM instead of connecting } \\
\text { COM to the negative output. }\end{array}$ \\
\hline $\begin{array}{l}\text { NI 9205 with } \\
\text { DSUB }\end{array}$ & $\begin{array}{l}\text { NI 9933 37-pin connector kit, } \\
\text { screw terminal connector and } \\
\text { DSUB shell }\end{array}$ & $\begin{array}{l}\text { Connect the positive output of the calibrator to } \\
\text { AI0 (pin 1) and the negative output of the } \\
\text { calibrator to AI8 (pin 20) and COM (pin 10). If } \\
\text { the calibrator has a guard connection, connect } \\
\text { that terminal to COM instead of connecting } \\
\text { COM to the negative output. }\end{array}$ \\
\hline
\end{tabular}

2. Set the calibrator voltage to the Test Point value indicated in Table 4 . NI recommends that you verify all values, although you can save time by verifying only the values used in your application.

3. If you use $\mathrm{C}$ function calls, create a task using DACmxCreateTask, as shown in the following table. If you use LabVIEW, skip this step. The task is created in step 4 in LabVIEW.

\begin{tabular}{|l|c|}
\hline \multicolumn{1}{|c|}{ NI-DAQmx Function Call } & \multicolumn{1}{c|}{ LabVIEW Block Diagram } \\
\hline \hline $\begin{array}{l}\text { Call DAQmxCreateTask with the } \\
\text { following parameters: }\end{array}$ & \\
$\begin{array}{l}\text { taskName: AIVerificationTask } \\
\text { taskHandle: \&taskHandle }\end{array}$ & LabVIEW does not require this step. \\
\hline
\end{tabular}


4. Create and configure an AI voltage channel through NI-DAQmx using DAQmxCreatesIVol tagechan (DAQmx Create Virtual Channel VI), as shown in the following table. Use Table 4 to deternine the minimum and maximum values for the device.

\begin{tabular}{|c|c|}
\hline NI-DAQmx Function Call & LabVIEW Block Diagram \\
\hline 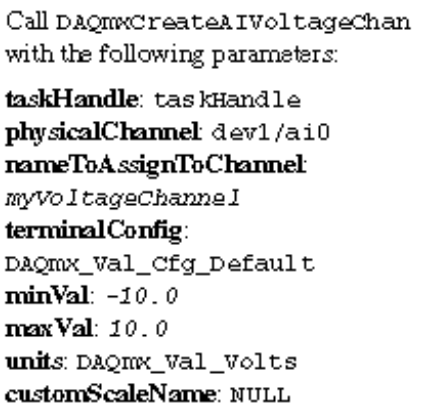 & 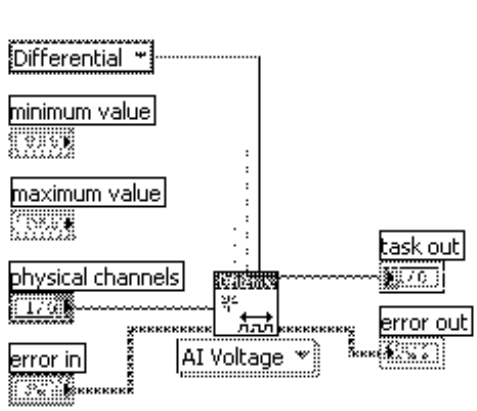 \\
\hline
\end{tabular}

5. Configure the timing properties for the voltage acquisition using DAQmxCfg Sampcl kTiming (DAQmx Timing VI), as shown in the following table.

\begin{tabular}{|c|c|}
\hline NI-DAQmx Function Call & LabVIEW Block Diagram \\
\hline $\begin{array}{l}\text { Call DAomc fgSampcl kTi ming } \\
\text { with the following parameters: } \\
\text { taskHandle: tas kHandle } \\
\text { source: NULL } \\
\text { rate: } 100000.0 \\
\text { activeEdge: } \\
\text { Dacmx_val_Rising } \\
\text { sampleMode: } \\
\text { Dagmx_val_FiniteSamps } \\
\text { sampsPerChan: } 10000\end{array}$ & 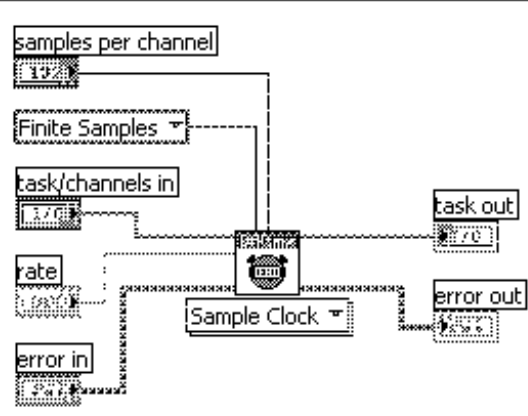 \\
\hline
\end{tabular}


6. Start the acquisition using DAQmxS tartTask (DAQmx Start Task VI), as shown in the following table.

\begin{tabular}{|l|c|}
\hline NI-DAQmx Functimn Call & LabV IEW Block Diagram \\
\hline \hline Call DAQmxStar tTask with & the following parameter: \\
taskHandle: tas kHandle & task out \\
& error in \\
\hline
\end{tabular}

7. Aoquire 10,000 points of woltage data using DAQnkReadanal $\propto \mathrm{gF} 64$ (DAQmx Read VI), as shown in the following table.

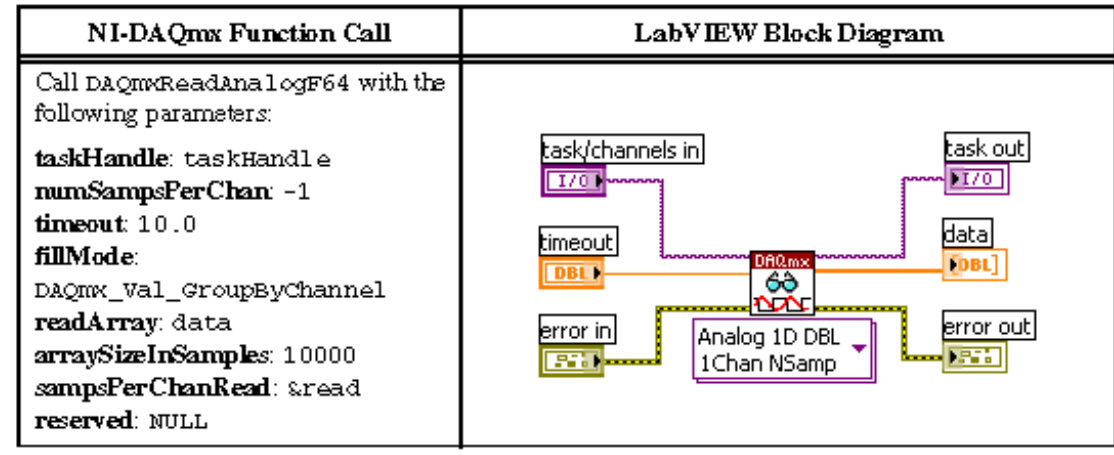

8. Average the voltage values that you acquired. Compare the resulting average to the Upper Limit and Lower Limit values in Table 4. If the result is between these values, the device pases the test.

9. Clear the acquisition using DAQmxclearTask (DAQmx Clear Task VI), as shown in the following table.

\begin{tabular}{|c|c|}
\hline NI-DAQTxx Functien Call & LabV IEW Block Diagram \\
\hline $\begin{array}{l}\text { Call DAQmol earTask with the following } \\
\text { parameter: } \\
\text { taskHandle: tas kHandle }\end{array}$ & Eask in \\
\hline
\end{tabular}

10. Repeat steps 3 through 9 for all ranges and all values.

11. Disonnect the calibr ator from the device or proceed to the Adjustment Procedure section to adjust the analog input calibration constants. 


\section{Adjustment Procedure}

The NI 9205/9206 adjustment procedure adjusts the analog input calibration constants. At the end of each adjustment procedure, the new constants are stored in the extemal calibration area of the device EEPROM. These values are password-protected, which ensures that you do not accidentally aocess or modify any calibration constants adjusted by the metrology labor atory. The default password is ivI.

Complete the following steps to adjust the device with a calibr ator:

1. Refer to Table 1 for a list of the NI connection accessories for the NI 9205/9206 and instructions on connecting the calibrator to the device.

2. Set the calibrator to output $4.0 \mathrm{~V}$.

3. Open a calibration session on the device using Daonxinitextcal (DAQmx Initialize Extemal Calibration VI), as shown in the following table.

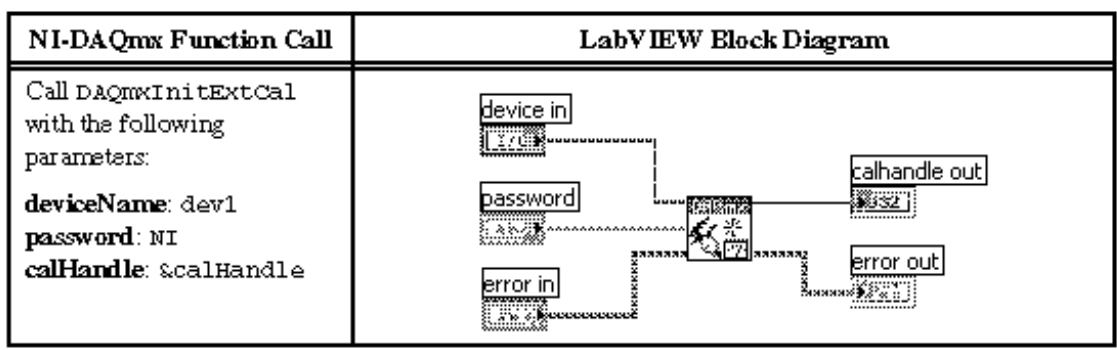


4. Perform an extemal adjustment using DAomomseriescaladjust (DAQnx Adjust M-Series Calibration VI), as shown in the following table.

\begin{tabular}{|c|c|}
\hline NI-DAQmx Function Call & LabVIEW Block Diagram \\
\hline $\begin{array}{l}\text { Call } \\
\text { Dagmxaseri escal Adjust } \\
\text { with the following } \\
\text { parameters: } \\
\text { calHandle: calHandle } \\
\text { referenceVoltage: } 4.0\end{array}$ & $\begin{array}{lll}\text { calhandle in } \\
\text { eference voltage (4.0 V) }\end{array}$ \\
\hline
\end{tabular}

5. Save the adjustment to the EEPROM using DagmxcloseExtCal (DAQmx Close External Calibration), as shown in the following table. This function also saves the date, time, and temper ature of the adjustment to the onboard memory.

8. Nole If the device fails the calibr ation procedure, no constants are written to the EEPROM.

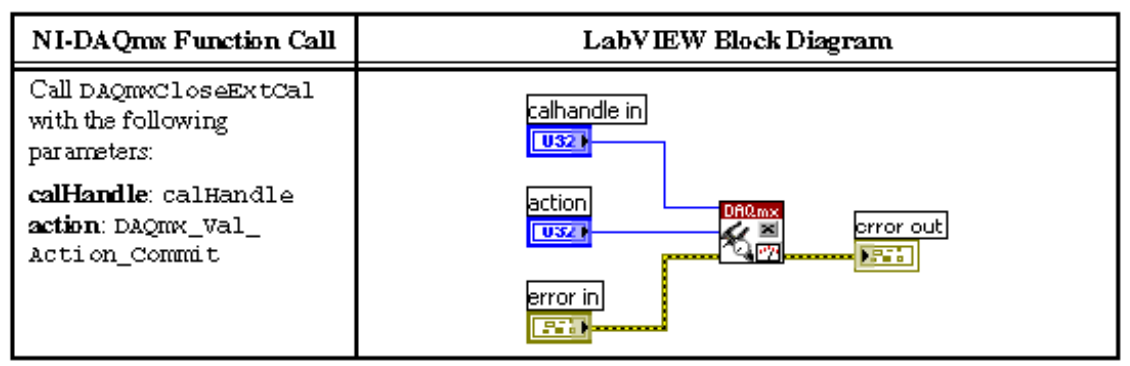

6. Disoonnect the calibrator from the device.

The device is now calibrated with respect to the external source. After calibrating the device, verify the analog input operation by repeating the Verificotion Procedure section 


\section{Al Absolute Accuracy Tables and Formulas}

The values in the following tables are based on calibrated scaling coefficients, which are stored in the onboard EEPROM. The following calibration specifications are for $23 \pm 10^{\circ} \mathrm{C}$.

Table 2. Accuracy Summary

\begin{tabular}{|c|c|c|c|}
\hline Nominal Range (V) & $\begin{array}{c}\text { Absolute Accuracy } \\
\text { at Full Scale }{ }^{\mathbf{1}}(\mu \mathbf{V})\end{array}$ & $\begin{array}{c}\text { Random Noise, } \\
\sigma(\mu \mathbf{V r m s})\end{array}$ & Sensitivity $^{\mathbf{2}}(\mu \mathbf{V})$ \\
\hline \hline \pm 10 & 3230 & 240 & 96.0 \\
\hline \pm 5 & 1730 & 116 & 46.4 \\
\hline \pm 1 & 390 & 26 & 10.4 \\
\hline \pm 0.2 & 114 & 10 & 4.0 \\
\hline \hline
\end{tabular}

Absolute accuracy values at full scale on the analog input channels assume the device is operating within $10^{\circ} \mathrm{C}$ of the last external calibration and are valid for averaging 100 samples immediately following internal calibration.

Sensitivity is the smallest voltage change that can be detected. It is a function of noise.

Table 3. Accuracy Details

\begin{tabular}{|c|c|c|c|c|c|c|}
\hline $\begin{array}{c}\text { Nominal } \\
\text { Range (V) }\end{array}$ & $\begin{array}{c}\text { Gain } \\
\text { Error } \\
\text { Reading) }\end{array}$ & $\begin{array}{c}\text { Gain } \\
\text { Tempco } \\
\left(\mathbf{p p m} /{ }^{\circ} \mathbf{C}\right)\end{array}$ & $\begin{array}{c}\text { Reference } \\
\text { Tempco }\end{array}$ & $\begin{array}{c}\text { Residual } \\
\text { Offet } \\
\text { Error } \\
(\mathbf{p p m} \text { of } \\
\text { Range) }\end{array}$ & $\begin{array}{c}\text { Offset } \\
\text { Tempco } \\
(\mathbf{p p m} \text { of } \\
\text { Range/ }{ }^{\circ} \text { C) }\end{array}$ & $\begin{array}{c}\text { INLError } \\
\text { (ppm of } \\
\text { Range) }\end{array}$ \\
\hline \hline \pm 10 & 115 & 11 & 5 & 20 & 44 & 76 \\
\hline \pm 5 & 135 & 11 & 5 & 20 & 47 & 76 \\
\hline \pm 1 & 155 & 11 & 5 & 25 & 66 & 76 \\
\hline \pm 0.2 & 215 & 11 & 5 & 40 & 162 & 76 \\
\hline
\end{tabular}




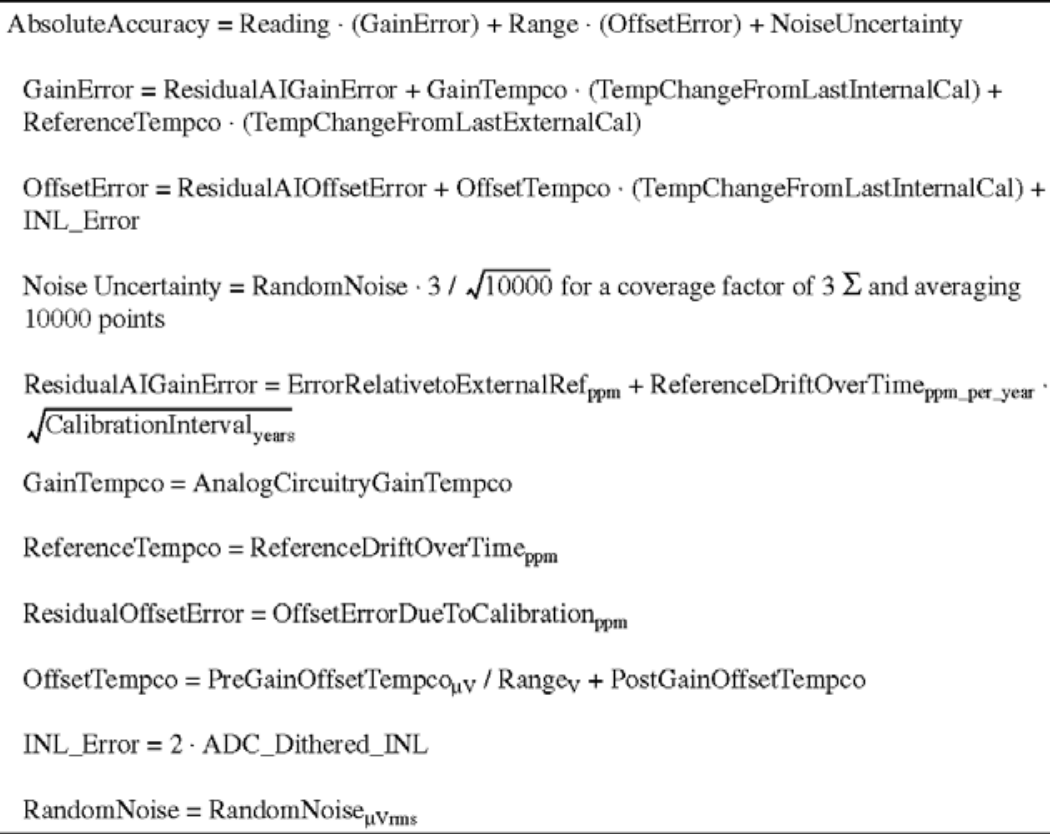

\section{Device Test Limits}

Table 4 lists the specifications that the NI 9205/9206 meets if it has been two years between calibrations.

\section{Using the Table}

The following definitions describe how to use the information from Table 4.

\section{Range}

Range refers to the minimum or maximum voltage range of an input signal. 


\section{Test Point}

The Test Point is the voltage value that is input or output for verification purposes. This value is broken down into two columns-Location and Value. Location refers to where the test value fits within the test range. Value refers to the voltage value to be verified. Max refers to maximum value, $\mathrm{Min}$ refers to minimum value, and $\mathrm{Mid}$ refers to mid-scale.

\section{2-Year Limits}

The 2-Year Limits column contains the Upper Limits and Lower Limits for the test point value. That is, when the device is within its 2-year calibration interval, the test point value should fall between these upper and lower limit values.

Table 4. NI 9205/9206 Analog Input Values

\begin{tabular}{|c|c|c|c|c|c|}
\hline \multicolumn{2}{|c|}{ Range (V) } & \multicolumn{2}{c|}{ Test Point } & \multicolumn{2}{c|}{ 2-Year Limits } \\
\hline Minimum & Maximum & Location & Value (V) & Lower Limit (V) & Upper Limit (V) \\
\hline \hline-10 & 10 & Max & 9.980000 & 9.976836 & 9.983164 \\
\hline-10 & 10 & Mid & 0.000000 & -0.001407 & 0.001407 \\
\hline-10 & 10 & Min & -9.980000 & -9.983164 & -9.976836 \\
\hline-5 & 5 & Max & 4.990000 & 4.988303 & 4.991697 \\
\hline-5 & 5 & Mid & 0.000000 & -0.000718 & 0.000718 \\
\hline-5 & 5 & Min & -4.990000 & -4.991697 & -4.988303 \\
\hline-1 & 1 & Max & 0.998000 & 0.997617 & 0.998383 \\
\hline-1 & 1 & Mid & 0.000000 & -0.000168 & 0.000168 \\
\hline-1 & 1 & Min & -0.998000 & -0.998383 & -0.997617 \\
\hline-0.200 & 0.200 & Max & 0.199600 & 0.199489 & 0.199711 \\
\hline-0.200 & 0.200 & Mid & 0.000000 & -0.000056 & 0.000056 \\
\hline-0.200 & 0.200 & Min & -0.199600 & -0.199711 & -0.199489 \\
\hline
\end{tabular}




\section{Where to Go for Support}

The National Instruments Web site is your complete resource for technical support. At $n i$. com/support you have access to everything from troubleshooting and application development self-help resources to email and phone assistance from NI Application Engineers.

National Instruments corporate headquarters is located at 11500 North Mopac Expressway, Austin, Texas, 78759-3504.

National Instruments also has offices located around the world to help address your support needs. For telephone support in the United States, create your service request at $\mathrm{ni}$.com/support and follow the calling instructions or dial 5127958248 . For telephone support outside the United States, contact your local branch office:

Australia 1800300800 , Austria 4306624579900 , Belgium 320275700 20, Brazil 551132623599 , Canada 800433 3488, China 862165557838 ,

Czech Republic 420224235 774, Denmark 4545762600 , Finland 3850972572511 , France 330148142424 , Germany 4908974131 30, India 918041190000 , Israel 972036393737 , Italy 3902 413091, Japan 8135472 2970, Korea 820234513400 , Lebanon 961013328 28, Malaysia 1800887710 , Mexico 018000100793 ,

Netherlands 310348433 466, New Zealand 0800553322 , Norway 47066907660 , Poland 48223390150 , Portugal 351210311210 , Russia 749578368 51, Singapore 18002265886 , Slovenia 38634254200 , South Africa 270118058197 , Spain 34916400085 , Sweden 460858789500 , Switzerland 41562005151 , Taiwan 886022377 2222, Thailand 6622786777 ,

Turkey 90212279 3031, United Kingdom 4401635523545

National Instruments, NI, ni.com, and LabVIEW are trademarks of National Instruments Corporation Refer to the Terms of Use section on ni . com/l egal for more information about National Instruments trademarks. Other product and company names mentioned herein are trademarks or trad names or thir rossoduct

appropnate location HelpwPatents in your software, the pacents. $t x t$ file on your $\mathrm{CD}$, or
$\mathrm{ni}$. com/patents.

2006 National Instruments Corporation. All rights reserved. 
OPERATING INSTRUCTIONS AND SPECIFICATIONS NI 9205

32-Channel, $\pm 200 \mathrm{mV}$ to $\pm 10 \mathrm{~V}, 16$-Bit Analog Input Module

\begin{tabular}{|ccc|}
\hline Français Deutsch 日本語 한국어 & 简体中文 \\
\hline ni.com/manuals \\
\hline
\end{tabular}
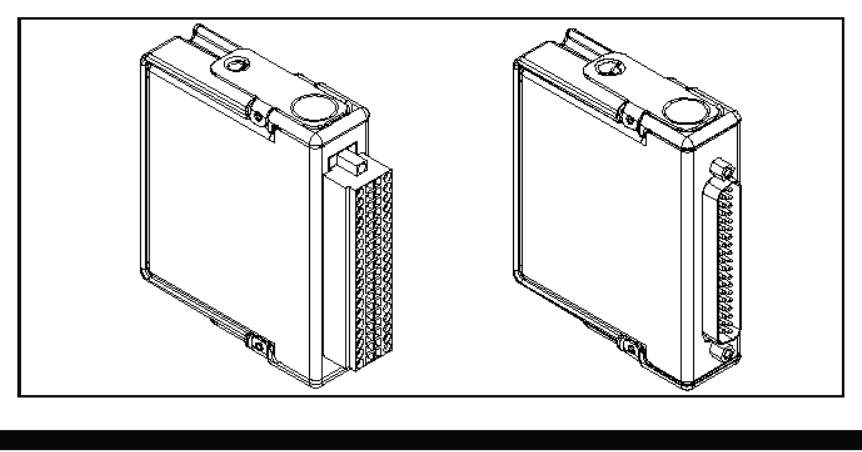

\section{TNATONAL INSTRUMENTS}

This document describes how to use the National Instruments 9205 and includes specifications and pin assignments for the NI 9205. In this document, the NI 9205 with spring terminal and the NI 9205 with DSUB are referred to inclusively as the NI 9205. Visit ni.com/info and enter rdsoftwareversion to determine which software you need for the modules you are using. For information about installing, configuring, and programming the system, refer to the system documentation. Visit ni.com/info and enter cseriesdoc for information about C Series documentation.

Note The safety guidelines and specifications in this document are specific to the NI 9205. The other components in the system might not meet the same safety ratings and specifications. Refer to the documentation for each component in the system to determine the safety ratings and specifications for the entire system. Visit ni.com/info and enter cseriesdoc for information about $\mathrm{C}$ Series documentation.

\section{Safety Guidelines}

Operate the NI 9205 only as described in these operating instructions. 


\begin{abstract}
Hot Surface This icon denotes that the component may be 1II hot. Touching this component may result in bodily injury.
\end{abstract}

\title{
Safety Guidelines for Hazardous Voltages
}

You can connect hazardous voltages only to the NI 9205 with spring terminal. Do not connect hazardous voltages to the NI 9205 with DSUB.

If hazardous voltages are connected to the module, take the following precautions. A hazardous voltage is a voltage greater than $42.4 \mathrm{~V}_{\mathrm{pk}}$ or $60 \mathrm{VDC}$ to earth ground.

A Caution Ensure that hazardous voltage wiring is performed only by qualified personnel adhering to local electrical standards. Caution Do not mix hazardous voltage circuits and
human-accessible circuits on the same module.

Caution Make sure that devices and circuits connected to the module are properly insulated from human contact.

Caution When module terminals are hazardous voltage LIVE ( $>42.4 \mathrm{~V}_{\mathrm{pk}} / 60 \mathrm{VDC}$ ), you must ensure that devices and circuits connected to the module are properly insulated from human contact. You must use the NI 9940 connector backshell kit to ensure that the terminals are not accessible. Figure 1 shows the NI 9940 connector backshell.

Note You can use the NI 9940 connector backshell only with the NI 9205 with spring terminal.

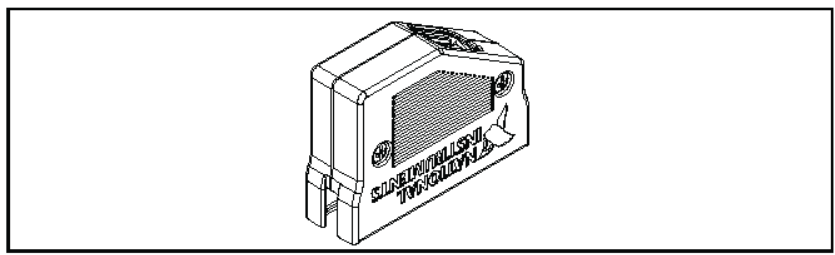

Figure 1. NI 9940 Connector Backshell

\section{Safety Guidelines for Hazardous Locations}

The NI 9205 is suitable for use in Class I, Division 2, Groups A, B, C, D, T4 hazardous locations; Class I, Zone 2, AEx nC IIC T4 and 
Ex nC IIC T4 hazardous locations; and nonhazardous locations only. Follow these guidelines if you are installing the NI 9205 in a potentially explosive environment. Not following these guidelines may result in serious injury or death.

A Caution Do not disconnect I/O-side wires or connectors unless power has been switched off or the area is known to be nonhazardous.

1. Caution Do not remove modules unless power has been switched off or the area is known to be nonhazardous.

1. Caution Substitution of components may impair suitability for Class I, Division 2.

Caution For Zone 2 applications, install the system in an enclosure rated to at least IP 54 as defined by IEC 60529 and EN 60529.

A Caution For Zone 2 applications, connected signals must be within the following limits:

Capacitance $0.2 \mu \mathrm{F} \max$

Special Conditions for Hazardous Locations Use in Europe This equipment has been evaluated as EEx nC IIC T4 equipment under DEMKO Certificate No. 03 ATEX 0324020X. Each module is marked $\varepsilon$ II $3 \mathrm{G}$ and is suitable for use in Zone 2 hazardous locations. If you are using the NI 9205 in Gas Group IIChazardous locations or in ambient temperatures of $-40^{\circ} \mathrm{C} \leq \mathrm{Ta} \leq 70^{\circ} \mathrm{C}$, you must use the device in an NI chassis that has been evaluated as EEx nC IIC T4, Ex nA IIC T4, or Ex nL IIC T4 equipment.

\section{Special Conditions for Marine Applications}

Some modules are Lloyd's Register (LR) Type Approved for marine applications. To verify Lloyd's Register certification, visit ni.com/certification and search for the LR certificate, or look for the Lloyd's Register mark on the module.

\footnotetext{
Caution To meet radio frequency emission requirements for marine applications, use shielded cables and install the system in a metal enclosure. Suppression ferrites must be installed on power supply inputs near power entries to modules and controllers. Power supply and module cables must be separated on opposite sides of the enclosure and must enter and exit through opposing enclosure walls.
} 


\section{Connecting the NI 9205}

The NI 9205 is a 32-channel single-ended/16-channel differential analog input module.

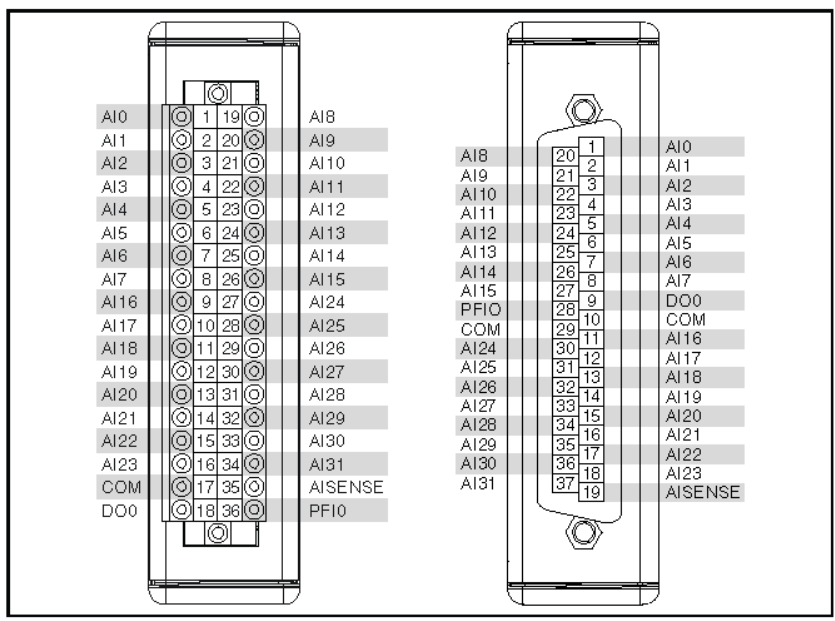

Figure 2. NI 9205 Terminal and Pin Assignments

The NI 9205 provides connections for the 32 single-ended or 16 differential analog input channels, as well as one digital input channel, one digital output channel, COM, and AI SENSE.

Q5 Note The digital output channel is supported only in CompactRIO systems.

The NI 9205 with spring terminal has a 36-terminal detachable spring-terminal connector, and the NI 9205 with DSUB has a 37-pin DSUB connector. Each analog input channel has an AI terminal or pin to which you can connect an analog output device. The NI 9205 is capable of an aggregate sampling rate of $250 \mathrm{kS} / \mathrm{s}$. The NI 9205 also supports triggering. Refer to the software help for information about input trigger modes.

The NI 9205 channels share a common ground that is isolated from the other modules in the system. All channels share a programmable gain instrumentation amplifier and are multiplexed to an ADC. Each channel also has $\pm 30 \mathrm{~V}$ overvoltage protection. For more information about overvoltage protection, refer to the Specifications section. Refer to Figure 3 for an illustration of the input circuitry for one analog input channel on the NI 9205. 


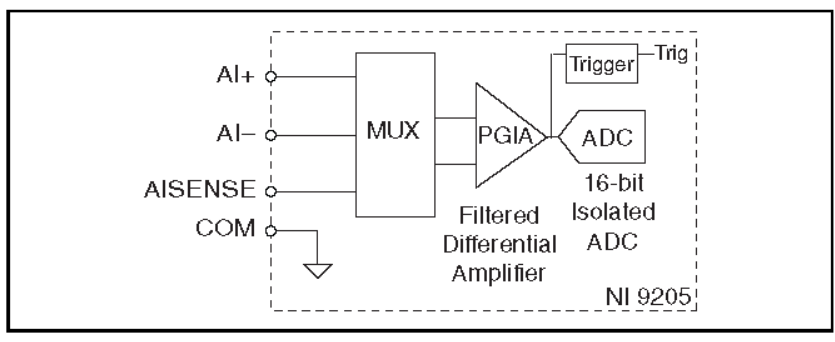

Figure 3. Input Circuitry for One Analog Channel on the NI 9205

\section{Connecting Wires to the NI 9205 Spring-Terminal Connector}

Use a flathead screwdriver with a blade smaller than $2.3 \times 1.0 \mathrm{~mm}$ $(0.09 \times 0.04 \mathrm{in}$. $)$ to connect wires to the detachable spring-terminal connector. Insert the screwdriver into a spring clamp activation slot and press a wire into the corresponding connector terminal, then remove the screwdriver to clamp the wire into the terminal. Refer to the Specifications section for more information about spring-terminal wiring. Refer to Figure 4 for an illustration of connecting wires to the NI 9205 with spring terminal.

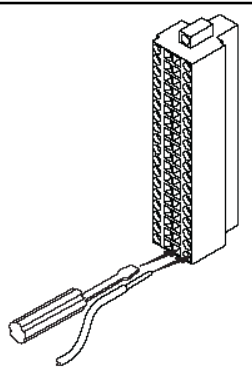

Figure 4. Connecting Wires to the NI 9205 with Spring-Terminal Connector

\section{Wiring for High-Vibration Applications}

If an application is subject to high vibration, National Instruments recommends that you use a backshell kit or shielded cable to protect the connections. For the NI 9205 with spring terminal, use the NI 9940 backshell to protect the connections. For the NI 9205 with DSUB, use a 37-pin shielded cable or the NI 9933 backshell to protect the connections. Refer to Figure 1 for an illustration of the NI 9940 connector backshell. Refer to Figure 5 for an illustration of the NI 9933 connector backshell. 


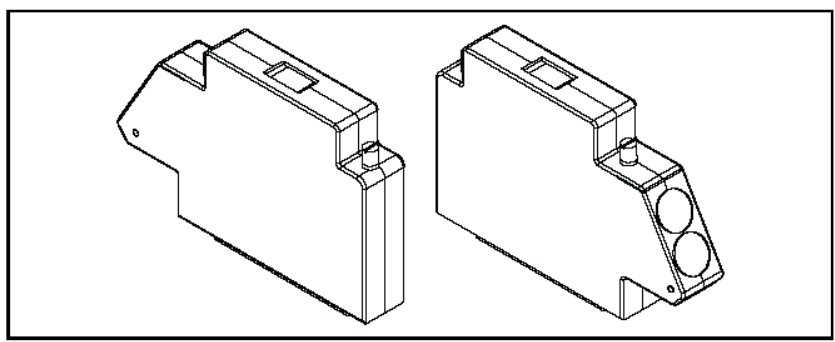

Figure 5. NI 9933 Connector Backshell

\section{Connecting Devices to the NI 9205}

You can connect the NI 9205 directly to a variety of devices and other signal sources. Make sure the devices you connect to the NI 9205 are compatible with the input specifications of the module. Refer to the Specifications section for more information about the input specifications.

When connecting various sources to the NI 9205 , you can use differential, single-ended, or a combination of differential and single-ended connections. Refer to Figures 6,7 , and 8 for diagrams of each connection type.

\section{Differential Measurements}

You can use a differential measurement configuration to attain more accurate measurements and less noise. A differential measurement configuration requires two inputs for each measurement, thus reducing the number of available channels on the NI 9205 to 16 . Table 1 shows the signal pairs that are valid for differential connection configurations with the NI 9205.

Table 1. Differential Pairs

\begin{tabular}{|c||c|c|c||c|c|}
\hline Channel & Signal+ & Signal- & Channel & Signal+ & Signal- \\
\hline 0 & AI0 & AI8 & 16 & AI16 & AI24 \\
\hline 1 & AI1 & AI9 & 17 & AI17 & AI25 \\
\hline 2 & AI2 & AI10 & 18 & AI18 & AI26 \\
\hline 3 & AI3 & AI11 & 19 & AI19 & AI27 \\
\hline 4 & AI4 & AI12 & 20 & AI20 & AI28 \\
\hline 5 & AI5 & AI13 & 21 & AI21 & AI29 \\
\hline 6 & AI6 & AI14 & 22 & AI22 & AI30 \\
\hline 7 & AI7 & AI15 & 23 & AI23 & AI31 \\
\hline
\end{tabular}


Refer to Figure 6 for an illustration of connecting a device to the NI 9205 using differential connections.

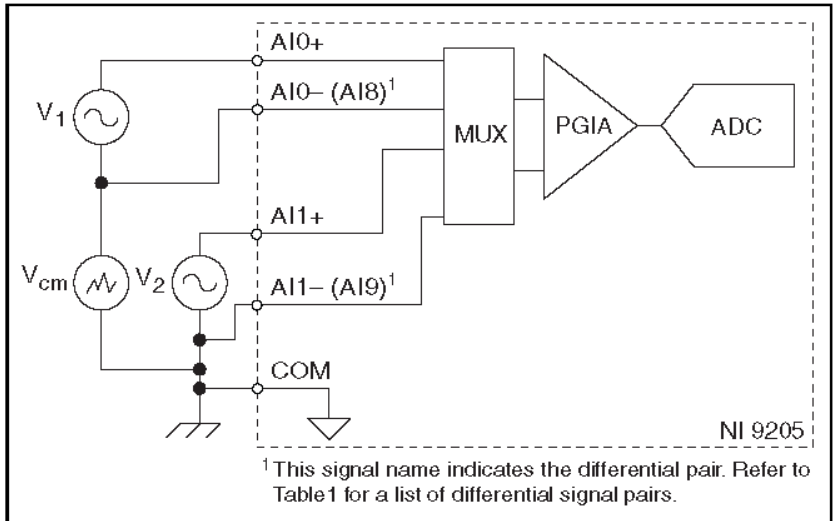

Figure 6. Connecting a Device to the NI 9205 Using Differential Connections

In a differential connection configuration, the NI 9205 rejects the common-mode noise voltage, $\mathrm{V}_{\mathrm{cm}}$, during the measurement of $\mathrm{V}_{1}$.

\section{Referenced Single-Ended (RSE) Measurements}

You can use an RSE measurement configuration to take measurements on 32 channels when all channels share a common ground. Refer to Figure 7 for an illustration of connecting a device to the NI 9205 using RSE connections.

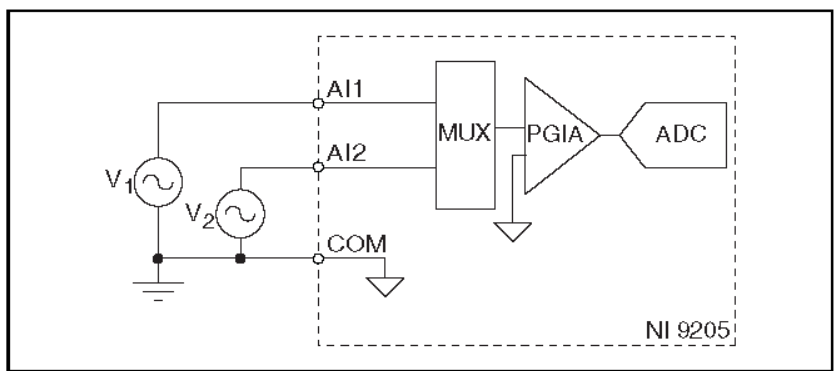

Figure 7. Connecting a Device to the NI 9205 Using RSE Connections

In an RSE connection configuration, the NI 9205 measures each input channel with respect to COM. 
3 Note If you leave COM unconnected, the signals float outside the working input range of the NI 9205. This may result in unreliable measurements because there is no way to ensure that the input signal is within $10 \mathrm{~V}$ of COM.

\section{Non-Referenced, Single-Ended (NRSE) Measurements}

You can use an NRSE measurement configuration to take measurements on all 32 channels while reducing noise more effectively than with an RSE connection configuration. This configuration provides remote sense for the negative (-) input of the PGIA that is shared by all channels configured for NRSE mode. The behavior of this configuration is similar to the behavior of RSE connections, but it provides improved noise rejection. Refer to Figure 8 for an illustration of connecting a device to the NI 9205 using NRSE connections.

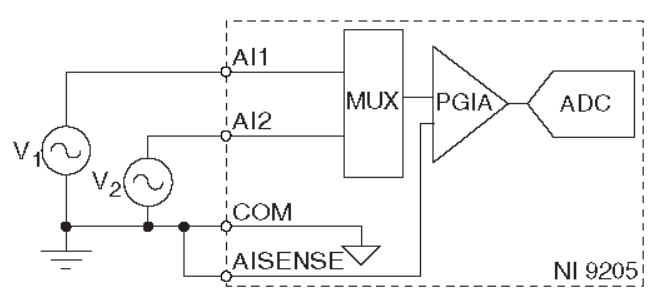

Figure 8. Connecting a Device to the NI 9205 Using NRSE Connections

In an NRSE connection configuration, the NI 9205 measures each input channel with respect to AI SENSE.

\section{Sleep Mode}

This module supports a low-power sleep mode. Support for sleep mode at the system level depends on the chassis that the module is plugged into. Refer to the chassis manual for information about support for sleep mode. If the chassis supports sleep mode, refer to the software help for information about enabling sleep mode. Visit ni.com/info and enter cseriesdoc for information about $\mathrm{C}$ Series documentation. 
Typically, when a system is in sleep mode, you cannot communicate with the modules. In sleep mode, the system consumes minimal power and may dissipate less heat than it does in normal mode. Refer to the Specifications section for more information about power consumption and thermal dissipation.

\section{Specifications}

The following specifications are typical for the range -40 to $70^{\circ} \mathrm{C}$ unless otherwise noted. All voltages are relative to COM unless otherwise noted.

\section{Analog Input Characteristics}

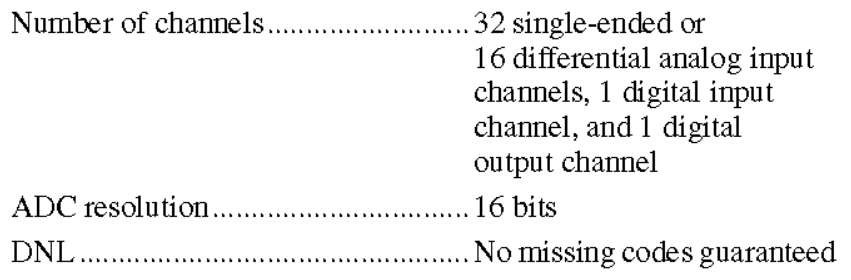

INL. Refer to the AI Absolute Accuracy Tables and Formulas

MTBF 775,832 hours at $25^{\circ} \mathrm{C}$; Bellcore Issue 6, Method 1, Case 3, Limited Part Stress Method

4 Note Contact NI for Bellcore MTBF specifications at other temperatures or for MIL-HDBK-217F specifications.

Conversion time

R Series Expansion chassis ....... $4.50 \mu \mathrm{s}(222 \mathrm{kS} / \mathrm{s})$

All other chassis $4.00 \mu \mathrm{s}(250 \mathrm{kS} / \mathrm{s})$

Input coupling. DC

Nominal input ranges $\pm 10 \mathrm{~V}, \pm 5 \mathrm{~V}, \pm 1 \mathrm{~V}, \pm 0.2 \mathrm{~V}$

Minimum overrange

(for $10 \mathrm{~V}$ range) $4 \%$

Maximum working voltage for analog inputs (signal + common mode) . Each channel must remain within $\pm 10.4 \mathrm{~V}$ of common 


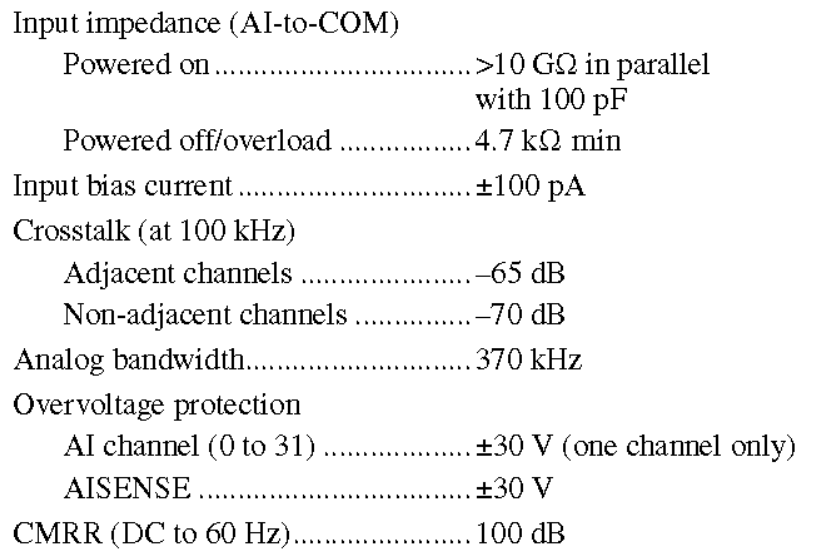

Typical AI+ to AI- CMRR graph

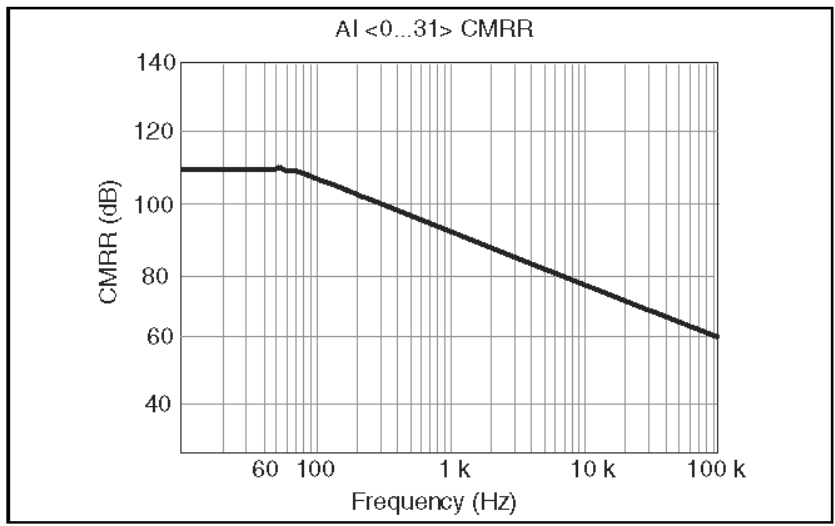

Settling time for multichannel measurements, accuracy, all ranges $\pm 120 \mathrm{ppm}$ of full-scale step $( \pm 8$ LSB $)$ $4 \mu$ s convert interval $\pm 30 \mathrm{ppm}$ of full-scale step $( \pm 2$ LSB $)$ $8 \mu$ s convert interval 
Accuracy details

\begin{tabular}{|c|c|c|c|c|c|c|}
\hline $\begin{array}{c}\text { Nominal } \\
\text { Range } \\
\text { (V) }\end{array}$ & $\begin{array}{c}\text { Residual } \\
\text { Gain } \\
\text { Error } \\
\text { (ppm of } \\
\text { Reading) } \\
\end{array}$ & $\begin{array}{c}\text { Gain } \\
\text { Tempoo } \\
\left(\mathbf{p p m} /{ }^{\circ} \mathbf{C}\right)\end{array}$ & $\begin{array}{c}\text { Reference } \\
\text { Tempoo }\end{array}$ & $\begin{array}{c}\text { Residual } \\
\text { Offset } \\
\text { Error } \\
\text { (ppm of } \\
\text { Range) } \\
\end{array}$ & $\begin{array}{c}\text { Offset } \\
\text { Tempoo } \\
\text { (ppme of } \\
\text { Range/ } /^{\circ} \mathrm{C} \text { ) } \\
\end{array}$ & $\begin{array}{c}\text { INL } \\
\text { Error } \\
\text { (ppm of } \\
\text { Range) }\end{array}$ \\
\hline \pm 10 & 115 & 11 & 5 & 20 & 44 & 76 \\
\hline \pm 5 & 135 & 11 & 5 & 20 & 47 & 76 \\
\hline \pm 1 & 155 & 11 & 5 & 25 & 66 & 76 \\
\hline \pm 0.2 & 215 & 11 & 5 & 40 & 162 & 76 \\
\hline
\end{tabular}

\section{Absolute accuracy formulas}

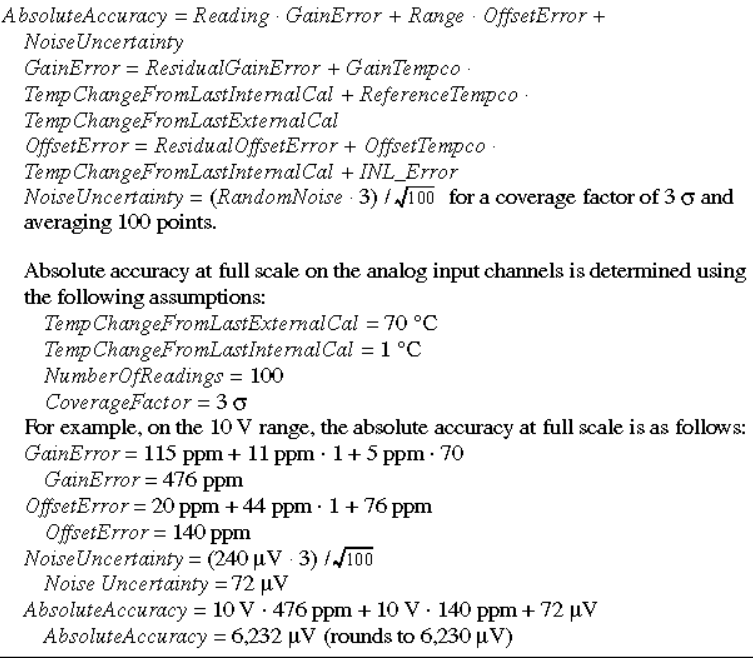




\section{Digital Characteristics}

Overvoltage protection .................... $\pm 30 \mathrm{~V}$

Digital input logic levels

\begin{tabular}{|l|c|c|}
\hline \multicolumn{1}{|c|}{ Level } & Min & Max \\
\hline \hline Input high voltage $\left(\mathrm{V}_{\mathrm{IH}}\right)$ & $2.0 \mathrm{~V}$ & $3.3 \mathrm{~V}$ \\
\hline Input low voltage $\left(\mathrm{V}_{\mathrm{IL}}\right)$ & $0 \mathrm{~V}$ & $0.34 \mathrm{~V}$ \\
\hline
\end{tabular}

Digital output logic levels

\begin{tabular}{|l|c|c|}
\hline Level & Min & Max \\
\hline \hline Output high voltage $\left(\mathrm{V}_{\mathrm{OH}}\right)$, sourcing $75 \mu \mathrm{A}$ & $2.1 \mathrm{~V}$ & $3.3 \mathrm{~V}$ \\
\hline Output low voltage $\left(\mathrm{V}_{\mathrm{OL}}\right)$, sinking $250 \mu \mathrm{A}$ & $0 \mathrm{~V}$ & $0.4 \mathrm{~V}$ \\
\hline
\end{tabular}

External digital triggers

Source. PFI0

Delay $100 \mathrm{~ns} \max$

\section{Power Requirements}

Power consumption from chassis

Active mode $625 \mathrm{~mW} \max$

Sleep mode $15 \mathrm{~mW}$

Thermal dissipation (at $70^{\circ} \mathrm{C}$ )

Active mode $625 \mathrm{~mW} \max$

Sleep mode $15 \mathrm{~mW}$

\section{Physical Characteristics}

If you need to clean the module, wipe it with a dry towel.

Spring-terminal wiring. 18 to 28 AWG copper conductor wire with $7 \mathrm{~mm}$ ( 0.28 in.) of insulation stripped from the end

Weight
NI 9205 with spring terminal .... $158 \mathrm{~g}(5.8 \mathrm{oz})$
NI 9205 with DSUB $148 \mathrm{~g}(5.3 \mathrm{oz})$ 


\title{
Safety
}

\section{Maximum Voltage ${ }^{1}$}

Connect only voltages that are within the following limits.
AI, PFI0, and DO-to-COM
$\pm 30 \mathrm{VDC}$

\section{NI 9205 with Spring Terminal Isolation Voltages}

\begin{tabular}{|c|c|}
\hline \multicolumn{2}{|c|}{ Channel-to-channel......................... None } \\
\hline Channel-to-earth ground & \\
\hline Continuous .................. & $\begin{array}{l}.250 \mathrm{~V}_{\text {rms }}, \text { Measurement } \\
\text { Category II }\end{array}$ \\
\hline Withstand....... & $\begin{array}{l}2,300 \mathrm{~V}_{\mathrm{rms}}, \text { verified by a } 5 \mathrm{~s} \\
\text { dielectric withstand test }\end{array}$ \\
\hline
\end{tabular}

Measurement Category II is for measurements performed on circuits directly connected to the electrical distribution system. This category refers to local-level electrical distribution, such as that provided by a standard wall outlet, for example, $115 \mathrm{~V}$ for U.S. or $230 \mathrm{~V}$ for Europe.

\footnotetext{
${ }^{1}$ The maximum voltage that can be applied or output between $\mathrm{AI}$ and COM without creating a safety hazard.

(c) National Instruments Corp.

$27 \quad$ N 9205 0perating Instructions and Specifications
}

\begin{abstract}
Caution Do not connect the NI 9205 with spring terminal to signals or use for measurements within Measurement Categories III or IV.
\end{abstract}

\section{NI 9205 with DSUB Isolation Voltages}

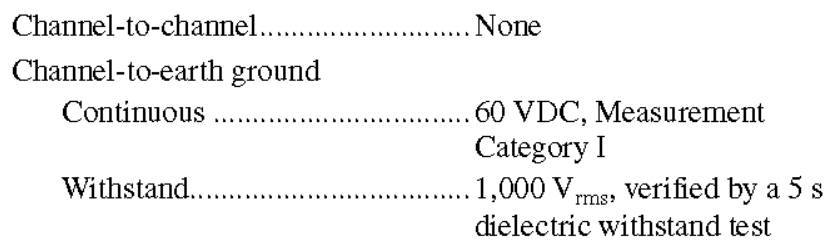

Measurement Category I is for measurements performed on circuits not directly connected to the electrical distribution system referred to as MAINS voltage. MAINS is a hazardous live electrical supply system that powers equipment. This category is for measurements of voltages from specially protected secondary circuits. Such voltage measurements include signal levels, special equipment, limited-energy parts of equipment, circuits powered by regulated low-voltage sources, and electronics. 


\begin{abstract}
A Caution Do not connect the NI 9205 with DSUB to signals or use for measurements within Measurement Categories II, III, or IV.
\end{abstract}

\title{
Safety Standards
}

This product is designed to meet the requirements of the following standards of safety for electrical equipment for measurement, control, and laboratory use:

- IEC 61010-1, EN 61010-1

- UL 61010-1, CSA 61010-1

Note For UL and other safety certifications, refer to the product label or visit ni.com/certification, search by module number or product line, and click the appropriate link in the Certification column.

\section{Hazardous Locations}

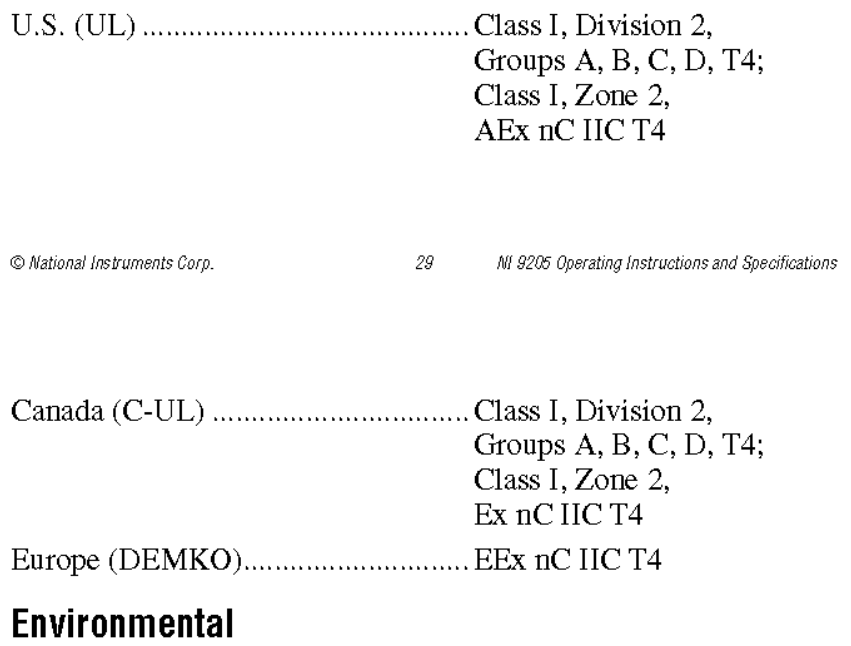

National Instruments C Series modules are intended for indoor use only but may be used outdoors if installed in a suitable enclosure. Refer to the manual for the chassis you are using for more information about meeting these specifications.

Operating temperature (IEC 60068-2-1, IEC 60068-2-2) .....-40 to $70^{\circ} \mathrm{C}$

Storage temperature (IEC 60068-2-1, IEC 60068-2-2) .....-40 to $85^{\circ} \mathrm{C}$ Ingress protection.......................... IP 40

Operating humidity (IEC 60068-2-56)...... 10 to $90 \% \mathrm{RH}$, noncondensing 


$\begin{aligned} & \text { Storage humidity } \\ & \text { (IEC 60068-2-56)......................... } 5 \text { to } 95 \% \text { RH, } \\ & \text { noncondensing }\end{aligned}$
Maximum altitude ......................... $2,000 \mathrm{~m}$
Pollution Degree (IEC 60664) ......... 2

\section{Shock and Vibration}

To meet these specifications, you must panel mount the system and use a backshell kit or shielded cable to protect the connections. Use the NI 9940 backshell for the NI 9205 with spring terminal and a 37-pin shielded cable or the NI 9933 backshell for the NI 9205 with DSUB.

Operating vibration

$$
\begin{aligned}
& \text { Random (IEC 60068-2-64)........ } 5 \mathrm{~g}_{\mathrm{rms}}, 10 \text { to } 500 \mathrm{~Hz} \\
& \text { Sinusoidal (IEC 60068-2-6) ....... } 5 \text { g, } 10 \text { to } 500 \mathrm{~Hz} \\
& \text { Operating shock } \\
& \text { (IEC 60068-2-27)...........................30 g, } 11 \mathrm{~ms} \text { half sine, } \\
& 50 \mathrm{~g}, 3 \mathrm{~ms} \text { half sine, } \\
& 18 \text { shocks at } 6 \text { orientations }
\end{aligned}
$$

\section{Electromagnetic Compatibility}

This product is designed to meet the requirements of the following standards of EMC for electrical equipment for measurement, control, and laboratory use:

- EN 61326 EMC requirements; Industrial Immunity

- EN 55011 Emissions; Group 1, Class A

- CE, C-Tíck, ICES, and FCC Part 15 Emissions; Class A

3 Note For EMC compliance, operate this device with shielded cabling.

\section{CE Compliance}

This product meets the essential requirements of applicable European directives, as amended for $\mathrm{CE}$ markings, as follows:

- 2006/95/EC; Low-Voltage Directive (safety)

- 2004/108/EC; Electromagnetic Compatibility Directive (EMC)
Note Refer to the Declaration of Conformity (DoC) for this product for any additional regulatory compliance information. To obtain the DoC for this product, visit


ni.com/certification, search by module number or product line, and click the appropriate link in the Certification column.

\section{Environmental Management}

National Instruments is committed to designing and manufacturing products in an environmentally responsible manner. NI recognizes that eliminating certain hazardous substances from our products is beneficial not only to the environment but also to NI customers.

For additional environmental information, refer to the NI and the Environment Web page at ni .com/environment. This page contains the environmental regulations and directives with which NI complies, as well as other environmental information not included in this document.

\section{Waste Electrical and Electronic Equipment (WEEE)}

EU Customers At the end of their life cycle, all products must be sent to a WEEE recycling center. For more information about WEEE recycling centers and National Instruments WEEE initiatives, visit ni .com/ environment/weee. htm.

\section{电子信息产品污染控制管理办法（中国 RoHS）}

(9) 中国客户 National Instruments 符合中国电子信息 产品中限制使用某些有害物质指令 (ROHS)。关于 National Instruments 中国 RoHS 合规性信息, 请登录 ni.com/environment/rohs_china。 (For information about China RoHS compliance, go to ni. com / environment/rohs_china.)

\section{Calibration (Analog Input)}

You can obtain the calibration certificate and information about calibration services for the NI 9205 at ni.com/calibration.

Calibration interval 2 years

\section{Where to Go for Support}

The National Instruments Web site is your complete resource for technical support. At ni. com/support you have access to everything from troubleshooting and application development self-help resources to email and phone assistance from NI Application Engineers.

National Instruments corporate headquarters is located at 11500 North Mopac Expressway, Austin, Texas, 78759-3504. 
National Instruments also has offices located around the world to help address your support needs. For telephone support in the United States, create your service request at ni. com/support and follow the calling instructions or dial 5127958248 . For telephone support outside the United States, contact your local branch office:

Australia 1800300800 , Austria $43662457990-0$, Belgium 32 (0) 2757 0020, Brazil 551132623599 , Canada 800433 3488, China 862150509800 ,

Czech Republic 420224235774 , Denmark 4545762600 , Finland 358 (0) 9725 72511, France 01576624 24, Germany 49897413130 , India 918041190000 , Israel 97236393737 , Italy 390241309277 , Japan 0120-527196, Korea 820234513400 , Lebanon 961 (0) 1332828 , Malaysia 1800887710 , Mexico 018000100793 , Netherlands 31 (0) 348433 466, New Zealand 0800553 322, Norway 47 (0) 66907660 , Poland 48223390150 , Portugal 351210311210 , Russia 74957836851 , Singapore 18002265886 , Slovenia 38634254200 , South Africa 27011805 8197, Spain 34916400085 , Sweden 46 (0) 8587895 00, Switzerland 41562005151 , Taiwan 886022377 2222, Thailand 6622786777 , Turkey 90212279 3031, United Kingdom 44 (0) 1635523545 


$\begin{array}{lr}\text { DNATIONAL } & \text { Technical Sales } \\ \text { United States } \\ \text { (866) 531-6285 } \\ \text { info@ni.com }\end{array}$

Requirements and Compatibility | Ordering Information | Detailed Specifications | Pinoutsif Front Panel Connections

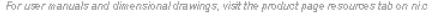

Last Revised: 2010-02-17 10:21:10.0

8-Channel, $\pm 60 \mathrm{~V}, 800 \mathrm{kS} / \mathrm{s}, 12-B i t \mathrm{C}$ Series Analog Input Module

NI 9221

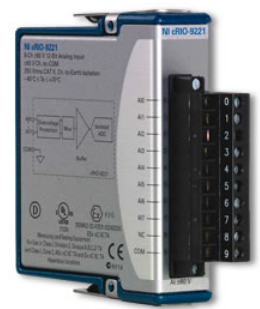

.8 analog inputs, $\pm 60 \vee$ input range

- $800 \mathrm{kS} / \mathrm{s}$ aggregate sampling rate

- Hot-swappable operation, overvoltage protection, isolation

-40 to $70^{\circ} \mathrm{C}$ operating rang

- NIST-traceable calibration

- 12-bit resolution, single-ended inputs, screw terminal or D-Sub connector type

Overview

The NI 9221 is a C Series module for high-speed measurements with a range of $\pm 60 \mathrm{~V}$. This wider yoltage range makes this module well-sulted for industrial-level, automotive, or even smaller-cell battery measurements. As with the majority of C Series modules, the $\mathrm{N} I 9221$ is protected from harmful voltage spikes of up to $2,300 \mathrm{Vrms}$. This means that no harmfiul volt age within the isolation rating can harm other modules in the system, the chassis, or any connected computer equipment. In addition to the absolute protection from the isolation, the module features up to $100 \mathrm{~V}$ of overvoltage protection for errant signal connection or unexpected outputs to the channels. The $\mathrm{N} / 9221$ features two connecto options - a 10-position screw-terminal connector for direct connectivity and a 25-postion D-Sub connector. The industry-standard 25-position D-Sub connector provides for low-cost cabling to a wide vanety of accessories from $\mathrm{Nl}$ or other vendors. Some vendors also offer custom D-sub cable tabrication services and provide cables with a pinout tha matches your exact appic ation needs. NI recommends the NI 9932 strain-relief connector accessory for the NI cRIO-g221 analog input module. The NI 9934 (or other 25-pin D-sub connector) is required to use the N. 9221 with a D-sub module. The NI 9934 includes a screw-terminal connector with strain reller as well as a D-Sub solder cup back

Back to Top

Requirements and Compatibility

os Information

- RearTime os

Driver Information

- NI-DAQmo

- NH-RIO
Software Compatibility

- LabVIEW

- LabWindowsicVI

- Measurement Studio

- SignalExpress

- Visual C++

- Visual Studio

- Visual Studio .NET

Comparison Tables

\begin{tabular}{|c|c|c|c|c|}
\hline Module & Signal Type & Channels & Sample Rate & Resolution (bits) \\
\hline 9201 & Voltage & 8 & $500 \mathrm{kS} / \mathrm{s}$ & 12 \\
\hline 9203 & Current & 8 & $200 \mathrm{kS} / \mathrm{s}$ & 16 \\
\hline 9205 & Voltage & $32 \mathrm{SE} / 16 \mathrm{DI}$ & $250 \mathrm{kS} / \mathrm{s}$ & 16 \\
\hline
\end{tabular}




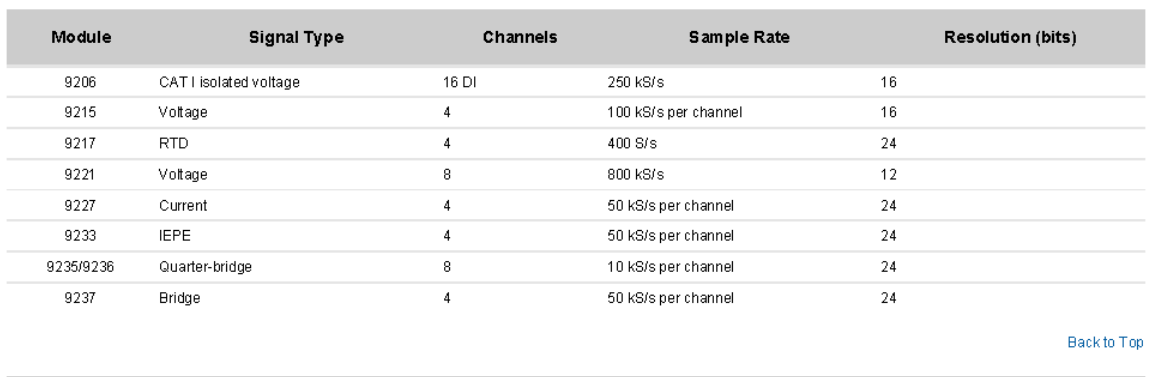

\section{Application and Technology}

High-accuracy NI C Series analog input modules for NI CompactDAQ and CompactRIO prowide high-performance measurements for a wide variety of industrial, in-vehicle, and laboratory sensors and signal types. Each module includes built-in signal conditioning and an integrated connector with screw terminal or cable options for flexible and low-cost signal wiring. All modules feature CompactRil0 Extreme Industrial Certifications and Ratings.

c Series Compatibility

The C Series hardware family features more than 50 measurement modules and several chassis and carriers for deployment. With this variety of modules, you can mix and match measurements such as temperature, acceleration, flow, pressure, strain, acoustic, voltage, current, digital, and more to create a custom system. Install the modules in one of several carriers to create a single module USB, Ethernet, or Wi-Fi system, or combine them in chassis such as NI CompactDAQ and CompactRIO to create a

mived-measurement sy stem with synchronized measurements. You can install up to eight modules in a simple, complete NI CompactDAQ USB data acquisition sy stem to synchronize all of the analog output, analog input, and digital IIO from the modules. For a system without a PC, CompactRIO holds up to eight modules and features a built-in processor, RAM, and storage for an embedded data logger or control unit. For higher-speed control, CompactRlO chassis incorporate a field-programmable gate array (FPGA) W

Advanced F eatures

When used with CompactRIO, C Series analog input modules connect directly to reconfigurable IIO (RIO) FPGA hardware to create high-performance embedded Systems. The reconfigurable FPGA hardware within CompactRIO provides a variety of options for custom timing, triggering, synchronization, filtering, signal processing, and high-speed decision making for all C Series analog modules. For instance, with CompactRIO, you can implement custom triggering for any analog sensor type on a per-channel basis using the flexibility and performance of the FPGA and the numerous arthmetic and comparison function blocks buit into LabVIEW FPGA.

Key Features

- High-accuracy, high-performance analog measurements for ary CompactRlO embedded System, R Series expansion chassis, or NI CompactDAQ chassis

- Screw terminals, ENC, D-Sub, spring terminals, strain relief, high voltage, cable, solder cup backshell, and other connectivity options

- Available channel-to-earth ground double-isolation barrier for safety, noise immunity, and high-common-mode voltage range

- CompactRIo Extreme Industrial Certifications and Ratings

- Built-in signal conditioning for direct connection to sensors and industrial devices

Visit ni. comicompactrio or ni.comicompactdaq for up-to-date information on module availability, example programs, application notes, and other developer tools

Connectivity Accessories

NII CompactDAQ and CompactRIO Systems are designed to provide flexible options for low-cost field wiring and cabling. Most $\mathrm{C}$ Series modules have a unique connector block option that offers secure connections to your $C$ Series system Table 2 contains all of the connector blocks for $C$ Series 10 modules

\begin{tabular}{|c|c|}
\hline Accessory & Description \\
\hline NI 9932 & 10-position strain relief and high-voltage screw-terminal connector kit \\
\hline NI 9933 & 37-pin D-Sub connector kit with strain relief and D-Sub shell \\
\hline NI 9934 & 25-pin D-Sub connector kit with strain relief and D-Sub shell \\
\hline NI 9935 & 15-pin D-Sub connector kit with strain relief and D-Sub shell \\
\hline NI 9936 & 10-position screw-terminal plugs (quantity 10) \\
\hline NI 9939 & 16-position connector kit with strain relief \\
\hline
\end{tabular}

Table 3 lists the recommended connector block accessories for each C Series analog input module.

\begin{tabular}{|c|c|}
\hline C Series Analog Input Module & Recommended Module Accessory \\
\hline NI 9201 & NI 9932, N1 9936 \\
\hline NI 9201 with D-Sub & NI 99341 \\
\hline NI 9211 & NI 9932, NI 9936 \\
\hline NI 9215 & NI 9932, NI 9936 \\
\hline N1 9217 & N1 9939 \\
\hline NI 9221 & NI 9932, NI 9936 \\
\hline NI 9221 with D-Sub & N1 99341 \\
\hline
\end{tabular}




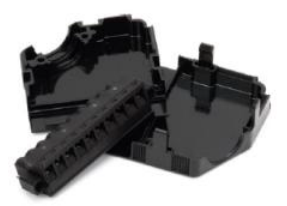

Figure 1. NI 9932 10-Position Strain Relief and High-Voltage Screw-Terminal Connector Kit

The NII 9933 includes a screw-terminal connector with strain relief as well as a D-Sub solder cup backshell for creating custom cable assemblies for ary module with a 37-pin D-Sub connector.

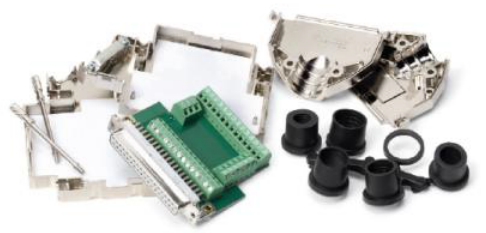

Figure 2. NI 9933 37-Pin D-Sub Connector Kit with Strain Relief and D-Sub Shell

The NII 9934 includes a screw-terminal connector with strain relief as well as a D-Sub solder cup backshell for creating custom cable assemblies for ary module with a 25-pin D-Sub connector

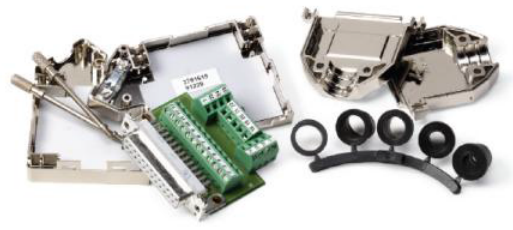

Figure 3. NI 9934 25-Pin D-Sub Connector Kit with Strain Relief and D-Sub Shell

The NI 9935 includes a screw-terminal connector with strain relief as well as a D-Sub solder cup backshell for creating custom cable assemblies for ary module with a 15-pin D-Sub connector.

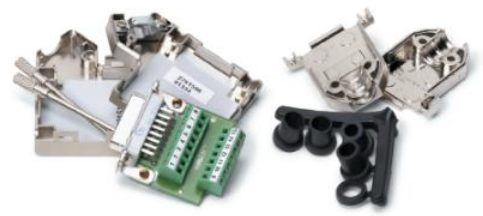

Figure 4. N 9935 15-Pin D-Sub Connector Kit with Strain Relief and D-Sub Shell

The NI 9936 consists of 10-position screw-terminal plugs for ary 10-position screw-terminal module 


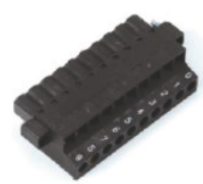

Figure 5. NI 9936 10-Position Screw-Terminal Plugs

\section{Ordering Information}

For a complete list of accessories, visit the product page on ni.com.

\begin{tabular}{|c|c|c|c|}
\hline Products & Part Number & Recommended Accessories & Part Number \\
\hline \multicolumn{4}{|l|}{ NI 9221 Voltage Input Module } \\
\hline $\begin{array}{l}\text { N1 } 9221 \text { with Screw Terminals } \\
\text { Requires: } 1 \text { Connectivity Accessorie }\end{array}$ & 779014-01 & Connectivity Accessories: screwTerminal - NI 9927 Strain relief, operator protection (qty 1) & $782715-01$ \\
\hline
\end{tabular}

Software Recommendations

LabVIEW Professional Development System for Windows

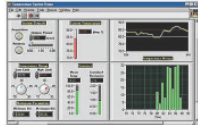

NI LabWindows ${ }^{\mathrm{m}} / \mathrm{CVI}$ for Windows

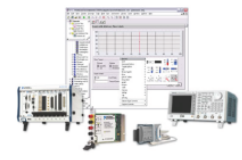

- Advanced software tools for large project

- Automatic code generation using DAQ
Assistant and Instrument I/O Assistant - Tight integardware signal processing - Open connectivity
NET objects - Capability to build DLLs, executables, and MSl installers

- Rea-time advanced $2 \mathrm{D}$ graphs and charts Complete hardware compatibility with IVI, , and seria - Analysis tools for array manipulation, signa stics, and curve fitting Simplifed cross-plattorm communication with - Simplified cross-p

- Measurement Studio. NET tools (included in LabWindows/CVI Full only) - The mark LabWindows is used under

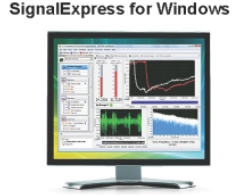

- Quickly configure projects without programming

- Control over 400 PC-based and stand-alone - Log data from more than 250 data acquisition
devices

- Pertorm basic signal processing, analysis,
and file $/ / O$

- Scale your application with automatic

LabVIEW code generation

- Create custom reports or easily export data
to LabVIEW, DIAdem or Microsoft Excel

NI Measurement Studio

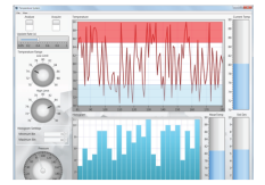

- Customizable graphs and charts for WPF, dows Forms, and ASP NET Web Forms Ul design

- Analysis libraries for array operations, signa generation, windowing, filters, signal processing - Hardware integration support with native
.NET data acquisition and instrument control braries

- Automatic code generation for all NI-DAQmX - Intelligent and efficient data-logging libraries
for streaming measurement data to disk - Suppot tor Microsof visuat sudo disk - Support for Microsoft Visual Studio. NET
2012/2010/2008

NI system assurance programs are designed to make it even easier for you to own an NI system. These programs include configuration and deployment services for your NI PXI, CompactRiO, or Compact FieldPoint system. The NI Basic System Assurance Program provides a simple integration test and ensures that your system is delivered completely assembled in one box. When you conigure your system with the Ni Standard System Assurance Program, you can select from avallable Ni system diver sets and application development environments to create customized, reorderable software configurations. Your system arrives fully assembled and tested in one box with your software preinstalled
When you order your system with the standard program, you also receive system-specific documentation including a bill of materials, an integration test report, a recommended 


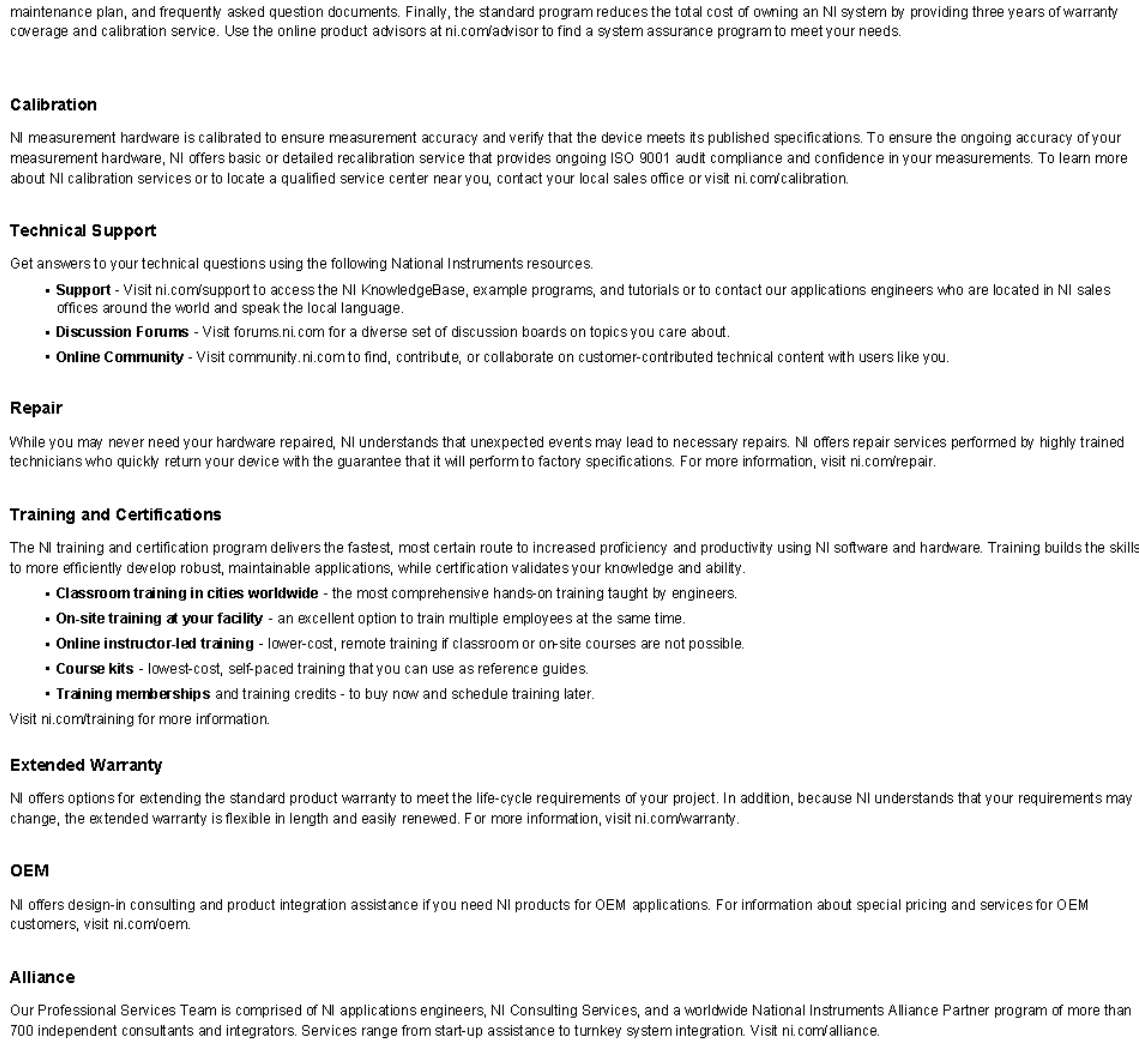

NI offers options for extending the standard product warranty to meet the life-cycle requirements of your project. In addition, because $\mathrm{Nl}$ understands that your requirements may change, the extended warranty is flexible in length and easily renewed. For more information, visit nicomiwarranty.

\section{OEM}

NI offers design-in consulting and product integration assistance if you need NI products for OEM applications. For information about special pricing and services for OEM customers, visit ni.comioem.

Alliance

Our Professional Services Team is comprised of $\mathrm{NI}$ applications engineers, NI Consulting Services, and a worldwide National Instruments Alliance Partner program of more than 700 independent consultants and integrators. Services range from start-up assistance to turnkey system integration. Visit ni. comvalliance.

Detailed Specifications

The following specifications are typical for the range -40 to $70^{\circ} \mathrm{C}$ unless otherwise noted. All voltages are relative to $\mathrm{COM}$ unless otherwise noted. The specifications are the same for the NI 9201 and the NI 9221 unless otherwise noted.

\section{Input Characteristics}

Number of channels

8 analog input channels

ADC resolution

12 bit

Type of ADC

Successive approximation register (SAR)

\begin{tabular}{|l|l|l|}
\hline \multicolumn{3}{|c|}{ Sample rate (aygreyate) } \\
\hline \multicolumn{1}{|c|}{ Module } & Maximum Sample Rate (R Series Expansion Chassis) & Maximum Sample Rate (All Other Chassis) \\
\hline N1 9201, single channel & $475 \mathrm{kS} / \mathrm{s}$ & $800 \mathrm{kS} / \mathrm{s}$ \\
\hline N 19201, scanning & $475 \mathrm{kS} / \mathrm{s}$ & $500 \mathrm{kS} / \mathrm{s}$ \\
\hline NN 9221 & $475 \mathrm{kS} / \mathrm{s}$ & $800 \mathrm{kS} / \mathrm{s}$ \\
\hline
\end{tabular}

Input range 


\begin{tabular}{|c|c|c|c|c|c|}
\hline \multicolumn{6}{|c|}{ Operating voltaye ranges 1} \\
\hline \multirow{2}{*}{ Module } & Measurem & nt Voltage, $\mathrm{C}$ & annel-to-com & \multicolumn{2}{|c|}{ Maximum Voltage, Channe-to-Earth Ground or COM-to-Earth Ground } \\
\hline & $\operatorname{Min} M$ & Typ M & $\operatorname{Max}(M$ & Screw Terminal & DSUB \\
\hline N1 9201 & \pm 10.3 & \pm 10.53 & \pm 10.8 & \multirow[t]{2}{*}{$250 \mathrm{~V}_{\text {rms }}$} & \multirow[t]{2}{*}{ $\pm 60 \mathrm{VDC}$} \\
\hline 9221$\}$ & \pm 61.4 & \pm 62.50 & \pm 63.8 & & \\
\hline
\end{tabular}

\begin{tabular}{|l|l|l|}
\hline \multicolumn{3}{|c|}{ N1 9201 accuracy (excludes noise) } \\
\hline Measurement Conditions & Percent of Reading (Gain Eror) & Percent of Range* (Offset Emor) \\
\hline Calibrated typ $\left(25^{\circ} \mathrm{C}, \pm 5^{\circ} \mathrm{C}\right)$ & $\pm 0.04 \%$ & $\pm 0.07 \%$ \\
\hline Calibrated $\max \left(-40\right.$ to $\left.70^{\circ} \mathrm{C}\right)$ & $\pm 0.25 \%$ & $\pm 0.25 \%$ \\
\hline Uncalibrated typ $\left(25^{\circ} \mathrm{C}, \pm 5^{\circ} \mathrm{C}\right)$ & $\pm 0.26 \%$ & $\pm 0.46 \%$ \\
\hline Uncalibrated $\max \left(-40\right.$ to $\left.70^{\circ} \mathrm{C}\right)$ & $\pm 0.67 \%$ & $\pm 1.25 \%$ \\
\hline${ }^{\prime}$ Range equals $10.53 \mathrm{~V}$ & \\
\hline
\end{tabular}

\begin{tabular}{|l|l|l|}
\hline \multicolumn{3}{|c|}{ N1 9221 accuracy (excludes noise) } \\
\hline Measurement Conditions & Percent of Reading (Gain Eror) & Percent of Range* (Offset Error) \\
\hline Calibrated typ $\left(25^{\circ} \mathrm{C}, \pm 5^{\circ} \mathrm{C}\right)$ & $\pm 0.04 \%$ & $\pm 0.07 \%$ \\
\hline Calibrated $\max \left(-40\right.$ to $\left.70^{\circ} \mathrm{C}\right)$ & $\pm 0.25 \%$ & $\pm 0.25 \%$ \\
\hline Uncalibrated typ $\left(25^{\circ} \mathrm{C}, \pm 5^{\circ} \mathrm{C}\right)$ & $\pm 0.26 \%$ & $\pm 0.43 \%$ \\
\hline Uncalibrated $\max \left(-40\right.$ to $\left.70^{\circ} \mathrm{C}\right)$ & $\pm 0.67 \%$ & $\pm 1.06 \%$ \\
\hline${ }^{*}$ Range equals $62.50 \mathrm{~V}$ & \\
\hline
\end{tabular}

Stability

Gain drift

Offset drift

N1 9201

N 9221

Input bandwidth $(-3 \mathrm{~dB})$

NI 9201

NI 9221

Input impedance

Resistance

Capacitance

Input noise (code-centered)

RMS

Peak-to-peak

No missing codes

DNL

INL

Crosstalk (at $10 \mathrm{kHz}$ )

Settling time (to 1 LSB)
$\pm 34 \mathrm{ppm} 7^{\circ} \mathrm{C}$
$\pm 100 \mathrm{w} / /^{\circ} \mathrm{C}$
$\pm 580 \mathrm{wVi}^{\circ} \mathrm{C}$
$690 \mathrm{kHz}$ min
$950 \mathrm{kHz}$ min
$1 \mathrm{M \Omega}$
$5 \mathrm{pF}$
$0.7 \mathrm{LSB}_{\text {Ims }}$
5 LSB
12 bits
-0.9 to 1.5 LSB
\pm 1.5 LSB
$-75 \mathrm{~dB}$ 


\begin{tabular}{|c|c|}
\hline NI 9201 & $2 \mu \mathrm{s}$ \\
\hline NI 9221 & $1.25 \mu \mathrm{s}$ \\
\hline MTEF & $\begin{array}{l}1,092,512 \text { hours at } 25^{\circ} \mathrm{C} \text {; Bellcore Issue } 2 \text {, Method } 1 \text {, Case } 3 \text {, Limited Part } \\
\text { Stress Method }\end{array}$ \\
\hline Q7. Note Contact NI for Bellcor & K-217F specifications. \\
\hline \multicolumn{2}{|l|}{ Power Requirements } \\
\hline \multicolumn{2}{|l|}{ Power consumption from chassis } \\
\hline Active mode & $1 \mathrm{~W} \max$ \\
\hline Sleep mode & $1 \mathrm{mWN} \max$ \\
\hline \multicolumn{2}{|l|}{ Thermal dissipation (at $70^{\circ} \mathrm{C}$ ) } \\
\hline Active mode & $1 \mathrm{~W} \max$ \\
\hline Sleep mode & $32 \mathrm{mw} \max$ \\
\hline \multicolumn{2}{|l|}{ Physical Characteristics } \\
\hline \multicolumn{2}{|c|}{ If you need to clean the module, wipe it with a dry towel. } \\
\hline \multicolumn{2}{|c|}{ Q. Note For two-dimensional drawings and three-dimensional models of the $\mathrm{C}$ Series module and connectors, visit ni.comidimensions and search by module number. } \\
\hline Screw-terminal wiring & $\begin{array}{l}12 \text { to } 24 \text { AWG copper conductor wire with } 10 \mathrm{~mm} \text { (0.39 in.) of insulation } \\
\text { stripped fromthe end }\end{array}$ \\
\hline Torque for screw terminals & 0.5 to $0.6 \mathrm{~N} \cdot \mathrm{m}(4.4$ to $5.3 \mathrm{lb} \cdot \mathrm{in})$ \\
\hline Ferrules & $0.25 \mathrm{~mm}^{2}$ to $2.5 \mathrm{~mm}^{2}$ \\
\hline \multicolumn{2}{|l|}{ Weight } \\
\hline NI $9201 / 9221$ with screw terminal & $150 \mathrm{~g}(5.3 \mathrm{oz})$ \\
\hline NI 920192221 with DSU日 & $145 \mathrm{~g}(5.1 \mathrm{oz})$ \\
\hline \multicolumn{2}{|l|}{ Safety } \\
\hline \multicolumn{2}{|l|}{ Satety Voltajes } \\
\hline \multicolumn{2}{|c|}{ Connect only voltages that are within the following limits. } \\
\hline Channelto-com & $\pm 60 \mathrm{VDC} \max$ \\
\hline \multicolumn{2}{|c|}{ N1 9201/9221 with Screw Terminal Isolation Voltajes } \\
\hline Channel-to-channel & None \\
\hline \multicolumn{2}{|l|}{ Channel-to-earth ground } \\
\hline Continuous & $250 \mathrm{~V}_{\text {Ims' }}$ Measurement Category II \\
\hline Withstand & $2,300 V_{\text {rms }}$ verfified by a $5 s$ dielectric withstand test \\
\hline
\end{tabular}

Measurement Categony II is for measurements performed on circults directly connected to the electrical distribution sy stem. This category refers to locarlevel electrical

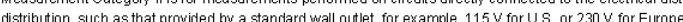

A Caution Do not connect the NI $9201 / 9221$ with screw terminal to signals or use for measurements within Measurement Categories III or IV.

NI 92019221 with DSUB Isolation Voltages

Channel-to-channel

None

Channel-to-earth ground

Continuous

$60 \mathrm{VDC}$, Measurement Category

Withstand

$1,000 \mathrm{~V}_{\text {rms' }}$ verfified by a $5 \mathrm{~s}$ dielectric withstand test

Measurement Category I is for measurements performed on circuits not directly connected to the electrical distribution system referred to as MAINS voltage. MAINS is a hazardous live electrical supply sistem that powers equipment This categony is for measurements of voltages from specially protected secondary circuits. Such voltage measurements include signal levels, special equipment, limited-energy parts of equipment, circuits powered by regulded lo

A Caution Do not connect the NI 9201/9221 with DSUB to signals or use for measurements within Measurement Categories II, III, or IV.

Hazardous Locations

U.S. (UL)

Class I, Division 2, Groups A, B, C, D, T4; Class I, Zone 2, AEx nC IIC T4

Canada (C-UL)

Class I, Division 2, Groups A, B, C, D, T4; Class I, Zone 2, Ex nC IIC T4

Europe (DEMKO)

EEx nC IIC T4

Sdety Standards

This product is designed to meet the requirements of the following standards of safety for electrical equipment for measurement, control, and laboratory use 
IEC 61010-1, EN 61010-1

- UL1010-1, ESA

Q Note For UL and other safety certifications, refer to the product label or the Onine Product Certification section.

Electrom agnetic Compatibility

This product meets the requirements of the following EMC standards for electrical equipment for measurement, control, and laboratory use:

- EN 61326 (IEC 61326): Class A emissions; Industrial immunity

- EN 55011 (CISPR 11): Group 1, Class A emissions

- ASINZS CISPR 11: Group 1, Class A emissions

- FCC 47 CFR Part 158: Class A emissions

- ICES-001: Class A emissions

Q. Note For the standards applied to assess the EMC of this product, refer to the Onine Product Cert fication section.

Q. Note For EMC compliance, operate this device with double shielded cables.

CE Compliance

This product meets the essential requirements of applicable European Directives, as amended for CE marking, as follows:

- 2006/95iEC; Low-Voltage Directive (safety)

- 2004/108/EC; Electromagnetic Compatibility Directive (EMC)

1. Note For the standards applied to assess the EMC of this product, refer to the Onime Product Certic $x$ ation section

Online Product Certification

Refer to the product Declaration of Conformity (DoC) for additional regulatory compliance information. To obtain product certifications and the DoC for this product, visit ni.comvcertification, search by module number or product line, and click the appropriate link in the Certification column.

\section{Shock and Vibration}

To meet these specifications, you must panel mount the system. If you are using the NI $9201 / 9221$ with screw terminal, you also must either affix ferrules to the ends of the terminal wires or use the $N \mid 1932$ backshell $k$ kit to protect the connections.

Operating vibration

\begin{tabular}{ll} 
Random (IEC 60068-2-64) & $5 \mathrm{~g}_{\mathrm{rms}}, 10$ to $500 \mathrm{~Hz}$ \\
\hline Sinusoidal (IEC 60068-2-6) & $5 \mathrm{~g}, 10$ to $500 \mathrm{~Hz}$ \\
\hline Operating shock (IEC 60068-2-27) & $30 \mathrm{~g}, 11 \mathrm{~ms} \mathrm{half} \mathrm{sine,} 50 \mathrm{~g}, 3$ ms half sine, 18 shocks at 6 orientations
\end{tabular}

\section{Environmental}

National Instruments $\mathrm{C}$ Series modules are intended for indoor use only but may be used outdoors if i installed in a suitable enclosure. Refer to the manual for the chassis you are using for more information about meeting these specifications

Operating temperature (IEC 60068-2-1, IEC 60068-2-2)

-40 to $70^{\circ} \mathrm{O}$

Storage temperature (IEC 60068-2-1, IEC 60068-2-2)

-40 to $85^{\circ} \mathrm{C}$

Ingress protection

IP 40

Operating humidity (IEC 60068-2-56)

10 to $90 \% \mathrm{RH}$, noncondensing

5 to $95 \% \mathrm{RH}$, noncondensing

Maximum attiude $2,000 \mathrm{~m}$

Pollution Degree

\section{Environmental Management}

National Instruments is committed to designing and manufacturing products in an environmentally responsible manner. NI recognizes that eliminating certain hazardous substances from our products is beneficial not only to the enwironment but also to $\mathrm{NI}$ customers.

For additional environmental information, refer to the $N /$ and the Envionment Web page at ni.com/environment. This page contains the environmental regulations and directives with which NII complies, as well as other environmental information not included in this document.

Waste Electrical and Electronic Equipment (WEEE)

EU Customers At the end of their life cycle, all products must be sent to a WEEE recycling center. For more information about WEEE recycling centers and Nationa

EU Customers At the end of their life cycle, all products must be 电子信息产品污染控制管理办法（中国 RoHS

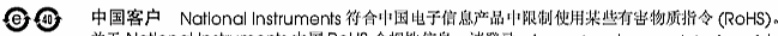

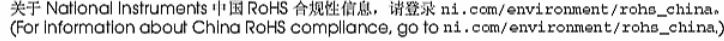


Development of the West Virginia University Small Microgravity Research Facility (WVU SMiRF)
Copyright $^{\odot} 2014$

Kyle G. Phillips 
Pinouts/Front Panel Connections

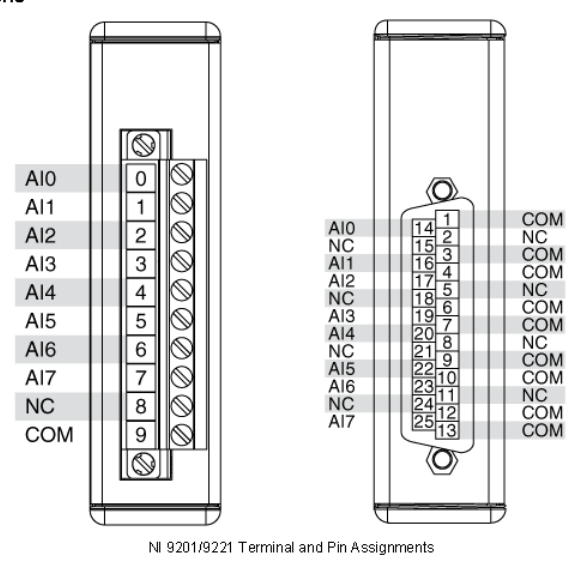

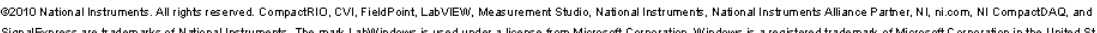

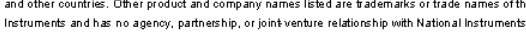

My Profile | RSS | Privacy | Legal | Contact N| $@ 2012$ National Instruments Corporation. All rights reserved. 


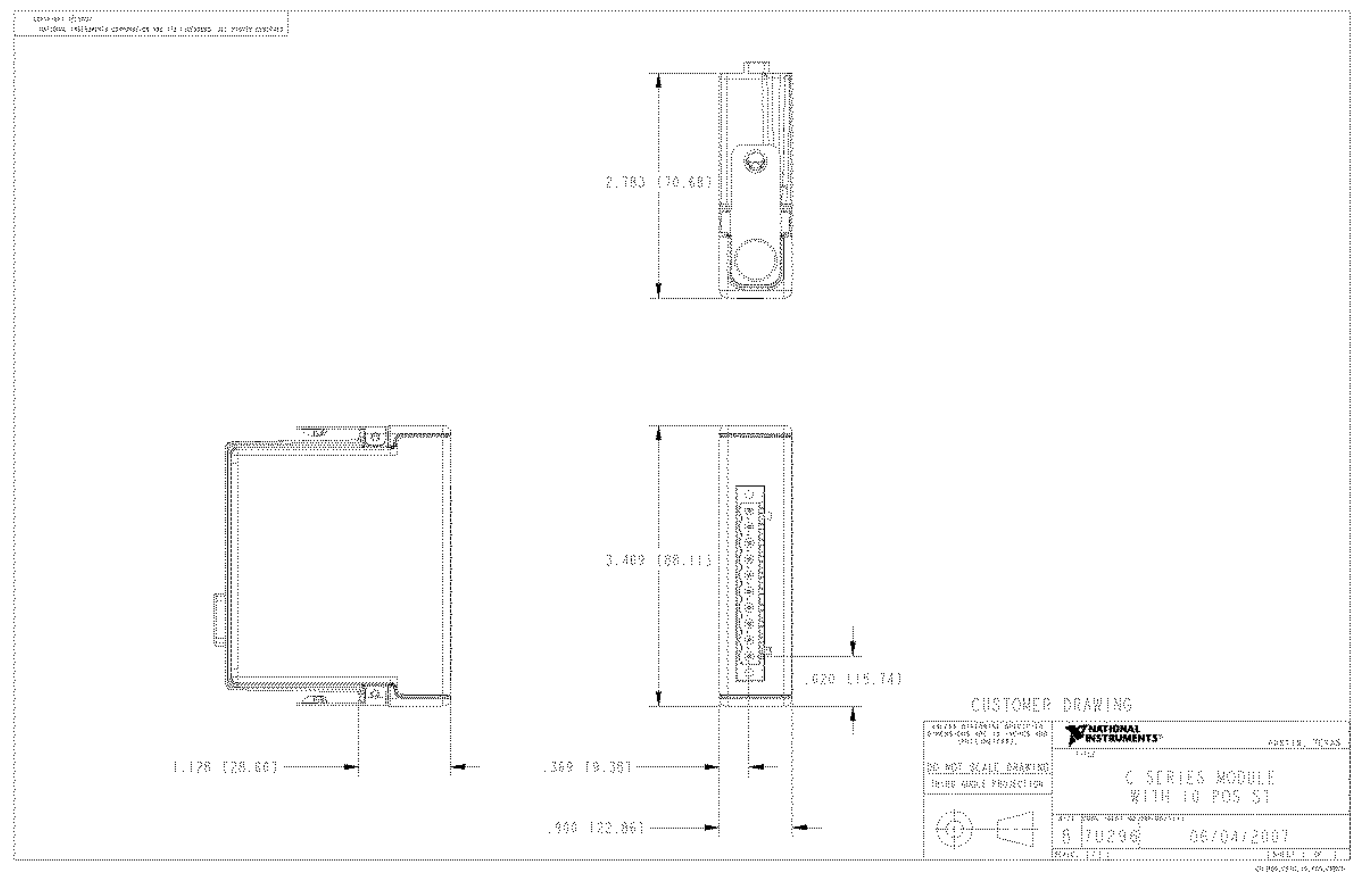




\section{CALIBRATION PROCEDURE NI 9201/9221}

\section{Français Deutsch 日本語 한국어 简体中文 \\ ni.com/manuals}

This document contains the verification and adjustment procedures for the National Instruments 9201 and National Instruments 9221. In this document, the NI 9201 and the NI 9221 are referred to inclusively as the NI 9201/9221. For more information about calibration solutions, visit ni.com/calibration.

\section{Contents}

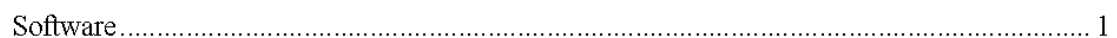

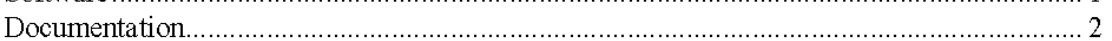

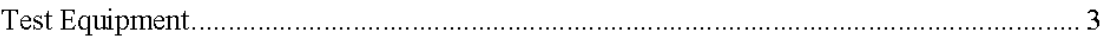

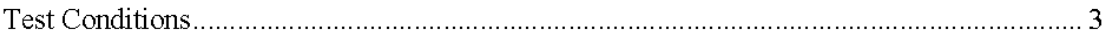

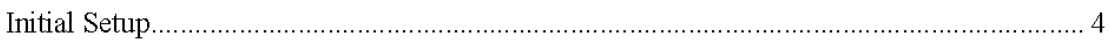

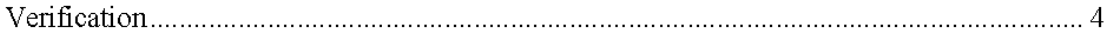

Accuracy Verification.......................................................................................... 4

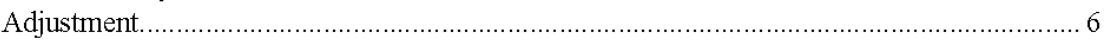

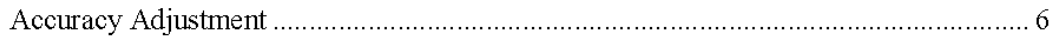

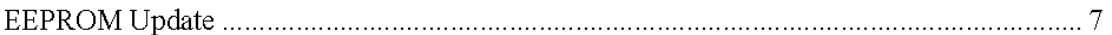

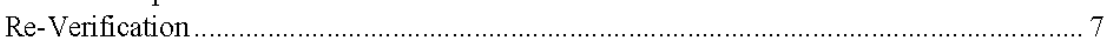

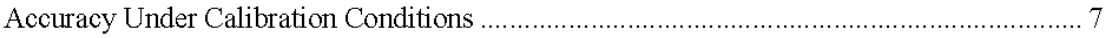

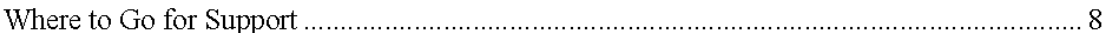

\section{Software}

Calibrating the NI 9201/9221 requires the installation of NI-DAQmx 9.2 or later on the calibration system. You can download NI-DAQmx from ni.com/downloads. NI-DAQmx supports LabVIEW, LabWindows ${ }^{\mathrm{TM}} / \mathrm{CVI}^{\mathrm{TM}}, \mathrm{C} / \mathrm{C}++, \mathrm{CH}$, and Visual Basic .NET. When you install NI-DAQmx, you only need to install support for the application software that you intend to use. 


\section{Documentation}

Consult the following documents for information about the NI 9201/9221, NI-DAQmx, and your application software. All documents are available on $\mathrm{ni}$. com and help files install with the software.

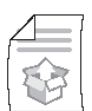

NI cDAQ-9174/9178 Quick Start

NI-DAQmx installation and hardware setup

NI 9201/9221 Operating Instructions and Specifications

NI 9201/9221 specific information, specifications, and calibration interval

NI-DAQmx Readme

Operating system and application software support in NI-DAQmx

LabVIEW Help

LabVIEW programming concepts and reference information about NI-DAQmx VIs and functions

NI-DAQmx C Reference Help

Reference information for NI-DAQmx C functions and NI-DAQmx C properties

NI-DAQmx.NET Help Support for Visual Studio

Reference information for NI-DAQmx .NET methods and NI-DAQmx .NET properties, key

concepts, and a $\mathrm{C}$ enum to .NET enum mapping table

2 | ni.com | NI 9201/9221 Calibration Procedure 


\section{Test Equipment}

Table 1 lists the equipment recommended for the performance verification and adjustment procedures. If the recommended equipment is not available, select a substitute using the requirements listed in Table 1.

Table 1. Recommended Equipment

\begin{tabular}{|l|l|l|}
\hline \multicolumn{1}{|c|}{ Equipment } & $\begin{array}{c}\text { Recommended } \\
\text { Models }\end{array}$ & \multicolumn{1}{c|}{ Requirements } \\
\hline \hline Calibrator & Fluke 5700A & $\begin{array}{l}\text { Use a high-precision voltage source with an } \\
\text { output impedance of less than or equal to } 50 \Omega \\
\text { and an accuracy that meets the following } \\
\text { requirement for your module: } \\
\text { (NI 9201) Use a high-precision voltage source } \\
\text { with an accuracy of less than or equal to 15 ppm. } \\
\text { (NI 9221) Use a high-precision voltage source } \\
\text { with an accuracy of less than or equal to 25 ppm. }\end{array}$ \\
\hline $\begin{array}{l}\text { Chassis } \\
\begin{array}{l}\text { Connection } \\
\text { Accessory }\end{array}\end{array}$ & NI cDAQ-9178 & - \\
\hline $\begin{array}{l}\text { Screw-Terminal } \\
\text { Connection } \\
\text { Accessory }\end{array}$ & NI 9932 & - \\
\hline
\end{tabular}

\section{Test Conditions}

The following setup and environmental conditions are required to ensure the NI 9201/9221 meets published specifications.

- Keep connections to the NI $9201 / 9221$ as short as possible. Long cables and wires act as antennae, picking up extra noise that can affect measurements.

- Verify that all connections to the NI 9201/9221 are secure.

- Use shielded copper wire for all cable connections to the NI 9201/9221. Use twisted-pairs wire to eliminate noise and thermal offsets.

- Maintain an ambient temperature of $23^{\circ} \mathrm{C} \pm 5^{\circ} \mathrm{C}$. The NI $9201 / 9221$ temperature will be greater than the ambient temperature.

- Keep relative humidity below $80 \%$.

- Allow a warm-up time of at least 10 minutes to ensure that the NI 9201/9221 measurement circuitry is at a stable operating temperature. 


\section{Initial Setup}

Complete the following steps to set up the NI 9201/9221.

1. Install NI-DAQmx.

2. Make sure the NI cDAQ-9178 power source is not connected.

3. Install the module in slot 8 of the NI cDAQ-9178 chassis. Leave slots 1 through 7 of the NI cDAQ-9178 chassis empty.

4. Connect the NI cDAQ-9178 chassis to your host computer.

5. Connect the power source to the NI cDAQ-9178 chassis.

6. Launch Measurement \& Automation Explorer (MAX).

7. Right-click the device name and select Self-Test to ensure that the module is working properly.

\section{Verification}

The following performance verification procedure describes the sequence of operation and test points required to check the NI 9201/9221 and assumes that adequate traceable uncertainties are available for the calibration references.

\section{Accuracy Verification}

Complete the following procedure to determine the As-Found status of the NI 9201/9221.

1. Set the calibrator to Standby mode (STBY).

2. Connect the channel you want to verify on the NI 9201/9221 to the calibrator. Refer to Figure 1 for a connection diagram.

Figure 1. Accuracy Connections for the NI $9201 / 9221$
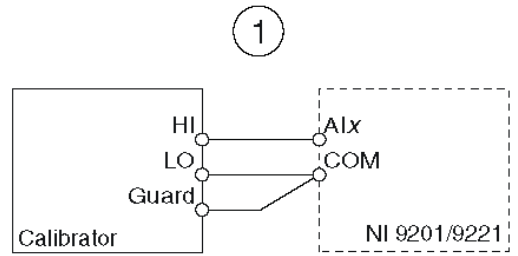

(2)

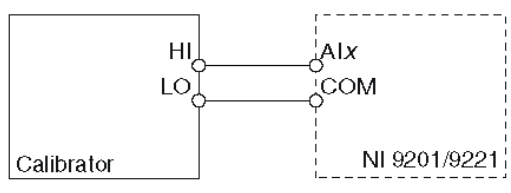

1 Connections when using a calibrator with a guard connection.

2 Connections when using a calibrator with no guard connection.

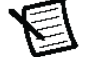

Note If the calibrator outputs are truly floating, connect the negative output to a quiet earth ground as well as COM to give the entire system a ground reference.

3. Set the calibrator to Operate mode (OPR).

4. Set the calibrator voltage to a Test Point value indicated in Table 4 or Table 5.

5. Acquire and average samples.

4 | ni.com | NI 9201/9221 Calibration Procedure 
a. Create and configure an AI voltage channel according to Table 2.

Table 2. NI 9201/9221 Al Voltage Channel Configuration

\begin{tabular}{|c|c|c|c|c|}
\hline \multirow{2}{*}{ Module } & \multicolumn{2}{|c|}{ Input Range } & \multirow{2}{*}{$\begin{array}{c}\text { Scaled } \\
\text { Units }\end{array}$} & Terminal Configuration \\
\cline { 2 - 3 } & Min & Max & Volts & RSE \\
\hline \hline NI 9201 & -10 & 10 & RSE \\
\hline NI 9221 & -60 & 60 & Volts & Ren \\
\hline
\end{tabular}

b. Configure the AI voltage channel timing according to Table 3.

Table 3. NI 9201/9221 Timing Configuration

\begin{tabular}{|c|c|c|}
\hline Samples Per Channel & Acquisition Mode & Rate (S/s) \\
\hline \hline $10 \mathrm{k}$ & Finite & $25 \mathrm{k}$ \\
\hline
\end{tabular}

c. Start the task.

d. Read samples from the NI 9201/9221.

e. Average the samples for each channel.

f. Clear the task.

6. Compare the average sample for each channel to the test limits in Table 4 or Table 5 .

Table 4. NI 9201 Voltage Test Limits

\begin{tabular}{|c|c|c|c|c|c|}
\hline \multicolumn{2}{|c|}{ Range (V) } & \multicolumn{2}{c|}{ Test Point } & \multicolumn{2}{c|}{ 1-Year Limits } \\
\hline Min & Max & Location & Value (V) & Lower Limit (V) & Upper Limit (V) \\
\hline \hline-10.000 & 10.000 & Max & 9.500000 & 9.483250 & 9.516750 \\
\hline-10.000 & 10.000 & Mid & 0.000000 & -0.012000 & 0.012000 \\
\hline-10.000 & 10.000 & Min & -9.500000 & -9.516750 & -9.483250 \\
\hline
\end{tabular}

Table 5. NI 9221 Voltage Test Limits

\begin{tabular}{|c|c|c|c|c|c|}
\hline \multicolumn{2}{|c|}{ Range (V) } & \multicolumn{2}{c|}{ Test Point } & \multicolumn{2}{c|}{ 1-Year Limits } \\
\hline Min & Max & Location & Value (V) & Lower Limit (V) & Upper Limit (V) \\
\hline \hline-60.000 & 60.000 & Max & 57.000000 & 56.900500 & 57.099500 \\
\hline-60.000 & 60.000 & Mid & 0.000000 & -0.071000 & 0.071000 \\
\hline-60.000 & 60.000 & Min & -57.000000 & -57.099500 & -56.900500 \\
\hline
\end{tabular}

Note The test limits listed in Tables 4 and 5 are derived using the values in Table 7. 
7. Repeat steps 4 through 6 for each Test Point in Table 4 or Table 5 .

8. Set the calibrator to Standby mode (STBY).

9. Disconnect the NI $9201 / 9221$ from the calibrator.

10. Repeat steps 2 through 9 for each channel on the NI 9201/9221.

\section{Adjustment}

The following performance adjustment procedure describes the sequence of operation required to adjust the NI $9201 / 9221$.

\section{Accuracy Adjustment}

Complete the following procedure to adjust the accuracy performance of the NI 9201/9221.

1. Set the calibrator to Standby mode (STBY).

2. Connect the channel you want to adjust on the NI 9201/9221 to the calibrator. Refer to Figure 1 for a connection diagram.

3. Adjust the NI 9201/9221.

a. Initialize a calibration session on the NI 9201/9221. The default password is NI.

b. Input the external temperature in degrees Celsius.

c. Call the NI 9201 or NI 9221 get C Series adjustment points function to obtain an array of recommended calibration voltages for the NI 9201/9221.

d. Set the calibrator to a reference value determined by the array of recommended calibration voltages.

e. Set the calibrator to Operate mode (OPR).

f. Call and configure the NI 9201 or NI 9221 adjustment function according to Table 6 .

Table 6. Adjustment Configuration

\begin{tabular}{|c|c|}
\hline Physical Channel & Reference Value \\
\hline \hline cDAQMod8/aix & The reference value from the array of adjustment points \\
\hline
\end{tabular}

g. Set the calibrator to Standby mode (STBY).

h. Repeat steps $d$ through $g$ for each calibration voltage in the array.

i. Close the calibration session.

4. Disconnect the NI 9201/9221 from the calibrator.

5. Repeat steps 2 through 4 for each channel on the NI 9201/9221.

6 | ni.com | NI 9201/9221 Calibration Procedure 


\section{EEPROM Update}

When an adjustment procedure is completed, the NI 9201/9221 internal calibration memory (EEPROM) is immediately updated.

If you do not want to perform an adjustment, you can update the calibration date and onboard calibration temperature without making any adjustments by initializing an external calibration, setting the $\mathrm{C}$ Series calibration temperature, and closing the external calibration.

\section{Re-Verification}

Repeat the Verification section to determine the As-Left status of the device.

Note If any test fails Re-Verification after performing an adjustment, verify that you have met the Test Conditions before returning your device to NI. Refer to Where to Go for Support for assistance in returning the device to NI.

\section{Accuracy Under Calibration Conditions}

The values in the following table are based on calibrated scaling coefficients, which are stored in the onboard EEPROM.

The following accuracy table is valid for calibration under the following conditions:

- Ambient temperature $23^{\circ} \mathrm{C} \pm 5^{\circ} \mathrm{C}$

- $\quad$ NI 9201/9221 installed in slot 8 of an NI cDAQ-9178 chassis

- Slots 1 through 7 of the NI cDAQ-9178 chassis are empty

Table 7. NI 9201/9221 Accuracy Under Calibration Conditions

\begin{tabular}{|c|c|c|}
\hline Module & Gain Error & Offset Error \\
\hline \hline NI 9201 & $0.05 \%$ & $12 \mathrm{mV}$ \\
\hline NI 9221 & $0.05 \%$ & $71 \mathrm{mV}$ \\
\hline
\end{tabular}

Note For operational specifications, refer to the most recent NI 9201/9221

Operating Instructions and Specifications online at $\mathrm{n}$. com/manuals. 


\section{Where to Go for Support}

The National Instruments Web site is your complete resource for technical support. At $\mathrm{ni} . \mathrm{com} /$ support you have access to everything from troubleshooting and application development self-help resources to email and phone assistance from NI Application Engineers.

National Instruments corporate headquarters is located at 11500 North Mopac Expressway, Austin, Texas, 78759-3504. National Instruments also has offices located around the world to help address your support needs. For telephone support in the United States, create your service request at $\mathrm{ni}$.com/support and follow the calling instructions or dial 5127958248 . For telephone support outside the United States, visit the Worldwide Offices section of ni .com/ $\mathrm{niglobal}$ to access the branch office Web sites, which provide up-to-date contact information, support phone numbers, email addresses, and current events.

CVI, LabVIEW, National Instruments, NI, ni.com, the National Instruments comorate logo, and the Eagle logo are trademarks of National Instruments Corporation. Refer to the Trademark Information at ni. com/trademarks for other National Instruments trademarks. The mark LabW Windows is used under a license from Microsoft Corporation. Windows is a registered trademark of Microsoft Co mo ration in the United States and other countries. Otherp roduct and company names mentioned herein are trademarks or trade names of their respective companies. For patents covering National Instruments products/technology, refer to the appropriate location: Help *Patents in your software, the patents. $t x t$ file on your media, or the National Instruments Patents Notice at $\mathrm{ni}$. com/patents. You can find info mation about end-user license agreements (EULAS) and third-party legal notices in the NI-DAOmx Readme. Refer to the Export Compliance Information at $\mathrm{ni}$. com/legal/ export-compliance for the National Instruments global trade compliance policy and how to obtain relevant HTS codes, ECCNs, and other importexport data

C. 2007-2013 National Instruments. All rights reserved. 


\section{OPERATING INSTRUCTIONS AND SPECIFICATIONS} NI 9201/9221

\section{8-Channel, 12-Bit Analog Input Modules}

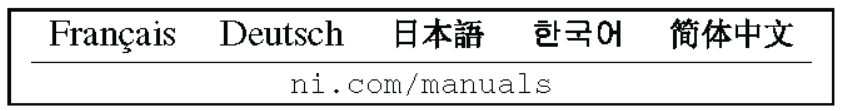
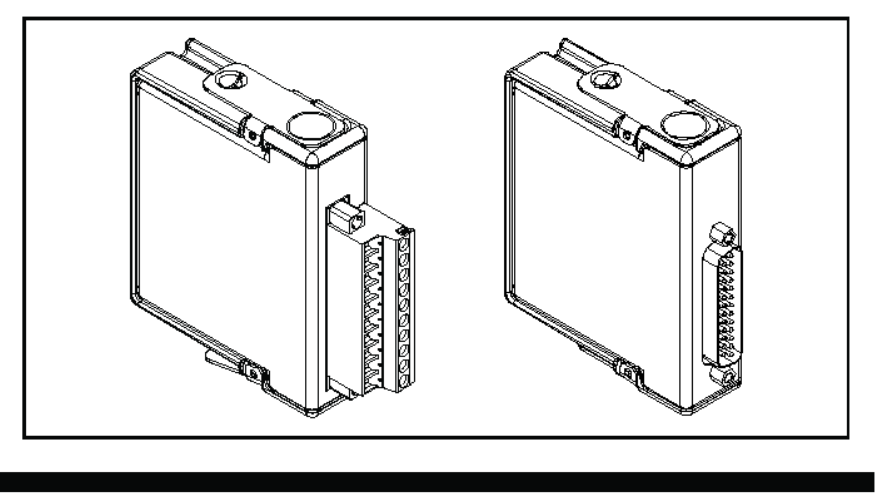

\section{TINSTRUMENTS}

This document describes how to use the National Instruments 9201 and National Instruments 9221 and includes specifications and terminal assignments for the NI 9201/9221. In this document, the NI 9201/9221 with screw terminal and NI 9201/9221 with DSUB are referred to inclusively as the NI 9201/9221. Visit ni.com/info and enter rdsoftwareversion to determine which software you need for the modules you are using. For information about installing, configuring, and programming the system, refer to the system documentation. Visit ni.com/info and enter cseriesdoc for information about C Series documentation.

Note The safety guidelines and specifications in this document are specific to the NI 9201/9221. The other components in the system might not meet the same safety ratings and specifications. Refer to the documentation for each component in the system to determine the safety ratings and specifications for the entire system. Visit ni.com/info and enter cseriesdoc for information about C Series documentation. 


\section{Safety Guidelines}

Operate the NI 9201/9221 only as described in these operating instructions.

Hot Surface This icon denotes that the component may be min hot. Touching this component may result in bodily injury.

\section{Safety Guidelines for Hazardous Voltages}

You can connect hazardous voltages only to the NI 9201/9221 with screw terminal. Do not connect hazardous voltages to the NI 9201/9221 with DSUB.

If hazardous voltages are connected to the module, take the following precautions. A hazardous voltage is a voltage greater than $42.4 \mathrm{~V}_{\mathrm{pk}}$ or $60 \mathrm{VDC}$ to earth ground.
$\triangle$
Caution Ensure that hazardous voltage wiring is performed only by qualified personnel adhering to local electrical standards.
Caution Do not mix hazardous voltage circuits and
human-accessible circuits on the same module.

Caution Make sure that devices and circuits connected to the module are properly insulated from human contact.

A Caution When module terminals are hazardous voltage LIVE (>42.4 $\mathrm{V}_{\mathrm{pk}} / 60 \mathrm{VDC}$ ), you must ensure that devices and circuits connected to the module are properly insulated from human contact. You must use the NI 9932 connector backshell kit to ensure that the terminals are not accessible.

Figure 1 shows the NI 9932 connector backshell.

Note You can use the NI 9932 connector backshell only with the NI $9201 / 9221$ with screw terminal. 


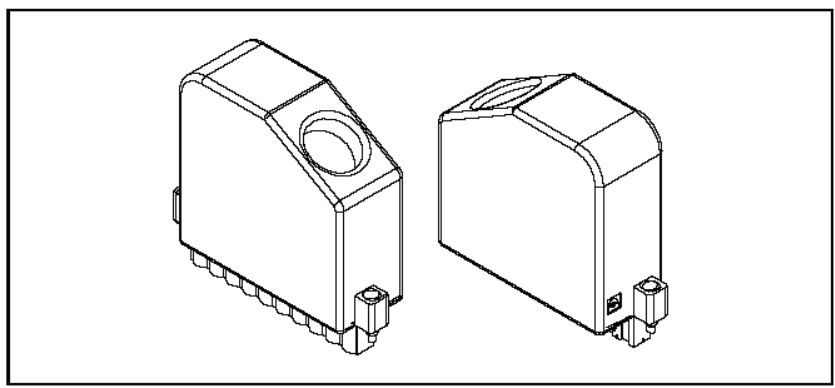

Figure 1. NI 9932 Connector Backshell

\section{Safety Guidelines for Hazardous Locations}

The NI 9201/9221 is suitable for use in Class I, Division 2, Groups A, B, C, D, T4 hazardous locations; Class I, Zone 2, AEx nC IIC T4, and Ex nC IIC T4 hazardous locations; and nonhazardous locations only. Follow these guidelines if you are installing the NI 9201/9221 in a potentially explosive environment. Not following these guidelines may result in serious injury or death.

Caution Do not disconnect I/O-side wires or connectors unless power has been switched off or the area is known to be nonhazardous.

1. Caution Do not remove modules unless power has been switched off or the area is known to be nonhazardous.

A Caution Substitution of components may impair suitability for Class I, Division 2.

A Caution For Zone 2 applications, install the system in an enclosure rated to at least IP 54 as defined by IEC 60529 and EN 60529

Caution For Zone 2 applications, install a protection
device between the input signal and the NI $9201 / 9221$
input terminal. The device must prevent the
channel-to-COM voltage from exceeding $85 \mathrm{~V}$ if there is
a transient overvoltage condition.

Special Conditions for Hazardous Locations Use in Europe This equipment has been evaluated as EEx nC IIC T4 equipment under DEMKO Certificate No. 03 ATEX 0324020X. Each module is marked \&x II $3 \mathrm{G}$ and is suitable for use in Zone 2 hazardous 
locations, in ambient temperatures of $-40^{\circ} \mathrm{C} \leq \mathrm{Ta} \leq 70^{\circ} \mathrm{C}$. If you are using the NI 9201/9221 in Gas Group IIC hazardous locations, you must use the device in an NI chassis that has been evaluated as Ex nC IIC T4, EEx nC IIC T4, Ex nA IIC T4, or Ex nL IIC T4 equipment.

\section{Special Conditions for Marine Applications}

Some modules are Lloyd's Register (LR) Type Approved for marine applications. To verify Lloyd's Register certification, visit ni.com/certification and search for the LR certificate, or look for the Lloyd's Register mark on the module.

Caution To meet radio frequency emission requirements for marine applications, use shielded cables and install the system in a metal enclosure. Suppression ferrites must be installed on power supply inputs near power entries to modules and controllers. Power supply and module cables must be separated on opposite sides of the enclosure and must enter and exit through opposing enclosure walls.

\section{Connecting the NI 9201/9221}

The NI 9201/9221 provides connections for eight analog input channels.

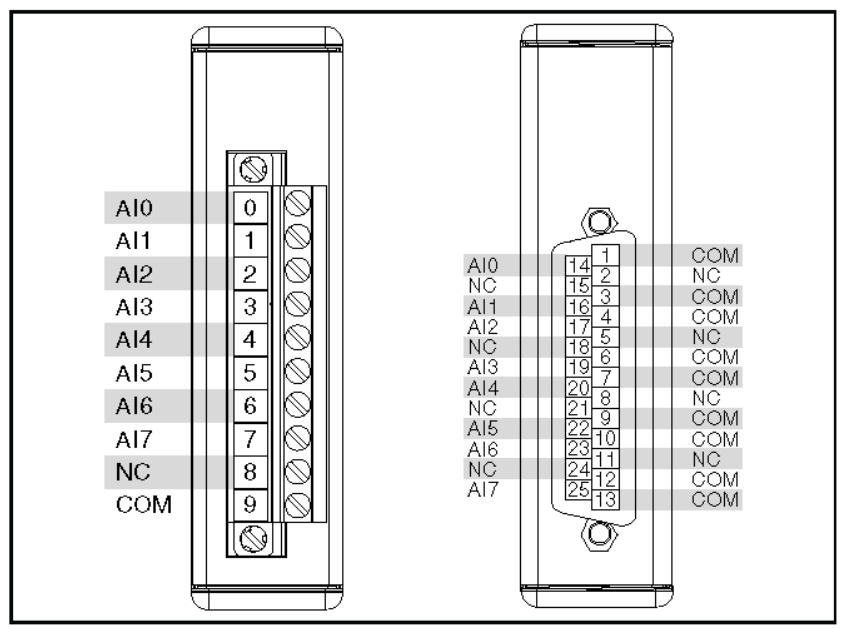

Figure 2. NI 9201/9221 Terminal and Pin Assignments 
The NI 9201/9221 with screw terminal has a 10-terminal, detachable screw-terminal connector. The NI 9201/9221 with DSUB has a 25-pin DSUB connector. Each channel has an AI terminal or pin to which you can connect a voltage signal. COM, the common terminal or pin, is internally connected to the isolated ground reference of the module.

The NI 9201/9221 channels are isolated from other modules in the system. The module protects each channel from overvoltages. Refer to the Specifications section for more information about overvoltage protection. The input signals are scanned, buffered, conditioned, and then sampled by a single 12-bit ADC. Refer to Figure 3 for an illustration of the input circuitry on the NI $9201 / 9221$.

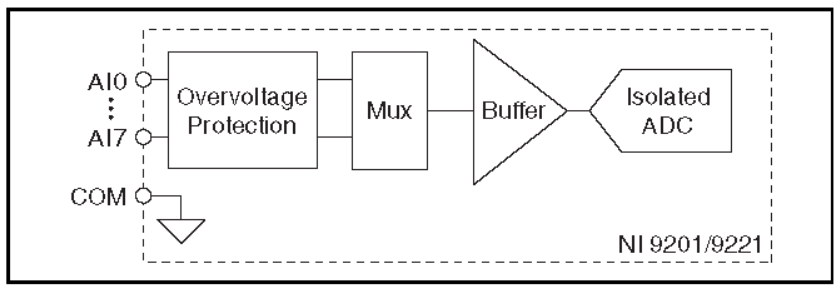

Figure 3. Input Circuitry on the NI 9201/9221

You can connect single-ended voltage signals to the NI 9201/9221. Connect the positive lead of the voltage signal to AI. Connect the ground signal to COM. Refer to Figure 3 for an illustration of connecting a single-ended voltage signal to the NI 9201/9221.

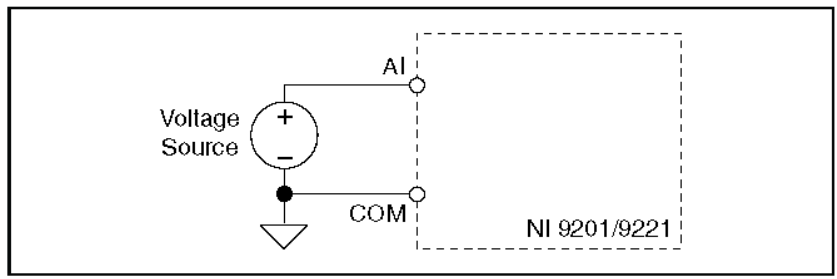

Figure 4. Connecting a Single-Ended Voltage Signal to the NI 9201/9221

8 Note You must use 2-wire ferrules to create a secure single terminal on the NI $9201 / 9221$ with screw terminal. 


\section{Wiring for High-Vibration Applications}

If an application using the NI 9201/9221 with screw terminal is subject to high vibration, National Instruments recommends that you either use ferrules to terminate wires to the detachable screw-terminal connector or use the NI 9932 backshell kit to protect the connections. Refer to Figure 5 for an illustration of using ferrules. Refer to Figure 1 for an illustration of the NI 9932 connector backshell.

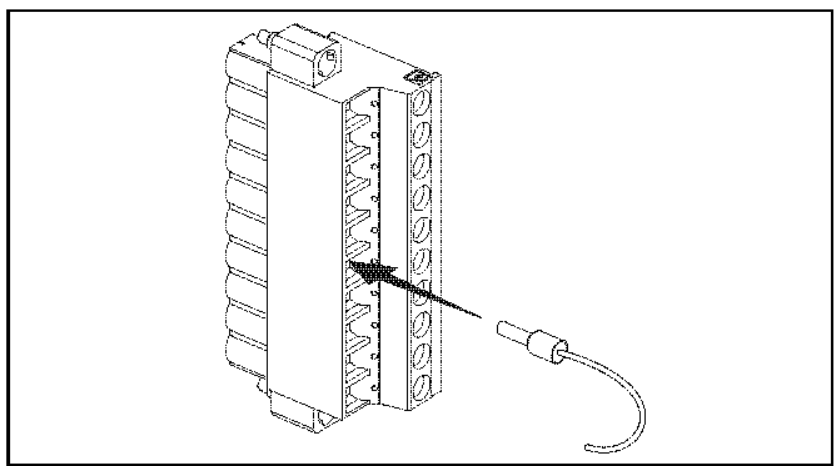

Figure 5. 10-Terminal Detachable Screw-Terminal Connector with Ferrule

\section{Sleep Mode}

This module supports a low-power sleep mode. Support for sleep mode at the system level depends on the chassis that the module is plugged into. Refer to the chassis manual for information about support for sleep mode. If the chassis supports sleep mode, refer to the software help for information about enabling sleep mode. Visit ni.com/info and enter cseriesdoc for information about C Series documentation.

Typically, when a system is in sleep mode, you cannot communicate with the modules. In sleep mode, the system consumes minimal power and may dissipate less heat than it does in normal mode. Refer to the Specifications section for more information about power consumption and thermal dissipation. 


\section{Specifications}

The following specifications are typical for the range -40 to $70^{\circ} \mathrm{C}$ unless otherwise noted. All voltages are relative to COM unless otherwise noted. The specifications are the same for the NI 9201 and the NI 9221 unless otherwise noted.

\section{Input Characteristics}

Number of channels........................ 8 analog input channels

$\mathrm{ADC}$ resolution 12 bits

Type of ADC Successive approximation register (SAR)

Sample rate (aggregate)

\begin{tabular}{|l|c|c|}
\hline \multicolumn{1}{|c|}{ Module } & $\begin{array}{c}\text { Maximum Sample } \\
\text { Rate (R Series } \\
\text { Expansion Chassis) }\end{array}$ & $\begin{array}{c}\text { Maximum } \\
\text { Sample Rate } \\
\text { (All Other Chassis) }\end{array}$ \\
\hline \hline $\begin{array}{l}\text { NI 9201, } \\
\text { single channel }\end{array}$ & $475 \mathrm{kS} / \mathrm{s}$ & $800 \mathrm{kS} / \mathrm{s}$ \\
\hline NI 9201, scanning & $475 \mathrm{kS} / \mathrm{s}$ & $500 \mathrm{kS} / \mathrm{s}$ \\
\hline NI 9221 & $475 \mathrm{kS} / \mathrm{s}$ & $800 \mathrm{kS} / \mathrm{s}$ \\
\hline
\end{tabular}

Input range

NI $9201 \ldots \ldots \ldots \ldots \ldots \ldots \ldots \ldots \ldots \ldots \pm 10 \mathrm{~V}$
NI $9221 \ldots \ldots \ldots \ldots \ldots \ldots \ldots \ldots \ldots \ldots \ldots \ldots \ldots \ldots \ldots \ldots \ldots$

Operating voltage ranges ${ }^{1}$

\begin{tabular}{|c|c|c|c|c|c|}
\hline \multirow{2}{*}{ Module } & \multicolumn{3}{|c|}{$\begin{array}{c}\text { Measurement Voltage, } \\
\text { Channel-to-COM }\end{array}$} & \multicolumn{2}{c|}{$\begin{array}{c}\text { Maximum Voltage, } \\
\text { Channel-to-Earth Ground or } \\
\text { COM-to-Earth Ground }\end{array}$} \\
\cline { 2 - 5 } & $\begin{array}{c}\text { Min } \\
\text { (V) }\end{array}$ & $\begin{array}{c}\text { Typ } \\
\text { (V) }\end{array}$ & $\begin{array}{c}\text { Max } \\
\text { (V) }\end{array}$ & $\begin{array}{c}\text { Screw } \\
\text { Terminal }\end{array}$ & DSUB \\
\hline \hline NI 9201 & \pm 10.3 & \pm 10.53 & \pm 10.8 & $250 \mathrm{~V}_{\text {rms }}$ & \pm 60 VDC \\
\hline NI 9221 & \pm 61.4 & \pm 62.50 & \pm 63.8 & & \\
\hline
\end{tabular}

Overvoltage protection

(channel-to-COM) $\pm 100 \mathrm{~V}$

\footnotetext{
${ }^{1}$ Refer to the Safety Guidelines section for more information about safe operating voltages. 
NI 9201 accuracy (excludes noise)

\begin{tabular}{|l|c|c|}
\hline \multicolumn{1}{|c|}{ Measurement Conditions } & $\begin{array}{c}\text { Percent of } \\
\text { Reading } \\
\text { (Gain Error) }\end{array}$ & $\begin{array}{c}\text { Percent of } \\
\text { Range* } \\
\text { (Offset Error) }\end{array}$ \\
\hline \hline Calibrated typ $\left(25^{\circ} \mathrm{C}, \pm 5^{\circ} \mathrm{C}\right)$ & $\pm 0.04 \%$ & $\pm 0.07 \%$ \\
\hline Calibrated max $\left(-40\right.$ to $\left.70^{\circ} \mathrm{C}\right)$ & $\pm 0.25 \%$ & $\pm 0.25 \%$ \\
\hline Uncalibrated typ $\left(25^{\circ} \mathrm{C}, \pm 5^{\circ} \mathrm{C}\right)$ & $\pm 0.26 \%$ & $\pm 0.46 \%$ \\
\hline Uncalibrated max $\left(-40\right.$ to $\left.70^{\circ} \mathrm{C}\right)$ & $\pm 0.67 \%$ & $\pm 1.25 \%$ \\
\hline${ }^{*}$ Range equals $10.53 \mathrm{~V}$ & \\
\hline
\end{tabular}

NI 9221 accuracy (excludes noise)

\begin{tabular}{|l|c|c|}
\hline \multicolumn{1}{|c|}{ Measurement Conditions } & $\begin{array}{c}\text { Percent of } \\
\text { Reading } \\
\text { (Gain Error) }\end{array}$ & $\begin{array}{c}\text { Percent of } \\
\text { Range* } \\
\text { (Offset Error) }\end{array}$ \\
\hline \hline Calibrated typ $\left(25^{\circ} \mathrm{C}, \pm 5^{\circ} \mathrm{C}\right)$ & $\pm 0.04 \%$ & $\pm 0.07 \%$ \\
\hline Calibrated $\max \left(-40\right.$ to $\left.70^{\circ} \mathrm{C}\right)$ & $\pm 0.25 \%$ & $\pm 0.25 \%$ \\
\hline Uncalibrated typ $\left(25^{\circ} \mathrm{C}, \pm 5^{\circ} \mathrm{C}\right)$ & $\pm 0.26 \%$ & $\pm 0.43 \%$ \\
\hline Uncalibrated $\max \left(-40\right.$ to $\left.70^{\circ} \mathrm{C}\right)$ & $\pm 0.67 \%$ & $\pm 1.06 \%$ \\
\hline${ }^{*}$ Range equals $62.50 \mathrm{~V}$ & \\
\hline
\end{tabular}

Stability

Gain drift .............................. $\pm 34 \mathrm{ppm} /{ }^{\circ} \mathrm{C}$

Offset drift

NI $9201 \ldots \ldots \ldots \ldots \ldots \ldots \ldots \ldots \ldots \ldots \ldots \ldots \ldots \ldots \ldots \ldots \ldots \ldots \ldots \ldots$
NI $9221 \ldots \ldots \ldots \ldots \ldots \ldots \ldots \ldots \ldots$

Input bandwidth $(-3 \mathrm{~dB})$

NI 9201 $690 \mathrm{kHz}$ min

NI 9221 $.950 \mathrm{kHz}$ min 


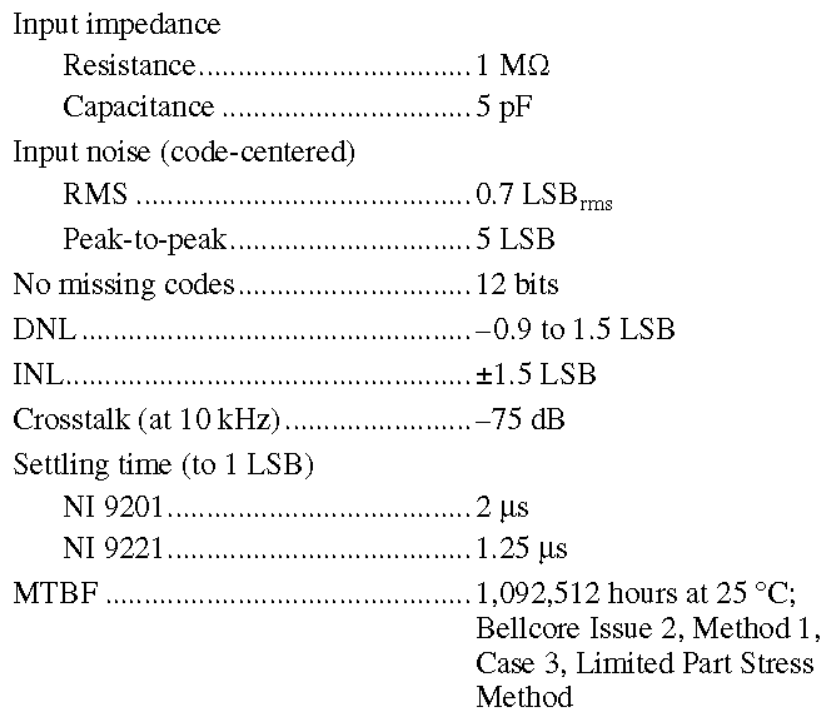

Note Contact NI for Bellcore MTBF specifications at other temperatures or for MIL-HDBK-217F specifications.

\section{Power Requirements}

Power consumption from chassis

Active mode $1 \mathrm{~W} \max$

Sleep mode $1 \mathrm{~mW} \max$

Thermal dissipation (at $70^{\circ} \mathrm{C}$ )

Active mode $1 \mathrm{~W} \max$

Sleep mode $32 \mathrm{~mW} \max$

\section{Physical Characteristics}

If you need to clean the module, wipe it with a dry towel.

3 Note For two-dimensional drawings and three-dimensional models of the C Series module and connectors, visit ni.com/dimensions and search by module number. 


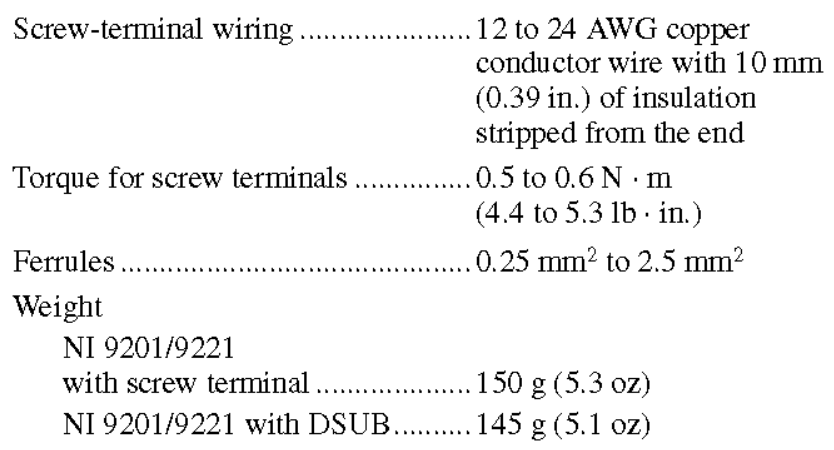

\section{Safety}

\section{Safety Voltages}

Connect only voltages that are within the following limits.

Channel-to-COM \pm 60 VDC $\max$

\section{NI 9201/9221 with Screw Terminal Isolation Voltages}

Channel-to-channel. None

Channel-to-earth ground

$$
\begin{aligned}
& \text { Continuous ........................... 250 } \mathrm{V}_{\mathrm{rms}} \text {, } \\
& \text { Measurement Category II } \\
& \text { Withstand.............................. 2,300 } \mathrm{V}_{\mathrm{rms}} \text {, verified by a } 5 \mathrm{~s} \\
& \text { dielectric withstand test }
\end{aligned}
$$

Measurement Category II is for measurements performed on circuits directly connected to the electrical distribution system. This category refers to local-level electrical distribution, such as that provided by a standard wall outlet, for example, $115 \mathrm{~V}$ for U.S or $230 \mathrm{~V}$ for Europe.

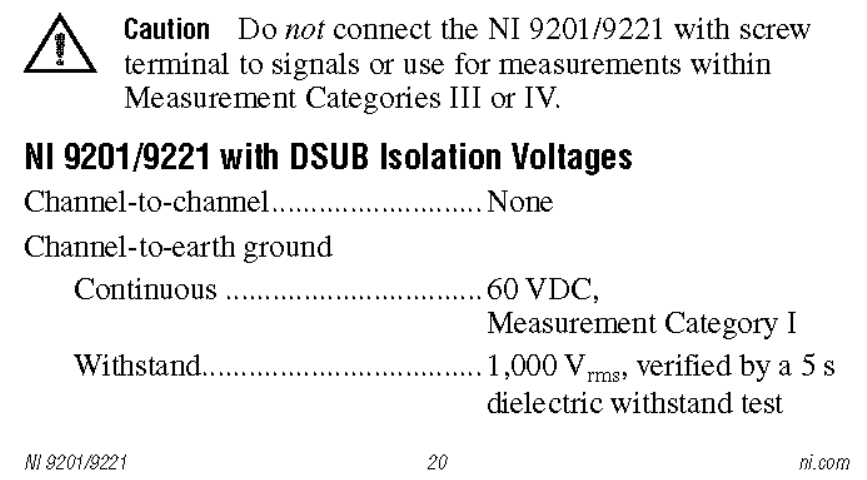


Measurement Category I is for measurements performed on circuits not directly connected to the electrical distribution system referred to as MAINS voltage. MAINS is a hazardous live electrical supply system that powers equipment. This category is for measurements of voltages from specially protected secondary circuits. Such voltage measurements include signal levels, special equipment, limited-energy parts of equipment, circuits powered by regulated low-voltage sources, and electronics.

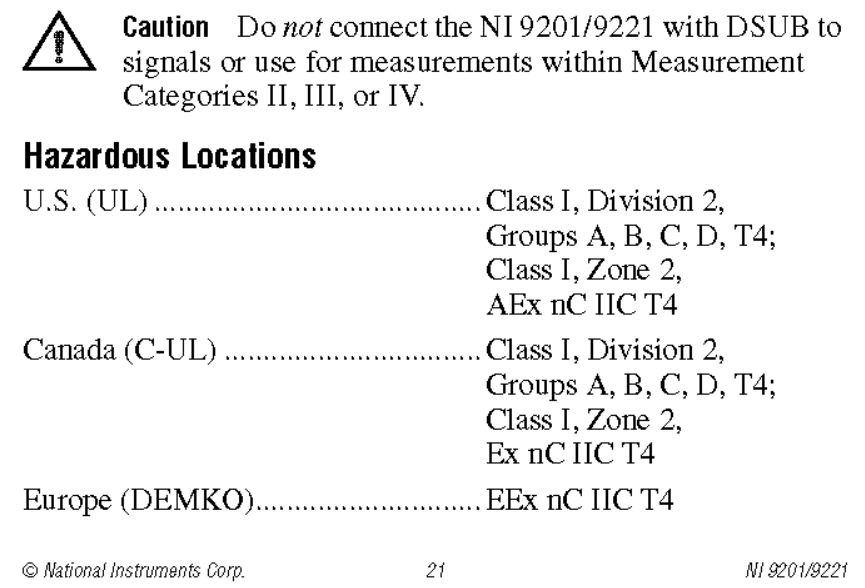

\section{Safety Standards}

This product meets the requirements of the following standards of safety for electrical equipment for measurement, control, and laboratory use:

- IEC 61010-1, EN 61010-1

- UL 61010-1, CSA 61010-1

7 Note For UL and other safety certifications, refer to the product label or the Online Product Certification section.

\section{Electromagnetic Compatibility}

This product meets the requirements of the following EMC standards for electrical equipment for measurement, control, and laboratory use:

- EN 61326 (IEC 61326): Class A emissions; Industrial immunity

- EN 55011 (CISPR 11): Group 1, Class A emissions

- AS/NZS CISPR 11: Group 1, Class A emissions

- FCC 47 CFR Part 15B: Class A emissions

- ICES-001: Class A emissions 
Note For the standards applied to assess the EMC of this product, refer to the Online Product Certification section.

Note For EMC compliance, operate this device with double-shielded cables.

\section{CE Compliance C $C$}

This product meets the essential requirements of applicable European Directives as follows:

- 2006/95/EC; Low-Voltage Directive (safety)

- 2004/108/EC; Electromagnetic Compatibility Directive (EMC)

\section{Online Product Certification}

Refer to the product Declaration of Conformity (DoC) for additional regulatory compliance information. To obtain product certifications and the DoC for this product, visit ni.com/ certification, search by module number or product line, and click the appropriate link in the Certification column.

\section{Shock and Vibration}

To meet these specifications, you must panel mount the system. If you are using the NI 9201/9221 with screw terminal, you also must either affix ferrules to the ends of the terminal wires or use the NI 9932 backshell kit to protect the connections.

Operating vibration

Random (IEC 60068-2-64)........ $5 \mathrm{~g}_{\mathrm{rms}}, 10$ to $500 \mathrm{~Hz}$

Sinusoidal (IEC 60068-2-6) ....... 5 g, 10 to $500 \mathrm{~Hz}$

Operating shock

(IEC 60068-2-27) ..........................30 g g, $11 \mathrm{~ms}$ half sine, $50 \mathrm{~g}, 3 \mathrm{~ms}$ half sine, 18 shocks at 6 orientations

\section{Environmental}

National Instruments C Series modules are intended for indoor use only but may be used outdoors if installed in a suitable enclosure. Refer to the manual for the chassis you are using for more information about meeting these specifications.

Operating temperature (IEC 60068-2-1, IEC 60068-2-2) .....-40 to $70^{\circ} \mathrm{C}$ 


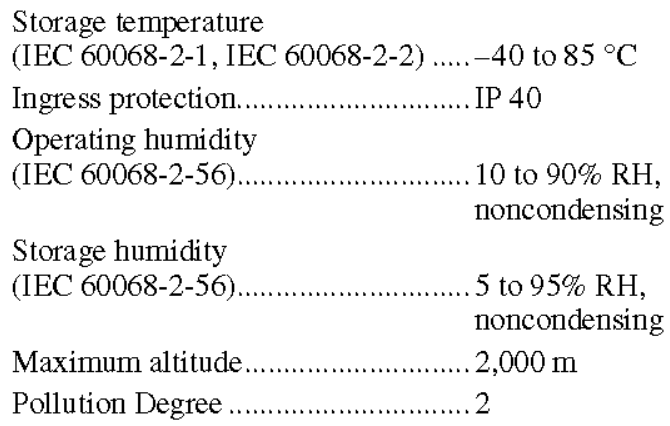

\section{Environmental Management}

National Instruments is committed to designing and manufacturing products in an environmentally responsible manner. NI recognizes that eliminating certain hazardous substances from our products is beneficial to the environment and to NI customers.

For additional environmental information, refer to the NI and the Environment Web page at ni.com/environment. This page contains the environmental regulations and directives with which NI complies, as well as other environmental information not included in this document.

\section{Waste Electrical and Electronic Equipment (WEEE)}

EU Customers At the end of the life cycle, all products must be sent to a WEEE recycling center. For more information about WEEE recycling centers and National Instruments WEEE initiatives, visit ni.com/ environment/weee.

\section{电子信息产品污染控制管理办法（中国 RoHS）}

(e) 中国客户 National Instruments 符合中国电子信息 产品中限制使用某些有害物质指令 $(R O H S)$ 。关于 National Instruments 中国 RoHS 合规性信息, 请登录 ni.com/environment/rohs_china。 (For information about China RoHS compliance, go to ni. com/ environment/rohs_china.) 


\section{Calibration}

You can obtain the calibration certificate and information about calibration services for the NI 9201/9221 at ni. com/

calibration.

Calibration interval ........................ 1 year

\section{Where to Go for Support}

The National Instruments Web site is your complete resource for technical support. At ni. com/support you have access to everything from troubleshooting and application development self-help resources to email and phone assistance from NI Application Engineers.

National Instruments corporate headquarters is located at 11500 North Mopac Expressway, Austin, Texas, 78759-3504. National Instruments also has offices located around the world to help address your support needs. For telephone support in the United States, create your service request at ni.com/support and follow the calling instructions or dial 5127958248 . For telephone support outside the United States, contact your local branch office:

Australia 1800300800 , Austria $43662457990-0$, Belgium 32 (0) 2757 0020, Brazil 551132623599 , Canada 800433 3488, China 862150509800 , Czech Republic 420224235 774, Denmark 4545762600 , Finland 358 (0) 972572511 , France 0157662424 , Germany 49897413130 , India 918041190000 , Israel 9723 6393737, Italy 390241309277 , Japan 0120-527196, Korea 820234513400 , Lebanon 961 (0) 1332828 , Malaysia 1800887710 , Mexico 018000100793 , Netherlands 31 (0) 348433466 , New Zealand 0800553 322, Norway 47 (0) 66907660 , Poland 48223289010 , Portugal 351210311 210, Russia 74957836851 , Singapore 18002265886 , Slovenia 38634254200 , South Africa 27011805 8197, Spain 34916400085 , Sweden 46 (0) 858789500 , Switzerland 41562005151 , Taiwan 886022377 2222, Thailand 6622786777 , Turkey 90212279 3031, United Kingdom 44 (0) 1635523545

\footnotetext{
National Instruments, NI, ni.com, and LabVIEW are trademarks of National Instruments Corporation. Refer to the Terms of Use section on $\mathrm{ni}$. com/legal for more inf ormation about National Instruments trademarks. Other product and company names mentioned herein are trademarks or trade names of their respective companies For patents covering National Instruments products/technology, reter to the appropriate location: Help $\rightsquigarrow$ Patents in your software, the patents. txt file on your media, or the National instruments Patent Notice at $\mathrm{ni}$. com/patents.

(c) 2004-2009 National Instruments Corp. All rights reserved 


$\begin{array}{lr}\text { DNATIONAL } & \text { Technical Sales } \\ \text { United States } \\ \text { (866) 531-6285 } \\ \text { info@ni.com }\end{array}$

Ordering Information | Detailed Specifications | PinoutsíFront Panel Connections

Forcuser manuals and tont

Last Revised: 2010-11-03 14:35:12.0

4-Channel, $\pm 5 \mathrm{~V}, 51.2 \mathrm{kS} / \mathrm{s}$ per Channel, 24-Bit IEPE

NI 9234

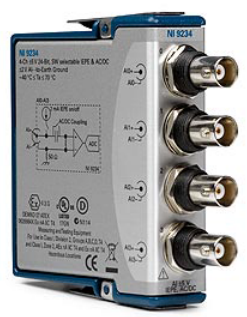

- 24-bit resolution

- $102 \mathrm{~d}$ 日 dynamic range

Antialiasing filters

- 4 simultaneous analog inputs

- TEDS read'wite

$\cdot \pm 5 \mathrm{~V}$ input range

Overview

The National Instruments 9233 and 9234 are four-channel dynamic signal acquisition modules for making high-accuracy measurements from IEPE sensors. The NI 9233 and $9234 \mathrm{C}$ Series analog input modules delver $102 \mathrm{~dB}$ of dynamic range and incorporate IEPE (2 mA constant current) signal conditioning for accelerometers and microphones. The four input channels simultaneously acquire at rates from 2 to $50 \mathrm{kHz}$ or, with the $\mathrm{Nl} 9234$, up to $51.2 \mathrm{kS} / \mathrm{s}$. In addition, the modules include built-in antialiasing filters that automatically adjust to your sampling rate. Compatible with a single-module USB carrier and NI CompactDAQ and CompactRIO hardware, the NI 9233 and 9234 are ideal for a wide variety of mobilepportable applications such as industrial machine condition monitoring and in-vehicle noise, vibration, and harshness testing

Comparison Tables

\begin{tabular}{|c|c|c|c|}
\hline Model & Max S ampling Rate & IEPE & Coupling \\
\hline NI 9233 & $50 \mathrm{kS} / \mathrm{s}$ & Always enabled ( $2 \mathrm{~mA}$ ) & AC coupling \\
\hline N 9234 & $51,2 \mathrm{kSIs}$ & Software selectable ( 0 or $2 \mathrm{~mA})$ & Software selectable $A C I D C$ coupling \\
\hline
\end{tabular}

Application and Technology

Hardware

Each simultaneous signal is buffered, analog prefittered, and sampled by a 24-bit deltasigma analog-to-digital converter (ADC) that performs digital filtering with a cutoff frequency that automatically adjusts to your data rate. The NN 9233 and 9234 feature a voltage range of $\pm 5 \mathrm{~V}$ and a dynamic range of more than $100 \mathrm{~dB}$. In addition, the modules include the chassis ground) for IEPE sensor connecto 作

The $\mathrm{N} N 233$ and 9234 use a method of $\mathrm{AID}$ conversion known as delta-sigma modulation. If, for example, the data rate is $25 \mathrm{kS} / \mathrm{s}$, then each ADC actually samples its input signal

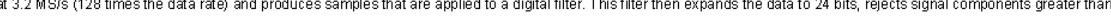
$12.5 \mathrm{kHz}$ (the Nyquist frequency), and digtally resamples the data at the chosen data rate of $25 \mathrm{kS} / \mathrm{s}$. This combination of analog and digital filtering prowides an accurate

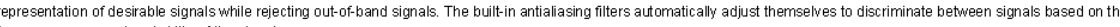

USB Platform 


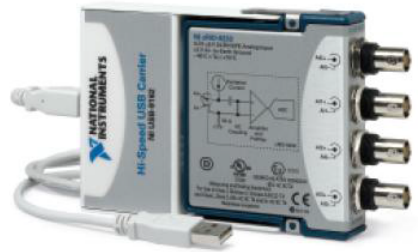

\section{NI CompactDAQ Plafform}

NI CompactDAQ delivers the simplicty of USB to sensor and electrical measurements on the benchtop, in the field, and on the production line. By combining the ease of use and low cost of a data logger with the performance and flexibility of modular instrumentation, NI CompactDAQ offers fast, accurate measurements in a small, simple, and affordable design can measure up to 256 channels of electrical, physical, mechanical, or acoustical signals in a single System. In addition, per-channel ADCs and indwidually isolated modules ensure fast, accurate, and safe measurements.

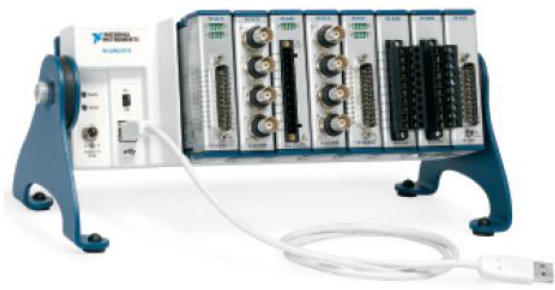

NI CompactRIO PIatform

When used with the small, rugged CompactRIO embedded control and data acquistion system, NI C Series analog input modules connect directly to reconfigurable VOO (RIO) field-programmable gate array (FPGA) hardware to create high-performance embedded systems. The reconfigurable FPGA hardware within CompactRlo provides a variety of options for custom timing, triggering, synchronization, fittering, signal processing, and high-speed decision making for all C Series analog input modules. For instance, with
CompactRIO, you can implement custom triggering for any analog sensor type on a per-channel basis using the flexibility and performance of the FPGA and the numerous arithmetic and comparison function blocks buit into NI LabVIEW FPGA.

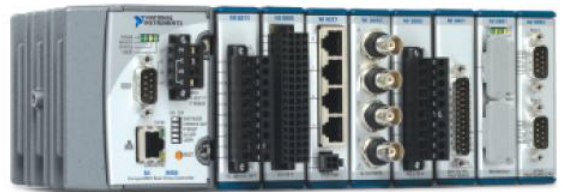

\section{Analysis Software}

The NI 9233 and 9234 are well-sulted for noise and vibration analysis applications. The NI Sound and Vibration Measurement Suite, which specfifically addresses these applications, has two components: the NI Sound and Vibration Assistant and LabVIEW analysis VIs (functions) for power spectra, frequency response (FRF), fractional octave analysis, sound-level measurements, order spectra, order maps, order extraction, sensor calibration, human vibration filters, and torsional vibration

\section{NI Sound and Vibration Assistant}

The Sound and Vibration Assistant is interactive software designed to simplify the process of acquiring and analyzing noise and vibration signals by offering:

- A drag-and-drop, interactive analysis and acquisition environmen

- Rapid measurement configuration

- Extended functionality through LabVIEW

Interactive Analysis Ervironment

The Sound and Vibration Assistant introduces an innovative approach to configuring your measurements using intuitive drag-and-drop steps. Combining the functionality of traditional noise and yibration analysis software with the flexibility to customize and automate routines, the Sound and Vibration Assistant can help you streamine your application.

Rapid Measurement Configuration

There are mary buit-in steps available for immediate use in the Sound and Vibration Assistant. You can instantly configure a measurement and analysis application with:

- Hardware $1 / 0$ - generation and acquistion of signals from a variety of devices, including data acquistition devices and modular instruments

- Signal processing - filtering, windowing, and averaging 


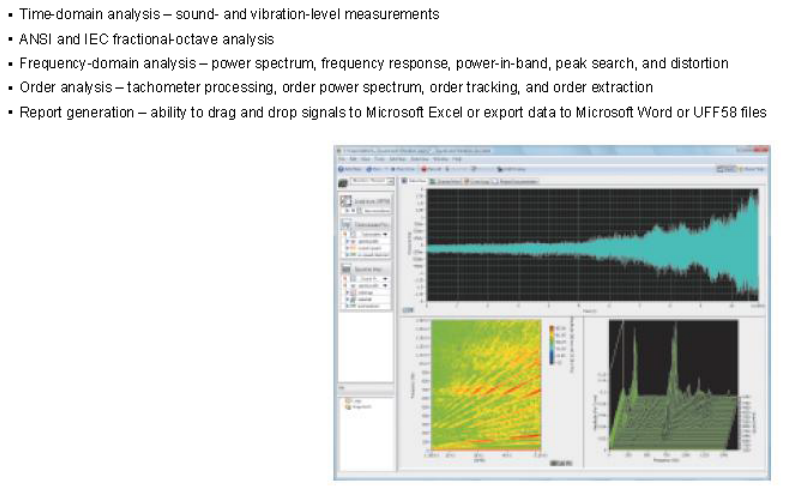

Figure 1. N/ Sound and Vibration Assistant Performing Engine Run-up Test

Extended Functionality through LabVIEW

Reuse your measurement applications developed with the Sound and Vibration Assistant in LabVIEW by corverting projects into LabVIEW block diagrams. With the LabVIEW full-featured graphical programming environment, you can further automate your application or customize your analysis.

Sound and Vibration A nalysis Vls for LabVIEW

With the sound and vibration analysis VIS in LabVIEW, you can develop a variety of custom audio, acoustic, and vibration applications. Functionality includes:

- Full, 1/3, 1/6, 1/12, and $1 / 24$ octave analysis with linear $A$, B, or $C$ weighting

- Baseband, zoom, and subset power spectrum

- Peak search and Power in band

- Frequency response (FRF)

- Fittering

- Swept sine

- Distortion analysis (THD, THD+N, IMD)

- Noise measurements (SNR)

- Human vibration weighting fitters

- Torsional vibration

- Tachometer signal processing

- Order tracking, spectrum, and Order extraction

- Waterfall display for power, octave, and order spectra

- Shaft centerline, orbit, Bode, and order spectra

- Shaft certerline, orbit, Bode, and polar plot format

- File input and output to UFF58

Recommended Hardware

The Sound and Vibration Measurement Suite includes more than 50 examples that work with both dynamic signal acquisition (DSA) and multifunction data acquistion devices Wevices. With 24 bit ADCs and diglatto-analog cornerters (DACS) and integrated alialiasing fitters, DSA devices are ideal for acoustic, noise, and vibration measurements.

There are numerous system requirements to consider when selecting data acquisition hardware for measuring or generating sound and vibration signals. From IEPE signal condtioning for accelerometers and microphones to high dynamic range (up to $118 \mathrm{~dB}$ ) and multichannel synchronization (up to 13,000 channels), National Instruments offers a wide range of hardware products for your applications.

\begin{tabular}{|c|c|c|c|c|c|c|c|c|c|c|}
\hline Product & Bus & $\begin{array}{l}\text { Input Resolution } \\
\text { (bits) }\end{array}$ & $\begin{array}{c}\text { Dynamic } \\
\text { Range (dB) }\end{array}$ & $\begin{array}{l}\text { Sampling Rate } \\
\text { per Channel }\end{array}$ & Analog Inputs & Input Range & Gain Settings & Coupling & $\begin{array}{c}\text { TEDS } \\
\text { Support }\end{array}$ & $\begin{array}{l}\text { Analog } \\
\text { Outputs }\end{array}$ \\
\hline \multicolumn{11}{|c|}{ High Performance } \\
\hline NI 4461 & $\begin{array}{l}\mathrm{PXI}, \mathrm{PCl} \\
\mathrm{PXI}\end{array}$ & ${ }_{24}^{24}$ & 118 & $204.8 \mathrm{kS} / \mathrm{s}$ & 2 & $\pm 42 \mathrm{~V}$ to $316 \mathrm{mV}$ & -20 to $30 \mathrm{~dB}$ in $10 \mathrm{~dB}$ increments & $A C / D C$ & 5 & 2 \\
\hline \multicolumn{11}{|l|}{ High Density } \\
\hline NI 4495 & PXI & 24 & 114 & $204.8 \mathrm{kS} / \mathrm{s}$ & 16 & \pm 10 to $1 \mathrm{~V}$ & 0 to $20 \mathrm{~dB}$ & $D C$ & - & - \\
\hline NI 4496 & PXI & 24 & 114 & $204.8 \mathrm{kS} / \mathrm{s}$ & 16 & \pm 10 to $1 \mathrm{~V}$ & 0 to $20 \mathrm{~dB}$ & AC & 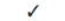 & - \\
\hline N1 4498 & PX] & 24 & 114 & $204.8 \mathrm{kS} / \mathrm{s}$ & 16 & $\pm 10 \mathrm{~V}$ to $316 \mathrm{mV}$ & 0 to $20 \mathrm{~dB}$ & $A C$ & $\checkmark$ & - \\
\hline \multicolumn{11}{|l|}{ Low Cost } \\
\hline NI 4472 & PXI, PCI & 24 & 110 & $102.4 \mathrm{kS} / \mathrm{s}$ & 8 & $\pm 10 \mathrm{~V}$ & - & $\mathrm{AC} / \mathrm{DC}$ & - & - \\
\hline \multirow{2}{*}{\multicolumn{11}{|c|}{ Ultraportable }} \\
\hline & & & & & & & & & & \\
\hline N1 9234 & USB & 24 & $\begin{array}{l}102 \\
102-x\end{array}$ & $51.2 \mathrm{kS} / \mathrm{s}$ & $\begin{array}{r}4 \\
4 \\
\end{array}$ & $\begin{array}{l} \pm 5 \mathrm{~V} \\
\pm 50\end{array}$ & $\begin{array}{lll}- & & \\
\end{array}$ & $A C / D C$ & $y$ & - \\
\hline
\end{tabular}

Tabie 2. Additional NI Dynamic Signai Acquisition Devices 
Ordering Information

For a complete list of accessories, visit the product page on ni.com.

\begin{tabular}{|c|c|c|c|}
\hline Products & Part Number & Recommended Accessories & Part Number \\
\hline \multicolumn{4}{|l|}{ N1 9234} \\
\hline NI 9234 with Sound and Vibration Measurement Sulte & $779680-02$ & No accessories required. & \\
\hline NI 9234 with BNC & $779680-01$ & No accessories required. & \\
\hline
\end{tabular}

Software Recommendations

LabVIEW Professional
Development System for
Windows

- Advanced software tools for large project
development
- Automatic code generation using DAQ
Assistant and Instrument I/O Assistant
- Tight integration with a wide range of
hardware
- Advanced measurement analysis and digital
signal processing
- Open connectivity with DLLs, ActiveX, and
-NET objects
- Capability to build DLLs, executables, and

- Capability to build DLLs, executables, and
MSI installers

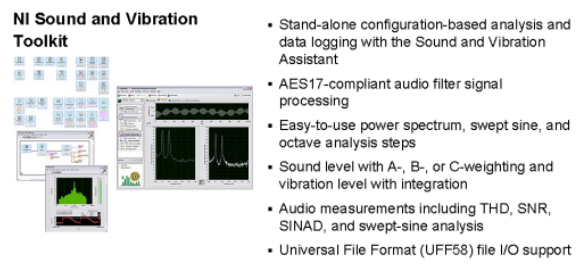

NI Sound and Vibration $\quad$ - Minimize development time with ready-to-run

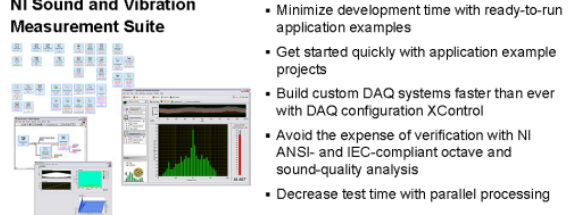

Back to Top

Support and Services

System Assurance Programs

NI system assurance programs are designed to make it even easier for you to own an NI system. These programs include configuration and deployment services for your NI PXI, CompactRIO, or Compact FieldPoint system. The NI Basic System Assurance Program provides a simple integration test and ensures that your system is delivered completely assembled in one box. When you configure your system with the $\mathrm{NI}$ Standard System Assurance Program, you can select riom avallable $\mathrm{N}$ system ariver sets and application development environments to create customized, Forderable somware conigurations. Your system arrives fully assembled and tested in one box with your sotware preinstalled When you order corerge and calbration

Calibration NI measurement hardware is calibrated to ensure measurement accuracy and verify that the device meets its published specifications. To ensure the ongoing accuracy of your about NI calibration services or to locate a qualified sevvice center near you, contact your local sales office or visit ni.com/calibration.

Technical Support

Get answers to your technical questions using the following National Instruments resources.

- Support - Visit nicom/support to access the NI KnowledgeBase, example programs, and tutorials or to contact our applications engineers who are located in NI sales

- Discussion Forums - Visit forums.ni.com for a diverse set of discussion boards on topics you care about 


\footnotetext{
Online Community - Visit community. ni.com to find, contribute, or collaborate on customer-contributed technical content with users like you

Repair

While you may never need your hardware repaired, NI understands that unexpected events may lead to necessary repairs. NI offers repair services performed by highly trained technicians who quickly return your device with the guarantee that it will perform to factory specifications. For more information, visit ni.comirepair.

Training and Certifications

The $\mathrm{N}$ training and certification program delivers the fastest, most certain route to increased proficiency and productivity using $\mathrm{NI}$ software and hardware. Training builds the skills to more efficiently develop robust, maintainable applications, while certification validates your knowledge and ability.

- Classroom training in cities worldwide - the most comprehensive hands-on training taught by engineers.

- On-site training at your facility - an excellent option to train multiple employees at the same time.

- Online instructor-led training - lower-cost, remote training if classroom or or-site courses are not possible

- Course kits - lowest-cost, self-paced training that you can use as reference guides.

- Training memberships and training credits - to buy now and schedule training later. Visit ni.comitraining for more information

Extended Warranty

NI offers options for extending the standard product warranty to meet the life-cycle requirements of your project. In addition, because NI understands that your requirements may change, the extended warranty is flexible in length and easily renewed. For more information, visit ni.comwwarranty.

OEM

NI offers design-in consulting and product integration assistance ifyou need NI products for OEM applications. For information about special pricing and sevvices for OEM customers, visit ni.comioem.

Alliance

Our Professional Services Team is comprised of NI applications engineers, NI Consulting Services, and a worldwide National Instruments Alliance Partner program of more than 700 independent consultants and integrators. Sevvices range from start-up assistance to turnkey system integration. Vist ni. comvalliance
}

Detailed Specifications

The following specifications are typical for the range -40 to $70^{\circ} \mathrm{C}$ unless otherwise noted

\begin{tabular}{ll}
\hline Input Characteristics & \\
\hline Number of channels & 4 analog input channels \\
\hline ADC resolution & 24 bits \\
\hline Type of ADC & Delta-Sigma (with analog prefiltering) \\
\hline Sampling mode & Simultaneous \\
\hline Type of TEDS supported & IEEE 1451.4 TEDS Class I \\
\hline Internal master timebase $\left(f_{M}\right)$ & \\
\hline Frequency & $13.1072 \mathrm{MHz}$ \\
\hline Accuracy & $\pm 50 \mathrm{ppm}$ max \\
\hline Data rate range $\left(f_{s}\right)$ using internal master timebase & \\
\hline Minimum & $1.652 \mathrm{kSis}$ \\
\hline Maximum & $51.2 \mathrm{kS} / \mathrm{s}$ \\
\hline Data rate range $\left(f_{s}\right)$ using external master timebase & \\
\hline Minimum & $0.391 \mathrm{kS} / \mathrm{s}$ \\
\hline Maximum & $52.734 \mathrm{kS} / \mathrm{s}$ \\
\hline Data rates ${ }^{1}\left(f_{s}\right)$ & $\frac{f_{M} \div 256}{n}, n=1,2, \ldots, 31$ \\
\hline Input coupling & $\mathrm{ACIDC}$ (software-selectable) \\
\hline AC cutoff frequency & \\
\hline$-3 \mathrm{~dB}$ & $0.5 \mathrm{~Hz}$ \\
\hline-0.1 dB & $4.6 \mathrm{~Hz}$ max \\
\hline AC cutoff frequency response & \\
\hline
\end{tabular}




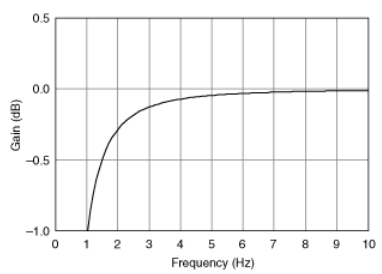

Input range

$\pm 5 \mathrm{~V}$

AC voltage full-scale range

Minimum

Typical

Maximum

Common-mode voltage range (Al-to earth ground)

IEPE excitation current (software selectable onjoff)

Minimum

Typical

Power-on glitch

$\pm 5.1 \mathrm{~V}_{\mathrm{pk}}$

$\pm 5.2 \mathrm{~V}_{\mathrm{pk}}$

$\pm 2 \vee \max$

IEPE compliance voltage

$2.0 \mathrm{~mA}$

$2.1 \mathrm{~mA}$

$90 \mu \mathrm{A}$ for $10 \mu \mathrm{s}$

an IEPE sensor, use the following equation to make sure your configuration meets the IEPE compliance voltage range.

must be 0 to 19 , where $V$ is the common-mode voltage applied to the $N 19234, V$, is the bias roltage of the IEPE sensor, and $V$ tull-scale is the full-scale voltage of the IEPE sensol.

Overvoltage protection (with respect to chassis ground)

For a signal source connected to $\mathrm{Al}+$ and $\mathrm{Al}-$

$\pm 30 \mathrm{~V}$

For a low-impedance source connected to $\mathrm{Al}+$ and $\mathrm{Al}-$

-6 to $30 \mathrm{~V}$

Input delay

$38.4 i f_{s}+3.2 \mu s$

Accuracy ${ }^{2}$

\begin{tabular}{|l|l|l|}
\hline \multicolumn{3}{|c|}{ Accuracy $^{2}$} \\
\hline Measurement Conditions & Percent of Reading (Gain Error) & Percent of Range ${ }^{3}$ (Offset Erron) \\
\hline Calibrated max $\left(-40\right.$ to $\left.70^{\circ} \mathrm{C}\right)$ & $0.34 \%, \pm 0.03 \mathrm{~dB}$ & $\pm 0.14 \%, 7.1 \mathrm{mV}$ \\
\hline Calibrated typ $\left(25^{\circ} \mathrm{C} \pm 5^{\circ} \mathrm{C}\right)$ & $0.05 \%, \pm 0.005 \mathrm{~dB}$ & $\pm 0.006 \%, 0.3 \mathrm{mV}$ \\
\hline Uncalibrated $\max \left(-40\right.$ to $\left.70^{\circ} \mathrm{C}\right)$ & $1.9 \%, \pm 0.16 \mathrm{~dB}$ & $\pm 0.27 \%, 13.9 \mathrm{mV}$ \\
\hline Uncalibrated typ $\left(25^{\circ} \mathrm{C} \pm 5^{\circ} \mathrm{C}\right)$ & $0.48 \%, \pm 0.04 \mathrm{d \theta}$ & $\pm 0.04 \%, 2.3 \mathrm{mV}$ \\
\hline
\end{tabular}

\begin{tabular}{|c|c|}
\hline \multicolumn{2}{|l|}{ Gain drift } \\
\hline Typical & $0.14 \mathrm{mdB} /)^{\circ} \mathrm{C}\left(16 \mathrm{ppm} v^{\circ} \mathrm{C}\right.$ \\
\hline Maximum & $0.45 \mathrm{mdB} /)^{\circ \mathrm{C}}\left(52 \mathrm{ppm} /{ }^{\circ \mathrm{C}}\right)$ \\
\hline \multicolumn{2}{|l|}{ Offset drift } \\
\hline Typical & $19.2 \mu \mathrm{V} /{ }^{\circ} \mathrm{C}$ \\
\hline Maximum & $118 \mu \mathrm{V} /{ }^{\circ} \mathrm{C}$ \\
\hline \multicolumn{2}{|c|}{ Channel-to-channel matching } \\
\hline \multicolumn{2}{|l|}{ Gain } \\
\hline Typical & $0.01 \mathrm{~dB}$ \\
\hline Maximum & $0.04 \mathrm{~d} \theta$ \\
\hline Phase $\left(f_{i n}\right.$ in $\left.\mathrm{kHz}\right)$ & $f_{i n} \cdot 0.045^{\circ}+0.04 \max$ \\
\hline
\end{tabular}




\begin{tabular}{ll}
\hline Frequency & $0.45 \cdot f_{\mathrm{s}}$ \\
\hline Flatness $\left(f_{\mathrm{s}}=51.2 \mathrm{kS} / \mathrm{s}\right)$ & $\pm 40 \mathrm{mdB}(\mathrm{pk}-\mathrm{to}-\mathrm{pk}$ max $)$ \\
\hline Phase nonlinearity $\left(f_{\mathrm{s}}=51.2 \mathrm{kS} / \mathrm{s}\right)$ & $\pm 0.45^{*} \mathrm{max}$ \\
\hline Stophand & \\
\hline Frequency & $0.55 \cdot f_{\mathrm{s}}$ \\
\hline Rejection & $100 \mathrm{~dB}$ \\
\hline Alias-free bandwidth & $0.45 \cdot f_{\mathrm{s}}$ \\
\hline Oversample rate & $64 \cdot f_{\mathrm{s}}$ \\
\hline Crosstalk $(1 \mathrm{kHz})$ & $-110 \mathrm{~dB}$ \\
\hline CMRR $\left(f_{\text {in }} \leq 1 \mathrm{kHz}\right)$ & \\
\hline Minimum & $40 \mathrm{~dB}$ \\
\hline Typical & $47 \mathrm{~dB}$ \\
\hline SFDR $\left(f_{\text {in }}=1 \mathrm{kHz},-60 \mathrm{dBFS}\right)$ & $120 \mathrm{~dB}$
\end{tabular}

\begin{tabular}{|c|c|c|c|}
\hline \multicolumn{4}{|c|}{ Idle channet noise and noise density } \\
\hline Idle Channel & $51.2 \mathrm{kS} / \mathrm{s}$ & $25.6 \mathrm{kS} / \mathrm{s}$ & $2.048 \mathrm{kS} / \mathrm{s}$ \\
\hline \multirow[t]{2}{*}{ Noise } & $97 \mathrm{dBFS}$ & $99 \mathrm{dBFS}$ & $103 \mathrm{dBFS}$ \\
\hline & $50 \mathrm{~N} \mathrm{rms}_{\mathrm{rms}}$ & $40 \mathrm{~N}_{\mathrm{rms}}$ & $25 \mathrm{~N}_{\mathrm{rms}}$ \\
\hline sity & $310 \mathrm{nV} / \mathrm{JHz}$ & $350 \mathrm{nV} / \mathrm{d} / \mathrm{z}$ & $780 n$ \\
\hline
\end{tabular}

Input impedance

Differential $305 \mathrm{k} \Omega$

Al- (shield) to chassis ground

Total harmonic distortion (THD)

\begin{tabular}{|l|l|l|}
\hline Input Amplitude & $1 \mathrm{kHz}$ & $\mathbf{8} \mathrm{kHz}$ \\
\hline-1 .
\end{tabular}

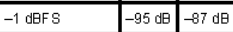

\begin{tabular}{|l|l|l|}
\hline$-20 \mathrm{dBFS}$ & $-95 \mathrm{~dB}$ & $-80 \mathrm{d \theta}$ \\
\hline
\end{tabular}

Intermodulation distortion $(-1 \mathrm{dBFS})$

\begin{tabular}{ll} 
DIN $250 \mathrm{~Hz} / 8 \mathrm{kHz}, 4: 1$ amplitude ratio & $-80 \mathrm{~dB}$ \\
\hline CCIF $11 \mathrm{kHz} / 12 \mathrm{kHz}, 1: 1$ amplitude ratio & $-93 \mathrm{~dB}$ \\
\hline MTEF & 390,362 hours at $25^{\circ} \mathrm{C}$; Bellcore Issue 2, Method 1, Case 3, Limited Part Stress Method
\end{tabular}

390,362 hours at $25^{\circ} \mathrm{C}$; Bellcore Issue 2, Method 1 , Case 3 , Limited Part Stress Method

Q. Note Contact NI for Bellcore MTEF specifications at other temperatures or for MIL-HDEK-217F specifications

Power Requirements

Power consumption from chassis

Activemote

$900 \mathrm{~mW}$ max

Sleep mode $\quad 25 \mu$ w max

Thermal dissipation (at $70^{\circ} \mathrm{C}$ )

Active mode

$930 \mathrm{~mW}$ max

Sleep mode

25 w ${ }^{\prime \prime}$ max

Physical Characteristics

Weight $173 \mathrm{~g}(6.1 \mathrm{oz})$

Safety 


\begin{tabular}{|c|c|}
\hline \multicolumn{2}{|l|}{ If you need to clean the module, wipe it with a dry towel. } \\
\hline \multicolumn{2}{|l|}{ Safety Voltages } \\
\hline \multicolumn{2}{|l|}{ Connect only voltages that are within the following limits. } \\
\hline Channel-to-earth ground & $\pm 30 \mathrm{~V}$ max, Measurement Categony 1 \\
\hline \multicolumn{2}{|l|}{ Isolation } \\
\hline Channerto-channel & None \\
\hline Channetto-earth ground & None \\
\hline \multicolumn{2}{|c|}{$\begin{array}{l}\text { Measurement Category I is for measurements performed on circuits not directly connected to the electrical distribution sy stem referred to as MAINS }{ }^{4} \text { voltage. This category is foc } \\
\text { measurements of voltages from specially protected secondary circuits. Such voltage measurements include signal levels, special equipment, limited-energy parts of equipment, } \\
\text { circuits powered by regulated low-yoltage sources, and electronics. }\end{array}$} \\
\hline \multicolumn{2}{|c|}{ 1. Caution Do not connect the NI 9234 to signals or use for measurements within Measurement Categories II, III, or IV. } \\
\hline \multicolumn{2}{|l|}{ Hazardous Locations } \\
\hline U.S. (UL) & Class I, Dwision 2, Groups A, B, C, D, T4; Class I, Zone 2, AEx nA IIC T4 \\
\hline Canada (C-UL) & Class I, Division 2, Groups A, B, C, D, T4; Class I, Zone 2, Ex nA IIC T4 \\
\hline Europe (DEMKO) & Ex nA IC T4 \\
\hline \multicolumn{2}{|l|}{ Safety Standards } \\
\hline \multirow{3}{*}{\multicolumn{2}{|c|}{$\begin{array}{l}\text { This product is designed to meet the requirements of the following standards of safety for electrical equipment for measurement, control, and laboratory use: } \\
\text { - IEC 61010-1, EN 61010-1 } \\
\text { - UL 61010-1, CSA 61010-1 }\end{array}$}} \\
\hline & \\
\hline & \\
\hline \multicolumn{2}{|c|}{ Q7 Note For UL and other safety certifications, refer to the product label or the Onine Product Certification section. } \\
\hline \multicolumn{2}{|l|}{ Electrom agnetic Compatibility } \\
\hline \multicolumn{2}{|c|}{ This product meets the requirements of the following EMC standards for electrical equipment for measurement, control, and laboratory use: } \\
\hline \multicolumn{2}{|l|}{$\begin{array}{l}\text { - EN } 61326 \text { (IEC 61326): Class A emissions; Basic immunity } \\
\text { - EN } 55011 \text { (CISPR 11): Group 1, Class A emissions } \\
\text { - ASINZZ CISPR 11: Group 1, Class A emissions } \\
\text { - FCC } 47 \text { CFR Part 15B: Class A emissions } \\
\text { - ICES-001: Class A emissions }\end{array}$} \\
\hline \multirow{2}{*}{\multicolumn{2}{|c|}{$\begin{array}{l}\text { Q7. Note For the standards applied to assess the EMC of this product, refer to the Online Product Cert tic ation section. } \\
\text { Note For EMC compliance, operate this device with shielded cables. }\end{array}$}} \\
\hline & \\
\hline \multicolumn{2}{|c|}{ CE Compliance } \\
\hline \multicolumn{2}{|c|}{ This product meets the essential requirements of applicable European Directives, as amended for CE marking, as follows: } \\
\hline \multicolumn{2}{|c|}{$\begin{array}{l}\text { - 2006/95/EC; Low-Voltage Directive (safety) } \\
\text { - 2004/108/EC; Electromagnetic Compatibility Directive (EMC) }\end{array}$} \\
\hline \multicolumn{2}{|c|}{ 8. Note For the standards applied to assess the EMC of this product, refer to the Onine Product Cert fic ation section. } \\
\hline \multicolumn{2}{|l|}{ Online Product Certification } \\
\hline \multicolumn{2}{|c|}{$\begin{array}{l}\text { Refer to the product Declaration of Conformity ( } \mathrm{DoC} \text { ) for additional regulatory compliance information. To obtain product certifications and the DoC for this product, visit } \\
\text { ni.comicertification, search by module number or product line, and click the appropriate link in the Certification column. }\end{array}$} \\
\hline \multicolumn{2}{|l|}{ Shock and Vibration } \\
\hline \multicolumn{2}{|l|}{ To meet these specifications, you must panel mount the system. } \\
\hline \multicolumn{2}{|l|}{ Operating vibration } \\
\hline Random (IEC 60068-2-64) & $5 \mathrm{~g}_{\text {rms }} 10$ to $500 \mathrm{~Hz}$ \\
\hline Sinusoidal (IEC 60068-2-6) & $5 \mathrm{~g}, 10$ to $500 \mathrm{~Hz}$ \\
\hline Operating shock (IEC 60068-2-27) & $30 \mathrm{~g}, 11 \mathrm{~ms}$ half sine, $50 \mathrm{~g}, 3$ ms half sine, 18 shocks at 6 orientations \\
\hline \multicolumn{2}{|l|}{ Environmental } \\
\hline \multicolumn{2}{|c|}{$\begin{array}{l}\text { National Instruments C Series modules are intended for indoor use only but may be used outdoors if in stalled in a suitable enclosure. Refer to the manual for the chassis you are } \\
\text { using for more information about meeting these specifications. }\end{array}$} \\
\hline Operating temperature (IEC 60068-2-1, IEC 60068-2-2) & -40 to $70^{\circ} \mathrm{C}$ \\
\hline Storage temperature (IEC 60068-2-1, IEC 60068-2-2) & -40 to $85^{\circ} \mathrm{C}$ \\
\hline Ingress protection & IP 40 \\
\hline Operating humidity (IEC 60068-2-56) & 10 to $90 \% \mathrm{RH}$, noncondensing \\
\hline
\end{tabular}


Development of the West Virginia University Small Microgravity Research Facility (WVU SMiRF)

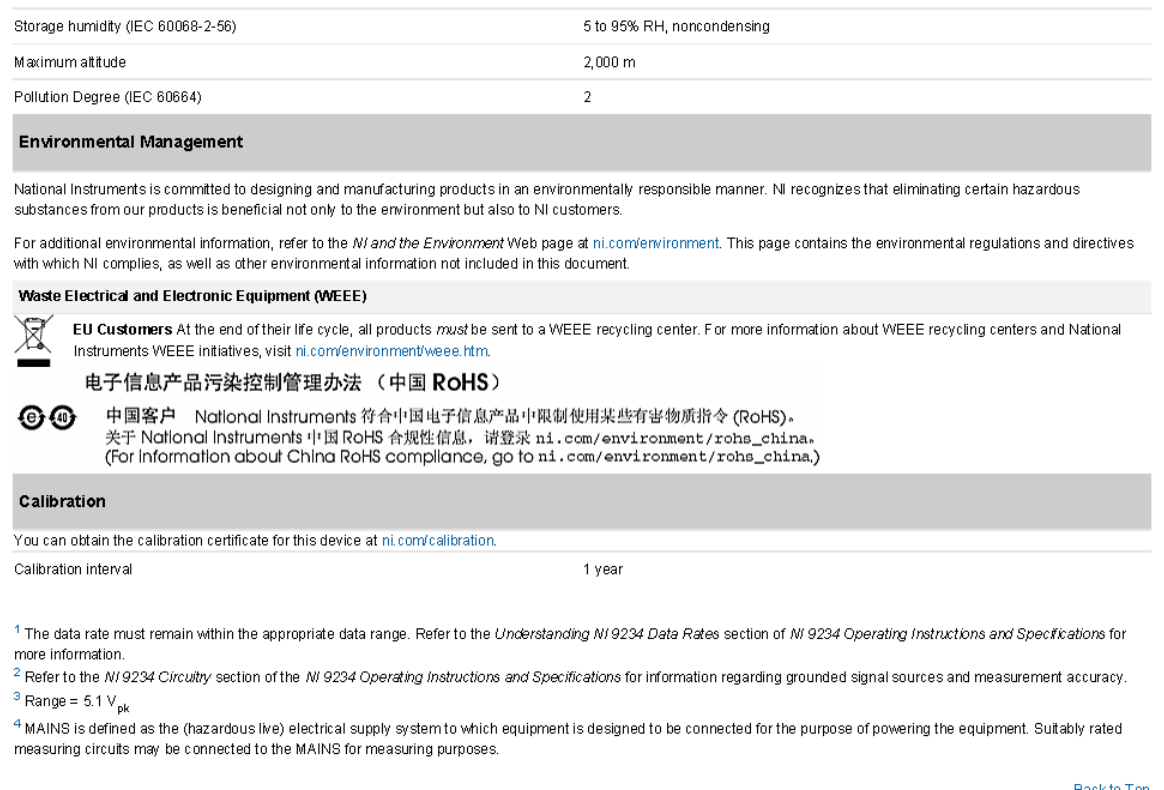


Development of the West Virginia University Small Microgravity Research Facility (WVU SMiRF)
Copyright $^{\odot} 2014$

Kyle G. Phillips

Pinouts/Front Panel Connections

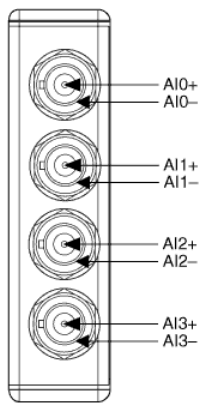

Back to To

Q2010 National Instruments. All rights reserved. CVI, Comp actRIO, FieldP oint, LabVIEW, Measurement Studio, National Instrument, National Instuments Allianoe Parther, N1, ni.com, NI CompactDAQ, N1-DAQ,

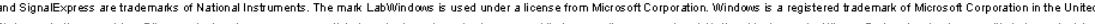

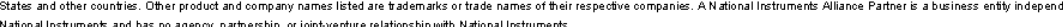

My Profile | RSS | Privacy | Legal | Contact N| 12012 National Instruments Corporation. All rights reserved. 
Development of the West Virginia University

Small Microgravity Research Facility (WVU SMiRF)
Copyright $^{\odot} 2014$

Kyle G. Phillips

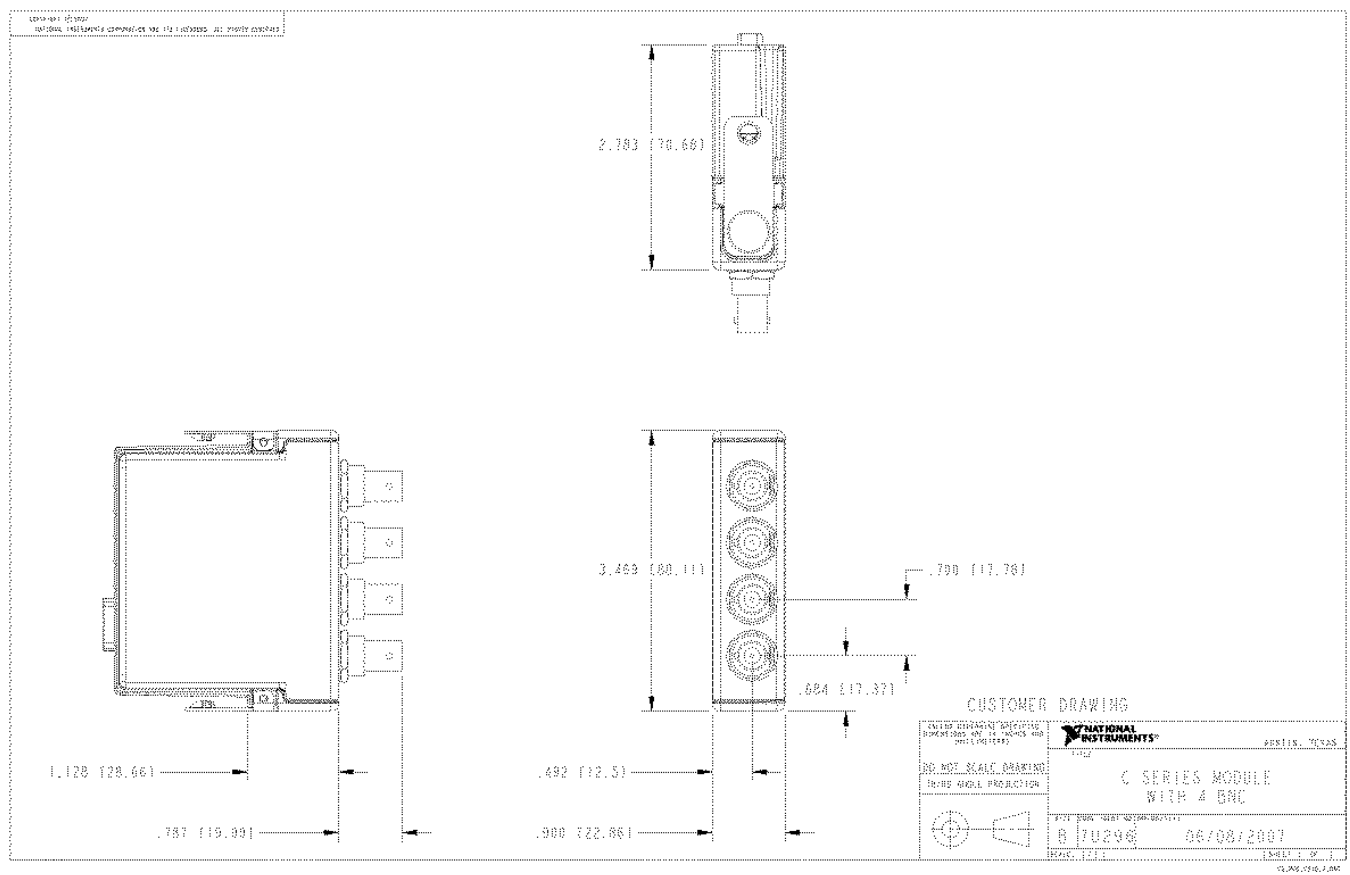




\section{CALIBRATION PROCEDURE NI 9234}

\section{4-Channel, \pm 5 V, 24-Bit Software-Selectable IEPE and AC/DC Analog Input Module}

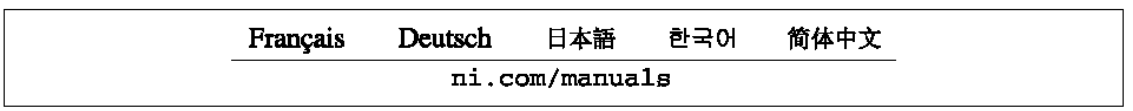

This document contains the verification and adjustment procedures for the National Instruments 9234 . For more information on calibration, visit $\mathrm{ni}$.com/calibration.

\section{Contents}

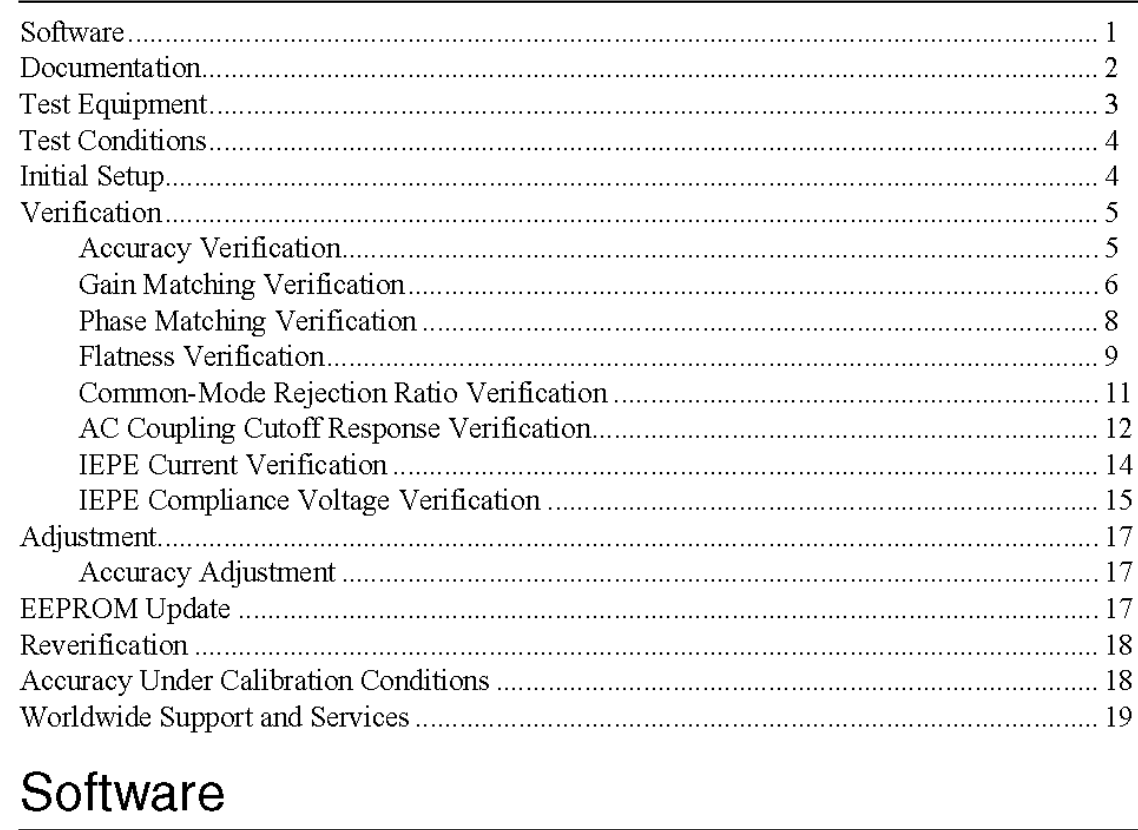

Calibrating the NI 9234 requires the installation of NI-DAQmx 9.2 or later on the calibration system. You can download NI-DAQmx from ni . com/downloads. NI-DAQmx supports LabVIEW, LabWindows ${ }^{\mathrm{TM}} / \mathrm{CVI}, \mathrm{C} / \mathrm{C}++, \mathrm{CH}$, and Visual Basic .NET. When you install NI-DAQmx you only need to install support for the ADE that you intend to use. 


\section{Documentation}

Consult the following documents for information about the NI 9234, NI-DAQmx, and your application software. All documents are available on $\mathrm{ni}$. com and help files install with the software.

NI CDAQ-9174/9178 USB Chassis Quick Start

NI-DAQmx installation and hardware setup

NI 9234 Operating Instructions and Specifications

NI 9234 specific information, specifications, and calibration interval

NI-DAQmx Readme

Operating system and application software support in NI-DAQmx

LabVIEW Help

LabVIEW programming concepts and reference information about NI-DAQmx VIs and functions

NI-DAQmx C Reference Help

Reference information for NI-DAQmx C functions and NI-DAQmx C properties

NI-DAQmx.NET Help Support for Visual Studio

Reference information for NI-DAQmx .NET methods and NI-DAQmx .NET properties, key concepts, and a $\mathrm{C}$ enum to .NET enum mapping table

2 | ni.com | NI 9234 Calibration Procedure 


\section{Test Equipment}

Table 1 lists the equipment recommended for the performance verification and adjustment procedures. If the recommended equipment is not available, select a substitute using the requirements listed in Table 1.

Table 1. Recommended Equipment

\begin{tabular}{|c|c|c|c|}
\hline Equipment & $\begin{array}{c}\text { Recommended } \\
\text { Model }\end{array}$ & Where Used & Requirements \\
\hline Calibrator & Fluke $5700 \mathrm{~A}$ & $\begin{array}{l}\text { Accuracy, } \\
\text { Adjustment }\end{array}$ & $\begin{array}{l}\text { Use a high-precision voltage source with an } \\
\text { accuracy } \leq 100 \text { ppm and an output that is } \\
\text { able to be not grounded (floating). }\end{array}$ \\
\hline \multirow[t]{5}{*}{$\begin{array}{l}\text { Function } \\
\text { Generator }\end{array}$} & \multirow[t]{5}{*}{ NI 4461} & $\begin{array}{c}\text { Gain } \\
\text { Matching }\end{array}$ & $\begin{array}{l}\text { Use a function generator capable of } \\
\text { sourcing } 1 \mathrm{kHz} \text { and } 10 \mathrm{kHz} \pm 4.5 \mathrm{Vpk} \mathrm{AC} \\
\text { sinusoidal signals with amplitude } \\
\text { uncertainty of } \pm 10 \% \text { or less. }\end{array}$ \\
\hline & & $\begin{array}{l}\text { Phase } \\
\text { Matching }\end{array}$ & $\begin{array}{l}\text { Use a function generator capable of } \\
\text { sourcing both } 1 \mathrm{kHz} \text { and } 10 \mathrm{kHz} \pm 4.5 \mathrm{~V}_{\mathrm{pk}} \\
\text { AC sinusoidal signals with amplitude } \\
\text { uncertainty of } \pm 10 \% \text { or less. }\end{array}$ \\
\hline & & Flatness & $\begin{array}{l}\text { Use a function generator capable of } \\
\text { sourcing } \pm 4.5 \mathrm{~V}_{\mathrm{pk}} \mathrm{AC} \text { sinusoidal signal } \\
\text { from } 100 \mathrm{~Hz} \text { to } 22.5 \mathrm{kHz} \text { with a gain over } \\
\text { frequency flatness of } \pm 8 \mathrm{mdB} \text { or less. }\end{array}$ \\
\hline & & CMRR & $\begin{array}{l}\text { Use a function generator capable of } \\
\text { sourcing } 1 \mathrm{kHz} \pm 4.5 \mathrm{Vpk} \mathrm{AC} \text { sinusoidal } \\
\text { signals with amplitude uncertainty of } \\
\pm 0.04 \mathrm{~dB} \text { or less. }\end{array}$ \\
\hline & & $\begin{array}{l}\text { AC Coupling } \\
\text { Response }\end{array}$ & $\begin{array}{l}\text { Use a function generator capable of } \\
\text { sourcing } \pm 0.5 \mathrm{~V}_{\text {pk }} \text { AC sinusoidal signals } \\
\text { from } 1 \mathrm{~Hz} \text { to } 10 \mathrm{~Hz} \text { with a gain over } \\
\text { frequency flatness of } \pm 8 \text { mdBor less and a } \\
\text { frequency uncertainty of } \pm 1 \% \text { or less. }\end{array}$ \\
\hline DMM & NI 4070 & IEPE Current & $\begin{array}{l}\text { Use DMM in current mode in the smallest } \\
\text { range to measure } 2 \mathrm{~mA} \mathrm{DC} \text { with a } \\
\text { measurement uncertainty } \leq 0.5 \% \text {. }\end{array}$ \\
\hline $\begin{array}{l}\text { Source } \\
\text { Measure Unit } \\
\text { (SMU) }\end{array}$ & $\begin{array}{l}\text { Keithley Model } \\
2400\end{array}$ & $\begin{array}{l}\text { IEPE } \\
\text { Compliance } \\
\text { Voltage }\end{array}$ & $\begin{array}{l}\text { Use an SMU capable of sourcing } 20 \mathrm{~V} \text { DC } \\
\text { with an accuracy } \leq 0.1 \% \text { while sinking } \\
>2 \mathrm{~mA} \text { DC. }\end{array}$ \\
\hline BNC Cable & - & All & $\begin{array}{l}\text { Use a length appropriate for your } \\
\text { application. }\end{array}$ \\
\hline Chassis & NI cDAQ-9178 & All & - \\
\hline
\end{tabular}




\section{Test Conditions}

The following setup and environmental conditions are required to ensure the NI 9234 meets published specifications

- Keep connections to the device as short as possible. Long cables and wires act as antennas, picking up extra noise that can affect measurements.

- Verify that all connections to the device are secure.

- Use shielded copper wire for all cable connections to the device. Use twisted-pairs wire to eliminate noise and thermal offsets.

- Maintain an ambient temperature of $23 \pm 5^{\circ} \mathrm{C}$. The device temperature will be greater than the ambient temperature.

- Keep relative humidity below $80 \%$.

- Allow a warm-up time of at least 10 minutes to ensure that the NI 9234 measurement circuitry is at a stable operating temperature.

\section{Initial Setup}

Complete the following steps to set up the NI 9234.

1. Install NI-DAQmx.

2. Make sure the NI cDAQ-9178 power source is not connected.

3. Connect the NI cDAQ-9178 to the system safety ground.

a. Attach a ring lug to a $14 \mathrm{AWG}(1.6 \mathrm{~mm})$ wire.

b. Connect the ring lug to the ground terminal on the side of the NI cDAQ-9178 using the ground screw.

c. Attach the other end of the wire to the system safety ground.

4. Install the module in slot 8 of the NI cDAQ-9178 chassis. Leave slots 1 through 7 of the NI cDAQ-9178 chassis empty.

5. Connect the NI cDAQ-9178 chassis to your host computer.

6. Connect the power source to the NI cDAQ-9178 chassis.

7. Launch Measurement \& Automation Explorer (MAX).

8. Right-click the device name and select Self-Test to ensure that the module is working properly. 


\section{Verification}

The following performance verification procedures describe the sequence of operation and test points required to verify the NI 9234 and assumes that adequate traceable uncertainties are available for the calibration references.

\section{Accuracy Verification}

Complete the following procedure to determine the As-Found status of the NI 9234.

1. Set the calibrator to Standby mode (STBY)

2. Connect the NI 9234 to the calibrator as shown in Figure 1.

Figure 1. Accuracy Verification Connections to the NI 9234

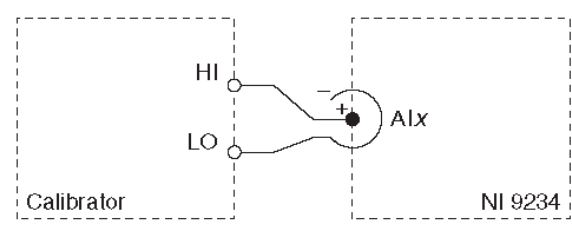

3. Verify that the calibrator output is not grounded (floating).

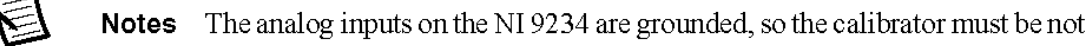
grounded (floating) to prevent ground loop measurement errors.

To float the output of the Fluke 5700A, enable External Guard mode (EX GRD).

4. Set the calibrator voltage to a Test Point value indicated in Table 4.

5. Set the calibrator to Operate mode (OPR).

6. Acquire and average samples.

a. Create an AI voltage task on the NI 9234 according to Table 2.

Table 2. NI 9234 Configuration for Accuracy Verification

\begin{tabular}{|c|c|c|c|}
\hline Min (V) & Max (V) & Scaled Units & Channel Configuration \\
\hline \hline-5 & 5 & Volts & DC Coupled \\
\hline
\end{tabular}

b. Configure the AI voltage task timing according to Table 3 .

Table 3. NI 9234 Timing Configuration

\begin{tabular}{|c|c|c|}
\hline Sample Mode & Samples Per Channel & Rate (Hz) \\
\hline \hline Finite Samples & 10240 & 10240 \\
\hline
\end{tabular}

c. Start the task. 
d. Average the readings and record the average.

e. Compare the average to the limits in Table 4.

f. Clear the task.

7. Set the calibrator to Standby mode (STBY).

8. Repeat steps 3 through 7 for each test point in Table 4.

Table 4. NI 9234 Verification Test Limits for Accuracy

\begin{tabular}{|c|c|c|c|}
\hline \multicolumn{2}{|c|}{ Test Point } & \multicolumn{2}{c|}{ 1-Year Limits } \\
\hline Location & Value (V) & Lower Limit (V) & Upper Limit (V) \\
\hline \hline Max & 4.0 & 3.9952 & 4.0048 \\
\hline Mid & 0.0 & -0.0012 & 0.0012 \\
\hline Min & -4.0 & -4.0048 & -3.9952 \\
\hline
\end{tabular}

$E$ Note The test limits listed in Table 4 are derived using the values in Table 28 .

9. Disconnect the calibrator from the device.

10. Repeat steps 1 through 9 for each channel on the NI 9234.

\section{Gain Matching Verification}

Complete the following procedure to determine the As-Found status of the NI 9234.

1. Set the calibrator to Standby mode (STBY).

2. Connect the NI 9234 to the calibrator. Refer to Figure 2 for a connection diagram.

Figure 2. Gain Matching Verification Connections to the NI 9234

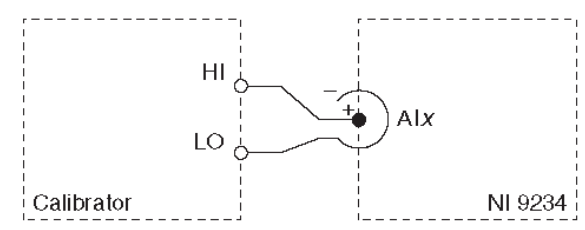

3. Verify that the calibrator output is not grounded (floating).

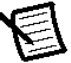

Notes The analog inputs on the NI 9234 are grounded, so the calibrator must be not grounded (floating) to prevent ground loop measurement errors.

To float the output of the Fluke 5700A, enable External Guard mode (EX GRD).

4. Set the calibrator voltage to $4.0 \mathrm{~V}$.

5. Set the calibrator to Operate mode (OPR).

6 | ni.com | NI 9234 Calibration Procedure 
6. Acquire and average samples.

a. Create an AI voltage task on the NI 9234 according to Table 5.

Table 5. NI 9234 Configuration for Gain Matching Verification

\begin{tabular}{|c|c|c|c|}
\hline Min (V) & Max (V) & Scaled Units & Channel Configuration \\
\hline \hline-5 & 5 & Volts & DC Coupled \\
\hline
\end{tabular}

b. Configure the AI voltage task timing according to Table 6 .

Table 6. Timing Configuration

\begin{tabular}{|c|c|c|}
\hline Sample Mode & Samples Per Channel & Rate (Hz) \\
\hline \hline Finite Samples & 10240 & 10240 \\
\hline
\end{tabular}

c. Start the task.

d. Average the readings and record the average as $V_{1}$.

e. Clear the task.

f. Set the calibrator to Standby mode (STBY).

g. Set the calibrator voltage to $-4.0 \mathrm{~V}$.

h. Set the calibrator to Operate mode (OPR).

i. Start the task.

j. Average the readings and record the average as $\mathrm{V}_{2}$.

k. Clear the task.

7. Set the calibrator to Standby mode (STBY).

8. Disconnect the calibrator from the device.

9. Perform the following calculation using the recorded $V_{1}$ and $V_{2}$ values.

$$
\operatorname{Gain}(d B)=\left(20 \times \log _{10}\left(\frac{V_{1}-V_{2}}{8}\right)\right)
$$

10. Repeat steps 1 through 9 for each channel.

11. Compare the channel-to-channel gain difference to the maximum limit of $\pm 40 \mathrm{mdB}$.

If the Gain Matching verification procedure determines that the NI 9234 is outside of the limits, refer to Worldwide Support and Services for assistance in returning the device to NI. 


\section{Phase Matching Verification}

Complete the following procedure to determine the As-Found status of the NI 9234.

1. Connect the NI 9234 to the function generator as shown in Figure 3.

Figure 3. Phase Matching Verification Connections to the NI 9234

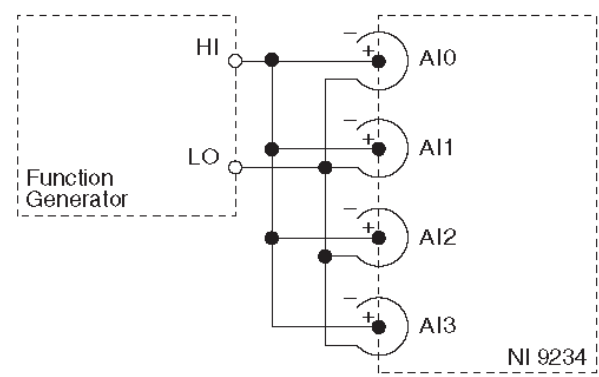

2. Configure function generator according to Table 7 .

Table 7. Function Generator Configuration for Phase Matching Verification

\begin{tabular}{|c|c|}
\hline Signal Type & Amplitude $\left(\mathrm{V}_{\mathrm{pk}}\right)$ \\
\hline \hline Sinewave & \pm 4.5 \\
\hline
\end{tabular}

3. Set the function generator frequency to a value indicated in Table 10.

4. Acquire and average samples.

a. Create an AI voltage task on the NI 9234 according to Table 8.

Table 8. NI 9234 Configuration for Phase Matching Verification

\begin{tabular}{|c|c|c|c|}
\hline Min (V) & Max (V) & Scaled Units & Channel Configuration \\
\hline \hline-5 & 5 & Volts & AC Coupled \\
\hline
\end{tabular}

$E$ Note Wait $20 \mathrm{~s}$ for the AC coupling filter on the NI 9234 to settle.

b. Configure the AI voltage task timing according to Table 9 .

Table 9. NI 9234 Timing Configuration

\begin{tabular}{|c|c|c|}
\hline Sample Mode & Samples Per Channel & Rate (Hz) \\
\hline \hline Finite Samples & 16384 & 51200 \\
\hline
\end{tabular}

c. Enable the function generator.

d. Start the task.

8 | ni.com | NI 9234 Calibration Procedure 
e. Disable the function generator.

f. Clear the task.

5. Calculate the phase for each channel on the NI 9234. NI recommends using the Extract Single Tone Information VI to calculate phase.

6. Compare the channel-to-channel phase difference to the limits in Table 10.

7. Repeat steps 2 through 6 for each test point in Table 10.

Table 10. NI 9234 Verification Test Limits for Phase Matching

\begin{tabular}{|c|c|}
\hline Generator Frequency $\mathbf{( k H z})$ & Phase Matching Channel-to-Channel Limit \\
\hline \hline 1 & $\pm 0.085^{\circ}$ \\
\hline 10 & $\pm 0.49^{\circ}$ \\
\hline
\end{tabular}

If the Phase Matching verification procedure determines that the NI 9234 is outside of the limits, refer to Worldwide Support and Services for assistance in returning the device to NI.

\section{Flatness Verification}

Complete the following procedure to determine the As-Found status of the NI 9234

1. Connect the NI 9234 to the function generator as shown in Figure 4.

Figure 4. Flatness Verification Connections to the NI 9234

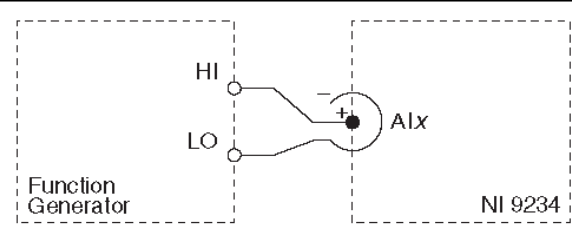

2. Configure the function generator according to Table 11 .

Table 11. Function Generator Configuration for Flatness Verification

\begin{tabular}{|c|c|c|}
\hline Signal Type & Amplitude $\left(\mathrm{V}_{\mathrm{pk}}\right)$ & Frequency $(\mathbf{k H z})$ \\
\hline \hline Sinewave & \pm 4.5 & 1 \\
\hline
\end{tabular}

3. Acquire a reference amplitude.

a. Create and configure an AI voltage task on the NI 9234 according to Table 12.

Table 12. NI 9234 Configuration for Flatness Verification

\begin{tabular}{|c|c|c|c|}
\hline Min (V) & Max (V) & Scaled Units & Channel Configuration \\
\hline \hline-5 & 5 & Volts & AC Coupled \\
\hline
\end{tabular}


Note Wait $20 \mathrm{~s}$ for the AC coupling filter on the NI 9234 to settle.

b. Configure the AI voltage task timing according to Table 13 .

Table 13. NI 9234 Timing Configuration

\begin{tabular}{|c|c|c|}
\hline Sample mode & Samples Per Channel & Rate (Hz) \\
\hline \hline Finite Samples & 16384 & 51200 \\
\hline
\end{tabular}

c. Enable the function generator.

d. Start the task.

e. Record the amplitude of the waveform as Amplitude Ref. $_{\text {. }}$

f. Disable the function generator.

g. Clear the task.

4. Set the function generator frequency to an appropriate value indicated in Table 14.

5. Enable the function generator.

6. Acquire samples.

a. Start the task.

b. Record the amplitude of the waveform as Amplitude Acq $_{\text {. }}$

c. Disable the function generator.

d. Clear the task.

7. Perform the following calculation using the recorded Amplitude ${ }_{\text {Ref }}$ and Amplitude Acq $_{\text {A }}$ values.

$$
\text { Flatness }=20 \times \log \left(\frac{\text { Amplitude }_{\text {Acq }}}{\text { Amplitude }_{\text {Ref }}}\right)
$$

8. Repeat steps 4 through 7 for each function generator frequency iteration in Table 14.

Table 14. NI 9234 Verification Test Limits for Flatness

\begin{tabular}{|c|}
\hline Function Generator Frequency $\mathbf{~ k H z})$ \\
\hline \hline 1 (Amplitude $_{\text {Ref }}$ ) \\
\hline 0.1 \\
\hline 0.4 \\
\hline 0.7 \\
\hline 2.5 \\
\hline 5 \\
\hline 7.5 \\
\hline 10 \\
\hline
\end{tabular}

10 | ni.com | NI 9234 Calibration Procedure 
Table 14. NI 9234 Verification Test Limits for Flatness (Continued)

\begin{tabular}{|c|}
\hline Function Generator Frequency $(\mathbf{k H z})$ \\
\hline \hline 12.5 \\
\hline 15 \\
\hline 17.5 \\
\hline 20 \\
\hline 22.5 \\
\hline
\end{tabular}

9. Perform the following calculation using the maximum and minimum calculated flatness from steps 7 and 8 .

$$
\text { Flatness }_{\text {peak }- \text { to }- \text { peak }}=\text { Flatness }_{\max }-\text { Flatness }_{\min }
$$

10. Compare the calculated Flatness peak-to-peak $_{\text {to }}$ the limit of $40 \mathrm{mdB}$.

11. Repeat steps 1 through 8 for each channel.

If the Flatness verification procedure determines that the NI 9234 is outside of the limits, refer to Worldwide Support and Services for assistance in returning the device to NI.

\section{Common-Mode Rejection Ratio Verification}

Complete the following procedure to determine the As-Found status of the NI 9234.

1. Connect the NI 9234 to the function generator and short the positive pin and negative shell of the NI 9234 channel.

2. Connect the function generator to the chassis ground as shown in Figure 5.

Figure 5. CMRR Verification Connections to the NI 9234

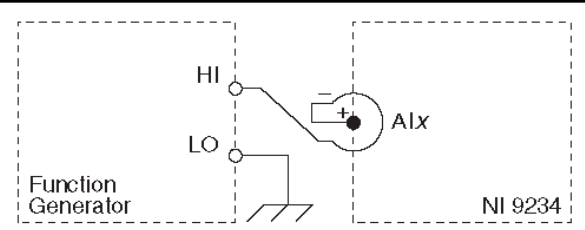

3. Configure the function generator according to Table 15 .

Table 15. Function Generator Configuration for CMRR Verification

\begin{tabular}{|c|c|c|}
\hline Signal Type & Amplitude $\left(\mathbf{V}_{\mathrm{pk}}\right)$ & Frequency $(\mathbf{k H z})$ \\
\hline \hline Sinewave & \pm 0.5 & 1 \\
\hline
\end{tabular}


4. Acquire a reference amplitude.

a. Create and configure an AI voltage task on the NI 9234 according to Table 16.

Table 16. NI 9234 Configuration for CMRR Verification

\begin{tabular}{|c|c|c|c|}
\hline Min (V) & Max (V) & Scaled Units & Channel Configuration \\
\hline \hline-5 & 5 & Volts & DC Coupled \\
\hline
\end{tabular}

b. Configure the AI voltage task timing according to Table 17.

Table 17. NI 9234 Timing Configuration

\begin{tabular}{|c|c|c|}
\hline Sample Mode & Samples Per Channel & Rate (Hz) \\
\hline \hline Finite Samples & 16384 & 51200 \\
\hline
\end{tabular}

c. Enable the function generator.

d. Start the task.

e. Record the measured amplitude as Amplitude $_{\text {Acq. }}$

f. Disable the function generator.

g. Clear the task.

5. Perform the following calculation using the recorded Amplitude $_{\text {Acq }}$.

$$
C M R R=-20 \times \log _{10}\left(\frac{\text { Amplitude }_{\text {Acq }}}{0.5}\right)
$$

6. Compare the calculation result to the minimum CMRR limit of $40 \mathrm{~dB}$.

7. Repeat steps 1 through 6 for each channel.

If the Common-Mode Rejection Ratio verification procedure determines that the NI 9234 is outside of the limits, refer to Worldwide Support and Services for assistance in returning the device to NI.

\section{AC Coupling Cutoff Response Verification}

Complete the following procedure to determine the As-Found status of the NI 9234.

1. Connect the NI 9234 to the function generator as shown in Figure 6.

Figure 6. AC Coupling Cutoff Response Verification Connections

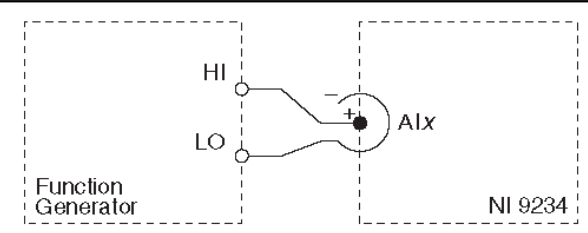

12 | ni.com | NI 9234 Calibration Procedure 
2. Configure the function generator according to Table 18 .

Table 18. Function Generator Configuration for AC Coupling Cutoff Response Verification

\begin{tabular}{|c|c|c|}
\hline Signal Type & Amplitude $\left(\mathbf{V}_{\mathrm{pk}}\right)$ & Frequency $(\mathrm{Hz})$ \\
\hline \hline Sinewave & \pm 4.5 & 20 \\
\hline
\end{tabular}

3. Acquire a reference amplitude.

a. Create and configure an AI voltage task on the NI 9234 according to Table 19.

Table 19. NI 9234 Configuration for AC Coupling Cutoff Response Verification

\begin{tabular}{|c|c|c|c|c|}
\hline Min (V) & Max (V) & Scaled Units & $\begin{array}{c}\text { Physical } \\
\text { Channels }\end{array}$ & $\begin{array}{c}\text { Channel } \\
\text { Configuration }\end{array}$ \\
\hline \hline-5 & 5 & Volts & AI0:3 & AC Coupled \\
\hline
\end{tabular}

b. Configure the AI voltage task timing according to Table 20 .

Table 20. NI 9234 Timing Configuration

\begin{tabular}{|c|c|c|}
\hline Sample mode & Samples Per Channel & Rate (Hz) \\
\hline \hline Finite Samples & 2048 & 2048 \\
\hline
\end{tabular}

c. Enable the function generator.

d. Start the task.

e. Record the amplitude of the waveform as Amplitude Ref $_{\text {. }}$

f. Clear the task.

4. Disable the function generator.

5. Set the function generator to $4.6 \mathrm{~Hz}$.

6. Acquire sample.
a. Create and configure an AI voltage task on the NI 9234 according to Table 19.
b. Configure the AI voltage task timing according to Table 20 .
c. Enable the function generator.
d. Start the task.
e. Record the amplitude of the waveform as Amplitude $_{\text {Acq }}$
f. Disable function generator.
g. Clear the task. 
7. Perform the following calculation using the recorded Amplitude ${ }_{\text {Ref }}$ and Amplitude Acq $_{\text {A }}$ values.

$$
(-100) \mathrm{mdB} \leq 20 \times \log _{10}\left(\frac{\text { Amplitude }_{\text {Acq }}}{\text { Amplitude }_{\text {Ref }}}\right)
$$

8. Repeat steps 1 through 7 for each channel.

If the AC Coupling Cutoff Response verification procedure determines that the NI 9234 is outside of the limits, refer to Worldwide Support and Services for assistance in returning the device to NI.

\section{IEPE Current Verification}

Complete the following procedure to determine the As-Found status of the NI 9234.

1. Connect the NI 9234 to the DMM as shown in Figure 7.

Figure 7. IEPE Current Verification Connections to the NI 9234

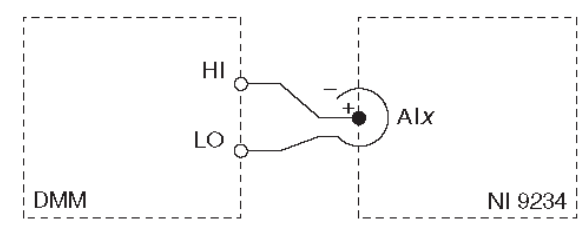

2. Configure the DMM for a DC current measurement in the lowest appropriate range to measure $2 \mathrm{~mA}$.

3. Measure IEPE Source short circuit current.

a. Create an AI voltage task on the NI 9234 according to Table 21.

Table 21. NI 9234 Configuration for IEPE Current Verification

\begin{tabular}{|c|c|c|c|c|}
\hline Min (V) & Max (V) & $\begin{array}{c}\text { Scaled } \\
\text { Units }\end{array}$ & $\begin{array}{c}\text { Channel } \\
\text { Configuration }\end{array}$ & IEPE Excitation \\
\hline \hline-5 & 5 & Volts & AC Coupled & Enabled \\
\hline
\end{tabular}

b. Configure the AI voltage task timing according to Table 22 .

Table 22. NI 9234 Timing Configuration

\begin{tabular}{|c|c|}
\hline Sample Mode & Rate $(\mathbf{H z})$ \\
\hline \hline Continuous Samples & 51200 \\
\hline
\end{tabular}

c. Start the task.

d. Record the DMM measurement.

e. Clear the task.

14 | ni.com | NI 9234 Calibration Procedure 
4. Compare the DMM measurement to the minimum current limit of $2.0 \mathrm{~mA}$.

5. Repeat steps 1 through 4 for each channel.

If the IEPE Current verification procedure determines that the NI 9234 is outside of the limits, refer to Worldwide Support and Services for assistance in returning the device to NI.

\section{IEPE Compliance Voltage Verification}

Complete the following procedure to determine the As-Found status of the NI 9234

1. Connect the NI 9234 to the DMM as shown in Figure 8.

Figure 8. IEPE Compliance Voltage Verification Connections to the NI 9234 (IEPE Source Short Circuit Current Measurement)

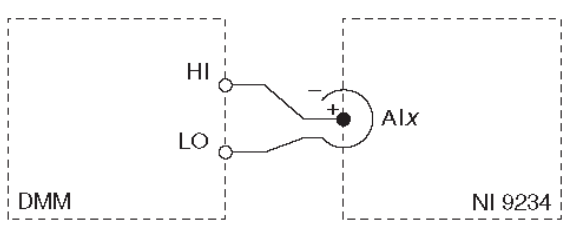

2. Configure the DMM for a DC current measurement in the lowest appropriate range to measure $2 \mathrm{~mA}$.

3. Measure IEPE Source short circuit current.

a. Create an AI voltage task on the NI 9234 according to Table 23.

Table 23. NI 9234 Configuration for IEPE Voltage Verification

\begin{tabular}{|c|c|}
\hline Channel Configuration & IEPE Excitation \\
\hline \hline AC Coupled with IEPE & Enabled \\
\hline
\end{tabular}

b. Configure the AI voltage task timing according to Table 24 .

Table 24. NI 9234 Timing Configuration

\begin{tabular}{|c|c|}
\hline Sample Mode & Rate (Hz) \\
\hline \hline Continuous Samples & 51200 \\
\hline
\end{tabular}

c. Start the task.

d. Record the DMM measurement DMM Current $_{\text {- }}$

e. Perform the following calculation using the recorded $\mathrm{DMM}_{\text {Current }}$ value.

$$
S M U_{\text {SourceCurrent }}=-\left(0.95 \times D M M_{\text {Current }}\right)
$$

4. Record the calculation result as $\mathrm{SMU}_{\text {SourceCurrent }}$ 
5. Connect the SMU to the channel you want to verify on the NI 9234. Refer to Figure 9 for a connection diagram.

Figure 9. IEPE Compliance Voltage Verification Connections to the $\mathrm{NI} 9234$ (IEPE Source Compliance Voltage Measurement)

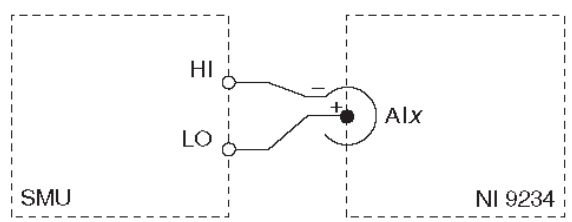

6. Configure the SMU as a current source and set the voltage limit to the lowest appropriate range to measure $24 \mathrm{~V}$.

7. Measure IEPE Source compliance voltage.

a. Create and configure an AI voltage task on the NI 9234 according to Table 25.

Table 25. IEPE Compliance Verification Equipment Configuration

\begin{tabular}{|c|c|}
\hline Channel Configuration & IEPE Excitation \\
\hline \hline AC Coupled with IEPE & Enabled \\
\hline
\end{tabular}

b. Configure the AI voltage task timing according to Table 26 .

Table 26. NI 9234 Timing Configuration

\begin{tabular}{|c|c|}
\hline Sample Mode & Rate $(\mathrm{Hz})$ \\
\hline \hline Continuous Samples & 51200 \\
\hline
\end{tabular}

c. Set the source current on the SMU to the recorded $\mathrm{SMU}_{\text {SourceCurrent }}$ value.

d. Start the task.

e. Enable the SMU.

f. Record the SMU measurement $\mathrm{SMU}_{\text {Voltage. }}$

g. Disable the SMU.

h. Clear the task.

8. Compare the voltage measurement from the SMU to the minimum voltage limit of $19 \mathrm{~V}$.

9. Repeat steps 1 through $\mathrm{h}$ for each channel.

If the IEPE Compliance Voltage verification procedure determines that the NI 9234 is outside of the limits, refer to Worldwide Support and Services for assistance in returning the device to NI.

16 | ni.com | NI 9234 Calibration Procedure 


\section{Adjustment}

The following performance adjustment procedure describes the sequence of operations required to adjust the NI 9234.

\section{Accuracy Adjustment}

Complete the following procedure to adjust the accuracy performance of the NI 9234.

1. Set the calibrator to Standby mode (STBY).

2. Connect the NI 9234 to the calibrator as shown in Figure 1.

3. Adjust the NI 9234.

a. Initialize a calibration session on the NI 9234. The default password is NI.

b. Input the external temperature in degrees Celsius.

c. Call the NI 9234 get C Series adjustment points function to obtain an array of recommended calibration voltages for the NI 9234.

d. Set the calibrator to a reference value determined by the array of recommended calibration voltages

e. Set the calibrator to Operate mode (OPR).

f. Call and configure the NI 9234 adjustment function according to Table 27.

Table 27. Adjustment Configuration

\begin{tabular}{|c|c|}
\hline Physical Channel & Reference Value \\
\hline \hline cDAQMod8/aix & The reference value from the array of adjustment points \\
\hline
\end{tabular}

g. Set the calibrator to Standby mode (STBY).

h. Repeat steps $d$ through $g$ for each calibration voltage in the array.

i. Close the calibration session.

4. Disconnect the NI 9234 from the calibrator.

5. Repeat steps 1 through 4 for each channel on the NI 9234.

\section{EEPROM Update}

When an adjustment procedure is completed, the NI 9234 internal calibration memory (EEPROM) is immediately updated.

If you do not want to perform an adjustment, you can update the calibration date and onboard calibration temperature without making any adjustments by initializing an external calibration, setting the $\mathrm{C}$ Series calibration temperature, and closing the external calibration. 


\section{Reverification}

Repeat the Verification section to determine the As-Left status of the device.

Note If any test fails Reverification after performing an adjustment, verify that you have met the Test Conditions before returning your device to NI. Refer to Worldwide Support and Services for assistance in returning the device to NI.

\section{Accuracy Under Calibration Conditions}

The following calibration specifications are valid under the following conditions:

- Ambient temperature $23 \pm 5^{\circ} \mathrm{C}$

- $\quad$ NI 9234 installed in slot 8 of an NI cDAQ-9178 chassis

- Slots 1 through 7 of the NI cDAQ-9178 chassis are empty

Note The test limits listed in Table 4 are derived using the values in Table 28.

Table 28. NI 9234 Accuracy Under Calibration Conditions

\begin{tabular}{|c|c|}
\hline \multicolumn{1}{|c|}{ Percent of Reading (Gain Error) } & Percent of Range ${ }^{*}$ (Offset Error) \\
\hline \hline $\pm 0.09 \%$ & $\pm 0.024 \%( \pm 1.2 \mathrm{mV})$ \\
\hline${ }^{*}$ Range equals $5.1 \mathrm{~V}$ \\
\hline
\end{tabular}

Note For operational specifications, refer to the most recent NI 9234 Operating Instructions and Specifications online at $\mathrm{n} i . \mathrm{com} / \mathrm{manuals.}$

18 | ni.com | NI 9234 Calibration Procedure 


\section{Worldwide Support and Services}

The National Instruments website is your complete resource for technical support. At ni .com/ support you have access to everything from troubleshooting and application development self-help resources to email and phone assistance from NI Application Engineers.

Visit $\mathrm{n}$ i.com/services for NI Factory Installation Services, repairs, extended warranty, and other services.

Visit ni.com/register to register your National Instruments product. Product registration facilitates technical support and ensures that you receive important information updates from NI

A Declaration of Conformity (DoC) is our claim of compliance with the Council of the European Communities using the manufacturer's declaration of conformity. This system affords the user protection for electromagnetic compatibility (EMC) and product safety. You can obtain the DoC for your product by visiting $\mathrm{ni} . \mathrm{com} / \mathrm{certification.} \mathrm{If} \mathrm{your} \mathrm{product} \mathrm{supports} \mathrm{calibration,}$ you can obtain the calibration certificate for your product at $\mathrm{ni}$. com/calibration.

National Instruments corporate headquarters is located at 11500 North Mopac Expressway, Austin, Texas, 78759-3504. National Instruments also has offices located around the world. For telephone support in the United States, create your service request at $\mathrm{ni}$. com/support or dial 512795 8248. For telephone support outside the United States, visit the Worldwide Offices section of $\mathrm{ni}$.com/niglobal to access the branch office websites, which provide up-to-date contact information, support phone numbers, email addresses, and current events.

\footnotetext{
Refer to the NI Trademarks and Logo Guidelines at ni. com/trademarks for more information on National Instruments trademarks. Other product and company names mentioned herein are trademarks or trade names of their respective companies. For patents covering National Instruments products/technology, refer to the app rop riate location: Help sPatents in your software, the patents. txut file on your media, or the National Instruments Patents Notice at $\mathrm{n}$ i . com/pa tents. You can find information about end-user license agreements (EULAS) and third-party legal notices in the readme file for your NI product. Refer to the Export Comoliance Information at $\mathrm{ni}$. com/legal/export-compliance for the National Instruments global trade compliance policy and how to obtain relevant HTS codes, ECCNs, and other importtexport data.

C 2009-2013 National Instruments. All rights resenved. 


\section{OPERATING INSTRUCTIONS AND SPECIFICATIONS}

\section{NI 9234}

4-Channel, \pm 5 V, 24-Bit Software-Selectable

IEPE and AC/DC Analog Input Module

\begin{tabular}{|ccc|}
\hline Français & Deutsch 日本語 한국어 & 简体中文 \\
\hline \multicolumn{4}{c}{$\mathrm{ni.com} / \mathrm{manuals}$} \\
\hline
\end{tabular}

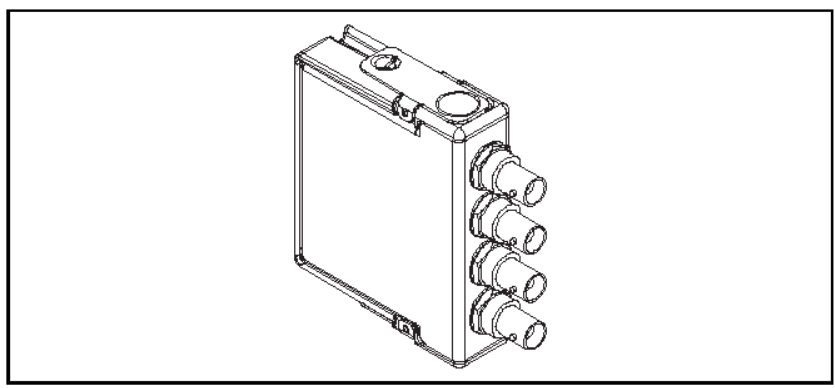

\section{TNATIONAL INSTRUMENTS}

This document describes how to use the National Instruments 9234 and includes specifications and connector assignments for the NI 9234. Visit ni.com/info and enter rdso ftwareversion to determine which software you need for the modules you are using. For information about installing, configuring, and programming the system, refer to the system documentation. Visit ni . com/info and enter cseriesdoc for information about C Series documentation.

Note The safety guidelines and specifications in this document are specific to the NI 9234. The other components in the system might not meet the same safety ratings and specifications. Refer to the documentation for each component in the system to determine the safety ratings and specifications for the entire system. Visit ni.com/info and enter cseriesdoc for information about $\mathrm{C}$ Series documentation. 


\section{Safety Guidelines}

Operate the NI 9234 only as described in these operating instructions.

Hot Surface This icon denotes that the component may be m hot. Touching this component may result in bodily injury.

\section{Safety Guidelines for Hazardous Locations}

The NI 9234 is suitable for use in Class I, Division 2, Groups A, B, C, D, T4 hazardous locations; Class I, Zone 2, AEx nA IIC T4, and Ex nA IIC T4 hazardous locations; and nonhazardous locations only. Follow these guidelines if you are installing the NI 9234 in a potentially explosive environment. Not following these guidelines may result in serious injury or death.
$\triangle$
Caution Do not disconnect I/O-side wires or connectors unless power has been switched off or the area is known to be nonhazardous.
Caution Do not remove modules unless power has been
switched off or the area is known to be nonhazardous.

\section{Caution Substitution of components may impair} suitability for Class I, Division 2.

Caution For Zone 2 applications, install the system in an enclosure rated to at least IP 54 as defined by IEC 60529 and EN 60529.

1. Caution For Zone 2 applications, connected signals must be within the following limit:

Capacitance $0.2 \mu \mathrm{F} \max$

\section{Special Conditions for Hazardous Locations Use in Europe} This equipment has been evaluated as Ex nA IIC T4 equipment under DEMKO Certificate No. 07 ATEX 0626664X. Each module is marked $\Theta_{x}$ II $3 \mathrm{G}$ and is suitable for use in Zone 2 hazardous locations. If you are using the NI 9234 in Gas Group IIChazardous locations or in ambient temperatures of $-40^{\circ} \mathrm{C} \leq \mathrm{Ta} \leq 70^{\circ} \mathrm{C}$, you must use the device in an NI chassis that has been evaluated as EEx nC IIC T4, Ex nA IIC T4, or Ex nL IIC T4 equipment. 


\section{Special Conditions for Marine Applications}

Some modules are Lloyd's Register (LR) Type Approved for marine applications. To verify Lloyd's Register certification, visit ni.com/certification and search for the LR certificate, or look for the Lloyd's Register mark on the module.
1. Caution To meet radio frequency emission requirements for marine applications, use shielded cables and install the system in a metal enclosure. Suppression ferrites must be installed on power supply inputs near power entries to modules and controllers. Power supply and module cables must be separated on opposite sides of the enclosure and must enter and exit through opposing enclosure walls.

\section{Connecting the NI 9234}

The NI 9234 has four BNC connectors that provide connections to four simultaneously sampled analog input channels.

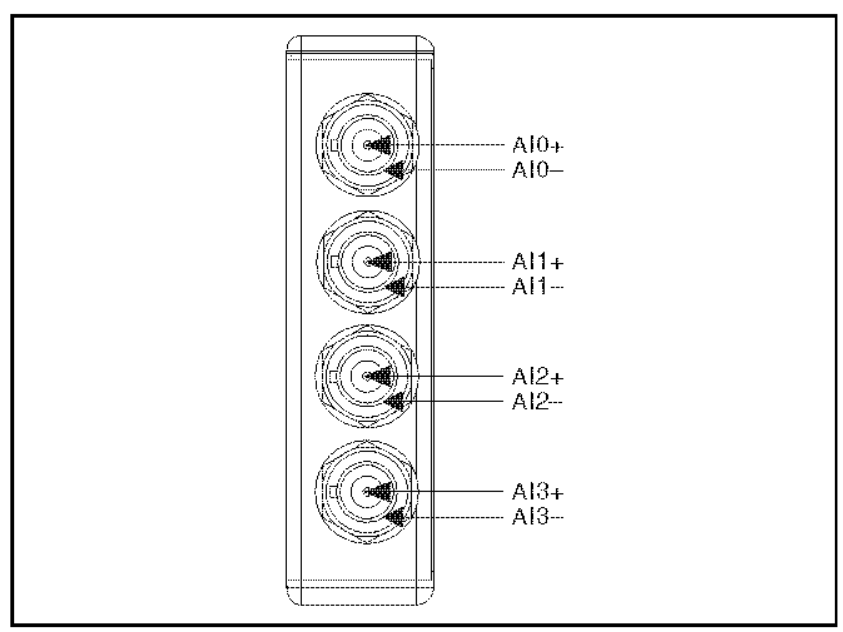

Figure 1. NI 9234 Connector Assignments 
Each channel has a BNC connector to which you can connect a signal source. You can also enable excitation current on a per-channel basis to connect Integrated Electronics Piezoelectric (IEPE) sensors. Refer to the NI 9234 Circuitry section for more information. The center pin of the connector, $\mathrm{AI}+$, provides the DC excitation, when enabled, and the positive input signal connection. The shell of the connector, $\mathrm{AI}-$, provides the excitation return path and the signal ground reference.

\section{Connecting Signal Sources to the NI 9234}

You can connect ground-referenced or floating signal sources to the NI 9234. To avoid picking up ground noise, use a floating connection. To further minimize ground noise, prevent the metal shells of the BNC connectors from coming in contact with each other, the modules, or the chassis.

If you make a ground-referenced connection between the signal source and the NI 9234, make sure the voltage on the AI- shell is in the common-mode range to ensure proper operation of the NI 9234. The AI- shell is protected against accidental contact with overvoltages within the overvoltage protection range. Refer to the Specifications section for more information about operating voltages and overvoltage protection.

Refer to Figures 2 and 3 for illustrations of connecting grounded and floating signal sources to the NI 9234.

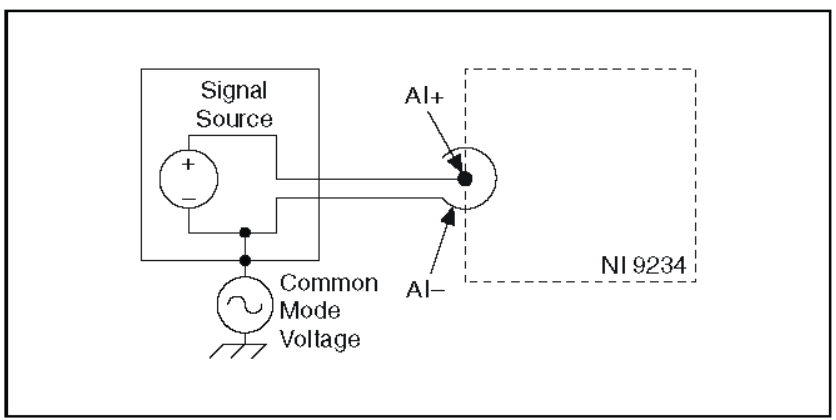

Figure 2. Connecting a Grounded Signal Source to the NI 9234 


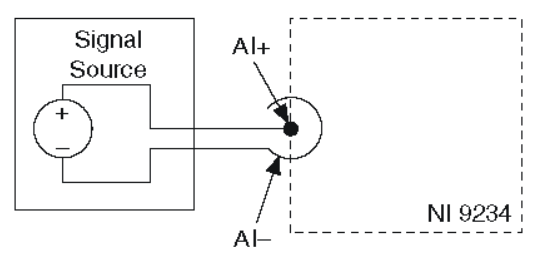

Figure 3. Connecting a Floating Signal Source to the NI 9234

The NI 9234 can also provide an IEPE excitation current for each channel to measure ground-referenced or floating IEPE sensors. Typical IEPE sensors have a case that is electrically isolated from the IEPE electronics, so connecting the sensor to the NI 9234 results in a floating connection even though the case of the sensor is grounded.

\section{NI 9234 Circuitry}

The NI 9234 analog input channels are referenced to chassis ground through a $50 \Omega$ resistor. To minimize ground noise, make sure the chassis ground is connected to earth ground. Each channel is protected from overvoltages. The input signal on each channel is buffered, conditioned, and then sampled by a 24-bit Delta-Sigma $\mathrm{ADC}$. You can configure each channel in software for AC or DC coupling. For channels set to AC coupling, you can turn the IEPE excitation current on or off. Refer to the software help for information about configuring channels on the NI 9234. 


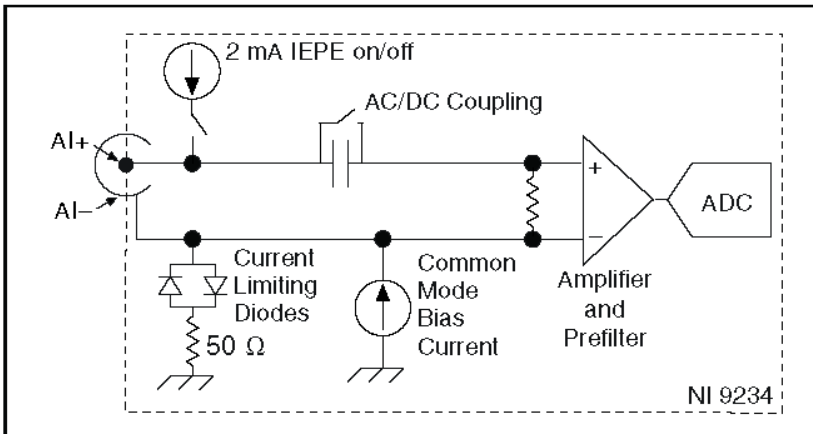

Figure 4. NI 9234 Input Circuitry for One Channel

The NI 9234 uses common-mode bias current to bias the current-limiting diodes when IEPE current is turned off. When the NI 9234 is using grounded signal sources, this current causes an error that is dependent on the AI- lead impedance. This error is approximately $50 \mathrm{ppm}$ of range and $15 \mathrm{ppm}$ of reading per $\Omega$ of $\mathrm{AI}-$ impedance. The common-mode bias current causes an error only with grounded sources and is not an issue with floating signal sources. For best accuracy, use a floating connection or use low-impedance leads when connecting grounded signal sources.

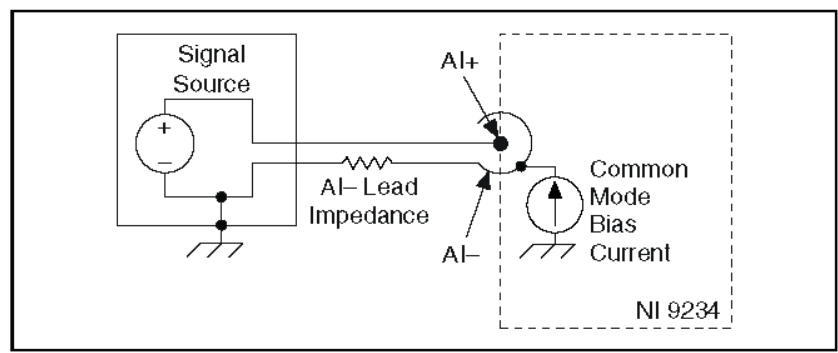

Figure 5. Measurement Error Introduced by Common-Mode Bias Current

The NI 9234 also has TEDS circuitry. For more information about TEDS, go to ni.com/info and enter rateds. 


\section{Understanding NI 9234 Filtering}

The NI 9234 uses a combination of analog and digital filtering to provide an accurate representation of in-band signals while rejecting out-of-band signals. The filters discriminate between signals based on the frequency range, or bandwidth, of the signal. The three important bandwidths to consider are the passband, the stopband, and the alias-free bandwidth.

The NI 9234 represents signals within the passband, as quantified primarily by passband flatness and phase nonlinearity. All signals that appear in the alias-free bandwidth are either unaliased signals or signals that have been filtered by at least the amount of the stopband rejection.

\section{Passband}

The signals within the passband have frequency-dependent gain or attenuation. The small amount of variation in gain with respect to frequency is called the passband flatness. The digital filters of the NI 9234 adjust the frequency range of the passband to match the data rate. Therefore, the amount of gain or attenuation at a given frequency depends on the data rate. Figure 6 shows typical passband flatness for the NI 9234.

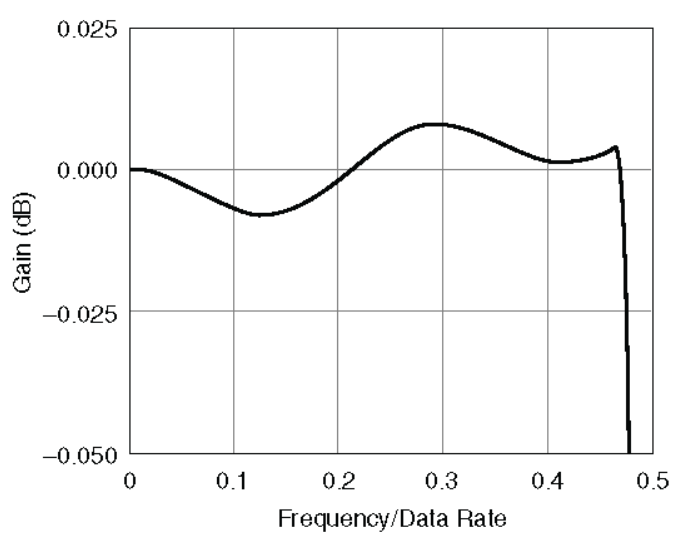

Figure 6. NI 9234 Typical Passband Flatness 


\section{Stopband}

The filter significantly attenuates all signals above the stopband frequency. The primary goal of the filter is to prevent aliasing. Therefore, the stopband frequency scales precisely with the data rate. The stopband rejection is the minimum amount of attenuation applied by the filter to all signals with frequencies within the stopband.

\section{Alias-Free Bandwidth}

Any signal that appears in the alias-free bandwidth of the NI 9234 is not an aliased artifact of signals at a higher frequency. The alias-free bandwidth is defined by the ability of the filter to reject frequencies above the stopband frequency, and it is equal to the data rate minus the stopband frequency.

\section{Understanding NI 9234 Data Rates}

The frequency of a master timebase $\left(f_{M}\right)$ controls the data rate $\left(f_{s}\right)$ of the NI 9234. The NI 9234 includes an internal master timebase with a frequency of $13.1072 \mathrm{MHz}$, but the module also can accept an external master timebase or export its own master timebase. To synchronize the data rate of an NI 9234 with other modules that use master timebases to control sampling, all of the modules must share a single master timebase source. Refer to the software help for information about configuring the master timebase source for the NI 9234. Visit ni.com/info and enter cseriesdoc for information about $\mathrm{C}$ Series documentation.

The following equation provides the available data rates of the NI 9234:

$f_{s}=\frac{f_{M} \div 256}{n}$

where $n$ is any integer from 1 to 31 .

However, the data rate must remain within the appropriate data rate range. Refer to the Specifications section for more information about the data rate range. When using the internal master timebase 
of $13.1072 \mathrm{MHz}$, the result is data rates of $51.2 \mathrm{kS} / \mathrm{s}, 25.6 \mathrm{kS} / \mathrm{s}$, $17.067 \mathrm{kS} / \mathrm{s}$, and so on down to $1.652 \mathrm{kS} / \mathrm{s}$, depending on the value of $n$. When using an external timebase with a frequency other than $13.1072 \mathrm{MHz}$, the NI 9234 has a different set of data rates.

Note The cRIO-9151 R Series Expansion chassis does not support sharing timebases between modules.

\section{Sleep Mode}

This module supports a low-power sleep mode. Support for sleep mode at the system level depends on the chassis that the module is plugged into. Refer to the chassis manual for information about support for sleep mode. If the chassis supports sleep mode, refer to the software help for information about enabling sleep mode. Visit ni.com/info and enter cseriesdoc for information about C Series documentation.

Typically, when a system is in sleep mode, you cannot communicate with the modules. In sleep mode, the system consumes minimal power and may dissipate less heat than it does in normal mode. Refer to the Specifications section for more information about power consumption and thermal dissipation.

\section{Specifications}

The following specifications are typical for the range -40 to $70^{\circ} \mathrm{C}$ unless otherwise noted.

\section{Input Characteristics}

Number of channels. 4 analog input channels

$\mathrm{ADC}$ resolution 24 bits

Type of ADC Delta-Sigma (with analog prefiltering)

Sampling mode Simultaneous

Type of TEDS supported IEEE 1451.4 TEDS Class I

Internal master timebase $\left(f_{M}\right)$

Frequency $13.1072 \mathrm{MHz}$

Accuracy \pm 50 ppm max

Data rate range $\left(f_{s}\right)$ using internal master timebase

Minimum $1.652 \mathrm{kS} / \mathrm{s}$

Maximum $51.2 \mathrm{kS} / \mathrm{s}$ 
Data rate range $\left(f_{s}\right)$ using external master timebase

Minimum

$0.391 \mathrm{kS} / \mathrm{s}$

Maximum $52.734 \mathrm{kS} / \mathrm{s}$

Data $\operatorname{rates}^{1}\left(f_{s}\right)$ $\frac{f_{M} \div 256}{n}, n=1,2, \ldots, 31$

Input coupling AC/DC (software-selectable)

$\mathrm{AC}$ cutoff frequency

$-3 \mathrm{~dB}$. $0.5 \mathrm{~Hz}$

$-0.1 \mathrm{~dB}$ $4.6 \mathrm{~Hz} \max$

\footnotetext{
${ }^{1}$ The data rate must remain within the appropriate data rate range. Refer to the Understanding NI 9234 Data Rates section for more information.

(C) National Instruments Corp

19 N/ 9234 Operating Instrustions and Specifications
}

$\mathrm{AC}$ cutoff frequency response

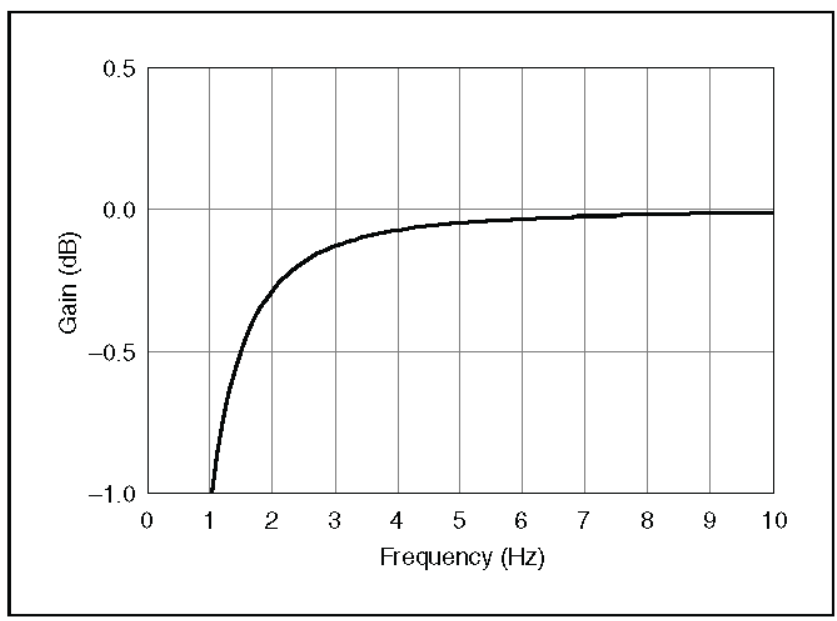




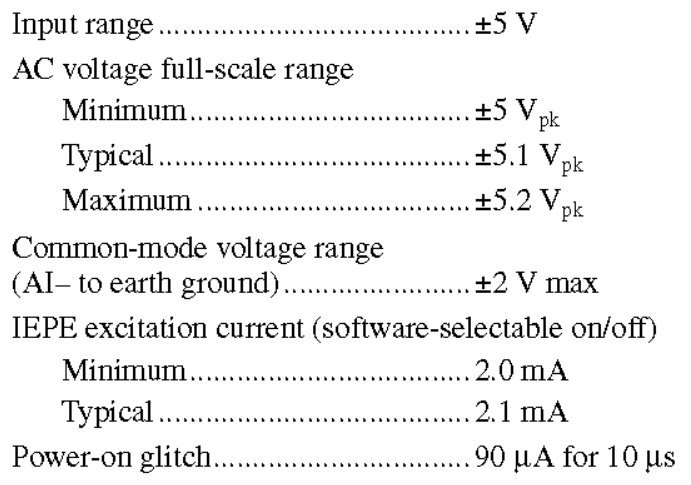

If you are using an IEPE sensor, use the following equation to make sure your configuration meets the IEPE compliance voltage range.

$$
\left(V_{\text {common-mode }}+V_{\text {bias }} \pm V_{\text {full-scale }}\right) \text { must be } 0 \text { to } 19
$$

where $\quad V_{\text {common-mode }}$ is the common-mode voltage applied to the NI 9234

$V_{\text {bias }}$ is the bias voltage of the IEPE sensor

$V_{\text {full-scale }}$ is the full-scale voltage of the IEPE sensor

Overvoltage protection (with respect to chassis ground)

For a signal source connected

to $\mathrm{AI}+$ and $\mathrm{AI}-$ $\pm 30 \mathrm{~V}$

For a low-impedance source

connected to $\mathrm{AI}+$ and $\mathrm{AI}-\ldots . . . . . .-6$ to $30 \mathrm{~V}$

Input delay $38.4 / f_{s}+3.2 \mu \mathrm{s}$ 
Accuracy $^{1}$

\begin{tabular}{|l|c|c|}
\hline Measurement Conditions & $\begin{array}{c}\text { Percent of } \\
\text { Reading } \\
\text { (Gain Error) }\end{array}$ & $\begin{array}{c}\text { Percent of } \\
\text { Range* } \\
\text { (Offset Error) }\end{array}$ \\
\hline \hline Calibrated max $\left(-40\right.$ to $\left.70^{\circ} \mathrm{C}\right)$ & $\begin{array}{c}0.34 \%, \\
\pm 0.03 \mathrm{~dB}\end{array}$ & $\begin{array}{c} \pm 0.14 \%, \\
7.1 \mathrm{mV}\end{array}$ \\
\hline Calibrated typ $\left(25^{\circ} \mathrm{C} \pm 5^{\circ} \mathrm{C}\right)$ & $0.05 \%$, & $\pm 0.006 \%$, \\
& $\pm 0.005 \mathrm{~dB}$ & $0.3 \mathrm{mV}$ \\
\hline Uncalibrated $\max \left(-40\right.$ to $\left.70^{\circ} \mathrm{C}\right)$ & $1.9 \%$, & $\pm 0.27 \%$, \\
& $\pm 0.16 \mathrm{~dB}$ & $13.9 \mathrm{mV}$ \\
\hline Uncalibrated typ $\left(25^{\circ} \mathrm{C} \pm 5^{\circ} \mathrm{C}\right)$ & $0.48 \%$, & $\pm 0.04 \%$, \\
& $\pm 0.04 \mathrm{~dB}$ & $2.3 \mathrm{mV}$ \\
\hline${ }^{*}$ Range $=5.1 \mathrm{~V}_{\mathrm{pk}}$ & \multicolumn{2}{|l}{} \\
\hline
\end{tabular}

\footnotetext{
${ }^{1}$ Refer to the NI 9234 Circuitry section for information regarding grounded signal sources and measurement accuracy.

( ) National instruments Corp

23 N/9234 Operating Instructions and Specifications
}

Gain drift

Typical ............................. $0.14 \mathrm{mdB} /{ }^{\circ} \mathrm{C}\left(16 \mathrm{ppm} /{ }^{\circ} \mathrm{C}\right)$

Maximum ............................ $0.45 \mathrm{mdB} /{ }^{\circ} \mathrm{C}\left(52 \mathrm{ppm} /{ }^{\circ} \mathrm{C}\right)$

Offset drift

Typical ............................. 19.2 $\mu \mathrm{V} /{ }^{\circ} \mathrm{C}$

Maximum .............................. $118 \mu \mathrm{V} /{ }^{\circ} \mathrm{C}$

Channel-to-channel matching

Gain

Typical............................... $0.01 \mathrm{~dB}$

Maximum........................ $0.04 \mathrm{~dB}$

Phase $\left(f_{\text {in }}\right.$ in $\left.\mathrm{kHz}\right)$........................ $f_{\text {in }} \cdot 0.045^{\circ}+0.04 \max$

Passband

Frequency .............................. $0.45 \cdot f_{s}$

Flatness $\left(f_{s}=51.2 \mathrm{kS} / \mathrm{s}\right) \ldots \ldots \ldots \ldots \pm 40 \mathrm{mdB}$ (pk-to-pk max)

Phase nonlinearity

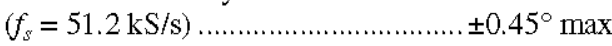

Stopband

Frequency .............................. $0.55 \cdot f_{s}$

Rejection............................... $100 \mathrm{~dB}$

N 9234 Operating instructions and Specifications 24 ni.com 
Alias-free bandwidth $0.45 \cdot f_{s}$

Oversample rate $64 \cdot f_{s}$

Crosstalk $(1 \mathrm{kHz})$ $-110 \mathrm{~dB}$

$\operatorname{CMRR}\left(f_{i n} \leq 1 \mathrm{kHz}\right)$

Minimum $40 \mathrm{~dB}$

Typical $47 \mathrm{~dB}$

$\operatorname{SFDR}\left(f_{i n}=1 \mathrm{kHz},-60 \mathrm{dBFS}\right) \ldots \ldots . .120 \mathrm{~dB}$

Idle channel noise and noise density

\begin{tabular}{|l|c|c|c|}
\hline Idle Channel & $\mathbf{5 1 . 2} \mathbf{~ k S / s}$ & $\mathbf{2 5 . 6} \mathbf{~ k S / s}$ & $\mathbf{2 . 0 4 8} \mathbf{~ k S / s}$ \\
\hline \hline Noise & $97 \mathrm{dBFS}$ & $99 \mathrm{dBFS}$ & $103 \mathrm{dBFS}$ \\
\cline { 2 - 4 } & $50 \mu \mathrm{V}_{\mathrm{rms}}$ & $40 \mu \mathrm{V}_{\mathrm{rms}}$ & $25 \mu \mathrm{V}_{\mathrm{rms}}$ \\
\hline Noise density & $310 \mathrm{nV} / \mathrm{Hz}$ & $350 \mathrm{nV} / \mathrm{Hz}$ & $780 \mathrm{nV} / \sqrt{\mathrm{Hz}}$ \\
\hline
\end{tabular}

Input impedance

Differential $305 \mathrm{k} \Omega$

$\mathrm{AI}-$ (shield) to chassis ground.... $50 \Omega$

Total harmonic distortion (THD)

\begin{tabular}{|c|c|c|}
\hline $\begin{array}{c}\text { Input } \\
\text { Amplitude }\end{array}$ & $\mathbf{1} \mathbf{~ k H z}$ & $\mathbf{8} \mathbf{~ k H z}$ \\
\hline \hline$-1 \mathrm{dBFS}$ & $-95 \mathrm{~dB}$ & $-87 \mathrm{~dB}$ \\
\hline$-20 \mathrm{dBFS}$ & $-95 \mathrm{~dB}$ & $-80 \mathrm{~dB}$ \\
\hline
\end{tabular}

Intermodulation distortion ( $-1 \mathrm{dBFS})$

DIN $250 \mathrm{~Hz} / 8 \mathrm{kHz}$

4:1 amplitude ratio .... $-80 \mathrm{~dB}$

CCIF $11 \mathrm{kHz} / 12 \mathrm{kHz}$

1:1 amplitude ratio $-93 \mathrm{~dB}$

MTBF 390,362 hours at $25^{\circ} \mathrm{C}$; Bellcore Issue 2, Method 1, Case 3, Limited Part Stress Method

2 Note Contact NI for Bellcore MTBF specifications at other temperatures or for MIL-HDBK-217F specifications. 


\section{Power Requirements}

Power consumption from chassis

Active mode $900 \mathrm{~mW} \max$

Sleep mode $25 \mu \mathrm{W} \max$

Thermal dissipation $\left(\right.$ at $70^{\circ} \mathrm{C}$ )

Active mode $930 \mathrm{~mW} \max$

Sleep mode $25 \mu \mathrm{W} \max$

\section{Physical Characteristics}

If you need to clean the module, wipe it with a dry towel.

Weight $173 \mathrm{~g}(6.1 \mathrm{oz})$

\section{Safety}

\section{Safety Voltages}

Connect only voltages that are within the following limits.

Channel-to-earth ground. $\pm 30 \mathrm{~V} \max$ Measurement Category I

Isolation

Channel-to-channel None

Channel-to-earth ground None

Measurement Category I is for measurements performed on circuits not directly connected to the electrical distribution system referred to as MAINS voltage. MAINS is a hazardous live electrical supply system that powers equipment. This category is for measurements of voltages from specially protected secondary circuits. Such voltage measurements include signal levels, special equipment, limited-energy parts of equipment, circuits powered by regulated low-voltage sources, and electronics.

\footnotetext{
A Caution Do not connect the NI 9234 to signals or use for measurements within Measurement Categories II, III, or IV.
} 


\section{Hazardous Locations}

$\begin{aligned} & \text { U.S. (UL) ................................. Class I, Division 2, } \text { Groups A, B, C, D, T4; } \\ & \text { Class I, Zone 2, } \\ & \text { AEx nA IIC T4 } \\ & \text { Canada (C-UL) ........................... Class I, Division 2, } \text { Groups A, B, C, D, T4; } \\ & \text { Class I, Zone 2, } \text { Ex nA IIC T4 } \\ & \text { Europe (DEMKO) ........................ Ex nA IIC T4 }\end{aligned}$

\section{Safety Standards}

This product is designed to meet the requirements of the following standards of safety for electrical equipment for measurement, control, and laboratory use:

- IEC 61010-1, EN 61010-1

- UL 61010-1, CSA 61010-1

8 Note For UL and other safety certifications, refer to the product label or the Online Product Certification section.

\section{Electromagnetic Compatibility}

This product meets the requirements of the following EMC standards for electrical equipment for measurement, control, and laboratory use:

- EN 61326 (IEC 61326): Class A emissions; Basic immunity

- EN 55011 (CISPR 11): Group 1, Class A emissions

- AS/NZS CISPR 11: Group 1, Class A emissions

- FCC 47 CFR Part 15B: Class A emissions

- ICES-001: Class A emissions

4 Note For the standards applied to assess the EMC of this product, refer to the Online Product Certification section.

4 Note For EMC compliance, operate this device with shielded cabling. 


\section{cE Compliance $C \in$}

This product meets the essential requirements of applicable European Directives as follows:

- 2006/95/EC; Low-Voltage Directive (safety)

- 2004/108/EC; Electromagnetic Compatibility Directive (EMC)

\section{Online Product Certification}

Refer to the product Declaration of Conformity (DoC) for additional regulatory compliance information. To obtain product certifications and the DoC for this product, visit ni.com/ certification, search by module number or product line, and click the appropriate link in the Certification column.

\section{Shock and Vibration}

To meet these specifications, you must panel mount the system.

Operating vibration

Random (IEC 60068-2-64)........ $5 \mathrm{~g}_{\mathrm{rms}}, 10$ to $500 \mathrm{~Hz}$

Sinusoidal (IEC 60068-2-6) ....... 5 g, 10 to $500 \mathrm{~Hz}$

Operating shock

(IEC 60068-2-27) $30 \mathrm{~g}, 11 \mathrm{~ms}$ half sine, $50 \mathrm{~g}, 3 \mathrm{~ms}$ half sine, 18 shocks at 6 orientations

\section{Environmental}

National Instruments C Series modules are intended for indoor use only but may be used outdoors if installed in a suitable enclosure. Refer to the manual for the chassis you are using for more information about meeting these specifications.

Operating temperature (IEC 60068-2-1, IEC 60068-2-2) .....-40 to $70^{\circ} \mathrm{C}$

Storage temperature (IEC 60068-2-1, IEC 60068-2-2) .....-40 to $85^{\circ} \mathrm{C}$

Ingress protection. IP 40 


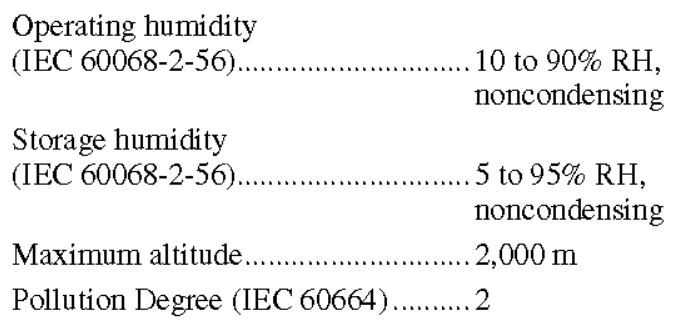

\section{Environmental Management}

$\mathrm{NI}$ is committed to designing and manufacturing products in an environmentally responsible manner. NI recognizes that eliminating certain hazardous substances from our products is beneficial to the environment and to NI customers.

For additional environmental information, refer to the $N I$ and the Environment Web page at ni.com/environment. This page contains the environmental regulations and directives with which NI complies, as well as other environmental information not included in this document.

\section{Waste Electrical and Electronic Equipment (WEEE)}

EU Customers At the end of their life cycle, all products must be sent to a WEEE recycling center. For more information about WEEE recycling centers and National Instruments WEEE initiatives, visit ni.com/ environment/weee.htm.

\section{电子信息产品污染控制管理办法（中国 RoHS）}

(2) 中国客户 National Instruments 符合中国电子信息 产品中限制使用某些有害物质指令 $(R O H S)$ 。关于 National Instruments 中国 RoHS 合规性信息, 请登录 ni.com/environment/rohs_china。 (For information about China RoHS compliance, go to ni. com/ environment/rohs_china.)

\section{Calibration}

You can obtain the calibration certificate and information about calibration services for the NI 9234 at ni.com/calibration. Calibration interval 1 year 


\section{Where to Go for Support}

The National Instruments Web site is your complete resource for technical support. At ni. com/support you have access to everything from troubleshooting and application development self-help resources to email and phone assistance from NI Application Engineers.

National Instruments corporate headquarters is located at 11500 North Mopac Expressway, Austin, Texas, 78759-3504. National Instruments also has offices located around the world to help address your support needs. For telephone support in the United States, create your service request at ni. com/support and follow the calling instructions or dial 5127958248 . For telephone support outside the United States, contact your local branch office:

Australia 1800300800 , Austria $43662457990-0$, Belgium 32 (0) 2757 0020, Brazil 551132623599 , Canada 800433 3488, China 862150509800 , Czech Republic 420224235774 , Denmark 4545762600 , Finland 358 (0) 972572511 , France 0157662424 , Germany 49897413130 , India 918041190000 , Israel 97236393737 , Italy 390241309277 , Japan 0120-527196,

Korea 820234513400 , Lebanon 961 (0) 1332828 , Malaysia 1800887710 , Mexico 018000100793 , Netherlands 31 (0) 348433 466, New Zealand 0800553 322, Norway 47 (0) 66907660 , Poland 48223390150 , Portugal 351210311210 , Russia 74957836851 , Singapore 18002265886 , Slovenia 38634254200 , South Africa 27011805 8197, Spain 34916400085 , Sweden 46 (0) 8587895 00, Switzerland 41562005151 , Taiwan 886022377 2222, Thailand 6622786777 , Turkey 90212279 3031, United Kingdom 44 (0) 1635523545

\footnotetext{
National Instruments, NI, ni.com, and LabVIEW are trademarks of National Instruments Corporation. Refer to the Tems of Use section on $\mathrm{ni}$. com/legal for more inf ormation about National Instruments trademarks. Other product and company names mentioned herein are trademarks or trade names of their respective companies. your software, the patents . txt file on your media, or the National instruments patent Notice at ni. com/patents.

(c) 2008 National Instruments Corp. All rights reserved.

$374238 \mathrm{C}-01$ Aug08
} 


$\begin{array}{lr}\text { DNATIONAL } & \text { Technical Sales } \\ \text { United States } \\ \text { (866) 531-6285 } \\ \text { info@ni.com }\end{array}$

Requirements and Compatibility | Ordering Information | Detailed Specifications | Pinoutsi'Front Panel Connections

For c ser manuals and dinim

Last Revised: 2012-11-13 13:01:50.0

NI 9472

$8 \mathrm{Ch}, 24 \mathrm{~V}$ Logic, $100 \mu$ s Sourcing C Series Digital Output Module

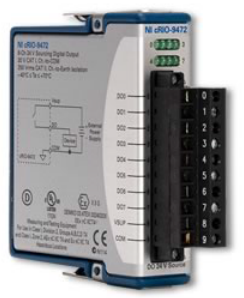

- \&channel, 100 us digital output

- Hot-swappable operation

- 6 to $30 \vee$ range, sourcing digital output

- D-Sub or screwterminal connector options

Overview

The $\mathbb{N} 19472$ is an eight-channel, 100 us sourcing digital output module for any $\mathrm{NI}$ CompactDAQ or CompactRl 0 chassis. Each channel is compatible with 6 to $30 \mathrm{~V}$ signals and features $2,300 \mathrm{~V}$ rms of transient overyoltage protection between the output channels and the backplane. Each channel also has an LED that indicates the state of that channel. With the N1 9472, you can connect directly to a variety of industrial devices such as motors, actuators, and relays. There are two connector options for the NI 9472 - a 10-position screw-terminal connector and a 25-position D-Sub connector. This industry-standard 25-position D-Sub connector provides for low-cost cabling to a wide variety of accessories from $\mathrm{NI}$ or other vendors. Many vendors with custom D-Sub cable fabrication services can deliver cables with a pinout that matches your exact application needs

Back to Top

Requirements and Compatibility

$\begin{array}{lll}\text { OS Information } & \text { Driver Information } & \text { Software Compatibility } \\ \text { - RearTime os } & \text { - N1-DAQmo } & \text { - LabVIEW } \\ \text { - Windows } & \text { - N1-RIO } & \text { - LabWindowsiCVI } \\ & & \text { - SignalExpress } \\ & \text { - Visual Studio } \\ & \text { - Visual Studio. NET }\end{array}$

Back to Top

Comparison Tables

\begin{tabular}{|c|c|c|c|c|c|c|}
\hline Product Name & Signal Levels & Number of Channels & Current $R$ ating & Speed & Connectivity & Special Features \\
\hline NN 9472 & 6 to $30 \mathrm{~V}$ & 8 & 750 mAich & $100 \mu \mathrm{s}$ & Screw-terminal, D-Sub & Sourcing digital output \\
\hline NI 9474 & 5 to $30 \mathrm{~V}$ & 8 & 1 Aich & $1 \mu \mathrm{s}$ & Screw terminal & Sourcing digital output \\
\hline NI 9475 & Up to $60 \mathrm{~V}$ & 8 & 1 Aich & 1 นs & 27-pin D-Sub & Sourcing digital output \\
\hline NN 9476 & 6 to $36 \mathrm{~V}$ & 32 & 250 maich & $500 \mu \mathrm{s}$ & 37-pin D-Sub & Sourcing digital output \\
\hline NI 9477 & 5 to $60 \mathrm{~V}$ & 32 & 1 Aich & $8 \mu \mathrm{s}$ & 37-pin D-Sub & Sinking digital output \\
\hline NN 9478 & 5 to $50 \mathrm{~V}$ & 16 & 1.2 Aich & $7 \mu \mathrm{s}$ & 37-pin D-Sub & Solenoid and valve drive modu \\
\hline
\end{tabular}

Back to Top 
Application and Technology

High-performance digital output and switching modules for NI CompactDAQ systems, CompactRIO embedded systems, and R Series expansion chassis provide extended voltage ranges and high-current-switching capacity for direct control of a wide array of industrial and automotive actuators. Each module features an integrated connector junction operating temperatures and 50 g shock

When used in CompactRIO, C Series digital output modules connect directly to reconfigurable I/O (RIO) field-programmable gate array (FPGA) hardware to create

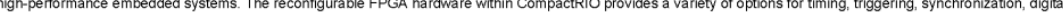
waveform generaton, or dighal comminicaton. For nstance, Wh

C Series Compatibility

The $\mathrm{C}$ Series hardware family features more than 50 measurement modules and several chassis and carriers for deployment. With this variety of modules, you can mix and match measurements such as temperature, acceleration, flow, pressure, strain, acoustic, voltage, current, digital, and more to create a custom system. Install the modules in one of (ive mixed-mis processor, RAM, and stora for an a LabVIEW software to achieve silicon-speed processing on I/O data from C Series modules.

Ordering Information

For a complete list of accessories, visit the product page on ni.com

\begin{tabular}{|c|c|c|c|}
\hline Products & Part Number & Recommended Accessories & Part Number \\
\hline
\end{tabular}

Software Recommendations

LabVIEW Professional
Development System for
Windows $\begin{aligned} & \begin{array}{l}\text { - Advanced software tools for large project } \\ \text { development } \\ \text { - Automatic code generation using DAQ } \\ \text { Assistant and Instrument I/O Assistant } \\ \text { - Tight integration with a wide range of } \\ \text { hardware }\end{array} \\ & \begin{array}{l}\text { - Advanced measurement analysis and digital } \\ \text { signal processing } \\ \text { - Open connectivity with DLLs, ActiveX, and } \\ \text {-NET objects } \\ \text { - Capability to build DLLs, executables, and } \\ \text { MSL installers }\end{array}\end{aligned}$

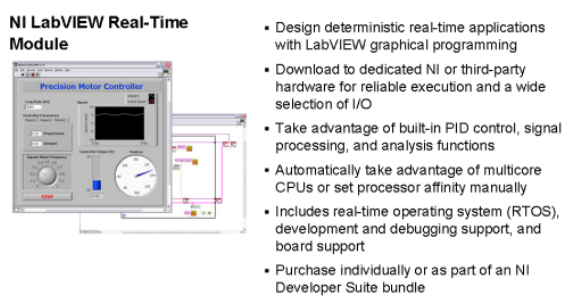

NILabVIEW FPGA Module

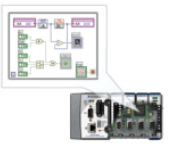

ifper.
- Create your own l/O har
coding or board design

Graphically configure FPGAs on NI

- Define your own control algorithms with loop

- Execute multiple tasks simultaneously and

-

- Implement custom timing and triggering logic,
digital protocols, and DSP algorithms

- Incorporate existing HDL code and third-party
IP including Xilinx CORE Generator functions 


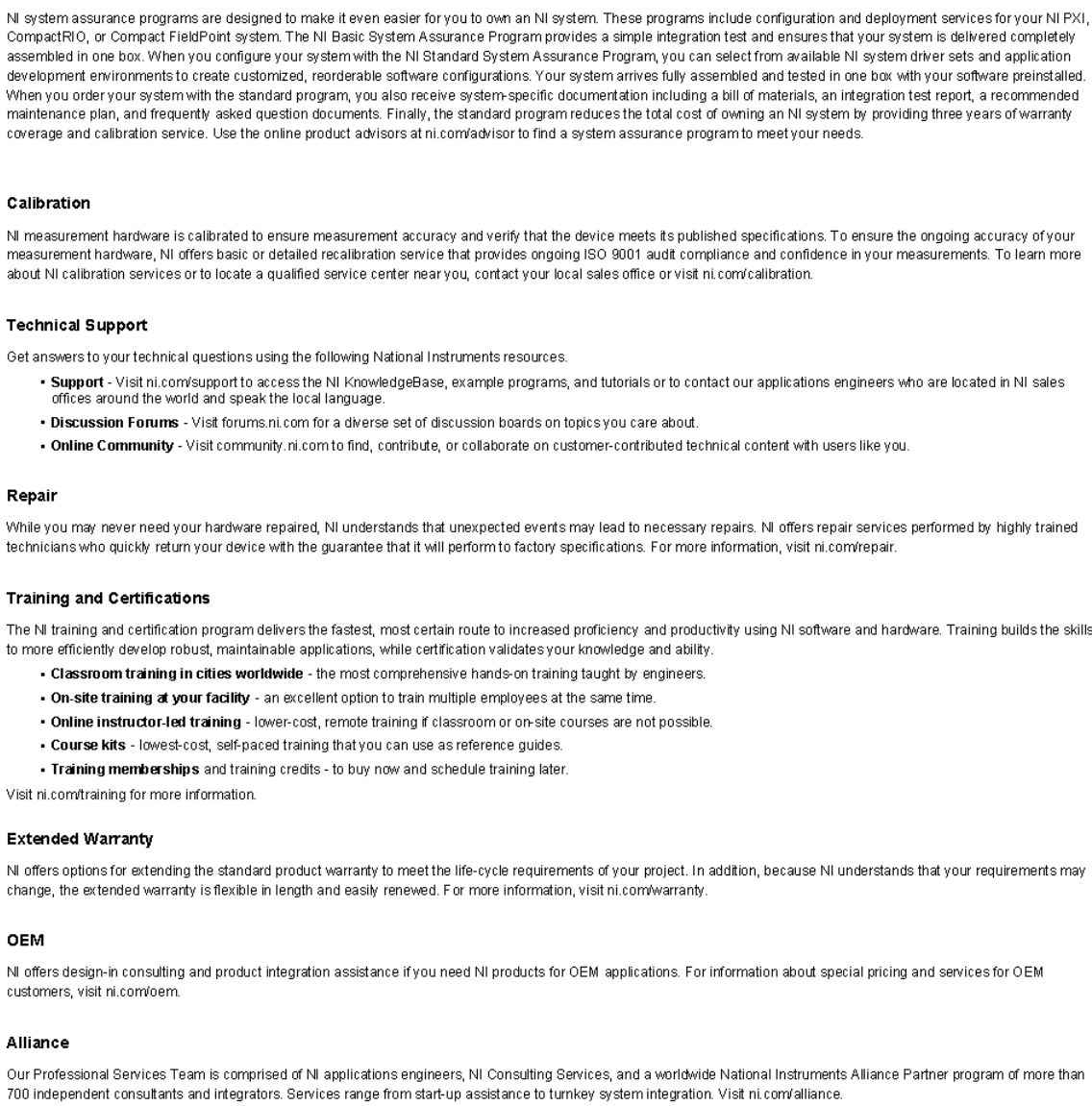

Detailed Specifications

The following specifications are typical for the range -40 to $70^{\circ} \mathrm{C}$ unless otherwise noted. All voltages are relative to $\mathrm{COM}$ unless otherwise noted. The specifications are the same for the NI 9472 and the N 9474 unless otherwise noted.

\section{Output Characteristics}

Number of channels

8 digital output channels

Output type

Power-on output state

Sourcing

External power supply voltage range $\left(V_{\text {sup }}\right)$

NN 9472

Channels of

NI 9474

6-30 VDC

5-30 VDC 


\begin{tabular}{|c|c|c|c|}
\hline \multicolumn{4}{|l|}{ Output impedance $\left(R_{\partial}\right)$} \\
\hline \multicolumn{3}{|l|}{ Typical } & $0.07 \Omega$ \\
\hline \multicolumn{3}{|l|}{ Maximum } & $0.13 \Omega$ \\
\hline \multicolumn{4}{|c|}{ Continuous output current $(\alpha)$, per channel } \\
\hline \multicolumn{3}{|l|}{ NI 9472} & $0.75 \mathrm{~A} \max$ \\
\hline \multicolumn{3}{|l|}{ N 19474} & $1 \mathrm{~A} \max$ \\
\hline \multicolumn{3}{|l|}{ Output voltage $\left(V_{\partial}\right)$} & $V_{\text {sup }}-\left(U_{0} \cdot R_{0}\right)$ \\
\hline \multicolumn{4}{|l|}{ Iro protection } \\
\hline \multicolumn{3}{|l|}{ Voltage } & 30 VDC $\max$ \\
\hline \multirow[t]{7}{*}{ Reversed voltage } & \multicolumn{3}{|r|}{ None } \\
\hline & \multicolumn{2}{|c|}{ Short-circuit behwior } & ehavior \\
\hline & Current & Channel Behavior & Module Protection \\
\hline & 0 to $1 \mathrm{~A}$ & Channel does not trip & Module is not damaged \\
\hline & 1 to $6 \mathrm{~A}$ & Channel does not trip & Module may be damaged \\
\hline & 6 to $13 \mathrm{~A}$ & Channel may trip & Module may be damaged \\
\hline & $=13 \mathrm{~A}$ & Channel trips & Module is not damaged \\
\hline Short-circuit trip time & & & $10 \mu \mathrm{s}$ at $13 \mathrm{~A}$ \\
\hline \multicolumn{4}{|l|}{ Output delay time (full load) } \\
\hline \multicolumn{3}{|l|}{ N 9472} & $100 \mu \mathrm{s} \max$ \\
\hline \multicolumn{3}{|l|}{ N 19474} & $1 \mu s \max$ \\
\hline \multicolumn{4}{|l|}{ MTEF } \\
\hline \multicolumn{3}{|l|}{ NN 9472} & $\begin{array}{l}1,113,301 \text { hours at } 25^{\circ} \mathrm{C} \text {; Bellcore Issue } 2 \text {, Method } 1 \text {, Case } 3 \text {, Limited Part } \\
\text { Stress Method }\end{array}$ \\
\hline NI 9474 & & & $\begin{array}{l}\text { 479,889 hours at } 25^{\circ} \mathrm{C} \text {; Bellcore Issue 2, Method } 1 \text {, Case 3, Limited Part } \\
\text { Stress Method }\end{array}$ \\
\hline Q6 Note Contact NI fo & ns at other te & emperatures or for MIL- - & HDEK-217F specifications. \\
\hline NI 9472 Power Requir & & & \\
\hline Power consumption from ch & & & \\
\hline Active mode & & & $230 \mathrm{~mW} \max$ \\
\hline Sleep mode & & & $0.4 \mathrm{~mW} \max$ \\
\hline Thermal dissipation (at $70^{\circ} \mathrm{C}$ & & & \\
\hline Active mode & & & $1.5 \mathrm{~W} \max$ \\
\hline Sleep mode & & & $55 \min \max$ \\
\hline NI 9474 Power Requir & & & \\
\hline Power consumption from ch & & & \\
\hline Active mode & & & $660 \mathrm{miN} \max$ \\
\hline Sleep mode & & & $0.6 \mathrm{~min} \max$ \\
\hline Thermal dissipation (at $70^{\circ} \mathrm{O}$ & & & \\
\hline Active mode & & & $1.5 \mathrm{~W}$ max \\
\hline Sleep mode & & & $0.6 \mathrm{mWN} \max$ \\
\hline Physical Characteristi & & & \\
\hline If you need to clean the moc & & & \\
\hline Screw-terminal wiring & & & $\begin{array}{l}12 \text { to } 24 \text { AWG copper conductor wire with } 10 \mathrm{~mm} \text { ( } 0.39 \mathrm{in} \text { ) of insulation } \\
\text { stripped from the end }\end{array}$ \\
\hline Torque for screw terminals & & & 0.5 to $0.6 \mathrm{~N} \cdot \mathrm{m}(4.4$ to $5.3 \mathrm{lb} \cdot \mathrm{in})$. \\
\hline Ferrules & & & $0.25 \mathrm{~mm}^{2}$ to $2.5 \mathrm{~mm}^{2}$ \\
\hline
\end{tabular}




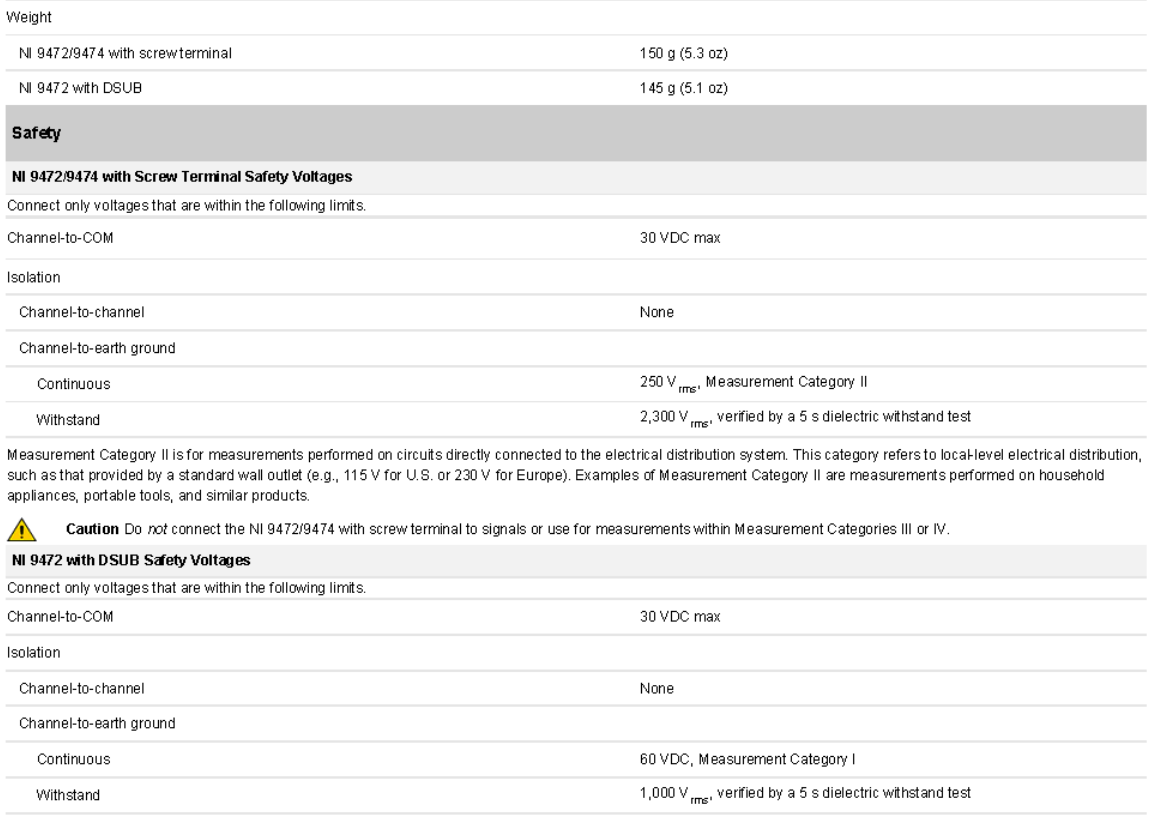

Measurement Category 1 is for measurements performed on circuits not directly connected to the electrical distribution system referred to as MAINS ${ }^{1}$ yoltage. This category is for measurements of voltages from specially protected secondary circuits. Such voltage measurements include signal levels, special equipment, limited-energy parts of equipment, circuits powered by regulated low-yoltage sources, and electronics.

1. Caution Do not connect the NI 9472 with DSUB to signals or use for measurements within Measurement Categories II, III, or IV

Sđety Standards

This product is designed to meet the requirements of the following standards of safety for electrical equipment for measurement, control, and laboratory use:

- IEC 61010-1, EN 61010-

- UL 61010-1, CSA 61010-1

E. Note For UL and other safety certifications, refer to the product label or the Onine Product Certification section

Hazardous Locations

U.S. (UL)

Class I, Division 2, Groups A, B, C, D, T4; Class I, Zone 2, AEx nC IIC T4

Canada (C-UL)

Class I, Division 2, Groups A, B, C, D, T4; Class I, Zone 2, Ex nC IIC T4

Europe (DEMKO)

EEx nC IICT4

Environmental

National Instruments $C$ Series modules are intended for indoor use only but may be used outdoors if installed in a suitable enclosure. Refer to the manual for the chassis you are using for more information about meeting these specific ations.

Operating temperature (IEC 60068-2-1, IEC 60068-2-2)

-40 to $70^{\circ} \mathrm{C}$

Storage temperature (IEC 60068-2-1, IEC 60068-2-2)

-40 to $85^{\circ} \mathrm{C}$

Ingress protection

Operating humidity (IEC 60068-2-56)

IP 40

Storage humidity (IEC 60068-2-56)

10 to $90 \% \mathrm{RH}$, noncondensing

Maximum altitude

5 to $95 \% \mathrm{RH}$, noncondensing

$2,000 \mathrm{~m}$

Pollution Degree (IEC 60664)

Shock and Vibration

To meet these specifications, you must panel mount the system. If you are using the NI $9472 / 9474$ with screw terminal, you also must either affix ferrules to the ends of the terminal wires or use the NI 9932 backshell kit to protect the connections 
Development of the West Virginia University Small Microgravity Research Facility (WVU SMiRF)

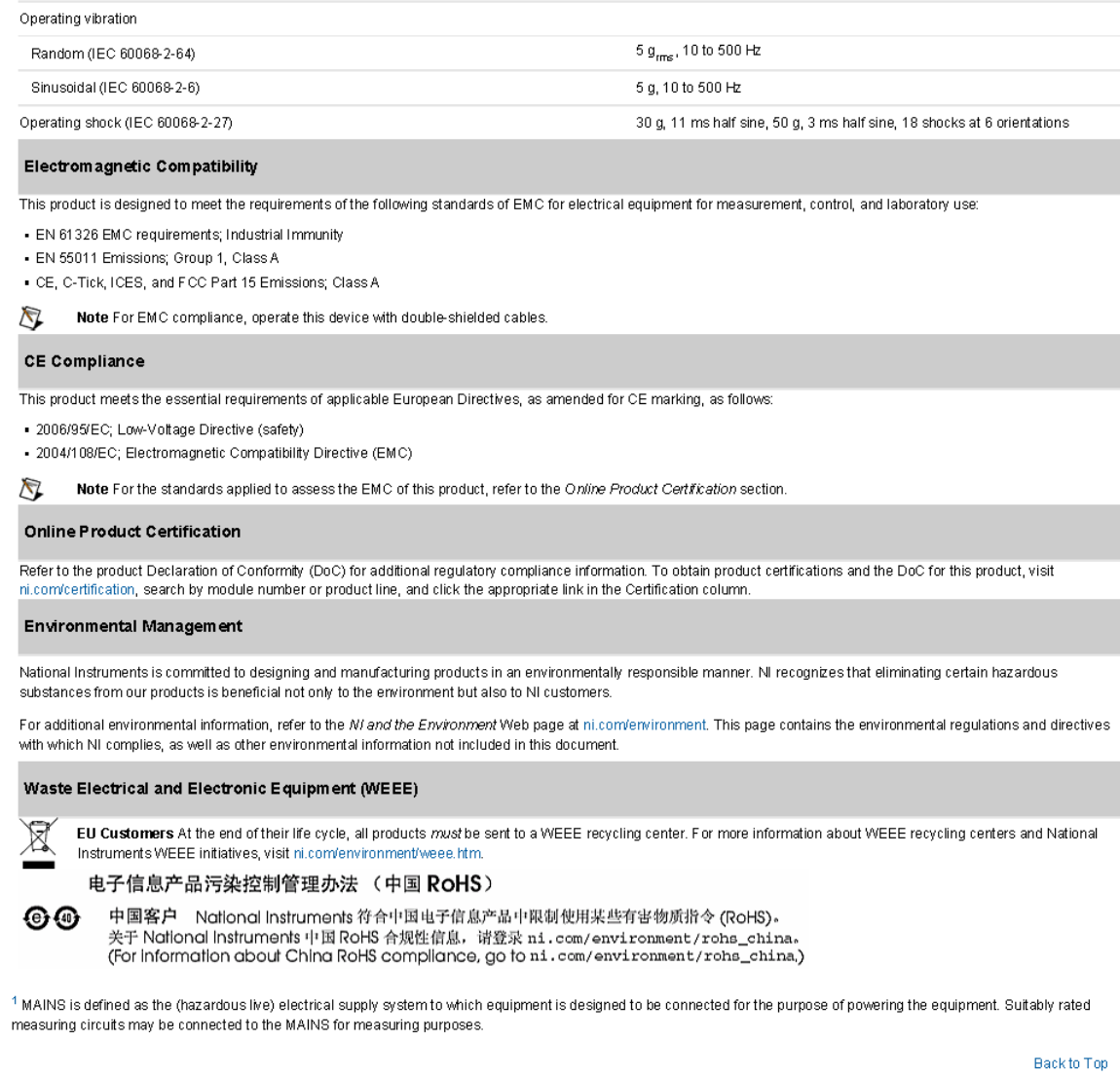

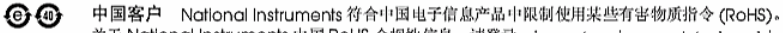

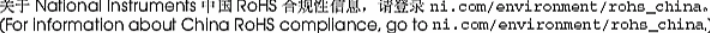

${ }^{1}$ MAINS is defined as the (hazardous INe) electrical supply system to which equipment is designed to be connected for the purpose of powering the equipment. Suttably rated measuring circuits may be connected to the MAINS for measuring purposes. 
Development of the West Virginia University

Small Microgravity Research Facility (WVU SMiRF)
Copyright $^{\odot} 2014$

Kyle G. Phillips

Pinouts/Front Panel Connections

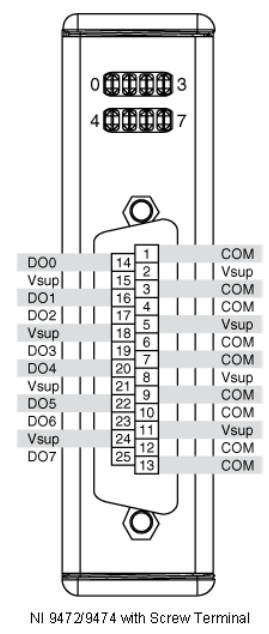




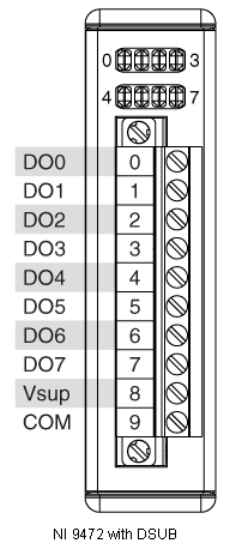

Back to Top

92010 National Instruments. All rights reserved. CompactRIO, CVI, FieldP oint, LabVIEW, National Instruments, National Instruments Alliance Parther, N1, ni.com, NI Comp actDAQ, and Signalexpress are

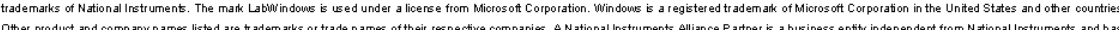

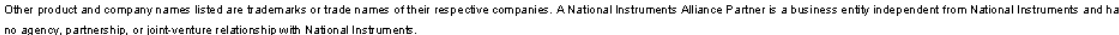

My Profile | RSS | Privacy | Legal | Contact NII 2012 National Instruments Corporation. All rights reserved. 


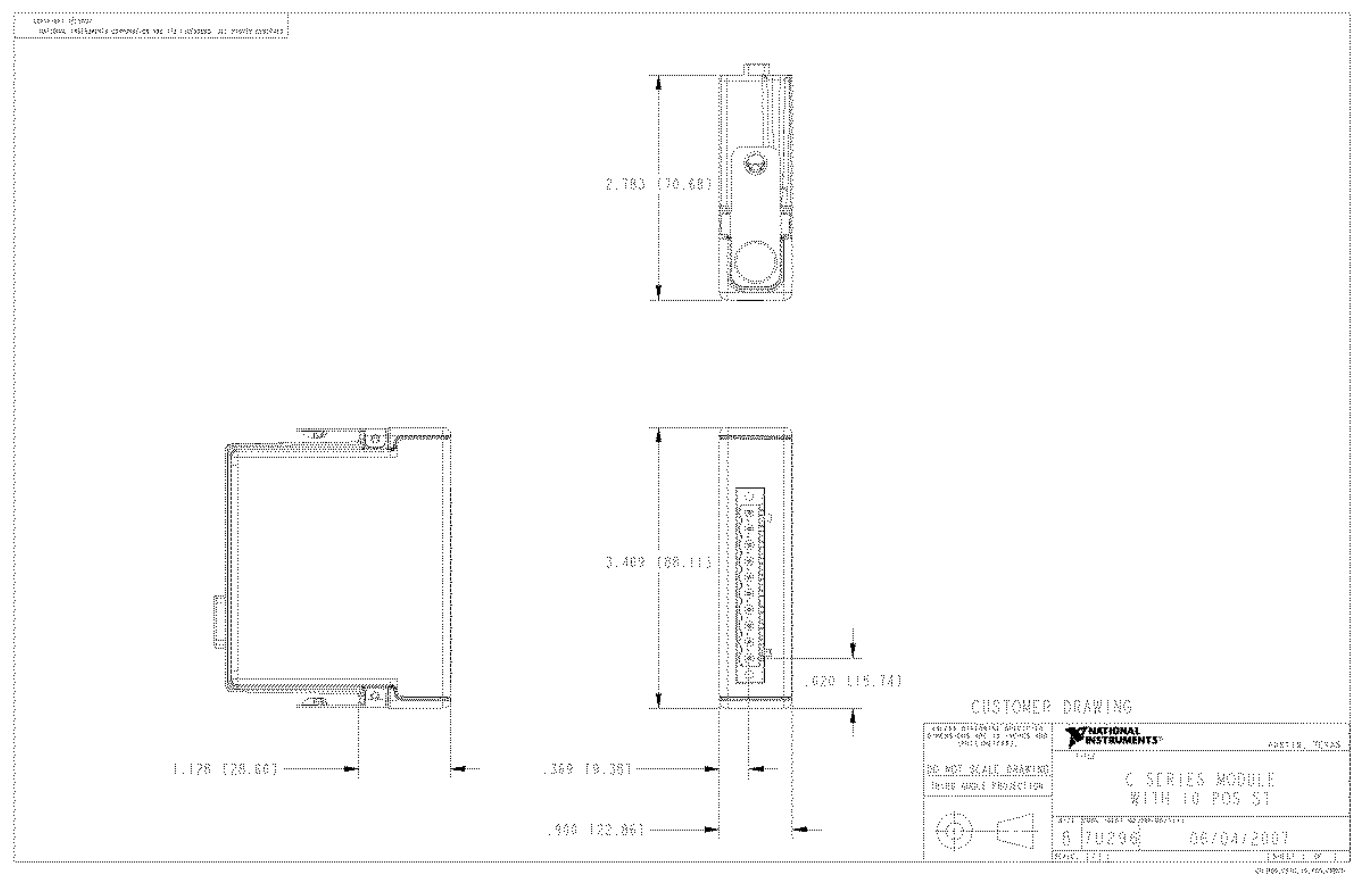

OPERATING INSTRUCTIONS AND SPECIFICATIONS

NI 9472/9474

8-Channel Digital Output Modules

\begin{tabular}{|c|c|c|c|c|}
\hline França1s & Deutscl & 日本語 & 한국어 & 简体中文 \\
\hline
\end{tabular}

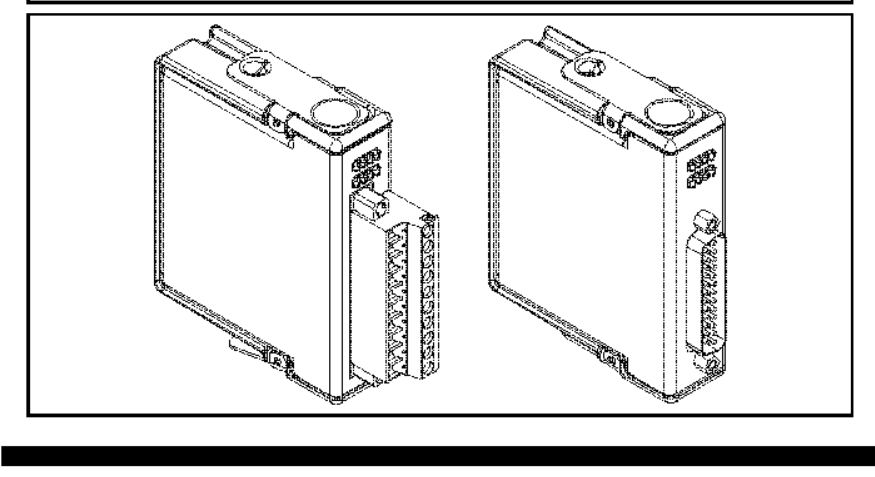

PNATIONAL

- INSTRUMENTS 
This document describes how to use the National Instruments 9472 and National Instruments 9474 and includes specifications and terminal or pin assignments. In this document, the NI $9472 / 9474$ with screw terminal and NI 9472 with DSUB are referred to inclusively as the NI 9472/9474. Visit ni.com/info and enter rdsoftwareversion to determine which software you need for the modules you are using. For information about installing, configuring, and programming the system, refer to the system documentation. Visit ni.com/info and enter cseriesdoc for information about $\mathrm{C}$ Series documentation.

Note The safety guidelines and specifications in this document are specific to the NI 9472/9474. The other components in the system might not meet the same safety ratings and specifications. Refer to the documentation for each component in the system to determine the safety ratings and specifications for the entire system. Visit ni.com/info and enter cseriesdoc for information about C Series documentation.

\section{Safety Guidelines}

Operate the NI $9472 / 9474$ only as described in these operating instructions.

Hot Surface This icon denotes that the component may be III hot. Touching this component may result in bodily injury.

\section{Safety Guidelines for Hazardous Voltages}

You can connect hazardous voltages only to the NI 9472/9474 with screw terminal. Do not connect hazardous voltages to the NI 9472 with DSUB.

If hazardous voltages are connected to the module, take the following precautions. A hazardous voltage is a voltage greater than $42.4 \mathrm{~V}_{\mathrm{pk}}$ or $60 \mathrm{VDC}$ to earth ground.

A Caution Ensure that hazardous voltage wiring is performed only by qualified personnel adhering to local electrical standards.

Caution Do not mix hazardous voltage circuits and human-accessible circuits on the same module.

Caution Make sure that devices and circuits connected to the module are properly insulated from human contact.

1 Caution When module terminals are hazardous voltage LIVE ( $>42.4 \mathrm{~V}_{\mathrm{pk}} / 60 \mathrm{VDC}$ ), you must ensure that devices 
and circuits connected to the module are properly insulated from human contact. You must use the NI 9932 connector backshell kit to ensure that the terminals are not accessible.

Figure 1 shows the NI 9932 connector backshell.

Not Note You can use the NI 9932 connector backshell only with the NI $9472 / 9474$ with screw terminal.

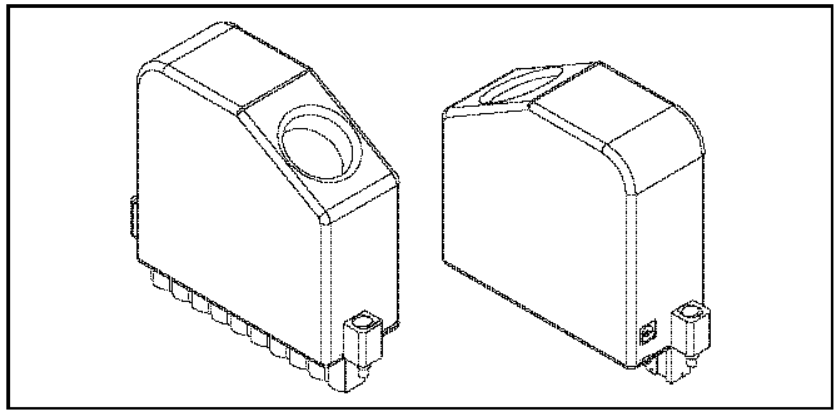

Figure 1. NI 9932 Connector Backshell

\section{Safety Guidelines for Hazardous Locations}

The NI 9472/9474 is suitable for use in Class I, Division 2, Groups A, B, C, D, T4 hazardous locations; Class I, Zone 2, AEx nC IIC T4 and Ex nC IIC T4 hazardous locations; and nonhazardous locations only. Follow these guidelines if you are installing the NI 9472/9474 in a potentially explosive environment. Not following these guidelines may result in serious injury or death.

1 Caution Do not disconnect I/O-side wires or connectors unless power has been switched off or the area is known to be nonhazardous.

A Caution Do not remove modules unless power has been switched off or the area is known to be nonhazardous.

1. Caution Substitution of components may impair suitability for Class I, Division 2.

Caution For Zone 2 applications, install the system in an and EN 60529. 


\begin{abstract}
Caution For Zone 2 applications, install a protection device between the $\mathrm{V}_{\text {sup }}$ and COM terminals on the NI 9472/9474. The device must prevent the input $\mathrm{V}_{\text {sup }}$-to-COM voltage from exceeding $42 \mathrm{~V}$ if there is a transient overvoltage condition.
\end{abstract}

Special Conditions for Hazardous Locations Use in Europe This equipment has been evaluated as EEx nC IIC T4 equipment under DEMKO Certificate No. 03 ATEX 0324020X. Each module is marked \&x II $3 \mathrm{G}$ and is suitable for use in Zone 2 hazardous locations. If you are using the NI $9472 / 9474$ in Gas Group IIC hazardous locations or in ambient temperatures of $-40{ }^{\circ} \mathrm{C} \leq \mathrm{Ta} \leq 70^{\circ} \mathrm{C}$, you must use the device in an NI chassis that has been evaluated as EEx nC IIC T4, Ex nA IIC T4, or Ex nL IIC T4 equipment.

\title{
Special Conditions for Marine Applications
}

Some modules are Lloyd's Register (LR) Type Approved for marine applications. To verify Lloyd's Register certification, visit ni.com/certification and search for the LR certificate, or look for the Lloyd's Register mark on the module.

\footnotetext{
1. Caution To meet radio frequency emission requirements for marine applications, use shielded cables and install the system in a metal enclosure. Suppression ferrites must be installed on power supply inputs near power entries to modules and controllers. Power supply and module cables must be separated on opposite sides of the enclosure and must enter and exit through opposing enclosure walls.
} 


\section{Connecting the NI $9472 / 9474$}

The NI $9472 / 9474$ provides connections for eight digital output channels.

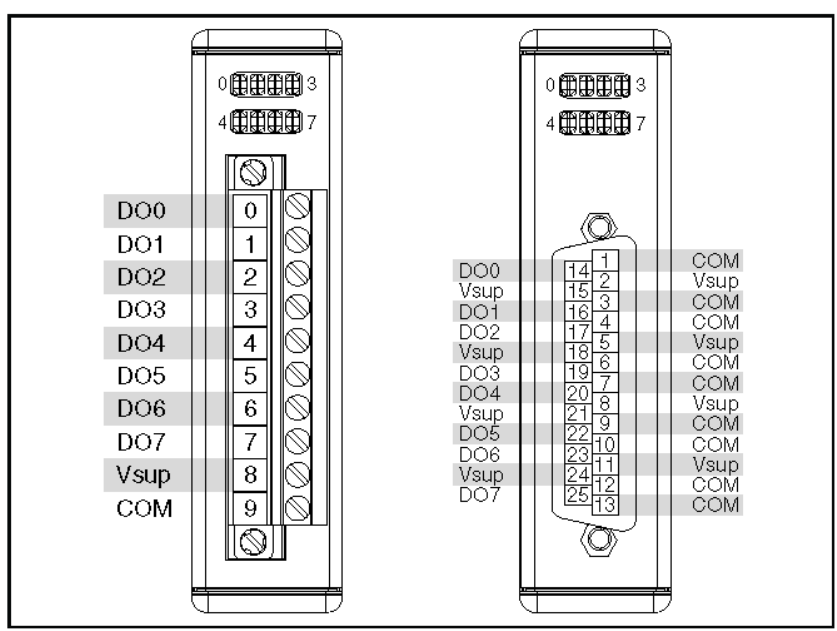

Figure 2. NI $9472 / 9474$ Terminal and Pin Assignments

The NI $9472 / 9474$ with screw terminal has a 10-terminal, detachable screw-terminal connector. The NI 9472 with DSUB has a 25-pin DSUB connector. Each channel of the NI 9472/9474 has a terminal or pin, DO, to which you can connect a device. The eight digital output channels are internally referenced to the common terminal or pin, COM. National Instruments recommends you provide independent $\mathrm{COM}$ and $\mathrm{V}_{\text {sup }}$ wiring for each channel of the NI 9472 with DSUB to minimize current flow in the COM and $\mathrm{V}_{\text {sup }}$ wiring. The COM pins on the NI 9472 with DSUB are internally connected..

Note You must use 2-wire ferrules to create a secure connection when connecting more than one wire to a single terminal on the NI $9472 / 9474$ with screw terminal.

Each channel has an LED that indicates the state of the channel. When a channel LED is lit, the channel is on. When the LED is dark, the channel is off. The LEDs are disabled when the chassis is in sleep mode. Refer to the Sleep Mode section for more information about sleep mode. 
You must connect an external power supply to the NI 9472/9474. This power supply provides the current for the devices you connect to the module. Connect the positive lead of the power supply to $\mathrm{V}_{\text {sup }}$ and the negative lead of the power supply to COM. The $\mathrm{V}_{\text {sup }}$ pins on the NI 9472 with DSUB are internally connected. You can connect only one external voltage supply to the device. Refer to the Specifications section for information about the power supply voltage range.
A Caution Do not remove or insert modules if the external power supply connected to the $\mathrm{V}_{\text {sup }}$ and COM pins is powered on.

The NI 9472/9474 has current sourcing outputs, meaning the DO terminal or pin is driven to $\mathrm{V}_{\text {sup }}$ when the channel is turned on.

You can directly connect the NI $9472 / 9474$ to a variety of industrial devices such as solenoids, motors, actuators, relays, and lamps. Make sure the devices you connect to the NI 9472/9474 are compatible with the output specifications of the module. Refer to the Specifications section for more information about the output specifications.

Connect the device to DO and connect the common of the device to COM. Refer to Figure 3 for an illustration of how to connect a device to the NI $9472 / 9474$.

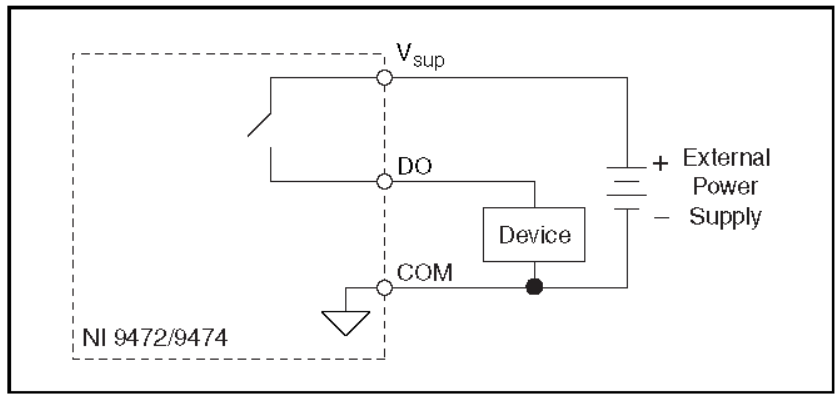

Figure 3. Connecting a Device to the NI $9472 / 9474$ 


\section{Increasing Current Drive}

Each channel of the NI 9472 has a continuous output current of $0.75 \mathrm{~A}$, and each channel of the NI 9474 has a continuous output current of $1 \mathrm{~A}$. If you want to increase the output current to a device, you can connect any number of channels together in parallel. For example, using the NI 9474, if you want to drive $4 \mathrm{~A}$ of current, connect DO $<0 \ldots 3>$ in parallel as shown in Figure 4. You must turn all parallel channels on and off simultaneously so that the current on any single channel cannot exceed the 1 A rating.

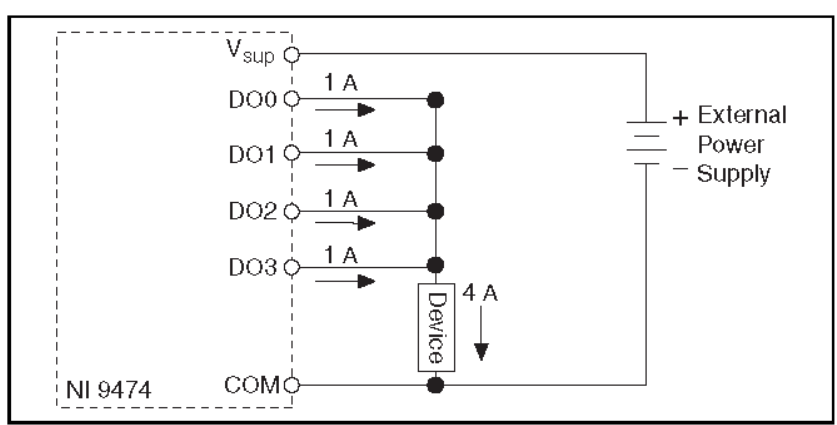

Figure 4. Increasing the Current to a Device Connected to the NI 9474

\section{Wiring for High-Vibration Applications}

If an application using the NI 9472/9474 with screw terminal is subject to high vibration, National Instruments recommends that you either use ferrules to terminate wires to the detachable screw-terminal connector or use the NI 9932 backshell kit to protect the connections. Refer to Figure 5 for an illustration of using ferrules. Refer to Figure 1 for an illustration of the NI 9932 connector backshell.

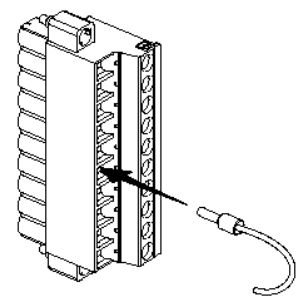

Figure 5. 10-Terminal Detachable Screw-Terminal Connector with Ferrule 


\section{I/0 Protection}

The NI $9472 / 9474$ is short-circuit proof in accordance with IEC 1131-2 and provides overcurrent protection.

\section{Understanding Short-Circuit-Proof Devices}

Each channel on the NI $9472 / 9474$ has circuitry that protects it from current surges resulting from short circuits. Whether the module suffers damage from these overcurrent conditions depends on the following factors:

- The amount of current through the channel

- The amount of time the current is above the current limit

- The frequency of the current surges

When the amount of current through the DO terminal or pin is greater than $13 \mathrm{~A}$, the channel trips and goes into an overcurrent state. In an overcurrent state, the channel turns off and the module is not damaged. If the current through DO is between $6 \mathrm{~A}$ and $13 \mathrm{~A}$, the state of the channel is indeterminate and depends on factors such as the current level, the temperature, and the power supply.

Higher inrush currents that exist for less than the trip time do not trip the protection circuitry. Refer to the Specifications section for

more information about the maximum continuous output current, short-circuit behavior, and short-circuit trip time. You also can refer to the IEC 1131-2 standard for more information about short-circuit-proof devices.

Tip Because the NI 9472/9474 module includes internal flyback diodes, you do not need to add external diodes when connecting to switching energy storing devices.

\section{Power Supplies and Overcurrent Conditions}

If a short-circuit occurs, the current through DO can exceed the current rating for the power supply and the maximum continuous output current for the NI 9472/9474.

If the power supply you are using with the NI $9472 / 9474$ cannot supply more than $13 \mathrm{~A}$, the module may be damaged if a short-circuit condition occurs.

\section{Detecting an Overcurrent Condition}

If a device connected to the module is not working while the channel is on, the module channel may be in an overcurrent state. Neither the software nor the module LEDs indicate if an overcurrent condition occurs. A channel LED may be on even if the channel is off because of an overcurrent condition. To determine if 
the channel is in an overcurrent state, measure the voltage between $\mathrm{DO}$ and $\mathrm{V}_{\text {sup. }}$. If the voltage is equal to the voltage of the external power supply connected to the module, the channel is in an overcurrent state.

\section{Resetting Channels After an Overcurrent Condition}

After you have determined and fixed the cause of the overcurrent condition, reset the channel by turning it off. Alternatively, you can disconnect the external power supply from the module. However, doing so disconnects power from all of the module channels. Normal operation can resume after you correct the overcurrent condition and reset the channel.

\section{Sleep Mode}

This module supports a low-power sleep mode. Support for sleep mode at the system level depends on the chassis that the module is plugged into. Refer to the chassis manual for information about support for sleep mode. If the chassis supports sleep mode, refer to the software help for information about enabling sleep mode. Visit ni.com/info and enter cseriesdoc for information about C Series documentation.

Typically, when a system is in sleep mode, you cannot communicate with the modules. In sleep mode, the system consumes minimal power and may dissipate less heat than it does in normal mode. Refer to the Specifications section for more information about power consumption and thermal dissipation.

\section{Specifications}

The following specifications are typical for the range -40 to $70^{\circ} \mathrm{C}$ unless otherwise noted. All voltages are relative to COM unless otherwise noted. The specifications are the same for the NI 9472 and the NI 9474 unless otherwise noted.

\section{Outp ut Characteristics}

Number of channels 8 digital output channels

Output type Sourcing

Power-on output state Channels off

External power supply voltage range $\left(V_{\text {sup }}\right)$

NI 9472 6-30 VDC

NI 9474 5-30 VDC 
Output impedance $\left(R_{0}\right)$

Typical

$0.07 \Omega$

Maximum

$0.13 \Omega$

Continuous output current $\left(I_{0}\right)$, per channel

NI 9472 $0.75 \mathrm{~A} \max$

NI 9474 $1 \mathrm{~A} \max$

Output voltage $\left(V_{0}\right)$ $V_{\text {sup }}-\left(I_{0} \cdot R_{0}\right)$

I/O protection

Voltage... 30 VDC max

Reversed voltage None

Short-circuit behavior

\begin{tabular}{|c|c|c|}
\hline Current & Channel Behavior & Module Protection \\
\hline \hline 0 to $1 \mathrm{~A}$ & Channel does not trip & Module is not damaged \\
\hline 1 to $6 \mathrm{~A}$ & Channel does not trip & Module may be damaged \\
\hline 6 to $13 \mathrm{~A}$ & Channel may trip & Module may be damaged \\
\hline$>13 \mathrm{~A}$ & Channel trips & Module is not damaged \\
\hline
\end{tabular}

Short-circuit trip time $10 \mu \mathrm{s}$ at $13 \mathrm{~A}$

Output delay time (full load)

NI 9472 .....

NI 9474 MTBF

NI 9472 (2)

NI 9474
$100 \mu \mathrm{s} \max$ $1 \mu \mathrm{s} \max$

$1,113,301$ hours at $25^{\circ} \mathrm{C}$; Bellcore Issue 2, Method 1, Case 3, Limited Part Stress Method 479,889 hours at $25^{\circ} \mathrm{C}$;

Bellcore Issue 2, Method 1, Case 3, Limited Part Stress Method

Note Contact NI for Bellcore MTBF specifications at other temperatures or for MIL-HDBK-217F specifications.

\section{NI 9472 Power Requirements}

Power consumption from chassis

Active mode $230 \mathrm{~mW} \max$

Sleep mode $0.4 \mathrm{~mW} \max$ 
Thermal dissipation (at $70^{\circ} \mathrm{C}$ )

Active mode

$1.5 \mathrm{~W} \max$

Sleep mode $55 \mathrm{~mW} \max$

\section{NI 9474 Power Requirements}

Power consumption from chassis

Active mode $660 \mathrm{~mW} \max$

Sleep mode $0.6 \mathrm{~mW} \max$

Thermal dissipation (at $70^{\circ} \mathrm{C}$ )

Active mode $1.5 \mathrm{~W} \max$

Sleep mode $0.6 \mathrm{~mW} \max$

\section{Physical Characteristics}

If you need to clean the module, wipe it with a dry towel.

Screw-terminal wiring 12 to 24 AWG copper conductor wire with $10 \mathrm{~mm}$ ( 0.39 in.) of insulation stripped from the end

Torque for screw terminals 0.5 to $0.6 \mathrm{~N} \cdot \mathrm{m}$ (4.4 to $5.3 \mathrm{lb} \cdot \mathrm{in}$.)

N $9472 / 9474$

Ferrules $0.25 \mathrm{~mm}^{2}$ to $2.5 \mathrm{~mm}^{2}$

Weight

NI 9472/9474 with

screw terminal $150 \mathrm{~g}(5.3 \mathrm{oz})$

NI 9472 with DSUB $145 \mathrm{~g}(5.1 \mathrm{oz})$

\section{Safety}

\section{NI 9472/9474 with Screw Terminal Safety Voltages}

Connect only voltages that are within the following limits.

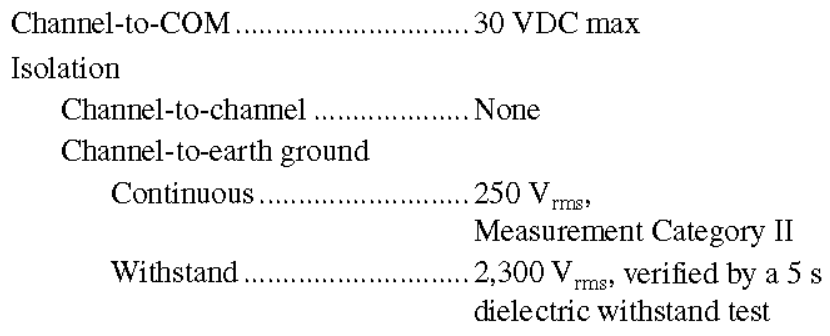

Measurement Category II is for measurements performed on circuits directly connected to the electrical distribution system. 
This category refers to local-level electrical distribution, such as that provided by a standard wall outlet, for example, $115 \mathrm{~V}$ for U.S. or $230 \mathrm{~V}$ for Europe.

\begin{abstract}
A Caution Do not connect the NI 9472/9474 with screw terminal to signals or use for measurements within Measurement Categories III or IV.
\end{abstract}

\title{
NI 9472 with DSUB Safety Voltages
}

Connect only voltages that are within the following limits.

Channel-to-COM ........................ 30 VDC max
Isolation
Channel-to-channel .................. None
Channel-to-earth ground
Continuous ........................ 60 VDC,
Measurement Category I
Withstand ......................... $1,000 \mathrm{~V}_{\text {rms }}$, verified by a $5 \mathrm{~s}$
dielectric withstand test

Measurement Category I is for measurements performed on circuits not directly connected to the electrical distribution system referred to as MAINS voltage. MAINS is a hazardous live electrical

supply system that powers equipment. This category is for measurements of voltages from specially protected secondary circuits. Such voltage measurements include signal levels, special equipment, limited-energy parts of equipment, circuits powered by regulated low-voltage sources, and electronics.

\section{A Caution Do not connect the NI 9472 with DSUB to signals or use for measurements within Measurement Categories II, III, or IV.}

\section{Safety Standards}

This product is designed to meet the requirements of the following standards of safety for electrical equipment for measurement, control, and laboratory use:

- IEC 61010-1, EN 61010-1

- UL 61010-1, CSA 61010-1

Note For UL and other safety certifications, refer to the product label, or visit ni.com/certification, search by module number or product line, and click the appropriate link in the Certification column. 


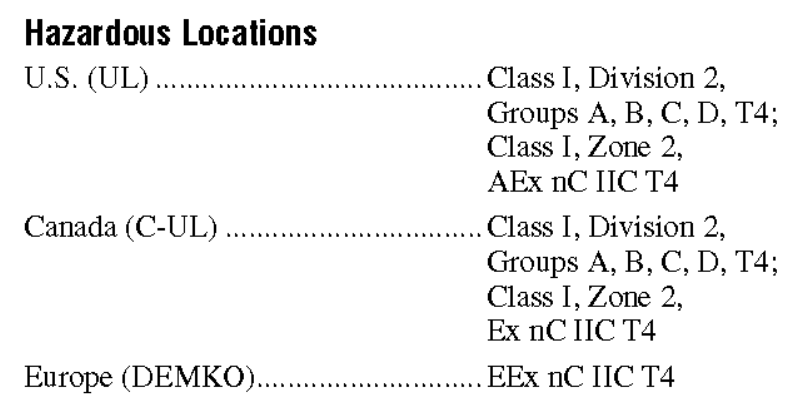

\section{Environmental}

National Instruments C Series modules are intended for indoor use only but may be used outdoors if installed in a suitable enclosure. Refer to the manual for the chassis you are using for more information about meeting these specifications.

Operating temperature (IEC 60068-2-1, IEC 60068-2-2) .....-40 to $70^{\circ} \mathrm{C}$

Storage temperature (IEC 60068-2-1, IEC 60068-2-2) .....-40 to $85^{\circ} \mathrm{C}$

Ingress protection. IP 40

Operating humidity (IEC 60068-2-56)... 10 to $90 \% \mathrm{RH}$, noncondensing

Storage humidity (IEC 60068-2-56)............................ 5 to $95 \%$ RH, noncondensing

Maximum altitude......................... 2,000 m

Pollution Degree (IEC 60664) .......... 2 


\section{Shock and Vibration}

To meet these specifications, you must panel mount the system. If you are using the NI 9472/9474 with screw terminal, you also must either affix ferrules to the ends of the terminal wires or use the NI 9932 backshell kit to protect the connections.

Operating vibration

Random (IEC 60068-2-64)........ $5 \mathrm{~g}_{\mathrm{rms}}, 10$ to $500 \mathrm{~Hz}$

Sinusoidal (IEC 60068-2-6) ....... 5 g, 10 to $500 \mathrm{~Hz}$

Operating shock

(IEC 60068-2-27) ...........................30 g, $11 \mathrm{~ms}$ half sine,

$50 \mathrm{~g}, 3 \mathrm{~ms}$ half sine,

18 shocks at 6 orientations

\section{Electromagnetic Compatibility}

This product is designed to meet the requirements of the following standards of EMC for electrical equipment for measurement, control, and laboratory use:

- EN 61326 EMC requirements; Industrial Immunity

- EN 55011 Emissions; Group 1, Class A

- CE, C-Tíck, ICES, and FCC Part 15 Emissions; Class A

$\begin{array}{lll}\text { N } 3472 / 9474 & 28 & \text { ni.com }\end{array}$

$\$$ Note For EMC compliance, operate this device with shielded cabling.

\section{CE Compliance}

This product meets the essential requirements of applicable European directives, as amended for CE markings, as follows:

- 2006/95/EC; Low-Voltage Directive (safety)

- 2004/108/EC; Electromagnetic Compatibility Directive (EMC)

2 Note Refer to the Declaration of Conformity (DoC) for this product for any additional regulatory compliance information. To obtain the DoC for this product, visit ni.com/certification, search by module number or product line, and click the appropriate link in the Certification column 


\section{Environmental Management}

National Instruments is committed to designing and manufacturing products in an environmentally responsible manner. NI recognizes that eliminating certain hazardous substances from our products is beneficial not only to the environment but also to NI customers.

For additional environmental information, refer to the $N I$ and the Environment Web page at ni . com/environment. This page contains the environmental regulations and directives with which NI complies, as well as other environmental information not included in this document.

\section{Waste Electrical and Electronic Equipment (WEEE)}

EU Customers At the end of their life cycle, all products must be sent to a WEEE recycling center. For more information about WEEE recycling centers and National Instruments WEEE initiatives, visit ni . com/

environment/weee. htm.

\section{电子信息产品污染控制管理办法（中国 RoHS）}

(9) 中国客户 National Instruments 符合中国电子信息

产品中限制使用某些有害物质指令 (ROHS)。关于 National Instruments 中国 RoHS 合规性信息, 请登录 ni.com/environment/rohs_china。 (For information about China RoHS compliance, go to ni. com / environment/rohs_china.)

\section{Where to Go for Support}

The National Instruments Web site is your complete resource for technical support. At ni.com/support you have access to everything from troubleshooting and application development self-help resources to email and phone assistance from NI Application Engineers.

National Instruments corporate headquarters is located at 11500 North Mopac Expressway, Austin, Texas, 78759-3504. National Instruments also has offices located around the world to help address your support needs. For telephone support in the United States, create your service request at ni . com/support and follow the calling instructions or dial 5127958248 . For 
telephone support outside the United States, contact your local branch office:

Australia 1800300 800, Austria $43662457990-0$, Belgium 32 (0) 2757 0020, Brazil 551132623599 , Canada 800433 3488, China 862150509800 , Czech Republic 420224235 774, Denmark 4545762600 , Finland 358 (0) 972572511 , France 01576624 24, Germany 49897413130 , India 918041190000 , Israel 9723 6393737, Italy 390241309277 , Japan 0120-527196, Korea 820234513400 , Lebanon 961 (0) 1332828 , Malaysia 1800887710 , Mexico 018000100793 , Netherlands 31 (0) 348433 466, New Zealand 0800553 322, Norway 47 (0) 66907660 , Poland 48223390150 , Portugal 351210311210 , Russia 74957836851 , Singapore 18002265886 , Slovenia 38634254200 , South Africa 27011805 8197, Spain 34916400085 , Sweden 46 (0) 8587895 00, Switzerland 41562005151 , Taiwan 886022377 2222, Thailand 6622786777 , Turkey 90212279 3031, United Kingdom 44 (0) 1635523545 
Development of the West Virginia University Small Microgravity Research Facility (WVU SMiRF)
Copyright $^{\odot} 2014$

Kyle G. Phillips

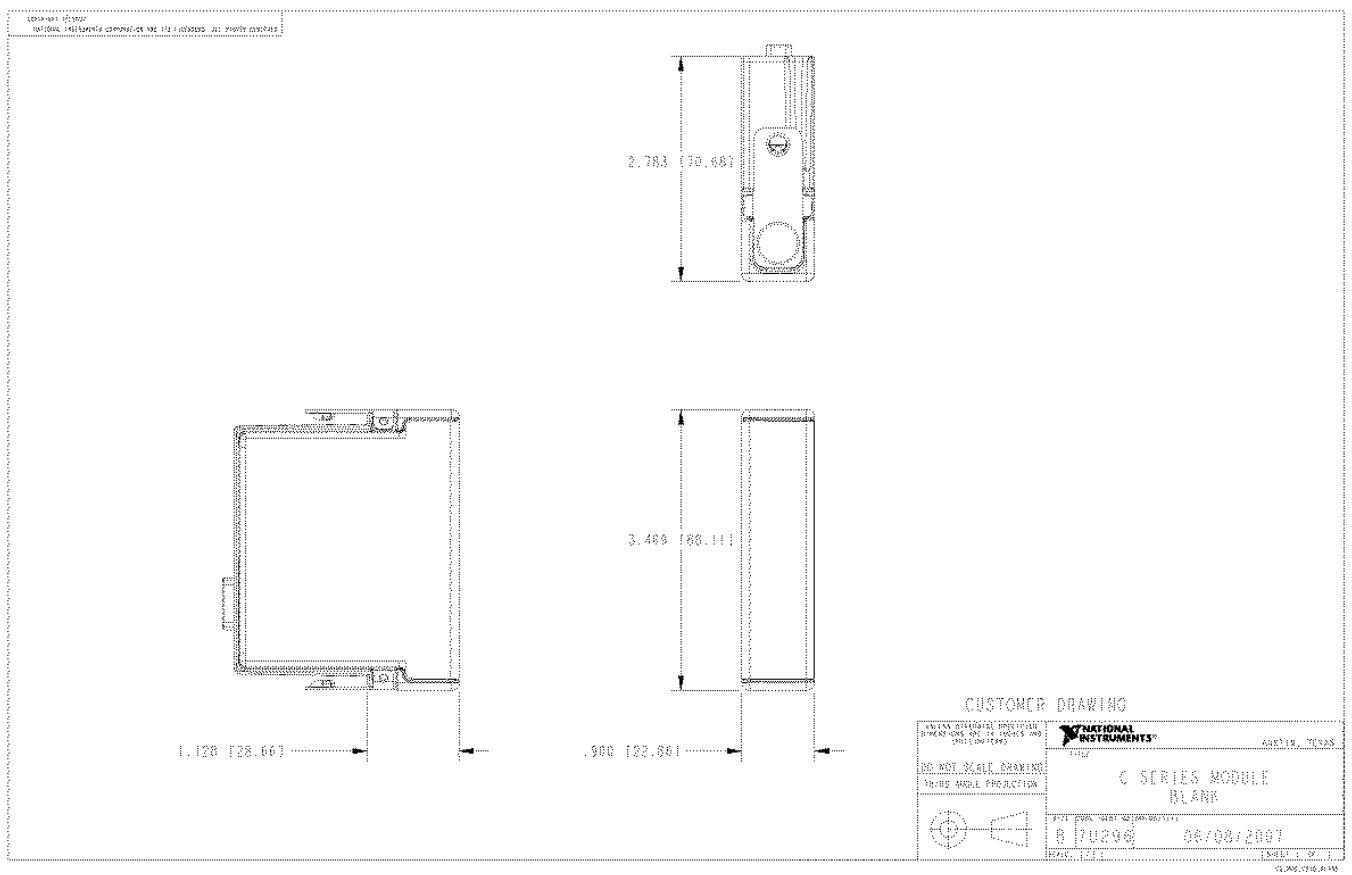


Development of the West Virginia University

Small Microgravity Research Facility (WVU SMiRF)
Copyright $^{\odot} 2014$

Kyle G. Phillips

\section{USER GUIDE}

\section{NI 9977 C Series Filler Module}

This document describes the National Instruments 9977 C Series Filler Module.

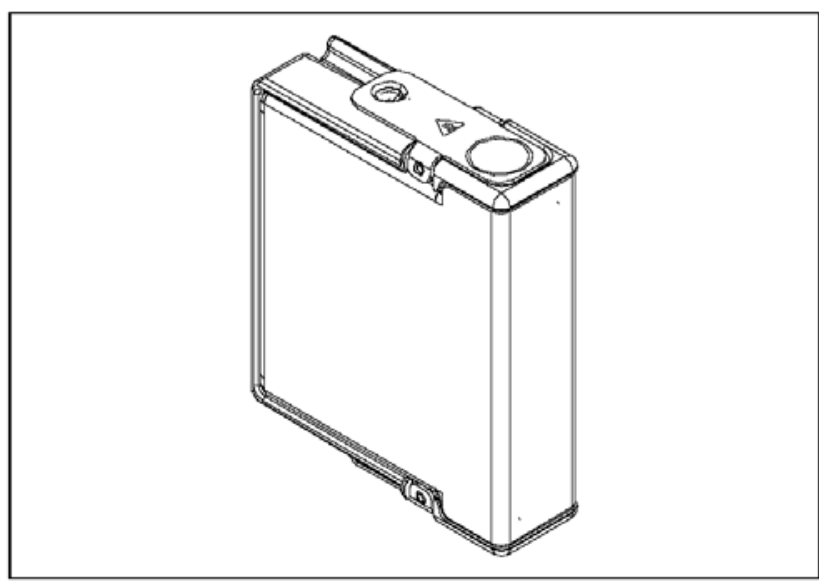

Figure 1. NI 9977 C Series Filler Module

\section{Introduction}

The NI 9977 C Series Filler Module is a blank, non-functioning C Series module. You can use the NI 9977 to fill empty slots in any chassis that accepts $\mathrm{C}$ Series modules.

\section{Physical Characteristics}




\section{Where to Go for Support}

The National Instruments Web site is your complete resource for technical support. At ni.com/support you have access to everything from troubleshooting and application development self-help resources to email and phone assistance from NI Application Engineers.

National Instruments corporate headquarters is located at 11500 North Mopac Expressway, Austin, Texas, 78759-3504.

National Instruments also has offices located around the world to help address your support needs. For telephone support in the United States, create your service request at $\mathrm{ni}$.com/support and follow the calling instructions or dial 5127958248 . For telephone support outside the United States, contact your local branch office:

Australia 1800300800 , Austria 4306624579900 , Belgium 32027570020 , Brazil 551132623599 , Canada 800433 3488, China 862165557838 ,

Czech Republic 420224235 774, Denmark 4545762600 , Finland 38509725725 11, France 330148142424 , Germany 490897413130 , India 918041190000 , Israel 972036393737 , Italy 3902413091 , Japan 81354722970 , Korea 820234513400 , Lebanon 961013328 28, Malaysia 1800887710 , Mexico 018000100793 , Netherlands 310348433 466, New Zealand 0800553322 , Norway 47066907660 , Poland 48223390150 , Portugal 351210311210 , Russia 749578368 51, Singapore 18002265886 , Slovenia 38634254200 , South Africa 270118058197 , Spain 34916400085 , Sweden 460858789500 , Switzerland 41562005151 , Taiwan 886022377 2222, Thailand 662278 6777, Turkey 902122793031 , United Kingdom 4401635523545 

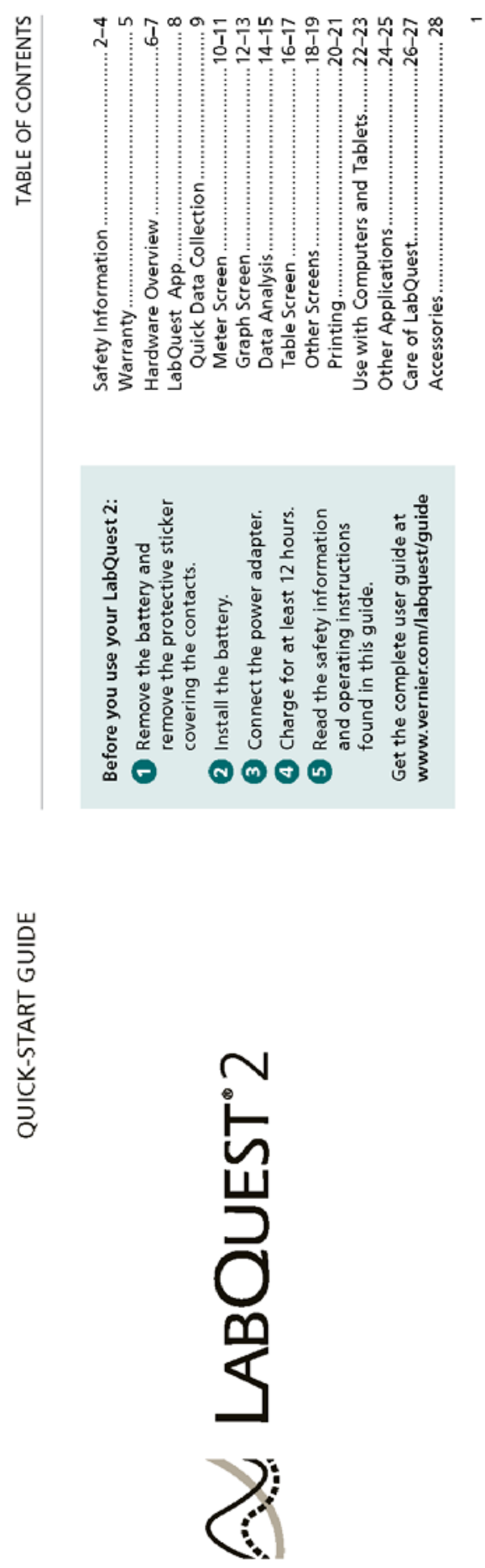

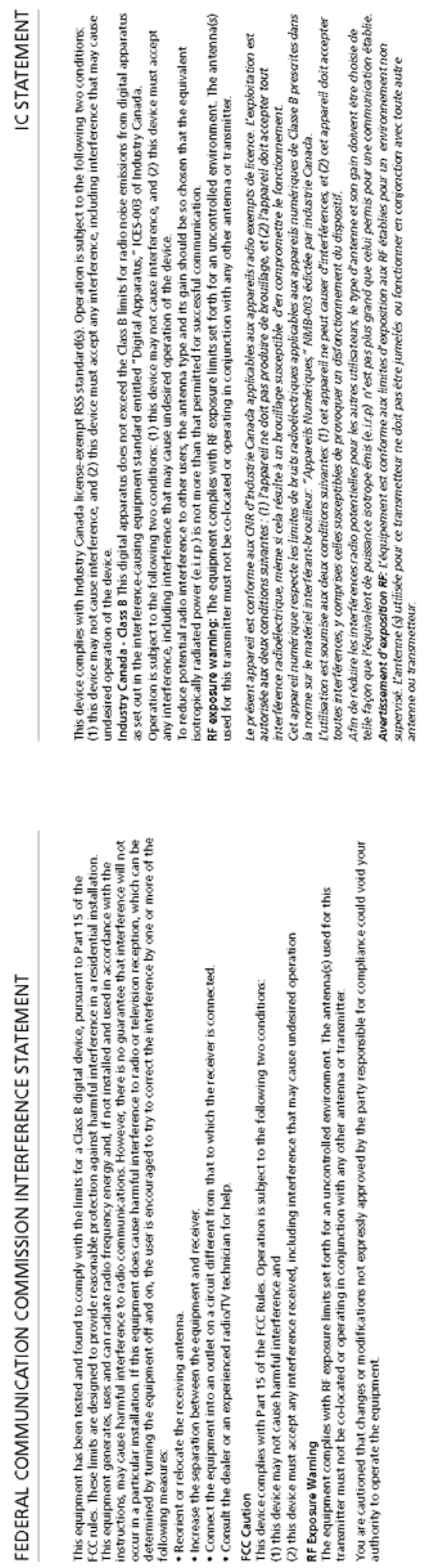

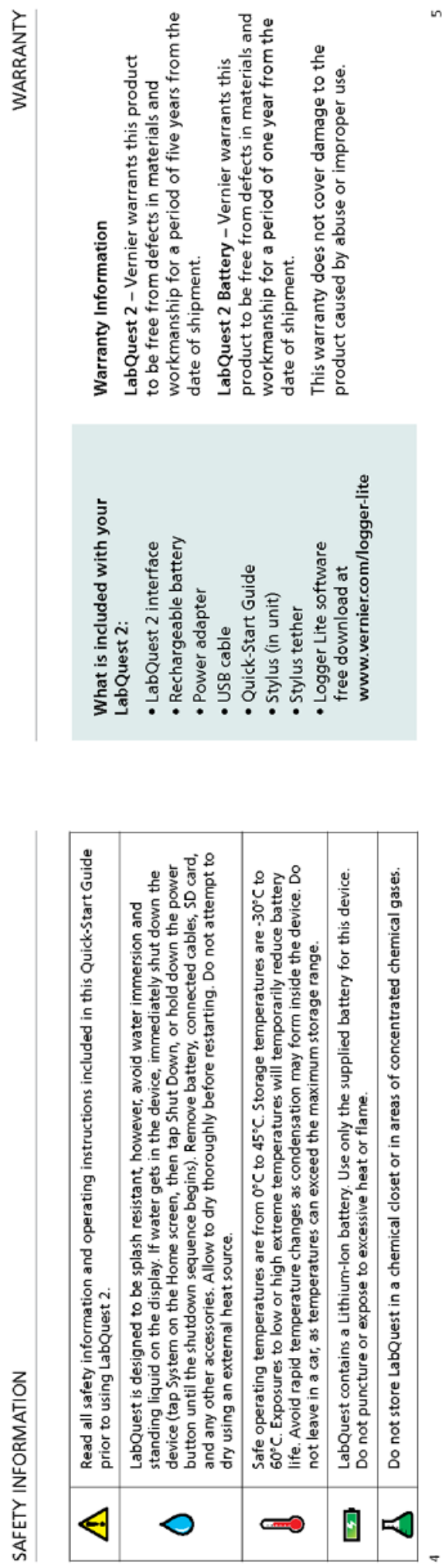

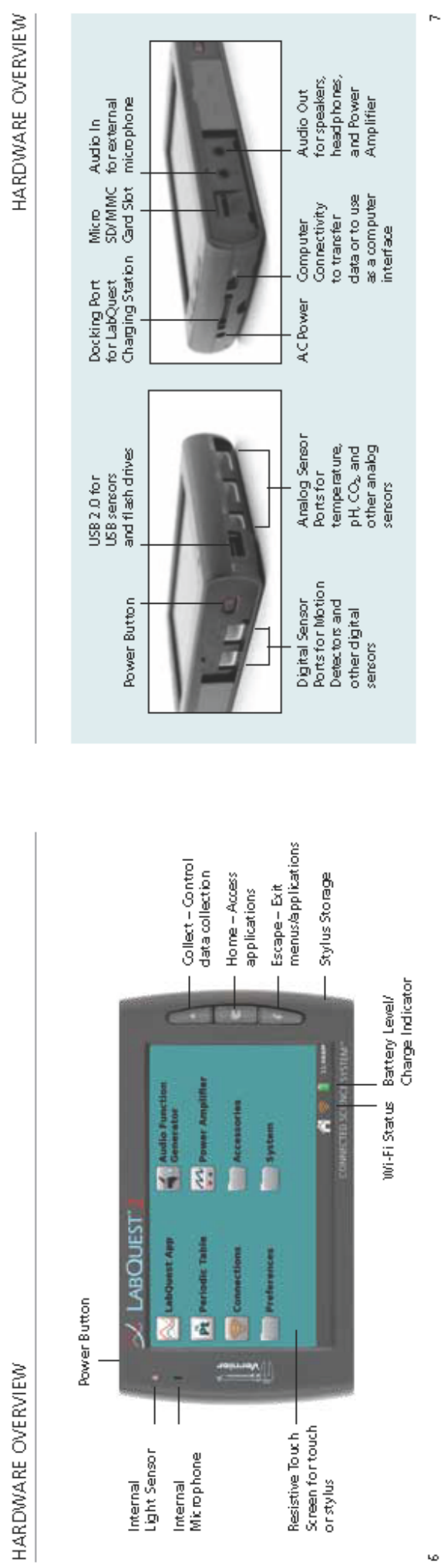

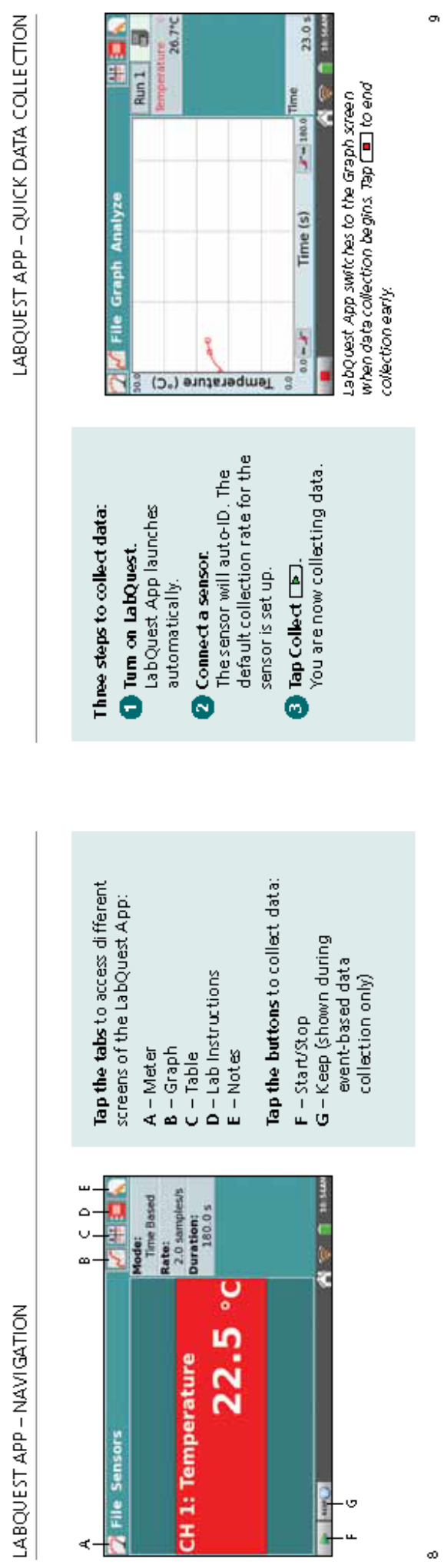

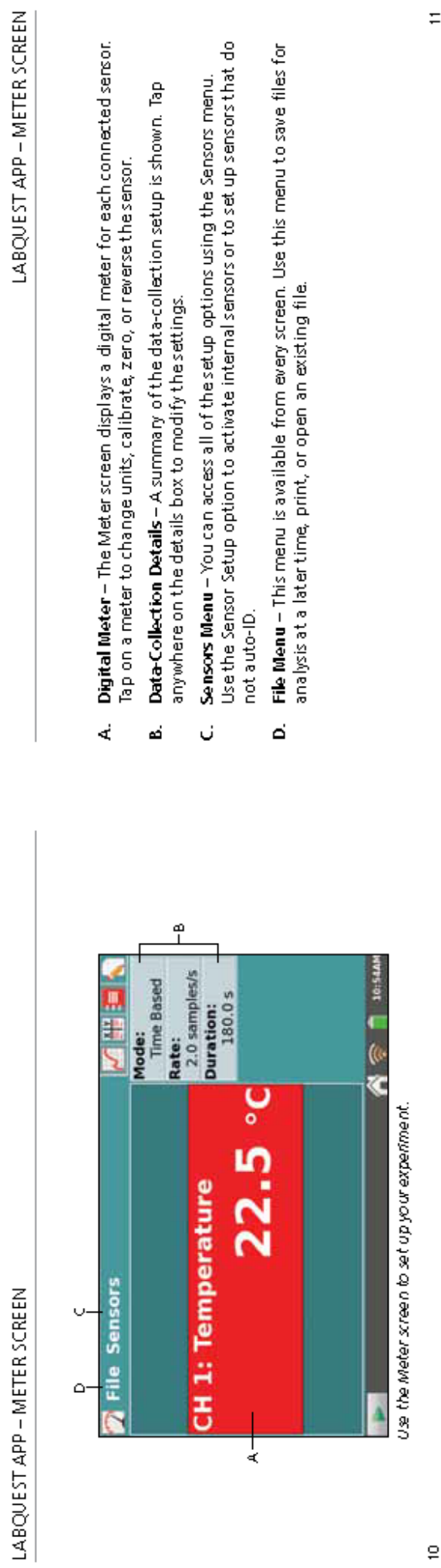

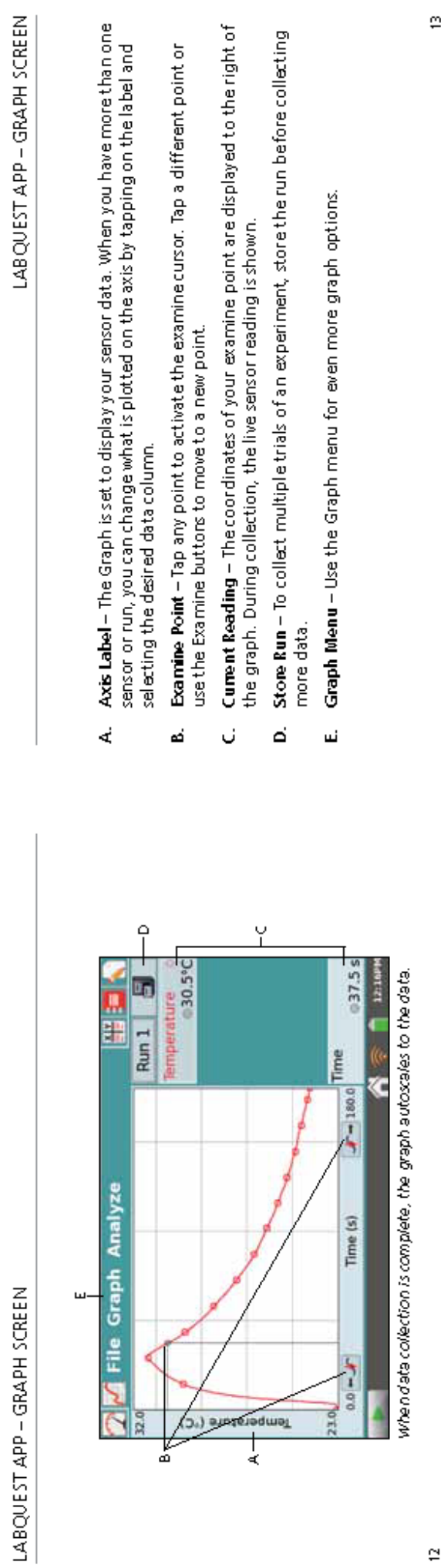

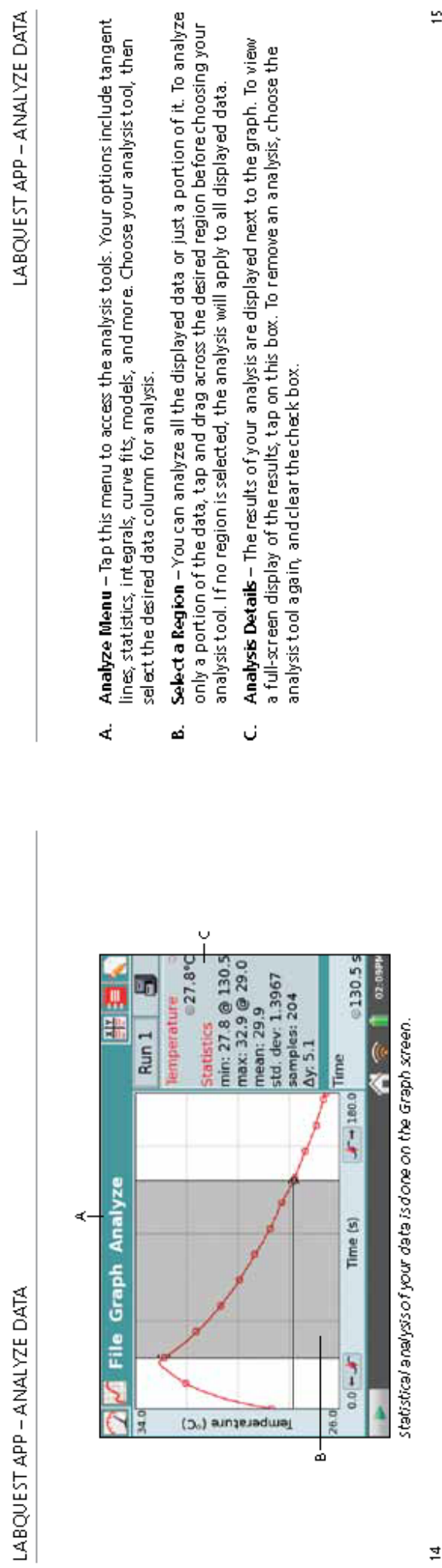

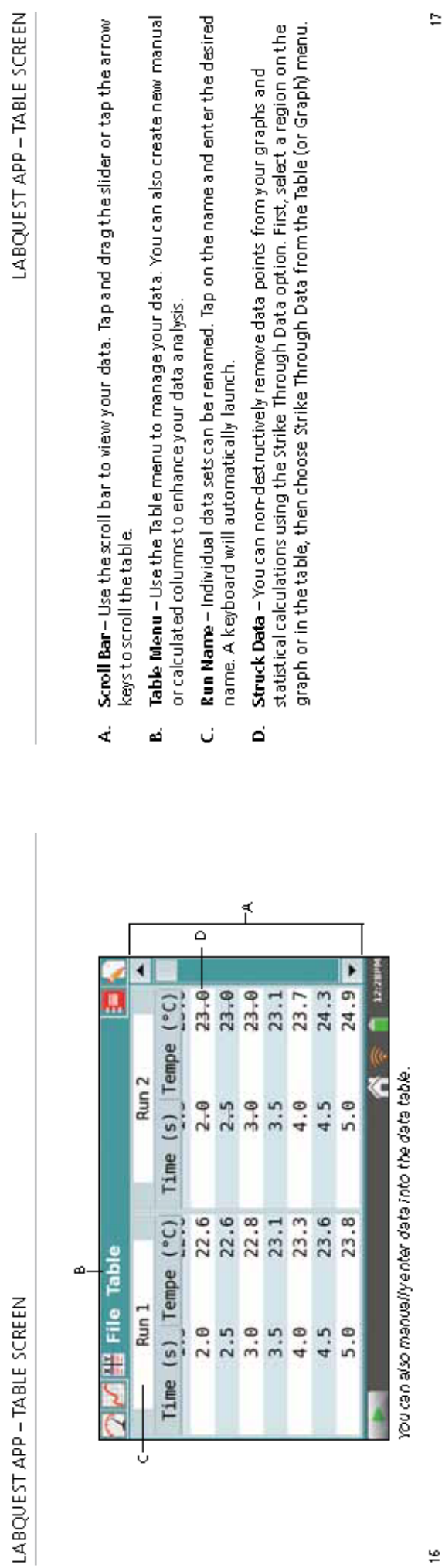

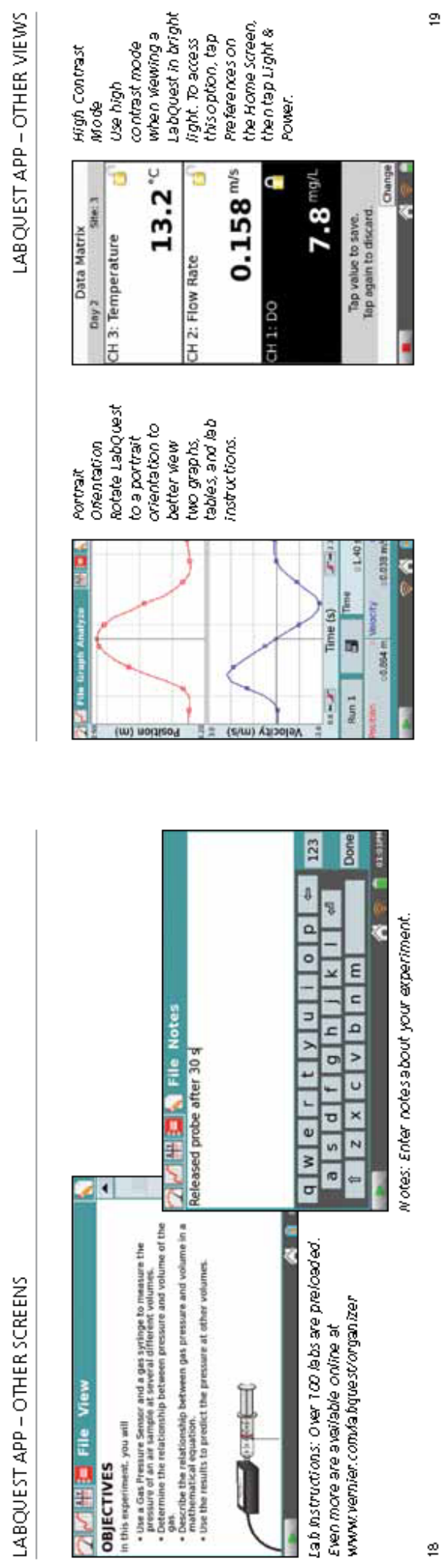

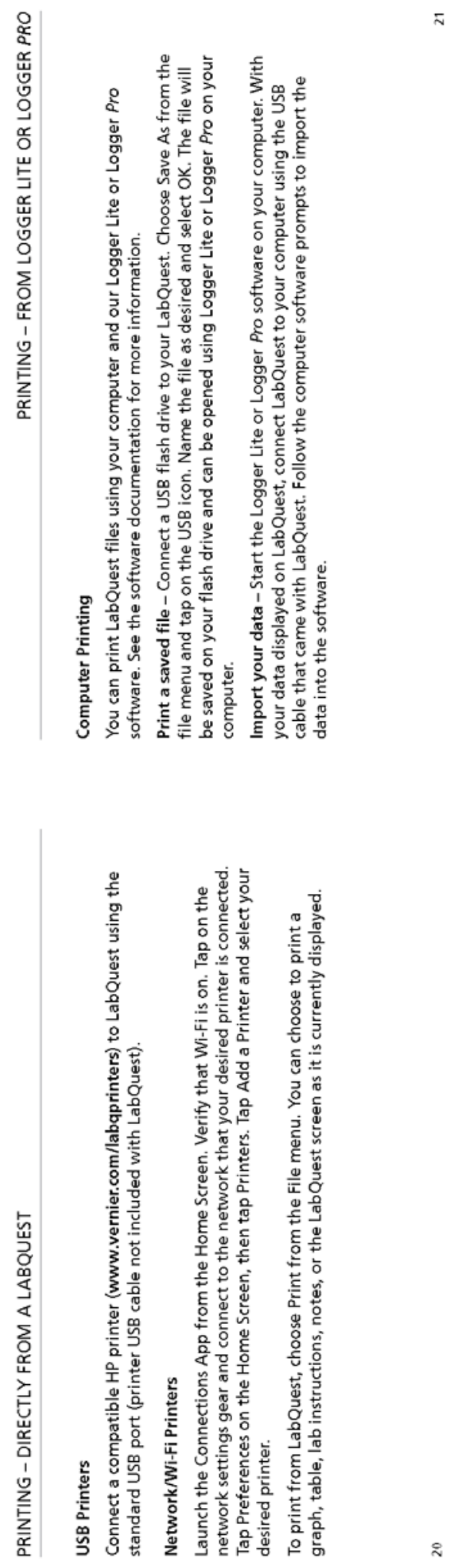

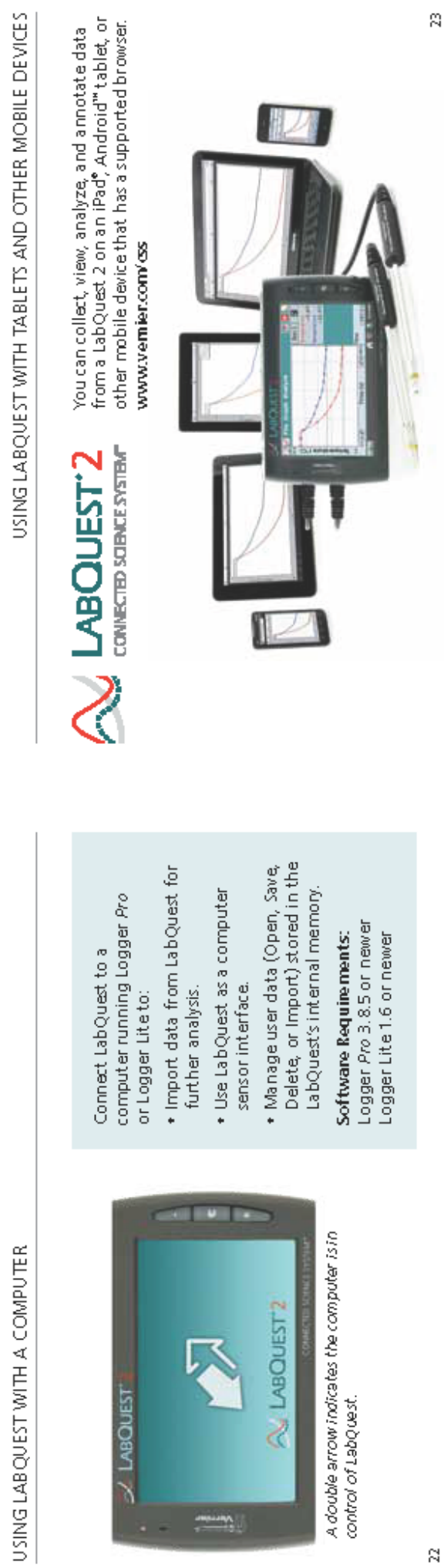

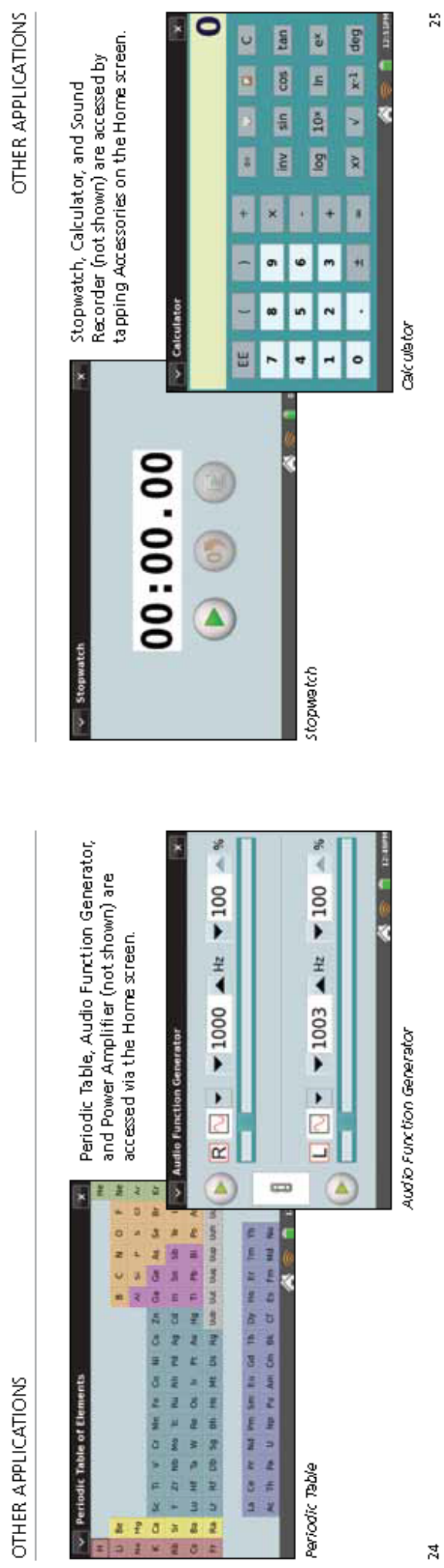

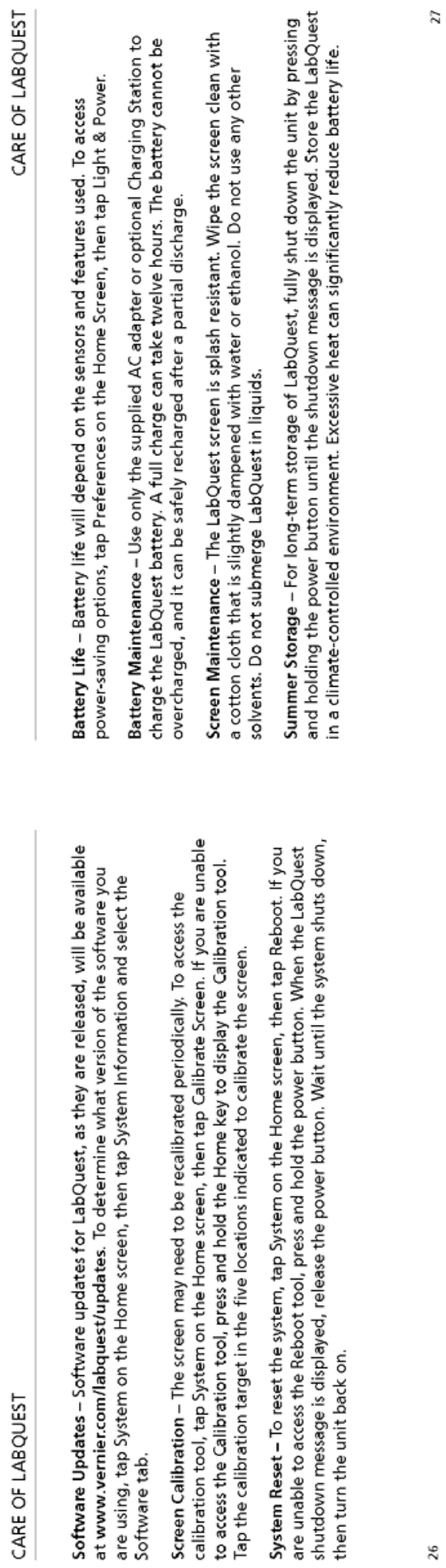

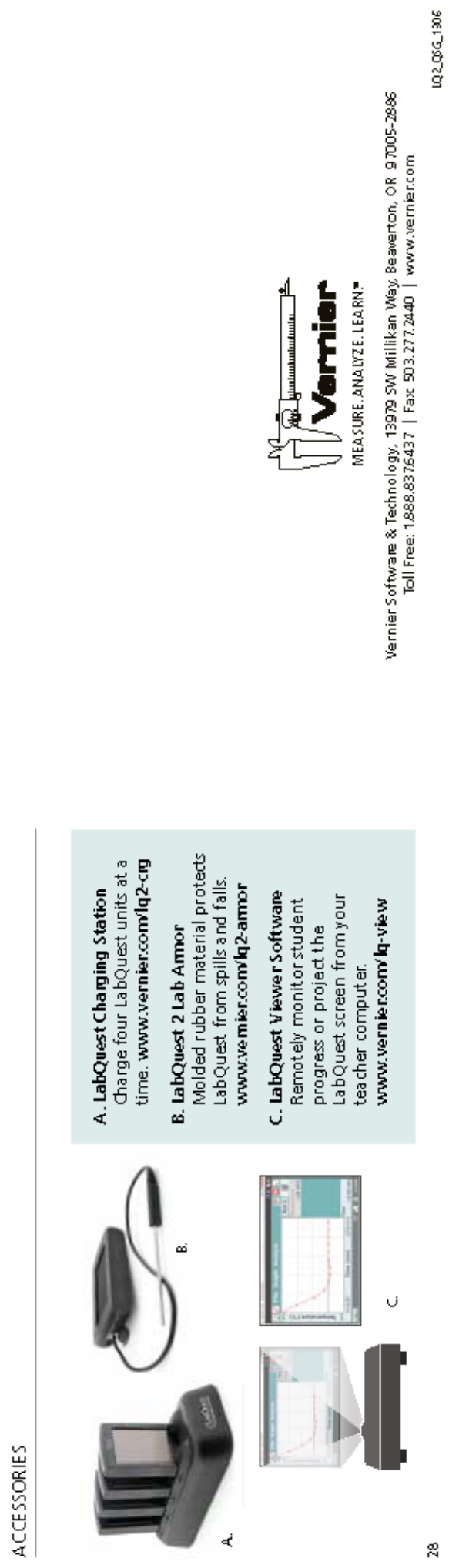


\section{. LABQUEST'2 USER MANUAL}




\section{SAFETY INFORMATION}

\section{Federal Communication Commission Interference Statement}

This equipment has been tested and found to comply with the limits for a Class B digital device, pursuant to Part 15 of the FCC rules. These limits are designed to provide reasonable protection against harmful interference in a residential installation. This equipment generates, uses and can radiate radio frequency energy and, if not installed and used in accordance with the instructions, may cause harmful interference to radio communications. However, there is no guarantee that interference will not occur in a particular installation. If this equipment does cause harmful interference to radio or television reception, which can be determined by turning the equipment off and on, the user is encouraged to try to correct the interference by one or more of the following measures:

- Reorient or relocate the receiving antenna.

- Increase the separation between the equipment and receiver.

- Connect the equipment into an outlet on a circuit different from that to which the receiver is connected.

- Consult the dealer or an experienced radio/TV technician for help.

FCC Caution

This device complies with Part 15 of the FCC Rules. Operation is subject to the following two conditions:

(1) this device may not cause harmful interference and

(2) this device must accept any interference received, including interference that may cause undesired operation

\section{RF Exposure Warning}

The equipment complies with RF exposure limits set forth for an uncontrolled environment. The antenna(s) used for this transmitter must not be co-located or operating in conjunction with any other antenna or transmitter.

You are cautioned that changes or modifications not expressly approved by the party responsible for compliance could void your authority to operate the equipment.

\section{IC Statement}

This device complies with Industry Canada license-exempt RSS standard(s). Operation is subject to the following two conditions: (1) this device may not cause interference, and (2) this device must accept any interference, including interference that may cause undesired operation of the device.

Industry Canada - Class B This digital apparatus does not exceed the Class B limits for radio noise emissions from digital apparatus as set out in the interference-causing equipment standard entitled "Digital Apparatus," ICES-003 of Industry Canada. Operation is subject to the following two conditions: (1) this device may not cause interference, and (2) this device must accept any interference, including interference that may cause undesired operation of the device. To reduce potential radio interference to other users, the antenna type and its gain should be so chosen that the equivalent isotropically radiated power (e.i.r.p.) is not more than that permitted for successful communication. 
RF exposure warning: The equipment complies with RF exposure limits set forth for an uncontrolled environment. The antenna(s) used for this transmitter must not be co-located or operating in conjunction with any other antenna or transmitter.

Le présent appareil est conforme aux CNR d'Industrie Canada applicables aux appareils radio exempts de licence. L'exploitation est autorisée aux deux conditions suivantes: (1) l'appareil ne doit pas produire de brouillage, et (2) l'appareil doit accepter tout interférence radioélectrique, même si cela résulte à un brouillage susceptible d'en compromettre le fonctionnement.

Cet appareil numérique respecte les limites de bruits radioélectriques applicables aux appareils numériques de Classe B prescrites dans la norme sur le matériel interférant-brouilleur:

"Appareils Numériques," NMB-003 édictée par industrie Canada. L'utilisation est soumise aux deux conditions suivantes: (1) cet appareil ne peut causer d'interférences, et (2) cet appareil doit accepter toutes interférences, y comprises celles susceptibles de provoquer un disfonctionnement du dispositif. Afin de réduire les interférences radio potentielles pour les autres utilisateurs, le type d'antenne et son gain doivent être choisie de telle façon que l'équivalent de puissance isotrope émis (e.i.r.p) n'est pas plus grand que celui permis pour une communication établie. Avertissement d'exposition RF: L'équipement est conforme aux limites d'exposition aux $R F$ établies pour un environnement non supervisé. L'antenne (s) utilisée pour ce transmetteur ne doit pas être jumelés ou fonctionner en conjonction avec toute autre antenne ou transmetteur.

\section{ABOUT THIS GUIDE}

LabQuest 2, released in March 2012, ships with a Quick-Start Guide ${ }^{2}$ to get you up and running with basic data collection and analysis. The LabQuest 2 User Manual is an extended guide detailing the features, hardware, and software of LabQuest 2. 


\section{CONTENTS}

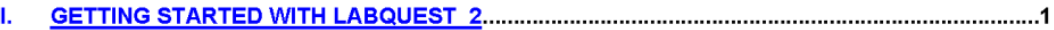

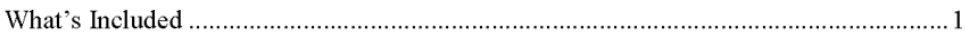

Using LabQuest for the First Time .......................................................................... 1

Quick Start to Data Collection ................................................................................. 2

II. LABQUEST 2 HARDWARE

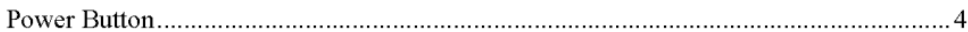

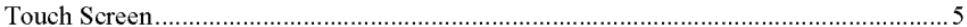

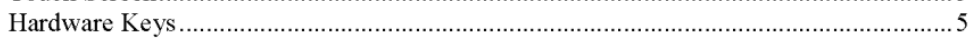

Sensor Ports ....

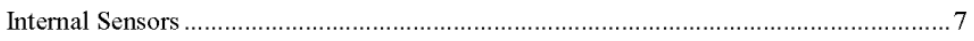

III. LABQUEST APP

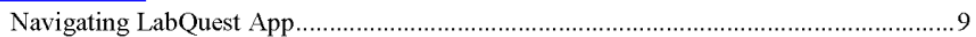

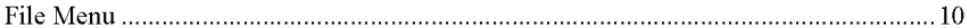

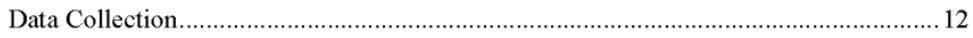

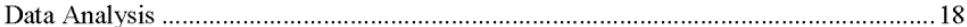

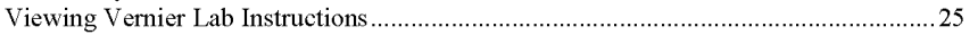

Adding Notes to Your Experiment................................................................................. 26

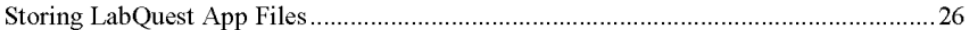

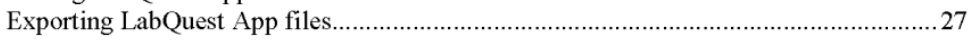

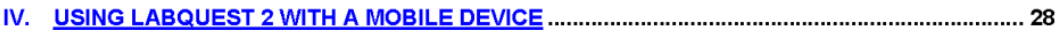

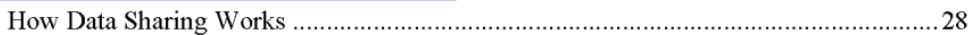

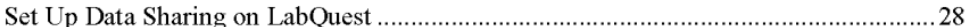

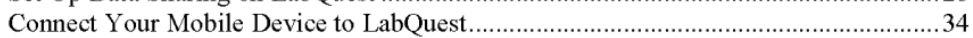

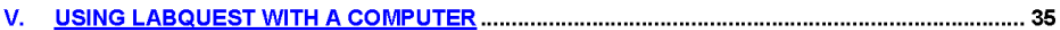

Collecting Data with Logger Lite or Logger Pro............................................................. 35

Transferring Data from LabQuest to a Computer .......................................................... 35

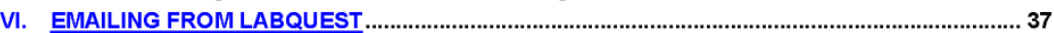

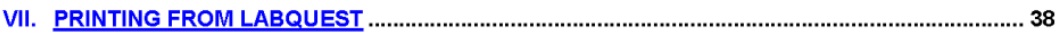

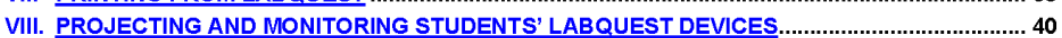

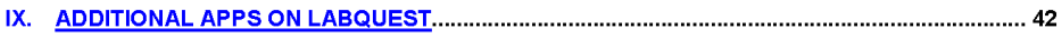

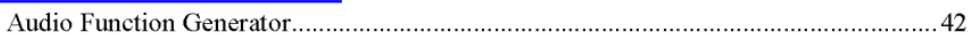

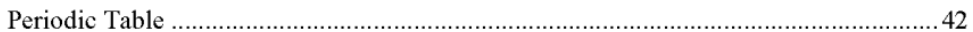

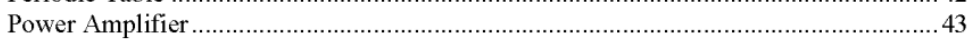

Calculator ..........4. 43

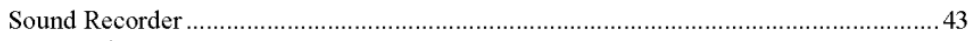

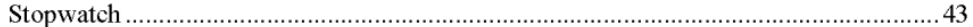

APPENDIX A. LABQUEST 2 TECHNICAL SPECIFICATIONS

APPENDIX B. LABQUEST MAINTENANCE

APPENDIX C. LABQUEST KEYBOARD

APPENDIX D. LICENSE INFORMATION

APPENDIX E. WARRANTY

APPENDIX F. ACCESSORIES, REPLACEMENT PARTS, AND RELATED PRODUCTS 


\section{GETTING STARTED WITH LABQUEST 2}

\section{What's Included}

- LabQuest 2 interface

- Rechargeable battery (in unit)

- AC power adapter

- Computer connection (USB) cable

- Quick-Start guide

- Stylus (in unit)

- Stylus tether

\section{Using LabQuest for the First Time}

Install the Battery

LabQuest ships with the battery in place; however, there is protective sticker covering the battery contacts that needs to be removed before the first use:

- Turn LabQuest over and open the battery door by sliding the door lock and lifting the battery cover.

- Remove the battery and remove the small protective sticker covering the battery contacts.

- Reinstall the battery with the label side up, ensuring the battery contacts align with the LabQuest contacts.

- Replace the battery door.

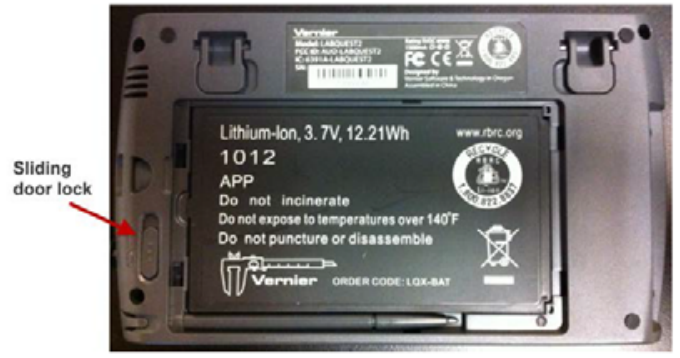

LabQuest with battery door removed

Charge Overnight

We recommend charging the battery for at least 12 hours prior to using LabQuest for the first time on battery power. To do this, connect the included power adapter to LabQuest and an AC power source. You can also charge LabQuest using the LabQuest 2 Charging Stationg (order code LQ2-CRG, sold separately). 


\section{Getting Started with LabQuest 2}

LabQuest uses a high-quality lithium-ion battery. This is the same chemistry used in premium laptop and cell phone batteries, and you can expect similar performance. There is never a need to condition the battery by regular full discharge/charge cycles.

It is safe to leave the battery charging indefinitely, and there is no need to fully discharge the battery before charging. Battery life will depend on the sensors used, but in most cases you can obtain six or more hours of use before recharging. We recommend charging LabQuest overnight to start the next day with a full charge. For more details about the battery, see LabQuest Battery.

\section{Quick Start to Data Collection}

Follow these steps to get up and running quickly with basic data collection. For more detailed instructions, see Data Collection.

1. Press and release the power button located on the top edge of LabQuest to turn on the unit. LabQuest App launches automatically.

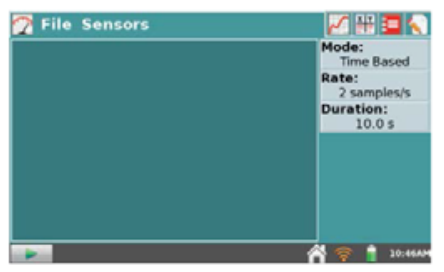

2. Connect an analog sensor to the $\mathrm{CH} 1$ port on LabQuest or connect a digital sensor to the DIG 1 port. LabQuest App will auto-ID the connected sensor and automatically set the default collection rate for the sensor.

Note: If your sensor is an older-style (DIN) sensor requiring an adapter, the sensor will not auto-ID. Instead, you will need to set up the sensor manually. To do this, choose Sensor Setup from the Sensors

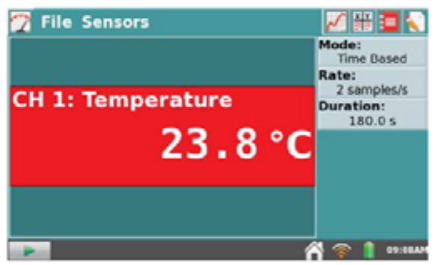
memu. Select the field labeled "No Sensor" adjacent to the appropriate port and choose your sensor from the list.

3. Next, tap Collect $\square$. Data collection begins and LabQuest graphs the data in real time.

You can stop collection early by tapping Stop

Once data collection is complete, the graph autoscales to the data.

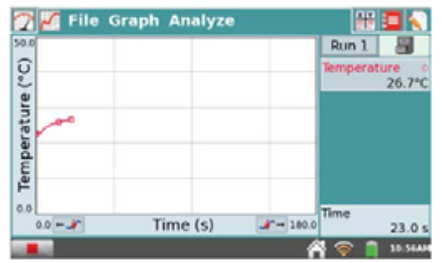


4. Tap the graph to examine a point of interest. The coordinates of the point are shown in the panel to the right of the graph. Tap another point or tap the Examine buttons to move the cursor left - irand right $\boldsymbol{H} \rightarrow$.

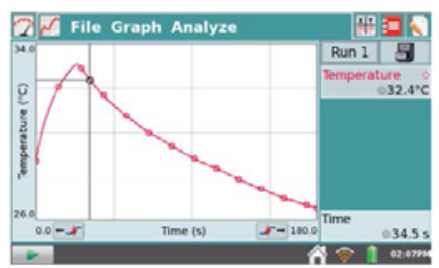

5. To select a region of data for analysis, tap-and-drag across the graph to highlight the desired region. Note: To analyze all data, you do not need to select a region.

\section{Statistics}

To view statistics for the selected data on the Graph screen, choose Statistics from the Analyze menu. Select the check box to select a data set. The statistics information is then displayed in the panel to the right of the graph.

To remove the displayed statistics, tap Analyze on the Graph screen, choose Statistics, and then select the checked box to clear the selection.

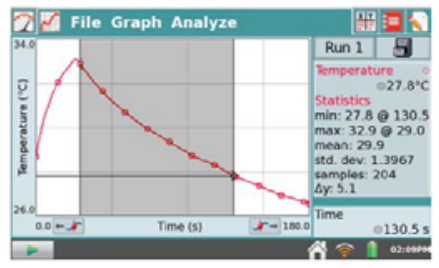

\section{Curve Fit}

To fit a curve to the selected data on the Graph screen, choose Curve Fit from the Analyze menu. Select the check box to select a data set. Tap Choose Fit and choose the desired fit equation. LabQuest automatically determines and displays the fit coefficients, and displays a preview of the curve fit on the graph. Tap OK to apply the fit and return to the Graph screen.

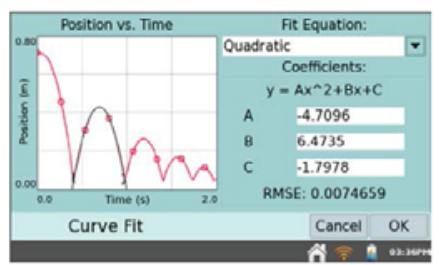

To remove the fit, choose Curve Fit from the Analyze Menu, and then select the checked box to clear the selection.

Note: The Curve Fit coefficients cannot be mamually adjusted. To enter your own parameters, see the Model tool within Data Analysis. 


\section{LABQUEST 2 HARDWARE}

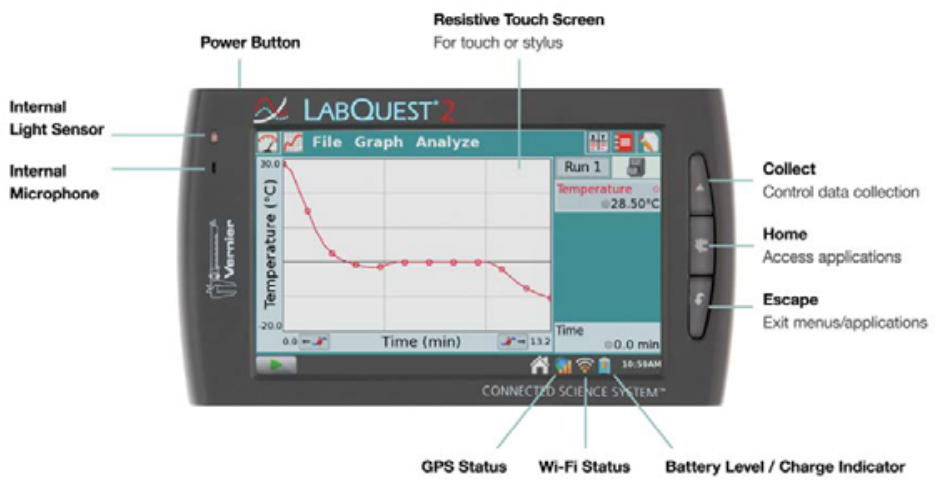

Once LabQuest is connected to $\mathrm{AC}$ power or the battery has been charged, press the power button located on the top of the unit, near the left edge. LabQuest will complete its booting procedure and automatically launch the LabQuest App by default, as shown above. If the screen does not light after a moment when used on battery power, connect the power adapter to LabQuest and to an $\mathrm{AC}$ power source, then try the power button again.

\section{Power Button}

- Power on - If the screen is off for any reason (LabQuest is off, asleep, or the screen has turned off to conserve battery power), press and release the power button to turn LabQuest back on. If LabQuest was off, LabQuest will also complete its booting procedure that takes about a minute and then display LabQuest App.

- Sleep/wake - When LabQuest is on, press and release the power button once to put LabQuest into a sleep mode. Note that the sleep mode does not start until you release the power button. In this mode, LabQuest uses less power but the battery can still drain. This mode is useful if you are going to return to data collection again soon, in which case waking LabQuest from sleep is quicker than restarting after shutdown. To wake LabQuest from sleep, press and release the power button.

- Shut down - To shut down LabQuest, hold the power button down for about five seconds. LabQuest displays a message indicating it is shutting down. Release the power button, and allow LabQuest to shut down. To cancel the shutdown procedure at this point, tap Cancel. You can also shut down LabQuest from the Home screen $\stackrel{2}{a}$. To do this, tap System and then tap Shut Down $\mathcal{O}$.

- Emergency shutdown - If you hold the power button down for about eight seconds, LabQuest powers off uncleanly. This is the same as pulling the battery out of the unit while it is running. This is not recommended unless LabQuest is frozen, as you may lose your data and potentially cause file system corruption. 


\section{Touch Screen}

LabQuest has an LED backlit resistive touch screen that quickly responds to pressure exerted on the screen. LabQuest is controlled primarily by touching the screen. The software is designed to be finger-friendly. In some situations, you may desire more control for precise navigation. In such cases, we recommend using the included stylus.

If you are having trouble viewing the color screen or are using LabQuest outside in bright sunlight, we recommend changing to the High Contrast mode. Tap Preferences on the Home screen, then tap Light \& Power. Select the check box for High Contrast to enable this mode.

\section{Hardware Keys}

In addition to using the touch screen, the three hardware keys can also be used to control your LabQuest.

- Collect - Start and stop data collection within LabQuest App

- Home - Launch the Home screen

- Escape - Close most applications, menus, and exit dialog boxes without taking action (i.e., cancel dialog boxes)

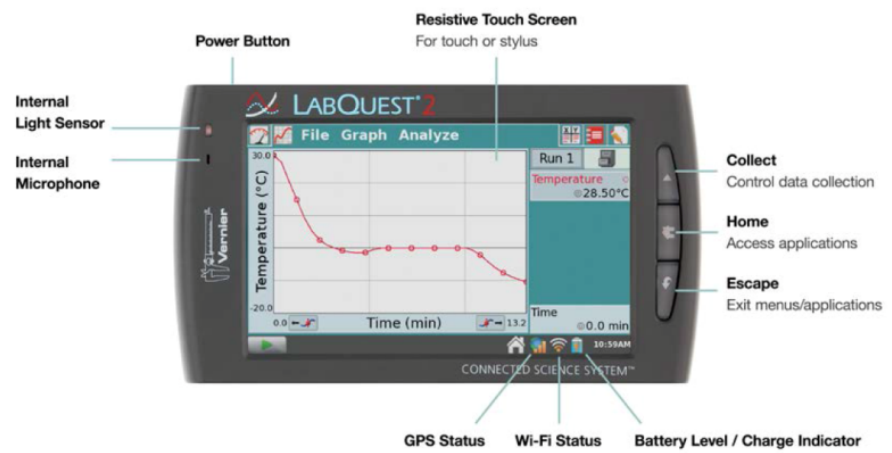

\section{Sensor Ports}

LabQuest has three analog sensor ports $(\mathrm{CH} 1, \mathrm{CH} 2$, and $\mathrm{CH} 3)$ for analog sensors such as our $\mathrm{pH}$ Sensor, Temperature Probe, and Force Sensor. Also included is a full-size USB port for USB sensors, USB flash drives, and USB printers. In addition to the power button, the top edge of LabQuest has two digital sensor ports (DIG 1 and DIG 2) for Motion Detectors, Drop Counters, and other digital sensors. 


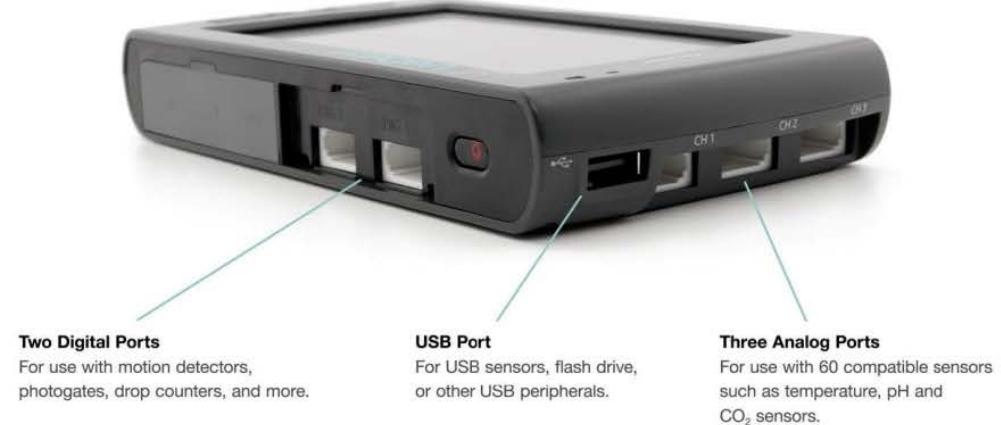

Audio ports are also located adjacent to the digital ports, as well as a microSD card slot for expanding disk storage. On the side opposite of the analog ports, there is a stylus storage slot, an AC power port for recharging the battery, and a mini USB port for connecting LabQuest to a computer. In between these ports, there is a serial connection for charging the unit in a LabQuest 2 Charging Station pr (order code LQ2-CRG, sold separately) and a stylus tether attachment point.

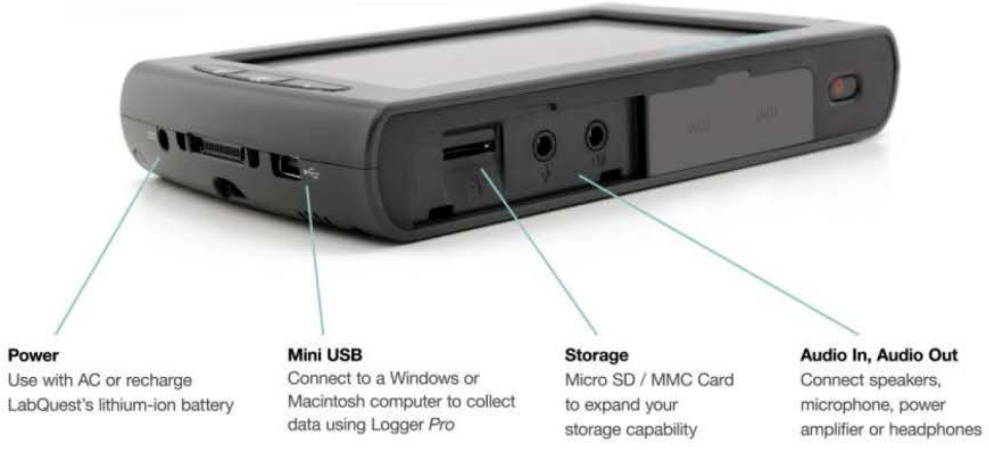




\section{Internal Sensors}

LabQuest also has several built-in sensors, including a GPS, microphone, three-axis accelerometer, temperature sensor, and relative light sensor.

To enable internal sensors within LabQuest App, choose Sensor Setup from the Sensors menu. Within the Sensor Setup dialog box, select a check box to enable the associated sensor. Then tap $\mathrm{OK}$ to return to the LabQuest App Meter screen.

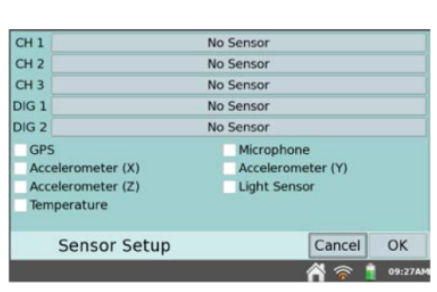

- GPS - The internal GPS collects latitude, longitude, and altitude readings, and may be used with other sensors. You can choose units of decimal degrees, degree minutes, or UTM. Note: It may take up to 15 minutes to acquire an initial signal outdoors. We do not recommend using GPS indoors.

- Accelerometers - The 3-axis accelerometer measures accelerations up to $\pm 2 g$ in the $x$-, $\mathrm{y}-$, and $\mathrm{z}$-axis directions. The sensor is located $72 \mathrm{~mm}$ from the docking connector and $32 \mathrm{~mm}$ from the digital port side of the plastic case.

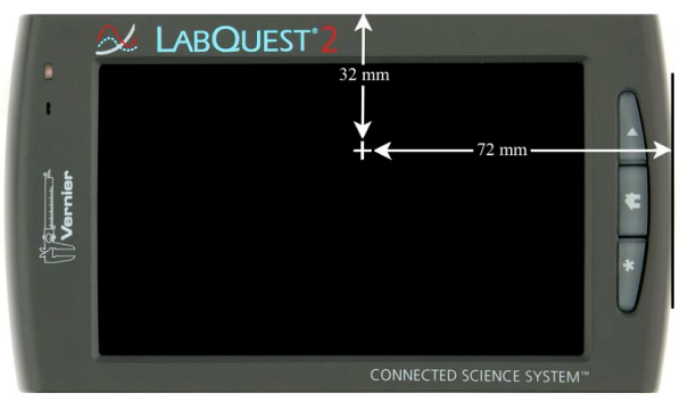

- Temperature - The internal temperature sensor monitors ambient temperature. The sensor is located between $\mathrm{CH} 2$ and $\mathrm{CH} 3$, in front of the BTA connectors.

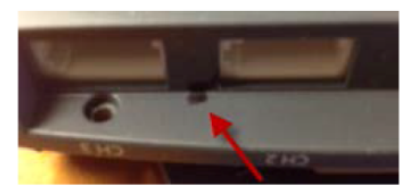

- Light Sensor - The internal light sensor is located on the front of LabQuest, to the left of the screen. This light sensor combines one exposed photodiode $(\mathrm{CH} 0)$, which is responsive to both visible and infrared light, and one filtered photodiode $(\mathrm{CH} 1)$, which is responsive primarily to infrared light. The $\mathrm{CH} 1$ reading is subtracted from the $\mathrm{CH} 0$ reading and then converted to a value in lux that represents illuminance (ambient light level) to approximate the human eye response. The spectral response range for this sensor is approximately $400-800 \mathrm{~nm}$. 


\section{LabQuest 2 Hardware}

- Microphone - The internal Microphone is located on the front of LabQuest to the left of the screen, as shown below, and measures waveforms. Note: This sensor cannot be used with external sensors.

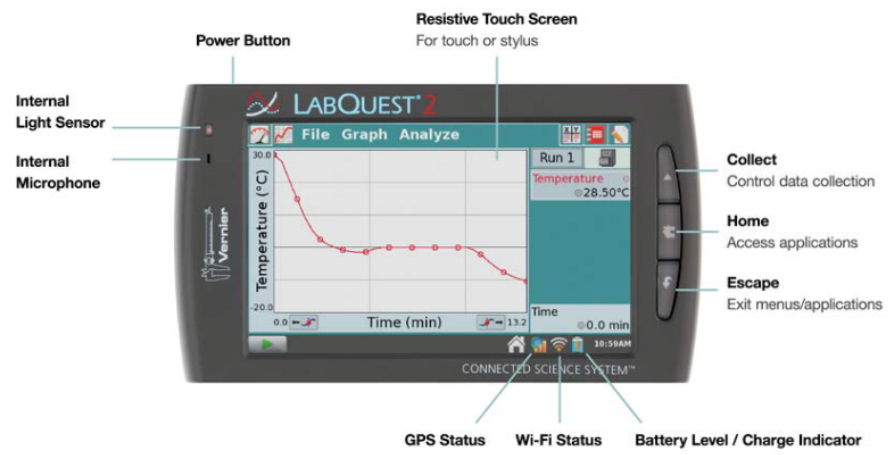




\section{LABQUEST APP}

The data-collection and analysis software, LabQuest App, is the heart of LabQuest. When you turn on LabQuest, the LabQuest App starts automatically. If the LabQuest App is not displayed on your screen, launch LabQuest App 2 from the Home screen.

\section{Navigating LabQuest App}

LabQuest App has five different screens. Tap on the desired tab to display the associated screen.

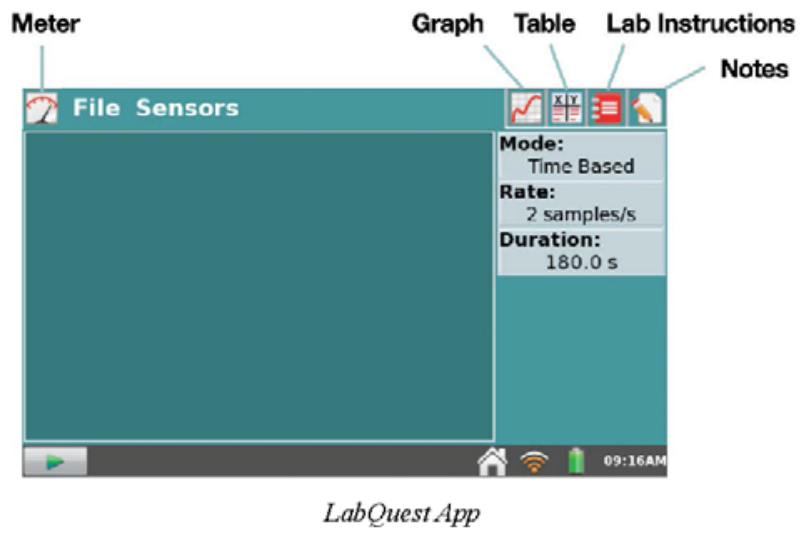

2 Meter screen - Set up sensors, set datacollection parameters, and see a digital meter for your connected sensors. For a detailed description of how the Meter screen is used for data collection, see Data Collection.

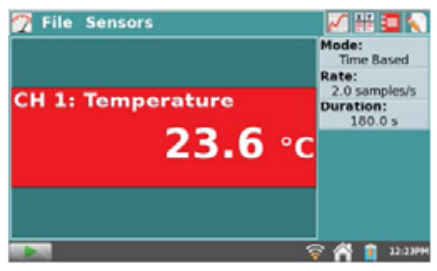

W Graph screen-See a graph of your data and perform analyses, including curve fits. For a detailed description of how the Graph screen is used for data analysis, see Data Analysis.

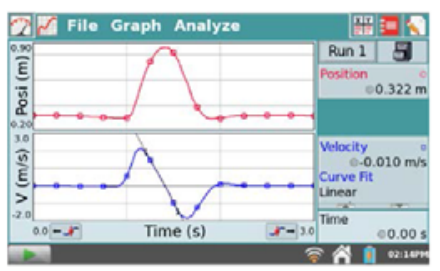


IIf Table screen - See a tabular representation of your data, create calculated columns, and manually enter data. For a detailed description of how the Table screen is used for data analysis, see Manipulating Data from the Table $\underline{\text { Screen. }}$

I Lab Instructions screen - Access one of over 100 preloaded experiments for paperless instructions (more experiments are available online). For detailed instructions on adding additional experiments to LabQuest, see Viewing Vernier Lab Instructions.
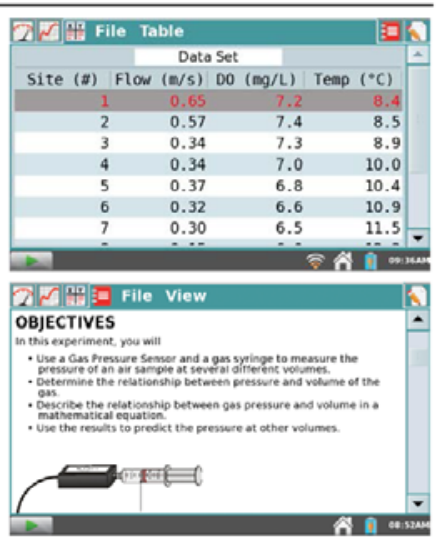

Notes screen - Record observations about your experiment. For more information, see Adding Notes to Your Experiment.

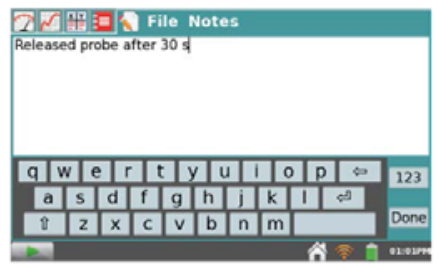

\section{File Menu}

A LabQuest App file can contain data-collection settings, graphs, data tables, analyses, and even notes. These files have a.$q m b l$ extension and can be saved to the internal storage space on LabQuest or to an external storage space such as a USB flash drive or microSD card LabQuest App files can also be opened and manipulated on a computer with Logger Pro software.

All five tabs within LabQuest App share the same File menu, which is similar to the File menu on a computer. From the File menu, you can perform a number of tasks related to LabQuest App files, such as opening, saving, and closing files, adjusting file settings, printing, and more. These are described in more detail as follows.

- New - Choose New from the File menu to reset all data-collection parameters and sensor calibrations back to default values. If you have

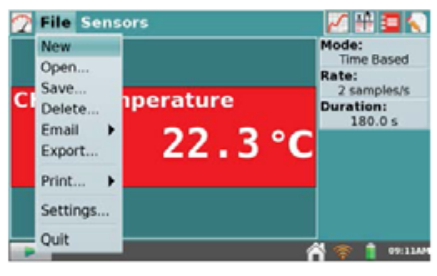
unsaved data, you will be prompted to either save or discard the data before continuing. 
- Open - Choose Open from the File menu to display a list of files that you have saved on LabQuest. To open a file, tap the file name, then tap Open. To open a file from an external storage source, tap the source icon (EUSB, QSD) to display the files available on that source, then tap on the file name, and tap Open.

- Save - Choose Save from the File menu to display the Save As dialog box where you can

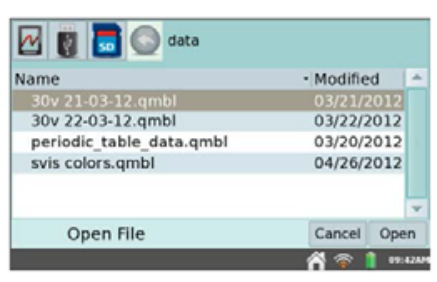
tap on a source icon (EUSB, $\mathrm{SD}$, LabQuest) to select a destination, then tap in the File name field to assign a name to the file.

- Delete - Choose Delete from the File menu to display a file list similar to that accessible by choosing open. Tap on a source icon (E USB, $\mathrm{SD}$, $\mathrm{U}$ LabQuest) to select the source where the file is located, tap the desired file name, then tap Delete. You can delete only one file at a time.

- Email - Choose Email from the File menu to email the Data File, Graph, Text File, or Screen Shot from the current file when LabQuest is connected to a wireless network with Internet. For detailed instructions on setting up this feature, see Emailing from LabOuest.

- Export-Choose Export from the File menu to export the current data file in a text format

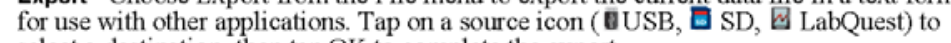
select a destination, then tap OK to complete the export.

A typical use of this feature is to export a text file to an SD card or a USB flash drive for further data manipulation on a computer with a spreadsheet program. The exported file contains all column values from all runs in the current session, delimited by tabs. For more detailed instructions on using this feature, see Storing LabQuest App Files.

- Print - Choose Print from the File menu to print the Graph, Table, Lab Instructions Notes, or Screen from the current file to a USB or Wi-Fi-enabled printer. For detailed instructions, see Printing from LabQuest.

- Settings - Choose Settings from the File menu to adjust file settings for the current session. These settings are specific to and saved within the LabQuest file. These settings return to the default upon choosing New from the File menu.

\section{- Angle Units for Trigonometric}

Calculcations - Calculated columns, curve fits, and modeled functions may use trigonometric calculations; choose Degrees or Radians here. The default is set to radians.

- Number of Points for Derivative

Calculations - Calculated columns, curve fits, modeled functions, and even the automatic setup for sensors (e.g., Motion Detectors) may use numerical derivatives.

The algorithm for such derivatives utilizes a user-defined number of points. The default value of seven points is good for many experiments, but you may want to choose a larger number for human-scale Motion Detector experiments (e.g., our

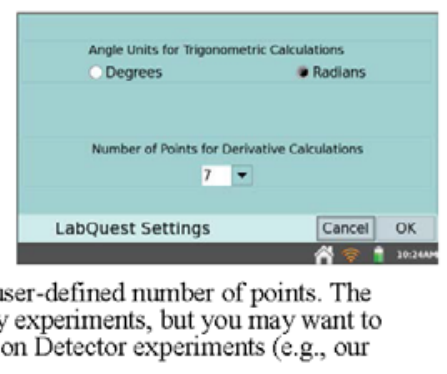


Motion Match lab), or a smaller number for cart-based Motion Detector experiments (e.g., our Impulse and Momentum lab).

- Quit - Choose Quit from the File menu to exit LabQuest App. Since other applications can run simultaneously with LabQuest App, there is typically no need to quit LabQuest App during standard use.

\section{Data Collection}

Data collection with LabQuest starts from the Meter Screen, which is the default screen for LabQuest App. From this screen, you can view sensor meters in real time as well as a summary of the data-collection settings. Additionally, you can access tools to modify and customize these settings for your particular experiment.

\section{Digital Meters}

A digital meter for each connected sensor is shown on this screen. Tap on a meter to change the sensor's settings. The available options will depend on the sensor and may include options to change the units, calibrate, zero, and reverse the sensor. These options can also be accessed from the Sensors menu.

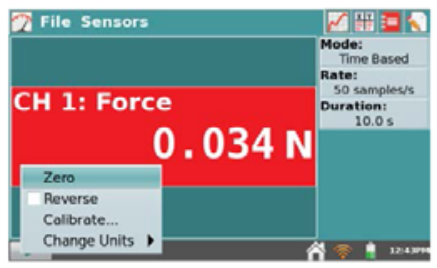

\section{Analog Meters}

To display an analog meter, choose Meter Setup from the Sensors menu, or as a shortcut, tap the digital meter on the Meter screen.

Within the Meter Setup dialog box, enter the minimum and maximum values for the meter range. If you want to view both the analog and digital readings for a sensor, select the Show digital reading check box in the Meter Setup dialog box.

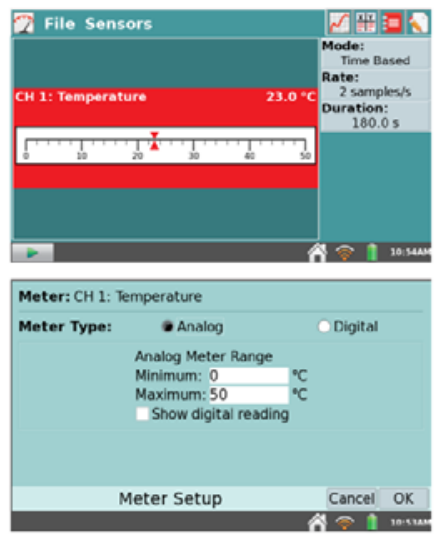

\section{Data-Collection Summary}

A summary of the data-collection settings (Mode, Rate, Duration) is shown in the panel to the right of the Meter screen. For most sensors, the default data-collection mode is Time Based. The default collection rate for the connected sensor is automatically set up when LabQuest identifies the sensor. To modify the data-collection settings, tap the summary box. Alternatively, you can choose Data Collection from the Sensors menu. For detailed descriptions of data-collection modes and parameters, see Data-Collection Settings. 


\section{Data-Collection Controls}

To start data collection from any LabQuest App screen, tap Collect $\square$. During data collection, tap Stop $=$ to end data collection early. In selected data-collection modes (e.g., Events with Entry), a Keep button $=$ will appear to the right of the Stop button. In this mode, you must tap win to record the data point in the data table. For more information, see Events with Entry.

\section{Data-Collection Settings}

The Sensors menu provides access to detailed setup controls. Use this menu to set up internal sensors, nonauto-ID sensors, and the Wireless Dynamics Sensor System (WDSS). You can also use this menu to change the data-collection parameters and adjust sensorspecific settings.

- Sensor Setup - Choose Sensor Setup from the Sensors menu to view connected sensors. To manually set up non-auto-ID sensors, tap the field displaying "No Sensor" for the channel to which your sensor is connected. Then choose the appropriate sensor from the list and tap $\mathrm{OK}$.

You can also activate (or deactivate) the internal sensors from the Sensor Setup dialog box. Select the check box next to the desired sensor to make that sensor available for data collection.

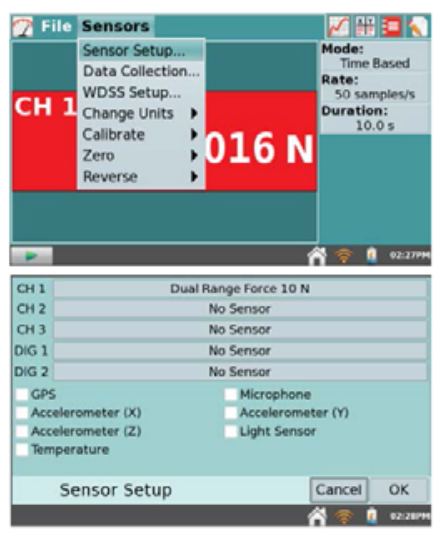

- Data Collection - Choose Data Collection from the Sensors menu to set the data-collection mode and associated parameters. The parameters listed will depend on the mode selected.

- Mode: Time-Based - Time-based data collection is the default data-collection mode for most sensors. In this mode, sensor readings are recorded at regular time intervals.

Adjustable parameters for this mode include the rate (or interval) and duration of data collection. The total number of samples to be collected based on these parameters will be displayed.

\section{Warning Messages}

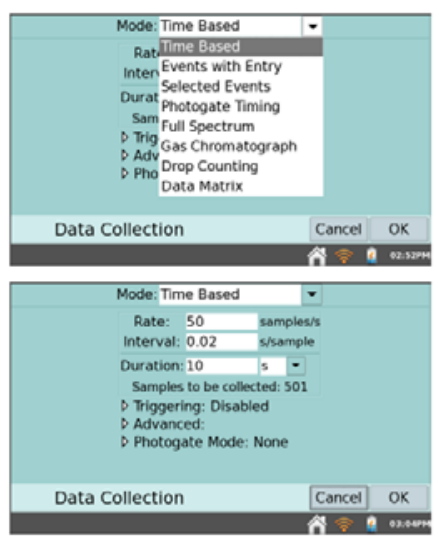

Under some circumstances, the Rate and Duration fields may be highlighted in yellow or red. The yellow warning indicates that the rate has been set to a value slower or faster than what is recommended for the connected sensor, or that the 
number of samples could lead to performance issues. You can still collect data using these settings; however, you may have undesirable results.

The red warning indicates one of the following, and you cannot exit the dialog box until the warning condition has been resolved:

- The rate has been set to a value slower or faster than what a connected device and/or sensor configuration can support

- The number of samples exceeds the storage available

- The number of samples exceeds 2000 (for rates faster than 80,000 samples/s)
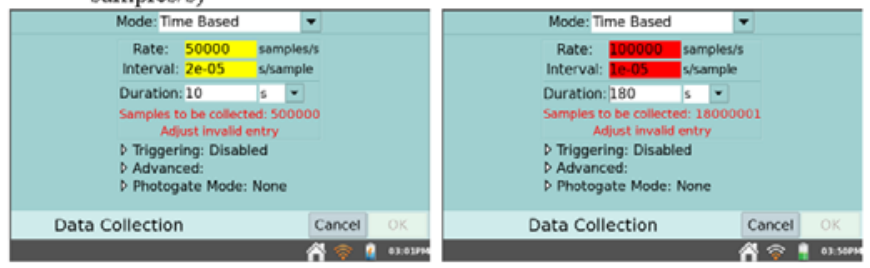

Triggering - Triggering is only available in the Time Based mode. When enabled, LabQuest waits for a trigger condition to be met before beginning data collection. To set the trigger condition, choose the sensor and set the threshold condition. You can also set the number of points to be saved before the trigger condition is met.

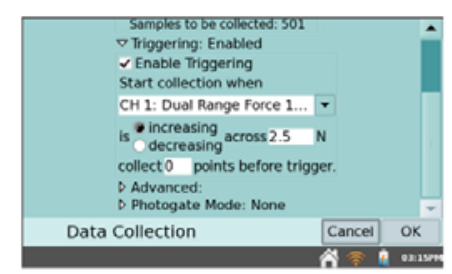

Advanced - The Advanced field has three options that can be independently enabled by selecting the associated check box.
Oversampling can be used with
data-collection rates less than ten
samples per second. When enabled,
the sensor will sample at a higher
rate than the number of samples per
second that you set, and then
LabQuest averages those readings and records the averages in the data table. This setting can be used to reduce measurement noise by

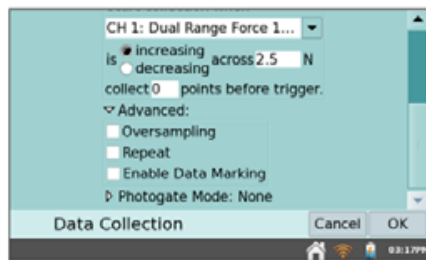
combining a burst of readings into one value. As an example, oversampling can sometimes reduce the influence of unseen but real variations, such as as those from a flickering light source.
Repeat can be used with data-collection rates less than 250 samples per second. When enabled, a new data-collection run is started as soon as the current run has ended. Data for each run is overwritten when a new run is started. This setting is helpful when doing exploratory investigations. 
Data Marking can be used to mark points of interest during a timebased data collection. When enabled, a Mark Data button $\odot$ will appear during data collection. Tap $\odot$ to mark that particular point as a point of interest. After completing data collection, data marks can be named using the panel to the right of the graph.

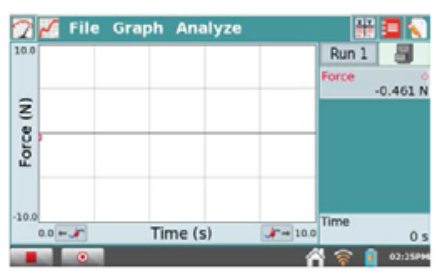

o Mode: Events with Entry - Sometimes experiments depend on a quantity other than time. For example, in a Boyle's law experiment, one wants to know the pressure as a function of the volume of gas. In Events with Entry mode, no time information is recorded.

Enter a name and units for each independent variable. You also have an option to average data over ten seconds a In Events with Entry mode, a Keep button $\cdots \longrightarrow$ appears during data collection. Tap Keep to record the sensor value (e.g., gas pressure in the Boyle's law experiment). In response, LabQuest prompts you to enter a value for the independent variable (e.g., gas volume in the Boyle's law experiment). Sensor data is plotted against the independent variable, as shown in the screenshot at the right.

- Mode: Selected Events - Selected Events mode is similar to Events with Entry, except that entries of $1,2,3 \ldots$ are automatically recorded as the independent variable. To record time information in place of an entry value, select the check box for Use Time Column.

- Mode: Photogate Timing - Photogates require a different set of timing options. When a photogate is detected, LabQuest enters the Photogate Timing mode.

You have two options for ending data collection in this mode: tap Stop, or end collection after a defined number of events. A block/unblock pair counts as

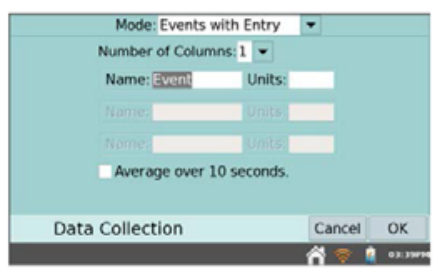

nd report the averaged reading.
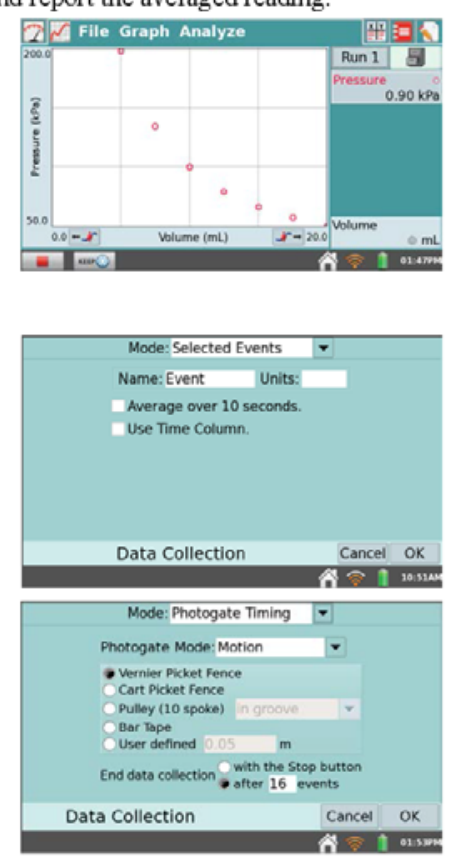
two events. Choose the proper Photogate Mode for your experiment. For more information, see www.vernier.com/til/1623/

- Mode: Full Spectrum - When a spectrometer is detected, LabQuest enters the Full Spectrum mode. In this mode, Intensity, Absorbance,

Fluorescence (SpectroVis Plus only), or \% Transmittance can be measured as a function of wavelength.

Detailed instructions for setting up data collection with spectrometers are included in each of our spectrometer labs모.

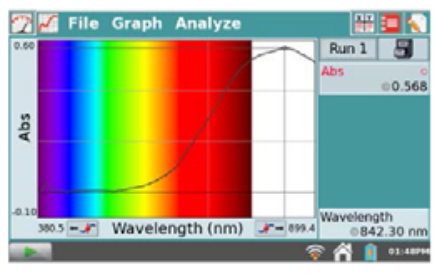

- Mode: Gas Chromatograph - When aVernier Mini Gas Chromatograph (Mini GC) is detected, LabQuest enters the Gas Chromatograph mode. In this mode, various parameters are defined for the user to establish a temperature and pressure profile adequate for the current experiment.

When the mode is first entered, a set of default parameters is displayed. Tap the parameter field to enter a new value, or adjust the default value using the arrows. These values will be reflected in the preview of the time-dependent temperature graph displayed at the right. After setting the parameters, tap $\mathrm{OK}$ to initiate the Mini GC warm up.

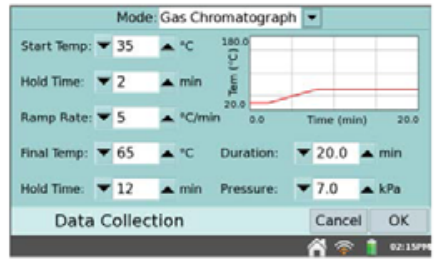

For more detailed information on the Vernier Mini Gas Chromatograph, see www.vernier.com/gc2-mini/

- Mode: Drop Counting - This mode is used with the Vernier Drop Counter. When using this mode, data points are recorded every time a drop is detected by the drop counter. For information on calibrating a drop counter, see www.vernier.com/files/manuals/vdc-btd.pdf

- Mode: Data Matrix - This mode is helpful for field work. It provides a way to collect data referenced to two parameters, such as the locations of your sampling sites and the dates they were sampled. You can collect data from an unlimited number of sensors by swapping the sensors in and out of LabQuest during data collection. For more detailed information, see www.vernier.com/til/2366/

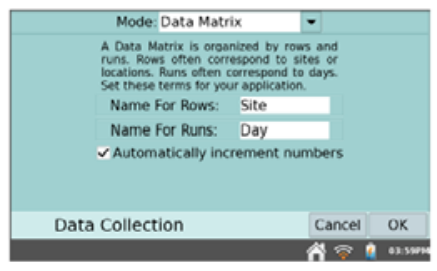




\section{LabQuest App}

- wDSs Setup-Choose WDSS Setup from the Sensors memu to scan for, select, and configure Wireless Dynamics Sensor Systems. Turn on the WDSS and note its name. Tap Scan to create a list of available WDSS devices. The scan may take 20 to 30 seconds and may have to be repeated several times to find your particular WDSS. Once a scan has found your WDSS, select its name in the list, then tap OK.

Select the check box(es) to enable the sensor(s) you want to use. Tap OK to complete the setup. Adjust the data collection parameters by choosing Data Collection from the Sensors menu. See Data-Collection Settings for more information.

Note: WDSS cannot be set up for remote data collection using LabQuest. Use Logger Pro on a computer to set up WDSS for remote use.

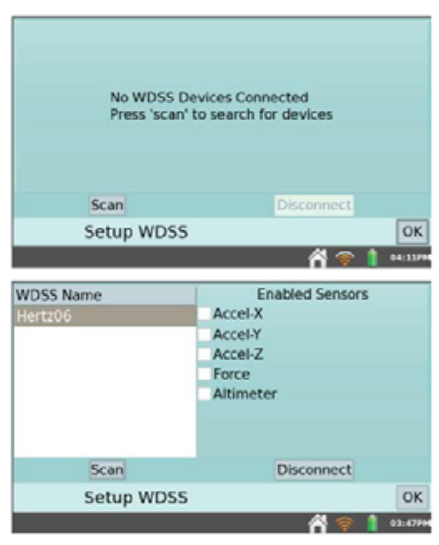

- Change Units - In some cases, you may have the option to display the sensor data in another set of units. Choose Change Units from the Sensors menu to select a different set of units. Choosing a new unit will change all existing runs for that sensor to the new unit, as well as any subsequent runs. If this feature is grayed-out in the menu, then the data for that particular sensor cannot be displayed in another set of units.

- Calibrate - Most sensors use a custom factory calibration identified by LabQuest. However, some sensors do require calibration, and you will use the Calibrate tool accessible from the Sensors menu. Follow the detailed calibration instructions provided in the booklet included with the sensor. Booklets are also available online at: www.vernier.com/support/manuals/

- Zero-Choose Zero from the Sensors menu to set the current sensor reading to zero. This adds an offset to the current reading. Not all sensors can be zeroed

- Reverse-Some sensors read both positive and negative values. For example, the DualRange Force Sensor reads positive values when pulled, and negative when compressed, by default. Choose Reverse from the Sensors menu to swap the sign of the readings with respect to the default settings. Not all sensors can be reversed.

\section{Graph Settings}

LabQuest App displays the Graph screen when data collection begins. From this screen, you can adjust graph settings during data collection. You can choose what is plotted, how the graph is scaled, and how the data is formatted.

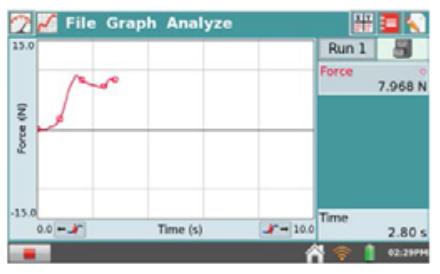




\section{LabQuest App}

- Graph Options - To control how the data are graphed, choose Graph Options from the Graph menu. Here you can choose the columns used for the $\mathrm{x}$ - and $\mathrm{y}$-axes and the axes limits. See Adjusting the Graph View for more information.

Select Autoscale to adjust to the data range after data collection ends. Autoscale from 0 does the same, but includes the origin. Manual scaling will respect values entered in the range limits, unless incoming data falls outside the range. In this case, the range will expand to include the data. To enter range limits, tap in each field and use the keyboard to enter numeric values.

The Point Symbols option is selected by default, and will surround some, but not all, of the points with a mark (e.g., a circle or triangle). This allows easy identification of a trace by the corresponding mark in the graph legend. Select the checked box to disable this feature.

The Connect Points option connects data points with straight-line segments. These lines help the eye follow the data trend, but in some cases are

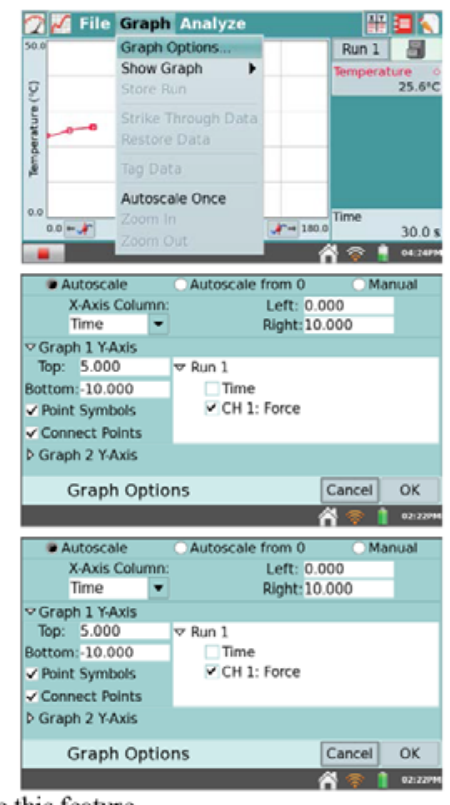
not appropriate. Select the checked box to disable this feature.

From this screen, you also select the data to be graphed. For the $\mathrm{x}$-axis, choose the desired column from the $\mathbf{X}$-Axis Column dropdown menu. For the $y$-axis, select one or more columns from the run(s) listed under the expanded Graph 1 Y-Axis field.

LabQuest can display a single graph, or two graphs sharing a common $\mathrm{x}$-axis and range. Tap the triangle next to Graph 1 Y-Axis or Graph 2 Y-Axis to show or hide the settings for that axis. If no column is selected for Graph 2, only one graph will be drawn.

When you are done setting Graph Options, tap OK to return to the Graph screen.

\section{Data Analysis}

You can analyze data from the Graph screen $\mathbb{N}$ or the Table screen $\mathbb{N}$. LabQuest analysis features are described in greater detail, below.

\section{Examining Data on the Graph Screen}

To examine data on the Graph screen, tap on a data point of interest. The Examine cursor jumps to the data point with the nearest $\mathrm{x}$-value. Cursor lines highlight the $\mathrm{x}$ - and $\mathrm{y}$-axis values, and the right-side readouts display the associated numerical values of the examined point. You can make 


\section{LabQuest App}

fine adjustments to the cursor location by using the Left Examine Button $-\boldsymbol{J}$ and Right Examine Button $\downarrow \rightarrow$ located on either side of the horizontal-axis label.

In some cases, you may want to examine data for a particular region on the graph. To select a portion for analysis, tap and drag across the desired region. You can adjust the trailing endpoint of the selected region using the Examine Buttons, if needed. For greater control, you can opt to use the stylus.

\section{Adjusting the Graph View}

You can adjust the graph view in real time during data collection by applying one of the following actions from the Graph menu.

- Graph Options - Tap Graph Options to manually adjust graph settings. To adjust the graph view, change the values in the Left and Right fields for the $\mathrm{x}$-axis and $\mathrm{y}$-axis.

- Show Graph - Choose Show Graph from the Graph menu to quickly jump between displaying one or two graphs. You can also choose the Full Width option to remove the data collection summary box and maximize the graphs on the screen.

When using spectrometers to collect intensity data, the spectrum is displayed on the graph background by default. Accordingly, Show Spectrum is selected under the Show Graph

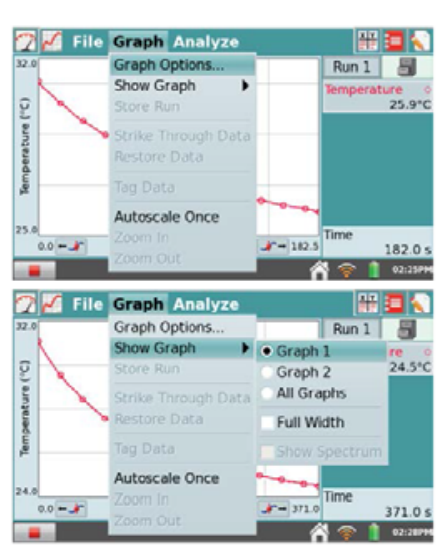
options. To hide the spectrum, choose Show Spectrum from Graph Options and select the checked box to remove the selection.

- Autoscale Once - Choose Autoscale Once from the Graph menu to scale the graph to the data you have collected so far. Note: The graph will automatically rescale larger to show data collected that would otherwise be off the graph.

- Tap on a graph axis label to change the data that is plotted on that axis.

Once data collection has ended, zoom options are also available.

- Zoom In - Select a region on the graph, then tap Zoom In to automatically adjust the axes for viewing the selected region.

- Zoom Out-Tap Zoom Out to undo a Zoom In and return the graph axes to the previous settings. If Zoom In is used several times, Zoom Out will undo each Zoom In, one at a time.

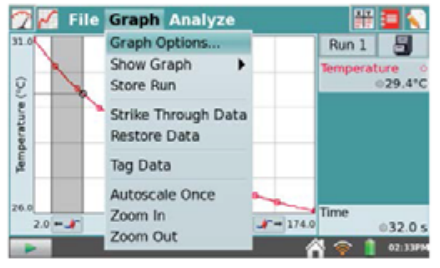




\section{Collecting multiple runs}

- Store Run - You can collect several runs for comparison. Choose Store Run from the Graph menu to save the current run and proceed with data collection. As a shortcut, tap the File Cabinet 回.

To collect another run, tap Collect. Your new run is displayed on the graph. To see your first run, tap the Run 2 button to the left of the Filing Cabinet, and select either Run 1 or All Runs. In this way, you can gather multiple runs for comparison, and view just the ones you want.

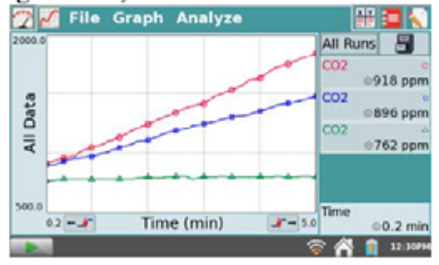

\section{Striking and Tagging Data from the Graph Screen}

To strike or tag data from the graph screen, tap on the data point or tap and drag to select a region of data. Then, choose the desired tool from the Graph menu and the action will be applied to the data.

- Strike Through Data and Restore Data - Use these tools to ignore/restore selected data. Struck data are ignored for analysis and graphing, and the graph will update accordingly. To restore all data, tap the Graph menu and choose Restore Data.

- Tag Data-Use this tool to tag a data point with a comment. After selecting the point and tapping Tag Data, a large mark (e.g., a circle or square) will be displayed on the graph to tag the data. To add a comment, tap in the panel to the right of the graph and enter a comment into the blank field.

\section{Analyzing Data from the Graph Screen}

The Analyze menu on the Graph Screen gives you access to additional tools such as tangent lines, integrals, statistics, and curve fits. To apply one of these tools, choose the desired tool from the Analyze menu. If prompted, select the desired column.

Upon enabling an analysis tool, a summary of analysis information is displayed in a panel to the right of the graph. Scroll arrows will appear, if needed. You can tap the summary to display the values on a detail dialog for ease of reading.

The following analysis tools are available from the Analyze menu.

- Tangent - The Tangent mode enhances the Examine cursor by adding a tangent line and numeric display of the slope as you tap different locations on the graph.
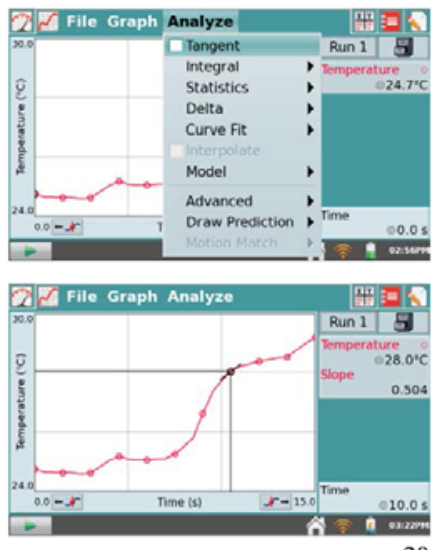
- Integral - The Integral tool numerically integrates graphed data. Select a region, if needed, and choose Integral from the Analyze menu. Enable the tool by tapping on the displayed sensor or column name. The integral is drawn and the numeric result is displayed in a panel to the right of the graph.

- Statistics - The Statistics tool displays statistics for graphed data. Select a region, if needed, and choose Statistics from the Analyze menu. Enable the tool by tapping on the displayed sensor or column name. Descriptive statistics are displayed in a panel to the right of the graph. If a region is selected, brackets are drawn to indicate the region used for calculations.

- Delta - The Delta tool opens a preview window where you can examine $\mathrm{x}$ - and $\mathrm{y}$-deltas. Choose Delta from the Analyze menu to open the preview window. Then, tap and drag to create a box overlaid on the graph. The vertical side of the box yields $\Delta y$, and the horizontal side of the box yields $\Delta x$. Tap OK to keep these values and display the box on the Graph screen. To exit the Delta tool without displaying the box on the Graph screen, tap Cancel.

- Curve Fit - The Curve Fit tool fits a chosen function to your data. If a region of the graph is selected, only that region is used for fitting. If there is no selection, the entire graph is used.

Choose Curve Fit from the Analyze menu. In the Choose Fit list, choose the desired equation. LabQuest displays the fit in the preview graph at the left. The fit coefficients and Root Mean Square Error (RMSE) are also displayed. Tap OK to keep this fit and display the curve on the Graph screen. To exit the Curve Fit tool without applying the curve, tap Cancel.

Tip: The RMSE (root mean square error) is a measure of how well the fit matches the data. The smaller the RMSE, the closer the data are to the fitted line. The RMSE has the same units as the $y$-axis data.

- Interpolate - Once you have performed a curve fit, you can use the Interpolate tool to read values from the fitted function. Choose
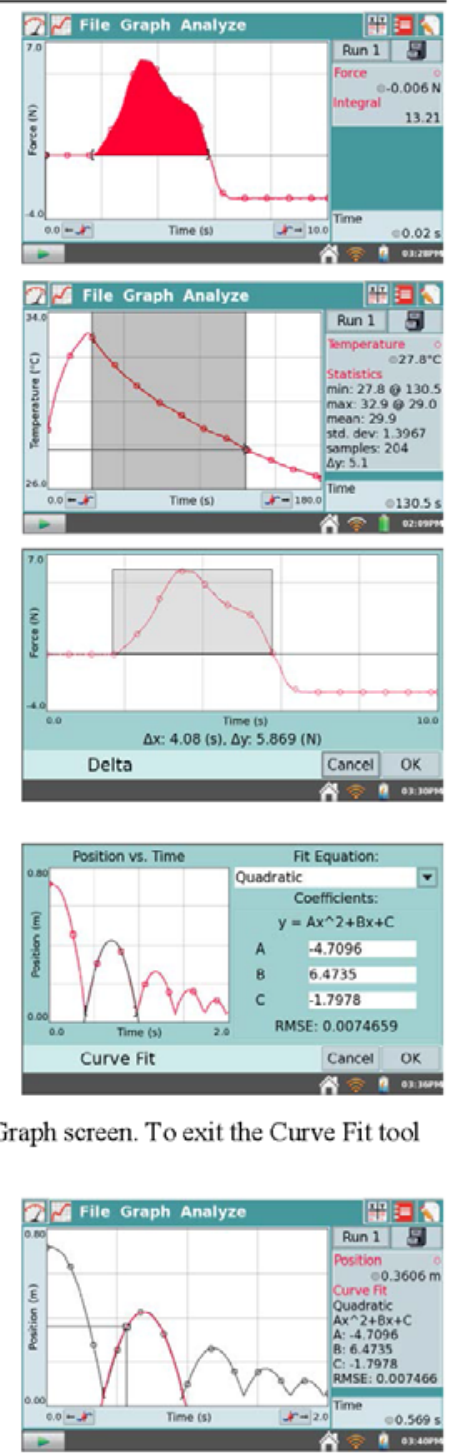


\section{LabQuest App}

Interpolate from the Analyze menu, then tap on the graph. The lines associated with the Examine cursor now locate a position on the fitted function. Coordinates along the fitted line are shown in the panel to the right of the graph. You can tell that LabQuest is in the Interpolation mode by the square Examine cursor.

- Model -The Model tool manually fits a chosen function to your data. Choose Model from the Analyze menu, then choose the desired model equation from the Model Equation list. LabQuest displays the modeled function in the preview graph at left.

The model parameters (e.g., A, B, and C) are adjustable. Change them by direct entry or by using the arrows.

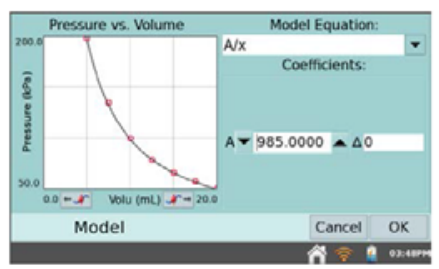

Tap OK to keep this function and display the modeled function on the Graph screen. To exit the Model tool without applying the function, tap Cancel.

Tip: If no function appears when modeling, your parameters are defining a curve that is outside of the plot window.

- Advanced-Choose Advanced from the Analyze menu to access advanced analysis tools including a Baseline Adjustment, Fast Fourier Transform (FFT), and Peak Integration.

- Baseline Adjustment - This tool applies a factor that raises or lowers the $\mathrm{x}$-axis. Because LabQuest App uses the $\mathrm{x}$-axis as its baseline when calculating an integral, adjusting the baseline may

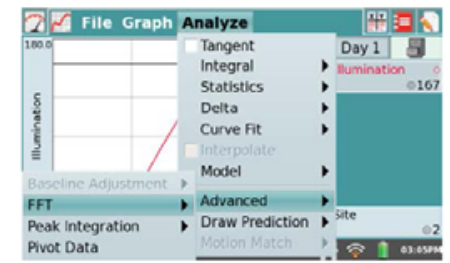
result in a better integral. This tool is typically used when analyzing Spirometer data.

- FFT - The FFT tool calculates a Fast Fourier Transform of the selected data. The FFT is displayed in a separate graph that can be analyzed. Tap OK to return to the main graph. The peak frequency will be displayed on the graph legend. The FFT tool is typically used when analyzing Microphone data. For more information, see: www.vernier.com/til/2310

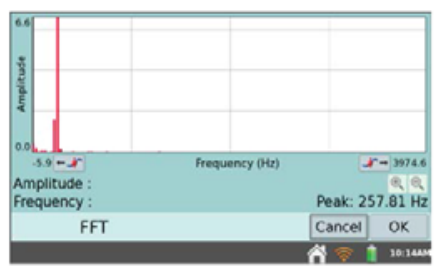

- Peak Integration - This tool calculates the integral for a selected portion of a graph. It is most commonly used with the Vernier Mini GC, but it can be applied to any data plot. Peak Integration differs from the Integral tool in that it does not use the $\mathrm{x}$-axis as the baseline. Instead, the integral for Peak Integration is evaluated from the minimum y-values to the left and right of a selected peak. 
- Pivot Data - This tool is only available in Data Matrix mode. It transposes the rows (usually graphed as the $\mathrm{x}$-axis and representing the sampling site) with the runs (usually selected by tapping on the button to the left of the Filing Cabinet and representing the sampling day). This allows field data to be viewed as the location changes, or pivoted and viewed as the day changes.

- Draw Prediction - The Draw Prediction tool is a free-hand sketch tool for drawing on the Graph screen. This can be used for a variety of purposes, but is most often used to sketch a prediction of how a graph will appear once data are subsequently collected.

Choose Draw Prediction from the Analyze menu. Then, tap and drag across the screen for smooth curves, or tap the screen to connect subsequent taps with straight-line segments. The Reset button removes your sketch if you need to start over. Tap OK to place your sketch on the main graph. To remove a prediction, choose Draw Prediction again from the Analyze menu.

- Motion Match - The Motion Match menu item is only available if a Motion Detector is connected. Choose between a new Position or Velocity match. In each case, LabQuest generates a random target graph for the matching exercise. Only the selected graph, Position or Velocity, is shown. You may collect data over the target graph as many times as you like using the Collect button. To see a new target graph, choose New Position Match or New Velocity Match. Remove Match removes the target graph
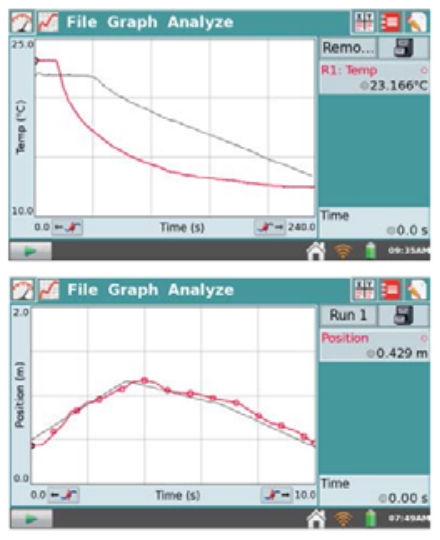

Tip: For additional information on using Motion Detectors with LabQuest, see www. vernier.com/til/s

Adjusting the Table View

In addition to viewing and manipulating data from the Graph screen, you can also access data from the Table screen. There are several shortcuts on this screen. Alternatively, you can also access the fields by choosing Data Column Options from the Table menu.

- Tap Run 1 in the name field to rename the run.

- Tap a column header (Time, Position, etc.) to change the column name, units, or displayed precision.

Manipulating Data from the Table Screen

The Table menu allows you to create, modify, or delete columns of data. The following tools are available from the Analyze menu:

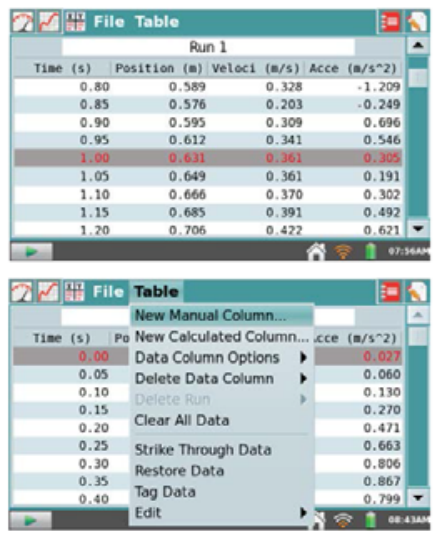

23 


\section{LabQuest App}

- New Manual Column - New Manual Column creates an empty column where you can enter or generate values directly. You can add a manual column to a data set that includes sensor data, or you can create a new data set consisting entirely of manually entered data.

- New Calculated Column - New Calculated Column creates a new column whose values are
based on other columns by a mathematical formula. Tap the Name field to enter a name

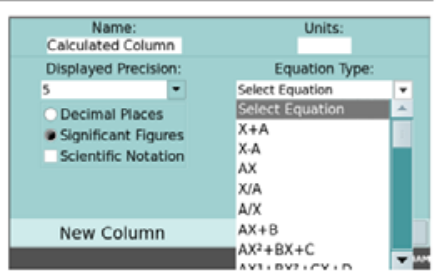
for the new column, tap the Units field to enter the units, and then select an equation from the Equation Type list. For example, you might define a calculated column as the inverse square of another column. After creating a calculated column, you can display the calculated data on a graph, or manipulate it further with additional calculated columns.

- Data Column Options - This allows you to access the fields for setting the column name, units, and displayed precision. Alternatively, you can access these fields by tapping on the column name from the Table screen.

- Delete Data Column - This allows you to delete a manual or calculated column of data. Note that you cannot delete data collected from a sensor; however, you can hide data using the Strike Through Data tool.

- Delete Run - If you have stored at least one run using the Store Run tool, this option will be available in the Table menu. Upon choosing Delete Run, tap the desired run name to delete the run. You will not be able to delete the last run created.

- Clear All Data - This action will clear all data in the table. Upon choosing this option, you will be prompted to confirm. This option is used when you wish to clear previously collected data without changing any of the data collection parameters or sensor setup information.

- Strike Through Data and Restore Data - Use these tools to ignore/restore selected data. Struck data are ignored for analysis and graphing, and the graph will update accordingly. To restore all data, choose Restore Data from the Table menu. Note that you can also access these tools from the Graph menu on the Graph screen.

- Tag Data - Use this tool to tag a data point with a comment. After selecting the data point within the table, choose Tag Data from the Table menu. A circle will be displayed on the Graph screen to "tag" the data. To add a comment, tap the Graph tab, then tap the info panel to the right of the graph. A list of Data Tags will be displayed. Tap in the blank field to enter a comment for the tag.

- Edit - This tool allows you to copy and paste values from one location to another. In particular, you might copy a range of values from the table and paste them into notes on the Notes screen.

Additionally, when LabQuest is in the Data Matrix mode, an Advanced Menu is displayed adjacent to the Table menu. Choose Pivot Data from the Advanced

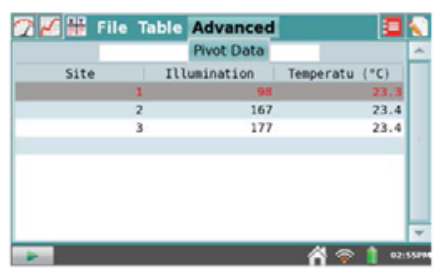




\section{LabQuest App}

menu to transpose the rows (usually graphed as the

$\mathrm{x}$-axis and representing the sampling site) with the runs (usually selected by tapping on the button to the left of the Filing Cabinet and representing the sampling day). This allows field data to be viewed as the location changes or pivoted to be viewed as the day changes.

\section{Viewing Vernier Lab Instructions}

The Lab Instructions screen, selectable by tapping the Lab Instructions tab $\Xi$, allows students to view lab instructions from Vernier lab books. LabQuest comes preloaded with over 100 experiments. You can easily download experiments from other books that you have purchased and load them onto LabQuest using our easy-to-use web tool, LabQuest Lab Organizer. For more information, see Adding Experiments to LabQuest. You can also create new content using our online LabQuest Lab Creator. For more information, see Creating Your Own Experiments.

\section{Opening Vernier Lab Instructions}

From the Lab Instructions screen, choose View Lab Instructions from the View menu. This displays a list of the lab books with experiments preloaded on LabQuest. Select the desired book and experiment, and then tap $\mathrm{OK}$.

In addition to scrolling through the instructions using the scrollbar at right, you can also zoom in on a particular portion of the instructions by choosing Zoom In from the View menu. Choose Zoom Out from the View menu to restore the previous view. Choose Reset from the View menu to restore the original view.

\section{Adding Experiments to LabQuest}

If you have purchased Vernier lab books and have experiments in those books that you would like to load onto LabQuest, you can do so using our online tool, LabQuest Lab Organizer. Follow these simple instructions:

1. Visit our web site: www.vernier.com/labquest/organizer/

2. Select the experiments that you wish to add. (Note that you can either select individual experiments or add entire books)

3. Follow the on-screen guide to create and download a LabQuest Library update and save it to a USB flash drive or SD card.

4. Follow the provided instructions to transfer the update from the flash drive or SD card to your LabQuest units.

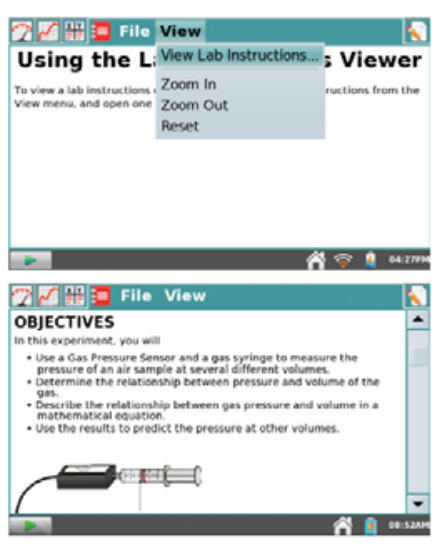
. 
LabQuest App

\section{Creating Your Own Experiments}

If you would like to create your own content, you can do so using our online tool, LabQuest Lab Creator. Follow these simple instructions:

1. Visit our web site: www.vernier.com/labquest/creator/

2. Choose your starting point. You can create a new experiment from scratch, start with a template, or paste an existing experiment into our Lab Creator.

3. Follow the on-screen guide to create your content. Note that you can save your content and finish another time. Click Save, and a web link will be displayed that you can bookmark from your browser for later access.

4. When your content is complete, follow the on-screen guide to preview, name, and download your experiment to a USB flash drive or SD card.

5. Follow the provided instructions to transfer the update from the flash drive or SD card to your LabQuest units.

\section{Adding Notes to Your Experiment}

The Notes screen, selectable by tapping the Notes tab 8 , allows students to enter notes as they perform an experiment. The menu provides access to standard edit commands of Cut, Copy, Paste, and Clear All.

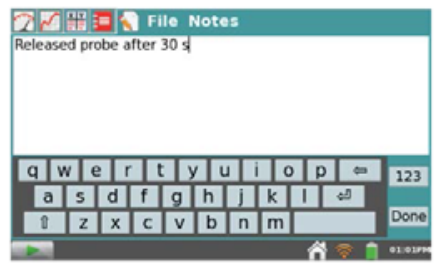

\section{Storing LabQuest App Files}

LabQuest files have a .qmbl extension and can be saved to the internal storage space on LabQuest, or to an external storage space such as a flash drive. LabQuest App files can also be opened and manipulated on a computer with Vernier Logger Pro software.

LabQuest can recognize additional storage space in a connected microSD (Secure Digital) card or a USB flash drive. The drive or card may be formatted in FAT16 or FAT32 (the most common Windows and Mac OS formats) for reading and writing. LabQuest cannot read NTFS or HFS+ formatted drives.

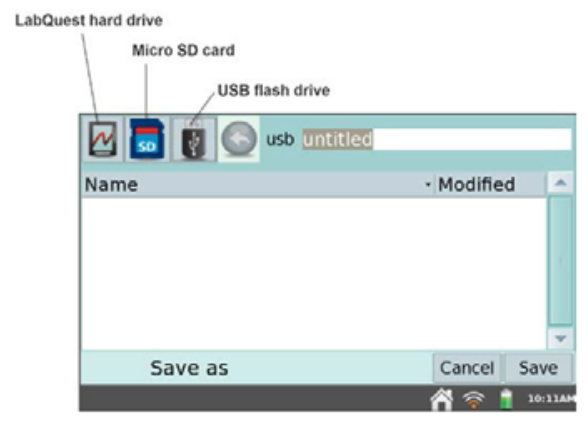


1. If saving to a microSD card or USB flash drive, make sure the drive is inserted in the appropriate port on LabQuest. Note: After inserting the drive, wait a few seconds while LabQuest recognizes the drive before proceeding.

2. Choose Save from the File menu. This opens a Save As dialog box.

3. Tap on the appropriate icon to select your storage destination.

四 - LabQuest internal hard drive

- microSD card

- USB flash drive

4. After selecting your destination, tap on the name field and enter the file name.

5. Tap OK to return to the Save As screen.

6. Tap Save to save the file.

Note: You cannot create directories within LabQuest App, but you can use directories that already exist on the SD card or USB drive. We recommend organizing your files on a USB drive or SD card by creating any needed folders on a computer before you use the drive or card with LabQuest.

\section{Exporting LabQuest App files}

In some cases, you may wish to export the LabQuest App file in a text format (.txt) for further analysis within a program other than Logger Pro.

To do this, choose Export from the File menu. Follow steps similar to those described in $\underline{\text { Storing }}$ LabQuest App Files, but choose Export from the File menu instead of Save As.

To open the exported .txt file in a spreadsheet program, confirm the program's file browser is set to look for all file types. Select your text file.

If you have access to a wireless network with Internet, you can email the data file, graph, text file, or screenshot. For detailed instructions, see Emailing from LabQuest. 


\section{USING LABQUEST 2 WITH A MOBILE DEVICE}

As part of the Connected Science System ${ }^{\star}$, LabQuest 2 serves as a Data Sharing source that can wirelessly stream sensor data to Data Sharing apps on mobile devices such as iPad ${ }^{\infty}$ and Android ${ }^{\mathrm{TM}}$ tablets.

\section{How Data Sharing Works}

- Students in a lab group set up an experiment with Vernier sensors and a Data Sharing source.

- Students use a Data Sharing app to wirelessly start data collection.

- Experiment data is streamed from the Data Sharing source to each student's Data Sharing app.

- Each lab group member performs an individual analysis on their Data Sharing app.

- Students take their data and analysis home on the Data Sharing app for further analysis and lab reports.

\section{Set Up Data Sharing on LabQuest}

To set up LabQuest as a Data Sharing source, you will need to connect LabQuest to a Wi-Fi Network and enable Data Sharing, described in detail as follows. Note: These instructions are for LabQuest 2.2 or newer.

\section{Connect LabQuest to a Wi-Fi Network}

1. Launch the Connections App from the Home screen.
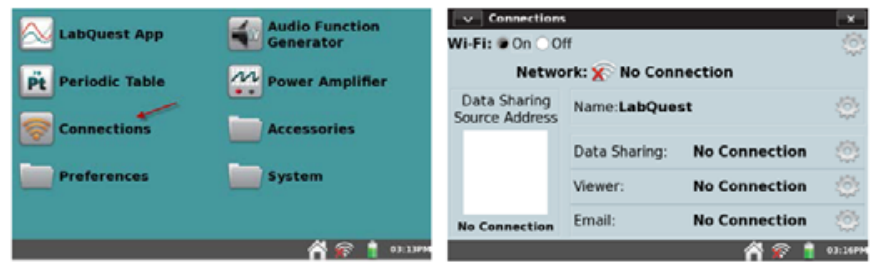

2. Verify Wi-Fi is enabled (On).

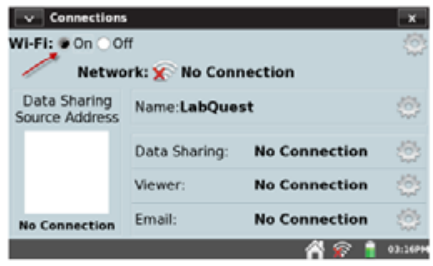




\section{Using LabQuest 2 with a Mobile Device}

3. Tap the Network Settings gear on the Connections App to open the Network Configuration dialog box. The Network Configuration dialog box lists any networks that LabQuest sees, as well as an option to join networks manually or create your own network.
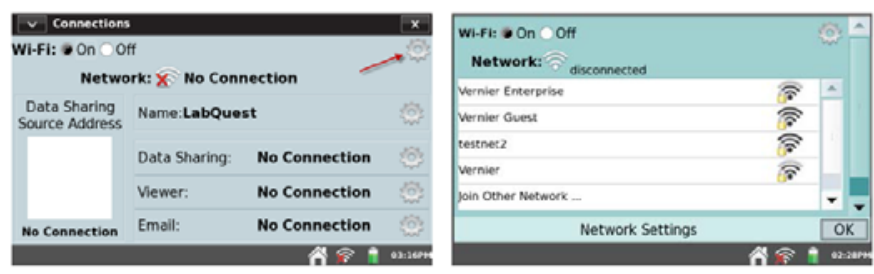

4. Select a network

Existing network (Infrastructure Network)

To connect to an existing Wi-Fi network, follow these instructions.

a. Locate the network within the list and tap on the network name to select it.

If prompted, enter your network's passphrase and tap Connect.
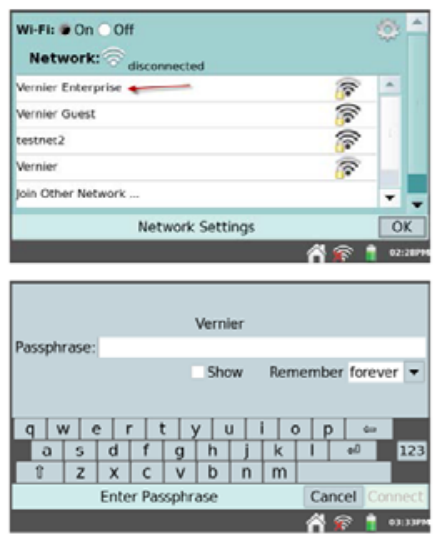

Note: If your network requires additional network authentication information or if you have an enterprise network that requires a network certificate, see our web site for more instructions:

www.vernier.com/til/2836

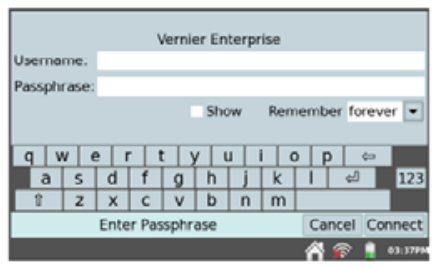




\section{Using LabQuest 2 with a Mobile Device}

b. Verify LabQuest is attempting to connect to the network. The network status should say "Connecting."

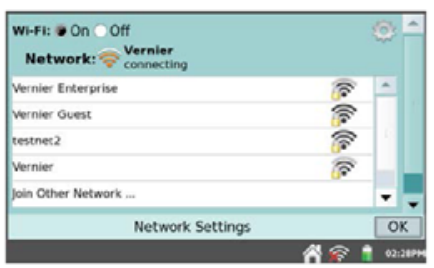

c. Verify LabQuest has successfully connected to the network. The network status should have changed to a numeric IP address.

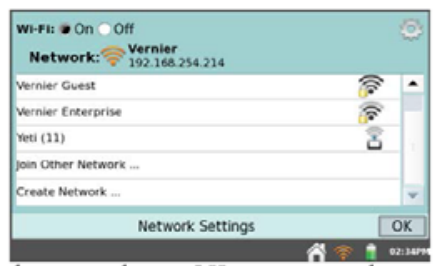

d. When LabQuest has successfully connected to the network, tap OK to return to the Connections app.

Create a new LabQuest Network (Ad-Hoc Network)

If you do not have access to an existing Wi-Fi network, or are not permitted to access your school's network, you can quickly and easily set up a network with LabQuest 2.

Note: Students can connect to a LabQuest Network with their mobile devices. This network will not support access to the Internet. The email function of LabQuest will not work with a LabQuest ad-hoc network.

a. Select Create Network.

Note: If you are in an area with several WiFi networks, you may need to scroll to the bottom of the network list to locate "Create Network."

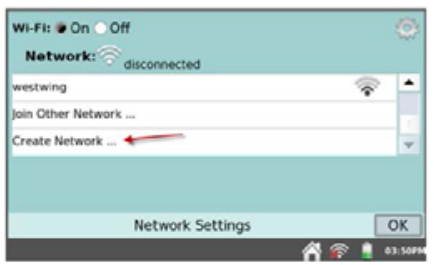

b. Tap the Network Name field and assign a name to this new network (e.g., Ms. Johnsons classroom). Then, tap Create.

Optional: Protect your network by assigning a password that you will provide only to your students. Select the check box and assign a password to your new network (e.g., Ifrog, scienceisfun!). The Passphrase must meet certain criteria. See our web site must meet certain criteria. See our web site
for additional information: www.vernier.com/til/2699/

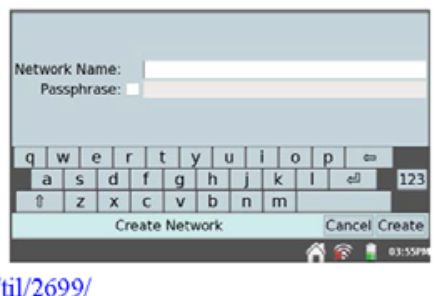




\section{Using LabQuest 2 with a Mobile Device}

c. Verify LabQuest is attempting to create a network. The network status should say "Connecting."

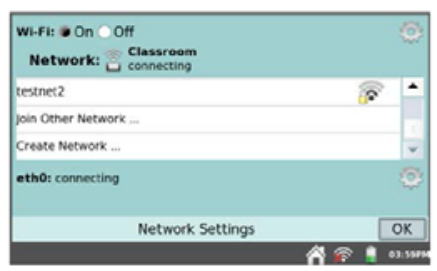

d. Verify LabQuest has successfully created a network. The network status should have changed to a numeric IP address.

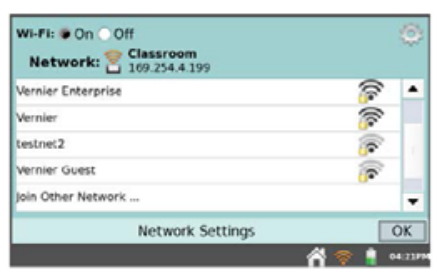

e. When LabQuest has successfully created network, tap OK to return to the Connections app.

Note: Some Android implementations seem to have trouble viewing and connecting to ad-hoc networks. These devices require the use of an existing (infrastructure) network.

Enable Data Sharing on LabQuest

In addition to connecting to a Wi-Fi network, you will need to set up LabQuest as a Data Sharing Source using the following instructions.

1. Launch the Connections App from the Home screen.
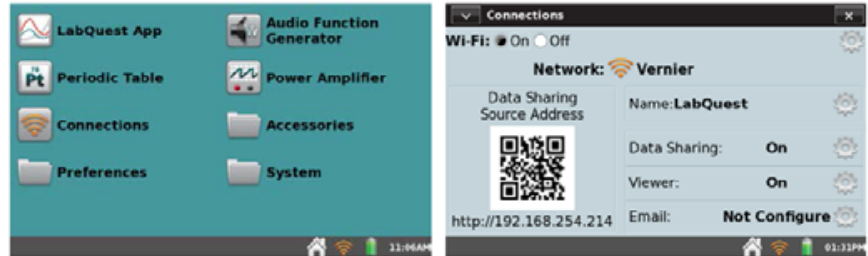

2. Optional: To easily identify this LabQuest unit, you can give the unit a unique name. To do this, tap the Name field gear.

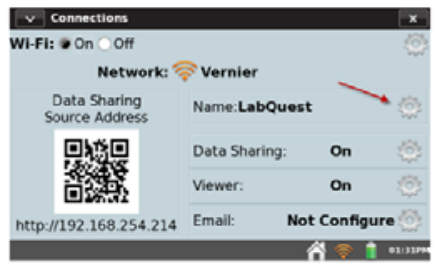


Tap the Name field to edit the LabQuest name (e.g., LabQuest A). Tap Done to save the name.

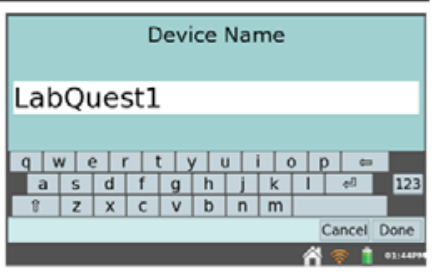

3. From the Connections App, tap the Data Sharing gear to open the Data Sharing Settings dialog box.

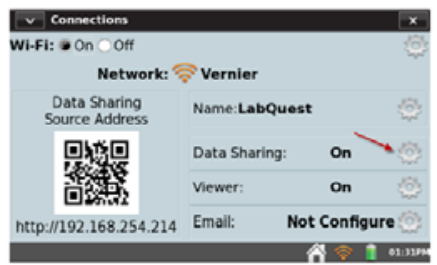

4. Verify Data Sharing is enabled (On).

Optional: If you want to allow Data Sharing apps on mobile devices to start and stop data collection, select the check box to enable this feature.

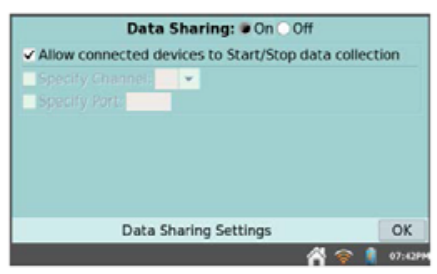

5. Tap OK to save the setup.

\section{Restricting Access to the Connections Setup}

Once your LabQuest is configured as desired, you can set the preferences to restrict access to the Connections setup information. This prevents students from changing your network and Data Sharing settings.

1. Tap Preferences from the home screen, then tap Advanced Preferences.
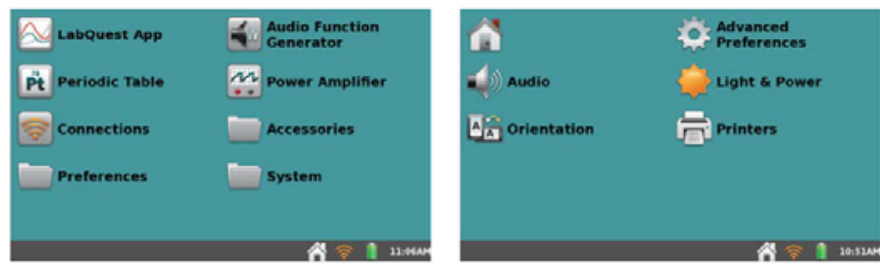
2. Within the Advanced Preferences dialog box, tap the Network tab.

3. Select lockdown-mode and then tap Edit.

4. Select the desired key value.

- Full access to all settings (Value $=\mathbf{0}$ ) Use this setting when you are configuring your Connections settings.

- Access to network settings only (Value $=1$ )

Use this setting when you what to restrict access to settings but want to allow access to different networks.

- Restrict access to all settings (Value $=2$ )

Use this setting when you want to restrict access to all settings, including the network settings.

Note: Changes to the Connections main screen will not show if the lockdown mode is changed while the Connections App is running. Tap $\mathrm{x}$ to close the application, then launch the Connections App again.
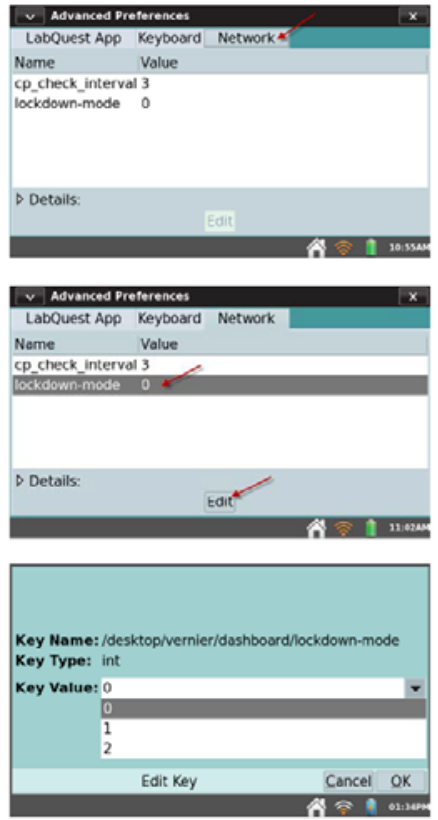
Using LabQuest 2 with a Mobile Device

\section{Connect Your Mobile Device to LabQuest}

After setting up LabQuest as a Data Sharing source, you can then connect to this source from your mobile device using a data sharing app (Data Share web app or Graphical Analysis for iPad) on that device.

Access LabQuest 2 Data Using the Data Share Web App모

1. Launch the Connections App from the Home screen.
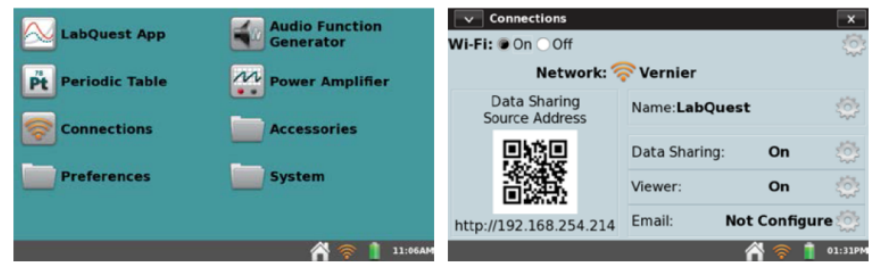

2. Connect your mobile device to the same Wi-Fi network to which you connected LabQuest 2.

3. On your mobile device, open a supported browser and enter the Data Sharing Source Address. Alternatively, if you have a QR code reader on your device, you can access this via the provided $Q R$ code.

\section{Access LabQuest 2 Data Using Graphical Analysis for iPad g}

1. Connect the iPad to the same Wi-Fi network to which you connected LabQuest 2.

2. Launch Graphical Analysis on the iPad.

3. In Graphical Analysis, tap the Source button in the top-left corner. Discovered Data Sharing Sources will be listed automatically.

4. Tap the name of the LabQuest 2 source to connect. If your LabQuest 2 source is not listed in Data Sharing Sources, you can connect by choosing Specify a Source or Scan Source QR Code.

For information on Graphical Analysis, see the User Manual모 on our web site. 


\section{USING LABQUEST WITH A COMPUTER}

Use LabQuest 2 with Logger Lite 1.6 (or newer) or Logger Pro 3.8.5 (or newer).

Logger Lite can be downloaded from our web site at: www.vernier.com/logger-lite/

If you already own a Logger Pro 3 license, free updates to Logger Pro 3 are available at: www.vernier.com/updates/logger-pro/

\section{Collecting Data with Logger Lite or Logger Pro}

To collect data in Logger Lite or Logger Pro with LabQuest:

1. Connect a sensor to LabQuest.

2. Connect LabQuest to your computer using the included USB cable. The mini end of the cable connects to LabQuest and the full-sized end connects to an available USB port on your computer.

3. Open Logger Lite or Logger Pro on your computer. The computer will detect LabQuest and the attached sensor, and display a graph ready for data collection. LabQuest will display a screen with two arrows indicating it is connected to the computer.

4. Click the Collect button in Logger Lite or Logger Pro to collect data.

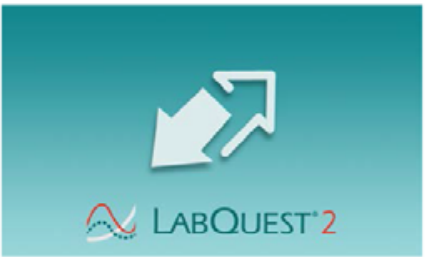

\section{Transferring Data from LabQuest to a Computer}

Automatically Transferring Data

If you collect data in LabQuest App and subsequently connect LabQuest to a computer, Logger Lite or Logger Pro will automatically detect the presence of the remote data and display a message indicating the data can be retrieved from LabQuest. Follow the onscreen instructions to download data to the computer.

\section{Manually Transferring Data}

To manually transfer saved data from LabQuest, open Logger Lite or Logger Pro on your computer and choose LabQuest Browser from the File menu, then choose Open. Choose the desired LabQuest file from the displayed list and click Open. Note that only one LabQuest file can be opened at a time. Once opened on the computer, you may then choose to save the file as a Logger Lite or Logger Pro file by choosing Save As from the File menu.

Tip: If sensors are still connected when a LabQuest file is opened in Logger Pro or Logger Lite, the sensors will be ignored. To enable the sensors, choose New from the File meru in Logger Lite or Logger Pro. 


\section{Using LabQuest with a Computer}

Another method for manually transferring data from LabQuest to Logger Lite or Logger Pro is to choose LabQuest Browser from the File menu in the computer software, then choose Import. Import differs from Open in that only the data in the LabQuest file are added to the current computer session. This allows you to compile data from multiple LabQuest sessions into a single computer session for graphing and comparison.

Tip: Compile class results from multiple LabQuest files (or multiple LabQuests) by repeatedly importing data into a single Logger Pro or Logger Lite file.

Manually Moving Data from Computer to LabQuest

Data and/or sensor configurations can be saved to LabQuest from your computer. To do this, open an existing Logger Lite or Logger Pro file or set up a new file with the desired data collection settings. Choose LabQuest Browser from the File menu, then choose Save As. Enter a descriptive filename. Any data, as well as the sensor configuration, will be stored on LabQuest as a LabQuest file. Any features in the computer file not existing on LabQuest (such as embedded images, video analysis, graph annotations, or most calculated columns) will be ignored on LabQuest.

\section{Deleting Data on LabQuest}

To delete data on LabQuest from Logger Lite or Logger Pro, choose LabQuest Browser from the file menu, then choose Delete. Choose the desired file for deletion from the displayed list and click Delete.

Tip: Use this feature to mass delete files from LabQuest by selecting multiple files from the displayed list. 


\section{EMAILING FROM LABQUEST}

If LabQuest is connected to a network with Internet access, you can email your data file, graph, text file, or screenshot.

To set this up, follow these instructions:

1. Connect LabQuest to a network with Internet access. For detailed instructions, see Connect LabQuest to a Wi-Fi Network.

Note: A LabQuest Network (Ad-Hoc Network) does not have Internet access. You will need to join an existing network with Internet.

2. Launch the Connections App from the Home screen.
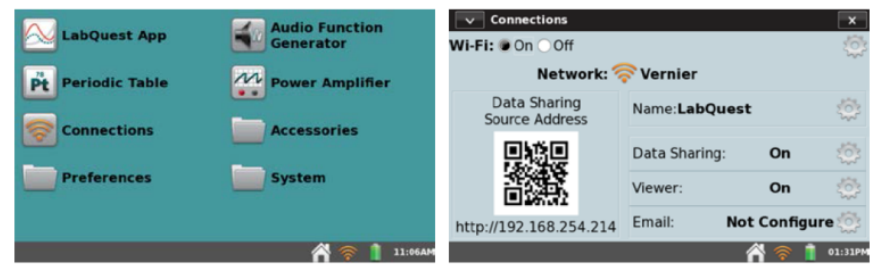

3. Tap the Email settings gear to open the Email Configuration dialog box.
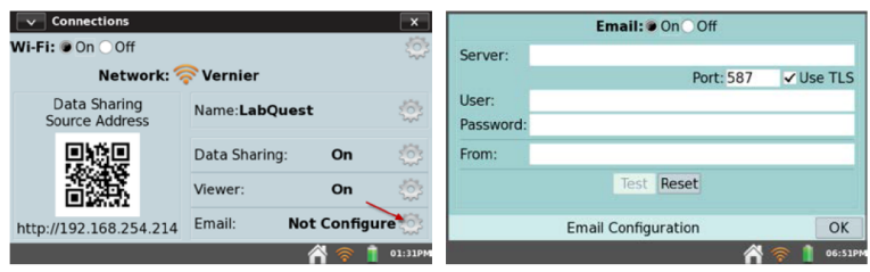

Note: If you are not able to access the Email Configuration dialog box, see Restricting Access to the Connections Setup for more information.

4. Verify email is enabled (On).

5. Enter your outgoing email server information, as well as your username and password, and tap OK. If you don't know your server information, check with your technology specialist.

Note: To test your email settings, tap Test. You should receive a message to your email address stating that you have successfully configured the mailer on your LabQuest. To clear existing setting, tap Reset.

For more information on setting up Gmail accounts, see: www.vernier.com/til/2643/ 


\section{PRINTING FROM LABQUEST}

You can print a graph, data table, lab instructions, your own notes, or the screen as it is currently displayed in LabQuest App. LabQuest can print to a compatible printer via a USB cable or via a Wi-Fi network. For a list of compatible printers see: www.vernier.com/til/1659/

To print to a compatible printer for the first time, follow the Printer Setup instructions below. This setup is only required for the first time you use a particular printer. Once a printer has been installed, LabQuest will store the drivers.

To print from LabQuest App to an installed printer, choose Print from the File menu. Then choose the item that you want to print (Graph, Table, Lab Instructions, My Notes, or Screen).

The Print Options dialog box appears where you can choose the installed printer from the dropdown list and set additional print settings (add a title, footer, or print in greyscale).
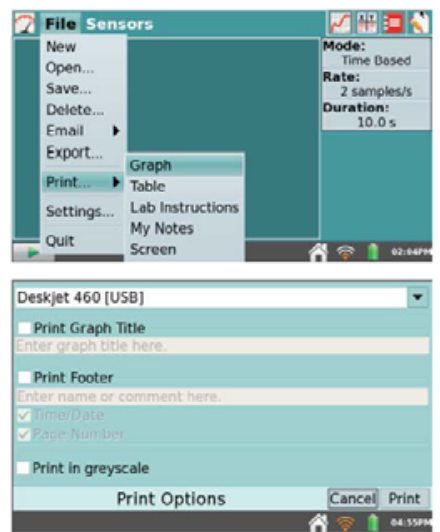

\section{Printer Setup}

1. If printing to a USB printer, connect the printer to the full-size USB port on LabQuest and turn on the printer.

If printing to a Wi-Fi-enabled printer, verify that LabQuest is connected to the same Wi-Fi network as the printer. See Connect LabQuest to a Wi-Fi Network for more information, if needed.

2. Tap Preferences from the Home screen, and then tap Printers to open the Printer Configuration dialog box.
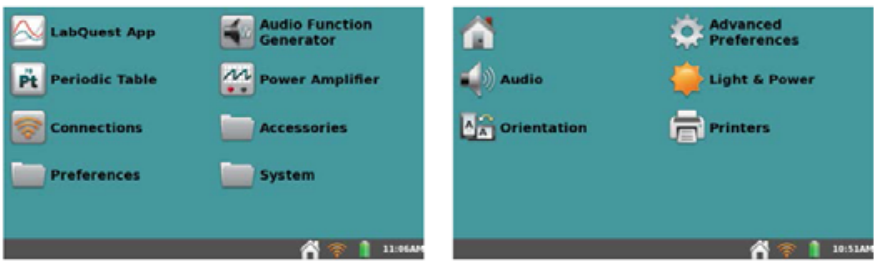
3. Tap Scan for Printers. In response, LabQuest will search for available printers.

Note: If LabQuest is unable to locate a desired network printer, try accessing it directly by tapping Enter IP Address.

4. Select the desired printer and tap Install to set up the drivers.

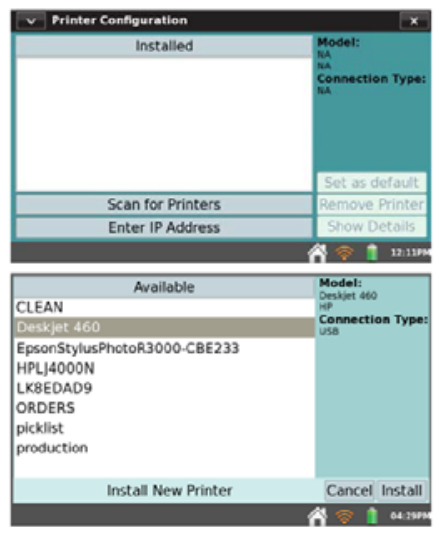

\section{Troubleshooting}

Even if a driver is found, printing may still fail due to an incompatible printer. If you are sure that the printer is compatible and you are still getting an error, it may be caused by one of the following conditions: out of paper, out of ink/toner, a door on the printer is ajar, a paper jam, or you may have selected the wrong printer on the Print Options screen. 


\section{PROJECTING AND MONITORING STUDENTS' LABQUEST DEVICES}

Use our LabQuest Viewer software (order code, LQ-VIEW) 모 to view and control LabQuest wirelessly from your Windows or Macintosh computer. When LabQuest is connected to a computer wirelessly or via USB, LabQuest Viewer can be used in conjunction with a projector to share the LabQuest 2 screen with the entire class.

This allows an instructor to demonstrate LabQuest 2 for a class, and it also allows students to share or present their work to fellow classmates. LabQuest Viewer can also be used with an original LabQuest (order code, LABQ) a connected wirelessly to a computer via a Wi-Fi adapter. LabQuest Viewer is part of the Connected Science System ㅁ.

With LabQuest Viewer, you can:

- View and control one or more LabQuests from a computer.

- Connect to a computer and projector or interactive white board for class demos or sharing.

- Monitor student progress on any LabQuest connected to your network.

- Create screenshots of the LabQuest screen to copy and paste into lab instructions.

- Set customizable permissions that permit viewing without control, or password-protect a connection.

- Install LabQuest Viewer on any computer in your school or college department with the included site license.

\section{System Requirements}

- Windows - Windows XP (32 bit only), Vista (32 and 64 bit), and Windows 7 (32 and 64 bit)

- Macintosh - Macintosh OS X 10.5 (Intel-based Macs only), 10.6, and 10.7

For additional information, see: www.vernier.com/lq-view

\section{LabQuest Setup}

To set up your LabQuest to communicate with LabQuest Viewer App for iPad, or LabQuest Viewer software on your Mac or Windows computer, follow these instructions.

1. Connect LabQuest to a network with Internet access. For detailed instructions, see Connect LabQuest to a Wi-Fi Network.

2. Launch the Connections App from the Home screen.
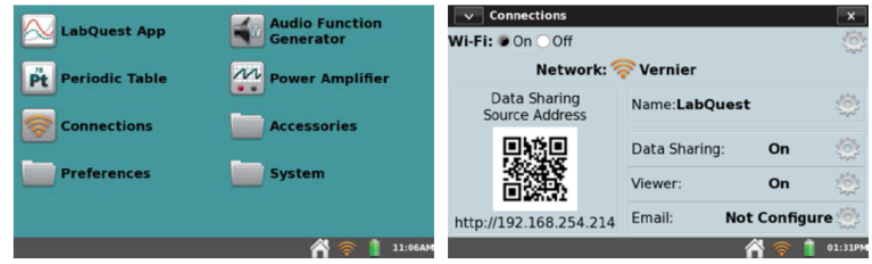
Projecting and Monitoring Students' LabQuest Devices

3. Tap the Viewer settings gear to open the Viewer Settings dialog box.
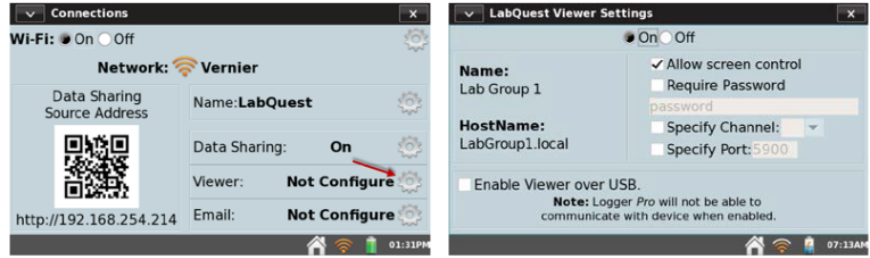

4. Enable LabQuest Viewer (On)

5. Select the check box to Allow screen control.

6. Tap OK to exit the LabQuest Viewer settings. 


\section{ADDITIONAL APPS ON LABQUEST}

Several accessory applications can be launched from the Home menu. It is not necessary to quit LabQuest App to use these accessories; to return to LabQuest App, either close the accessory using the close button in the upper right corner of the screen, or switch to the LabQuest App by tapping LabQuest App on the Home screen.

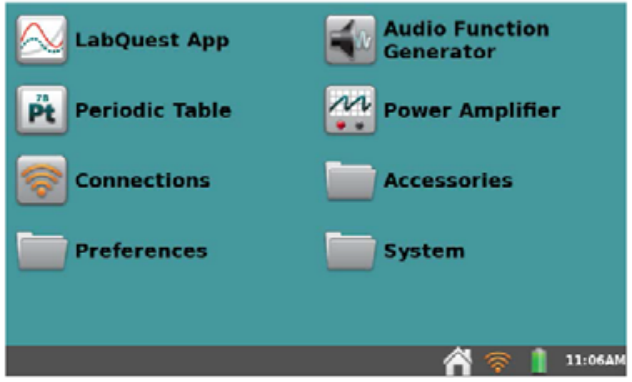

Access additional apps and accessories from the Home screen

\section{Audio Function Generator}

From the Home screen, tap Audio Function Generator to launch this app. The function generator is used to create waveforms in the audio frequency range. Select a waveform, frequency, and volume for each channel. Start and stop using the buttons at left. The link between left and right channels is on by default, so that both channels start and stop together. Tap the link icon to control the channels independently.

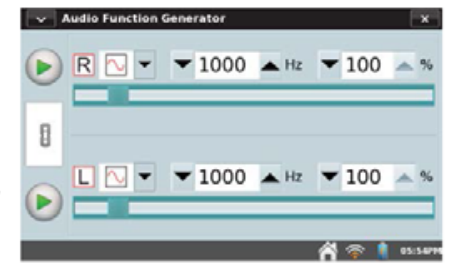

The sliders can also be used to control frequency. Tapping left or right of the slider will halve or double the frequency.

A common use of the Audio Function Generator is to create tones and beats for waveform study using a microphone. For the best waveform quality, connect a powered computer speaker to the audio output jack of LabQuest.

\section{Periodic Table}

From the Home screen, tap Periodic Table to launch this app. The Periodic Table contains standard reference information on the elements. Tap an element to see details.

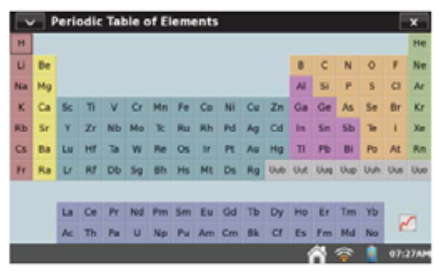




\section{Power Amplifier}

From the Home screen, tap Power Amplifier to launch this app. The Power Amplifier App controls the Vernier Power Amplifier (order code PAMP) 모 used to create waveforms with up to $10 \mathrm{~V}$ amplitude and currents of $1 \mathrm{~A}$. Connect the Power Amplifier to the LabQuest audio output.

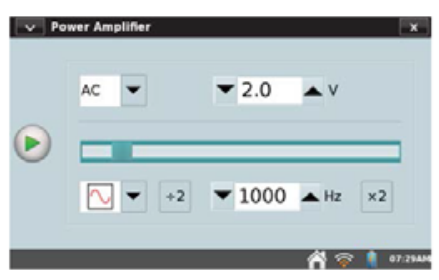

Select the desired output (AC or DC). DC output levels are limited to $0.2 \mathrm{~V}$ steps. AC waveforms include sine, square, sawtooth, and ramps. Select an amplitude and frequency using the controls. The frequency can be changed by factors of two using either the buttons, or in small steps using the slider. Start and stop the output using the control at left.

\section{Calculator}

From the Home screen, tap Accessories, and then tap Calculator to launch this app anytime. The calculator is a standard scientific calculator that uses algebraic notation. You can also copy a calculator result and paste it into the Notes tab.

\section{Sound Recorder}

From the Home screen, tap Accessories, and then tap Sound Recorder to launch this app. The sound recorder is used to capture short audio clips, typically for voice notes. To record a clip, tap the record button 0 . To stop, tap the square red stop button. Play the clip back using the green play button. The disk button allows you to save the clip, which can later be opened using the open file folder icon. The blank page icon clears out any current audio clip.

Tip: Use Sound Recorder to quickly make audio notes on experiments.

\section{Stopwatch}

From the Home screen, tap Accessories, and then tap Stopwatch to launch this app. The Stopwatch is a simple timer. Tap the start button to begin timing; tap it again to stop. Subsequent taps will continue to start and stop the timer. Tap the middle reset button to return the timer to zero. The copy button will place the current time on the clipboard for pasting into the Notes screen of LabQuest App, or into the calculator.
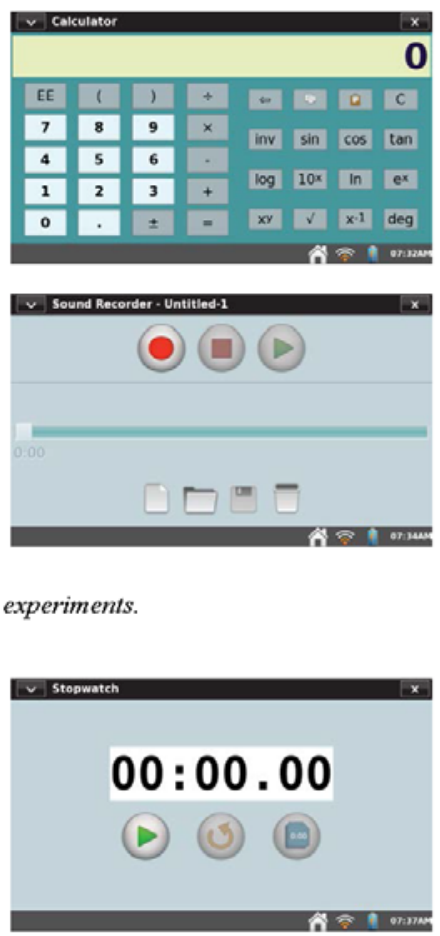


\section{APPENDIX A. LABQUEST 2 TECHNICAL SPECIFICATIONS}

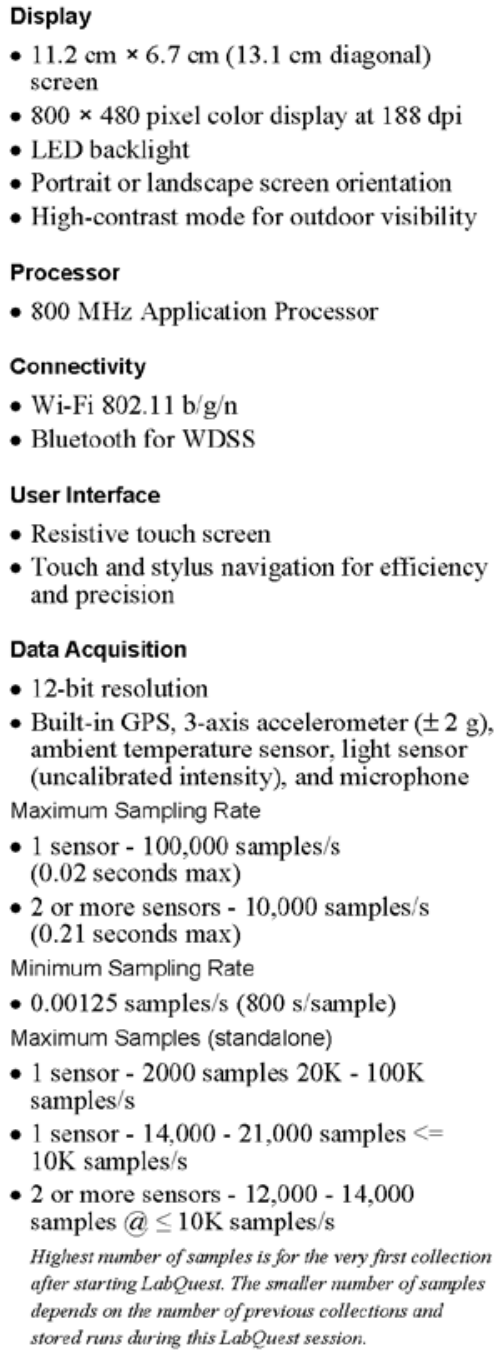

\section{Environmental Durability}

- Operating Temperature: $0-45^{\circ} \mathrm{C}$

- Storage Temperature: $-30-60^{\circ} \mathrm{C}$

- Splash resistant

- Rugged enclosure designed to withstand a fall from lab bench

Size and Weight

- Size: $8.8 \mathrm{~cm} \times 15.4 \mathrm{~cm} \times 2.5 \mathrm{~cm}$

- Weight: $350 \mathrm{~g}$

Ports

- 5 sensor channels

- USB port for sensors, flash drives, and peripherals

- USB mini port

- DC power jack

- microSD/MMC slot

- Audio in and out

Storage

- $200 \mathrm{MB}$

- Expandable with microSD and USB flash drive

Power

- Rechargeable, high-capacity battery

- DC charging/powering through external adapter (included)

Non-compatible Sensors

LabQuest 2 does not support the following sensors at this time:

- Digital Control Unit" (DCU)

- Heat Pulser

- Ohaus balance

* Not supported with LabQuest 2 as a standalone device. The DCU can be used with Logger Pro software and LabQuest 2. 


\section{APPENDIX B. LABQUEST MAINTENANCE}

\section{LabQuest Battery}

LabQuest uses a high-quality lithium-ion battery. This is the same chemistry used in premium laptop and cell phone batteries, and you can expect similar performance. There is never a need to condition the battery by regular full discharge/charge cycles.

Use only the supplied AC adapter or optional LabQuest 2 Charging Station 모 (order code LQ2-CRG, sold separately) to charge the LabQuest battery. A replacement adapter fas can be purchased from our web site (order code LQ-PS).

The battery takes about eight hours to completely charge. It is safe to leave the battery charging indefinitely, and there is no need to fully discharge the battery before charging. Battery life will depend on the sensors used, but in most cases you can obtain six or more hours of use before recharging. We recommend charging LabQuest overnight to start the next day with a full charge.

For use with a computer, either the battery must be charged or LabQuest must be connected to AC power. LabQuest cannot operate on USB power alone. When LabQuest is running and connected to the computer, the USB connection will, however, slowly charge the battery.

When using LabQuest as a standalone device, the screen will dim after a few minutes of no use, even during data collection. However, LabQuest will not turn itself off until the battery is almost discharged. Data loss due to a loss of power is minimized through periodic saving of a backup fíle.

Battery life depends on the sensors and features used. To optimize day-to-day battery life, set the screen brightness to the minimum acceptable level, and turn off LabQuest when it is not in use. Also disconnect any sensors you are not actively using. To access power-saving options, tap Preferences on the Home Screen, then tap Light \& Power.

The long-term life of the battery will vary, but you can expect about three hundred to four hundred full charge/discharge cycles before the battery will need to be replaced. In this count, a charge from half-way to a full charge would count as half of a charge cycle. In typical school use, the battery can last three years or more. Exposure to temperatures over $35^{\circ} \mathrm{C}$ will significantly reduce battery life.

As a battery reaches the end of its useful life, the run time will become shorter and shorter. Eventually the run time will be too short for your application, and you will want to replace the battery. Rechargeable batteries are considered a consumable, and as such, are warranted for one year. A replacement battery모 can be ordered from our web site (order code LQ2-BAT). Recycling information is available at www.call 2 recycle.orge日.

\section{LabQuest Case and Screen}

LabQuest is water and shock resistant. Do not submerge LabQuest in liquids. Wipe it clean with a damp cloth only; do not use any solvents including ammonia or glass cleaners. 
Appendix B - LabQuest Maintenance

\section{Stylus Tether}

LabQuest includes a stylus and a tether. If desired, you can attach the tether to the stylus and LabQuest. Additional styluses are available from Vernier.

\section{LabQuest Software}

LabQuest arrives with its own software, LabQuest App, preloaded. The LabQuest App will be updated from time to time to introduce new features and to improve performance. Most users will want to run the latest version available.

Free updates with step-by-step instructions are available on our web site: www.vernier.com/downloads/

\section{Screen Calibration}

To calibrate the screen, tap System on the Home screen. Then choose Calibrate Screen.

Follow the on-screen instructions for tapping the plus sign with the stylus.

If you are unable to access the Calibration tool, press and hold the Home key until the Calibration tool is displayed.

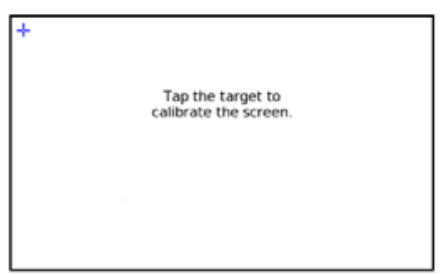

\section{Getting Additional Help}

For access to user manuals, forums, and our technology information library, please visit our web site at:

www.vernier.com/labq2

If you are located in the United States or Canada, you may also contact Vernier directly.

888.837 .6437

info(a) vernier.com

www.vernier.com

Otherwise, please contact Vernier International:

941.349 .1000

info $a$ vernier-intl.com

www.vernier-intl.com 


\section{APPENDIX C. LABQUEST KEYBOARD}

LabQuest App automatically displays the keyboard when text can be entered.

\section{Standard}

The standard keyboard displays lowercase letters. To capitalize letters, use the Shift key $\because$. To delete the previous entry, use the Backspace key $\square$. To start a new line, use the Carriage Return $\square$. Note that the spacebar is located to the right of the letter $m$.

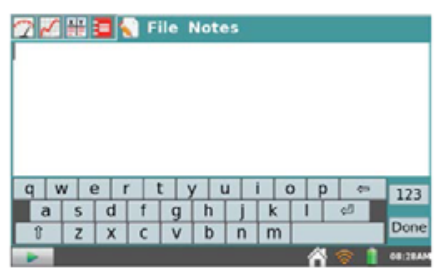

\section{Numeric and special characters}

To access the numeric keyboard from the standard keyboard, tap 123

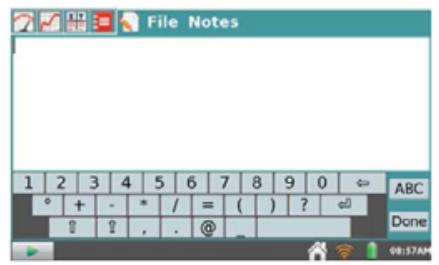

Special characters

To access symbols from the numeric keyboard, $\operatorname{tap} i$.

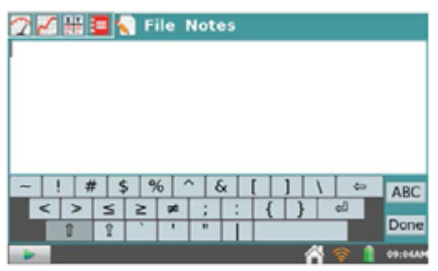

Symbols

To access Greek characters, superscripts, subscripts, and additional operators from the numeric keyboard, $\operatorname{tap} i$.

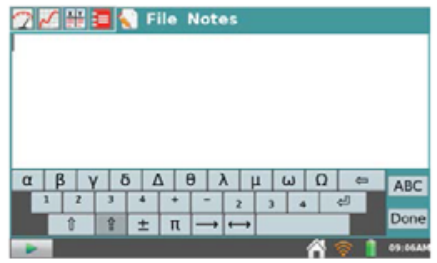

$\mathrm{C}-1$ 


\section{APPENDIX D. LICENSE INFORMATION}

This product contains certain open source software originated by third parties that is subject to the GNU General Public License as published by the Free Software Foundation, GNU

Library/Lesser General Public License (LGPL) and different and/or additional copyright licenses, disclaimers or notices. These licenses give you the right to redistribute and/or modify the software.

The software is distributed in the hope that it will be useful, but WITHOUT ANY

WARRANTY; without even the implied warranty of MERCHANTABILITY or FITNESS FOR A PARTICULAR PURPOSE. See the GNU General Public License for more details.

Complete source code for the open source software is available on request from Vernier Software \& Technology. Contact us at info@vernier.com, or by writing to

Source Code Request

Vernier Software \& Technology

13979 SW Millikan Way

Beaverton OR 97005

USA

Source code will be made available for download, or you may request a CD-ROM of the code. A shipping and handling fee will be charged for a CD-ROM.

The exact terms of GPL, LGPL and some other licenses are provided to you with the source code distribution. You may also read the license at www.gnu.org/licenses. 
APPENDIX E. WARRANTY

Vernier warrants this product (with the exception of the battery) to be free from defects in materials and workmanship for a period of five years from the date of shipment to the customer. This warranty does not cover damage to the product caused by abuse or improper use.

The LabQuest battery is a consumable, and as such, Vernier warrants this product to be free from defects in materials and workmanship for a period of one year from the date of shipment to the customer. For more information about the LabQuest battery, see LabQuest Battery. 
APPENDIX F. ACCESSORIES, REPLACEMENT PARTS, AND RELATED PRODUCTS

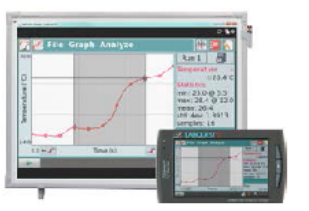

LabQuest Viewer

Display and control LabQuest on a computer to teach

students how to use LabQuest, demonstrate experim ents, or

share class data

www.vernier.com/lq-view
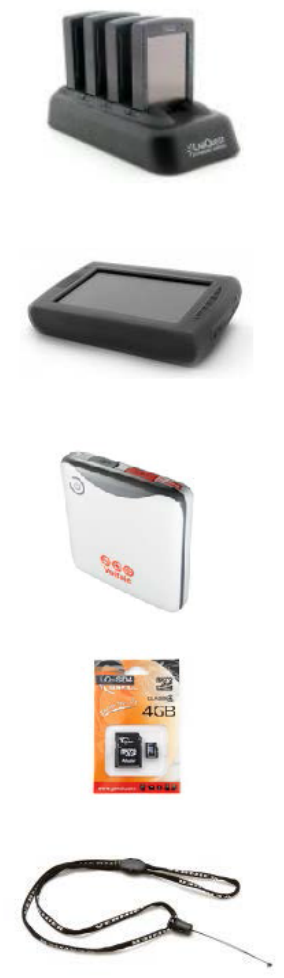

\section{LabQuest 2 Charging Station}

Charge and store up to four LabQuest 2 interfaces with this compact and affordable station.

www.vernier.com/la2-crg

\section{LabQuest 2 Lab Armor}

Add extra protection from spills and falls. www.vernier.com/lq2-armor

\section{LabQuest Battery Boost 2}

With the added power of an external battery, data can be collected for extended periods in the field where $\mathrm{AC}$ power is not available.

www.vernier.com/lq-boost2

\section{LabQuest 4 GB SD Card}

Easily add storage to your LabQuest or move files between LabQuests or a computer.

www.vernier.com/lq-sd4/

\section{Vernier Lanyard}

A LabQuest neck strap to prevent accidental drops during

www.vernier.com/lq-lan field studies. 

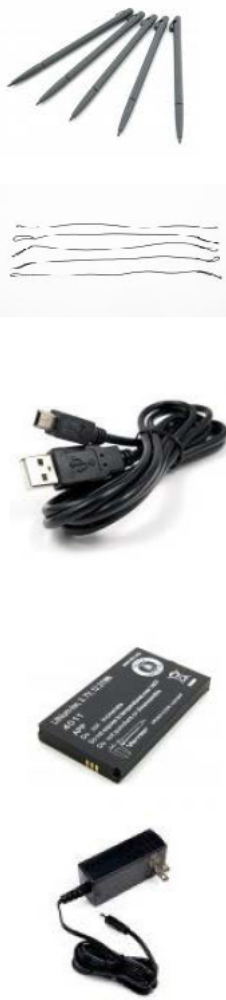

LabQuest 2 Stylus (Set of 5)

Replacement LabQuest 2 styluses ( 5 pack). One is included with each LabQuest 2.

www.vernier.com/lq2-styl-5

\section{LabQuest Stylus Tethers (Set of 5)}

Replacement LabQuest tethers ( 5 pack). One is included

with each LabQuest 2.

www.vernier.com/lq-teth-5

\section{LabQuest USB Computer Cable}

Replacement LabQuest-to-computer USB cable. The cable has a USB mini-B plug and USB standard-A plug. One cable is included with each LabQuest 2. www.vernier.com/lq-cb-usb

\section{LabQuest 2 Battery}

Replacement high-capacity, rechargeable, lithium-ion battery for the Vernier LabQuest 2. One battery is included with each LabQuest 2.

www.vernier.com/lq2-bat

\section{LabQuest Power Supply}

Replacement power supply for the LabQuest. One power supply is included with each LabQuest 2.

www.vernier.com/lq-ps

\section{Curriculum Resources from Vernier Software \& Technology}

Vernier offers a complete set of lab books for elementary through college. For more information, see www.vernier.com/books. 
Development of the West Virginia University

Copyright $^{\odot} 2014$

Small Microgravity Research Facility (WVU SMiRF)

Kyle G. Phillips

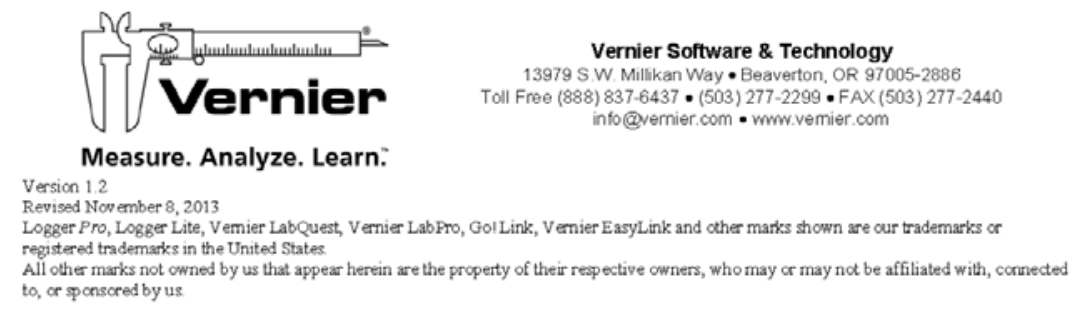



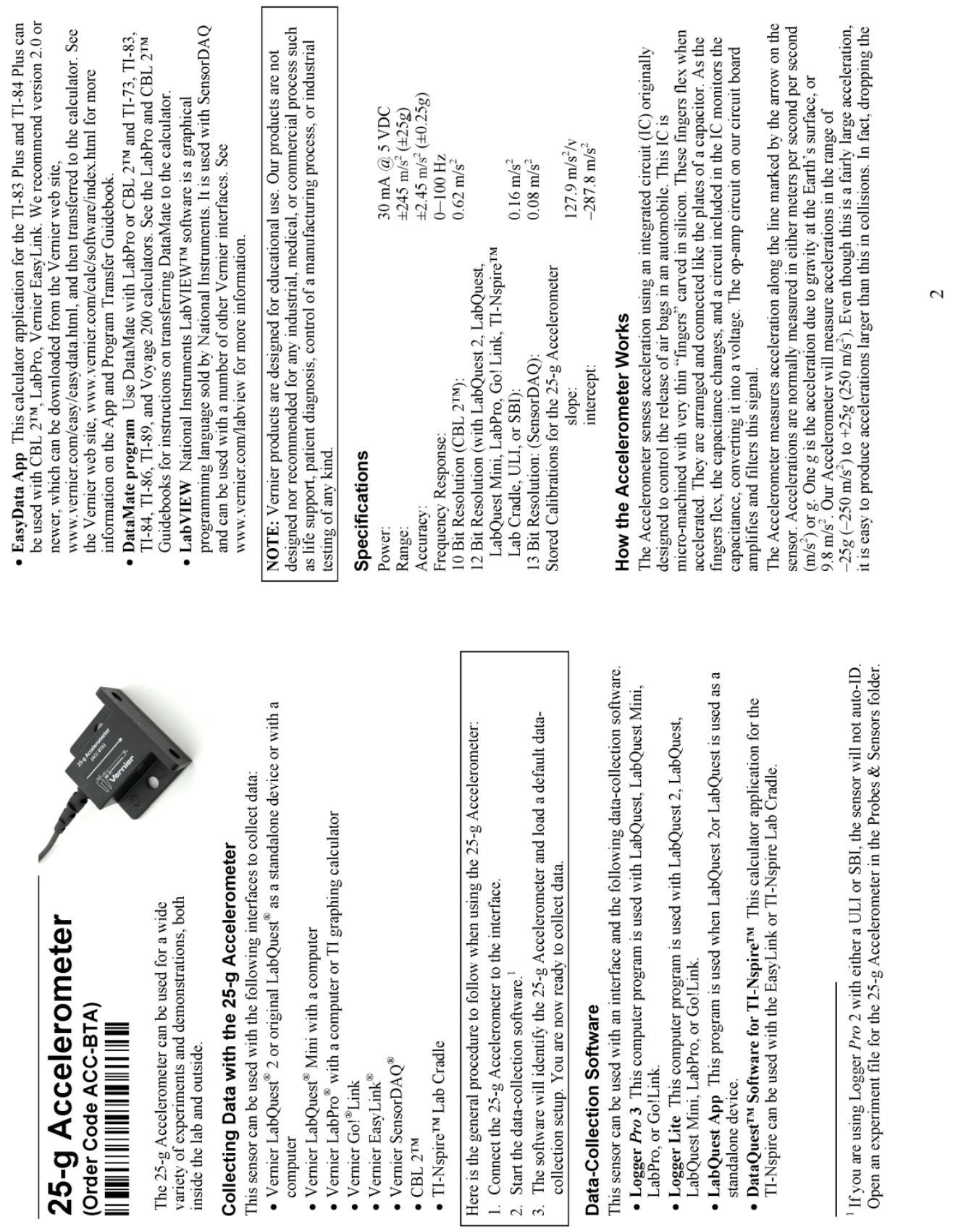

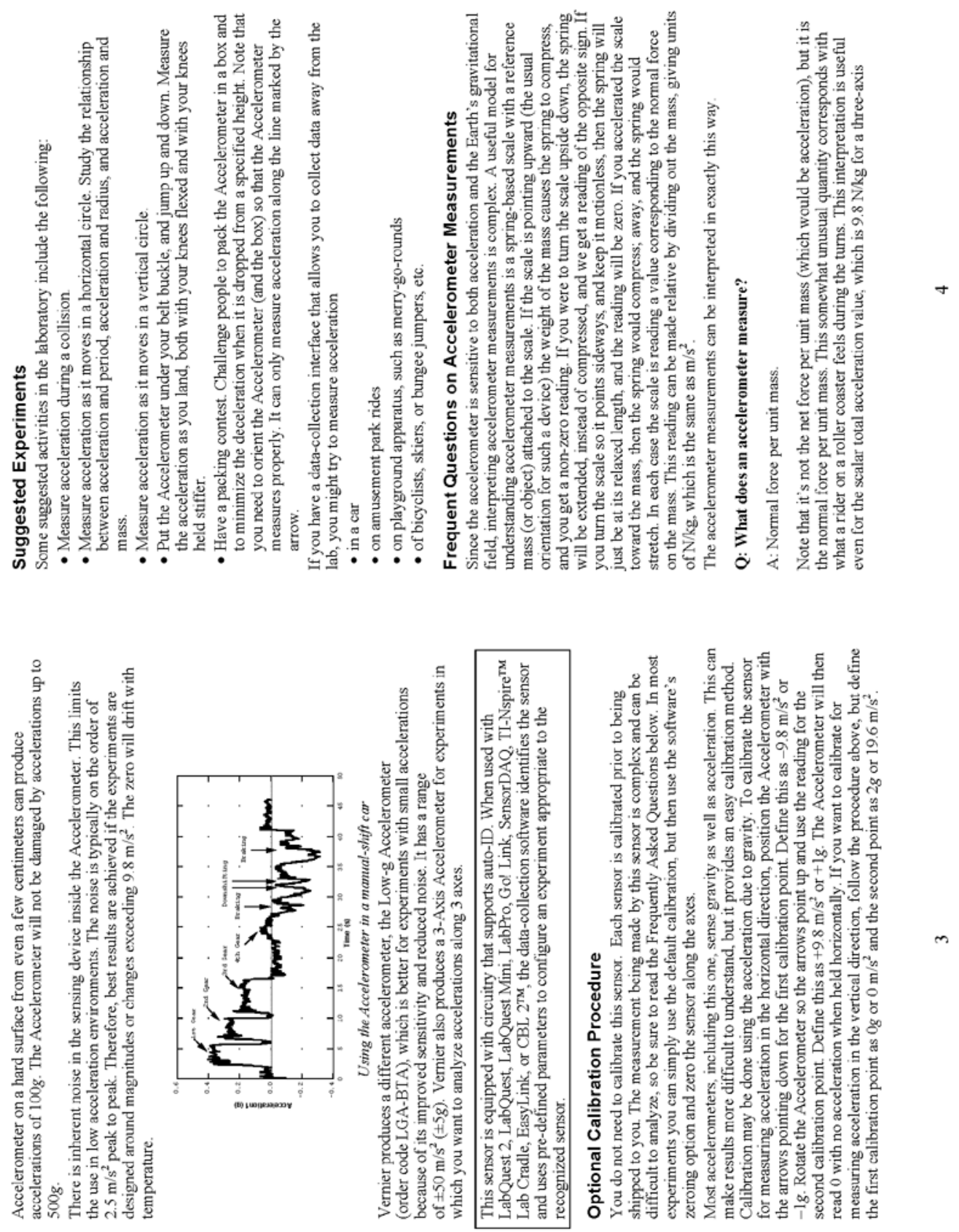

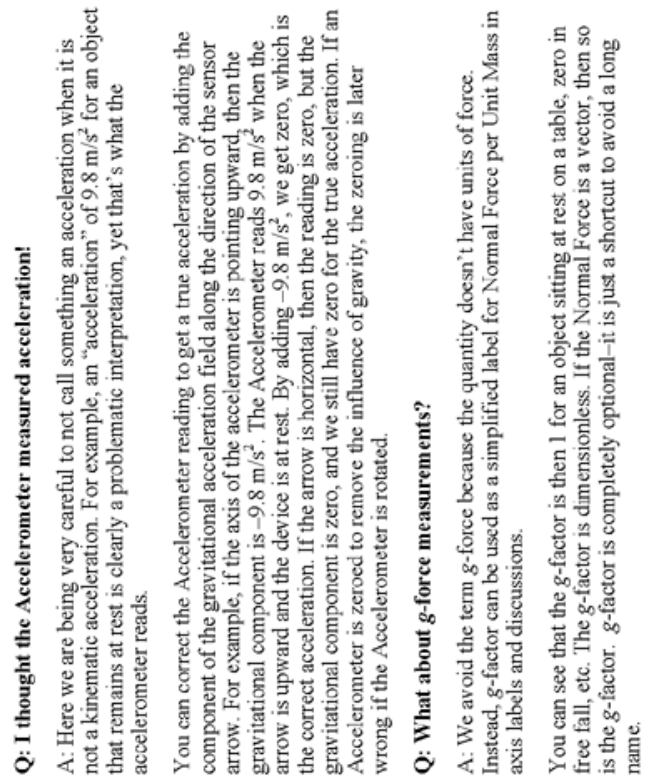

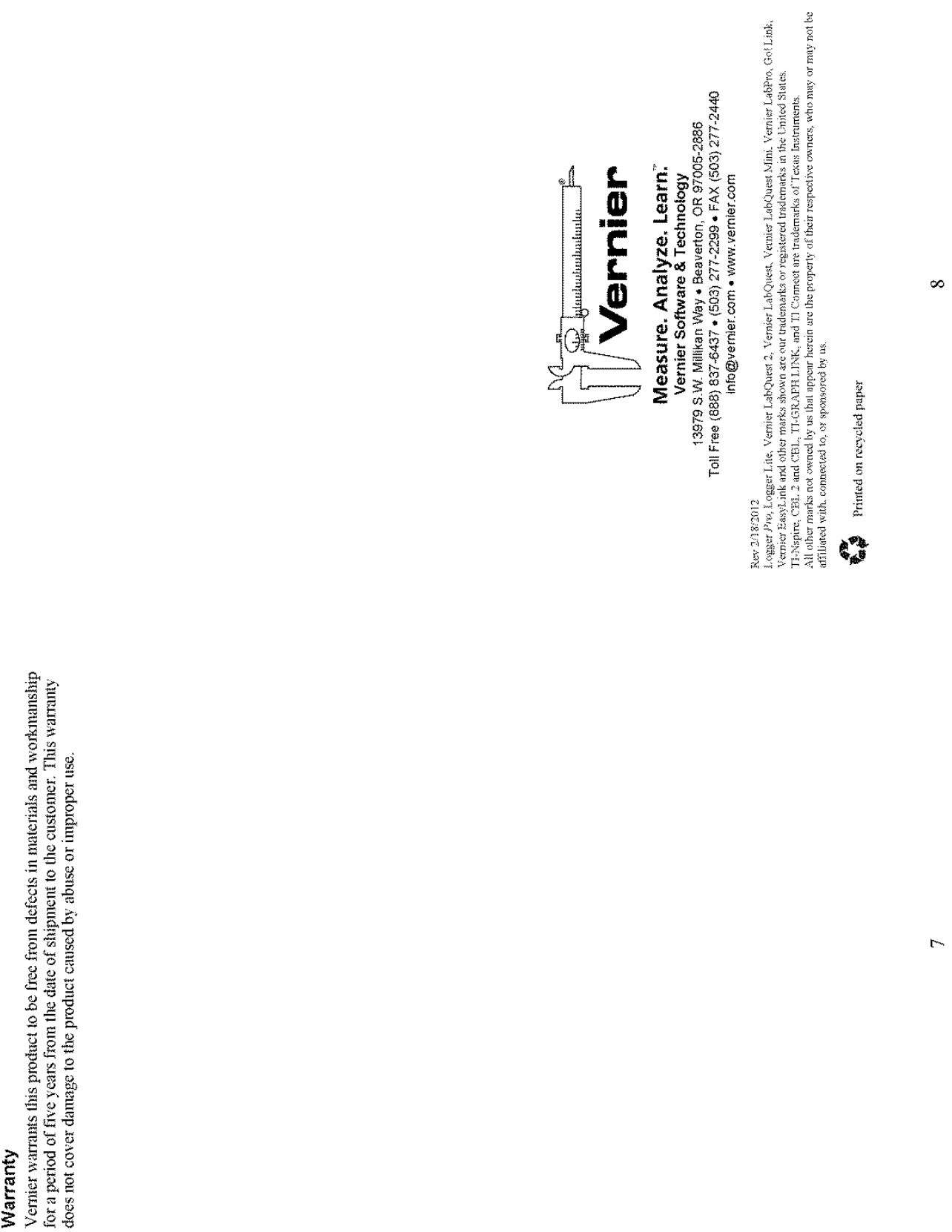

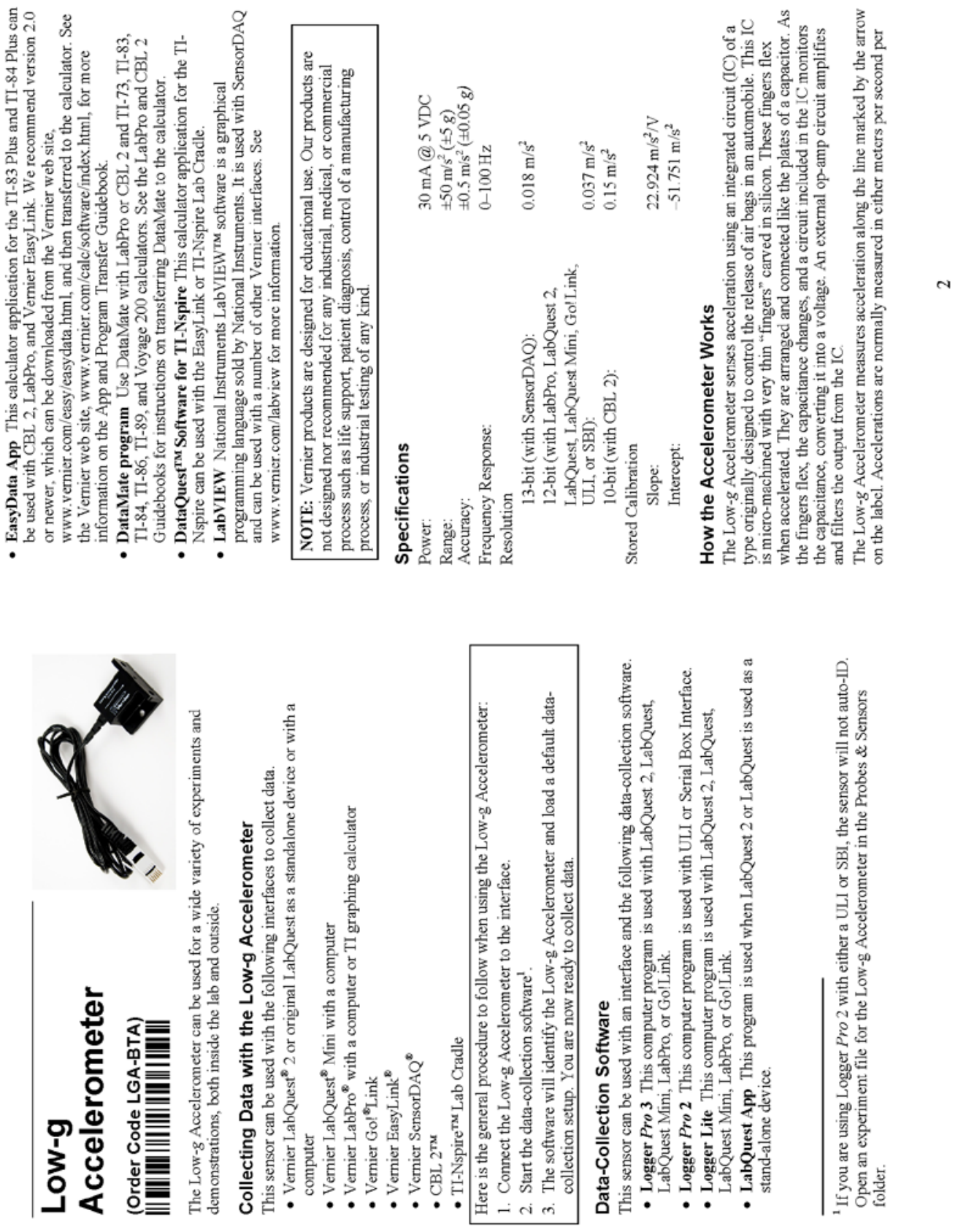

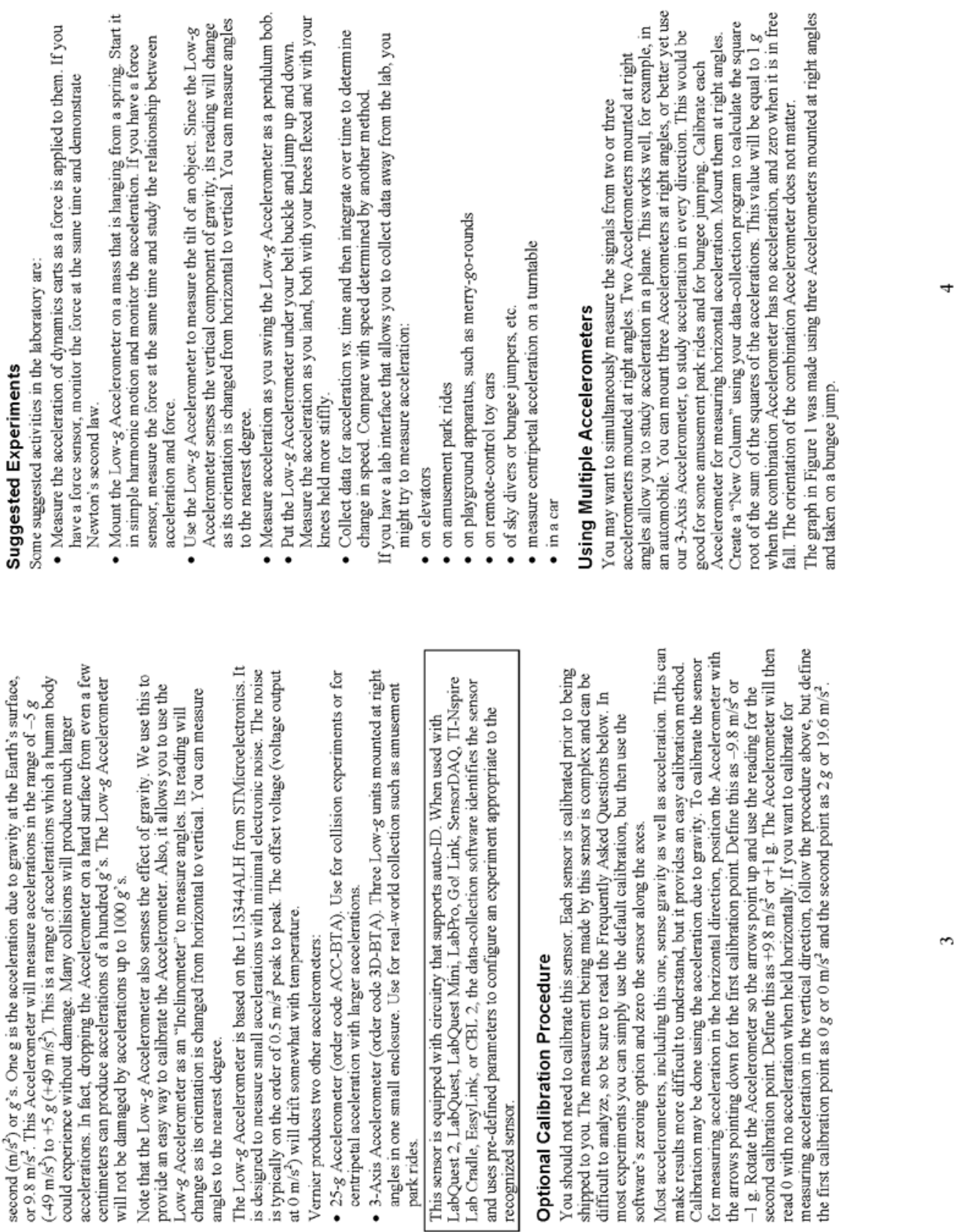

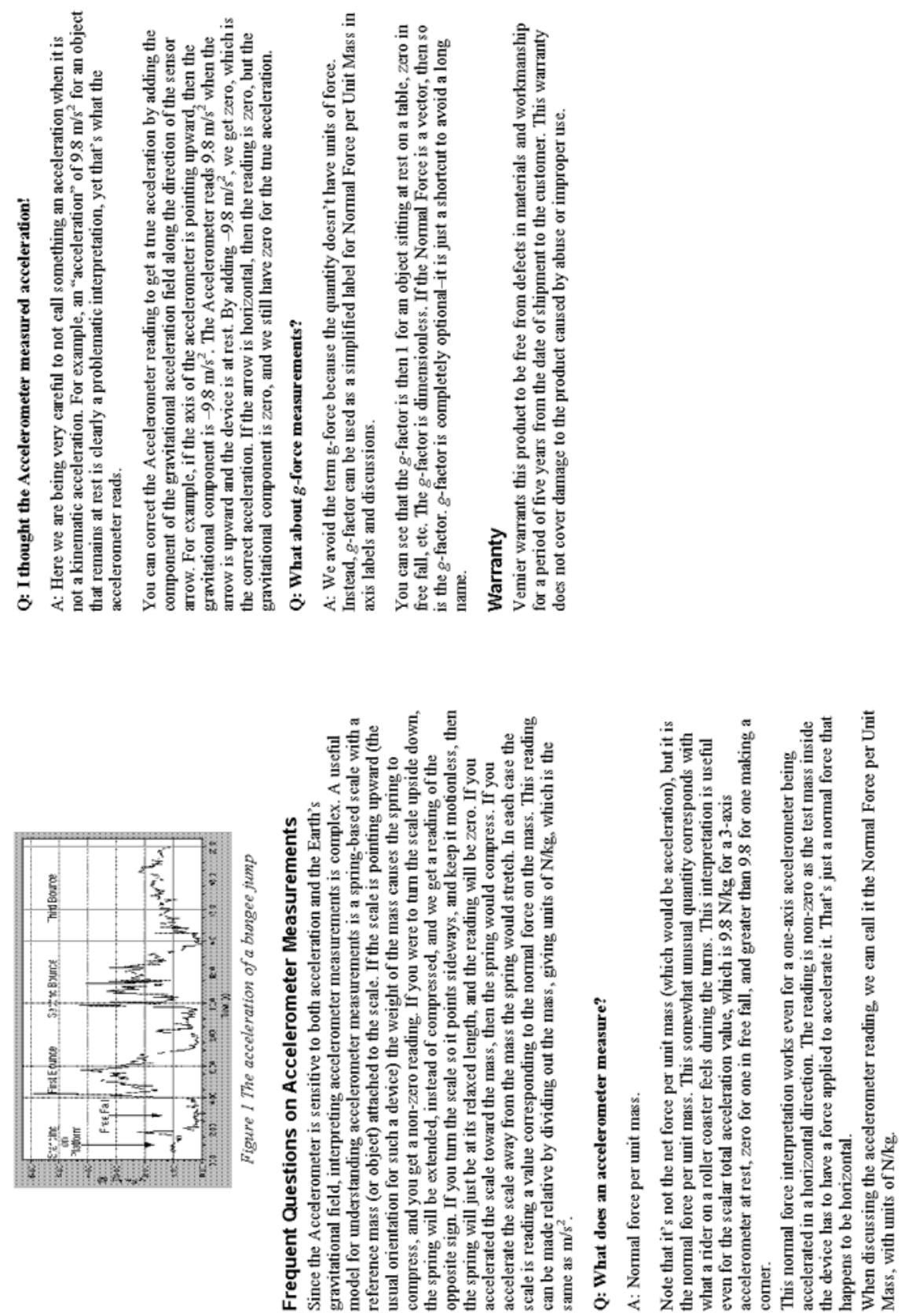


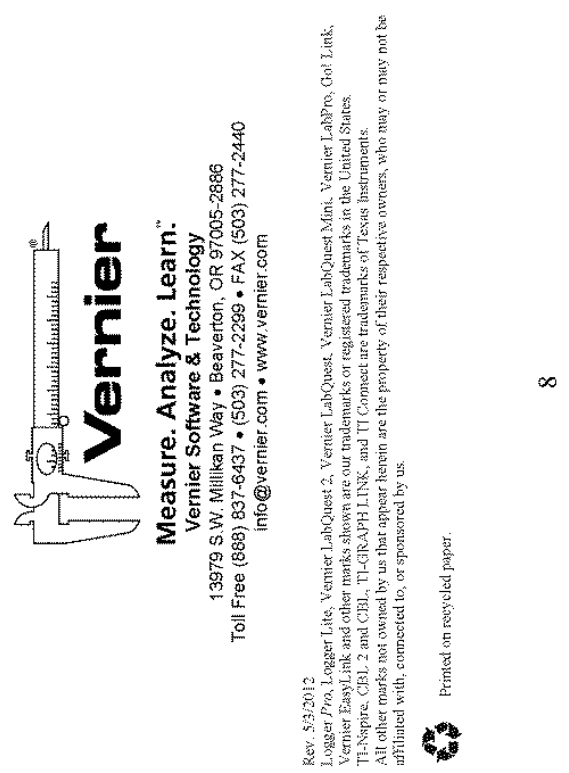




\subsection{Appendix E}

\section{Structure Information}

Appendix C: Structure Information presents the final bill of materials that formed the WVU SMiRF drop tower structure. The BilJax scaffolding was purchased through Grainger. This appendix also presents costing from Grainger and erection recommendations from BilJax. Information provided herein is provided for comprehension of the project documentation and reflects past costing from Grainger and copyrighted material belonging to BilJax to which all rights apply for their copyright material. 


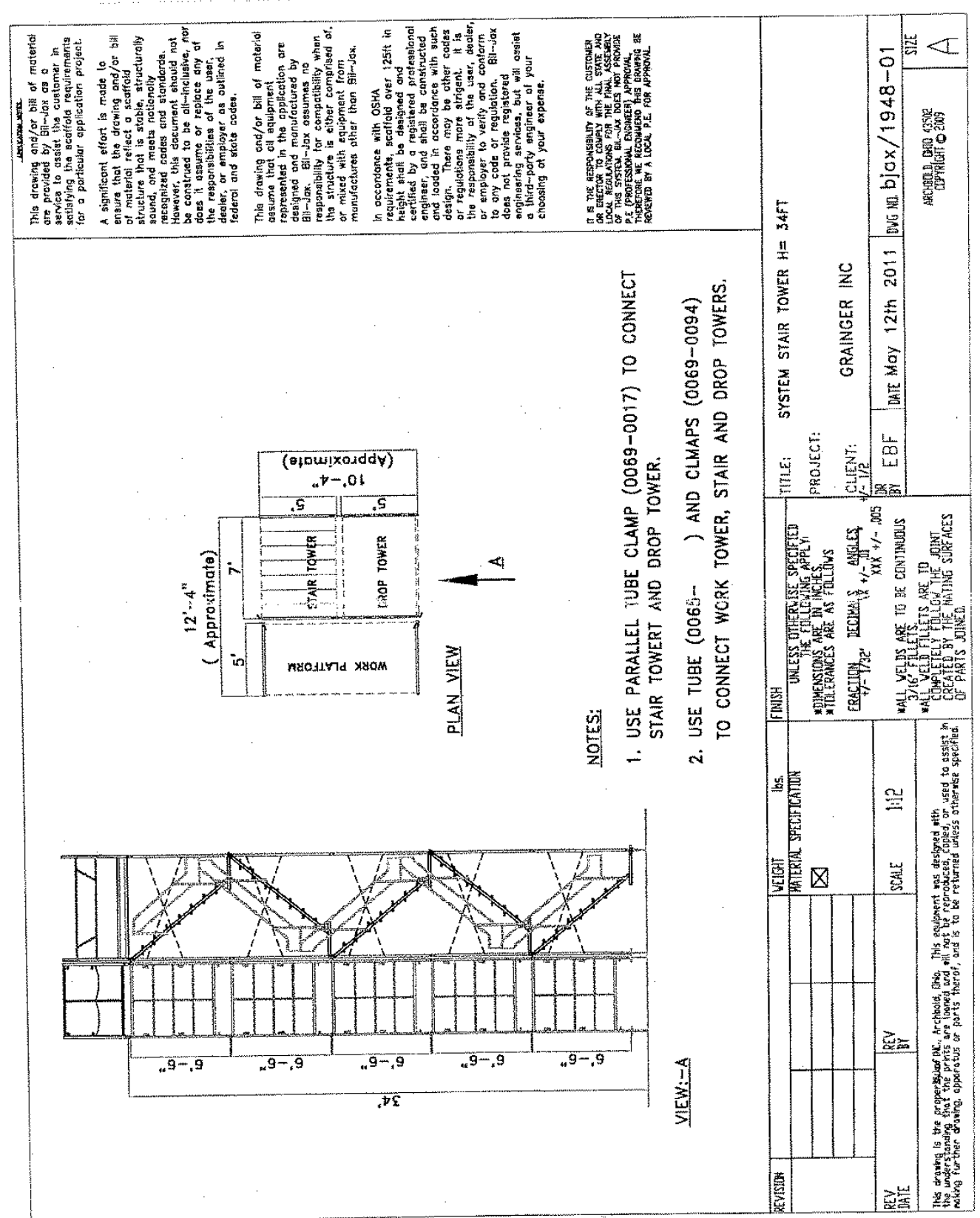




\section{STAIR TOWERS FOR INTERMITTENT USE}

Bil-Jax offers a heavy duty stair tower for worker access to butidings, bridges, observation towers, or renovation done inside or outside. The stairs can also be used for access in a scalfold set-up.

Stair Tower Packages include frames, braces, leveling jacks, stair sections with handrails, stair rests, full top plattorm and top pane guard.

NOTE: Tie-ins not included in Stair Tower Packages. See Page 17 to orde Scaffold Tie Braces.

FOR APPLICATIONS UP TO 100:

Each tower package contains a top section with guard railing and a bottom section, totaling $13^{x}$ in height. To figure cente units needed, subtract 13 from the total height of the tower and divide the balance by 6.5 .
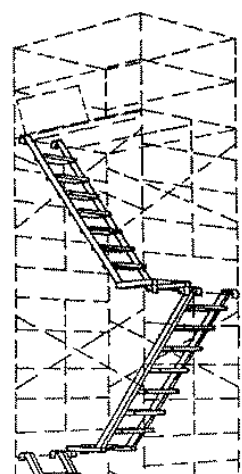

$\rightarrow+1,-1,-1=7$

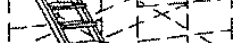

$F=2 x-7<$
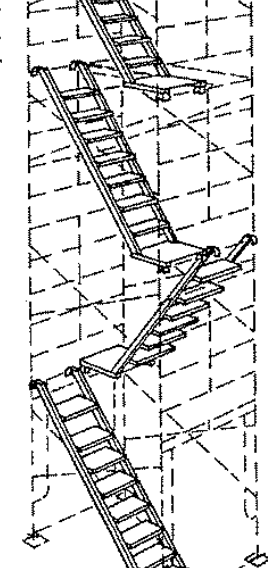

HANDRAALS AND DIAGONAL RRACING MUST BE USED STAF TOWERS. HANDRALS AND SOME BRACING

STAR TOWERS EORMIERMIITENT USE UP TO 100

\begin{tabular}{|c|c|c|}
\hline Part No. & Description & Wo \\
\hline$s \mathrm{~T}-260 \cdot 64 s-(7)$ & $\begin{array}{l}13 \text { stair ower Unit Package } \\
\text { (notudes top and Base Sections) }\end{array}$ & 878 \\
\hline $5 \mathrm{~T}-26064 \mathrm{C}(7)$ & $6^{6} 5$ Stail tower Unit Package & 342 \\
\hline $\mathrm{st}-280-66 \mathrm{~s}-(5,6)$ & $\begin{array}{l}13 \text { stair Towe Uni Package } \\
\text { (trcludes Top and Base Sections) }\end{array}$ & 974 \\
\hline$s T-260660-(5,6)$ & $\begin{array}{l}67 \text { stair rower Unit package } \\
\text { (Center Section Only) }\end{array}$ & 344 \\
\hline
\end{tabular}

STAIR TOWER COMPONENTS
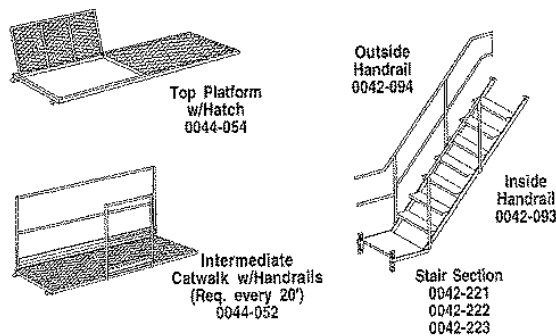

O2

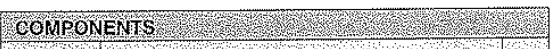

\begin{tabular}{|c|c|c|}
\hline Part No. & Description & wto \\
\hline $0042=019$ & Tube Size 5 Stail Platform hest & 7 \\
\hline 0042027 & Tube Stze o star Ptafform Rest: & 8. \\
\hline 0042035 & Trbo Sizo 7 star Platron Rest & 85 \\
\hline $0042=047$ & Open frame salety Guard & 5 \\
\hline 0042.094 & Outside tandrali & 28 \\
\hline 0042.093 & Inside Y tandrail & 15 \\
\hline $0042 \div 85$ & Outside Handrall (rop Section onyy) & 25 \\
\hline 0042175 & Inside Handrail ( op secitor Ony) & 14 \\
\hline $0042-221$ & 24 Width stair Section 65 & 74 \\
\hline 0042.222 & 24 Wdth Stair Section $66^{\prime}$ & 84 \\
\hline $6042-223$ & $24^{\prime}$ Wdh stair section 67 & 64 \\
\hline 0044052 & Internediate Cathalk 28 W $\times 7$ w/ Guard fal & 91 \\
\hline $0044-054$ & Hinged Plattorm $28 \mathrm{~W} \times 7$ exp steel & $60 \%$ \\
\hline O0044-235 & $28 \mathrm{~W} \times 7$ Exp steel Gatwalk, 75 pst $\mathrm{Ca}$ & 56 \\
\hline
\end{tabular}

\section{HINGED LADDER BOARD}

FEATURING:

- 28" Wide hinged platform (aluminum frame with
plywood deck) in $7^{\prime}$ and $10^{\prime}$ lengths.

- Aluminum ladder attached to platform folds down providing an access ladder.

- Ladder stores under plafform, out of the way when not in use.

- Pequires frames that are 6 to $6^{\prime} 6^{n}$ high.

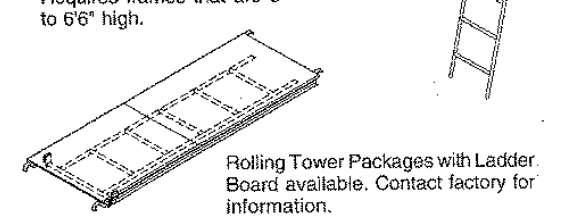

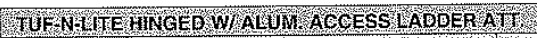
Pan No

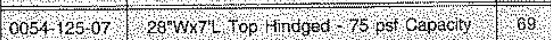

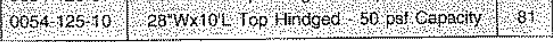

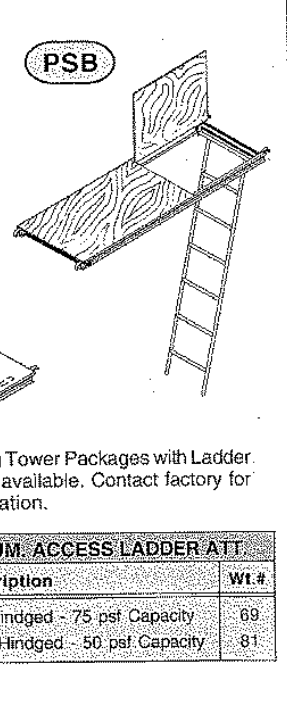

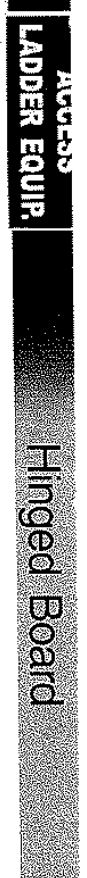




\section{Tips On SCAFFold SAFETY}

The following safety tips are offered by the engineering department of Bil-Jax, inc. as guidelines in avoiding job-site situations that could prove dangerous to scaffold workmen.

CHECK SAFETY CODES:

OSHA, state and local safety codes should be consulted; and approval obtained from Building Safety and Industrial Commission on scaffold tips.

INSPECT AND CHECK:

Take no chances. Inspect the scaffold set up atter erection and daly white in use. Don't remove or alow removat of any parts withous the OK from the proper authorities. When wire rope is used, inspect it on each job.

BEGIN WITH GOOD FOOTING: Use base plates, sills or footers for scaffold is leveled or plumbed.

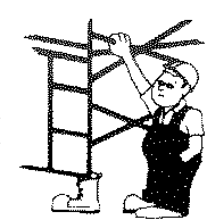

REJECT DAMAGED PARTS Bent or otherwise damaged end trames or braces should not be used. Put them aside for replacement. For repair, call Bil-dax, Inc.

DON'T SHORT-CHANGE BRACING:

Use bracing at als points provided; adid extra braces if needed to insure stability.

TIE SCAFFOLD TO THE BUILDING: Scaffolding should be tied to the struc ture using heavy wire or tie-in devices. the first vertical tie should be af the maximum height of 4 times the narrowest base dimension. Additional ties are not to axceed 26 feet vertically Maximum horizontal distance belween ties is not to exceed 30 feet.

DON'T OVERLOAD SCAFFOLDING: Follow the safe load capacities as given by the scartold manufacturer. There's a 4to-t sefety factor must be fipted scaffolding

USE METAL CATWALKS, PLATFORMS; Where avaliable. If wood plank is used, it must be scaffold grade or betfer inspect thoroughly before every job to make sure it is free from breaks, knots, cracks or warpage. Decking should be full width.

\section{DONT RIDE MOVING SCAFFOLD; and remember scaffold units ane limited and remember scatro units are thilied dimencion (unless base is widened by outrigers or more end frames; or tied into building) Always keep casters}

*Exceptions: 3 times in $\mathrm{CA}, \mathrm{OH}, \mathrm{OR}, \mathrm{MT}, \mathrm{ME}$
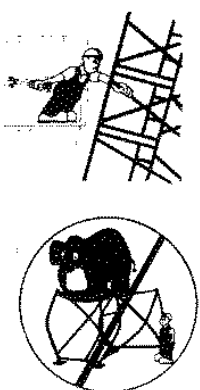
Courtesy of $2=12$ Archbold, Ohio

POST WHERE WORKMEN CAN SEE

\begin{tabular}{|c|c|}
\hline NAME & PHONE \\
\hline & \\
\hline & \\
\hline & \\
\hline & \\
\hline & \\
\hline & \\
\hline & \\
\hline & \\
\hline & \\
\hline & \\
\hline & \\
\hline & \\
\hline & \\
\hline & \\
\hline & \\
\hline FIRE & \\
\hline POLICE & \\
\hline AMBULANCE & \\
\hline
\end{tabular}

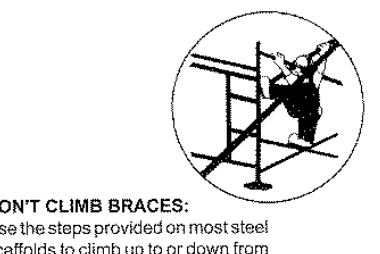
Use the steps providet on most stee work leveis. Use scaffold climbing ladders where required.

PROTECT WORKING LEVELS

Use overhead canopies to protect workers on lower work levels when work is being done overhead. Rope off unsafe areas

Page 1 of 2

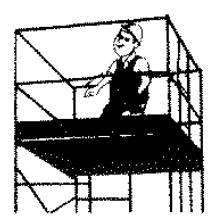

USE DOUBLE GUARD RALLS; platform heights of 6 feet or more.

Fring Date 08-2005 and toeboards on exposed sides at 
Reprinted from the August 30, 1996 Federal Registar Department of Labor

Occupational Safety and Health Administration 29 CFR Part 1926

Safety Standards for Scaffolds Used in the Construction Industry; Final Rule The effective date for this Standard is November 29 1996 with some provisions effective September 27, 1997

125 Taylor Parkway, Archbold, OH 43502-9309

PART 1926 SUBPAFTL SCAFFOLOS

1926.450 SCOPE, APPLICATION AND DEFINITIONS APPLICABLE TO

(a) scope and application. This subpart appiles to all scaffolds used in workplaces covered by this Part, it does not apply to crane of derrick suspended persomel plations, which are covered by Sec. $1926.550 / \mathrm{g}$ ). The criteria for aerial li

Adjustable suspension scatfold means a suspension scaftold equipped with a hoist)s) that cati be operated by an enployee(s) on the scaffold.

Bearer (putiog) means a horizontal transverse scafiold member (which ay be supported by ledgers of runners\} upon which the scaffold plattorn Boatswains' chair means a sing fig-point actijustable stspension scaffoid con Boatswains chair means a singe-point adustable stspension scafold con-
sisting of a seat or sling designed to suppot one employee in a sitting posi Body belt (saiety bett) means a strap with means both for securing it aboul the waist and for attaching it to a lanyard, lifeline, or deceleration device. Body harness means a design of straps which may be secured about the employee in a manner to distribute the fall arrest forces over ait least the thighs, nents of a personal fall arrost system.

Brace means a rigid connection that holds one scafiold member in a fixed position with respeci to another member, or to a butding or siructure.

Bricklayers' square scaffold means a supported scatiold composed of framed souares which support a platform.

Carpenters' bracket scaffold means a supported scaffoid consisting of a Chatiorm supporied by brackets attached to butding or structural walls. suported by two essentialy horizontal and parallel ropes atta of a platorm tural members of a building or other structure. Additional support may be

provided by vertical pickups.
Chimney hoist means a multi-point adjustable suspension scaffold used to provide access to work inside chinneys. (See "Multi-point adjustable suspenion scaifold.")

Cleat means a structural block used at the end of a platform to prevent the plattorm trom sipping oft its supports. Cleats are also used to provide footing Competent person means one who is cap

Codictable hazards in the surrounding is capable of identifying existing and sanitary, hazardous, or dangerous to employees, and who has authorization

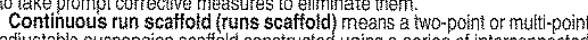
adjustable suspension scaffold constructed using a series of interconnected fold. Coupler
scaffold. Crawing board (chicken ladder) means a supoorted scaffold consisting of a plank with cleats spaced and secured to provide footing, for use on sloped suraces such as roots.

Deceleration device means any mechanism, such as a rope grab, rip-stitch lanyard, specially-woven sanyard, tearing or deforming lanyard, or autornatic ergy during a fall arrest or fimits the eneroy imposed on an employee during tall arrest.

Double pole (independent pole) scaffold means a supported scattold consisting of a platiom(s) resting on cross beams (bearers) suppoted by ledgers and a double row of uprights independent of support (except ties, gutys, braces) fiom an, stucture.

Equivalent means atternative designs, materiass or methods to protect agalist a hazard which the employer can demonstrate will provide an equal o sions spectied of safey for enpl

Exposed power lines means electrical power fines which are accessible to employees and which are not shielded from contact. Such lines do not inchde extension cords or power tool cords.
Eye or eye splice means a loop with or without a thimble at the end of a wire Fabricated decking and planking means manufactured plattorms made of wood (including laminated wood and solid sawn wood planks), metal or Fabricated frame scaftold (tubular weided frame scaffold) means a scaf-
fold consisting of a plattom (S) supported on fabricated end frames with integral posts, horizontal bearers, and intermediate members.

Failure means load retusal, breakage, or separation of component parts. coad retusals the point where the ulunate strength is exceeded par

pom scaffold means a supported scaffoid consisting of a platiom supGorted by brackets attached to fortiwork. topralls, midrats, and posts, erected to prevent employees from falling off a scafiold platform on walkway to lower tevels.

Hoist means a manual of power-operated mectianical device to raise of ower a suspended scafiold

Horse scaffold means a supported scaffold consisting of a platiorm supHotal consiruction horses (saw horses). Hors

independent pole scatrold (see "Double pole scaztold")

interior hung scaffold means a suspension scaffold consishting of a plat

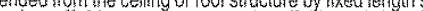
Ladder jack scaffold means a supported scaffold consisting of a platorn esting on brackets altached to ladders

Lo wide ftan treans a mosile, fixed-size, self-supporting ladder consisting anding means patarm at the ond stairs.

Large area scaffold means a pole scaffold, tube and coupler scaffold, systems scafiold, or fabricated treme scaffold erected over sutsstantially the entire work area. For example: a scafiold erected over the entire floor area of room.

Lean-to scaffold means a supporied scaffold which is kept erect by thiting it Ward and resting it against a building or structure.

Line means a component consisting of a fexibje line that connects to an anchorages a t both ends to stretch horizontally (thorizontal itelinem serves a mears for connecting other components of a personal fall arrest

system to the anchorage. and to which an employee can tall. Such areas include, but are not fimited to grou nevels, toors, fooss, ramps, runways, excavations, pits, tanks, mater an's adjustable supp scaffold ous run suspension scatod designed antused tor masominy operalions. Maximum intended load means the total load of ail persons, equipment lools, materials, transmilted loads, and other loads reasonably anticipated to Mobile scaffold mins dupported scaffold

Muiti-level suspended scaffoid means a two-point or multi-point adjustcommon stirturs Multi-point adjustable suspension scaffold means a suspension scatthen tho ropes rom ovenead suppors and equipped with means to raise and lower the Needie beam scattol't means a platiorm suspended from ney holsts. Open sides and ends means the edges of a platiorm that are more thans. inches $(36 \mathrm{~cm}$ ) away horizontally from a sturdy continuous, vertical surf 14 (such as a building wall) or a sturdy, continuous, horizontal surface (Such as a toot), or a point of access. Exception: For plastering and lathing operations the horzontal witestiold distance is 18 inches (46cm)

Outrigger means the sturctural member of a supported scaffold used to in order to provide suppot for and increased stability of the scaftolo

Outrigger beam (Thrustout) means the structural member of a suspenextending the scaffold point of attachment to a point out and away from the structure or building

Outrigger scaffold means a support scaffold consisting of a platform rest ing on oulrigger beams (thrustouts) projecting beyond the wall or face of the building or structure, the inboard ends of which are secured inside the building or siructure.

uverhand bricklaying means the process of laying bricks and masont unifs such that the surface of the wall to be jointed is on the opposile side o plete the work. It includes mason tending and electrical instalation incorporated into the brick wath during the overhand bricklaying process. 


\section{Development of the West Virginia University Small Microgravity Research Facility (WVU SMiRF)}

(6) On scaffolds where scaffold planks are abutted to create a long plattorm each abutted end shall rest on a separate support surface. This provision does not preclude the use of common support members, such as "T" sec-
tions, to support atututing planks, or hook on platforms designed to rest on common supposts.

(7) On scafolds where plattorms are overlapped to create a long platform, the overlap shall occur only over supporis, and shall not be less than in inches $(30 \mathrm{~cm})$ Uniess the
preven movement

(8) At all points of a scaffold where the platorm changes direction, such as furning a corner, any platiorm that yests on a bearer at an ange oner than a right angle shall be laid first, and plattorms which rest at right angles over the same bearer shall be laid second, on top of the first plation

(9) Wood platiorms shall not be covered with opaque finishes, except that plattorm edges may be covered or marked for identitication. Plattorms may cilo-resistant finishes; however the coating may not obscure the iop or bot tom wood suffaces.

(10) Scafifold components manufactured by different manufacturers shail not be intermixed unjess the components fut together without force and the scatfold's structuraf interity is maintanted by the user. Scafiold components manufactured by difserent manufacturers shall not be modfied in order to intermix them uniess a competent person deternines the resuliting scaftold is struckarally sound.

(11) Scaftold components not be used togeither unjess a

competent person has deter-

mined that galvanic action will not reduce the strengith of any component to a level below (1) of this section.

(c) Criteria for supported scaffolds.

(1) Supported scafolds with a height to base width (including outrigger supports, if used) ratio of more than four to one (4:1) stal be restrained from tipping by guying, tying, bracing, or equivalent means, as folows: members suppont both inner and outer legs.

(ii) Guys, tres, and braces shall be installed according to the scaffold manuiacture's recommenciations or at the closest horizontad member to the 20 teet $6 . \mathrm{m}$ ) or less thereatter for scaffolds 3 feet $10.91 \mathrm{~m}$ ) wide or less and every 26 feat $(7.9 \mathrm{~m})$ or less thereafter ior scaftolds greater than 3 feet $40.91 \mathrm{~m})$ wide. The top guy, tie or brace of completed scatfolds shall be placed no further than the 4:t height from the top. Such ouys, ties, and braces shall be installed at each end of the scaffold and at horizontal intervals not to exceed 30 feet (9.1 mi) (measured from one end [not bothl towards the other).

(iii) Ties, guys, braces, or outriggers shal be used to prevent the tipping of cantilat (2) Supported scaffodd poles lers, posts, frames, and worights shall bear on base pates and mud sils or other adequate firm foundation.

(i) Footings shall be level, sound, rigid, and capable of supporting the loaded

scaltold without settlitis or displacement.
(i) Unstable objects shall not be used to support scaffolds or plattorm units. il) Unstable objects shats not be used as working piattorm

v) Front-end loaders and similar pieces of equipment shal not be used to manufacturer for such use

(v) Fork-lifis shal not be used to support scaftold platiorms unless the entire plattorm is attached to the fork and the fork-lift is not moved horizontally whtie the platiorm is occupied.

(3) Support scaffold poles, legs, posts, frames, and uprights shall be plumb and braced to prevent swaying and displacement

(e) Access. This paragrapt appties to scatfold access for all employees. Access requirements ad employees erecing or dismanthing suppon

(1) When scafifold platforms are more than 2 feet $(0.6 \mathrm{~m})$ above or below point of access, portabje ladders, hook-on ladders, attachable ladders, stair towers (scaffold stairways/towers), stairway-type ladders (such as ladder stands), ramps, wakkways, integral presabficated scaffold access, or direct access trom another scattold sinucture, personne hoist, of sinnar surface (2) Portable, hook-on, and attachable ladders (Additional requirements for $X$ of this part - Stairways and Ladters

(1) Portable hook-on, and attachable ladders shall be positioned so as not to tip the scaffold.

(ii) Hook-on and attachable ladders shall be positioned so that their bottom rung is not more than 24 inches ( $6 \mathrm{tcm}$ ) above the scaffold supporting level: (ii) When hook-on and attachable ladders are used on a supported scaffold
more than 35 feet (10.7m) high, they shall have rest plattorms at 35 -foot $(30.7 \mathrm{~m})$ maximum vertical intervais.
(iv) Hook-on and attachable ladders shal be specifically designed for use with the type of scaffold used: $1-1 / 2$ inches $(29 \mathrm{~cm})$; and
(vi) Hook-on and attachable ladders shail have uniformly spaced rungs with a maximum spacing beiween rungs of $16-3 / 4$ inches.

(3) Stainway-type ladders shall: (ii) Be provided with rest plattorms â 12 foot $(3.7 \mathrm{~m})$ maximum vertical inter-

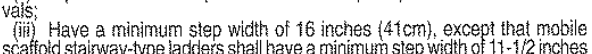
scatiold stainway-type ladders shan have a minimum step width of $11-1 / 2$ inches (30 cm); and

(iv) have sip-resistant treads on all steps and landings.

4) Stainowers (ccatiold stairway/towers) bottom step is not more than 24 inches $(61 \mathrm{~cm})$ above the scaftold supporting

level A stairrali consisting of a toprail and a midrail shal be provided on each

side of each scalfodd stainway.
(ii) The toprail of each stailfail system shal atso be capable of sewing as a

(iii) Handaais, and topralis that serve as hancirails, shail provide an ad-

equate handhold for employees grasping them to avoid falling.
(iv) Stairbail systems and handrails shall be surfaced to prevent injury to (v) The ends of stairrail systems and handrails shahl be constructed so that they do not constitute a prosection hazaro.

(vi) Handrails, and loprails that are used as handrails, shall be ai least 3 inches $(7.6 \mathrm{~cm})$ from other objects.

(vii) Stairrails shatl be not less than 28 inches $(71 \mathrm{~cm})$ nor more than 37 inches $94 \mathrm{~cm}$, srom the upper surface of the starral to the surface of the (viil) A landing platiorm at least 18 inches (45.7cm) wide by at least 18 (ix) Each scaftold stainway shall be at least 18 inches $(45.7 \mathrm{~cm})$ wide be-

tween staisrails.

(xi) Stairways shall be installed boiween 40 degrees and 60 degrees from the horizontal

(xil) Guardrails meeting the requirements of paragraph (g) (4) of this section shat be provided on the open sides and ends of eact lanang. stairs. Greater variations in riser height are allowed for the top and hot of steps of the entive system, not for each fight of stairs.

(xiv) Tread depth shall be uniform, within $1 / 4$ inch, for each flight of staiss.

(5) Ramps and walkways. guardrail systems which comply wal be inclined more than a slope of one (1) vertical to three (3) horizontal (20 degrees above the horizontal).

(jil) If the siope of a ramp orwakway is steeper than one (1) vertical in eight 8) horizontal the ramp orwalkway shal have cieats not more than tourteen (14) inches (35cm) apart which are securely iastened to the planks to provide rooting.

6) Integral prefabricated scaffold access frames shal:

(1) Be specitically designed and constructed for use as ladder rungs;

iij Have a rung length of at least 8 inches (20cm); (1il) Not be used as work piatiorns when nungs are less than $17-1 / 2$ inches device, which complies with Sec. 1926.502.

(vi) Be uniformly spaced within each frame section

(v) Be provided with rest plattorms at $35-100$ l $(10.7 \mathrm{~m}$ ) maximum vertical intervals on all supported scaftolds move than 35 feet (10.7m) high; and (vi) Have a maxmum spacing between rungs of $16-3 / 4$ inches (43cm). Nonunitorm rung spacing caused by joining end frames together is allowed, pro(7) Steps and yungs of fadder and stainway type access shall tine up vertically with eact other between rest piattorms.

(8) Direct access to of from another surface shal be used only when the scaffold is not more than 14 inches (36cm) horizontally and not more than 24

(9) Eftective September 2,1997 , access for employees erecting or dismanung supported scaffolds shat be in accordance with the following:

(3) The employer shas! provide sate means of access for each employee sibte and does not creaze a creater hazard the employer sha katave a com. petent person determine whether it is feastble or would pose a greater hazar to provide and have employees use a sale means of access. This determiflation shal be based on site conditions and the lype of scaffold being erected or dismantled.

(ii) Hook-on or attachable fadders shall be installed as soon as scaffold erection has progress to a point that permits sate installation and use. 
(iii) When erecting or dismantling tubular welded frame scaffolds, (end) frames, with horizontal members that are paraliel, level and are not more than 22 inches apart verticaly may be used as climbing devices jor access, pro-
vided they are erected in a manner that creates a usable ladder and provides good hand hold and foot space.

(iv) Cross braces on tubular welded frame scaffoids shail not be used as a means of access or egress.

(f) Use. Scaffolds and scaffold components shail not be loaded in excess of their maximum intended loads or rated capacities, whichever is less.

(2) The use of shore or lean'to scaftolds is prohibited. by a competent person before each work shifi, and a after any occurrence which could affect a scaffotd's structural integrity.

(4) Any part of a scaffold damaged or weakened such that its strength is less than that required by paragraph (a) of this section shall be immediately repaired or replaced, braced to meet those provisions; or removed from service untib repaired.

(5) Scaiflolds shall not be moved horizontalyy while employees are on them, unless they have been designed by a registered protessional engineer speSec. 1926.452 (w) are followed.

(6) The clearance between scaffolds and power lines shal be as follows: Scaftolds shall not be erected, used, dismantied, aliered or moved such that wey or any conduche materlat handed on them might cone closer to exposed and energized power fines than as follows:

\begin{tabular}{|c|c|c|}
\hline 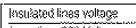 & 3inimims 9istanch & Al:emaives \\
\hline 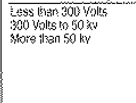 & 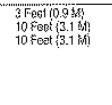 & 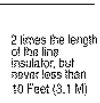 \\
\hline 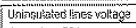 & Bsinimus Distarces & Alleantives \\
\hline $\begin{array}{l}\text { Lass than } 50 \text { iny } \\
\text { More than } 50 \text { k }\end{array}$ & 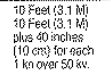 & 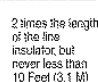 \\
\hline
\end{tabular}

Exception to paragraph (f)(6): Scaftolds and materials may be closer to power lines than specitied above where such clearance is necessary for performance of work, and only ater the uthy company. or electhical system opelectrical system operator has deeneroized the ines, relocated the lines, or installed protective coverings to preveri accidental contact with the lines. (7) Scaftolds shall be erected, moved, dismanted, or altered only under the supervision and direction of a competent person qualined in scaffocd erection, moving, dismanting or aterations. Such activitiss shal be performed only by .

(8) Employees shall be prohibited from working on scaffolds covered with stow, lce
materials.

(9) Where swinging loads are being hoisted onto or near scaffolds such that the loads might contact the scattold, tag lines of equivalent measures to control the loads shat be used.

(10) Suspension ropes supporting adjustable suspension scaffolds staal be of a dernele large enough to provide sufficient surface area for the functioning of brake and hoist mechanisms. When acids or other corrosive substances are used on a scatifid the rope shall be shielded, treated to protect against the corrosive substances, or shat be of materiat that wil not be damaged by the substance being used.

(12) Work on of from scaffolds is prohibited during storms or high winds unless a competent person has cotermined that it is safe for employees to be on the scaftod and those employees are protected by a personal fall arres is secured against the anticipated wind forces imposed.

(13) Debris shall not be allowed to accumblate on platiorms.

74. Makeshitt devices such as but not limited to boxes and barrels, shal not be used on top of scafiold platforms to increase the working level height of

(15) Ladders shail not be used on scaffolds to increase the working level height of employees, except on large area scaffolds where employers have

(i) When the ladder s placed against a structure which is not a part of the encured against the sideways thrust exerted by

(ili) The ladder legs shall be on the same plattorm or other means shall be

provided to stabilize the ladder against unequal platform deftection, and (iv) The ladder legs shall be secured to prevent them from slip(16) Plattorms shall not deflect more than $1 / 60$ of the span when

loaded. the suspension wire rope when performing welding from suspended scatilds, the followng precaulons shall be laken, as applcable: (1) An insulated thishe shal be used to attach each susperision ger) Excess suspension wire rope and any additional independent lines from orounding shall be insulazed

(ii) The suspension wire rope shall be covered with insulating material axtending at least 4 teet $(1,2 \mathrm{~m}$ ) above the hoist. if there is a sall line below the hoist, it shat be mistuated to prevent contac with the platiorm. The portion of the tall line that hangs free below the scaliolu shall be guided or retained, or both, so that is does no

(ii) Each hoist shall be covered with insutated proiective covers in addition to a work lead attachment required by the weilding process, a grounding conductor shels be connected from the scalsiz to the structure. The size of this conductor shal be at leasi the size of the welding process work lead, and this conductor

(v) if the scaffodd orouncing lead is disconnected at ary time, the widing machine shall be stht of: and

(vi) An active welding rod or uninsulated weiding lead shall not be allowed to contact the scafiold or its suspension system.

(g) Fail protection. a lower level shal be protected from ank tong that lower level, Para graphs (g)(1)(1) through (Vil) of this section establish the types of fall protection to be provided to the employees on each type of for scaffold erectors and dismantlers. for scatiold erectors and dismanters
Note to paragraph (g)(1): The fall protection requirements for employees instalang suspension scaffold support systems on floors roofs, and other elevated surfaces

(1) Each employee on a boatswains' chair, catenary scaffold, float scatfold, neede beam scafiold, of ladder jack scattold shall be proched by a personal fall arrest system;

(19) Each employee on a single-point or two-point adjustable sussystem and guardrail system

(iil) Each employee on a crawing board (chick ladder) shall be protected by a personal fall arrest system, a guardrail system : with minimum 200 pound toprail capacity) or by a threesourth inch (1.9cm) diameter grabline or equivalen (handhold securely fastened beside each crawing board

(iv) Each emproyee on a seli-contained adjustabie scaftold shal . and by both a personal fall arrest system and a guardrail system (with minimum 200 pound toprail capacity) when the plation is

supported by ropes:
(v) Each employee on a walkway located within a scaftold shal be protected by a quardiail system (with minimum 200 pound topra capacily) instated within $9-1 / 2$ inches $924.1 \mathrm{~cm}$ ) of and along a (wit the side of the walkway

(vi) Each employee petiorming overhand bricklaying operations open sides and ends of the scaffold (exceot at the side next to the wall being laidj by the use of a personal fall arresi system or guardrail system (with minimum 200 pound toprail capacity).

(vii) For all scaffolds not otherwise specified in paragraphs (g)/ t)(t) through (g) (1)(vi) of this section, each employee shall be protected my use or personal lall arrest systems or guardralls system (2) Ef fective Sentember? 1097 the employer shal have a com petent person determine the feastolity and safety of providing ta protection for employees erecting or dismanting supported scatsolds. Employers are requiled to provide fall protection for employees erecting or dismanting supported scaltods where the installa tion and use of such protection is feasible and doss not create

(3) In addilition to meeting the sequirements of Sec. 1926.502 (d) personal fail arrest systerns used on scafoldds shal be attached by member. Vertical lifelines shal not be used when overhead components, such as overhead olection or additonal platiorm levels, are part of a single-point or two-pont? adjustable suspension scafold. When vertical lifelines are used, they shail be fastened to a fixed sate point of anchorage, shall be independent of the scaffold 


\section{Recommended Scaffolding Erection Procedure}

\section{INTRODUCTION}

This guide has been prepared by the Scaffolding, Shoring \& Forming Institute to assist contractors, architects, engineers, dealers, erectors, and users, etc. for the proper use of scaffolding equipment. Scaffolding Safety Rules published by the lnstitute should be used in conjtintion with this publication, as well as the instructions for the use of scaffolding provided by the manufacturer. Safety precautions and requirenents prescribed by local, state, and federal agencies, including OSHA, must be followed at all times and persons working with scaffolding systems should be equipped with requisite safety devices.

\section{WOMENCLATURE:}

1. Accessories - Those items other than frames and braces used to facilitate the construction of scaffolding towers and structures.

2. Adjustment Screw-Device composed of a threaded screw and an adjusting bandle used for the vertical adjustment of the scaffolding.

3. Base Plate-A device used to distribute the leg load.

4. Climbing Ladders - -A separate ladder attached to the scaffolding structure or built into the scaffold frame.

5. Casters Wheels of a suitable dimension and unit designed to attach to the base of a tower and containing a brake to prevent the wheels from rotating.

6. Coupling Pin-Device used to align and connect lifts or tiers together vertically.

7. Cross-bracing-System of members connecting frames or panels of scaffolding to make a tower structure.

8. Extension Device Any device used to obtain vertical adjustment of scaffolding other than an adjustment screw.

9. Factor of Safety - the ratio of ultimate load to the at+ towable load.

10. Frame or Pane ${ }^{*}$---the principal prefabricated, welded structural unit.

11. Guardrail-A rail secured to uprights and erected along the exposed sides and ends of platforms.

12. Horizental Diagonal Bracing-Diagonal braces running horizontally between frames of scaffolding.

13. Lifts or Tiers* The number of frames stacked one above each other in a direction.

14. Locking Device Adevice used to secure the cross brace to the panel.

15. Putlog or Truss - A separate horizontal load carrying member.

16. Rolling Towers A composite structure of frames, braces, platforms, guardrails, and accessories supported by casters.

17. Safe Leg Load . That load which can safely be directly imposed on the frame leg.

18. Safe Scaffold Frame Horizontal Member Load-That load which can safely be directly imposed on a horizontal member.

19. Scaffolding Layout - An engineered drawing prepared prior to erection showing arrangement of equipment for proper scaffolding use.
20. Side Bracket -A cantilevered arm unit, supported by the scaffolding frame.

21. Sill or Mud Sill * A footing, usually wood, which distributes the vertical leg loads to the ground.

22. Ties-A tension compression member used to securely attach scaffold to a structure.

23. Toeboard-A barrier secured along the sides and ends of a platform, to guard against the falling of material.

24. Towers - A composite structure of frames, braces, and accessories.

25. Ultimate Load- The maximum load which may be placed on the scaffolding causing failute by buckling of column members or yielding of some component.

* These terms can be used synonymously.

\section{MNSPECTION ON SEAFTOLDNE}

EQUIPMENT PRIOR TO ERECTHON

The three main areas of inspection are for corrosion, straighmess of members and welds. This applies to all components of a scaffolding system.

1. CORROSION--Heavily rusted or eroded scaffolding equipment is a telltale sign of abuse or neglect.

2. STRAIGHTNESS OF MEMBERS-Mishandling, trucking and storing may cause damage to scaffolding equipment. All scaffolding components should be straight and free from bends, kinks or dents.

3. WELDS-Equipment should be checked before use for damaged welds and any piece of equipment showing damaged welds or rewelding beyond the original factory weld should not be used. The factory weld reference pertains to location and quality of rewelds. While CORROSION, STRAIGHTNESS, and WELDS are of primary concem other component parts should be checked.

4. Locking devices on frames and braces shall be in good working order, and if not, must be repaired or replaced prior to use.

5. Coupling pins must effectively align the frame or panel legs.

6. Pivoted cross braces must have the center pivot securely in place.

7. Caster Brakes shall be in good working order and if not must be repaired or replaced prior to use.

Safe Bearing Loads for Solls

Considering that the allowable loads (bearing) on various soils and rock range from less than 1,000 p.s.f. to more than 50,000 p.s.f. care should be exercised in determining the capacity of the soil for every scaffolding job, realizing that weather conditions can turn an otherwise suitable ground condition into a hazardous situation. As an example, dry clay with an allowable bearing capacity of 8,000 p.s.f. could become very plastic after a rainfall and drop to less than 2,000 p.s.f.

Psint Date 03-2007

PAGE 1 OF 4 
9. Check to be certain all guard rails are in place.

10. If scaffolding is enclosed, check to see that additional precautions have been taken as noted in Section of Erection. Recheck periodically ties, clamps, etc., for movement.

11. Insure that safe access to work platform(s) is provided.

\section{Dismanthang of SCAFrolding}

The work of dismantling scaffolding should be under the supervision of an individual with proper experience and aptitude. The following should be observed while dismantling,

1. Check to see if scaffolding has been structurally altered in any way which would make it unsafe, and if so reconstruce where necessary before commencing with the dismantling procedures.

2. Dismantle scaffold from the top down. Begin by renoving all accessories from that lift being dismantled at the time.

3. Always work from a minimum of two plank placed on the tier of frames below those being removed. Move the planking down as dismantling progresses.

4. Do not remove ties until dismantling has reached the tier to which they are attached.

5. Always stay within the inside of the scaffotd. Do not climb on the outside for any reason when dismantling. Do not climb on ties, braces or unbraced frames.

6. Only remove fastening devices from bottom of frames being removed.

7. Lower scaffolding components in a safe manner as they are dismantled. Avoid dropping or throwing the components as this could result in damage to the equipment, or injury to personnel below.

\section{EREction of Rolling Towers}

When erecting rolling scaffolding towers, the following additional items apply. These items are in addition to the application portions of the preceding seetion.

1. Caster should be of adequate load capacity and size in relation to the height of the tower, the surface over which the tower is to be used and in accordance with all government, state, and local codes, ordinances, and regulations.

Casters with plain stems shall be attached to the panel or adjustment screw by pins or other suitable means.

2. Do not extend adjusting serews on rolling towers more than 12".

3. The platform height shall not exceed* four (4) times the smallest base dimension unless guyed or otherwise stabilized.

4. Horizontal diagonal braces should be used near the botw tom, top and at $20^{\top}$ intervals measured from the rolling surface.

5. When side brackets are used, consideration should be given to the overturning effect these brackets will have upon the stability of the tower.

6. Cross brace every lift-both sides

7. Install guardrails.

8. Plank according to Plank and Accessories Section of Erection Procedure.

Q Bil-Jax, Inc. 20094

Printed it the U.S.A.

PAGE 3 OF 4
* EXCEPTIONS: Three times in Califomia, Ohio, Oregon, Montana, Maine; $3+1 / 2$ times in Washington.

\section{Final Inspection of Roluing Towers}

The following additional points should be checked when making a final inspection of rolling scaffold towers prior to their use. These points are in addition to the applicable items covered under the preceding section entitled, "Final Inspection of Erected Scaffolding."

1. Check to see that the platform beight does not exceed four (4) times the smallest base dimension unless the tower is properly guyed or otherwise stabilized.

2. Check to see that, if adjusting screws have been ased, they are not extended more than $12^{\prime \prime}$.

3. Check to make sure the caster brakes are in good working condition and are applied when tower is not being moved.

4. Inspect to make sure horizontal diagonal bracing has been placed near the bottom, top, and at 20 intervals measured from the rolling surface. A hook on manufactured platform properly attached to the top frame may be equivalent to the top horizontal diagonal brace.

5. Cross bracing has been installed on both sides of every lift.

6. Check the area in which the tower is to be used to insure there are no obstructions either in, on, or above the floor which will interfere with the proper and safe use of the rolling tower.

7. Check for guardrails

8. Check to see that all planks and fabricated platforms are properly installed.

9. Insure that safe access to work platform(s) is provided.

REFER TO SCAFFOLDING, SHORING \& FORMING INSTITUTE SCAFFOLDING SAFETY RULES BEFORE USING SCAFFOLDING.

The procedures outlined in this Guide describe conventional procedures for erecting and dismantling scaffolding systems. However, equipment and systems differ and, accordingly, reference must always be made to the instructions and procedures of the manufacturer or supplier of the equipment. Since field conditions vary and are beyond the control of the Institute and its members, safe and proper use of this equipment is the responsibility of the user and not the Institute or its members.

\section{SCAFFOLDING AND SHORING INSTTTUTE 1230 KEITH BUILDING} CLEVELAND, OHIO 44115

\section{BIL-JAX 125 TAYLOR PARKWAY ARCHBOLD, OHIO 43502}

Form No. SSI- $83-80 \cdots 68$ 


\section{INSTRUCTIONS FOR ERECTING ROLLING OR STATIONARY TOWERS}

Bil-Jax, Inc. recommends that all users of this equipment be supplied with safety information and OSHA rules and regulations. This information must be thoroughly read and understood before using the equipment. If you have not been provided with OSHA regulations or safety literature, please call 419/445-8915 before use.

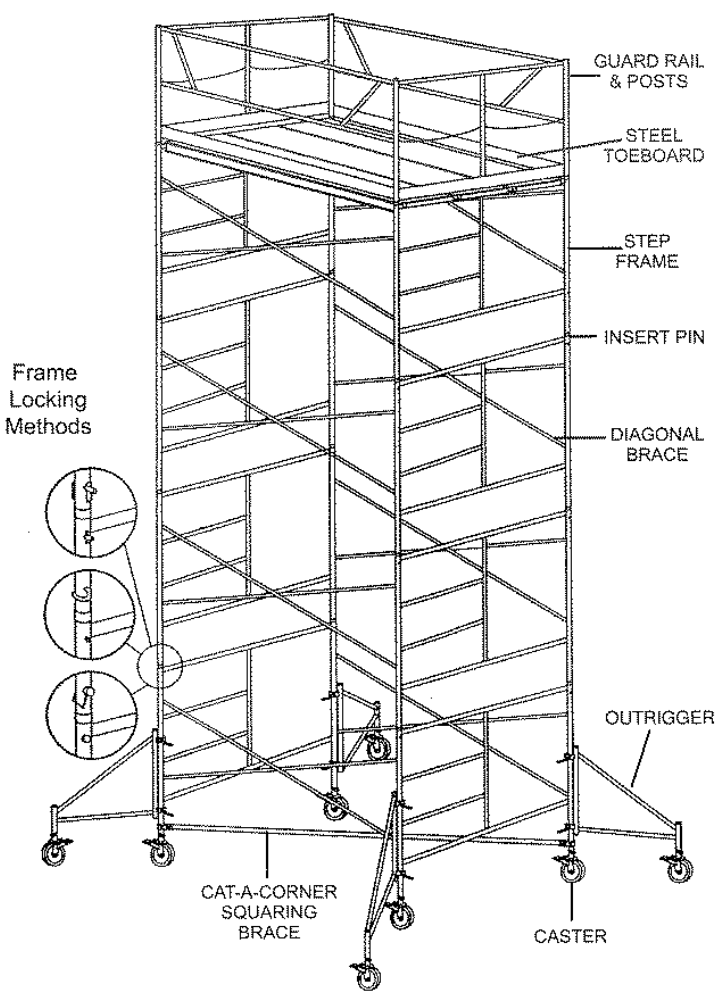

NOTE: Some areas may require separate access ladders or stairs. Consult your local authorities for information before beginning scaffold erection.
Bil-Jax Rolling Towers offer largeworkplatformareas and adjustable platform levels, while providing workers with a quick, easy and safe assembly.

A Rolling Tower should only be used on solid, level and clear floor areas. Do not use on soil or unlevel surfaces. Checkforoverhead obstructions which may interfere with the tower.

1. Before starting scaffold erection, check all parts for damage, making sure that they are in proper working order. Any part that does not look to be in good working condition or is damaged in any way should not be used.

PAGE 1 OF 4 


\section{QUTRIGGERS}

5. Determine tower height required to complete the job. If height exceeds 3 times the minimum base dimension, (see Free Standing Tower Height section below) install outriggers to increase minimum base dimension or tie in to permanent structure as codes require. Pin caster to outrigger and attach outrigger to scaffold leg. Adjust to desired angle and tighten clamp firmly to prevent movement. Set caster brakes.

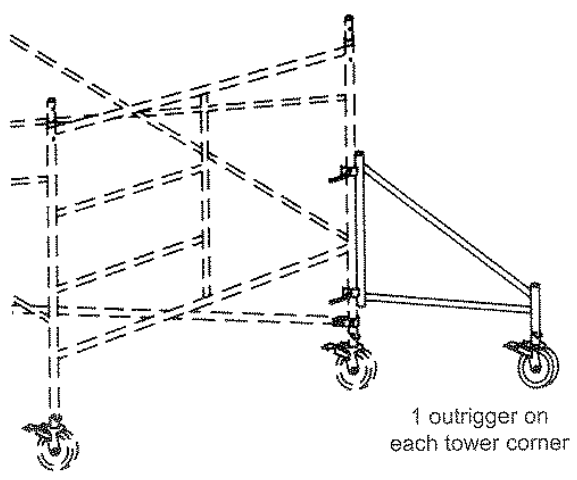

Bil-Jax, Inc, recommends the maximum free-standing tower height permitted for a Rolling Tower be 3 times the minimum base dimension, although OSHA codes allow 4 to 1 in most states. Consult your state and loca codes to insure your tower complies with all applicable regulations.

\section{A $\times 3=B$ \\ (smallest (some states (maximum \\ base allow $3-1 / 2$ tower height \\ dimension) or 4 -check permitted) \\ your local \&}

(use smallest of the base dimensions)

\section{ADDING FRAME SECTIONS}

6. Hoist end frames and braces to next level by means of rope and tag line. Install diagonal bracing. Secure frames together using inserts and insert locking pins. Be sure to erect step frames so that the steps continue above one another on the same side of the frame. Full decking should be instalied on completed tier before attempting to assemble next tier, and should be moved up as each additional tier is completed.

NOTE: When required, access ladder or stair sections must be installed at the same time as each scaffold section is added.

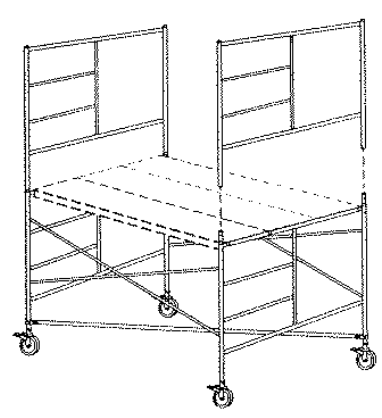

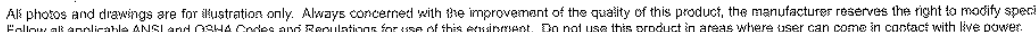

PAGE 3 OF 4 


\begin{tabular}{|c|c|c|c|c|c|c|}
\hline \multirow{2}{*}{\multicolumn{3}{|c|}{ 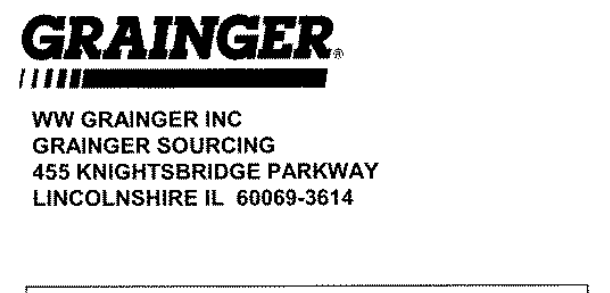 }} & \multicolumn{4}{|c|}{ Sourcing Request } \\
\hline & & & \multirow{3}{*}{\multicolumn{4}{|c|}{ 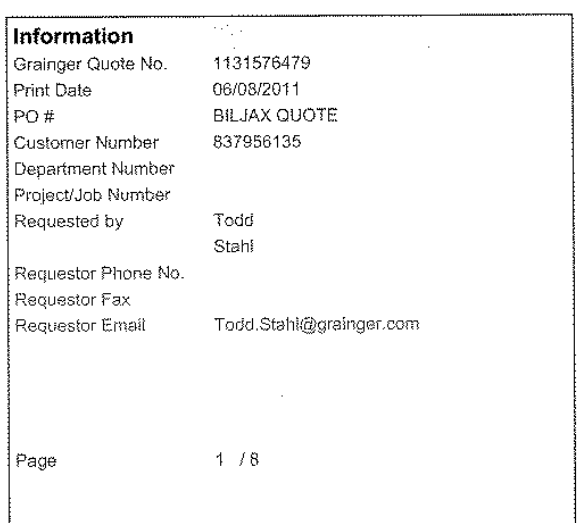 }} \\
\hline \multicolumn{3}{|c|}{$\begin{array}{l}\text { Sold To } \\
\text { Name: WVU DEPT OF MAE } \\
\text { Account th: } 837956135 \\
\text { Phore: } 3042932419 \\
\text { Fasx: }\end{array}$} & & & & \\
\hline \multicolumn{3}{|c|}{$\begin{array}{l}\text { Ship To } \\
\text { Nam: WVU OEPT OF MAE } \\
\text { 359 EVANSDALE DR } \\
\text { MORGANTOWN, WV 26506-0000 } \\
\text { US } \\
\text { Shipper ACCOUNt: }\end{array}$} & & & & \\
\hline \multicolumn{7}{|c|}{ 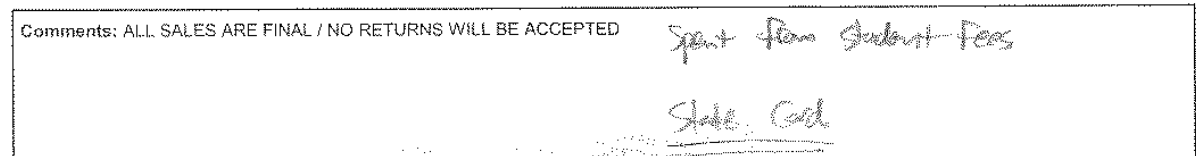 } \\
\hline & $\begin{array}{l}\text { Item } \\
\text { PO-Line }\end{array}$ & Material/Description & $\therefore$ & uantity & Unit & Unit Price \\
\hline$\sqrt{2}$ & 1000000 & 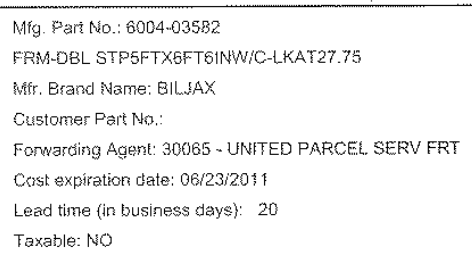 & & 10 & & 106.06 \\
\hline 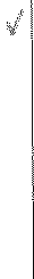 & 20 & 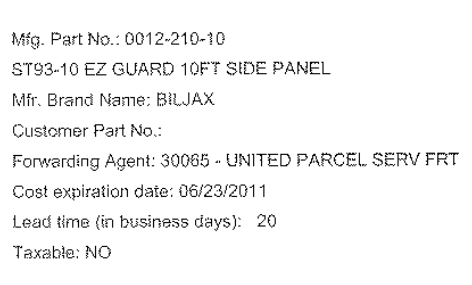 & & 2 & EA & 40.54 \\
\hline 4 & 300 & $\begin{array}{l}\text { MFg. Fart No.: 0010-01-10 } \\
\text { BRG-DIA-H27 3/4 X 7FT } \\
\text { Mif. Brand Name: BlLAAX } \\
\text { Customer Parl No: }\end{array}$ & & 10 & EA & 23.76 \\
\hline
\end{tabular}




\begin{tabular}{|c|c|c|c|c|c|c|}
\hline & \multicolumn{2}{|c|}{$\begin{array}{l}\text { WW GRAINGER INC } \\
\text { GRAINGER SOURCING } \\
\text { 455 KNIGHTSBRIDGE PARKWAY } \\
\text { LINCOLNSHIRE IL 60069-3614 }\end{array}$} & $\begin{array}{l}\text { Information } \\
\text { Grainger Quote Number } \\
\text { Print Date } \\
\text { Custorner Number } \\
\text { Page }\end{array}$ & $\begin{array}{l}1131 \\
05 / 0 \\
8370 \\
21\end{array}$ & $\begin{array}{l}576479 \\
8 / 2011 \\
56135 \\
8\end{array}$ & \\
\hline & $\begin{array}{l}\text { Item } \\
\text { PO-Line }\end{array}$ & Material/Description & Quan & & Unit & Unit Price \\
\hline W & $\begin{array}{c}40 \\
000000\end{array}$ & 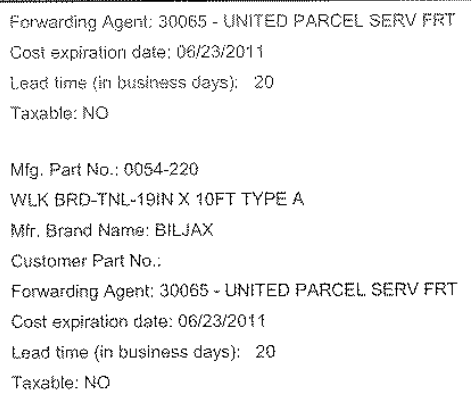 & & 3 & EA & 153.79 \\
\hline$d$ & $\begin{array}{l}50 \\
000000\end{array}$ & $\begin{array}{l}\text { Mig. Part No: 0068-061-50 } \\
\text { ST2SFi50 2IN SNAP PIN PKG50 C-1008 PIN } \\
\text { MFf. Brand Name: BiLJAX } \\
\text { Customer Part No.: } \\
\text { Forwarding Agent: } 30065 \text { - UNITED PARCEL SERV FFT } \\
\text { Cost expiralion date: 06i23/2011 } \\
\text { Lead time (in business days): } 20 \\
\text { Taxable: NO }\end{array}$ & & 1 & EA & 21.06 \\
\hline$\sqrt{V}$ & $\begin{array}{l}60 \\
0000000\end{array}$ & 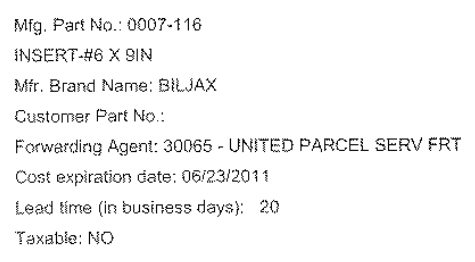 & & 20 & EA & 2.09 \\
\hline 18 & $\begin{array}{c}70 \\
0000000\end{array}$ & 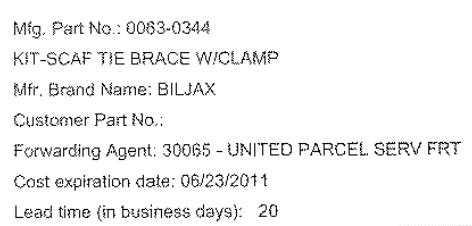 & & $\sqrt{4}$ & EA & 21,60 \\
\hline
\end{tabular}




\section{GRAINGER. \\ IIIIIII}

WW GRAINGER INC

GRAINGER SOURCING

455 KNIGHTSBRIDGE PARKWAY

LINCOLNSHIRE IL 60069-3614

\section{Sourcing Request}

\begin{tabular}{|ll|}
\hline Information & \\
Grainger Quote Number & 1131576479 \\
Print Date & $06 / 08 / 2011$ \\
Customer Number & 837956135 \\
Page & 318 \\
& \\
\hline
\end{tabular}

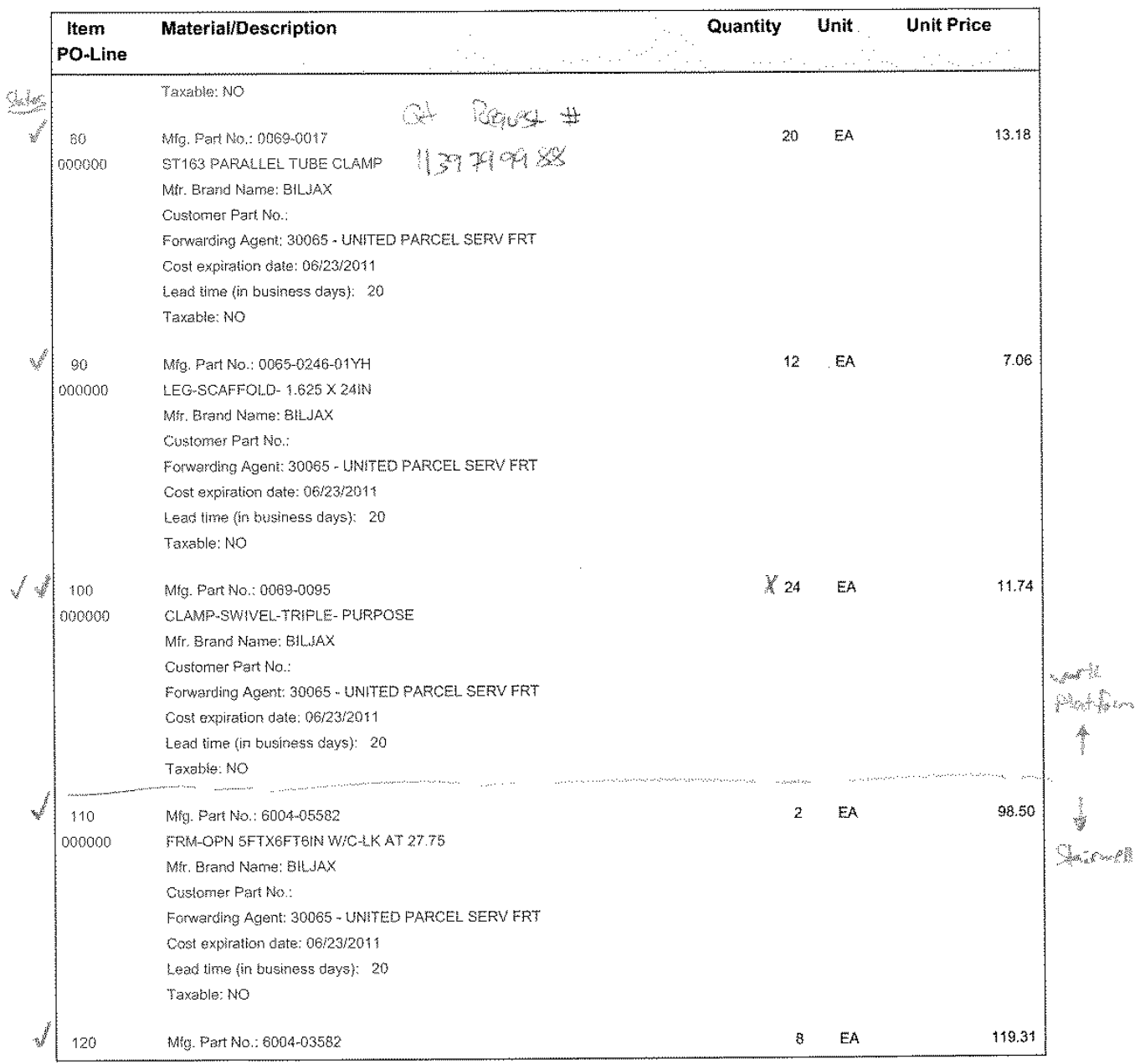




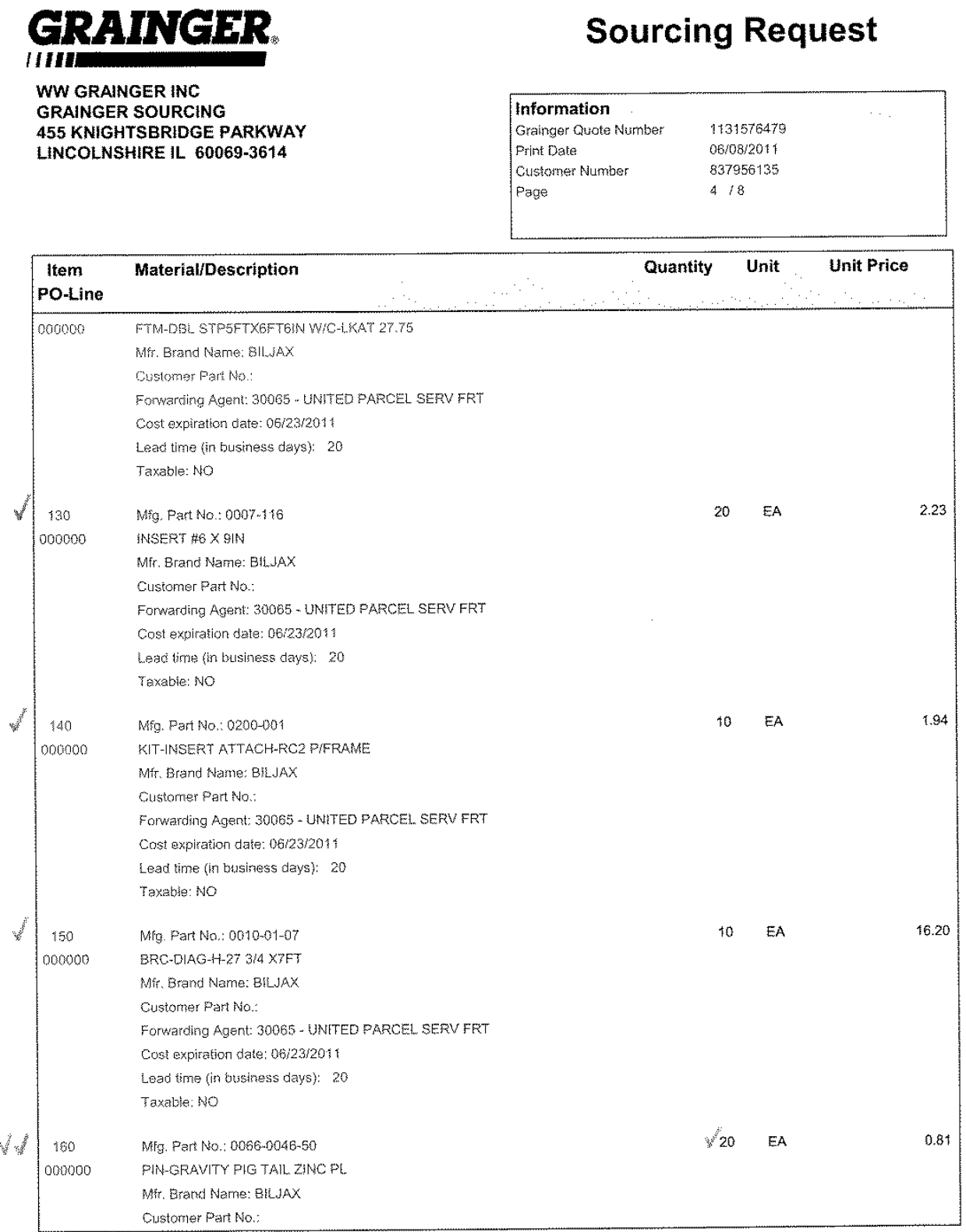




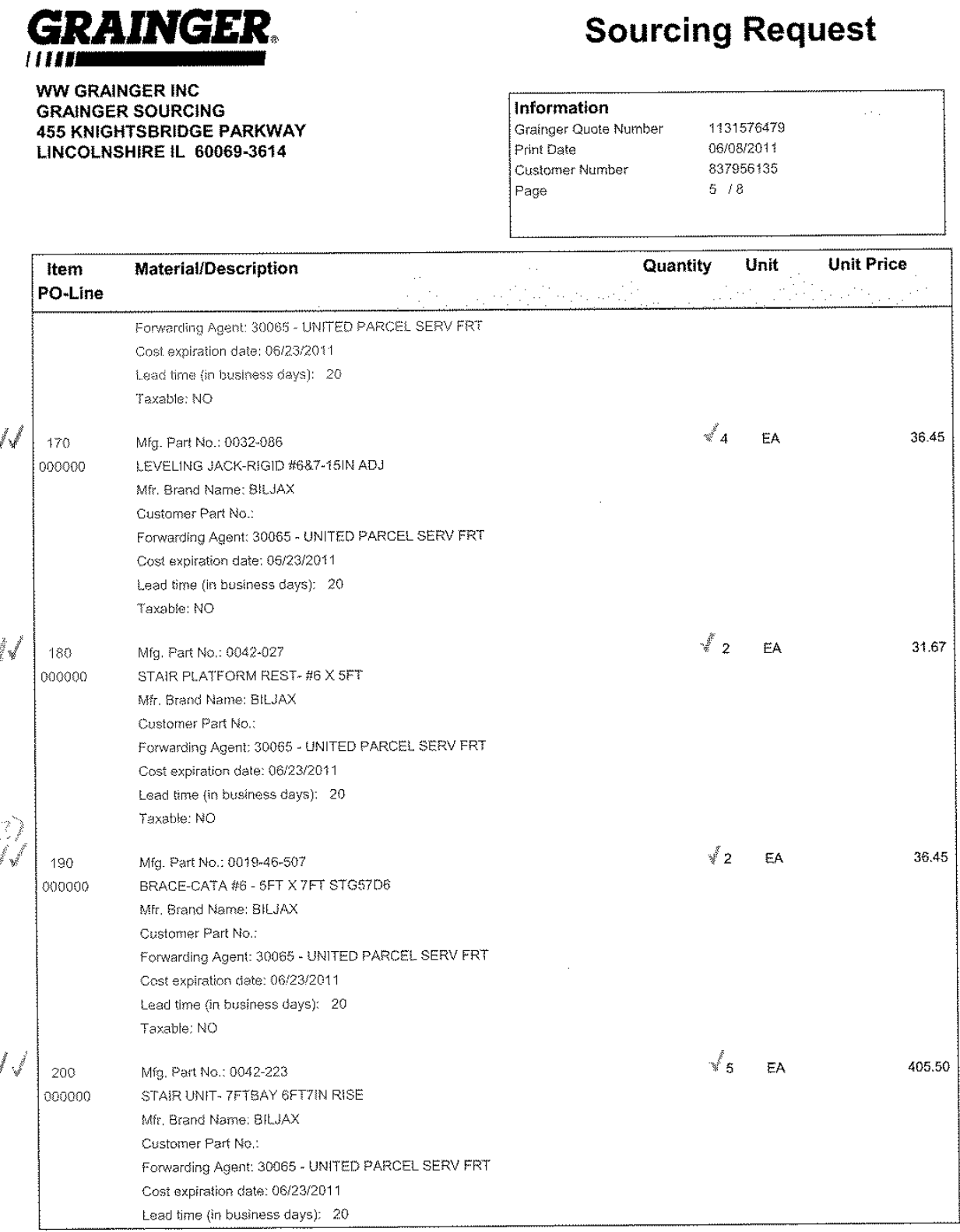




\section{GRAINGER.}

IIIIIII

WW GRAINGER INC

GRAINGER SOURCING

455 KNIGHTSBRIDGE PARKWAY

LINCOLNSHIRE IL 60069-3614

\section{Sourcing Request}

\begin{tabular}{|ll}
\hline Information & \\
Grainger Quote Number & 1131576479 \\
Print Date & $06 / 08 / 2011$ \\
Customer Number & 837956135 \\
Page & $6 / 8$
\end{tabular}

678

\begin{tabular}{|c|c|c|c|c|}
\hline $\begin{array}{c}\text { Item } \\
\text { Po-Line }\end{array}$ & Material/Description & Quantity & Unit & Unit Price \\
\hline & Taxeble: NO & & & \\
\hline 210 & Mig. Fart No.: $0042-083$ & 4 & EA & 71.52 \\
\hline \multirow[t]{7}{*}{600000} & FANDRAIL. INSIDE 7FT STARR UNIT & & & \\
\hline & Mr. Band Name: BHJAX & & & \\
\hline & Customer Part No.: & & & \\
\hline & Fonwarding Agen:: 30065 - UNITED PARCEL SERV FRT & & & \\
\hline & Cost expiration date: $06 / 23 / 2011$ & & & \\
\hline & Lead time (irt business days): 20 & & & \\
\hline & Taxable: NO & & & \\
\hline 220 & Mig. Pars No.: 0042-094 & 4 & EA & 82.70 \\
\hline \multirow[t]{7}{*}{000000} & HANDRALL OUTSIDE 7FT STAIR UNIT & & & \\
\hline & Mfr. Brand Name: BiLJAX & & & \\
\hline & Customer Part No: & & & \\
\hline & Forwarcing Agent: 30065 - UNETED PARCEL SERV FRT & & & \\
\hline & Cost expiration date: 06/23/2011 & & & \\
\hline & Lead time (in business days): 20 & & & \\
\hline & Taxable: NO & & & \\
\hline 230 & Mrg. Part No: :0042-175 & 1 & EA & 71.77 \\
\hline \multirow[t]{7}{*}{000000} & HANDRAIL INSIDE END 7FT STAIR UNIT & & & \\
\hline & Mfs: Brand Name: $B \biguplus 3 A X$ & & & \\
\hline & Customer Part No: & & & \\
\hline & Fonwarding Agent: 30055 - UNITED PARCEL SERV FRT & & & \\
\hline & Cost expiration date: $06 / 23 / 2011$ & & & \\
\hline & Lead tàme (in business days): 20 & & & \\
\hline & Taxable: NO & & & \\
\hline 240 & Mig. Part No: $0042-185$ & 1 & EA & 80.68 \\
\hline \multirow[t]{7}{*}{000000} & HANDRALL OUTSIDE END 7ET STR UNT & & & \\
\hline & Mfr. Brand Name: BiL.JAX & & & \\
\hline & Customer Part No: & & & \\
\hline & Forwarding Agent: 30065 - UNTTED PARCEL SEFV FRT & & & \\
\hline & Cost expiration date: $06 / 23 / 2011$ & & & \\
\hline & Lead time (in business days): 20 & & & \\
\hline & Taxable: NO & & & \\
\hline 250 & Mig. Part No.: 0012-226.06 & $\sqrt{2}$ & EA & 76.95 \\
\hline
\end{tabular}




\section{GRAINGER}

IIIIII

WW GRAINGER INC

GRAINGER SOURCING

455 KNIGHTSBRIDGE PARKWAY

LINCOLNSHIRE IL 60069-3614

\section{Sourcing Request}

\begin{tabular}{ll}
\hline Information & \\
Grainger Quote Number & 1131576479 \\
PFint Date & $06 / 08 / 2011$ \\
Customer Number & 837956135 \\
Page & $7 / 8$
\end{tabular}

Page

$7 / 8$

\begin{tabular}{|c|c|c|c|c|}
\hline $\begin{array}{l}\text { Item } \\
\text { PO-Line }\end{array}$ & Material/Description & Quantity & Unit & Unit Price \\
\hline 000000 & 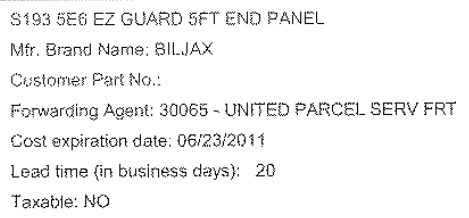 & & & \\
\hline $\begin{array}{c}260 \\
000000\end{array}$ & $\begin{array}{l}\text { Mog. Part No.: 0012 209-07 } \\
\text { S193-7 EZ-GUARD 7fT SIDE PANEL } \\
\text { Mfs. Brand Name: BiLJAX } \\
\text { Customer Part No: } \\
\text { Forwarding Agent: } 30065 \text { - UNITED PARCEL SERV FRT } \\
\text { Cost expiration date: 06/23/2011 } \\
\text { tead time (in business days): } 20 \\
\text { Taxable: No }\end{array}$ & $\sqrt{2}$ & EA & 28.35 \\
\hline $\begin{array}{c}270 \\
000000\end{array}$ & $\begin{array}{l}\text { Mfg. Part No: 0012-202-07 } \\
\text { ST90-7 PANFEL GUARD 7FT WITH TOEBOARO } \\
\text { Mf. Brand Name: BILJAX } \\
\text { Customer Part No.: } \\
\text { Fonarding Agent: } 30065 \text { - UNITED PARCEL SEFV FRT } \\
\text { Cost expiration date: 06/23/2013 } \\
\text { Lead time (in business days): } 20 \\
\text { Taxable: NO }\end{array}$ & $/ 2$ & EA & 28.35 \\
\hline $\begin{array}{c}280 \\
000000\end{array}$ & 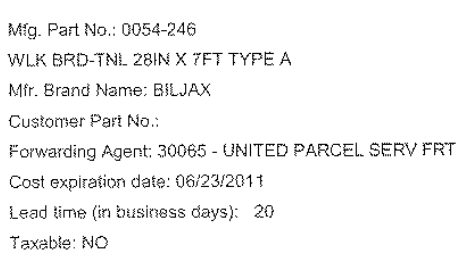 & $\sqrt{2}$ & EA & 121.50 \\
\hline $\begin{array}{c}290 \\
000000\end{array}$ & 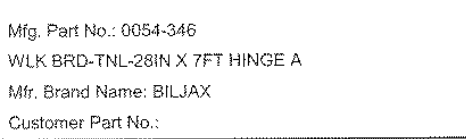 & $y$ & EA & 186.22 \\
\hline
\end{tabular}




\section{GRAINGER IIIII)}

WW GRAINGER INC

GRAINGER SOURCING

455 KNIGHTSBRIDGE PARKWAY

LINCOLNSHIRE IL. 60069-3614

\section{Sourcing Request}

\begin{tabular}{|ll}
\hline Information & \\
Grainger Quote Number & 1131576479 \\
Print Date & $06 / 08 / 2011$ \\
Customer Number & 837956135 \\
Page & $8 / 8$
\end{tabular}

page

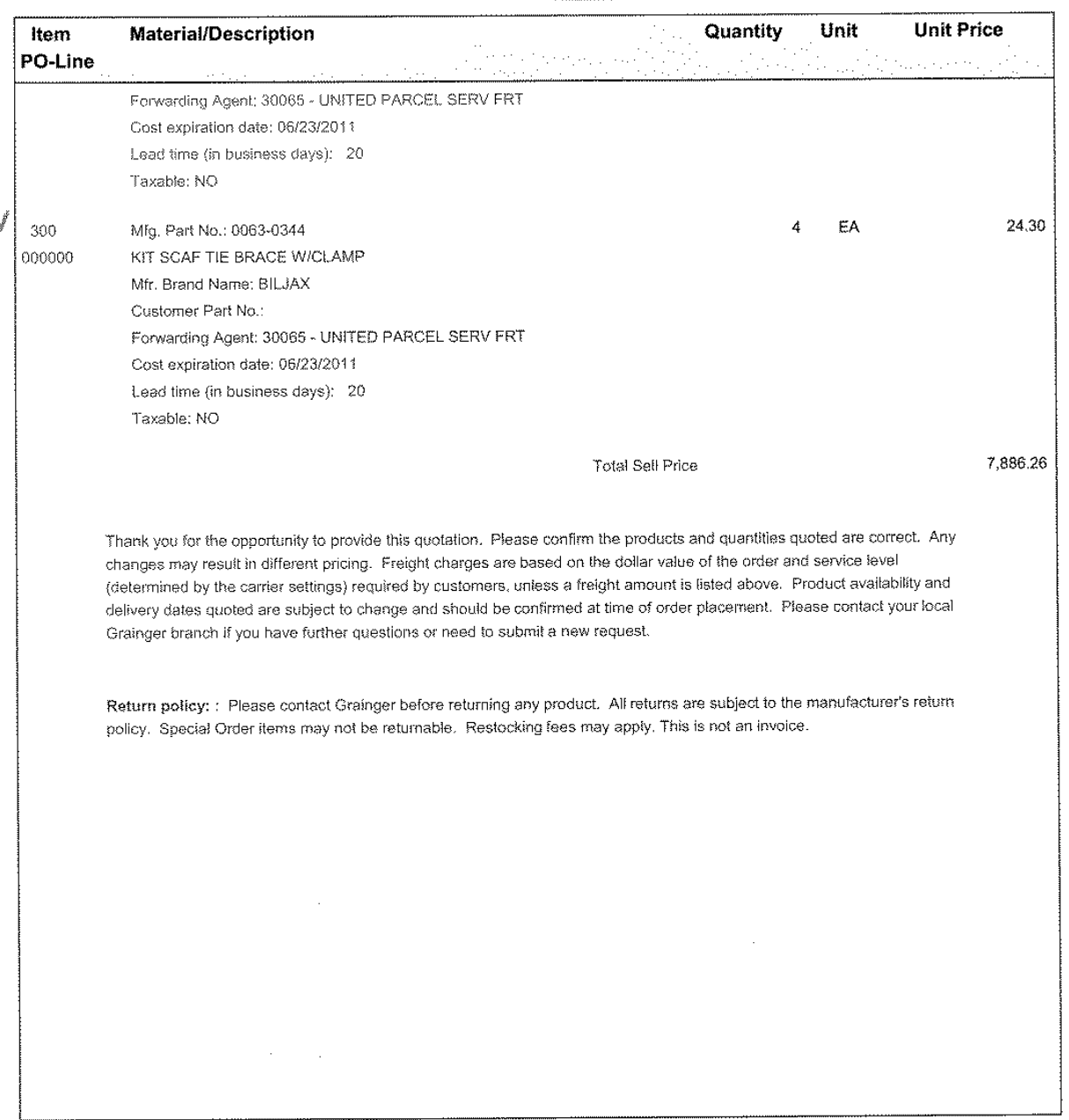

9 Copyright 1997-2005 Grainger. All rights reserved. 


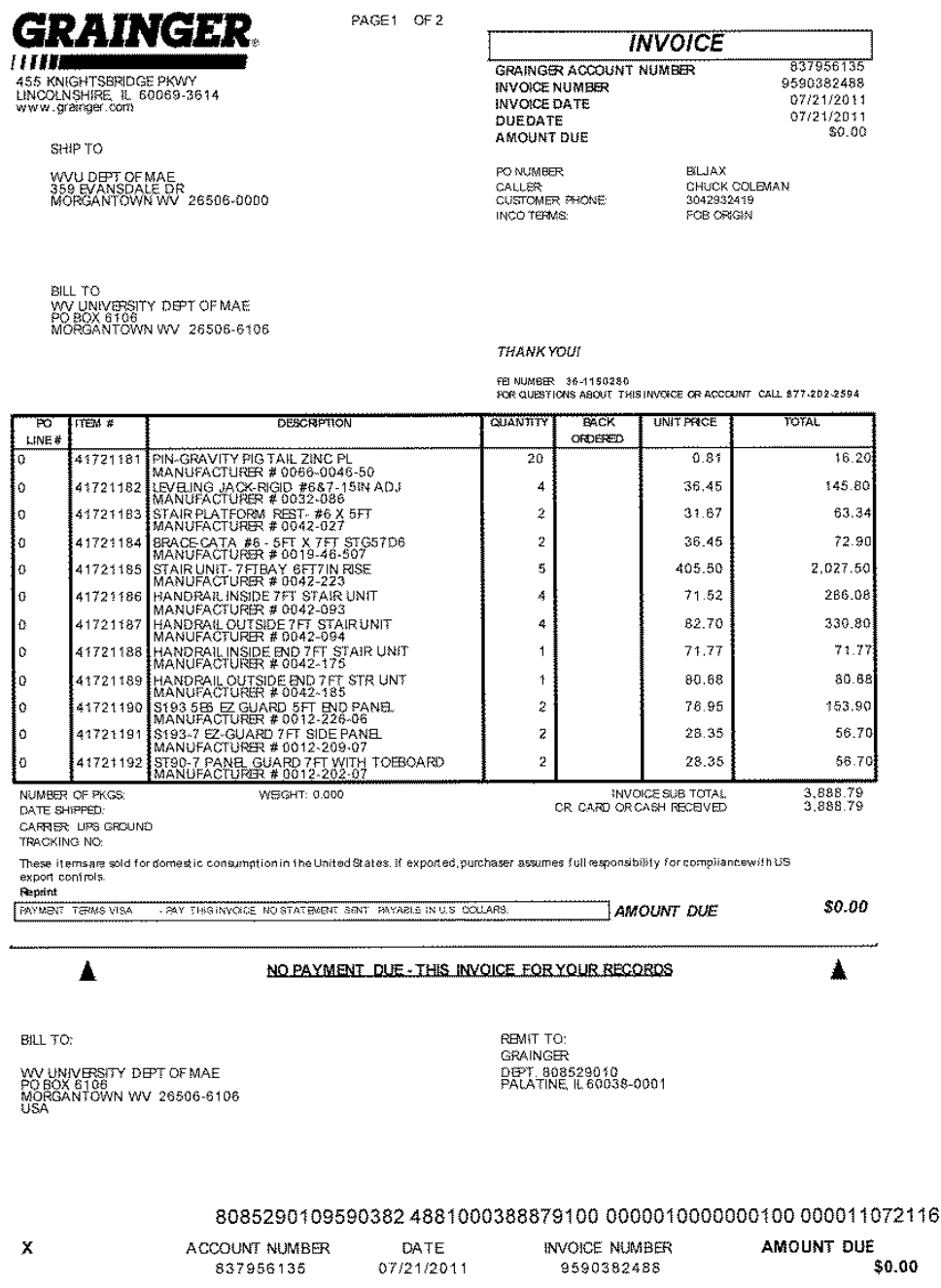


Development of the West Virginia University

Small Microgravity Research Facility (WVU SMiRF)
Copyright $^{\odot} 2014$

Kyle G. Phillips

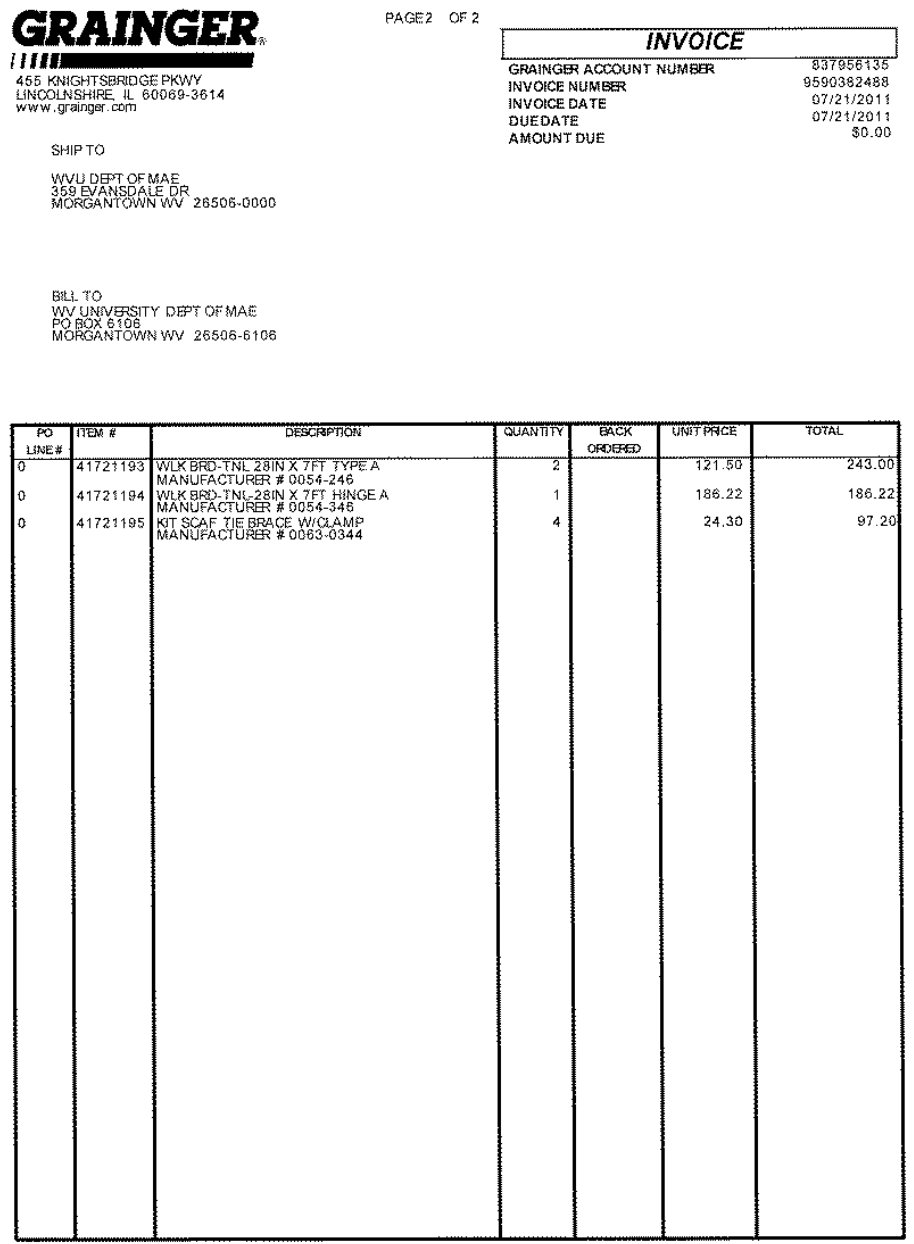




\section{Haulotte \. BIL $\overline{\mathbf{A X}} \quad$ Phone: $419-445-8915$ \\ Fax: $419-445-0367$}

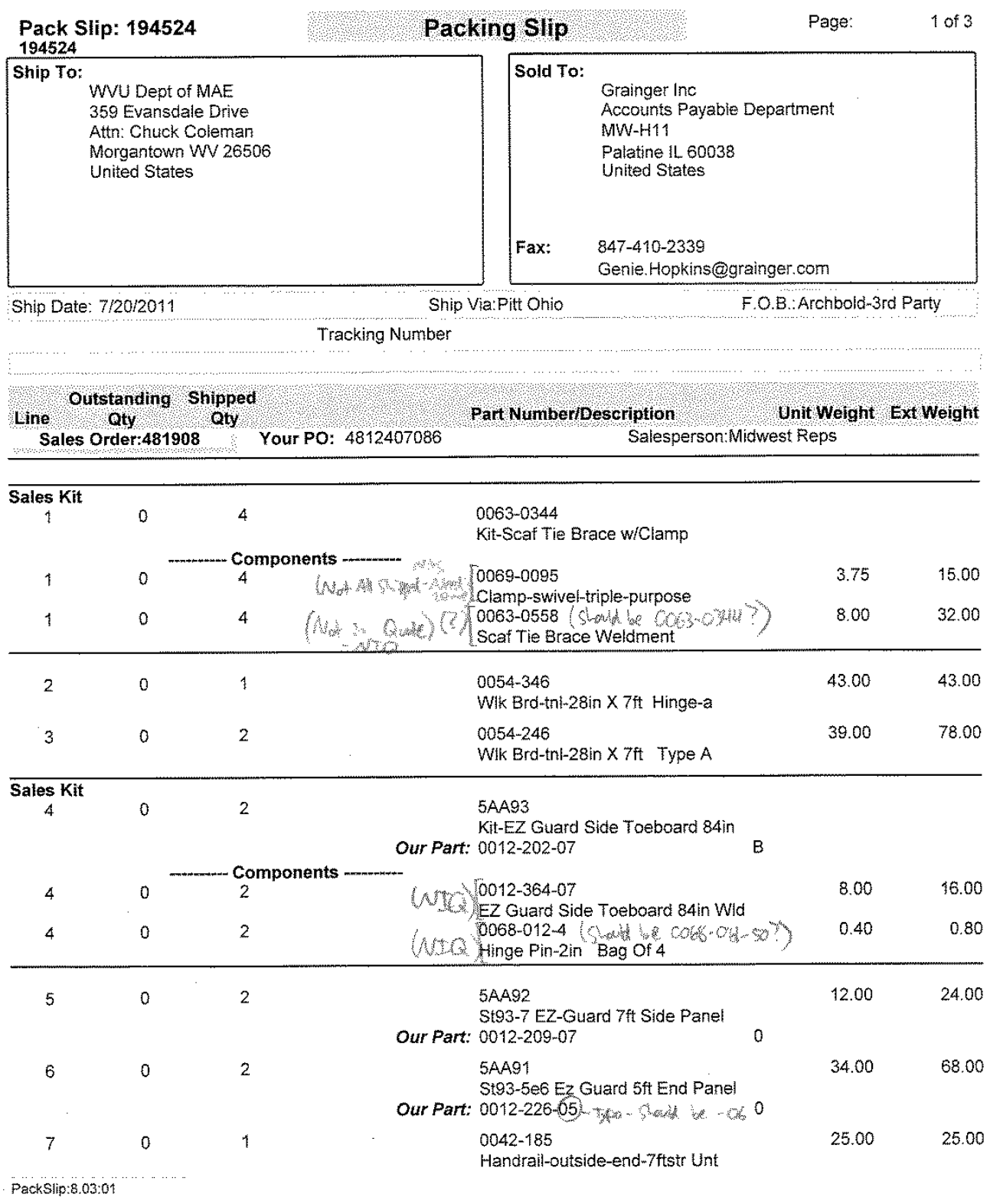




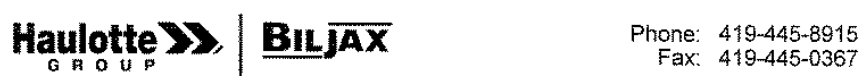

\begin{tabular}{|c|c|c|c|c|c|c|}
\hline \multicolumn{3}{|c|}{$\begin{array}{l}\text { Pack Slip: } 194524 \\
194524\end{array}$} & \multicolumn{2}{|c|}{ Packing Slip } & Page: & \multirow{3}{*}{ Ext Weight } \\
\hline Line & $\begin{array}{l}\text { Outstanding St } \\
\text { Qty }\end{array}$ & Shippec & & Part NumberiDescription & Unit Weight & \\
\hline & es Order:481908 & 8 & Your PO: 4812407086 & \multicolumn{2}{|c|}{ Salesperson:Midwest Reps } & \\
\hline 8 & 0 & 1 & & $\begin{array}{l}\text { 0042-175 } \\
\text { Handrail-Inside-End-7ft Stair Uniêt }\end{array}$ & 14.00 & 14.00 \\
\hline 9 & 0 & 4 & & $\begin{array}{l}0042-094 \\
\text { Handrail-outside-7ftstair Unit }\end{array}$ & 28.00 & 112.00 \\
\hline 10 & 0 & 4 & & $\begin{array}{l}0042-093 \\
\text { Handrail-inside -7ftstair Unit }\end{array}$ & 15.00 & 60.00 \\
\hline 11 & 0 & 5 & & $\begin{array}{l}\text { 0042-223 } \\
\text { Stair Unit-7ftbay-6ft7in Rise }\end{array}$ & 74.00 & 370.00 \\
\hline 12 & 0 & 2 & Our Part & $\begin{array}{l}\text { 5AA95 } \\
\text { Brace-Cata \#6- 5ftx 7ft Stg57d6 } \\
0019-46-507\end{array}$ & 15.75 & 31.50 \\
\hline 13 & 0 & 2 & & $\begin{array}{l}0042-027 \\
\text { Stair Platform Rest-\#6 X 5ft }\end{array}$ & 8.18 & 16.36 \\
\hline 14 & 0 & 4 & Our Part & $\begin{array}{l}\text { 5AA97 } \\
\text { Leveling Jack-Rigid } \# 6 \& 7-15 \text { in Adj } \\
0032-086\end{array}$ & 10.20 & 40.80 \\
\hline 15 & 0 & 20 & Our Part: & $\begin{array}{l}\text { 5AA98 } \\
\text { Pin-Gravity Pig Tail-Zine PI } \\
0066-0046\end{array}$ & 0.18 & 3.64 \\
\hline
\end{tabular}

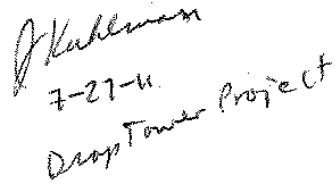

Packslip:8,03:01 


\section{Haulotte \) BILJAX}

Phone: $419-445-8915$

Fax: $419-445-0367$

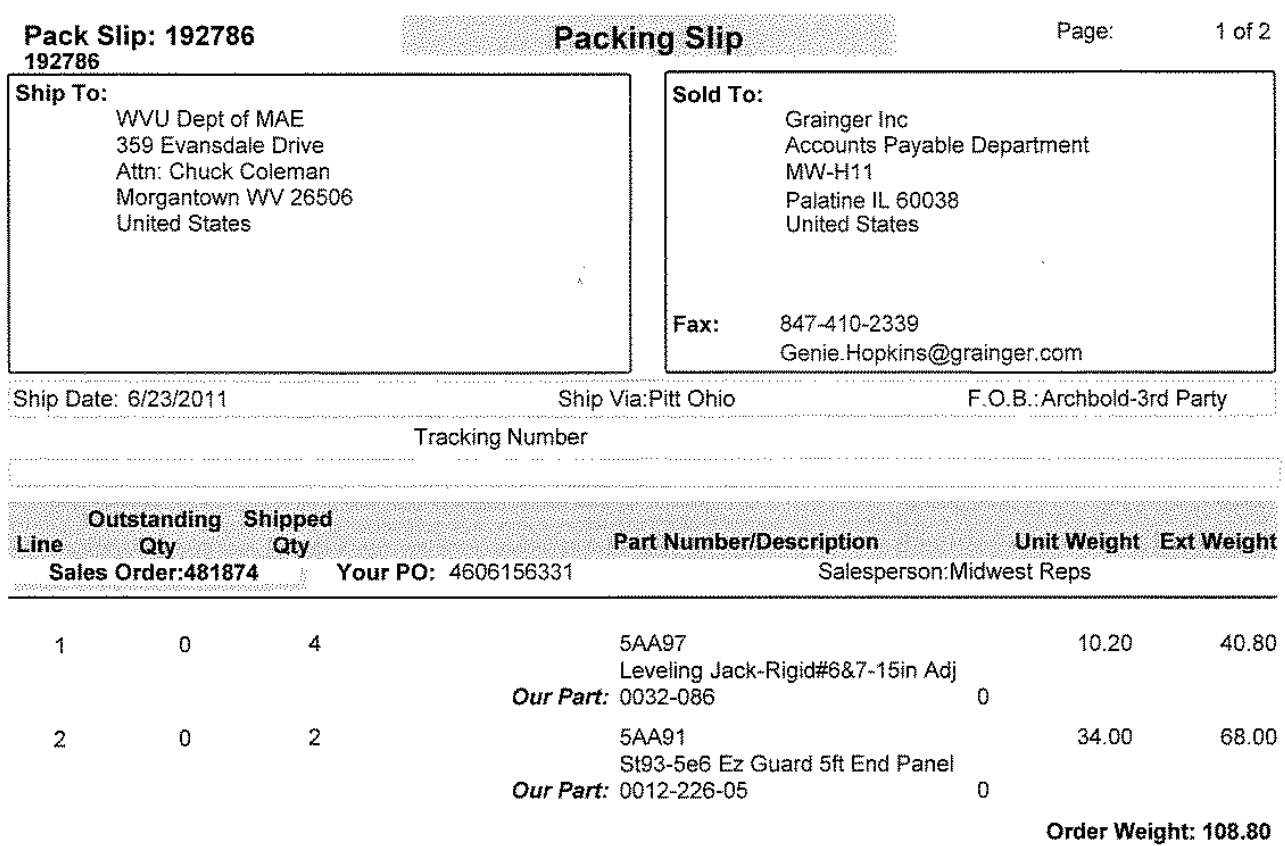

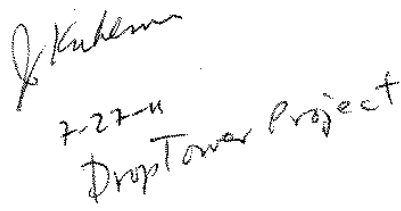

PackSlip:8.03:01 


\section{$\underset{G}{\text { Haulotte } \$ \text { B. BILJAX }}$}

Packing Slip

Pack Slip: 192786

Part Numberioescription

Line

Utstanding Shipped

CONDITIONS:

The seller shall not be liable for any delay in manufacture of delivery due to fires, strikes, differences with workmen, accidents, delays in transportation, shortages of cars, shortage of fuet or other material, shortage of labor, inabilily to procure supplies, and all other causes beyond reasonable control of

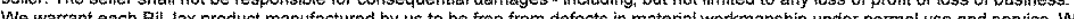

We warrant each Bif ax product manufactured by us to be free from defects himatenal workmanship under normal use and service. We will repair or

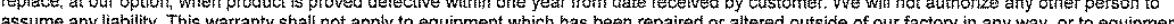
which has been subject to misure return. Units must be returned pepaid

HABIUITY OF PURCHASER

tis expressiy understood and agreed that the selier shall not be liable for damages or losses of any kind whatsoever, whether to persons, property or for any other loss arising from the use of, transportation of, or in any way connected with the said equipment or any part thereof, from whatsoever cause arising, and the purchaser agrees to indemnify and to hotd seller harmless from all such liabilities. Purchaser agrees that seller shall not be liable for any aste loss of profit, or loss of time in the use of the equipment from whatsoever reasons.

Purchaser agrees to distribute and ensure compliance with safety literature supplied with this order and to all persons buying, leasing or using this

The use of purchaser's purchase order number in this purchase is for customer's convenience and identification only. This invoice and the conditions set therein constitutes the sole agreement between Bi-Jax and the purchaser and supersedes any purchase order provisions whether sent to, received prior to, or subsequent to this invoice. Absence of purchase order number shall not constitute grounds for nonpayment of invoice charges. in case of loss or damage in transit of fallure to receive shipment, the consignee must immediately notify the carrier's agent at destination in witing in order to substantiate formal ctaim when presented to the carrier.

Claims for shortage or deductions for erroneous charges must be promptly presented or they will not be allowed

If our goods appear damaged, discontinue their use and notify us immediately so the matter may be investigated without delay. Requests for order cancellation which calis for specially designed equipment will not be considered if the manufacture of the material has been

This is to certify that the merchandise represented by this invoice was produced in accordance with the Fair Labor Standards Act of 1938, as amended. ORDERACCEPTANCE CONDITIONS:

Term and conditions on this page constitute our entire obligation on acceptance of any order, notwithstanding date of order or stipuiations thereon. Orders are subject to acceptance at the Corporate Office, Archbold, OH. Prices, discounts, terms and specifications are subject to change without notice and shall be those fective at the of shipment. Delivery is subject to appllable laws and regut the and to all are

REQUIREMENTS FOR RETURN MERCHANDISE POLICY

Al merchandise must be returned within 60 days of the invoice date. All returned merchandise must be approved by the Archbold, $\mathrm{OH}$ office the RMA and be shipped prepaid not shipped prepaid, in addition to a handling charge. All merchandise must be returned as originally packaged and in new, unutsed, and clean condition. No returns will be accepted on merchandise that has been used. Any credit allowed will be subject to handling inspection, und reconditioning charges. No freight charges will be credited due to the ordering of incorrect merchandise by the purchaser. Any cost incurred due to unauthorized returns will be debited to the purchaser.

RESTOCKING CHARGES

We will accept return of any merchandise up to 60 days from the date of invoice subject to the following

All Standard Merchandise-Minimum $15 \%$ yestocking fee

Special Order Merchandise- $50 \%$ restocking fee up to 30 days. No retums after 30 days.

BACK-ORDERED ITEMS:

Shipped when avaitable unless cancellation received prior to shipment. Regular freight terms apply to back-order shipments.

PREPAID FREIGHT:

All prepaid shipments, UPS or otherwise, will have the appropriate surcharge included on the invoice

DAMAGES AND /OR SHORTAGES:

Merchandise is inspected and counted before loading. Any shortages or overages in packaging must be reported to Bit-Jax within 5 days after receipt. Damages or shortages must be noted by consignee on carrier's delivery receipt before signing, and claim filed promptly with delivering carrier. Claims are

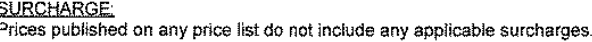

PackSip:8.03:01 


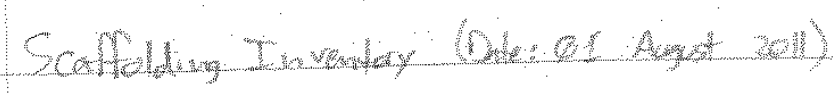

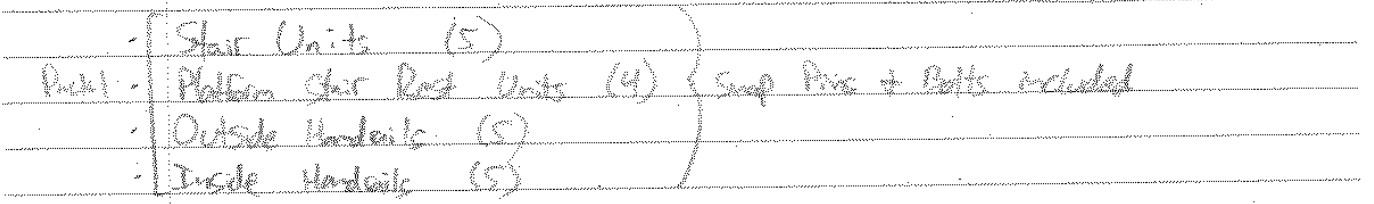

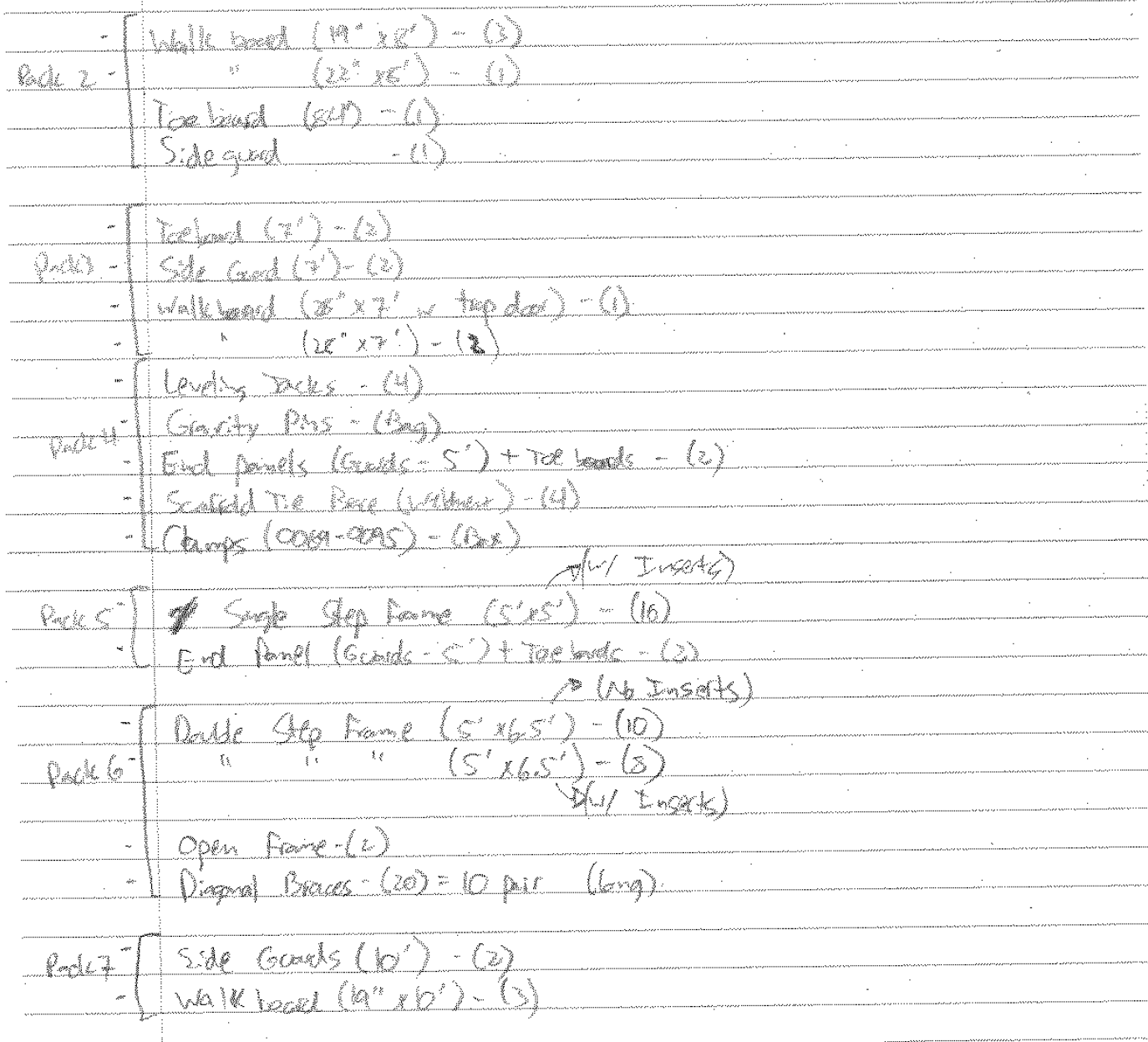


Development of the West Virginia University

Small Microgravity Research Facility (WVU SMiRF)
Copyright $^{\circledR} 2014$

Kyle G. Phillips

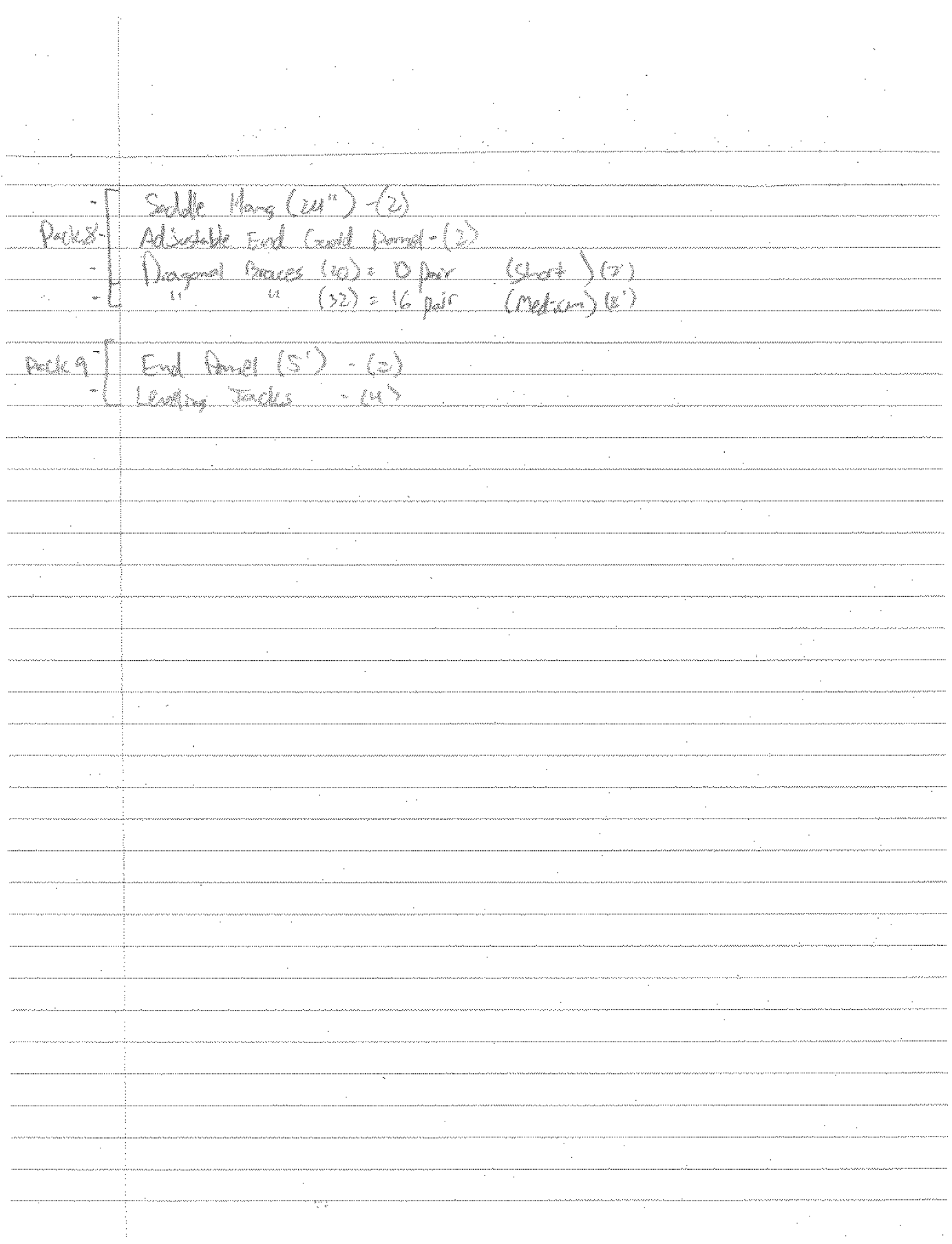




\subsection{Appendix F}

\section{Release System Information}

Appendix D: Release System Information presents cost and technical information provided by the manufacturer of the release system electromagnet, MagneTool, in addition to safety and operations instructions. Information provided herein is provided for comprehension of the project documentation and reflects past costing and copyrighted material belonging to MagneTool to which all rights apply for their copyright material. 
MAGMETOOLINC.

505 EL.MWOOD

(248) 589-5400 FAX (248) 588-5710

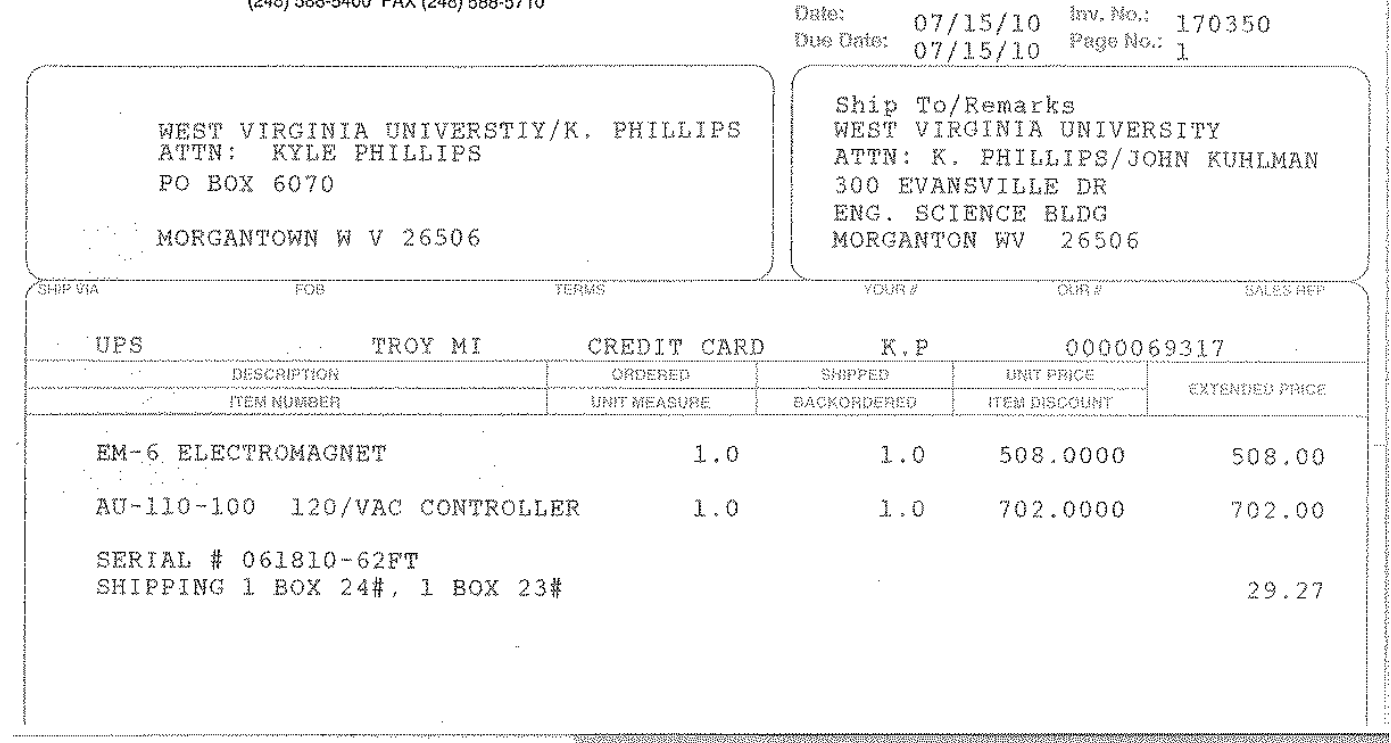

MUOLO

A. IA UNTVERSTIY

ILLitis DH

HNG SCIENCW BTM

MORCANTSON WV 26506
ATTW: $K$, RHIESTPS/JOUN KUHLMAN

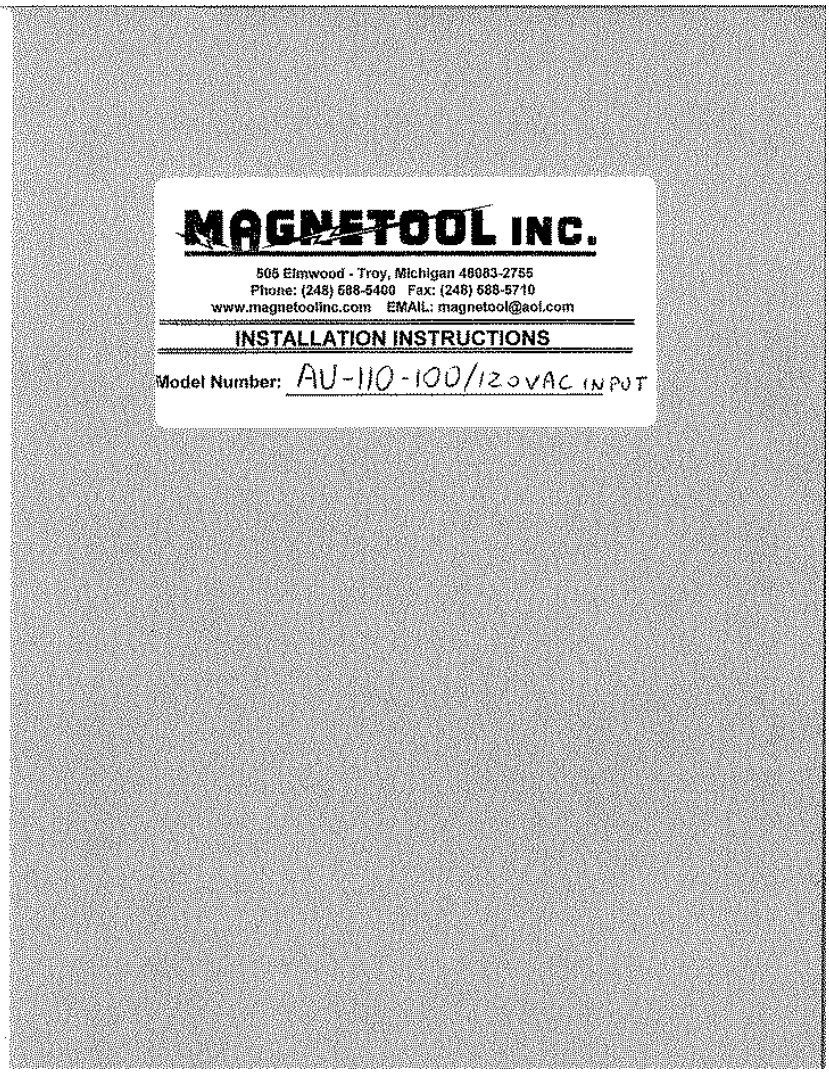




\section{AU SERIES WIRING AND ADJUSTMENTS}

TRANSFORMER PRIMARY TAPS: Unless otherwise requested by customer, all standard AU series controllers are preset at the factory for the-law yoltage input connection. All source inputvoltrges are single phase $50 / 60$ Hertz. Standard 12 and 24 VDC output models are-onnected for 120 VAC input. Standard 110 and 220 VDC output models are connected for $240-\mathrm{VAC}$ inpit $\mathrm{T}$ thin $\mathrm{f}$ kmor taps must be changed for alternate input voltage connections (See figure H1).

CONTROL TERMINALS; Terminals (T1) through (T4) are supplied with a pre-connected jumper for limit switch or normally open relay contact operation (See figure \#2). For 120VAC PLC operation, remove jumper between (T3) and (T4) and supply a 120VAC signal to (T2) and (T4) (see figure \#3). Optional PLC input voltages are available, consult factory for details.

MAGNET CONNECTION: Magnet(s) must be connected to (M1), (M2), and ground (GND). Additional magnets are wired in parallel (see figure \#4). Total wattage of magnet or magnets in parallel is not to exceed maximum rated wattage of magnet controller. Suitable wire size to minimize voltage drop and rated for full amperage draw should be used.

RELEASE ADJUSTMENT: Release adjustment (1P) controls release pulse voltage. The control is normally located on the enclosure door. However, for AU-series with 12 and 24 VDC output or sub-panel (SP) models, (1P) will be located on the sub-panel. See parts layout drawing for location.

Control (1P) is preset at the factory to provide the best release possible for a wide variety of conditions. If release is not acceptable, adjustment of control (1P) will be necessary. To properly adjust (1P) use the following procedure.

1. Install magnet and place part in "work" position.

2. Set magnet approximately $1 / 8^{* \prime}$ above part.

3. Turn $\mathrm{AC}$ power on.

4. Energize and de-energize magnet manually (using (T1) through (T4) inputs), while slowly turning release adjustment (1P) from one stop to the other. This is a very fine adjustment. Be sure to turn (1P) very slowly while at the same time cycling the magnet on and off. You will find the point at which the magnet will pick up and drop the part without hesitation. Some additional fine tuning may be required when using during actual application. Also keep in mind that some parts may become magnetized after several cycles. For this reason it may be necessary to use several parts during setup to obtain actual release setting.

Note: Some AU-series controls utilize a locking release potentiometer with screwdriver adjustment. Be sure to loosen the locknut before adjusting and re-tighten the locknut when the adjustment is complete.

5. If release cannot be obtained, contact our engineering staff. Please provide controller model number, serial number, size of magnet, workpiece size, and type of material. 


\section{AU SERIES WIRING DIAGRAMS}

(STANDARD UNIT'S)

EICURE \#1

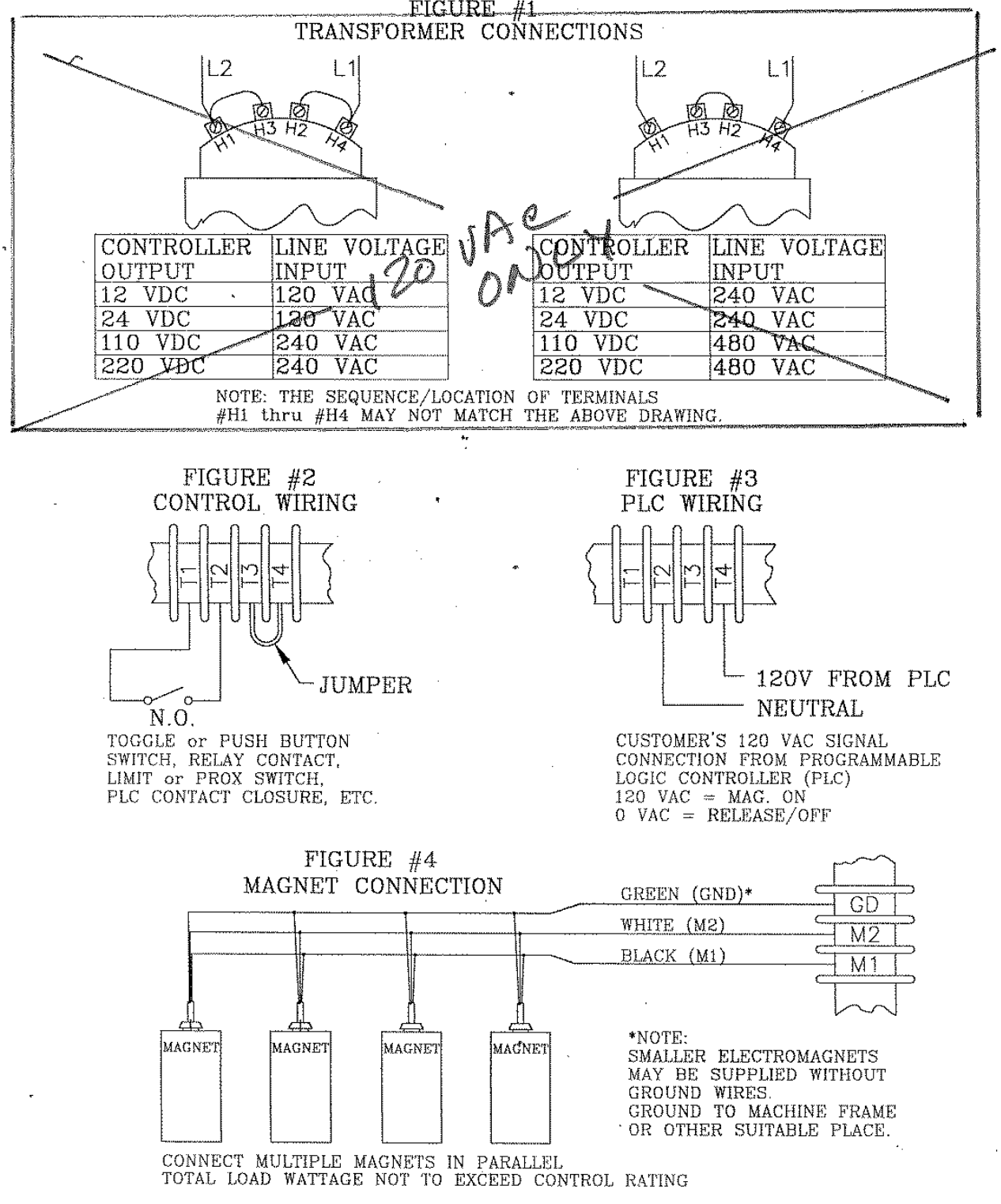

O4AUG99 FLE:AU DISK 4/AUHOOKUP, dwg 


\section{MAGNETOOL INC.}

\section{WARRANTY:}

The seller will warrant for one (1) year from date of invoice that all products will be free from manufacturing defects that hinder the products proper operation. If defects appear within the warranty period, the customer is to return the unit to the manufacturer via prepaid freight for inspection. If the inspection proves the claim, the manufacturer will repair or replace the product at its discretion to bring the product to proper working order. The seller is not required to make repairs to units abused or modified. The seller's only liability is that of product repair or replacement: The seiler is not fiable for any damages caused by fire, supply shortage, installation difficulties, or conditions beyond the seller's control. 


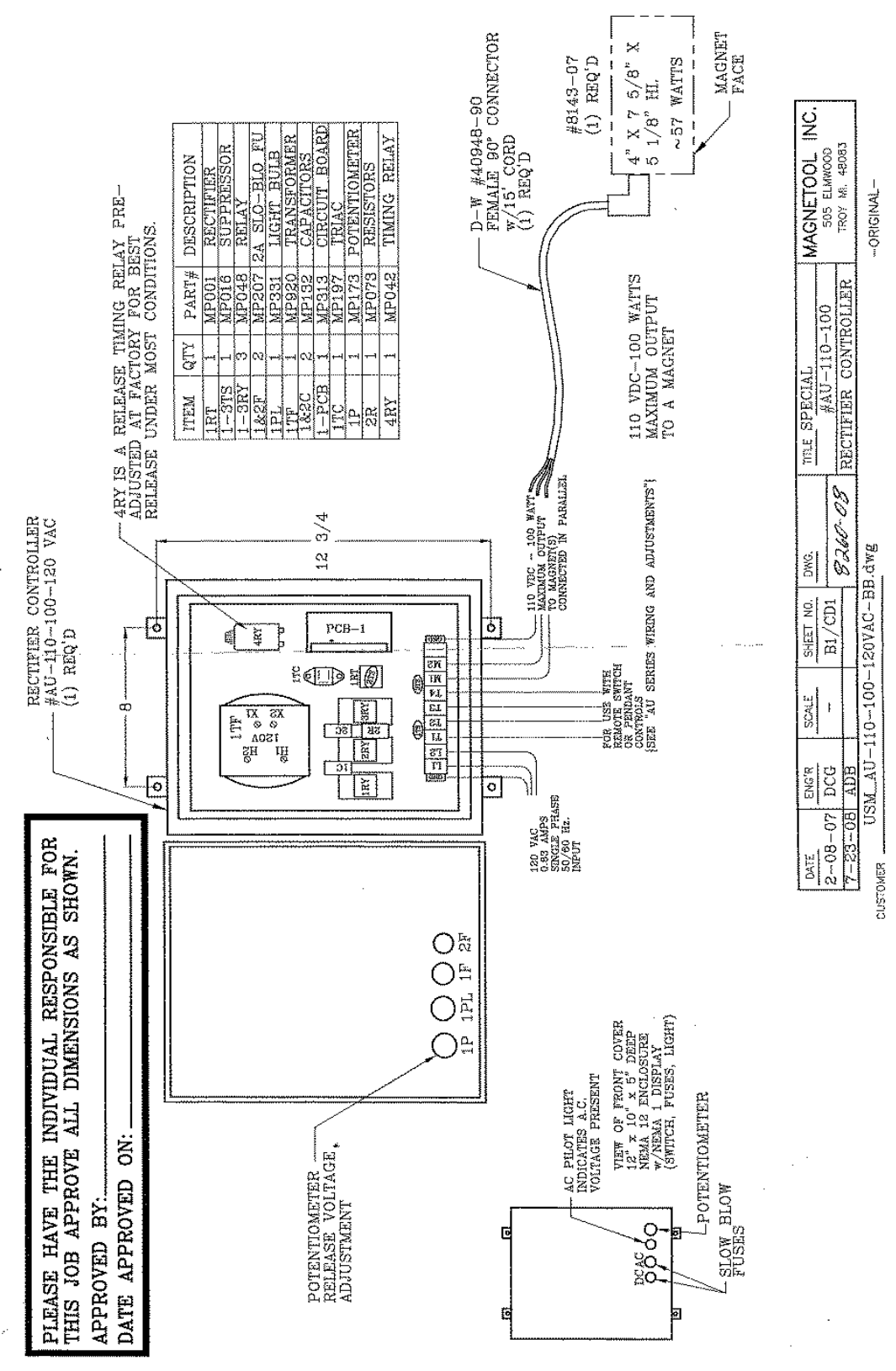




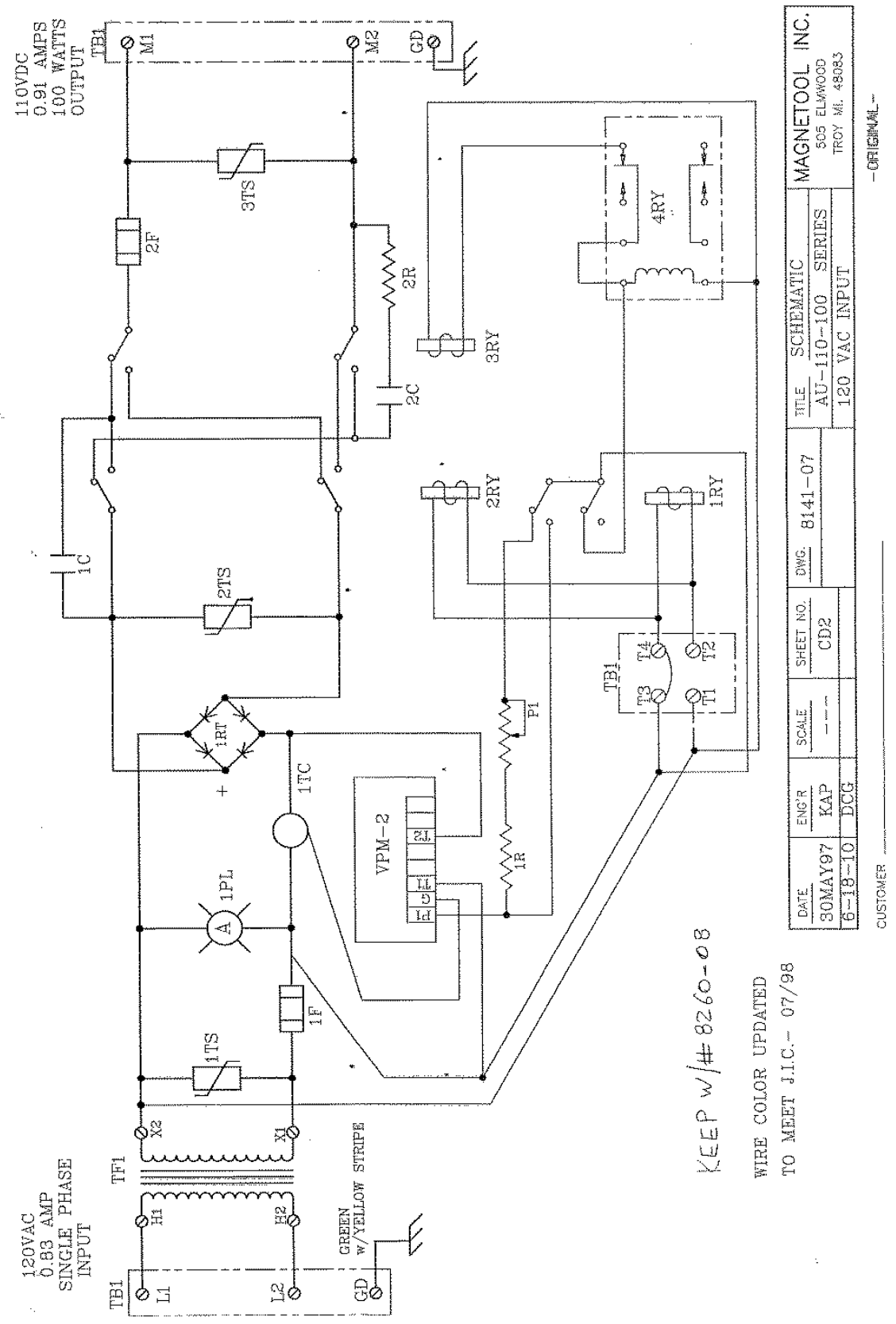




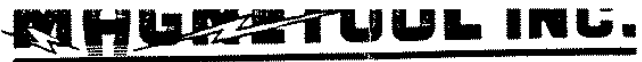

\section{whit oe
248}

Phone: (9.40) 588.5400 Telecopier: (240) 588-5710

505 Elmwood

Troy, Michigan 48083-2755*

P.D. Box 2556

Sun City, CA 9238 4610 Warehouse Lane

Latedo, Texas 78040
(210) $725-0333$

Ave. Insurgentes 4230

Monterrey, N.L. Mexico
48-06-07

\section{LIFTING MAGNET OPERATING PRACTICES}

1) Operator shall carry out an Every Lift Inspection, as follows:

1) Inspect magnet face for foreign material \& smoothness.

2) Inspect condition of lifting bail or eye bolt.

2) Operator shall make sure load is clean in area of magnet contact.

3) Operator shall check load weight and dimension to insure that load falls within lifting magnet's rated load capacity or application load capacity and the crane rated capacity.

4) Operator shall position lifting magnet for a balenced lift.

5) Operator shall confirm that hoist ropes or chain are not kinked or twisted.

6) Operator shall not ride or allow others to ride suspended loads.

7) Operator or other personnel shall maintain safe clearance around suspended loads and shall not allow any part of their bodies or other personnel's bodies beneath a suspended load.

8) Operators should push, not pull suspended loads.

9) operator shall not allow load to contact any obstruction.

10) Operator shall affix magnet suspension device (s) securely to crane hook.

11) Operator should perform a preliminary lift with load suspended only several inches to establish a safe lift prior to moving load higher.

12) Operator shall accelerate and decelerate load smoothly.

\section{Inifting Abilities}

The factors listed below are those that limit the flow of the magnetic lines of force from the magnet into the load which in turn reduces the magnet's lifting ability:

\section{Load Surface Conditions}

Magnetic lines of force do not flow easily through air. they need iron in order to flow freely. Therefore anything that creates an need lro load limits the flow of magnetic air gap between a magnet and the load limity of a nagnet. paper. dirt. force and thus reduces the lifting capacity of a magnet. a rough surface rust, paint and scale act the same as air. Also, a roug between the finish is the sacke

Load Length and Width

When the length or width of a load increases it ceases to lie flat and the load begins to droop at the edges. This arooping or sagging of the load can create an air gap between the load and the magnet. If this ocours then the liftina capacitv of the mannet is roillnat 
Magnetic lines of force are more effective when they flow through iron instead of air. The thicker the load is the more lines of magnetic force are able to flow. After a certain thickness of load no more lines of force will flow because the magnet has reached its full capacity.

Thin material (load) means less iron available and thus fewer lines of magnetic force flow from the magnet into the load. Therefore the lifting ability of the magnet is reduced.

Most magnets or magnet systems are rated to tell the user what minimum thickness of load is required to reach full lifting capacity. Below such thickness of load the user must derate the lifting capacity of the magnet.

\section{Load Alloy}

Low carion steels, such as 1020 steel, are nearly as good. conductors of magnetic lines of force as pure iron. However, many other alloys contain non-magnetic materials which reduce the ability of magnetic lines of force to flow into the load. An alloy such as 300 series of stainless steel is a poor conductor of magnetic force and no lift should be attempted.

Type 416 stainless steel is considered magnetic, but it centains enough chromium so that it can only conduct one half as wuch magnetic force as 1020 steel. The carbon content of cast iron reduces the flow to one half of the magnetic force of 1020 steel.

\section{Attitude of Load}

As the attitude of the surface of the load to which the magnet is a tached (Iifting surface) changes from horizontal to vertical, the lifting ability of the magnet decreases. When the lifting surface of the load is vertical, the ability of the magnet to support the load is dependent upon the coefficient of fxiction of the lifting surface as well as the weight of the load.

\section{Portion of Maqnet Face in Contact with Load}

The full face of the magnet must contact the load if the magnet is to achieve maximum capabilities. Holes in that portion of the surface of the load upon which the magnet is placed will reduce the magnet's lifting capability. If the load is narrower or shorter than the magnet face, the magnet's lifting ability will also be reduced.

\section{Load Temperature}

The temperature of the load can cause damage to the magnet and if high enough, even change the magnetic characteristics of the load. 


\section{DO'S AND DON'TS OF USING LIFT MAGNETS}

DO: Check and clean magnet face for dirt, welts, flatness. Make sure magnet face is making $100 \%$ contact with work. Check that material to be lifted is flat and clean.

Check lifting bails for wear or defects.

Check that the load does not exceed the rated capacity of the magnet.

Make a trial lift of $I^{\prime \prime}$ to $2 "$ when handing any load.

Move load smoothly.

Prevent excessive swinging of load.

DON'T: Use any magnet that has excessively rounded poles, welts or uneven poles.

Exceed rated capacity for any reason.

Try to lift thin, large sheets unless magnet (or magnet system) is designed for that purpose.

Lift unbalanced loads.

Litt loacs over personnel.

Have feet, hands or any other part of the body under the load at any time.

Jerk load.

Lift round bars with a flat magnet.

Try to lift the following:

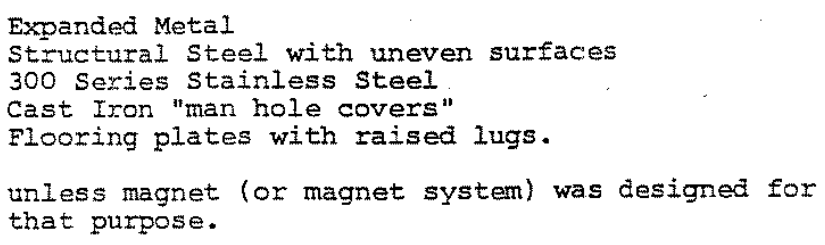




\section{WARNING}

Lifting capacity is determined by lifting flat, smooth, non flexible, low carbon steel of sufficient thickness that no magnetism can be felt on the opposite side of the steel.

The entire face of the magnet must be in "direct contact with the work and the load be properly balanced. Any deviation from the above will reduce the pickup capacity and the lift must be txied with extreme caution. The magnet face must be kept clean and free from welts, " gouges and be checked for uneven wear. If any of these conditions axist, the magnet face must be re-ground. Move load sinoothly and keep as low as possible. Do not move load over personnel at any time. Do not have any part of the body under the load at any time. Do not use the magnet for lifting large flexible sheets. Do not use the magnet if there are any broken, loose or worn components. Do not use the magret if it has not been inspected befare each lixt. Do not use magnet without reading instruction manual.

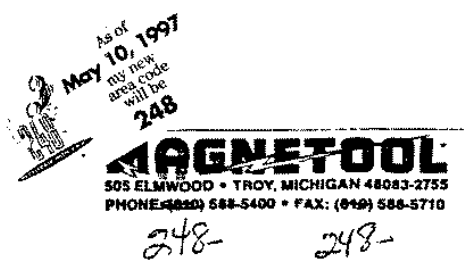




\title{
17.0 Appendix G
}

\section{Deceleration System Analysis \& Information}

\begin{abstract}
Appendix E: Deceleration System Analysis \& Information provides the reader with the material test results and the computer code used to perform the deceleration system design analysis, as well as the costing and material sourcing for the deceleration system memory foam. The information provided herein is provided for comprehension of the project documentation and reflects past costing and copyrighted material belonging to Dynamic Systems, Inc. to which all rights apply for their copyright material.
\end{abstract}




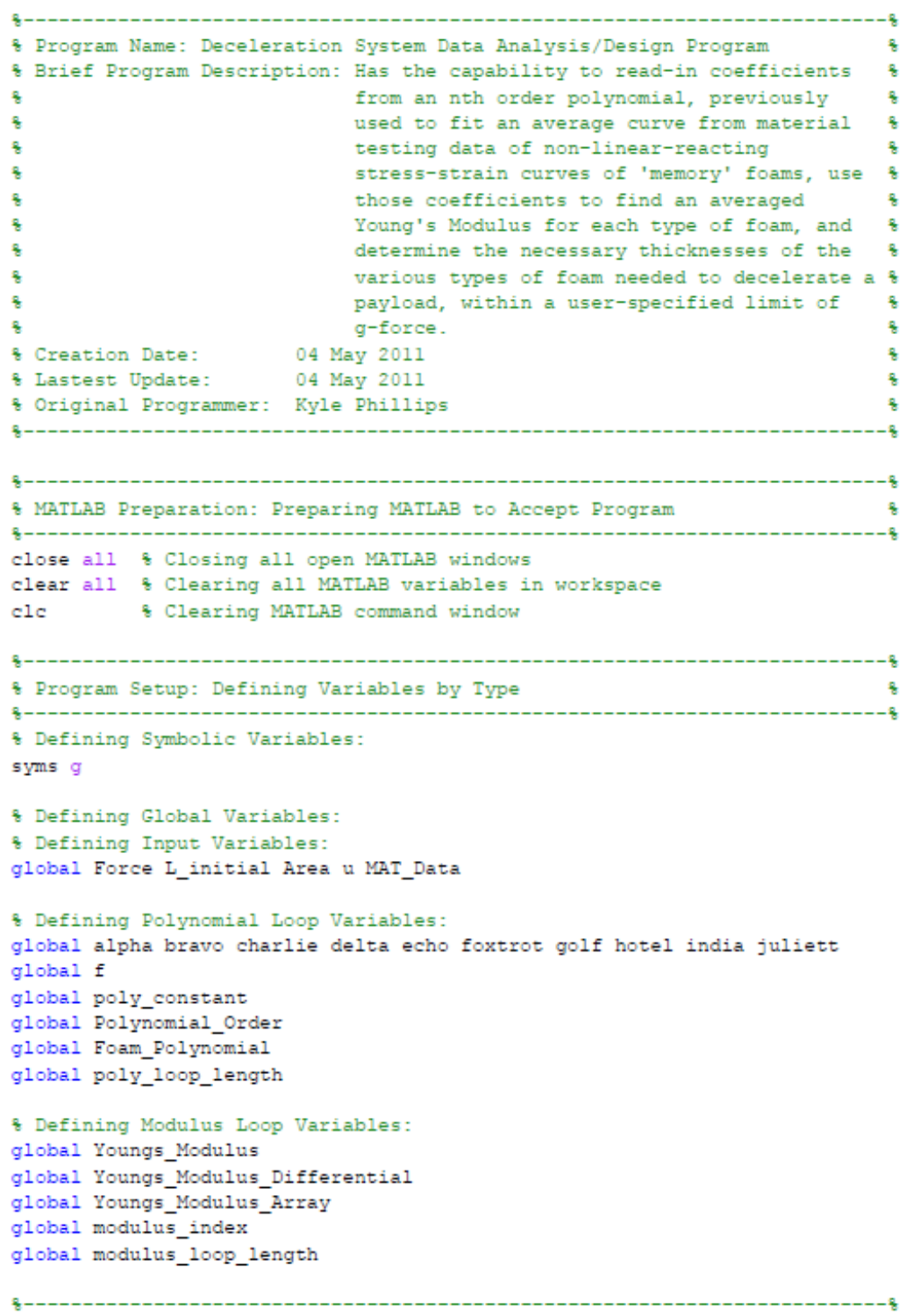




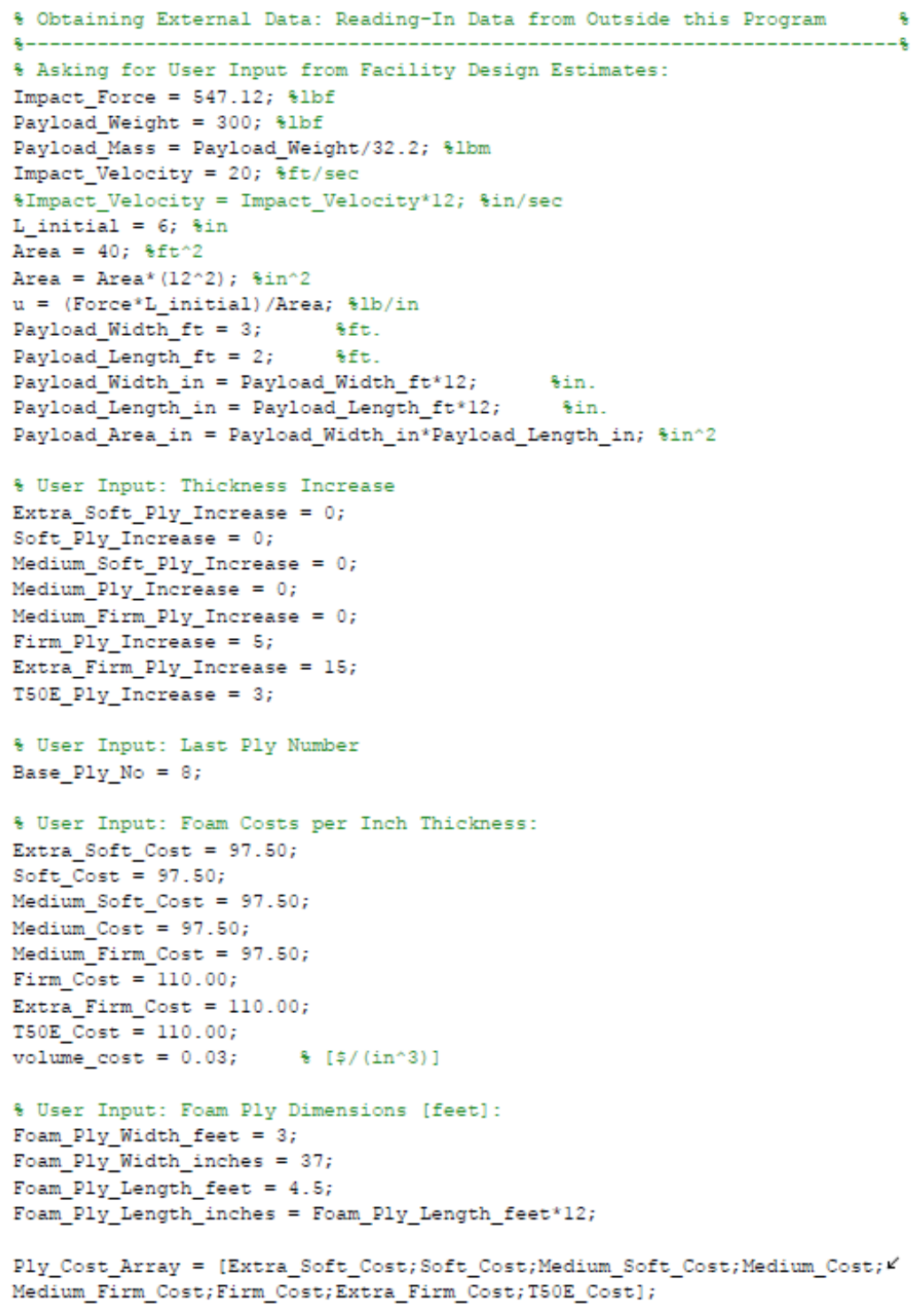


\& disp('What are the projected values for the following variables?');

\& Impact Force = input ('Initial Payload Impact Force at Deceleration System [1bf]:");

\& Payload_Weight = input ("Payload Weight [1bf]:");

\& Impact_Velocity = input ('Initial Payload Impact Velocity at Deceleration

\& System $[\mathrm{ft} / \mathrm{s}]:$ ') :

\& Loading the averaged material testing coefficient matrix:

MAT_Eile_Read_In = load('Mike_Files/Material_Polyfits.mat', 'material_poly');

\& Re-formatting the data from structure to cell to array:

MAT_Cell = struct 2 cell (MAT_File_Read_In);

MAT_Array $=\operatorname{cel} 12 \operatorname{mat}($ MaI_Cel1);

\& Determining Indexing:

[MAT_row, MaT_column] = size (MAT_Array);

MAI_orig_index $=$ MAI_row;

\& Changing Order of Foam Types from (Stiffest to Softest) to (Softest to

\& Stiffest)

for row_index = 1:MaT_row

for col_index = 1:MAT_column

end

MAT_Data (row_index, col_index) = MAT_Array (MAT_orig_index, col_index);

end

MaI_orig_index $=$ MaI_orig_index -1 ;

\& Assigning Coefficient Data to Foam Type Variables:

Extra_Soft_Coefficients = MAT_Data $(1,:)$;

Soft_Coefficients = MAT_Data $(2,:)$;

Medium_Soft_Coefficients $=$ MAI_Data $(3,:)$;

Medium_Coefficients = MAT_Data $(4,:)$;

Medium_Firm_Coefficients = MAT_Data $(5,:)$;

Firm_Coefficients = MAI_Data $(6,:)$;

Extra_Firm_Coefficients = MaI_Data $(7,:)$;

T50E_Coefficients $=$ MAT_Data $(8,:)$;

\& Storing Coefficients for Future Use

Polynomial_Cell_Array = [Extra_Soft_Coefficients; Soft_Coefficients; $K$

Medium_Soft_Coefficients; Medium_Coefficients; Medium_Firm_Coefficients; $\swarrow$

Firm_Coefficients; Extra_Firm_Coefficients; T50E_Coefficients\};

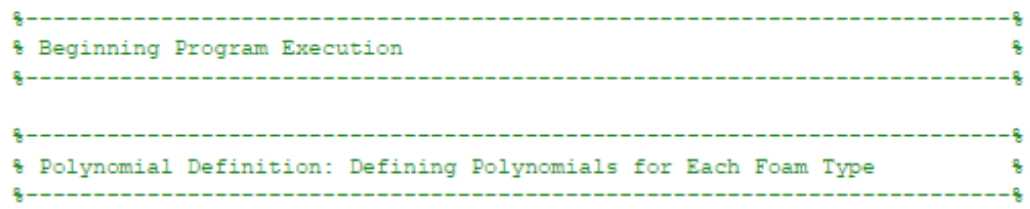




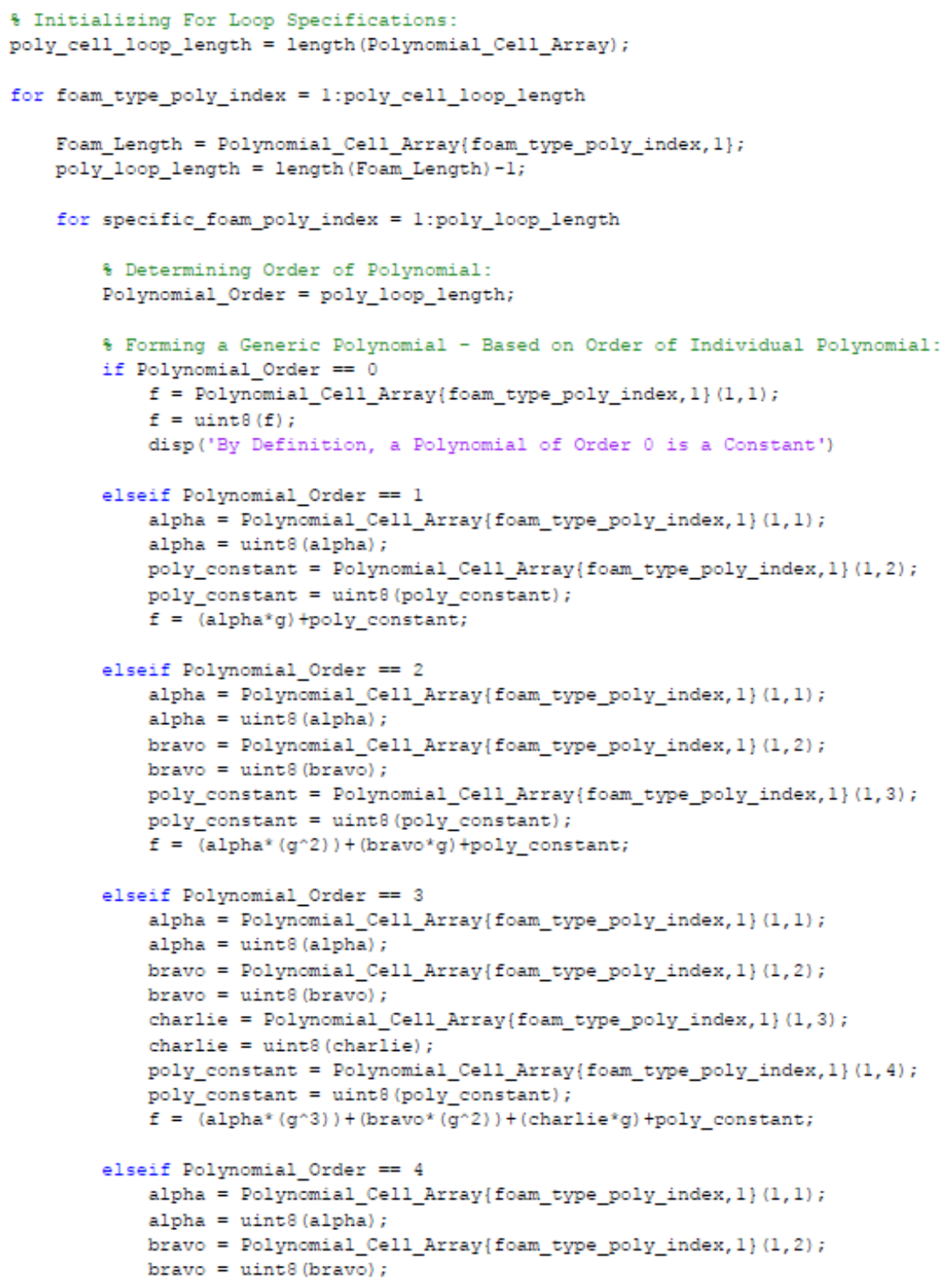




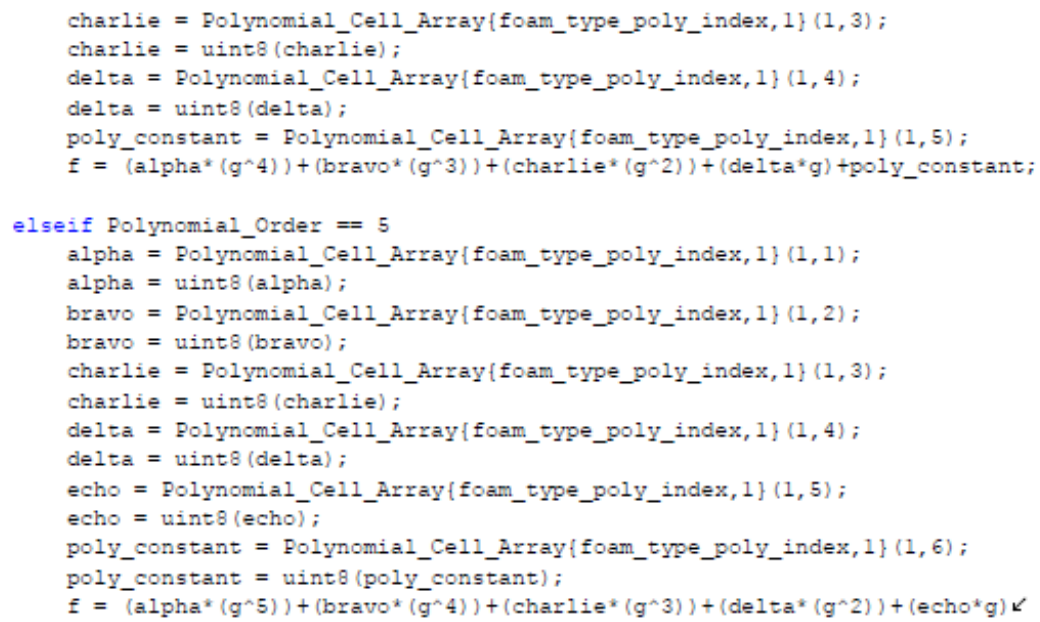




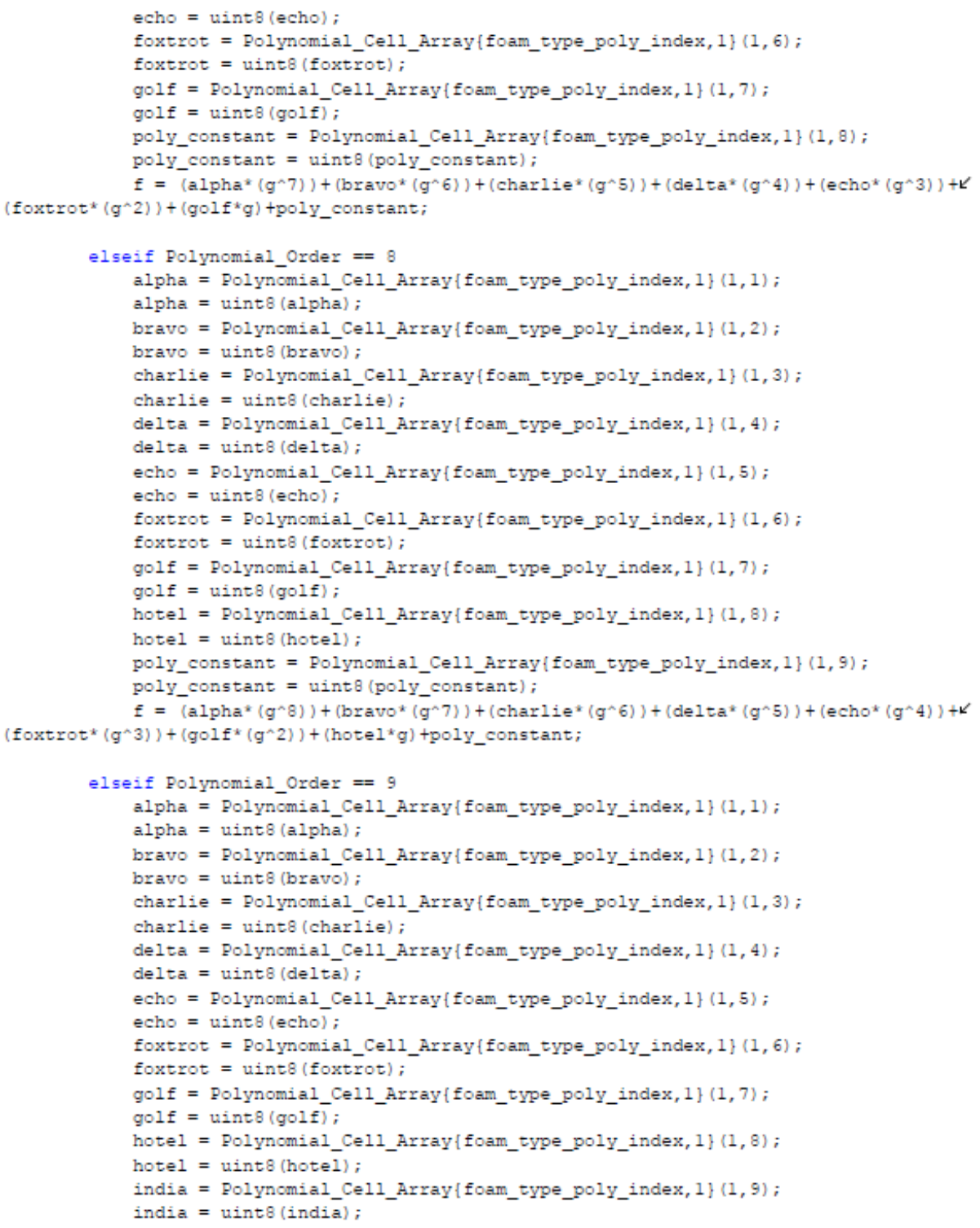




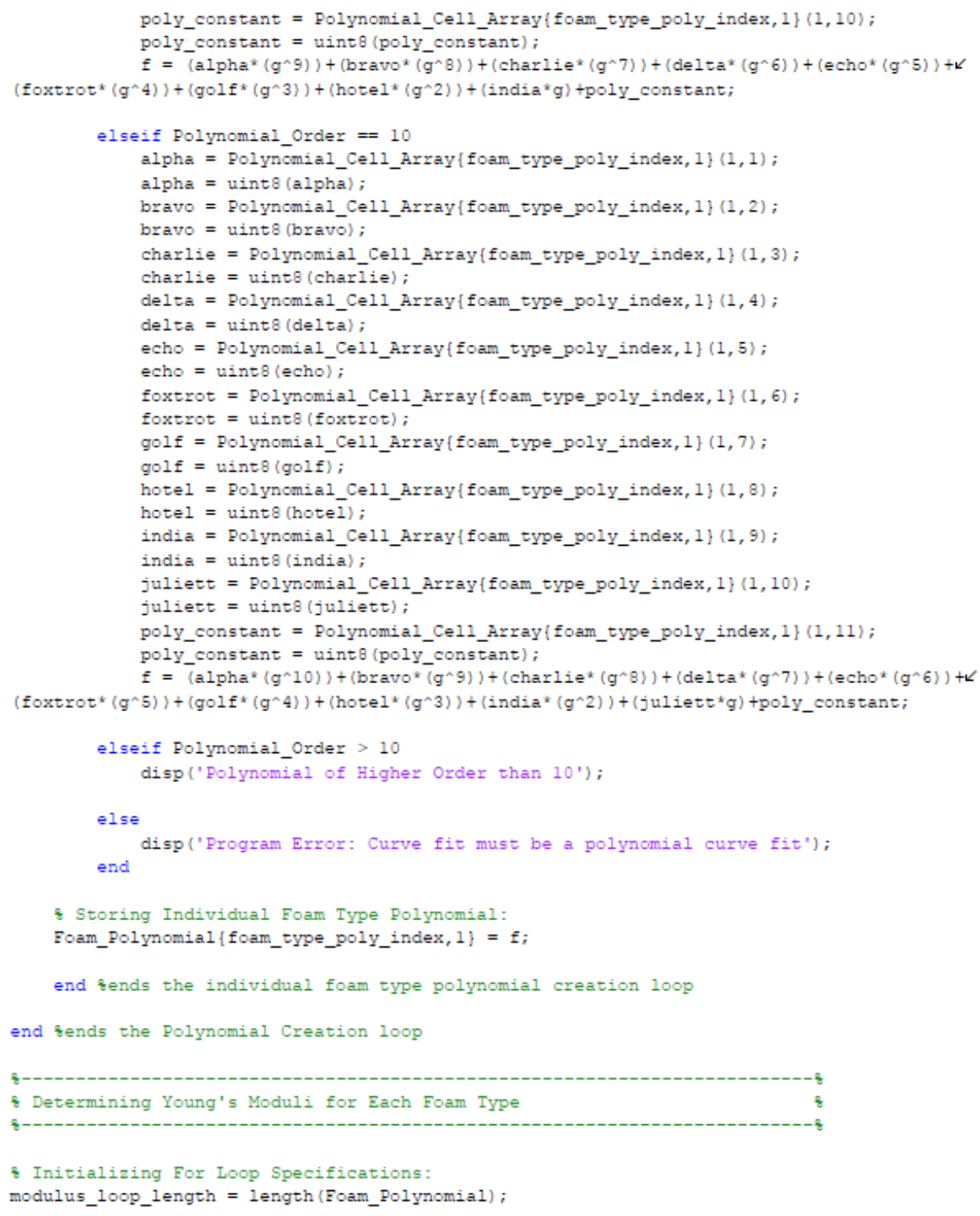




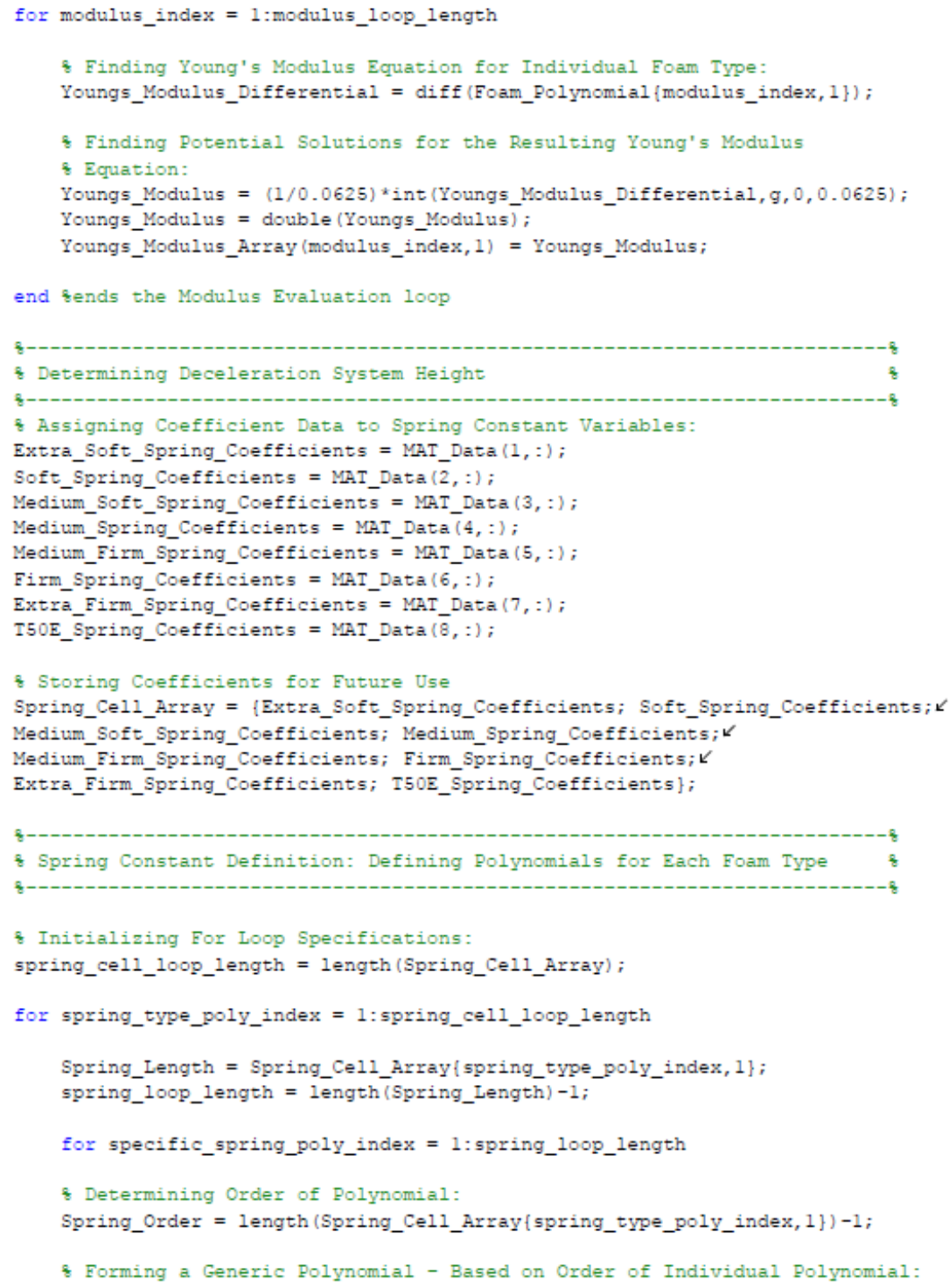




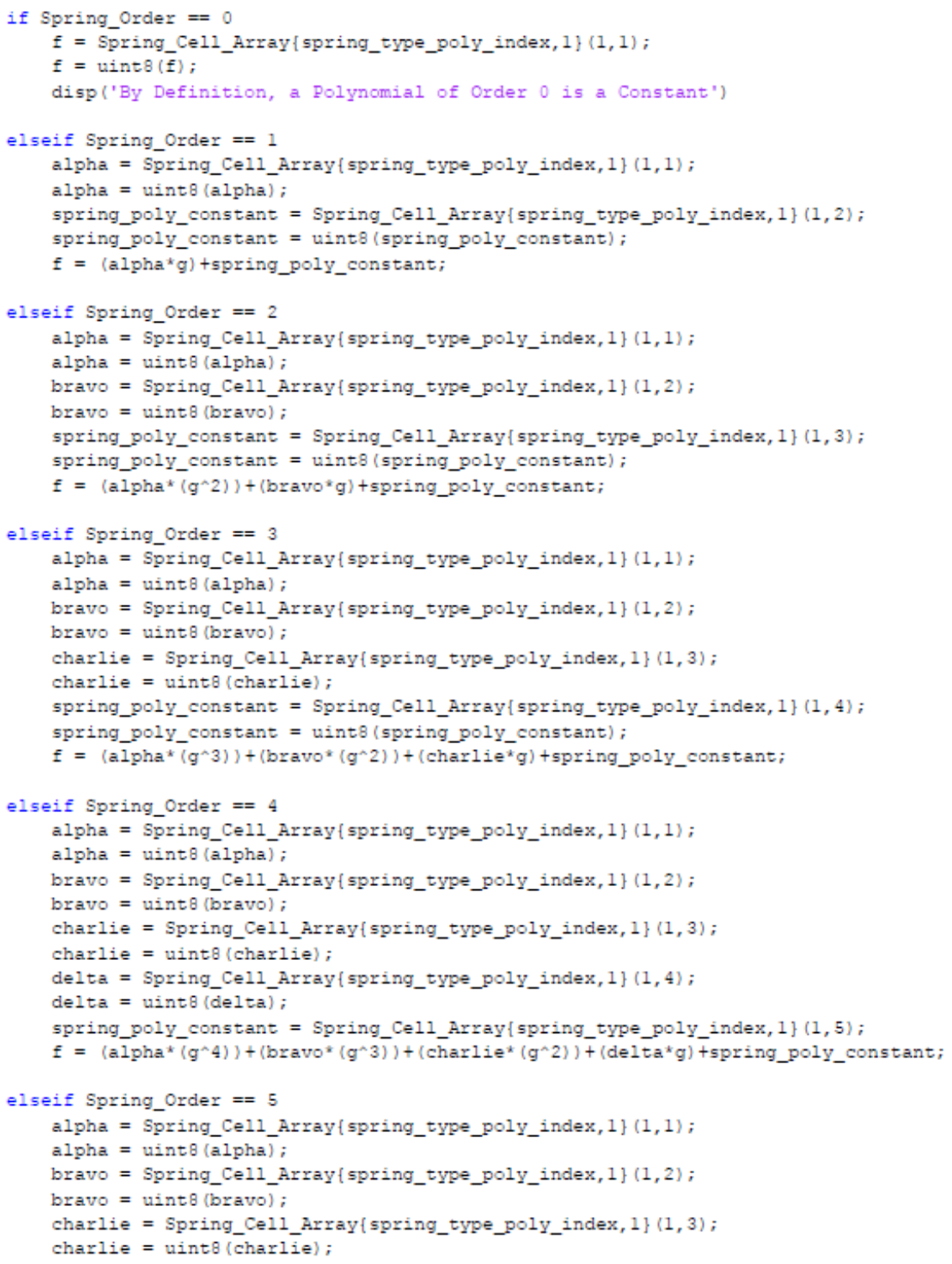




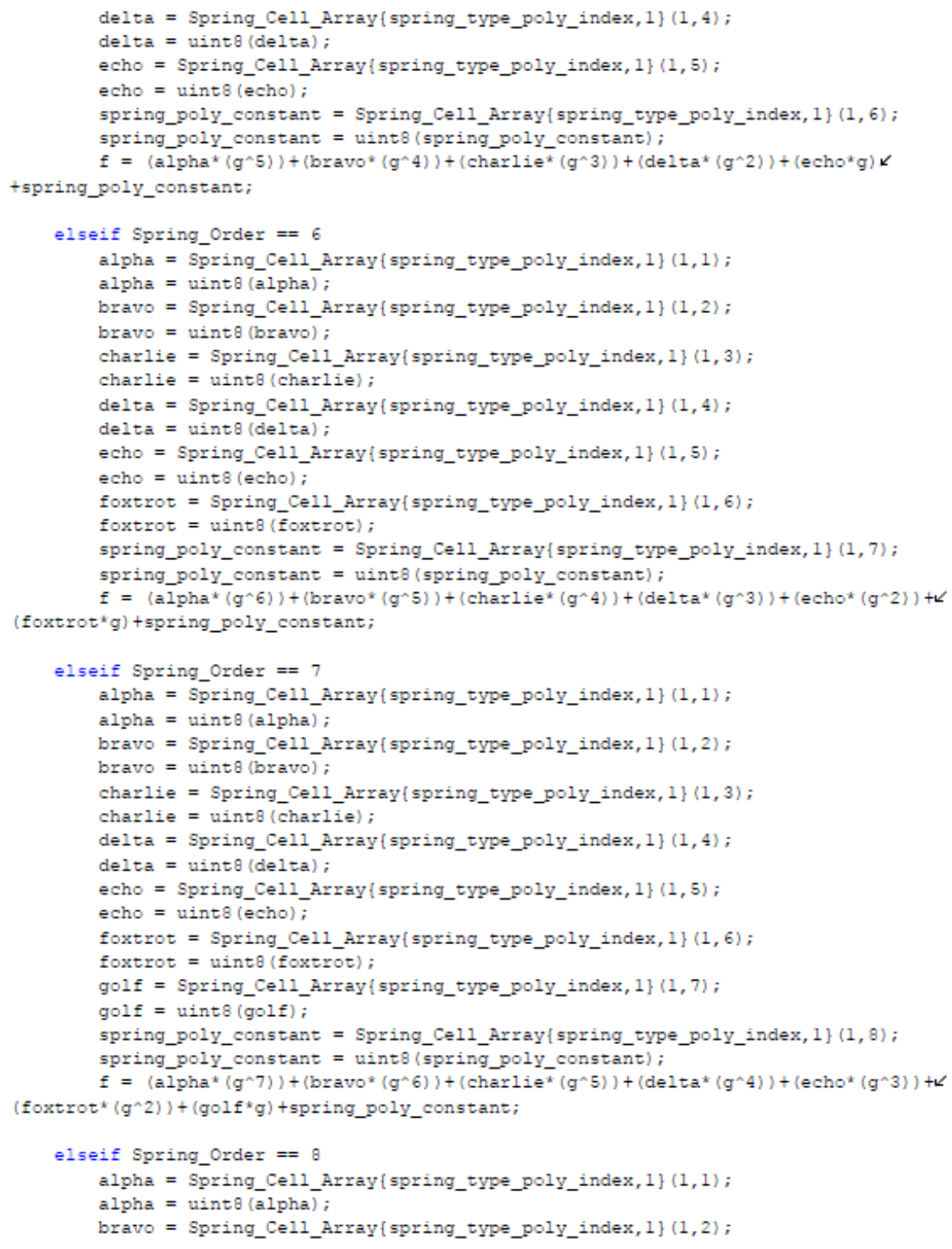




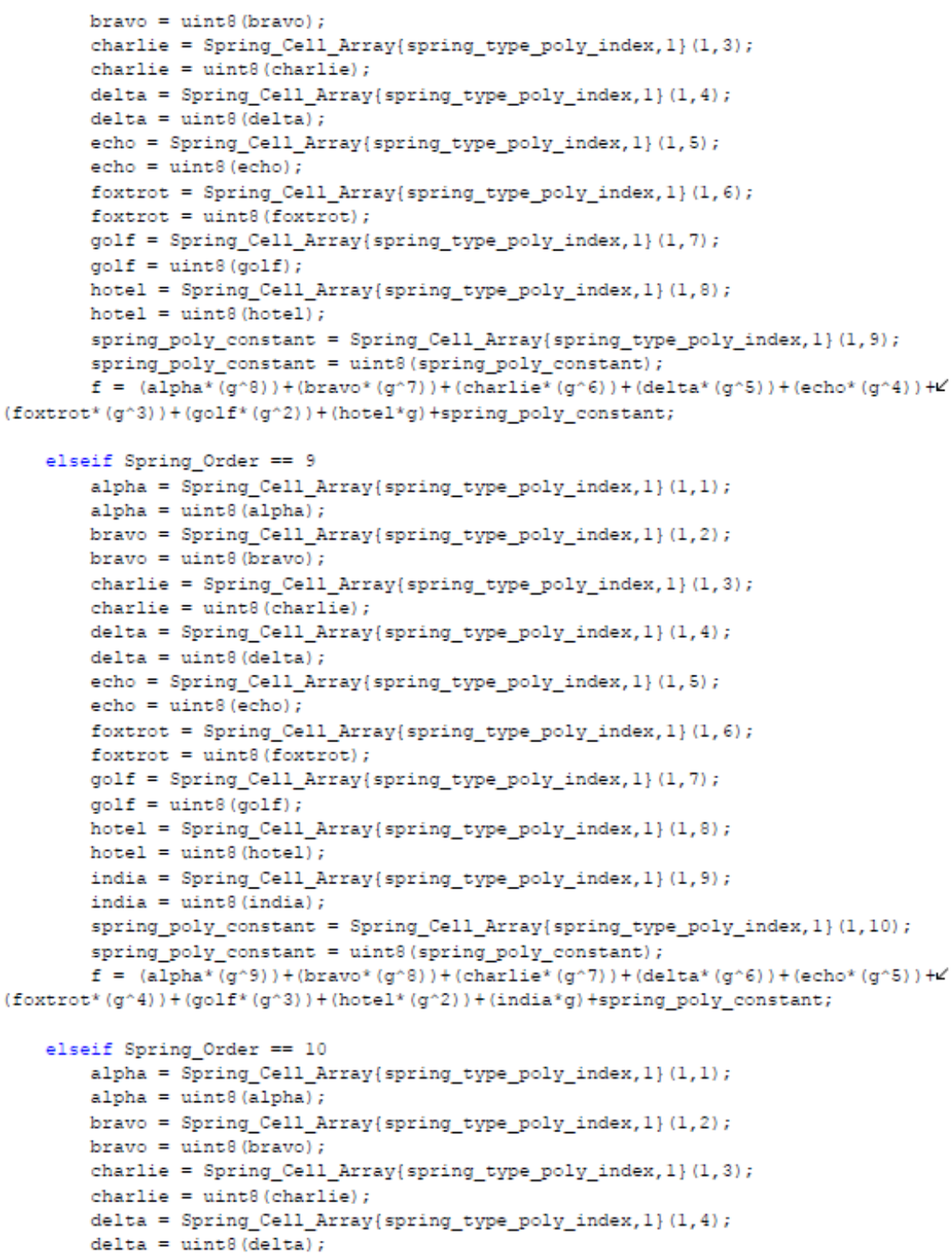




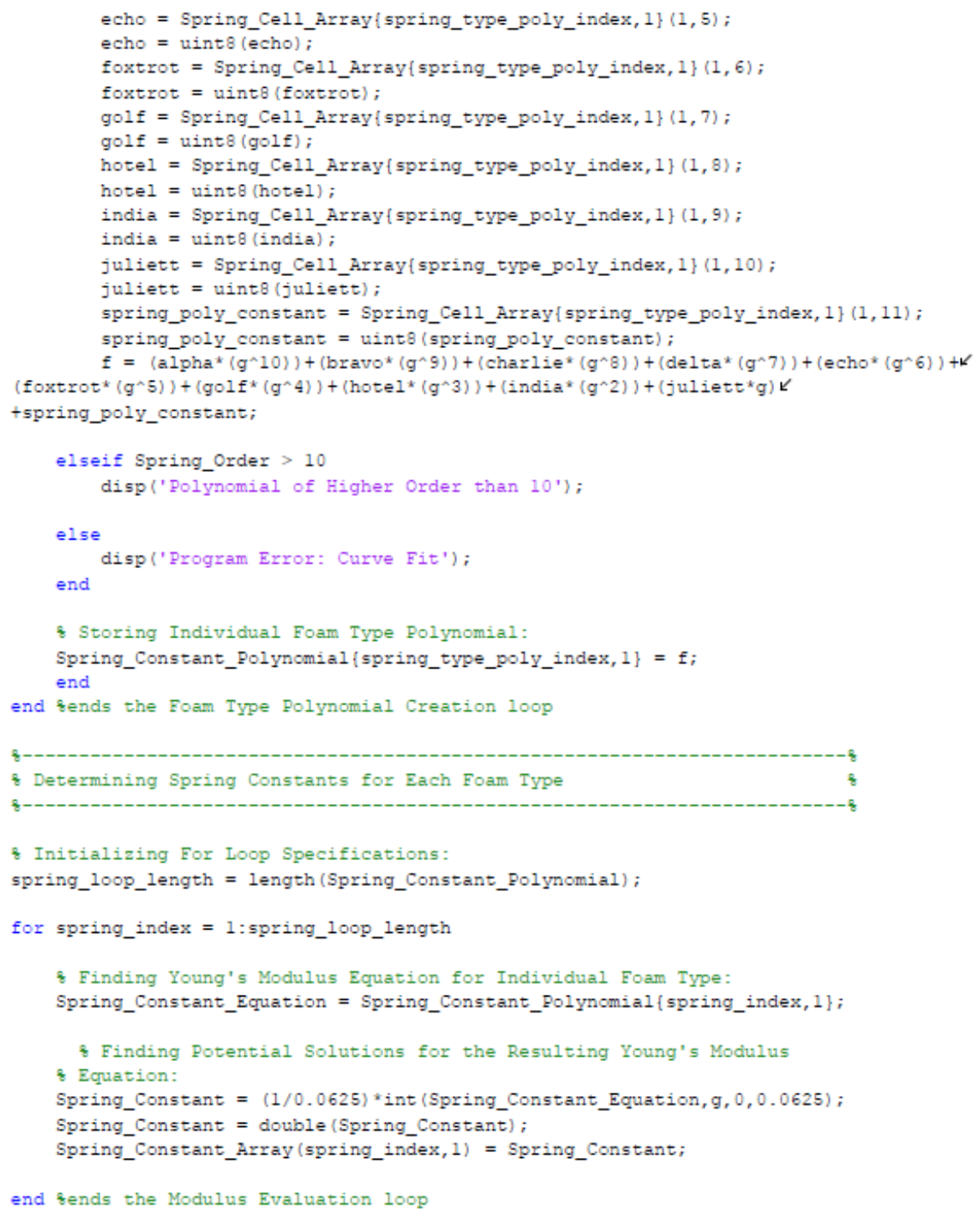




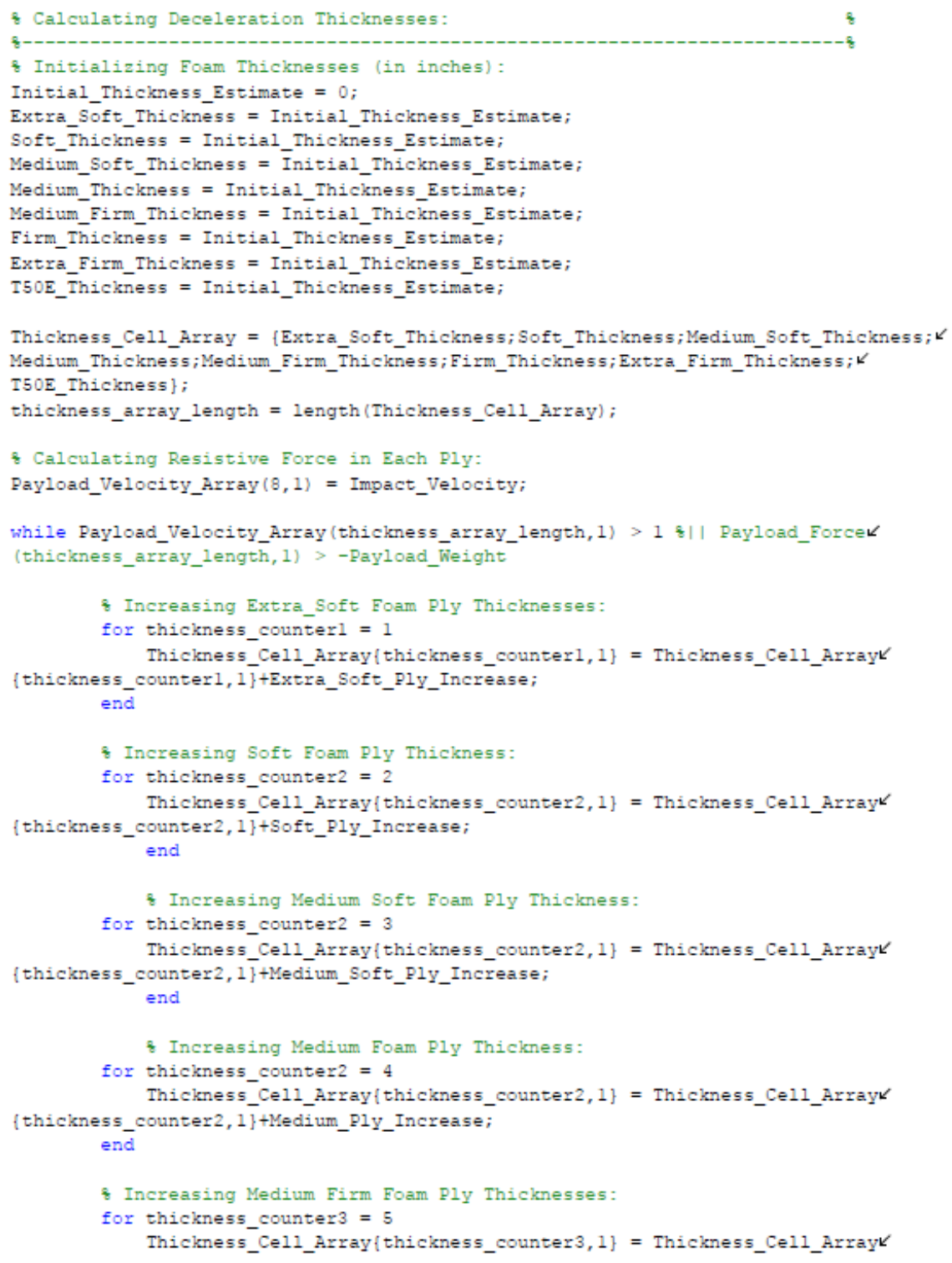




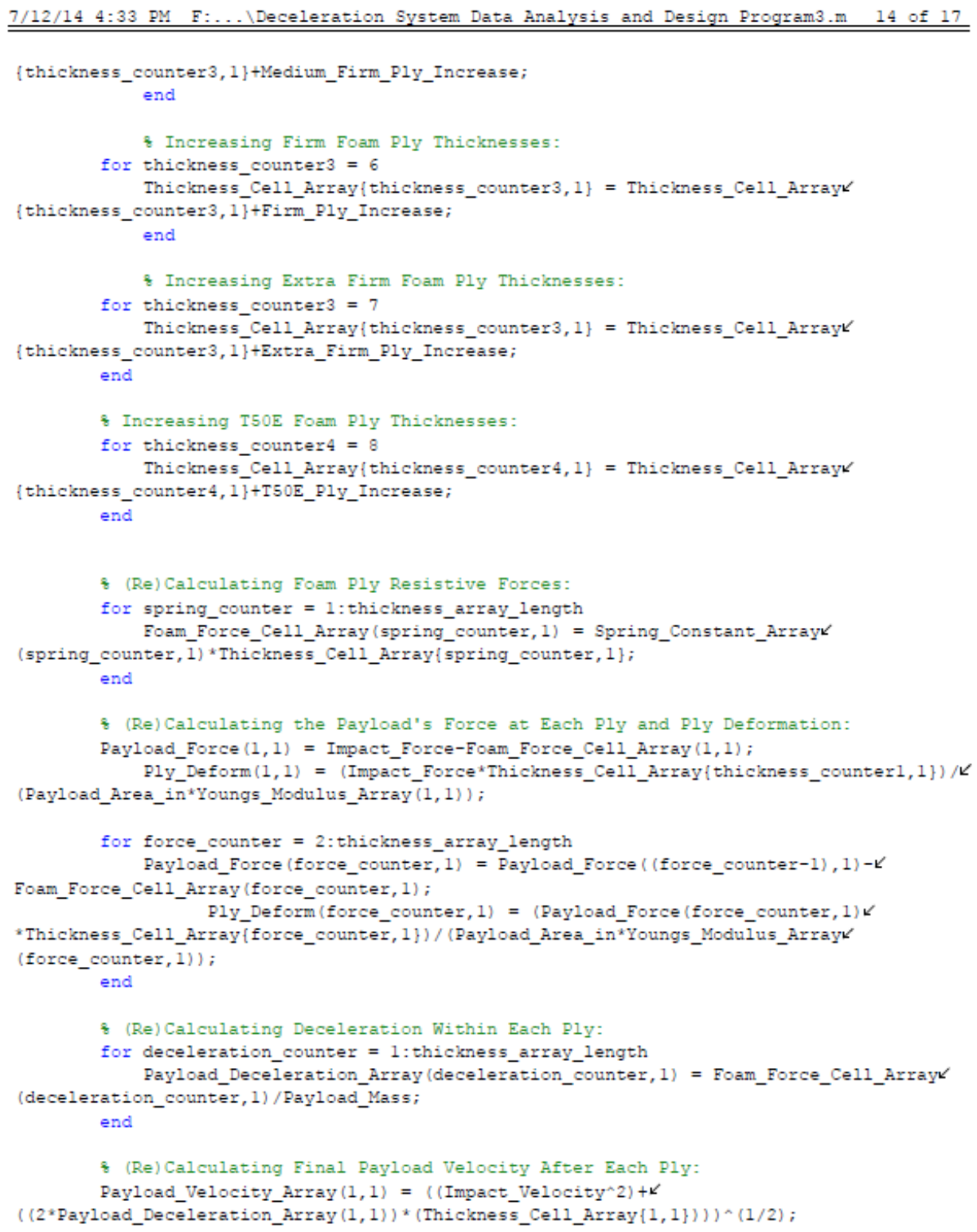




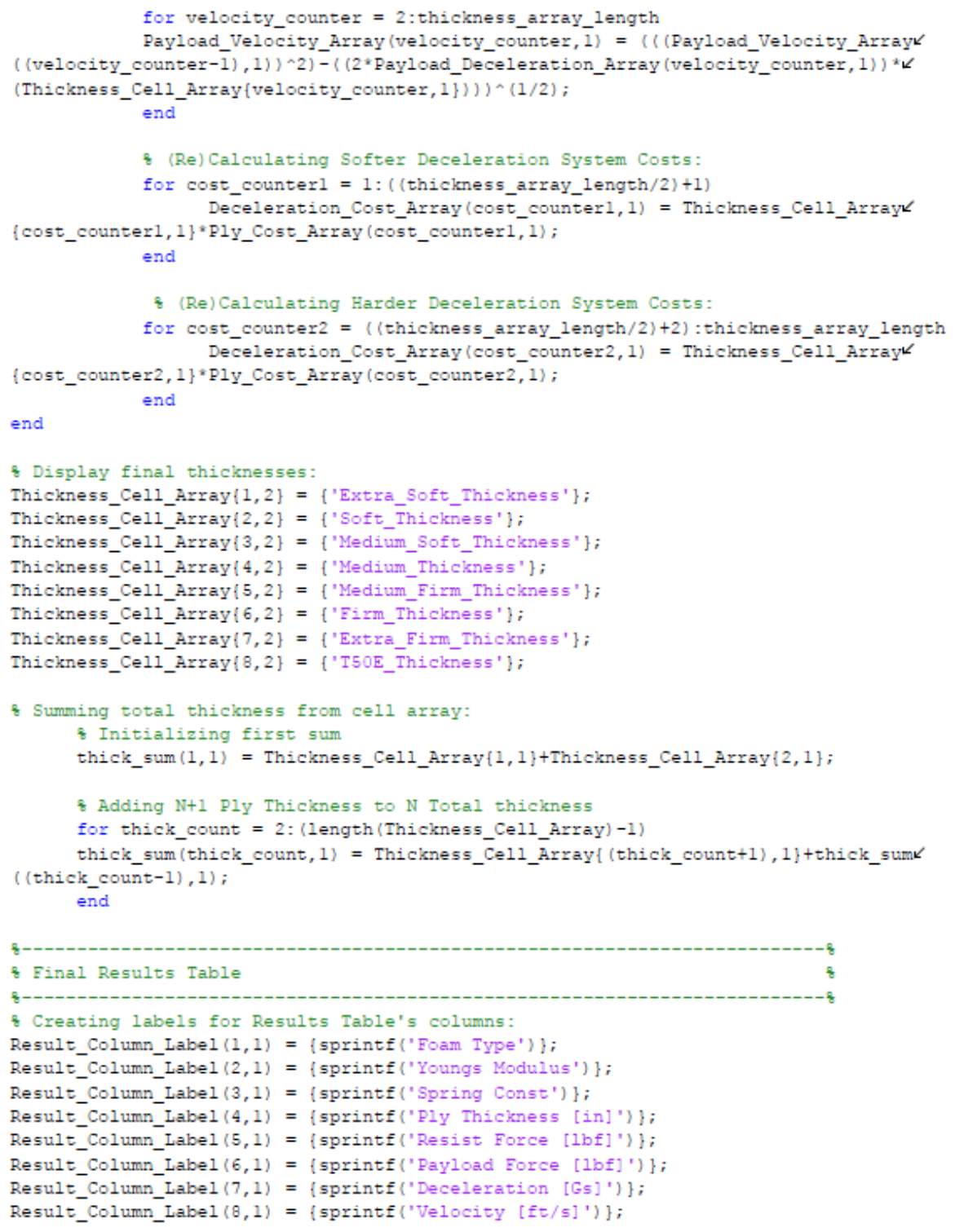




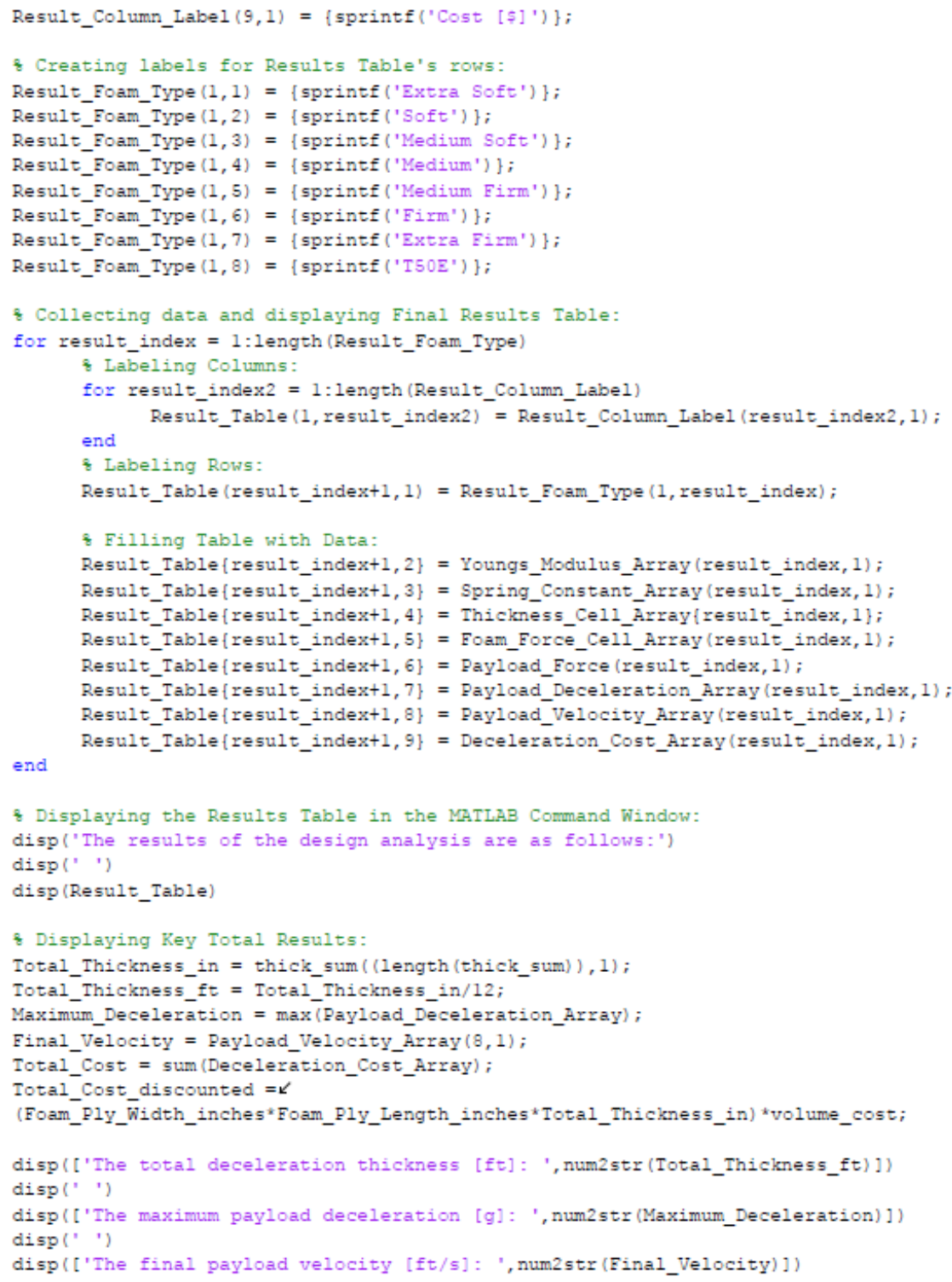




\section{7/12/14 4:33 PM F:... Deceleration System Data Analysis and Design Program3.m 17 of 17}
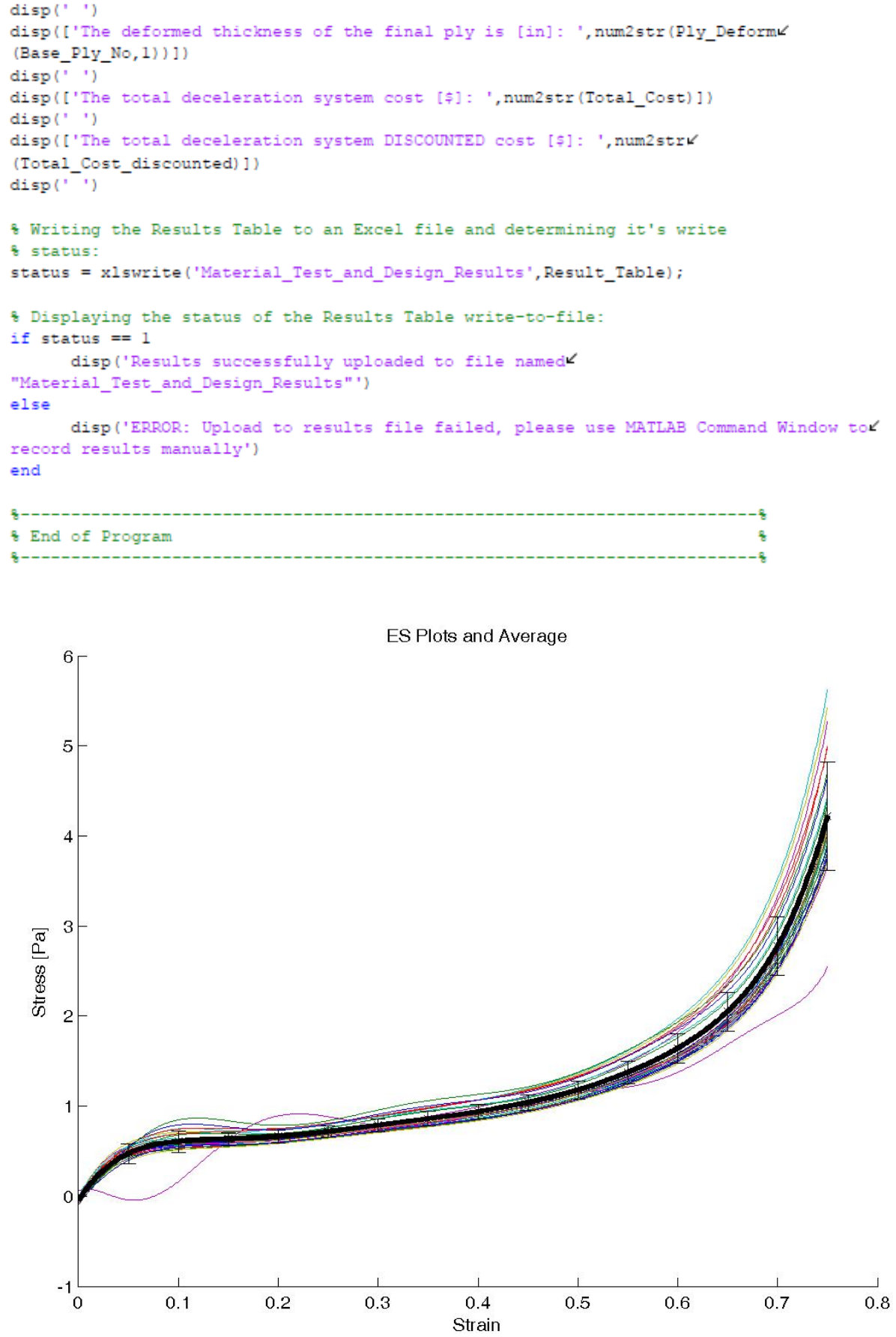

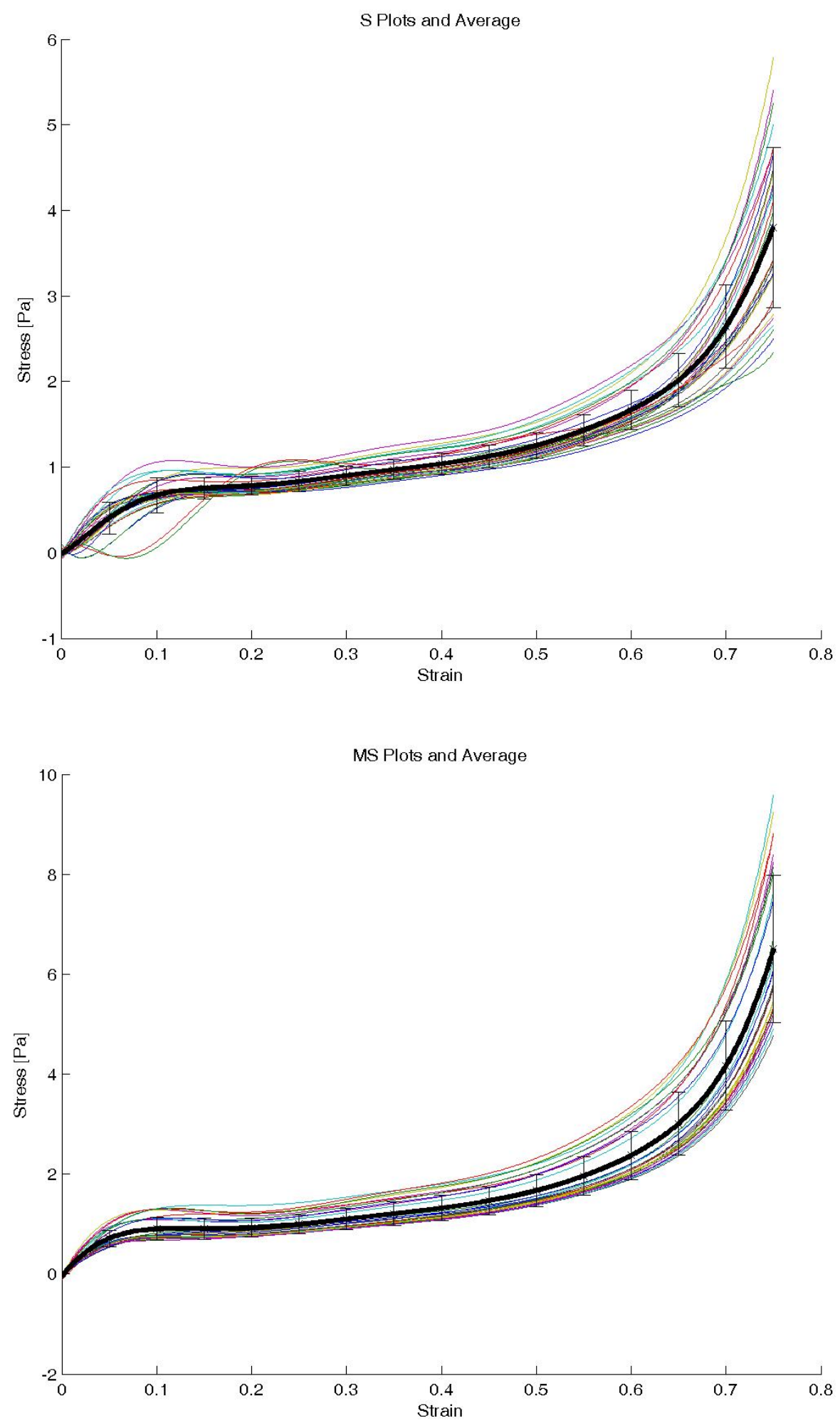

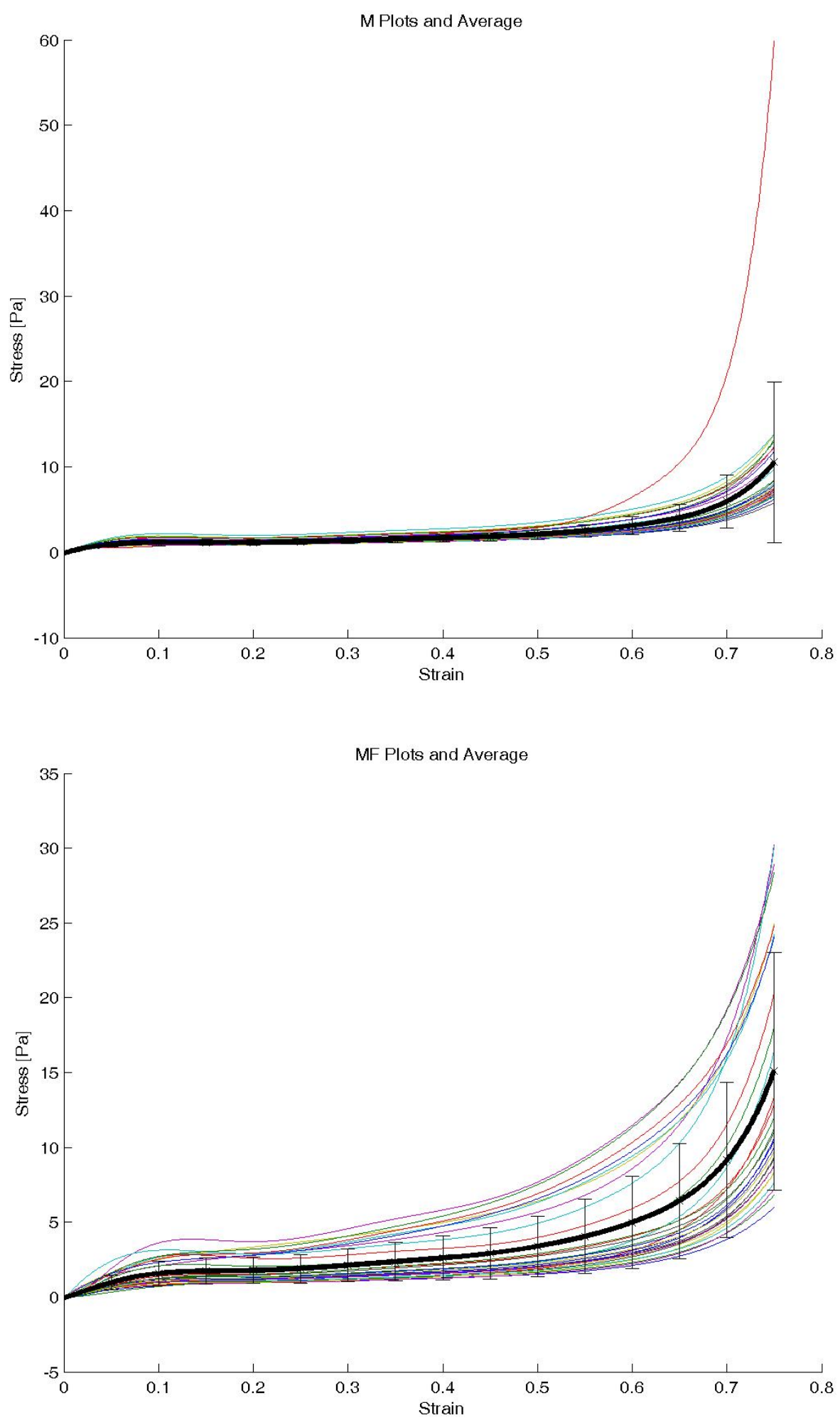

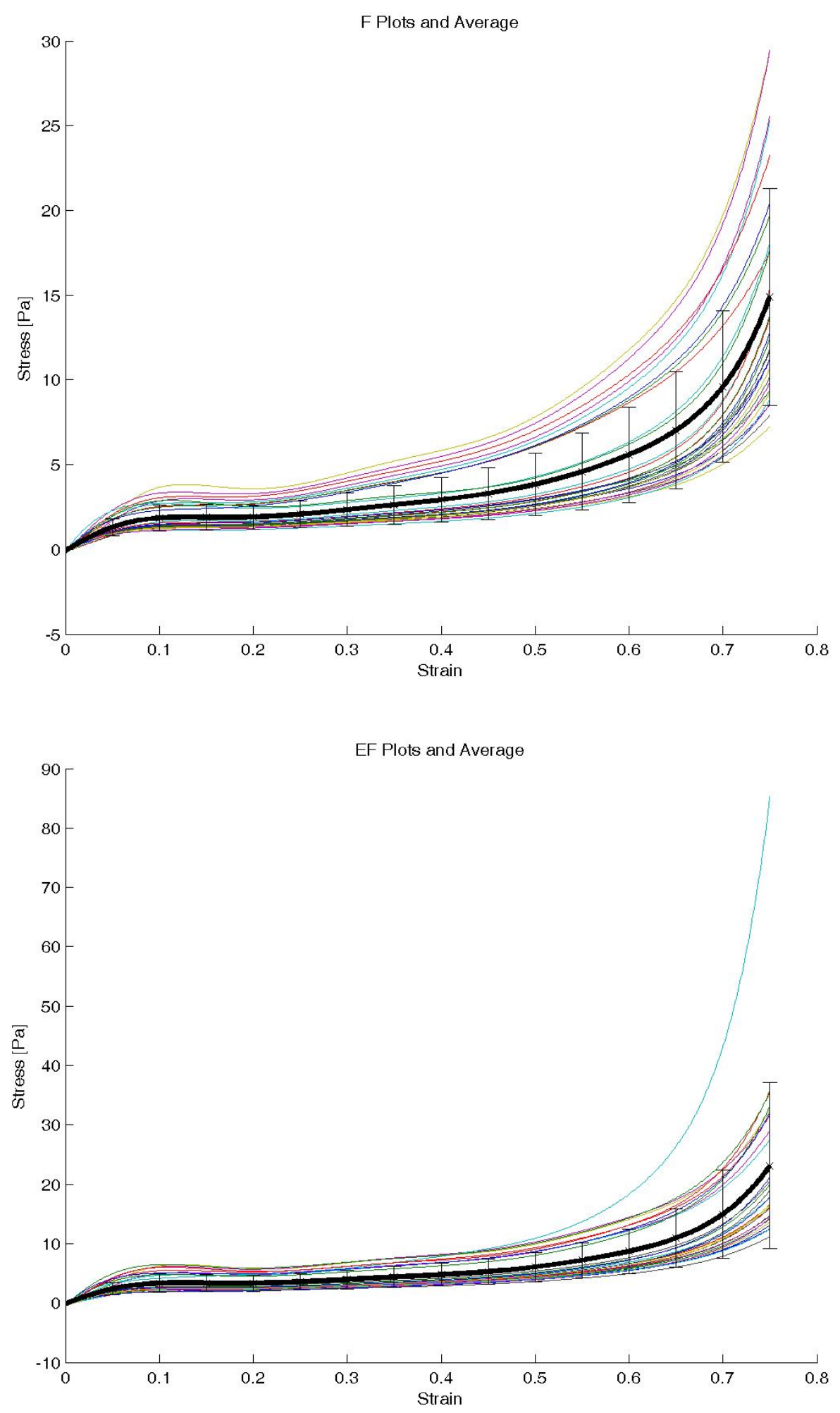

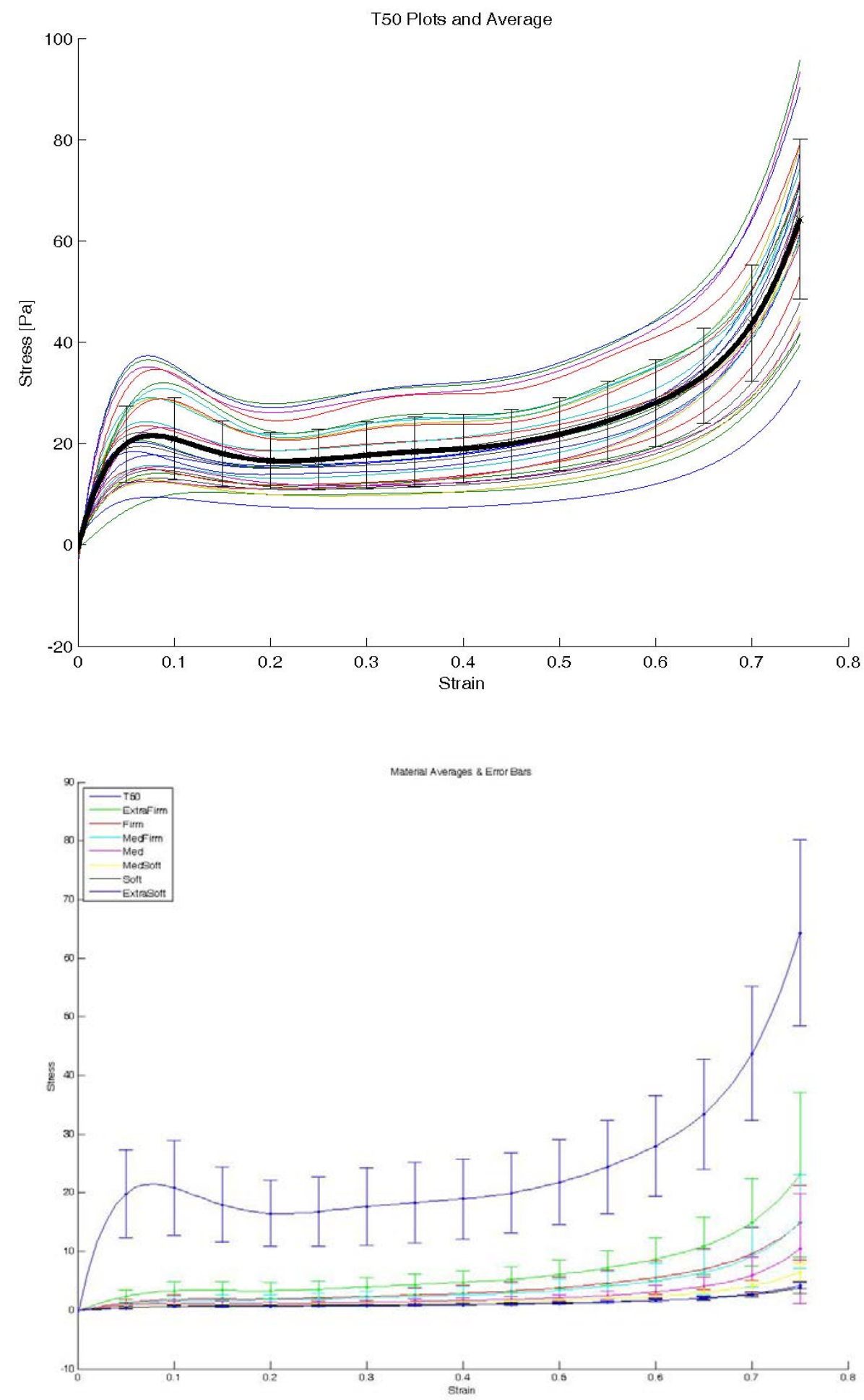


\subsection{Appendix $\mathrm{H}$}

\section{Lifting System Information}

Appendix F: Lifting System Information presents technical information about the components used for the lifting system of the WVU SMiRF. This appendix also presents the design of the support system used to mount the lifting system and integrate the system with the drop tower structure. The information provided herein is provided for comprehension of the project documentation and reflects past costing and copyrighted material belonging to Columbia Winch and Hoist and Allied Power Products, Inc. to which all rights apply for their copyright material. 


\section{ALLIED POWER PRODUCTS, IWC. Alled Power Product, , ho}

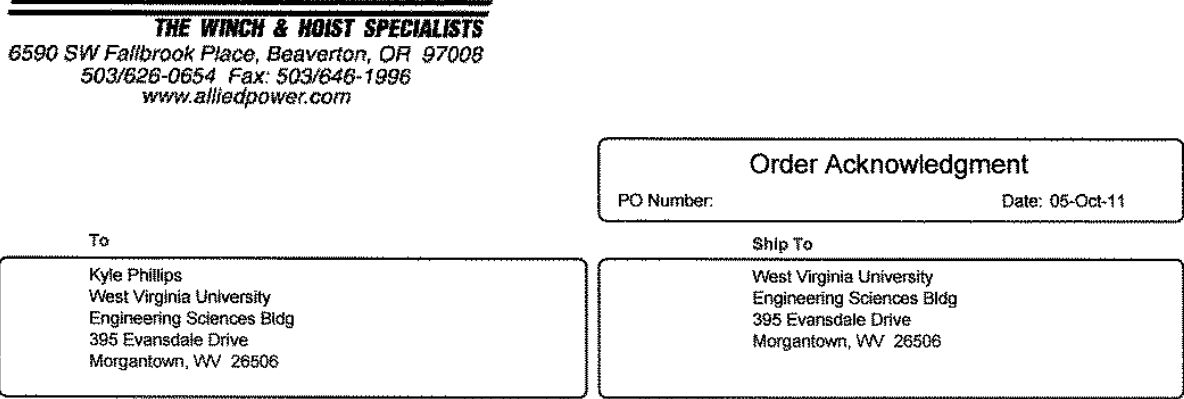

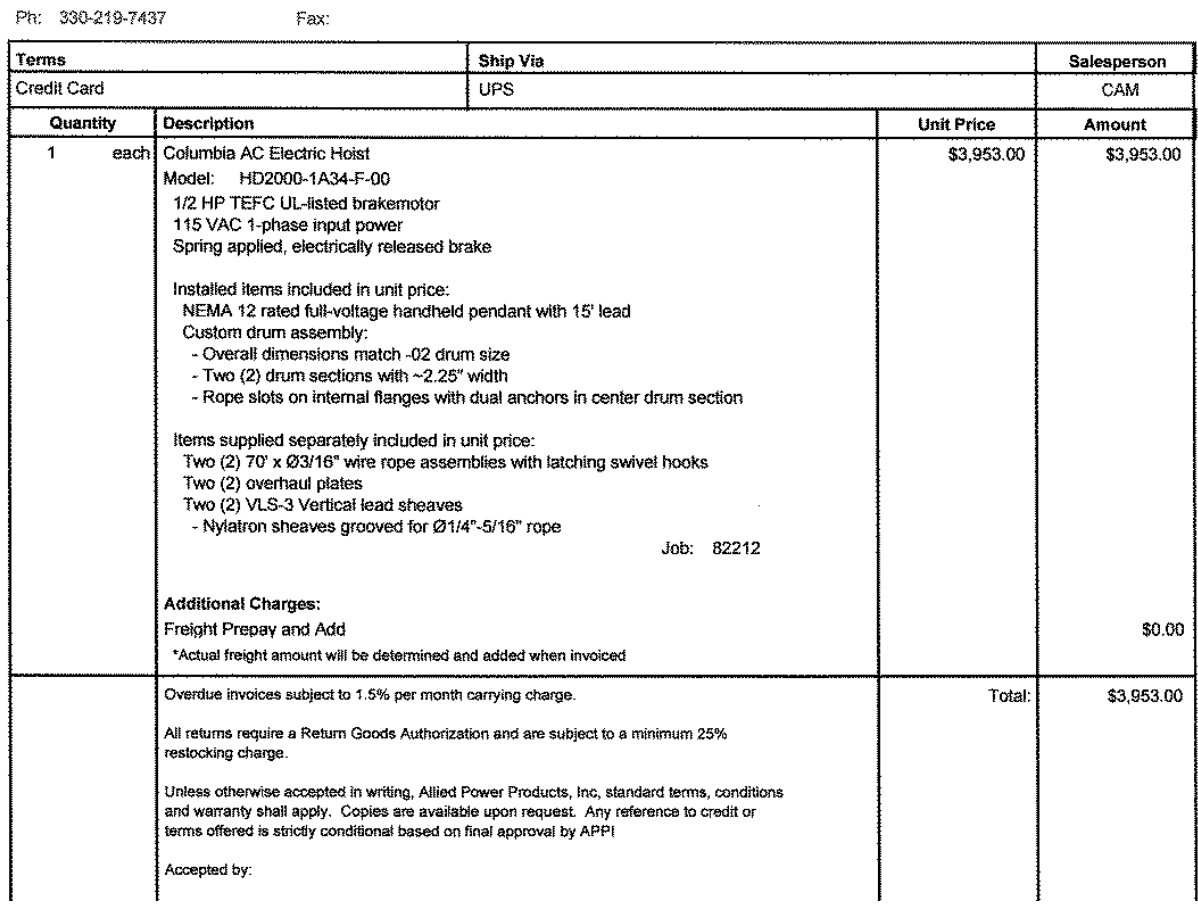




\section{Frame 1 AC Electric Hoist OLUMIBIA Parts Breakdown

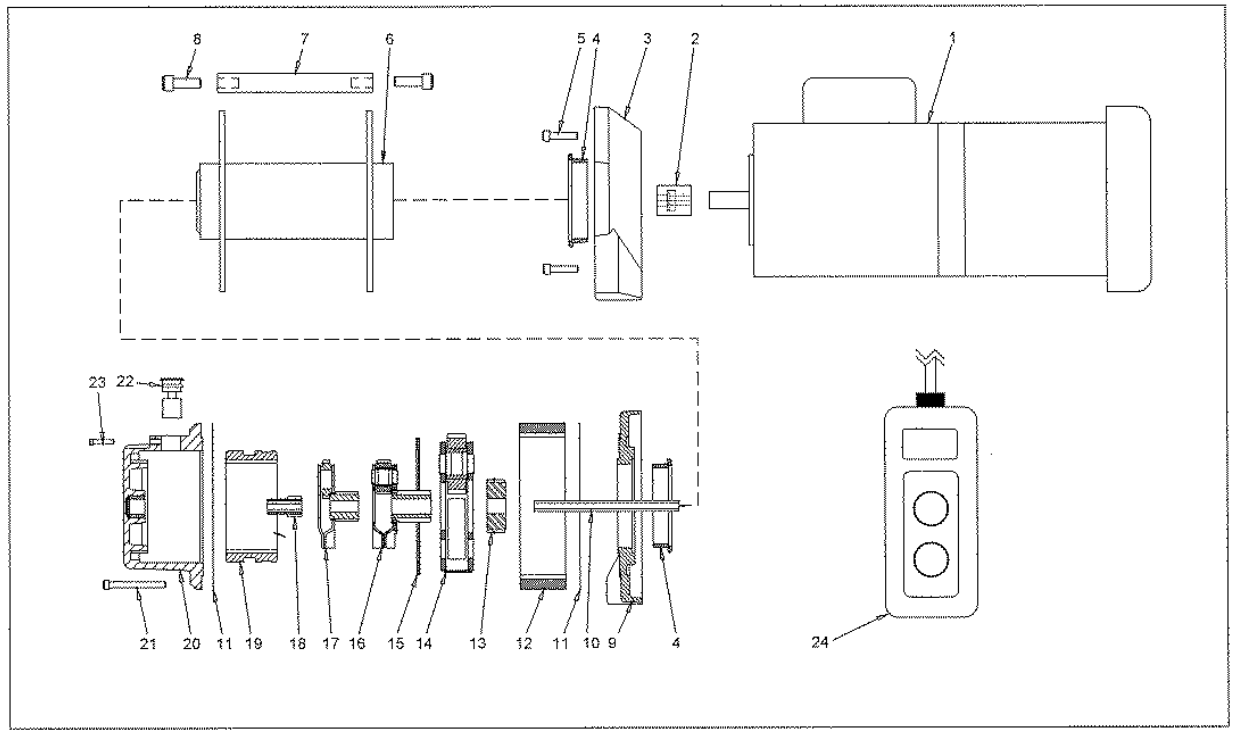

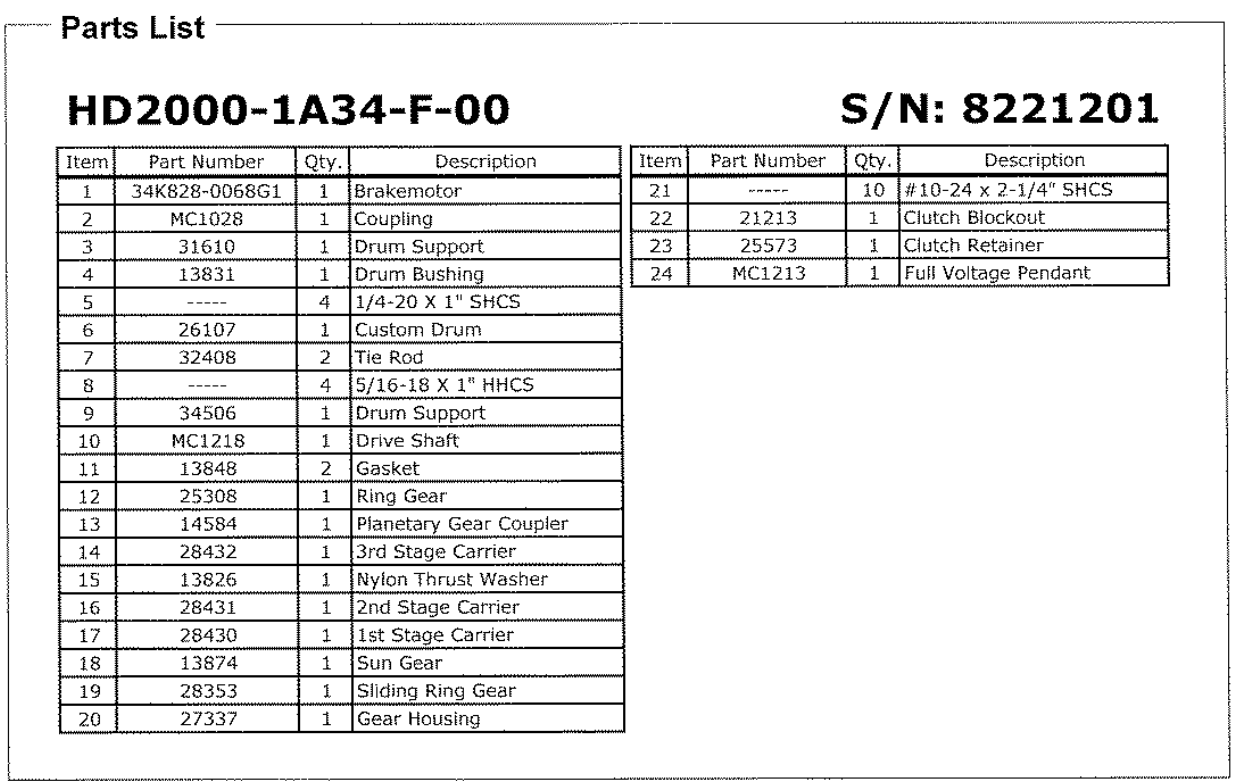




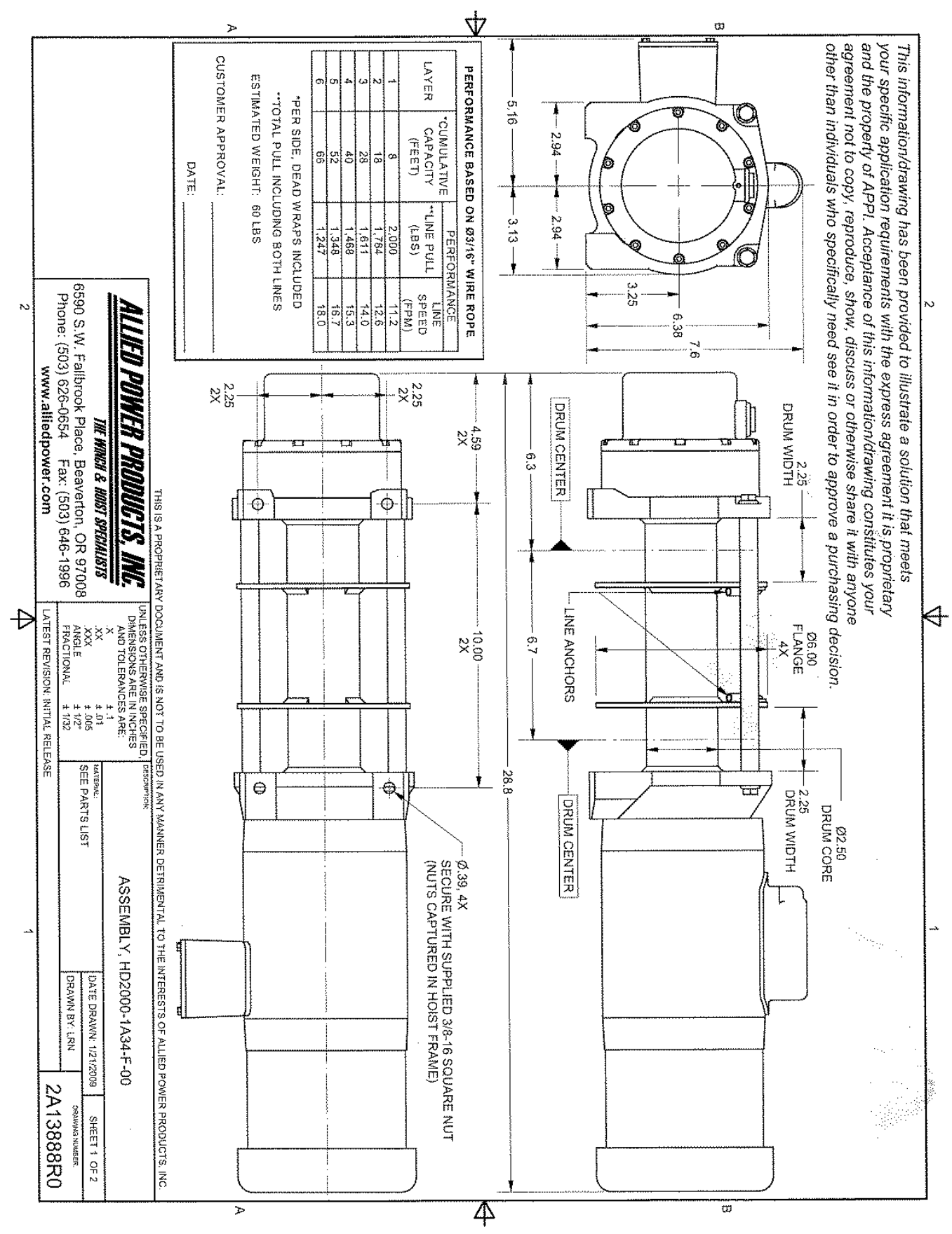




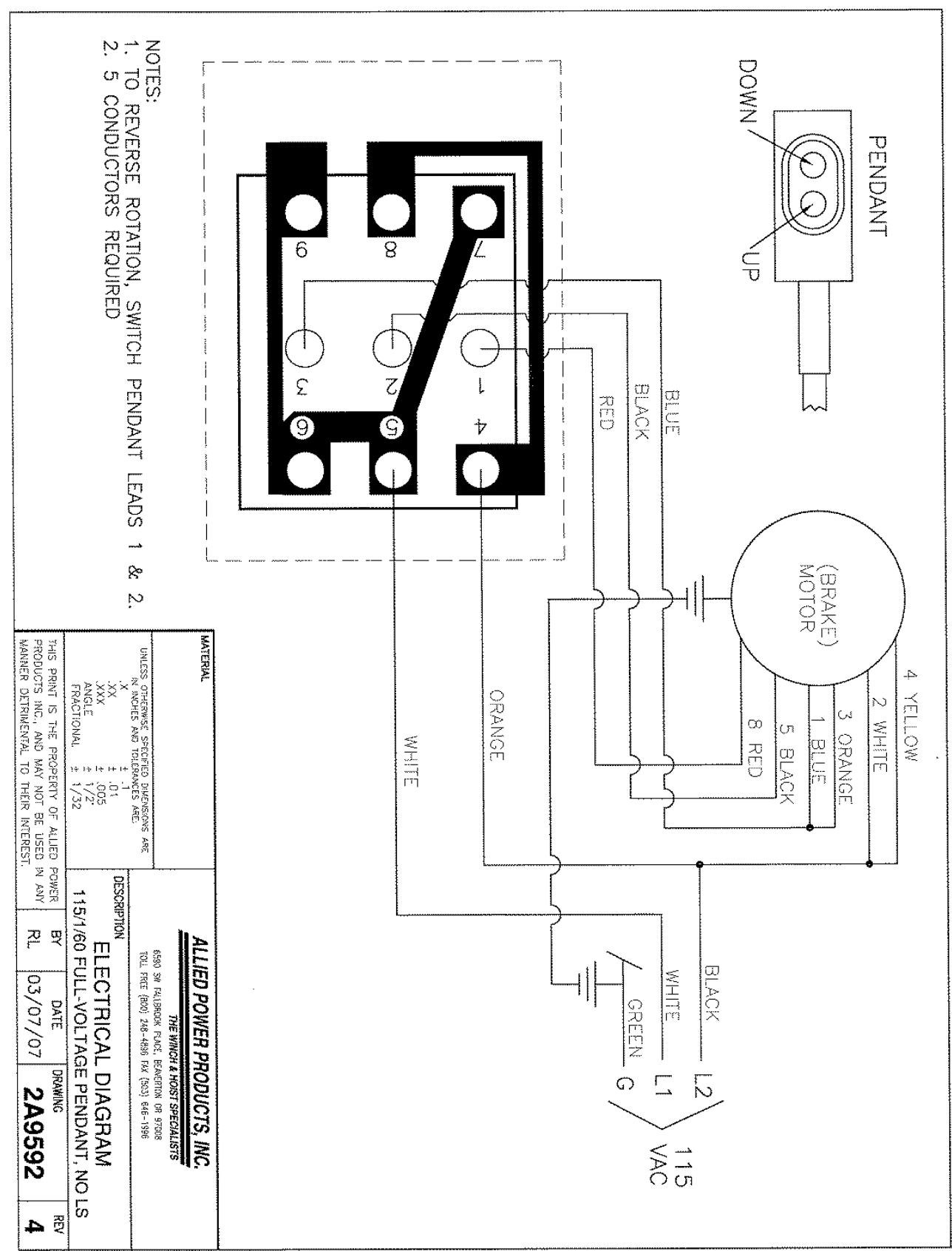




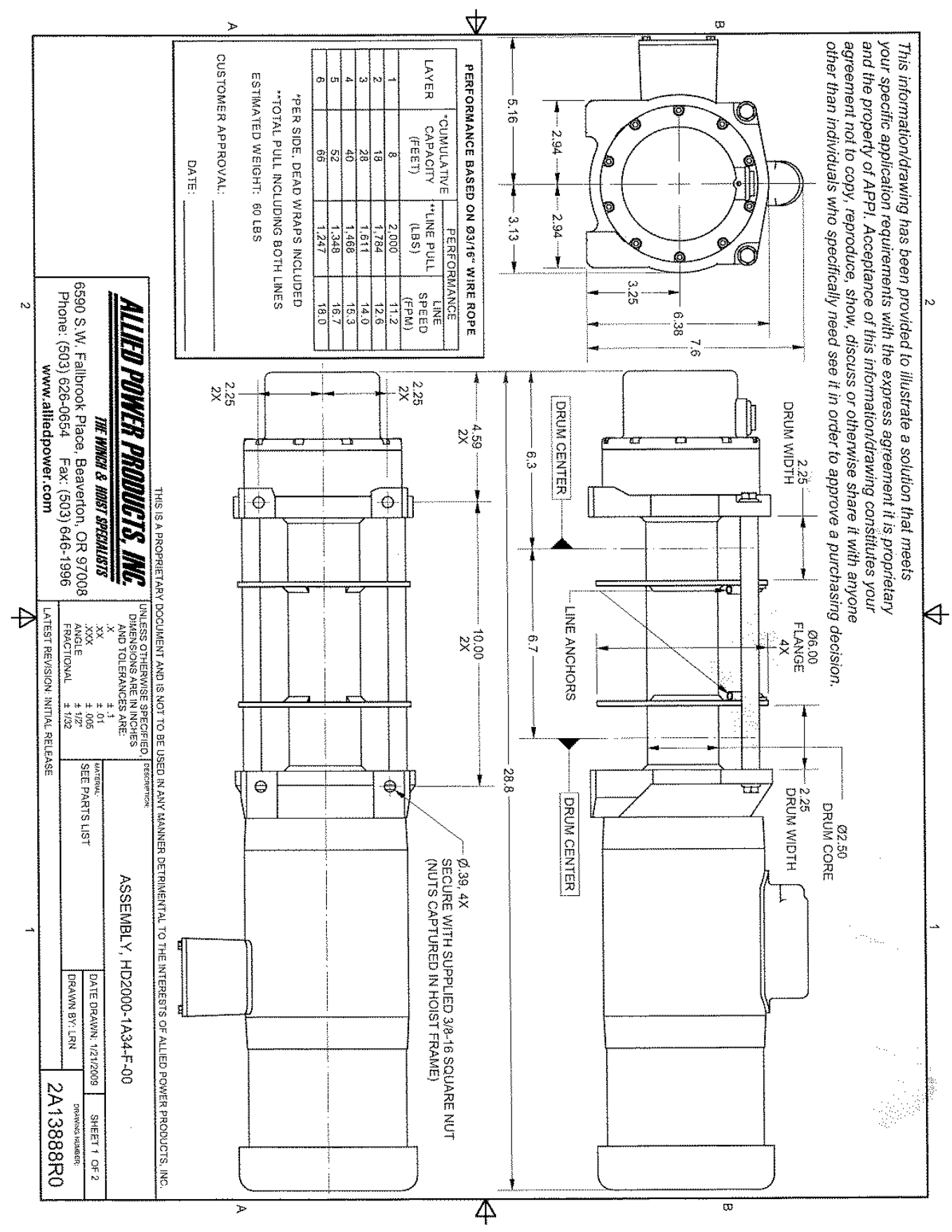


Development of the West Virginia University

Small Microgravity Research Facility (WVU SMiRF)

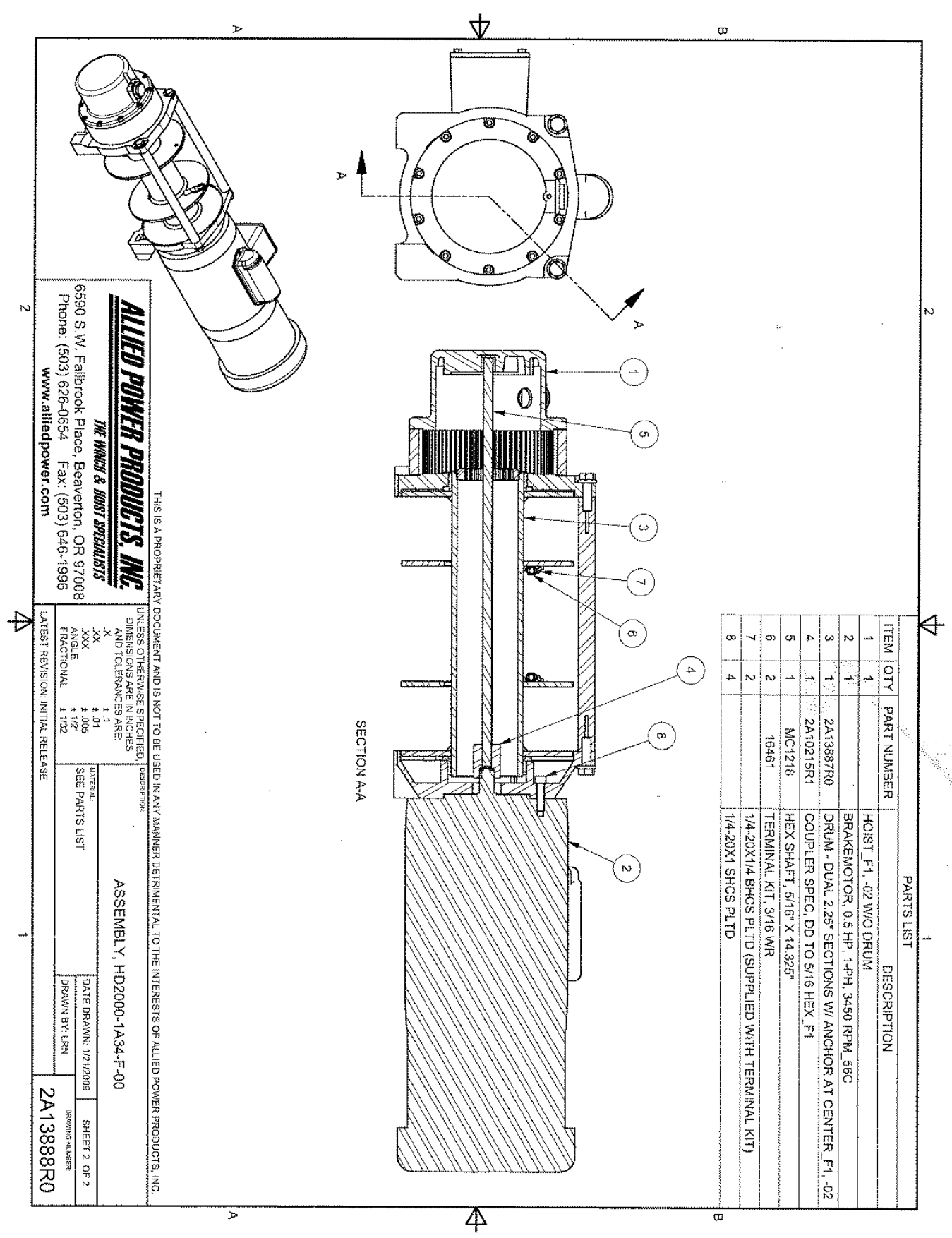


Development of the West Virginia University

Small Microgravity Research Facility (WVU SMiRF)
Copyright $^{\odot} 2014$

Kyle G. Phillips

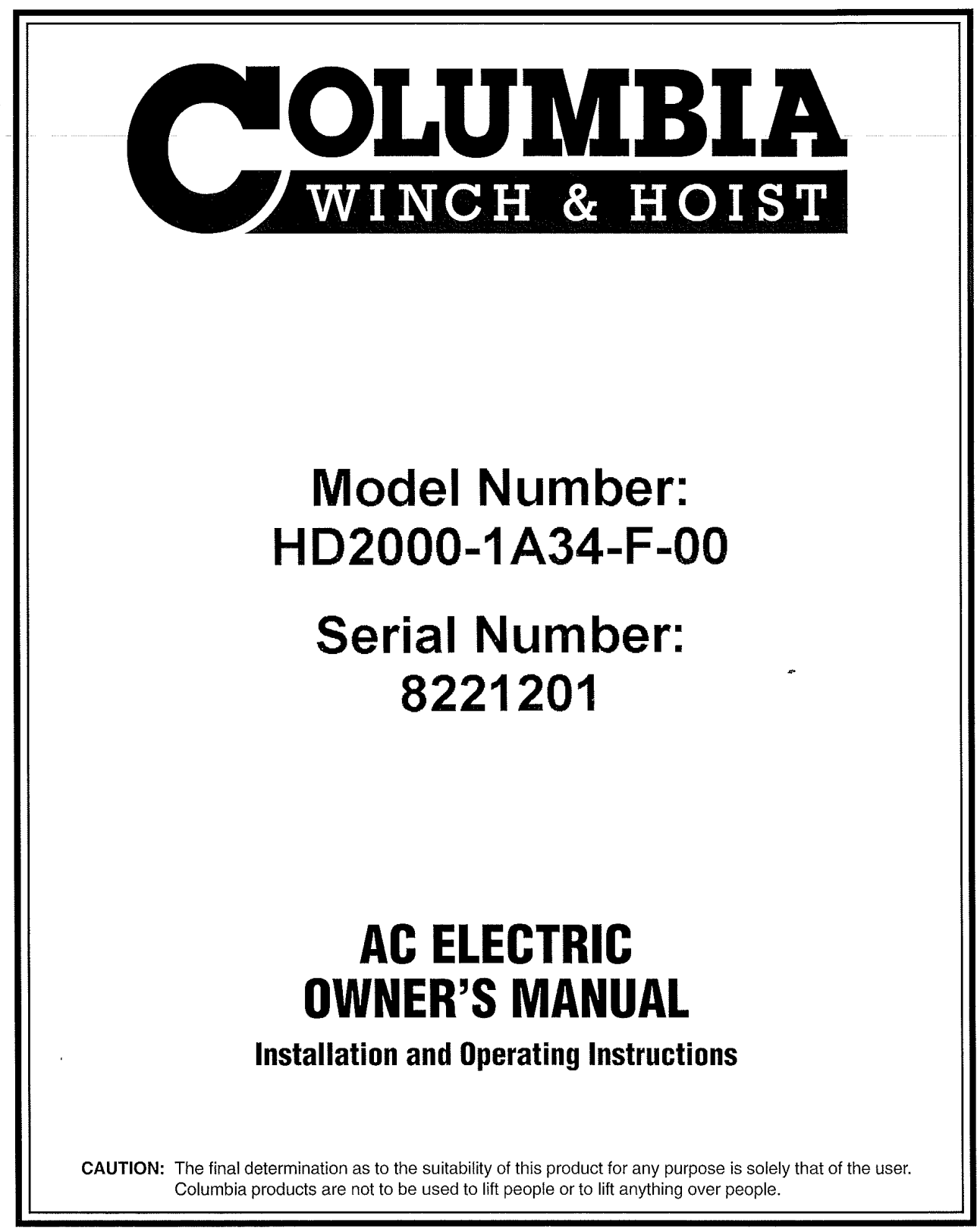




\section{READ THIS FIRST}

The information in this manual is intended to help ensure your Columbia product is properly installed, operated and maintained.

Although every effort has been made to make this manual complete and understandable, it is not a definitive guide to every possible situation or circumstance.

The proper installation, operation and maintenance of this product is solely the responsibility of the owner.

Safe operation of this product is directly dependent on the operator's skill, knowledge and judgment before, during and after the use of the product.

To avoid hazardous situations, every operator must be knowledgeable about appropriate safety guidelines, codes and regulations related to rigging, wire rope, and winch / hoist usage. Remember that an uninformed or careless operator can make the operation of any equipment dangerous.

Ultimately, the owner / operator must make the final decision as to how this product will be used and whether that intended use is safe.

If, after reading this manual, you have any questions regarding the installation or use of this Columbia product, contact your dealer or the Customer Service Manager of Allied Power Products, Inc. for an answer to your question.

Replacement manuals are available free of charge by writing:

Allied Power Products, Inc. 6590 SW Fallbrook Place Beaverton, OR 97008

THIS MANUAL CONTAINS EXTREMELY IMPORTANT INFORMATION ABOUT THE INSTALLATION AND OPERATION OF YOUR COLUMBIA PRODUCT. FOR YOUR OWN SAFETY, READ THIS MANUAL COMPLETELY PRIOR TO PRODUCT INSTALLATION AND / OR OPERATION.

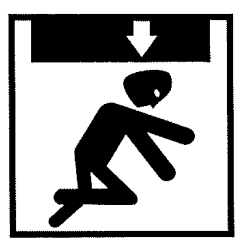

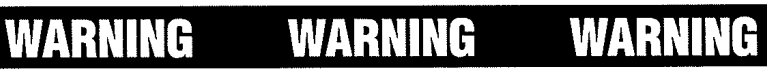

COLUMBIA PRODUCTS ARE NOT TO BE USED FOR LIFTING PEOPLE OR THINGS OVER PEOPLE 


\section{Table of Contents}

Section 1

Description Motor

Drum

Controls

Ratings and Suitability Factors of Operation

Section 2 .... Installation Guidelines Location Mounting Electrical Wire Rope

Section 3 .... Operating Instructions Preparing for Operation Operating the Unit Remote Pendant Control Duty Cycle Braking System Operating Safety Precautions

Section 4..... General Maintenance

Section 5 Warranty

\begin{tabular}{|c|}
\hline Appendix \\
\hline Mounting Template \\
Wiring Diagram \\
Parts Breakdown \\
\hline
\end{tabular}

\section{Model Number Information}

When instructions in this manual refer to specific specifications on different models, identify your unit by referring to the MODEL NUMBER as shown on the unit nameplate and the front of this manual.

Sample Model Number:

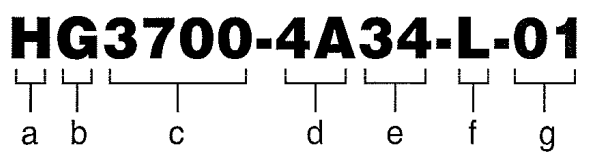

\begin{tabular}{|c|c|c|}
\hline & Frame 1 & Frame $2 / 3$ \\
\hline $\mathbf{a}$ & \multicolumn{2}{|c|}{$\begin{array}{l}H=\text { Hoist }, \text { rated for hoisting } \\
W=\text { Winch }, \text { rated for pulling }\end{array}$} \\
\hline b & $\begin{array}{l}C=\text { Frame } 1 \\
D=\text { Frame } 1\end{array}$ & $\begin{array}{l}F=\text { Frame } 2 \\
G=\text { Frame } 3\end{array}$ \\
\hline C & \multicolumn{2}{|c|}{ Model Specification } \\
\hline d & \multicolumn{2}{|c|}{$\begin{array}{l}1 A=115 \mathrm{VAC} 1 \varnothing \\
2 A=230 \mathrm{VAC} 1 \varnothing \text { or } 230 \mathrm{VAC} 3 \varnothing \\
3 A=380 \mathrm{VAC} 3 \varnothing \\
4 A=460 \mathrm{VAC} 3 \varnothing \\
5 A=575 \mathrm{VAC} 3 \varnothing\end{array}$} \\
\hline e & \multicolumn{2}{|c|}{$\begin{array}{l}17=1725 \text { RPM motor speed } \\
25=2500 \text { RPM motor speed } \\
34=3450 \text { RPM motor speed }\end{array}$} \\
\hline f & \multicolumn{2}{|c|}{$\begin{array}{l}\mathrm{L}=\text { Low-voltage control model } \\
\mathrm{F}=\text { Full-voltage control model } \\
\mathrm{X}=\text { Unit supplied without controls }\end{array}$} \\
\hline \multirow[t]{2}{*}{ g } & $\begin{array}{l}-01=5^{\prime \prime} \text { drum } \\
-02=9^{\prime \prime} \text { drum }\end{array}$ & $\begin{array}{l}-01=8 " \text { drum } \\
-02=10^{\prime \prime} \text { drum } \\
-03=12.5^{\prime \prime} \text { drum } \\
-05=25^{\prime \prime} \text { drum }\end{array}$ \\
\hline & \multicolumn{2}{|c|}{$-00=$ Custom Drum Configuration } \\
\hline
\end{tabular}

An ' $S$ ' at the end of the model number indicates a custom configuration. 


\section{Description}

Columbia AC winch and hoist assemblies consist of a frame with motor and drum, and controls.

\section{Motor}

Standard motors are Totally Enclosed Fan Cooled (TEFC). Duty ratings differ for each model; see 'Operating Instructions' for more information about your unit's duty cycle.

\section{Drum}

The drum is attached to the motor through planetary gear reductions to deliver the required pulling capacity and performance.

\section{Controls}

\section{Full-Voltage Control Models}

Full-voltage control models include a remote pendant control with "power-in" and "power-out" buttons, allowing flexible operation of the unit.

The remote pendant control is rated NEMA $4 \mathrm{X}$ for indoor or outdoor use.

\section{Low-Voltage Control Models}

Low-voltage control models include a control box which converts $A C$ input power to a lower voltage for the remote pendant control.

The control box and remote pendant control are rated NEMA $4 \mathrm{X}$ for indoor or outdoor use.

\section{Ratings and Suitability}

Columbia winches and hoists are not to be used to lift, pull, support or otherwise transport people or loads over people.

Line pull ratings represent the maximum load that can be placed on a new unit. This load rating varies with the amount of wire rope wound on the drum and is affected by the age and condition of the unit.

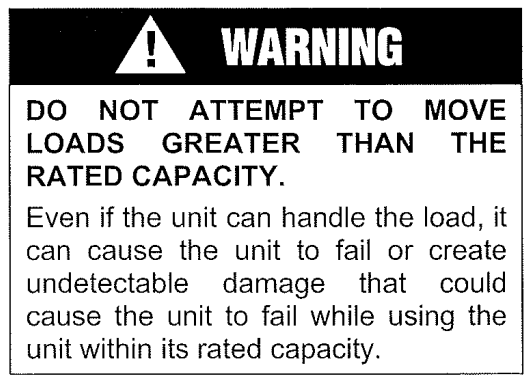

\section{Factors of Operation}

Harsh environments decrease the load rating of the unit and make it more susceptible to damage. Avoid installation in extreme temperatures, dirty surroundings and wet environments.

Improper installation can create excessive wear or failure of any of the component parts or fasteners in the unit. Be sure that the unit is properly installed, the fasteners checked for tightness and the mounting inspected for damage on a periodic basis.

Use of the unit will create wear in the components. The more frequently it is used and the heavier the load, the 
greater the strain and wear on the components. This may result in damage that causes a failure over a period of time. Periodic inspection of the unit is the only way to ensure its continued operating capability.

The amount of wire rope on the drum
affects the performance of the unit.
Line-pull decreases with each
additional layer of wire rope wound on
the drum. Line-speed increases with
each additional layer of wire rope
wound on the drum.

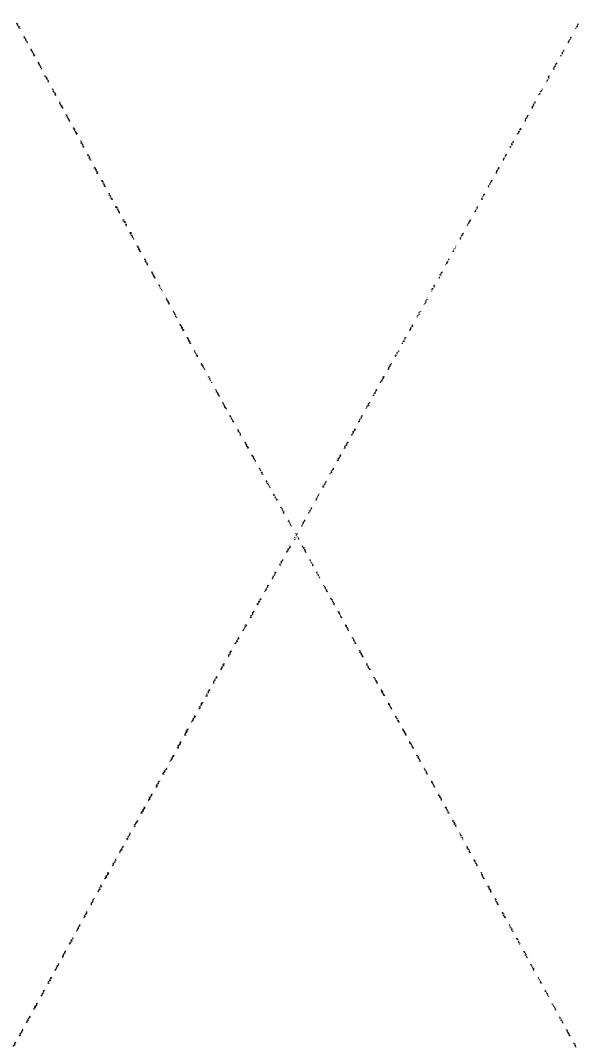

\section{Installation Guidelines}

The safe installation of this product is solely dependent upon the owner and/or operator's skill, knowledge and judgment. Installation must be done only by those qualified and familiar with all operating guidelines, safety codes and regulations related to rigging, wire rope and winch and/or hoist usage.

The determination of suitability for this product for any specific use is the responsibility of the owner and/or operator.

The following guidelines are to be used only as a reference.

\section{Location}

When selecting a location for the hoist, the following guidelines must be met:

1. An unobstructed area that keeps the operator and others out of the path of the unit and the path of broken wire rope. Broken wire rope can snap back and cause serious injury to personnel and machinery.

2. A location where it will not interfere with or be damaged by other objects or machinery.

3. A location with adequate lighting and heat for both the operator and unit. Although the unit is rated for operation in temperatures ranging from $-50^{\circ}$ to 
$+125^{\circ}$ Fahrenheit, extremes in temperature will affect the performance of the unit.

4. A position near an appropriate power source. Do not install the unit in any area defined as hazardous by the National Electric Code.

5. A location that permits proper fleet angle. Maintaining proper fleet angle allows the wire rope to spool uniformly onto the drum and prevents damage to the wire rope.
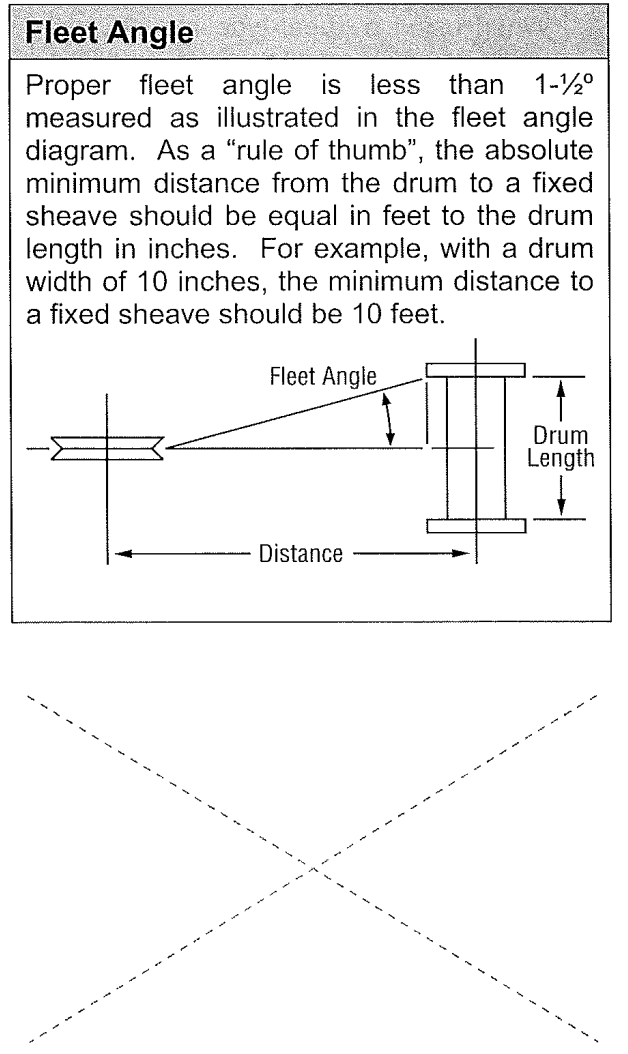

Possible Mounting Locations:
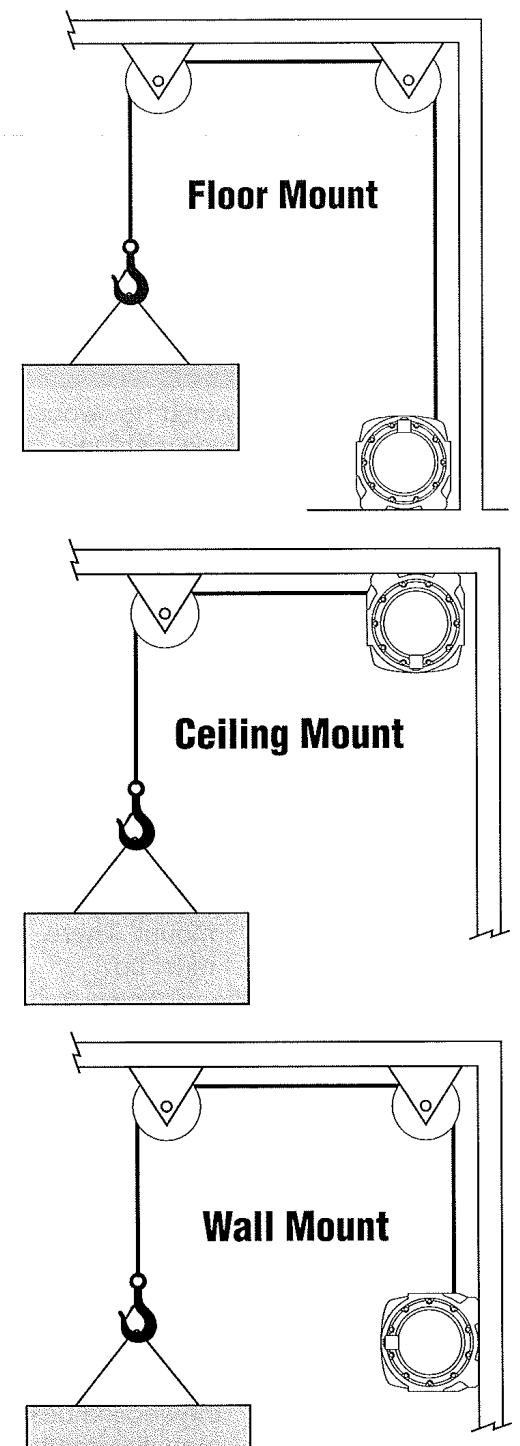


\section{Mounting}

The hoist must be attached to a rigid and level foundation or support structure that is adequate to support the hoist and its load under all load conditions, including shock-loads, without flexing.

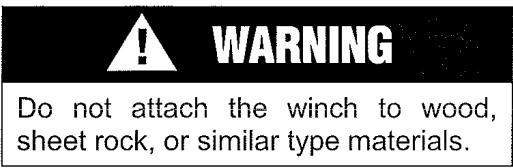

Three different mounting positions are shown.

\section{Compression-Type Mount}

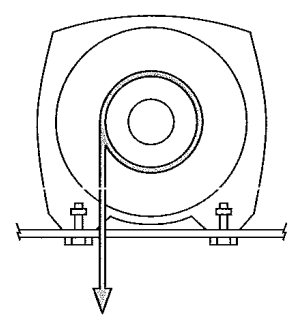

If possible, a compression-type mount (unit on top of the mounting plate) should be used This is the strongest and safest type of mount. There must be

adequate clearance for the wire rope to pass without touching the mounting plate.

\section{Tension-Type Mount}

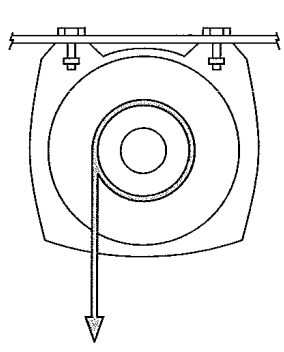

Use a tension type mount (unit below the mounting plate) when a compression mount is not possible. This is the second best mounting method.

\section{Shear-Type Mount}

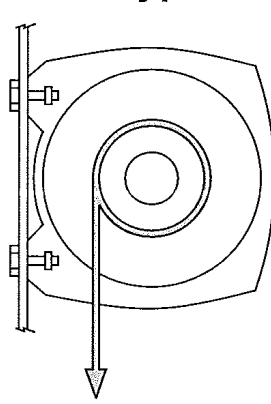

A shear type mount (unit mounted in front of the plate) is the least desirable mount. While the strength of this mount exceeds the unit's rated capacity, it has far less tolerance to shock loads that exceed the rated capacity.

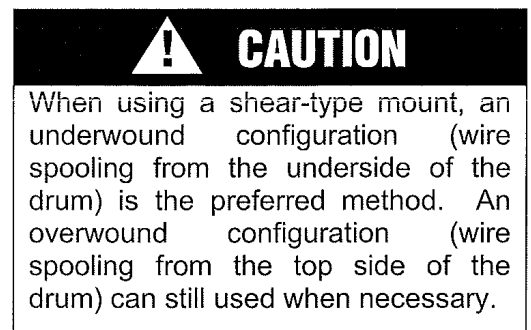

Regardiess of mounting position, the wire rope must be spooled on the drum according to the "Direction of Rotation". Failure to spool rope properly on the drum can result in catastrophic failure of the unit.

\section{Tie Bars}

\begin{tabular}{l} 
Frame 1 Units (HD, WD) \\
\hline $\begin{array}{l}\text { Tie bars on these units are not to be } \\
\text { removed or altered under any } \\
\text { circumstances. }\end{array}$ \\
\hline
\end{tabular}

\section{Frame 2/3 Units (HF, HG, WF, WG)}

Tie bars may be moved to alternate locations for wire rope clearance. At least three tie bars must be used at all times. 


\section{Mounting Holes}

The unit mounting hole pattern is of critical importance to the strength of the unit. Mounting holes MUST match the template and/or drawings supplied with this owner's manual. Any deviation from the mounting dimensions or instructions can cause the drum supports to break under load. This can create an unsafe working condition causing serious injury or property damage.

The unit base MUST be flat in all directions within +0.020 inches. An uneven base can cause binding, twisting, excessive wear or catastrophic failure of the gear train and ultimately the unit. If necessary, use shim stock to ensure even mounting.

Use the fasteners provided to mount the unit. Tighten all nuts and/or bolts to $33 \mathrm{ft}$. Ibs (4.6 KgM.) of torque.

\section{WARIIING}

Do not weld any fastener used for attaching the unit or mounting plate. Welding makes the fasteners brittle and subject to failure even in a no-load situation.

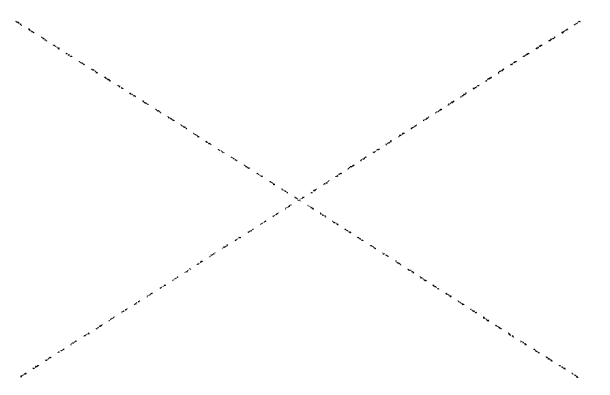

\section{Electrical Guidelines}

This Columbia unit is driven by $\mathrm{AC}$ power. Input voltage is listed on the front of this manual.

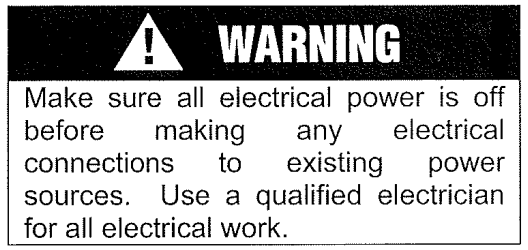

The use of extension cords may adversely affect the performance of the hoist.

\begin{tabular}{l}
\hline Full-Voltage Control Models \\
\hline These units are shipped pre-wired and \\
"ready to run." Refer to the supplied \\
electrical diagram.
\end{tabular}

\section{Low-Voltage Control Models}

Make all electrical connections according to the supplied wiring diagram. All applicable local codes and regulations must be followed during installation of the unit.

These units are shipped pre-wired to the $A C$ motor and the low-voltage pendant control.

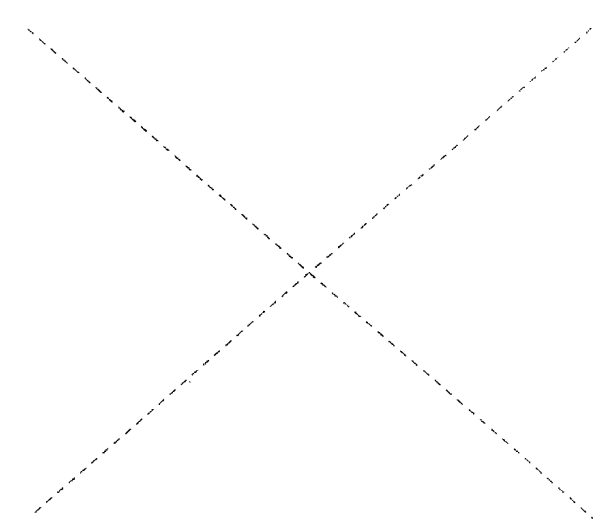




\section{General Safety Precautions}

1. Never put any type of cutoff device between the motor and controls. This could result in equipment failure or severe personal injury.

2. Install proper branch circuits, disconnect devices, protection and grounding in accordance with all applicable electric codes and regulations. Amperage and voltage information is listed on the motor name plate.

3. A power shut-off switch must be visible, clearly marked and within 25 feet of the operator. If the unit is permanently wired into a junction box the installation of a master power shut-off is required.

4. Never open the control box with electrical power on or manually operate the relays inside the box. This could cause equipment failure or serious personal injury.

5. Never put any type of cutoff device between the motor, control box and/or pendant control. This could result in equipment failure or severe personal injury.

6. The use of extension cords may adversely affect the function of the unit.

\section{Wire Rope}

The wire rope must be spooled on the drum according to the direction or rotation label on the unit or the drum brake will not function.

The wire rope anchor, which attaches the first wrap of the wire rope to the drum, is not designed to hold rated loads. Keep a minimum of five wraps of wire rope on the drum at all times to achieve rated load.

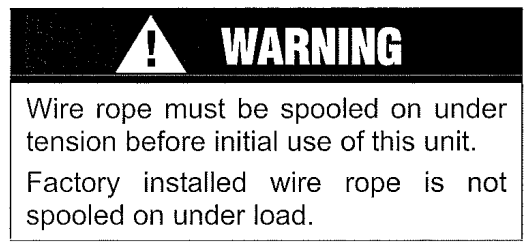

To correctly spool the wire rope, it is necessary to unwind the wire rope and then rewind the wire rope under load. It is recommended that during the initial tensioning of the wire rope a load of approximately $15 \%$ of the rated first layer line puil be used. in the event that tension is taken off the line, the wire rope must be retensioned according to the above guidelines.

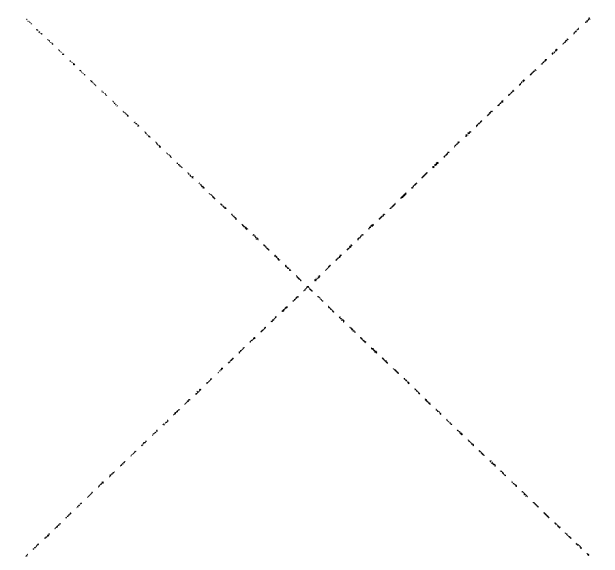




\section{Operating Instructions}

The safe operation of this product is solely dependent upon the owner and/or operator's skill, knowledge and judgment. Only those qualified and familiar with all operating guidelines, safety codes and regulations related to rigging, wire rope and winch and/or hoist usage should operate this unit.

The determination of suitability for this product for any specific use is the responsibility of the owner and/or operator.

The following guidelines are to be used only as a reference.

\section{Preparing for Operation}

Inspect the condition of all components, including mounting bolts, electrical connections, sheaves, wire rope, hook, rigging, etc.

Verify the line pull required to move your desired load and make sure you do not exceed the load rating of the unit.

Test for proper forward and reverse operation without a load.

\section{Operating the Unit}

Ensure that the load is free of objects around it.

Connect the load to hook with a sling or other approved device. Never hook the wire rope around a load and onto itself.
Use the control switch on the supplied pendant intermittently to take up wire rope slack to avoid shock loads that can momentarily exceed the unit and wire rope rating. Move the load slowly at first to make sure the load is securely attached to the hook.

Do not allow the hook to be any closer than two feet from the drum to prevent damage to the unit and wire rope.

Always inspect and carefully rewind the wire rope after each use. Improperly spooled, kinked or tangled wire rope will damage and shorten the life of the wire rope.

\section{Remote Pendant Control}

The remote pendant control is used to "power in" and "power out" the wire rope. Depressing the power in button will cause cable to wind on the drum and depressing the power out button will cause cable to wind off the drum. Each button is springloaded and will automatically return to the 'OFF' position when released. Do not attempt to push both buttons at the same time. If the buttons do not work properly, STOP USING THE UNIT IMMEDIATELY and contact your dealer. DO NOT OPERATE THE UNIT until the problem is corrected. 


\section{Duty Cycle}

\begin{tabular}{l} 
Frame 1 Units (HD, WD) \\
\hline Do not use these units continuously under \\
any circumstances. Recommended use is \\
15 minutes out of each hour on an \\
intermittent basis. \\
Continuous duty usage can cause \\
undetectable damage and lead to failure of \\
the brake and motor.
\end{tabular}

\section{Frame 2/3 Units (HF, HG, WF, WG)}

These units are rated for continuous duty and may be operated up to 40 minutes out of each hour.

Factors such as altitude, ambient temperature, air quality, fluctuations in voltage, motor cleanliness and the frequency of start/stop cycles all affect the duty cycle.

\section{Braking System}

Winches (WD, WF, WG)

Any time the winch is stopped, the drum brake will automatically engage against the load.

\section{Free-spool Control}

The free-spooling control allows wire rope to be pulled off the drum manually.

DO NOT attempt to disengage the freespool control when there is a load on the wire rope.

DO NOT use the load to assist in pulling cable off the drum.

DO NOT attempt to re-engage the freespool control while the drum is turning.

DO NOT attempt to use the free-spool control to stop the drum from turning.

Any of these actions can cause severe damage to or failure of the winch.

\section{Hoists (HD, HF, HG)}

Any time the hoist is stopped, the brake will automatically engage against the load.

The manual release on the motor/brake assembly will disengage the brake in emergency situations.

Never leave any load unattended without properly securing it.

\section{Operating Safety Precautions}

1. Never touch the wire or rigging while another person is at the control switch or at anytime while operating the unit.

2. Never attempt to puil a load with less than five wraps of wire rope around the drum. Fewer wraps could cause the wire rope anchor to break and release the cable and load.

3. Koop the operator, assistants and spectators at a safe distance from the wire rope and rigging and out of the path of the load during the lifting operation. If the wire rope breaks or pulls loose under load, it can lash back with enough force to cause property damage, injury or even death.

4. Keep hands away from wire rope and hook while operating the unit. Never let the wire rope slide through your hands. Always wear heavy leather gloves when handling wire rope.

5. Never touch the wire rope or hook while they are under load. 


\section{General Maintenance}

Maintenance of this product is the sole responsibility of the owner and/or operator.

The determination of suitability for this product for any specific use is the responsibility of the owner and/or operator.

The following guidelines are to be used only as a reference.

Periodically inspect the unit for general wear and tear. Immediately determine the cause of any change in performance or sound during operation and take corrective action as required.

Keep unit, wire rope and remote control free from contaminants. Use a clean rag or towel to remove any dirt and debris. If necessary, unwind unit completely, wipe clean, and rewind properly before storage.

Inspect the remote control and/or pendant for damage. Store remote control and/or pendant in protected, clean, dry area whenever possible.

This unit is of sealed construction. No internal lubrication by the owner is needed for the life of the unit.

\section{Wire Rope}

Inspect the wire rope before and after each operation. The life of the wire rope is directly related to the care and use it receives. Wire rope must be replaced when it has one or more of the following defects as defined in the American National Standards Industry Handbook A10.5 or as defined by OSHA:

- Corrosion

- Frayed or broken wire

- Abrasions

- Kinking

- Heat damage

- Any apparent reduction of rope diameter.

STOP USING THE UNIT AND REPLACE THE WIRE ROPE if any of the defects listed above are discovered.

Using light oil on the wire rope and hook can keep rust and corrosion from forming.

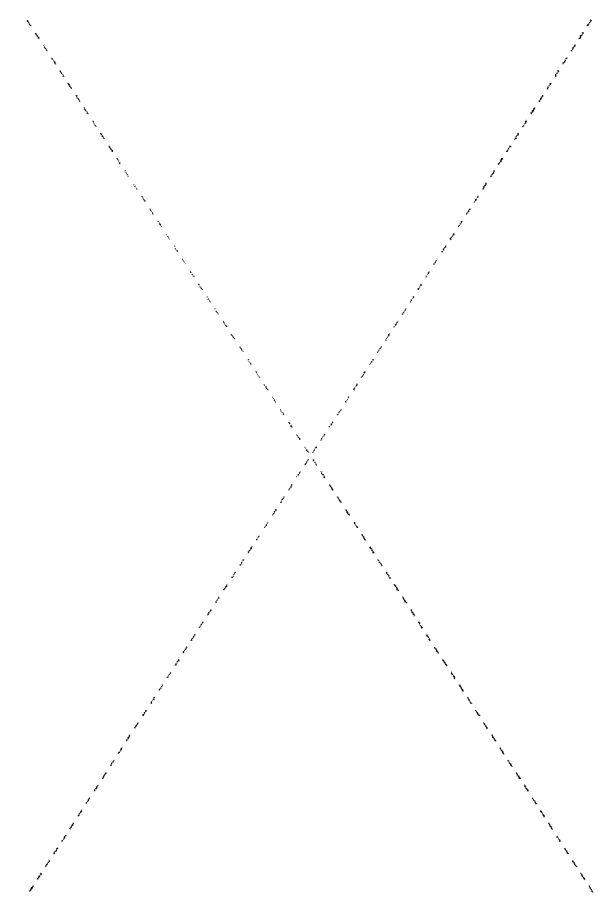




\section{Trouble-Shooting Guide}

\begin{tabular}{|c|c|}
\hline Problem & Possible Cause \\
\hline $\begin{array}{l}\text { Unit will not produce line pull/line speed } \\
\text { as listed }\end{array}$ & $\begin{array}{l}\text { a) Unit is mounted to an uneven surface } \\
\text { b) Cable sheaves or block purchase not turning freely } \\
\text { c) Load is constrained } \\
\text { d) Inadequate voltage }\end{array}$ \\
\hline Unit overheats prematurely & $\begin{array}{l}\text { a) Environmental factors (altitude, ambient temperature, air } \\
\text { quality, fluctuations in voltage, motor cleanliness) } \\
\text { b) Start/stop cycles too frequent to allow proper spool-up of } \\
\text { unit } \\
\text { c) Load beyond rated capacity }\end{array}$ \\
\hline Unit does not respond to control input & $\begin{array}{l}\text { a) Limit switch contacts are OPEN } \\
\text { b) Unit is not correctly connected to power source }\end{array}$ \\
\hline Drum brake does not hold & a) Wire rope wound onto the drum in the wrong direction \\
\hline Line stacks against flanges & $\begin{array}{l}\text { a) Unit is not level } \\
\text { b) Line leads to a point not in line with the drum } \\
\text { c) Distance to cable sheaves, block purchase or load } \\
\text { inadequate for proper fleet angle }\end{array}$ \\
\hline Line spools poorly & $\begin{array}{l}\text { a) Line tension was lost } \\
\text { b) Wire rope not installed properly } \\
\text { c) Tension not adequate to spool wire rope on tightly } \\
\text { d) Distance to cable sheaves, block purchase or load } \\
\text { inadequate for proper fleet angle }\end{array}$ \\
\hline Line stacks against flanges & $\begin{array}{l}\text { d) Unit is not level } \\
\text { e) Line leads to a point not in line with the drum }\end{array}$ \\
\hline Line spools poorly & $\begin{array}{l}\text { e) Line tension was lost } \\
\text { f) Tension not adequate to spool wire rope on tightly } \\
\text { g) Distance to cable sheaves, block purchase or load } \\
\text { inadequate for proper fleet angle }\end{array}$ \\
\hline Unit will lower load, but will not raise load & $\begin{array}{l}\text { a) Load is beyond rated capacity } \\
\text { b) Load is constrained } \\
\text { c) Upper limit switch contact is OPEN }\end{array}$ \\
\hline \multicolumn{2}{|l|}{ Units with Motor Brakes } \\
\hline Motor brake does not hold & $\begin{array}{l}\text { a) Wire rope wound onto the drum in the wrong direction } \\
\text { b) Manual release lever is engaged } \\
\text { c) Brake lining is worn } \\
\text { d) Improper gap adjustment }\end{array}$ \\
\hline Motor brake drags/overheats & $\begin{array}{l}\text { a) Unit operated beyond suggested duty cycle } \\
\text { b) Solenoid which releases brake gets inadequate voltage } \\
\text { (use of extension cords is a common cause) } \\
\text { c) Solenoid which releases brake has failed } \\
\text { d) Improper gap adjustment } \\
\text { e) Damage to fan or fan cover }\end{array}$ \\
\hline
\end{tabular}




\section{LIMITED WARRANTY}

Allied Power Products, Inc. (APPI) warrants the products it manufactures to be free from defects in material and workmanship to the original buyer for a period of 12 MONTHS from the date of shipment from APPI. All warranties for products sold but not manufactured by $\mathrm{APPI}$ are solely that of the manufacturer.

This warranty and liability of APPI is limited to the replacement or repair of any product manufactured by APPI if the product is found - upon examination at our facility - defective due to materials or workmanship. All freight, removal and/or installation charges shall be borne by the Buyer.

This warranty does not cover failures or malfunctions found by APPI to result from:

- Improper installation, operation and/or maintenance of the product.

- Replacements, repairs and/or alterations made by or on behalf

of the buyer without written approval from APPI.

- Use of accessories and/or other components in conjunction with the product without written approval from APPI.

APPI SHALL NOT IN ANY EVENT BE HELD LIABLE FOR ANY CONSEQUENTIAL OR INCIDENTAL DAMAGE OR FOR EXPENSES OR DELAY CAUSED BY DEFECTIVE MATERIAL OR WORKMANSHIP.

Except for the above warranty, APPI makes no other express or implied warranties and NO WARRANTY OF MERCHANTABILITY OR FITNESS FOR A PARTICULAR PURPOSE.

This warranty shall be governed by and construed in accordance with the laws of the State of Oregon and enforced in Oregon courts. If any portion of this limited warranty and limitation on damages is determined to be invalid or unenforceable, the remainder of the warranty shall remain in full force and effect.

All warranty claims must be submitted to APPI in writing to:

Allied Power Products, Inc.

6590 SW Fallbrook Place

Beaverton, OR 97008

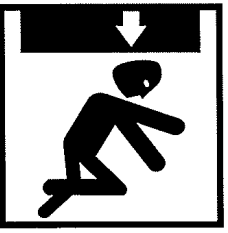

WABNWE WARNING WARNING

COLUMBIA PRODUCTS ARE NOT TO BE USED FOR LIFTING PEOPLE OR THINGS OVER PEOPLE 
Development of the West Virginia University

Small Microgravity Research Facility (WVU SMiRF)
Copyright $^{\odot} 2014$

Kyle G. Phillips

\section{ALLIED POWER PRODUCTS, INC.}

THE WINCH \& HOIST SPECIALISTS

6590 SW Fallbrook Place, Beaverton, OR 97008

Toll Free (800) 248-4896 Fax (503) 646-1996

wWw. alliedpower.com

(C) February 2008 


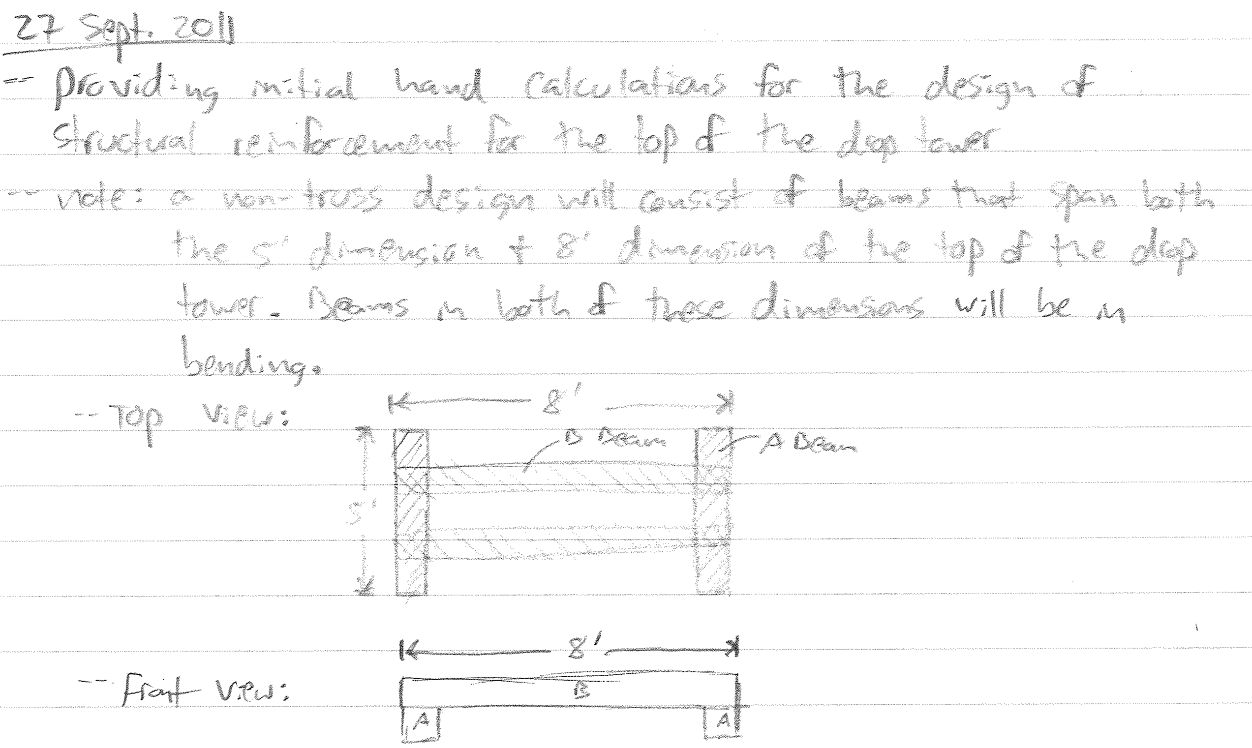

-cousdering the A-Beaw:

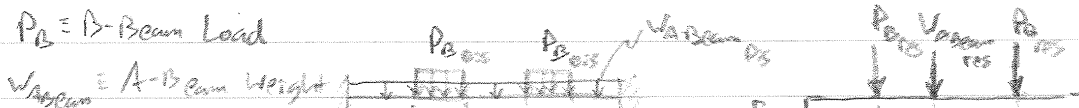

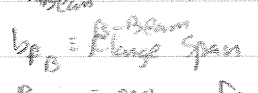

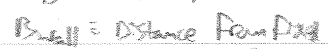
Find be is them b. Wroptame to ower

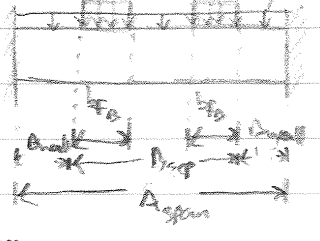
$\Rightarrow \rightarrow$

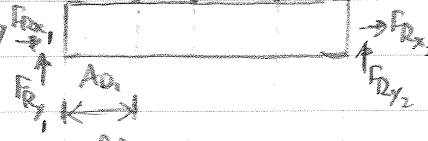

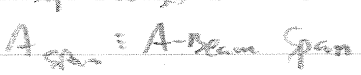

$$
\begin{aligned}
& A D_{1}=B \text { mant }+\angle b_{F_{B}}
\end{aligned}
$$

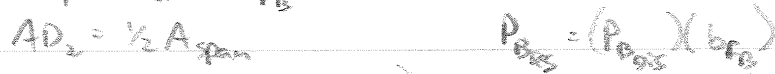

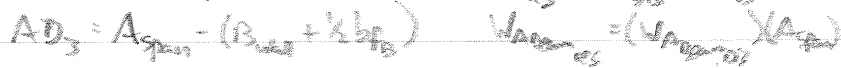

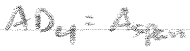

$$
\begin{aligned}
& F_{R_{x_{1}}}=-F_{R_{x_{2}}}=0
\end{aligned}
$$

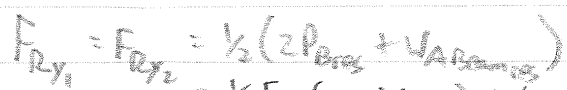

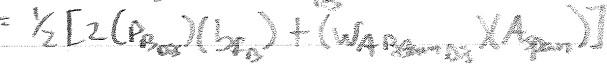




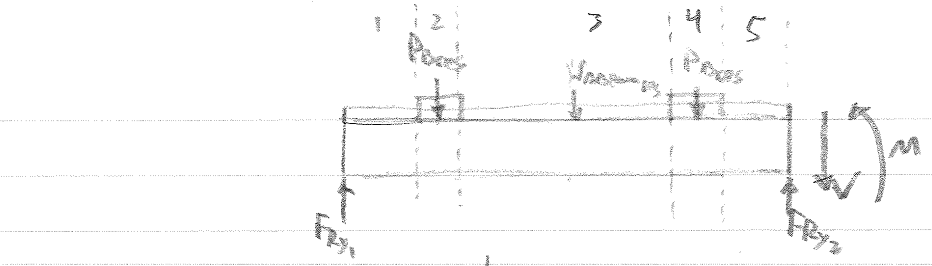

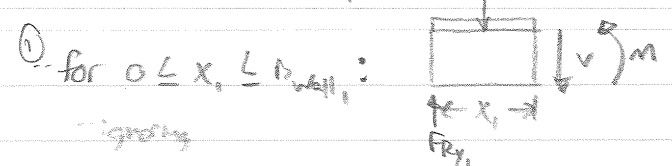

$$
\begin{aligned}
& v=f_{R_{y_{1}}}-\left(w_{\text {A Beandis }}\right)\left(x_{1}\right)
\end{aligned}
$$

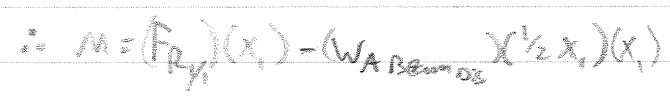

$$
\begin{aligned}
& M=-(1 / 2)\left(\text { waBam }_{\text {A }}\right)\left(x_{1}^{2}\right)+\left(f_{R_{y_{1}}}\right)\left(x_{1}\right)
\end{aligned}
$$

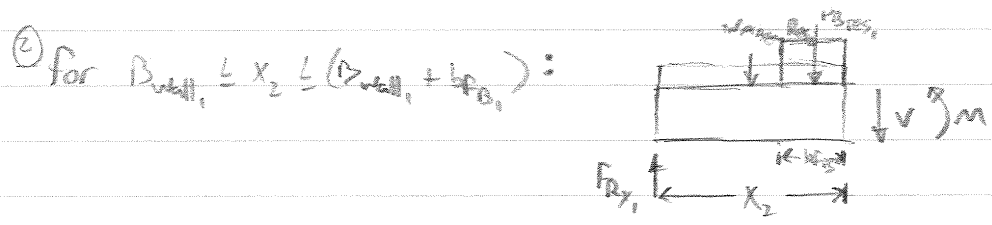

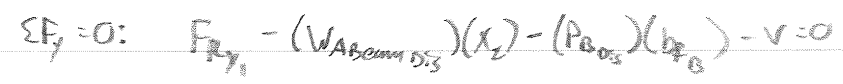

$$
\begin{aligned}
& V=F_{B_{1}}-\left(W_{A B \operatorname{sen}}\right)\left(x_{2}\right)-\left(P_{B_{B}}\right)\left(h_{A_{B}}\right)
\end{aligned}
$$

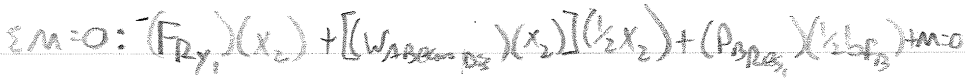

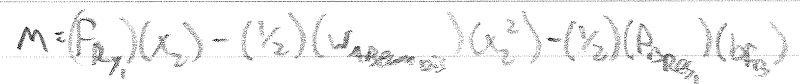

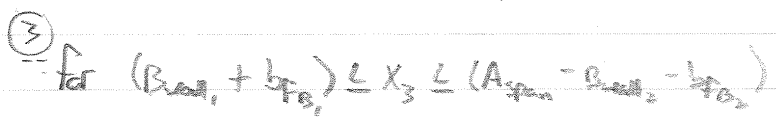

$$
\begin{aligned}
& \sum F_{y}=0:
\end{aligned}
$$

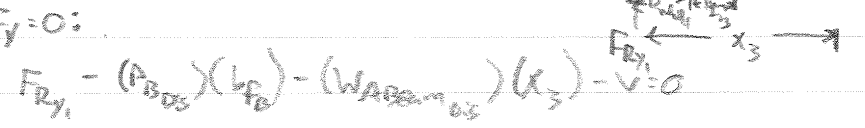

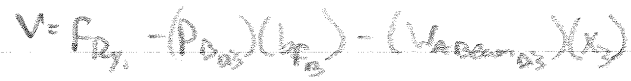




$$
\begin{aligned}
& \sum M=0:
\end{aligned}
$$

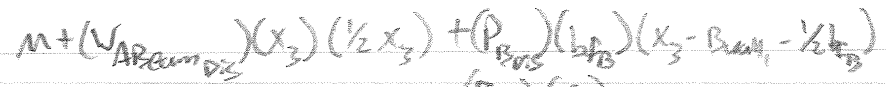

$$
\begin{aligned}
& -\left(f_{2 y}\right)\left(x_{3}\right)=0
\end{aligned}
$$

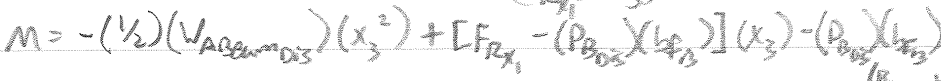

$$
\begin{aligned}
& \text { (4) }
\end{aligned}
$$

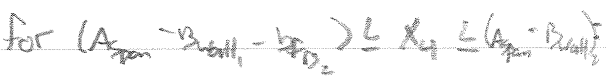

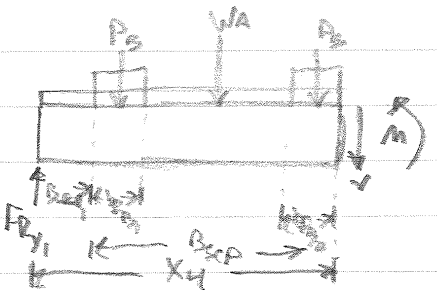

$$
\begin{aligned}
& \sum F=0
\end{aligned}
$$

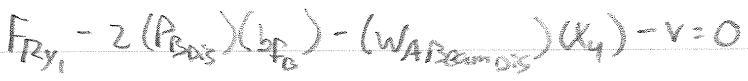

$$
\begin{aligned}
& V=F_{R_{1}}-2\left(P_{R_{0}}\right)\left(b_{f_{B}}\right)-\left(W_{A} \cos _{\text {as }}\right)\left(x_{4}\right)
\end{aligned}
$$

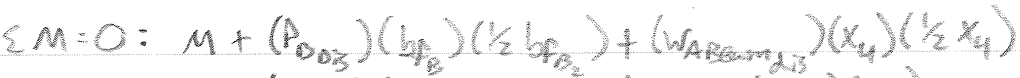

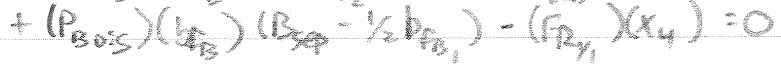

$$
\begin{aligned}
& \text { - Since } b_{\mathrm{F}_{3}}=b_{\mathrm{F}_{\mathrm{B}, 2}} \text {, } \\
& M=(1 / 2)^{2}\left(W_{A B c a m b i}\right)\left(x_{4}^{2}\right)+\left(F_{A_{1}}\right)\left(x_{4}\right)-\left(P_{B D 3}\right)\left(b_{f_{B}}\right)\left(B_{B_{9}}\right) \\
& \text { (5)... For }\left(A_{\text {gan }}-B_{\text {wotl }}\right) \leq x_{5} \leq A_{\text {sian }} \text { : }
\end{aligned}
$$

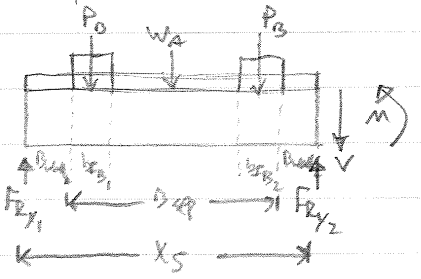

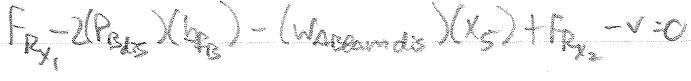

$$
\begin{aligned}
& v=F_{x_{1}}+F_{R_{12}}-2\left(P_{B_{d s}}\right)\left(b_{R_{B}}\right)-\left(w_{\text {Adrom }}\right)\left(x_{5}\right)
\end{aligned}
$$




$$
\begin{aligned}
& \Sigma M=0:
\end{aligned}
$$

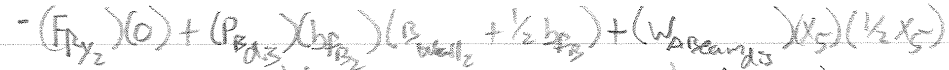

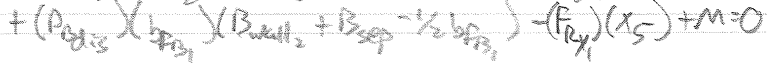

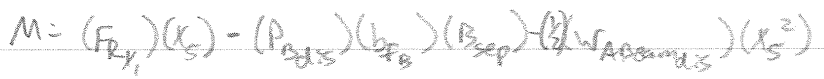

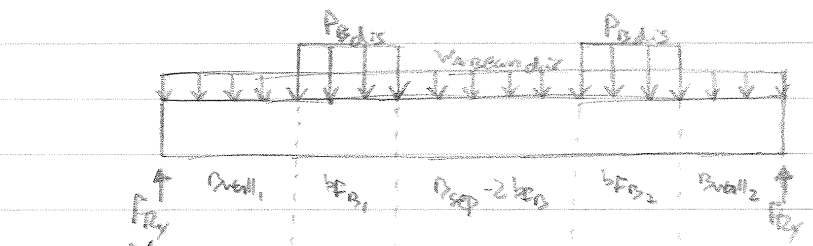

$F_{R y_{1}}=1000$
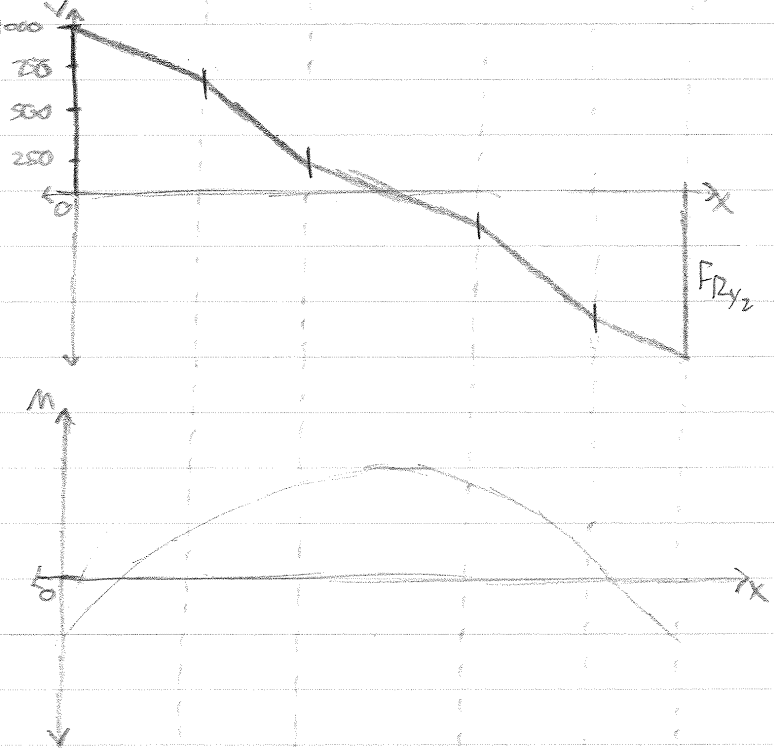
$\varepsilon M=0$ :

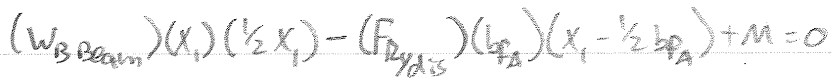

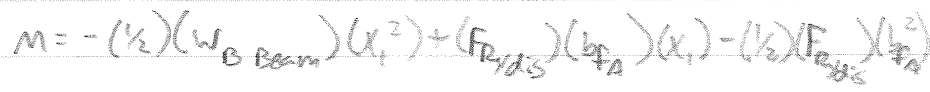

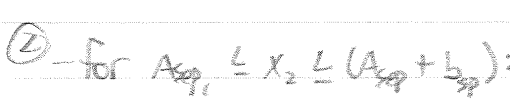

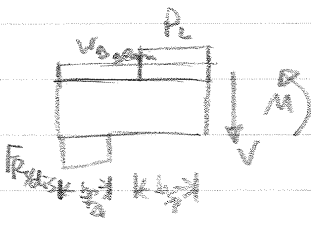

$$
\sum F_{y}=0:
$$$$
1+x_{2} \rightarrow
$$

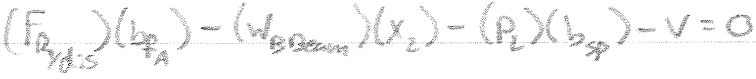

$$
\begin{aligned}
& V=\left(f_{R_{y d s}}\right)\left(f_{f_{A}}\right)-\left(w_{B \text { andin }}\right)\left(x_{2}\right)-\left(P_{2}\right)\left(l_{\text {ap }}\right)
\end{aligned}
$$

$\sum M=0:$

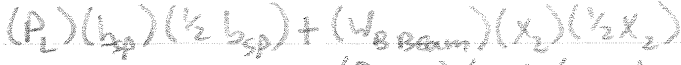

$$
\begin{aligned}
& -\left(f_{R_{13}}\right)\left(b_{B_{A}}\right)\left(x_{2}-1 / L_{f_{A}}\right)+M=0
\end{aligned}
$$




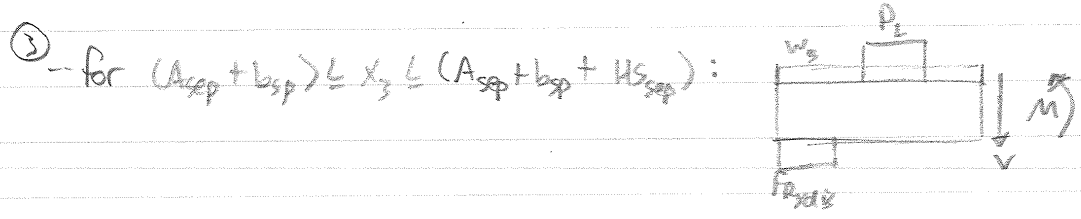

$$
\begin{aligned}
& \Sigma F=0 \text { : }
\end{aligned}
$$

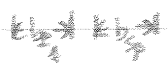

$$
\begin{aligned}
& \left(F_{R_{\text {d d B }}}\right)\left(b_{f_{A}}\right)-\left(u_{B \text { Bam }}\right)\left(x_{3}\right)-\left(P_{L}\right)\left(b_{S P}\right)-r=0 \\
& v=\left(F_{R_{\text {dis }}}\right)\left(b_{f_{A}}\right)-\left(w_{B \text { bram }}\right)\left(x_{3}\right)-\left(P_{L}\right)\left(b_{\text {sp }}\right)
\end{aligned}
$$

$\sum M=0:$

$$
\begin{aligned}
& \left(w_{\text {g pram }}\right)\left(x_{3}\right)\left(1 / 2 x_{3}\right)+\left(p_{2}\right)\left(b_{s p}\right)\left(x_{3}-A_{s p p}-1 / 2 b_{s p}\right) \\
& -\left(r_{R_{d i}}\right)\left(b_{B P_{A}}\right)\left(x_{3}-1 / 2 b_{P_{A}}\right)+M=0 \\
& M=-(1 / 2)\left(w_{\text {B Beam }}\right)\left(x_{3}^{2}\right)-\left[\left(P_{2}\right)\left(b_{\text {pp }}\right)+\left(F_{\text {pdis }}\right)\left(\text { b }_{\text {A }}\right)\right]\left(x_{3}\right)
\end{aligned}
$$

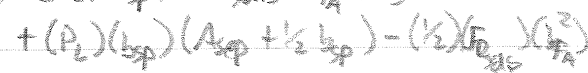

(4)

$$
\begin{aligned}
& \operatorname{fr}\left(A_{\text {spp }}+b_{s p}+H S_{s p p}\right) \leq x_{4} \leq\left(A_{s p p}+b_{s p}+U S_{s p}+b_{\text {sp }}\right):
\end{aligned}
$$

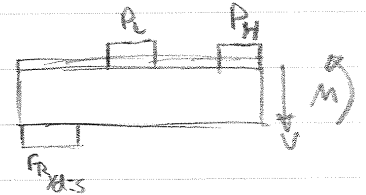

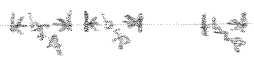

$$
\begin{aligned}
& \text { HA हैता सH: } \\
& +x_{4} \rightarrow \\
& \Sigma F_{y}=0
\end{aligned}
$$

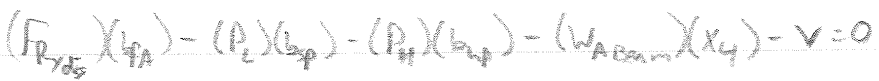

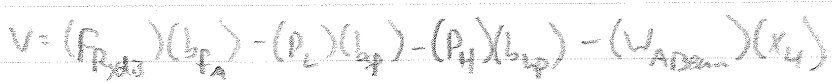




$$
\begin{aligned}
& \sum M=O \text { : }
\end{aligned}
$$

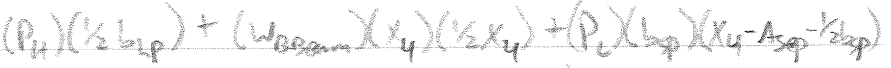

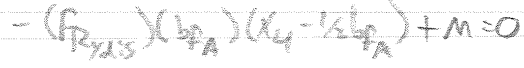

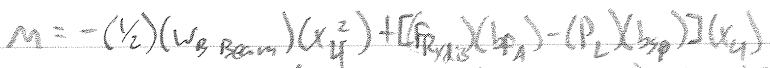

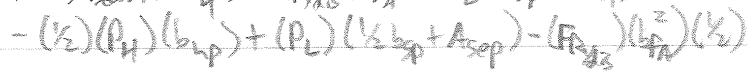

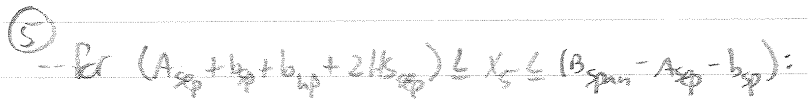

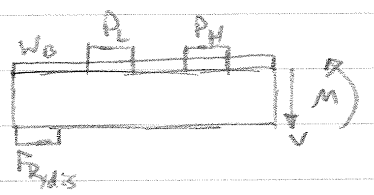

$$
\begin{aligned}
& \Sigma F=0
\end{aligned}
$$

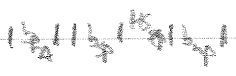

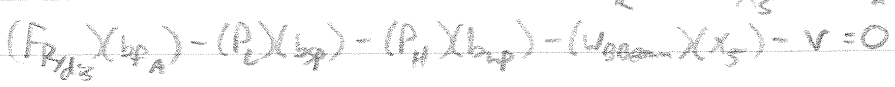

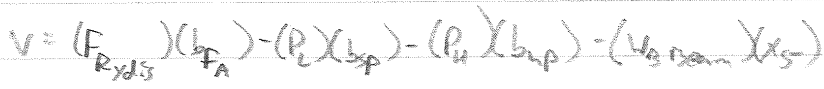

$$
\begin{aligned}
& \sum M=O
\end{aligned}
$$

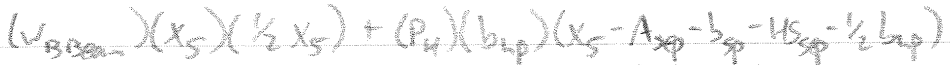

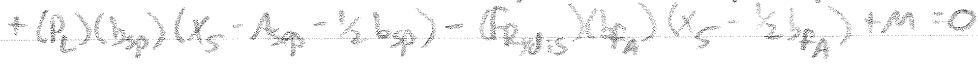

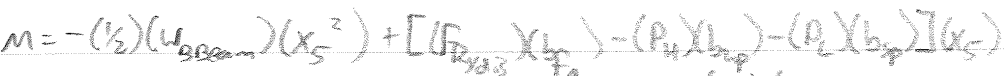

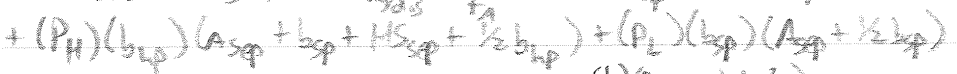

$$
\begin{aligned}
& -(1 / 2)\left(\rho_{\text {meds }}\right)\left(L_{p_{A}}^{2}\right)
\end{aligned}
$$

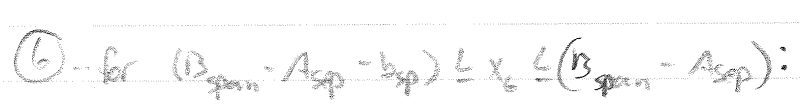

$$
\begin{aligned}
& \sum P_{Y}=0
\end{aligned}
$$

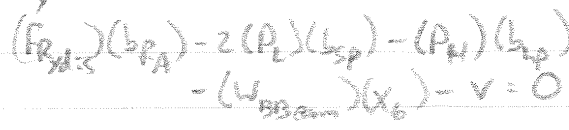

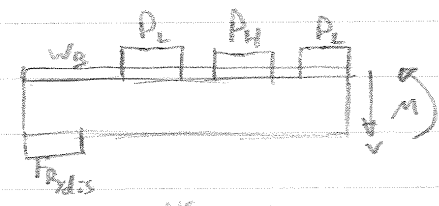

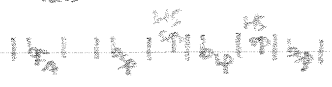

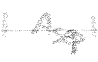

$$
\begin{aligned}
& \text { Herom }-X_{0} \longrightarrow
\end{aligned}
$$




$$
\begin{aligned}
& v=\left(F_{R_{y d i s}}\right)\left(b_{F_{A}}\right)-2\left(P_{L}\right)\left(b_{s p}\right)-\left(P_{A}\right)\left(b_{b_{p}}\right)-\left(w_{B \text { Brem }}\right)\left(x_{G}\right) \\
& \Sigma M=0: \\
& \left(p_{2}\right)\left(b_{p p}\right)\left(1 / b_{p p}\right)+\left(P_{4}\right)\left(b_{u_{p}}\right)\left(b_{x_{p}}+1 / s_{s_{p}}+1 / 2 b_{4 p}\right)
\end{aligned}
$$

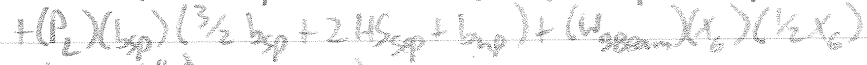

$$
\begin{aligned}
& \left.-\left(f_{R_{\text {dis }}}\right) x_{0}-z_{2} b_{A}\right)+M=0
\end{aligned}
$$

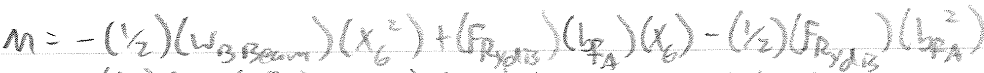

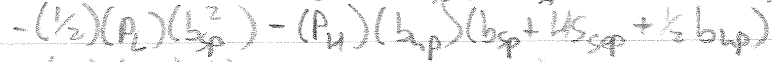

$$
\begin{aligned}
& -\left(p_{2}\right)\left(b_{p p}\right)\left(3 b_{\text {op }}+2 H L_{\text {op }}+b_{\text {np }}\right) \\
& \text { (7) for }\left(B_{\text {span }}-A_{\text {pp }}\right) \leq x_{7} \leq B_{\text {span }} \text { : }
\end{aligned}
$$

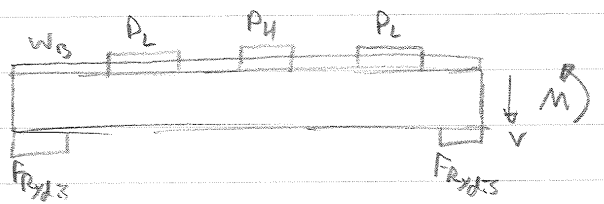

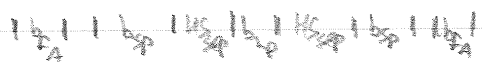

$$
\begin{aligned}
& \text { Asep } \mid \text { AAsal } \\
& \Sigma F_{y}=0: \\
& \text { 近 } \rightarrow \\
& z\left(f_{R_{y d: s}}\right)\left(b_{f_{A}}\right)-2\left(P_{L}\right)\left(b_{s_{p}}\right)-\left(P_{3}\right)\left(b_{L p}\right)-\left(u_{B \text { B seam }}\right)\left(x_{7}\right)-v=0
\end{aligned}
$$

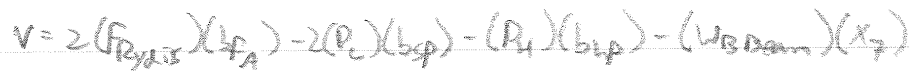

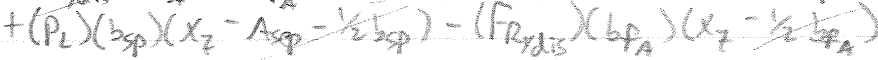

$$
\begin{aligned}
& +\left(\omega_{3} \operatorname{sen}\right)\left(x_{3}\right)\left(1 / 2 x_{7}\right)+n=0
\end{aligned}
$$

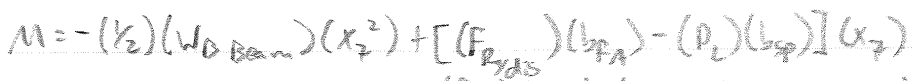

$$
\begin{aligned}
& -\left(P_{H}\right)\left(b_{u p}\right)\left(A_{\text {spp }}+b_{\text {p }}+4 S_{\text {sp }}+1 / 2 b_{\text {pp }}\right)
\end{aligned}
$$


Development of the West Virginia University Small Microgravity Research Facility (WVU SMiRF)
Copyright $^{\odot} 2014$

Kyle G. Phillips

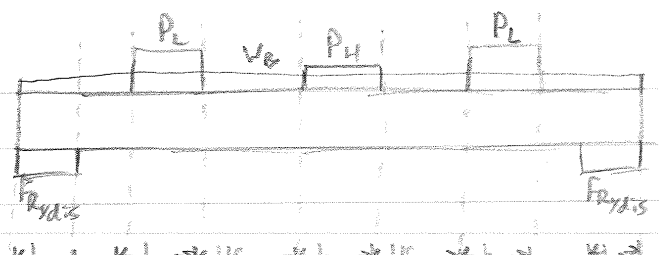

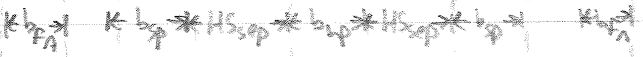

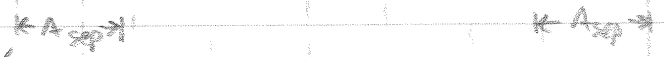

$\mathrm{F}_{4}$

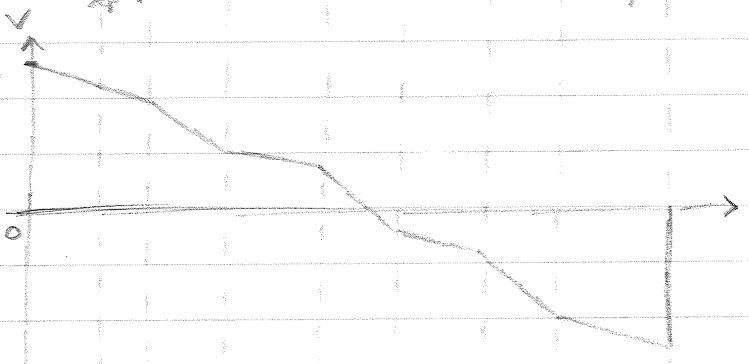

.

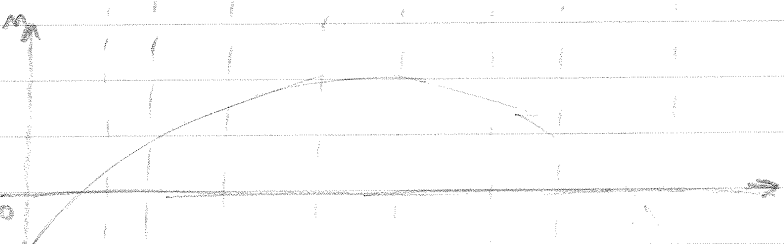




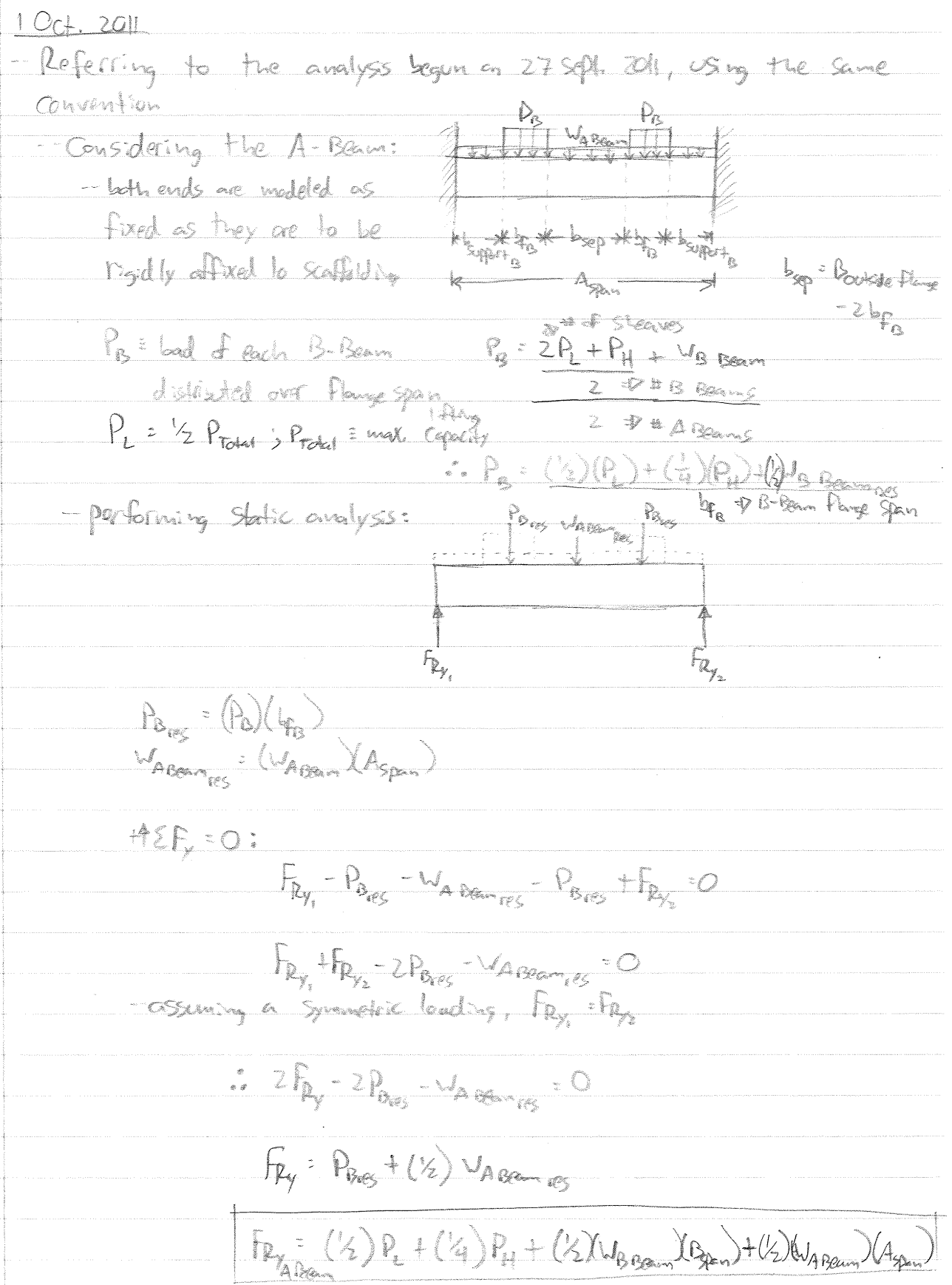




$$
\begin{aligned}
& \text { performing Shear }+ \text { Moment Analysis on ABseam: }
\end{aligned}
$$

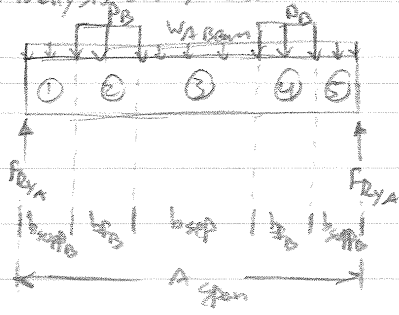

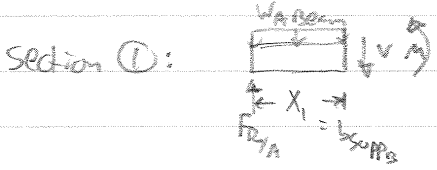

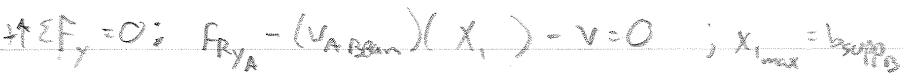

$$
\begin{aligned}
& v_{1}=\left(w_{A \text { Beama }}\right)\left(x_{1}\right)-F_{R_{\text {A }}}
\end{aligned}
$$

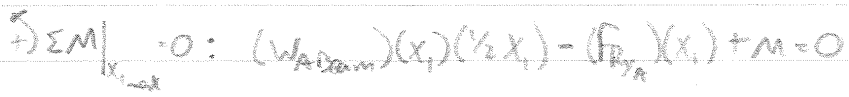

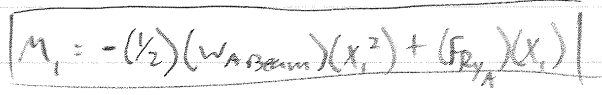

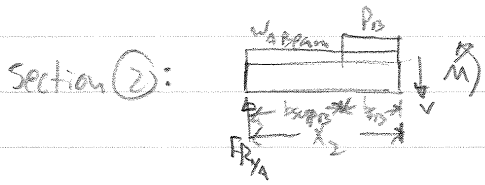

$$
\begin{aligned}
& A \varepsilon_{F_{y}}=0: F_{R_{y}}-\left(W_{A \text { Bram }}\right)\left(x_{2}\right)-\left(P_{B}\right)\left(b_{F_{B}}\right)-v=0
\end{aligned}
$$

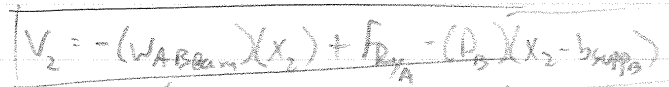

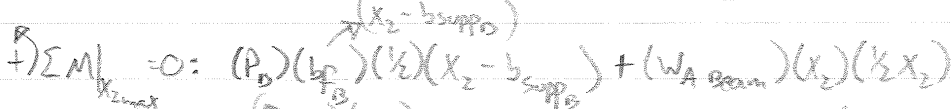

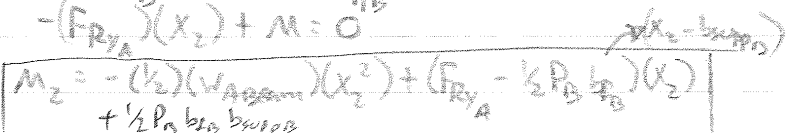




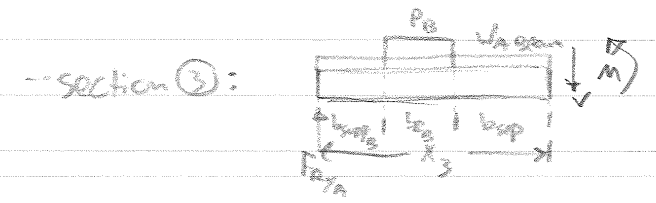

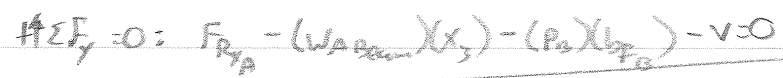

$$
\begin{aligned}
& V_{3}=-\left(w_{A_{\text {Beam }}}\right)\left(x_{3}\right)-\left(P_{B}\right)\left(b_{P_{B}}\right)+F_{R_{Y_{4}}}
\end{aligned}
$$

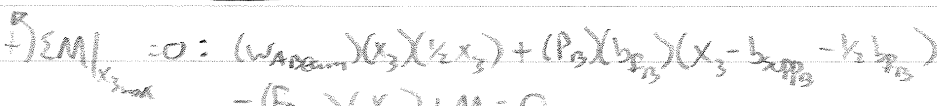

$$
\begin{aligned}
& -\left(f_{Q_{4}}\right)\left(x_{3}\right)+M=0 \\
& \text { - ide: postable sublicution for be }
\end{aligned}
$$

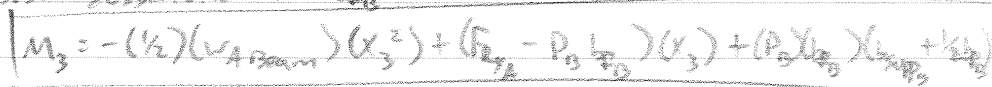

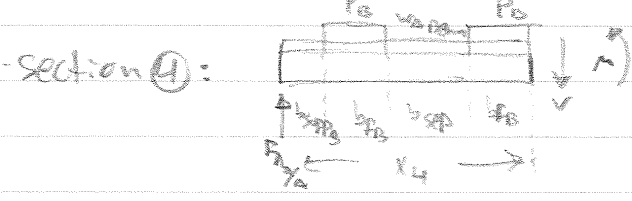

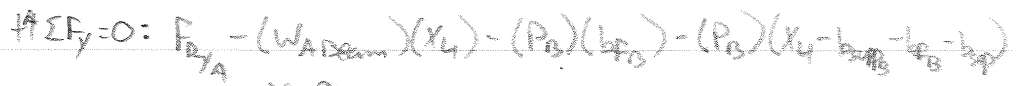

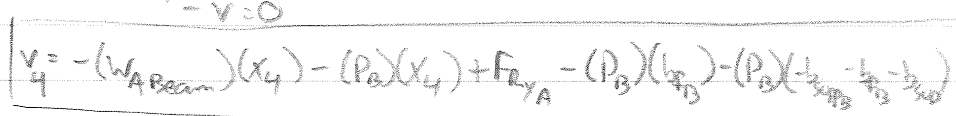

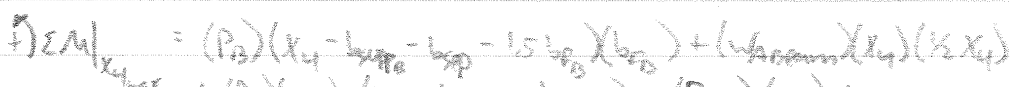

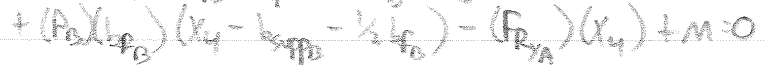

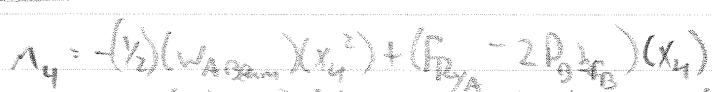

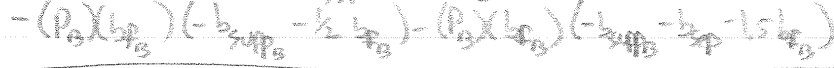




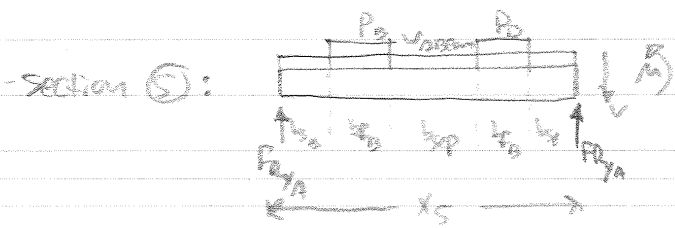

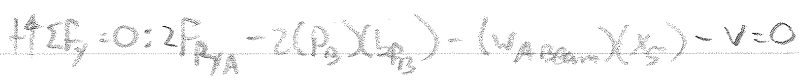

$$
\left|V_{5}=-\left(w_{A B \text { Bam }}\right)\left(X_{5}\right)+2 f_{B_{A}}-2\left(P_{B}\right)\left(L_{B}\right)\right|
$$

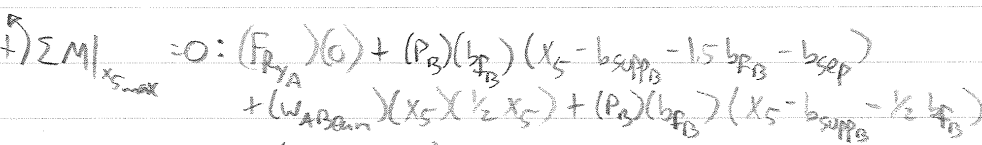

$-\left(f_{R_{x_{A}}}\right)\left(x_{5}\right)+m=0$

$$
\begin{aligned}
& M_{5}=-(1 / 2)\left(w_{A \text { Baw }}\right)\left(x_{5}^{2}\right)+\left(f_{B_{A}}-2 P_{B} b_{B}\right)\left(x_{5}\right)
\end{aligned}
$$

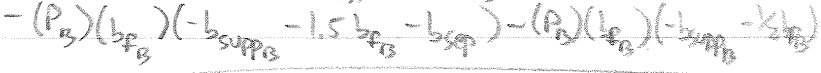

- Please note: possible $b_{B}$ substitutions to be a function of $X$

Note: Graphs Should look similar to those derived on 27 ept. 2011

- Performing shear + Mownowt Analyss an B-Bewant?

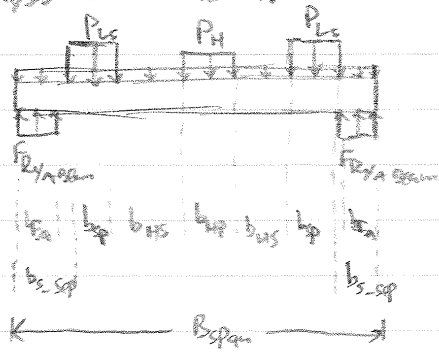




$$
\begin{aligned}
& \text { Porforming talic cunalysis: } \\
& +4 \sum F_{y}=0:
\end{aligned}
$$

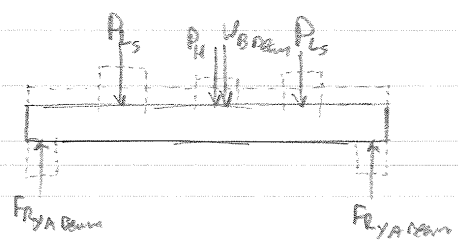

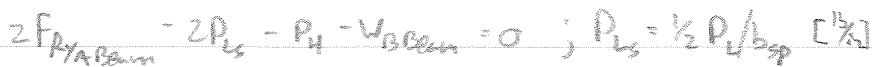

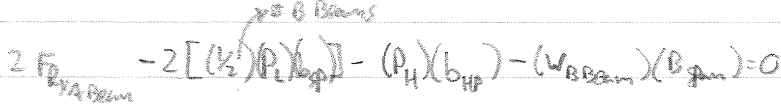

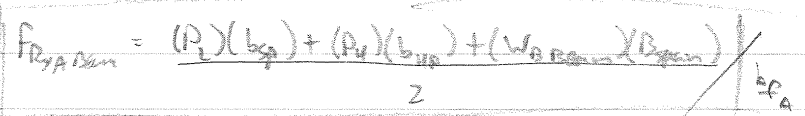

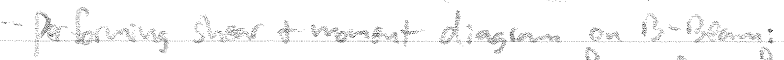

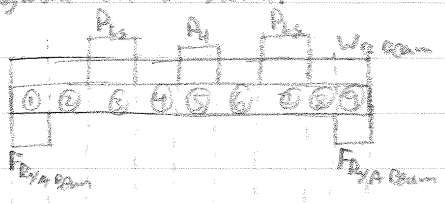

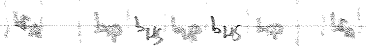

$$
\begin{aligned}
& \text { bxal } \\
& \longleftrightarrow \text { gan } \longrightarrow
\end{aligned}
$$

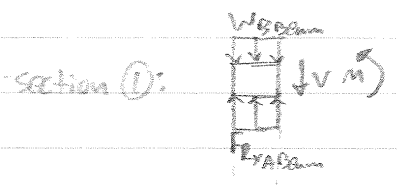

$$
\begin{aligned}
& +\sum f_{y}=0 \text { : } \\
& -\left(w_{B \text { Batant }}\right)\left(x_{p}\right)+\left(f_{R_{\text {YA Beam }}}\right)\left(x_{1}\right)-v=0
\end{aligned}
$$

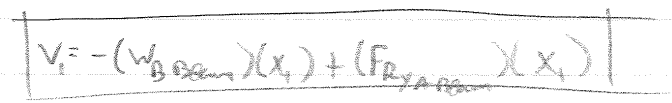

$$
\begin{aligned}
& +\sum \sum M_{x_{1-6 x}}=0 \text { : }
\end{aligned}
$$

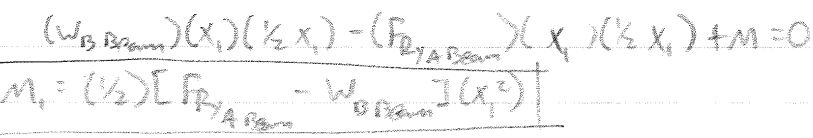




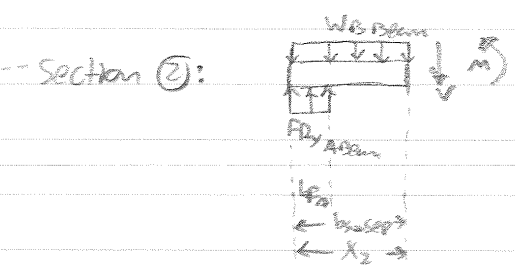

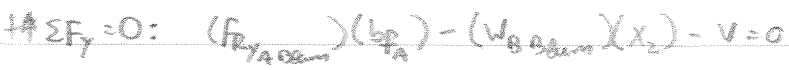

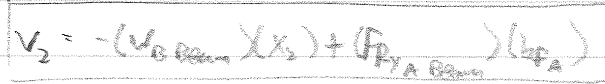

$+\sum_{\Sigma M_{z_{\text {max }}}=0}$

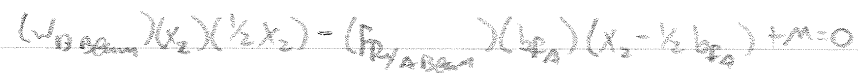

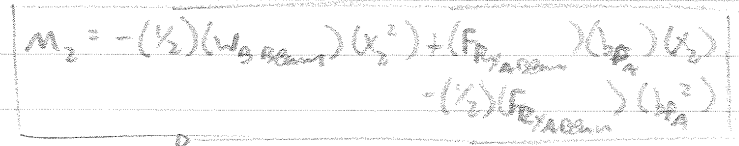

- Section (3):

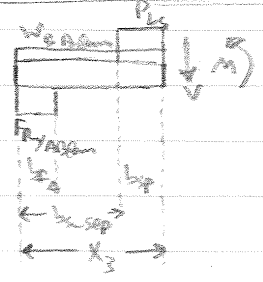

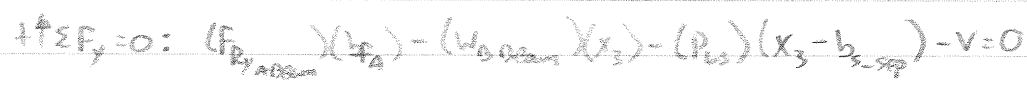

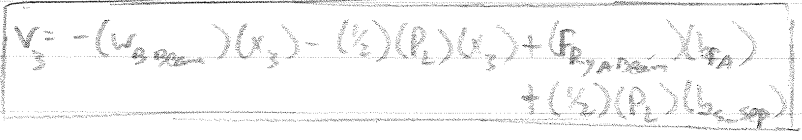

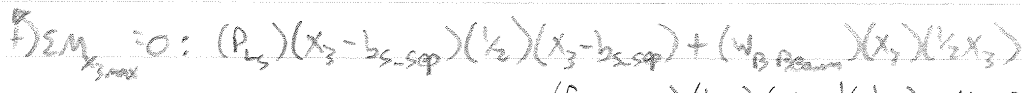

$$
-\left(f_{R_{1} \text { A Beam }_{\text {B }}}\right)\left(b_{P_{A}}\right)\left(x_{3}-1 / 2 b_{A}\right)+M=0
$$

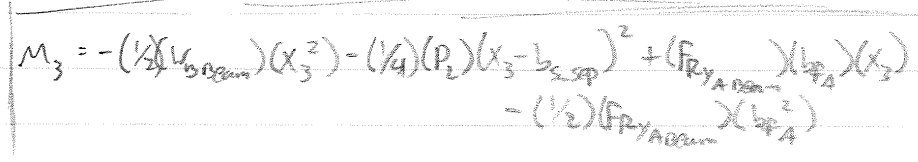




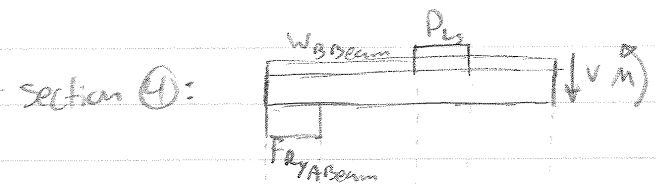

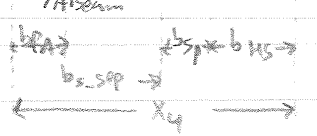

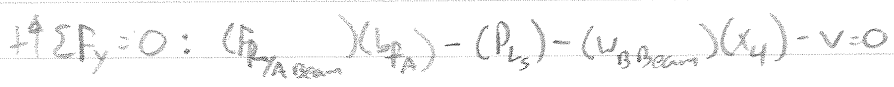

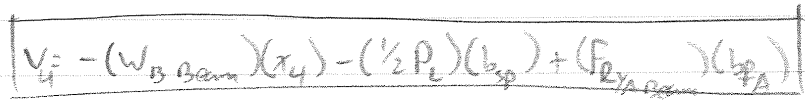

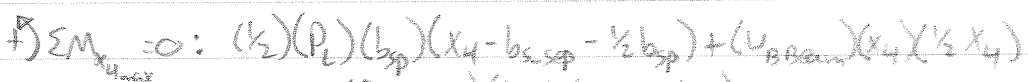

$$
\begin{aligned}
& -\left(h_{y_{A}}\right)\left(b_{A}\right)\left(X_{4}-b_{3} b_{A}\right)+M=0
\end{aligned}
$$

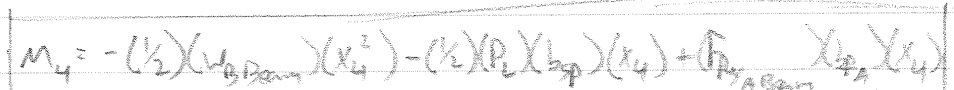

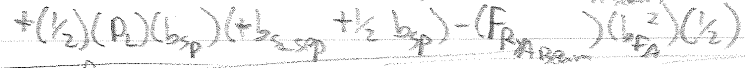

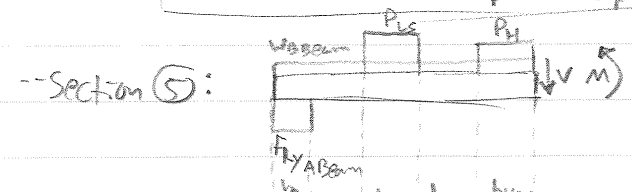

$$
\begin{aligned}
& \text { is the the but } \\
& 2 b_{2}=x_{0} \text {. }
\end{aligned}
$$

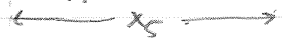

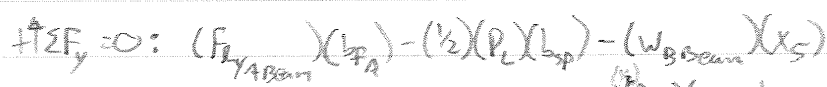

$$
\begin{aligned}
& -\left(P_{11}\right)\left(x_{5}-b_{25}-b_{2}-b_{4}\right) \cdot v_{0}
\end{aligned}
$$

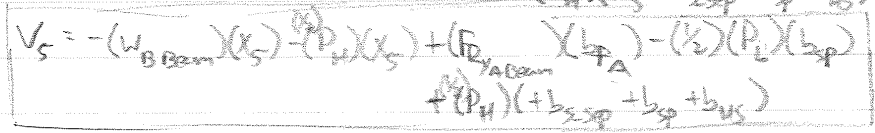

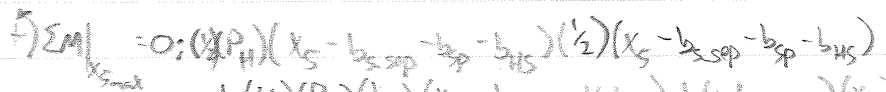

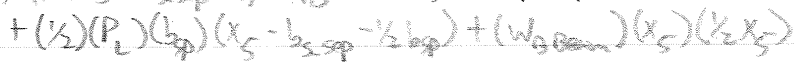

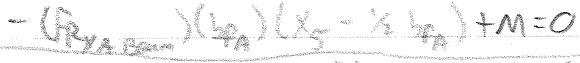

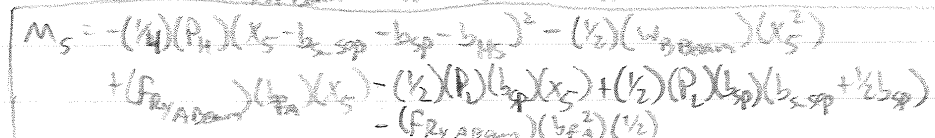




$$
\begin{aligned}
& \text { - section (6): } \\
& \text { Pryations } \\
& \text { by ktsp* bus * but wost } \\
& x_{6} \rightarrow
\end{aligned}
$$

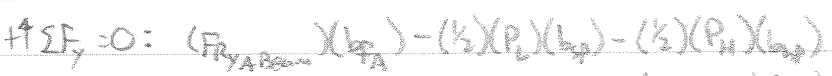

$$
\begin{aligned}
& -\left(w_{3}+x_{0}\right)-v=0
\end{aligned}
$$

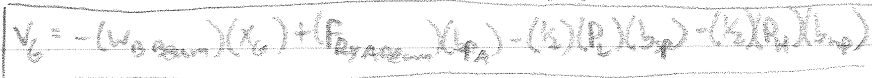

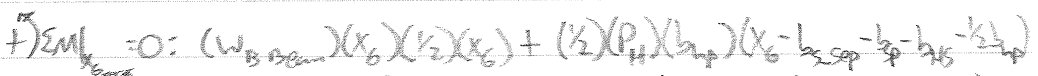

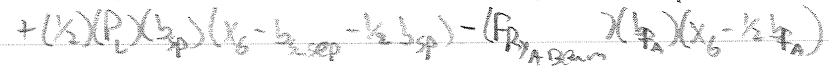

$$
\begin{aligned}
& M_{6}=-(1 / 2)\left(w_{6},\left(x_{6}^{2}\right)-(1 / 2)\left(P_{4}\right)\left(b_{4}\right)\left(x_{6}\right)-(1 / 2)\left(P_{2}\right)\left(b_{4}\right)\left(x_{6}\right)\right.
\end{aligned}
$$

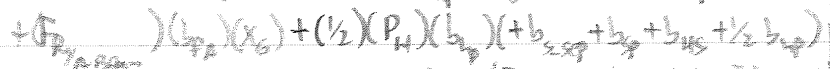

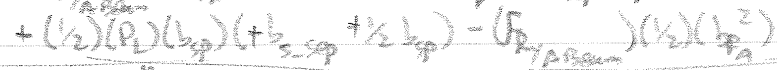

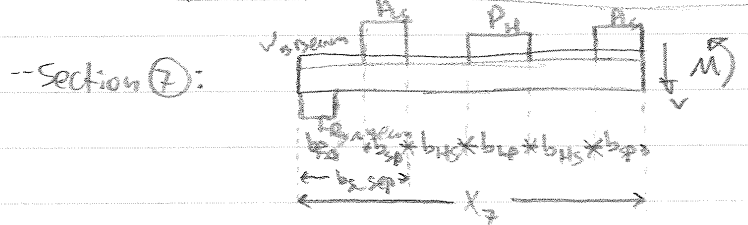

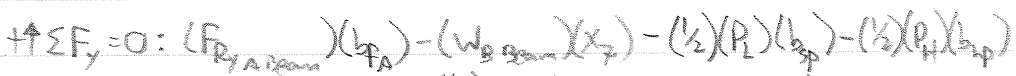

$$
\begin{aligned}
& -(1 / 2)\left(p_{1}\right)\left(x_{7}-b_{2} \operatorname{ses}-2 b_{45}-b_{4 p}-b_{p}\right)-v=0
\end{aligned}
$$

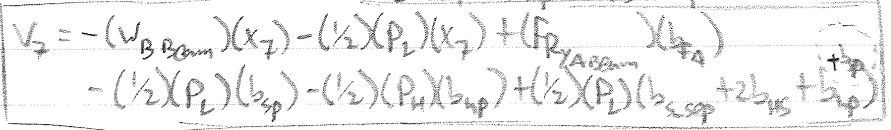

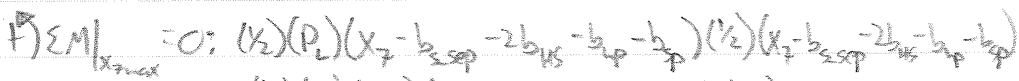

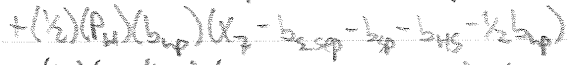

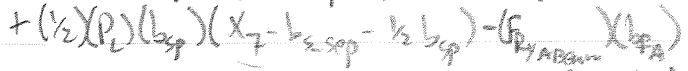

$$
\begin{aligned}
& +\left(\operatorname{waram}_{1}\right)\left(x_{7}\right)\left(1 / 2 x_{7}\right) \\
& \left(x_{3}-b_{2} b_{n}\right) \sin 0
\end{aligned}
$$




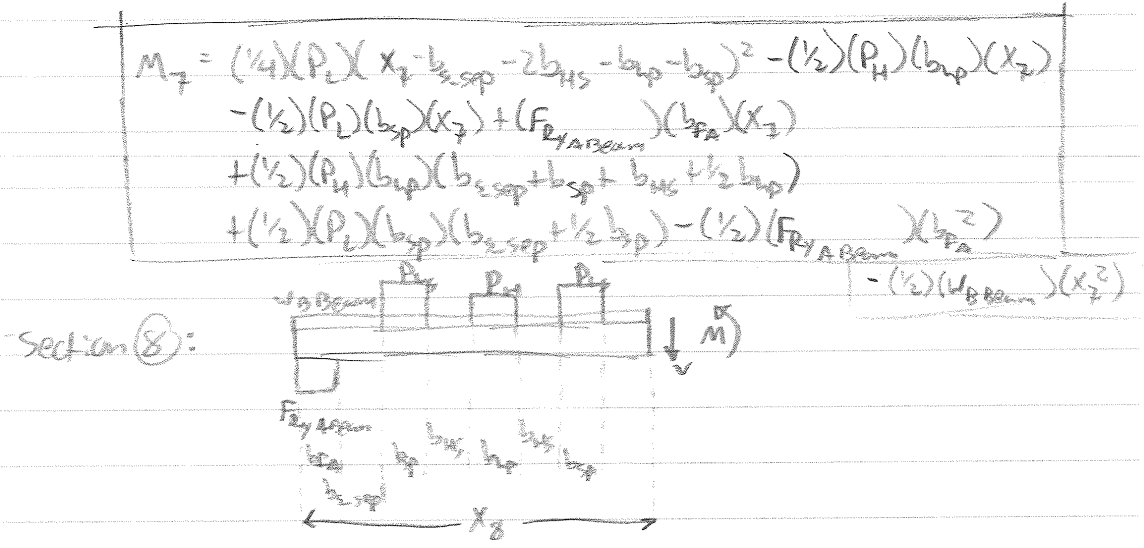

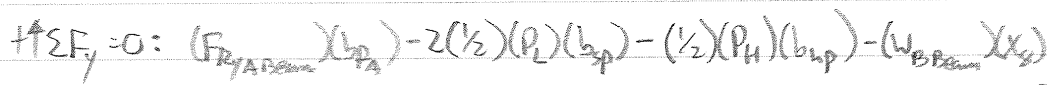

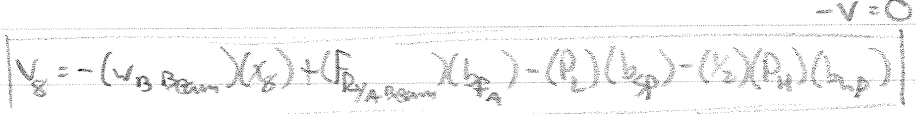

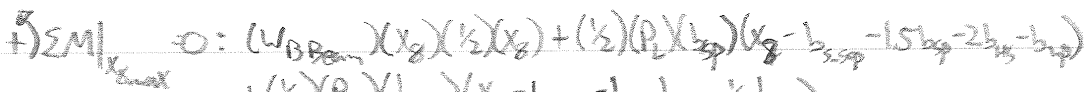

$$
\begin{aligned}
& +(1 / 2)\left(P_{H A}\right)\left(b_{4 P}\right)\left(x_{8}-b_{\Sigma S P Q}-b_{S P}-b_{H S}-3 / 2 b_{4 P}\right)
\end{aligned}
$$

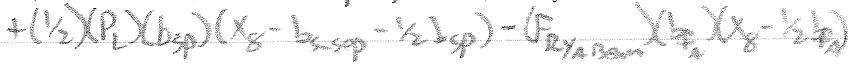

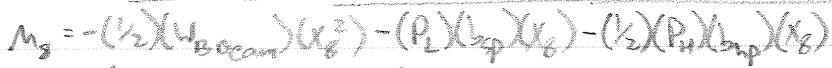

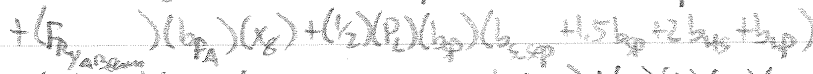

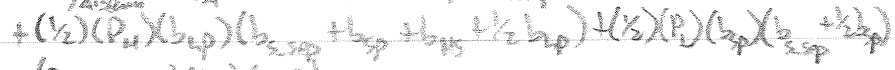

$$
\begin{aligned}
& -\left(R_{R_{A B}}\right)(K)\left(G_{H_{A}}^{2}\right)
\end{aligned}
$$

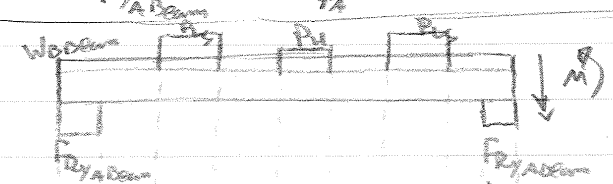

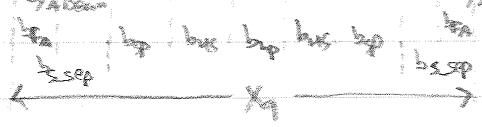

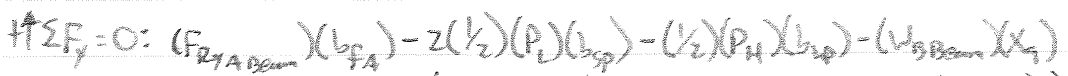

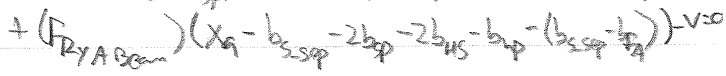




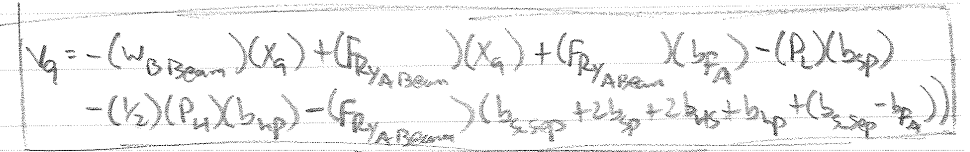

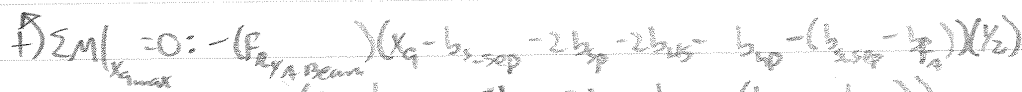

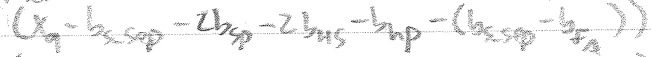

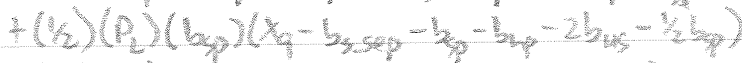

$$
\begin{aligned}
& +(1 / 2)\left(P_{4}\right)\left(b_{4 p}\right)\left(x_{4}-b_{s-s_{p}}-b_{4}-b_{4}-1 / 2 b_{4}\right) \\
& +(1 / 2)\left(p_{1}\right)\left(b_{3 p}\right)\left(x_{q}-b_{s, s o p}-1 / 2 b_{s p}\right)+\left(w_{B \text { Bsem }}\right)\left(x_{q}\right)\left(z_{2} x_{q}\right)
\end{aligned}
$$

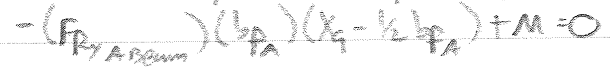

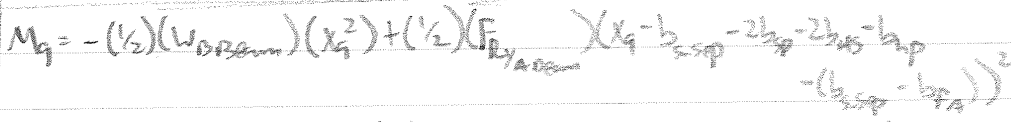

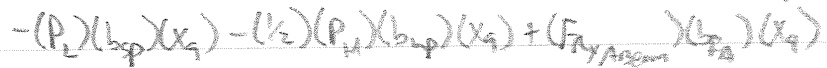

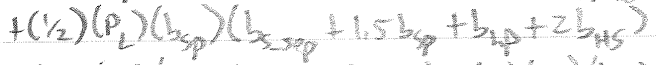

$$
\begin{aligned}
& +(1 / 2)\left(p_{2}\right)\left(b_{s p}\right)\left(b_{s p_{p}}+1 / 2 b_{s p}\right)+(1 / 2)\left(P_{11}\right)\left(b_{p p}\right)\left(b_{s p p}+h_{p p}+b_{n p}+/ 2 h_{p}\right) \\
& -(1 / 2)\left(F_{R_{A B}}\right)\left(b_{B}^{2}\right)
\end{aligned}
$$


West Virginia University

Small Microgravity Research Facility

Lift Support Hand Calculation Analysis

Created By: Kyle Phillips

Date: 01 Oct. 2011

\begin{tabular}{|c|c|}
\hline \multicolumn{2}{|l|}{ Pre-Defined Variables } \\
\hline General & \\
\hline Maximum Lifting Capacity $\left(\mathrm{P}_{\text {total }}\right)\left[\mathrm{b}_{\mathrm{f}}\right]$ & 2000 \\
\hline Hoist Weight $\left(\mathrm{W}_{\text {Hoist }}\right)\left[\mathrm{lb}_{\mathrm{f}}\right]$ & 80 \\
\hline \multicolumn{2}{|l|}{ A Beam } \\
\hline$\overline{A-B e a m ~ S p a n ~}\left(A_{\text {span }}\right)[$ in] & $\overline{60}$ \\
\hline A-Beam Flange Span $\left(\mathrm{b}_{\mathrm{fA}}\right)[\mathrm{in}]$ & 4.1 \\
\hline A-Beam Weight $\left(\mathrm{W}_{\text {ABeam }}\right)[\mathrm{lbff} / \mathrm{in}]$ & 1.08333 \\
\hline Distace from Support $\left(A_{\text {sep }}\right)[$ in] & 12 \\
\hline Moment of Inertia $\left(l_{x x_{-} A}\right)\left[i^{4}\right]$ & 11 \\
\hline Neutral Axis Distance $\left(\mathrm{C}_{\mathrm{A}}\right)[$ in] & 2.1 \\
\hline \multicolumn{2}{|l|}{ B Beam } \\
\hline B-Beam Span $\left(B_{\text {span }}\right)[$ in] & 96 \\
\hline B-Beam Flange Span $\left(\mathrm{b}_{\mathrm{fB}}\right)$ [in] & 4.1 \\
\hline Distance to Outer Flanges of B-Beams ( $\left.B_{\text {sep }}\right)$ [in] & 24 \\
\hline Distance to Inner Flanges of B-Beams $\left(\mathrm{b}_{\text {sep }}\right)$ [in] & 15.8 \\
\hline Distance from Support $\left(\mathrm{b}_{\text {support_B }}\right)$ [in] & 18 \\
\hline Sheave Plate Span $\left(\mathrm{b}_{\mathrm{sp}}\right)$ [in] & 7 \\
\hline Hoist Plate Span $\left(\mathrm{b}_{\mathrm{hp}}\right)$ [in] & 6 \\
\hline Hoist-Sheave Void Distance $\left(\mathrm{b}_{\mathrm{HS}}\right)$ [in] & 26 \\
\hline Sheave Plate Standoff Distance $\left(\mathrm{b}_{\left.\text {s_sep }_{\text {sep }}\right)[\mathrm{in}]}\right.$ & 12 \\
\hline B-Beam Load $\left(P_{B_{-} A}\right)[\mathrm{bff} / \mathrm{in}]$ & 139.512 \\
\hline B-Beam Weight ( $\left.\mathrm{W}_{\text {Bieam }}\right)[\mathrm{lbf} / \mathrm{in}]$ & 1.08333 \\
\hline Payload Load (PL_A) [lbf] & 1000 \\
\hline Payload Load $\left(P_{L_{-} B}\right)[\mathrm{bff} / \mathrm{in}]$ & 142.857 \\
\hline Hoist Load $\left(P_{H \_A}\right)[\mathrm{lbf}]$ & 80 \\
\hline Hoist Load $\left(\mathrm{P}_{\mathrm{H}_{-} \mathrm{B}}\right)[\mathrm{lbf}]$ & 13.3333 \\
\hline Moment of Inertia $\left(l_{x_{0} \_}\right)\left[i^{4}\right]$ & 11 \\
\hline Neutral Axis Distance $\left(C_{B}\right)$ [in] & 2.1 \\
\hline
\end{tabular}

Calculation Constants
\begin{tabular}{|c|c|}
\hline \multicolumn{2}{|c|}{ A Beam } \\
\hline$F_{\text {RyA }}[\mathrm{bf}]$ & 604.5 \\
\hline \multicolumn{2}{|c|}{ B Beam } \\
\hline$F_{\text {RyE }}[\mathrm{bt} / / \mathrm{n}]$ & 193.171 \\
\hline
\end{tabular}

\begin{tabular}{|l|l|}
\hline $\mathrm{F}_{\text {RyB }}[\mathrm{Ibf} / \mathrm{in}]$ & 193.171 \\
\hline
\end{tabular} 


\begin{tabular}{|c|c|c|c|c|c|c|c|c|}
\hline & \multirow[b]{2}{*}{ Generic D } & \multicolumn{3}{|c|}{ Section 1} & \multicolumn{3}{|c|}{ Section 2 } & \multirow[b]{2}{*}{$\mathrm{V}[\mathrm{lbf}]$} \\
\hline & & $V[\mathrm{~b} f]$ & M [bf'in] & न न[lbf/in2] & $\nabla[\mathrm{b} f]$ & M [ libf in & | & \\
\hline \multirow{19}{*}{ r } & 0 & 604.5 & 0 & 0 & 3115.72 & 5148 & 982.8 & 32.5 \\
\hline & 1 & 603.417 & 603.958 & \begin{tabular}{|l|l|} 
& 115.301 \\
\end{tabular} & 2975.12 & 5465.96 & 1043.5 & 31.4167 \\
\hline & 4 & 602.333 & 1206.83 & 230.395 & 2834.53 & 5782.83 & 1104 & 30.3333 \\
\hline & 3 & 601.25 & 1808.63 & \begin{tabular}{|l|l|}
345.283 \\
\end{tabular} & 2693.93 & 6098.63 & 1164.28 & 29.25 \\
\hline & 4 & 600.167 & 2409.33 & 459.964 & 2553.34 & 6413.33 & 1224.36 & 28.1667 \\
\hline & 5 & 599.083 & 3008.96 & 574.438 & 2412.74 & 6726.96 & 1284.24 & 27.0833 \\
\hline & 6 & 598 & 3607.5 & 688.705 & 2272.15 & 7039.5 & 1343.9 & 26 \\
\hline & T & 596.917 & 4204.96 & 802.765 & 2131.55 & 7350.96 & 1403.36 & 24.9167 \\
\hline & 8 & 595.833 & 4801.33 & 916.618 & 1990.96 & 7661.33 & 1462.62 & 23.8333 \\
\hline & 9 & 594.75 & 5396.63 & 1030.26 & 1850.36 & 7970.63 & 1521.66 & 22.75 \\
\hline & 10 & 593.667 & 5990.83 & 1143.7 & 1709.76 & 8278.83 & 1580.5 & 21.6667 \\
\hline & 11 & 592.583 & 6583.96 & 1256.94 & 1569.17 & 8585.96 & 1639.14 & 20.5833 \\
\hline & 12 & 591.5 & 7176 & 1369.96 & 1428.57 & 8892 & 1697.56 & 19.5 \\
\hline & 13 & 590.417 & 7766.96 & 1482.78 & 1287.98 & 9196.96 & 1755.78 & 18.4167 \\
\hline & 14 & 589.333 & 8356.83 & 1595.4 & 1147.38 & 9500.83 & 1813.8 & 17.3333 \\
\hline & 15 & 588.25 & 8945.63 & 1707.8 & 1006.79 & 9803.63 & 1871.6 & 16.25 \\
\hline & 16 & 587.167 & 9533.33 & 1820 & 866.191 & 10105.3 & 1929.2 & 15.1667 \\
\hline & 17 & 586.083 & 10120 & 1931.99 & 725.596 & 10406 & 1986.59 & 14.0833 \\
\hline & 18 & 585 & 10705.5 & 2043.78 & 585 & 10705.5 & 2043.78 & 13 \\
\hline \multirow{6}{*}{ 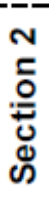 } & 19 & $\overline{583.917}$ & $\overline{11290}$ & $\overline{2} \overline{15} \overline{5} . \overline{36}$ & $\overline{4} \overline{4} 4 . \overline{4} \overline{04}$ & $-\overline{11004}$ & $\overline{21} \overline{0} \overline{0} \overline{7} \overline{6}$ & 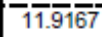 \\
\hline & 20 & 582.833 & 11873.3 & 2266.73 & 303.809 & 11301.3 & 2157.53 & 10.8333 \\
\hline & 21 & 581.75 & 12455.6 & 2377.89 & 163.213 & 11597.6 & 2214.09 & 9.75 \\
\hline & 22 & 580.667 & 13036.8 & 2488.85 & 22.6179 & 11892.8 & 2270.45 & 8.66667 \\
\hline & 23 & 579.583 & 13617 & 2599.6 & -117.978 & 12187 & 2326.6 & 7.58333 \\
\hline & 24 & 578.5 & 14196 & 2710.15 & -258.573 & 12480 & 2382.55 & 6.5 \\
\hline \multirow{12}{*}{ ڤొ } & 25 & 577.417 & $\overline{14774}$ & $2820 . \overline{48}$ & $-\overline{399} . \overline{169}$ & $\overline{12772}$ & $\overline{24} \overline{38} . \overline{28}$ & $5 . \overline{4} \overline{167}$ \\
\hline & 26 & 576.333 & 15350.8 & 2930.61 & $|-539.764|$ & 13062.8 & 2493.81 & 4.33333 \\
\hline & 27 & 575.25 & 15926.6 & 3040.54 & -680.36 & 13352.6 & 2549.14 & 3.25 \\
\hline & 28 & 574.167 & 16501.3 & 3150.25 & -820.955 & 13641.3 & 2604.25 & 2.16667 \\
\hline & 29 & 573.083 & 17075 & 3259.76 & -961.551 & 13929 & 2659.16 & 1.08333 \\
\hline & 30 & 572 & 17647.5 & 3369.07 & -1102.15 & 14215.5 & 2713.87 & 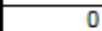 \\
\hline & 31 & 570.917 & 18219 & 3478.16 & -1242.74 & 14501 & 2768.36 & -1.08333 \\
\hline & 32 & 569.833 & 18789.3 & 3587.05 & -1383.34 & 14785.3 & 2822.65 & -2.16667 \\
\hline & 33 & 568.75 & 19358.6 & 3695.74 & -1523.93 & 15068.6 & 2876.74 & -3.25 \\
\hline & 34 & 567.667 & 19926.8 & 3804.21 & -1664.53 & 15350.8 & 2930.61 & -4.33333 \\
\hline & 35 & 566.583 & 20494 & 3912.48 & -1805.12 & 15632 & 2984.28 & -5.41667 \\
\hline & 36 & 565.5 & 21060 & 4020.55 & -1945.72 & 15912 & 3037.75 & -6.5 \\
\hline
\end{tabular}

\begin{tabular}{|c|c|c|c|c|c|c|c|c|}
\hline \multirow{6}{*}{ 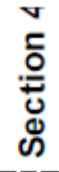 } & 37 & 564.417 & 21625 & 4128.4 & -2086.32 & 16191 & 3091 & -7.58333 \\
\hline & 38 & 563.333 & 22188.8 & 4236.05 & -2226.91 & 16468.8 & 3144.05 & -8.66667 \\
\hline & 39 & 562.25 & 22751.6 & 4343.49 & -2367.51 & 16745.6 & 3196.89 & -9.75 \\
\hline & 40 & 561.167 & 23313.3 & 4450.73 & -2508.1 & 17021.3 & 3249.53 & -10.8333 \\
\hline & 41 & 560.083 & 23874 & 4557.76 & -2648.7 & 17296 & 3301.96 & -11.9167 \\
\hline & 42 & 559 & 24433.5 & 4664.58 & -2789.29 & 17569.5 & 3354.18 & -1 \\
\hline \multirow{18}{*}{ 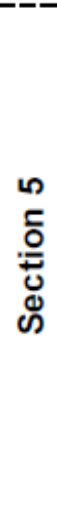 } & $\overline{43}$ & -557.917 & $\overline{249 \overline{9}}$ & $\overline{4} 77 \overline{19}$ & -2929.89 & $\overline{1784}$ & $-\overline{4} 4 \overline{06} \overline{19}$ & $-1 \overline{4} . \overline{08} \overline{33}$ \\
\hline & 44 & 556.833 & 25549.3 & 4877.6 & -3070.48 & 18113.3 & 3458 & -15.1667 \\
\hline & 45 & 555.75 & 26105.6 & 4983.8 & -3211.08 & 18383.6 & 3509.6 & -16.25 \\
\hline & 46 & 554.667 & 26660.8 & 5089.8 & -3351.67 & 18652.8 & 3561 & -17.3333 \\
\hline & 47 & 553.583 & 27215 & 5195.58 & -3492.27 & 18921 & 3612.18 & -18.4167 \\
\hline & 48 & 552.5 & 27768 & 5301.16 & -3632.87 & 19188 & 3663.16 & -19.5 \\
\hline & 49 & 551.417 & 28320 & 5406.54 & -3773.46 & 19454 & 3713.94 & -20.5833 \\
\hline & 50 & 550.333 & 28870.8 & 5511.7 & -3914.06 & 19718.8 & 3764.5 & -21.6667 \\
\hline & 51 & 549.25 & 29420.6 & 5616.66 & -4054.65 & 19982.6 & 3814.86 & -22.75 \\
\hline & 52 & 548.167 & 29969.3 & 5721.42 & -4195.25 & 20245.3 & 3865.02 & -23.8333 \\
\hline & 53 & 547.083 & 30517 & 5825.96 & -4335.84 & 20507 & 3914.96 & -24.9167 \\
\hline & 54 & 546 & 31063.5 & 5930.3 & -4476.44 & 20767.5 & 3964.7 & -26 \\
\hline & 55 & 544.917 & 31609 & 6034.44 & -4617.03 & 21027 & 4014.24 & -27.0833 \\
\hline & 56 & 543.833 & 32153.3 & 6138.36 & -4757.63 & 21285.3 & 4063.56 & -28.1667 \\
\hline & 57 & 542.75 & 32696.6 & 6242.08 & -4898.23 & 21542.6 & 4112.68 & -29.2 \\
\hline & 58 & 541.667 & 33238.8 & 6345.6 & -5038.82 & 21798.8 & 4161.6 & -30.3333 \\
\hline & 59 & 540.583 & 33780 & 6448.9 & -5179.42 & 22054 & 4210.3 & -31.4167 \\
\hline & 60 & 539.5 & 34320 & 6552 & -5320.01 & 22308 & 4258.8 & -32.5 \\
\hline
\end{tabular}


Development of the West Virginia University Small Microgravity Research Facility (WVU SMiRF)

\begin{tabular}{|c|c|c|c|c|c|c|c|}
\hline \multirow{2}{*}{\multicolumn{2}{|c|}{$\begin{array}{l}\text { A Beam } \\
\text { Section } 3 \\
\end{array}$}} & \multirow{2}{*}{\multicolumn{3}{|c|}{ Section 4}} & \multirow{2}{*}{\multicolumn{3}{|c|}{ Section 5}} \\
\hline & & & & & & & \\
\hline & & [ibf] & M [lbftin] & 2] & Ibf] & M [bffin] & बाibfin \\
\hline t68.6 & 89.46 & 5320.01 & 320 & 6552 & -539.5 & 320 & \\
\hline 11500.6 & प्र5.56 & 79.42 & 80 & 48.9 & .540 .583 & 3780 & \\
\hline 11531.4 & 001. & 38.82 & 238.8 & 45.6 & -541.667 & 238.8 & 4 \\
\hline 11561.2 & 207.14 & 4898.23 & 696.6 & 242.08 & -542.75 & 696.6 & 242. \\
\hline 11589.9 & 2212.62 & 757.63 & 153.3 & 38.36 & -543.833 & 2153.3 & 138. \\
\hline 11617.6 & 2217.9 & \begin{tabular}{|l|l|}
517.03 \\
\end{tabular} & 1609 & 034.44 & -544.917 & 31609 & 6034 . \\
\hline 11644.1 & 2222.96 & $\begin{array}{l}76.44 \\
\end{array}$ & 063.5 & 930.3 & -546 & 1063.5 & 5930 \\
\hline 11669.6 & 7. & 35.84 & 517 & 25.96 & 47.083 & 0517 & 825.9 \\
\hline & 32.48 & 95.25 & 969.3 & 21.42 & -548.167 & 969.3 & 721. \\
\hline & & 44.65 & & & -549.25 & & 516. \\
\hline & 2 & 14.06 & & & -550.333 & & \\
\hline & 45.2 & 3.46 & 20 & 54 & -551.417 & 20 & 400 \\
\hline & 99.02 & $2.87]$ & 68 & & -552.5 & 7768 & \\
\hline & 52.64 & 92.27 & |5 & & 553.583 & 15 & 195 \\
\hline & 6.06 & 11.67 & & & -554.667 & & 085 \\
\hline & 59.26 & 1.08 & & & -555.75 & & \\
\hline & 52.2 & 0.48 & 9.3 & 6 & -556.833 & 9.3 & 877 \\
\hline & 65.05 & 29.89 & 22 & 19 & -557.917 & 92 & 771. \\
\hline & 22 & 89.29 & & & -559 & & \\
\hline & - & $\overline{4} \overline{8} . \overline{7}$ & & & $\overline{60} . \overline{8} 3$ & & 55 \\
\hline & 72. & 508.1 & 3 & & -561.167 & & \\
\hline & 74.15 & 57.51 & & & -562.25 & & \\
\hline & 75 . & 26.91 & 8.8 & & -563.333 & 22188.8 & \\
\hline & 77 & 86.32 & & & -564. & & \\
\hline & & 1 & & & -565.5 & & \\
\hline & $\overline{2} 2 \overline{7} \overline{9} . \overline{94}$ & 55.12 & & & $\overline{6} \overline{6} . \overline{583}$ & $20 \overline{494}$ & \\
\hline & 80 . & 4.53 & & & -567.667 & & \\
\hline & & & & & & & \\
\hline & & & & & & & \\
\hline & & & & & 570.911 & & \\
\hline & & & & & & & \\
\hline & & & & & & & \\
\hline & 2282 & 1.955 & & & -574.167 & & \\
\hline & & & & & & & \\
\hline & 2200 & 9.104 & 이 & & -576.333 & 15350.8 & \\
\hline & & & & & -577.417 & 14774 & \\
\hline & & 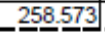 & & & & & \\
\hline
\end{tabular}




\begin{tabular}{|r|r|r|r|r|r|r|r|}
11929.6 & 2277.46 & 117.978 & 13617 & 2599.6 & -579.583 & 13617 & 2599.6 \\
\hline 11921.4 & 2275.91 & -22.6179 & 13036.8 & 2488.85 & -580.667 & 13036.8 & 2488.85 \\
\hline 11912.2 & 2274.15 & -163.213 & 12455.6 & 2377.89 & -581.75 & 12455.6 & 2377.89 \\
\hline 11901.9 & 2272.19 & -303.809 & 11873.3 & 2266.73 & -582.833 & 11873.3 & 2266.73 \\
\hline 11890.6 & 2270.02 & -444.404 & 11290 & 2155.36 & -583.917 & 11290 & 2155.36 \\
\hline 11878.1 & 2267.64 & -585 & 10705.5 & 2043.78 & -585 & 10705.5 & 2043.78 \\
\hline 118664.6 & 2265.05 & -725.596 & 10120 & 1931.99 & -586.083 & 10120 & 1931.99 \\
\hline 11849.9 & 2262.26 & -866.191 & 9533.33 & 1820 & -587.167 & 9533.33 & 1820 \\
\hline 11834.2 & 2259.26 & -1006.79 & 8945.63 & 1707.8 & -588.25 & 8945.63 & 1707.8 \\
\hline 11817.4 & 2256.06 & -1147.38 & 8356.83 & 1595.4 & -589.333 & 8356.83 & 1595.4 \\
\hline 11799.6 & 2252.64 & -1287.98 & 7766.96 & 1482.78 & -590.417 & 7766.96 & 1482.78 \\
\hline 11780.6 & 2249.02 & -1428.57 & 7176 & 1369.96 & -591.5 & 7176 & 1369.96 \\
\hline 11760.6 & 2245.2 & -1569.17 & 6583.96 & 1256.94 & -592.583 & 6583.96 & 1256.94 \\
\hline 11739.4 & 2241.16 & -1709.76 & 5990.83 & 1143.7 & -593.667 & 5990.83 & 1143.7 \\
\hline 11717.2 & 2236.92 & -1850.36 & 5396.63 & 1030.26 & -594.75 & 5396.63 & 1030.26 \\
\hline 11693.9 & 2232.48 & -1990.96 & 4801.33 & 916.618 & -595.833 & 4801.33 & 916.618 \\
\hline 11669.6 & 2227.82 & -2131.55 & 4204.96 & 802.765 & -596.917 & 4204.96 & 802.765 \\
\hline 11644.1 & 2222.96 & -2272.15 & 3607.5 & 688.705 & -598 & 3607.5 & 688.705 \\
\hline 11617.6 & 2217.9 & -2412.74 & 3008.96 & 574.438 & -599.083 & 3008.96 & 574.438 \\
\hline 11589.9 & 2212.62 & -2553.34 & 2409.33 & 459.964 & -600.167 & 2409.33 & 459.964 \\
\hline 11561.2 & 2207.14 & -2693.93 & 1808.63 & 345.283 & -601.25 & 1808.63 & 345.283 \\
\hline 11531.4 & 2201.46 & -2834.53 & 1206.83 & 230.395 & -602.333 & 1206.83 & 230.395 \\
\hline 11500.6 & 2195.56 & -2975.12 & 603.958 & 115.301 & -603.417 & 603.958 & 115.301 \\
\hline 11468.6 & 2189.46 & -3115.72 & 0 & 0 & -604.5 & 0 & \\
\hline
\end{tabular}

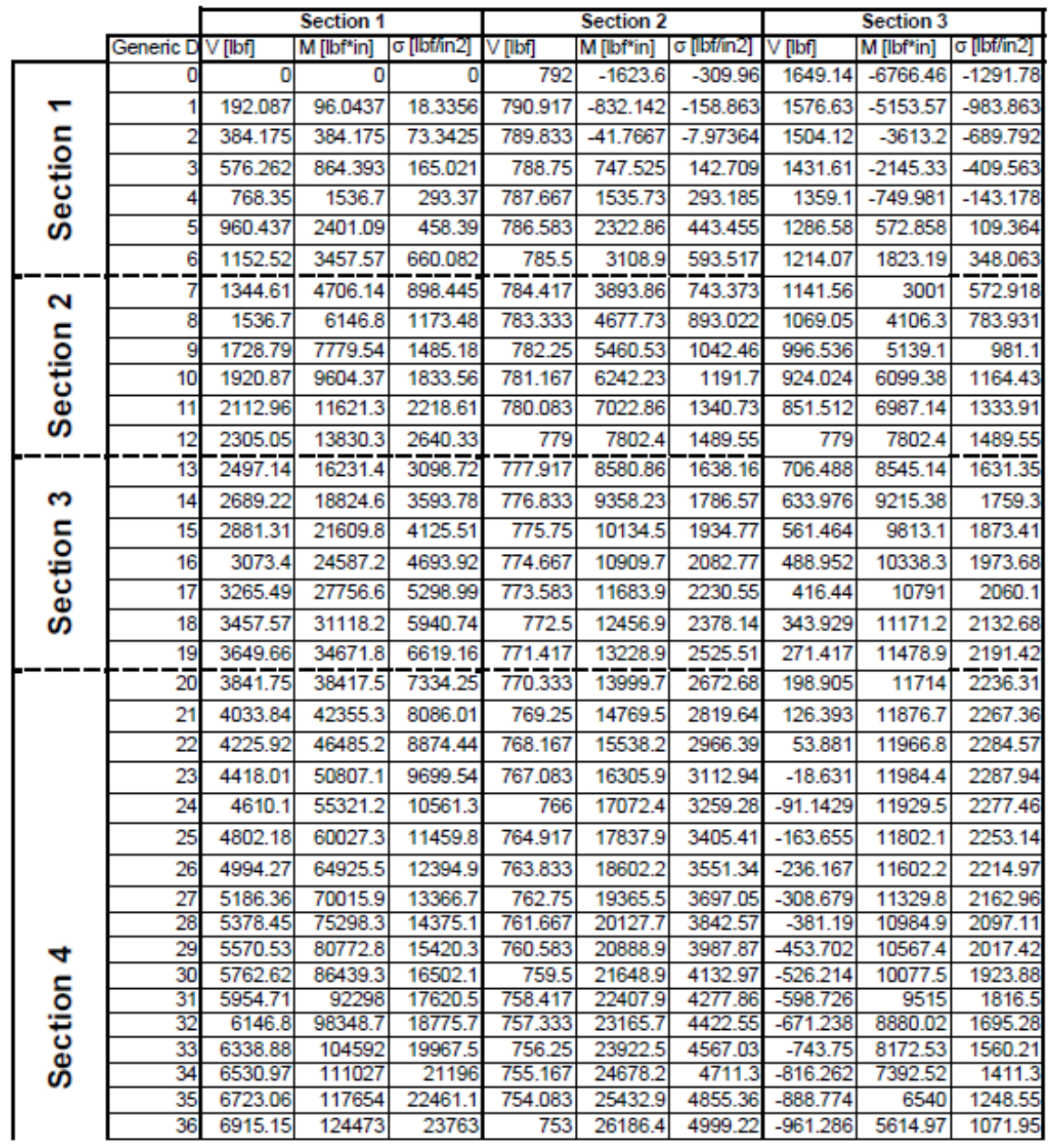




\begin{tabular}{|c|c|c|c|c|c|c|c|c|c|c|}
\hline & 37 & 7107.23 & 131484 & 25101.5 & 751.917| & 26938.9 & 5142.87 & -1033.8 & 4617.43 & \\
\hline & 38 & 7299.32 & 138687 & 26476.6 & 750.833 & 27690.2 & 5286.32 & -1106.31 & 3547.38 & 677 \\
\hline & 39 & 7491.41 & 146082 & 27888.5 & \begin{tabular}{|c|}
749.75 \\
\end{tabular} & 28440.5 & 5429.55 & -1178.82 & 2404.81 & 455 \\
\hline & 40 & 7683.5 & 153670 & 29337 & 748.667 & 29189.7 & 5572.59 & -1251.33 & 1189.73 & 227.1 \\
\hline & 41 & 7875.58 & 161449 & 30822.2 & 747.583 & 29937.9 & 5715.41 & & -97.856 & \\
\hline & 42 & 8067.67 & 169421 & 32344 & 746.5 & 30684.9 & 5858.03 & -1396.36 & -1457.96 & -278.3 \\
\hline & 43 & 8259.76 & 177585 & 33902.6 & 745.417 & 31430.9 & & & -2890.57 & -551.8 \\
\hline & 44 & 8451.85 & 185941 & 35497.8 & 744.333 & 32175.7 & 6142.64 & -1541.38 & -4395.7 & -839.178 \\
\hline & 45 & 8643.93 & 194488 & 37129.6 & 743.25 & & & & & \\
\hline \multirow{6}{*}{$\begin{array}{l}\text { 등 } \\
\text { 엃 } \\
\text { હે }\end{array}$} & 46 & 8836.02 & 203228 & 38798.2 & $7 \overline{42.167}$ & 33662.2 & 6426.43 & $\begin{array}{r}-1686.4 \\
\end{array}$ & -7623.48 & -1455.3 \\
\hline & 47 & 9028.11 & 212161 & 40503.4 & 741.083 & 34403.9 & 6568.01 & -1758.92 & -9346.14 & \\
\hline & 48 & 9220.2 & 221285 & 42245.3 & 740 & 35144.4 & 6709.39 & -1831.43 & -11141.3 & -2126 \\
\hline & 49 & 9412.28 & 230601 & 44023.8 & 738.917 & & & & -13009 & \\
\hline & 50 & 9604.37 & 240109 & 45839 & 737.833 & 36622.2 & 6991.52 & 5.45 & -14949.2 & -2853.9 \\
\hline & 51 & 9796.46 & 249810 & 47690.9 & 736.75 & & & & & \\
\hline \multirow{25}{*}{ 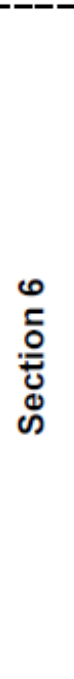 } & 52 & $\overline{9988.54}$ & $\overline{25970} \overline{2}$ & $4 \overline{9579.5}$ & $\overline{7} \overline{35} . \overline{67}$ & 5.7 & 7272.82 & 48 & 47.1 & $-3 \overline{63}$ \\
\hline & 53 & 10180.6 & 269787 & 51504.7 & 734.583 & 38830.9 & 7413.16 & & -21204.9 & 40 \\
\hline & 54 & 10372.7 & 280063 & 53466.7 & 733.5 & 39564.9 & 7553.3 & -2266.5 & -23435.1 & -4473 \\
\hline & 55 & 10564.8 & 290532 & 55465.2 & 732 & 40 & 7693.23 & -2339.01 & 37.9 & \\
\hline & 56 & 10756.9 & 301193 & 57500.5 & 731.333 & 99.7 & 7832.95 & 1.52 & -28113.1 & -53 \\
\hline & 57 & 10949 & 312046 & 59572.4 & 730.25 & 41760.5 & 7972.46 & -2484.04 & -30560.9 & -58 \\
\hline & 58 & 11141.1 & 323091 & 61681 & 729 & 42 & 1.77 & .55 & -33081.2 & \\
\hline & 59 & 11333.2 & 334328 & 63826.3 & 728.083 & 43218.9 & 8250.87 & -2629.06 & -35674 & -6810 \\
\hline & 60 & 11525.2 & & 660 & 727 & & & & -38339.3 & -731 \\
\hline & 61 & 11717.3 & & 682 & 725 & & & & & \\
\hline & 62 & 119 & & 704 & & & & & 87.5 & \\
\hline & 63 & 12101.5 & & 727 & & & & & & \\
\hline & 64 & 12293.6 & 393 & 75102.7 & 722.667 & & 8943.28 & & & \\
\hline & 65 & 124 & & & & & & & -527 & \\
\hline & 66 & 12677.8 & 418366 & 79869.9 & 720.5 & 48288.9 & 9218.79 & -3136.64 & -55854 & -1066 \\
\hline & 67 & 12869.9 & 431140 & 823 & 719. & & & & -59026.9 & \\
\hline & 68 & 13061.9 & 444106 & 8478 & 718.333 & & 9493.48 & -3281.67 & -62272.3 & -118 \\
\hline & 69 & 13254 & 457264 & 87295.9 & 717.25 & 50445.5 & 9630.51 & -3354.18 & -65590.2 & -12521 \\
\hline & 70 & 13446.1 & 470614 & 89844.5 & 716.167 & & 9767.34 & -3426.69 & -68980.6 & \\
\hline & 71 & 13638.2 & 484156 & 92429.8 & 715.083 & & 9903.95 & $\begin{array}{l}-3499.2 \\
\end{array}$ & $\begin{array}{l}-72443.6 \\
\end{array}$ & \\
\hline & 72 & 1383 & & 950 & & & & -357 & & \\
\hline & 73 & 14022.4 & & 97710 . & & & & -3644.23 & $-79587 \mid$ & \\
\hline & 74 & 14214.5 & 525935 & 100406 & 711. & 54018.2 & 10312.6 & -3716.74 & -83267.5 & \\
\hline & 74 & 14406. & 540246 & 103 & & 547 & & & -870 & \\
\hline & & 14598.6 & 554748 & 105907 & 709.667 & 55439.7 & 10583.9 & -3861.76 & -90846 & -170 \\
\hline
\end{tabular}

\begin{tabular}{|c|c|c|c|c|c|c|c|c|c|c|}
\hline \multirow{8}{*}{ 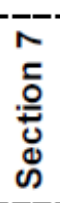 } & $=1$ & & & & & & & & $94 / 4$ & \\
\hline & 78 & $\overline{149} 8 \overline{8}$. & $\overline{584330}$ & $1115 \overline{5}$ & $\overline{7} \overline{07} .5$ & 56856.9 & $10 \overline{854} . \overline{5}$ & -4006.79 & -98714.5 & -1884 \\
\hline & 79 & 15174.9 & 599409 & 114433 & & & 10989.5 & -4079.3 & -102758 & \\
\hline & 80 & 15367 & 614680 & 117348 & 705.333 & & & & & \\
\hline & 81 & 1555 & 630143 & 120300 & 704 & 589 & 11258.8 & -422 & -11 & \\
\hline & 82 & 1575 & 6457 & 123 & 703. & 596 & 11 & & & \\
\hline & 83 & 159 & 6616 & 126314 & 702.083 & & 11527.3 & & 55 & \\
\hline & 84 & 161 & 6776 & 129 & 701 & & 116612 & -444 & -124060 & \\
\hline \multirow{6}{*}{ 옹 } & & $16 \overline{3}$ & $\overline{69} \overline{391}$ & $-\overline{132}$ & $\overline{699} .9 \overline{17}$ & & & & & \\
\hline & 86 & 16519.5 & 710 & 135 & 698.8 & & & & & \\
\hline & 87 & 16711.6 & 7269 & & 697 & & & -46 & & \\
\hline & 88 & 16903. & 743 & 141 & 696. & & & & -14 & \\
\hline & & 17095.8 & & 145 & 695.5 & & & & & \\
\hline & & 17287 & 77795 & 148 & & & 12460 & -4876.93 & -152017 & \\
\hline
\end{tabular}




\begin{tabular}{|c|c|c|c|c|c|c|c|c|c|c|}
\hline \multirow{5}{*}{ 모 } & & 1 & & & & & & & & \\
\hline & 92 & 17672 & & 93 & 692.333 & & 12725.2 & -50 & & \\
\hline & 9 & 178 & & 358 & & & & & & \\
\hline & 94 & 18056.2 & 848642 & & 690.167 & 68038.2 & 12 & -5166.98 & -172105 & \\
\hline & 95 & 18248 & 866794 & & 689.083 & & & -5239.49 & & \\
\hline
\end{tabular}

B Beam

\begin{tabular}{|c|c|c|c|c|c|c|c|c|}
\hline \multicolumn{3}{|c|}{ Section 4} & \multicolumn{3}{|c|}{ Section 5} & \multicolumn{3}{|c|}{ Section 6} \\
\hline [lbf] & $M\left[b f^{*}\right.$ in] & G & [ [bf] & M [bffin] & | $\mid[\mid b f / n 2]$ & [lbf] & $M$ [lbf & जाlbtin \\
\hline 292 & 6126.4 & 1169.59 & 592 & -623.6 & -119.051 & 252 & 8046.4 & 1536.13 \\
\hline 290.917 & 6417.86 & 1225.23 & 584.25 & -35.475 & -6.7725 & 250.917 & 8297.86 & 1584.14 \\
\hline 289.833 & 6708.23 & 1280.66 & 576.5 & 544.9 & 104.026 & 249.833 & 8548.23 & 1631.94 \\
\hline 288.75 & 6997.53 & 1335.89 & 568.75 & 1117.53 & 213.346 & 248.75 & 797.53 & 1679.53 \\
\hline 287.667 & 7285.73 & 1390.91 & 561 & 1682.4 & 321.185 & 247.667 & 9045.73 & 1726.91 \\
\hline 286.583 & 7572.86 & 1445.73 & 553.25 & 2239.53 & 427.546 & 246.583 & 9292.86 & 1774.09 \\
\hline 285.5 & 7858.9 & 1500.34 & 545.5 & 2788.9 & 532.426 & 245.5 & 9538.9 & 1821.06 \\
\hline 284.417 & 8143.86 & 1554.74 & 537.75 & 30.53 & 635.828 & 244.417 & 9783.86 & 1867.83 \\
\hline 283.333 & 8427.73 & 1608.93 & 530 & 864.4 & 737.749 & 243.333 & 027.7 & 1914.39 \\
\hline 282.25 & 8710.53 & 1662.92 & 522.25 & 4390.53 & 838.191 & 242.25 & 270.5 & 1960 \\
\hline \begin{tabular}{|l|}
281.167 \\
\end{tabular} & 8992.23 & 1716.7 & 514.5 & 4908.9 & 937.154 & 241.167 & 0512.2 & 2006.88 \\
\hline 280.083 & 9272.86 & 1770.27 & 506.75 & 5419.53 & 1034.64 & 240.083 & 10752.9 & 2052.82 \\
\hline 279 & 9552.4 & 1823.64 & 499 & 5922.4 & 1130.64 & 239 & 10992.4 & 2098.55 \\
\hline 277.917 & 9830.86 & 1876.8 & 491.25 & 6417.53 & $\overline{1225} \overline{16}$ & 237.917 & 11230.9 & $21 \overline{44} .07$ \\
\hline 276.833 & 10108.2 & 1929.75 & 483.5 & 6904.9 & 1318.21 & 236.833 & 468.2 & 2189.39 \\
\hline 275.75 & 10384.5 & 1982.5 & 475.75 & 7384.53 & 1409.77 & 235.75 & 1704.5 & 2234.5 \\
\hline 274.667 & 10659.7 & 2035.04 & 468 & 356.4 & 1499.86 & 234.667 & 939.7 & 2279.4 \\
\hline 273.583 & 10933.9 & 2087.37 & 460.25 & 8320.53 & 1588.46 & 233.583 & 12173.9 & 2324.1 \\
\hline 272.5 & 11206.9 & 2139.5 & 452.5 & 8776.9 & 1675.59 & 232.5 & 12406.9 & 2368.59 \\
\hline 271.417 & 11478.9 & 2191.42 & 444.75 & 9225.53 & 1761.24 & 231.417 & 12638.9 & 2412.87 \\
\hline 270.333 & 11749.7 & $\overline{22} \overline{43} .13$ & 437 & 9666.4 & $18 \overline{45.4}$ & 230.333 & 12869.7 & $2456 . \overline{9} 5$ \\
\hline 269.25 & 12019.5 & 2294.64 & 429.25 & 10099.5 & 1928.09 & 229.25 & 13099.5 & 2500.82 \\
\hline 268.167 & 12288.2 & 2345.94 & 421.5 & 10524.9 & 2009.3 & 228.167 & 13328.2 & 2544.48 \\
\hline 267.083 & 12555.9 & 2397.03 & 413.75 & 10942.5 & 2089.03 & 227.083 & 13555.9 & 2587.94 \\
\hline 266 & 12822.4 & 2447.91 & 406 & 11352.4 & 2167.28 & 226 & 13782.4 & 2631.19 \\
\hline 264.917 & 13087.9 & 2498.59 & 398.25 & 11754.5 & 2244.05 & 224.917 & 14007.9 & 2674.23 \\
\hline 263.833 & 13352.2 & 2549.06 & 390.5 & 12148.9 & 2319.34 & 223.833 & 2.2 & 2717.06 \\
\hline 262.75 & 15 & 25 & 382.75 & 8.5 & 23 & 222.75 & 5.5 & 2759.69 \\
\hline 261.667 & 13877.7 & 2649.39 & 375 & 14.4 & 2465.48 & 221.667 & & 2802.11 \\
\hline 260.583 & 14 & & 367.25 & & 2536.33 & 220.583 & 8.9 & 2844.33 \\
\hline 259.5 & 14 & 2748.88 & 359.5 & 8.9 & 2605.7 & 219.5 & 8.9 & 2886.34 \\
\hline 258.417 & & & 1.75 & & 2673.59 & 218.417 & & 2928. \\
\hline 257.333 & 14915.7 & 2847.55 & 344 & 352.4 & 2740 & 217.333 & 55.7 & 2969.73 \\
\hline 256.25 & & & 62 & & & 216 & & $\overline{3011}$ \\
\hline 255.167 & 15428.2 & 2945.39 & 328.5 & 15024.9 & 2868.39 & 215.167 & 15988.2 & 3052.3 \\
\hline 254.083 & 15682.9 & 994 & 320.75 & & 2930.36 & 214.083 & 22.9 & 3093.2 \\
\hline 253 & 15936.4 & 3042.4 & 313 & 15666.4 & 2990.86 & 213 & 16416.4 & 3134.04 \\
\hline
\end{tabular}




\begin{tabular}{|c|c|c|c|c|c|c|c|c|}
\hline & 3.9 & 3090.6 & 305.25 & 15975.5 & 3049.87 & 211.917 & 16628.9 & 3174. \\
\hline 833 & 440.2 & 3138.59 & 297.5 & 16276.9 & 3107.41 & 210.833 & 16840.2 & 3214.95 \\
\hline 249.75 & 16690.5 & 3186.37 & 289.75 & 16570.5 & 3163.46 & 209.75 & 17050.5 & 325 \\
\hline 248.667 & 16939.7 & 3233.95 & 282 & 16856.4 & 3218.04 & 208.667 & 17259.7 & 3295.04 \\
\hline 247.583 & 17187.9 & 3281.32 & 274.25 & 17134.5 & 3271.14 & 207.583 & & \\
\hline 246.5 & 17434.9 & 3328.48 & 266.5 & 17404.9 & 3322.75 & 206.5 & 17674.9 & 3374.3 \\
\hline 245.417 & 17680.9 & 3375.44 & 258.75 & 17667.5 & 3372.89 & 205.417 & & \\
\hline 244.333 & 17925.7 & 3422.19 & 251 & 17922.4 & 3421.55 & 204.333 & 18085.7 & 3452.73 \\
\hline 243.25 & & & 243.25 & & & 203.25 & & \\
\hline 242.167 & 18412.2 & 3515.06 & 235.5 & 18408.9 & 3514.43 & 202.167 & 18492.2 & 3530.34 \\
\hline 241.083 & 18653.9 & 3561.19 & 227.75 & 18640.5 & 3558.65 & 201.083 & & \\
\hline 240 & 18894.4 & 3607.11 & 220 & 18864.4 & 3601.39 & 200 & 18894.4 & 3607.11 \\
\hline 238.917 & 19133.9 & 365 & 212.25 & & & 198.917 & & \\
\hline 237.833 & 19372.2 & 3698.34 & 204.5 & 19288.9 & 3682.43 & 197.833 & 19292.2 & 3683.06 \\
\hline 236.75 & 19609.5 & 3743.64 & 196.75 & & & 196.75 & 19489.5 & \\
\hline 235.667 & 19845.7 & $-\overline{3788.73}$ & 189 & 19682.4 & $\overline{3757.55}$ & 195.667 & 35.7 & 37 \\
\hline 234.583 & 20080.9 & 3833.6 & 181.25 & 19867.5 & 379 & 194.583 & 19880.9 & \\
\hline 233.5 & 20314.9 & 3878.3 & 173.5 & 20044.9 & 3826.75 & 193.5 & 20074.9 & 3832.48 \\
\hline 232.417 & 20547.9 & 3922.77 & 165.75 & 20214.5 & 3859.14 & 192.417 & 20267.9 & \\
\hline 231.333 & 20779.7 & 3967 & 158 & 20376.4 & 3890.04 & 191.333 & 20459.7 & 39 \\
\hline 230.25 & 21010.5 & 4011.1 & 150.25 & 20530.5 & 3919.46 & 190.25 & 20650.5 & 394 \\
\hline 229.167 & 21240.2 & 4054.95 & 142.5 & 20676.9 & 3947.41 & 189.167 & 20840.2 & 3978 \\
\hline 228.083 & 21468.9 & 4098.6 & 134.75 & 20815.5 & 3973.87 & 188.083 & 21028.9 & 4014.6 \\
\hline 227 & 21696.4 & 4142 & 127 & & & 187 & & \\
\hline 225.917 & 21922.9 & 4185 & 9.25 & 21069.5 & 402 & 185.917 & 21402.9 & \\
\hline 224.833 & 22148.2 & 422 & 111.5 & & & 184.833 & 21588.2 & \\
\hline 223.75 & 22372.5 & 4271.12 & 103.75 & 21292.5 & 4064.94 & 183.75 & 21772.5 & \\
\hline 222.667 & 22595.7 & 4313.73 & 96 & 21392.4 & 4084 & 182.667 & 21955.7 & \\
\hline 221.583 & 22817.9 & & 88.25 & & 4101.59 & 181.583 & 22137.9 & \\
\hline 220.5 & 23038.9 & 4398.34 & 80.5 & 21568.9 & 4117.7 & 180.5 & 22318.9 & 4260. \\
\hline 219.417 & 23258.9 & 4440 & 72.75 & 21645.5 & 4132.33 & 179.417 & 22498.9 & \\
\hline 218.333 & 23477.7 & 448 & & 217 & 414 & 178.33 & & \\
\hline 217.25 & 23695.5 & 452 & 57.25 & 217 & 415 & 177.25 & 22855.5 & \\
\hline 216.167 & 23912.2 & 4565 & 49.5 & & 416 & 176.16 & 23032.2 & \\
\hline 215.083 & 24127.9 & 4606 & 41.75 & 21874.5 & 4177 & 175.083 & 23207.9 & \\
\hline 214 & 24342.4 & 464 & 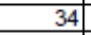 & & & & & \\
\hline 212.917 & 24555.9 & 4687 & 26.25 & 21942.5 & 4189.0 & 172.917 & 23555.9 & \\
\hline 21 & & & 18.5 & & 4193.3 & 171.833 & 8.2 & \\
\hline 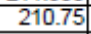 & & 476 & & & & & & \\
\hline 209 . & 25189.7 & 4808 & & 21986.4 & 4197.4 & 169.667 & 24069.7 & 4595 \\
\hline
\end{tabular}

\begin{tabular}{|r|r|r|r|r|r|r|r|r|}
208.583 & 25398.9 & 4848.87 & -4.75 & 21985.5 & 4197.24 & 168.583 & 24238.9 & 4627.42 \\
\hline 207.5 & 25606.9 & 4888.59 & -12.5 & 21976.9 & 4195.59 & 167.5 & 24406.9 & -4659.5 \\
\hline 206.417 & 25813.9 & 4928.1 & -20.25 & 21960.5 & 4192.46 & 166.417 & 24573.9 & 4691.37 \\
\hline 205.333 & 26019.7 & 4967.4 & -28 & 21936.4 & 4187.86 & 165.333 & 24739.7 & 4723.04 \\
\hline 204.25 & 26224.5 & 5006.5 & -35.75 & 21904.5 & 4181.77 & 164.25 & 24904.5 & 4754.5 \\
\hline 203.167 & 26428.2 & 5045.39 & -43.5 & 21864.9 & 4174.21 & 163.167 & 25068.2 & 4785.75 \\
\hline 202.083 & 26630.9 & 5084.07 & -51.25 & 21817.5 & 4165.16 & 162.083 & 25230.9 & 4816.8 \\
\hline 201 & 26832.4 & 5122.55 & -59 & 21762.4 & 4154.64 & 161 & 25392.4 & 4847.64 \\
\hline 199.917 & 27032.9 & 5160.82 & -66.75 & 21699.5 & 4142.64 & 159.917 & 25552.9 & $-\frac{4878.27}{4} .7$ \\
\hline 198.833 & 27232.2 & 5198.88 & -74.5 & 21628.9 & 4129.15 & 158.833 & 25712.2 & 4908.7 \\
\hline 197.75 & 27430.5 & 5236.74 & -82.25 & 21550.5 & 4114.19 & 157.75 & 25870.5 & 4938.92 \\
\hline 196.667 & 27627.7 & 5274.39 & -90 & 21464.4 & 4097.75 & 156.667 & 26027.7 & 4968.93 \\
\hline 195.583 & 27823.9 & 5311.83 & -97.75 & 21370.5 & 4079.83 & 155.583 & 26183.9 & 4998.74 \\
\hline 194.5 & 28018.9 & 5349.06 & -105.5 & 21268.9 & 4060.43 & 154.5 & 26338.9 & 5028.34 \\
\hline
\end{tabular}

\begin{tabular}{|r|r|r|r|r|r|r|r|r|}
193.417 & 28212.9 & 5386.09 & -113.25 & 21159.5 & 4039.55 & 153.417 & 26492.9 & 5057.73 \\
\hline 192.333 & 28405.7 & 5422.91 & -121 & 21042.4 & 4017.19 & 152.333 & 26645.7 & 5086.91 \\
\hline 191.25 & 28597.5 & 5459.53 & -128.75 & 20917.5 & 3993.35 & 151.25 & 26797.5 & 5115.89 \\
\hline 190.167 & 28788.2 & 5495.94 & -136.5 & 20784.9 & 3968.03 & 150.167 & 26948.2 & 5144.66 \\
\hline 189.083 & 28977.9 & 5532.14 & -144.25 & 20644.5 & 3941.23 & 149.083 & 27097.9 & 5173.23 \\
\hline 188 & 29166.4 & 5568.13 & -152 & 20496.4 & 3912.95 & 148 & 27246.4 & 5201.59 \\
\hline
\end{tabular}




\begin{tabular}{|c|c|c|c|c|c|c|c|c|}
\hline \multicolumn{3}{|c|}{ Section 7} & \multicolumn{3}{|c|}{ Section 8} & \multicolumn{3}{|c|}{ Section 9} \\
\hline $\mathrm{V}[\mathrm{l} b \mathrm{~b}]$ & M [loffin] & [O/lbf/in2] & V [bf] & IM [bf'in] & [ब[lbffine] & $V$ [lbf] & M [bffin] & | $\sigma[$ lbfin2] \\
\hline 5752 & 219796 & 441961.1 & -248 & 48296.4 & 9220.22 & -18000.4 & 864018.7 & 164949 \\
\hline 5679.49 & 214584 & 40966 & -249.083 & 48047.9 & 77 & \begin{tabular}{|l|l}
-17808.3 \\
\end{tabular} & 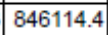 & 515 \\
\hline 5606.98 & 209441 & 39984.2 & -250.167 & 47798.2 & 9125.12 & $\mid-17616.2$ & 828402.1 & 58149 \\
\hline 5534.46 & 204369 & 39015.9 & -251.25 & 47547.5 & 9077.25 & \begin{tabular}{|l|l|l}
-17424.1 \\
\end{tabular} & 810882 & 54805 \\
\hline 5461.95 & 199367 & 38061 & -252.333 & 47295.7 & 9029.19 & -17232 & 793553.9 & 51497 \\
\hline 5389.44 & 194436 & 37119.5 & -253.417 & 47042.9 & 8980.91 & -17040 & 776417.9 & 148225 \\
\hline 5316.93 & 189575 & 36191.5 & -254.5 & 46788.9 & 8932.43 & 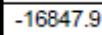 & 759474 & 144990 \\
\hline 5244.42 & 184784 & 35276.9 & -255.583 & 46533.9 & 8883.74 & -16655.8 & 742722.1 & 141792 \\
\hline 5171.9 & 180063 & 34375.7 & -256.667 & 46277.7 & 8834.84 & $\mid-16463.7$ & 726162.4 & 138631 \\
\hline 5099.39 & 175413 & 33488 & -257.75 & 20.5 & 8785.74 & \begin{tabular}{|l|l|}
-16271.6 \\
\end{tabular} & 4.8 & 5506 \\
\hline 5026.88 & 170834 & 32613.7 & -258.833 & 62.2 & 8736.43 & -16079.5 & 693619.2 & 2418 \\
\hline 4954.37 & 166324 & 31752.8 & -259.917 & 45502.9 & 8686.91 & -15887.4 & \begin{tabular}{|l|l|}
677635.7 \\
\end{tabular} & 129367 \\
\hline 4881.86 & 161885 & 30905.4 & -261 & 45242.4 & 8637.19 & -15695.3 & 661844.3 & 126352 \\
\hline 4809.35 & 157517 & 30071.3 & -262.083 & 44980.9 & 8587.25 & -15503.3 & 646245 & 374 \\
\hline 4736.83 & 153218 & 29250.8 & -263.167 & 44718.2 & 8537.12 & -15311.2 & 630837.8 & 12043 \\
\hline 4664.32 & 148990 & 28443.6 & -264.25 & 44454.5 & 8486.77 & -15119.1 & 615622.7 & 11752 \\
\hline 4591.81 & 144833 & 27649.9 & -265.333 & 44189.7 & 8436.22 & -14927 & 599.7 & 114660 \\
\hline 4519.3 & 140745 & 26869.6 & -266.417 & 43923.9 & 8385.46 & -14734.9 & 585768.7 & 11829 \\
\hline 4446.79 & 136728 & 26102.7 & -267.5 & 43656.9 & 8334.5 & -14542.8 & 129.9 & 03 \\
\hline 4374.27 & 132782 & 25349.2 & -268.583 & 43388.9 & 8283.33 & $\mid-14350.7$ & 556683.1 & 10627 \\
\hline 4301.76 & 128905 & 24609.2 & -269.667 & 43119.7 & 8231.95 & 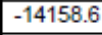 & 542428.4 & 103555 \\
\hline 4229.25 & 125100 & 23882.6 & -270.75 & 42849.5 & 8180.36 & -13966.6 & 528365.8 & 100870 \\
\hline 4156.74 & 121364 & 23169.5 & -271.833 & 42578.2 & 8128.57 & -13774.5 & 514495.3 & 98221.8 \\
\hline 4084.23 & 117699 & 22469.8 & -272.917 & 42305.9 & 8076.57 & -13582.4 & 500816.9 & 95610. \\
\hline 4011.71 & 114104 & 21783.5 & -274 & 42032.4 & 8024.37 & -13390.3 & 487330.5 & 93035.8 \\
\hline 3939.2 & 110579 & 21110.6 & -275.083 & 41757.9 & 7971.95 & $\mid-13198.2$ & \begin{tabular}{|l|l}
474036.3 \\
\end{tabular} & 90497.8 \\
\hline 3866.69 & 107125 & 20451.2 & -276.167 & 2 & 7919.34 & $\mid-13006.1$ & 460934.1 & \\
\hline 3794.18 & & 19 & -277.25 & & & -12814 & & \\
\hline 3721.67 & 0428 & 19172.6 & -278.333 & & 7813.48 & \begin{tabular}{|l|l|}
12621.9 \\
\end{tabular} & 435306.1 & 831 \\
\hline 3649.15 & & 18553.4 & -279.417 & & & $\mid-12429.9$ & & \\
\hline 3576.64 & 11.8 & 17947.7 & -280.5 & & 7706.79 & $\mid-12237.8$ & & 783 \\
\hline 3504.13 & & & 281.583 & & 7653.14 & -120 & & \\
\hline 3431.62 & & 16776.5 & -282.667 & & 7599.28 & \begin{tabular}{|l|l|}
-11853.6 \\
\end{tabular} & 86355 & 73758.7 \\
\hline 3359.11 & & & -28375 & & & 1 & \begin{tabular}{|l|l|}
374597.4 \\
\end{tabular} & \\
\hline 3286.6 & 82023.9 & 15659.1 & -284.833 & 39238.2 & 7490.94 & \begin{tabular}{|l|l|}
-11469.4 \\
\end{tabular} & 363032 & 69306.1 \\
\hline 3214.08 & & 15120.5 & -285.917 & & 7436.45 & \begin{tabular}{|l|l|l} 
\\
\end{tabular} & \begin{tabular}{|l|l|}
351658.6 \\
\end{tabular} & \\
\hline 3141.57 & 76452.1 & 14595.4 & -287 & 38666.4 & 7381.77 & -11085.2 & \begin{tabular}{|l|l|}
340477.3 \\
\end{tabular} & 65000.2 \\
\hline
\end{tabular}




\begin{tabular}{|c|c|c|c|c|c|c|c|c|}
\hline & $1.7 \mid$ & 3.7 & 83 & 8.9 & & 4 & & 2.3 \\
\hline 2996.55 & \begin{tabular}{|l|}
71161.7 \\
\end{tabular} & 13585.4 & -289.167 & 38090.2 & 7271.77 & -10701.1 & 318691 & \\
\hline 2924.04 & 68622 & 13100.6 & -290.25 & 37800.5 & 7216.46 & -10509 & 308086 & 58816.4 \\
\hline 2851.52 & 66152.6 & 12629.1 & -291.333 & 37509.7 & 7160.95 & -10316.9 & 297673 & 8828.5 \\
\hline 2779.01 & 63753.6 & 12171.1 & -292.417 & 37217.9 & 7105.23 & -10124.8 & 287452.2 & 5487 \\
\hline 2706.5 & 61424.9 & 11726.6 & -293.5 & 36924.9 & 7049.3 & -9932.72 & 277423.4 & 9627 \\
\hline 2633.99 & 59166.6 & 11295.4 & -294.583 & 36630.9 & 6993.16 & -9740.63 & 267586.8 & 51084.7 \\
\hline 2561.48 & 56978.6 & 10877.7 & -295.667 & & 6936.82 & -9548.54 & & \\
\hline 2488.96 & 54861 & 10473.5 & -296.75 & 36039.5 & 6880.27 & -9356.46 & 248489.7 & 47438.9 \\
\hline 2416.45 & 52813.7 & $\overline{100} 8 \overline{2} . \overline{6}$ & -297.833 & 35742.2 & $\overline{6} \overline{82} \overline{3} . \overline{52}$ & -9164.37 & 239229.2 & \\
\hline 2343.94 & 50836.7 & 9705.19 & -298.917 & 35443.9 & 6766.55 & -8972.28 & 230160.9 & 43939.8 \\
\hline 2271.43 & 48930.1 & 9341.2 & -300 & $\begin{array}{l}35144.4 \\
\end{array}$ & 6709.39 & -8780.2 & 221284.7 & \\
\hline 2198.92 & 47093.9 & 8990.65 & -301.083 & 34843.9 & 6652.01 & -8588.11 & 212600.5 & 40587.4 \\
\hline 2126.4 & 45327.9 & 8653.52 & -302.167 & & 6594.43 & -8396.02 & 204108.5 & \\
\hline 2053.89 & 43632.4 & 8329.82 & -303.25 & 34239.5 & 6536.64 & -8203.93 & 195808.5 & \\
\hline 1981.38 & 42007.2 & -8019.55 & -304.333 & & $\overline{6478.64}$ & -8011.85 & 187700.6 & \\
\hline 1908.87 & 40452.3 & 7722.71 & -305.417 & 33630.9 & 6420.44 & -7819.76 & 179784.8 & 3432 \\
\hline 1836.36 & 38967.8 & 7439.3 & -306.5 & 33324.9 & 6362.03 & -7627.67 & 172061.1 & \\
\hline 1763.85 & 37553.6 & 7169.32 & -307.583 & 33017.9 & 6303.41 & -7435.58 & 164529.5 & 3141 \\
\hline 1691.33 & 36209.7 & 6912.77 & -308.667 & 32709.7 & 6244.59 & -7243.5 & 157189.9 & \\
\hline 161 & 34936.2 & 6669.65 & -309.75 & & 6185.55 & -7051.41 & 150042.5 & 28 \\
\hline 1546.31 & 33733.1 & 6439.95 & -310.833 & 32090.2 & 6126.32 & -6859.32 & 143087.1 & \\
\hline & 326 & & -311 & & & -666 & 136 & 2602 \\
\hline 1401.29 & 31537.8 & 6020.86 & -313 & 31466.4 & 6007.22 & -6475.15 & 129752.6 & \\
\hline & 30545.7 & & 314.0 & & 594 & & & \\
\hline 1256.26 & 29623.9 & 5655.48 & -315.167 & 30838.2 & 5887.3 & -6090.97 & 117186.5 & \\
\hline 1183.75 & 28772.5 & & & & 5827.03 & -5898 & & \\
\hline 1111.24 & 27991.4 & 5343.82 & -317.333 & 30205.7 & 5766.55 & -5706.8 & 105388.7 & \\
\hline & 27280.7 & & -318.417 & 29887.9 & 5705.86 & -5514.7 & 99777.99 & \\
\hline 966 & 266 & & & & 564 & -532 & 943 & \\
\hline 893.702 & 26070.3 & 4977.05 & -320.583 & 29248.9 & 5583.87 & -5130.53 & 89132.75 & \\
\hline 821.19 & 25570.6 & 4881.66 & -321.667 & & 5522.57 & & 84098.26 & \\
\hline 748.679 & 25141.2 & & -322.75 & & & 47 & & \\
\hline 676.11 & 24782.2 & & -323.8 & 28282.2 & 5399.34 & $-45^{5}$ & 74605.54 & \\
\hline & 2449 & & -324.9 & & 533 & -4362 & & \\
\hline 531.143 & 24275.3 & 4634.37 & -326 & 27632.4 & 5275.28 & -4170.1 & 65881.17 & 125 \\
\hline & & & -327.083 & & & & & \\
\hline 386.11 & 24049.7 & 4591.3 & -328.1 & 26978.2 & 5150 & -378 & 57925.15 & \\
\hline & 24042.4 & & -329.25 & 26649.5 & 5087.64 & -359 & 54235.27 & \\
\hline & 24 & & 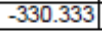 & 26319.7 & 502 & -340 & 50737.48 & \\
\hline
\end{tabular}

\begin{tabular}{|r|r|r|r|r|r|r|r|r|}
168.583 & 24238.9 & 4627.42 & -331.417 & 25988.9 & 4961.51 & -3209.66 & 47431.78 & -9055.16 \\
\hline 96.0714 & 24442.6 & 4666.32 & -332.5 & 25656.9 & 4898.14 & -3017.57 & 44318.16 & -8460.74 \\
\hline 23.5595 & 24716.7 & 4718.65 & -333.583 & 25323.9 & 4834.55 & -2825.49 & 41396.63 & 7902.99 \\
\hline-48.9524 & 25061.2 & 4784.4 & -334.667 & 24989.7 & 4770.77 & -2633.4 & 38667.19 & 7381.92 \\
\hline-121.464 & 25476 & 4863.59 & -335.75 & 24654.5 & 4706.77 & -2441.31 & 36129.83 & 6897.51 \\
\hline-193.976 & 25961.1 & 4956.21 & -336.833 & 24318.2 & 4642.57 & -2249.22 & 33784.57 & 6449.78 \\
\hline-266.488 & 26516.6 & 5062.25 & -337.917 & 23980.9 & 4578.16 & -2057.14 & 31631.39 & 6038.72 \\
\hline-339 & 27142.4 & 5181.73 & -339 & 23642.4 & 4513.55 & -1865.05 & 29670.29 & 5664.33 \\
\hline-411.512 & 27838.6 & 5314.64 & -340.083 & 23302.9 & 4448.73 & -1672.96 & 27901.29 & 5326.61 \\
\hline-484.024 & 28605.1 & 5460.97 & -341.167 & 22962.2 & 4383.7 & -1480.87 & 26324.37 & 5025.56 \\
\hline-556.536 & 29442 & 5620.74 & -342.25 & 22620.5 & 4318.46 & -1288.79 & 24939.54 & 4761.18 \\
\hline-629.048 & 30349.2 & 5793.93 & -343.333 & 22277.7 & 4253.02 & -1096.7 & 23746.8 & 4533.48 \\
\hline-701.56 & 31326.7 & 5980.55 & -344.417 & 21933.9 & 4187.37 & -904.612 & 22746.14 & 4342.45 \\
\hline-774.071 & 32374.6 & 6180.61 & -345.5 & 21588.9 & 4121.52 & -712.524 & 21937.57 & 4188.08 \\
\hline
\end{tabular}


Development of the West Virginia University Small Microgravity Research Facility (WVU SMiRF)
Copyright $^{\odot} 2014$

Kyle G. Phillips

\begin{tabular}{|r|r|r|r|r|r|r|r|r|}
-846.583 & 33492.9 & 6394.09 & -346.583 & 21242.9 & 4055.45 & -520.437 & 21321.09 & 4070.39 \\
\hline-919.095 & 34681.4 & 6621 & -347.667 & 20895.7 & 3989.19 & -328.35 & 20896.7 & 3989.37 \\
\hline-991.607 & 35940.4 & 6861.35 & -348.75 & 20547.5 & 3922.71 & -136.262 & 20664.39 & 3945.02 \\
\hline-1064.12 & 37269.7 & 7115.12 & -349.833 & 20198.2 & 3856.03 & 55.8252 & 20624.17 & 3937.34 \\
\hline-1136.63 & 38669.3 & 7382.32 & -350.917 & 19847.9 & 3789.14 & 247.913 & 20776.04 & 3966.34 \\
\hline-1209.14 & 40139.3 & 7662.95 & -352 & 19496.4 & 3722.04 & 440 & 21120 & 4032 \\
\hline
\end{tabular}
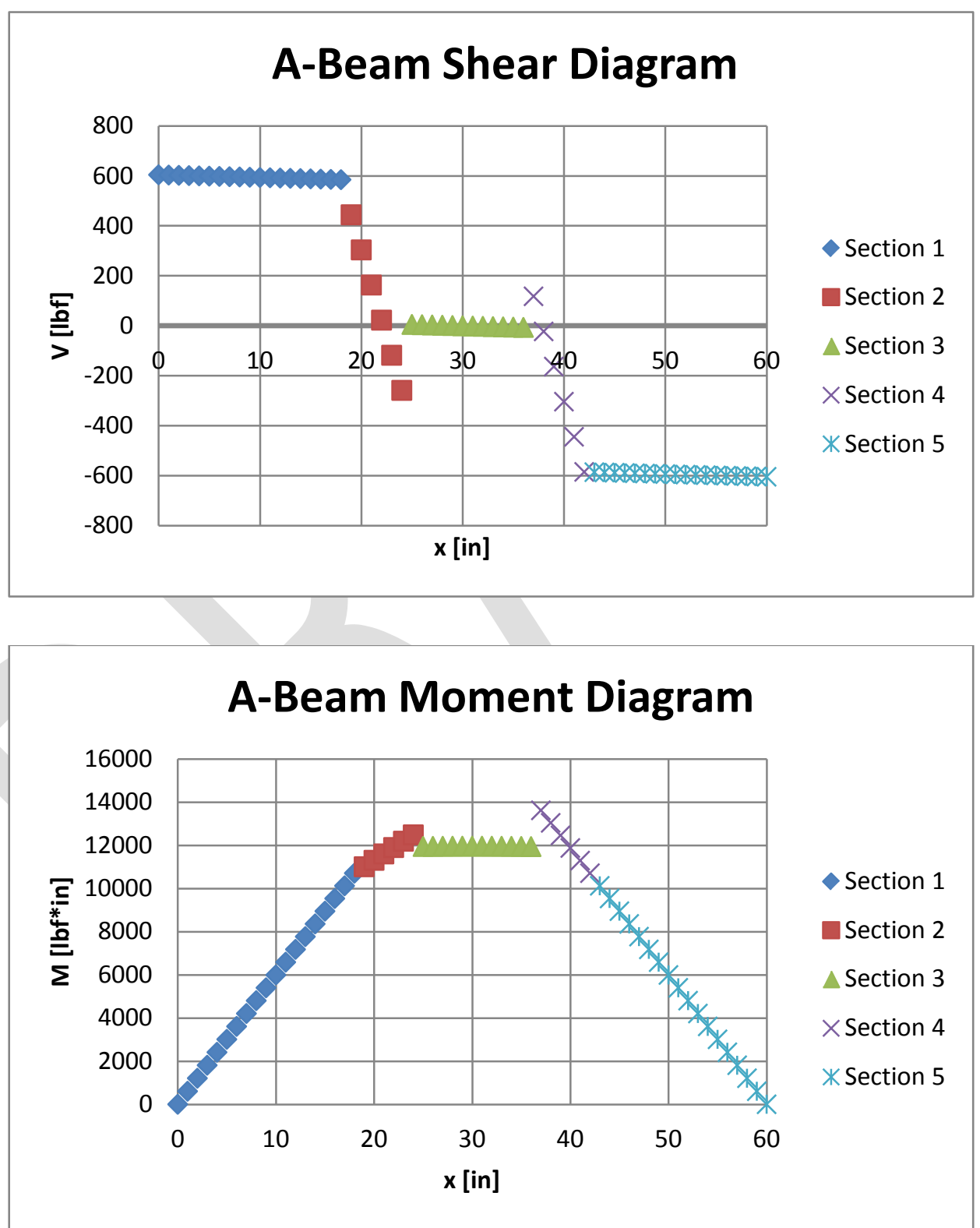

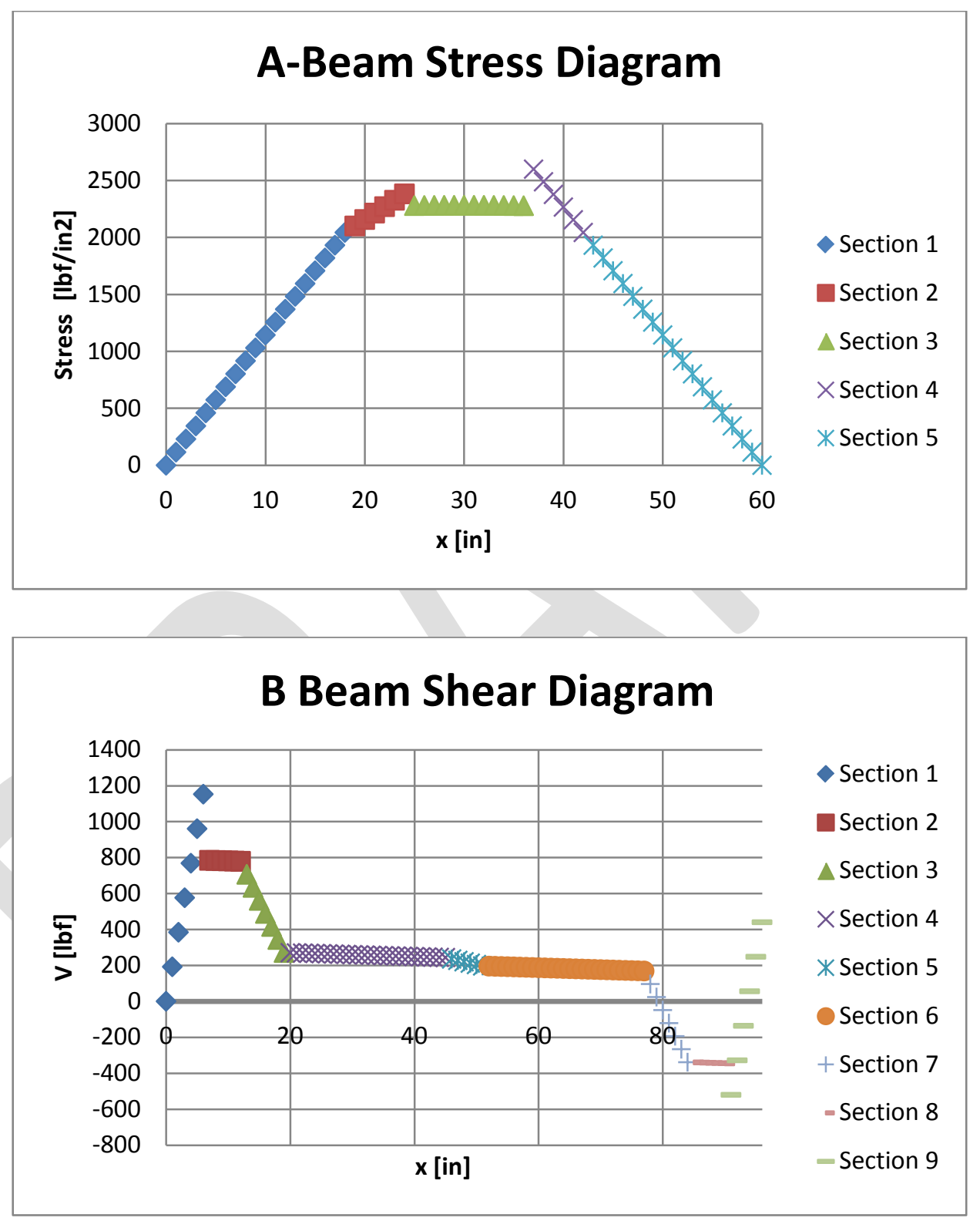


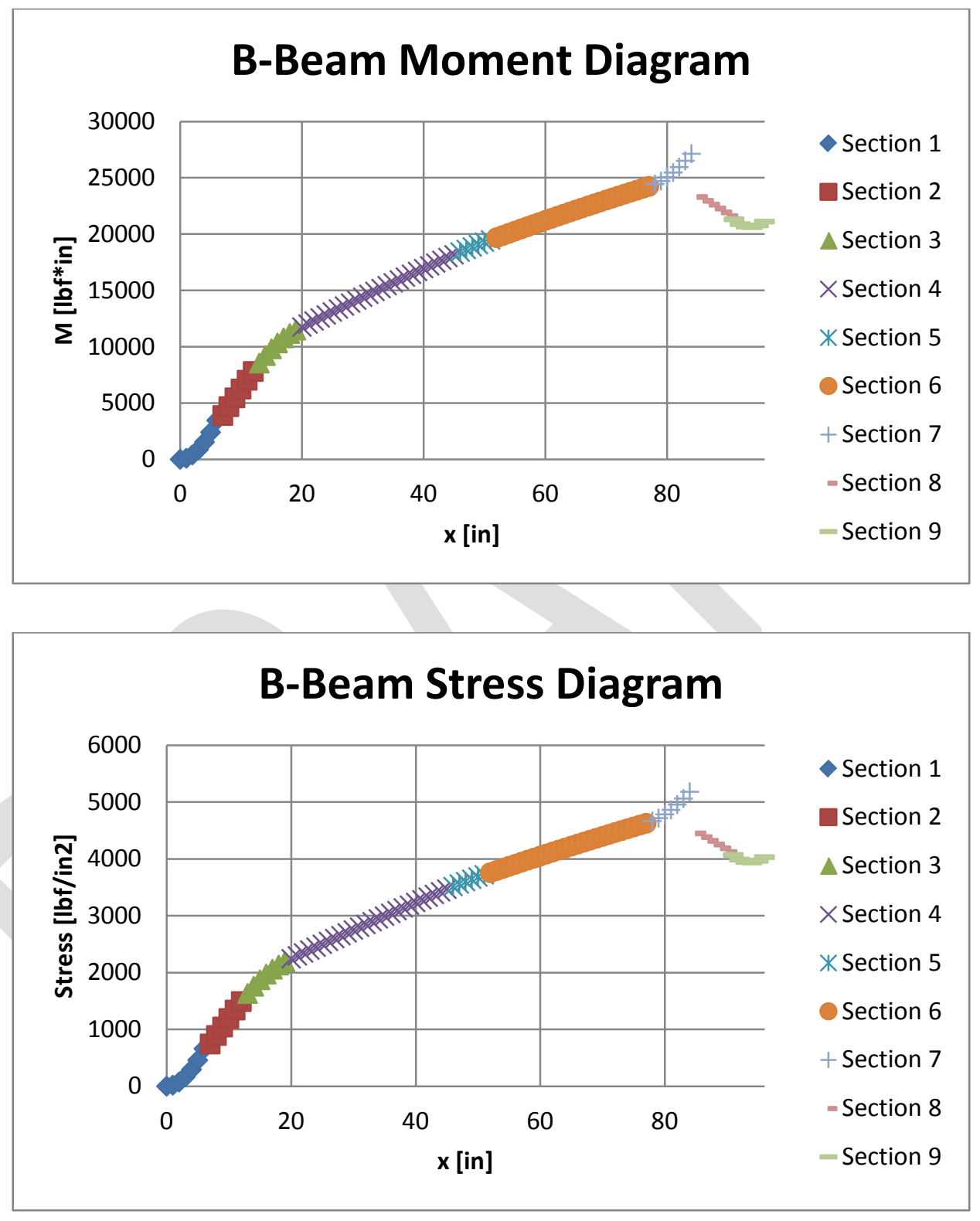


West Virginia University

Small Microgravity Research Facility

Lifting System Support Design Comparison

Created By: Kyle Phillips

Date: 20 Sept. 2011

--> Grayed areas represent dimensions too large for the given space (A-Bea

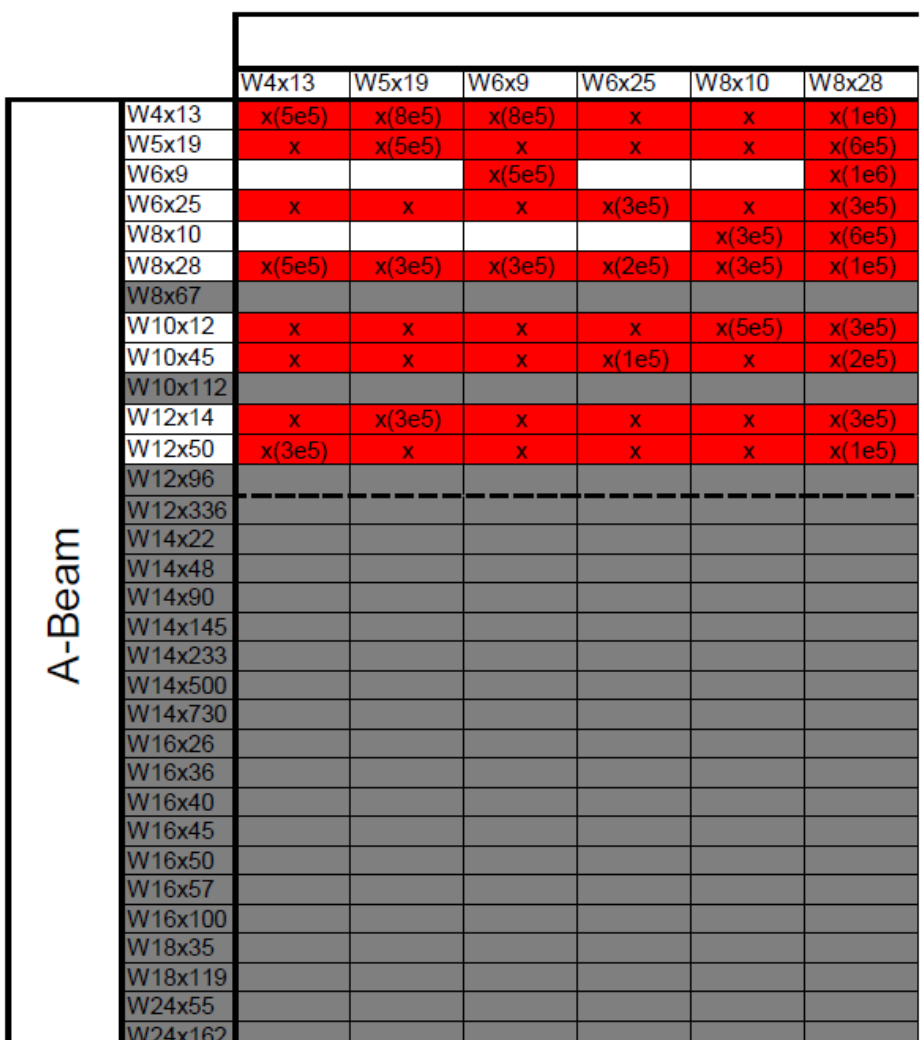

am limited by sprinkler lines (13.5"), B-Beam limited by structural ceiling beam (24"); natural 8" space for hoist

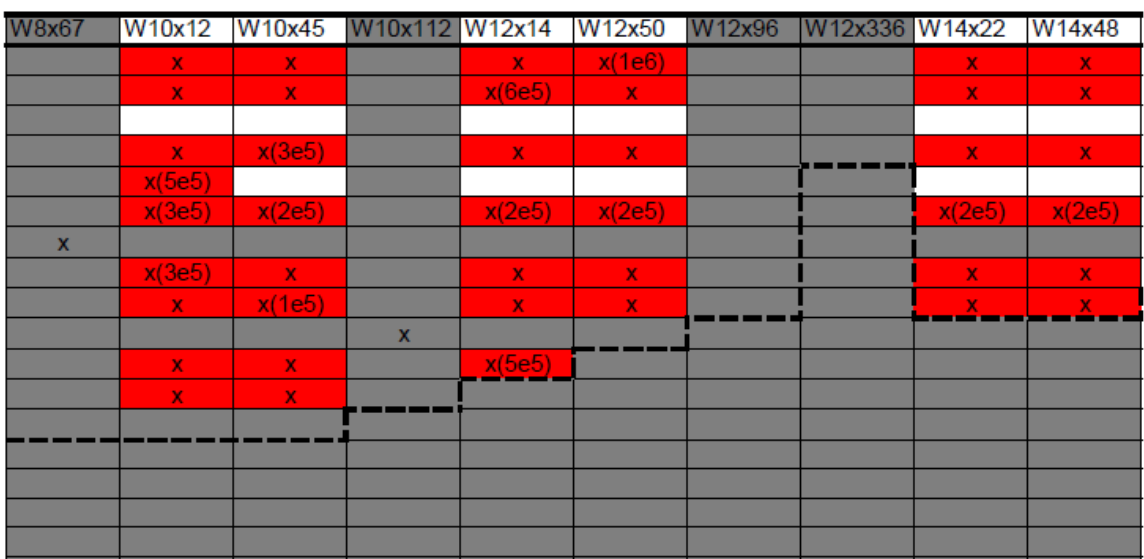


Development of the West Virginia University Small Microgravity Research Facility (WVU SMiRF)
Copyright $^{\circledR} 2014$

Kyle G. Phillips

)

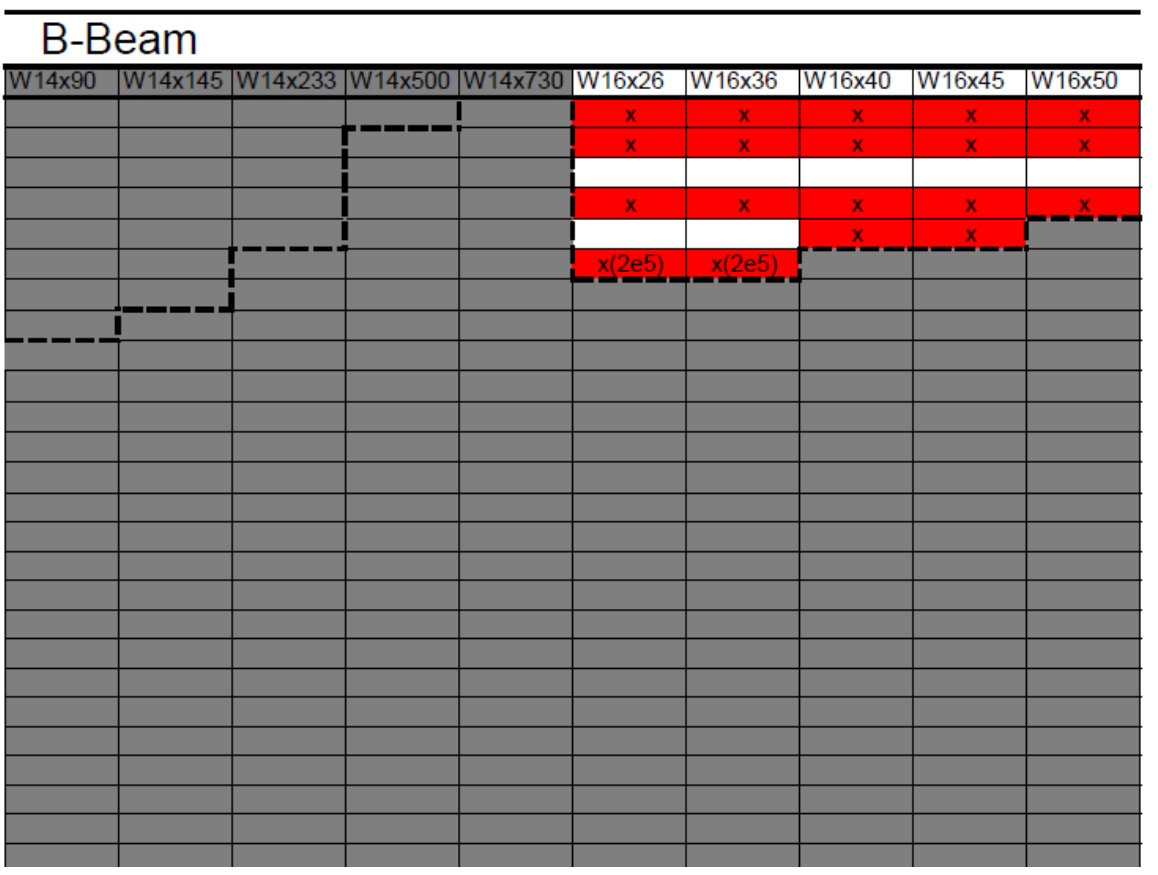

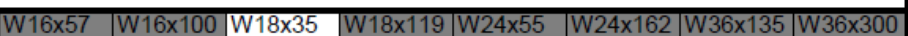

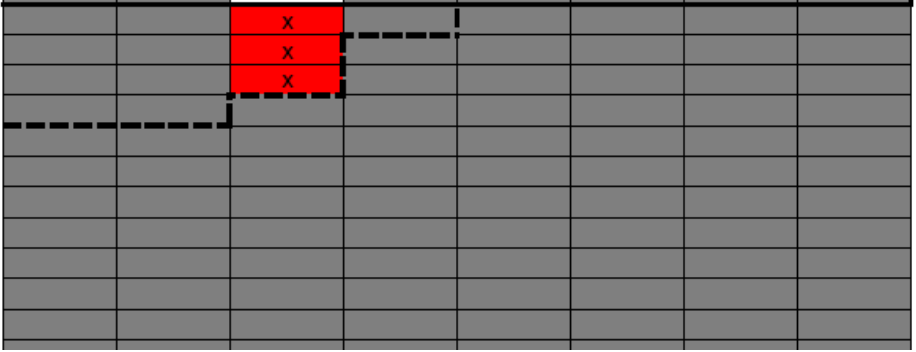


West Virginia University

Small Microgravity Research Facility

Lifting System Support Design Comparison

Created By: Kyle Phillips

Date: 20 Sept. 2011

\begin{tabular}{|c|c|c|c|c|c|c|c|}
\hline \multirow[b]{2}{*}{$\begin{array}{c}\text { A Beam } \\
\text { Classificatio } \\
n\end{array}$} & \multirow[b]{2}{*}{$\begin{array}{c}\text { B Beam } \\
\text { Classificatio } \\
n\end{array}$} & \multicolumn{6}{|c|}{ A Beam } \\
\hline & & $\begin{array}{c}\text { Beam } \\
\text { Height (d) } \\
\text { [in] }\end{array}$ & $\begin{array}{c}\text { Web } \\
\text { Thickness }\left(\mathrm{t}_{\boldsymbol{\alpha}}\right) \\
\text { [in] }\end{array}$ & $\begin{array}{c}\text { Flange } \\
\text { Width (b.) } \\
\text { [in] }\end{array}$ & $\begin{array}{c}\text { Flange } \\
\text { Thickness (t) } \\
\text { [in] }\end{array}$ & Weight [lb/ft] & $\begin{array}{c}\text { Moment of } \\
\text { Inertia }\left(l_{x_{x}}\right)\left[\text { in }^{4}\right]\end{array}$ \\
\hline \multirow{18}{*}{ W $4 \times 13$} & W4x13 & 4.2 & 0.28 & 4.1 & 0.35 & 13 & 11 \\
\hline & W5x19 & 4.2 & 0.28 & 4.1 & 0.35 & 13 & 11 \\
\hline & W6x9 & 4.2 & 0.28 & 4.1 & 0.35 & 13 & 11 \\
\hline & W6x25 & 4.2 & 0.28 & 4.1 & 0.35 & 13 & 11 \\
\hline & W8x10 & 4.2 & 0.28 & 4.1 & 0.35 & 13 & 11 \\
\hline & W8x28 & 4.2 & 0.28 & 4.1 & 0.35 & 13 & 11 \\
\hline & W10x12 & 4.2 & 0.28 & 4.1 & 0.35 & 13 & 11 \\
\hline & W10x45 & 4.2 & 0.28 & 4.1 & 0.35 & 13 & 11 \\
\hline & W12x14 & 4.2 & 0.28 & 4.1 & 0.35 & 13 & 11 \\
\hline & W12x50 & 4.2 & 0.28 & 4.1 & 0.35 & 13 & 11 \\
\hline & W14x22 & 4.2 & 0.28 & 4.1 & 0.35 & 13 & 11 \\
\hline & W14x48 & 4.2 & 0.28 & 4.1 & 0.35 & 13 & 11 \\
\hline & W16×26 & 4.2 & 0.28 & 4.1 & 0.35 & 13 & 11 \\
\hline & W16×36 & 4.2 & 0.28 & 4.1 & 0.35 & 13 & 11 \\
\hline & W16x40 & 4.2 & 0.28 & 4.1 & 0.35 & 13 & 11 \\
\hline & W16x45 & 4.2 & 0.28 & 4.1 & 0.35 & 13 & 11 \\
\hline & W16×50 & 4.2 & 0.28 & 4.1 & 0.35 & 13 & 11 \\
\hline & W18x35 & 4.2 & 0.28 & 4.1 & 0.35 & 13 & 11 \\
\hline \multirow{18}{*}{ W5x19 } & $\begin{array}{l}\text { W } 4 \times 13 \\
\end{array}$ & 5.2 & 0.27 & 5 & 0.43 & 19 & 26 \\
\hline & W5x19 & 5.2 & 0.27 & 5 & 0.43 & 19 & 26 \\
\hline & W6x9 & 5.2 & 0.27 & 5 & 0.43 & 19 & 26 \\
\hline & W6x25 & 5.2 & 0.27 & 5 & 0.43 & 19 & 26 \\
\hline & W8x10 & 5.2 & 0.27 & 5 & 0.43 & 19 & 26 \\
\hline & W8x28 & 5.2 & 0.27 & 5 & 0.43 & 19 & 26 \\
\hline & W10x12 & 5.2 & 0.27 & 5 & 0.43 & 19 & 26 \\
\hline & W10x45 & 5.2 & 0.27 & 5 & 0.43 & 19 & 26 \\
\hline & W12×14 & 5.2 & 0.27 & 5 & 0.43 & 19 & 26 \\
\hline & W12x50 & 5.2 & 0.27 & 5 & 0.43 & 19 & 26 \\
\hline & W14x22 & 5.2 & 0.27 & 5 & 0.43 & 19 & 26 \\
\hline & W14x48 & 5.2 & 0.27 & 5 & 0.43 & 19 & 26 \\
\hline & W16x26 & 5.2 & 0.27 & 5 & 0.43 & 19 & 26 \\
\hline & W16×36 & 5.2 & 0.27 & 5 & 0.43 & 19 & 26 \\
\hline & W16x40 & 5.2 & 0.27 & 5 & 0.43 & 19 & 26 \\
\hline & W16x45 & 5.2 & 0.27 & 5 & 0.43 & 19 & 26 \\
\hline & W16x50 & 5.2 & 0.27 & 5 & 0.43 & 19 & 26 \\
\hline & W18x35 & 5.2 & 0.27 & 5 & 0.43 & 19 & 26 \\
\hline \multirow{10}{*}{ W6x9 } & W4x13 & 5.9 & 0.17 & 3.9 & 0.22 & 9 & 16 \\
\hline & W5519 & 5.9 & 0.17 & 3.9 & 0.22 & 9 & 16 \\
\hline & W6x9 & 5.9 & 0.17 & 3.9 & 0.22 & 9 & 16 \\
\hline & W6x25 & 5.9 & 0.17 & 3.9 & 0.22 & 9 & 16 \\
\hline & W8x10 & 5.9 & 0.17 & 3.9 & 0.22 & 9 & 16 \\
\hline & W8x28 & 5.9 & 0.17 & 3.9 & 0.22 & 9 & 16 \\
\hline & W10x12 & 5.9 & 0.17 & 3.9 & 0.22 & 9 & 16 \\
\hline & W10x45 & 5.9 & 0.17 & 3.9 & 0.22 & 9 & 16 \\
\hline & W12x14 & 5.9 & 0.17 & 3.9 & 0.22 & 9 & 16 \\
\hline & W12x50 & 5.9 & 0.17 & 3.9 & 0.22 & 9 & 16 \\
\hline
\end{tabular}



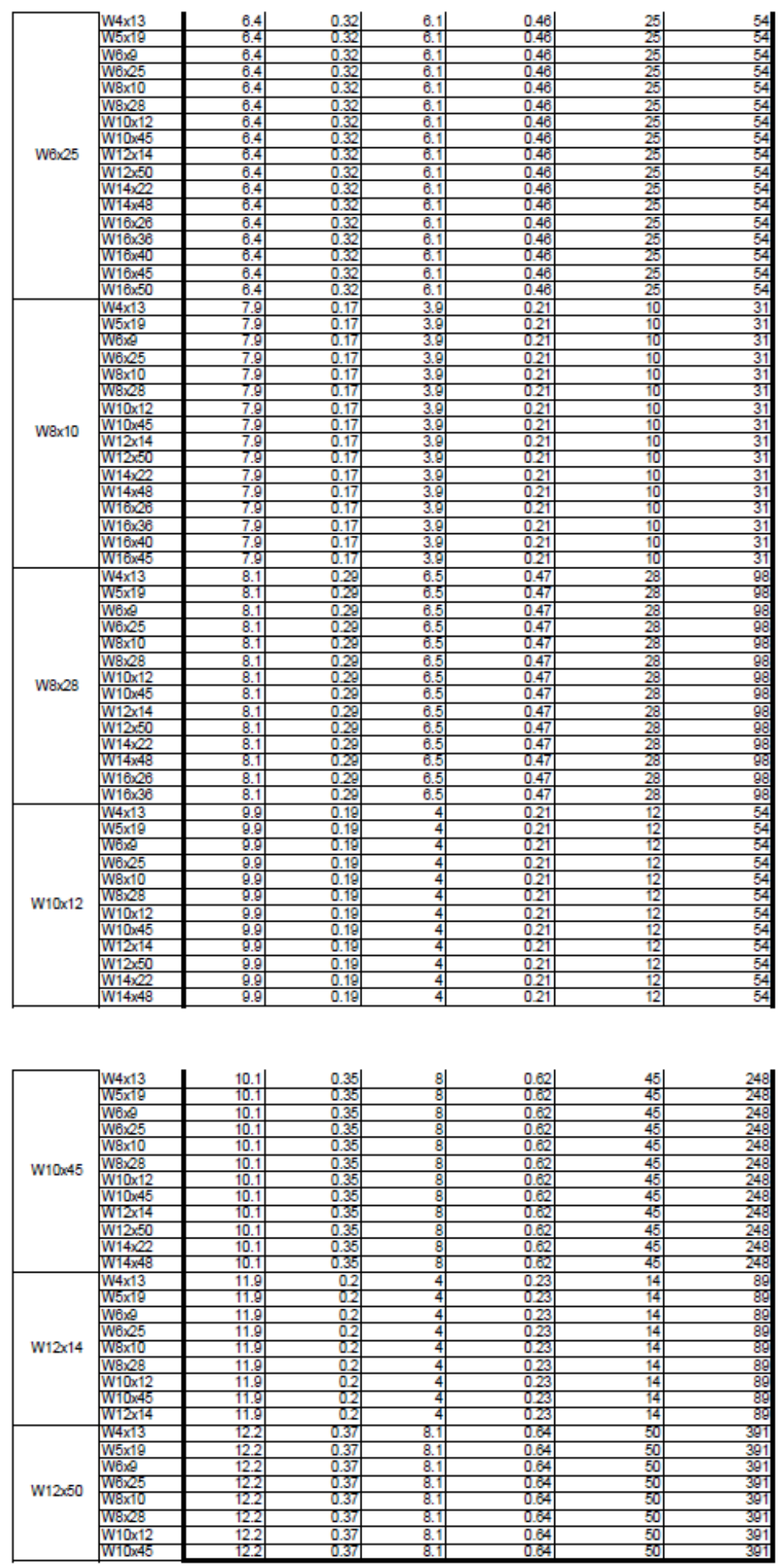


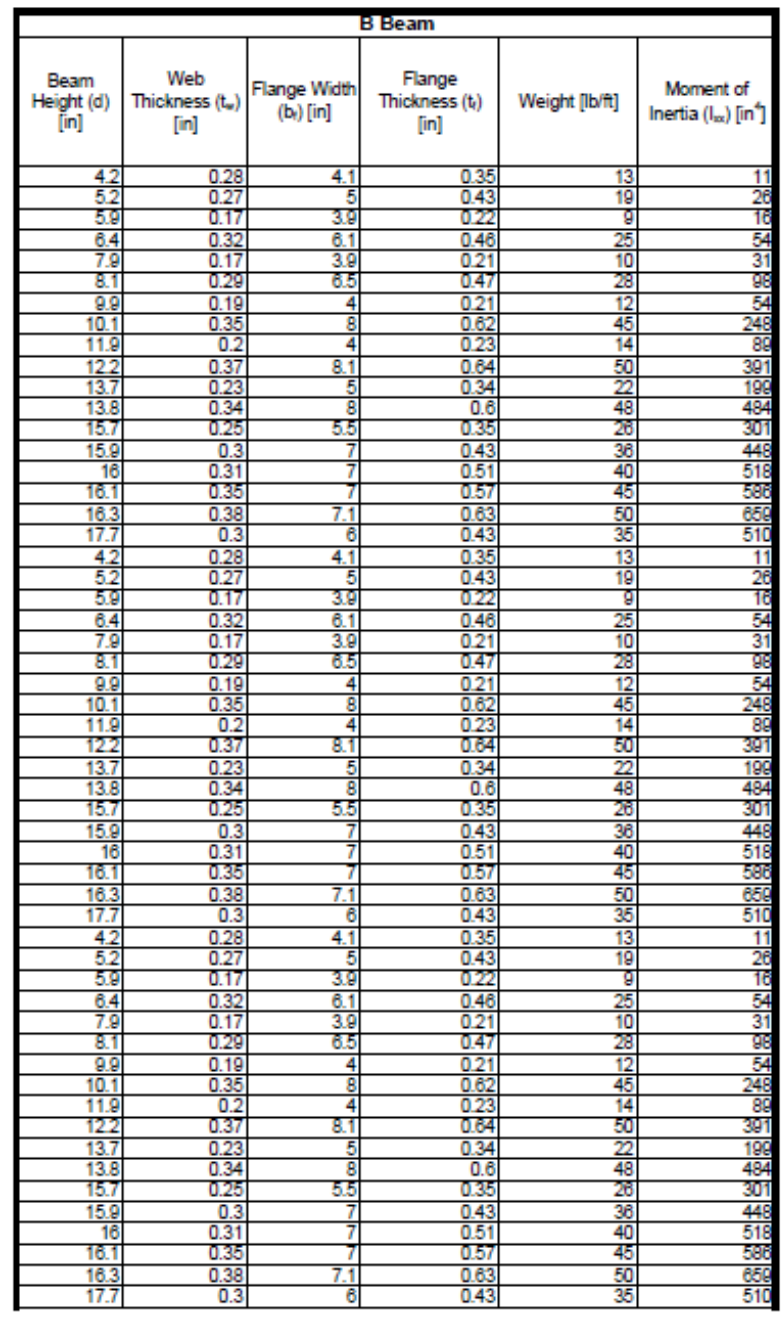




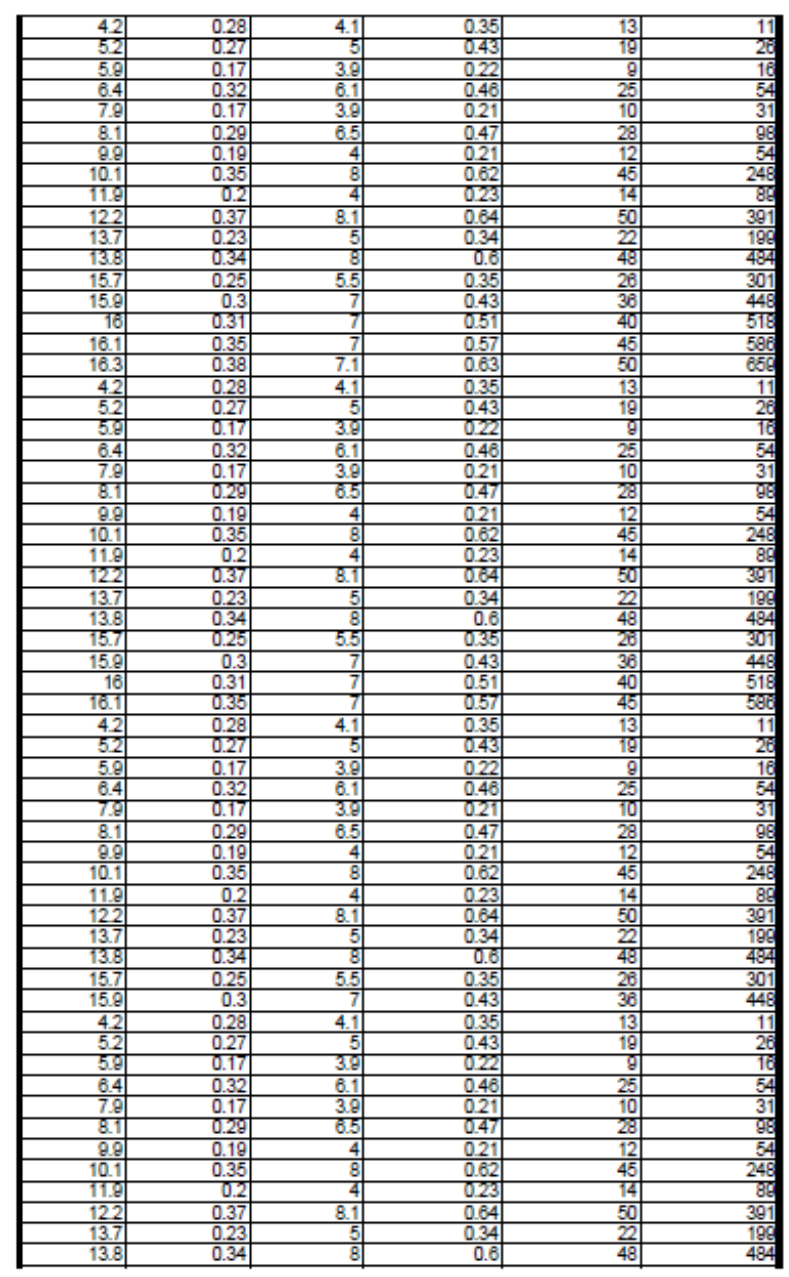



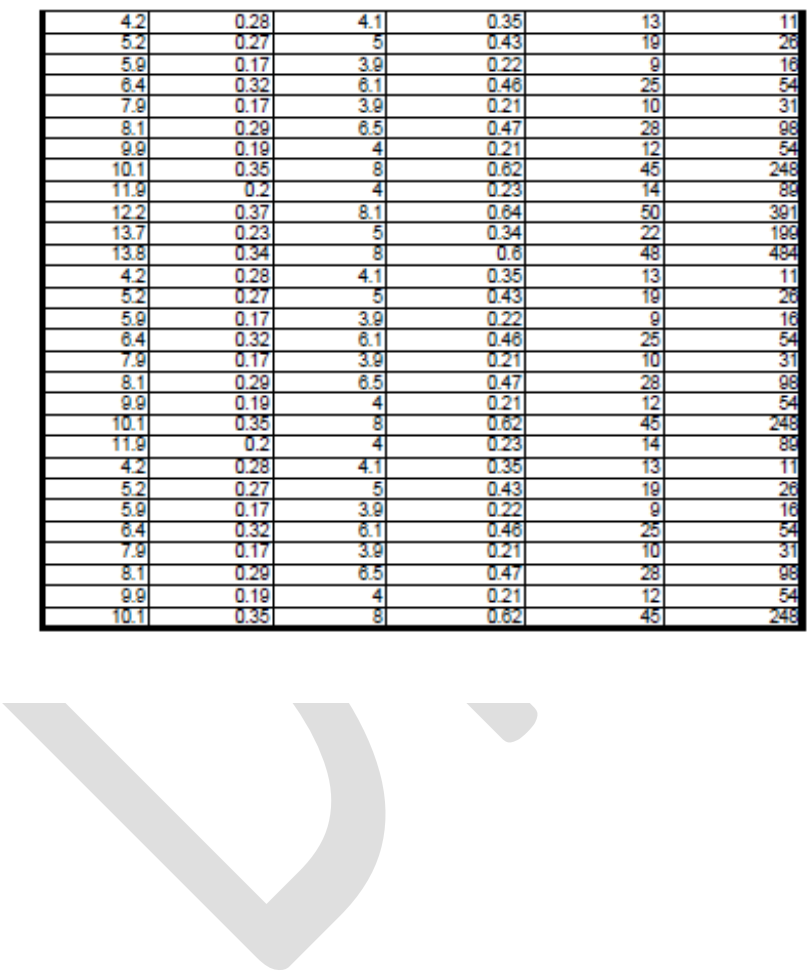


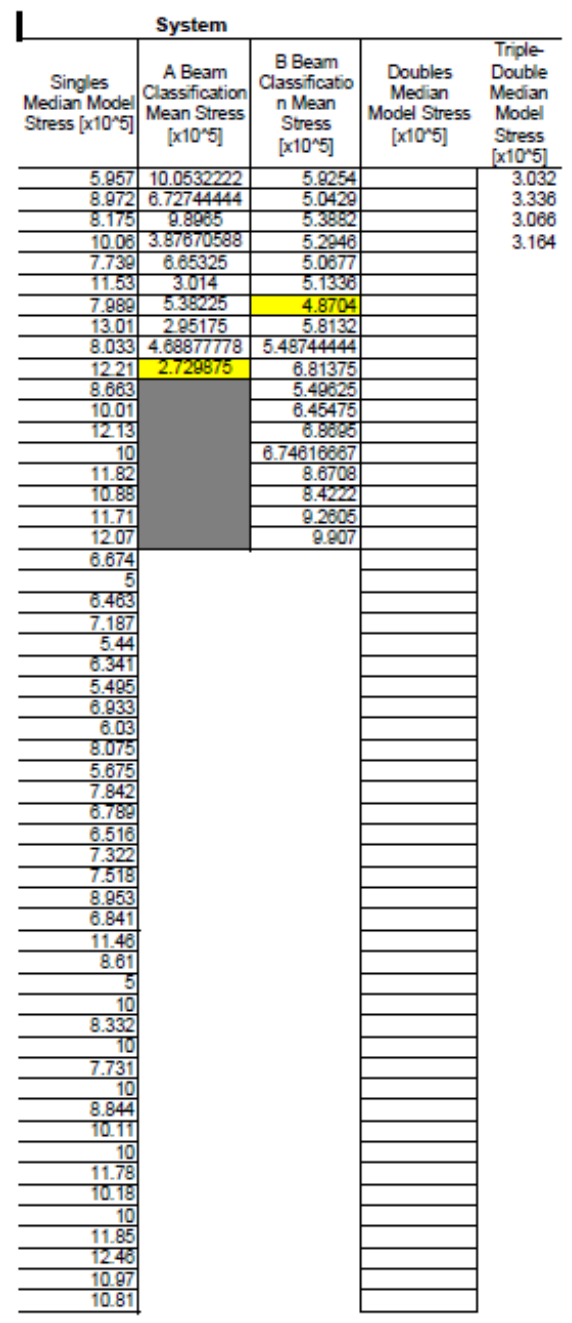


Development of the West Virginia University

Small Microgravity Research Facility (WVU SMiRF)
Copyright $^{\odot} 2014$

Kyle G. Phillips
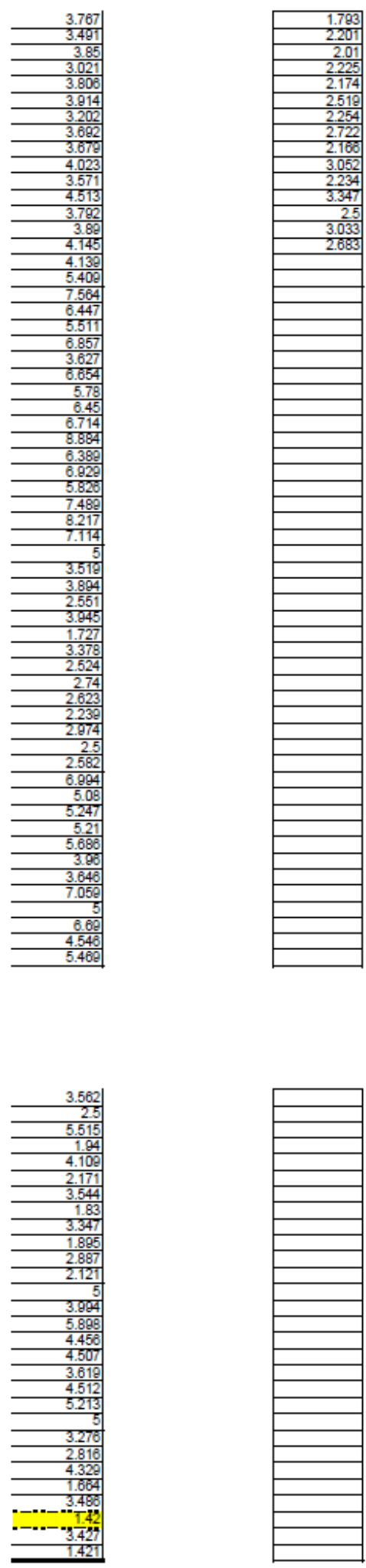
Development of the West Virginia University

Small Microgravity Research Facility (WVU SMiRF)
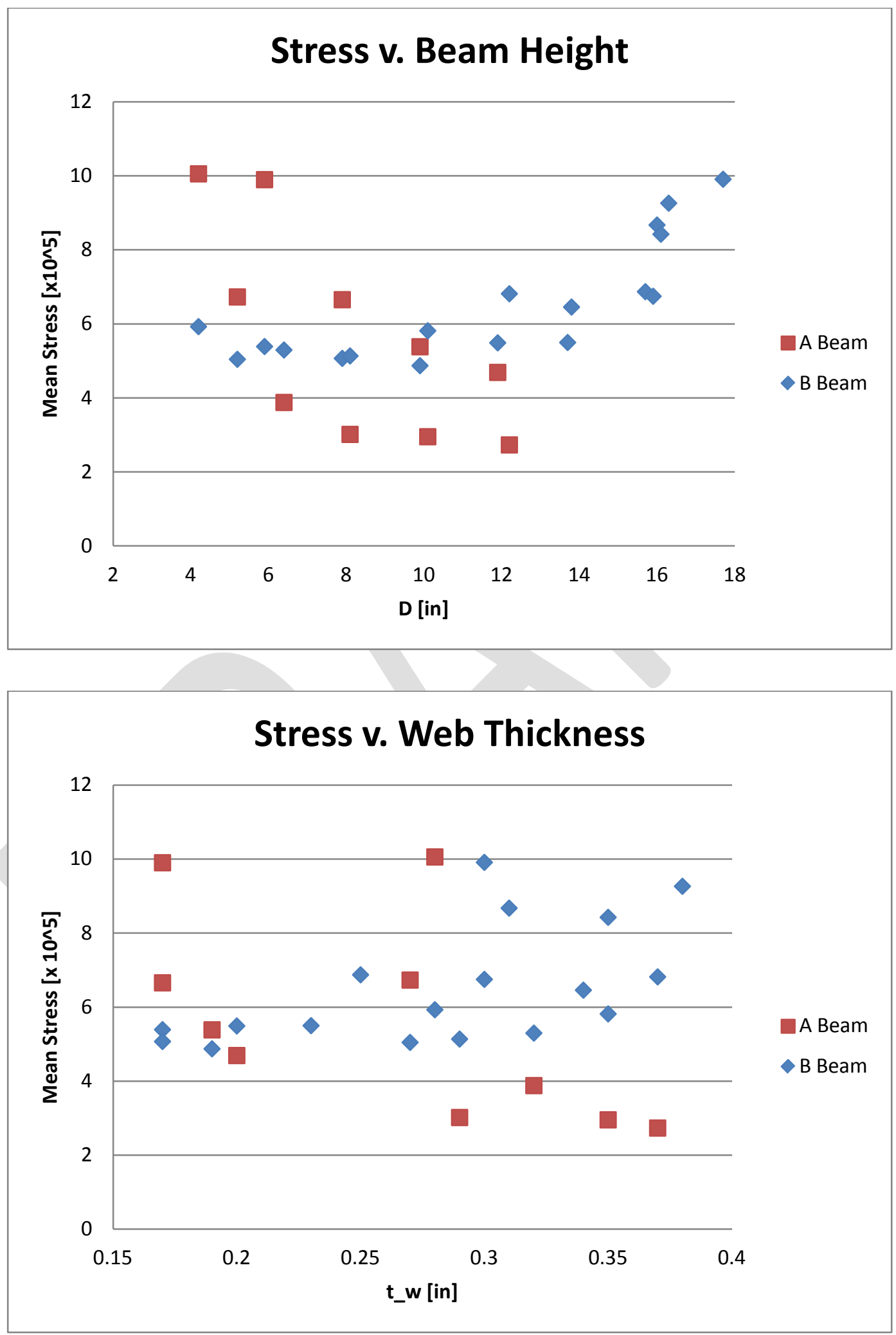
Development of the West Virginia University

Small Microgravity Research Facility (WVU SMiRF)

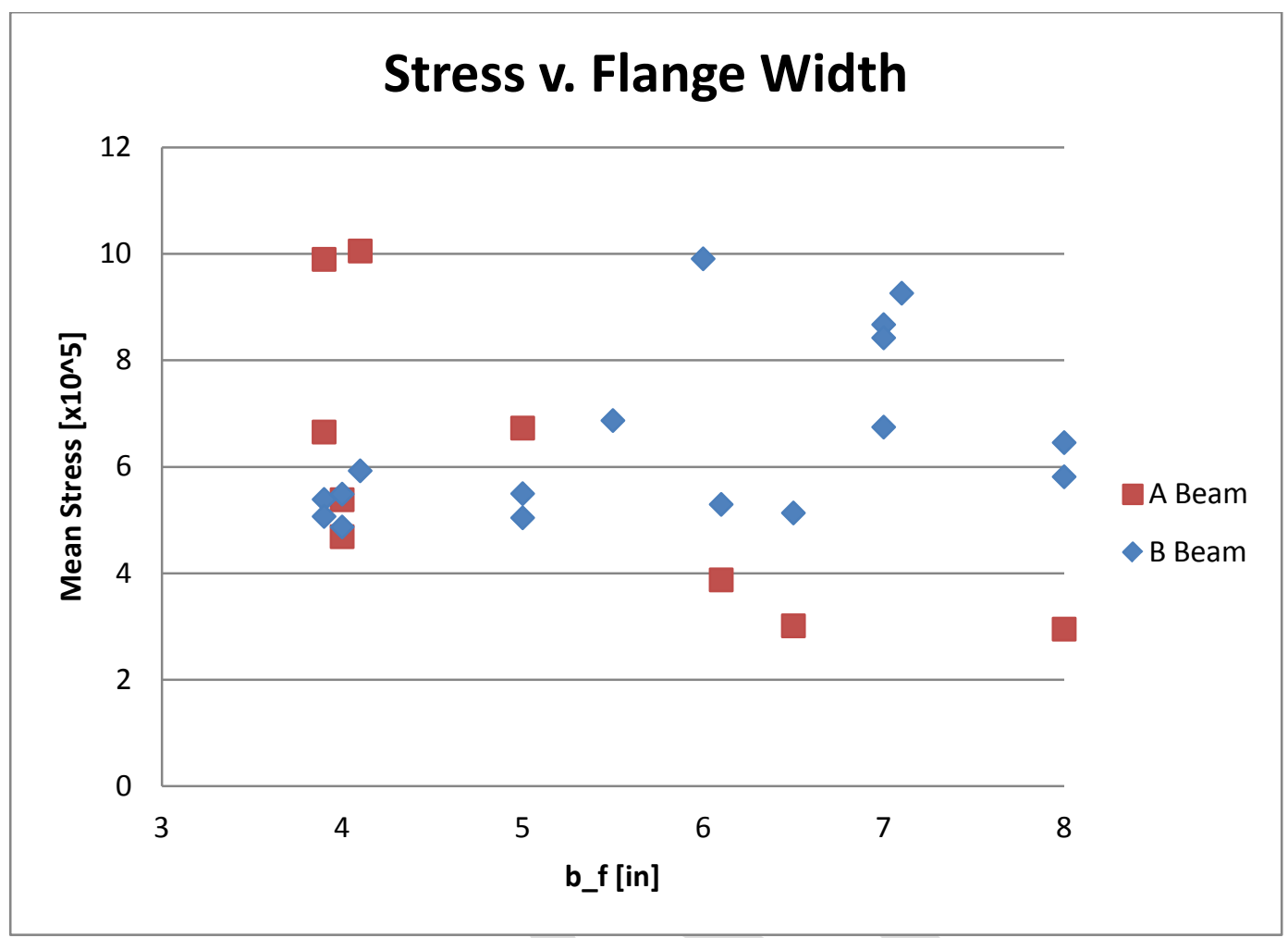

Stress v. Flange Thickness

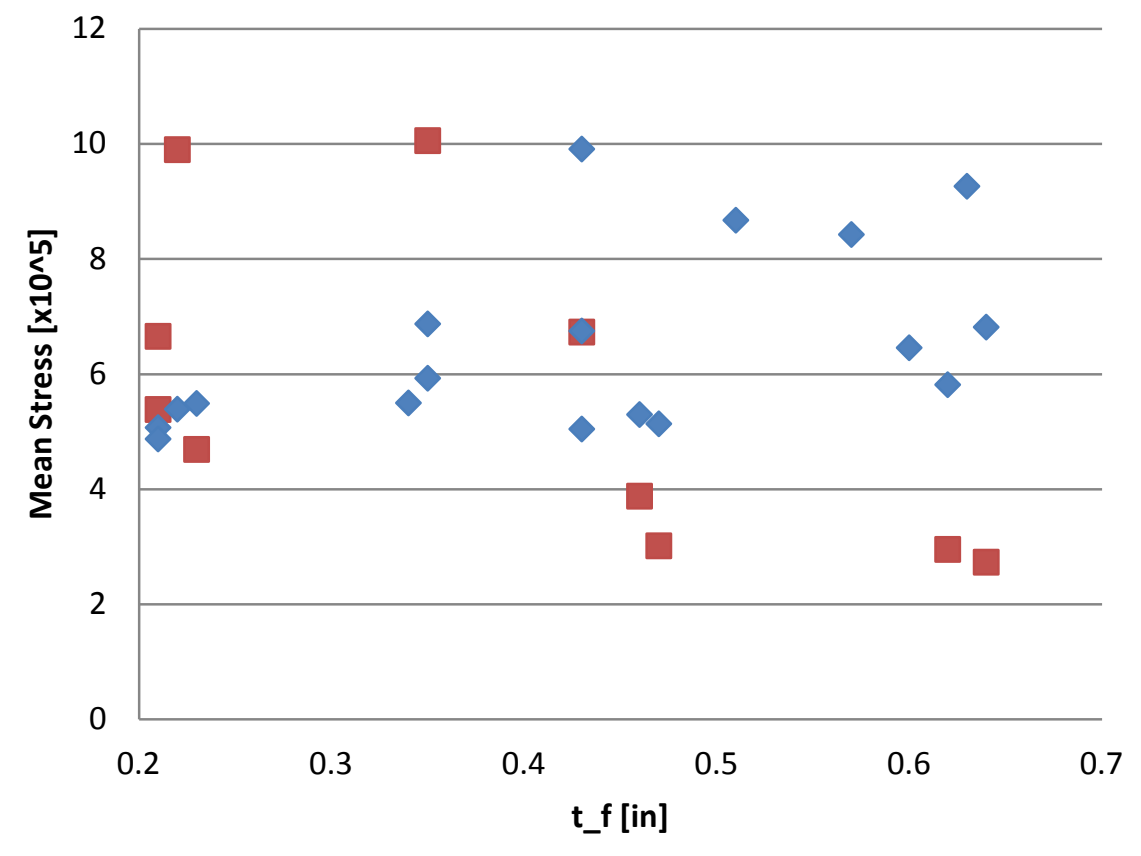




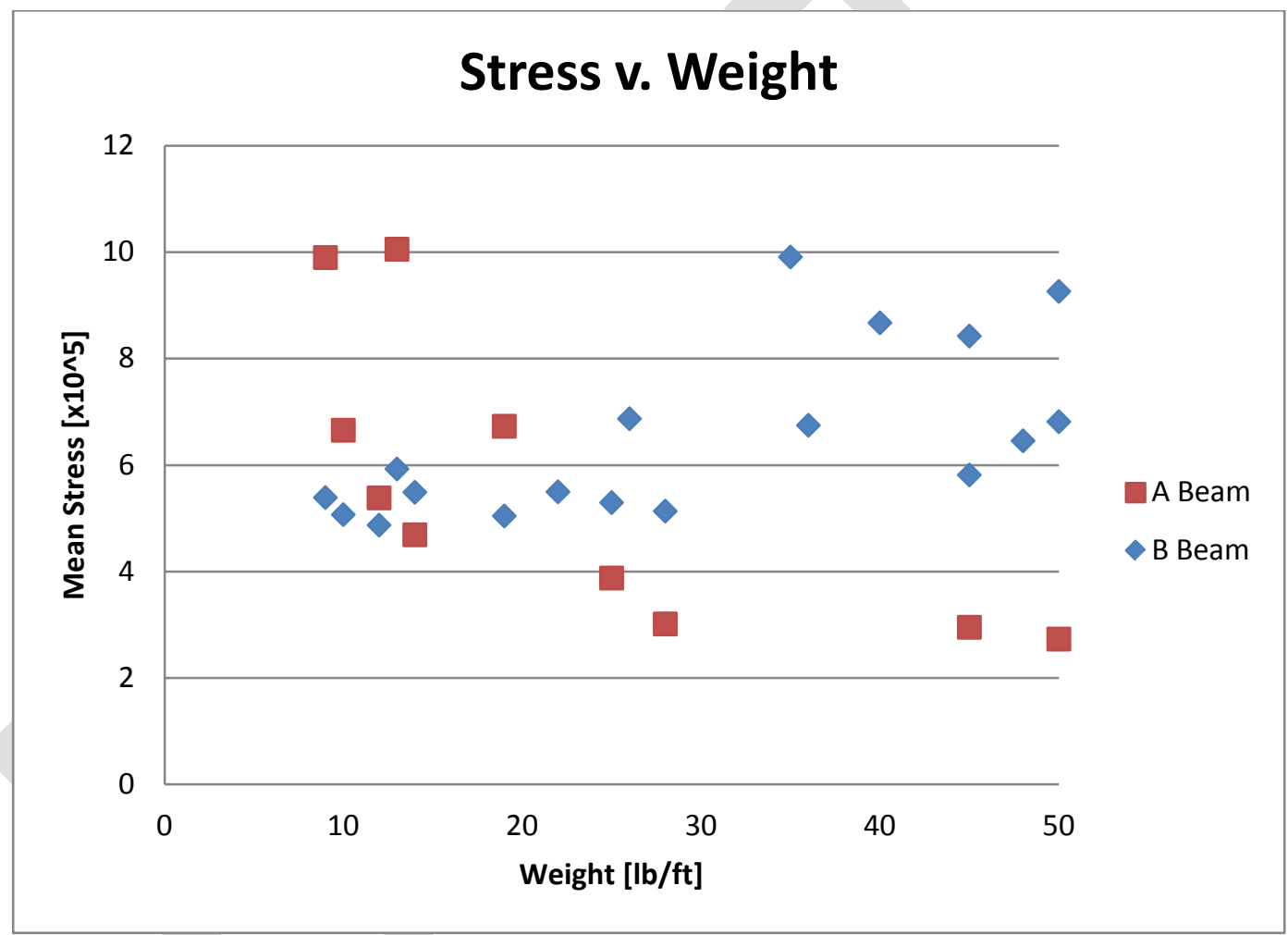




\section{Lifting System Structural Design}




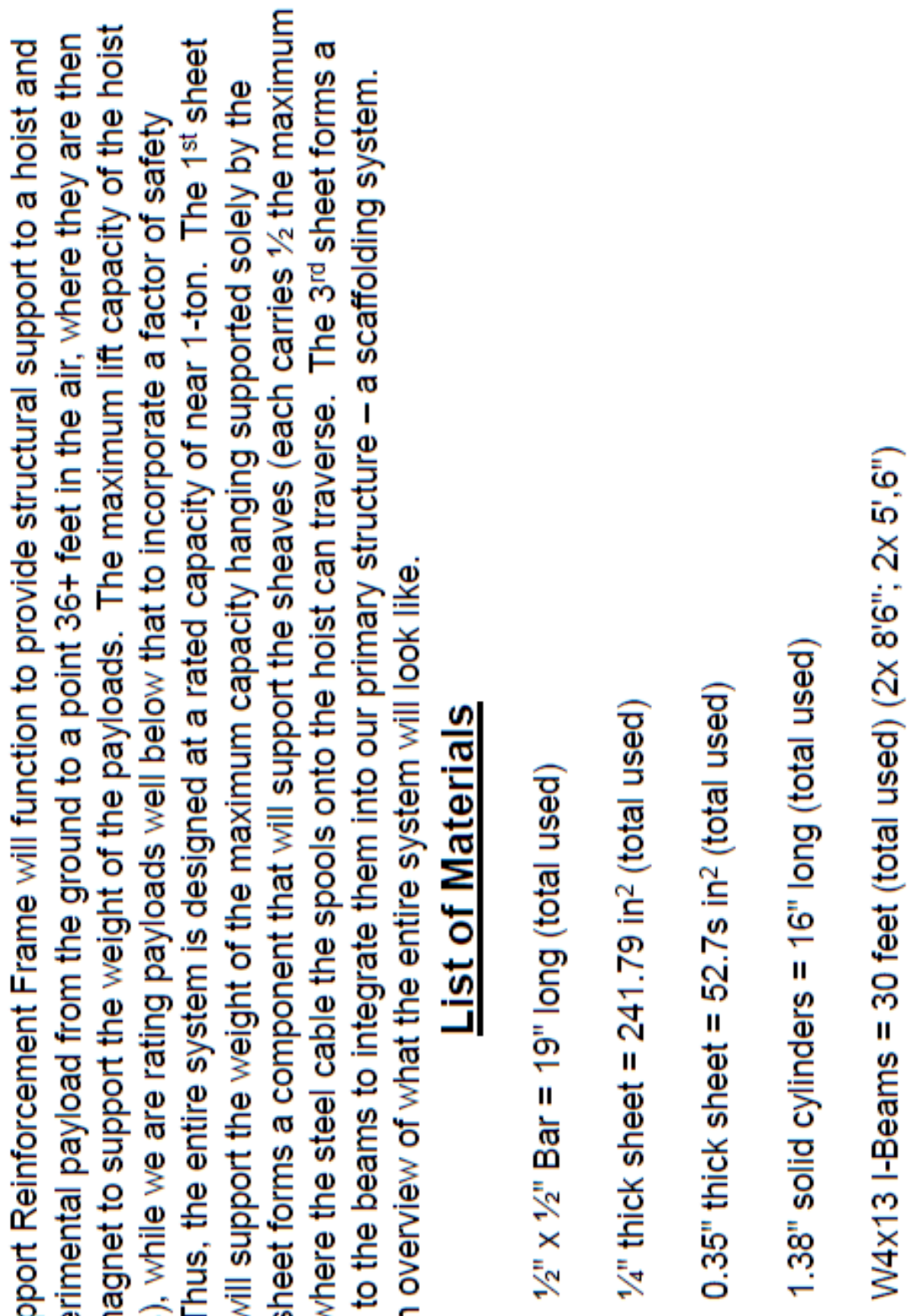


Development of the West Virginia University Small Microgravity Research Facility (WVU SMiRF)
Copyright $^{\odot} 2014$

Kyle G. Phillips

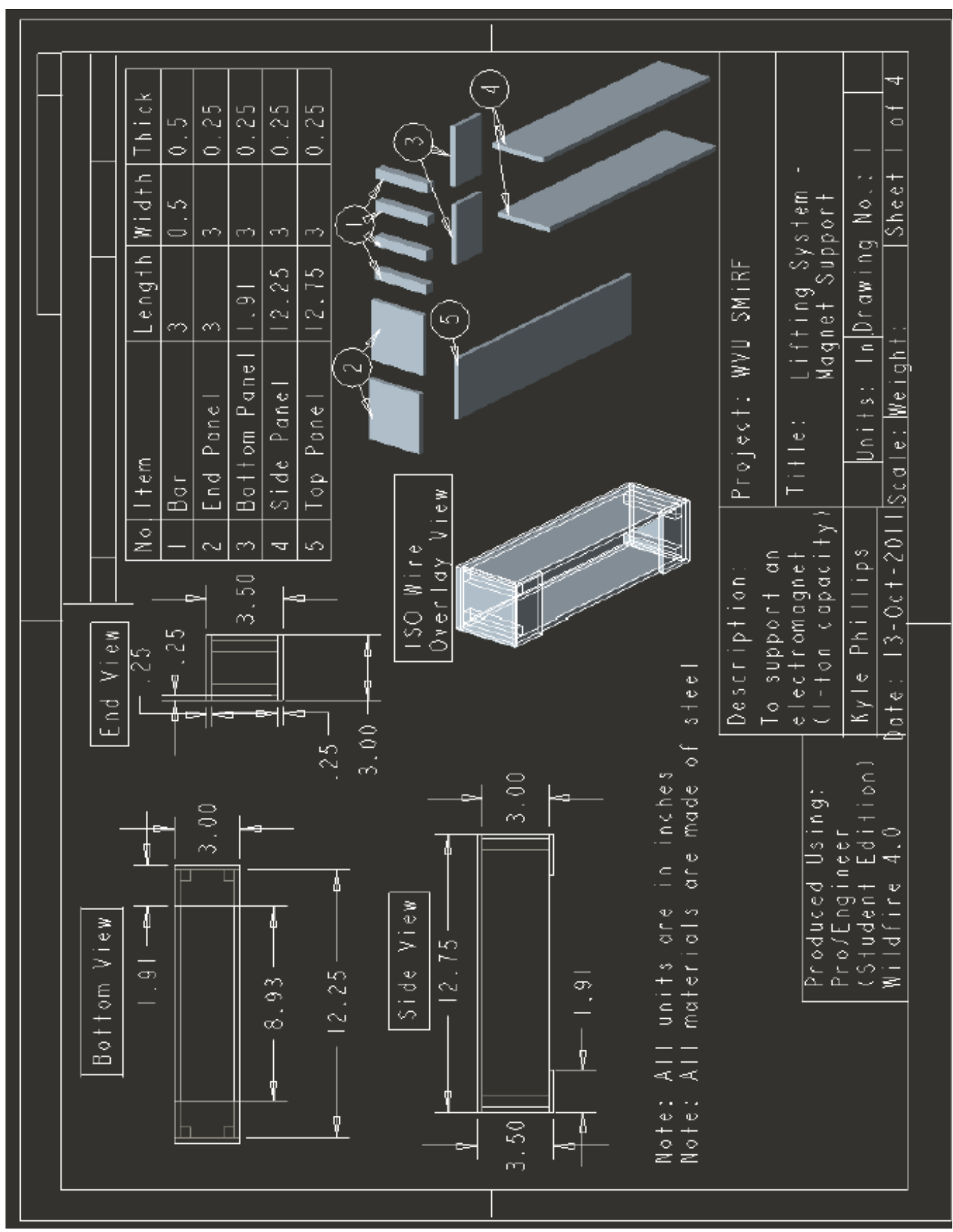


Development of the West Virginia University Small Microgravity Research Facility (WVU SMiRF)
Copyright $^{\odot} 2014$

Kyle G. Phillips

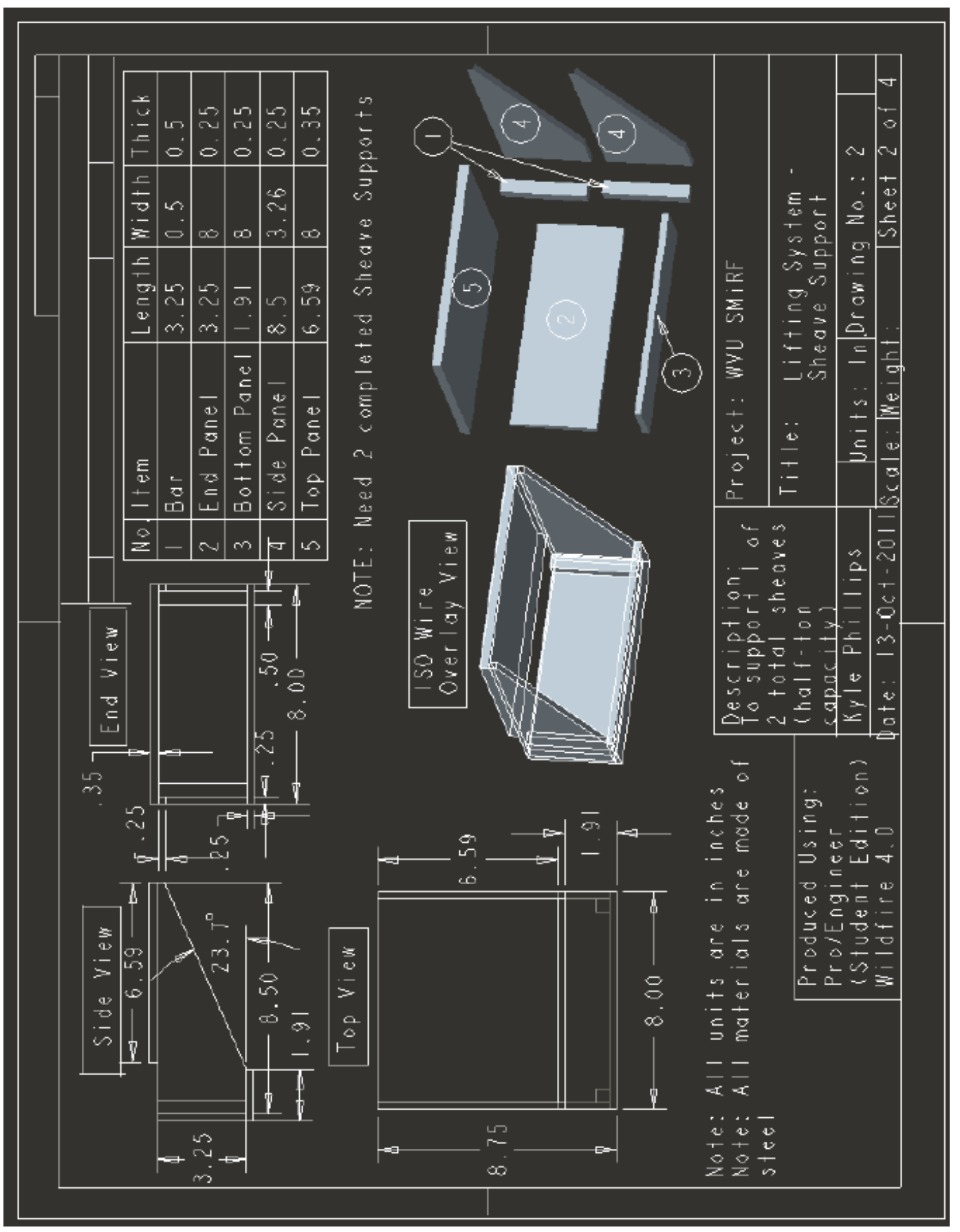


Development of the West Virginia University

Small Microgravity Research Facility (WVU SMiRF)
Copyright $^{\odot} 2014$

Kyle G. Phillips

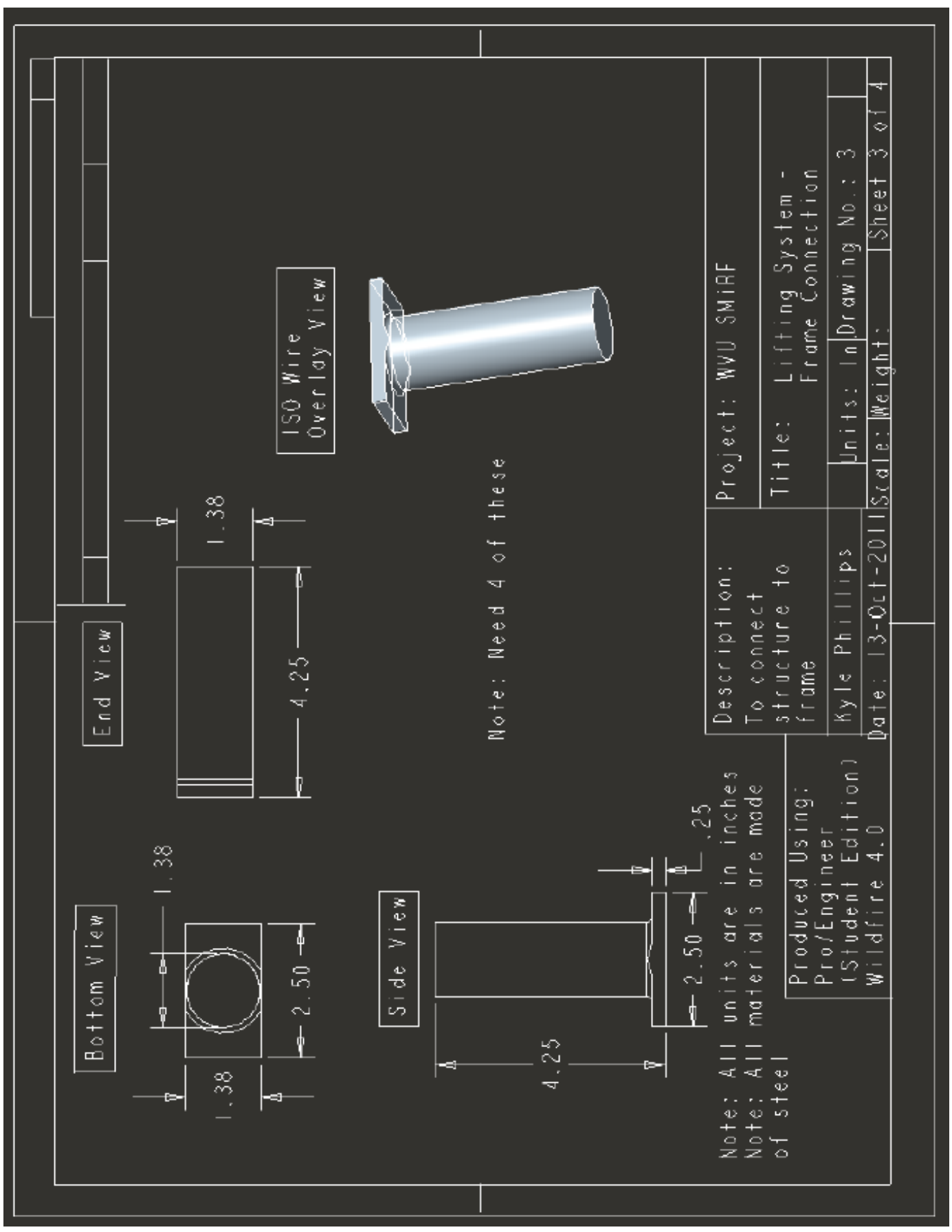


Development of the West Virginia University

Small Microgravity Research Facility (WVU SMiRF)
Copyright $^{\odot} 2014$

Kyle G. Phillips

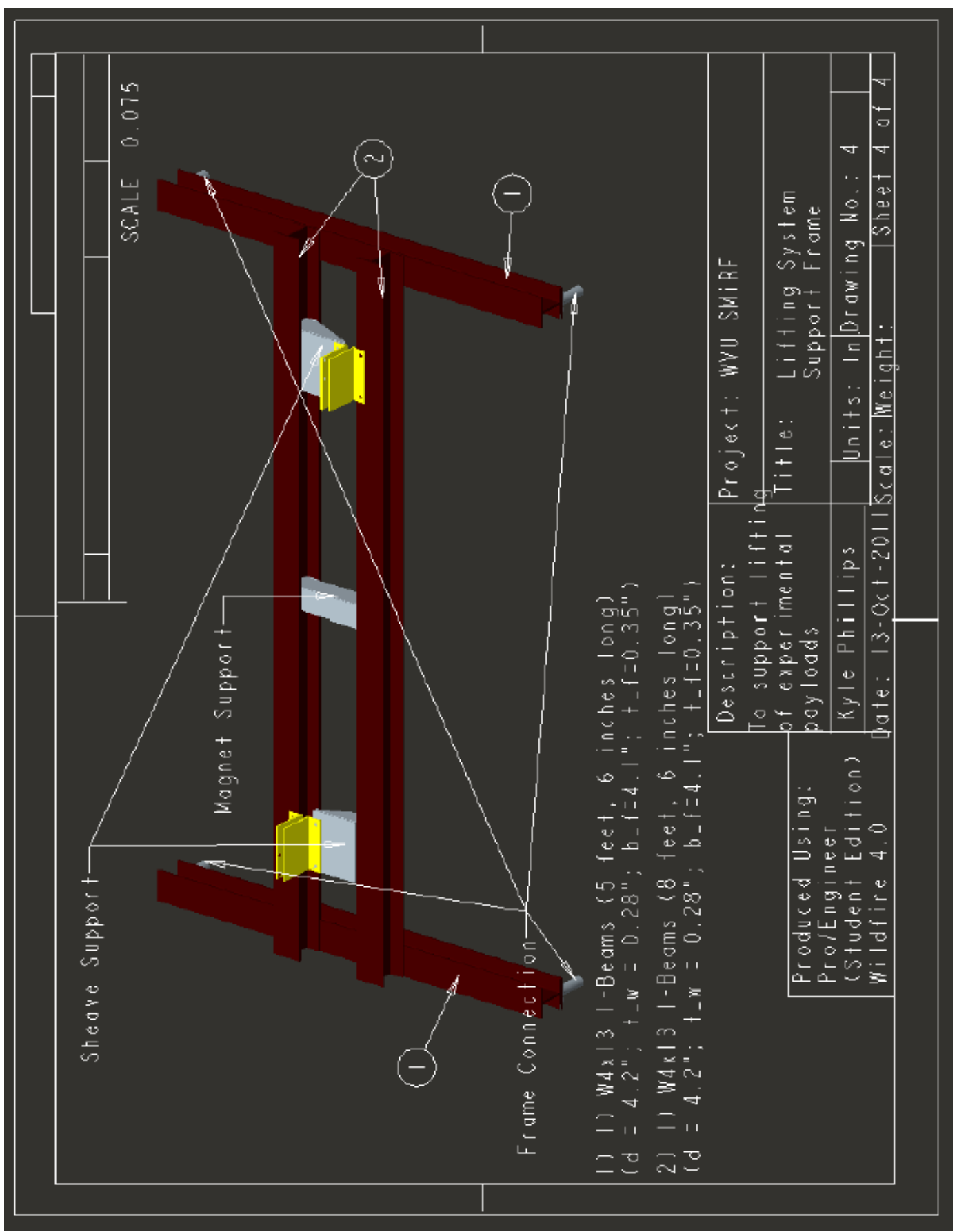




\subsection{Appendix I}

\section{WVU SMiRF Operations}

Appendix I: WVU SMiRF Operations presents operational flowcharts for:

- Initial checkout experiment focusing on Die Swell

- Data logging procedure for the Vernier LabQuest2 initial checkout data acquisition system

- Drop Test procedure to be used by all future operators to safely and successfully operate the WVU SMiRF

- Accelerometer Data Analysis procedure that should be employed by all future researchers to use Excel to analyze test data and provide legacy information for the WVU SMiRF 


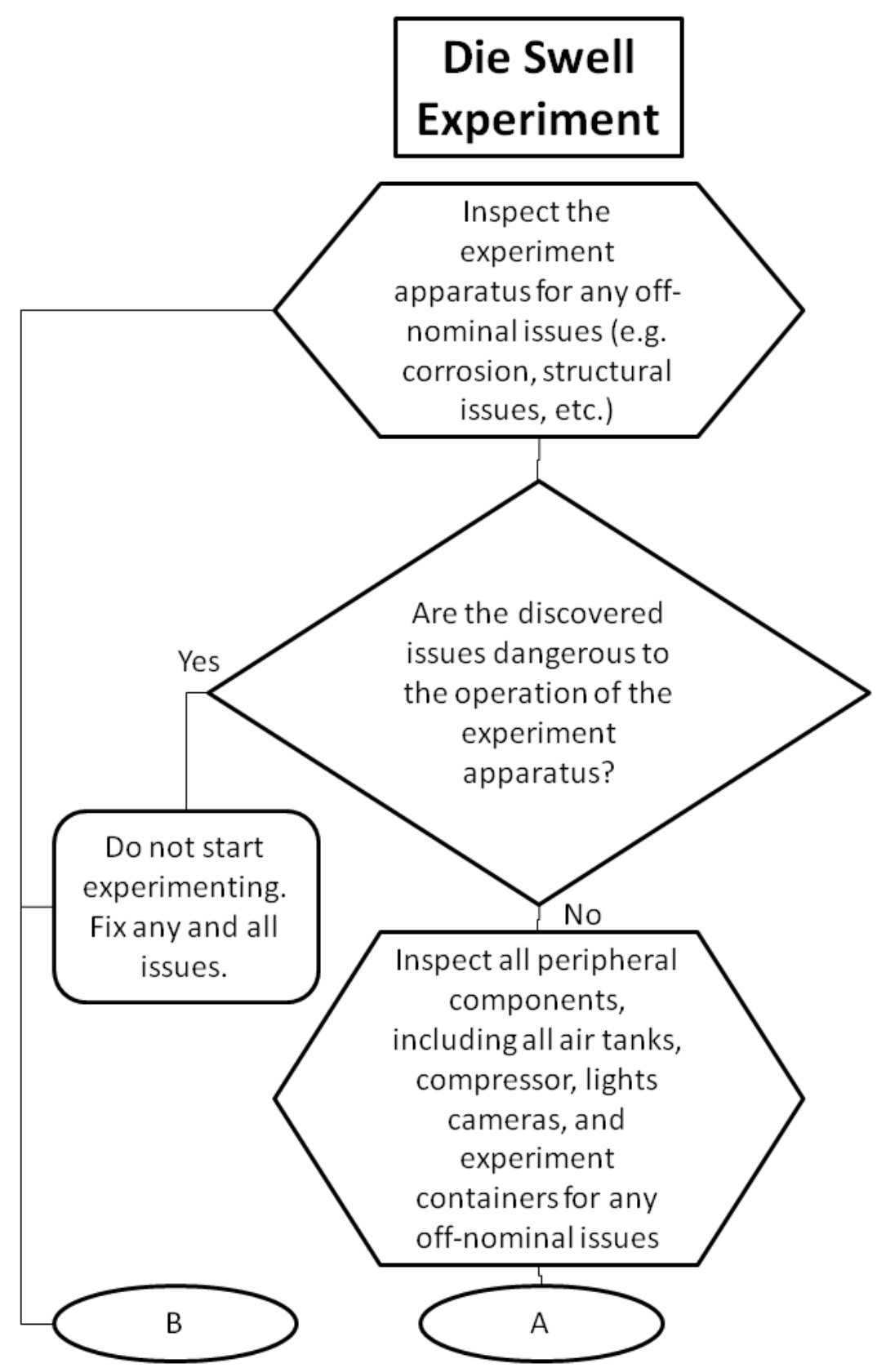




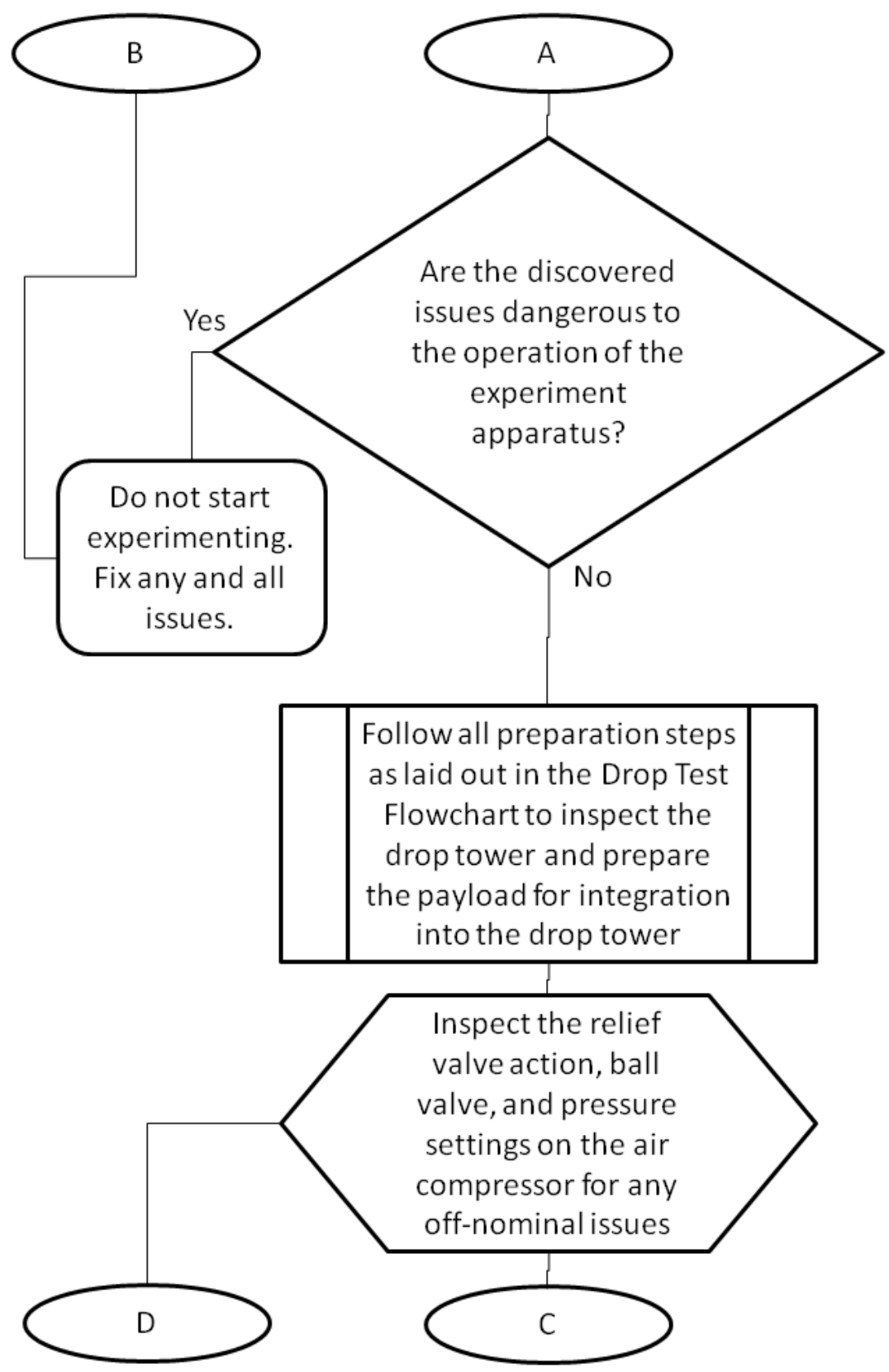




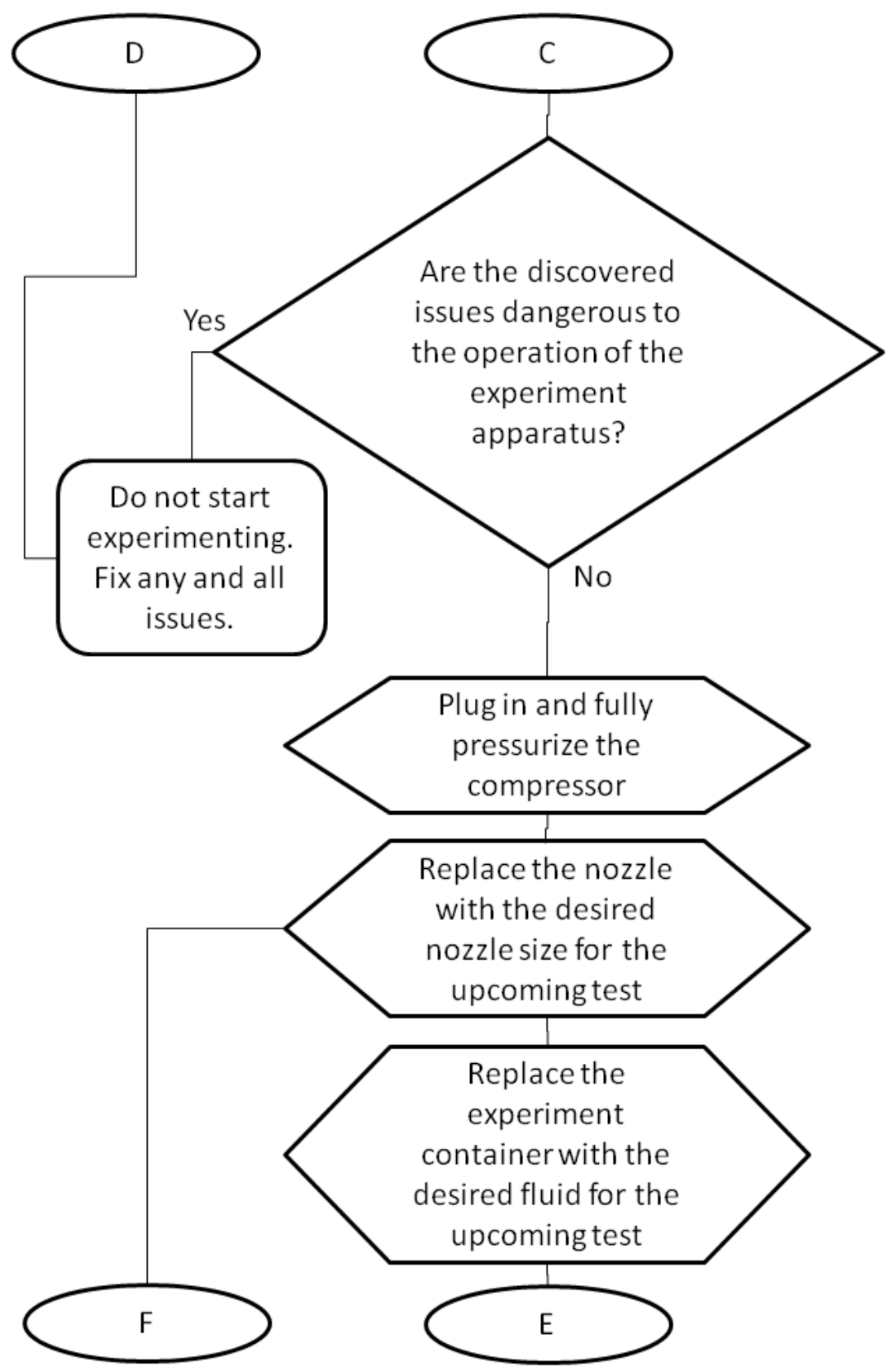




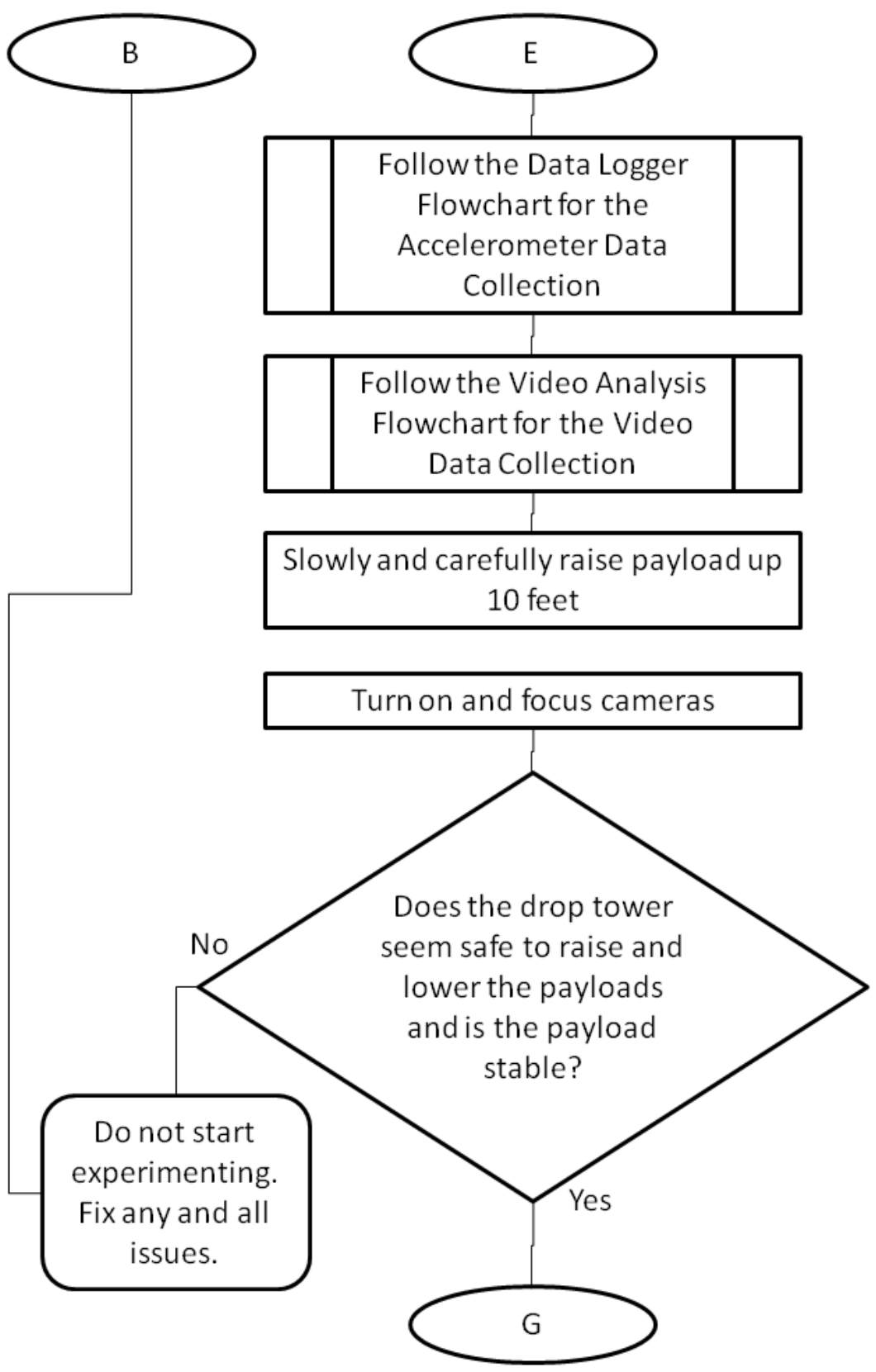




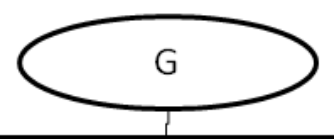

\section{Carefully remove drop tower} preparation surface

Lower payload onto deceleration pad

Charge 1 air tank

(remember to take it with you to the top of the tower)

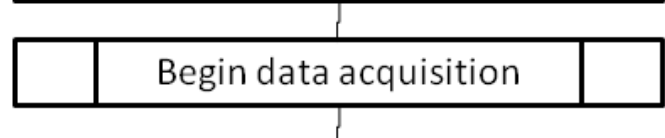

Raise payload

Turn on lighting

\begin{tabular}{|c|}
\hline Turn on lighting \\
\hline $\begin{array}{c}\text { Check camera focuses and adjust, if } \\
\text { necessary }\end{array}$ \\
\hline Open ball valve above nozzle \\
\hline
\end{tabular}

Attach air tank and stow for drop

\begin{tabular}{|l|c|l|}
\hline & $\begin{array}{c}\text { Transfer payload to } \\
\text { electromagnet }\end{array}$ & \\
\hline
\end{tabular}

Turn pressure valve to preset

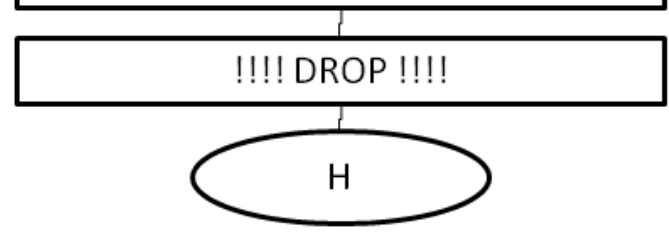




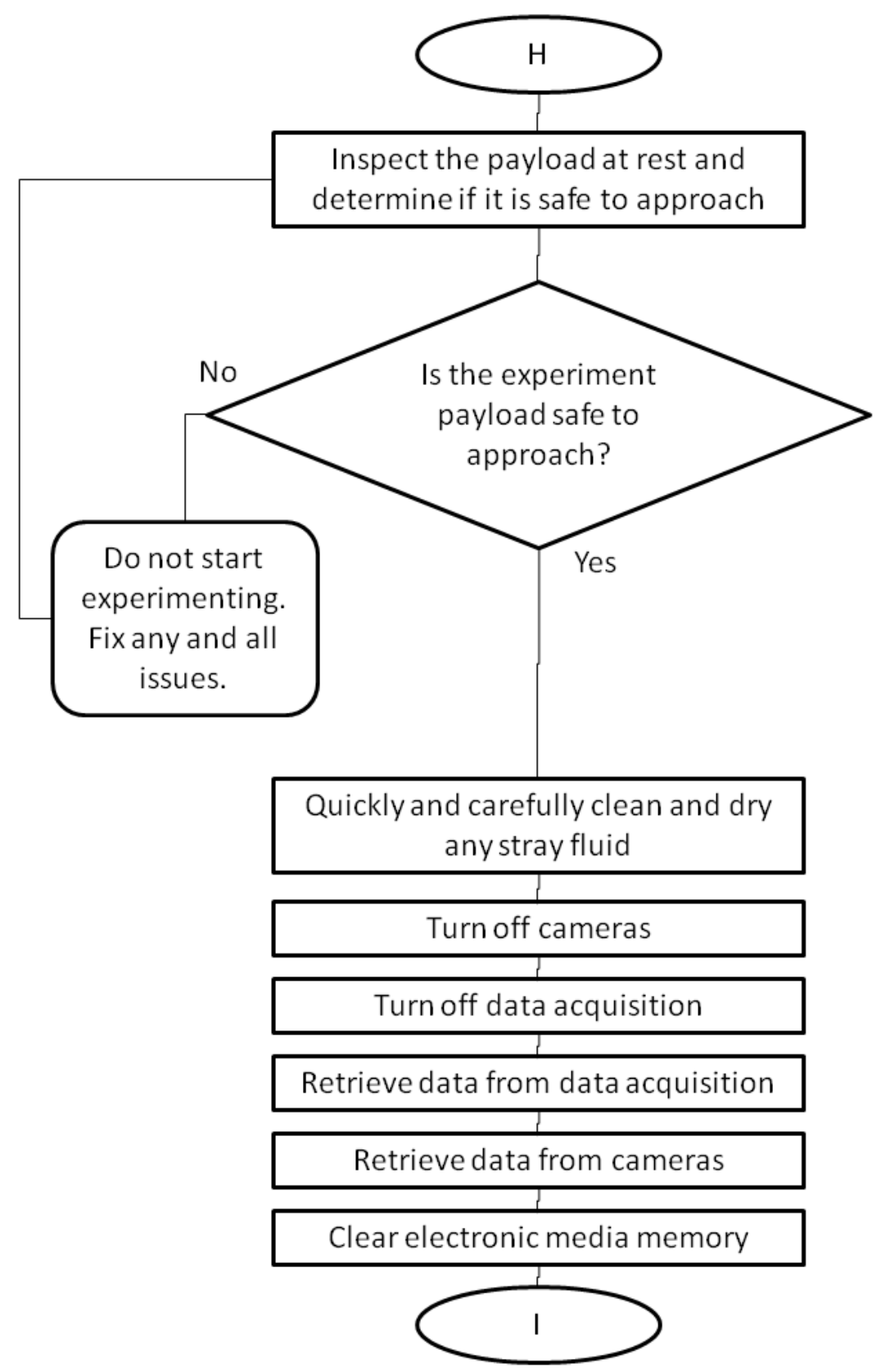




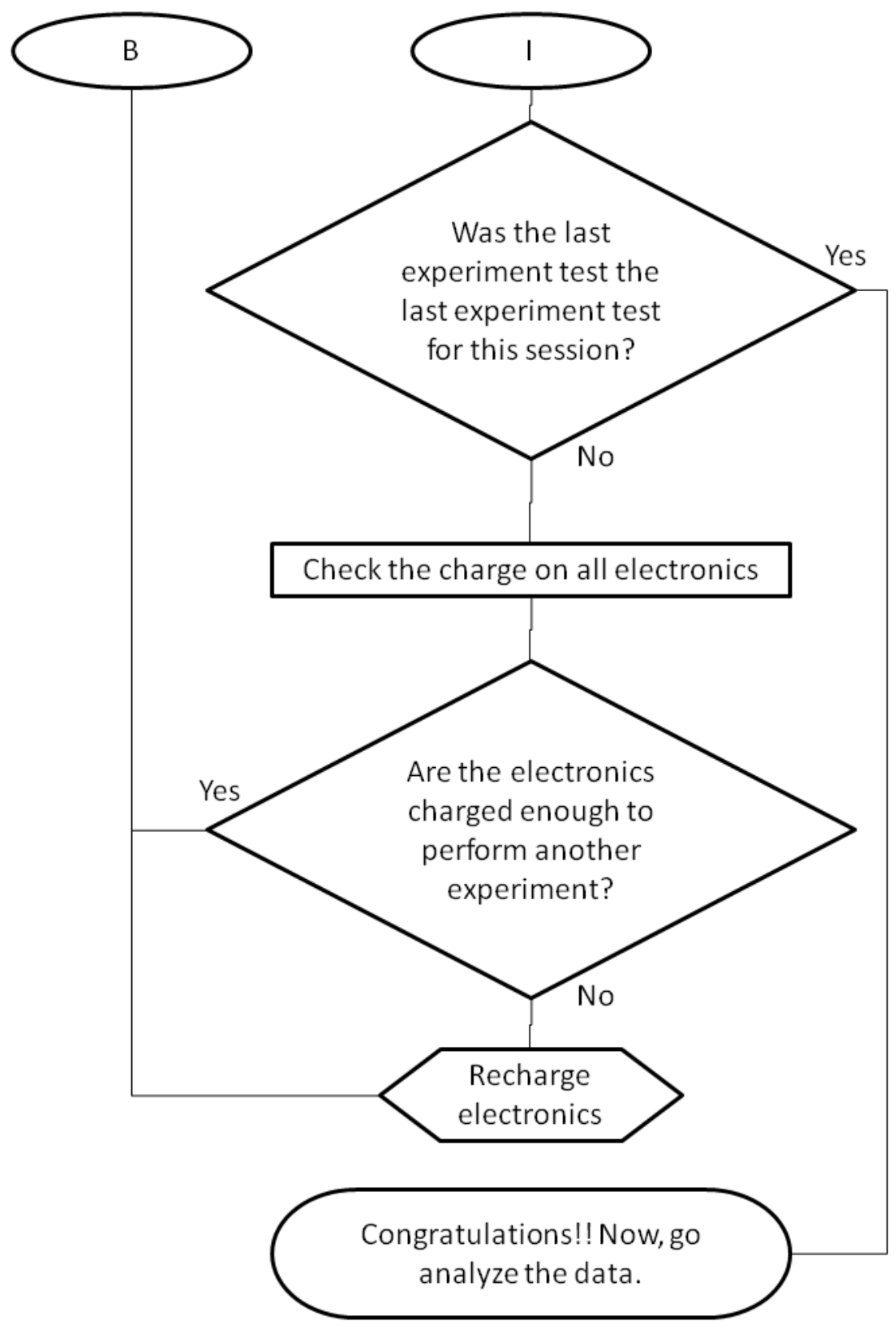




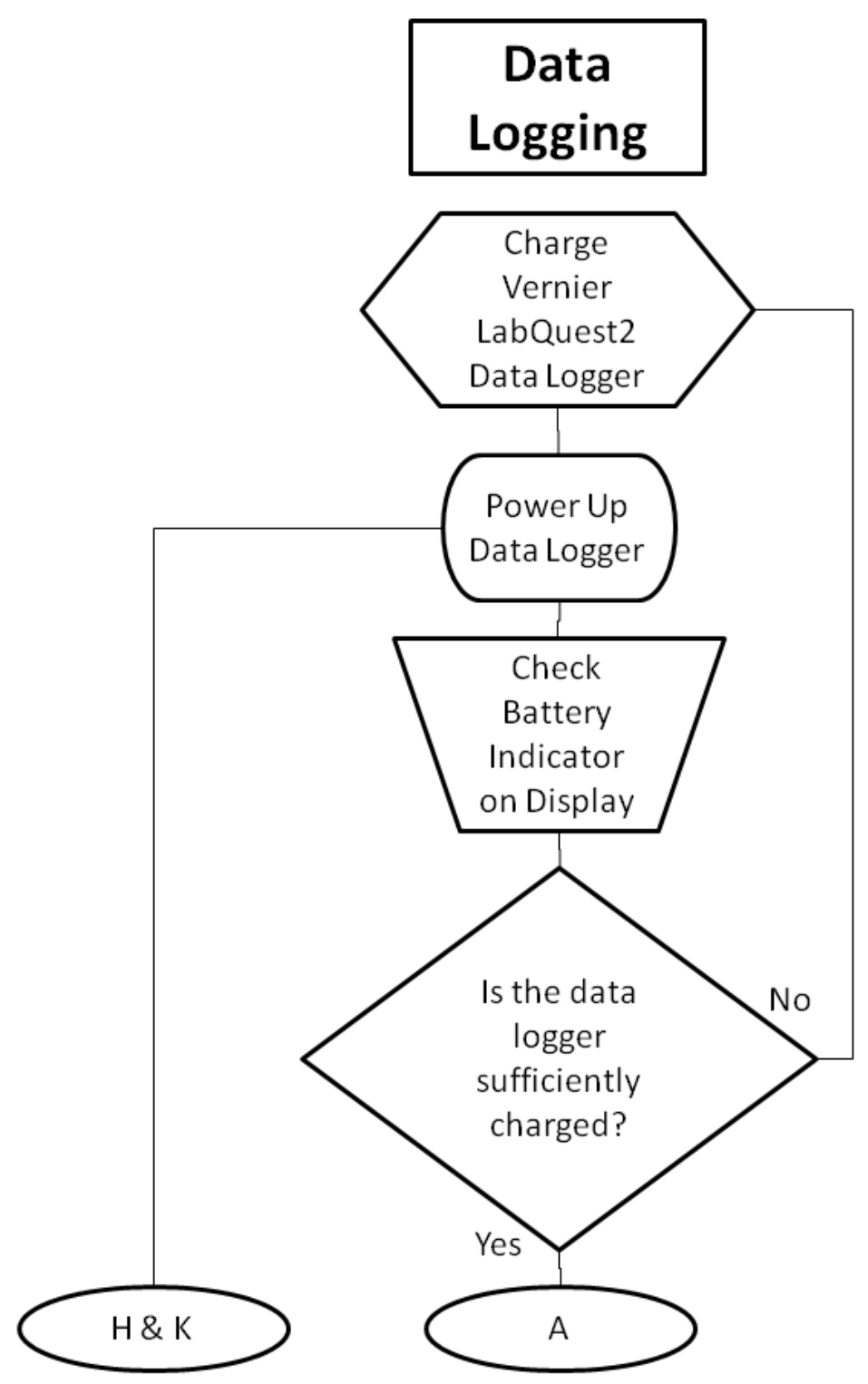




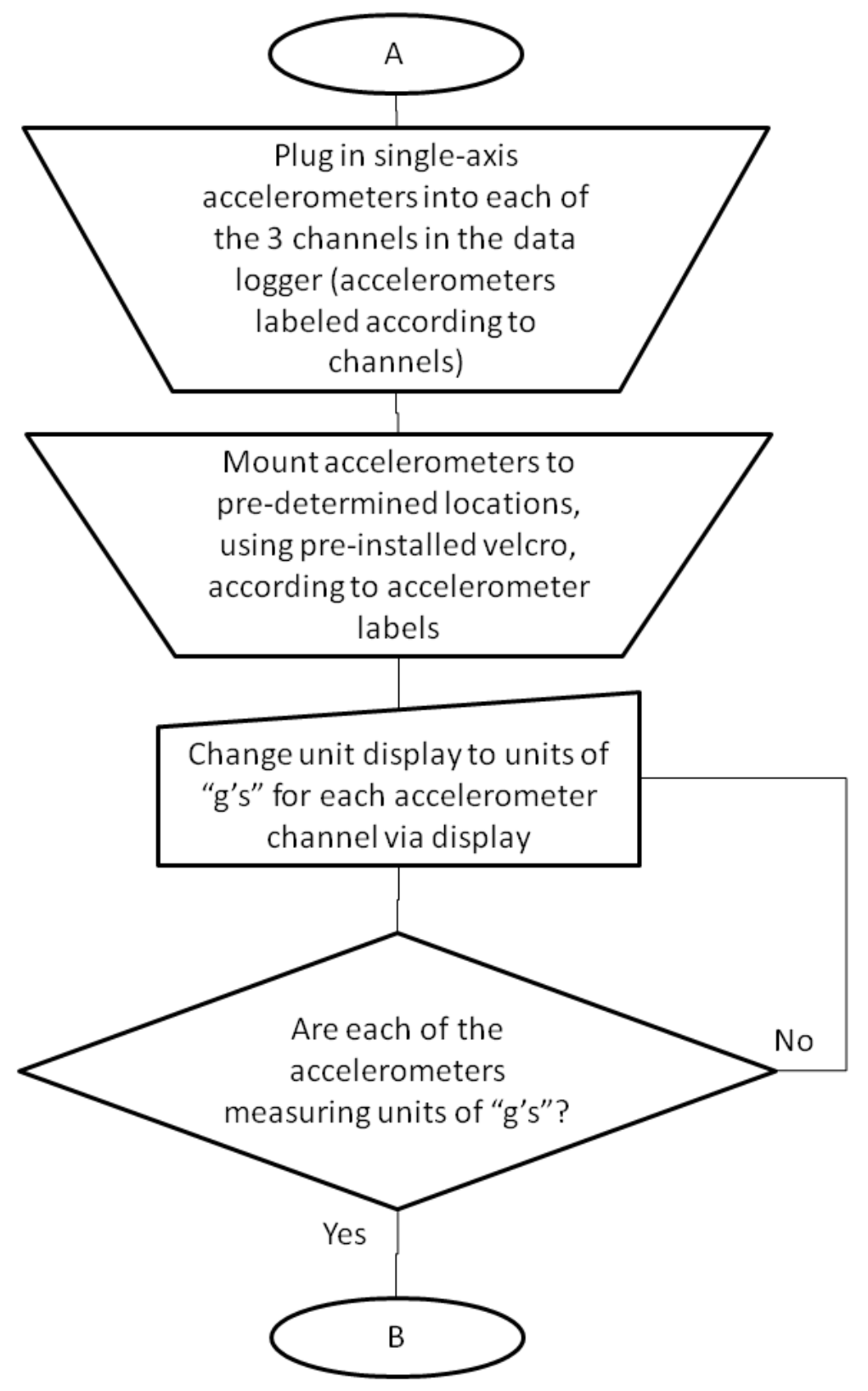




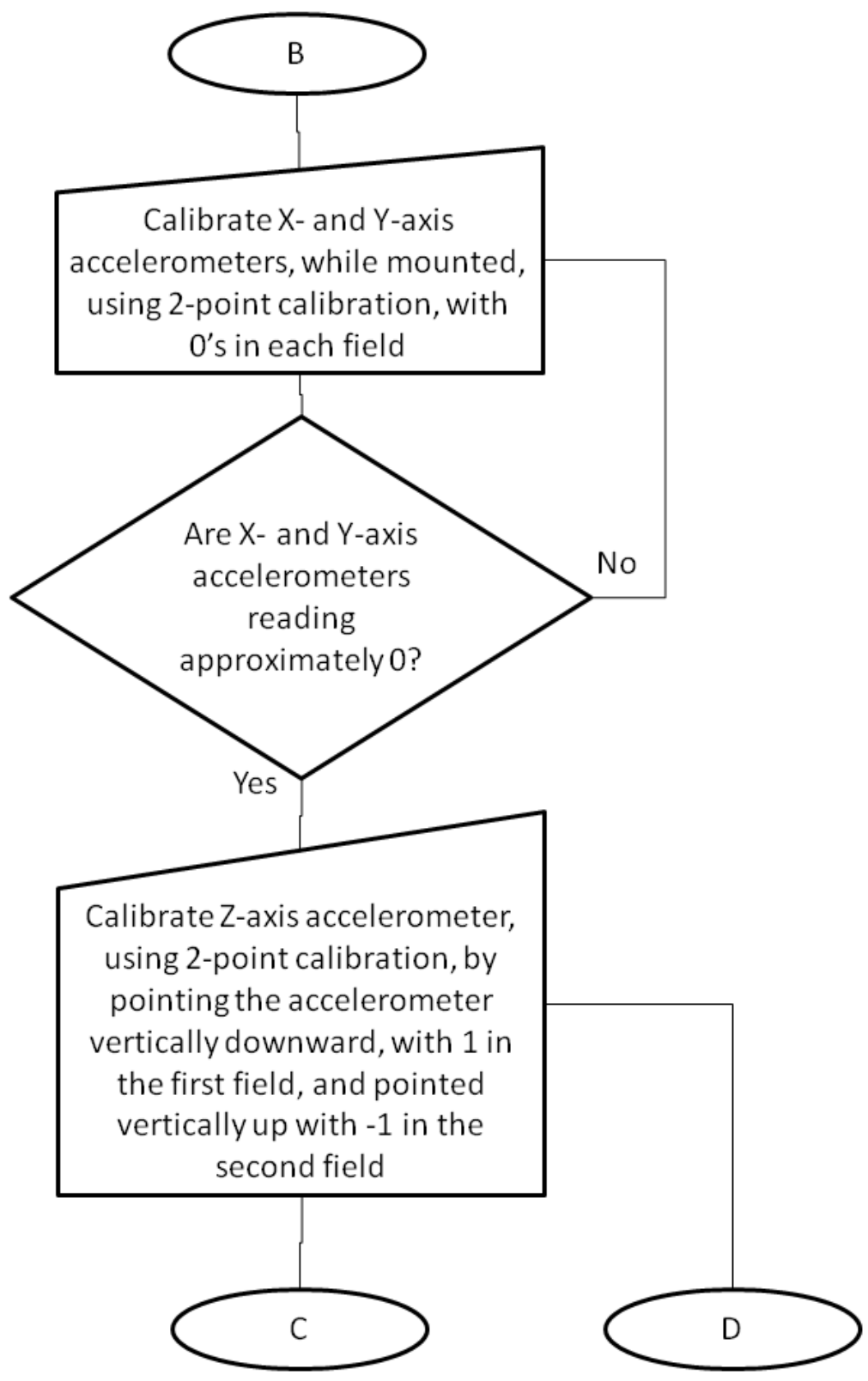




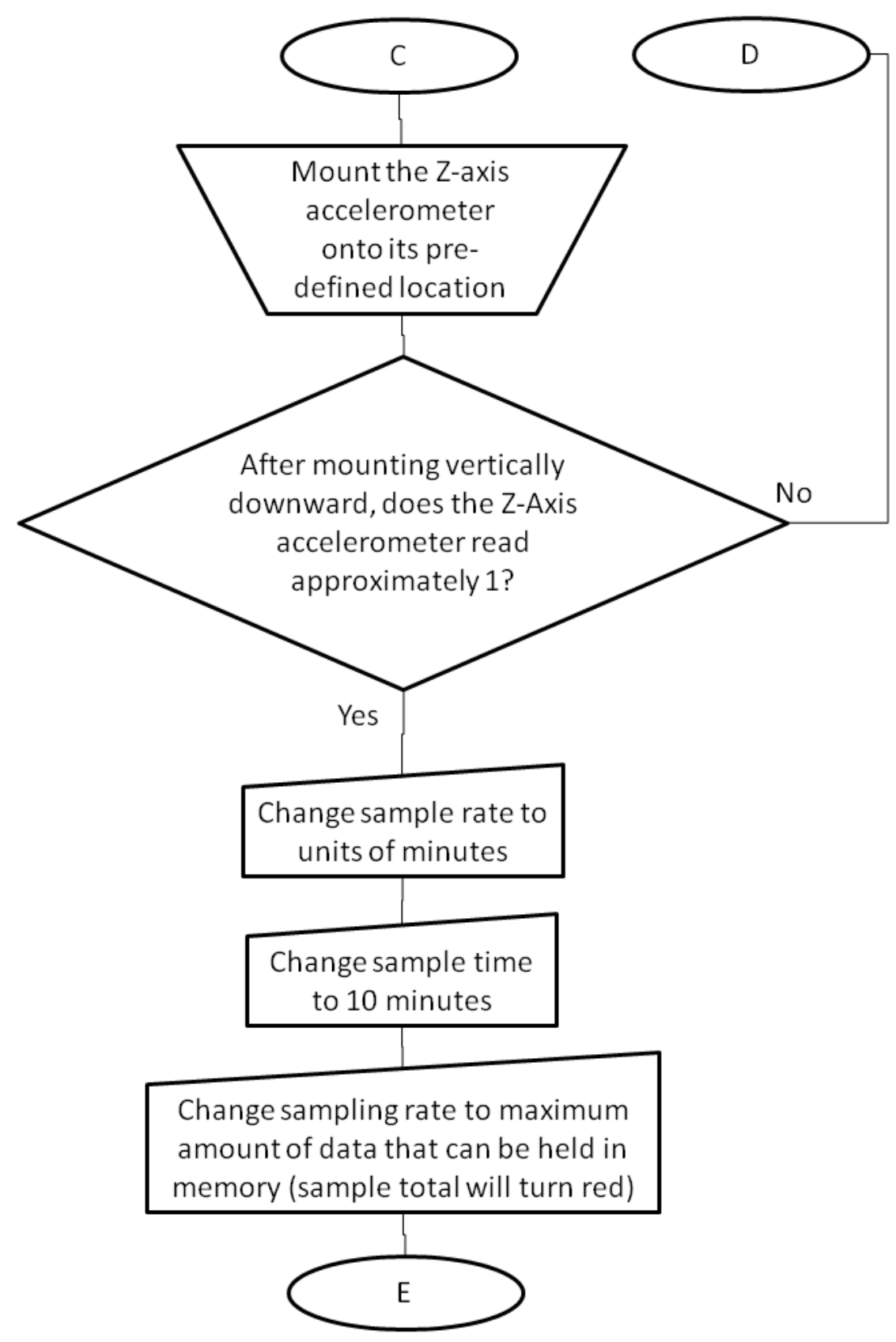




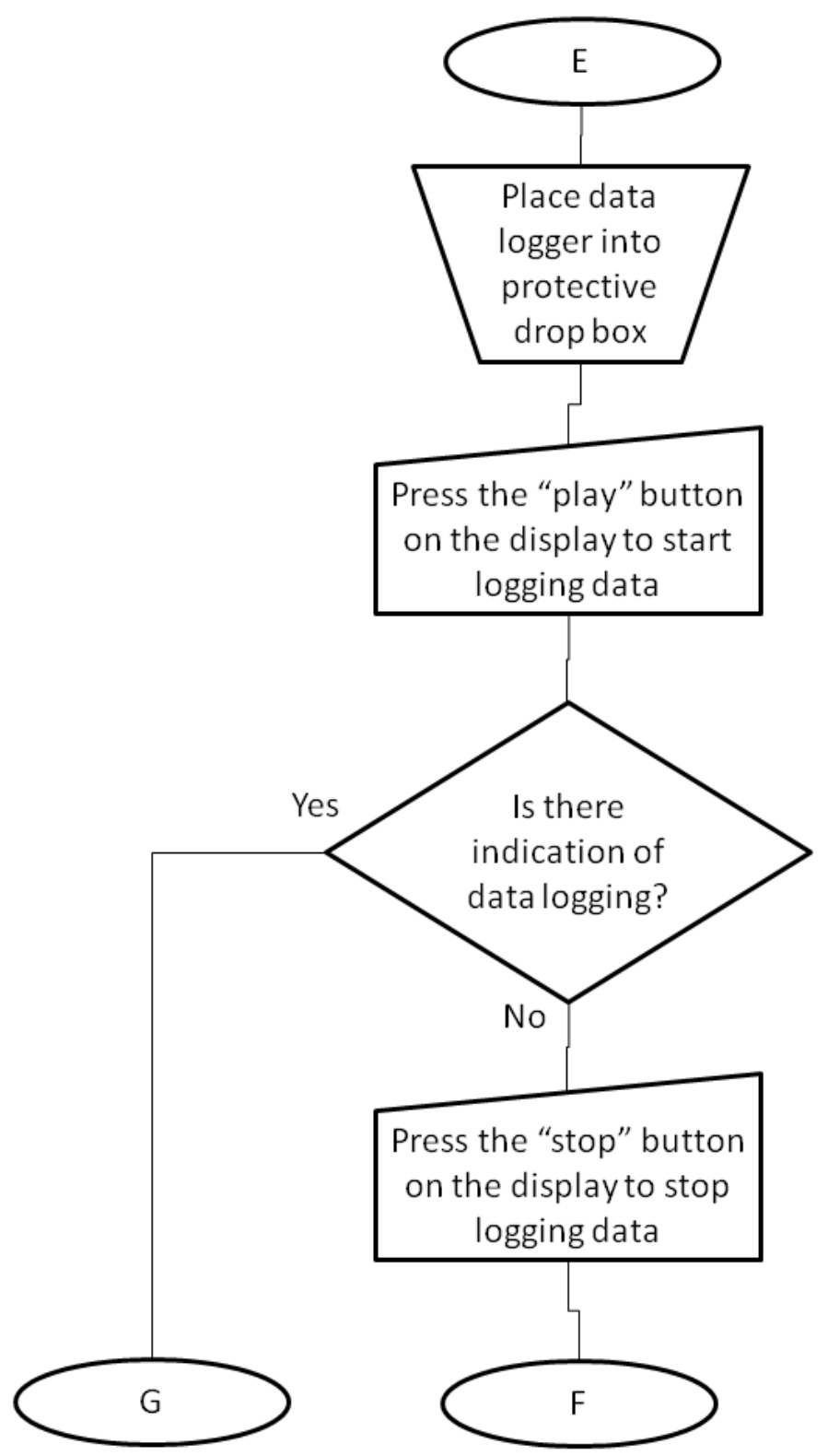




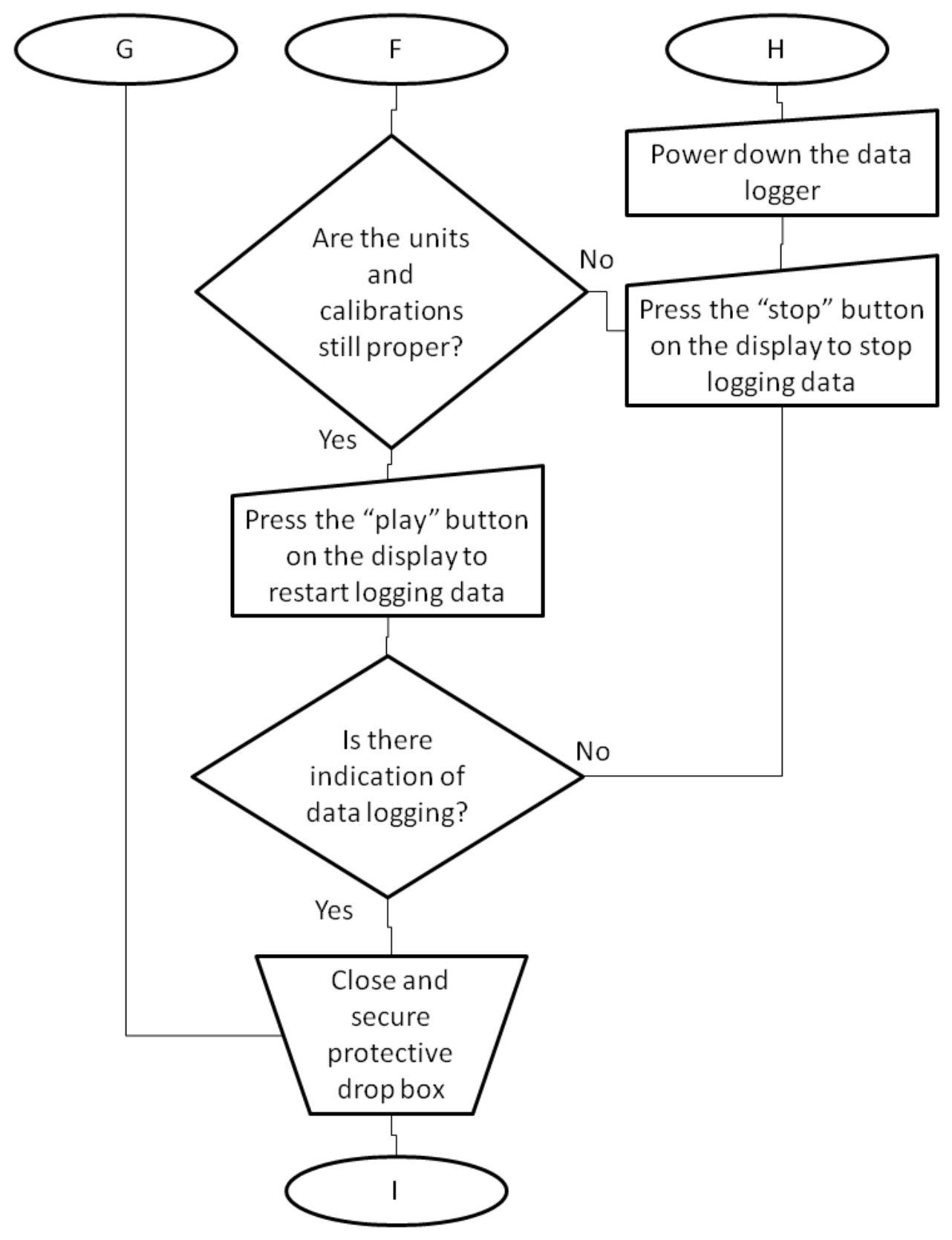




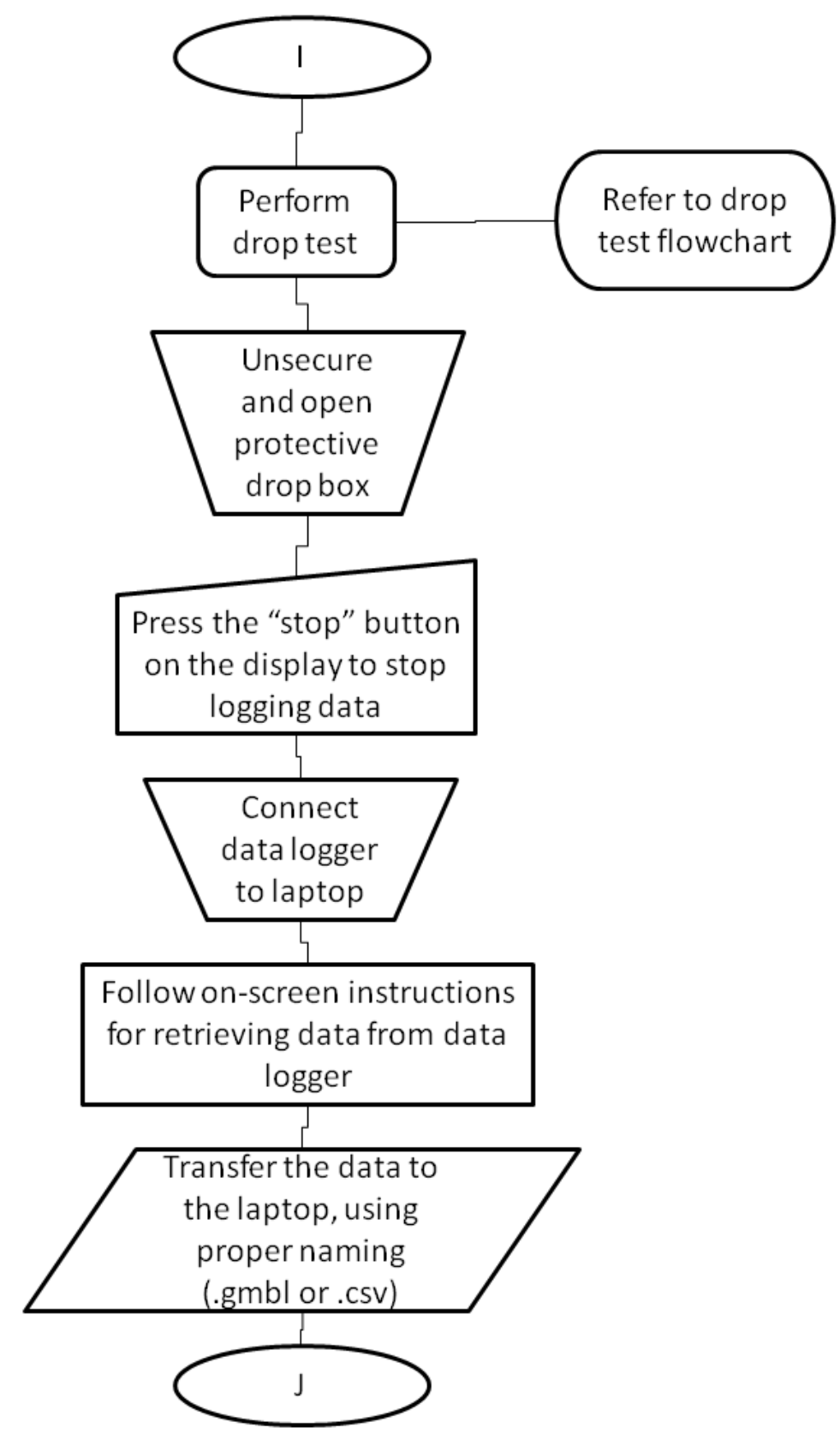




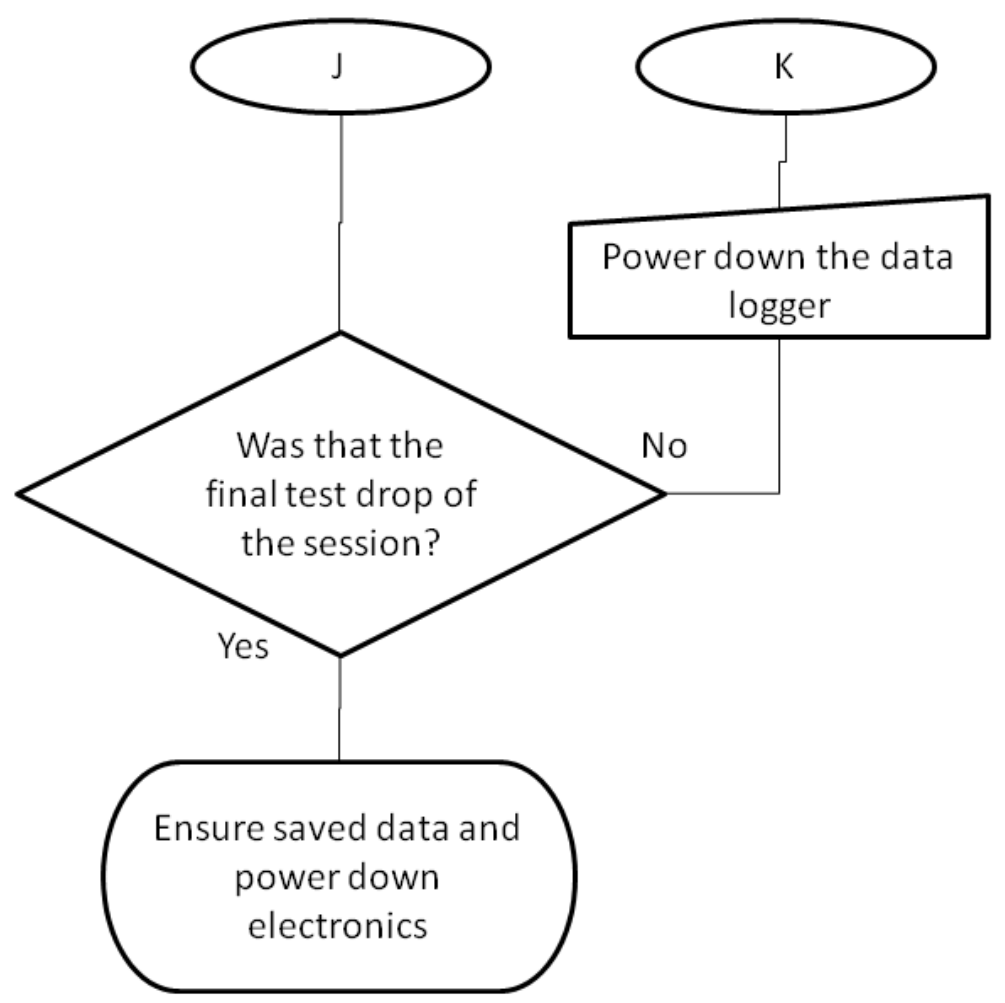




\section{Drop Test}

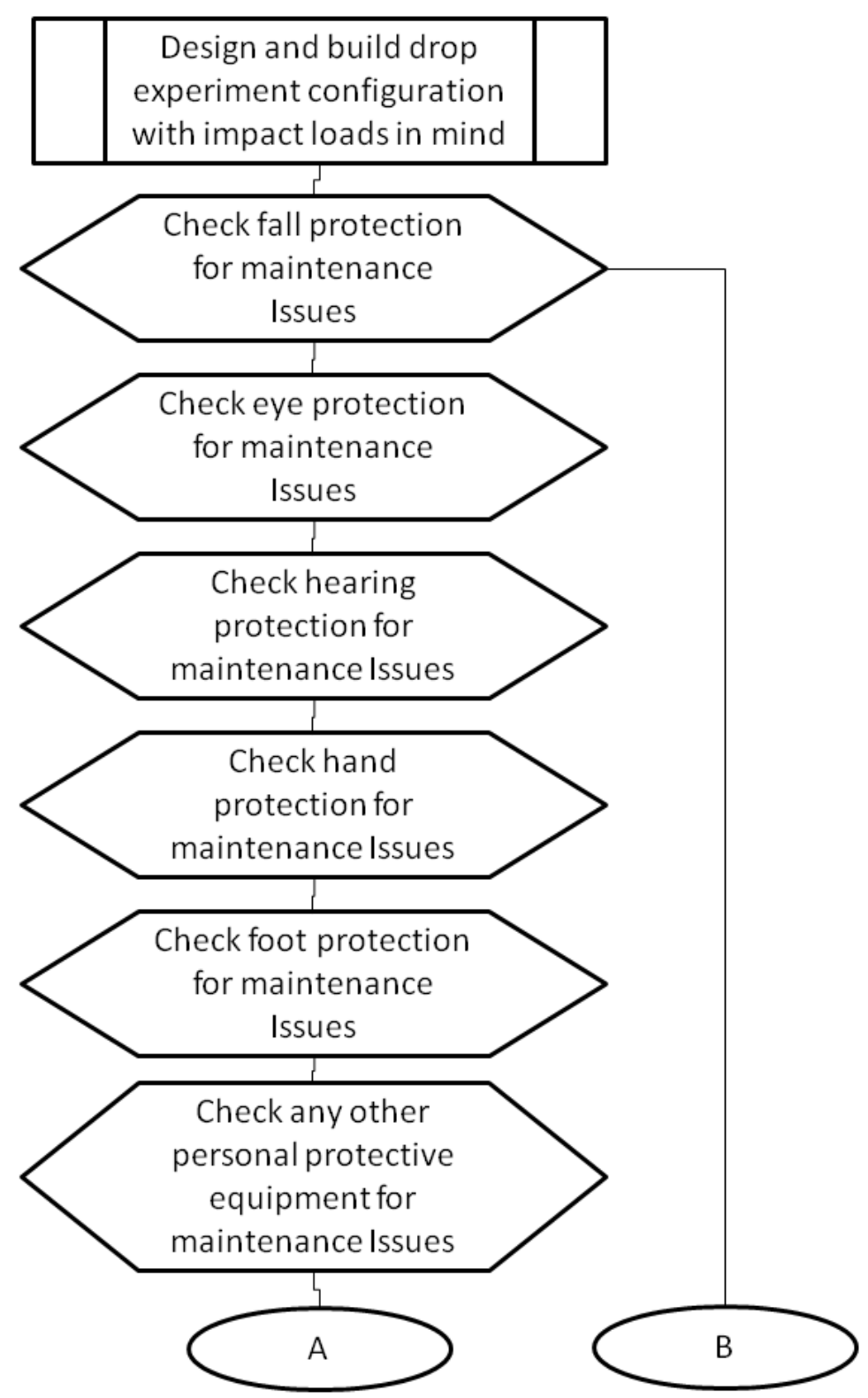




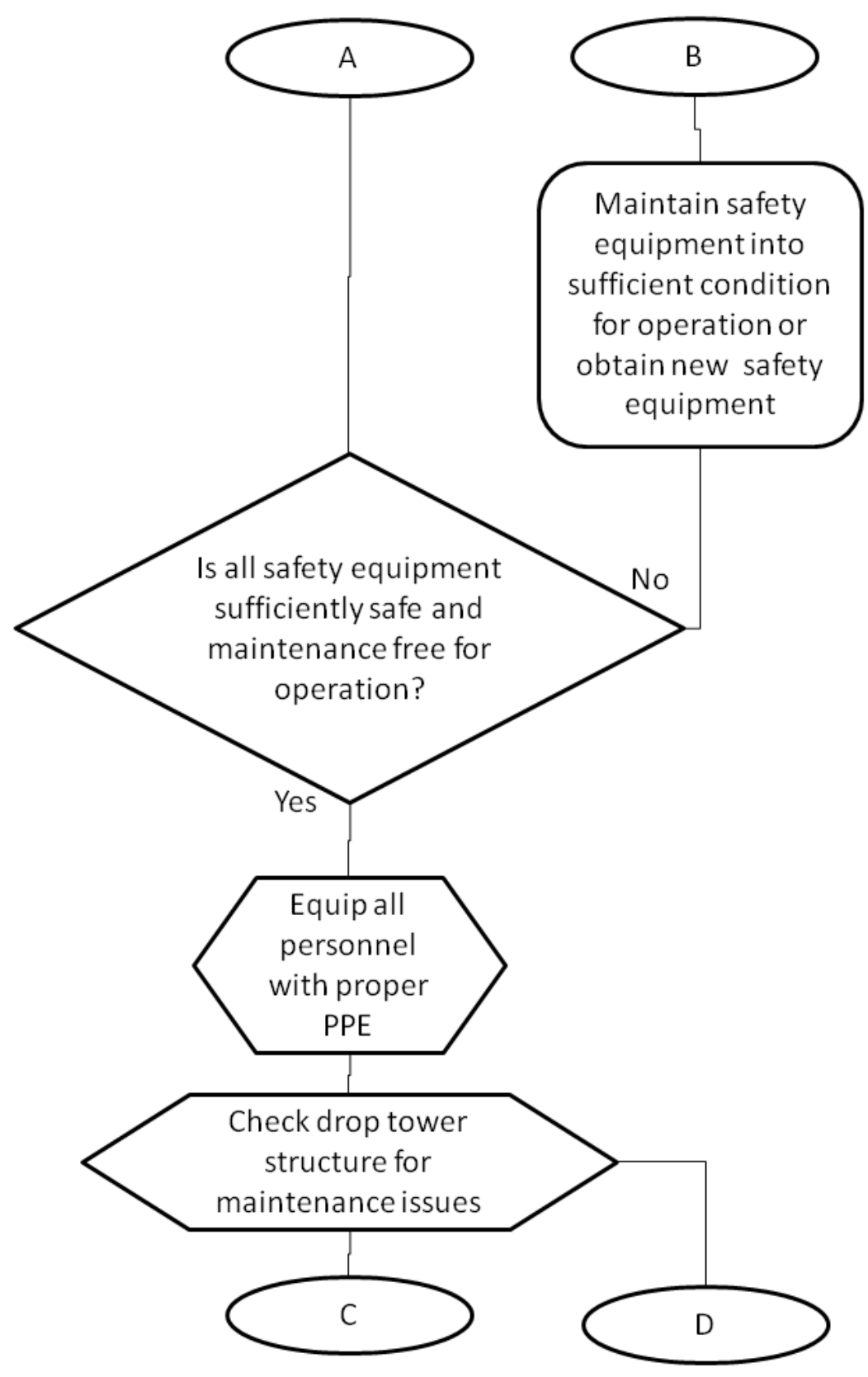




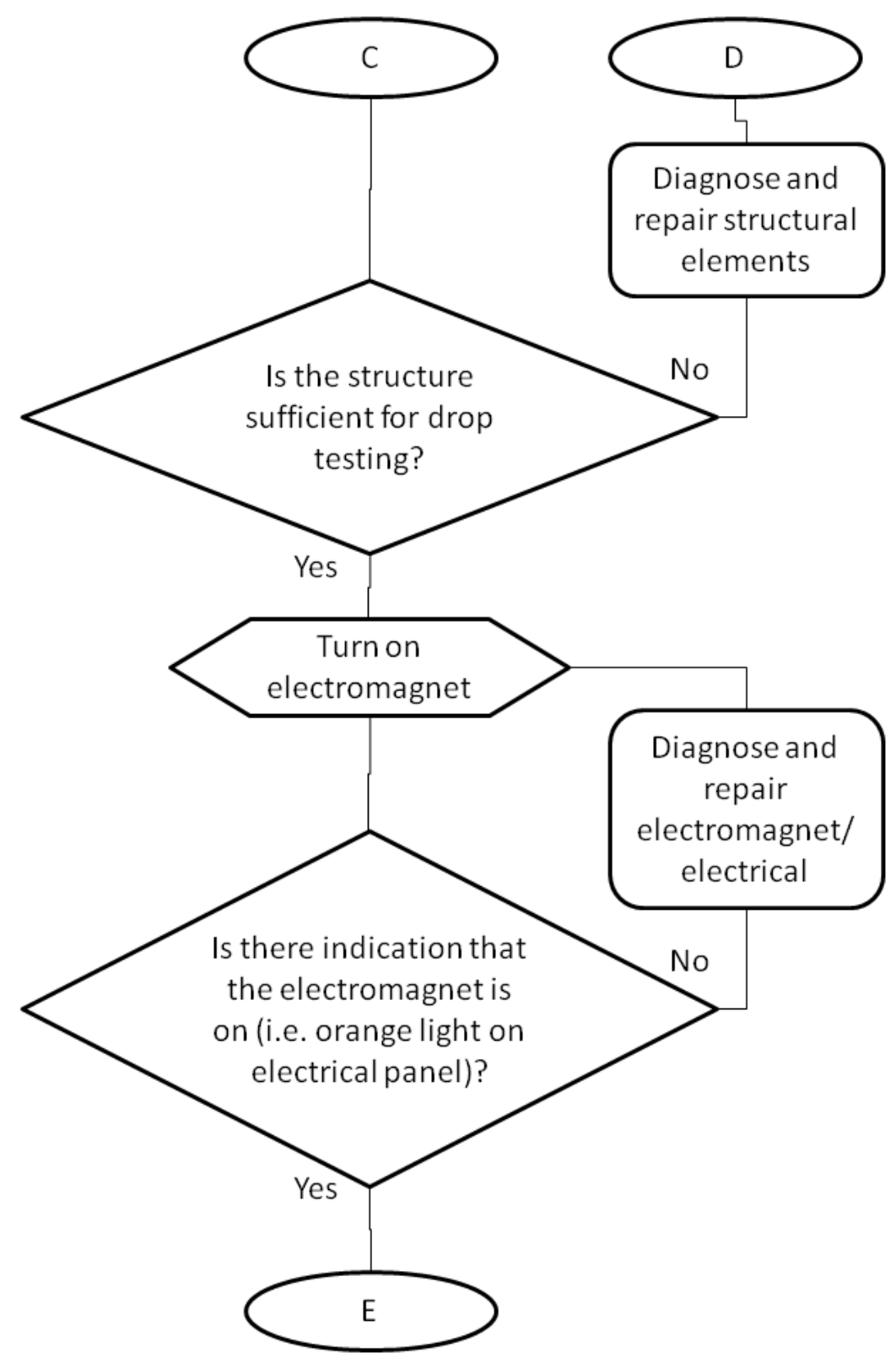




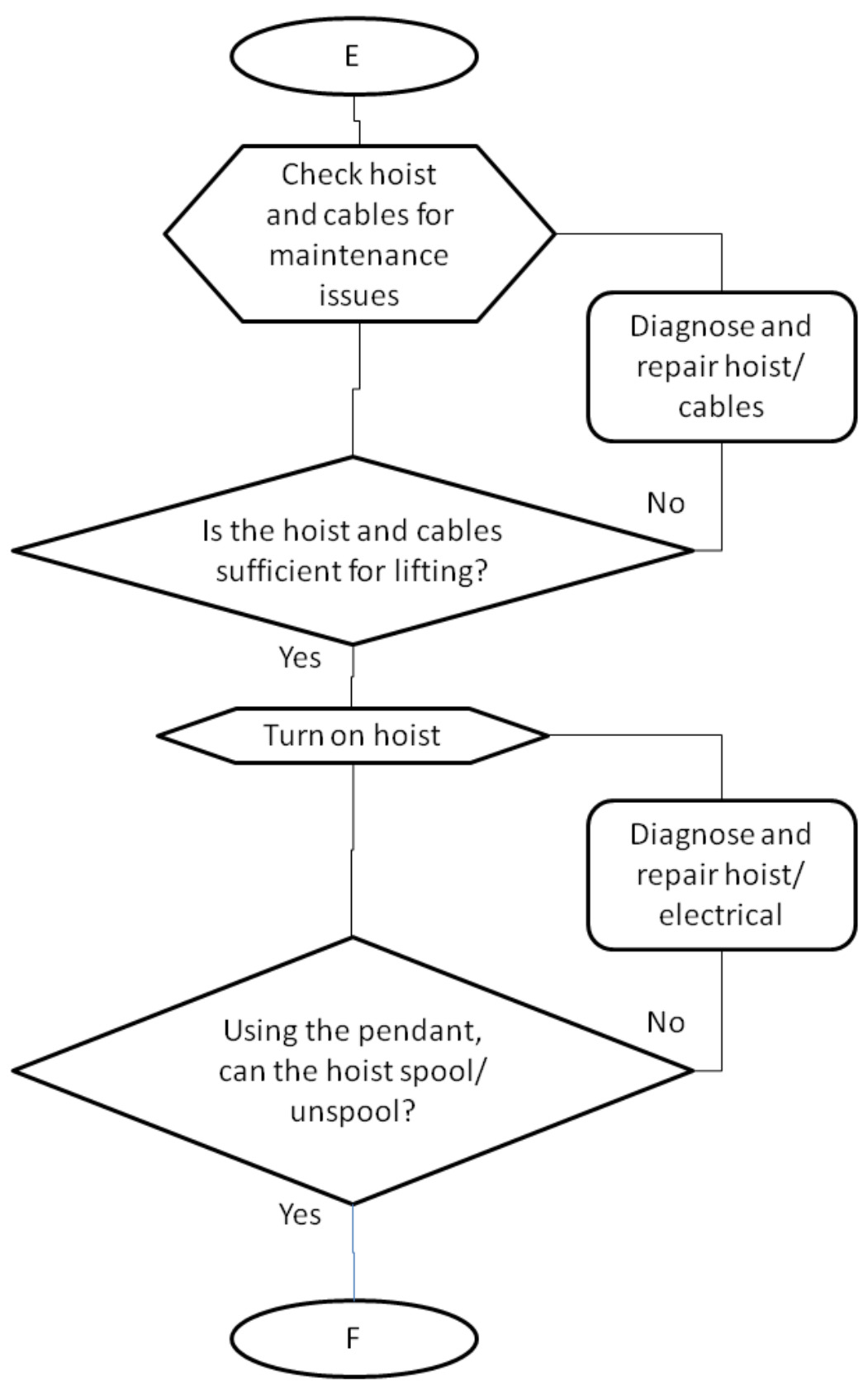




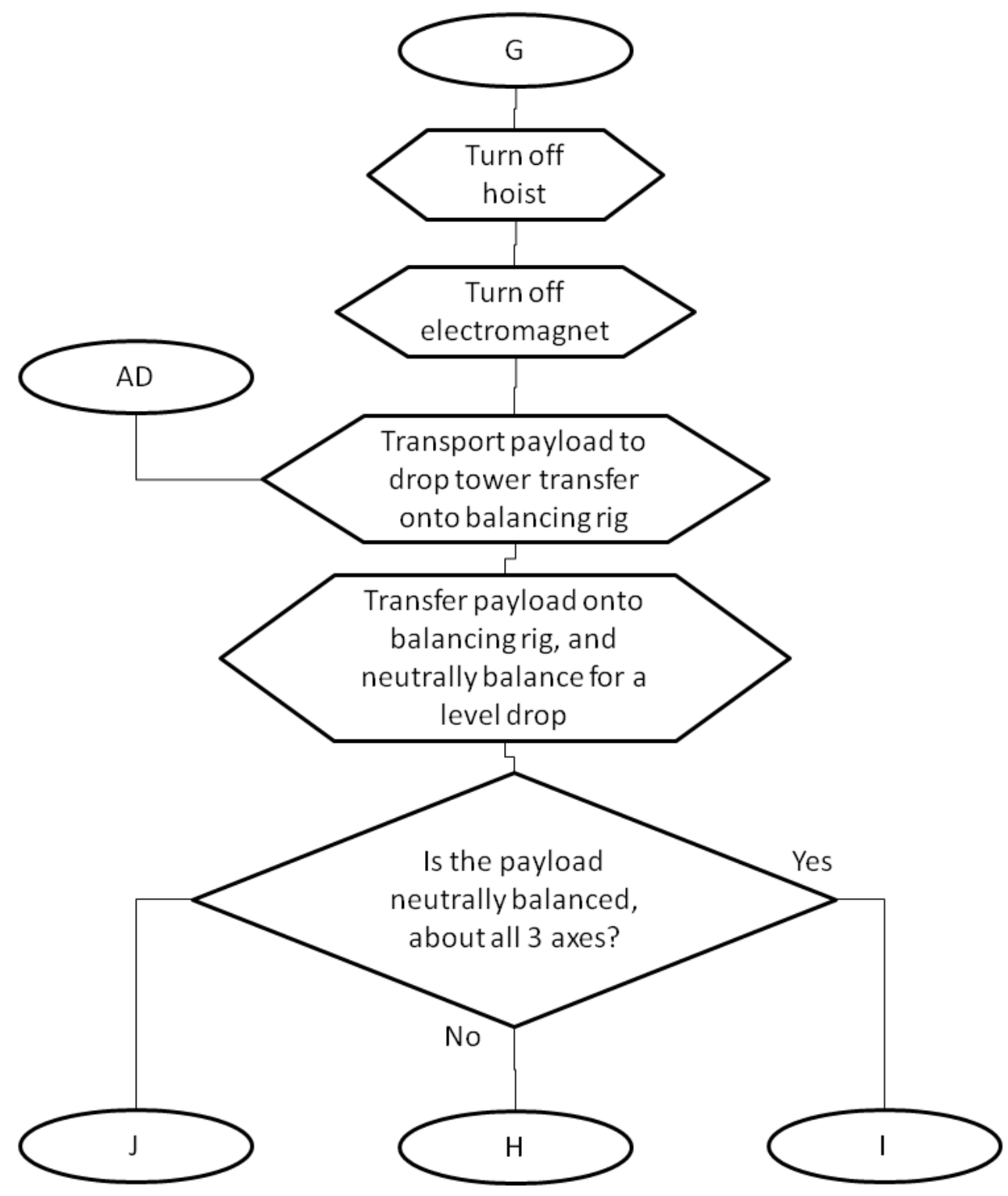




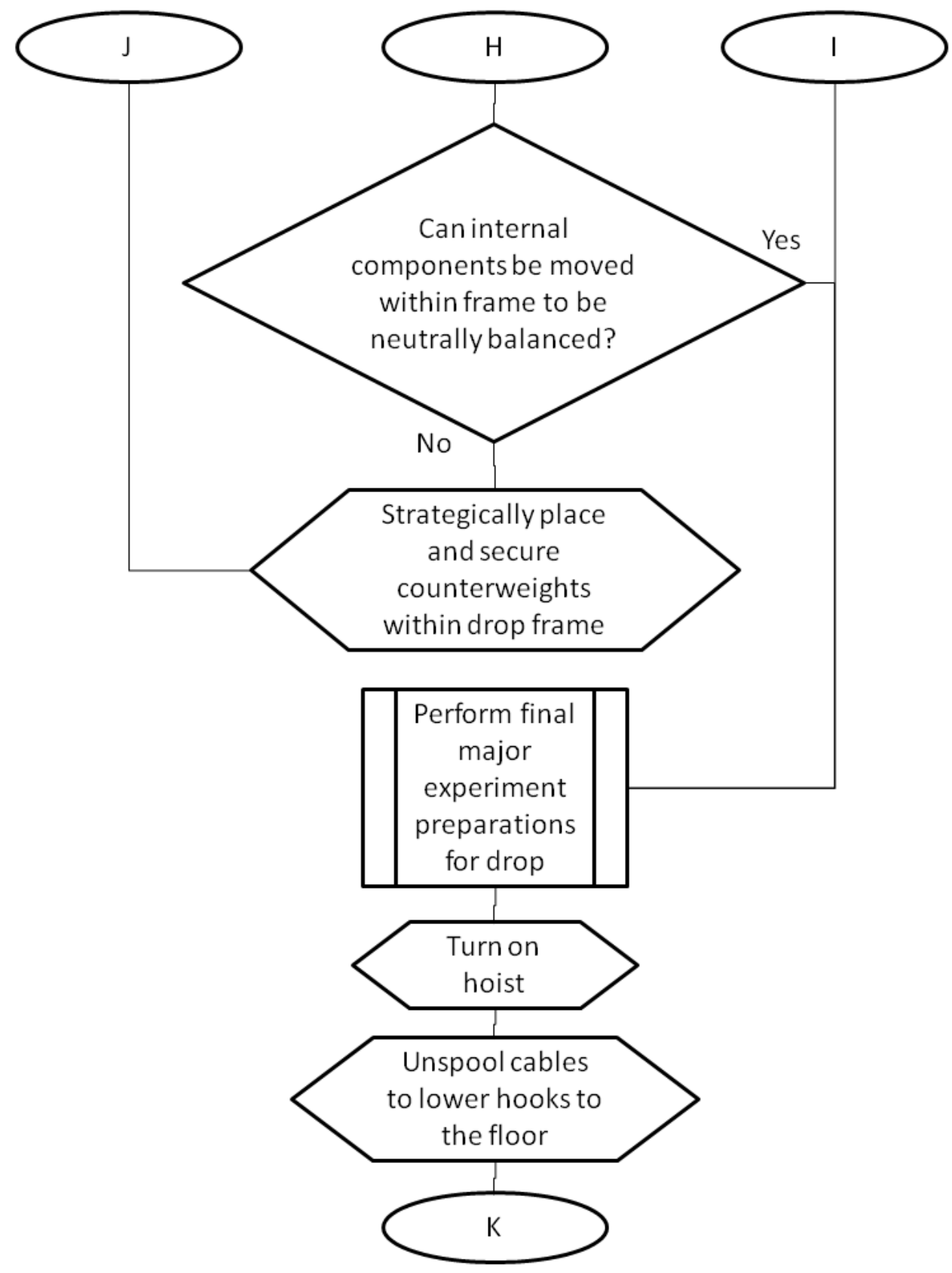




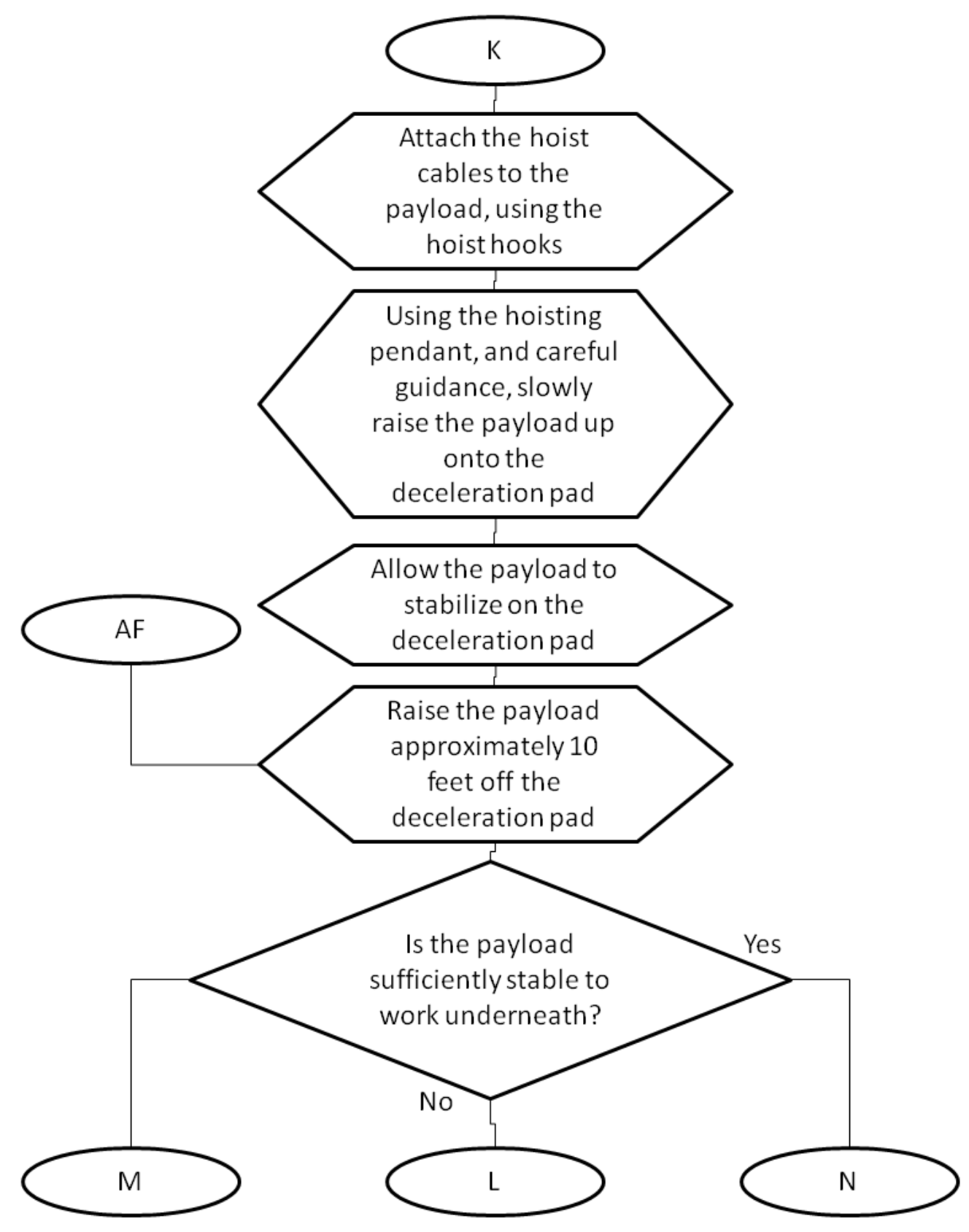




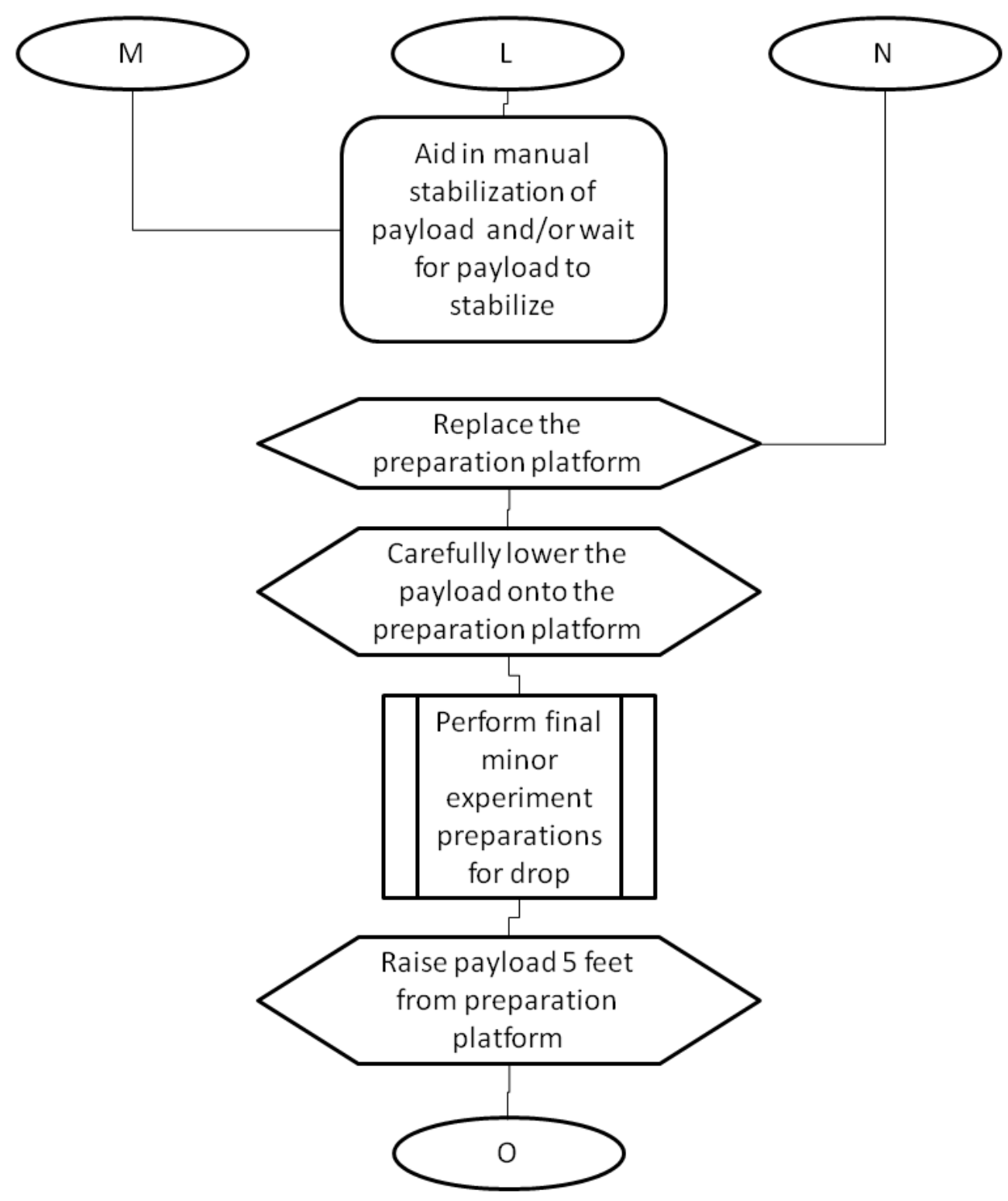




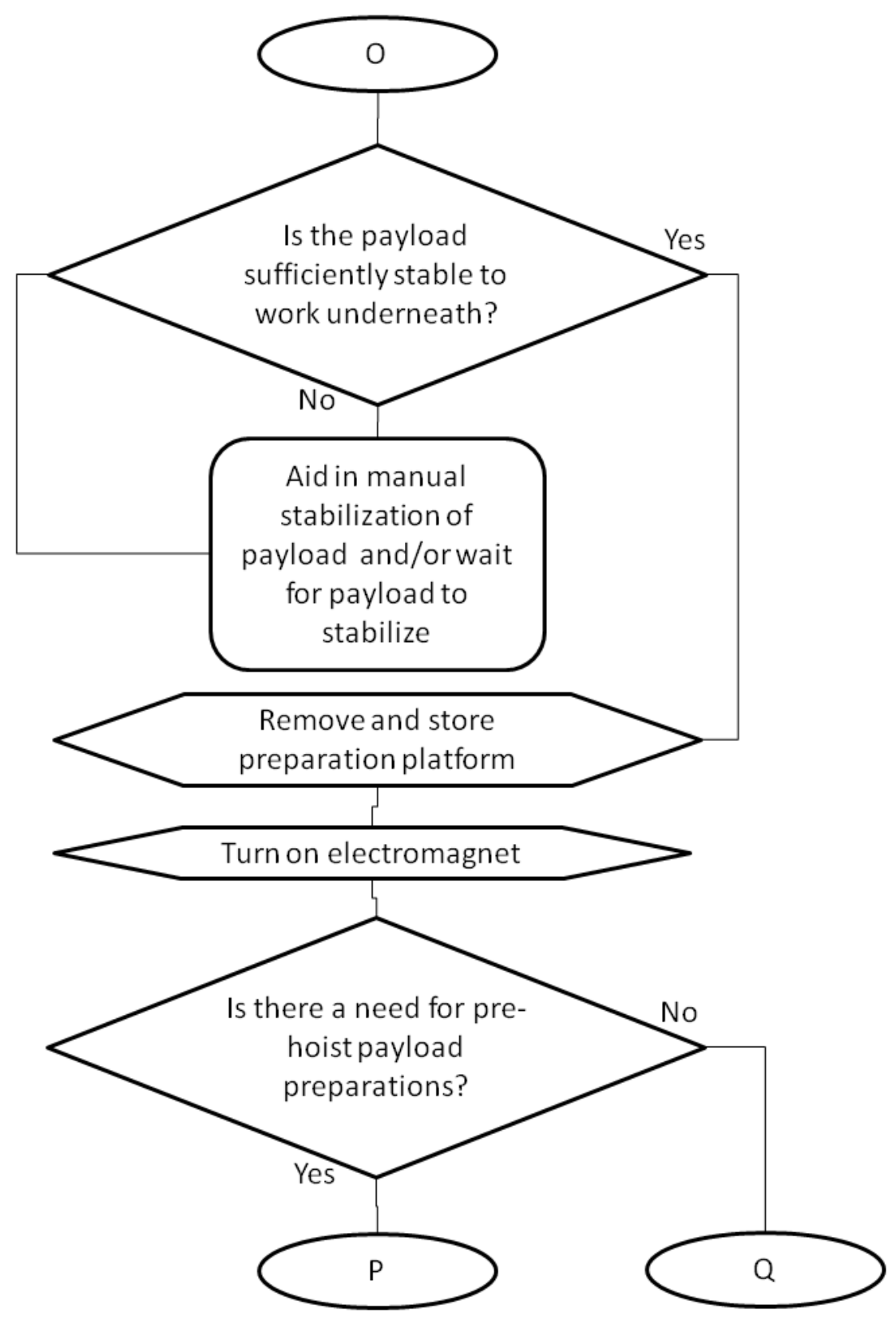




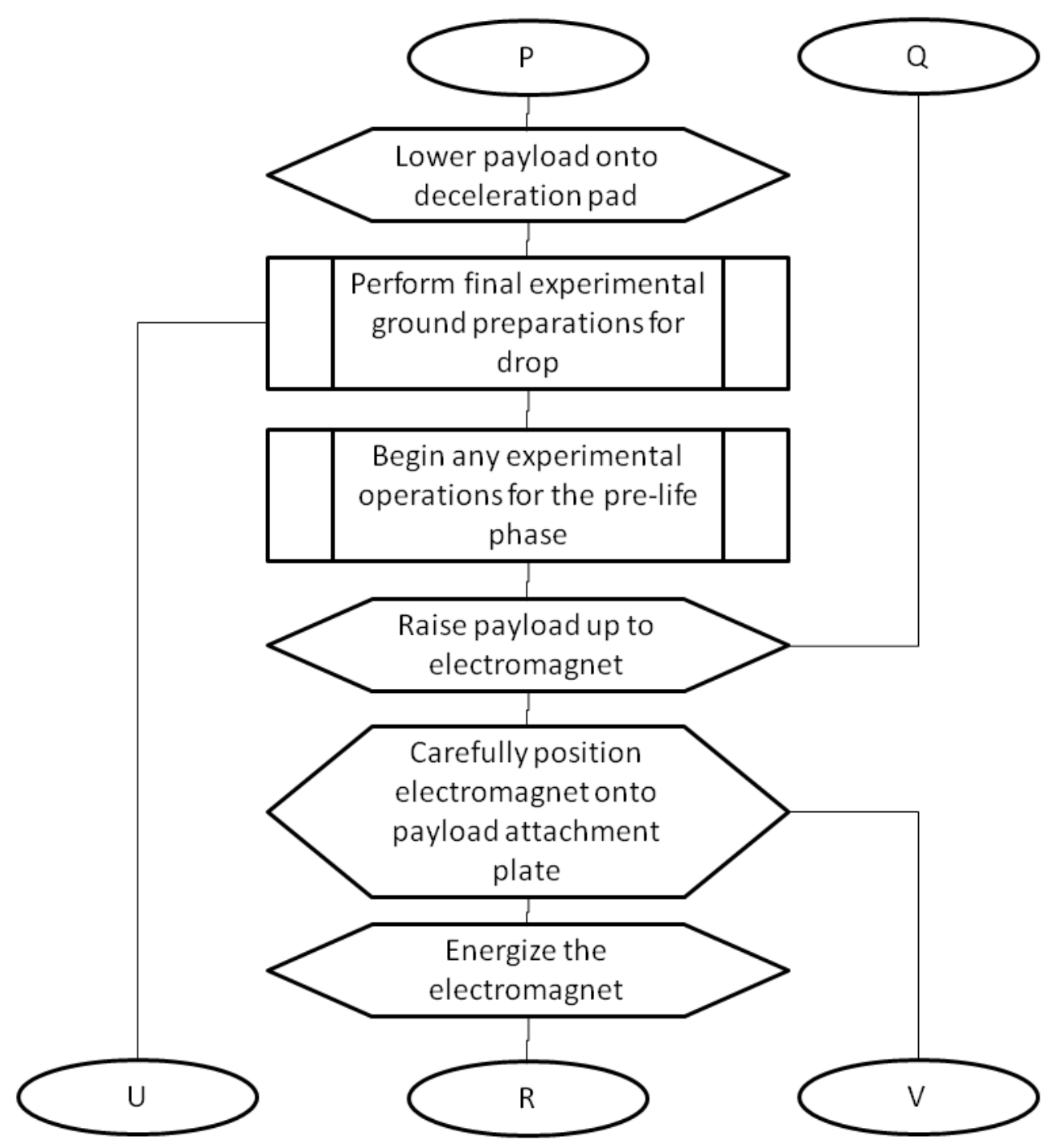




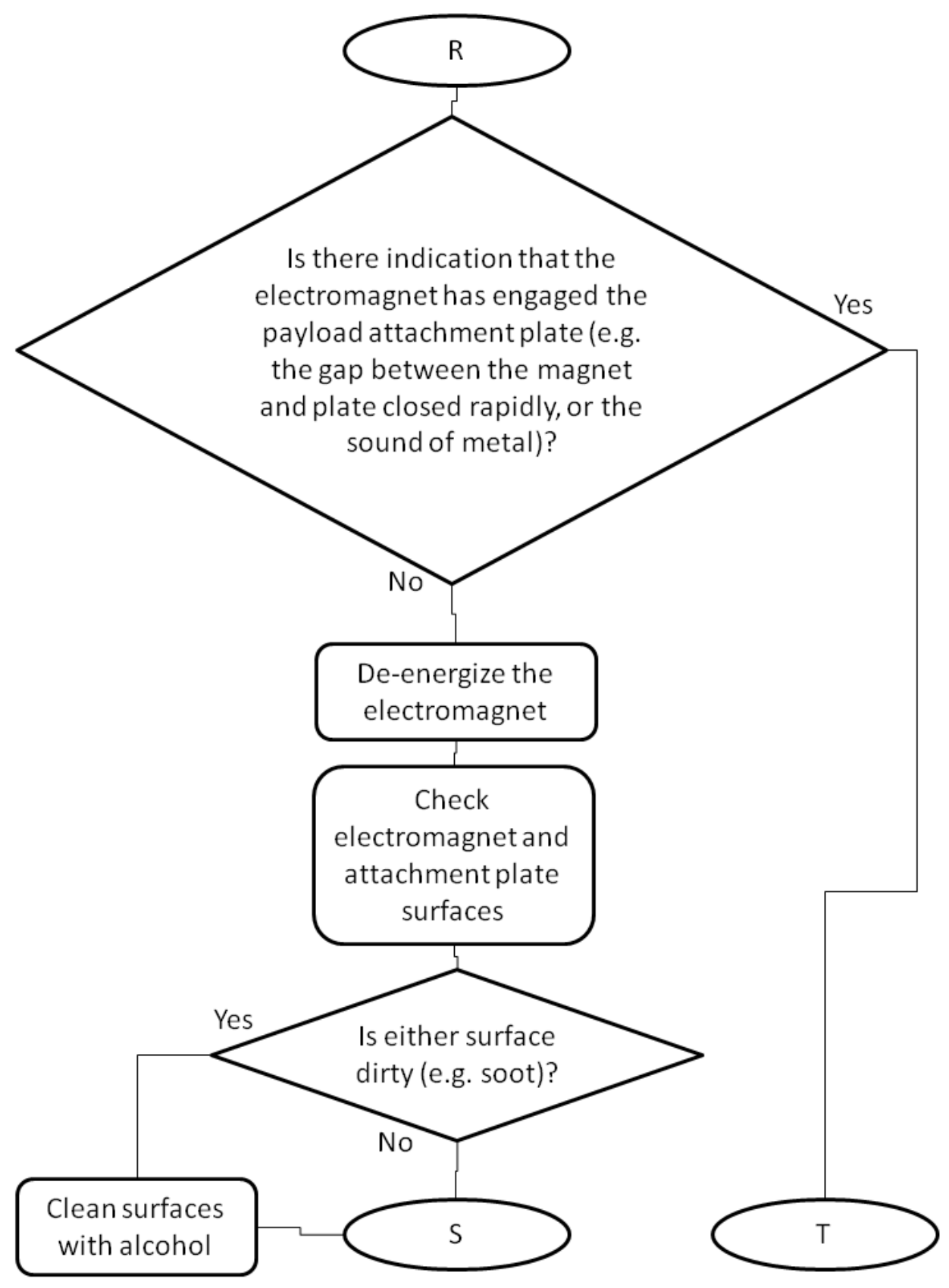




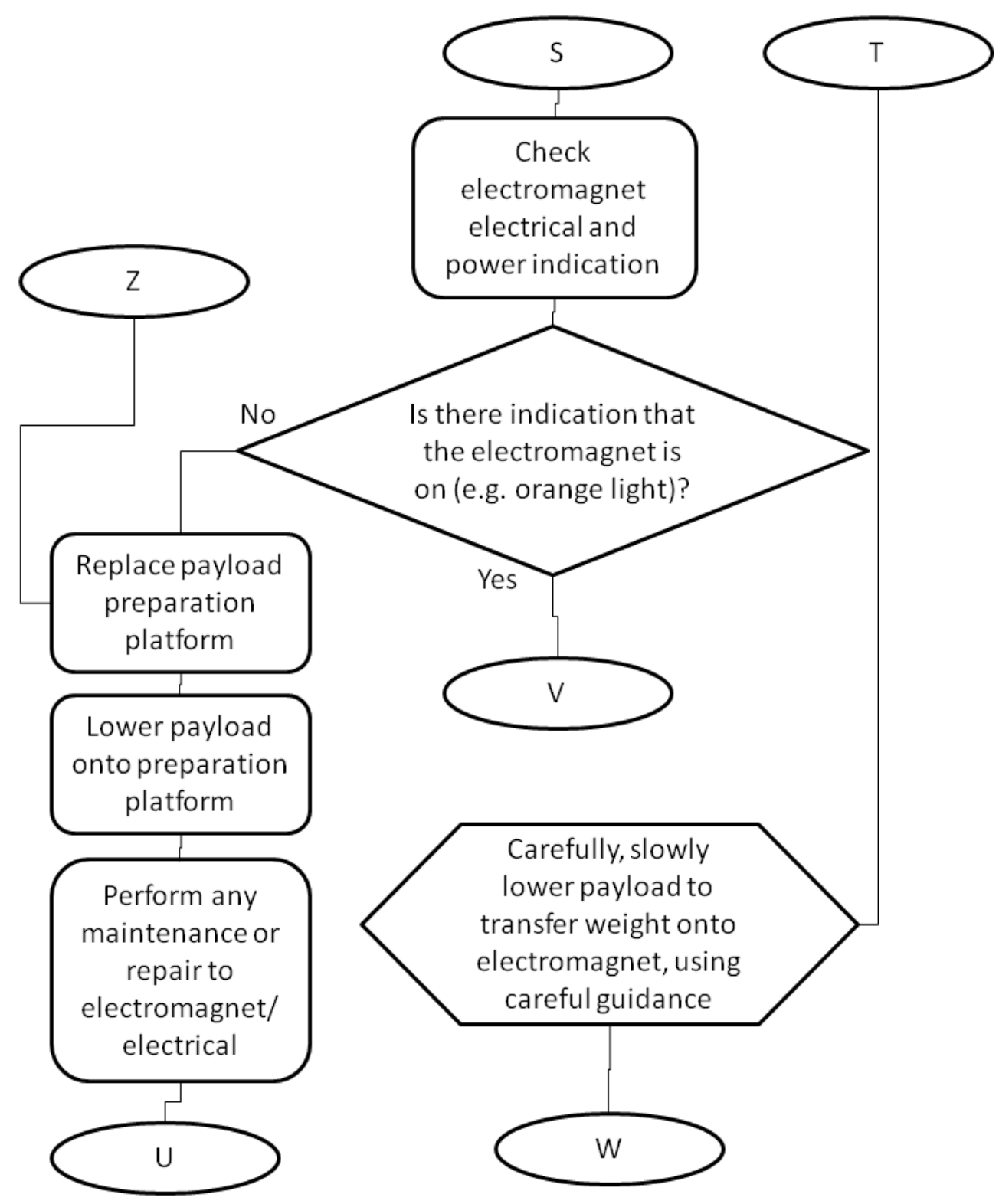




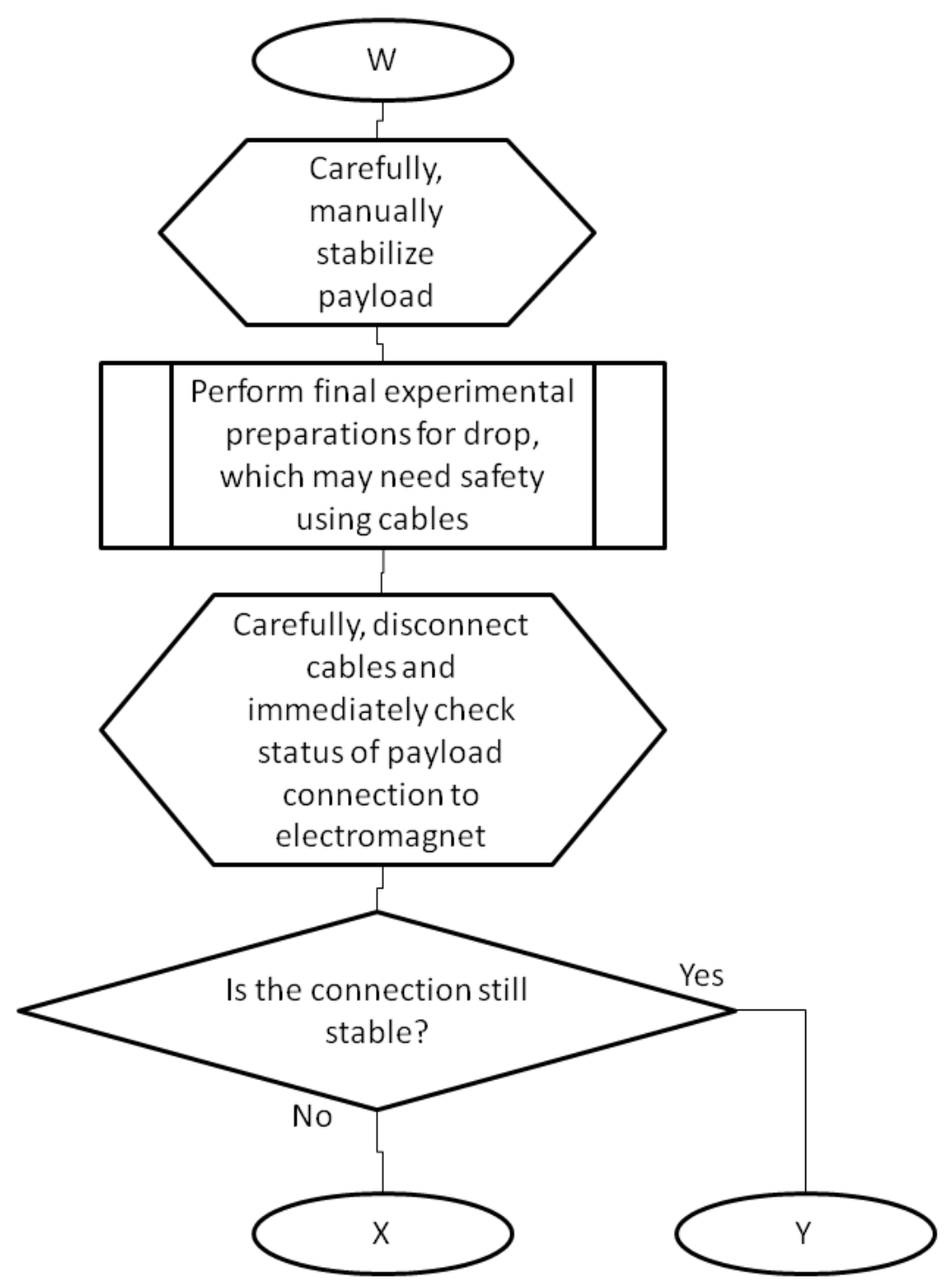




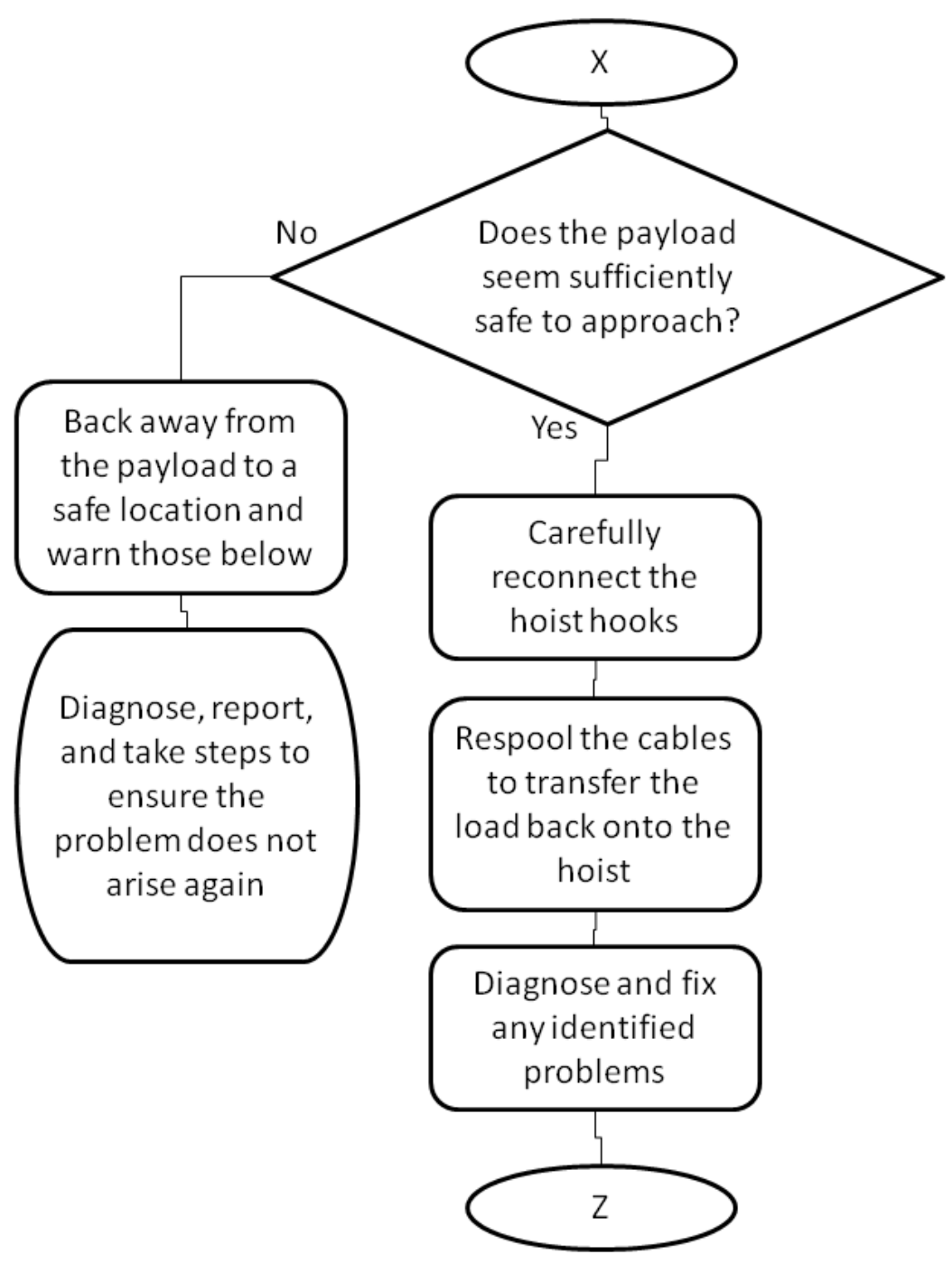




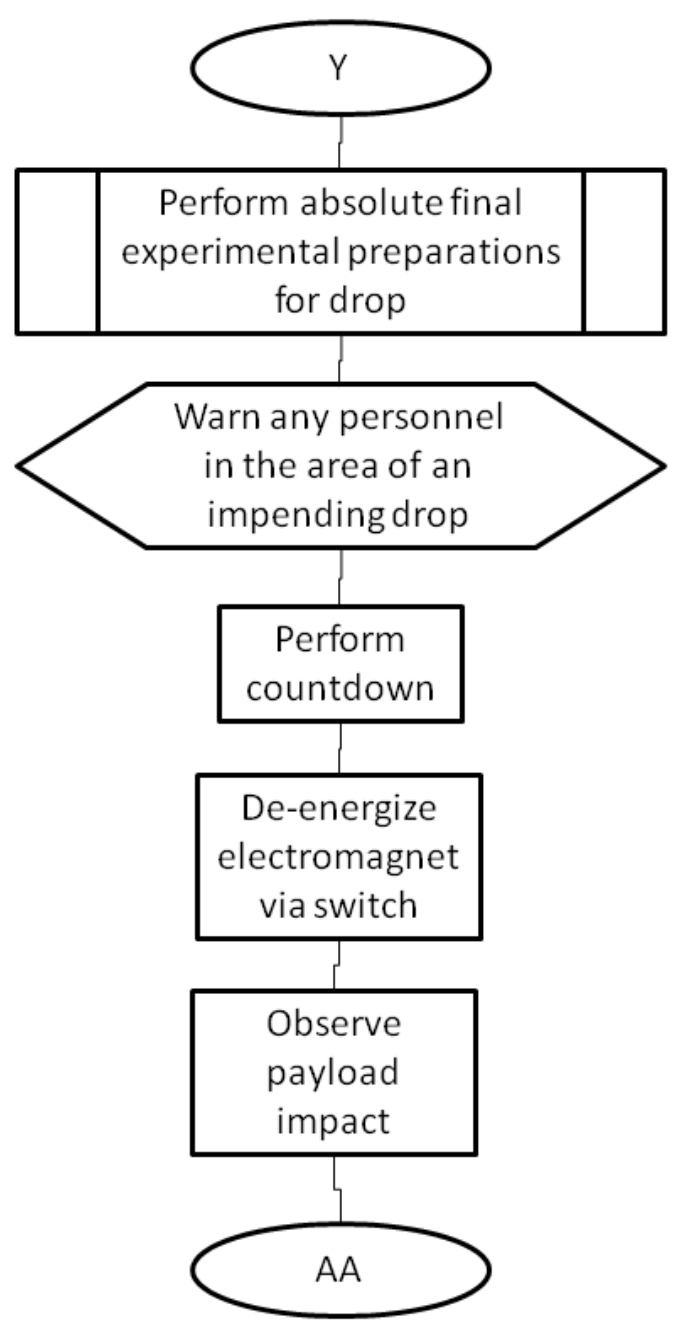




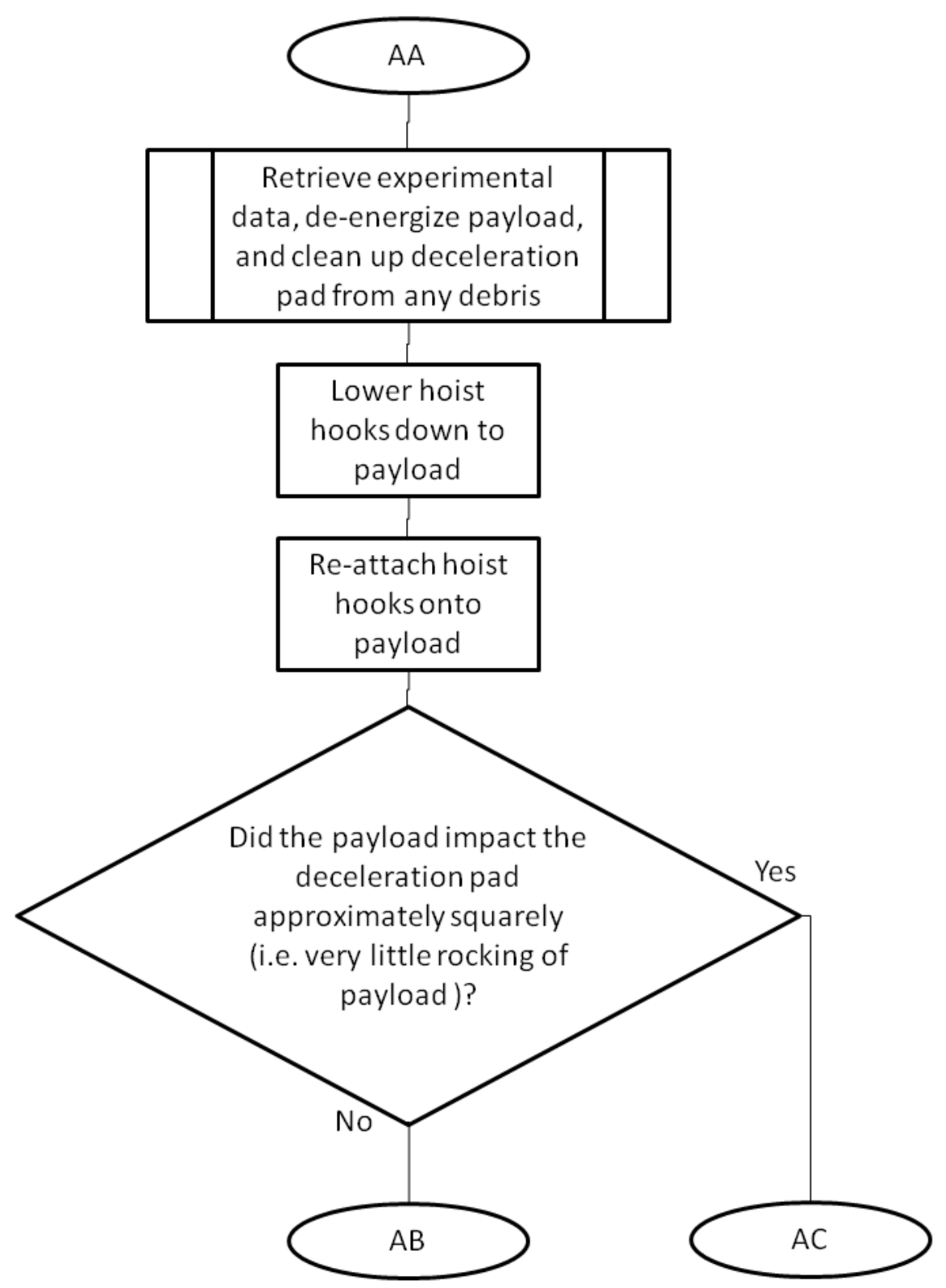




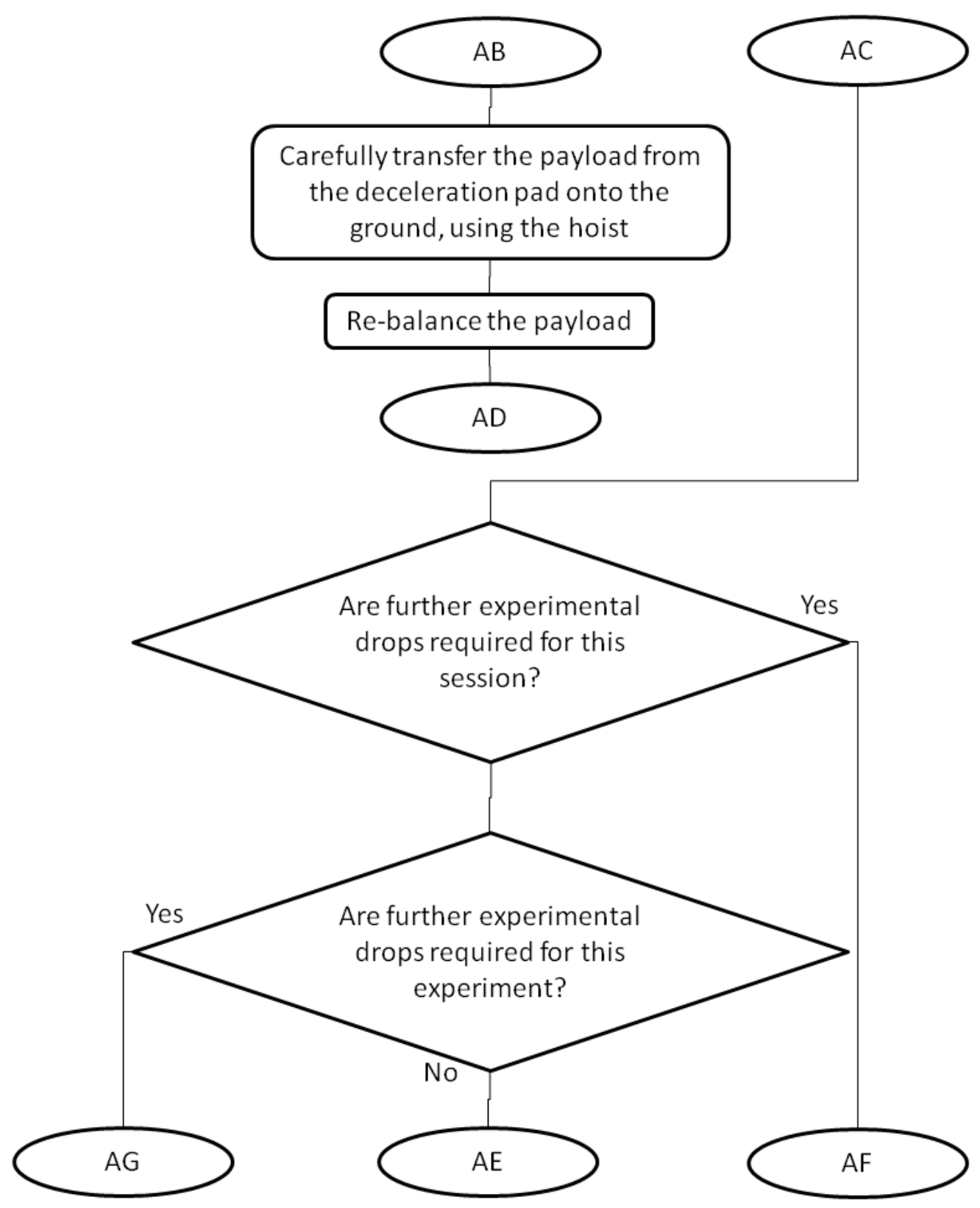




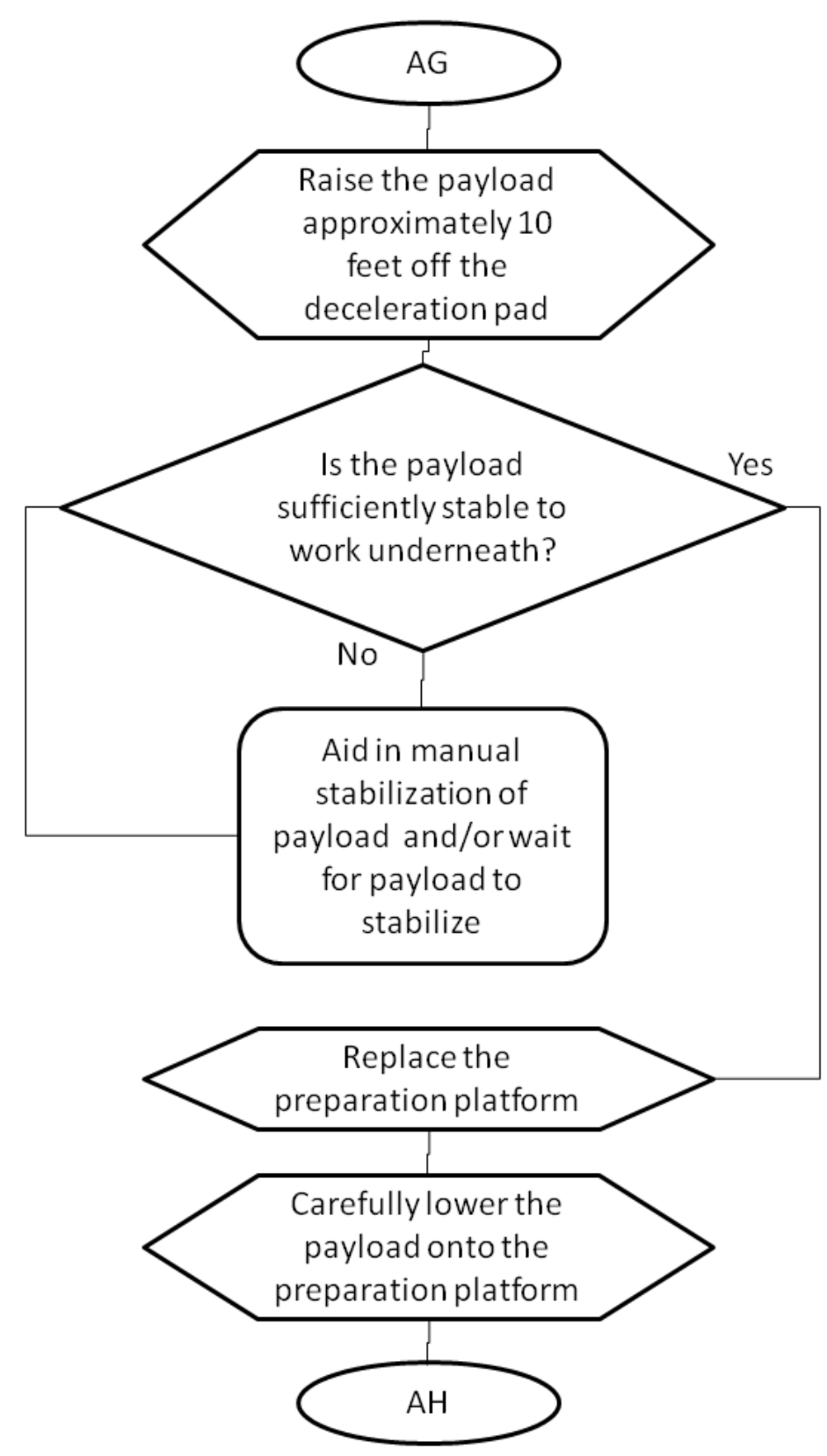




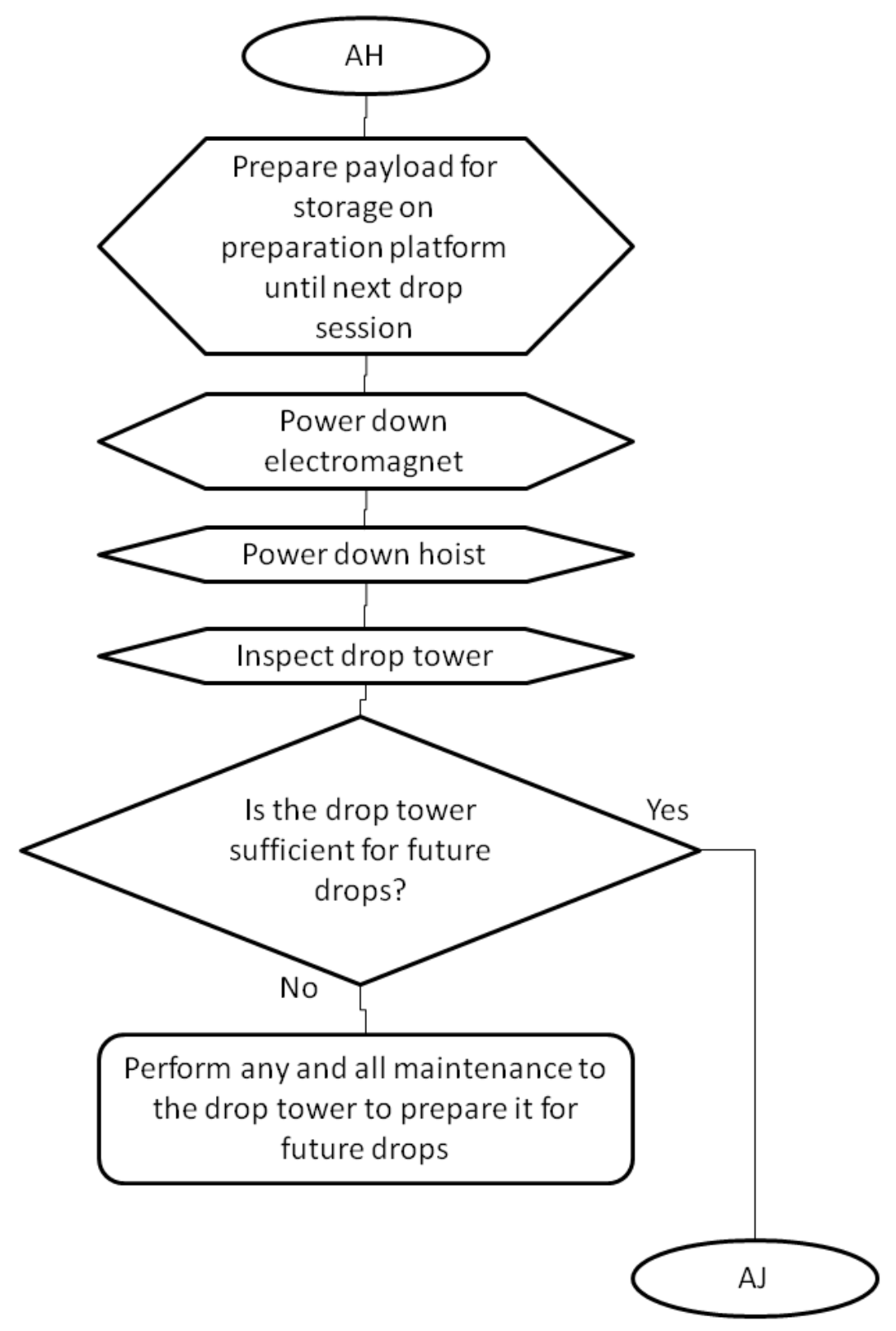




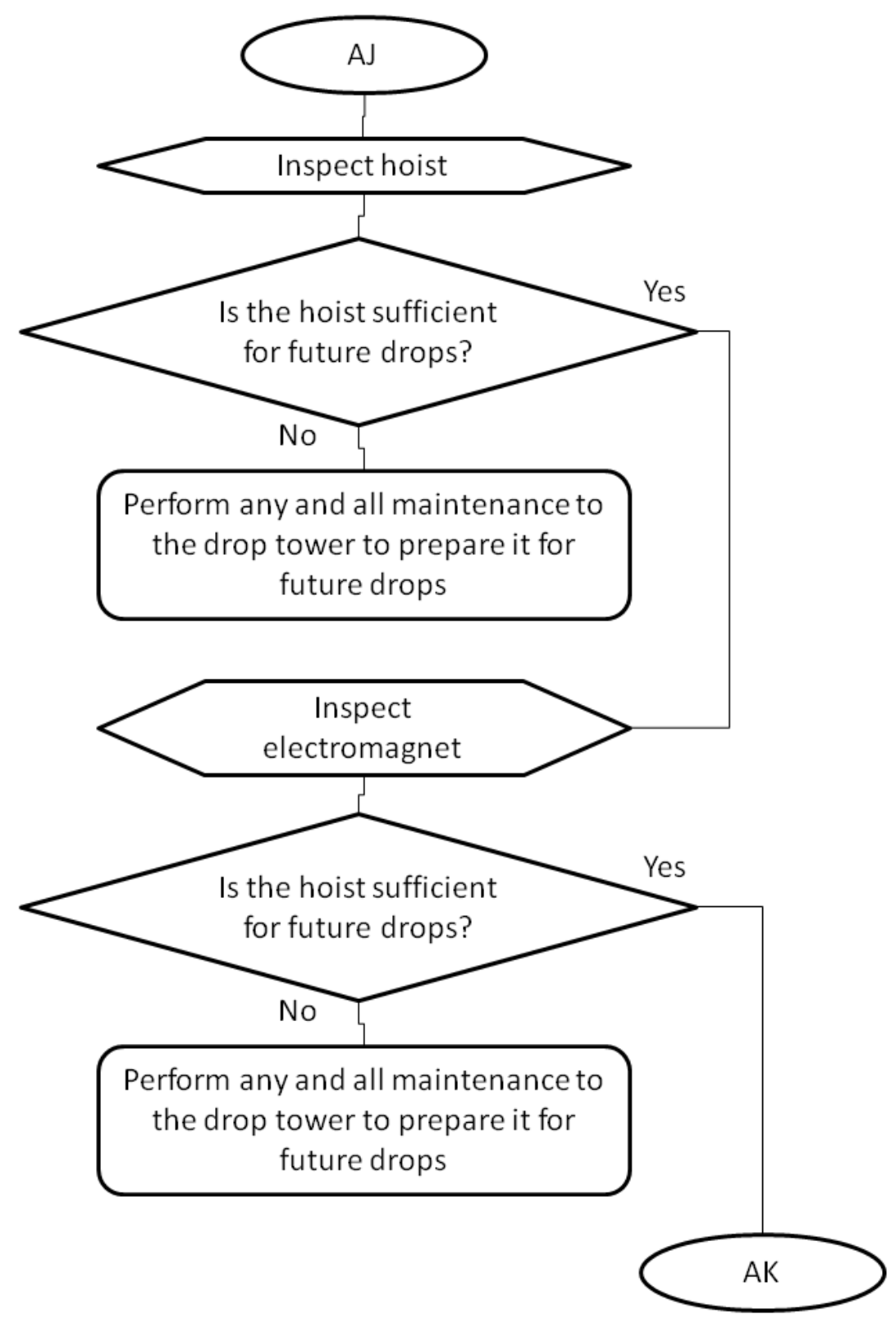




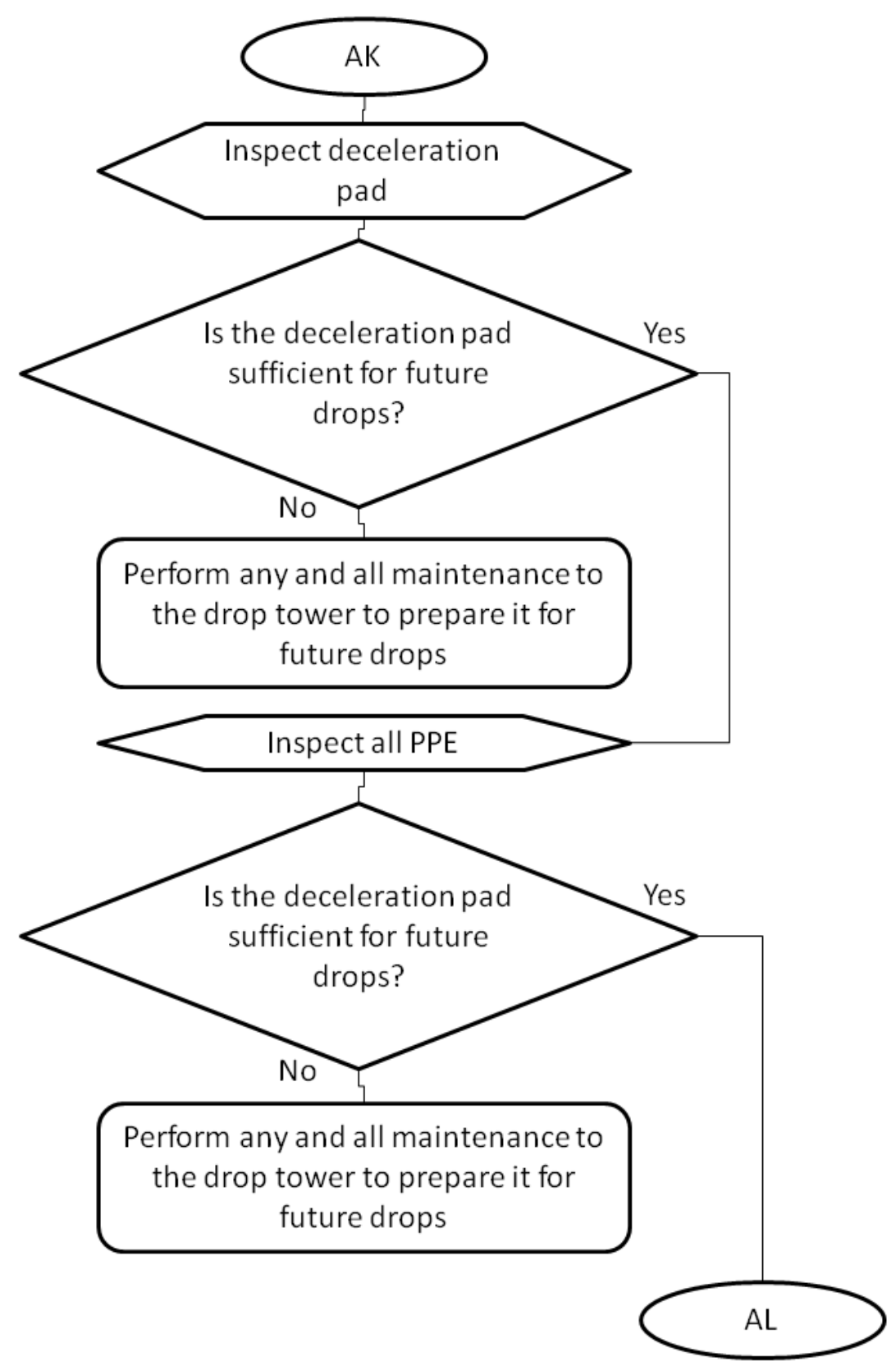




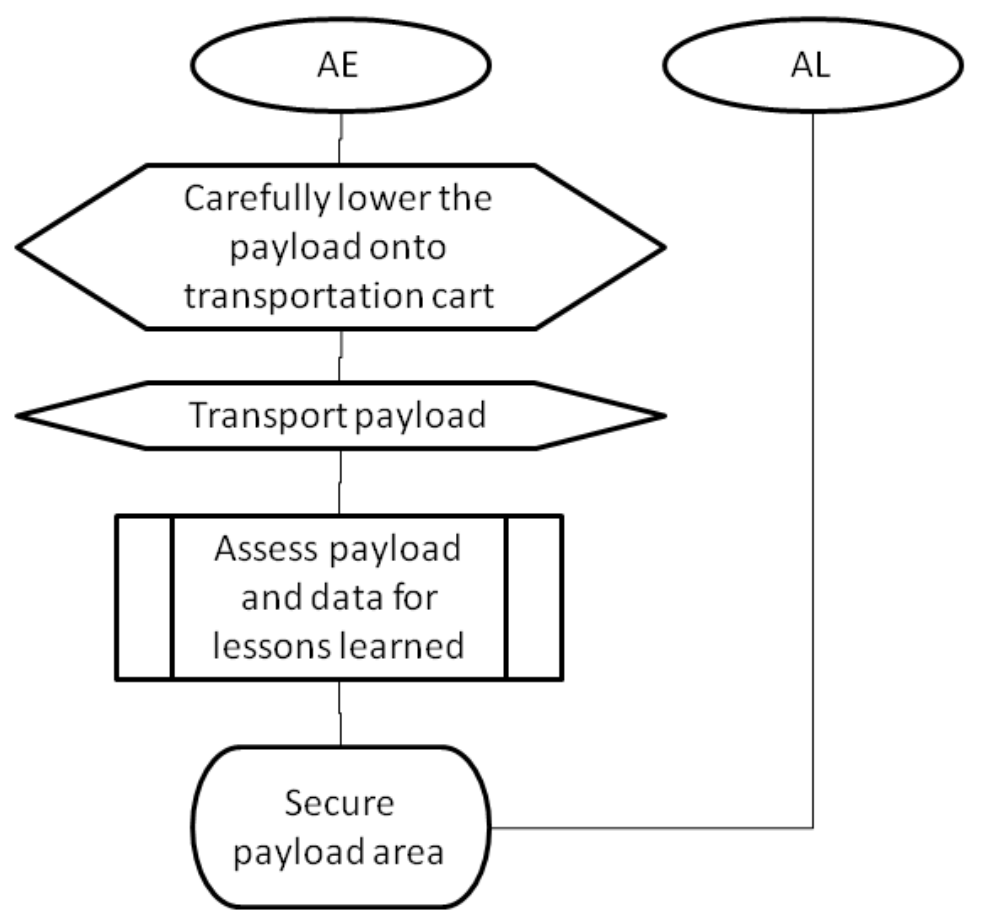




\section{Accelerometer Data Analysis}

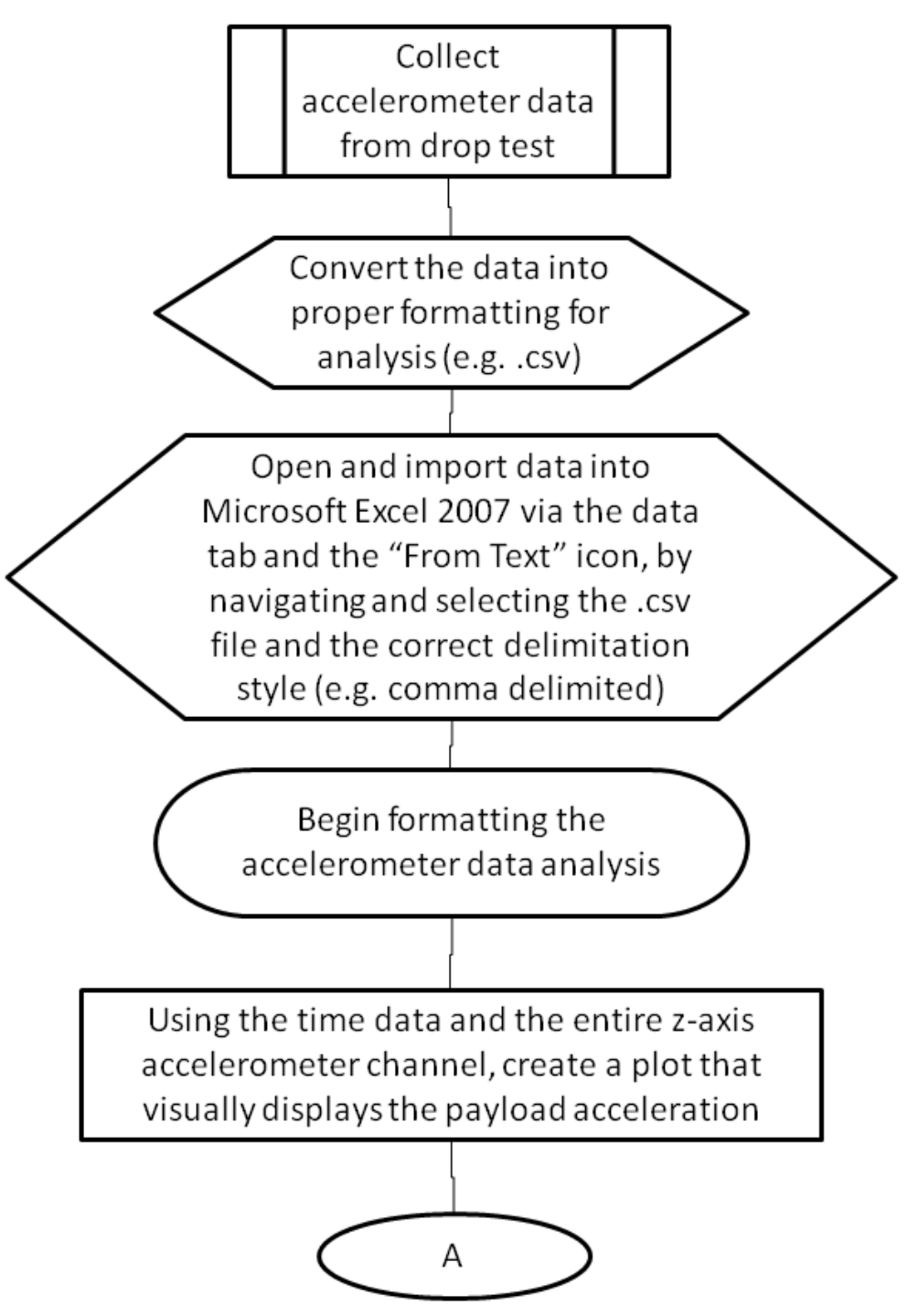




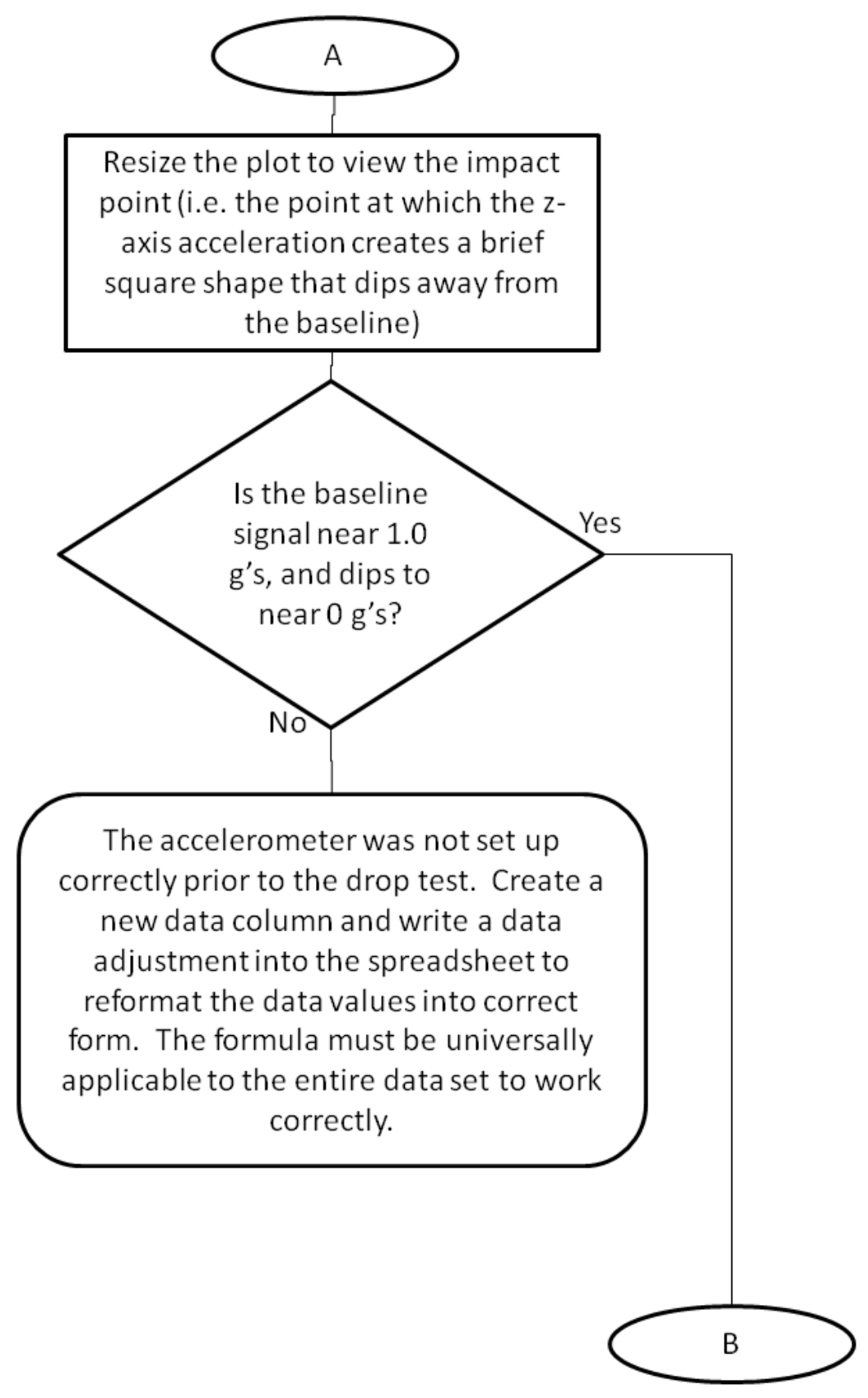




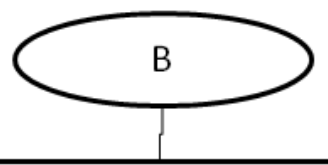

Find the maximum accelerometer data point (represents maximum measured point of impact - happens in every drop)

In reverse time-lapse, find the period ending just prior to the impact event, where the measurements were consistently below $0.2 \mathrm{~g}$ 's (i.e. the ending of the microgravity period)

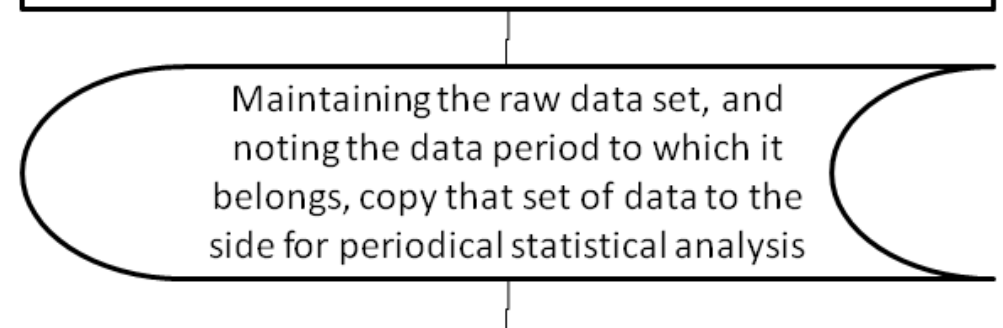

Continuing in reverse time-lapse, find the period ending just prior to the drop event, where the measurements transition from measurements consistently below $0.2 \mathrm{~g}$ 's to measurements consistently around $1.0 \mathrm{~g}$ 's (i.e. the end of the prep period and the beginning of the microgravity period)

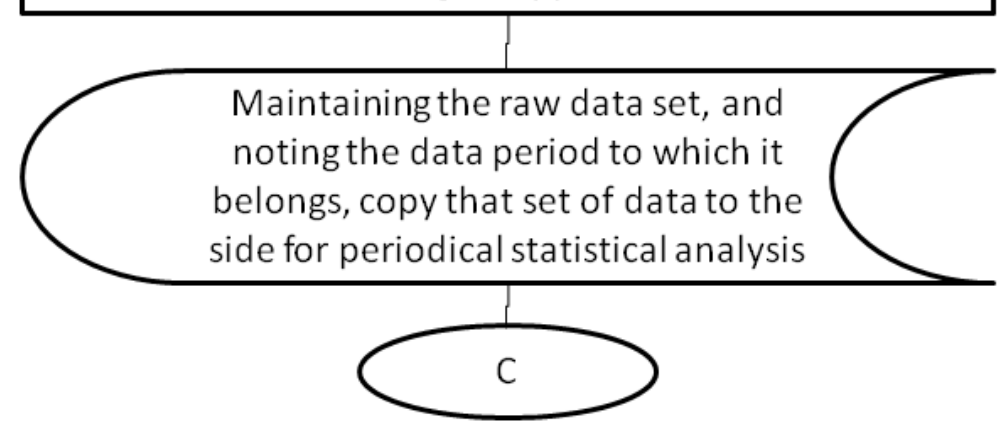




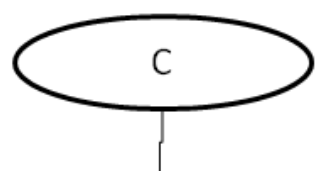

In forward time-lapse, from the data point that marks the beginning of the impact event (i.e. the data point after the ending of the drop period), where the measurements transition from consistently below $0.2 \mathrm{~g}$ 's to consistently varying above $0.2 \mathrm{~g}$ 's find the point at which the measurements transition to measurements consistently around $1.0 \mathrm{~g}$ 's (i.e. the end of the impact period and the beginning of the recovery period)

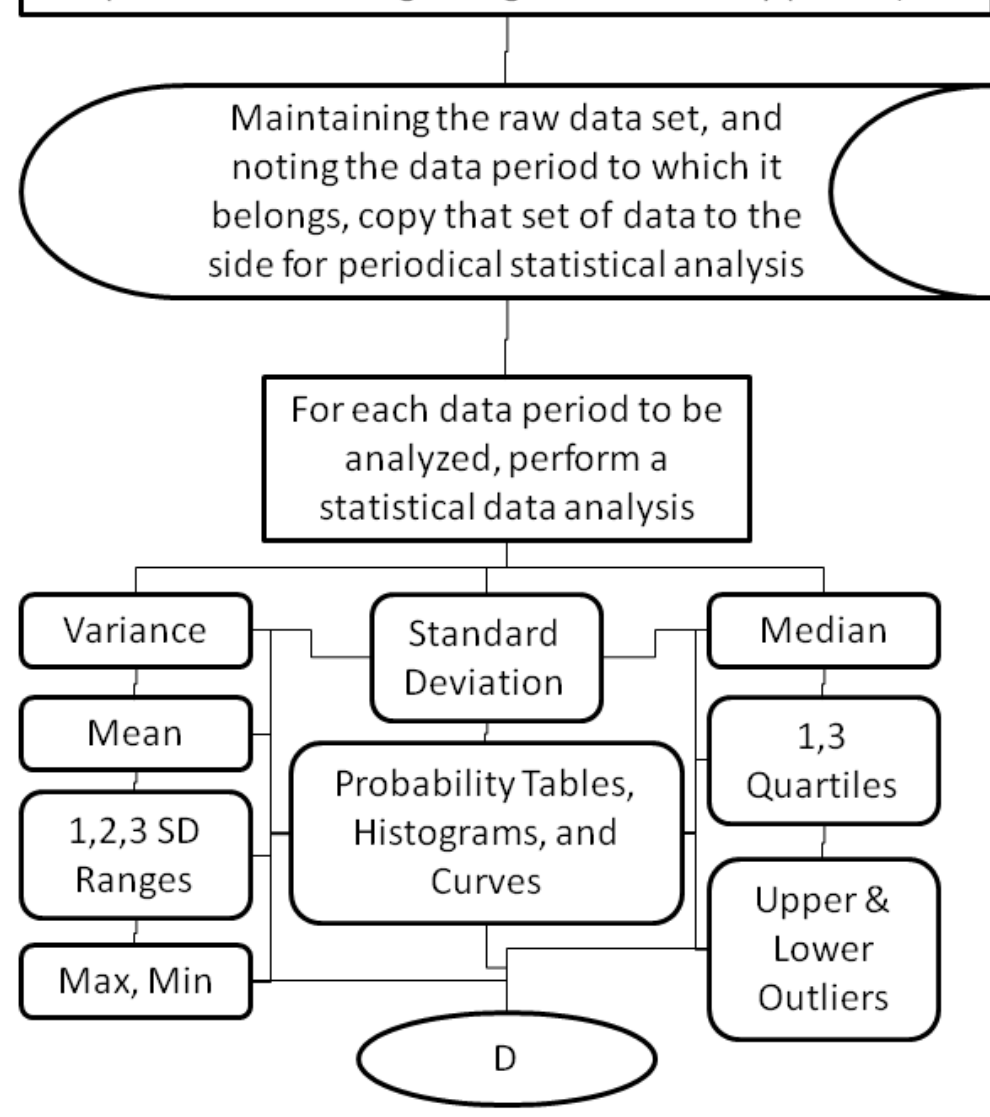




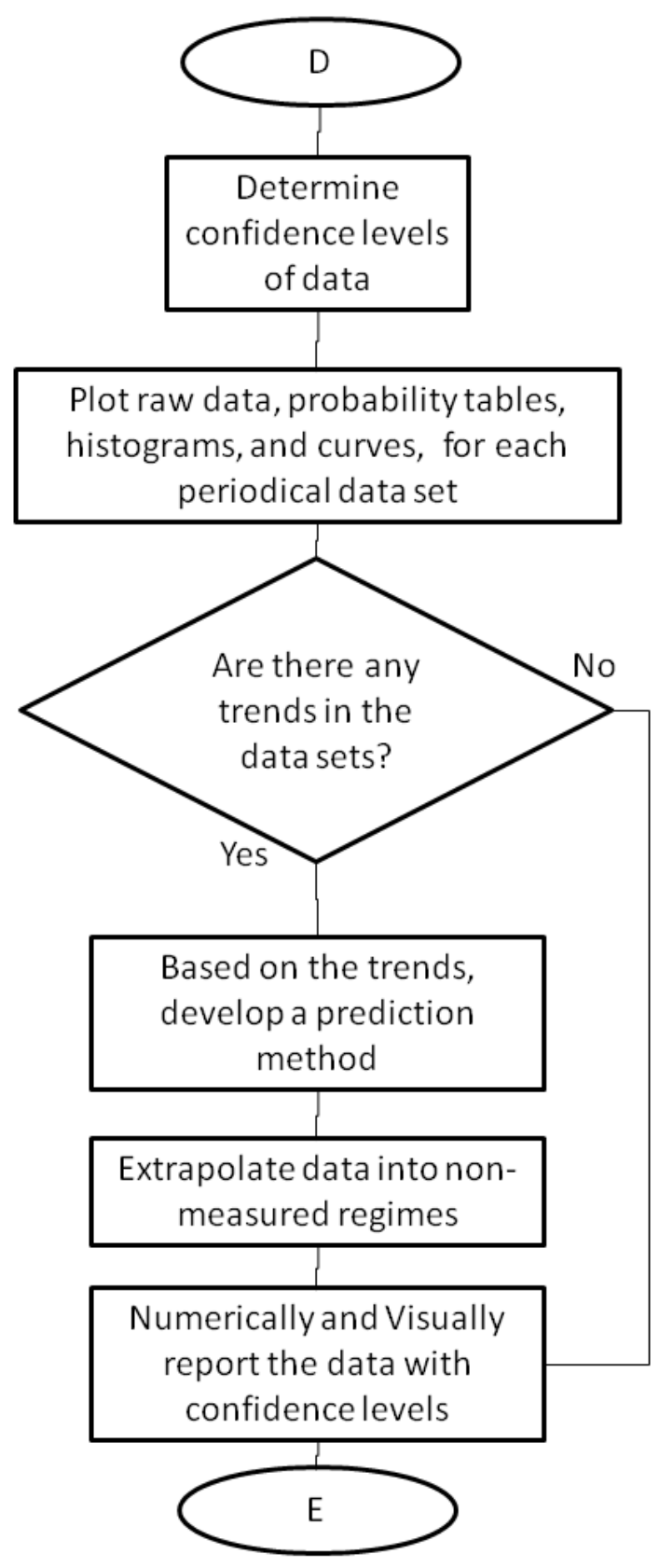




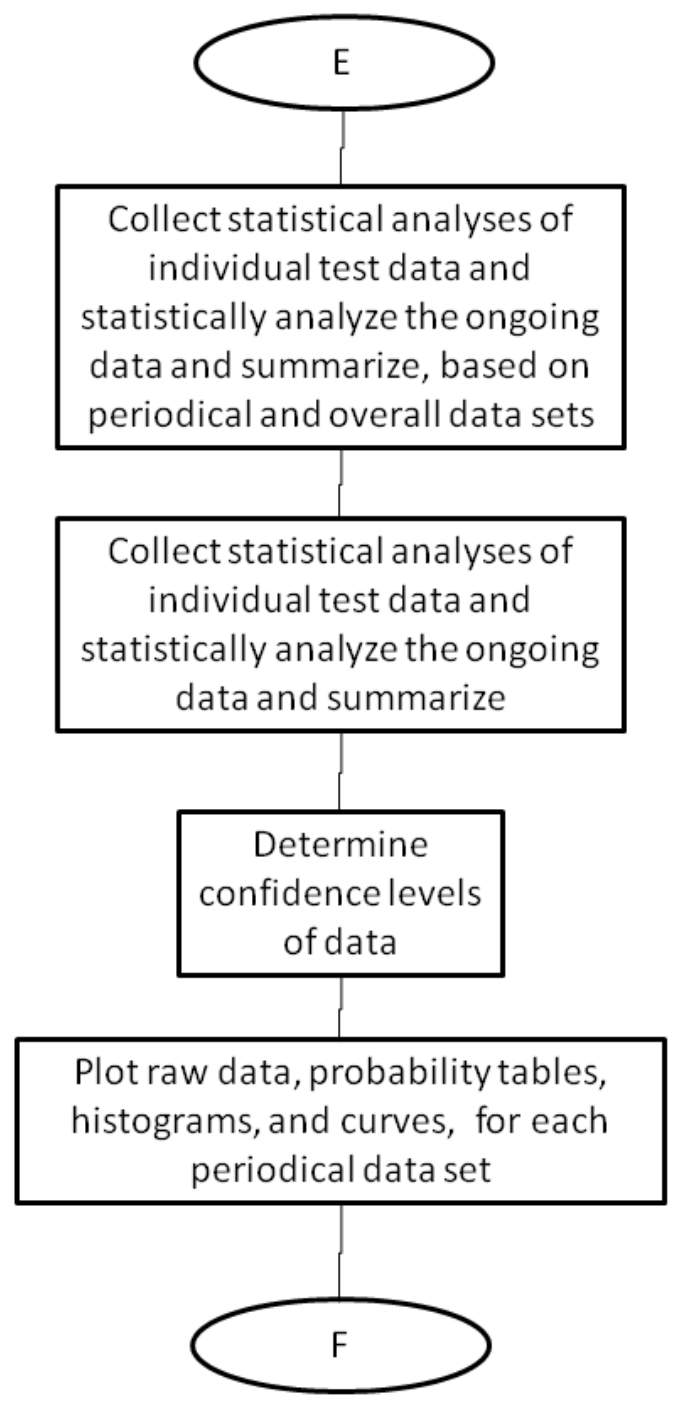




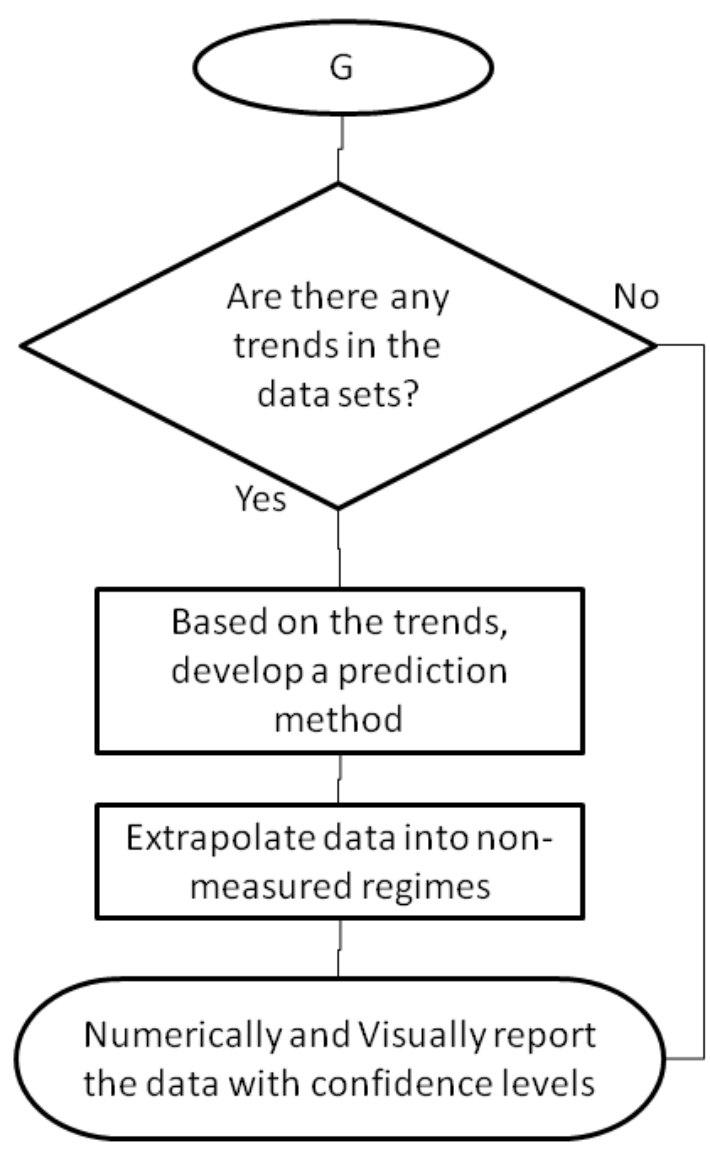




\subsection{Appendix J}

\section{Ongoing Operational Accelerometer Analysis}

Appendix M: Ongoing Operational Accelerometer Analysis presents the initial compilation of comprehensive accelerometer data produced from drops performed within the WVU SMiRF. The intent was to provide a common piece of software, widely accessible to researchers that intend to use the WVU SMiRF, to aid them in accelerometer data analysis. The other primary intention is to use the accelerometer data from the drop performed within the WVU SMiRF to create comprehensive compilation of data used for ongoing characterization of the facility as it changes over time to become more accurate for future drop tests. 


\section{Lift \& Prep Comp Data}
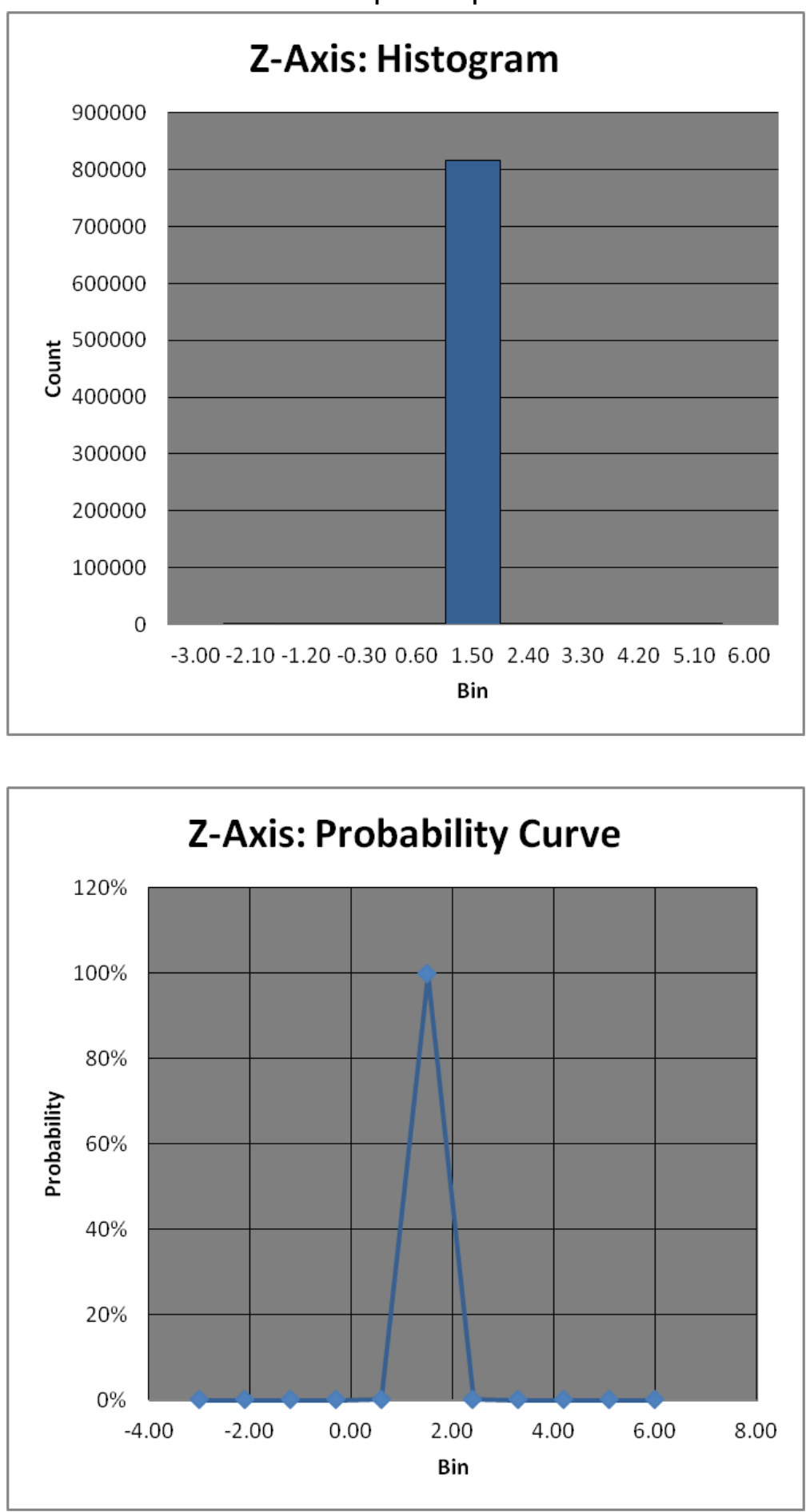
Low-G Data

\begin{tabular}{|c|c|c|}
\hline \multicolumn{3}{|c|}{ Drop Time Tables } \\
\hline Drop & Drop Time [s] & Variance \\
& & \\
\hline Drop 1 (20Oct2013) & 1.24 & 0.00 \\
\hline Drop 2 (200ct2013) & 1.28 & 0.00 \\
\hline Drop 3 (20Oct2013) & 1.30 & 0.00 \\
\hline Drop 4 (26Oct2013) & 1.25 & 0.00 \\
\hline Drop 5 (26Oct2013) & 1.18 & 0.01 \\
\hline Drop 6 (26Oct2013) & 1.28 & 0.00 \\
\hline Drop 7 (26Oct2013) & 1.21 & 0.00 \\
\hline Drop 8 (26Oct2013) & 1.26 & 0.00 \\
\hline Drop 9 (26Oct2013) & 1.22 & 0.00 \\
\hline Drop 10 (27Oct2013) & 1.29 & 0.00 \\
\hline Drop 11 (27Oct2013) & 1.20 & 0.00 \\
\hline Drop 12 (27Oct2013) & 1.20 & 0.00 \\
\hline Drop 13 (27Oct2013) & 1.23 & 0.00 \\
\hline Drop 14 (03Nov2013) & 1.27 & 0.00 \\
\hline Drop 15 (03Nov2013) & 1.31 & 0.00 \\
\hline Drop 16 (03Nov2013) & 1.30 & 0.00 \\
\hline Drop 17 (03Nov2013) & 1.30 & 0.00 \\
\hline Drop 18 (19Jan2014) & 1.25 & 0.00 \\
\hline Drop 19 (19Jan2014) & 1.35 & 0.01 \\
\hline Drop 20 (20Jan2014) & 1.29 & 0.00 \\
\hline Drop 21 (20Jan2014) & 1.26 & 0.00 \\
\hline Drop 22 (20Jan2014) & 1.32 & 0.00 \\
\hline
\end{tabular}


Development of the West Virginia University Small Microgravity Research Facility (WVU SMiRF)
Copyright $^{\odot} 2014$

Kyle G. Phillips

\begin{tabular}{|c|c|c|c|c|c|c|c|}
\hline \multicolumn{8}{|c|}{ Z-Axis } \\
\hline Variance & 0.01 & \multirow{2}{*}{\begin{tabular}{|l|} 
Lower Out \\
MIN
\end{tabular}} & 0.00 & & Bin MIN & -1.00 & \\
\hline Std. Dev. & 0.09 & & -0.93 & & Bin MAX & 2.00 & \\
\hline$-3 S D$ & -0.20 & 1st Q & 0.04 & & Bin Range & 3 & \\
\hline$-2 S D$ & -0.11 & Median & 0.07 & & No. of Bins & 10 & \\
\hline$-1 S D$ & -0.02 & $3 r d Q$ & 0.09 & & Bin Size & 0.3 & \\
\hline Mean & 0.06 & MAX & 1.16 & Bin No. & Bins & Frequency & Probability \\
\hline$+1 \mathrm{SD}$ & 0.15 & Upper Out & 0.15 & 1 & -1.00 & 0 & $0 \%$ \\
\hline$+2 \mathrm{SD}$ & 0.24 & IQR & 0.05 & 2 & -0.70 & 1.00 & $0 \%$ \\
\hline$+3 \mathrm{SD}$ & 0.33 & Range & 2.09 & 3 & -0.40 & 1.00 & $0 \%$ \\
\hline 1SD Range & 0.17 & & & 4 & -0.10 & 92.00 & $3 \%$ \\
\hline 2SD Range & 0.35 & & & 5 & 0.20 & 2772.00 & $95 \%$ \\
\hline 3SD Range & 0.52 & & & 6 & 0.50 & 51.00 & $2 \%$ \\
\hline & & & & 7 & 0.80 & 8.00 & $0 \%$ \\
\hline & & & & 8 & 1.10 & 2.00 & $0 \%$ \\
\hline & & & & 9 & 1.40 & 2.00 & $0 \%$ \\
\hline & & & & 10 & 1.70 & 0.00 & $0 \%$ \\
\hline & & & & 11 & 2.00 & 0.00 & $0 \%$ \\
\hline & & & & & Totals: & 2929.00 & $100 \%$ \\
\hline
\end{tabular}




\section{Low-g Charts}
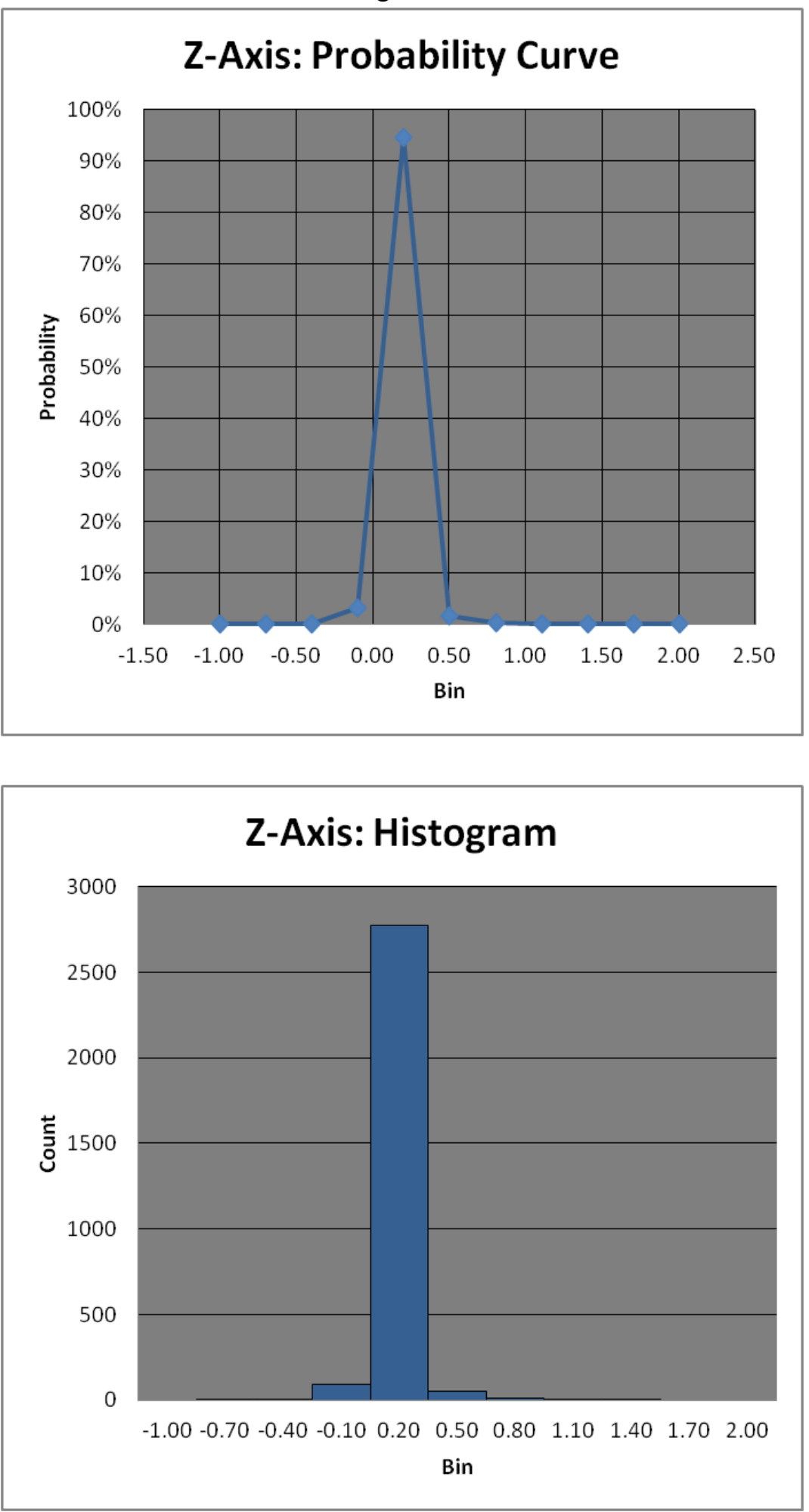
Impact Data
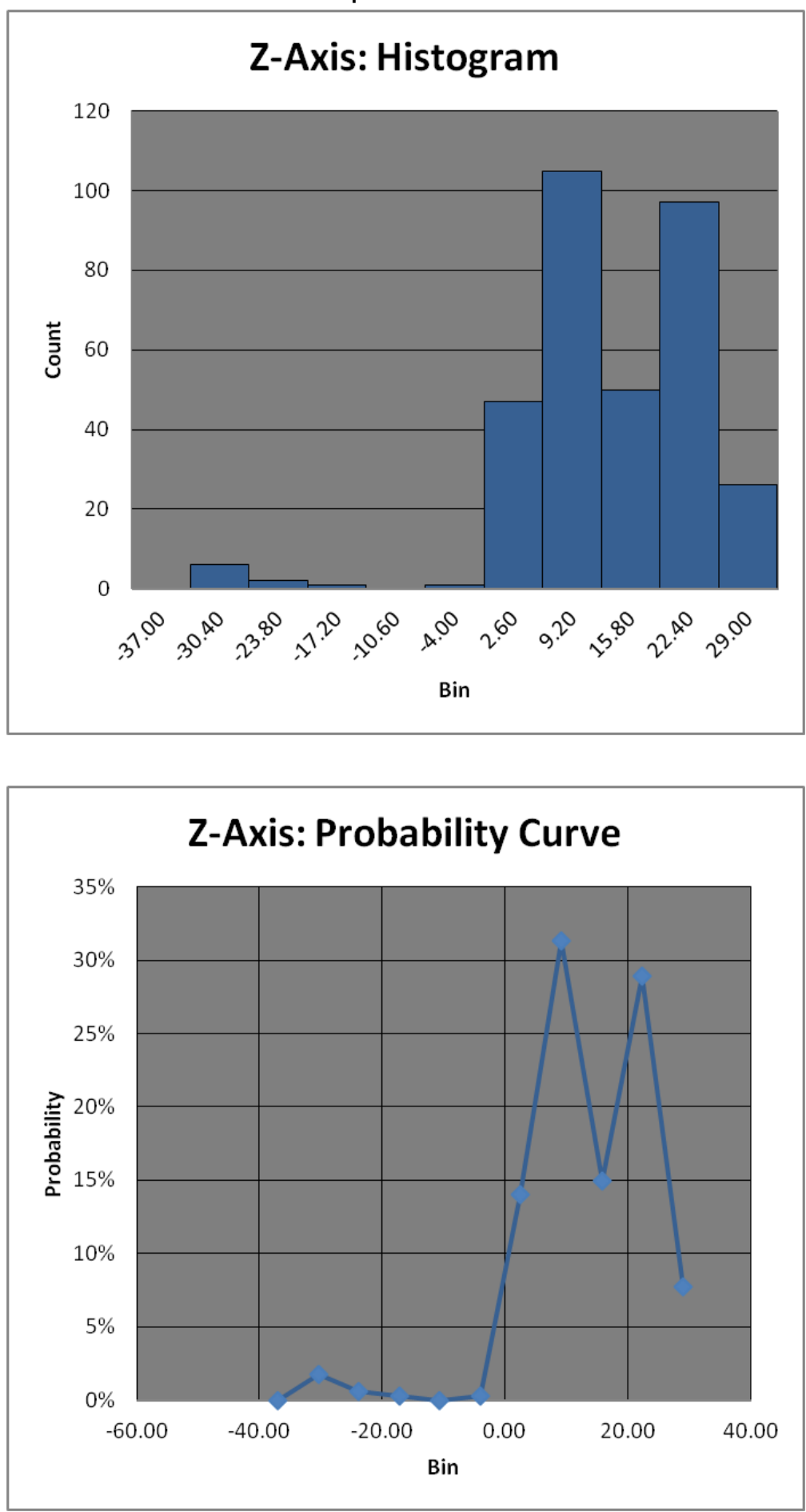


\section{Rebound Data}
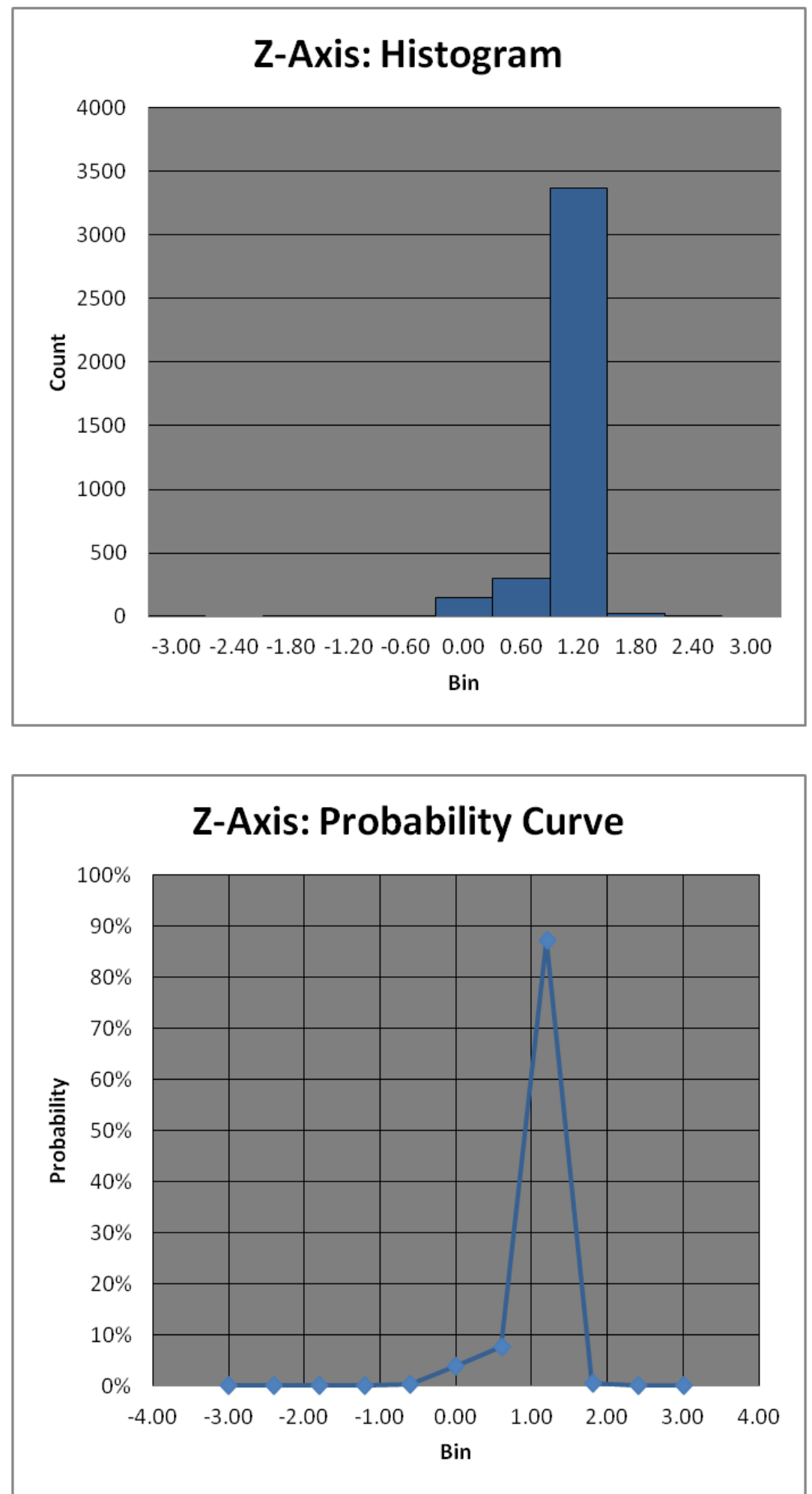

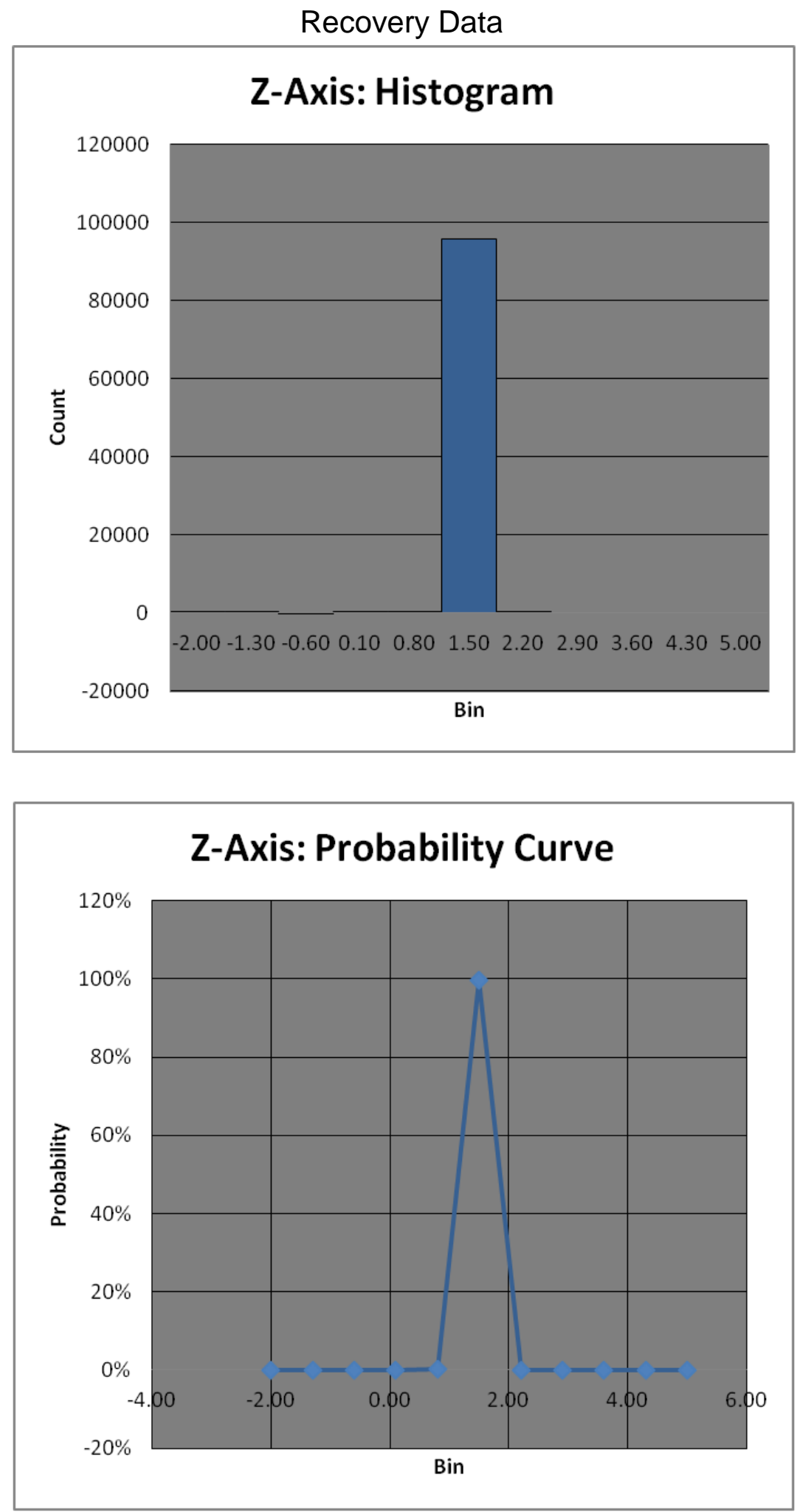


\subsection{Appendix K}

\section{WVU SMiRF Initial Calibration}

Appendix J: WVU SMiRF Initial Calibration presents the data compiled from the initial calibration drops at increasing weights used to characterized the facility and the compiled data from the initial experiment used to enhance that data and provide reliability data leading to averages for drop times, etc. 


\section{Lift \& Prep Comp Data}
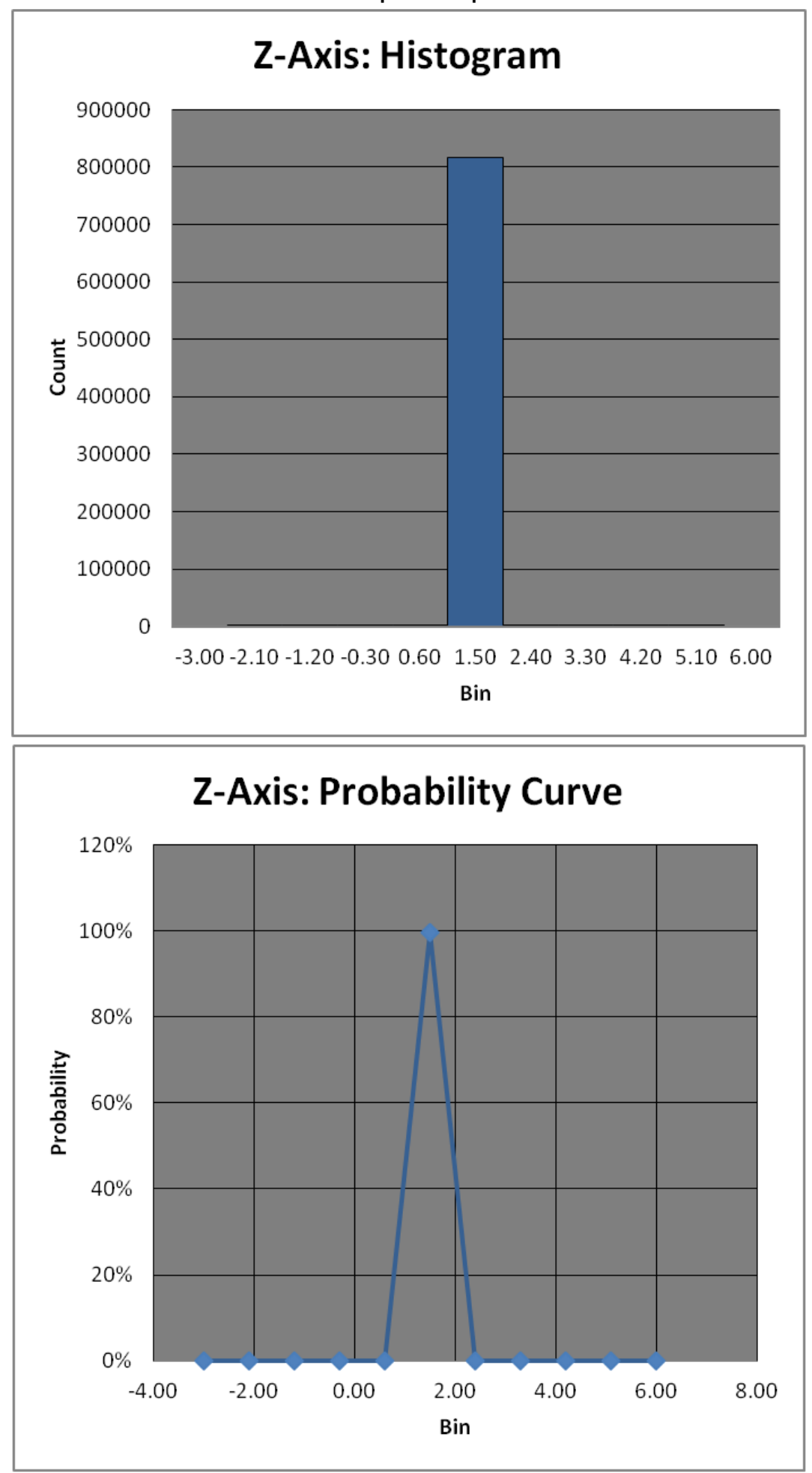

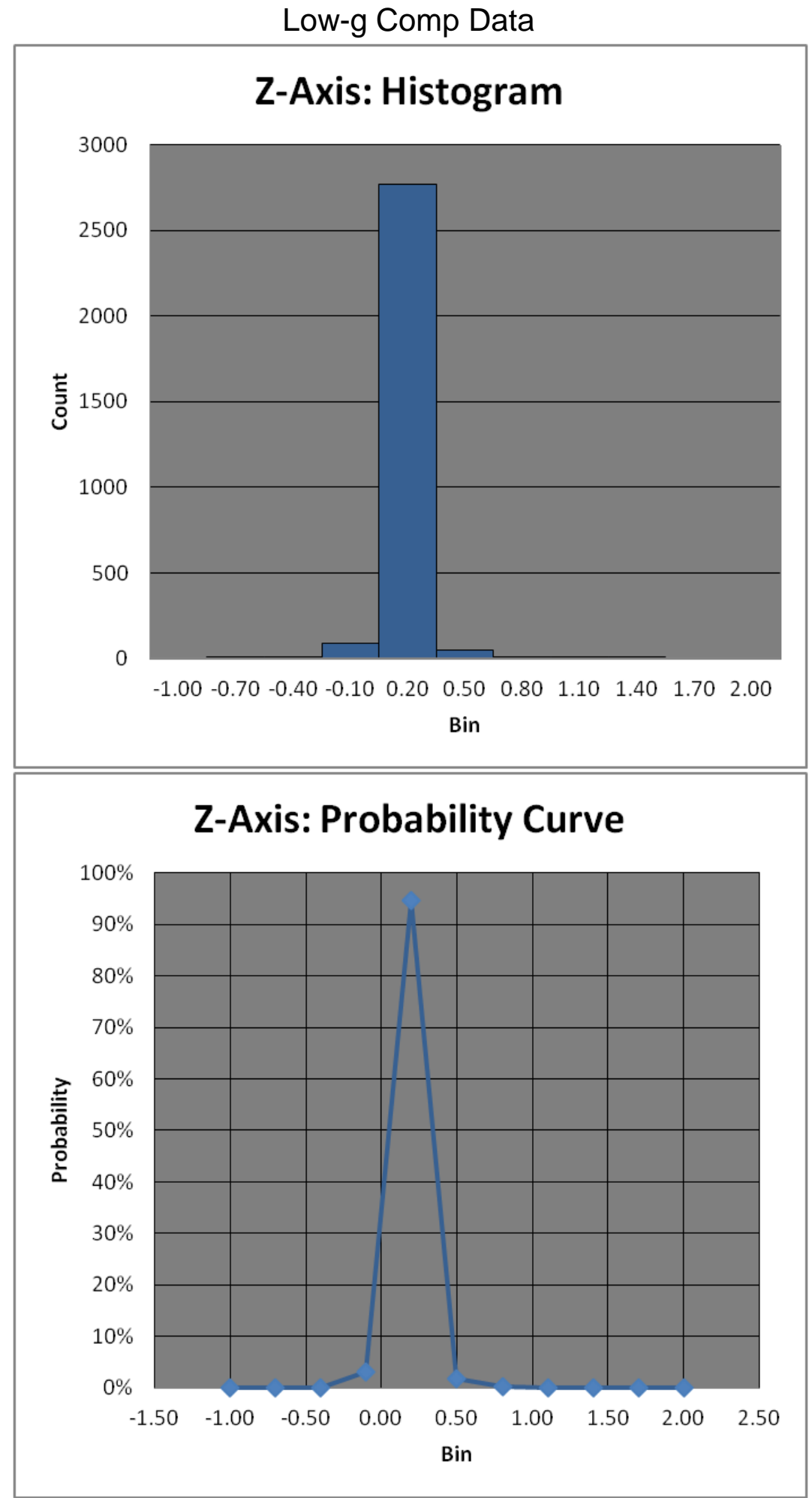


\begin{tabular}{|c|c|c|}
\hline \multicolumn{3}{|c|}{ Drop Time Tables } \\
\hline Drop & Drop Time [s] & Variance \\
& & \\
\hline Drop 1 (20Oct2013) & 1.24 & 0.00 \\
\hline Drop 2 (200ct2013) & 1.28 & 0.00 \\
\hline Drop 3 (20Oct2013) & 1.30 & 0.00 \\
\hline Drop 4 (260ct2013) & 1.25 & 0.00 \\
\hline Drop 5 (26Oct2013) & 1.18 & 0.01 \\
\hline Drop 6 (26Oct2013) & 1.28 & 0.00 \\
\hline Drop 7 (26Oct2013) & 1.21 & 0.00 \\
\hline Drop 8 (26Oct2013) & 1.26 & 0.00 \\
\hline Drop 9 (26Oct2013) & 1.22 & 0.00 \\
\hline Drop 10 (27Oct2013) & 1.29 & 0.00 \\
\hline Drop 11 (27Oct2013) & 1.20 & 0.00 \\
\hline Drop 12 (27Oct2013) & 1.20 & 0.00 \\
\hline Drop 13 (27Oct2013) & 1.23 & 0.00 \\
\hline Drop 14 (03Nov2013) & 1.27 & 0.00 \\
\hline Drop 15 (03Nov2013) & 1.31 & 0.00 \\
\hline Drop 16 (03Nov2013) & 1.30 & 0.00 \\
\hline Drop 17 (03Nov2013) & 1.30 & 0.00 \\
\hline Drop 18 (19Jan2014) & 1.25 & 0.00 \\
\hline Drop 19 (19Jan2014) & 1.35 & 0.01 \\
\hline Drop 20 (20Jan2014) & 1.29 & 0.00 \\
\hline Drop 21 (20Jan2014) & 1.26 & 0.00 \\
\hline Drop 22 (20Jan2014) & 1.32 & 0.00 \\
\hline
\end{tabular}


Development of the West Virginia University Small Microgravity Research Facility (WVU SMiRF)
Copyright $^{\odot} 2014$

Kyle G. Phillips

\begin{tabular}{|c|c|c|c|c|c|c|c|}
\hline \multicolumn{8}{|c|}{ Z-Axis } \\
\hline Variance & 0.01 & \multirow{2}{*}{\begin{tabular}{|l|} 
Lower Out \\
MIN
\end{tabular}} & 0.00 & & \multirow{2}{*}{\begin{tabular}{|l} 
Bin MIN \\
Bin MAX \\
\end{tabular}} & -1.00 & 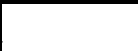 \\
\hline Std. Dev. & 0.09 & & -0.93 & & & 2.00 & \\
\hline$-3 S D$ & -0.20 & 1st Q & 0.04 & & Bin Range & 3 & \\
\hline$-2 S D$ & -0.11 & Median & 0.07 & & No. of Bins & 10 & \\
\hline$-1 S D$ & -0.02 & $3 \mathrm{rd} \mathrm{Q}$ & 0.09 & & Bin Size & 0.3 & \\
\hline Mean & 0.06 & MAX & 1.16 & Bin No. & Bins & Frequency & Probability \\
\hline$+1 \mathrm{SD}$ & 0.15 & Upper Out & 0.15 & 1 & -1.00 & 0 & $0 \%$ \\
\hline$+2 S D$ & 0.24 & IQR & 0.05 & 2 & -0.70 & 1.00 & $0 \%$ \\
\hline$+3 \mathrm{SD}$ & 0.33 & \begin{tabular}{|l|} 
Range \\
\end{tabular} & 2.09 & 3 & -0.40 & 1.00 & $0 \%$ \\
\hline 1SD Range & 0.17 & & & 4 & -0.10 & 92.00 & $3 \%$ \\
\hline 2SD Range & 0.35 & & & 5 & 0.20 & 2772.00 & $95 \%$ \\
\hline 3SD Range & 0.52 & & & 6 & 0.50 & 51.00 & $2 \%$ \\
\hline & & & & 7 & 0.80 & 8.00 & $0 \%$ \\
\hline & & & & 8 & 1.10 & 2.00 & $0 \%$ \\
\hline & & & & 9 & 1.40 & 2.00 & $0 \%$ \\
\hline & & & & 10 & 1.70 & 0.00 & $0 \%$ \\
\hline & & & & 11 & 2.00 & 0.00 & $0 \%$ \\
\hline & & & & & Totals: & 2929.00 & $100 \%$ \\
\hline
\end{tabular}


Impact Data
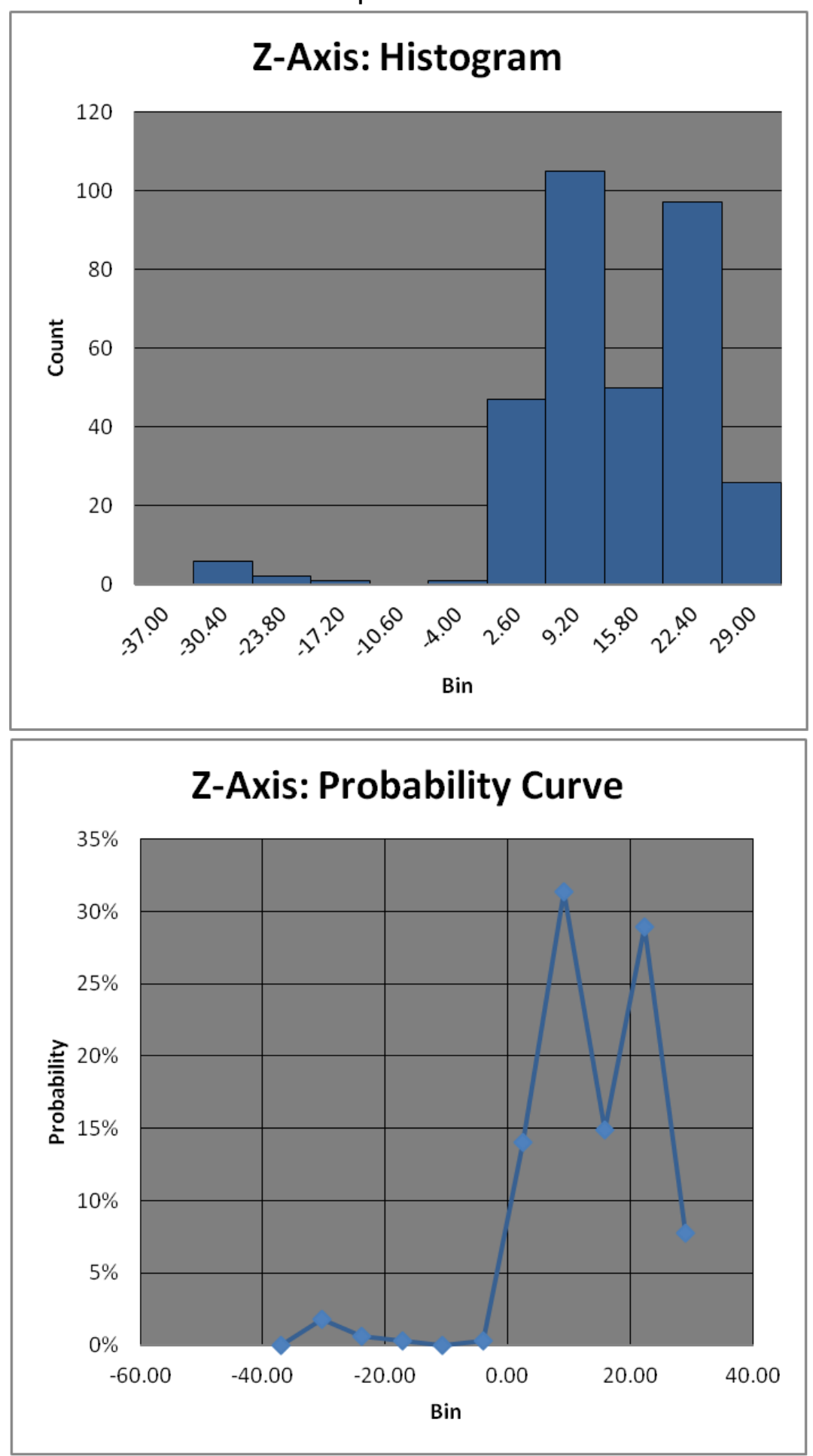


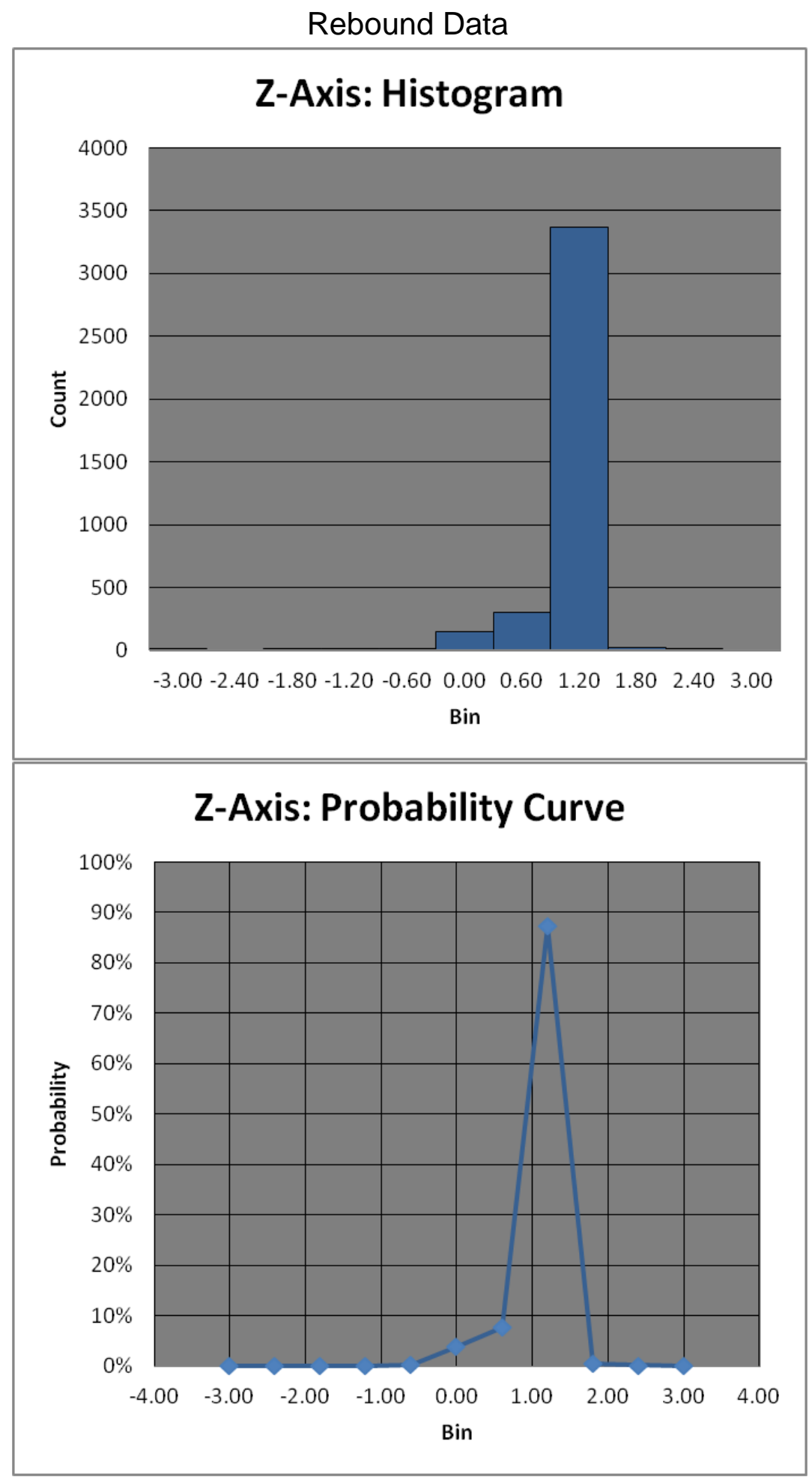




\section{Recovery Data}

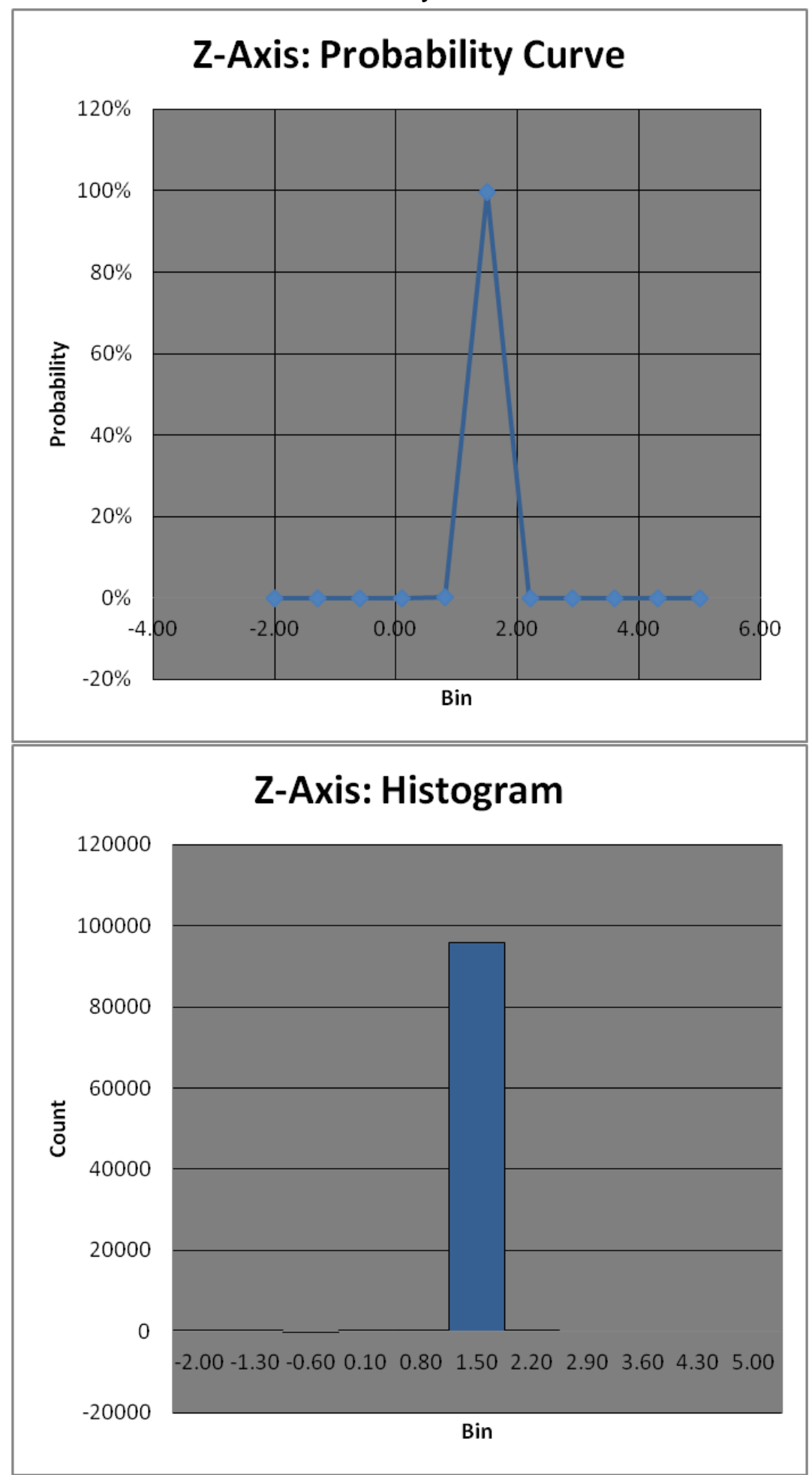




\subsection{Appendix L}

\section{Non-Newtonian Navier-Stokes Derivation}

Appendix K: Non-Newtonian Navier-Stokes Fluids Derivation presents the long form of the derivation of equation 6.1-1. The intent of this derivation and the resultant equation is to attempt to incorporate non-Newtonian fluid behavior into the Navier-Stokes equations, which is currently the leading mathematical model by which a large portion of fluid behavior is described. The researcher's current philosophy is that the vast majority of fluid behavior is non-Newtonian, and that Newtonian behavior is a unique subset that can afford simplification to such behavior. The scientific community must strive to create ever-more comprehensive mathematical models that describe the universe around us. This is simply an attempt toward that endeavor and serves as the beginning to a long discussion to incorporate non-Newtonian fluid behavior in future mathematical models. 
$21 \operatorname{Jan} 2012$

- attempting to derive governing equatiuns for visco-elastic Nuids from

basic proviples

- assumption 1: Each (flend) particle vill be assuned to be a contraweus

(Gluid) wateried w/ hamagenas paperties

-asumplim "2: Each (fluid) material is ascumad to be conslituted of a very buge amant (ecentically unfivite) number of infinitecisadly smatl (fluid) partides (ire. its dimensions approach o in the hase)

- bref list of petential influemes an the (Awid) putide:

-. in ternal influoncs will be maily due to (fluid) malerial propaties

idecriptols of these prowlies

- external influences will be dedraldel to thoe Afeds acturg on the

(flud) partive from is survounlings

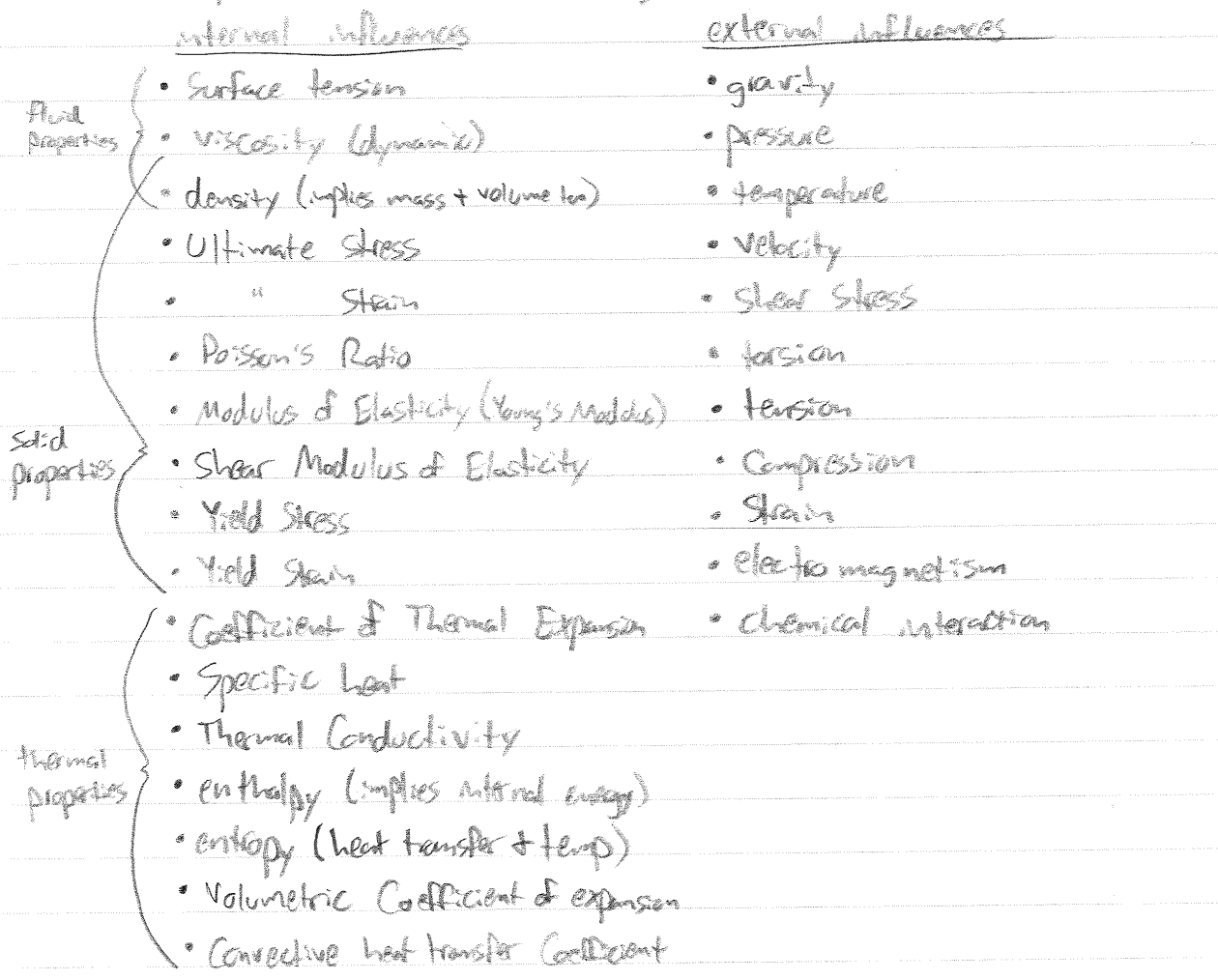




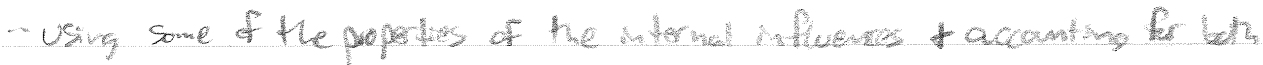

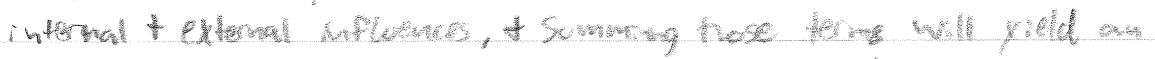
equatian (or set of equations) that (an be cred to dzeribe the behaver of the masority of common (Preudo-sild fluds) moletials

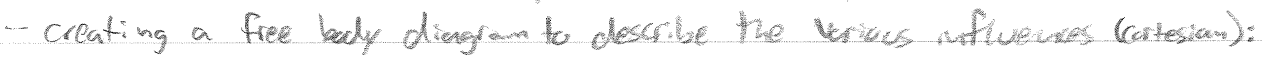

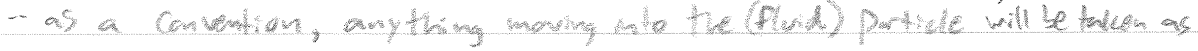

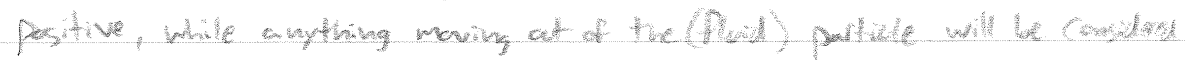

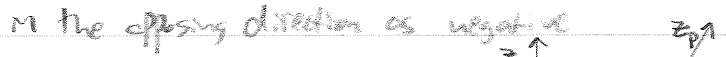

- gravitational influence:

the "g" subeript molubles an undrangingly-ariented glakel refrence Nowne the "P" subsript wiveles the particle reference fownes gavity will be ascumed bo at along the negaline $z_{3}$ forestion

the nhouce of grawity ats at the carts of owity (... (c)

of has (N.d) perbe the pait at whick the 2 reforence parmes wab arbitanly down fo Cra wornence + cold he re-oriented at the frtide 66 8 - to prosty nelue has the pride

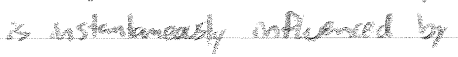

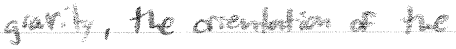
pulte whert bo the globl retronte parat wust be - due to ascuntition "2, all of the debromined to mircate whin

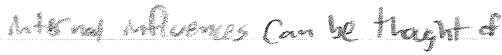

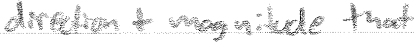
as constow t throughet the pricle gewty acts wh the protue t the 6 mall be than of the the maghintudes in ench roy,

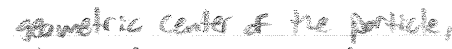

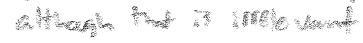




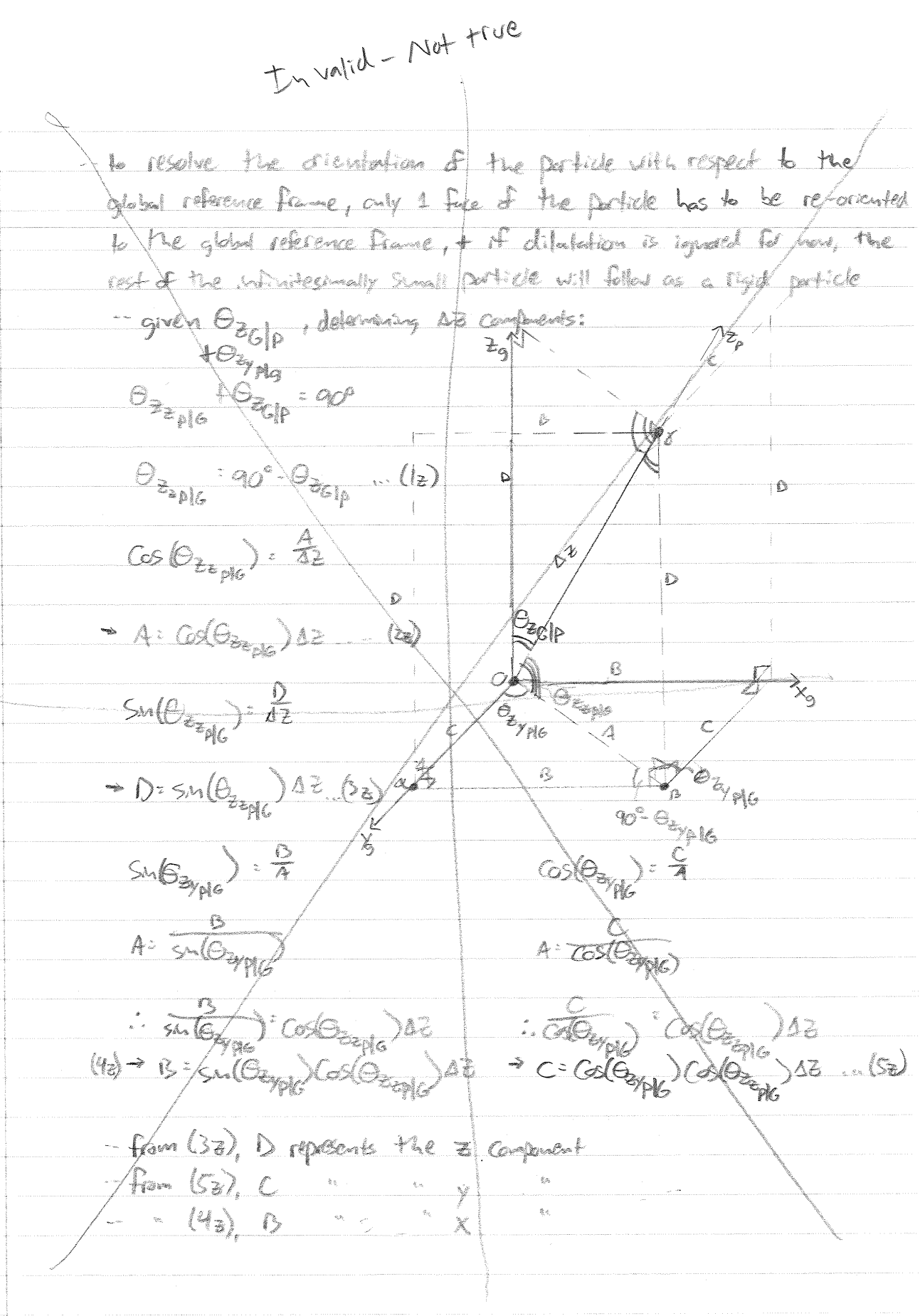




$$
\text { Invalid-Net proe }
$$

$\therefore$ given 2 angles of the (flud) protide refrence frome relup we to the global refrence frome, and the mass of the (fluid) ynatrial, one may determane the intwence of gravity on the (fled) portide - from Neutan's $2^{\text {nd }}$ Low:

$$
\begin{aligned}
& \vec{F}=m \vec{a} \\
& \sum F=m\left(a_{x}+g /+a_{y}\right)
\end{aligned}
$$

-hr this case, where our facus is on gavily, it acts as the accelration:

$$
\Sigma F_{g}=m\left(g / g_{y}+g_{z}\right)
$$

- the total gravity foue (weight) "s simply:

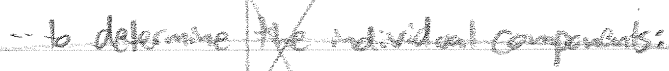

$$
\begin{aligned}
& 9 x / g\left[\sin \left(\theta_{z_{p u g}}\right) \cos \left(\theta_{z_{n}}\right) \Delta z\right] \\
& g_{y}=g\left[\cos \left(\theta_{z / p)}\right) \cos \left(\theta_{z_{z p l}}\right) d z\right] \\
& \rho_{z}=g\left[\sin \left(\theta_{z_{z}}\right) \Delta z\right]
\end{aligned}
$$


- to resolve the (fluid) partide orientation w/ referance to the global refereve frome, the angles betwen the the reprence fromes must be resulvel (or givin)

$$
\begin{aligned}
& \text { its passible that those angles way clange our the as the } \\
& \text { pirticle translates + retates } \\
& \text { the particle's tronglation + olation over the anay evion howe } \\
& \text { an equation which gooerus that indion }
\end{aligned}
$$

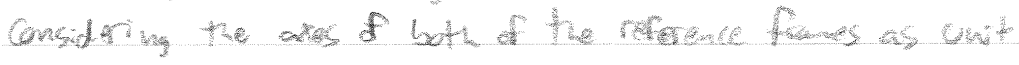

- the only componout necessery to resolve

is the componeants is the $-\vec{z}_{\mathrm{g}}$ direction

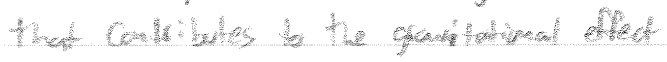

-. the given andes will delbrame the

(flud) particles orientalion on Guace

- due to ascumpliontl, the couter of

gavity $\left(C_{6}\right)$ lins at its gamentic

Center (C.e. Conkod) of the (fluid)

porticke

grasty ads in the - zing dinedkens at that past t the oriandation

of the protile con be dind

by the 3 ander in Catesan

Pase

se Stction $9.2(90.439)$ - Sthtes by Hobler to detrmane Cost concols

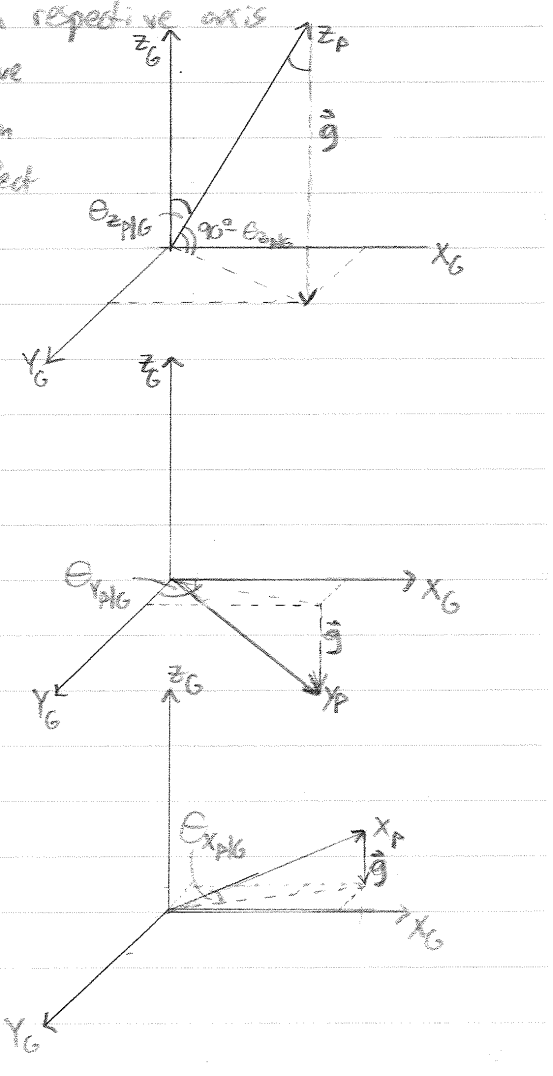


- the bae of garity will at at the $C_{C}$ of the (Puid) particle in the $-z_{g}$ direction $t$ is known as weight from Newton's $2^{\text {st }}$ Lant:

$$
\vec{F}=w^{2}
$$

- Wre, if the fous is on growity (if cher bods wre completely ignord), then the cully arcellation is dee the the grawitatomal athostion:

$$
\vec{F}=\operatorname{mog} ; \vec{f}=\text { weght }
$$

-once the dimensions of the particle are taken asto account, the amaust of weight po volune is linewn As specific weight, whore of the whas por volume is thimentin as density:

$$
\begin{aligned}
& y+\frac{m}{7}=p_{g} ; p=\frac{0}{7} \text {, wrimess } \\
& y^{2} \cos x+y \\
& \text { * a vilume }
\end{aligned}
$$

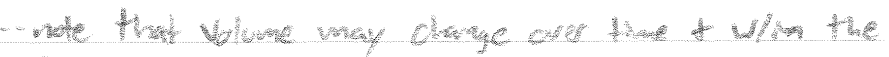
flow pathern $t$ therefore dewsity of the (Muid) purtele may chave wh respect to thme \& spatial bation

- this trpe of phemomenon is known as Compressiluility of the (fluid) material 


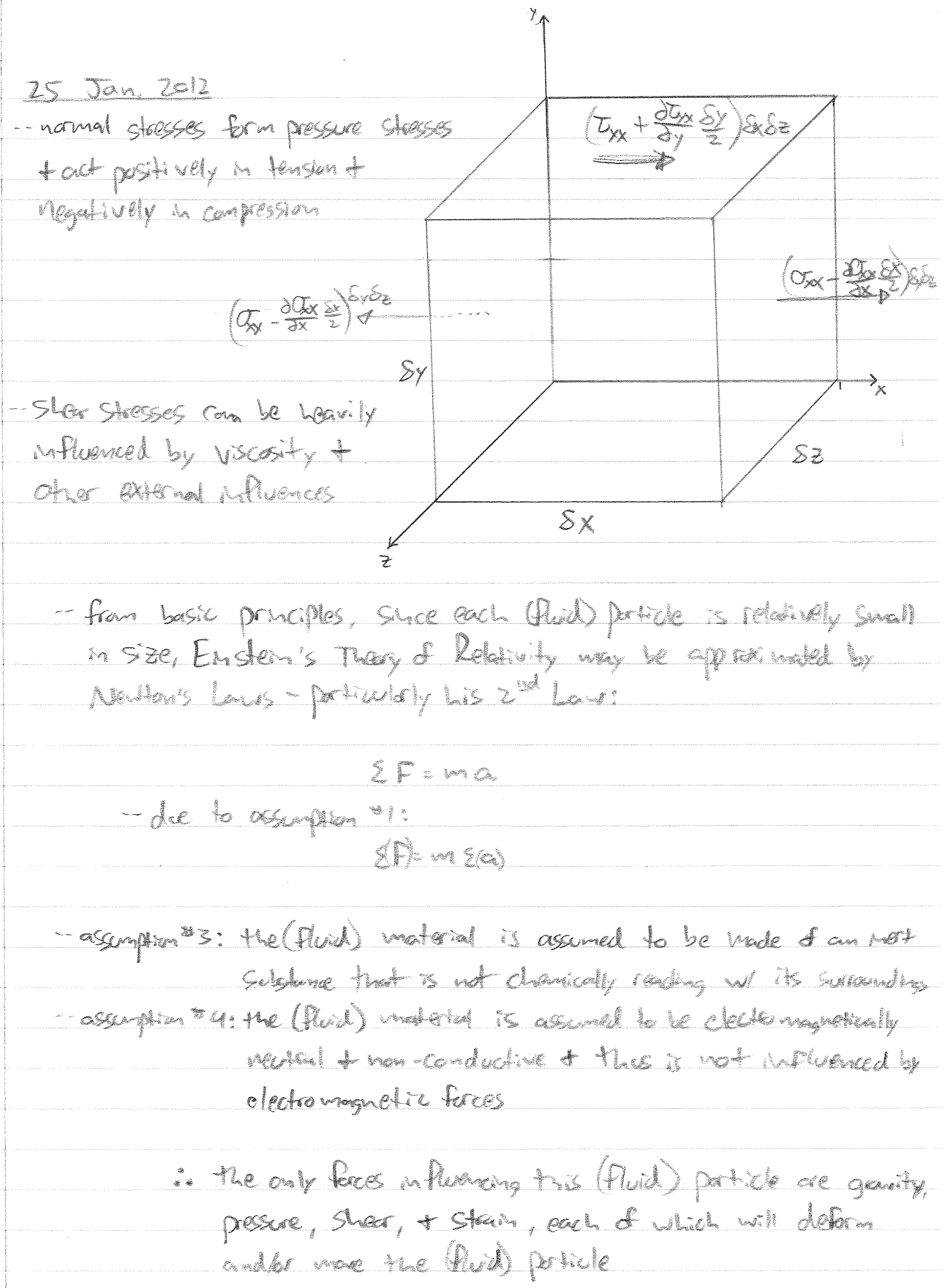

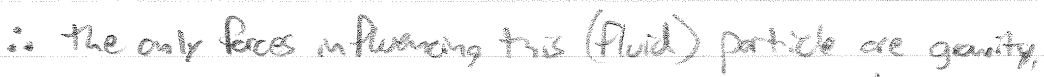
pressure, sherr, + stain, each of which will deform andibr mave the (lid) poticle 
- each normal I Sher stess 8 astomed to act at the center on which it acts (amplyig am avernge adhy over the fuce on which it emates)

- each drection has 3 directional budy forces liee 1 normal

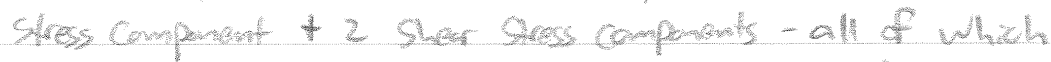

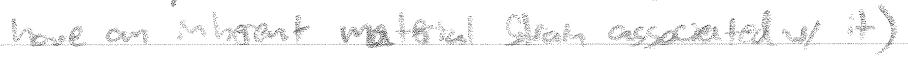

$\therefore$ elastic mataral properties shald be mcluded in the corrent Navier-stakes equations to better govern generol Puid flows + take mb account the elatic proptes of fivds

- thus, each hormal / Shear stress heeds to accumodote a version of Molke's Lan:

$$
\begin{aligned}
& \sigma=E E ; \sigma \equiv \text { cless } \\
& \epsilon \equiv \text { chain } \\
& \text { EE Elache Mdulus (AuA Vuns) }
\end{aligned}
$$

- $\mu$ its grem form, Houkes Law assumes a herly elastic (Newtanion) matarial w/ the Stres-Stican. diagrams elatic mion intercepting the $x$ ty axis at the orgh. However, the slope (claslic Modulus) may be non-linear, t the y-intercegt may be non-zord, bath of which ore probully wide Common them the idenl Mokian Materat.

- Modification of Hooke's Land to accomedate for non-Hodceivan Materials may be dase the following procedure may then be followed 


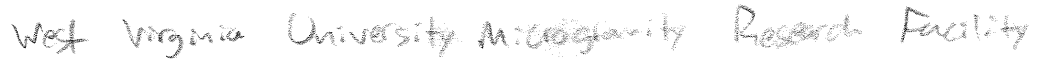

Drop Tower Development + Tecting Broble 2 )

25 Jan, 012

- Continuing the modificalian to the Naver. Steles equations

- traditionally, the viscos vesion of prtide deformation in the $x$-dired sere, twhes the fermes:

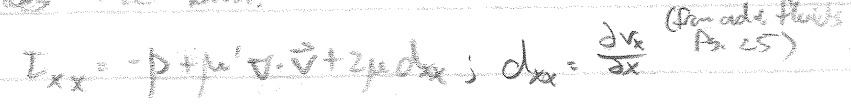

- Rem Hadee's Law, $\tau_{x x}=E E_{x x}$

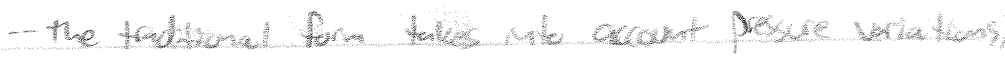

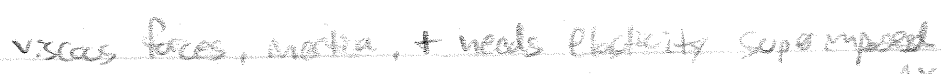

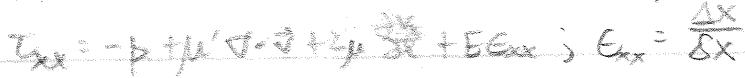

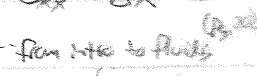

$$
\begin{aligned}
& \left.\tau_{x x}=-p+\mu \nabla \cdot \vec{v}+2 \mu \frac{\partial v_{x}}{\partial x}+E\left(\frac{\Delta x}{\partial x}\right) ; \Delta x=\frac{\partial y}{\partial x} d x\right) \delta t
\end{aligned}
$$

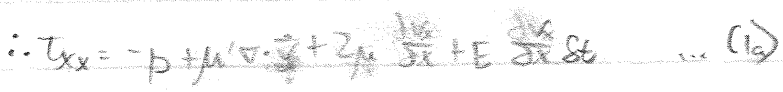

- Sinitlorly:

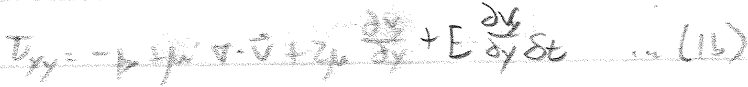

$$
\begin{aligned}
& \tau_{z z}=p+\mu v \cdot \vec{v}+q_{\mu} \frac{\partial v_{2}}{\partial z}+\epsilon \frac{\partial v_{3}}{\partial z} \delta t \quad . \quad(c)
\end{aligned}
$$

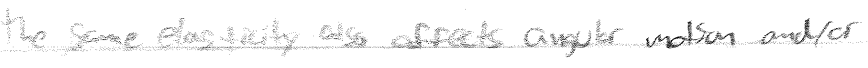
deformalion

since ga ascuesed bo be in smal the meremants, equations $1.75+1.76$ (p. 22 -alu flwits)

romain appoimately areuroule

-. If mare accuracy is requird, complumant onl

$E \frac{\partial V_{t}}{\partial t}$ ate trin in numedor to yreld 


$$
\begin{aligned}
& \text { (adu, Pivids) } \\
& \text { - fram equations 18.1, 1.727,1.7.8,1.79, we cbtam: } \\
& t_{x y}=t_{y x}=2 \mu d d_{x y} ; d_{x y}=1 / 2\left(\frac{\partial v_{y}}{\partial x}+\frac{\partial v_{y}}{\partial y}\right) \\
& t_{x y}=\mu\left(\frac{d v}{d x}+\frac{d v_{y}}{d y}\right) \ldots(1 d) \\
& \tau_{y z}=\mu\left(\frac{\partial v}{\partial z}+\frac{\partial v_{z}}{\partial y}\right) \\
& \tau_{z x}=\mu\left(\frac{\partial v_{z}}{\partial x}+\frac{\partial v_{x}}{\partial z}\right)
\end{aligned}
$$

$29 \operatorname{Jan} .2012$

refining the supermposition of Hooke's Law in to the normal stress Camponants of the Navior-Stokes Equations

- agarm vole thot the sher confoneds reman aprorimately

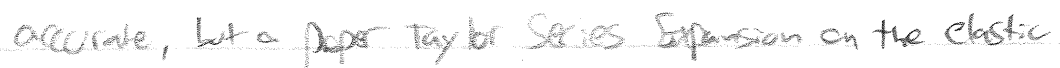
terms colld incrase the accuracy as previouly metiand, Hode's law can be written as folbos

$$
\begin{aligned}
& \sigma=E \varepsilon ; \sigma \equiv \text { normal stress. } \\
& \varepsilon \equiv \text { "stain }
\end{aligned}
$$

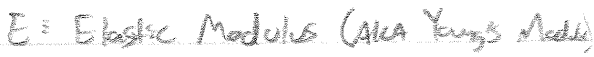

n the x-diretion:

$$
\sigma_{x x}-E \varepsilon_{x x}
$$

with diferent nothin:

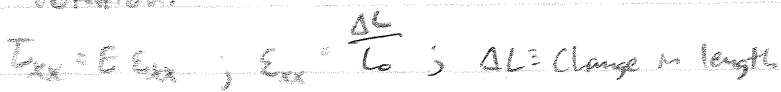

$$
\begin{aligned}
& \text { Lo Erighal dimatrow }
\end{aligned}
$$


for thear debrindion the type of deformetion de to normal stress), the k-component of the velocity gradint could be expessed by the equation: $u+\left(\frac{d u}{d x}\right) \delta x \quad(\beta, 20$ - Into to fluds $)$ while the stretahing of the volume in the x-dicediun Merensey by and amownt $\left[\left(\frac{d e}{d x}\right) \delta x\right] \delta t$ doring the chat the intrust bt, gring rise to the blat clange in valume of the (flud) puticle $\left.S \forall=\left(\frac{d x}{d x}\right) 8 \times 8 \times 52\right] 2 t$

- nde thet $\delta V_{0}=S x_{0} S y_{0} 8 z_{0}$ -. If the change on the $y$ - $+z$-drections is temprarity halted/agnared, the change in the $x$-dredion is considered.

$\therefore$ to sdate the Arange in the X-drection, combine

the previsus 2 equations.

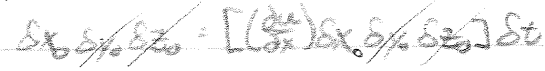
$\delta x_{0} \Rightarrow\left(\frac{d x}{3 x}\right) \delta x_{2} \delta t$ 
$\therefore$ the ratio of those terms is the stroin

$$
\varepsilon_{x x}=\frac{\Delta L}{L_{0}}=\frac{\left(\frac{\partial u}{\partial x}\right) \delta_{6} \delta_{t}}{\delta x_{0}} \cdot\left(\frac{\partial u}{\partial x}\right) \varepsilon_{t}=\frac{\partial u}{\partial x} \delta t
$$

$\therefore$ Prom Howke's law:

$$
\begin{aligned}
& E_{x x}=E E_{x x} \\
& E_{x y}=E\left(\frac{\partial v_{y}}{\partial x}\right) \delta_{t}
\end{aligned}
$$

to increase the accuracy of the grain term, a

Tayler Series Expmsion can be poformed onl reypet

to the velocity componont

by defintion, the it degree Taybr formula is:

$f(x)=\frac{f(a)}{a !}(x-a)^{0}+\frac{f^{\prime}(a)(x-a)^{\prime}}{1 !}+\frac{f^{\prime \prime}(a)(x-a)^{2}}{2 !}$

$+\frac{f^{(n)}(a)(x-a)^{3}}{3 !}+\cdots+\frac{f^{(n)}(a)}{n !}(x-a)^{n}$

$+\frac{f^{(n+1)}(z)}{(n+1) !}(x-a)^{(n+1)} ; R_{n}=\frac{f^{(n+1)}(z)}{(n+1) !}(x-a)^{(n+1)}$

Usually an erpansion to the $2^{\text {nd }}$ of $3^{\text {rd }}$ dricutive

is sefficiently acuide, but further expmension car

be conducted by emolally the same procss

$$
\begin{aligned}
& \epsilon_{x x^{2}}=\frac{\partial v_{x}}{\partial x} \delta t+\frac{\partial}{\partial x}\left[\frac{\partial v}{\partial x} \&\right](x)+\frac{\partial^{2}}{\partial x^{2}}\left[\frac{\partial v_{x}}{\partial x} \delta t\right] x^{2}+\cdots \\
& \text { assuming } a=0 \text { lasures the graph of } y \text { wa } x \\
& \text { poses Hough the arigh) }
\end{aligned}
$$




$$
\begin{aligned}
& \epsilon_{x x}=\frac{\partial v_{x}}{\partial x} \delta t+\frac{\partial^{2} V_{x}}{\partial x^{2}} \frac{\partial(x)}{d x} x+\frac{\partial^{3} V_{x}}{\partial x^{3}} \frac{\partial \delta(t)}{\partial x^{3}} x^{2}
\end{aligned}
$$

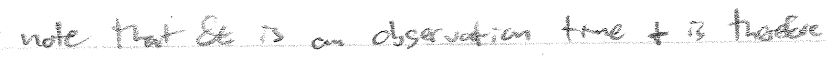

$$
\begin{aligned}
& \text { a cousthant, the derivalive of which is } 0 \\
& \therefore \varepsilon_{x x}=\frac{\partial v_{x}}{d x} \delta t
\end{aligned}
$$

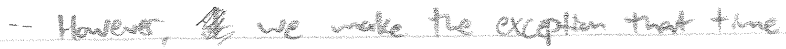

$$
\begin{aligned}
& \text { does not change wh such small increvinents of } \\
& \text { sace las an accuate appionivalian) } \\
& \text { - Thus, to increase the acuracy of the elastic term, one may } \\
& \text { prform the Taylo Caries Expansion on the Eladic Molulus } \\
& \text { to account for non-linearly behawn (fluid) materials } \\
& \text { (i.e. inou-Nedtaniona) } \\
& \text { foun Vooke's Law: } \\
& D_{x p}=E \varepsilon_{x x} \\
& E=\frac{Z_{x}}{\operatorname{mix}} \\
& \text { - note: the Elastic Madulus is nothing mare tham the } \\
& \text { stpe to a (Puid) material's Stress-Strain } \\
& \text { Corve } \\
& \text { * Hodke's Lan is nothing mere than a } \\
& \text { Wear apponathen to the (flud) } \\
& \text { material's stress-stain curve } \\
& y=m x+b ; y=\sigma \\
& x=\varepsilon \\
& m=E \\
& b=0
\end{aligned}
$$




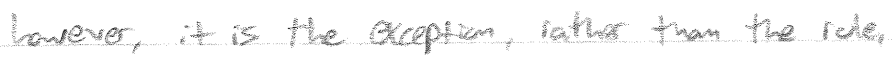
that a material's elastie region is strictly Mat N

a y-itacept an 0

$\therefore$ to increase the courcy of the Eladio Moddos, a Tayb series exparsion can be peffrencel ow its lenms

$$
\begin{aligned}
& E=\frac{t_{x x}}{\varepsilon_{x} x} ; t_{x x}=\frac{F}{A}=\frac{F}{\left.R_{x}\right)} \\
& E_{X}=\frac{D L}{L_{0}}=\frac{K_{-}-X}{K_{0}}
\end{aligned}
$$

$E=f(a)+f^{\prime}(a)(x-a)+\frac{f^{2}(a)}{2 !}(x-a)^{2}+\frac{f^{m}(a)}{3 !}(x-a)^{3}+a$ - Se sumplicity, we cscume a y-utropt do, although, hase woy by re-chanded based on $\alpha+Q$

$E=\frac{\left(\frac{1}{x}\right)\left(\frac{F}{y_{0}}\right)}{\frac{x_{x}-x}{x_{0}}}$

$E=\frac{\left(\frac{F}{X_{6}}\right) x_{0}}{\left(x_{0}-x\right) x}$

$E=(F)\left(x_{0}\right)$ $y \cdot\left(x-x^{2}\right)$

$E=\left(\frac{F_{0}}{y_{0}}\right)\left[\frac{1}{\left(x_{0}-x\right) x}\right]$ 


$$
\begin{aligned}
\therefore E= & \left(\frac{F x_{0}}{y_{0}}\right)\left[\frac{1}{\left(x_{0}-x\right)}\right]+\frac{d}{d x}\left\{\left(\frac{x_{0}}{y_{0}}\right)\left[\frac{1}{(x-x)}\right]\right\}(x-0) \\
& +\frac{\frac{d^{2}}{d x^{2}}\left\{\left(\frac{F x_{0}}{y_{0}}\right)\left[\frac{1}{\left.x_{0}-x\right) x}\right]\right\}(x-0)^{2}}{2 !} \\
& +\frac{\frac{d^{3}}{d x^{3}}\left\{\left(\frac{F x_{0}}{y_{0}}\right)\left[\frac{1}{\left(x_{0}-x\right)}\right]\right\}(x-0)^{3}+\cdots}{3 !}
\end{aligned}
$$$$
\text { - reducing the } 2^{\text {and }} \text { tem: }
$$$$
\frac{d}{d x}\left\{\left(\frac{F x_{0}}{y_{0}}\right)\left[\frac{1}{\left(f_{0}-x\right)}\right]\right\}=\left(\frac{F x_{0}}{y_{0}}\right) \frac{d}{d x}\left[\frac{1}{\left(x_{a}-x x\right.}\right]
$$$$
\left.=\left(\frac{F x_{0}}{y_{0}}\right) \frac{d}{d x}[\underbrace{\frac{1}{x_{0}-x}}_{T=\infty})\left(\frac{1}{x}\right)\right]
$$

Recpocal Rule:

$$
D_{x}\left[\frac{1}{f(x)}\right]=\frac{f(x)}{[f(x)]^{2}}
$$

$$
\text { Boduct Rule: } D_{x}[f(x) g(x)]=f(x)_{g}(x)+f(x) g(x)
$$$$
\therefore D_{x}[A(x) B(x)]=A^{\prime}(x) B(x)+A(x) B^{\prime}(x)
$$

$$
\left.=\frac{A^{\prime}(x)}{\left[A(x)^{2}\right.}\right\} B(x)+A(x)\left\{\frac{B^{\prime}(x)}{\left[B(x]^{2}\right.}\right\}
$$

$\operatorname{Trm} A: D_{x}\left[x_{0}-x\right]=D_{x}\left(x_{0}\right)-D_{x}(x): 0-1=-1$

$\operatorname{rem}$ B: $D_{x}[x]=1$ 


$$
\begin{aligned}
\therefore D_{x}[A(x) B(x)] & \left(\frac{1}{\left(x_{0}-x\right)^{2}}\right)\left(\frac{1}{x}\right)+\left(\frac{1}{x_{0}-x}\right)\left(\frac{1}{x^{2}}\right) \\
& =\left[\frac{1}{\left(x_{0}-x\right)^{2} x}\right]+\left[\frac{1}{\left(x_{0}-x\right) x^{2}}\right] \\
& =\frac{\frac{x_{0}-x}{\left(x_{0}-x\right)^{2} x^{2}}-\frac{x}{\left(x_{0}-x\right) x^{2}}}{x_{0}} \\
& =\frac{\left(x_{0}-x\right)^{2} x^{2}}{\left(x_{0}-x\right) x^{2}}
\end{aligned}
$$$$
\text { : Ne } 2^{\text {ud }} \text { hom rolues b: }
$$$$
\left(\frac{f x_{0}^{2}}{f_{0}}\right)\left[\left(x_{0}-x\right) x\right]^{-2}(x)
$$

$$
=\left[\frac{f}{x_{y_{0}}}\left[\frac{x_{0}}{\left(x_{0}-x\right)}\right]^{*}\left(\frac{1}{x}\right)^{(x)}\right.
$$$$
=\operatorname{Lix}_{x}\left(\frac{1}{\varepsilon_{x x}}\right)^{2}\left(\frac{1}{x}\right)(\infty)
$$$$
=\left(\frac{t_{x}}{\varepsilon_{x x}}\right)(0)(x)
$$

\section{- reducing the $3^{\text {d }}$ term:}

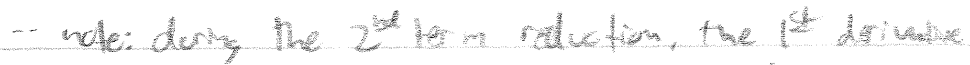

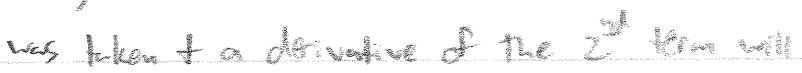
give the $3^{\text {di }}$ ter derivents 


$$
\begin{aligned}
& \text { - the } 3^{d} \tan \sin \frac{d^{2}}{d x^{2}}\left\{\left(\frac{f_{0}}{y_{0}}\right)\left[\frac{1}{\left(x_{0}-x\right)}\right]\right\} x^{2} \\
& \text { by proluct rue: }
\end{aligned}
$$

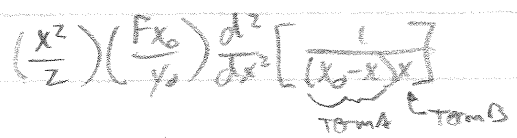

$$
\begin{aligned}
& \text { - as seen in the der vation of the } 2^{\text {nd tom: }} \\
& D_{x}(A)=-1 \\
& D_{x}(B)=1 \\
& D_{x}[A(x) B(x)]=\frac{x_{0}}{\left[\left(x_{0}-x\right) x\right]^{2}}=\left(x_{0}\right) \frac{1}{\left[\left(x_{0}-x\right) x\right]^{2}} \\
& =\left(R_{0}\right)\left[\frac{1}{\left(1+x^{2}\right)^{2} x^{2}}\right] \\
& \left.\ln \frac{1}{(x-y)\left(x_{0}-x\right) x^{2}}\right]
\end{aligned}
$$

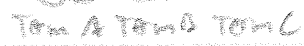

$$
\begin{aligned}
& \therefore \text { By lalky the derivative of he derivatue of the }
\end{aligned}
$$

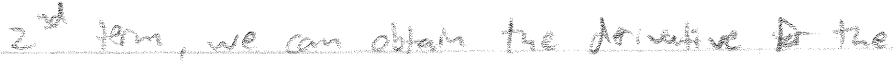

$$
\begin{aligned}
& 3^{n+16 m} \\
& \text {-Dy the chan role: } \\
& \text { D. }[A(x) B(x) C(x)]=A(x) B(x)(C x)+A(x) B^{\prime}(x)(x)+A(x) B C(x) \\
& A^{\prime}(x)=-1 \\
& 3(x)=-1 \\
& C^{\prime}(x)=2 x
\end{aligned}
$$


Dy Recprocal Rule:

$$
\begin{aligned}
D_{x}[A(x) B(x) C(x)]=\left\{\frac{A^{\prime}(x)}{[A(x)]^{2}}\right\} B(x)(x) & +A(x)\left\{\frac{B^{\prime}(x)}{\left(x_{x}\right]^{2}}\right\} C(x) \\
& +A(x) B(x)\left\{\frac{C(x)}{[C(x)]^{2}}\right\}
\end{aligned}
$$$$
\therefore D_{x}[A(x) B(x)(x)]=-\left(\frac{1}{\left(u_{0}-x\right)^{2}}\right)\left(x_{0}-x\right) x^{2}+
$$

- Rese dervalives hove bou balem ncorretly t must be

relowe starting fem the Taybr faves Expansion

- The $1^{\text {L }}$ termin the Expansion:

$$
\left(\frac{B x_{0}}{y_{4}}\right)\left[\frac{1}{\left(x_{0}-x\right) x}\right]
$$

$$
\begin{aligned}
& =\left(\frac{f}{y^{x}}\right)\left[\frac{x}{x^{x}}\right] \\
& E_{0}=\frac{t_{0}}{\varepsilon_{x x}} \quad \ldots \text { Term }
\end{aligned}
$$

$$
\begin{aligned}
& \text { The } 2^{\text {nd }} \text { tam in the Expinsan: } \\
& \qquad E=\frac{d}{d x}\left\{\left(\frac{F x_{0}}{y_{0}}\right)\left[\frac{1}{\left(x_{a}-x\right)}\right]\right\} x
\end{aligned}
$$

- by praduat role:

$$
E=\left(\frac{E x}{1}\right)\left(x_{0}\right) \frac{d}{d x}\left[\frac{1}{6-x)}\right]
$$

$$
=\left(\frac{F x}{y_{0}}\right)\left(x_{0}\right) \frac{d}{d x}[\underbrace{\left.\left(x_{-}-x\right)^{-1} x^{-1}\right]}_{\text {Term } A} \tau_{T \text { Tem }}^{T}
$$

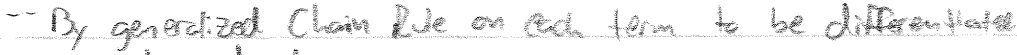
$\frac{d y}{d x}=\frac{d u}{d u} d y=u^{2}+a x$ the subtitute 
-appling the clan rule in convinkter wh the product role:

$$
\begin{aligned}
& \therefore d x\left[\left(x_{0}-x\right)^{-1} x^{-1}\right]=d\left[L\left(x_{0}-x\right)^{-1}\right] x^{-1}+\left(x_{0}-x\right)^{-1} \frac{d}{d x}\left(x^{-1}\right) \\
& D_{x}[f(x) g(x)]=f(x) g(x)+f(x) g(x) \\
& \text { - Pef tem A: let } u=x_{0}-x \text {. lef } y^{*} u^{-1}
\end{aligned}
$$$$
\frac{d u}{d x}=1 \quad \frac{d y}{d x}=-1 a^{-2}
$$

Dy dam Rue: $\frac{d y}{d x}+\frac{d y}{d a} d x$

$$
\begin{aligned}
\left.d\left[f_{0}-x\right)^{-1}\right] & =-\left(x_{0}-x\right)^{-2}(-1) \\
& =\left(x_{0}-x\right)^{-2}
\end{aligned}
$$$$
\therefore d\left[\left(x_{0}-x\right)^{-1} x\right]:(x-x)^{-2} x^{-1}-\left(x_{0}-x\right)^{-3} x^{-2}
$$$$
=\frac{1}{\left(x_{0}-x\right)^{2} x}-\frac{1}{\left(x_{0}-x\right)^{2}}
$$

$$
\frac{x-\left(x_{0}-x\right)}{\left(x_{0}-x\right)^{2} x^{2}}
$$

$=2 x-x_{0}$

$$
\left[\left(x_{0}-x\right) \times\right]^{2}
$$

$$
\begin{aligned}
& \frac{2}{(x-x)^{2} x}-\frac{x_{0}}{\left(x_{0}+\right)^{2} x^{2}} \\
& \text { Thus, the } 2^{\text {wh }} \text { terwa becures: } \\
& E=\left(\frac{E}{y}\right)\left[\frac{2 x_{0} x}{(x) x}-\frac{x^{2} x}{(x+x)^{2}}\right]
\end{aligned}
$$


Development of the West Virginia University Small Microgravity Research Facility (WVU SMiRF)
Copyright $^{\oplus} 2014$

Kyle G. Phillips

$$
\begin{aligned}
& E=\left(\frac{F}{y_{0} x}\right)\left[(2 x)\left(\frac{x_{0}}{x_{0}-x}\right)-\left(\frac{f_{0}}{x_{0}-x}\right)^{2}\right] \\
& E_{2}\left(\frac{T_{x}}{\varepsilon_{x}}\right)\left(2 x-\frac{1}{\varepsilon_{x}}\right) \quad \text { Term }{ }_{2}
\end{aligned}
$$

- The $3^{\text {nd }}$ term an the expansion:

$$
E=\frac{\frac{d^{2}}{d x^{2}}\left\{\left(\frac{P x_{0}}{f_{0}}\right)\left[\frac{1}{2 !\left(e_{0} x\right)}\right] x^{2}\right.}{2 !}
$$

- by pandect rule:

$$
E=\left(\frac{1}{2}\right)\left(\frac{F}{\%}\right) x_{2} x^{2} \frac{d}{d x}\left[\frac{1}{2 x}+x\right]
$$

boston the $2^{\text {nd }}$ lativilive, one can the the devalue of the result of the fit derivative

$$
\begin{aligned}
\therefore \frac{d^{2}}{d x^{2}}\left[\frac{1}{\left(x_{0}-x\right) x}\right] & =\frac{d}{d x}\left[\frac{2 x-x_{0}}{\left[\left(x_{0} x\right)\right]^{2}}\right] \\
& =\frac{d}{d x}\left[\frac{2 x}{\left(x_{0}-x\right)^{2} x^{2}}-\frac{x_{0}}{\left(x_{0}-x\right)^{2} x^{2}}\right] \\
& =\frac{d}{d x}\left[\frac{2}{\left(x_{0}-x\right)^{2} x}=\frac{x_{0}}{\left(x_{0}-x\right)^{2} x^{2}}\right] \\
& =(2) \frac{d}{d x}\left[\frac{1}{\left(x_{0}-x\right)^{2} x}\right]-\left(x_{0}\right) \frac{d}{d x}\left[\frac{1}{\left(x_{0}-x\right)^{2} x^{2}}\right]
\end{aligned}
$$

- for mem d:

(a) $d x\left[(x-x)^{2} x^{-1}\right]$

608 
Development of the West Virginia University Small Microgravity Research Facility (WVU SMiRF)
Copyright $^{\circledR} 2014$

Kyle G. Phillips

by product rue on Term $I$ :

$$
\text { Dr[Dug(x)] }=\frac{d}{d x}\left[\left(x_{0}-x\right)^{-2}\right] x^{-1}+\left(x_{0}-x\right)^{-2} d x\left(x^{-1}\right)
$$

- fer Term ta:

$$
\begin{aligned}
- \text { let } u & =x_{0}-x \quad y=u^{-2} \\
\frac{d u}{d x} & =-1 \quad \frac{d y}{d u}=-2 u^{-3}
\end{aligned}
$$

by chain we: $\frac{d y}{d x}=\frac{d y}{d x} \frac{d y}{2 x}$

$$
\begin{aligned}
& =-2(6-x)^{-3}(-1) \\
& =2\left(x_{0}-x\right)^{-3}
\end{aligned}
$$

$\therefore$ Term I becomes:

$$
\text { (2) }\left\{\left[\frac{2}{\left(x_{0}-x\right)^{3} x}\right]-\left[\frac{1}{\left(x_{0}-x\right)^{2} x^{2}}\right]\right\}
$$

$$
\begin{aligned}
& =\frac{4 x-2\left(x_{0}-x\right)}{\left(x_{0}-x\right)^{3} x^{2}} \\
& =\frac{2 x-2 x_{0}}{\left(x_{0}-x\right)^{3} x^{2}}=\frac{2 x-2 x_{0}}{\left[\left(x_{0}-x\right) x\right]^{2}\left(x_{0}-x\right)} \\
& =\frac{2 x}{\left(x_{0}-x\right)^{3} x^{2}}-\frac{2 x_{0}}{\left(x_{0}-x\right)^{3} x^{2}}
\end{aligned}
$$

Thor, the $3^{\text {at }}$ term becomes:

$$
\begin{aligned}
& E=\left(\frac{1}{2}\right)\left(\frac{E}{x_{0}}\right) x_{0} x^{2}\left[\frac{2 x}{\left(x_{0}-x\right)^{3} x^{2}}-\frac{2 x_{0}}{\left(x_{0}-x\right)^{3} x^{2}}\right] \\
& E=\left(\frac{1}{2}\right)\left(\frac{E}{y_{0}}\right)\left[\frac{x_{0}(2 x)}{\left.x_{0}-x\right)^{3}}-\frac{x_{0}^{2}(2)}{\left(x_{0}-x\right)^{3}}\right]
\end{aligned}
$$

609 


$$
\begin{aligned}
& E=\left(\frac{f}{y_{0}}\right)\left\{\left(\frac{x_{0}}{x_{0}-x}\right)\left[\frac{x}{\left(x_{0}-x\right)^{2}}\right]-\left(\frac{x_{0}}{x_{0}-x}\right)^{2}\left(\frac{1}{x_{0}-x}\right)\right\} \\
& E_{3}\left(\varepsilon_{x x}\right)\left[\left(x_{0}-x\right)^{-1}-\varepsilon_{x y}^{-1}\right]\left(x_{0}-x\right)^{-1} x^{-1} \ldots \text { Term } \# \\
& \text { - the } 4^{\text {th }} \text { tem in the Expansion: } \\
& E=\frac{\frac{d^{3}}{d x^{3}}\left\{\left(\frac{E_{0}}{y_{0}}\right)\left[\frac{1}{\sqrt{x} \times x}\right]\right.}{3 !} \\
& \text {-by product rele: } \\
& E=\left(\frac{1}{b}\right)\left(\frac{F}{y_{0}}\right) x_{0} x^{3} \frac{d^{3}}{d x^{3}}\left[\frac{1}{\left(x_{0}-x\right) x}\right] \\
& \text {-. to oblah the } 3^{\text {of }} \text { drivative, one can take the } \\
& \text { derivative of the result of the second dervative } \\
& \frac{d^{3}}{d x^{3}}\left[\frac{1}{\left(x_{0}-x\right)}\right] \div \frac{d}{d x}\left[\frac{2\left(x-x_{0}\right)}{\left(x_{0}-x\right)^{3} x^{3}}\right] \\
& =(2) \frac{d}{d x}\left[\left(x_{0}-x\right)^{-3} x^{-1}\right]-\left(x_{0}\right) \frac{d}{d x}[\underbrace{}_{0}\left[(x-x)^{-3} x^{-2}\right]) \\
& \text { - for remat } \\
& \text { lot } a=x^{-}-x \quad y=u^{-3} \\
& \frac{d x}{d x}=-1 \quad y=-3 x^{-4}
\end{aligned}
$$

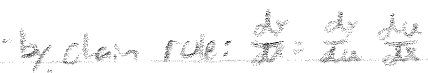

$$
\begin{aligned}
& \frac{1}{d x}\left[\left(x_{0}-x\right)^{-3} x^{-1}\right]=3\left(x_{0}-x\right)^{-4} x^{-1}-\left(x_{0}-x\right)^{-3} x^{-2}
\end{aligned}
$$




$$
\begin{aligned}
& \frac{d}{d x}\left[\left(x_{0}-x\right)^{-3} x^{-1}\right]=\frac{3}{(x-x)^{4} x^{2}}-\frac{1}{\left(x_{0}-x\right)^{3} x^{2}} \\
& \text { - term E becones: } \\
& \frac{d}{d x}\left[\left(x_{0}-x\right)^{-3} x^{-2}\right]=3(x-x)^{-4} x^{-1}-2\left(x_{0}-x\right)^{-3} x^{-3} \\
& =\frac{3}{\left(x_{0}-x\right)^{4} x}-\frac{2}{\left(x_{0}-x\right)^{3} x^{3}} \\
& \therefore \frac{d^{3}}{d x^{3}}\left[\frac{1}{\left(x_{0}-x\right) x}\right]=\frac{6}{\left(x_{0}-x\right)^{4} x}-\frac{2}{\left(x_{0}-x\right)^{3} x^{2}}-\frac{3 x_{0}}{\left(x_{0}-1\right)^{4} x}+\frac{2 x_{0}}{\left(x_{0}+x^{3} x^{3}\right.} \\
& =\frac{b-3 x_{0}}{\left(x_{0}-x\right)^{4} x}+\frac{46-x}{\left(x_{0}-x\right)^{3} x^{2}} \\
& =\left(6-3 x_{0}\right) x+2(6-x)^{2} \\
& (6-x)^{4} x^{2} \\
& =6 x-2 x_{0} x+2(x-x)(x-x) \\
& \left(x_{0}-x\right)^{4} x^{2} \\
& =6 x-3 x+2\left(x^{2}-2 x+x^{2}\right) \\
& \left(x_{0}-x\right)^{4} x^{2} \\
& =6 x-3 x_{0} x+2 x^{2}-4 x_{0} x+2 x^{2} \\
& (x-x)^{4} x^{2} \\
& =2 x^{2}+6 x-7 x_{0}+2 x_{a}^{2} \\
& \left(x_{0}+x\right)^{4} x^{2}
\end{aligned}
$$




$$
\begin{aligned}
& \frac{d^{3}}{d x^{3}}\left[\frac{1}{\left(x_{0}-x\right) x}\right]=\frac{2}{\left(x_{0}-x\right)^{4}}+\frac{6}{\left(x_{0}-x\right)^{4} x}-\frac{7 x}{\left(x_{0}-x\right)^{4} x}+\frac{2 x^{2}}{\left(x_{0}-x\right)^{4} x^{2}} \\
& \text { - Thus the } 4^{\text {th }} \text { taren becomes: } \\
& E=\left(\frac{1}{6}\right)\left(\frac{F}{10}\right) x_{0}\left[\frac{2}{\left(x_{0}-x\right)^{4}}+\frac{6}{\left(x_{0}-x\right)^{4} x}-\frac{7 x}{\left(x_{0}-x\right)^{2} x}+\frac{x^{2}}{\left(x_{0}-x\right)^{2} x^{2}}\right] \\
& E=\left(\frac{1}{6}\right)\left(\frac{E}{y_{0} x}\right)\left[\frac{2 x^{4} x_{a}}{\left(x_{0}-x\right)^{4}}+\frac{6 x^{4} x_{0}}{\left(x_{0}-x\right)^{4} x}-\frac{2 x^{4} x^{2}}{\left(x_{0}-x\right)^{4} x}+\frac{2 x^{4} x^{3}}{\left(x_{0}-x^{4} x^{2}\right.}\right. \\
& \left.E=\left(\frac{1}{6}\right) \frac{F}{y_{a} x}\right)\left\{\left(\frac{x_{a}}{x_{0}-x}\right)\left[\frac{2 x^{4}}{\left(x_{0}-x\right)^{3}}\right]+\left(\frac{x_{0}}{6-x}\left[\frac{6 x^{3}}{\left(x_{0}-x\right)^{3}}\right]\right.\right. \\
& -\left(\frac{x}{0}-x\right)^{2}\left[\frac{7 x^{3}}{\left(x_{0}-x^{2}\right.}\right]+\left(\frac{5}{x_{0}-x}\right)\left[\frac{2 x^{2}}{x_{0}^{2}-2}\right]
\end{aligned}
$$

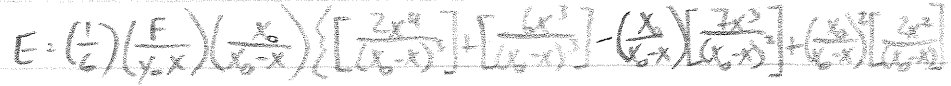

$$
\begin{aligned}
& \left.\left.E=\left(\frac{1}{3}\right)\left(\frac{x_{x}}{\varepsilon_{x}}\right)\left(\frac{x^{2}}{x_{0}-x}\right)\left\{\frac{x^{2}}{\left(x_{0}-x\right)^{2}}\right]+\left[\frac{3 x}{\left(x_{0}-x\right)^{2}}\right]-\left[\frac{(7) x}{\left(x_{0}-x\right.}\right] \frac{1}{s_{x}}\right)+\left(\frac{1}{E_{x}}\right)\right\} \\
& E_{4}=\left(\frac{1}{3}\right)\left(\frac{D_{x} x}{\varepsilon_{x x}}\right)\left(\frac{x^{2}}{x_{0}-x}\right)\left(\left(\frac{1}{\varepsilon_{x}}\right)^{2}-\left[\frac{\left(\frac{7}{2}\right) x}{\left(x_{0}-x\right)}\right]\left(\frac{1}{\varepsilon_{x}}\right)+\left[\frac{(x+3) x}{\left(x_{0}-x\right)^{2}}\right)\right\}-18 \text { - }
\end{aligned}
$$

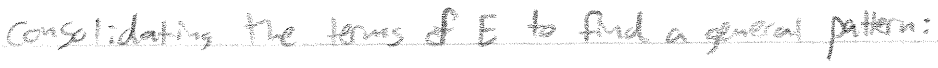

$$
\begin{aligned}
& (\text { constant }) \quad E_{1}=\left(\frac{\tau_{x X}}{\varepsilon_{\text {xX }}}\right) \\
& \text { (war) } \left.E_{z}=\left(\frac{b_{x y}}{\varepsilon_{x x}}\right) / 2 x-\frac{1}{\varepsilon_{x x}}\right)
\end{aligned}
$$

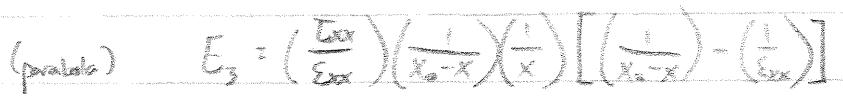

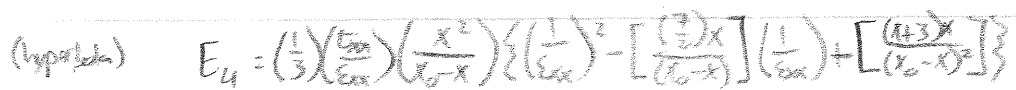


-. at least 2 mare tems would be wocessary to discower. the patien

- instead, for better accumacy durius deswetion, to confrim prenous deriverives $t$ to accurately derive futue equallows. attomphing ba derive derientive equedors sequentrally.

after revewing the fundamentals of Taybor Series, it is

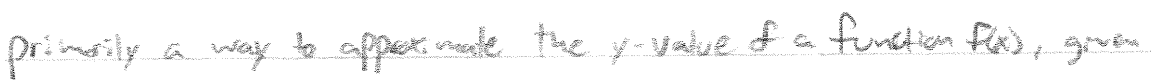
a point $x$

A ther wads, guen $x$. Whet is $y$ ?

-.. The taylor seres is a way to Let apprax mate an

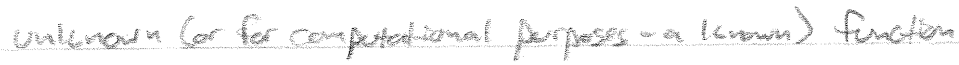

-in this case, we wish to apporimimate the clastic

componat of the stresses experienced by the (Aluid) puticle $\therefore$ we do nat vish to apockimate the Eloctic Modulus but rather the Shan, given the Elatic Molulos a prot

- from Mocke's Lown:

$$
\begin{aligned}
& \tau_{x x}=E \varepsilon_{x x} ; \tau_{x y} \equiv \text { Normal stress } * \frac{E}{A} \\
& \text { E E Elastic Moduls } \\
& \varepsilon_{x x} \equiv \text { Normal Stain }=\frac{\Delta L}{L}
\end{aligned}
$$

as previasly unded the Elagtic Modulus is just an equalim for a straght the - if the mothat is now labely elastic, the Elaste Madulus will hove its own equation (pertaxps croted by its own Taylor Serres Exponsion) 


$$
\begin{aligned}
& \text { also, as pervously nded, } \\
& \varepsilon_{x x}=\frac{\Delta x}{x_{0}}, \Delta x=\left(\frac{\partial v_{x}}{\partial x} x_{0}\right) \delta t \\
& \text { by dinition } \Delta x=x-x_{0} \\
& x=\Delta x+x_{0} \\
& x=\left(\frac{d}{\partial x}+0\right) d x
\end{aligned}
$$

that substitution may be convenient post-derivatian of the staita

$\Rightarrow$-Ascumption 4: Ascuming the Elatic Modulus (whether constant, lwear, of won-liman) is known + given for the (Nwid) meterial (via the constant or an equation for the Modolus)

$$
\begin{aligned}
& \therefore \text { we aim to expand } \varepsilon_{x}=\frac{x-x_{0}}{x_{0}} ; x_{0} \equiv \text { constunt } \\
& x+\text { furchen of surmandins }
\end{aligned}
$$

3 general concepts are needed for the Taybr Sories Expusion -Taybe's Formeta:

$$
\begin{aligned}
& f(x)=\frac{f(a)(x-a)}{a !}+\frac{f(a)(x-a)}{1 !}+\frac{f^{\prime \prime}(a)(x-a)}{2 !} \\
& +\frac{f(a)(x-a)^{3}}{3 !}+\frac{f^{(a)}(a)(x a)^{4}}{4 !}+\frac{f^{(5)}(a)(x-a)}{5 !} \\
& +\frac{f^{(b)}(0)(x-a)^{6}}{6 !}+\frac{f^{(n)}(a)(x-a)^{n}}{n !}+\cdots \\
& \text { Poduct Rule: } D_{x}[f(x) g(x) h(x) m(x) \cdots]= \\
& f^{\prime}(x) g(x) h(x) \operatorname{mon}(x)+f(x) g^{7}(x) h(x) \operatorname{mon}(x) \\
& +f(x) g(x) h^{\prime}(x) m(x)+f(x) g(x) h(x) m^{\prime 2}(x)+\cdots
\end{aligned}
$$




$$
\begin{aligned}
& \text { - Chain Rule: } \frac{d y}{d x}=\left(\frac{d y}{d w}\right)\left(\frac{d x}{d x}\right) \\
& \text { or } \left.h^{\prime}(x)=D_{x}[f(g(x))]=f^{\prime}(g(x)) g^{\prime} x\right)
\end{aligned}
$$

- Using Taylor's Polynomial to apprarinate the Stan

d) respect to stres based on the scrounding:

$$
\begin{aligned}
\varepsilon_{x x}= & \frac{x-x_{0}}{x}+\frac{d}{d x}\left\{\frac{x-x_{0}}{x_{0}}\right\}(x-a)+\frac{d^{2}}{d x^{2}}\left\{\frac{x-x_{0}}{x_{0}}\right\} \frac{(x-a)^{2}}{2} \cdots \\
& +\frac{d^{3}}{d x^{3}}\left\{\frac{x-x_{0}}{x_{0}}\right\} \frac{(x-a)^{3}}{3 !}+\frac{d 4}{d x^{4}}\left\{\frac{x+x_{0}}{x_{0}}\right\} \frac{(x-a)^{4}}{4} \\
& +\frac{d^{5}}{d x^{5}}\left\{\frac{x-x_{0}}{x_{0}}\right\} \frac{(x-a)^{5}}{5}+\frac{d^{6}}{d x^{6}}\left\{\frac{x_{0}-x_{0}}{x_{0}}\right\} \frac{(x-a)^{6}}{6 !}
\end{aligned}
$$

- taking Sequential dervatives to incoporate it bo the

Tayb Seres Expansion:

$$
\begin{aligned}
& \text {-Thes, the } 1^{2} \tan B \text { : } \\
& \varepsilon_{x x_{1}}=\left(x_{0}\right)\left(x-x_{0}\right)=\frac{x-x_{0}}{x_{0}}=\varepsilon_{x_{1}} \ldots \operatorname{Tam}^{4}
\end{aligned}
$$

$$
\text { Thes the } z^{\text {the tem is: }}
$$

$$
\sum_{x y_{2}}=(x-a) \frac{d}{d x}\left\{\frac{x-x_{0}}{x}\right\} ; x_{0}=\text { consthent }
$$

by prolud role:

$$
\begin{aligned}
\sum_{\operatorname{tin}_{2}} & \left(\frac{x-a}{x_{0}}\right) \frac{d}{d x}\left\{x-x_{0}\right\} \\
& \left(\frac{x-a}{x_{0}}\right)\left\lfloor\frac{d}{d x}(x)-\frac{d^{1}}{d x}\left(x_{0}\right)\right]
\end{aligned}
$$




$$
\begin{aligned}
& \therefore \varepsilon_{x x_{2}}=\left(\frac{1}{x_{0}}\right)(x-a) \\
& \text { Thus with the idgondent vrialle wow a } \\
& \text { Constant, all further tems will be zero } \\
& \therefore \varepsilon_{x x}=\varepsilon_{x x}+\left(\frac{1}{x}\right)(x-a) \\
& \text {-if the pust of mlegt is the corrent } \\
& \text { epanded fow } a: x+\varepsilon_{x y}=\varepsilon_{x x} \\
& \text { - If the point of miterest is the diginal } \\
& \text { position } a=x_{0}+\varepsilon_{x x}=2 \varepsilon_{x x} \text { ! } \\
& \text { - that dasn't make seuse } \\
& \text { : We have proved that the reaction of the stam, } \\
& \text { thes the elasticity of the (Pluid) material, is due to } \\
& \text { the Elastic Modulus, the measurament of which would } \\
& \text { - Thus, we con morpolete the Elatic Madulus into } \\
& \text { the equation for } \tau_{x x}+\text { must apporinute it using a } \\
& \text { Tayler Serves Expansion } \\
& \text { agat, by Holve's law: } \\
& \tau_{x y}=E \xi_{x x}
\end{aligned}
$$

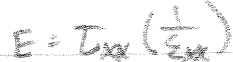

$$
\begin{aligned}
& E=\tau_{x x}\left(\frac{x}{x-x_{0}}\right) \\
& E=(\operatorname{Ex} x)(x-6)^{-1}
\end{aligned}
$$$$
\text { be very difficut for a flud }
$$ 


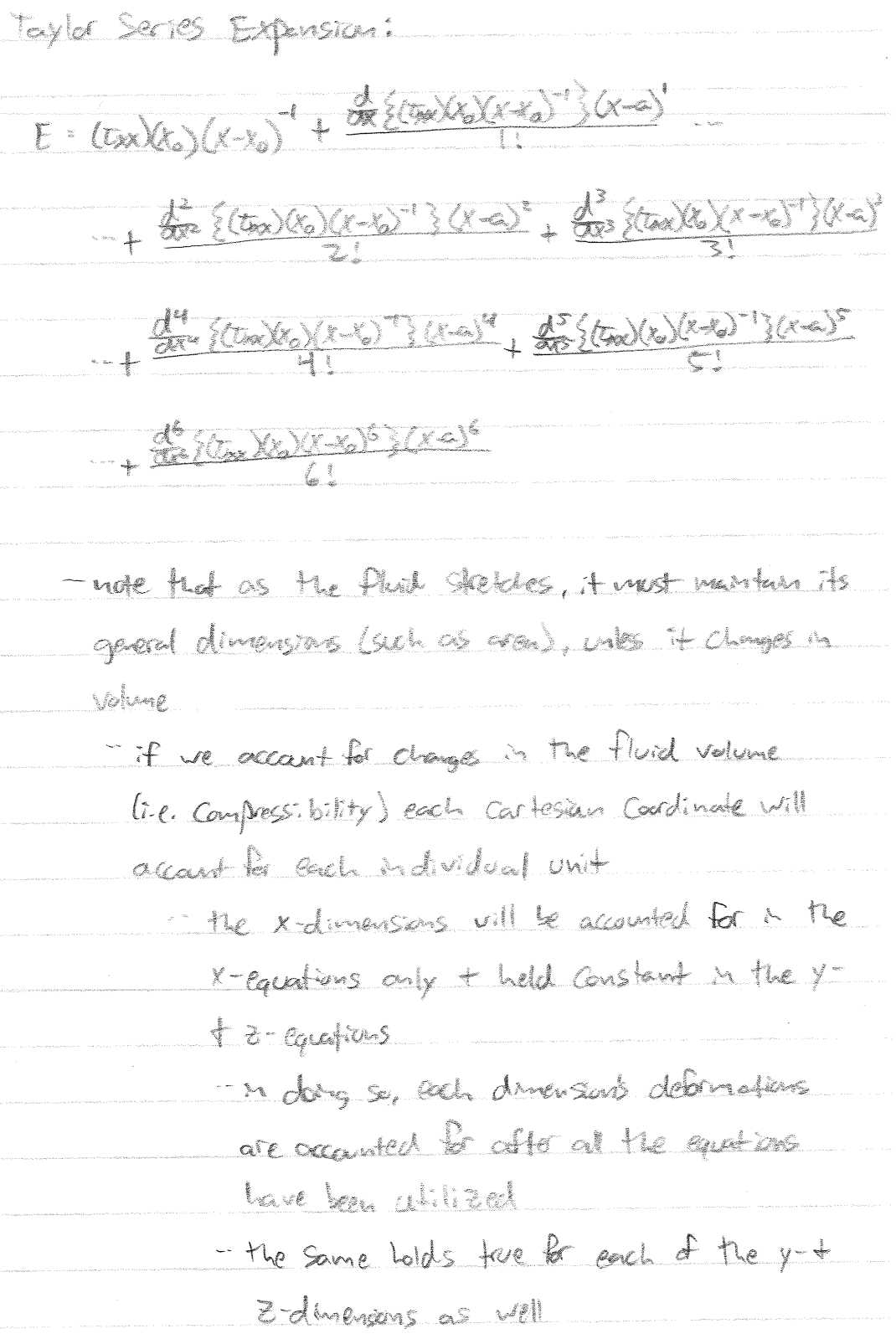




$$
\begin{aligned}
& \text { - thus, presure, density, shear streges, I } \\
& \text { thus viscosity change based upen the } \\
& \text { cartesian dinewion in quetion. } \\
& \text { the Naver-stoles equations oprate } \\
& \text { on the flutd this why + thendere } \\
& \text { fotue odditims b it mut also ac } \\
& \text { in this manner bo conpatible } \\
& \text { - Gingen thase mgights, + thee about the prewouly dor ved } \\
& \text { Shem cheses t Elastic Molulus, the flowing is a derivatom } \\
& \text { to Le uncluded bn the curbut Nawier-Stoles equations }
\end{aligned}
$$

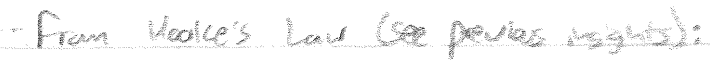

$$
\begin{aligned}
& t_{x x}=E \varepsilon_{x x} \\
& E=\frac{E_{x x}}{\varepsilon_{x k}} ; E_{x x}=\frac{F}{A_{x x}}=\frac{F}{X y_{0}}=\left(\frac{F}{x_{0}}\right)\left(\frac{E}{x}\right) \cdot\left(\frac{E}{y_{0}}\right) x^{-1} \\
& \varepsilon_{x x}=\frac{A x}{b}=\frac{x-x_{0}}{x_{0}}=\left(\frac{1}{x_{0}}\right)\left(x-x_{0}\right)=\left(x+x_{0}\right)_{0}^{-1} \\
& \therefore E=Z_{x x} \varepsilon_{x x}^{-1} \\
& E=\left[\left(\frac{E}{y_{0}}\right) x^{-1}\right]\left[\left(x_{0}\right)\left(x-x_{0}\right)^{-1}\right] \\
& E=\left(\frac{F}{y_{0}}\right)\left(x_{0}\right)\left(x-x_{0}\right)^{-1} x^{-1} ; x \text { is the variable of } \\
& \text { in whest } \\
& \text { - this ascomes an "Whtan twores". }
\end{aligned}
$$

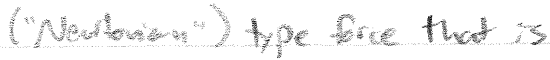

$$
\begin{aligned}
& \text { coudont with the dimpurand cheme }
\end{aligned}
$$


- therefore the Taylor Senes Expmansion on the Elastic Madulus:

$$
\begin{aligned}
& E=\left(\frac{F}{y_{0}}\right) x_{a}\left(x-x_{0}\right)^{-1} x^{-1}+\frac{\frac{d}{a x}\left\{\left(\frac{F}{y_{0}}\right) x_{0}\left[\left(x-x_{0}\right)^{-1} x^{-1}\right]\right\}}{I !}(x-a)^{1} \cdots \\
& +\frac{d^{2}\left\{( \frac { E } { x } ) x _ { 0 } \left[\left(x-x_{0}\right)^{-1}+x^{3}\right.\right.}{2 !}(x-a)^{2}+\frac{d^{3}\left\{\left(\frac{E}{x^{2}}\right) x_{0}\left[(x-x)^{-4} x^{+1}\right]\right.}{3 !}(x-a)^{3}
\end{aligned}
$$

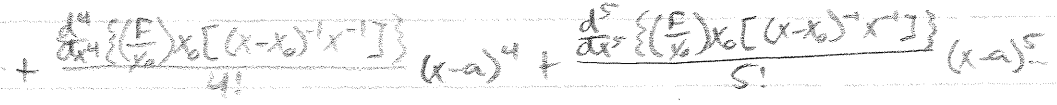

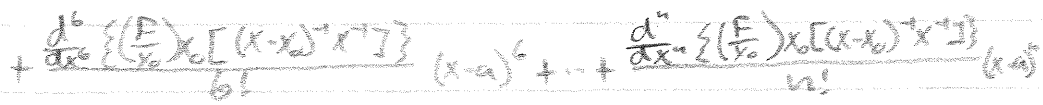

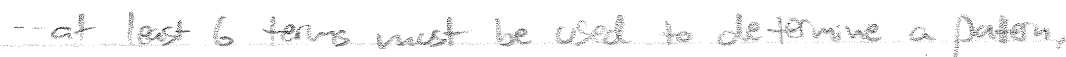
suce the it term is a condant, the $2^{\text {th }}$ is liner, the $3^{\text {int }}$

is prablic, the $4^{\text {th }}$ is tuprbolic, the next 3 may be

compored against the $4^{\text {th }}$ term to delenwhe a pattern. shce the first 3 tems are ind similor to poly noatals

- by the poduct roke, all constents multiplied by terms w/ variables may be moved atside of the derivative, suce it would have bo be summed as zera to the drivatue ary hay

- thus, the Taplor Seres Expansion becoms: 
Development of the West Virginia University Small Microgravity Research Facility (WVU SMiRF)
Copyright $^{\oplus} 2014$

Kyle G. Phillips

$$
\begin{aligned}
& E=\left(\frac{F}{x_{0}}\right) x_{0}\left(x-x_{0}\right)^{-1} x^{-1}+\left(\frac{F}{y_{0}}\right) x_{0}[(x-a)] \frac{d}{d x}\left\{\left(x-x_{0}\right)^{-1} x^{-3}\right\} \cdots \\
& +\left(\frac{E}{y_{0}}\right) x_{0}\left[\frac{(x-a)^{2}}{2}\right] \frac{d^{2}}{d x^{2}}\left\{\left(x-x_{0}\right)^{-1} x^{-1}\right\}+\left(\frac{E}{y_{0}}\right) x_{0}\left[\frac{(x-a)^{3}}{3 !}\right] \frac{d^{3}}{d x^{3}}\left\{\left(x-x_{0}\right)^{-1} x^{-1}\right\} \\
& +\left(\frac{E}{y_{0}}\right) x_{0}\left[\frac{(x a)^{4}}{4 !}\right] \frac{d^{4}}{d x^{4}}\left\{\left(x-\infty x^{-1}\right\}+\left(\frac{E}{y_{0}}\right) x_{0}\left[\frac{(x-5)}{5}\right] \frac{d^{5}}{d x^{5}}(x-x)^{4} x^{-1}\right\}
\end{aligned}
$$

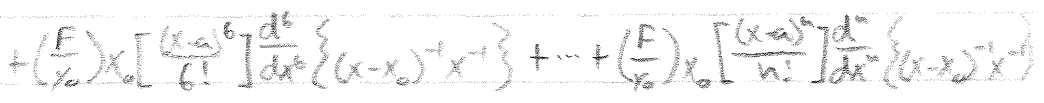

$$
\begin{aligned}
& E_{0}=\left(\frac{F}{x_{0}}\right) x_{0}\left[\frac{(x-a)}{0}\right] \frac{d^{0}}{d x^{0}}\left\{\left(x-x_{0}\right)^{-1} x^{-1}\right\} \\
& E,=\left(\frac{E}{y}\right) \cdot\left[\frac{(x-)^{2}}{1 !}\right] \frac{d}{d x}\left\{(x-x)^{-1} x^{-1}\right\} \\
& E_{2}=\left(\frac{F}{y_{0}}\right) \times\left[\frac{(x-a)^{2}}{2 !}\right] \frac{d^{2}}{d x^{2}}\left\{\left(x-x_{0}\right)^{-1} x^{-1}\right\} \\
& E_{3}=\left(\frac{F}{x_{0}}\right) x_{0}\left[\frac{(x-a)^{3}}{3 !}\right] \frac{d^{3}}{d x^{3}}\left\{\left(x-x_{0}\right)^{-1} x^{-1}\right\} \\
& \left.E_{u_{1}}=\left(\frac{f}{y_{0}}\right) x_{0}\left[\frac{(x-a)^{4}}{4 !}\right] \frac{d^{4}}{d x^{4}}\left(x-x_{0}\right)^{-1} x^{-1}\right\}
\end{aligned}
$$

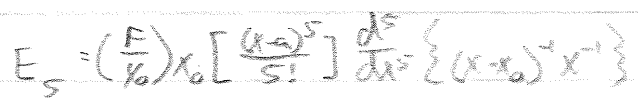

$$
\begin{aligned}
& E_{6}=\left(\frac{F}{y_{0}}\right) x_{0}\left[\frac{(x-a)^{6}}{6 !}\right] \frac{d^{6}}{d x^{6}}\left\{\left(x x_{0}\right)^{-4} x^{-1}\right\}
\end{aligned}
$$

-Thus, it is seen that each terms derivative need any be sequentially determined + substituted back in te the Taylor sere expansion to obtain the aprobimation

620 


$$
\begin{aligned}
& \text { produt Rule: } D_{x}[g(x) h(x) m(x) n(x)]=g^{\prime}(x) h(x) m(x) n(x)+\cdots \\
& +g h^{\prime}(x)(x) n(x)+g(x) h(x) \ln (x) \ln (x)+g(x) h(x) \operatorname{mon}(x) \\
& \text { chain De: } \frac{d y}{d x}=\left(\frac{d y}{d a}\right)\left(\frac{d u}{d x}\right) \\
& w^{\prime}(x)=D, i f[g(x)]:\left[F \left(g(x)\left[g^{\prime}(x)\right]\right.\right.
\end{aligned}
$$




$$
\begin{aligned}
\text { - let: } \quad u & =x-x_{6} \quad y=-u^{-2} \\
\frac{d u}{d x} & =1 \quad \frac{u}{d u}=2 u^{-3} \\
\frac{d y}{d x} & =2\left(x-x_{0}\right)^{-3}
\end{aligned}
$$

"also note that the other smiler dervative $\left.\frac{d}{d x}\left\{-(x-6)^{-1}\right\}=-d x(x-y)^{-1}\right\}+(x-10)^{-2}$ from

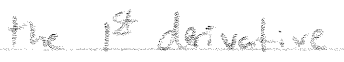

$$
\begin{array}{rl}
\therefore \frac{d^{2}}{d x^{2}}\left\langle\left(x-x_{0}\right)^{-1} x^{-3}\right\}^{2} & 2\left(x-x_{0}\right)^{-3} x^{-1}+\left(x-x_{0}\right)^{-2} x^{-2} \\
& +\left(x-x_{0}\right)^{-2} x^{-2}+2\left(x-x_{0}\right)^{-1} x^{-3}
\end{array}
$$

$-3^{d}$ den wative:

$\frac{d^{3}}{d x^{3}}\left\{\left(x-x_{0}\right)^{-1} x^{-1}\right\}=\frac{d}{d x}\left\{\frac{d^{2}}{d x^{2}}\left[\left(x+x_{0}\right)^{-1}+1\right]\right\}$

$$
\begin{aligned}
& =2 \frac{d}{d x}\left\{(x-x)^{-3}\right\} x^{-1}+2\left(x+x_{0}\right)^{-3} \frac{d}{d x}\left\{x^{-1}\right\}+\frac{d}{d x}\left\{\left(x-x_{0}\right)^{-2}\right\} x^{-2} \\
& \left.+\left(x-x_{0}\right)^{-2} d x\left\{x^{-2}\right\}+d x\right\}\left(x-x^{-2}\right) x^{-2}+\left(x-x_{0}\right)^{-2} \frac{1}{2 x}\left\{x^{2}\right. \\
& +2 d x\left\{\left(x+x_{0}\right)^{-1}\right\} x^{-3}+2\left(x-x_{0}^{-1} d x<x^{-3}\right.
\end{aligned}
$$

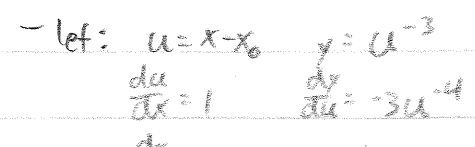
$d x=-3(x-y)-4$

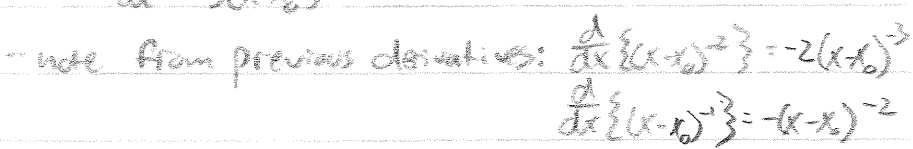




$$
\begin{aligned}
\therefore \frac{d^{3}}{d x^{3}}\left\{\left(x-x_{0}\right)^{-1} x^{-1}\right\}= & -6\left(x-x_{0}\right)^{-4} x^{-1}-2\left(x-x_{0}\right)^{-3} x^{-2} \\
& -2\left(x-x_{0}\right)^{-3} x^{-2}-2\left(x-x_{0}\right)^{-2} x^{-3} \\
& -2\left(x-x_{0}\right)^{-3} x^{-2}-2\left(x-x_{0}\right)^{-2} x^{-3} \\
& -2\left(x-x_{0}\right)^{-2} x^{-3}-6\left(x-x_{0}\right)^{-1} x^{-4}
\end{aligned}
$$

- th $^{\text {th }}$ dralive:

$\frac{d^{4}}{d x^{2}}\left\{\left(x-x_{0}\right)^{-1} x^{-7}\right\}=\frac{d}{d x}\left[\frac{d^{3}}{d x^{3}}\left\{(x-x)^{-1} x^{-1}\right\}\right]$

$=-6 \frac{d}{d x}\left\{\left(x-x_{0}\right)^{-4}\right\} x^{-1}-6\left(x-x_{0}\right)^{-4} \frac{d}{d x}\left\{x^{-1}\right\}-2 \frac{d}{d x}\left\{\left(x-x_{0}\right)^{-3}\right\} x^{-2}$

$-2\left(x-x_{0}\right)^{-3} \frac{d}{d x}\left\{x^{-2}\right\}-2 \frac{d}{d x}\left\{\left(x-x_{0}\right)-3 x^{-2}-2\left(x-x_{0}\right)^{-3} \frac{d}{d x}\left\{x^{-2}\right\}\right.$

$-2 d x\left\{\left(x-x_{0}\right)^{-2}\right\} x^{-3}-2\left(x-x_{0}\right)^{-2} d x\{x-3\}-2 \frac{d}{d x}\left\{\left(x-x_{0}\right)^{-3}\right\} x^{-2}$

$-2\left(x-x_{0}\right)^{-3} \frac{d}{d x}\left\{x^{-2}\right\}-2 \frac{d}{d x}\left\{\left(x x_{0}\right)^{-2}\right\} x^{-3}-2\left(x-x_{0}\right)^{-2} d x\left\{x^{-3}\right\}$

$\left.-2 \frac{d}{d x}\left\{(x-6)^{-2}\right\} x^{-3}-2(x-x)^{-2} d x\left\{x^{-3}\right\}-6 \frac{d x}{d x}\left(x-x_{0}\right)^{-1}\right\} x^{-4}$

$-6\left(x-x_{0}\right)^{-1} \frac{d}{d x}\{x-4\}$

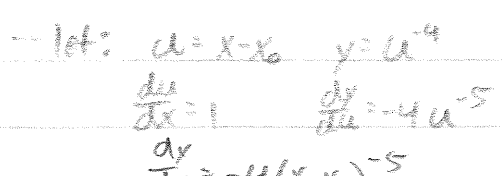

$$
\frac{d y}{d x}=-4\left(x-x_{0}\right)^{-5}
$$

-also note from ther derivolives:

$$
\begin{aligned}
& \frac{d}{d x}\left\{\left(x-x_{0}\right)^{-3}\right\}=-3\left(x-x_{0}\right)^{-4} \\
\frac{d}{d x}\left\{\left(x-x_{0}\right)^{-2}\right\} & =-2\left(x-x_{0}\right)^{-3} \\
\frac{d}{d x}\left\{\left(x-x_{0}\right)^{-1}\right\} & =-1\left(x-x_{0}\right)^{-2} \\
\therefore \frac{d}{d x}\left\{\left(x-x_{0}\right)^{-k}\right\} & =-k\left(x-x_{0}\right)^{-(k+1)} \text {... Relation at }
\end{aligned}
$$




$$
\begin{aligned}
\frac{d^{4}}{d x^{4}}\left\{\left(x-x_{0}\right)^{-1} x^{-1}\right\} & 24\left(x-x_{0}\right)^{-5} x^{-1}+6\left(x-x_{0}\right)^{-4} x^{-2} \\
& +6\left(x-x_{0}\right)^{-4} x^{-2}+4\left(x-x_{0}\right)^{-3} x^{-3} \\
& +6\left(x-x_{0}\right)^{-4} x^{-2}+4\left(x-x_{0}\right)^{-3} x^{-3} \\
& +4\left(x-x_{0}\right)^{-3} x^{-3}+6\left(x-x_{0}\right)^{-2} x^{-4} \\
& +6\left(x-x_{0}\right)^{-4} x^{-2}+4\left(x-x_{0}\right)^{-3} x^{-3} \\
& +4\left(x-x_{0}\right)^{-3} x^{-3}+6\left(x-x_{0}\right)^{-2} x^{-4} \\
& +4\left(x-x_{0}\right)^{-3} x^{-3}+6\left(x-x_{0}\right)^{-2} x^{-4} \\
& +6\left(x-x_{0}\right)^{-2} x^{-4}+24\left(x-x_{0}\right)^{-1} x^{-5}
\end{aligned}
$$

- $5^{\text {th }}$ derivative:

$$
\frac{d^{5}}{d x^{5}}\left\{\left(x-x_{0}\right)^{-5} x^{3}\right\}=\frac{d}{d x}\left[\frac{d^{4}}{d x^{4}}\left\{\left(x-x_{0}\right)^{-1} x^{+}\right\}\right]
$$$$
=24 \frac{d}{d x}\left\{\left(x-x_{0}\right)^{-5}\right\} x^{-1}+24\left(x-x_{0}\right)^{-5} \frac{d}{d x}\{x-1\}
$$$$
+6 \frac{d}{d x}\left\{\left(x-x_{0}\right)^{-4}\right\} x^{-2}+6\left(x-x_{0}\right)^{-4} \frac{d}{d x}\left\{x^{-2}\right\}
$$$$
+6 \frac{d}{d x}\left\{\left(x-x_{0}\right)^{-4}\right\} x^{-2}+6\left(x-x_{0}\right)^{-4} \frac{d}{d x}\left\{x^{-2}\right\}
$$$$
+4 \frac{d}{d x}\left\{\left(x-x_{0}\right)^{-3}\right\} x^{-3}+4\left(x-x_{0}\right)^{-3} \frac{d}{d x}\left\{x^{-3}\right\}
$$$$
+6 \frac{d}{d x}\left\{\left(x-x_{0}\right)^{-4}\right\} x^{-2}+6\left(x-x_{0}\right)^{-4} d x
$$$$
+4 \frac{d}{d x}\{(x-x)-3\} x^{-3}+4\left(x-x_{0}\right)^{-3} \frac{d}{d x}\left\{x^{-3}\right\}
$$$$
+4 \frac{d}{d x}\left\{\left(x-x_{0}\right)^{-3}\right\} x^{-3}+4\left(x-x_{0}\right)^{-3} \frac{d}{d x}\left\{x^{-3}\right\}
$$$$
+6 \frac{d}{d x}\left\{\left(x-x_{0}\right)^{-2}\right\} x^{-4}+6(x-x)^{-2} \frac{d}{d x}\left\{x^{-4}\right\}
$$$$
+6 \frac{d}{d x}\left\{(x-x)^{-4} 3 x^{-2}+6(x-\pi)^{-4} \frac{d}{d x}\left\{x^{-2}\right\}\right.
$$$$
+4 \frac{d}{d x}\left\{(x-6)^{-5} 3 x^{-3}+4\left(x-x_{0}\right)^{-3} \frac{d}{d x}\left\{x^{-3}\right\}\right.
$$$$
+4 \frac{d}{d x}\left\{\left(x-x_{0}\right)^{-3} x^{-3}+4\left(x x_{0}\right)^{-3} d x x^{-3}\right\}
$$$$
\left.+6 \frac{d}{d x}(x-x)^{-2}\right\} x^{-4}+6(x-x)^{-2} \frac{d}{d x}\left\{x^{-4}\right\}
$$ 


$$
\begin{aligned}
& +4 \frac{d}{d x}\left\{\left(x-x_{0}\right)^{-3}\right\} x^{-3}+4\left(x-x_{0}\right)^{-3} \frac{d}{d}\left\{x^{-3}\right\} \\
& +6 \frac{d}{d x}\left\{\left(x-x_{0}\right)^{-2}\right\} x^{4}+6(x+6)^{-2} \frac{d}{d x}\left\{x^{-4}\right\} \\
& +6 \frac{d}{d x}\left\{\left(x-x_{0}\right)^{-2}\right\} x^{-4}+6\left(x x_{0}\right)^{-2} \frac{d}{d x}\left\{x^{-4}\right\} \\
& +24 \frac{d}{d x}\left\{(x-6)^{-1}\right\} x^{-5}+24(x-y)^{-3} \frac{d}{d x}\left\{x^{-5}\right\} \\
& \text { emploing relation } 1 \text { : } \frac{d}{d x}\left\{\left(x-x_{0}\right)^{-k} z^{2}-k\left(x-x_{0}\right)^{-(k+1)}\right. \\
& \frac{d^{5}}{d x^{5}}\left\{\left(x-x_{0}\right)^{-1} x^{-1}\right\}=-120\left(x-x_{0}\right)^{-6} x^{-1}-24\left(x-x_{0}\right)^{-5} x^{-2} \cdots \\
& -24\left(x-x_{0}\right)^{-5} x^{-2}-12\left(x-x_{0}\right)^{-4} x^{-3} \cdots \\
& -24(x-10)^{-5} x^{-2}-12\left(x-x_{0}\right)^{-4} x^{-3} \\
& -12\left(x-x_{0}\right)^{-4} x^{-3}-12\left(x-x_{0}\right)^{-3} x^{-4} \\
& -24\left(x-x_{0}\right)^{-5} x^{-2}-12\left(x-x_{0}\right)^{-4} x^{-3} \cdots \\
& -12\left(x-x_{0}\right)^{-4} x^{-3}-12\left(x-x_{0}\right)^{-3} x^{-4} \cdots \\
& -12\left(x-x_{0}\right)^{-4} x^{-3}-12\left(x-x_{0}\right)^{-3} x^{-4} \cdots \\
& -12\left(x-x_{0}\right)^{-3} x^{-4}-24\left(x-x_{0}\right)^{-2} x^{-5} \cdots \\
& -24\left(x-x_{0}\right)^{-5} x^{-2}-12\left(x-x_{0}\right)^{-4} x^{-3} \ldots \\
& \left(2\left(x-x_{0}\right)^{-24} x^{-3}-12\left(x-x_{0}\right)^{-3} x^{-4} \cdots\right. \\
& -12\left(x-x_{0}\right)^{-4} x^{-3}-12\left(x-x_{0}\right)^{-3} x^{-4} \\
& -12\left(x-x_{0}\right)^{-3} x^{-4}-24\left(x-x_{0}\right)^{-2} x^{-5} \\
& -12\left(x-x_{0}\right)^{-4} x^{-3}-12\left(x-x_{0}\right)^{-3} x^{-4} \cdots \\
& -12\left(x-x_{0}\right)^{-3} x^{-4}-24\left(x-x_{0}\right)^{-2} x^{-5} \cdots \\
& -12\left(x-x_{0}\right)^{-3} x^{-4}-24\left(x-x_{0}\right)^{-2} x^{-5} \cdots \\
& -24\left(x-x_{0}\right)^{-2} x^{-5}-120\left(x-x_{0}\right)^{-1} x^{-6} \cdots
\end{aligned}
$$


- $6^{\text {th }}$ derivative:

$\frac{d^{6}}{d x^{6}}\left\{\left(x-x_{0}\right)^{-1} x^{-1}\right\}=\frac{d}{d x}\left[\frac{d^{5}}{d x}\left\{(x-1)^{-1} x^{-1}\right\}\right]$

$=-120 \frac{d}{d x}\left\{\left(x x_{0}\right)^{-6} 3 x^{-1}-120\left(x-x_{0}\right)^{-6} \frac{d}{d x}\left\{x^{-1}\right\}\right.$

$-24 \frac{d}{d x}\left\{(x-6)^{-5}\right\} x^{-2}-24(x-6)^{-5} d x\left\{x^{-2}\right\}$

$-24 d\left\{\left(x-x_{0}\right)^{-5}\right) x^{-2}-24(x+6)^{-5} d x\left\{x^{-2\}}\right.$

$-12 \frac{d}{d x}\left\{(x+0)^{-4}\right\} x^{-3}-12\left(x-x_{0}\right)^{-1} \frac{d}{d x}\left\{x^{-3}\right\}$

$-24 d x\left(x-x_{0}\right)^{-5} 3 x^{-2}-24\left(x-x^{-5} d x\left\{x^{-2}\right\}\right.$

$-12 \frac{d}{d x}\left\{(x-6)^{-4} 3 x^{-3}-12(x-5)^{-4} \frac{d}{d x}\left\{x^{-3}\right\}\right.$

$-12 \frac{d}{d x}\left\{(x-x)^{4}\right\} x^{3}-12\left(x-x_{0}\right)^{-4} \frac{d}{d x}\left\{x^{-3}\right\}$

$-12 \frac{d}{d x}\left\{\left(x-x_{0}\right)^{-3}\right\} x^{-4}-12\left(x-x_{0}\right)^{-3} \frac{d}{d x}\{x-4\}$

$-24 \frac{d}{d x}\left\{\left(x-x_{0}\right)^{-5} 3 x^{-2}-24\left(x-x_{0}\right)^{-5} d x\left\{x^{-2}\right\}\right.$

$\left.-12 \frac{d}{d x}\left\{\left(x-x_{0}\right)^{-4}\right\} x^{-3}-12\left(x-x_{0}\right)^{-4} \frac{d}{d x} x^{-3}\right\}$

$-12 \frac{d}{d x}\left\{(x-x)-4 x^{-3}-12(x-6)^{-4} \frac{d}{d x}\left\{x^{-3}\right\}\right.$

$-12 \frac{d}{d x}\left\{\left(x-x_{0}\right)^{-3} 3 x^{-4}-12\left(x-x^{-3} d x\left\{x^{-4}\right\}\right.\right.$

$-12 d x\left\{(x-6)^{-4} 3 x^{-3}-12(x-x)^{-4}\right.$ dx $\left\{x^{-3}\right\}$

$-12 \frac{d}{d x}\left\{(x-0)^{-3}\right\} x^{-4}-12(x-x)^{-3} d x\{x-4\} \cdots$

$-12 \frac{d}{d x}\left\{\left(x-x_{0}\right)^{-3}\right\} x^{-4}-12\left(x-x_{0}\right)^{-3} d x\left\{x^{-4}\right\}$.

$-24 \frac{d}{d x}\left\{(x-6)^{-2} 3 x^{-5}-24(x-6)^{-2} d\left\{x^{-5}\right\} \cdots\right.$

$-24 \frac{d}{d x}\left\{(x-6)^{-5} 3 x^{-2}-24(x-1)^{-5} \frac{d}{d x}\left\{x^{-2}\right\}\right.$.

$-12 \frac{d}{d x}\left\{\left(x-x_{0}\right)^{-4}\right\} x^{-3}-12\left(x-x_{0}\right)^{-4} \frac{d}{d x}\left\{x^{-3}\right\}$

$-12 \frac{d}{d x}\left\{\left(x-x_{0}\right)^{-4}\right\} x^{-3}-12\left(x-x_{0}\right)-4 \frac{d}{d x}\left\{x^{-3}\right\}$

$-12 \frac{d}{d x}\left\{(x-6)^{-3} 3 x^{-4}-12(x-6)^{-3} d x\left\{x^{-4}\right\}\right.$

$-12 \frac{d}{d x}\left\{\left(x-x_{0}\right)^{-4} 3 x^{-3}-12(x+6)^{-4} d x\left\{x^{-3}\right\}\right.$

$-12 \frac{d}{d x}\left(x-x_{0}\right)-3 x^{-4}-12\left(x-x_{0}\right)-3 \frac{d}{d x}\{x-4\}$ 


$$
\begin{aligned}
& -12 \frac{d}{d x}\left\{\left(x-x_{0}\right)^{-3}\right\} x^{-4}-12\left(x-x_{0}\right)^{-3} \frac{d}{d x}\{x-4\} \\
& -24 \frac{d}{d x}\left\{\left(x-x_{0}\right)^{-2} 3 x^{-5}-24\left(x-x_{0}\right)^{-2} \frac{d}{d x}\{x-5\} .\right. \\
& -12 \frac{d}{d x}\left\{\left(x-x_{0}\right)^{-3}\right\} x^{-4}-12\left(x-x_{0}\right)^{-3} \frac{d}{d x}\left\{x^{-4}\right\} \text {." } \\
& -24 \frac{d}{d x}\left\{\left(x-x_{0}\right)^{-2}\right\} x^{-5}-24(x-6)^{-2} \frac{d}{d x}\{x-5\} \text {." } \\
& -24 \frac{d}{d x}\left\{\left(x-x_{0}\right)^{-2} 3 x^{-5}-24(x-6)^{-2} \frac{d}{d x}\{x-5\}\right. \\
& -120 d x\left\{\left(x+x_{0}\right)^{-1} x^{-6}-12 \alpha\left(x+x_{0}+\frac{d}{d x}\left\{x^{-6}\right\}\right.\right. \\
& -12 \frac{d}{d x}\left\{\left(x-x_{0}\right)-43 x^{-3}-12\left(x x_{0}\right)^{-4} \frac{d}{d x}\left\{x^{-3}\right\} .\right. \\
& -12 \frac{d}{d x}\left\{\left(x-x_{0}\right)^{-3} 3 x^{-4}-12\left(x x_{0}\right)^{-3} \frac{d}{d x}\left\{x^{-4}\right\} \cdots\right. \\
& -12 \frac{d}{d x}\left\{(x-6)^{-3} 3 x^{-4}-12(x-x)^{-3} \frac{d}{d x}\left\{x^{-4}\right\} .\right. \\
& -24 \frac{d}{d x}\left(x-x_{0}\right)^{-2} 3 x^{-5}-24(x-6)^{-2} d x\left\{x^{-5}\right\}
\end{aligned}
$$

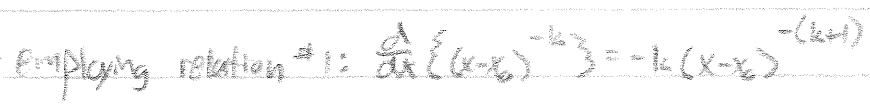

$$
\begin{aligned}
\frac{d^{6}}{d x^{6}}\left\{(x-x)^{-1} x^{-3}=\right. & 720\left(x-x_{0}\right)^{-1} x^{-1}+120\left(x-x_{0}\right)^{-6} x^{-2} \\
& +120\left(x-x_{0}\right)^{-6} x^{-2}+48\left(x-x_{0}\right)^{-5} x^{-3}
\end{aligned}
$$$$
+120\left(x-x_{0}\right)^{-6} x^{-2}+48(x-6)^{-5} x^{-1}
$$$$
+48\left(x-x_{0}\right)^{-5} x^{-3}+36\left(x-x_{0}\right)^{-4} x^{-4}
$$$$
+120(x-10)^{-6} x^{-2}+48(x-x)^{-5} x^{-3}
$$$$
+48\left(x-x_{0}\right)^{-5} x^{-3}+36\left(x-x_{0}\right)^{-4} x^{-4}
$$$$
+48\left(x-x_{0}\right)^{-5} x^{-3}+36\left(x-x_{0}\right)^{-4} x^{-4}
$$$$
+36\left(x-x_{0}\right)^{-4} x^{-4}+48\left(x-x_{0}\right)^{-3} x^{-5} .
$$$$
+120\left(x-x_{0}\right)^{-6} x^{-2}+48\left(x-x_{4}\right)^{-5} x^{-3} .
$$$$
+48\left(x-x_{0}\right)^{-5} x^{-3}+36\left(x-x_{0}\right)^{-4} x^{-4} .
$$$$
+48\left(x-x_{0}\right)^{-5} x^{-3}+36\left(x-x_{0}\right)^{-4} x^{-4}
$$$$
+36\left(x-x_{0}\right)^{-4} x^{-4}+48\left(x-x_{0}\right)^{-3} x^{-5} \text {. }
$$ 


$$
\begin{aligned}
& +48\left(x-x_{0}\right)^{-5} x^{-3}+36\left(x-x_{0}\right)^{-4} x^{-4} \\
& +36\left(x-x_{0}\right)^{-4} x^{-4}+48\left(x-x_{0}\right)^{-3} x^{-5} \\
& +36\left(x-x_{0}\right)^{-4} x^{-4}+48\left(x-x_{0}\right)^{-3} x^{-5} \\
& +48\left(x-x_{0}\right)^{-3} x^{-5}+120\left(x-x_{0}\right)^{-2} x^{-6} \\
& +120\left(x-x_{0}\right)^{-6} x^{-2}+48\left(x-x_{0}\right)^{-5} x^{-3} \\
& +48\left(x-x_{0}\right)^{-5} x^{-3}+36\left(x-x_{0}\right)^{-4} x^{-4} \\
& +48\left(x-x_{0}\right)^{-5} x^{-3}+36\left(x-x_{0}\right)^{-4} \\
& +36\left(x-x_{0}\right)^{-4} x^{-4}+48\left(x-x_{0}\right)^{-3} x^{-5} \\
& +48\left(x-x_{0}\right)^{-5} x^{-3}+36\left(x-x_{0}\right)^{-4} x^{4} \\
& +36\left(x-x_{0}\right)^{-4} x^{-4}+48\left(x-x_{0}\right)^{-3} x^{-5} \\
& +36\left(x-x_{0}\right)^{-4} x^{-4}+48\left(x-x_{0}\right)^{-3} x^{-5} \\
& +48\left(x-x_{0}\right)^{-3} x^{-5}+120\left(x-x_{0}\right)^{-2} x^{-6} \\
& +36\left(x-x_{0}\right)^{-4} x^{-4}+48\left(x-x_{0}\right)^{-3} x^{-5} \\
& +48\left(x-x_{0}\right)^{-3} x^{-5}+120\left(x-x_{0}\right)^{-2} x^{-6} \\
& +48\left(x-x_{0}\right)^{-3} x^{-5}+120\left(x-x_{0}\right)^{-8} x^{-6} \\
& +120\left(x-x_{0}\right)^{-2} x^{-6}+720\left(x-x_{0}\right)^{-1} x^{-7} \\
& +48\left(x-x_{0}\right)^{-5} x^{-3}+36\left(x-x_{0}\right)^{-4} x^{-4} \\
& +36\left(x-x_{0}\right)^{-4} x^{-4}+48\left(x-x_{0}\right)^{-3} x^{-5} \\
& +36\left(x-x_{0}\right)^{-4} x^{-4}+48\left(x-x_{0}\right)^{-3} x^{-5} \\
& +48\left(x-x_{0}\right)^{-3} x^{-5}+120\left(x-x_{0}\right)^{-2} x^{-4}
\end{aligned}
$$


- from the epmizons. Its noted that expowents of terms

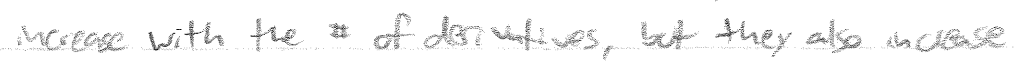

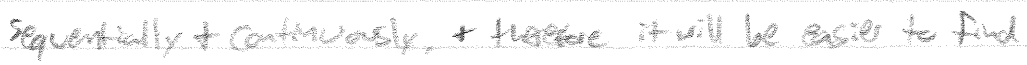
the paten of tars are simplified sumpifytos exch term:

$$
\begin{aligned}
& \text { aeroth term: } \frac{d^{2}}{d x}\left\{(x-6)^{+1} x^{-1}\right)=\left(x-x_{0}\right)^{-1} x^{-1} \\
& \cdots 12 \tan : \frac{d}{d x}\left\{(x-x)^{-1} x^{-1}\right\}=-(1)\left(x-x_{0}\right)^{-2} x^{-1}+(1)(x-1) x^{-1} \\
& =(1)\left(x-x_{0}\right)^{-2} x^{-1}+(1)(x-6)^{-2} x^{-2} \\
& =(+2)\left(x-x_{0}\right)^{-3} x^{-1}+(+2)\left(x-x_{0}\right)^{-2} x^{-2}+(+2)\left(x-x_{0}\right)^{-1} x^{-3}
\end{aligned}
$$

$-3^{d}$ ferm:

$\frac{d^{3}}{d x^{3}}\left\{(x-6)^{-1} x^{-1}\right\}=(-6)(x-x)^{-4} x^{-1}+(-2)(x-x)^{-3} x^{-2}$ $+(-2)\left(x-x_{0}\right)^{-3} x^{-2}+(-2)(x+6)^{-2} x^{-3}$ $+(-2)\left(x-x_{0}\right)^{-3} x^{-2}+(-2)(x-4)^{-2} x^{-3}$ $+\left(2 x\left(x-x_{0}\right)^{2} x^{2}+(-6)\left(x+x_{0}\right)^{-3} x^{-4}\right.$ 


$$
\begin{aligned}
& =(-6)\left(x-x_{0}\right)^{-4} x^{-1}+(-6)\left(x-x_{0}\right)^{-3} x^{-2} \\
& (-6)\left(x-x_{0}\right)^{-2} x^{-3}+(-6)(x-8)^{-1} x^{-4} \\
& -4^{\text {th }} \text { term. } \\
& \frac{d}{d x}\left\{\left(x-x_{0}\right)^{-1} x^{-1}\right\}=24\left(x-x_{0}\right)^{-5} x^{-1}+6\left(x-x_{0}\right)^{-4} x^{-2} \\
& +6\left(x-x_{0}\right)^{-4} x^{-2}+4\left(x-x_{0}\right)^{-3} x^{-3} \\
& +6\left(x-x_{0}\right)^{-4} x^{-2}+4\left(x-x_{0}\right)^{-3} x^{-3} \\
& f\left(x-x_{0}\right)^{-3} x^{2}+6\left(x-x_{0}\right)^{-2} x^{-4} \\
& +\left(\left(x-x_{0}\right)^{-4} x^{-2}+4\left(x-x_{0}\right)^{-3} x^{-3}\right. \\
& +4\left(x-x_{0}\right)^{-3} x^{-2}+b\left(x-x_{0}\right)^{-2} x^{-4} \\
& +4(x-x)^{-3} x^{-3}+6\left(x-x_{0}\right)^{-2} x^{-4} \\
& +\left(6 x-x_{0}\right)^{-2} x^{4}+24(x-6)^{-1} x^{-5} \\
& =(-24)\left(x-x_{0}\right)^{-5} x^{-1}+(+24)\left(x-x_{0}\right)^{-4} x^{-2}+(+24)\left(x-x_{0}\right)^{-3} x^{-3} \\
& +(+24)\left(x-x_{0}\right)^{-2} x^{-49}+(+24)\left(x-x_{0}\right)^{-1} x^{-5} \\
& -5^{\text {th }} \text { tarm: } \\
& d^{5}\{(x-y)+x\}=-126\left(x-x_{0}\right)^{-6} x^{-1}-24\left(x-x_{0}\right)^{-5} x^{-2} \\
& -24\left(x-x_{0}\right)^{-5} x^{-2}-12(x-x)^{-4} x^{-3} \\
& -24\left(x-x_{0}\right)^{5} x^{-2}-12\left(x-x_{0}\right)^{-4} x^{-3} \\
& -12(x-6)^{-4} x^{-3}-12(x-x)^{-3} x^{-4} \\
& -24\left(x x_{0}\right)^{-5} x^{-2}-12(x-6)^{-4} x^{-3} \\
& -12\left(x+x_{0}\right)^{-4} x^{-3}-12\left(x-x_{0}\right)^{-3} x^{-4} \\
& -12\left(x-x_{0}\right)^{-4} x^{-3}-12(x-10)^{-3} x^{-4}
\end{aligned}
$$




$$
\begin{aligned}
& -12\left(x-x_{0}\right)^{-3} x^{-4}-24\left(x-x_{0}\right)^{-2} x^{-5} \\
& -24\left(x-x_{0}\right)^{-5} x^{-2}-12\left(x-x_{0}\right)^{-4} x^{-3} \\
& -12\left(x-x_{0}\right)^{-4} x^{-3}-12(x-x)^{-3} x^{-4} \\
& -12\left(x-x_{0}\right)^{-4} x^{-3}-12(x-6)^{-3} x^{-4} \\
& -12\left(x-x_{a}\right)^{-3} x^{-4}-24\left(x+x_{0}\right)^{-2} x^{-5} \\
& -12(x-10)^{-4} x^{-3}-12(x+6)^{-3} x^{-4} \\
& -12\left(x+x_{0}\right)^{-3} x^{-4}-24\left(x_{0}\right)^{-2} x^{-5} \\
& -12\left(x+x_{0}\right)^{-3} x^{-4}-24(x-x)^{-2} x^{-5} \\
& -24(x+6)^{-2} x^{-5}-120(x+6)^{-1} x^{-6} \\
& \left.\left.=(-120)\left(x-x_{0}\right)^{-6} x^{-1}+120\right)\left(x-x_{0}\right)^{-5} x^{-2}+120\right)\left(x-x_{0}\right)^{-4} x^{-3} \\
& +(-120)(x-10)^{-3} x^{-4}+(-120)\left(x-x_{0}\right)^{-2} x^{-5}+(-120)(x-6)^{-1} x^{-6} \\
& \text { - } 6 \text { ten } \operatorname{lor}: \\
& \left.\frac{d^{6}}{d x^{6}}\left\{\left(x-x_{0}\right)^{-1} x^{-1}\right\}=720\left(x x_{0}\right)^{-7} x^{-1}+12 d x-x_{0}\right)^{-6} x^{-2} \\
& 4\left(200(x-1)^{-6} x^{-2}+4 x(x-6)^{-5} x^{-3}\right. \\
& 1120\left(x x_{0}\right)^{-6} x^{-2}+48(x-6)^{-5} x^{-3} \\
& 44(x-6)^{-5} x^{-3}+36(x-x)^{-4} x^{-1} \\
& +120\left(x-x_{0}\right)^{-6} x^{-6}+4\left(x-x_{0}\right)^{-5} x^{-3} \\
& +48\left(x-x_{0}\right)^{-5} x^{-3}+36\left(x-x_{0}\right)^{-4} x^{-4} \\
& +48\left(x-x_{0}\right)^{-5} x^{-3}+36(x-x)^{-4} x^{-4} \\
& +36\left(x-x_{0}\right)^{-4} x^{-4}+48(x-6)^{-3} x^{-5} \\
& +12 d\left(x-x_{0}\right)^{-6} x^{-2}+48\left(x-x_{0}\right)^{-5} x^{-3}
\end{aligned}
$$




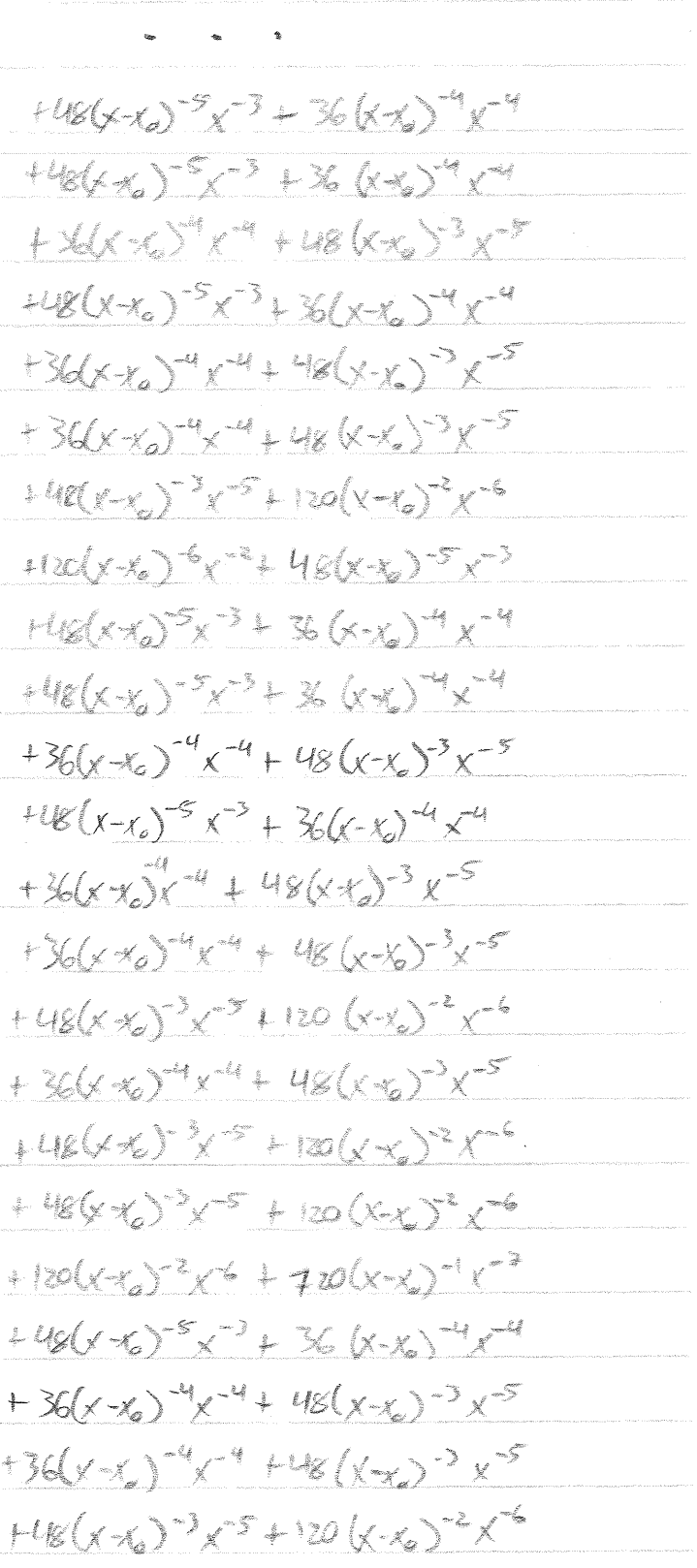




$$
\begin{aligned}
& =720\left(x-x_{0}\right)^{-7} x^{1}+720\left(x-x_{0}\right)^{-6} x^{-2}+720\left(x-x_{0}\right)^{-5} x^{-3} \\
& +720\left(x-x_{0}\right)^{-4} x^{-4}+720\left(x-x_{0}\right)^{-3} x^{-5}+720\left(x-x_{0}\right)^{-2} x^{-6} \\
& +720(x-6)^{-1} x^{-7} \\
& \text { Conglideating toms: } \\
& N_{0}=\left(x-x_{0}\right)^{-1} x^{-1} \\
& N_{1}=(d)\left(-x_{0}\right)^{-2} x^{-1}+(-1)\left(x-x_{0}\right)^{-1} x^{-2} \\
& N_{2}=(2)\left(x-x_{0}\right)^{-3} x^{-1}+(2)(x-4)^{-2} x^{-2}+\left(2 x-x_{0}\right)^{-1} x^{-3} \\
& N_{2}=(-6)\left(x-x_{0}\right)^{-4} x^{-1}+(-6)(x-6)^{-3} x^{-2}+(-6)(x-6)^{-2} x^{-3} \\
& 4(-6)\left(x-x_{0}\right)^{-1} x^{-4} \\
& N_{4}=(24)(x-6)^{-5} x^{-1}+(24)(x-6)^{-44} x^{-2}+(24)\left(x-x_{6}\right)^{-3} x^{-3} \\
& +(24)(x-6)^{-2} x^{-4}+(24)\left(x-x_{0}\right)^{-1} x^{-5} \\
& N_{5}=(-120)\left(x-x_{0}\right)^{-6} x^{-3}+(-120)(x-10)^{-5} x^{-2}+(-120)\left(x-x_{0}\right)^{-4} x^{-3} \\
& 4(-120)\left(x-x_{0}\right)^{-3} x^{-4}+(-120)\left(x-x_{0}\right)^{-2} x^{-5}+(-120)\left(x+x_{0}\right)^{-3} x^{-6} \\
& N_{6}=(720)\left(x-x_{0}\right)^{-7} x^{-1}+(720)(x-10)^{-6} x^{-2}+(720)\left(x-x_{0}\right)^{-5} x^{-3} \\
& +(2 x a)(6 x)^{-4} x^{-4}+(200)(x-y)^{-1} x^{-5}+(70)(x+6)^{-12} x^{-6} \\
& +(720)(x+y)^{-1} x^{-7}
\end{aligned}
$$




$$
\begin{aligned}
& \left.N_{0}=(0)[(x-x)]^{-1} x^{-1}\right] \\
& N_{1}=(-1)(11)\left[\left(x-x_{0}\right)^{-2} x^{-3}+\left(x-x_{0}\right)^{-1} x^{-2}\right] \\
& N_{2} *(+1)(2)\left[(x-8)^{-3} x^{-1}+(x-6)^{-2} x^{-2}+\left(x-x_{0}\right)^{-1} x^{-3}\right] \\
& M_{3}=(-1)(3)\left[(x+6)^{-4} x^{-1}+(x-6)^{-3} x^{-2}+(x-x)^{-2} x^{-3}+\left(x-x_{0}\right)^{-1} x\right] \\
& N_{4}=(4)(4 !)\left[\begin{array}{l}
(x-x)^{-5} x^{-1}+(x-6)^{-4} x^{-2}+\left(x-x_{0}\right)^{-3} x^{-3} \\
+\left(x-x_{0}\right)^{-2} x^{-4}+\left(x-x_{0}\right)^{-1} x^{-5}
\end{array}\right] \\
& \left.N_{5}=(-1)(5)\right]\left[\begin{array}{l}
\left(x-x_{0}\right)^{-6} x^{-1}+(x-x)^{-5} x^{-2}+\left(x-x_{0}\right)^{-4} x^{-3} \\
+\left(x-x_{0}\right)^{-3} x^{-4}+\left(x-x_{0}\right)^{-2} x^{-5}+\left(x-x_{0}\right)^{-1} x^{-6}
\end{array}\right] \\
& N_{6}=(+1)(6)\left[\begin{array}{l}
\left(x-x_{0}\right)^{-7} x^{-1}+\left(x-x_{0}\right)^{-6} x^{-2}+\left(x-x^{-5} x^{-3}\right. \\
+(x-6)^{-4} x^{-4}+(x-x)^{-3} x^{-5}+\left(x-x_{0}\right)^{-2} x^{-6} \\
+(x-x)^{-1} x^{-7}
\end{array}\right]
\end{aligned}
$$

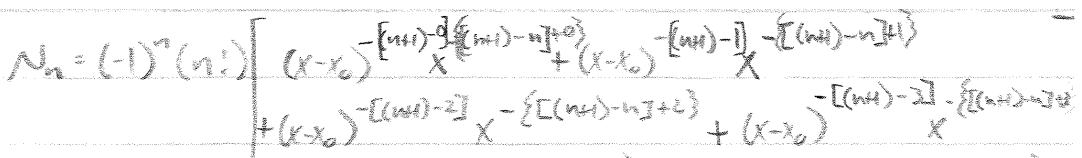

$$
\begin{aligned}
& +\left(x-x_{0}\right)^{-[(n+1)-4]} x^{-\{(n+1)-n]+4\}}+\left(x-x_{0}\right)^{-[(n+1)-5]} x^{-\{[n+1)-4]+5\}} \\
& {\left[\left(x-x^{-[(n+1)-6]} x^{-\{[(n+1)-3]+6\}}\right.\right.} \\
& N_{n}=(-1)^{n}(n !) \sum_{i=0}^{n}\left[(x-1)^{-[(n+1)-i]} x^{-(1+i)}\right]
\end{aligned}
$$




$$
N_{n}=(-1)^{n}(n) \sum_{i=0}^{n}\left[(x+\infty)^{[(n+1)-i]} x^{(1+i)}\right]^{-1}
$$

- it will be convenient to ellect $1 \frac{1}{\left(x-x_{0}\right)}$ tam for substitation bock inte the elstic modus -nole, ge geneal, that

$$
\left(x^{n-1} y^{n}\right)=\left(x^{n+1} y^{n-1}\right)\left(x^{m} y^{n}\right)
$$

- to ensure the preper pattern, I use the enpand ed versien:

$\Lambda_{0}=(-1)^{0}(0)\left[\left(x-x_{0}\right)(x)^{3}\right]^{-1}\left[\left(x-x_{0}\right)^{1-1} x^{1-1}\right]^{-1}$

$N_{0}=(1)^{0}\left(0^{1}\right)\left[\left(x-x_{0}\right) x\right]^{-1}\left[\left(x-x_{0}\right)^{0} x^{0}\right]^{-1}$

$$
N_{1}=(-1)^{1}(1)\left[\left(x-x_{0}\right) x^{-1}\left[\left(x-x_{0}\right)^{3} x^{0}+\left(x-x_{0}\right)^{2} x^{1}\right]^{-1}\right.
$$

$$
N_{2}=(1)^{2}(21)\left[\left(x-x_{0}\right)\right]^{-1}\left[\left(x-x_{0}\right)^{2} x^{a}+\left(x-x_{0}\right)^{3}+\left(x-x_{0}\right)^{2} x^{2}\right]^{-1}
$$

$$
N_{3}=(-1)^{2}(31)\left[\left(x-x_{0}\right) x\right]^{-1}\left[\left(x-x_{0}\right)^{3} x^{0}+\left(x-x_{0}\right)^{2} x^{1}+\left(x-x_{0}\right)^{2} x^{2}+\left(x-x_{0}\right)^{3} x^{3}\right]
$$

$$
N_{4}=(-1)^{4}(4)\left[\left(x-x_{0}\right) x\right]^{-1}\left[\begin{array}{l}
\left(x-x_{0}\right)^{4}+\left(x-x_{0}\right)^{3} x^{3}+\left(x-x_{0}\right)^{2} x^{2} \\
+\left(x-x_{0}\right) x^{3}+\left(x-x_{0}\right)^{-1} x^{4}
\end{array}\right]^{-1}
$$

$$
N_{5}=(-1)^{5}(5)\left[\left(x-x_{0}\right) x^{7}\left[\begin{array}{l}
\left(x-x_{0}\right)^{5} x^{4}+\left(x-x_{0}\right)^{4} x^{1}+\left(x-x_{0}\right)^{3} x^{2}+\left(x-x_{0}\right)^{2} x^{3} \\
+\left(x-x_{0}\right)^{1} x^{4}+\left(x-x_{0}\right)^{9} x^{5}
\end{array}\right]^{-1}\right.
$$




$$
\begin{aligned}
& N_{6}=(1)^{6}(6)\left[\left(x-x_{0}\right) x\right]^{-1}\left[\begin{array}{l}
\left(x-x_{0}\right)^{6} x^{3}+\left(x-x_{0}\right)^{5}+\left(x-x_{0}\right)^{4} x^{2} \\
\left(x-x_{0}\right)^{3} x^{3}+\left(x-x_{0}\right)^{2} x^{4}+(x-6) x^{6} \\
\left(x-x_{0}\right)^{6} x^{6}
\end{array}\right] \\
& N_{n}=\left[\left(x-x_{0}\right) x\right]^{-1}(-1)^{n}(n) \sum_{0}^{6}\left[\left(x-x_{0}\right)^{n-i} x^{3}\right]^{-1}
\end{aligned}
$$

Subtituthy back int the Elatic Molulus equations:

$E_{0}=\left(\frac{E}{y_{0}}\right) x_{0}\left[\frac{y+a g}{0}\right] N_{0}$

$E=\left(\frac{F}{f_{0}}\right) x_{0}\left[\frac{\mathrm{s} a}{\mathrm{~L}}\right] N$

$E_{2}=\left(\frac{E}{y_{0}}\right) K_{2}\left[\frac{(x+2)^{2}}{2}\right] N_{2}$

$E_{3} *\left(\frac{E}{x_{0}}\right) x_{0}\left[\frac{(x-a)^{3}}{2}\right] N_{3}$

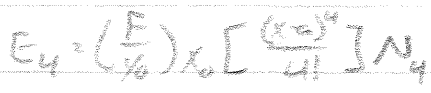

$E_{5}\left(\frac{E}{6}\right)_{x}\left[\frac{(x a y}{5}\right] N$

$E_{6}=\left(\frac{E}{y_{0}}\right) x_{0}\left[\frac{(x-a)^{6}}{6 !}\right] N_{6}$

$\therefore E_{n}=\left(\frac{E}{y_{0}}\right) X_{0}\left[\frac{\left(x_{0}\right)^{n}}{n !}\right] N_{n}$ 


$$
\begin{aligned}
& E_{n}=\left(\frac{E}{f_{0}}\right) x_{0}\left[\frac{(x a)}{n !}\right]\left[\left(x-x_{0} x\right]^{-1}(x)(n) \sum_{i=0}^{n}\left[(x-6)^{n} x^{n}\right]^{-1}\right.
\end{aligned}
$$

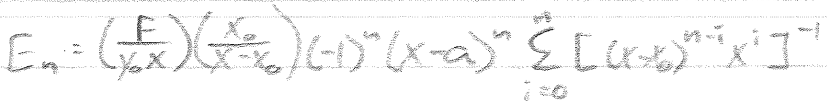

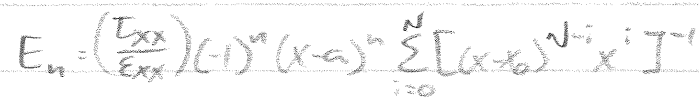

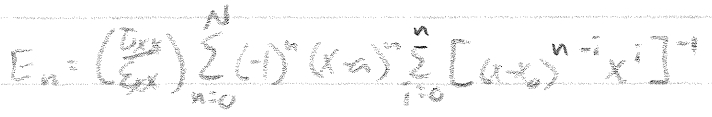

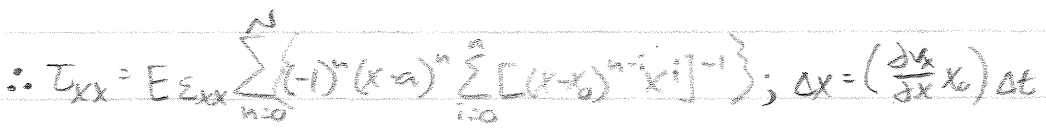

$$
\begin{aligned}
& \left.x \rightarrow \frac{d x}{d x} x_{0}\right) \Delta t \\
& x=\left(\frac{\sec }{d x} \Delta+1\right) x_{0} \\
& \text { - Subltutu thase rebtans M: }
\end{aligned}
$$

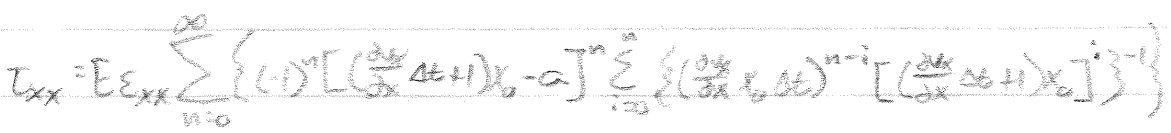

$$
\begin{aligned}
& \text {-nate fram previous: } \varepsilon_{x y}=\frac{\partial w}{\partial x} \Delta t
\end{aligned}
$$

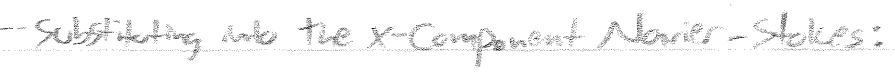

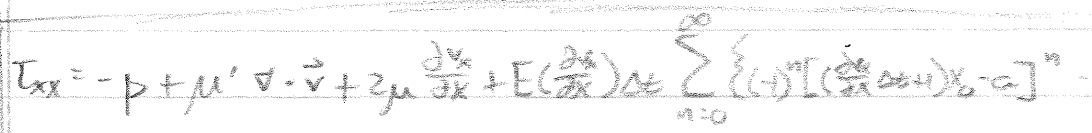

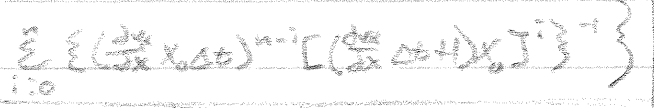

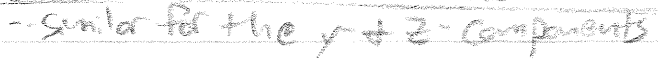




\subsection{Appendix M}

\section{Die Swell Experiment System Information}

Appendix L: Die Swell Experiment System Information presents further detail about the components used in the development and implementation of the die swell experiment apparatus and operations. All proprietary data attributed to various entities herein are reserved to those entities. 


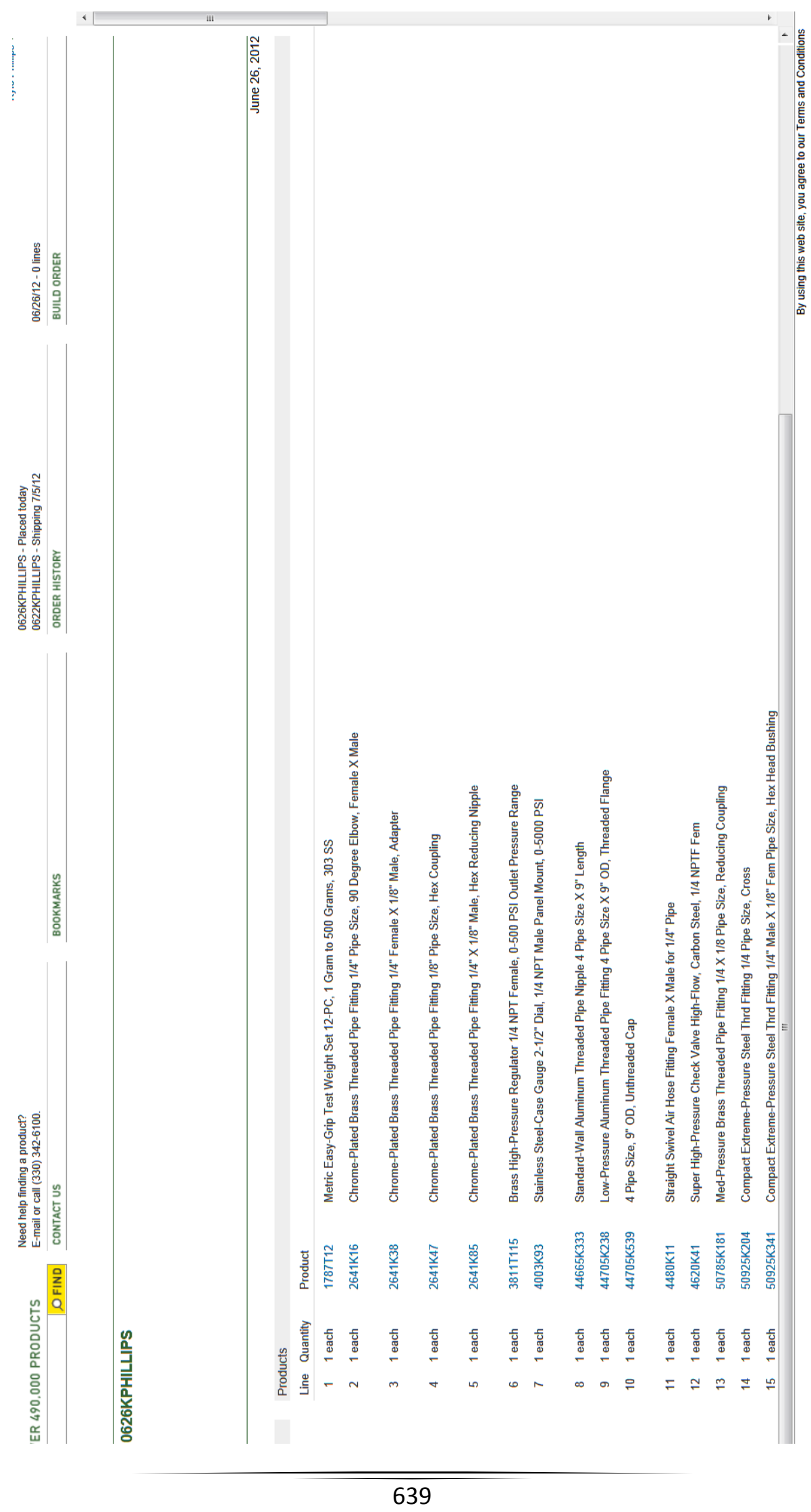




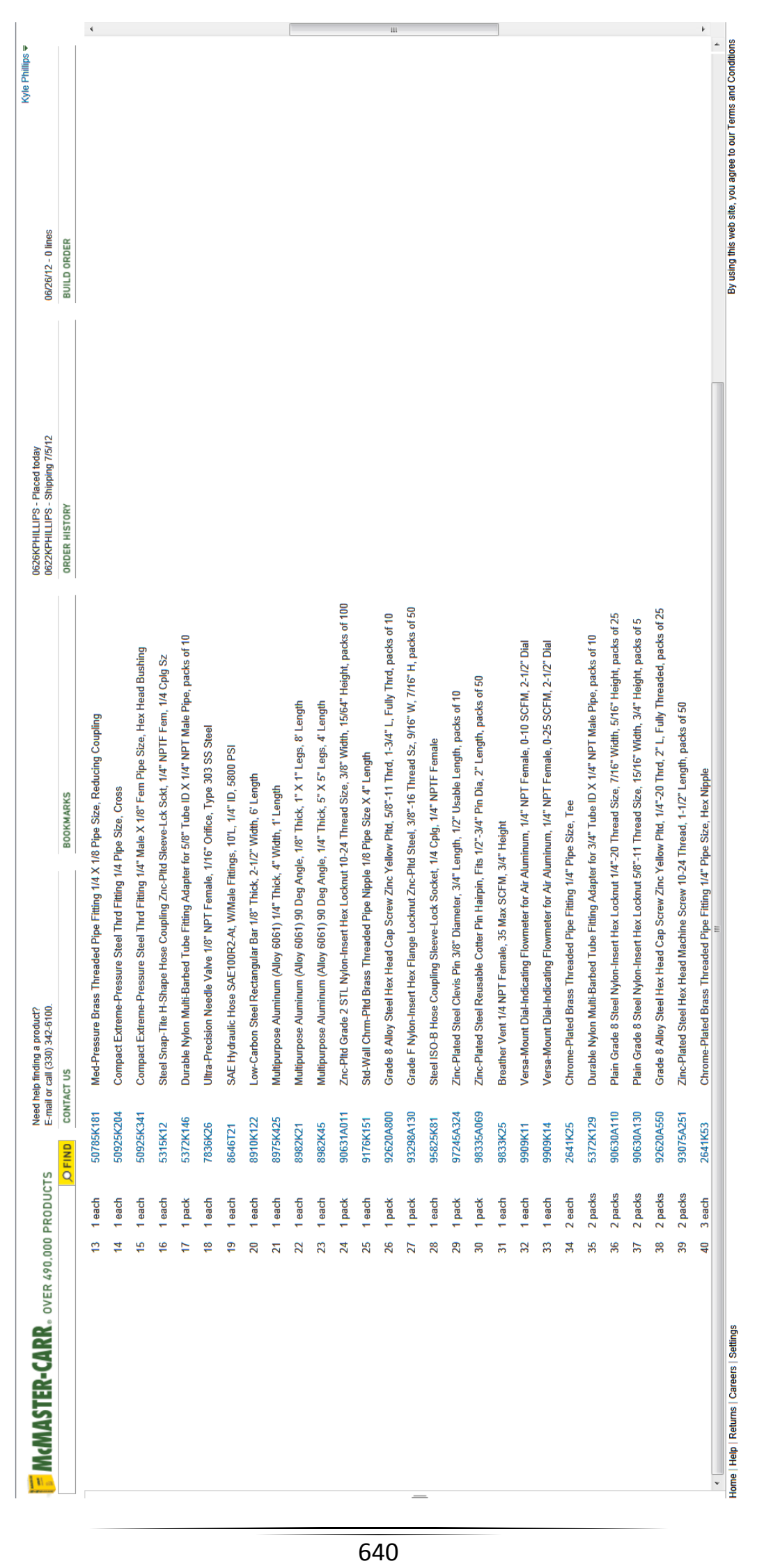




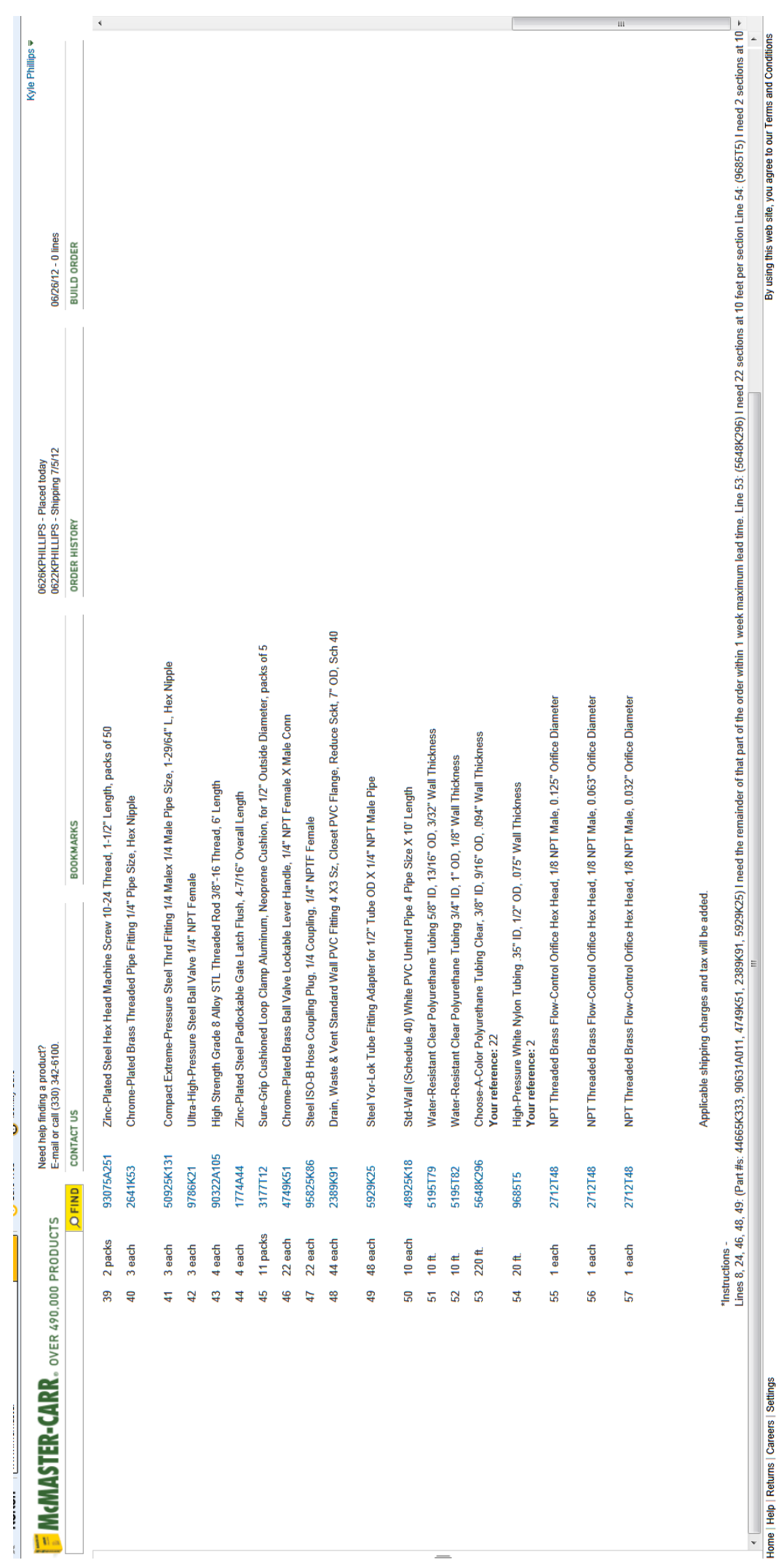




\subsection{Appendix N}

\section{Budget Model}

Appendix N: Budget Model presents the financial model employed during the development of the WVU SMiRF. This appendix presents both an estimated budget and the actual costs, as broken down by system. For system details, the reader is referred to the appropriate appendices that present such data. The reader is also encouraged to employ as many discounts and negotiation leverage during cost negotiations with vendors. Many of those techniques were employed during the development of the WVU SMiRF to drive costs down. The primary intent of this appendix is to prove that relatively large drop tower research facilities can be developed affordably and do not have to be cost-prohibitive. The intent here is to inspire other to take on the challenge of drop tower development in order to increase access to reduced gravity research to spur more technology development by way of more fundamental scientific understanding and the inherent contribution to scientific knowledge. 


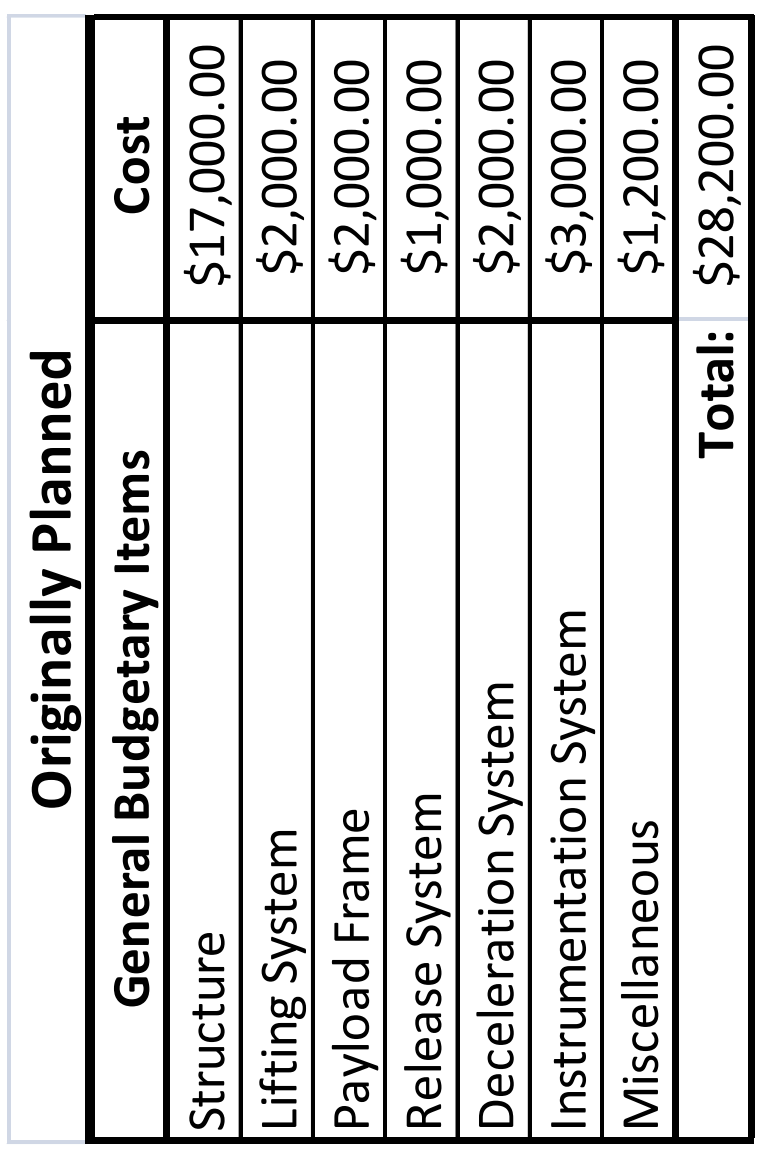




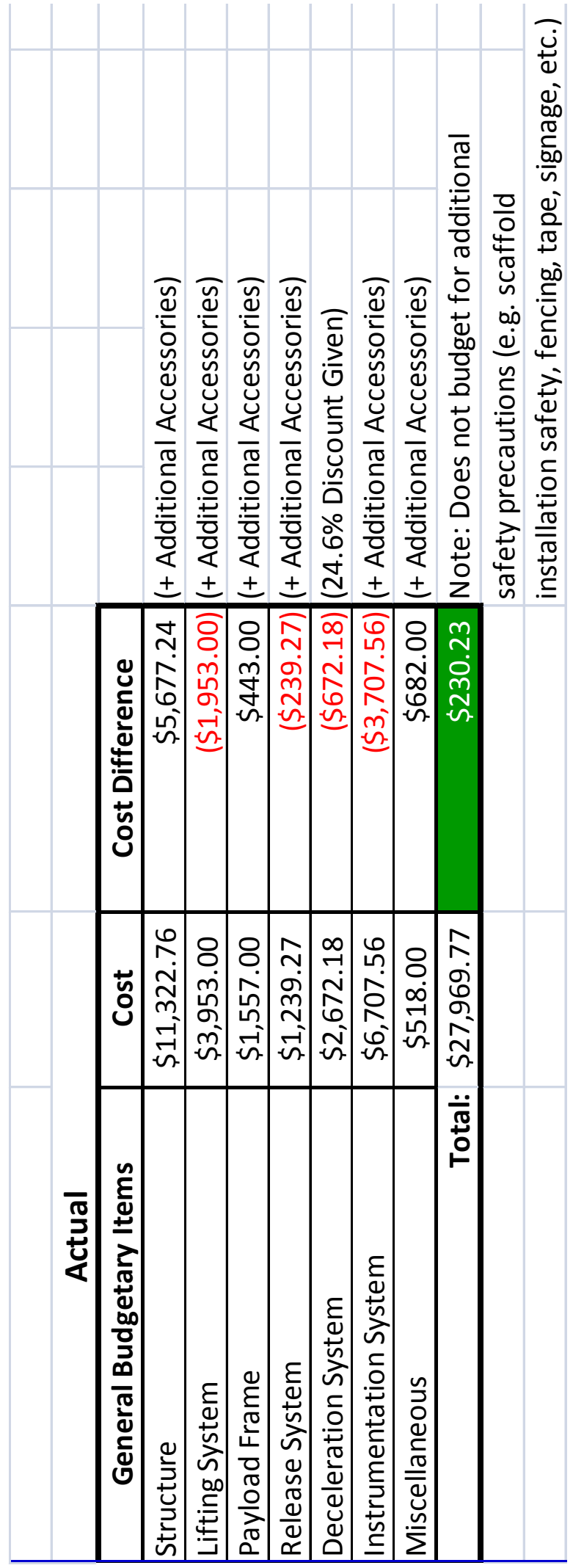


Development of the West Virginia University

Small Microgravity Research Facility (WVU SMiRF)
Copyright $^{\odot} 2014$

Kyle G. Phillips

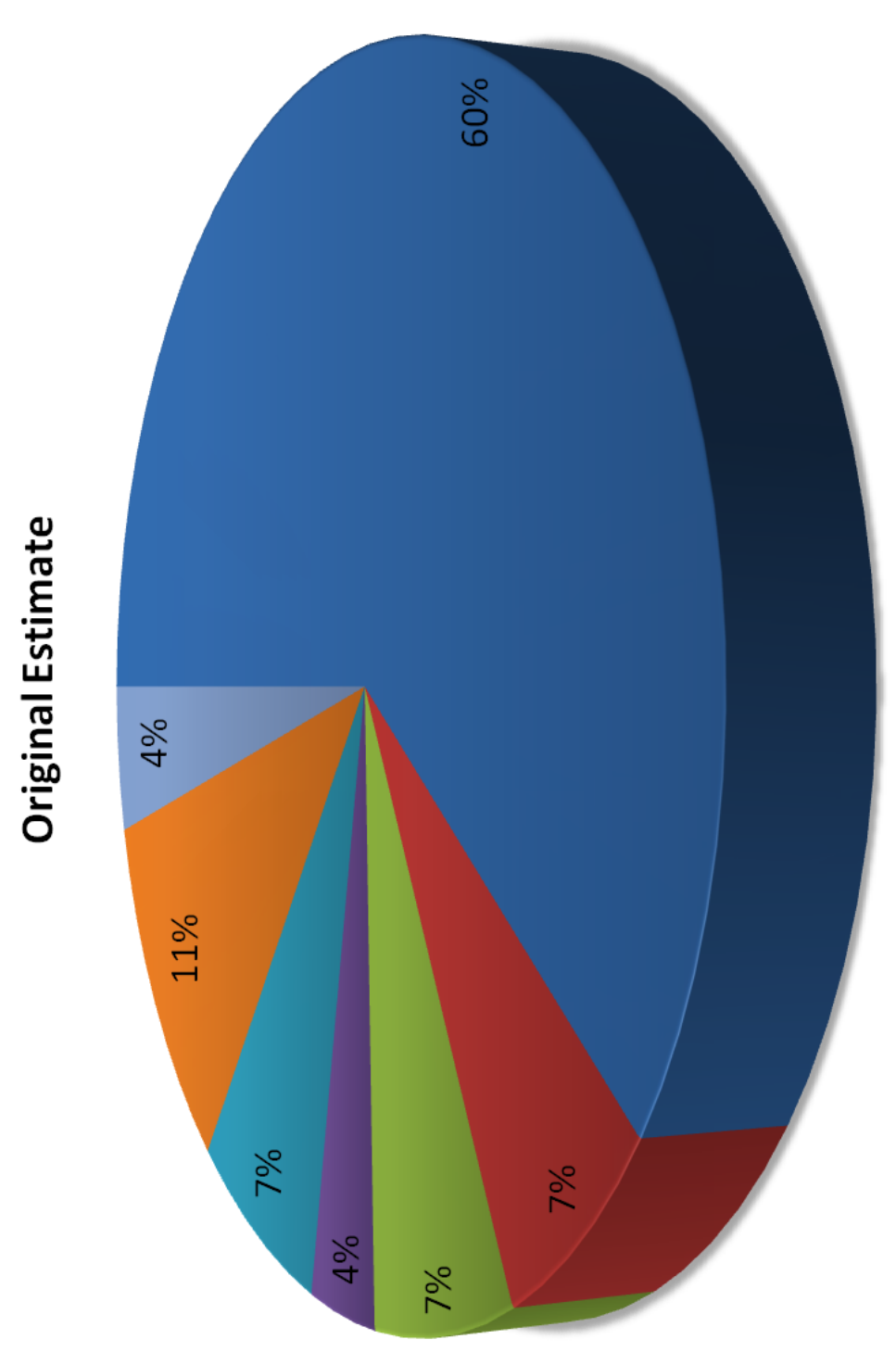

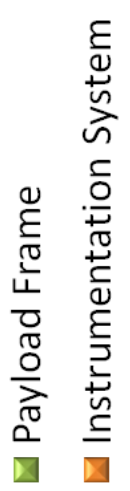

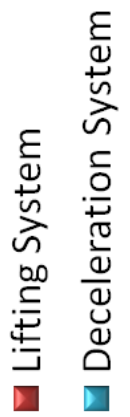

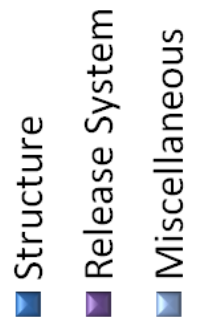


Development of the West Virginia University

Small Microgravity Research Facility (WVU SMiRF)
Copyright $^{\odot} 2014$

Kyle G. Phillips

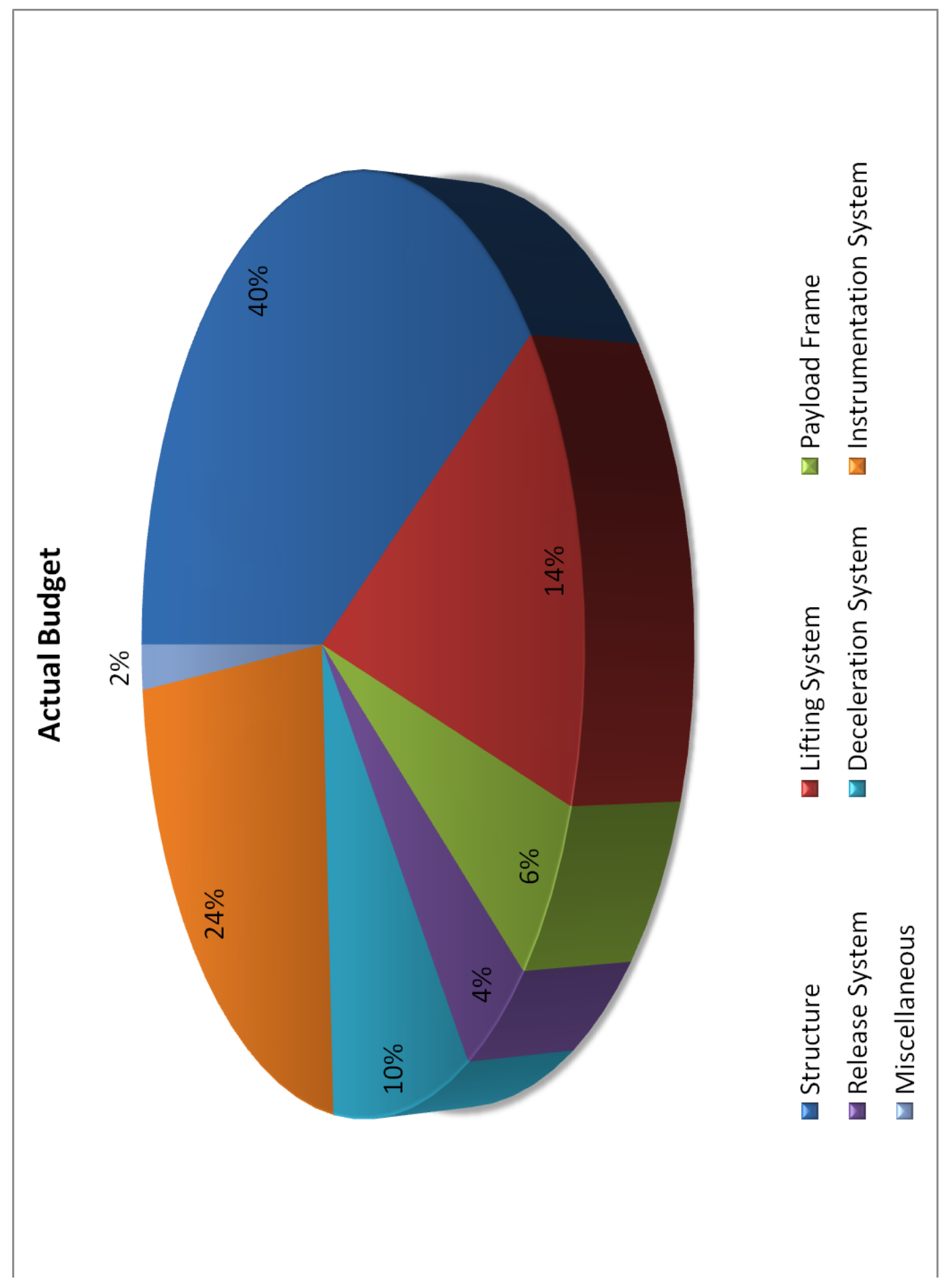




\subsection{Appendix O}

\section{Drop Tower Comparison - 2014}

Appendix O: Drop Tower Comparison - 2014 presents a pair of tables summarizing the modern research drop towers presented in Appendix A, as of the year 2014. In an attempt for both comprehension and comparison, government-sponsored drop towers and those residing at other institutions outside of the United States of America are compiled in one table, while drop tower facilities residing at academic institutions residing within the United States of America are compiled in a separate table, as the focus of this thesis was on the development of a drop tower that resides at a US academic institution. From Appendix A, one may notice that drop towers that had humble beginnings have now spread across the world, increasing our scientific understanding and aiding in the development of advanced technologies that have a wide array of applications in everything from everyday life to future space exploration. 
Development of the West Virginia University

Small Microgravity Research Facility (WVU SMiRF)
Copyright $^{\circledR} 2014$

Kyle G. Phillips

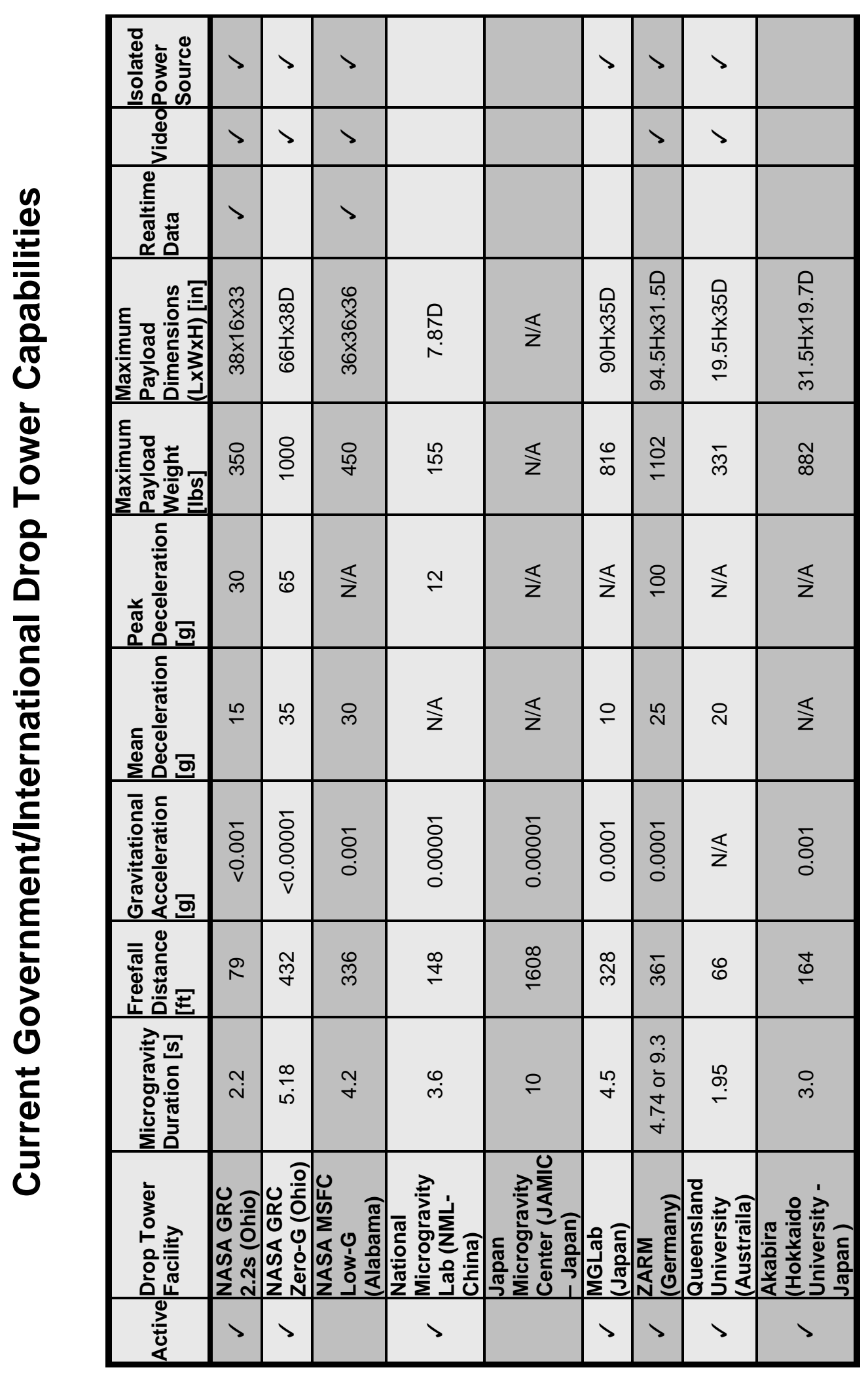


Development of the West Virginia University

Small Microgravity Research Facility (WVU SMiRF)
Copyright $^{\odot} 2014$

Kyle G. Phillips

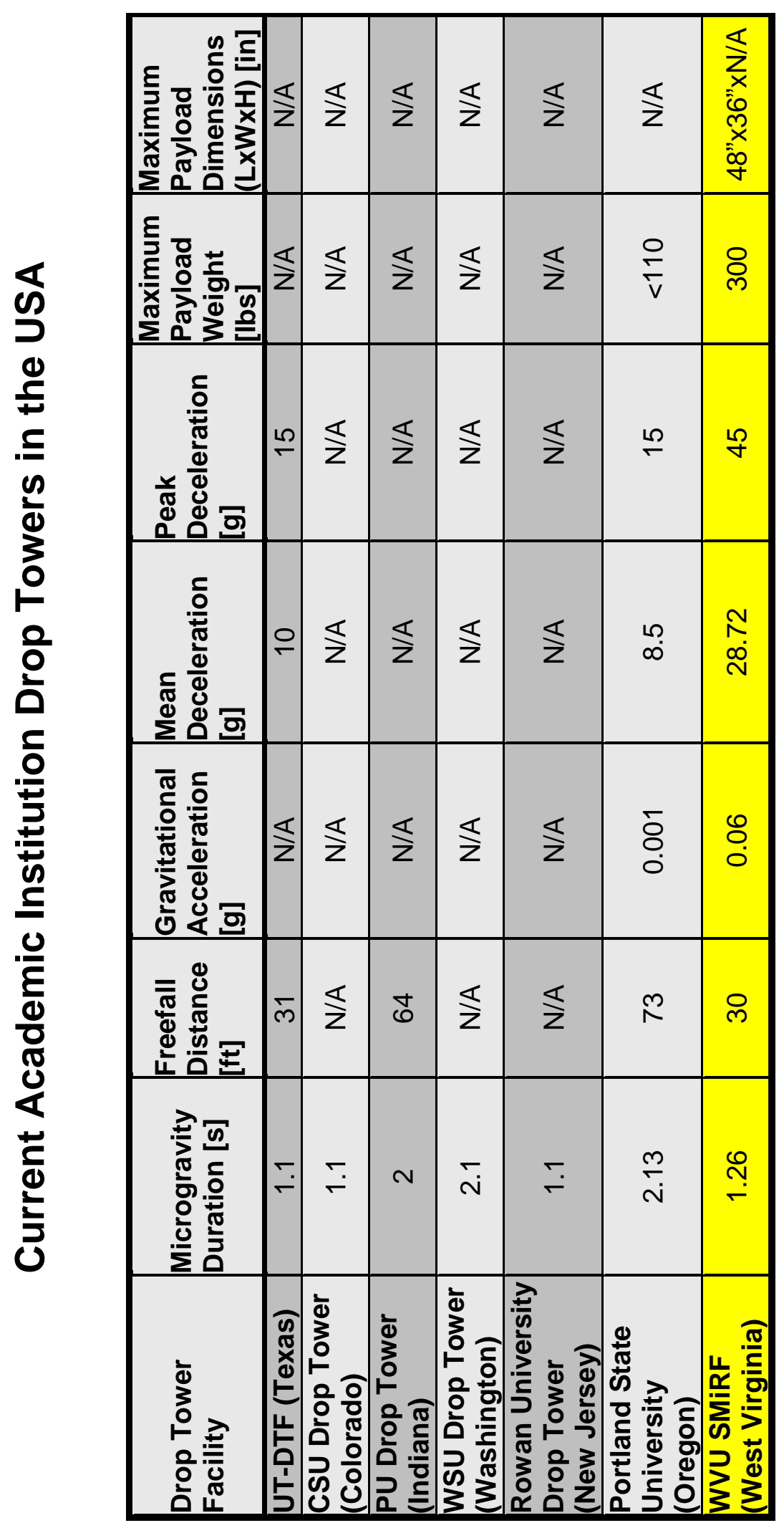




\subsection{Appendix P}

\section{Potential Website Layout}

Appendix P: Potential Website Layout presents one potential option for the layout of a WVU SMiRF website. The website should be both informative, but interactive with future expandability into restricted access to data storage and remote drop tower observation and potentially controls. 
Development of the West Virginia University

Small Microgravity Research Facility (WVU SMiRF)

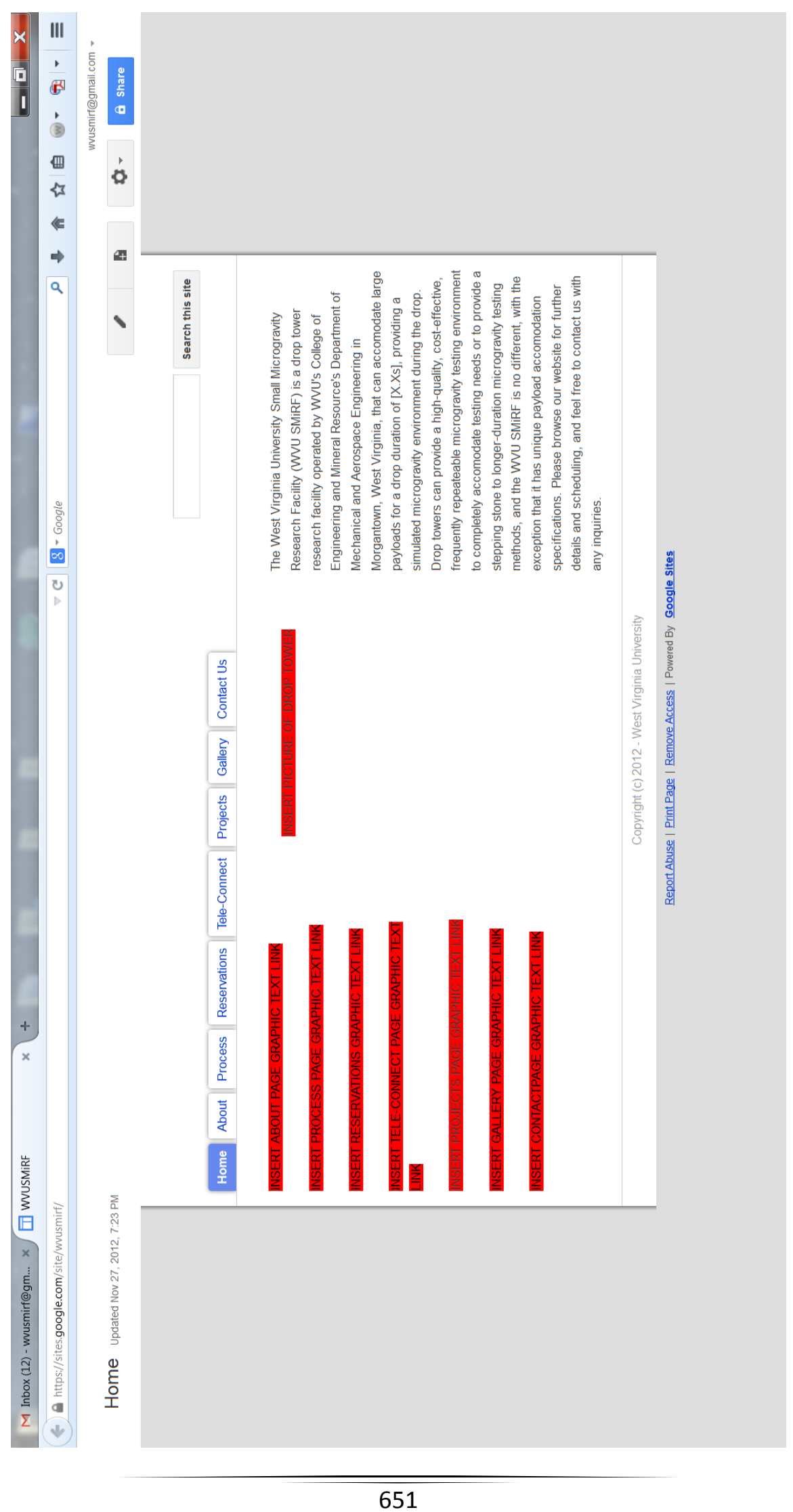


Development of the West Virginia University

Small Microgravity Research Facility (WVU SMiRF)
Copyright $^{\odot} 2014$

Kyle G. Phillips

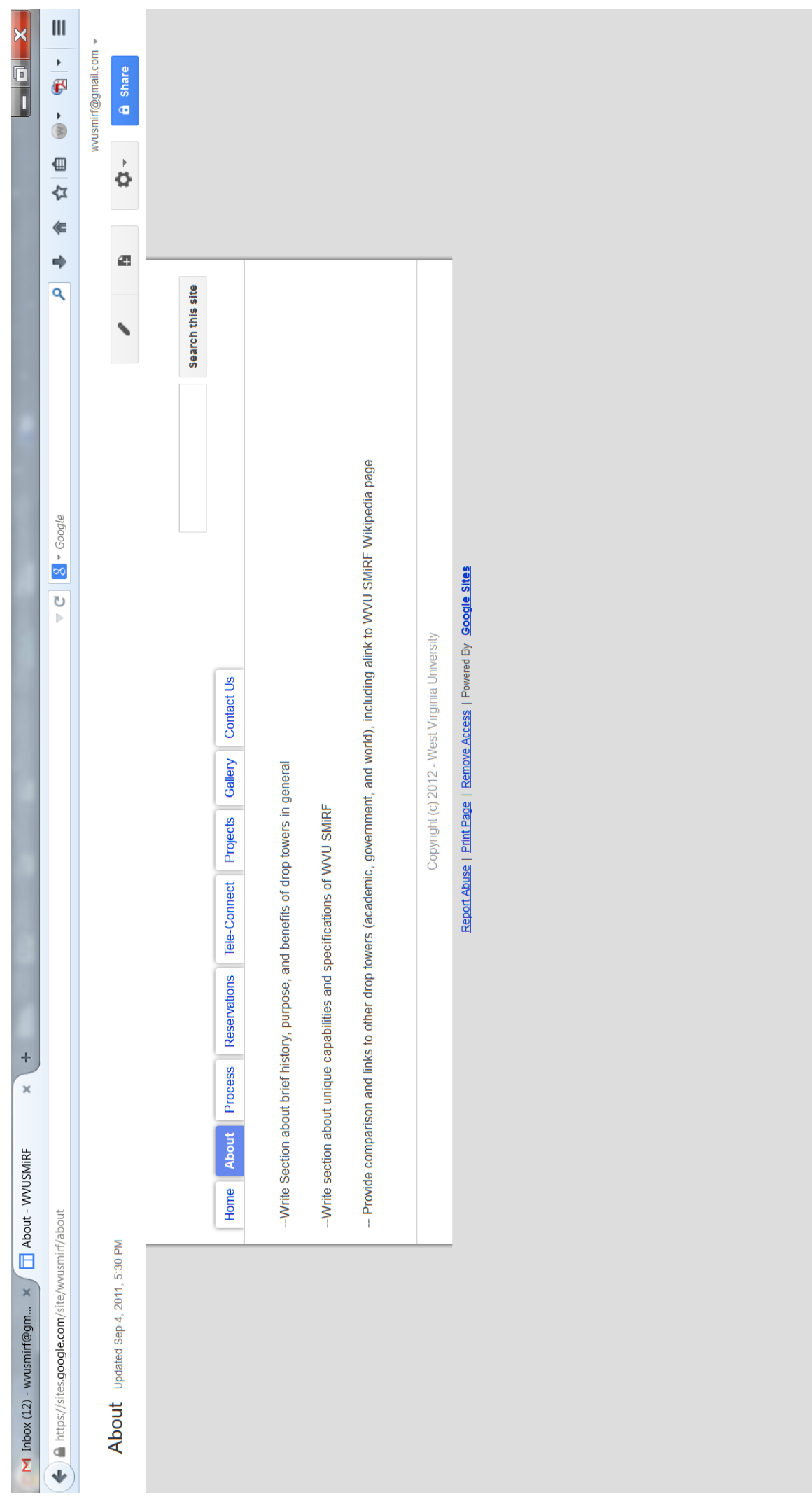


Development of the West Virginia University

Small Microgravity Research Facility (WVU SMiRF)
Copyright $^{\odot} 2014$

Kyle G. Phillips

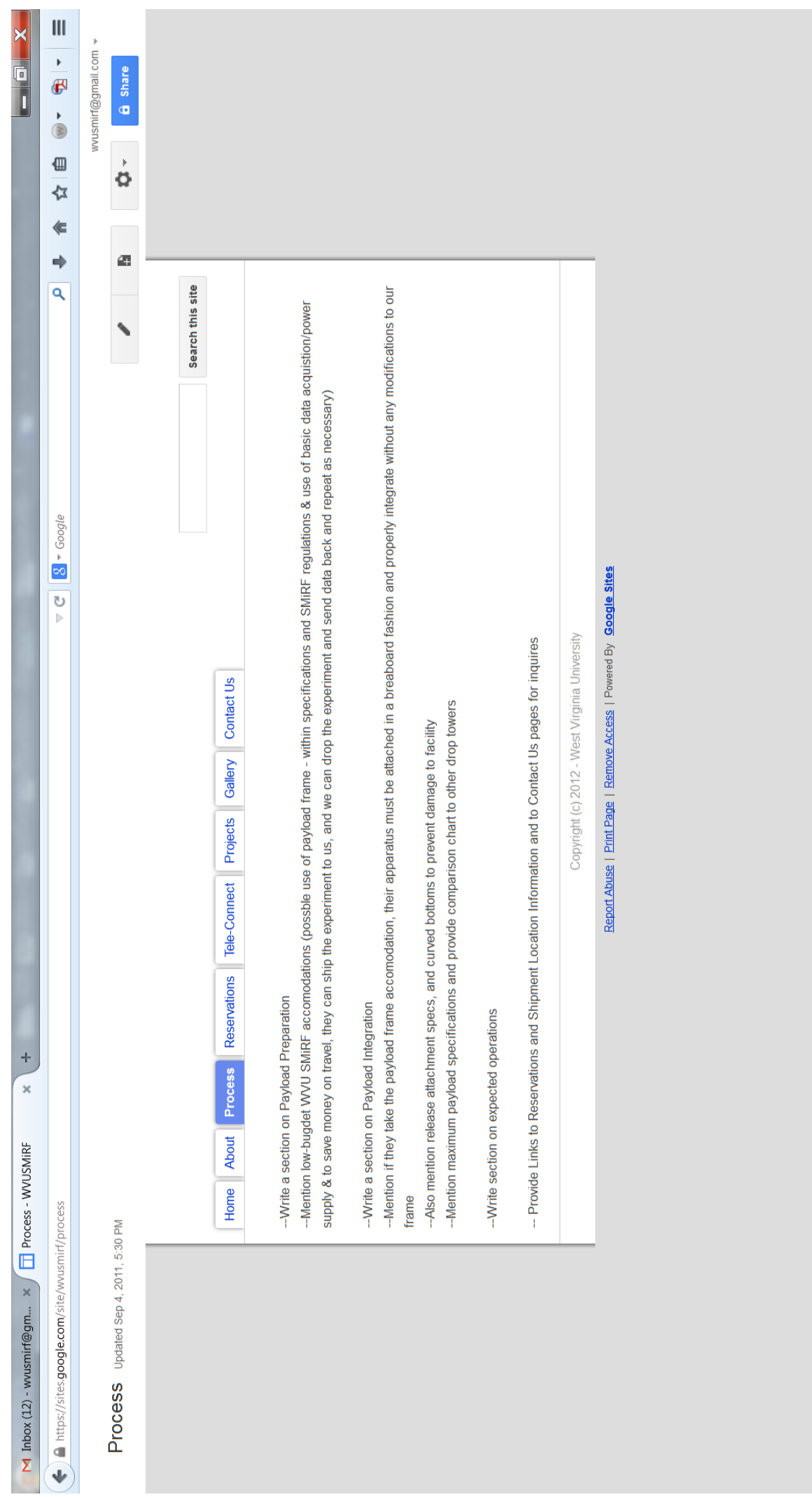


Development of the West Virginia University

Small Microgravity Research Facility (WVU SMiRF)
Copyright $^{\oplus} 2014$

Kyle G. Phillips
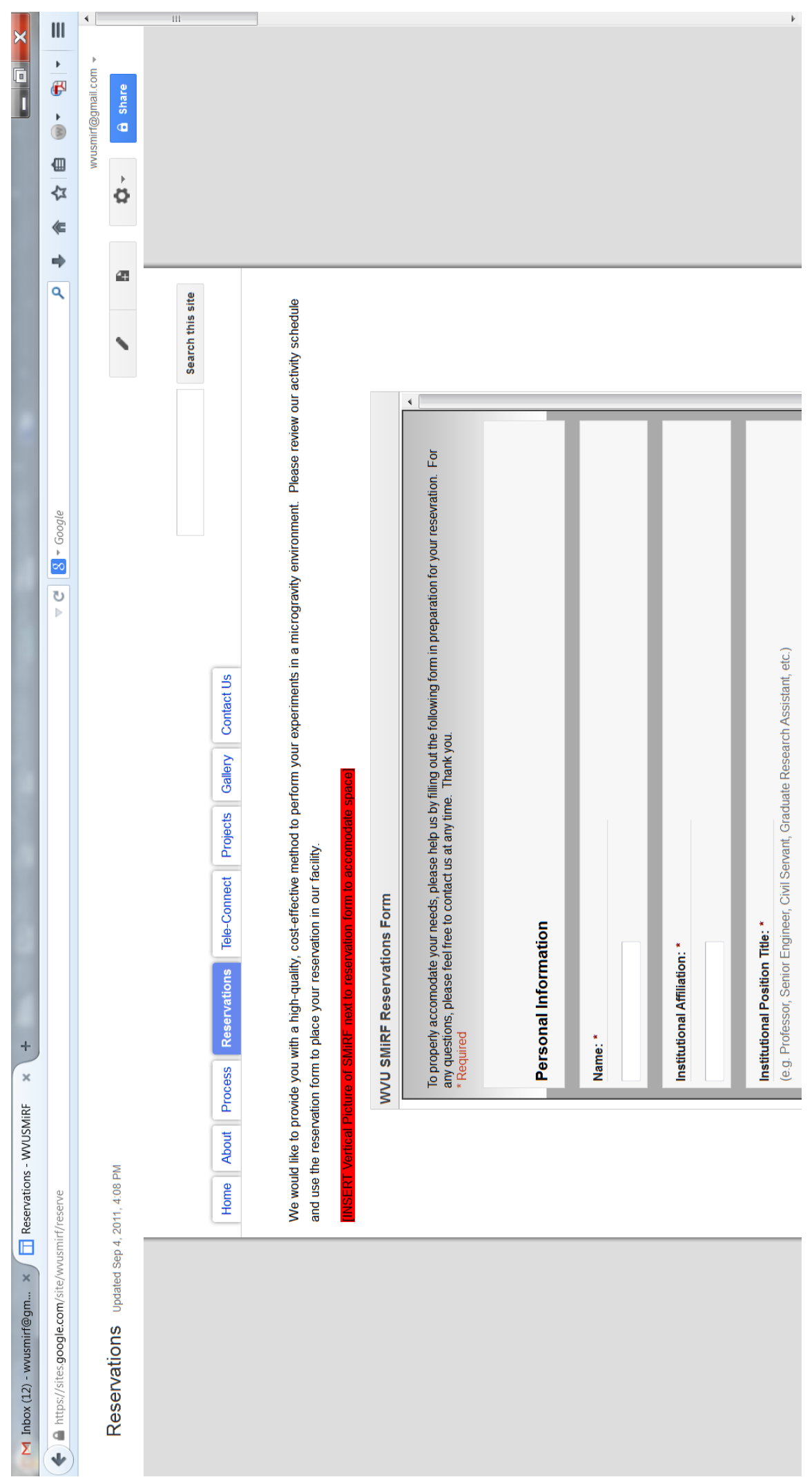
Development of the West Virginia University

Small Microgravity Research Facility (WVU SMiRF)
Copyright $^{\odot} 2014$

Kyle G. Phillips

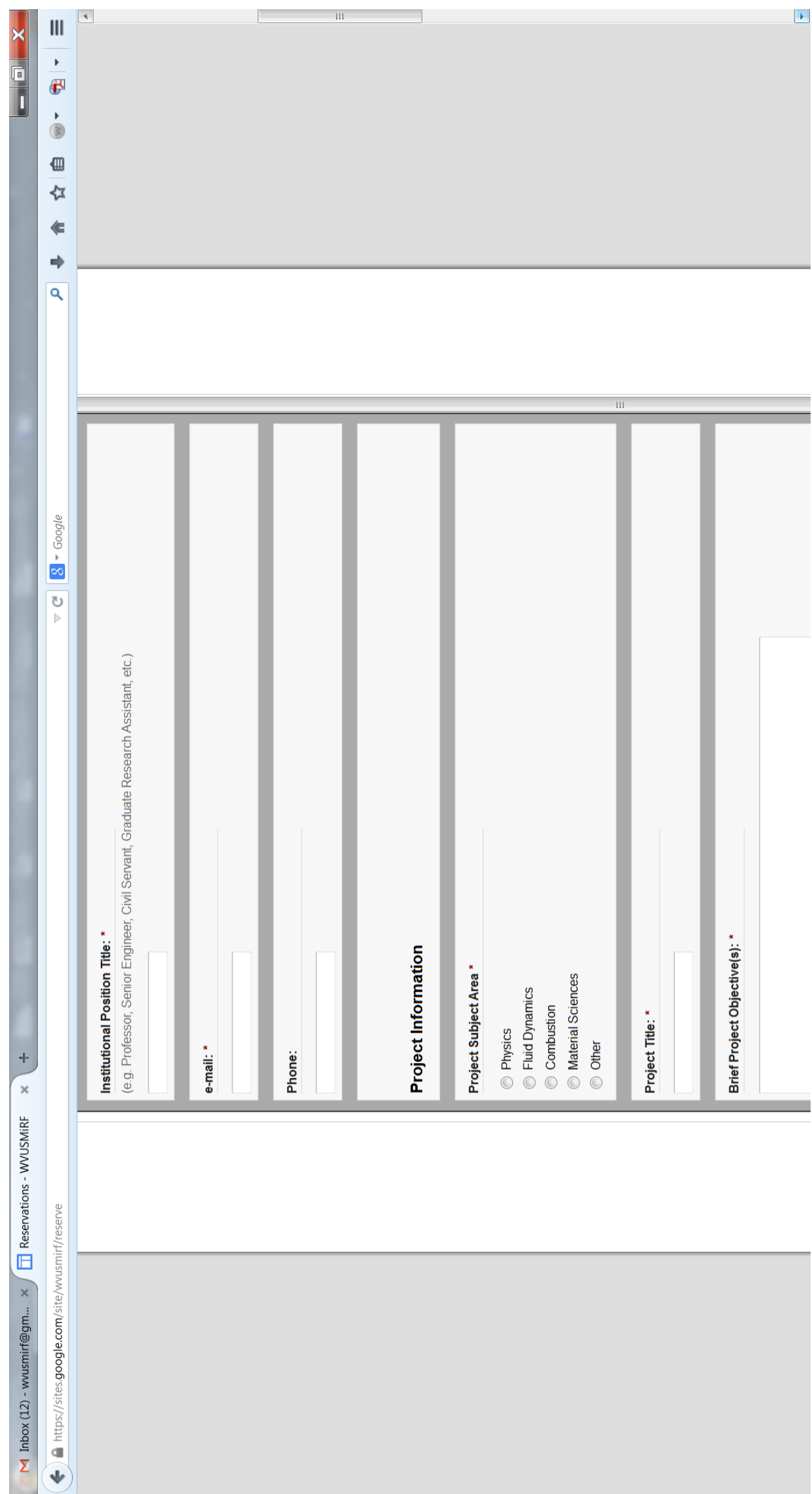


Development of the West Virginia University

Small Microgravity Research Facility (WVU SMiRF)
Copyright $^{\odot} 2014$

Kyle G. Phillips

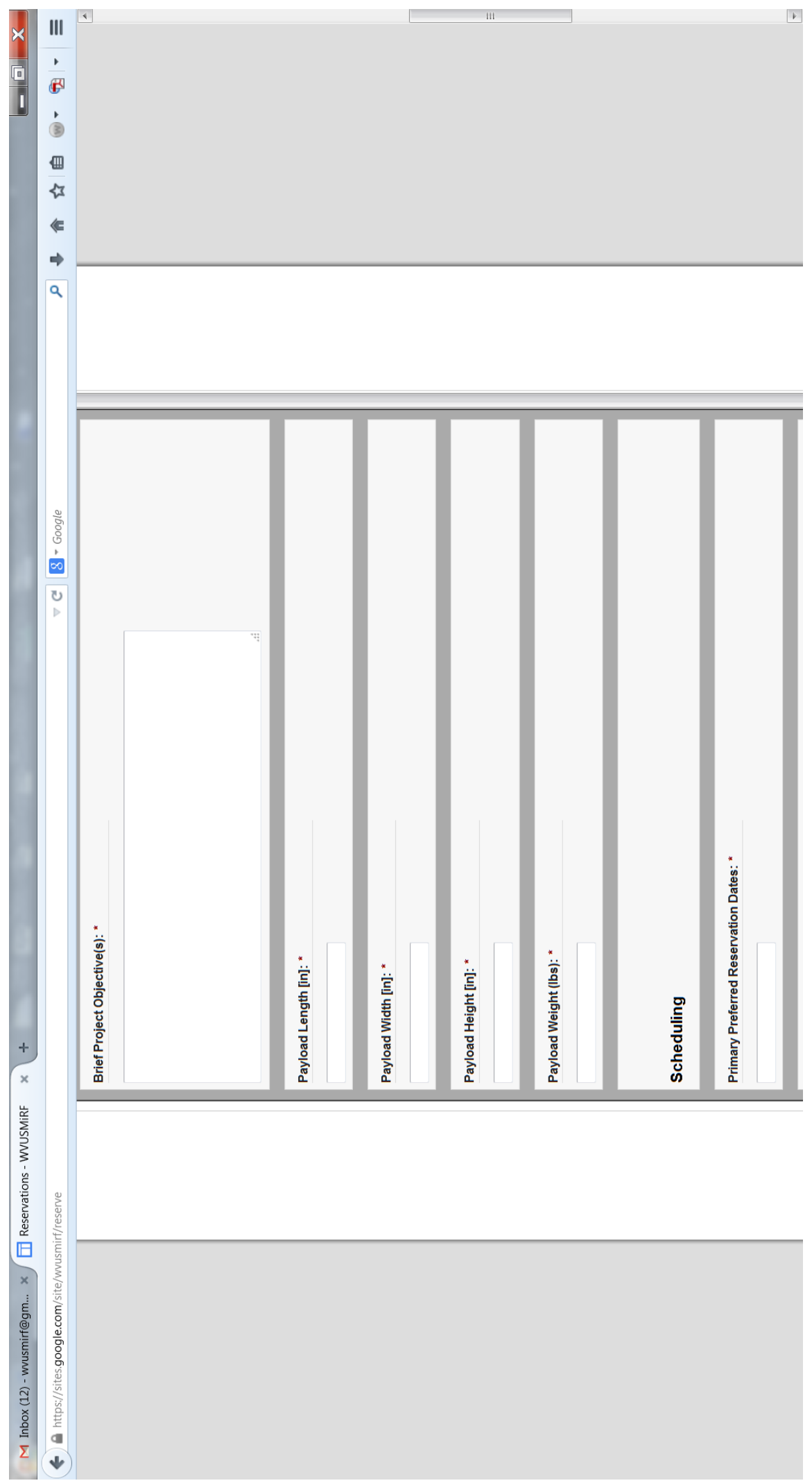


Development of the West Virginia University

Small Microgravity Research Facility (WVU SMiRF)
Copyright $^{\odot} 2014$

Kyle G. Phillips

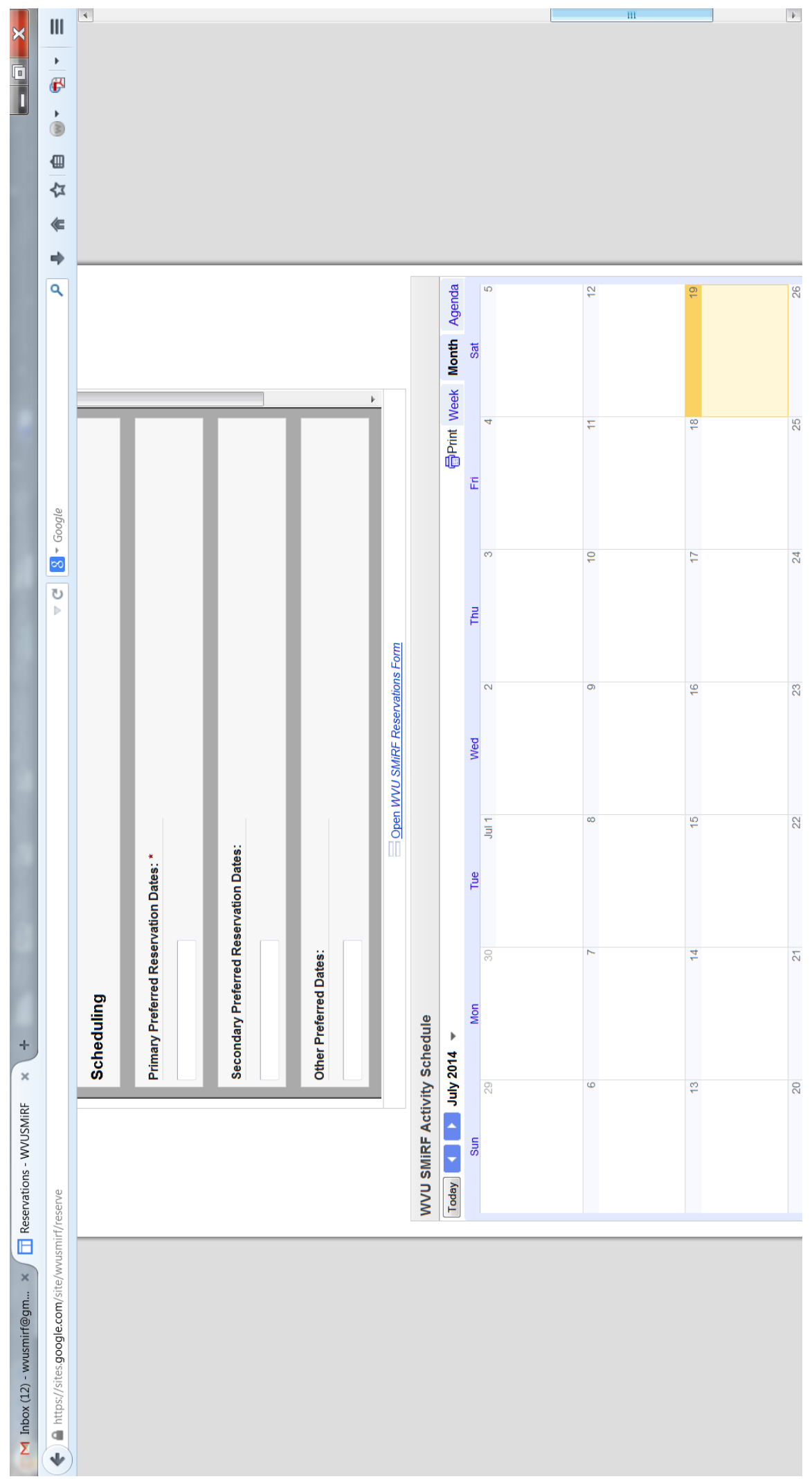


Development of the West Virginia University

Small Microgravity Research Facility (WVU SMiRF)
Copyright $^{\odot} 2014$

Kyle G. Phillips

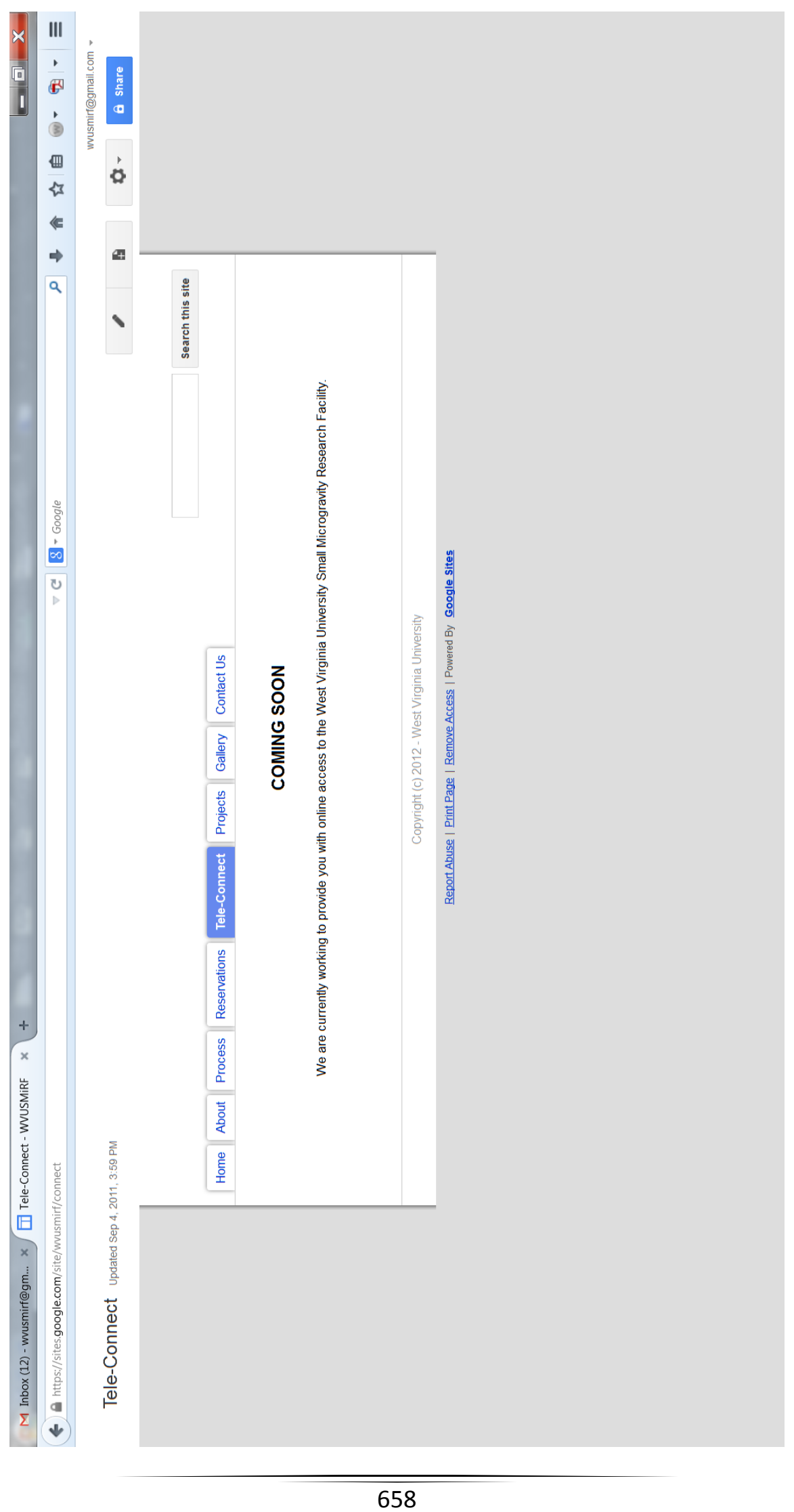


Development of the West Virginia University

Small Microgravity Research Facility (WVU SMiRF)
Copyright $^{\odot} 2014$

Kyle G. Phillips

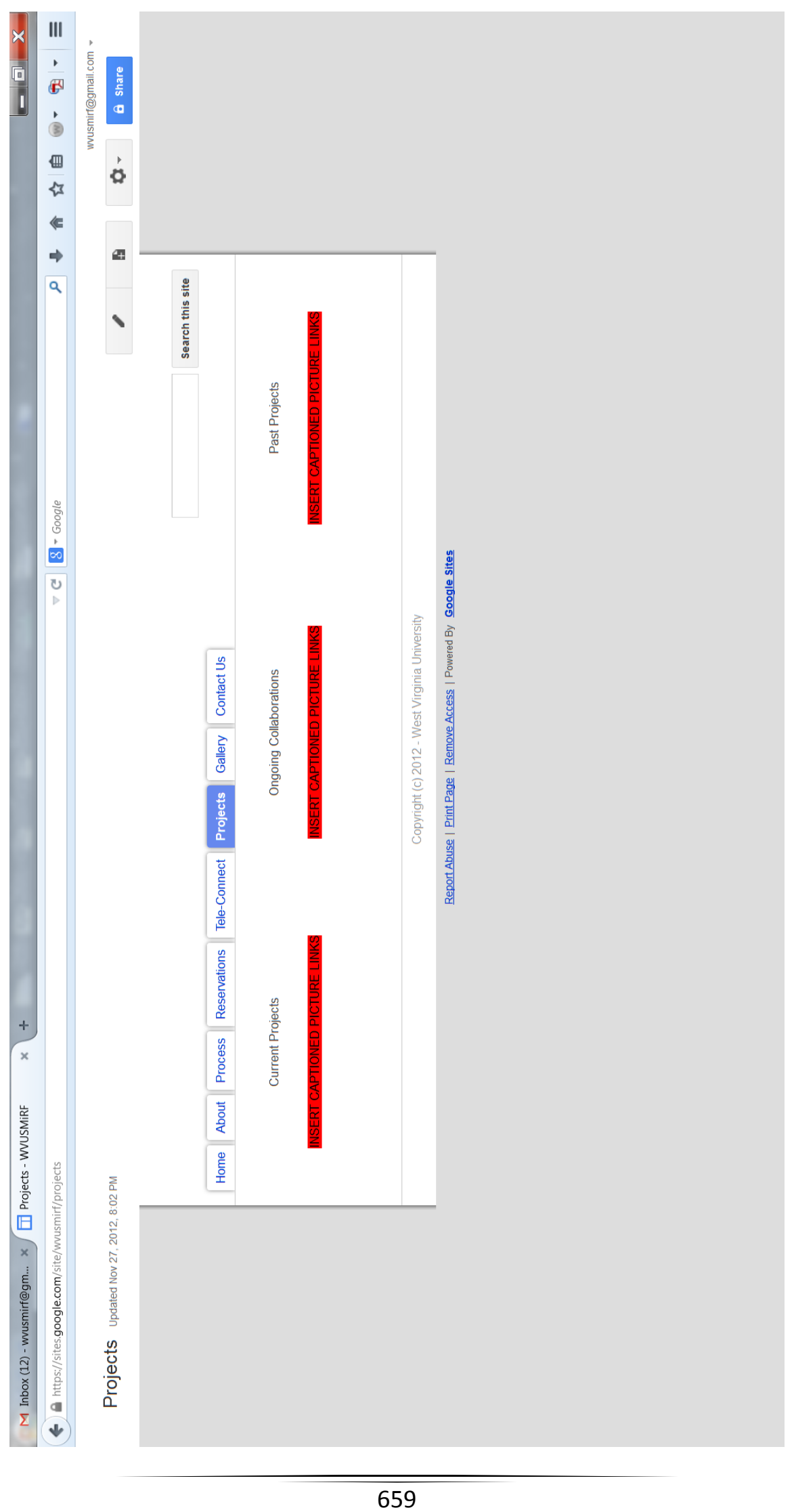


Development of the West Virginia University

Small Microgravity Research Facility (WVU SMiRF)
Copyright $^{\odot} 2014$

Kyle G. Phillips

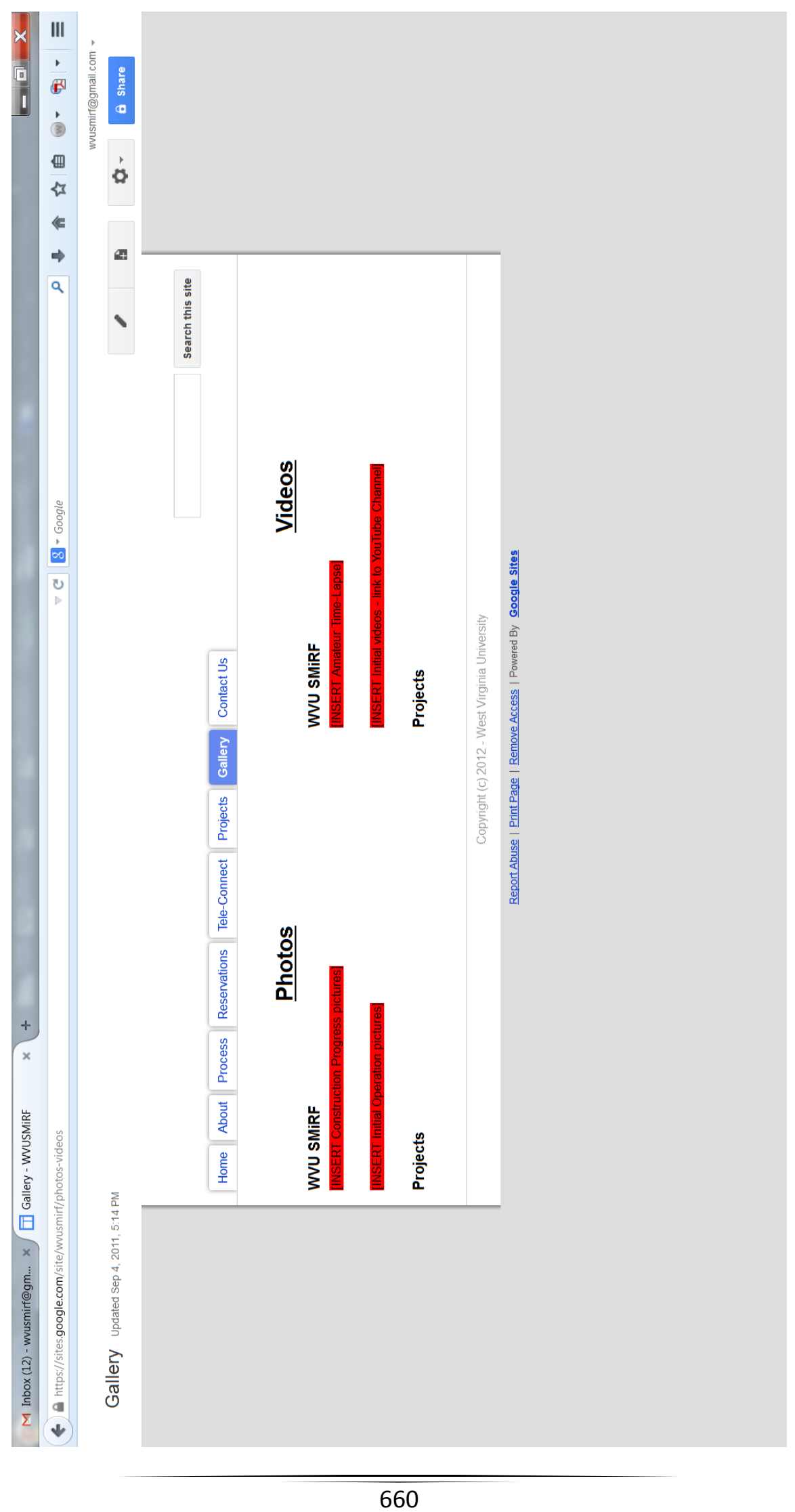


Development of the West Virginia University

Small Microgravity Research Facility (WVU SMiRF)
Copyright $^{\odot} 2014$

Kyle G. Phillips

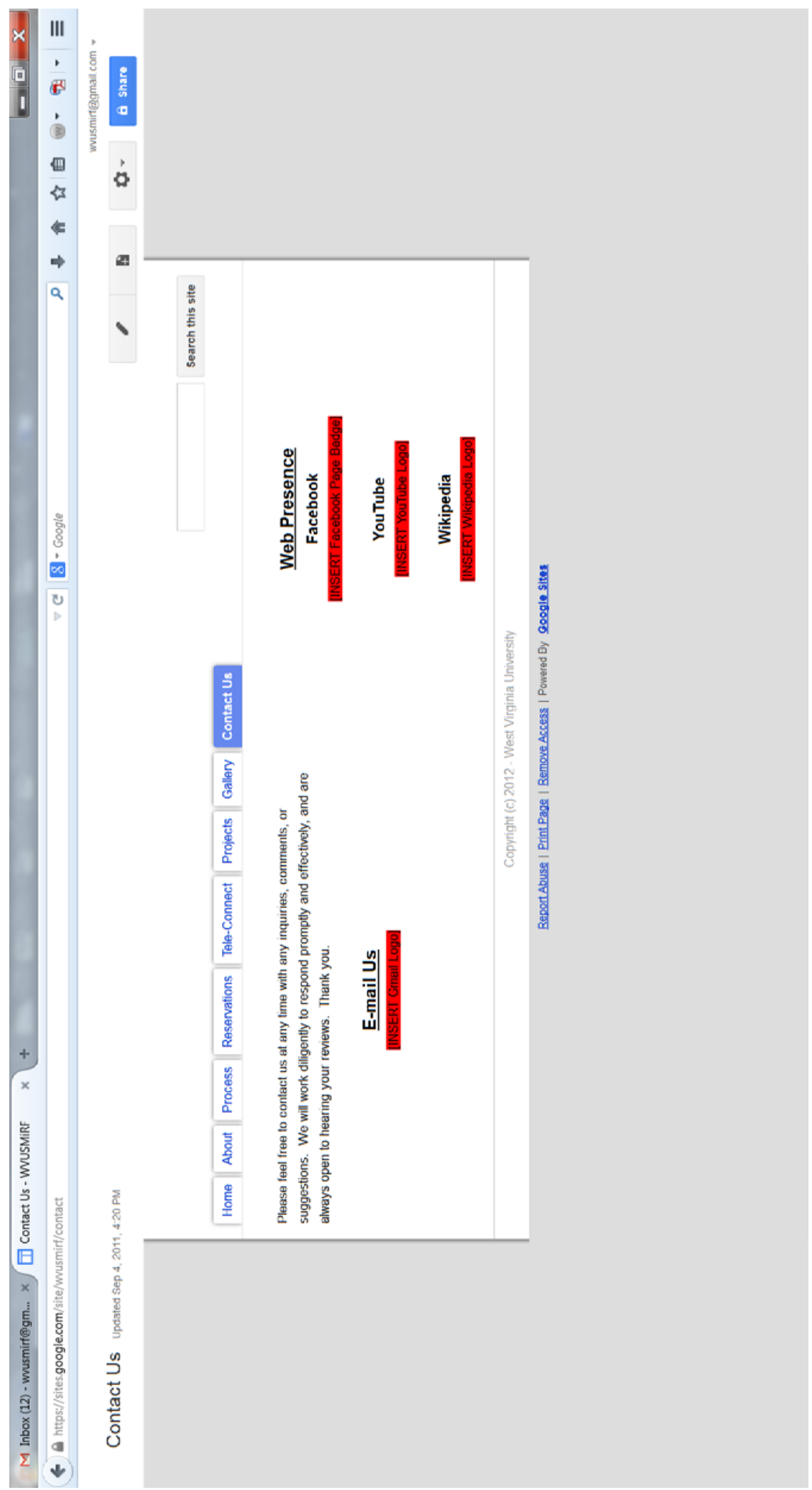

